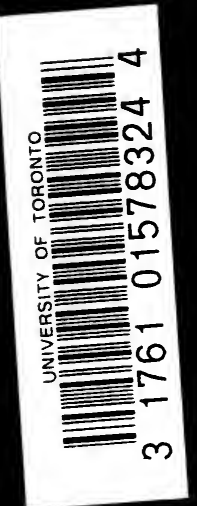





Digitized by the Internet Archive in 2007 with funding from Microsoft Corporation 

PRACTICE OF UROLOGY-CHETWOOD
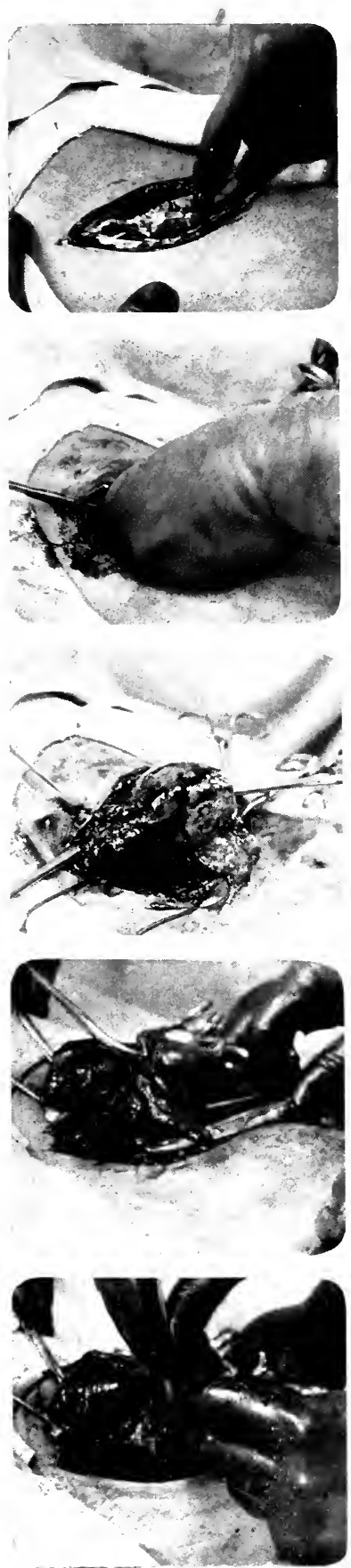
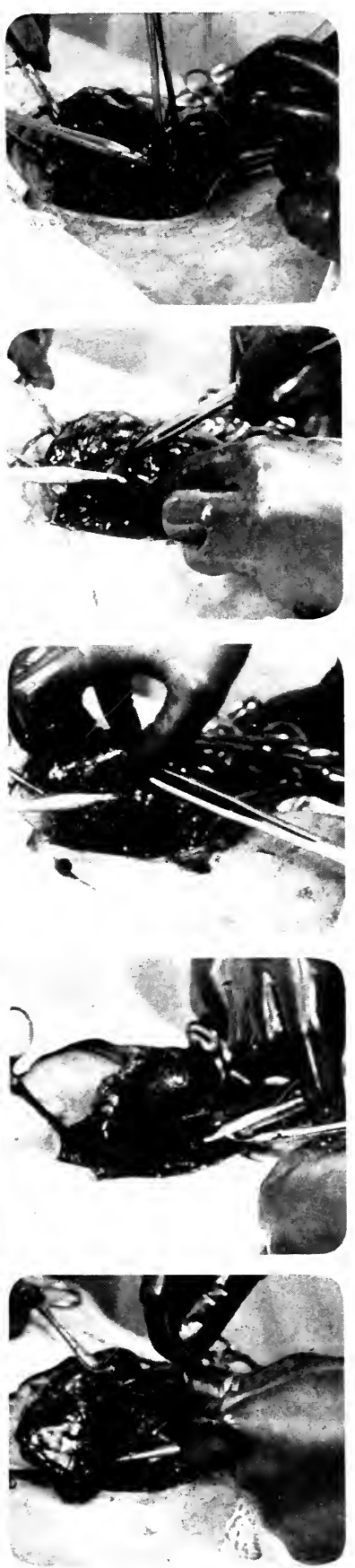

PLATE I
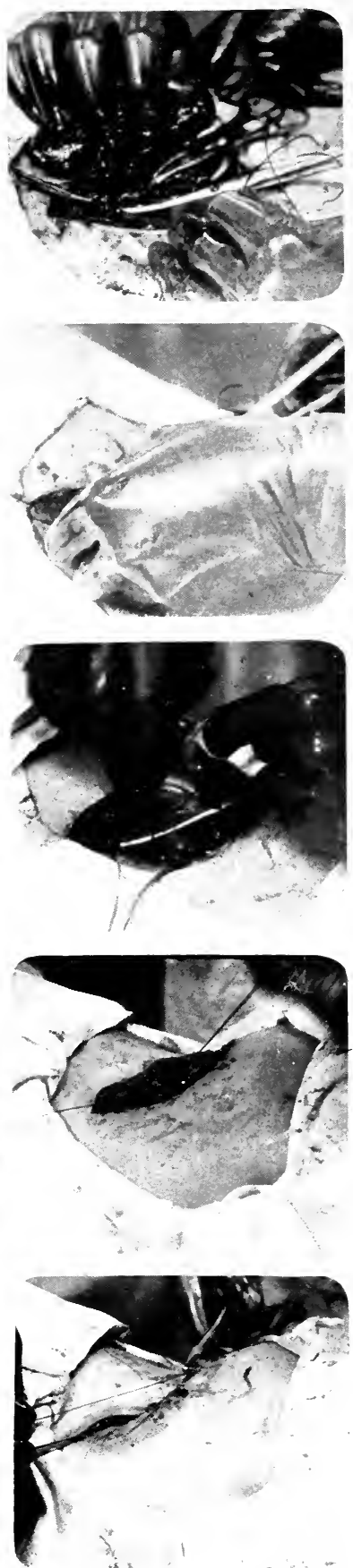

Nephrolithotomy and Nephrectomy 
Fra. 1. -Incioion of $\mathrm{ak}$ in and muscular wall beneath. the last rib.

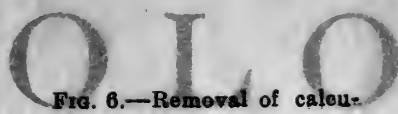

lus from pelvis.

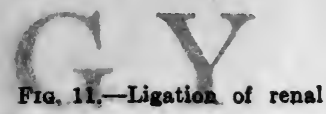
artery.

\section{A SURGICAT TKEATISK.}

\section{GENITO-URINARY DISFASISS}

Fra. 2.-Freeing the kidney with the gloved hand, after opening perirenal fatty capsule.
Fig. 7.-Removal of another

calculus from calix.
Frg. 12.-Removal of kidney.

\section{ISY}

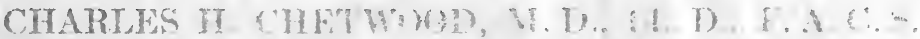

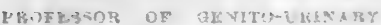

$\therefore 5, n$

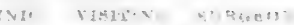

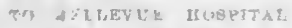

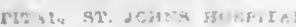

Fig. 3.-Elevation of kidney upon the loin with special retractor.
Fra. 8.-Delivering I a r ge calculus from substance of kidney, and removal through pelvis.

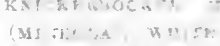

EN?

Frg. 13.-Final ligature applied to pedicle.
Frg. 4.-Exposure of posterior surface of pelvis and adjacent ureter.
Frg. 9.-Beginning of nephrectomy; cauterisation of ureter, after division of same.
Fıa. 14.-Reunion by suture of muscular wall.
FIG. 5.-Incision for posterior pyelotomy.
Fra. 10.-Ligation of veins and mobilization of kidney.
FıG. 15.-Suture of skin. 

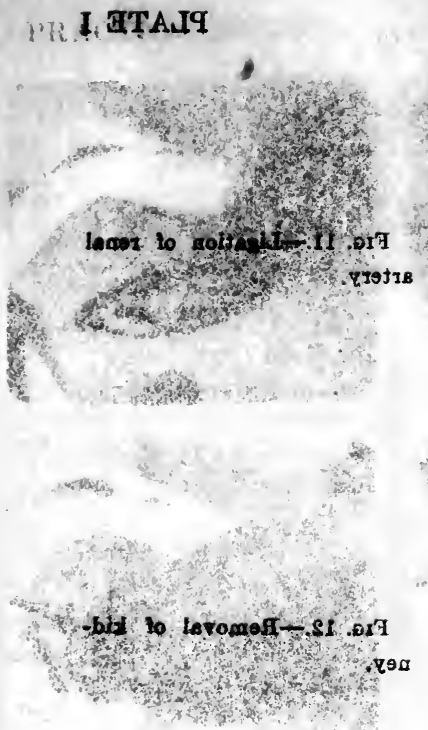

-qa ozusezil laair-.81 .01' .

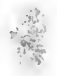

$$
\begin{aligned}
& \text { 19disome lo levomg9-. . or't } \\
& \text { zilao mot aulvolso } \\
& \text { (nintis is }
\end{aligned}
$$

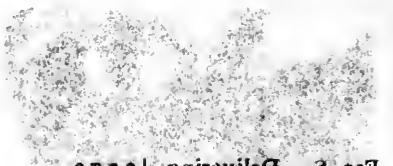

9 g T\& / gairovilo $\alpha-.3 .017$ to ovasfedne ofort enluoles dguordf levomer bns, venbi-d .eivlog

-dqor to gnirsaizag-e ort to soitcrivajuas ivin osog .

\footnotetext{
Illow raluseum to
}

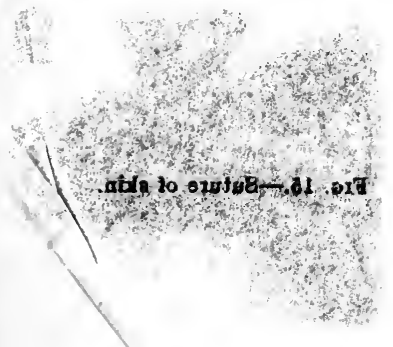

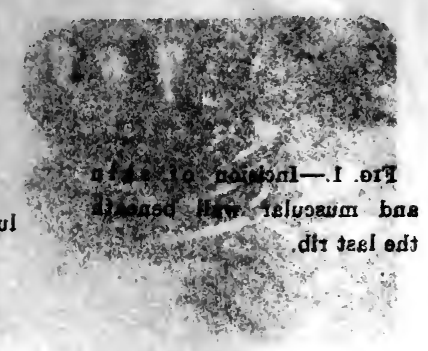

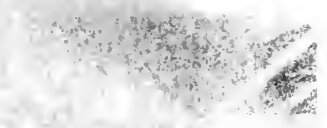

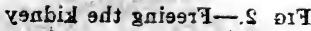
Igfls ,basd bovoly odt dtio -qeo रffBt lengrirgq gaingqo glve

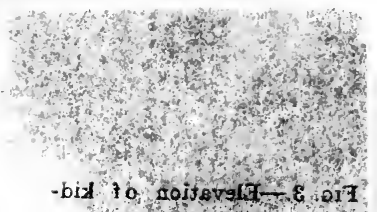
Isisoge dfin alol ods noun zon

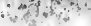

. TOtכBSTII

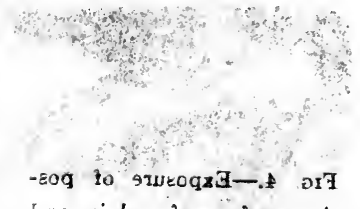

bns eivloq to 95simu toirgt

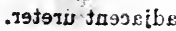

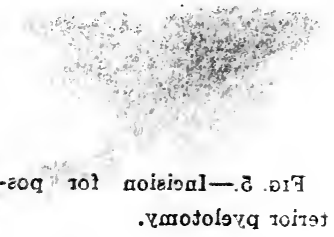




\section{THE PRACTICE OF \\ $\mathrm{U}$ \\ $\mathbf{R}$
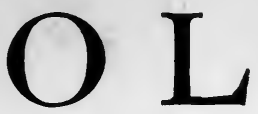 \\ $\mathrm{O}$ \\ G \\ Y}

A SURGICAL TREA'TISE

ON

\section{GENITO-URINARY DISEASES INCLUDING SYPHILIS}

BY

CHARLES H. CHETWOOD, M. D., LL. D., F. A. C. S. PROFESSOR OF GENITO-URINARY SURGERY, NEW YORK POLYCLINIC; VISITING SURGEON tO BELLEVUE hOSPITAL; SPECIAL CONSULTING SURGEON TO KNICKERBOCKER HOSpital, st. JohNS hospital (L. I. CITY), NASSAU hos pital (Mineola), white pLAINS hospital; member american association of Genito-trinary SURGEONS, AMERICAN UROLOGICAL ASSOCIATION; L'ASSOCIATION INTERNATIONALE D'UROLOGIE, ETC.

Drofuselp Illustrateo

second Edition

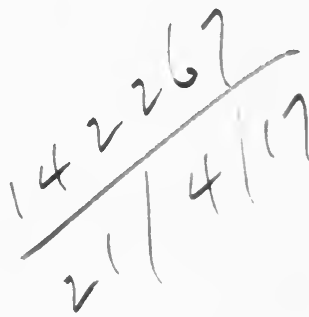

NEW YOR K

WILLIAM WOOD AND COMPANY

M D C C C C X V I 
Copyright, 1916

BY WILLIAM WOOD AND COMPANY

THE MAPLE PRESS YORK PA 


\section{PREFACE TO THE SECOND EDITION}

Since the first publication of this work, the author finds that, in the main, there is not reason to make great changes, and that his views upon the various topics considered remain, for the most part, the same as in the previous issue. Some of the questions then under discussion have been solved; others are still the subject of differing opinions.

The changes and additions involve: First, the department of cystoscopy, wherein the method of teaching this branch has been expanded and set forth in greater detail. In this, the author has been assisted by his Chief in the Division of Cystoscopy, Dr. H. B. Reece, and some of the illustrations used are his. Second, there have been some additions to the operative technic, but only where it is deemed to be of practical advantage. An example is the simple method, devised by the author's associate and Chief of Staff, Dr. Sinclair, of surmounting the difficulties encountered in impassable stricture, by suprapubic puncture and retrovesical urethrotomy. Third, there has been added a section on local anesthesia, so far as it lends itself effectively to this department of surgery. Other minor changes appear here and there throughout the different chapters.

Finally, the use of salvarsan, new and old, has been given additional attention, having become a fixed and indispensable factor in the treatment of all cases of syphilis. This particular malady, which in reality holds no place in urological surgery, is still a part of this treatise, not only because there is a continued demand for such combination in undergraduate colleges, but because the author's investigations and teachings, which cover a period of over twenty years, have been in the sphere of genito-urinary diseases, including syphilis; and if this work stands for anything, it would seem that the resulting experience in both departments may be recorded properly in one volume.

If this second edition retains the place that the original work has succeeded in making for itself, the author will have reason to feel gratified.

25 Park Avenue. 



\section{PREFACE TO THE FIRST EDITION}

It is not possible to complete a book of this scope without building upon many other works that have gone before, and it is, therefore, largely contributed to by the labors of others, not only in their writings and opinions expressed in medical meetings, but by their operative methods.

To the numerous contributors in this respect the author is glad to accord recognition here, and especially to refer to such international authorities as have been the leaders in the advancement of urological science in their respective countries, namely: Sir Henry Thompson of England, Felix Guyon of France, Leopold Casper of Germany, and Edward L. Keyes of America.

The last, by virtue of long association and teaching, more than any other, is responsible for whatever may be found of merit in this volume. To him, therefore, the author must especially dedicate this work, and for its faults and deficiencies, own entire responsibility.

This treatise is designed to propagate what the author considers to be orthodox teaching upon the various subjects contained therein, and has been undertaken only after an experience of over twenty years devoted to this specialized field of surgery.

Realizing that the consideration of any one of the important departments comprised might readily fill a volume by itself and still leave much unsaid, the writer hopes that this book will be one of the many sources of reading upon the subjects.

So far as the maladies treated of are the same in men and women, they are naturally included, but so far as they affect the organs peculiar to women, they are not. There is a tendency for some urologists to embrace the field of gynecology, but that is no reason why a book devoted to urology should advocate such a union by treating the subject in an inadequate manner, as is entailed, for instance, in including gonorrhea in women and omitting what is of prime importance-its complications and sequelæ-which indisputably belong to the domain of gynecology.

The subject matter herein is aligned as seemed to be most practical and desirable both from a surgical and clinical aspect, beginning with diagnosis and general technic, and following with the various abnormalities and diseases of the different anatomical regions, subdividing the operative surgery in like manner. 
A section on syphilis is also included. This is done because of the demand for it: for while this far-reaching disease is equally claimed by all departments of medicine, its treatment at the present day is surgical, and cannot be undertaken properly, except upon careful surgical principles. Therefore, the main part of the subject will be found somewhat condensed, sufficient detail being given, however, to afford a comprehension of the nature and action of the lesions in the various parts of the body, and particular attention is devoted to the consideration of the latest measures of treatment.

The characteristic features embodied in this volume, which give to it a modern status, are the present local treatment of gonorrhea; the application of serum diagnosis and serum therapy; advancement in cystoscopic and functional renal diagnosis; various features in surgical technic, and the latest on salvarsan therapy.

The author takes much pleasure in acknowledging also the assistance received from the able members of his staff, who, by this aid, have augmented their claim upon his devotion. First and foremost, his chief of staff, Dr. D. A. Sinclair, has been a constant and unfailing support in every imaginable detail; without such help, this work would still be uncompleted. To Dr. Faxton E. Gardner he is indebted for willing and competent assistance in preparing some of the pages on anatomy and embryology, general operative technic and neosalvarsan therapy; to Dr. H. G. Bugbee, for assistance in constructing the section on functional renal diagnosis; and the other members of his staff have rendered aid in many ways.

The excellence of the mechanical part of the production is due to the painstaking efforts of the publishers and the illustrators' skill.

As already stated, this work represents only a part of that which must be completed by others, but if it serves in any one particular to assist in the successful practice of urological diagnosis and technic, it will more than repay for the extensive labor and time devoted to its preparation.

25 Park Avenue, New York City,

Charles H. Chetwood. January, 1913. 


\section{TABLE OF CONTENTS}

\section{CHAPTER I}

Anatomy and Embryology of the Urogenital Tract PAGE $^{\circ}$

CHAPTER II

The Diagnosis of Sexual and Urinary Maladies-The Urologist's Armamentarium and General Technic .. . . . . . . . . . . . . .

CHAPTER III

Diagnosis (continued)-Physical Examination of the Patient, Oral, Manual and Instrumental-Clinical Examination of Urine . . . . . . . . . . 60

CHAPTER IV

Diagnosis (continued) - Examination of Urine, Chemical and MicroscopicBacteriology of the Urine

CHAPTER V

Sero-diagnosis and Sero-therapy

CHAPTER VI

Urethroscopic and Cystoscopic Diagnosis and Treatment

CHAPTER VIİ

Functional Renal Diagnosis-Roentgenography

CHAPTER VIII

Maladies of the Penis-Injuries-Inflammation-Growths . . . . . . . 170 CHAPTER IX

Malformations and Wounds of the Urethra 185

CHAPTER X

Urethritis, Gonorrhea-Bacteriology-Pathology-Clinical Course . . . . 191 CHAPTER XI

Treatment of Acute Urethritis, Internal and Local

\section{CHAPTER XII}

Chronic Urethritis-Causation-Pathology-Clinical Course-Diagnosis-

Treatment 


\section{CHAPTER XIII}

Complications of Acute and Chrognic Urethritis-Extragenital Complications of Gonorrhea

\section{CHAPTER XIV}

Stricture of the Urethra, Spasmodic-Organic or True Stricture, Traumatic and Conorrheal-Complications and Sequele-Treatment of Stricture .

\section{CHAPTER XV}

Operative Surgery of the Urethra

\section{CHAPTER XVI}

Maladies of the Scrotum and Contents, the Testicles and Spermatic CordWounds and Growths of the Scrotum-Anomalies and Injuries of the

Testicle-Orchitis and Epididymitis . . . . . . . . . . . . . . . . 299

\section{CHAPTER XVII}

Hydrocele-Varicoeele-Tumors of the Testicle and Epididymis . . . . . . 317

\section{CHAPTER XVIII}

Maladies of the Seminal Vesicles-Mbnormalities-Injuries-Concretions-

Acute and Chronic Seminal Vesiculitis . . . . . . . . . . . . . . 330

\section{CHAPTER XIX}

The Operative Surgery of the External Genitals . . . . . . . . . . . . . 340

\section{CHAPTER XX}

Maladies of the Prostate-Atrophy-Injuries-Ncoplasms-Calculi-Acute and Chronic Prostatitis-Prostatorrhea . . . . . . . . . . . . . . 365

\section{CHAPTER XXI}

Prostatic Iypertrophy-Causation-Pathology-Clinical Course . . . . . 385

\section{CHAPTER XXII}

Prostatic Hypertrophy (continued)-Diagnosis and Treatment . . . . . . 397

\section{CHAPTER XXIII}

Maladies of the Bladder-Malformations-Diverticula-Injuries-Acute and Chronic Cystitis-Vesical Atony-Contracture . . . . . . . . . . . 415

\section{CHAPTER XXIV}

Maladies of the Bladder (continued)-Stone in the Bladder-Tumors of the Bladder 
CHAPTER XXV

Operative Surgery of the Bladder, Prostate and Seminal Vesicles . . . . . 458

\section{CHAPTER XXVI}

Functional Disorders of the Urinary and Sexual Organs-The Neuroses . 502

\section{CHAPTER XXVII}

Congenital Anomalies and Wounds of the Kidney and Ureter . . . . . . . 520

\section{CHAPTER XXVIII}

Circulatory Changes in the Kidney-Acute and Chronic Nephritis-Hemorrhagic Infarction (non-suppurative)-Essential Hematuria . . . . . . 534

\section{CHAPTER XXIX}

Tumors of the Kidney and Ureter . . . . . . . . . . . . . 550

\section{CHAPTER XXX}

Renal and Ureteral Calculi-Calculus Anuria . . . . . . . . . . . . . 562

\section{CHAPTER XXXI}

Hydronephrosis-Causation-Morbid Changes-Clinical Features-Diagnosis -Treatment . . . . . . . . . . . . . 57

\section{CHAPTER XXXII}

Nephroptosis, Prolapsed or Movable Kidney-Causation-Clinical Features-

Diagnosis-Treatment . . . . . . . . . . . . . . . 588

\section{CHAPTER XXXIII}

Bacterial Inflammation of the Kidney-Pyelitis-Pyelonephritis-Causation-Morbid Changes-Clinical Course-Diagnosis-Treatment . . . 599

\section{CHAPTER XXXIV}

Metastatic Infection of the Kidney-Unilateral Septic Infarcts-CausationMorbid Changes-Clinical Course-Diagnosis-Treatment . . . . . 611

\section{CHAPTER XXXV}

Pyonephrosis and Perinephritis-Causation-Morbid Changes-Clinical Course —Diagnosis-Treatment . . . . . . . . . . . . 620

\section{CHAPTER XXXVI}

Operative Surgery of the Kidney and Ureter . . . . . . . . . . . . 632 
Chancroid-Causation-Pathology-Symptoms-Diagnosis-Treatment . . 681

\section{CHAPTER XXXIX}

Syphilis-Definition-Origin-General Pathology-Transmission-The Primary Sore . . . . . . . . . . . . . . . . . . . 695

\section{CHAPTER XL}

Syphilis (continued)-Clinical Course-Regional Syphilis, Skin-Mucous Membranes-Muscles-Tendons-Joints and Bones-Respiratory Tract -Alimentary Tract-Circulatory System-Genito-urinary OrgansOcular and Aural Syphilis . . . . . . . . . . . . . . . . . . . 707

\section{CHAPTER XLI}

Syphilis of the Nervous System, Cerebrospinal-General Paralysis and Locomotor Ataxia-The Hereditary Disease . . . . . . . . . . . . . . 743

\section{CHAPTER XLII}

The General Treatment of Syphilis-Internal Administration of MercurialsInunctions-Hypodermic Medication-Iodides-Modern Synthetic Preparations-Salvarsan . . . . . . . . . . . . . . . . 765

Tables Giving Equivalents of Metric System and Standarl Measures . . . 796 


\title{
THE PRACTICE OF UROLOGY
}

\author{
CHAPTER I \\ ANATOMY AND EMBRYOLOGY OF THE \\ UROGENITAL TRACT
}

\section{ANATOMY}

The Urinary and Genital Secretory Organs of the Male (Kidneys, Testicles) are bilateral and continue to be so for the greater extent of their Excretory Ducts (Ureters, Vasa Deferentia, Seminal Vesicles); but they unite in a common outlet (Bladder, Prostate, Urethra) before expulsion of their respective secretions.

A small part of the urogenital tract is visible outside the body. The greater portion is hidden within, being behind or below the peritoneum. In the male, the scrotum and the penis are the external genitals.

\section{Anatomy of the External Genitals}

The Scrotum is a cutaneous pouch which contains the testicles and a part of the spermatic cord. In the median line it shows a raphé, which is continued forward to the under surface of the penis and backward in the middle of the perineum to the anus, dividing the scrotum into two halves, one for each testicle. The scrotum varies greatly in different individuals as to appearance and flaccidity.

The Penis is both a genital and a urinary organ, containing the canals which conduct the flow of semen and urine. It is composed of vascular erectile bodies, ensheathed in skin and fascia. The extreme anterior portion of the penis presents the shape of a cone flattened from above downward, and longer on the upper than on the under surface. This is the glans penis, on the summit of which is the meatus urinarius, a vertical fissure 6 to $8 \mathrm{~mm}$. long.

The meatus is often found abnormally narrow. In such cases, it is also situated too high, and must be enlarged downward, that is, toward the under surface of the penis.

The glans is partially covered by a fold of skin continuous forward 
from the skin of the penis, called the prepuce. The length of the prepuce and the size of the orifice which it encircles at the top of the glans vary considerably in different individuals. The prepuce is attached in the midline of the lower aspect of the glans by the frenum. The posterior edge of the glans forms a rounded projecting eminence, the corona glandis, behind which is the coronal sulcus. Behind the latter begins the body of the penis, which is almost cylindrical in the flaccid state, but assumes a hemicylindrical shape when erect, the flattened portion being the upper surface or dorsum. The root of the penis is attached to the lower border of the symphysis by an elastic sling which passes around its base. This is the suspensory ligament (Fig. 2(1)), which is an upward prolongation of the scrotal dartos.

The penis is formed by a mass of erectile tissue divided into three parts-the cavernous bodies and the spongy body of the urethra. The cavernous bodies are two fibrous cylinders, juxtaposed in their

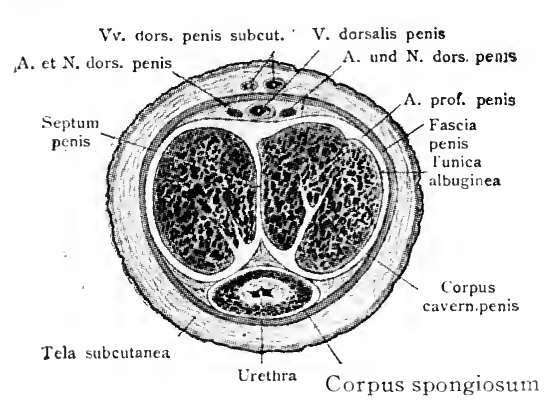

Fig. 1.-Cross section of Penis. anterior three-fourths as are the barrels of a shotgun, and occupying the dorsal region of the penis, of which they form the major part (Fig. 1). In front of the tuberosity of the ischium, where these cavernous bodies begin, they diverge backward by a tapering crus (Fig. 2); at their anterior extremity they form a single rounded end, which fits into a hollow of the pase of the glans. The dorsal vein, two arteries and nerves of the penis run in the groove between the cavernous bodies (Fig. 1).

The urethral spongy body is a sheath of erectile tissue surrounding the penile portion of the canal, situated in a groove below the cavernous bodies. It begins anteriorly by an expansion which constitutes the glans; its middle portion is narrower and cylindrical: behind, between the diverging crura of the cavernous bodies, it again expands to form the bulb, a most important landmark in all perineal operations (Fig. 2).

\section{The Urethra}

The Urethra is a canal extending from the external meatus to the bladder. As previously stated, it follows the entire length of the under surface of the penis in the midline, surrounded by its spongy body. When the penis is flaccid, this portion of the urethra hangs vertically downward, and the suspensory ligament of the penis 
forms the urethral angle, after which the urethra changes its direction, becomes oblique backward and downward, passes through the pelvic floor, then becomes oblique upward and backward, and finally opens into the neck of the bladder. (See Fig. 2.)

Therefore, the urethra is divided into three portions-spongy, membranous, prostatic. The spongy portion is the anterior urethra, and the membranous and prostatic portions are the posterior urethra. The movable urethra extends from the meatus to the suspensory ligament; beyond that portion to the bladder is the fixed urethra.

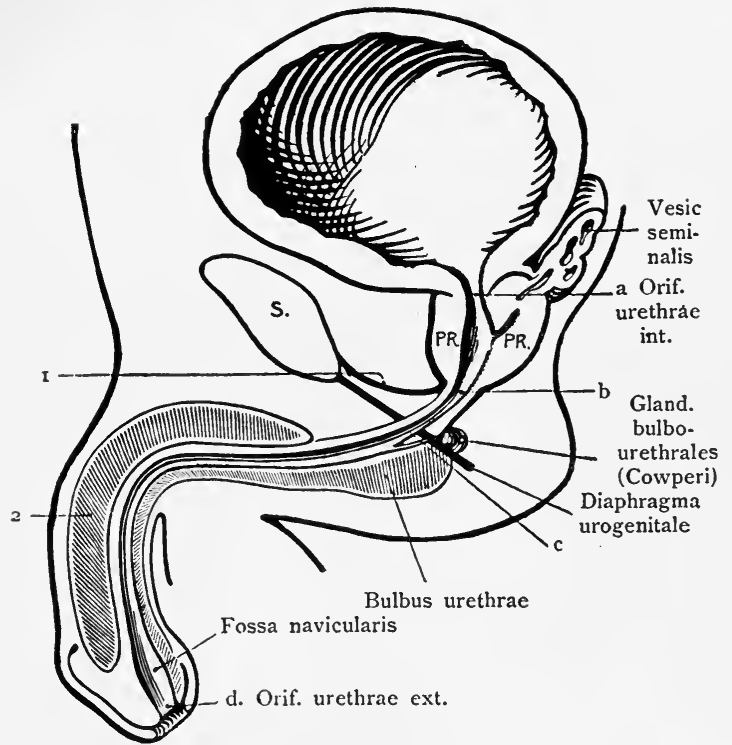

FIG. 2.- Side section of anterior and posterior urethra and adnexa. (Corning.)

(1) Suspensory ligament, (2) Corpus spongiosum.

When the penis is flaccid, the urethra exhibits two curves, one from the meatus to the suspensory ligament, which is concave downward and backward, and the other from the suspensory ligament to the neck of the bladder, which is concave upward and forward.

The urethra remains constantly in the median sagittal plane of the body; lateral deviations are rare, and it varies considerably in length in the pendulous portion.

The relations of the urethra surgically considered are:

In the penis, the urethra runs along the under surface in the midline, covered by skin and fascia, below the cavernous bodies.

In the perineum, it is still quite superficial and may be easily reached by the direct thrust of a knife into the groove of a staff, at 
the junction of the bulbus and the membranous portions whence it is directed further away from the perineum. The membranous portion passes through the triangular ligament. While it may be said that the urethra perforates this ligament, embryologically, the urethra is developed first and the triangular ligament later; more correctly, the urethra slides between the layers of the preprostatic and the retroprostatic fasciæ, which are attached to the triangular ligament in front and diverge to surround the prostate behind. The sharp edge of the ligament plays an important rôle in ruptures of the canal.

Behind, the urethra is connected with the rectum. The relations between these organs are much more intimate than is generally appre-

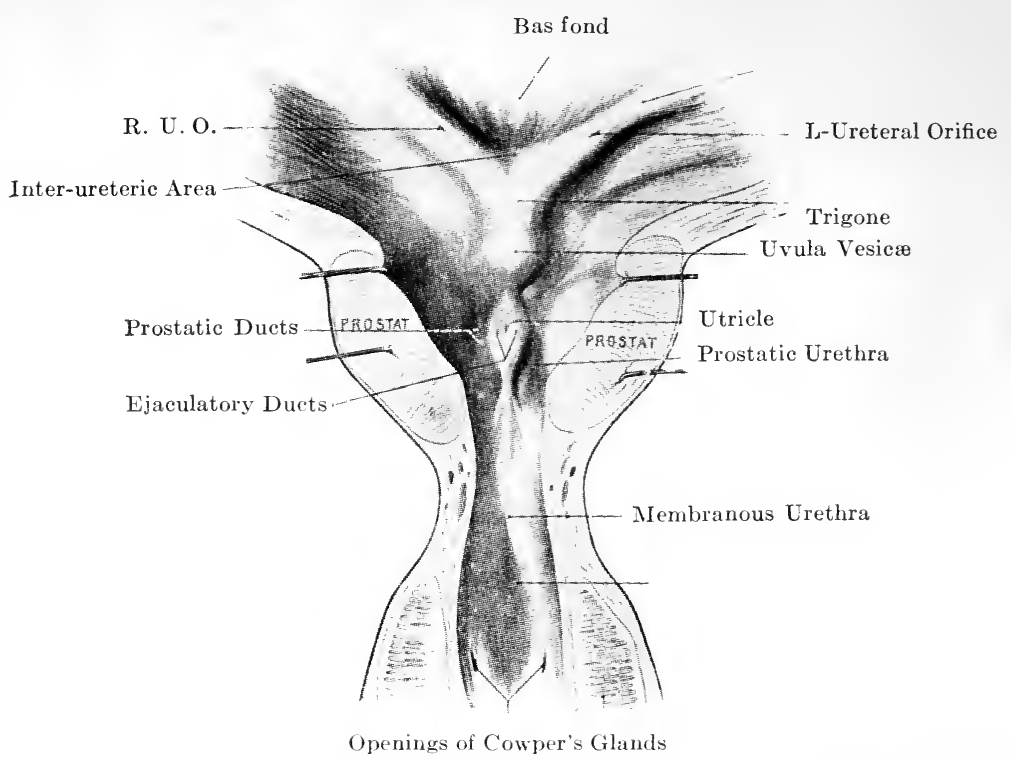

FIG. 3.-Surface view of floor of bladder and deep urethra.

ciated. The rectum projects forward considerably, and is bound to the urethra by the recto-urethral muscle, a layer of unstriped fibres most important in perineal prostatectomy; as long as this muscle is not severed, the rectum cannot be drawn backwards, and is in danger.

The relations of the urethra with the prostatic segment are those of the prostate itself, and will be referred to later.

Throughout its entire length, the urethra appears as a canal of very uneven calibre, a succession of narrow and dilated portions, studded with small apertures of glands. The meatus is a narrow and inextensible point, owing to its fibrous ring. Just behind the meatus is found a dilatation, the navicular fossa, on the roof of which is a small 
mucous fold, Guerini valve. The greater part of the penile portion is cylindrical. It shows the foramina, openings of Littre's glands. The bulb in front of the triangular ligament is a dilated area, and contains the orifices of Cowper's glands (Fig. 2). Behind the bulb lies the membranous portion which is normally constricted by the tonus of the thick muscular layer, and which gives a very characteristic sensation of tightness around the examining instrument. The prostatic portion (Fig. 3) is wider, and on its floor are seen several ridges, arrayed parallel with the long axis of the canal; the verumontanum appears as a projection in the midline. On top of the latter, in the median line, is the opening of the prostatic utricle, and on each side of this are the openings of the ejaculatory ducts. In the depression existing on either side of the verumontanum are the orifices of the prostatic glandules (Fig. 3).

The urethra is composed of mucosa with stratified polygonal epithelium, with no submucous fat, so it does not slide, and of a muscular coat arranged in an internal longitudinal and an external circular layer. The internal sphincter of the neck of the bladder is a thickening of the circular layer. The striped muscular fibres reinforce this circular layer, here as well as in the membranous portion (external sphincter).

The urethra has considerable extensibility, so that it can be much dilated, except the meatus, which must be cut. An almost incredible power of repair and considerable elasticity make it possible to bridge over large defects by freeing and approximating both ends.

\section{The Bladder}

The Bladder is a musculo-mucous reservoir, situated in the pelvis, behind the pubes, above the perineal floor, below the peritoneal cavity, before and above the rectal ampulla. The shape of the bladder and its relations to its surroundings vary greatly, according to the age of the subject and the degree of distention. In the fetus the bladder is cylindrical, and extends almost to the navel; in children it is conical, and still projects upward above the symphysis. In adults, when empty, it is generally flattened and triangular, sometimes thick and spherical ${ }^{1}$ and does not bulge very markedly above the pelvic floor, as is easily observed when a laparotomy is performed, under which circumstances it does not reach any higher than the lower border of the symphysis. When the bladder distends, it becomes rounded,

\footnotetext{
1 The spherical shape of the bladder, formerly described as normal, seems to be noted only in hose showing old lesions of cystitis with hypertrophy of the muscular coats.
} 
the transverse diameter being the first to reach its maximum; later, the organ becomes ovoid. The apex rises and is inclined forward, because the posterior portion permits of more distention than does the anterior. The shape of the bladder is never absolutely regular, especially in individuals over forty years of age.

The capacity of the bladder, that is, the quantity of liquid it will contain before the desire to urinate is created, is, in the living, an unstaple quantity, depending on the sensitiveness of the mucosa. It varies from 100 to 500 c.c. (3 to 16 ounces), with 250 to 400 c.c. ( 8 to 12 ounces) as an average. The average dimensions are: anteroposterior diameter, $71 / 2 \mathrm{~cm}$. (3 inches); transverse diameter, $9 \mathrm{~cm}$. (3 1/2 inches); vertical diameter, $10 \mathrm{~cm}$. (4 inches). The bladder may acquire an almost incredible capacity, retaining 4 or 5 liters (quarts) as a result of slow distention in cases of urinary obstruction.

In the standing position of the body, the antero-posterior axis of the filled bladder is almost horizontal, and the urethral opening or neck of the bladder is then the lowest point.

As all organs constantly alternating between vacuity and repletion require a wide range of mobility, the bladder is connected but loosely with the surrounding organs. It is maintained in position at its apex by the urachus, which in the adult effectively suspends the empty bladder; at its base, it is supported by the continuity of the urethra, and by the prostate gland, as well as by two bands of unstriped muscle fibres, extending from the lower part of the anterior surface of the bladder to their insertion into the posterior aspect of the symphysis (anterior ligaments of the bladder or pubo-prostatic ligaments; laterally, the lateral prostatic fasciæ, sometimes called the lateral ligaments of the bladder, and the fibrous cords representing the obliterated umbilical arteries, hold the bladder in position. When distended, the organ is still held, in addition, by the peritoneum. All these means of fixation are weak, and the bladder is therefore a freely movable organ. The neck, long considered as a fixed point, is lowered when the bladder is full and raised when it is empty. The distention of the rectum by means of a rubber bag, as practised in former times, will bring the neck of the bladder upward fully an inch and a quarter.

The relations of the bladder will be stated as found when the viscus is moderately distended, as this is the condition which exists at all operative procedures. In the bladder there are an anterior, a posterior and two lateral surfaces, an apex or summit, a base and a neck to be considered. ${ }^{1}$

\footnotetext{
1 The term "fundus" some authors apply to the apex, some to the base.
} 
The peritoneum covers the posterior surface, the apex, and part of the anterior and lateral surfaces of the bladder. On a vertical midline section of the pelvis the parietal peritoneum, which lines the posterior surface of the urachus and of the umbilical arteries, is shown passing from the anterior abdominal wall to the apex of the bladder, after forming the prevesical cul-de-sac; thence it spreads over the apex, and lines the posterior surface of the bladder, and finally is reflected upward along the anterior aspect of the rectum and pelvic colon, forming another cul-de-sac, the vesico-rectal, or Douglas' pouch. The prevesical cul-de-sac is furthest from the symphysis in the midline; laterally, the bottom of the pouch becomes progressively deeper toward the lateral surface of the organ. The height above the symphysis, which averages $3 \frac{1}{2} \mathrm{~cm}$. with a distention of 300 c.c. was formerly a point of extreme importance for the surgeon, but is of less consequence now that there is no fear of opening the bladder through the peritoneum, when it is done deliberately.

The peritoneum is reflected from the lateral aspect of the bladder, over the lateral wall of the pelvis, forming a cul-de-sac which is continued forward with the prevesical and behind with the vesicorectal pouch. One-third of the lateral surface of the bladder is covered with peritoneum. The Douglas cul-de-sac usually goes down to the base of the prostate, but sometimes stops $2 \mathrm{~cm}$. higher. The bottom of this cul-de-sac is 6 to $8 \mathrm{~cm}$. from the perineal skin, 1 to $2 \mathrm{~cm}$. from the base of the prostate, and is the remains of the much larger embryonic organ. The base is limited forward by the outlet and behind by a plane passing by the upper border of the seminal vesicles. The neck and interseminal space are the triangular spaces below the peritoneum and between the seminal vesicles, where the bladder and rectum are directly in contact.

The anterior surface of the bladder is in relation to the symphysis, Santorini plexus, and pubo-prostatic ligaments.

The lateral surface is crossed by the vas deferens and umbilical artery. The vas passes in an arched direction from before backward, crossing the umbilical artery and passing along the inner side of the ureter.

The apex of the bladder is that portion to which is attached the urachus or remains of the allantois. It lies behind the symphysis and is the superior eminence of the bladder in the flaccid state. It possesses special surgical significance in connection with the enclosed urachus with the existence of cysts, fistulæ, etc.

The interior surface of the bladder, except the region corresponding to the base, is smooth in children and adults; but in older subjects 
it is trabeculated, owing to the thinning of the muscular layer in points, and to its thickening in others. Sometimes the trabeculation develops real columns, with compartments, or even deep diverticula, between them.

In the region corresponding to the base there is a triangular space which is always smooth, called the trigone (Figs. 3 and 4), the anterior apex of which is formed by the urethral outlet, the two posterior apices being the ureteral openings. The urethral outlet is, properly speaking, the neck of the bladder. There is normally no constriction, however, and the orifice is on a level with the surrounding parts of the base of the bladder and the adjacent prostatic urethra. Any projection in this region is of pathological origin. The bladder outlet is closed by the tonicity of the internal sphincter.

The ureteral openings lie behind and external to the neck of the bladder. They are situated on a transverse line passing $21 / 2 \mathrm{~cm}$. behind the urethral outlet and $8 \mathrm{~mm}$. (1/3 inch) on each side of the median line. Their position is remarkably fixed; barring cases of extreme distention, the distance between the two does not change noticeably. They measure about $3 \mathrm{~mm}$. (1/8 inch) in diameter, and generally appear as oblique slits (see Fig. 3). Between the two openings is stretched the interureteric ridge or muscle, the posterior boundary of the trigone, while each of the two other sides is indicated by the less accentuated relief of the uretero-urethral muscular fascicle.

Behind the interureteric ridge, the base of the bladder shows a depression, the bas fond. Normally shallow, it becomes a deep pouch in which urine, and possibly calculi accumulate whenever the trigone is raised by the hypertrophy of the subadjacent prostate.

The structure of the bladder is composed mostly of muscular formation. This is divided into two layers, an external of longitudinal fibres and an internal circular. The latter surrounds the outlet to form the internal sphincter. Exterior to the muscular coat is a fibrous covering and interior is the mucous coat or lining membrane of the bladder. The arteries of the bladder are derived from the internal iliacs, the superior middle and inferior vesical branches.

The veins, distributed like the arteries, are derived from the large Santorini plexus which lies anterior to the bladder behind the symphysis.

There are no lymphatics in the muscosa, but they are abundant in the muscular tissue.

The principal nerves are the sacral and hypogastric plexuses. 


\section{The Ureters}

The bladder and the secreting portion of the kidney are connected by a set of tubular membranous organs: from below upward, the ureter, the renal pelvis and the calices. This is the embryological succession, and that followed by the ureteral catheter when used for exploratory purposes, and in the reverse order of the circulation of the urine.

The Ureter from its vesical opening follows a long curve upward, inward and forward, reaches the pelvic wall, courses upward along the latter, describing at the same time a curve forward, passes in front of the iliac vessels and the sacro-iliac synchondrosis, then ascends in the lumbar region up to about the transverse process of the first lumbar, where it forms a funnel-shaped expansion, the renal pelvis. These two curves give an $\mathrm{S}$ shape visible in a radiograph of ureteral catheters that have been left in situ for any length of time. During its entire course the ureter is sub- or retroperitoneal.

The calibre of the ureter is not even. The very common changes due to congenital irregularities, or the very slight degree of pathological distention particularly frequent in elderly individuals make it difficult to recognize this unevenness. It is not always easy to find normal ureters for anatomical study. Some anatomists who have injected hundreds of ureters with water, wax or gelatin, state they are at a loss to describe the normal shape and diameter. However, it is known that the vesical ending is always one of the narrowest, if not the narrowest point (1.5 to $3 \mathrm{~mm}$.); that the pelvic and lumbar portions each show an irregular fusiform dilatation (5 to $6 \mathrm{~mm}$.) and that the origin of the ureter always exhibits a marked constriction (2.5 to 3 $\mathrm{mm}$.). At the brim of the pelvis, the diameter is usually $4 \mathrm{~mm}$. The length of the ureter in situ is about $30 \mathrm{~cm}$. (11 to 12 inches).

From the vesical orifice, the ureters travel obliquely through the vesical wall, beneath the mucosa, then perforate the muscular wall. The ureteral fibres are not blended with the bladder fibres, but, on the contrary, are isolated by a connective sheath. This oblique intramural part exerts a marked valvular action and effectively prevents the reflux of urine into the ureter under normal conditions.

After leaving the bladder, for a length of $2 \mathrm{~cm}$. the ureter remains applied against the posterior bladder wall, from which it is separated by loose connective tissue, so that it can be exposed there by a transvesical incision. The ureter lies in front of the seminal vesicle which is crossed anteriorly by the vas deferens and the deferential artery; then the duct enters the posterior ligament of the bladder, that is, 
the fold limiting Douglas' pouch laterally. It reaches the lateral aspect of the rectum, too high, however, to be felt by rectal examination. On a level with the greater sacro-sciatic notch, it is related to the upper edge of the pyramidal and the posterior border of the internal obturator muscles; higher up, it follows the internal iliac artery and reaches the iliac fossa, where it rests in front of the common iliac vessels and the sacro-iliac joint. There it lies directly against the posterior pelvic wall but is also nearest to the anterior abdominal wall, through which it may be palpated at a point situated at the intersection of a horizontal line passing through the anterosuperior iliac spine and a vertical line drawn through the pubic spine. The spermatic vessels pass on the outer side of the ureter, and it lies $21 / 2 \mathrm{~cm}$. distant from the promontory of the sacrum. In the lumbar region, the ureter rests on the psoas muscle behind, and is situated 5 to $10 \mathrm{~mm}$. ( $1 / 5$ to $2 / 5$ inch) inward from the tip of the transverse processes. Medially, it is related to the vena cava inferior on the right side, and, not so intimately, to the aorta on the left. Between the large blood-vessels and the ureter is found the lumbar chain of lymph glands.

Externally, here, the ureter is in relation to the vertical portions of the colon. The relation is much more intimate between the ascending colon and the right ureter than between the descending colon and the left ureter. The outer edge of the ureter is united in its upper portion to the lower pole of the kidney by connective tissue containing fat and veins common to both kidney and ureter. This connective tissue varies considerably, and hence there are corresponding variations in the adhesions between the kidney and ureter.

Forward, the ureter is related to the peritoneum, which it raises in a ridge, without, however, forming any "meso". The ureter is strongly attached to the peritoneum, loosely to the retroureteral connective tissue, and when the lumbar peritoneum is stripped from the underlying tissues without special precaution, the ureter comes with it. In the lumbar portion, the ureter is loosely connected with the duodenum (second or fourth segments, according to side), and is crossed anteriorly by the spermatic vessels.

In its iliac portion, the ureter is exposed by an incision similar to that used for ligation of the iliac vessels; in the lumbar region, it is readily accessible through the curved and oblique lumbar incisions used for kidney surgery, lengthened downward and forward, as necessary. (See Op. Surg.)

The renal pelvis is the confluence where the ureter and its branches, the calices, meet. It varies considerably in shape and size, but two types are predominant (see Fig. 4). 
The first is the ampullar type, in which the pelvis is a funnel the base of which is in the kidney, the apex continuous with the ureter, and the great axis inclined downward and inward.

The second is the ramified type, in which the pelvis proper is very small, and the long calices seem to unite directly to form the ureter.

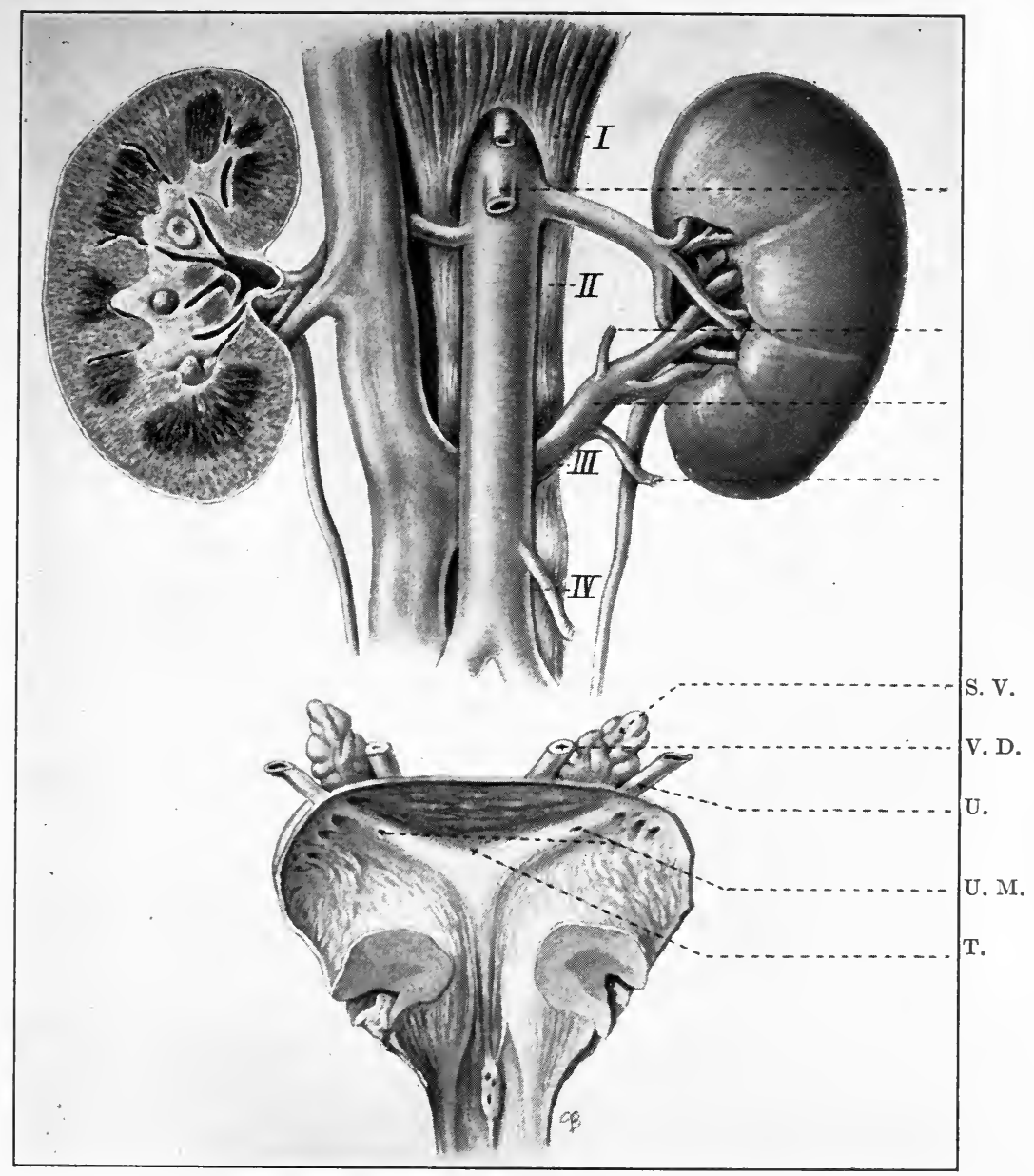

Fig. 4.-Anatomy of upper urinary tract. (After Poirier.)

The ampullar type is much exaggerated beyond the normal as a result of a chronic distention.

The anterior aspect of the pelvis is directly imposed upon the renal vessels; the posterior is separated from the psoas by an abundant 
fatty tissue, and is on a level with the transverse process of the first lumbar. The upper border is partially hidden under the upper branch of the renal artery. The lower border follows the lower half of the internal border of the kidney.

The calices are the highest segment of the excretory channels of urine. There are three great calices (see Fig. 4), each of which subdivides into smaller calices, the other end of which applies around the tip of a renal pyramid. The greater calices are situated in a plane passing through the convex border, and an incision there reaches them all. The smaller calices diverge in several directions, and many escape any incision that is used.

\section{The Kidneys}

The Kidneys are situated retroperitoneally, lying in the renal fossæ, one on each side of the spinal column, in the upper part of the lumbar region, and are limited above by the twelfth rib, inward by the spine, and downward by the iliac crest. Their upper border is on a level with the eleventh rib, their lower with the transverse process of the third lumbar. The right kidney is always slightly lower than the left.

The direction of the kidneys is not vertical, as the long axis is tilted outward and downward.

The shape of the kidney always resembles that of a bean, with a posterior and anterior convex surface, a convex long external border, a shorter notched concave inner border, and an upper and lower pole. But as the relations of the diameters to one another vary, there are elongated, spherieal and elliptical kidneys. On an average, the length is between 12 and $13 \mathrm{~cm}$. (about 5 inches), the breadth 5 to $6 \mathrm{~cm}$. (2 to $21 / 2$ inches), and the thickness $3 \mathrm{~cm}$. (1 $1 / 4$ inches). The weight varies from 100 to $200 \mathrm{gms}$. (3 to 6 ounces), being about $1 / 250$ of the body weight. The kidney parenchyma is dark red, more resistant, owing to its fibrous capsule, than the hepatic or splenic parenchyma. Normally it is insensitive, so that every kidney in which tenderness is elicited is pathological.

The relations of the kidney with the lumbar wall, most important of all, since the kidney is generally approached by the lumbar route, are represented in Fig. 5, which show that the kidney is so placed that half of it is below and half above the twelfth rib. In case the twelfth rib is short and horizontal, it is prolonged outward by the lumbo-costal ligament, so that the same proportion of kidney projects below that ligament as would project below the twelfth rib, 
were the latter long and oblique, as is usually the case. Consequently, the lower half of the kidney is accessible below the bony parts; the upper half is hidden behind these bony parts and always must be brought out. The relations of the kidney with the strong transverse process of the first lumbar are also worthy of note. This bony prominence is responsible for most of the ruptures of the kidney, which conse quently begin near the hilum. In Fig. 5 are indicated the outlines of the kidneys and quadratus lumborum muscles. It will be seen that the outer border of the kidney projects slightly beyond the lateral

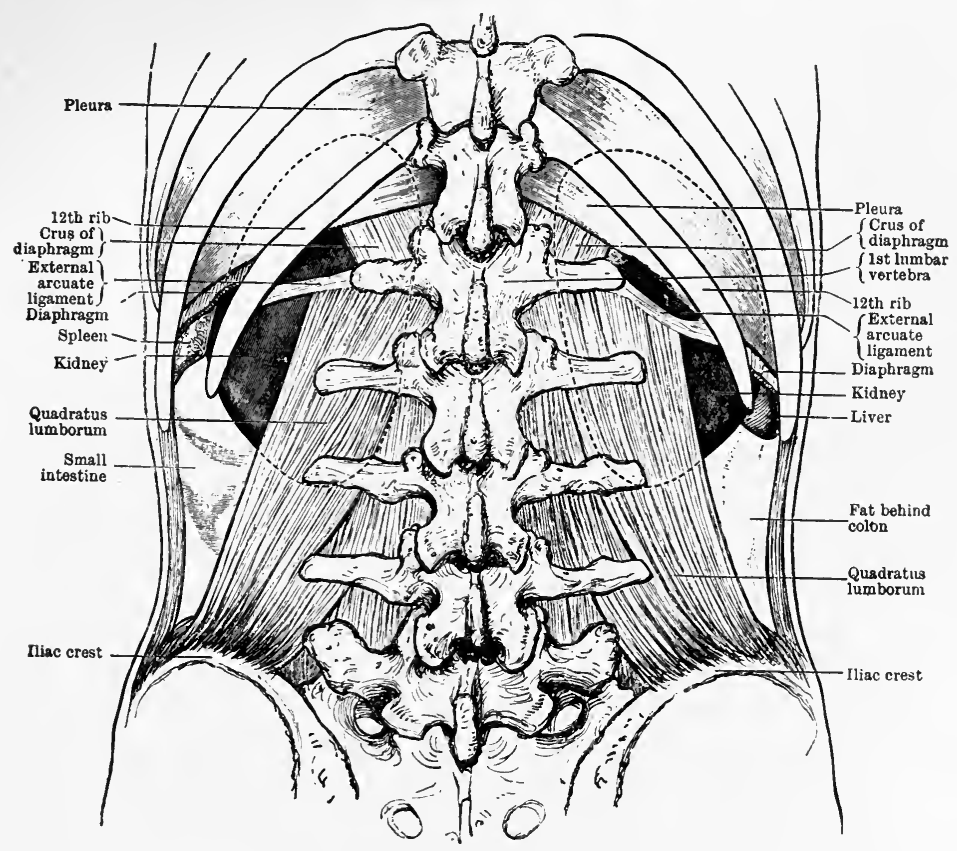

FIg. 5.-Anatomical relations of the kidney. (Cunningham.)

edge of those muscles, and is, therefore, easily reached by an incision made along their outer border. Between the two muscles, the aponeurosis of the transverse muscle of the abdomen is inserted into the transverse process of the abdomen. Each muscle is enclosed in a special fascial sheath. The upper part of the kidney rests upon the posterior insertion of the diaphragm. The pleural costo-diaphragmatic sinus does not descend lower than the twelfth rib, if the latter is long, but if it be short, the sinus extends 1 or $1.5 \mathrm{~cm}$. below it, and might be easily wounded in lumbar incision for exposure of the kidney. The last thoracic, the ileo-hypogastric and the ileo-inguinal nerves, 
which are seen crossing the operative field obliquely outward and downward may be included in the posterior relations of the kidney, beneath the quadratus muscle.

Anteriorly, the kidney lies behind the peritoneum. On the right side, it is connected from below upward with the hepatic flexure of the colon, the duodenum, more particularly the internal border, and the liver, upon which the kidney leaves its imprint in the shape of a shallow depression. On the left side, the kidney is related to the spleen, particularly its convex border, to the tail of the pancreas, the splenic flexure of the colon, and the ascending portion of the duodenum.

The medial border of the kidney is excavated by the sinus, and on this border the kidney receives its blood-vessels, lymphatics and nerves. The hilum of the kidney is covered by the duodenum (descending portion on the right and ascending portion on the left side). Its lower part is passed by the ureter. The upper pole of the kidney is covered by the suprarenal body. There is only $5 \mathrm{~cm}$. ( $2 \mathrm{in}$.) distance between the upper poles of the two kidneys; in this narrow space the aorta and inferior vena cava pass; hence, the frequency of adhesions between these large blood-vessels and the upper pole of the kidney in case of new growths.

The kidney is surrounded by a mass of fat, enclosed in a fascial sheath (perirenal fascia) which is the main support of the gland, the other means of suspension being the renal blood-vessels and the parietal peritoneum. On a horizontal cross-section of the body, the subperitoneal fascia is seen to divide, when it reaches the external border of the kidney, into two layers-the anterior, or prerenal lines the parietal peritoneum; the posterior or retrorenal ${ }^{1}$ passes between the kidney and the quadratus lumborum, to which it is attached by numerous strong fibrous tracts, and over the psoas, and becomes inserted in the midline into the anterior aspect of the vertebral bodies. Thus the perirenal fascial compartment is closed inward and outward. On a vertical sagittal section, the two layers are seen to enclose also the suprarenal body; above the latter they meet and become strongly adherent to the under surface of the diaphragm. This effects a strong fixation of the kidney. Downward, the two fascial leaves do not coalesce. The anterior always lines the parietal peritoneum, while the posterior divides into leaflets that blend progressively with the connective strata of the iliac fossa. Therefore, the renal compartment is opened downward. This, and the renal vessels,

1 The retrorenal leaf (Zuckerkandl's fascia) is much thicker than the anterior, which is reinforced by a connective layer (Toldt's fascia) resulting from the fusion of the left layer of the primitive mesocolon with the parietal peritoneum. 
whose insertion is naturally fixed, limit and determine the displacements of a movable kidney (see nephroptosis).

Within the perirenal fascia is the adipose capsule of the kidney, more or less abundant, according to the individual case, and forming a thick pad behind the posterior surface, around the hilum and both poles of the kidney.

The renal arteries arise from the sides of the abdominal aorta, between the superior mesenteric and the spermatic arteries, on a level with the intervertebral disk separating the first from the second lumbar vertebræ. The renal arteries are very large, averaging from 6 to $8 \mathrm{~mm}$. (1/4 to $1 / 3 \mathrm{inch})$ in diameter, and the right artery is about $25 \mathrm{~mm}$. (1 inch) longer than the left. Both renal arteries are oblique downward from their origin to the kidney. The length of these arteries is important surgically, as it determines the ease or difficulty which will be met with when delivering the kidney outside the body. A normal kidney has always a sufficiently long and elastic pedicle to permit of bringing it into the wound, after thorough freeing. The renal arteries lie behind the peritoneum. The right passes in front of the spine and behind the inferior vena cava; the left goes directly to the kidney.

Relative Positions of the Organs.- In the sinus, the renal artery divides into four principal branches, three anterior $^{1}$ going to the anterior three-fourths of the kidney, one posterior for the posterior fourth. This division may occur before the entrance into the sinus, on the inner border of the pelvis; the latter is then taken between the anterior branches and the posterior as between the prongs of a fork. In case of still more premature division, the first branch detached from the trunk of the renal (which always goes to the lower pole of the kidney) may cross the origin of the ureter anteriorly or posteriorly when it forms a band upon which the duct can become kinked.

In the kidney itself the arteries are of a distinctly terminal type. There are no peripheral anastomoses, as were formerly described. Each artery has its own independent territory. It even seems that the arteries of the poles are independent from those supplying the anterior and posterior aspects. These facts are plainly shown in a prepared specimen after artificial digestion when the arteries have been injected with a paraffine mass. It follows that it is not permissible to cut any branch of the renal artery, for necrosis of the corresponding portion of the parenchyma would necessarily ensue. As

\footnotetext{
${ }^{1}$ It follows that the most avascular zone in the kidney is not the convex border, but a line situated at the junction of the anterior three-fourths and the posterior fourth, that is, a line about $8 \mathrm{~mm}$. behind the convex border.
} 
a matter of fact, there is an anastomotic circle between the collaterals of the renal artery, the inferior capsular artery and branches of the spermatic artery, but this circle is altogether insufficient to save any part of a kidney when its artery has been severed. The same rule holds good for any of the abnormal supernumerary renal arteries. which are very frequent.

The veins of the kidney have numerous anastomoses which permit the passage of the blood through other than the regular channels. In cases of chronic congestion with compression of the parenchyma, effort to increase these ways of outlet may be made by decapsulation of the kidney and production of adhesions with neighboring organs.

The lymphatics of the kidney go to the prevertebral glands, which it has sometimes been attempted to remove, together with the kidney, in case of malignant tumors.

The kidney proper is formed by a fibrous capsule, easily stripped off under normal circumstances, a secreting cortex with Malpighian glomerules and convoluted tubules and excretory ducts grouped in pyramids, and opening in small calices through the "area cribrosa," which exists on top of each papilla (see Fig. 4). The fibrous capsule, is very important surgically; it alone gives the parenchyma enough resistance to allow of a suture being passed to it. It therefore must always be included in a kidney suture. A suture passing through points stripped of capsule invariably cuts through.

The ducts and reservoirs of the genito-urinary tract are all formed of three coats: an internal mucous, around which is a middle muscular, comprising a circular inner and outer longitudinal set of unstriped fibres, surrounded itself by a third adventitial connective coat. Except for the testicle, which has a very special sensibility, all genito-urinary organs have a very obtuse sensibility. Every organ that causes pain, either spontaneously or under examination, is the seat of pathological lesions.

All hollow organs resist sudden distention by spasm, accompanied by violent pain-renal colic, acute retention of urine, acute epididymitis. Contrasted with these is the painlessness of slow and gradual distention, allowing, for instance, the latent development of huge hydronephroses or an enormous dilatation of the bladder in prostatic hypertrophy.

\section{The Prostate}

The confluence of the urinary and genital tracts is surrounded by a gland which, physiologically, belongs only to the genital system, but which, on account of its situation, has an etiological bearing upon both 
tracts. This is the Prostate. Its dual nature is demonstrated in prostatic hypertrophy, the symptomatology of which is purely urinary in character, as is that of prostatitis, but these conditions are, at the same time, the opening wedges of all deep genital infections.

The prostate surrounds the urethral segment nearest to the bladder; it is situated beneath the latter, at a certain distance behind the symphysis. before the rectum, above the triangular ligament, between the internal border of the levatores ani. It has about the shape and size of a horse-chestnut, with base directed almost horizontally and apex pointing downward and forward.

The anterior surface is almost an inch behind the symphysis. It is almost vertical, or slightly oblique downward and forward, and is covered by the half ring of the striped urethral sphincter (see Embryology, p. 27), and connected by the lower fibres of the anterior ligament with the Santorini plexus of the bladder and the preprostatic fascia emanating from the triangular ligament. The posterior surface is bifid in shape, and is markedly oblique forward and downward. In the midline it shows a shallow, vertical depression, easily felt with the finger by rectum. It is related to the rectal ampulla, from which it is separated by the prostato-peritoneal fascia. This fascia results from the coalescence of the two layers of the embryonic peritoneal genito-rectal cul-de-sac, which primarily extended downward to the apex of the prostate. Between the blended layers of said cul-de-sac, that is, within the prostato-peritoneal fascia, is the proper plane of cleavage in perineal prostatectomy.

The lateral surfaces are in contact with the lateral prostatic fasciæ, which separate these surfaces from the internal borders of the levatores ani, and from the anterior recess of the ischio-rectal fossæ. These fasciæ contain the rich lateral venous plexuses of the prostate.

The base of the prostate adheres to the base of the bladder. When the bladder is separated from the prostate, the following are revealed from behind: the urethral orifice surrounded by the internal sphincter; second, a transversal crescentic isthmus which is the middle lobe ; third, the cross-section of the vasa and seminal vesicles and prostatic utricle, behind which is the posterior commissure; fourth, the rounded projection of the lateral lobes.

The apex of this gland rests on the triangular ligament, on a level with the lower border of the symphysis.

The prostate is surrounded by a musculo-connective tissue capsule, constituted, forward, by the preprostatic fascia, which is inserted below into the posterior edge of the transverse ligament of the pelvis, and ascends in front of the prostate, to unite with the anterior longitudinal 
fibres of the bladder; behind, by the prostato-peritoneal fascia; laterally, by the lateral prostatic fasciæ. This capsule does not exist above, where only connective tissue separates the prostate from the base of the bladder, nor downward, but here the apex of the prostate rests on the tough triangular ligament. Hence, the line of least resistance followed by the hypertrophying prostate is always upward, toward the bladder cavity or backward toward the rectum, a fact of great importance in the operation of prostatectomy. This capsule does not belong to the prostate proper; it adheres loosely to the glandular tissue.

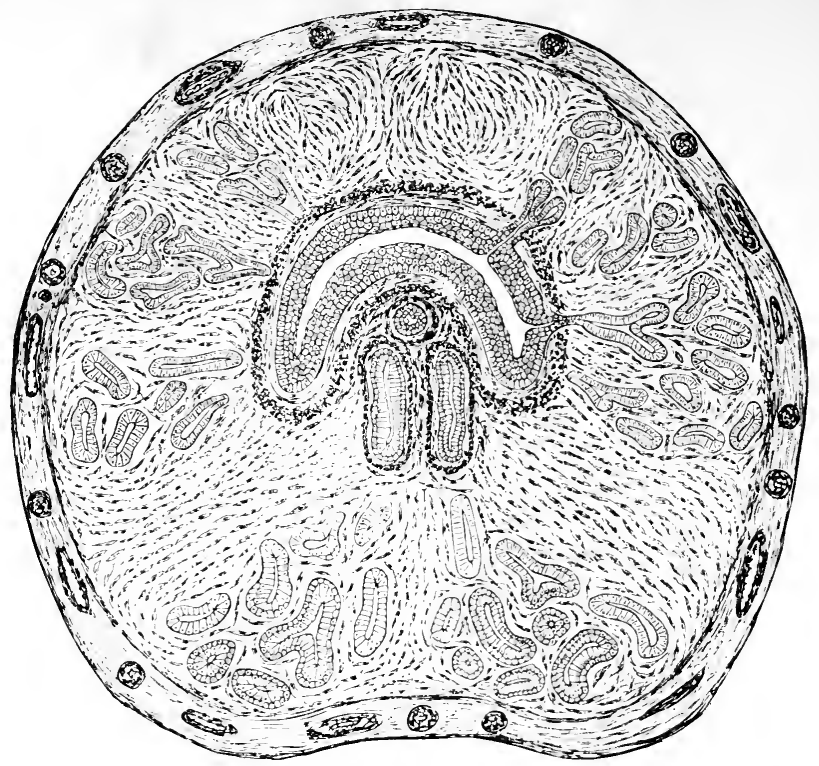

FIG. 6.-Transverse section of prostate, showing ejaculatory ducts in central portion.

\&:- The glandular tissue occupies a comparatively small area in the anatomical region occupied as the prostate. It is disposed in two lateral lobes on each side of the urethra, and an anterior preurethral commissure, more or less thick, sometimes absent. The posterior commissure has already been spoken of. One of the most interesting group of glands, surgically speaking, is situated beneath the vesical mucosa, and above the ejaculatory ducts, because it is often affected by prostatic hypertrophy median isthmus.

The lymphatics of the prostate are so numerous that the gland has been termed a lymphatic sponge.

The Ejaculatory Ducts are two small canals (formed by the juncture of the vas deferens with the seminal vesicle; they extend from 
the neck of the seminal vesicle to the prostatic urethra. They are about $25 \mathrm{~mm}$. ( 1 inch) long; oblique at 45 degrees, backward and upward, and are imbedded in prostatic tissue. They are separated at their vesicular extremities and converge at the urethral end; hence, the possibility of sparing them in perineal prostatectomy by leaving a median bridge of tissue (Fig. 6).

\section{The Seminal Vesicles}

The Seminal Vesicles are musculo-membranous pouches annexed to the ejaculatory ducts and the vas deferens. The length is on an average $5 \mathrm{~cm}$. (2 inches), the width about $12 \mathrm{~mm}$. (1/2 inch). They are oblique downward, inward and slightly forward. They mark out on the base of the bladder the triangle between the vasa deferentiæ, on which their antero-superior aspect is applied. The postero-inferior surface is applied on the rectum. It is always easy to palpate a pathological seminal vesicle by rectum; a normal vesicle cannot be felt unless distended.

The seminal vesicles are formed by the coiling of a single tube, the kinks being united by strong connective tissue. Unraveled, the vesicle tribe measures 10 to $12 \mathrm{~cm}$.

The Vas Deferens goes from the neck of the seminal vesicle to the tail of the epididymis. Its length is about $45 \mathrm{~cm}$. (18 inches). It is first applied on the posterior surface of the bladder, between the two seminal vesicles (see Fig. 4), then bends outward, follows the lateral wall of the bladder and reaches the internal inguinal ring, where it meets the nerves and vessels of the testicle, and with these forms the spermatic cord. It passes through the inguinal canal, issues through the outer ring, and goes through the scrotum finally to reach the testicle. Its first portion, near the bladder, is called the ampulla, because it is dilated and irregular in shape, not unlike the adjoining seminal vesicle. There it is also situated between the bladder and the rectum. The opening of the vas deferens in the seminal vesicle is so disposed that fluids injected into the vas first fill and distend the vesicle before passing into the ejaculatory duct. On the lateral surface of the bladder, the vas crosses the ureter, then the umbilical artery, finally the external iliac vessels and the epigastric artery. In the inguinal canal and in its funicular portion, it occupies the centre of the spermatic cord. The latter is composed of cellular connective tissue, surrounded by a fibrous sheath. It contains, besides the vas, the arteries, veins and nerves of the testicle and epididymis, plus the remnants of the fœtal funicular process. 
The vas, along which $\bullet$ runs the deferential artery, divides the spermatic cord into two parts. The anterior, which is the larger, contains more veins (those affected in the ordinary form of varicocele) surrounding the spermatic artery, lymphatics and nerve branches. The posterior portion contains a few veins and unstriped muscular fibres.

In the scrotum, the vas follows the internal border of the epididymis; it remains outside of the tunica vaginalis.

All the spermatic channels, up to this point, are formed by a mucosa, a muscular coat and an adventitial layer. The muscular coat of the deferens is enormously thick, as compared to the lumen of the

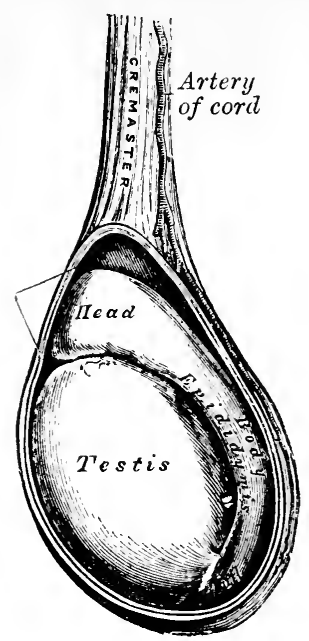

FIG. 7.-Testicle and appendages. canal, which is really small; hence, the ease with which the vas is palpated and recognized in all its accessible portions (hence, also, the difficulty of making a patulous anastomosis of the vas). The epithelium of the vas is cylindrical and ciliated.

\section{The Testicles}

The Epididymis is a small, elongated organ which rests upon the upper and posterior border of the testicle, as a crest rests upon a helmet. Its anterior is the large portion, and is called the head or globus major, and reaches to, or extends beyond the posterior pole of the testicle (see Fig. 7). The lower portion is called the globus minor, or tail (though in fact it does not taper) and is continued directly without line of demarcation, but simply with a change of direction, with the vas. This portion is outside the tunica vaginalis, and connected with the scrotum by the scrotal ligament. The epididymis is a tube coiled many times upon itself; uncoiled, it measures about 66 meters (20 feet) in length.

In the fully developed individual, the testicles are situated in the scrotum; they are ovoid in shape, partly covered by the epididymis (Fig. 7) suspended by the spermatic cord, of variable consistency and volume and have a very special sensibility.

Surgically, the layers covering the testicle are much more interesting than the testicle itself. From without, inward, are found: the skin of the scrotum, lined by a few muscular fibres; a layer of unstriped muscular fibres, the dartos, which also contains elastic fibres. Upward, the dartos is continued by the suspensory ligament of the 
penis (Fig. 2) and through the latter is inserted into the pubis. There is only one cutaneous scrotum; but interiorly the dartos is divided into two envelopes, one for each testicle, and each of the enveloping layers forms a distinct sheath around each testicle. The two testicular bags are united in the midline (median septum of the scrotum). The layer of unstriped muscle fibres is followed by a connective-tissue layer continuous with the fascia (Cooper's fascia) of the abdomen. This is very thin, and through it may be seen the cremaster muscle, a dependency of the internal oblique of the abdomen. This is followed by a fibrous sheath continuous with the fibrous sheath of the spermatic cord, and with the fascia transversalis of the abdomen. Behind and below the testicle this fibrous sheath forms the scrotal ligament which binds the testicle at the bottom of the scrotum. Finally, the last layer is composed of the tunica vaginalis, a serous membrane enveloping the testicle and part of the epididymis. The parietal layer is everywhere applied on the under surface of the fibrosa, and is smooth and even. The visceral layer covers the internal surface of the testicle, its anteroinferior border, then ascends toward the postero-superior border, where it finds the epididymis. It penetrates the middle portion of the epididymis, there forms a cul-de-sac, after which it lines the outer surface of the epididymis; thence, it ascends slightly along the cord, to be reflected into the parietal layer. The tail of the epididymis and the scrotal ligament remain outside of the vaginal cavity.

The testicle itself is surrounded by a tough fibrous layer, the albuginea. The arteries of this organ are the spermatic, which anastomose with the artery of the vas and the funicular artery. This explains why the testicle does not always atrophy after the spermatic has been severed.

\section{The Perineum}

The Perineum composes the whole of the soft parts closing the outlet of the pelvic cavity. It does not belong to the urogenital tract, but is a most important route of access to all of its parts, except the kidney, ureter and testicle. It is best exposed with the patient in the lithotomy position. It is lozenge shaped, is limited forward by the ischio-pubic rami and the ischia, laterally and behind by the greater sacro-sciatic ligaments, backward by the cocyx. The bi-ischiatic line divides it into posterior perineum (rectal) and anterior (urogenital), only the latter of which will be referred to here.

Proceeding by dissection, are met successively:

1. The skin, subcutaneous fat and fascia superficialis. 
2. A layer of muscles, all adnexa of the copulative organs, shown in Fig. 8.

3. The urogenital diaphragm, formed essentially by a tough fibrous lamina filling in the space between the pubes and through which pass the dorsal vein of the penis and the urethra (see Fig. 9). This is the triangular ligament. Behind its posterior border is the deep perineal transverse muscle (Fig 9), a flat muscular belly inserted

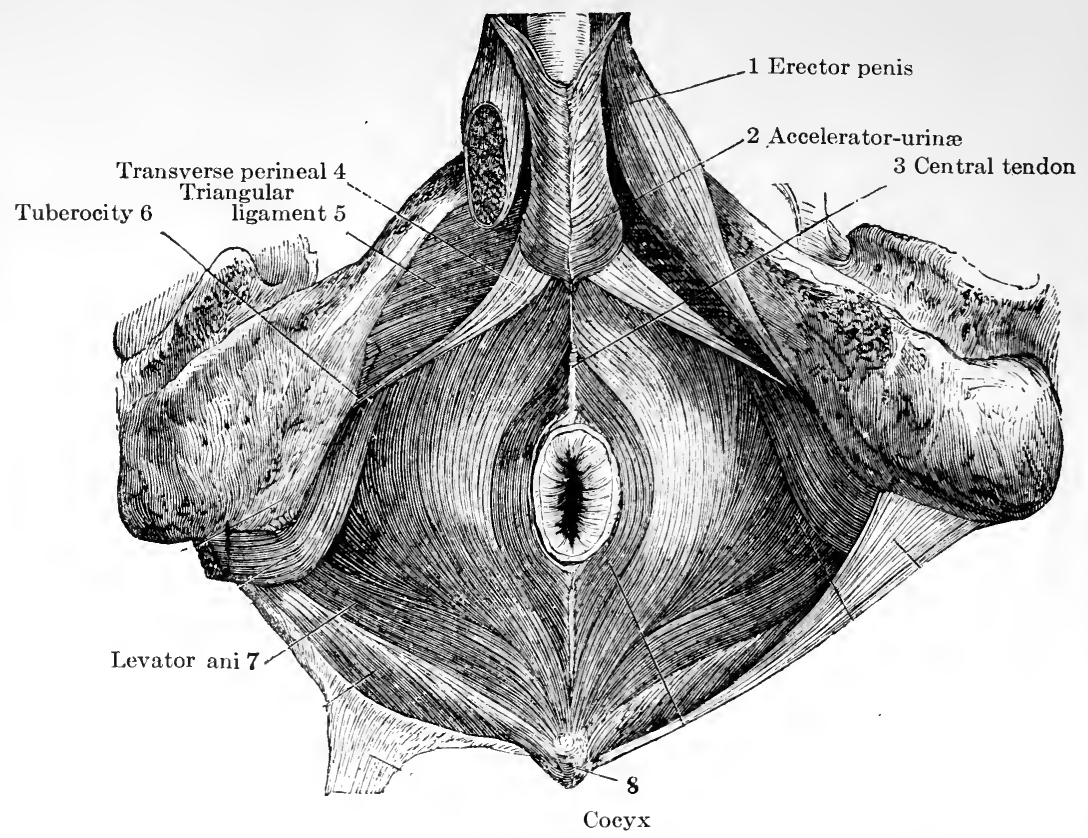

FIG. 8.-Dissection of perineum; superficial muscles. (Beaunis et Bouchard.)

outward into the ischii and ischio-pubic rami, and in the midline into the fibrous centrum. The triangular ligament is composed of two layers. ${ }^{1}$

Around the whole posterior urethra exists a system of striped fibres constituting the urethral sphincter, derived from the primitive sphincter (see Embryology, p. 26). In its upper part the prostate divides the sphincter, a half of which is applied over the anterior

\footnotetext{
1 The disposition of the layers of the triangular ligament and of the deep perineal muscles has been the subject of many recent remarkable studies, which have modified considerably the oldtime conception of these organs. But, however fascinating from a philosophical standpoint, these studies have little practical value. Note that the deep transverse here described is not the classical deep transverse which is the same as Guthrie's muscle or compressor urethre, part of the striped urethral sphincter.
} 
surface of the prostate, and the membranous portion, where the fibres are very well developed and form a flat layer, commonly described as situated between the two layers of the triangular ligament (Guthrie's muscle, compressor urethræ, cut-off). Above the triangular ligament are found the prostate, the base of the bladder and the endopelvic fascia.

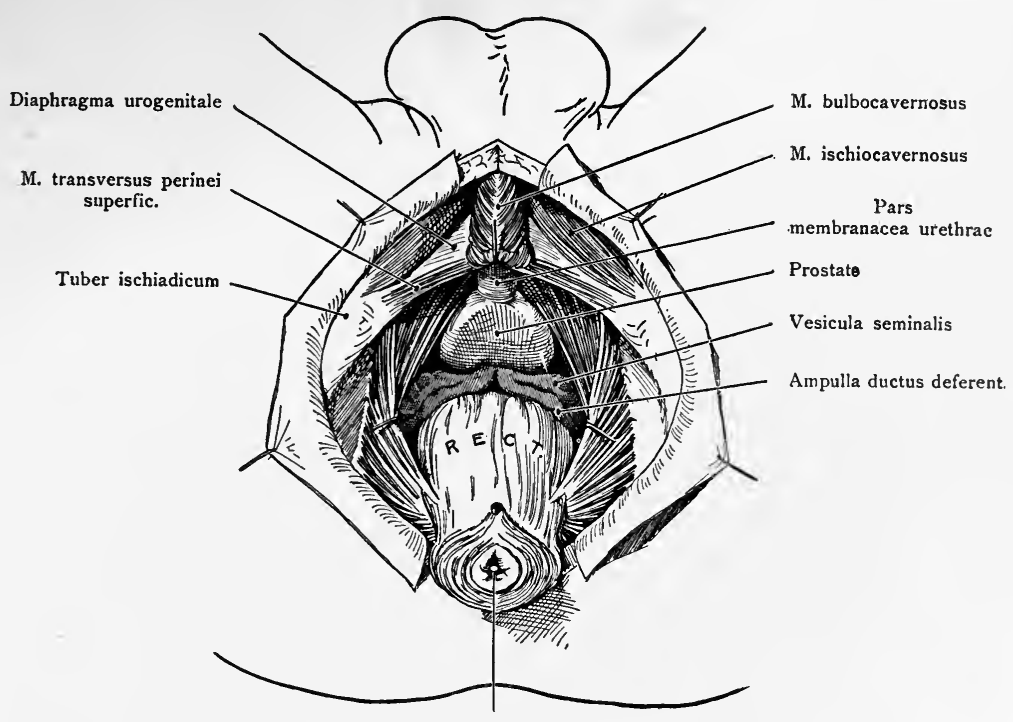

Fig. 9.-Deep perineal anatomy.

\section{EMBRYOLOGY}

A comparison of the genital and urinary tracts reveals the fact that both are developed de novo upon the same general plan. Each has a gland which secretes a specific product (testicle, kidney); a long, slender duct (epididymis and vas deferens, ureter) through which the secretion passes into a musculomembranous reservoir (seminal vesicles, bladder) where it accumulates between the intervals of expulsion; and each ends in a canal which extends from the reservoir to the surface (ejaculatory ducts and urethra). This canal is common to both tracts during its greater length (see Fig. 10), but central to this confluence the tracts diverge.

The outer opening of the urethra is the physiological end of both systems; surgically, however, it may be considered their beginning, since it is the portal of entry of most genital infections, and these infections are a prevalent cause of urogenital disease, which may extend for a greater or lesser distance along one or both of the urinary or 
genital tracts. All surgical explorations are also begun at this opening, and are carried as far as the kidney.

The development of the segmenting ovum commences with its sepa-
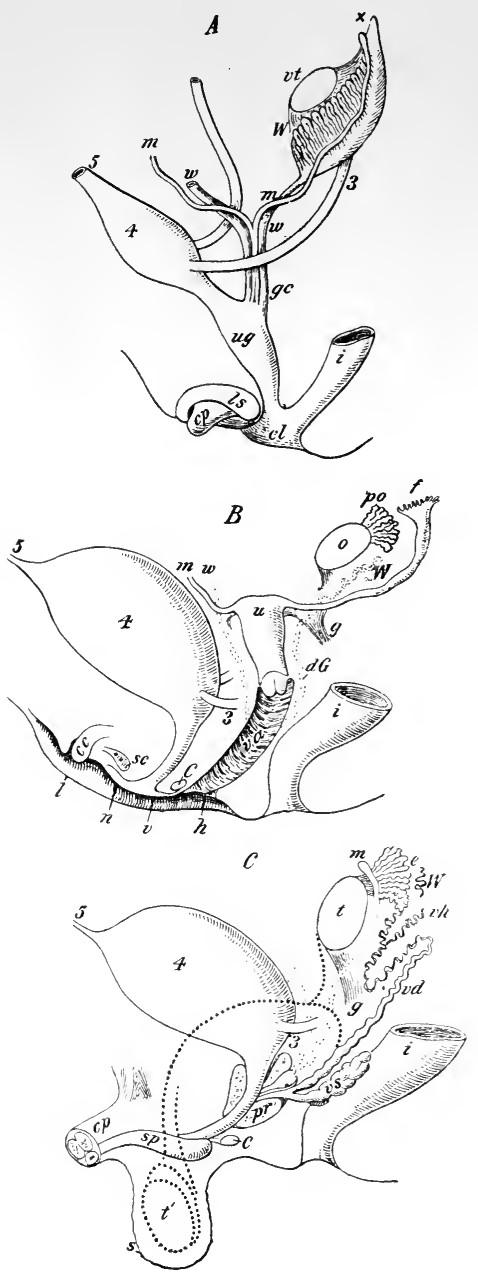

Fig. 10.-Embryological development of urogenital tract. (Quain.) $A$, Common to both sexes up to seven weeks; $B$, female type, thereafter; $C$, male type. assumes a ventral position, and appears as an organ distinct from the hind-gut, although both end in a common cavity, called the cloaca.

The developing allantois sends a prolongation, which participates derm. The space between these germ cells is known as the segmentation cavity, and the cells forming therein are designated the mesoderm. When the mesoderm divides into two layers, enclosing the pair of cavities called the cœlom, the external layer, with the epiblast, constitutes the somatopleure, and the internal, with the hypoblast, forms the splanchnopleure. From the splanchnopleure is evolved a primitive gut, the posterior segment of which, the hindgut, consists of a depression called the cloacal fossa. At this point the anus will form later, but in its embryonic stage, the bottom of the depression consists of entodermic and ectodermic epithelial cells only, without mesoblastic interposition. This cell mass is the cloacal membrane, behind which the hind-gut is prolonged by the caudal gut; the latter ends in a blind recess, the first trace of the allantois.

The urogenital germ, also called the intermediate cell mass, because of its position at the junction of the somatopleure and splanchnopleure, lies in the innermost part of the cœlom, beside the protovertebræ.

Between the third and fifth weeks, the incurvation of the embryo occurs, and the allantois, which has been turned at an acute angle, ration into the ectoderm and ento- 
in the formation of the fotal membranes, through the umbilicus into the extraembryonic cœlom. The portion contained within the abdomen is known as the stalk.

Meanwhile, interesting transitory organs are growing in the intermediate cell mass. From each embryonic segment a solid cellular cord branches into the somatopleure. These progressively lengthen backward and outward, finally bulging under the epidermis; subsequently they become hollowed out in tubules, communicating with the cœlomic cavity through nephrostomes, which, however, soon close. The external ends of these cords form a longitudinal duct which extends along several embryonic segments, and becomes the pronephros or head-kidney. This is the first segmental organ, and atrophies quickly. Caudal to the pronephros, a similar organ, called the metanephros (Wolffian body), grows in the same manner. It is visible in the, third week, but reaches its greatest development between the fifth and seventh weeks, when it extends along the entire pleuroperitoneal cavity. Each mesonephric tube connects with a vascular loop, whose afferent branch arises from the aorta and whose efferent empties into the posterior vein, making a typical Malpighian corpuscle. The Wolffian duct is the mesonephric excretory canal, and opens laterally and very high into the cloaca.

The Müllerian duct now appears near the Wolffian duct. It is not known whether the former emanates from the latter, or develops independently and borrows the persisting excretory duct of the already atrophied pronephros.

At the end of the fourth week there begins the development of the permanent kidney, when an evagination arises from the lower end of the Wolffian duct, near the cloaca. This is the ureter, and it ascends backward and upward along the vertebral column, its upper extremity dilating to form the future pelvis, and then dividing into four secondary pouches, which will eventually become the calices. The branching continues, and the offshoots penetrate the surrounding nephrogenic blastema, in which numerous blood-vessels already preëxist, evolving first the straight, then the convoluted tubes, and finally the glomerules, the fundamental element of the kidney, on which depends the final disposition of the organ. No fundamental kidney substance can develop where there are no preëxisting blood-vessels, and where such blood-vessels are abnormal, the corresponding part of the renal substance will also develop abnormally. Generally speaking, kidney anomalies are vascular in origin. The fœetal kidney is well formed in the third month, and from this time to the period of extra-uterine life, has a marked lobulation. 
On the mesial side of each mesonephros, the cœlomic epithelium thickens into the genital ridge or external germ epithelium. The epithelium also projects inward to form the internal germ epithelium. Up to the seventh week there is no sexual differentiation (Fig. 10, $A$ ). If the evolution is of the male type, the Müllerian duct plays an insignificant rôle at this time. The fused lower ends of both ducts remain to form the prostatic utricle (Fig. 10,C); and Morgagni's hydatid may also be of Müllerian origin. The posterior portion of the mesonephros atrophies, leaving as traces Giralde's body and Haller's vas aberrans, while its anterior part and duct are converted into spermatic channels.

There are in the germ epithelium some larger cells which sink in the depth toward the Wolffian duct. They are primordial spermatomeres, and are parent elements of spermatozoa. Cellular columns arise from the duct, creep between the spermatomeres, and later are changed into tubes, which in turn become excretory canals, tubuli recti, rete, and efferent cones of the testicle. The Wolffian duct engenders the epididymis, which is straight at first, but coils upon itself when it becomes elongated. From this same duct emanate the vas deferens and the ejaculatory ducts. In the third month a hollow tube springs from the latter, and as it grows to a considerable length, coils upon itself and becomes the seminal vesicle.

As it develops, the testicle bulges more and more beneath the peritoneum. At first it is continuous with the atrophying mesonephros, but it gradually becomes pinched off from the latter, remaining connected with it, however, by a peritoneal fold, the mesorchium. The mesorchium is prolonged upward by a peritoneal fold stretching toward the diaphragmatic region (the diaphragmatic ligament), and downward by a similar fold (the inguinal ligament) containing unstriped muscle fibres, which constitute the gubernaculum testis.

Contemporaneous with the above, important changes occur in the cloaca. At the beginning of the third month the perineal spur descends and divides its cavity into a posterior intestinal and an anterior (urogenital sinus) compartment. The latter carries the mouths of the ureters and of the Wolffian and Müllerian ducts, and is continued upward by the allantoidian stalk. From the lower part of the stalk and the upper part of the sinus the primitive bladder is developed. The upper part of the stalk remains slender, and becomes the urachus; the lower part of the sinus elongates and forms the posterior urethra.

The descent of the perineal spur, which brings with it the large peritoneal genito-rectal cul-de-sac, splits the primitive cloacal sphincter into a posterior ring around the anus and an anterior ring around the 
urethra. Therefore, the striped urethral sphincter is primarily a complete sheath enveloping the entire posterior urethra. This regular arrangement is complicated during the third month by the development of the prostate. Epithelial buds come from the mucosa of the initial portion of the urethra, just beneath the unstriped vesical sphincter, and grow wherever there is room. Reaching backward, they burst the sphincteric ring, and reduce it to a half-ring, concave backward, applied on the anterior surface of the urethra; after filling the free spaces between the urethra and the peritoneal cul-de-sac, they can increase only by the extension of the lateral cell masses forward and of the posterior cell masses upward. The former produce the lateral lobes which turn around the urethra and surround it completely; the latter are the origin of the median lobe.

The prostate originates from five separate groups of tubules (Lowsley). (1) The middle lobe, an independent structure although it is not separated from the lateral lobes by capsule. (2) and (3) The lateral lobes separated from each other by anterior and middle lobes. (4) The posterior lobe separated from the lateral lobes by a fibrous capsule. It is the portion palpated per rectum. Seldom is hypertrophied, and generally the primary site of prostatic carcinoma (Geraghty). (5) The anterior lobe, largest at development and smallest at birth.

Then the two layers of the peritoneal cul-de-sac, which at first extended downward to the point of the prostate, coalesce up to the level of the upper border of the seminal vesicles. The fused peritoneal layers constitute what is spoken of as Denonvillier's prostatoperitoneal fascia.

The perineal spur contains mesodermic tissue in its lower part and unstriped muscle fibre in the upper portion. From the former is evolved a central fibrous perineal membrane and from this latter, the system of unstriped fibres binding the urethra to the rectum, important in perineal prostatectomy. The outer fibres of the cloacal sphincter acquire secondary insertions into the ischio-pubic rami, and from these is developed the perineal musculature.

The Anterior Urethra is evolved between the seventh and fourteenth weeks, from a single and median genital tubercle, and from two lateral genital folds parallel with the median tubercle, with the edges of which they unite. The genital tubercle grows in front of the pubis, and becomes the penis. The glans appears during the third month, the prepuce and corpora cavernosa during the fourth. A cylinder, excavated on its inferior aspect, is formed by the tubercle and folds. This is the genital furrow, and is filled by an epithelial cell mass 
emanating from the cloacal membrane. The lips of the furrow unite in the lower midline, and the solid cord of cloacal epithelium becomes hollowed out in a tube which connects backward with the posterior urethra. The balanic portion develops independently from a separate epithelial mass, and later unites with the penile urethra. These successive stages of epithelial thickening, epithelial bud, solid cellcord secondarily converted into a tube by the hollowing out of a lumen within, form the fundamental process of development of all ducts of the urogenital tract. Some, notably the urethra, even develop by the reunion of several segments, each of which is formed by the abovementioned process. The complexity of the evolution explains the frequent abnormalities observed in this region.

In the fourth month, all permanent organs are differentiated. Henceforth, in most organs there will be only an increase in size; in a few there will be slight modifications of shape, and in others, changes of position.

At first the bladder is cylindrical, as it has been developed from a slender pedicle, and extends up to the umbilicus; later it becomes fusiform, and seemingly descends into the pelvis. In reality, the unequal growth of the bladder and of the abdominal walls causes the appearance of a descent, and as a consequence the layers of the peritoneal duplications which existed at first between the bladder and the abdominal walls fuse and leave no trace except the fascix limiting the prevesical space.

The testicle is first found in the lumbar region. The gubernaculum fibres attached to its inferior pole enter the inguinal canal, and are inserted into the deep aspect of the aponeurosis of the external oblique, marking out in a thinner spot the future external inguinal ring. Then the testicle, dragging along its blood-vessels and the vas deferens, leaves its first bed, travels through the iliac fossa and the inguinal canal, and at birth has reached the bottom of the scrotum. The causes of the descent at first seem to be only the unequal growth of the trunk and the inguinal ligaments; later, an active attraction is apparently exerted, which at the same time draws the peritoneum before the testicle and forms the funicular process. When the testicle reaches the scrotum, it is entirely surrounded by a peritoneal diverticulum, the future tunica vaginalis, which communicates widely with the peritoneal cavity by the peritoneo-vaginal canal. The latter closes spontaneously during the first year of life, but disturbances of this complicated migratory process are responsible for many malformations and lesions observed in this region. 


\section{CHAPTER II \\ THE DIAGNOSIS OF SEXUAL AND URINARY MALADIES}

\section{THE UROLOGIST'S ARMAMENTARIUM AND GENERAL TECHNIC}

Instruments used in genito-urinary surgery may be classified, from a practical point of view, as essential and not essential. Such division, however, will depend largely upon individual practice. A full equipment or armamentarium will naturally include all of the general outfit of the operating surgeon. The special instruments are those employed by the urologist for diagnosis and treatment in his specialized field of work. These, for the most part, comprise the different types of urethral sounds and catheters, syringes and irrigators, urethroscopes and cystoscopes, and some extra instruments of necessity in certain operations or peculiar to individual operators.

\section{SYRINGES AND IRRIGATORS}

Irrigator jar for anterior and deep urethral irrigations.

Urethral nozzles and eut-off (Chetwood). (Figs. 18 and 19.)

Urethral clamp (Chetwood). (Fig. 31.)

Anterior urethral syringes-plunger type. (Fig. 11.)

Anterior urethral syringe with bulbous ejector. (Fig. 12.)

Instillator-Keyes-Ultzmann type. (Fig. 14.)

Instillator-bulbous ejector type. (Fig. 17.)

100 c.c. syringe-Janet (Gentil). (Fig. 13.)

\section{Catheters, Bougies and Sounds}

Soft-rubber catheters (Nélaton) (No. 10 to 20 French). (Figs. 35 and 36.)

Soft-rubber catheters, Mercier or elbowed end, small and large. (Fig. 36.)

Woven catheters, bougie or olivary end, various sizes. (Figs. 37 and 38.)

Woven catheters, Mercier or elbowed end-blunt-various sizes. (Fig. 37.)

Woven catheters, Mercier or elbowed end-pointed-various sizes. (Fig. 37.) 
Woven catheters, double elbowed (bi-coudé)-various sizes. (Fig. 37.)

Elbowed obturator. (Fig. 40.)

Curved obturator. (Fig. 40.)

Silver catheters. (Fig. 49.)

Conical steel sounds (double taper) - sizes 15 to 32 French (Fig. 24.)

Blunt steel sounds-sizes 20 to 30 French. (Fig. 23.)

Kollmann dilators. (Fig. 34.)

Bulbous bougies - sizes 10 to 30 French. (Fig. 27.)

Olivary bougies-sizes 6 to 25 French. (Fig. 26.)

Filiform bougies. (Fig. 28.)

Tunneled metal catheters-several sizes. (Fig. 123, p. 288.)

Tunneled metal sounds-several sizes. (Fig. 123, p. 288.)

Guyon steel sounds and filiform guides-three or four sizes. (Fig. 29.)

Stone searcher. (Fig. 187, p. 447.)

For description of urethroscopes, cystoscopes and attachments, see index.

\section{Operative Outfit and Special Instruments}

General surgical equipment-knives, hemostatic forceps, etc.

Aspirator.

Aspiratory syringes (for mercurial injections).

Intravenous injection outfit. (See index.)

Litholapaxy instruments. (See index.)

Prostatic operative instruments. (See index.)

Galvano cautery prostatic incisor (Chetwood). (See index.)

Urethrotomes. (See Stricture.)

Other instruments will be mentioned in sections on operative surgery following each subject.

The examining room should be light and well aired, with conveniences for temporary darkening when the cystoscope is used. The artificial light is best produced by electricity. The floor and walls should be constructed of material that can be washed. Two reservoirs, one of hot and the other of cold, sterilized water for irrigation; a boiler for instruments, a full-sized utensil sterilizer, and a large sterilizer for dressings, sheets, gowns, etc., complete the sterilizing outfit.

A useful table of simple design, that is suitable for cystoscopy and for urethral and vesical lavage is a modification of the Isaacs' table shown in Figs. 20 and 21.

Scales.-Various scales exist to distinguish the gradation of the calibre of urethral instruments, known as French, English and Ameri- 
can. The scale was first accurately fixed in France, and there are now two French scales in use, the Charrière, known as the French scale, and the Béniqué. The former is more generally used in this country, while in France the new Béniqué scale is preferred by many urologists.

The French or Charrière scale expresses its measurement in onethird of a millimeter diameters. No. 1 indicates a diameter of $1 / 3$ $\mathrm{mm}$.; No. 2 indicates a diameter of $2 / 3 \mathrm{~mm}$. The number of an instrument is divided by 3 to ascertain its diameter; that is, a No. 30 French sound has a diameter of $10 \mathrm{~mm}$. or one-third of $30 \mathrm{~mm}$. The number of an instrument in the Béniqué scale is double that of the same instrument in the Charrière; a No. 60 Béniqué sound equals a No. 30 French.

The American scale measures in half millimeters to indicate its diameter. Its numbers equal two-thirds of the French, 20 American equals 30 French $\left(\frac{.020}{.0}\right.$ or $\frac{.030}{3}=10 \mathrm{~cm}$.)

The English scale averages about 2 less than the American; 18 English equals 20 American.

Lubrication of Instruments. - The question of a suitable lubricant for instruments is an important one. Oil has many disadvantages, for boiling alone sterilizes it, and this process is both difficult and dangerous. It smears the prism of the cystoscope, and so cannot be used in cystoscopy. Vaseline and lanolin are open to the same objection, although the former is an excellent lubricant and much better than many of the more modern varieties, but its serious disadvantage is the difficulty of removing the oily coating from the instrument after use. The oil also coats the mucous membrane and thus prevents the action of any medicament employed. Furthermore, an oily coating on a catheter adds to the length of time required to sterilize by boiling.

Among the lubricants commonly used, besides oil, are various combinations of sterilized gum tragacanth or Irish moss, glycerin, and boroglycerid. There are a number of such preparations upon the market, put up in sterile tubes. To be satisfactory, the lubricant should not be lumpy. It should be thin and smooth, but not watery. The preparation adopted in my clinic, is known as "K.Y." lubricant, or the following formula:

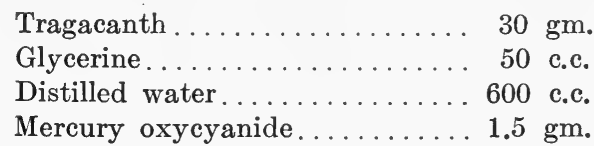


Note.-This lubricant is similar to one that has been employed in Europe (Guyon and Kraus). In some preparations carbolic acid, instead of oxycyanide of mercury, is used as an antiseptic. It has been found that after being exposed to the air it remains sterile eight days (Casper). Investigations have also shown that microörganisms are destroyed or their virulence inhibited by direct contact with the lubricant.

The antiseptic preserves the lubricant for some time, and possesses the additional advantage of not irritating the urethra.

In comparison with oily lubricants, the advantages in favor of this preparation are that the former coats instruments with a film that offers a nidus for microbes, and is not dissolved during the process of sterilization, while the latter lubricant, which is soluble in water, offers no impediment to sterilization, is also readily soluble in fluids used for irrigations, and thus does not interfere with their therapeutic action.

The ordinary method of lubrication is to spread the preparation freely upon the instrument to be used by compressing the containing tube. It is sometimes a good plan to distend the urethra with the lubricant as a means of separating the inflamed walls or of dilating an irregular constriction, before introducing the examining bougie. The introduction of the lubricant is made with the ordinary urethral syringe (Fig. 32 ).

Syringes and Nozzles.-The usual type of urethral syringe or injector is a 12 to 16 c.c. (5iii-iv) piston syringe with cone nozzle made

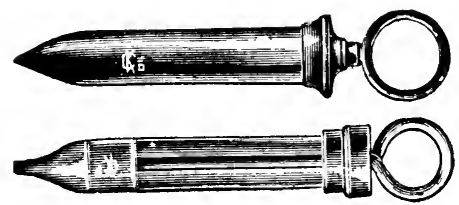

Fig. 11.-Urethral syringes. (Plunger type.) of glass or hard rubber. The nozzle is sometimes of soft rubber (Fig. 11). As a substitute for the ordinary urethral syringe, especially for the purpose of washing the urethra before using the sound, for cocainizing the urethra, or for injections of any kind, the bulbous syringe (Fig. 12) is most useful. It is easily cleansed, and may be boiled continually without detriment. It also makes a good hand syringe for patients' use, and obviates the difficulty, often encountered with the piston syringe, of leakage backward.

Injecting the Anterior Urethra.-For this purpose, one or the other of the syringes described (Figs. 11 and 12) is employed. The lips of the meatus being separated, the nozzle of the syringe is gently, but firmly, thrust into the opening so as to completely fill the orifice and prevent leakage alongside, and the fluid is then slowly injected by compressing the bulb or pushing the piston until the canal is comfortably dis- 
tended. The thumb and index-finger of the other hand meanwhile gently squeeze the meatus against the syringe to avoid leakage, and after the canal is filled, compress the lips together to retain the fluid, the syringe being then withdrawn.

The patient should be instructed in the method of injecting the anterior urethra, when it is required to be done by him. Otherwise, it may be improperly performed and valuable time lost.

When it is desired to retain liquids or lubricant jelly in the anterior urethra, the author's urethral clamp is of assistance, as, while it completely blocks the meatus, it may be left on at will, and does not exert harmful pressure (Fig. 31).

A syringe of generous capacity (100 to 150 c.c.) (Fig. 13) is an important accessory to the urologist's outfit for bladder and urethral

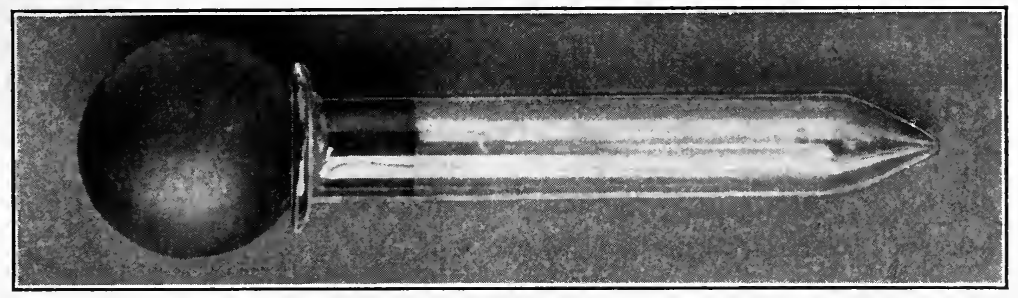

FIg. 12.-Anterior urethral syringe. Bulb ejector.

lavage and for cystoscopic examinations. The Janet syringe, having a capacity of from 100 to 150 c.c. is a very satisfactory instrument of this kind. One with a glass barrel is preferable, as the metal does not stand the action of the chemical solutions commonly used. I have had best satisfaction with the French (Gentil's) graduated glass syringe, in metal frame, with rubber plunger (Fig. 13). This instrument is easily utilized by one hand, and is sterilized by boiling. A metallic tip lessens the danger of breakage, of so common occurrence. The rubber plunger stands long usage and boiling, and the replacing, when necessary, is very simple. There are a number of other Janet syringes made in this country.

Deep urethral syringes are employed for instillations of small quantities of medicaments to the posterior urethra or neck of the bladder.

The Keyes-Ultzmann instillator (Fig. 14) is a short-curve silver canula of small calibre, with a 2 c.c. syringe attached in one piece, or fitted with a standard thread, when it may be utilized with a hypodermic syringe. The length of the shaft is 15 or $18 \mathrm{~cm}$. $(6$ or 7 inches), the calibre 15 to 18 French. It is well to be provided with a number of canulas, of different diameters. 
The canula of the Bangs' modification of the deep urethral syringe is as large as a fair-sized steel sound (25 F.) and distends the canal when making an application.

The Guyon canula, a flexible woven instrument with bulbous end, is introduced less painfully in some individuals. To this canula may be attached the bulbous and glass syringe for instillation, or the latter may be attached to the different sized silver canulas by having the
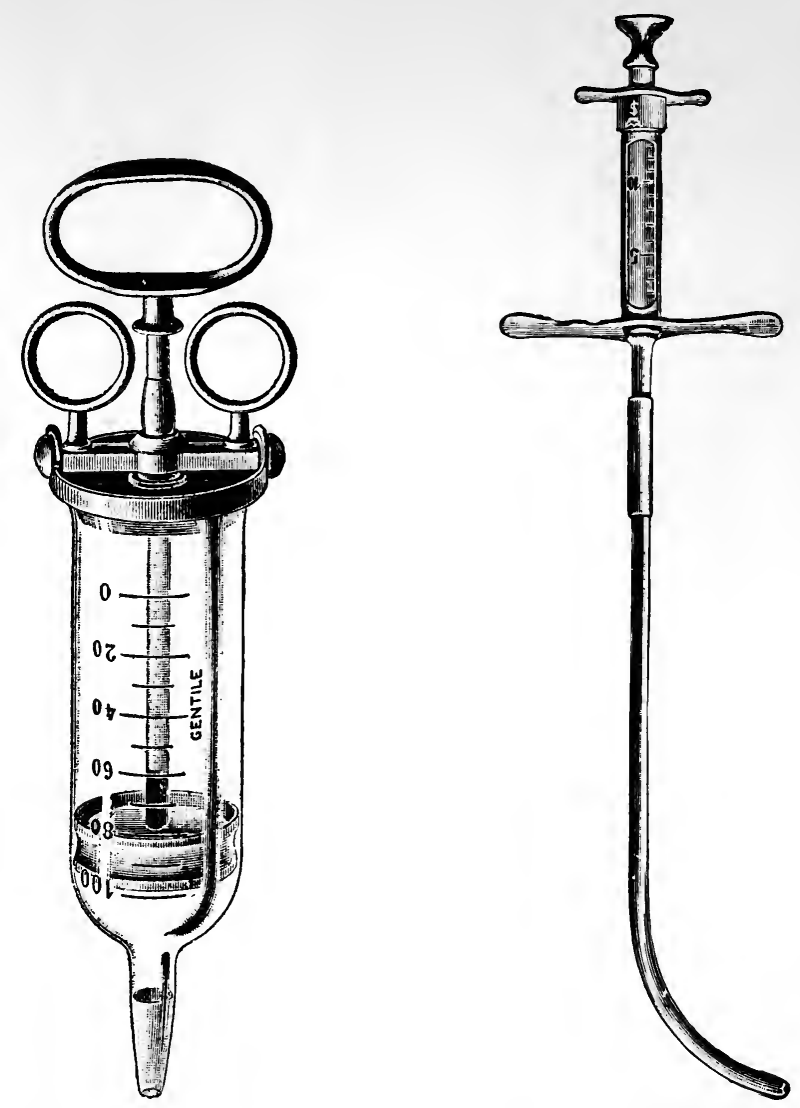

Fig. 13.-100 c.c. Bladder syringe. Fig. 14.-Keyes-Ultzmann deep urethral syringe.

thread ground down and a hard rubber coupling fitted. These instruments are easily portable, and can be sterilized by boiling or by being kept in an antiseptic solution (Figs. 15, 16 and 17).

Instillation or Injection of the Posterior Urethra.-When this procedure is conducted with the solid canula, the instrument is introduced like an ordinary sound until the end reaches the curved urethra or 
bulbo-membranous junction, where it often finds resistance. Great gentleness should be practised at this point, and the instrument must be made to enter without force, or not at all, as this is the location where damage is so often done to the mucous membrane and false passage produced by rough handling. When the deep urethra is

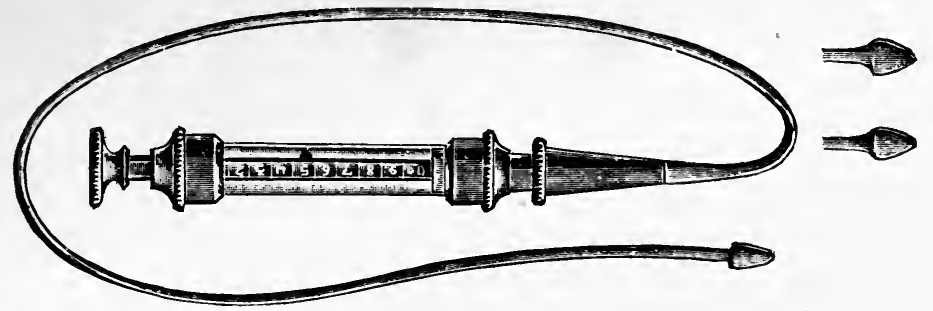

FIG. 15.-Guyon instillator.

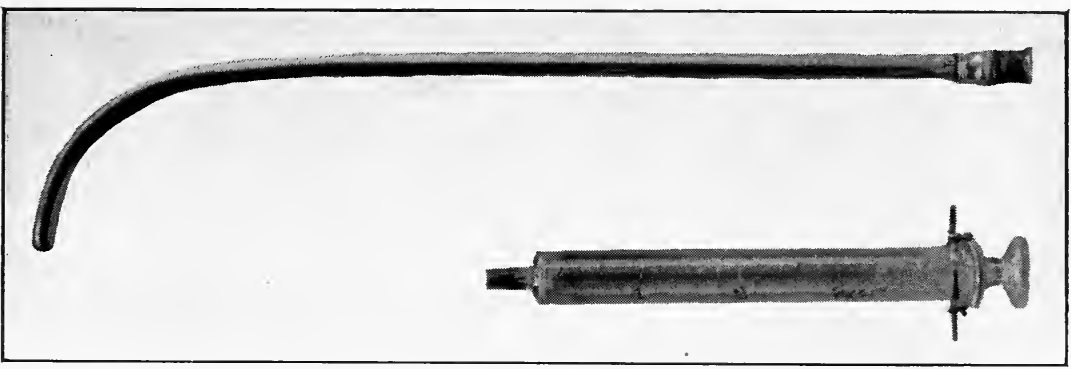

FIG. 16.-Aseptic deep urethral syringe.

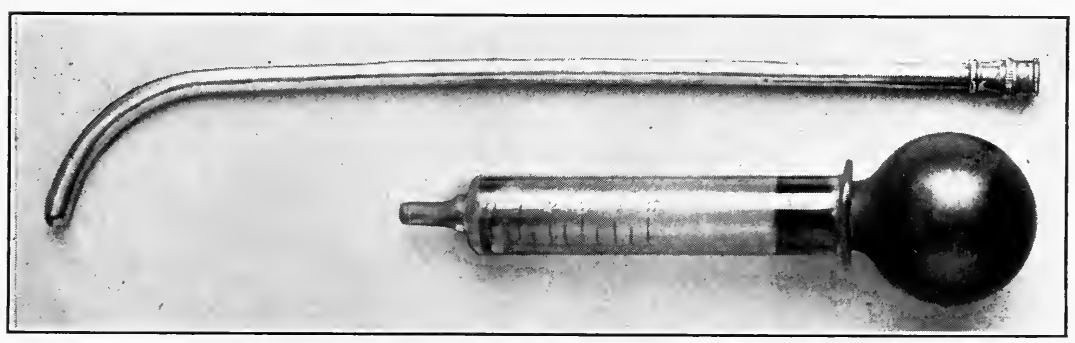

FIG. 17.-Deep urethral syringe with bulb ejector.

entered, the handle of the injector is depressed to an angle of about 45 degrees with the plane of the table, the full contents of the syringe or a few drops of the medicament being then deposited, according to the nature of treatment.

The flexible or Guyon canula for instillation is introduced until the olivary end is felt to pass the cut-off muscle, when it is known that it is 
in the posterior canal; if there is doubt of this, it is pushed further in, to the point where urine is first obtained, which marks the neck of the bladder, and then withdrawn from 2.5 to $4 \mathrm{~cm}$. (1-1 $1 / 2$ ins.) before injecting.

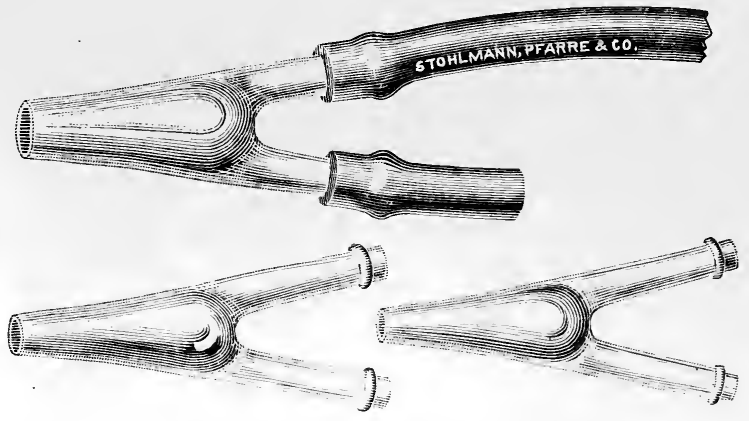

FIG. 18.-Urethral nozzles. (Chetwood.)

Irrigators.-Irrigators are employed for anterior and posterior urethral irrigation and bladder lavage. For this procedure are required a glass tank, so suspended that it may be easily shifted up or down, proper length of tubing, nozzles and cut-off or interrupter.

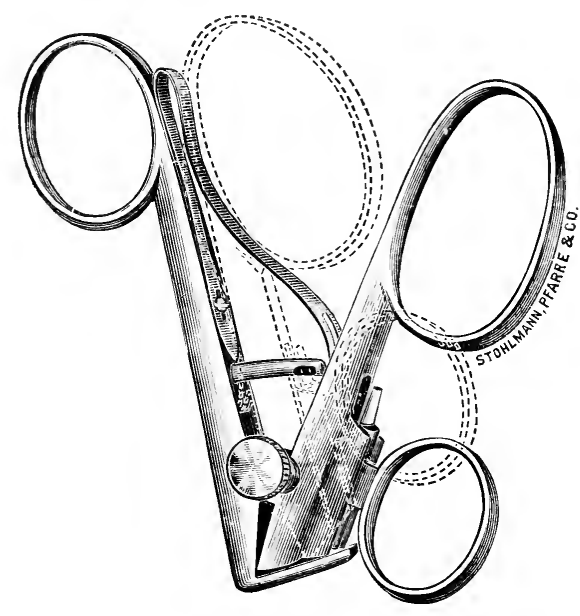

FIg. 19.-Author's alternating urethral irrigation clamp. The author's two-way bluntpointed glass nozzle and cutoff scissors will be found convenient and useful for both anterior and posterior irrigation, more especially after a little practice (Figs. 18 and 19). This method is not so clumsy, and allows less splashing than the larger interrupters employed with the Janet nozzles (such as Valentine's and Swinburne's). Many, however, prefer the latter patterns.

Anterior urethral irrigation (Fig. 20) is one of the methods of treating the front urethra when it is the seat of inflammatory trouble (see index); it is also employed as a measure of prevention against infection. The procedure may be accomplished with an ordinary cone-shaped nozzle attached to the tubing of an irrigator jar, in which case the nozzle is partially withdrawn with each inflation of 
the canal, to allow the escape of the fluid before refilling. In such case it is well to have attached a protecting bell to catch the splash, as is found with the Valentine and Swinburne instruments. I prefer, and
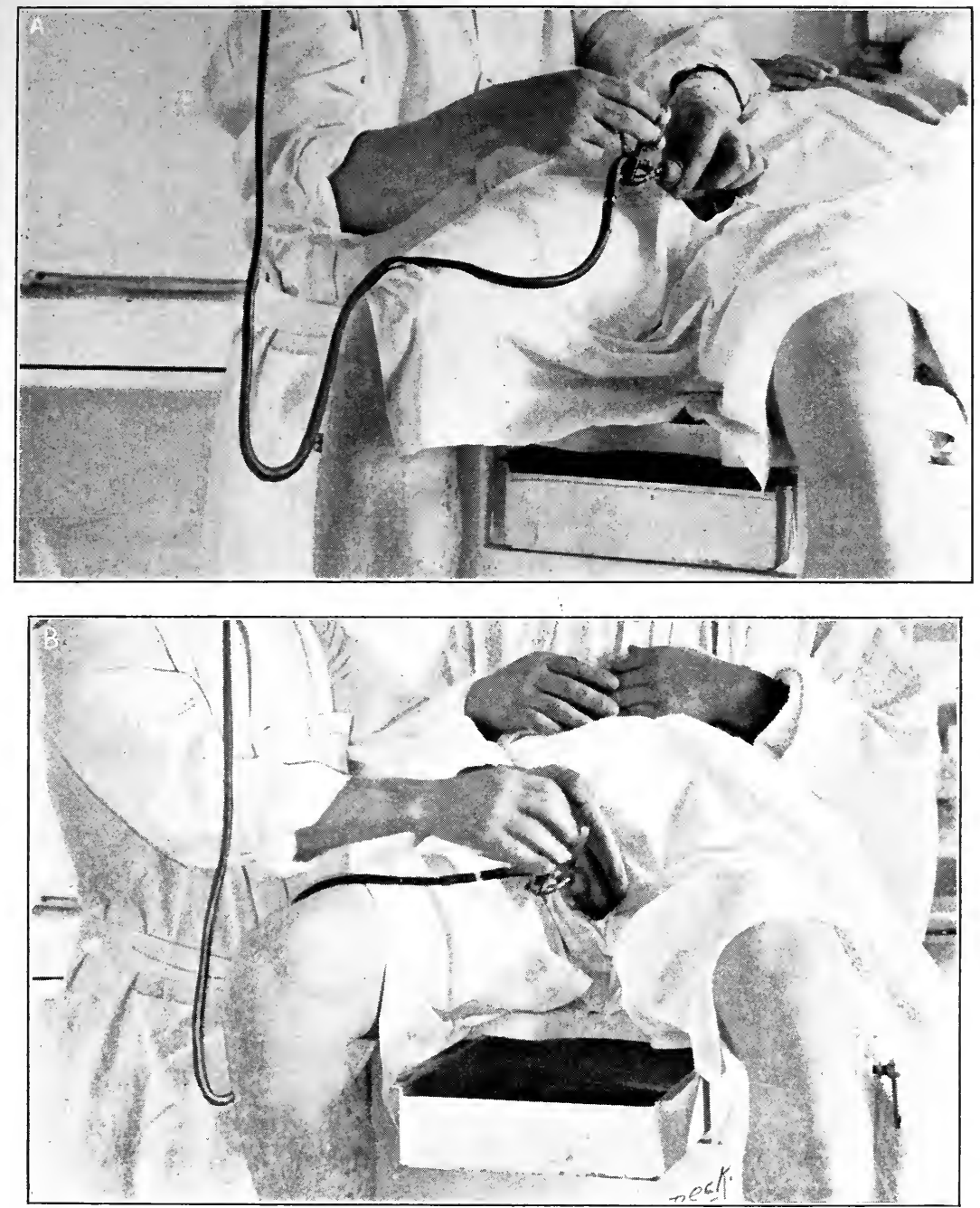

FIg. 20.-Anterior urethral irrigation. A. (above) Inflow-outlet closed. B. (below) outflow-inlet closed.

have used satisfactorily, the apparatus shown in Fig. 20. This apparatus consists of a reservoir with proper length of tubing. The reservoir is arranged to be elevated or lowered at will and attached to the end of the tubing is a scissors-like shut-off (Fig. 19) which allows, when in 
use, the opening and closing of the inflow tube by means of which the urethra is alternately filled and emptied with the irrigating fluid. This is accomplished by having a urethral nozzle, with one opening at the penile and two at the other extremity (Fig. 18). One of the latter
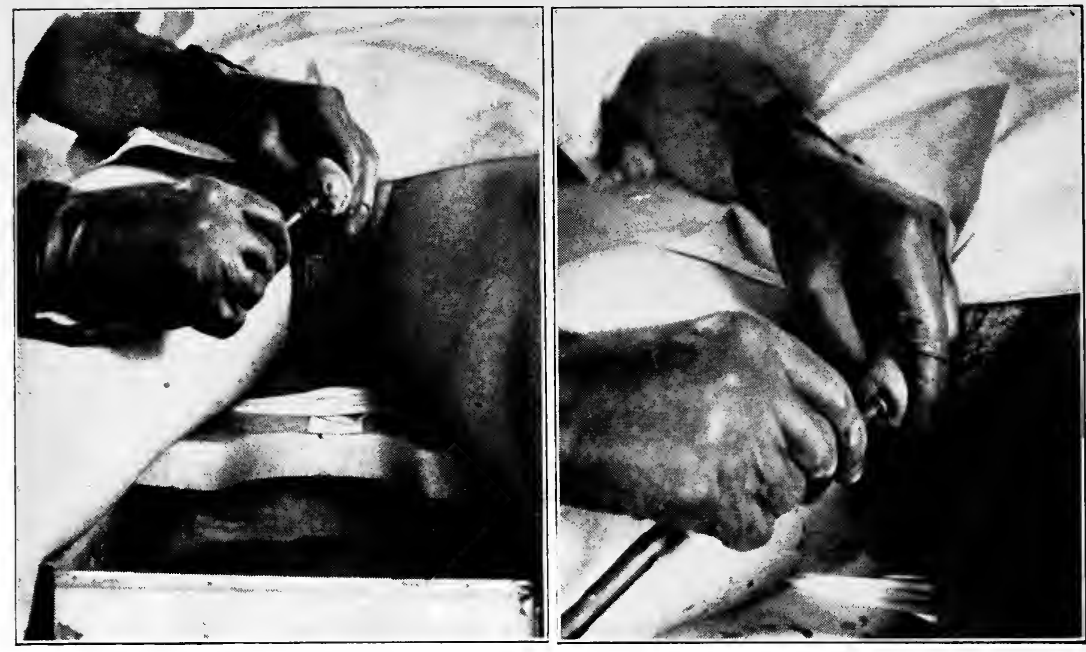

A

B

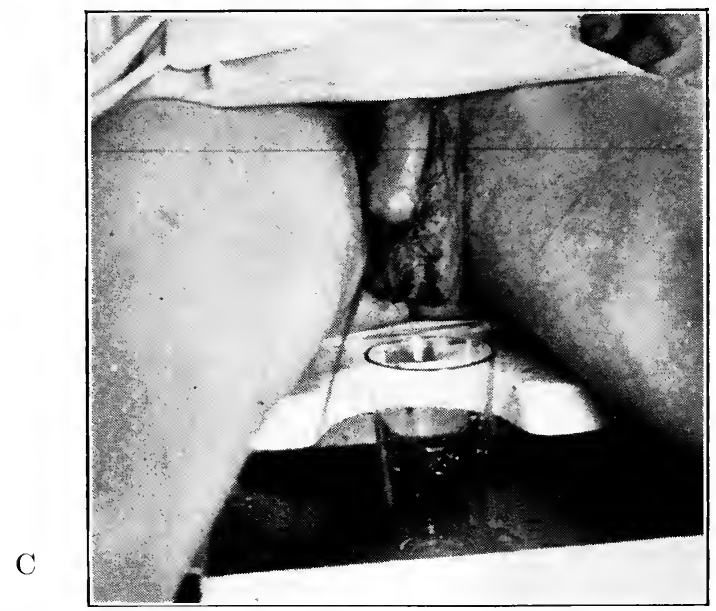

FIG. 21.-(A, B, C) Deep urethral and vesical irrigation without a catheter.

is connected with the irrigator inflow tube, and the other with a short tube for outflow. In operating this apparatus, the patient stands over the basin or sits upon the operating-table with thighs flexed. By alternately opening and closing the inflow tube, the urethra is flushed 
out with as much of the fluid contents of the jar as may be desired, and during this process the folds of the canal are opened and the mouths of the ducts and follicles cleaned.

Posterior urethral and bladder lavage without the catheter (Fig. 21) may be conducted at the same time as irrigation of the anterior canal by raising the tank the sufficient height (2 to 2.5 meters or 7 to 8 feet), with or without local anesthesia of the antero-posterior urethra, by distending the front section of the canal until the fluid escapes by the cut-off muscle through the posterior portion. Great care should be exercised not to inflict undue irritation by this procedure, and pressure should be relieved at once by reversing the flow with the alternating cut-off, or by withdrawing the nozzle if the patient complains unduly. This method of deep urethral irrigation without the catheter cannot always be successfully performed without producing irritation, sometimes to an extent that unnecessary pain results and even such complications as swollen testicle or greater activity of the inflammation in the deep urethra and neck of the bladder. It requires coöperation on the part of the patient, or his passive assistance, which means relaxation of the parts and total absence of resistance. This is best accomplished by having the patient in a comfortable semi-recumbent position, with the knees bent and feet supported on properly adjusted stirrups (Fig. 21). By employing one of the methods of anesthetizing the urethra before a cystoscopic examination (see page 28), the treatment is more easily accomplished and the element of resistance being removed thereby, there is much less likelihood of causing irritation and harm. In suitable cases this procedure is capable of exceptional benefit, especially when the patient as well as the operator becomes trained in its use by progressive practice. In irritable patients it is well for the first one or two treatments to desist when only a small quantity of fluid passes into the bladder, and it will be found on subsequent trial that a gradually increasing amount will pass the cut-off muscle and that by such patient persistence the entire quantity (250 to 500 c.c. -8 to 16 ounces) will enter with little difficulty.

The soft-rubber nozzle of the ordinary anterior urethral syringe attached to a 100 c.c. bladder syringe is sometimes used for forced vesical irrigation, but this plan should not be adopted in irritable conditions, as the force that can be exerted in this manner to overcome the resistance is very great.

The catheter is often a safer means of deep urethral or bladder instillation and irrigation, in which case, before the introduction of the catheter, the anterior urethra should be cleansed, either by irrigation or by gently flushing out the canal with a bulbous or piston urethral 
syringe. When the bladder is the part to be irrigated, the eatheter is introduced all the way, and after the contents are evacuated, the irrigating fluid is allowed to flow in from an irrigator jar (Fig. 22), or is injected by means of the 100 c.c. or 150 c.c. syringe, until the bladder is comfortably filled, when it is emptied and refilled as often as may be necessary to cleanse it; or, in some forms of treatment, the catheter is withdrawn and the patient is told to retain the fluid for a few minutes, or an hour or more. When it is intended to irrigate the posterior urethra, after injecting a small amount of fluid into the bladder, the

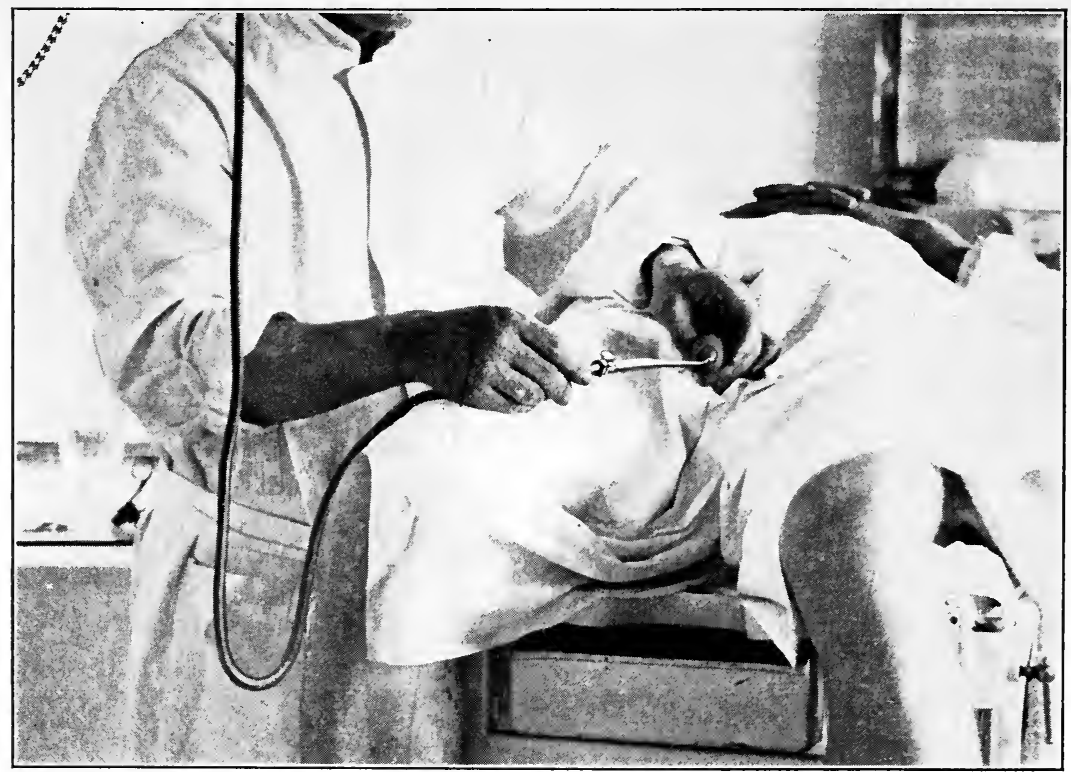

FIG. 22.-Vesical irrigation with catheter.

catheter is withdrawn about $2.5 \mathrm{~cm}$. (1 in.), or until the fluid does not return through the catheter, showing that the eye is in the posterior urethra and not in the bladder; then the desired amount of fluid is introduced, and after the catheter is withdrawn, the patient is told to pass the fluid out voluntarily, or it is retained for a variable period, according to the object of the treatment and the nature of the fluid.

\section{Exploratory and Dilating Instruments}

Sounds are inflexible solid instruments, used for exploration and dilatation of the urethra. Those made of nickled copper are superior 
to those of steel, the former being less likely to rust. The solid metal sounds (Figs. 23 and 24) are blunt or conical at the extremity. The former are used for diagnostic purposes, the latter for dilatation. The reasons for this are obvious; the shoulder of a blunt instrument can more readily detect the presence of urethral obstruction, while the tapered sound can be more easily engaged in a narrowing of the canal

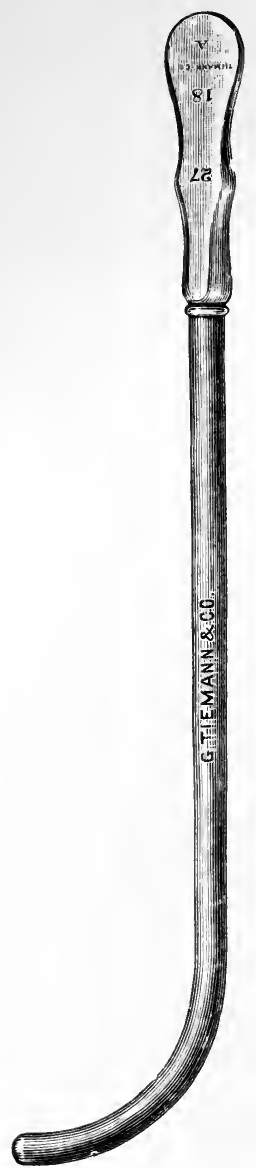

Fig. 23.-Blunt steel sound.

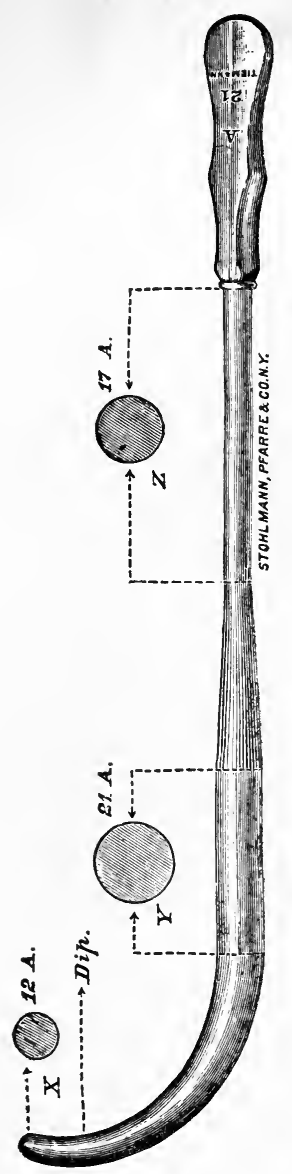

Fig. 24.-Double taper conical steel sound.

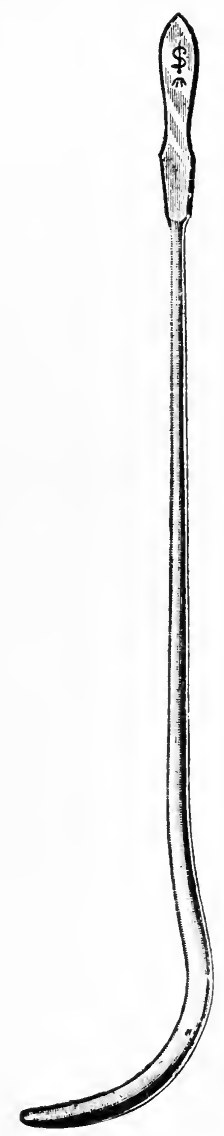

FIG. 25.-Conical steel sound Béniqué curve.

and is therefore better for dilating purposes. In dilating the urethra, it is preferable to employ a sound which is tapered in both directions, toward the handle as well as toward the extremity, the object of which is to avoid the continued distention of the meatus, especially when this part is constricted, while the deeper portion of the canal is being 
subjected to the required amount of distention. Careful calculation has been used in the development of the curve and taper of the metal sound. The larger sizes, therefore (above 24 or $25 \mathrm{~F}$.), should taper in size toward the handle. The blunt sound has its full size at the tip, which is maintained for $7.5 \mathrm{~cm}$. (about 3 inches), when it tapers down

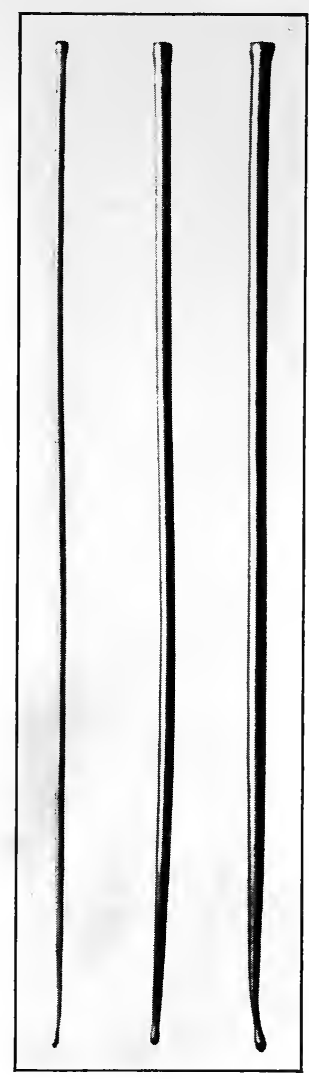

FIg. 26.

Olivary bougie.

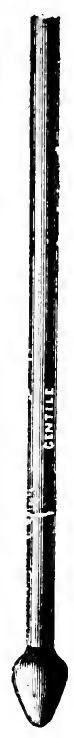

FIG. 27.

Bulbous bougie.

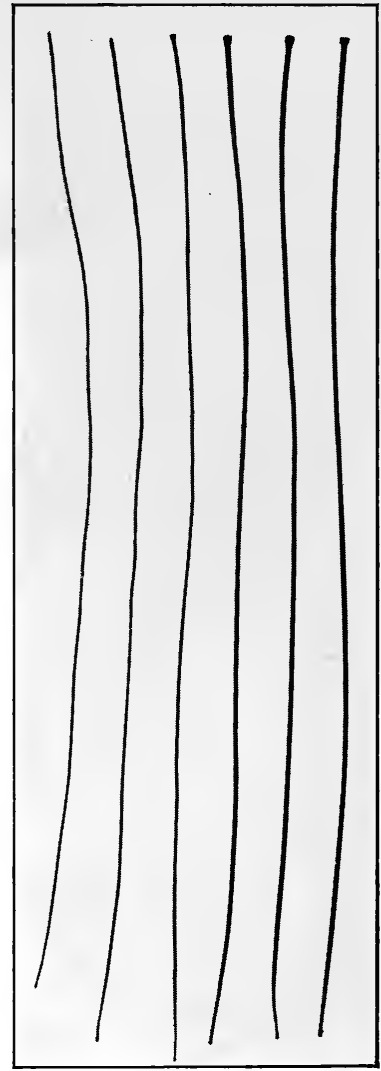

FIG. 28.

Filiform bougie.

several sizes toward the handle. The conical sound should taper in both directions, toward the handle and tip (Fig. 24). At the beginning of the straight portion the full size is reached, and continued for $4.5 \mathrm{~cm}$. (about $13 / 4$ inches) (Fig. 24-Y). Thence it tapers toward the handle about six sizes French. From the straight portion to the tip it tapers $7 \mathrm{~cm}$. (2 $3 / 4$ inches) about fourteen sizes French scale. The curve from straight portion to tip should be short, with an extra short- 
ness of the curve near the beak (Fig. 24-X), since this extra curve greatly facilitates introduction, especially at the hole in the triangular ligament, by keeping the point of the instrument against the roof of the canal. In sizes smaller than 20 , the sound, unless in the hands of an expert, does not compare in safety with an olivary bougie.

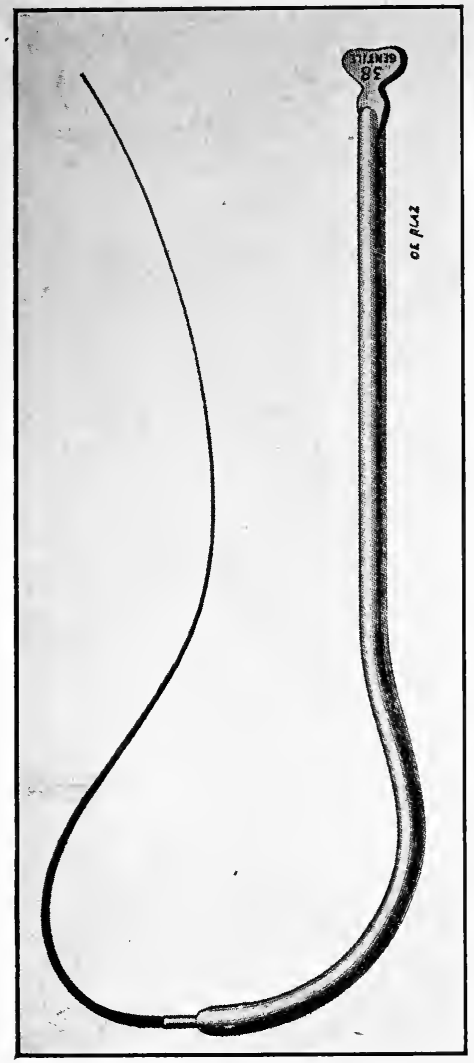

Fig. 29.-Guyon sound with conductor.

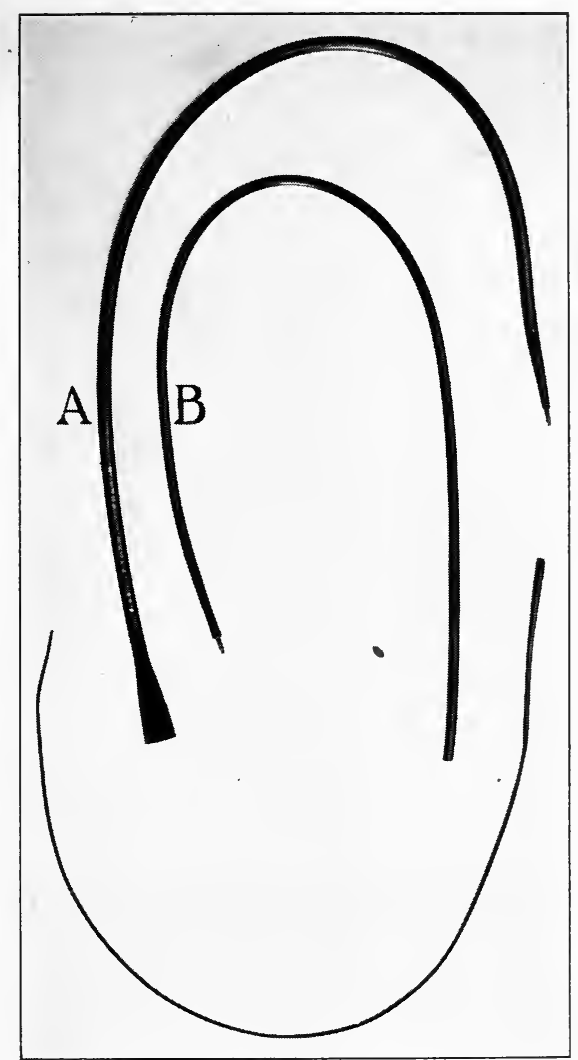

Fig. 30.-A, Filiform catheter. B, Filiform bougie. (Philips modification of Banks.)

The single-curved sound is largely used, although the double-curved or so-called Béniqué type of instrument is preferred by some urologists. Both styles of instruments have their uses. I find that it is better to adopt the form which seems suited to the individual case according to the facility of introduction. The Bénique sound (Fig. 25) is iniended on account of the pitch given to it by the double curve to find readily the entrance to the posterior urethra and surmount any obstacle in the prostatic portion or at the bladder orifice. 
Woven conical bougies are made with flexible neck, and may be tapered or olivary tipped. They are used both for diagnostic purposes and for dilatation. These instruments serve the purpose of dilating strictures up to the limit where steel sounds may be used. There

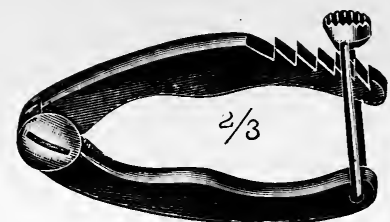

Fig. 31.-Author's urethral clamp. should be a complete set, from 10 to 25 French size, in every urological equipment (Fig. 26).

Bulbous bougies, Bougie à Boule, as they are called, appear in both types, flexible and metallic (Fig. 27). The French flexible bougies are preferable and quite durable enough. This bougie has two parts, a cylindrical rod of uniform size and a knob that tips one end of the staff. These instruments are called "stricture searchers" because they detect the presence of strictured areas on entering the canal, and upon withdrawal will carry upon the shoulder a drop of blood or pus as evidence of the inflamed area behind the constriction. It may be demonstrated, however, that even in a perfectly healthy urethra, resistance is felt on the passage of the knob from the bulbous into the narrower membranous part. Such a perfectly normal condition is unfortunately sometimes treated as stricture by those who are too prone to regard every point of narrowing as unwarranted.

Filiform bougies (Fig. 28) are made of woven silk or whalebone, with tiny bulbous tips and straight or cork-screw ends. They are the smallest instruments (sizes 1 to 4 French) employed for urethral investigation. The greatest care must be exercised in regard to the condition of these instruments, for whether of whale

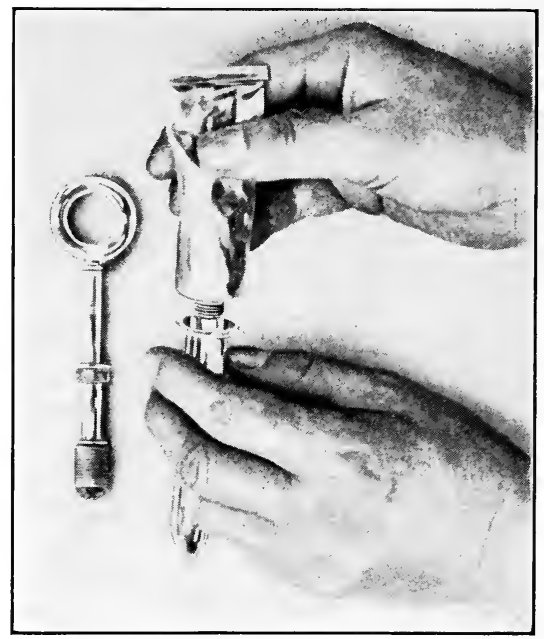

FIG. 32.-Filling syringe for anterior urethral anesthesia or lubrication. bone or woven silk, they are fragile, and the possibility of the bougie breaking off in the urethra must always be borne in mind. An imperfect filiform should never be used. Bougie tortillée is a type of filiform bougie, which, although originally straight, has been bent at one end. In order to make this curve practical for use, the tip is 
soaked in collodion and then dried. This bent extremity will sometimes find the aperture of a tortuous stricture better than the straight variety. The introduction of the filiform bougie is often the beginning of a satisfactory and complete dilatation of the urethra, even though at first it cannot be passed through the strictural area at all, or only after great patience. The passage of one filiform may be followed up by the introduction of one or several others alongside of the first, or it may be used as a guide for a tunneled sound (Fig. 123 , p. 288), an instrument that has been used largely for this purpose, but which cannot always be handled with that facility and gentleness. which are so important in urethral investigation. Indeed, it is far better to desist after the passage of a single filiform and to resume a day or so later, than to subject the canal to rough handling. And, often, by such conservative course, it will be found at the subsequent sitting, that the reintroduction of the filiform is much easier, and increase in the size and number of the instruments can then be accomplished by gradual progression.

Another type of filiform bougie is known as bougie

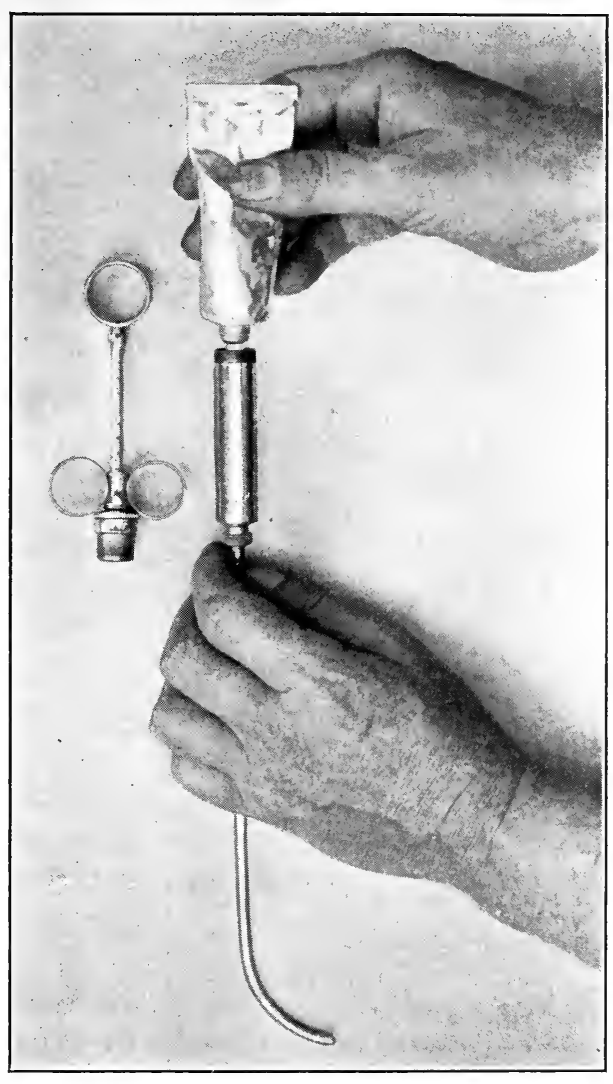

FIG. 33.-Filling solid piston syringe for posterior urethral anesthesia. armée. This instrument is provided at the extremity with a metallic tip, threaded so that it may be attached to a solid instrument of larger diameter to serve as an entering wedge for the dilator, or attached to the shaft of the urethrotome as a guide. The Guyon sound is an instrument with the Béniqué curve, with this woven filiform attachment, and may be had in various sizes. It is very useful in starting the dilatation of tight strictures (Fig. 29). 
In conducting the examination or practising dilatation of these small and difficult constrictions of the urethra, it is desirable to
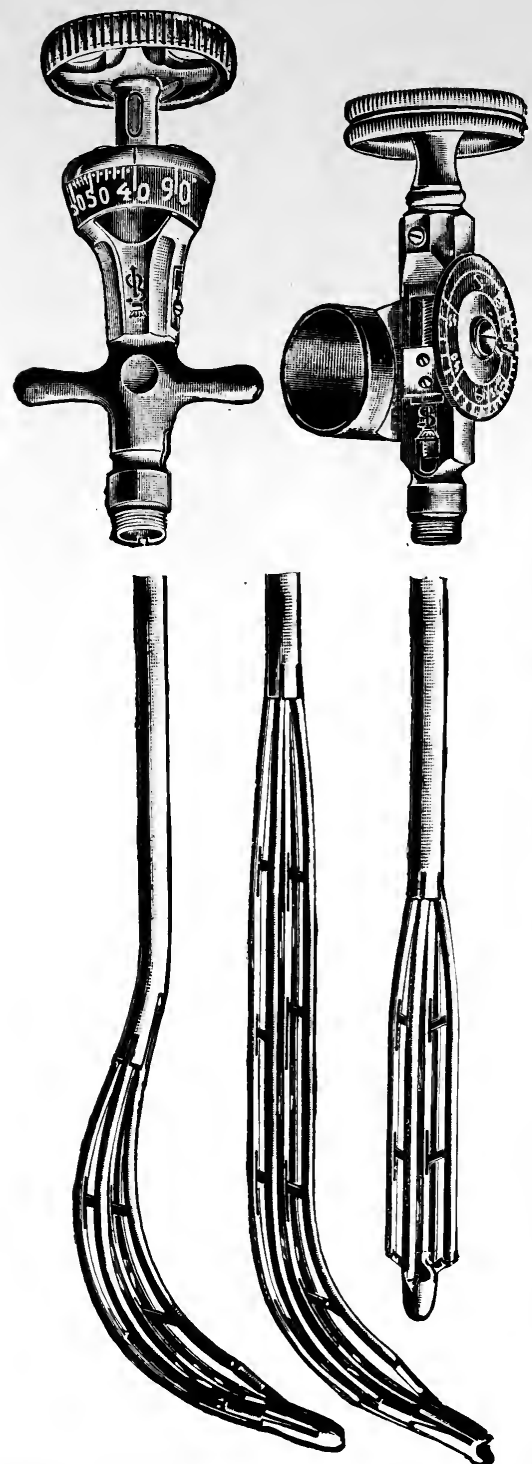

Fig. 34.-Kollmann's dilators: straight, curved, and Béniqué patterns. anesthetize the canal before beginning, and by so doing it will be found that not only more can be accomplished without pain and resistance on the part of the patient, but, of greatest importance, with less likelihood of damage to the parts.

The Banks bougie, a whalebone filiform, or better the Philips modification, a bougie with long distance filiform (Fig. 30) which rapidly enlarges to a size about 12 French, should be in the urological outfit, and will prove of great value for immediate dilatation above the filiform size. It is always a source of great satisfaction to master these tight and irregular obstructions, for which it is not necessarily expedient or best to resort to cutting or urethrotomy. And as each case has its own peculiarities, it is important to make careful observation of the condition at the point of constriction and of the particular manner in which the successful introduction of the instrument is accomplished. Record should be kept systematically of such details as are of moment in this respect, as well as of the size and number of instruments employed.

Anesthetization of the Urethra. -For anesthetizing the anterior urethra, a solution of cocaine or one of the substitutes may be employed, which can be retained by compressing the meatus for two or three minutes, or by means of the urethral clamp (Fig. 31). These solutions are 2 per cent. or 3 per 
cent. beta eucaine, 4 per cent. novocaine, or 5 per cent. alypin. I prefer the last one, and as a vehicle, instead of an aqueous medium, have used for some time the same lubricant jelly that is employed for lubricating instruments (see p. 31). In other words, 5 per cent. alypin is added to the preparation, and then it is put into collapsible tubes. This anesthetic jelly is expressed into a urethral syringe of the plunger variety (Fig. 32) and is then forced into the canal, and retained by means of the clamp. It will be found that this method renders great assistance in the urethral examination. To anesthetize the deep urethra, it is often only necessary to press or massage the anesthetic lubricant in the anterior canal backward, although this is not always possible. Otherwise, the preparation is put in the barrel of a deep urethral syringe, with solid piston, and deposited there (Fig. 33). This is the method employed for obtaining local anesthesia prior to cystoscopic examination.

Kollmann dilators (Fig. 34) serve the purpose of dilating the urethra, especially when it is desirable to carry this to an extreme degree. The necessity of enlarging the meatus is sometimes avoided by using these dilators, as the urethra is distended by expanding the blades of the instrument after introduction, by means of a wheel on the handle. They are about 20 French, so that introduction is possible through a right meatus, and they can be expanded to 45 French. One straight and one curved instrument fulfill most requirements. Among the various designs displayed are some which have attachments for irrigation. With all their advnatages, however, dilators can never entirely supplant sounds, as some individuals find the use of the latter less painful.

\section{Instruments for Evacuation}

Catheters are instruments that have both a diagnostic and therapeutic use. It is hardly necessary to state that it is never permissible to use any but a perfectly smooth instrument, for the urethra, when abraded by a lack of gentleness, or by the rough and jagged surface of an instrument, is much more prone to become inflamed. The importance of using the minimum amount of force, and of exercising gentleness, combined with skill, in urethral instrumentation, cannot be too frequently emphasized. The catheters of the best manufacture are readily sterilized by boiling and are durable. The eye, like the surface, should be smooth, and the other extremity should have a funnel end.

For the female, a glass catheter is of service, but this instrument cannot be used for irrigation. Only a slight curvature is necessary 
in catheters for the female urethra, and the same types as used for the male can be employed. More damage is done with the metal catheter

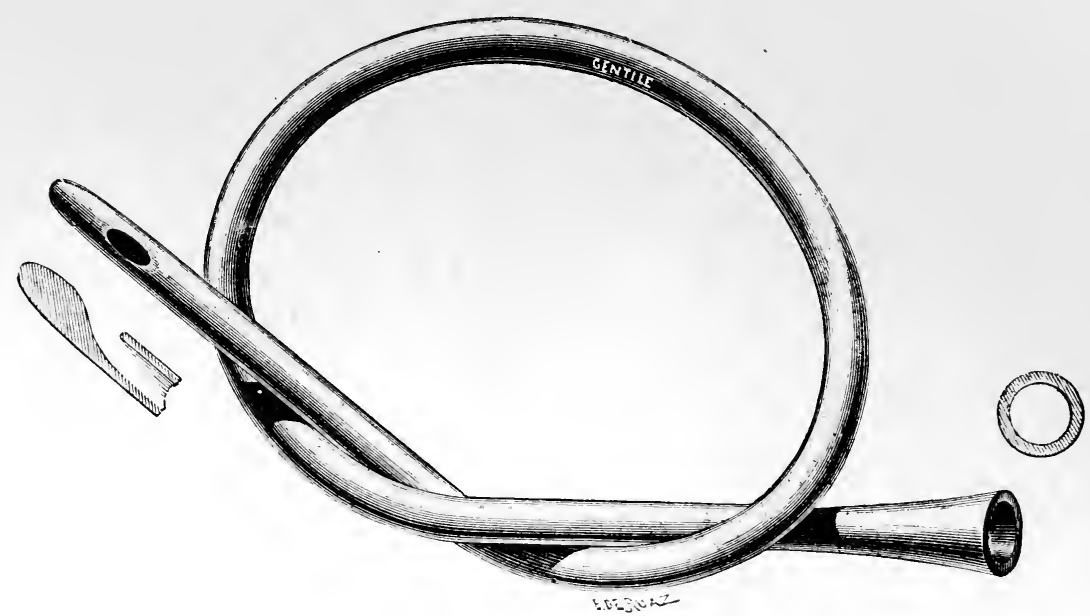

Fig. 35.-Nélaton soft-rubber catheter.

than with any other variety, on account of the ease with which it can produce false passages, especially in the hands of the inexperienced, when undue leverage is exerted in order to overcome resistance. The silver catheter really serves little purpose that cannot be fulfilled by the superior types of instruments about to be described, although practically every physician's outfit contains a silver catheter. (Fig. 39.)

Soft-rubber catheters (Figs. 35

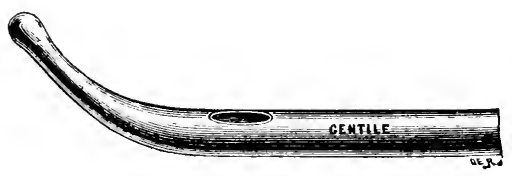
and 36) are the commonest type of catheters, and the safest. They should be provided with a funnel end and a velvet eye. The eye should be close to the extremity, and the terminal end should be solid and blunt, not usually pointed.

FIG. 36.--Soft-rubber catheters: blunt, pointed, and elbowed.

The calibre of the tube, as well as of the eye, should be as large as the size of the instrument will allow. Those between the sizes of 10 and 20 French are in common usage, the smaller sizes for applications and irrigations in sensitive patients, the larger sizes when the catheter is used more or less habitually. 
The soft-rubber elbow catheter (Fig. 36) is a modification of the more common form which will be found a useful addition to have on hand, in small and medium sizes (12 to 18 French), both tapered and blunt ends; and it is not infrequent to find that this type will enter more readily and comfortably than any other. The soft-rubber "bout coupé" catheter, which is open at the extremity instead of

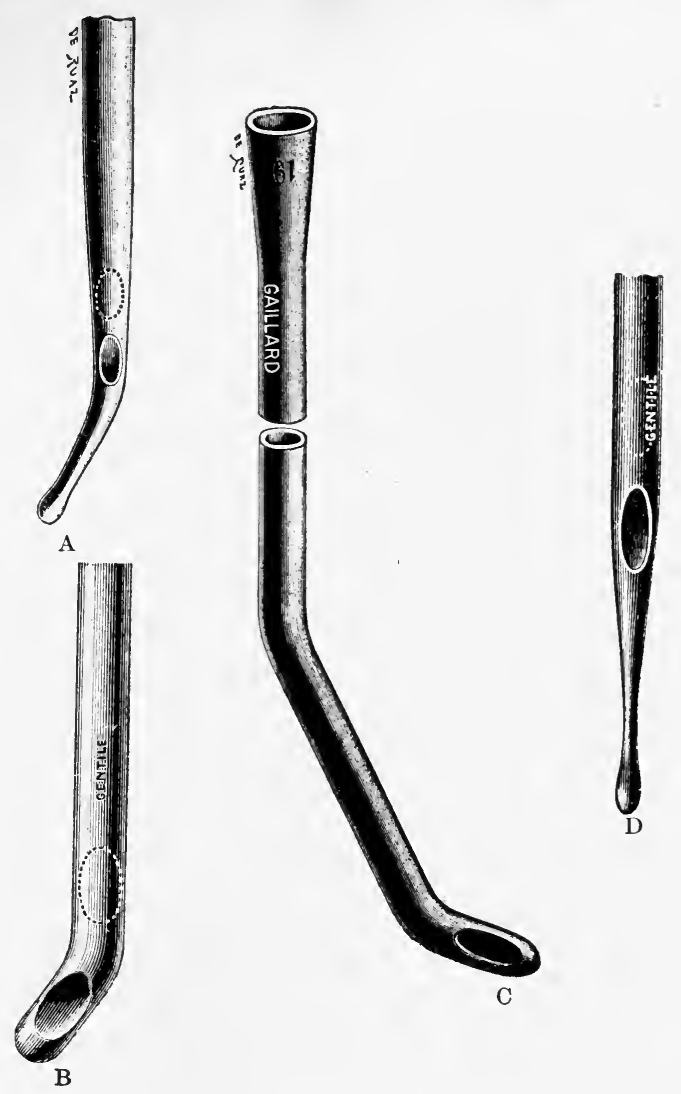

FIG. 37.-Silk woven catheters: elbowed, olivary (A), and blunt (B); double elbowed (C), and straight olivary (D).

the side, is designed to be retained or tied in the bladder, when such is required, but I find the ordinary and elbowed forms fully as useful for this purpose.

Woven olivary catheters (Fig. 37) are of use when the Iumen of the urethra is contracted as in the case of spasm or stricture, too resistant to be passed by the more flexible soft-rubber catheters. 
Woven single elbow (coudé) catheters (Fig. 37) when passed with the tip directed upward glide over obstacles on the floor of the urethra, especially in the prostate and neck of the bladder, and like the double elbowed are used more frequently in prostatic obstructions.

Woven double elbow (bi-coudé) catheters (Fig. 37) glide over some obstacles impassable to the single-elbow instrument, especially prostatic enlargements with extensive middle lobe elevation. (See p. 399.)

Woven olive-tipped elbow catheters combine the special advantages possessed by the olivary and elbowed types, respectively, and conse-

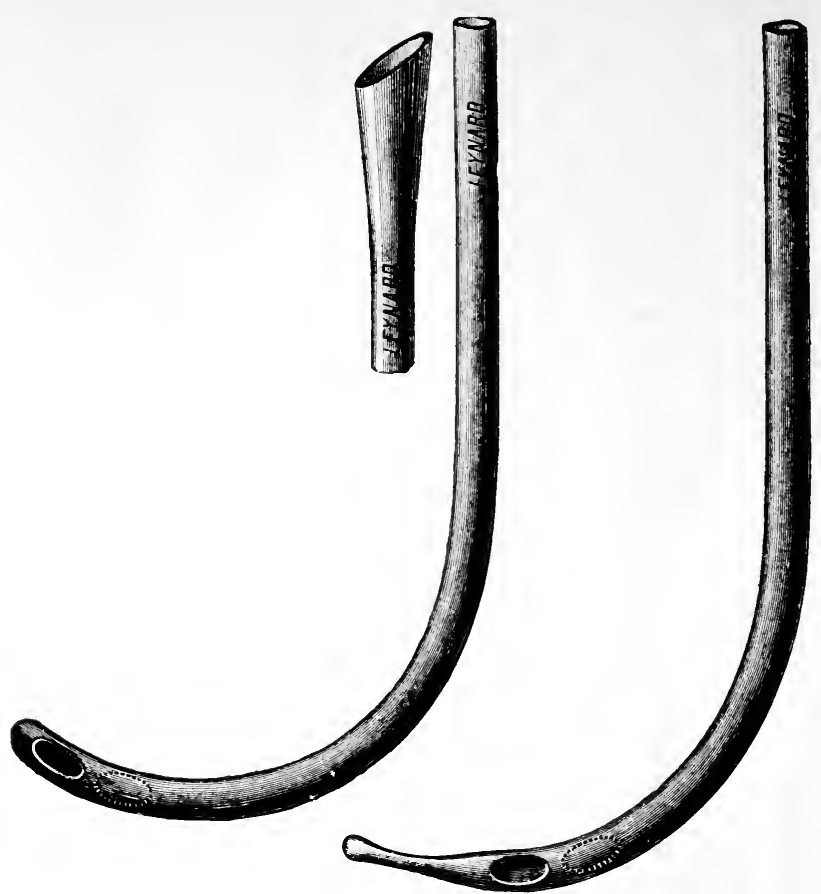

FIG. 38.- Silk woven prostatic catheters.

quently in some cases reach the bladder more readily, unless, as in spasmodic conditions, the blunt-pointed instrument finds less resistance. There is also made a woven catheter with the long prostatic curve and with the double curve or Béniqué shape (Fig. 38) which is found useful in some cases and is much safer than the silver or steel catheter of the same pattern. (Fig. 39.)

In selecting a catheter to relieve retention of urine, whether from stricture or prostatic obstruction, the soft rubber is preferable and should be tried first, either the ordinary form or the elbowed (Mercier) 
end, as they are less likely to irritate the sensitive canal that has not before been invaded. But if these instruments fail, recourse must be had to one of the woven forms, the olivary usually for stricture, and the Mercier or elbowed for prostatic retention. The calibre of these woven instruments is relatively larger than the soft rubber, hence, it is sometimes possible when circumstances demand, such as the exist-

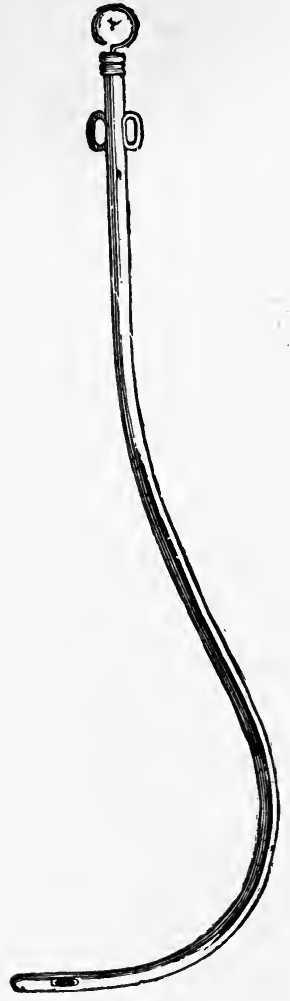

Fig. 39.-Metal catheter, Béniqué curve.

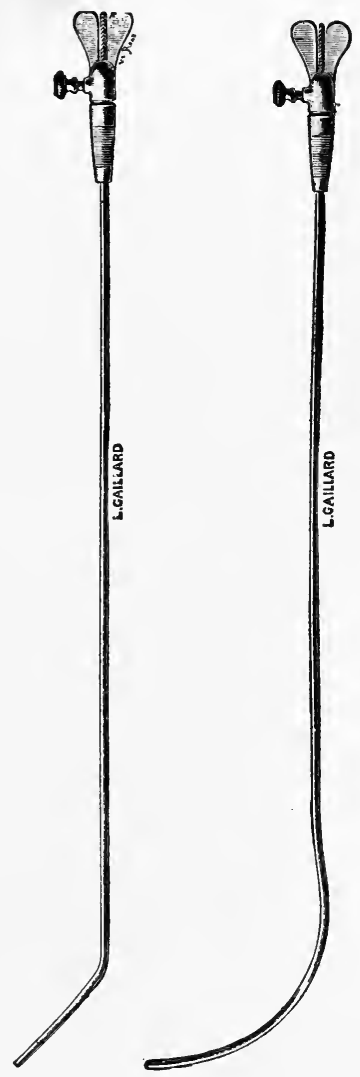

FIG. 40.-Catheter directors. A, Elbowed shape; B, Béniqué shape.

ence of obstructions impassable to larger instruments, to obtain the urine with an exceedingly small catheter of this variety.

Added to the catheter outfit should be the elbowed (Mercier) and Béniqué curved obturators of Guyon (Fig. 40) which, when introduced into the catheters, give to them their characteristic shape and make the soft rubber ones stiff and the flexible ones more rigid. They should be used with much care and gentleness and under such precautions may 
prove of great value in difficult situations when other means have been tried and failed. Atter the bladder is reached the obturator is withdrawn, leaving the catheter in place long enough to draw the urine and cleanse the bladder, or, in some cases, the catheter may be retained to drain the bladder for a longer period.

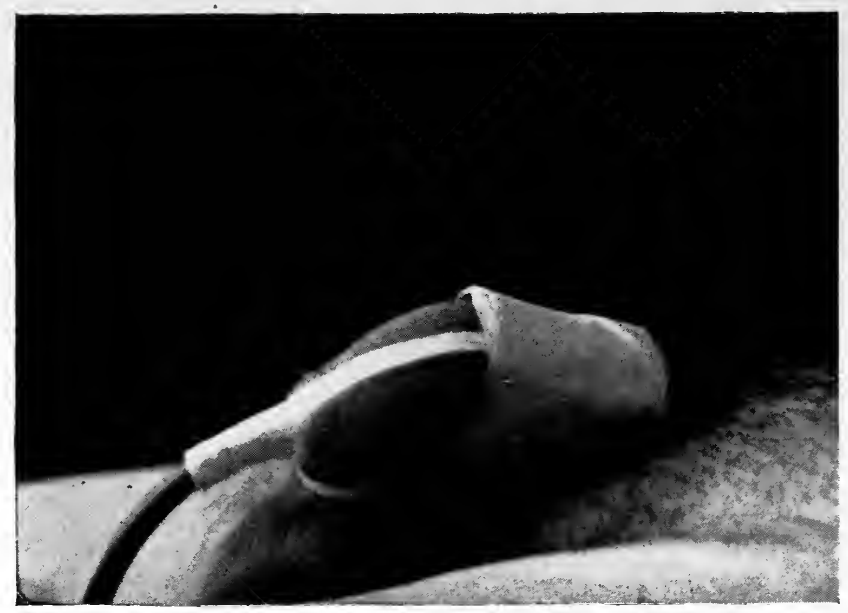

FIG. 41.-Self-retaining catheter. (Sinclair.)

Essential qualities of catheters may be summed up as follows: The eye of the soft-rubber catheter and the single or double elbow catheter of Mercier should be placed at the extreme end of the instrument, leaving no blind pouch beyond. The smooth eye to which reference

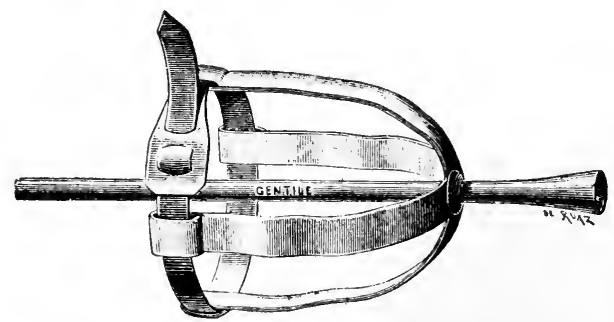

FIG. 42.-Gum rubber catheter retainer.

has already been made should be as large as possible in comparison with the calibre of the tube.

The eye of the olivary catheter (sometimes there are two eyes) is placed so as not to interfere with the tapered portion and neither the 
bougie end nor the neck should be too large, and the latter should be flexible.

The surface of all woven instruments must be smoothly polished. The best are the cheapest and all that are damaged in any particular should be discarded. Cracked and frayed woven instruments are a source of danger in the hands of either the surgeon or patient, not only because of possible trauma to the urethra, but because they are less likely to be properly sterilized.

The Indwelling or Retained Catheter.-In certain conditions of chronic cystitis, and in some cases that have to be catheterized at frequent intervals (see index) it is deemed advisable to leave the catheter in place to drain the bladder continuously and thus provide for frequent cleansing as well. This end is cleverly accomplished by using adhesive strips (Fig. 41). There is also manufactured for the same purpose a convenient rubber device which may be rapidly applied and removed and which is easily cleaned and sterilized (Fig. 42).

Urethroscopes, cystoscopes, as well as operative instruments, will be described later in their appropriate chapters.

\section{Antisepsis in the Preparation for Examination}

The strictest antiseptic precautions are demanded in conducting the instrumental examination which usually involves the introduction of urethral instruments. The preparation for examination consists of :

I. Antisepsis of the surgeon's hands.

2. Antisepsis of the patient.

3. Antisepsis of the instruments.

Antisepsis of the Surgeon's Hands.-There are the usual precautions to be observed in the surgeon's preparation. His hands are properly washed and immersed in a solution of corrosive sublimate (1-2000). The use of rubber gloves is an advisable method, as in the handling of many patients they are more readily cleansed than the bare hands and can be changed as often as may be desired. In spite of such provision, it is always proper to avoid touching that portion of the instrument that will reach the posterior urethra or interior of the bladder. This practice should become a habit with the surgeon.

Antisepsis of the Patient.-The importance of proper cleansing of the urethra prior to examination applies more particularly to cystoscopy and other intravesical exploration, on account of the 
possibility of carrying infection backward into the otherwise sterile regions of the deep urethra and bladder from the external meatus and microbe-laden anterior canal. The failure to observe such antiseptic precautions may result in the unnecessary occurrence of sharp urethral chill and urinary fever. While this is the case, an equally potent factor in producing such an after-occurrence is the rough handling of instruments and the laceration of the tissues, if the former have not a smooth surface. This applies to the anterior section of the urethra, as well as the posterior. Neglect to observe such precautions may result in the production, from undue violence, of periurethral inflammation or prostatitis leading to a follicular or prostatic abscess. The glands and prepuce should always be cleansed in any case. Flushing of the , anterior canal with 2 per cent. boric or a mild permanganate (1-8000) solution is a good preliminary measure. For the same purpose the oxycyanide of mercury (1-6000) is also recommended. This suffices for ordinary urethral exploration. In the case of cystoscopy or more protracted examination, the external genitals should be washed with liquid green soap and water and disinfected with corrosive sublimate solution (1-5000), following which the surrounding regions are covered with a sterile sheet, perforated for the exposure of the penis.

It is important to avoid, in the preliminary irrigation of the urethra, the use of too strong or astringent disinfectant solutions, as in such case the passage of instruments is rendered more difficult. In acute inflammatory conditions it is well to avoid instrumental interference, except when unavoidable, as in the case of retention of urine, for the more inflamed the urethra happens to be, the more susceptible it becomes to increase in inflammation from additional irritation. In chronic inflammation, the regular introduction of instruments is better tolerated and the objection outweighed by the benefit of local treatment properly directed. The occurrence of irritation, however, is always an indication that the treatment is misdirected or the agent too severe. Conservative measures will sometimes accomplish the desired relief, where active local measures only serve to prolong the trouble. This may mean to desist entirely from any local interference, or to postpone such action until, under the internal use of disinfectants and sedatives, a decline of the irritation supervenes.

Antisepsis of Instruments.-This entails complete sterilization of the instruments before and while they are in usage, and aseptic lubrication. The best urological technic demands that all instruments be thoroughly cleaned mechanically, as well as sterilized, as when the former has been accomplished, the latter is more readily effected. Therefore, after use, all instruments, metal or otherwise, 
should be cleansed immediately, preferably with warm water and soap, and in the case of instruments that cannot be boiled, should be wiped off with an antiseptic solution-metallic instruments with alcohol or 2 per cent. phenol (carbolic, lysol or creolin) and non-metallic instruments with phenol, 1 per cent. or bichloride, 1-1000. When, however, the composition of instruments is such that they will not be impaired

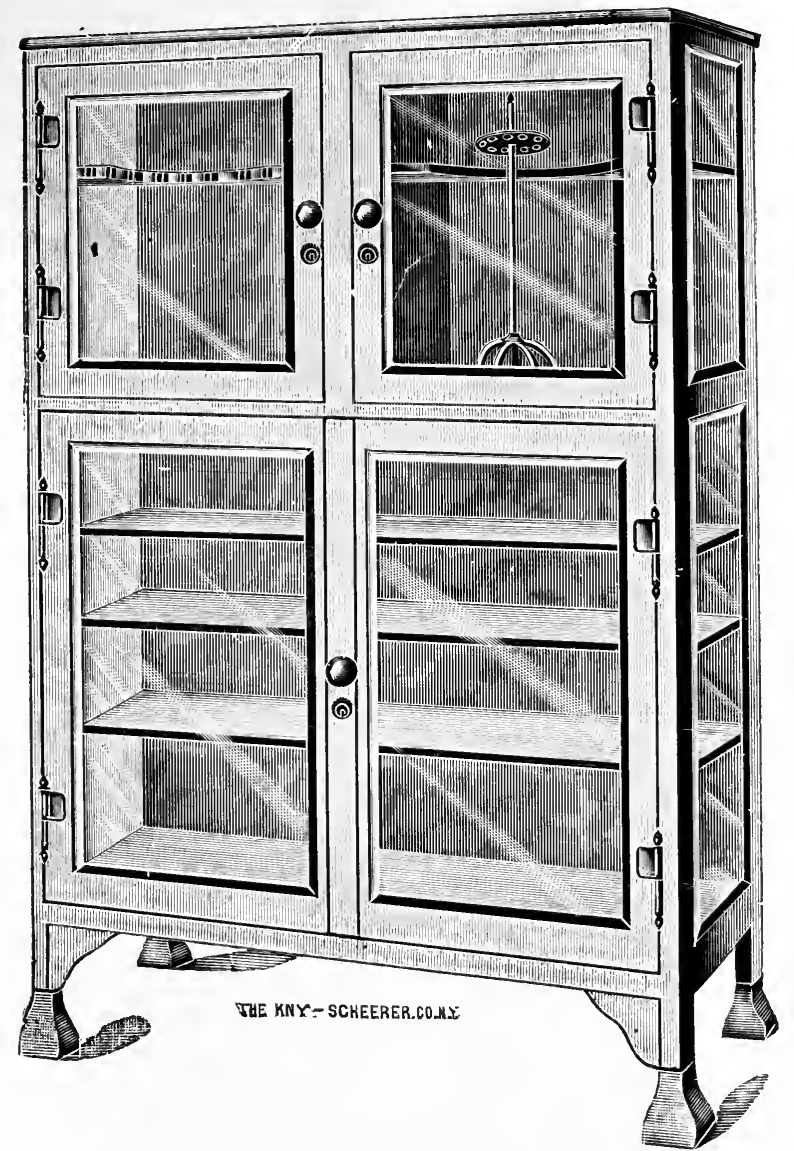

FIG. 43.--Instrument cabinet for formaldehyd fumigation.

by boiling, such instruments should be boiled again after use, and thoroughly dried in a sterile towel before being put away.

When instruments are not in use, it is desirable to store them in an antiseptic cabinet (Fig. 43) in which a powerful formaldehyd lamp is burned for at least one-half hour each day. Thus it is aimed to keep the urological armamentarium in an antiseptic condition and ready 
for use. Such instruments ${ }^{\circ}$ as are removed from the cabinet or case should be cleansed again as above, and when returned should be kept in a separate tray until the next.fumigation, unless they have been previously boiled.

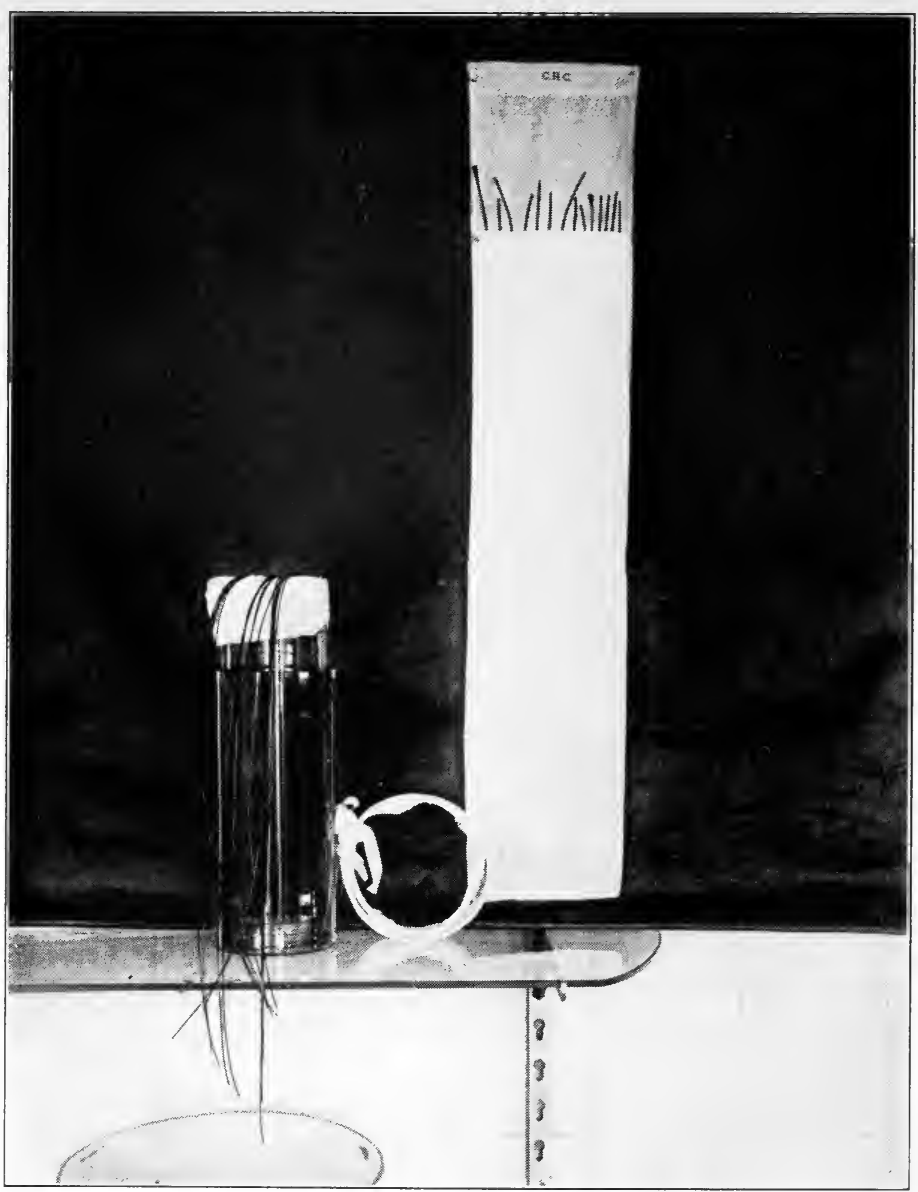

FIG. 44.-Method of cleansing by siphonage, and special folder for storing ureteral catheters.

Practically the same methods are observed as in general surgery. All instruments should be kept in a good state of repair, and it is especially important to be particular that the surfaces of urethral instruments contain no irregularities or roughness, and are free from cracks and joints. 
The sterilization of metallic instruments is most satisfactorily and thoroughly accomplished by continuous boiling for from five to fifteen minutes. There is no better method of treating metallic instruments, the only objection being the tendency to rust, and that of the cutting instruments to become dull. To obviate the tendency to rust, it is customary to boil in a strong solution of sodium chloride, and to keep in the instrument cabinet a tray containing calcium chloride to absorb moisture that may settle upon them.

Cutting Instruments.- These are treated as above after use; that is, are thoroughly cleaned mechanically with soap and water, wiped with a non-corrosive antiseptic solution, and stored in a fumigating cabinet, and then, immediately before use, they are immersed in alcohol, or a strong phenol solution, in which case it is necessary to remove the latter with sterile water.

Catheters of all kinds are treated with boiling water. This is invariably the case with the soft-rubber or metallic instruments, and is also true of the best makes of the woven silk. The soft-rubber catheters are boiled in plain water for about five minutes, removed with a pair of antiseptic forceps and placed in a formaldehyd cabinet. The metallic catheters are treated in the same manner as other metallic instruments. If they are solid silver or copper nickelled, they have not the same tendency to rust as have the nickelled steel instruments; all should be thoroughly dried with a sterile towel, or by being passed through an alcohol or Bunsen flame.

Flexible Woven Instruments.-Catheters or bougies, when of first class manufacture, may be immersed in boiling water, following careful mechanical cleansing, after which they are cooled with sterile water and wiped with an antiseptic solution of phenol or bichloride, and finally placed in the formaldehyd cabinet or vaporizer (Fig. 44), precaution being taken to keep them separated from each other • while they are still moist, and in consequence, soft. When the method of steam sterilization is employed for these instruments, it is necessary to expose them to the steam for at least one hour to make it effective; it is therefore essential to keep them apart during this process, preferably in separate wrappings.

Ureteral Catheters (See Cystoscopy).-These slender and delicate instruments are difficult to sterilize satisfactorily without damage, and yet there are no instruments requiring this precaution to a greater degree, as they are introduced into the pelvis of the kidneys and at times are employed to determine the functionating capacity of a healthy organ. Then, again, they are frequently used in the case of tuberculous and other infectious lesions, demanding great 
antiseptic cleanliness following their employment. It is a good habit with regard to these catheters, besides exercising the usual rules of cleanliness and antisepsis, to separate a certain number to be employed in purulent and infectious cases; and in the examination of an uninfected kidney, to employ an entirely new catheter, or one that has never been used in a suppurative case.

The care of the exterior of the ureteral catheters may be conducted first, by the usual mechanical cleansing with gauze wipes saturated with soap and water, then submerging them for one minute in a 2 per cent. formalin solution.

It is, however, the interior of these instruments, which is difficult to cleanse, but yet, of great importance. It is advisable, therefore, always to flush out the interior of these catheters with an antiseptic solution, such as a 10 per cent. formalin, and then to place the catheters over the edge of a sterile glass jar, containing a 2 per cent. boric-acid solution, placing the distal ends well into the jar, and the proximal ends hanging outside at a level much below the bottom of the jar. Each catheter should then be siphoned by starting the solution through them with a hypodermic syringe and needle, and allowing mechanical dripping to take place for at least a half-hour. Boric solution has tendency to crystallize, and is apt to block the interior of the eatheter, so it is necessary to follow boric siphonage with sterile water for about fifteen minutes (Fig. 44). After drying the catheters with a sterile gauze wipe, and placing them in a special folder, they should be stored in a formaldehyd cabinet, until used.

Sounds and bougies, like other metallic instruments, are best sterilized by boiling. If of copper nickel plate, they do not rust readily; if of steel nickel plate, they should be boiled in a strong salt solution and immediately dried in a sterile towel or passed through an alcohol or Bunsen flame.

Dilators and urethrotomes and other such complex instruments, which are constructed with joints and other working parts, should be cared for by the same method as is employed for the care of the cystoscope, special precaution being necessary in the case of such instruments after cleansing, to dry thoroughly the joints and working parts by treating the same with alcohol or warming over a Bunsen burner or alcohol flame.

Cystoscopes and endoscopes being such delic ately constructed instruments, and having lenses and cement subject to damage by the usual methods of sterilization, require special treatment. After usage, they should be thoroughly cleansed with liquid soap and warm water and a soft camel's hair brush. A stream of water should be allowed to 
pass through the interior of the sheath. The exterior should be cleansed with 1 or 2 per cent. carbolic or lysol solution, some of which is injected through the sheath. The entire instrument is then dried out with antiseptic gauze or cotton and placed in the formaldehyd fumigator cabinet, where they are fumigated for an hour or more each day before and after usage.

Sterilization of Syringes, Irrigators, Basins, Etc.-A properly equipped operating room should contain a utensil sterilizer large enough to receive vessels of all sizes that are used for general examination or cystoscopy. Such a sterilizer is equipped with a tray, and a foot treadle which lifts both the lid and the tray at the same time. All the syringes employed in the genito-urinary technic should be constructed of material that can be boiled. This requires that the piston or plunger be constructed of solid glass, metal, rubber or asbestos. A 100 c.c. syringe for bladder irrigation is described on page 33 ; also instillators and urethral syringes. Hypodermic syringes are of solid metal or glass, with piston of like material, and may be boiled.

Needles should have some antiseptic solution injected through their lumen after usage, and then be boiled with a wire in the interior, after which they should be kept in the formaldehyd fumigator cabinet. As in spite of these precautions, the ordinary steel needles rust in a short period, an ample supply of them should be kept on hand in the fumigator, ready for use, and capable of fitting the various types of syringes used. Generally speaking, there are three types of needles applicable to the different syringes used: one with a universal thread, another with a slide or sleeve union, and a third with an unusually large thread made necessary by the use of glass syringes, the universal thread being too small for the construction of glass.

Solutions are always made up of sterile water supplied by a tank of boiled water which is previously filtered.

All dressings, sheets and gowns are sterilized in the usual manner in a sterilizer of live steam which requires a period of two hours, or in a pressure sterilizer which requires a much shorter period. 


\section{CHAPTER III}

\section{DIAGNOSIS (Continued)}

\section{PHYSICAL EXAMINATION OF THE PATIENT}

Oral Examination.-Careful note should be taken of the patient's history, both family and personal. In investigating the family history, of the usual attention is directed to the inheritance of lithiasis, tuberculosis, rheumatic and gouty affections, on account of the possible rôle played by heredity in such predispositions.

As to the patient's personal or previous history, syphilis, gonorrh ea, tuberculosis and scarlet fever have all more or less significance as causative factors of the different genito-urinary maladies to be hereinafter described. The age of the patient has a bearing upon both the diagnosis and prognosis, e.g., prostatic hypertrophy, malignant disease, etc. The present weight of the patient and any marked change that may have occurred should be observed, as well as the condition of the circulatory, pulmonary and digestive systems. If the kidneys are affected, the state of the circulation must be noted. The existence of pulmonary tuberculosis, besides suggesting the causative agency, if the case is operative, is a serious complication. The condition of the alimentary tract may have an influence upon the selection of medicine and the dose thereof. Digestive derangement, as a part of defective metabolism, may furnish explanation of an aggravation of symptoms and a hint as to the proper direction of treatment. In other words, the urologist, to be successful to the highest degree, cannot be too narrow in his field of observation, but must add to a skilful and thorough examination a broad-minded interpretation of all important factors that may have any direct or indirect relation to the case; and to be able to afford the relief that the best skill can give, it is necessary to have both wide knowledge and correct judgment. Many genito-urinary conditions are curable, not infrequently by simple means, if the treatment be appropriately applied; treatment, on the other hand, may cause irremediable injury.

Great advancement has been made in both diagnosis and treatment in urological technic, and to be a master of the subject, one must know and be able to make use of such new developments.

A careful history of the present disease, its beginning, course and duration, should be obtained. 
Attention is particularly directed to the presence of certain symptoms significant of disease of the urogenital tract. With a knowledge of such symptoms the physician is able to elicit the essential facts by a line of pertinent questioning, which information it is difficult to obtain otherwise, or by allowing the patient too much latitude in the recital of his case. In other words, by proper interrogation, a tentative diagnosis may be made that will be confirmed often by complete examination. To this end it is necessary to keep in mind the importance of certain characteristic subjective symptoms, as follows:

(a) Pain.-The symptom of pain is always significant and should be investigated.

(b) Urgency and frequency of urination.

(c) Painful and difficult urination-denoting irritability or inflammation of the lower urinary tract; or objective symptoms :

1. Alteration of the urinary stream, suggesting some obstruction in the canal or at the bladder outlet.

2. The admixture of free pus or blood in the urine, which requires careful investigation to determine their source from the upper or lower urinary tract (see Cystoscopy).

Frequency of Urination.-In many instances, increased amount of urine is a notable symptom. Wide variations may exist, however, in regard to this tendency. Thus, it is apparent that the amount of urine voided is a distinctive feature, differentiating two classes of cases: those in which the quantity is increased with no change in the bladder capacity, as in chronic interstitial nephritis and diabetes; and those in which, although the frequency of micturition is increased, owing to inflammatory conditions, the quantity of urine remains normal, as in cystitis and urethro-cystitis. In such cases the frequency of urination is likely to be the same, night and day. In nervous patients it is much more evident by day, which is so in sexual neurasthenia, etc., involving neurosis of the bladder. Generally speaking, the frequency of urination is governed by the capacity of the bladder which is diminished by irritability and inflammation and is increased more often with unusual activity of the kidneys. Normal urination occurs about twice in the morning, twice during the afternoon and evening and again before retiring, not at all during the night, under ordinary conditions.

The average daily quantity of urine is about 1500 c.c. or 50 ounces. The mean capacity of the bladder varies in different individuals, being 300 to 500 c.c. (10 to 16 ounces) and some retain considerably more without much difficulty.

In questioning the patient relative to frequency and urgency, it 
should be learned to what extent frequency of urination is increased; when the urgency is most marked-by day or night-and what influences, such as rest and exercise, have any noteworthy effect. Thus, it will be found in the case of stone in the bladder or neoplasm that the frequency is greater under the disturbing influence of body movements, and hence, less during rest; while in prostatic conditions it may be more active during the night-time, owing to increased hyperemia.

Dysuria-Size, Force and Continuity of Urinary Stream.-These features are most significant, and sometimes pathognomonic, as is the case in stricture, when the stream is very small, or when it may cease altogether, the urine passing in drops. Alteration of the projectile force may be observed in lessened vesical tonus, in prostatic hypertrophy or any obstruction of long standing, and in disturbance of the vesical nerve centres. Sudden interruption of the flow may take place, when the neck of the bladder becomes occluded by a small stone, and more irregular changes in the flow and inconstancy of the stream occur, with prostatic enlargement and other obstruction at the neck of the bladder.

To sum up the crucial questions: (1) Has the urinary stream decreased in size, (2) lost projectile power, or (3) suddenly stopped? Further investigation as to coexistent features which combine to form the "symptom-complex" should follow.

\section{Pain in General}

Pain is of considerable diagnostic importance, because it is often the first sign which calls the patient's attention to his malady. Pain, swelling, and tenderness are usually united as cardinal symptoms of inflammation in one or the other regions of the urinary tract.

Nervous patients are apt to complain of pain of an intensity out of proportion to other symptoms and of a peculiar and migratory character; whereas, pain which is persistently stationary should always arouse suspicion. Special types of pain are peculiar to different regions and to certain maladies: for example, ardor or burning urination, chordee or painful erection associated with inflammations of the urethra, tenesmus or strangury pain peculiar to the bladder at the end of urination, caused by inflammation of the neck of the viscus; dragging pain in the groin due to inflammation of the cord or to impending swollen testicle. In the kidney and ureteral regions the symptom of pain has special significance and should be carefully investigated. Such investigation is made first, upon the patient's description and location of it and second, by an attempt to exaggerate or reproduce it by pressure upon the region in which it is located. The occurrence of pain 
in such instances is not an absolutely reliable guide as it may be felt some distance from the kidneys when they are affected, even to the extent of being on the opposite side.

Pain associated with the kidney and ureter usually occurs in zones. That of the kidney region is pyramidal in contour with its base posteriorly between the inferior margin of the ribs, the spinal column and the pelvis, and its apex anteriorly below the free margin of the ribs. The patient complaining of kidney pain usually locates the same in some part of this area and bi-manual palpation will elicit a corresponding region of tenderness. The area of ureteral pain extends from the kidney zone directly downward to the inguinal region and thence to the penis or testicle on the same side. When the investigation of a suspected kidney or ureteral lesion is carried on to the more precise method of cystoscopy, etc., the same characteristic pain which is complained of may be produced by ureteral catheterism and injection with a distending antiseptic fluid.

Painful urination should be investigated as to its location and duration, and time of occurrence with respect to the urinary flow, as in many cases it affords a substantial foundation for diagnosis. The location must first be ascertained as exactly as possible, whether in the region of the bladder or urethra; if in the urethra, what part. Again, the effects of urination, rest and exercise on the occurrence of such pain. Pain independent of urination may be located in all of the organs, including the kidney, from various causes. It is projected down the ureters into the testicle and to the end of the penis in renal colic. A dull ache in the perineum and rectal region suggests hypertrophy of the prostate, or prostatitis. Pain on urination only is the rule in cases of stricture. A diffused soreness and pain over the summit of the bladder are felt in general cystitis. When the neck of the bladder alone is the seat of trouble, urination is apt to be followed by spasmodic pain. Urethritis is accompanied by pain during urination, and pain during and after urination suggests urethrocystitis.

Hematuria.-Blood may occur in the urine in copious quantity, so as to give the entire urine the characteristic bloody appearance, or it may be only slightly mixed with it, either fluid or in clots, when it may come either before, during, or following the urinary flow. A copious admixture of blood may come either from the bladder or the kidney; a small amount, when it appears before urination, is usually from the urethra, and when following the urine, from the neck of the bladder. Although a detailed interrogation of the patient concerning the appearance of the urine is not necessary, on account of accurate laboratory methods, it is always wise to inquire 
concerning blood in the urine, since hemorrhage may occur infrequently, and the patient may supply valuable information that could be overlooked.

The most frequent conditions in which hematuria is noted are gonorrheal and other inflammations of the neck of the bladder (tuberculosis, calculi, tumors), and tuberculosis, tumors, calculi of the kidney. Again comes the question of exercise and rest. While rest is of the greatest benefit in hemorrhage due to stone, it may have little effect when the cause is renal tumor, and may increase the hemorrhage when the cause is renal calculus. Terminal urinary hemorrhage, that is, the appearance of hemorrhage at the end of micturition, is most suggestive of gonorrheal inflammation of the vesical neck. It occurs also in stone in the bladder. Such information gleaned from the foregoing facts provides provisional knowledge of the case, preliminary to the more exact methods of examination to be described in the sections on urethroscopy, cystoscopy and laboratory investigation.

Physical Examination.-Inspection takes the lead in the logical arrangement of methods of physical examination. By inspection, the different regions are examined for any visible enlargement. This applies not only to the external genitals but also to the internal organs, which may enlarge to a sufficient extent to be seen on the outside of the body.

Thus, enlargement may be seen along the course of the urethra on account of periurethral swelling and follicular abscess, which in like manner may also involve the perineum. The edema of inflammation and extravasation of urine may encroach upon the same region and be seen. Inspection of the lower abdominal region will recognize the globular swelling of an over-distended bladder which may reach the umbilicus. Cellulitis and extravasation of urine will also produce enlargement of a more diffuse character in this region.

Inspection of the renal region may recognize a considerable enlargement but more particularly where the enlargement is not sufficient to be obvious will appreciate the difference in mobility of one side as compared with the other in affections of the kidney of an acute inflammatory character.

Palpation.-Palpation is applied to different regions to elicit the character of any particular swelling-whether it be dense, elastic, or movable. It is employed as a preliminary measure of examination in diseases of the kidneys, prostate, testicles and epididymis. Fluctuation is sought for in any of the different regions as evidence of the existence of pus.

In the bladder region, the swelling of a distended bladder is 
differentiated from the more diffuse swelling of extravasation by its globular contour and dull percussion note, by its deep extension and by the production upon pressure of an intense desire to urinate.

Palpation of the kidneys is usually bi-manual (Fig. 45), and will often detect renal enlargements caused by neoplasm, calculi, hydronephrosis, or pyonephrosis, and prolapse of the organ. Muscular spasm and contraction in the presence of acute affections of the organ are elicited by light palpation. Such muscular spasm may affect the lateral abdominal wall, the lumbar muscles or the entire affected side. The region of the quadratus lumborum muscles is of importance, as muscular rigidity there may be present as one of the

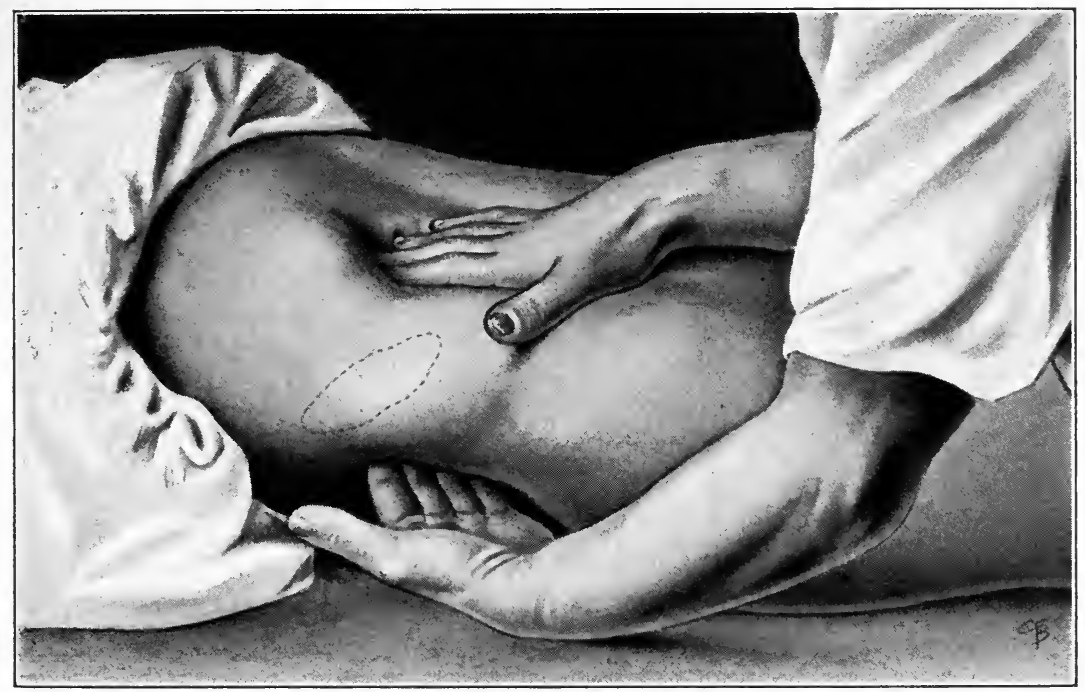

FIG. 45.-Bi-manual palpation of kidney.

signs of lesions in the kidney which are not detected by more general palpation. Lumbar tenderness is a sign of acute inflammation of the kidney. In some instances, the entire lumbar region is so affected; in other cases there is a small area of lumbar tenderness in the region of the last three ribs or in the costovertebral area.

Palpation per Rectum (Fig. 46).- This may be digital alone or bimanual. When the finger enters the rectum, the prostate is well within reach and felt anteriorly about $5 \mathrm{~cm}$. (2 inches) inside the sphincter. In normal conditions the prostate feels about the size of a horsechestnut with a median sulcus or groove. Anterior to the prostate is felt the membranous urethra, about $2 \mathrm{~cm}$. ( $\frac{3}{4} \mathrm{in}$.) in length, between the 
expanded portion of the corpus spongiosum or bulb in front and the prostate behind. On either side of the membranous urethra, Cowper's glands are recognized when they are indurated or enlarged; otherwise they are not palpable (Fig. 47). Above the prostate on either side, spreading upward and outward on the base of the bladder, are.felt the seminal vesicles. The entire length of these organs cannot be reached with the examining finger, and often they are so flat as to be indistinguishable. When, however, they are distended with secre-

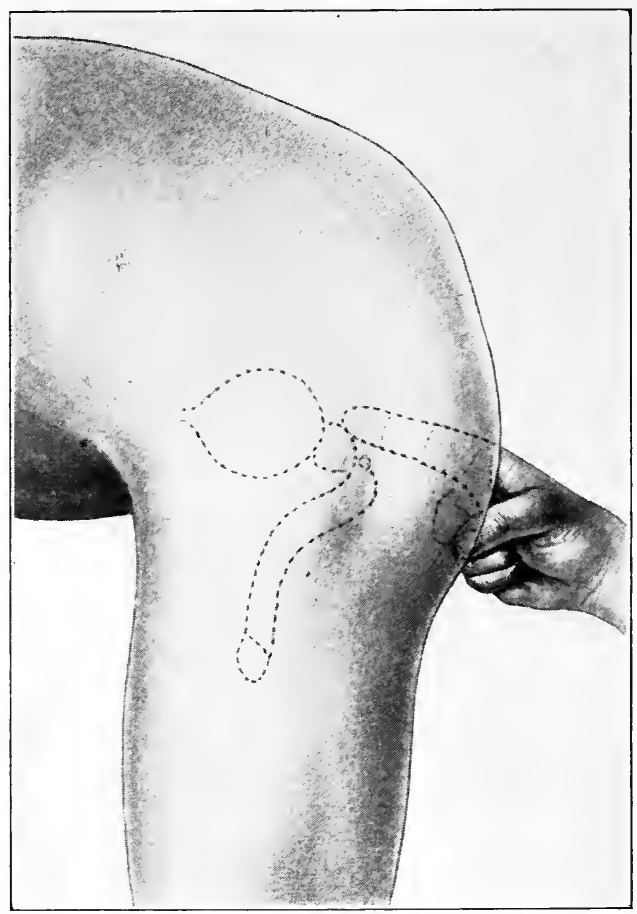

FIG. 46.-Palpation of prostate per rectum.

tions, inflamed, or otherwise enlarged, it is easy to recognize and palpate their lower portion and by massage, express the contents for examination or for treatment. Between the seminal vesicles and above the prostate is felt the base of the bladder. There is nothing conspicuous about this area except when inflamed and thickened or when it is filled in with an enlargement of the median isthmus of the prostate or occupied by a good-sized vesical stone. Stone in the ureter also may be felt through the rectum when it is located at the urethral entrance into the bladder just internal to the site of the seminal 
vesicles. The rectal palpation may be conducted with the patient in the recumbent position, but a more satisfactory position is to have him stand on a slight elevation, the upper portion of the body flexed upon the pelvis and bent as far forward as possible. When the patient is in bed, he may more easily assume the knee-chest position, which is also satisfactory.

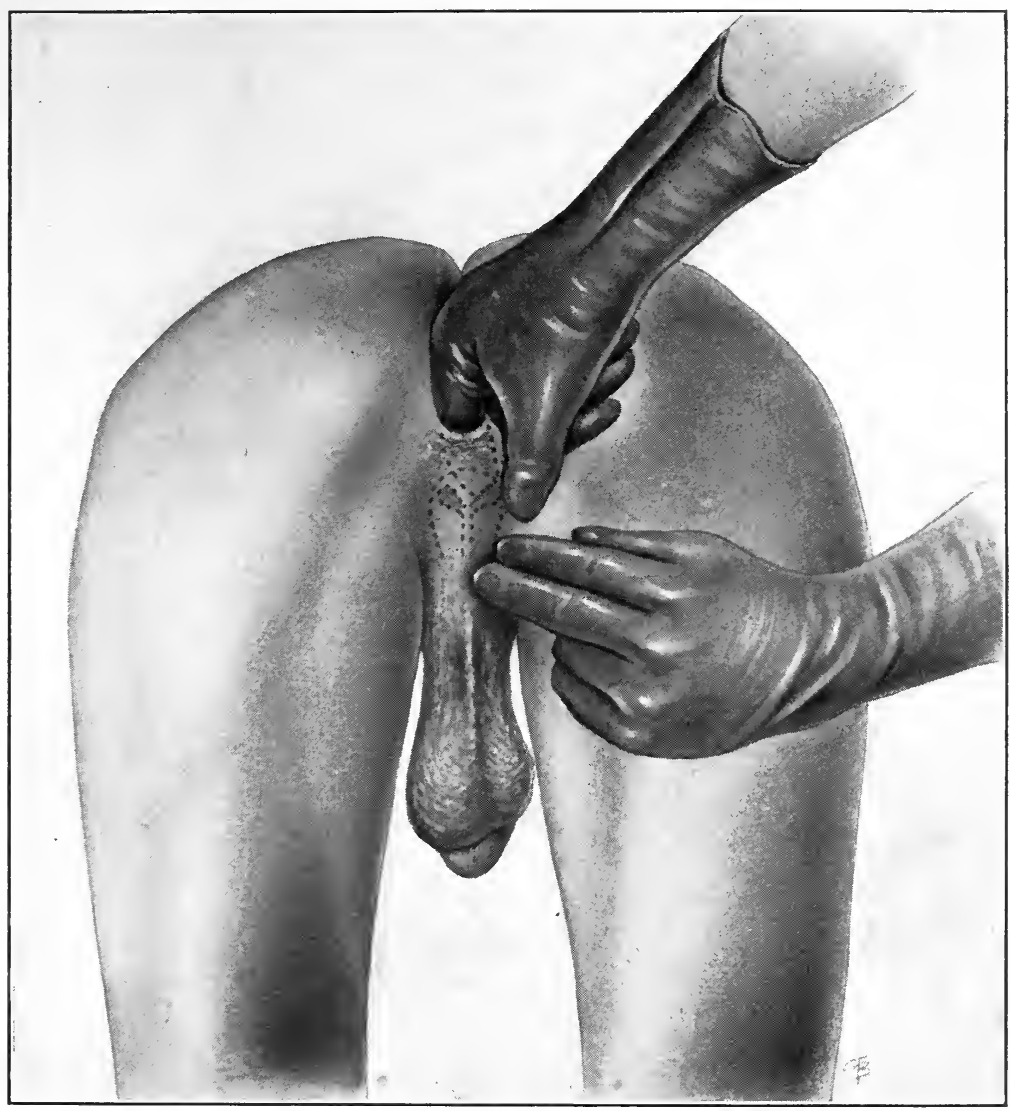

FIG. 47.-Palpation of Cowper's glands.

Bi-manual palpation is conducted by placing one hand over the abdomen as an adjunct to the examining finger of the rectum. The abdominal pressure sometimes aids in the rectal exploration by relaxing the parts and bringing them in closer reach of the finger. This may be aided by partially filling the bladder with fluid. There is nothing special about the palpation of the other organs, the penis, the 
urethra, the vas deferens, except the necessity of noting indurations, irregularities and sensitive areas.

Percussion.-By this means may be recognized tympany or dullness of the abdomen, a helpful factor in the diagnosis of tumors and of vesical retention.

Percussion of the kidney region, will elicit a variable degree of information, according to experience in utilizing such method. Generally speaking, the extent of the area of dullness or flatness in combination with tympanites may be detected in the kidney region; however, it requires considerable experience to outline these organs by the percussion note beyond the area of an inflated colon.

\section{Instrumental Exploration}

Urethral Exploration.-Examination of the urethra having already been conducted by outward inspection and clinical observation of the urine (q.v.), the more detailed instrumental exploration is next in order. Antiseptic precautions with respect to the care and disinfection of instruments and preparation of the patient (p. 53) should be observed; a due regard for the delicacy of the parts to be examined and the dangers of the harsh usage of instruments cannot be too frequently referred to.

The anterior urethra, which extends from the meatus to the triangular ligament is explored by means of sound or bougie, to determine any reduction from its normal calibre, congenital or acquired. The posterior urethra, consisting of the membranous and prostatic portions, is examined in like manner. The entire length of the canal is more thoroughly explored by anterior and posterior urethroscopy in more obscure conditions, and to clear up an uncertainty of diagnosis. (See index.)

The length of the normal urethra is between 18 and $20 \mathrm{~cm}$. (7 or 8 inches); 13 to $15 \mathrm{~cm}$. (5 1/4 to $53 / 4$ inches) in the anterior or pendulous portion, 20 to $25 \mathrm{~mm}$. (3/4 to $1 \mathrm{inch}$ ) in the membranous, and 25 to $30 \mathrm{~mm}$. (1 to $11 / 4$ inches) in the prostatic section.

The Calibre.- The urethra, when not in use, is a closed tube, the walls of which lie in contact, and in the flaccid state of the penis, arranged in folds. Its calibre is not entirely uniform, there being several points of physiological narrowing, which vary more or less in different individuals. The narrowest part is at the orifice, and here there is the greatest amount of variation. This contraction is often so great that in order to examine the rest of the canal or explore the bladder, it may be necessary to enlarge the opening. If the meatus is unduly 
small, it is better to cut it at once, as a part of the examination, to allow proper exploration at the time and adequate dilatation of the rest of the canal later, if stricture be present. Careful antisepsis should accompany this procedure. Within the meatus the urethra is enlarged, the fossa navicularis; then comes another point of narrowing, the internal meatus. Beyond this, down to the bulb, the calibre normally is of uniform size, and at the bulb, which is just in front of the membranous portion, is the widest part of the canal. Another point of narrowing occurs at the bulbo-membranous junction, and another point of dilatation is the prostatic sinus. It is impossible, therefore, to lay down a hard and fast rule with regard to the urethral calibre, the dimensions of which in no sense bear a relation to size and circumference of the penis. Generally speaking, we may take as a normal standard for comparative observation size 28 to 31 French scale (18 to 21 American) and any contractions within these sizes may be caused by organic stricture or congenital stenosis.

The two sections-anterior and posterior-of the urethra should be explored separately with the olivary bougie or sound (see Description of Instruments). And for the detection of deviations in calibre, it is well to employ the largest size that the meatus will admit, with a bulbous or blunt end. When an obstruction is met with such an instrument, the distance within the canal is noted, and the size reduced until its passage down the entire length of the anterior urethra is possible, careful note being taken of the size and number of the obstructions met, and whether they be linear, long or tortuous. When the bulbous or blunt end of the instrument passes by the strictured area, a characteristic resistance is detected, which is either that of a narrow band-like constriction, or one of a more extensive area. The patient may complain of extra-sensitiveness at the point of narrowing, and when the exploring instrument is withdrawn, the same points of resistance are again marked by the bulbous end, which often contains a tinge of blood or a drop of pus obtained from the inflamed area behind the stricture. If a conical bougie is employed, and a size introduced which fits the stricture closely, a steady and tight gripping of the instrument is felt, when pulling it out, that yields reluctantly, and this feature distinguishes a true stricture from one of the spasmodic variety.

Perhaps no instrument of any size will pass, yet the fact that the patient is able to urinate, even in a small stream or by drops, is an indication that an opening exists, although it has not been possible to enter it with the point of the instrument. Under these circumstances it is proper first to obtain good local anesthesia of the parts by the 
method already described (p. 46) and then make an endeavor to find the opening in the contracted area by means of a filiform bougie, the finest of instruments made for exploration. If success follows this expedient, dilatation may be accomplished then and there of this point of greatest contraction, to permit of further exploration of the balance of the canal; or if the examination has been prolonged, in order to avoid undue reaction, local and general, it may be desirable to desist until a subsequent sitting, two or three days later, when it will generally be found that entrance will be easier than at the first trial, provided due gentleness has been exercised. Sometimes, when failure is experienced in finding the opening of a stricture with one of the filiform bougies, success will follow the introduction of several at a time, which expand the canal and cover the entire face of the strictured area. Then each one is tried in rotation, in the hope that the minute aperture may present itself to the tip of the slender instrument. When this method fails, it is sometimes possible to find the opening by means of the endoscope (see index). The posterior urethra is explored in the same manner as the anterior, and special caution should be practised in gaining the entrance to the former, for it is at this point in the dilated part of the bulb that false passage is most likely to occur. (Fig. 50.)

Stricture of wide calibre at the bulbo-membranous junction may be detected with a blunt steel sound of large size. If there be spasmodic stricture, such an instrument will generally go in if gentle and steady pressure is exerted, but great care should be practised not to use unnecessary force. If this fails, local anesthesia may be employed. The size of the instrument is then gradually reduced, until one that will pass through the membranous canal is found. Small metal instruments are dangerous, except in experienced hands; and when it is found that the size of the obstruction requires an instrument below 24 to 25 French, it is wiser to have recourse to the flexible woven bougies, which are much safer. If even the smallest sizes of these instruments fail, the filiform bougie is employed, and may furnish the opening wedge for a much larger instrument, if the examination has not already been protracted. But if such is the case, when the patient has the ability to urinate, by not forcing the dilatation too rapidly, it may be found that after an interval of rest for twenty-four or fortyeight hours, the subsequent entrance is distinctly easier. In some cases, where entrance has been very difficult and complete retention of urine exists, the retention of the filiform in situ for twenty-four hours, by tying it in place, may be practised with good result. The subsequent instrumentation may then prove much more successful. 
The Introduction of Instruments.-The urethra, in the flaccid state of the penis, describes two curves, one in the penile portion, with its convexity upward, beginning at the meatus and ending beneath the pubic arch; the other curve, starting from this point, has its convexity downward, comprises the posterior urethra, and ends at the bladder orifice (Fig. 48).

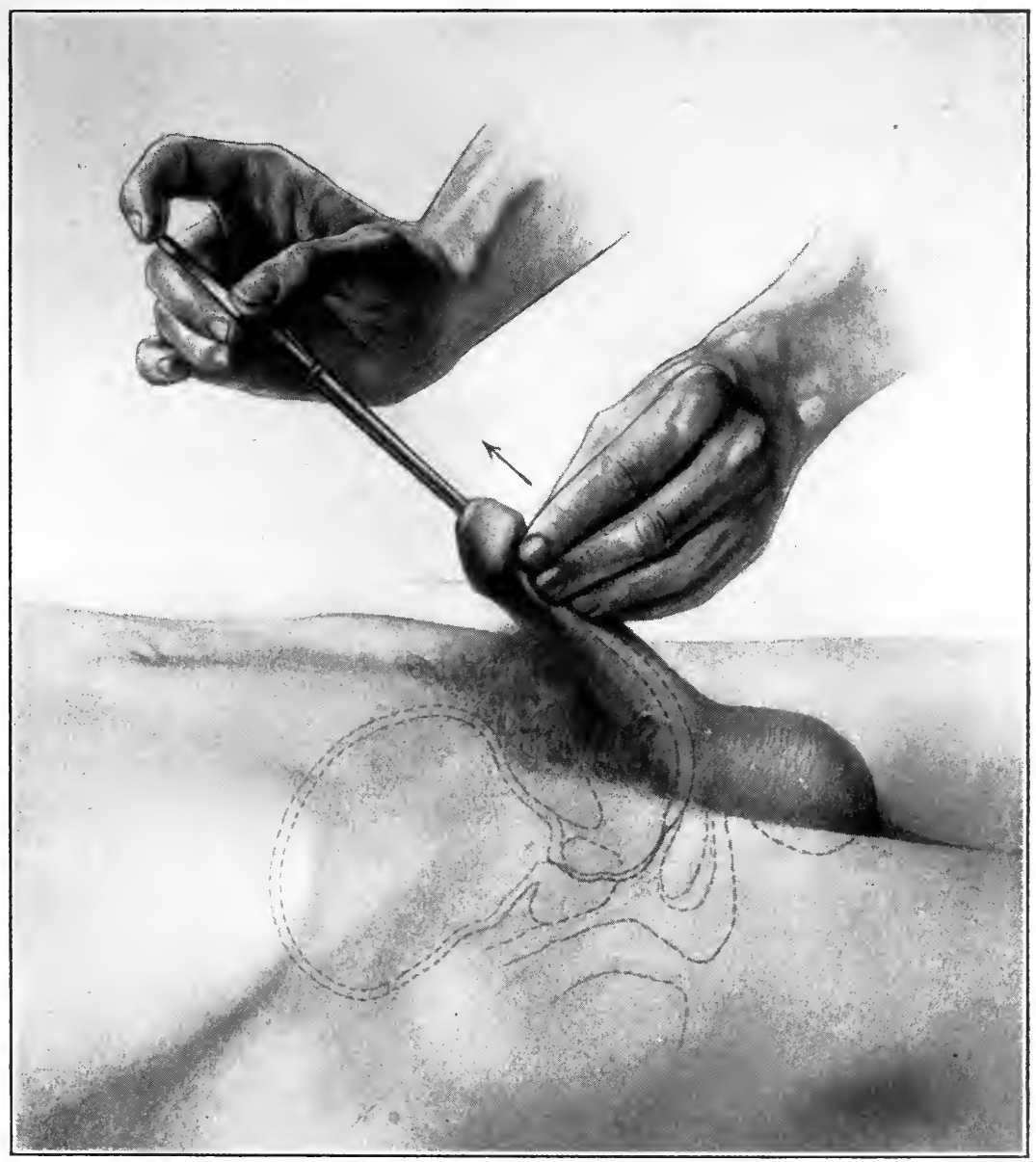

FIG. 48.-Passage of urethral sound drawing the organ up over the instrument.

In the introduction of instruments an appreciation of these curves is important. The anterior curve readily changes its form to suit that of the instrument, but not so the posterior. The instrument must bend itself to the course of this curve, or, as is the case 
with the solid steel sound, is designed in shape to suit the urethral curve. In pathological conditions, i.e., enlargements of the prostate, the urethra sometimes describes an additional curve in the prostatic urethra, and again at the neck of the bladder. (See Fig. 399, C and D.) These abnormal deviations of the canal require specially shaped instruments to reach the bladder, such as the coudé or bi-coudé catheters (p. 49). In passing the flexible instrument, whether it be a soft-rubber catheter or a woven elastic catheter or bougie, by lifting up the penis with the left hand the anterior urethra is made straight from meatus to bulb, and the instrument, well lubricated, is pushed directly downward to the latter point, which is recognized by the slight resistance met at the triangular ligament. This is overcome by gentle pressure, and by drawing the penis upward over the instrument, which then, under normal conditions, passes onward into the bladder. In the introduction of solid or rigid instruments, whether it is the ordinary steel sound, the Béniqué curved sound or catheter, the surgeon stands on the left of the patient; the handle of the instrument is depressed to the level of the abdomen, near the crest of the ilium, and the penis in the left hand, drawn over the curved end of the instrument as far as possible. The handle is then rotated to the median line; mean while, the left hand continues to draw the penis upward, over the instrument (Fig. 48). The handle is then elevated to the perpendicular, and the instrument is permitted to gravitate downward by its own weight, rather than by pressure, until its tip is felt in the bulbous portion against the triangular ligament. When the point is reached, the penis is again drawn well upward over the encircled shaft, to make sure that all folds have become obliterated, and then by depressing the handle well between the thighs, the deep canal is traversed and the bladder is reached. Sometimes this is assisted by gentle pressure on the perineum with the left hand, while the right hand supports the instrument (Fig. 49), or by pressure downward upon the abdomen with the right hand, while the left guides the passage of the sound or catheter. The single curved solid instrument is designed to favor its passage through the natural curve of the posterior urethra, and the double curve of the Béniqué sound or catheter is supposed to meet this requirement even to a more satisfactory degree. This is more likely in cases where the prostatic curve is elevated by enlargement. Otherwise, I find that individual cases differ; some receive the single curved and others the Bénique the more readily. The instrument is withdrawn in the reverse manner to its introduction.

Faulty technic, undue force, and lack of the antiseptic precautions laid down are likely to lead to those accidents and complications 
which sometimes accompany the use of sounds and catheters: namely, traumatism of the canal, producing general or systemic reaction by septic absorption and evidenced by urethral chill and fever, periurethral abscess, prostatitis, retention of urine, cystitis and false passages.

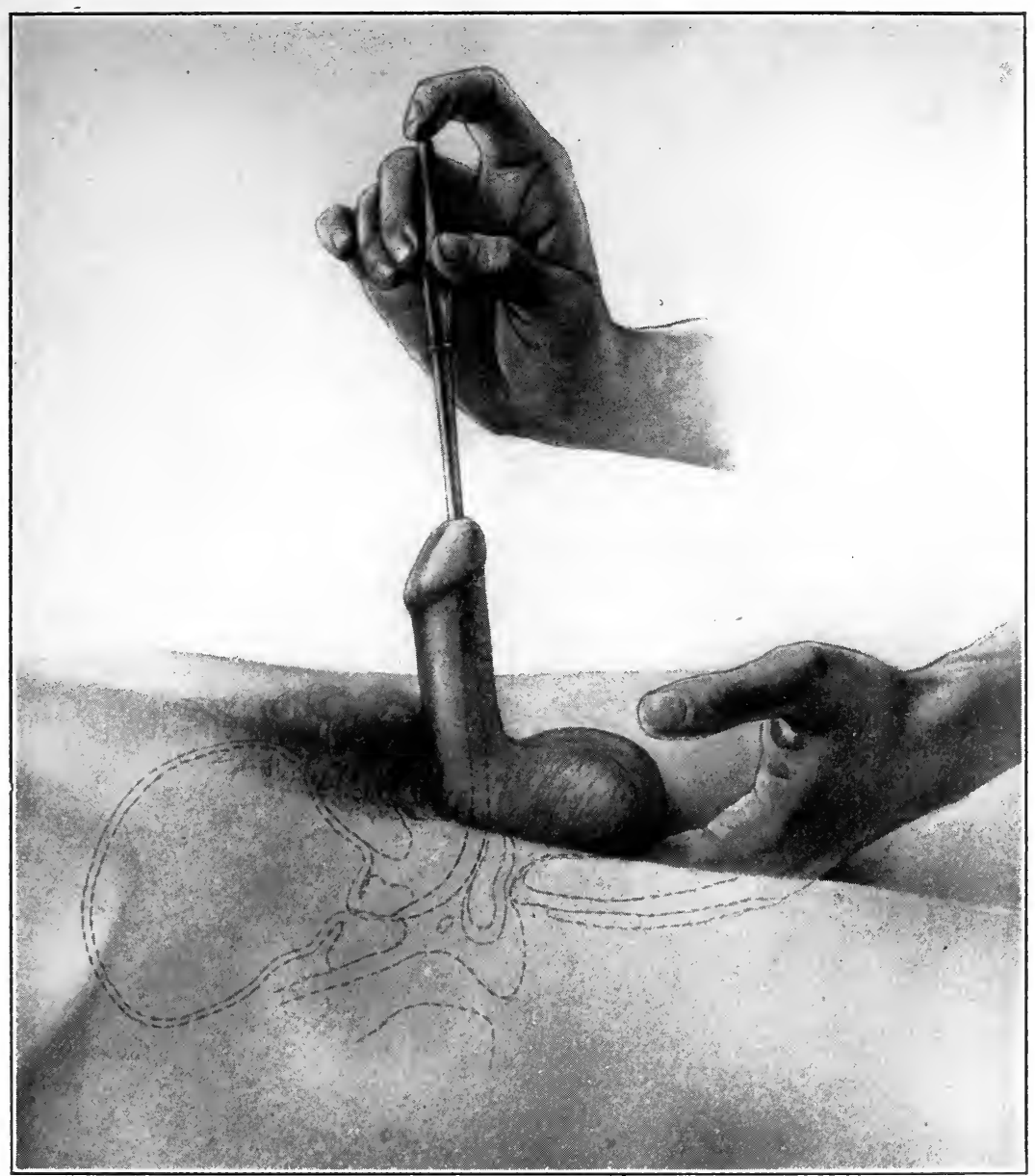

FIG. 49.--Passage of sound. Fingers in perineum directing the course through triangular ligament.

False Passages.-False passages occur more commonly in the dilated portion of the bulbous and prostatic urethra or where a false entrance is made through any strictured area. They are favored, so far as the urethra is concerned, by congenital pouches or dilated follicles, 
by undue dilatation of the bulb and by encroachments upon the prostatic canal of enlargements of the gland or constriction of the bladder orifice. They are produced by the operator by the use of unsuitable instruments, by a failure to recognize abnormal pouches or unobliterated folds, by employing too much force against urethral spasm of the "cut-off" muscle at the bulbo-membranous junction (Fig. 50) or by roughly piercing with the point of the instrument the projection of a prostatic lobe or a contracted vesical orifice. The solid

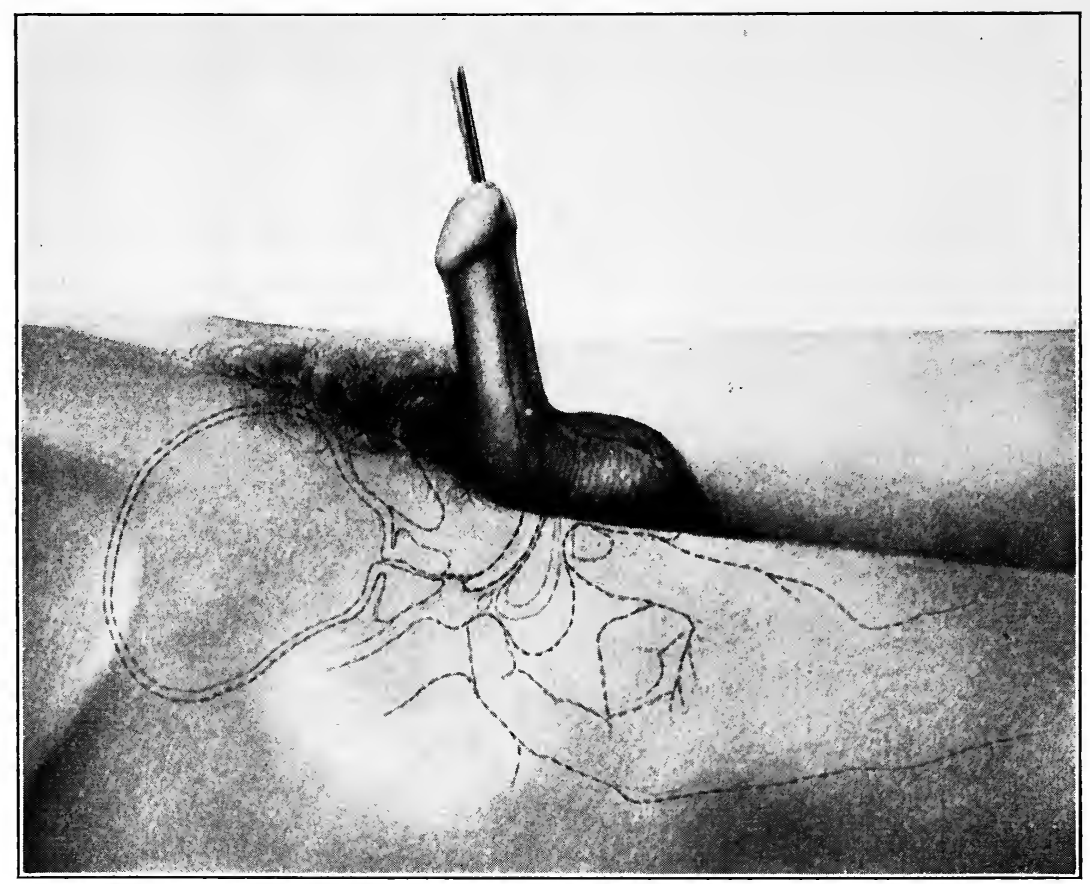

FIG. 50.- The finger in the rectum may aid the passage of a sound caught at the bulbo-membranous junction.

instrument is more likely to do damage than the flexible, and the pointed bougie will more readily penetrate the delicate mucosa. It is the floor of the urethra that is subject to this sort of damage, and upon which obstacles should be carefully avoided. Sometimes false passages heal themselves, but they are always a source of possible danger as a point of septic absorption, followed immediately by urinary fever, or secondarily by local suppuration of moderate or severe degree in the occurrence of periurethral inflammation, follicular or prostatic abscess. 


\section{Bladder Exploration}

Examination of the interior of the bladder by palpation is conducted with the aid of a short beak metal instrument, the Thompson searcher (Fig. 187, p. 447). This instrument is passed much in the same manner as the ordinary steel sound (p. 72), great care being taken in its introduction at the beginning of the curved urethra and in the prostate. No undue force should be employed. As the instrument is made to penetrate the prostatic urethra by downward pressure of the handle any marked deviation from the normal in general direction of this portion of the canal or elevation of the prostate as the sound enters the bladder is appreciated. After the bladder is reached the instrument is first held in the median line with the beak turned upward. If the neck of the bladder is free, that is to say, if there is no intravesical prostatic enlargement or bladder neoplasm, it can be readily rotated from side to side without obstruction.

The interior of the bladder is now examined systematically by rotating the instrument with abrupt motion, tapping the bladder from base to summit on one side, and then turning from summit to base on the other (Fig. 186, p. 446). When the neck of the bladder is reached again the instrument is turned with the beak downward toward the base and rotated gently from side to side. In this manner the presence of a foreign body or growth is detected, and in the case of a calculus the size and number can be generally appreciated as well as the soft or hard texture thereof. The intravesical bulging of the prostate may also be palpated and a rough measurement of the growth taken account of. By this means is accomplished a satisfactory preliminary observation that should be supplemented by cystoscopy, for definite and satisfactory knowledge of the intra-vesical condition (See p. 129).

\section{Urethral Chill and Urinary Fever}

There are two recognized conditions that come under this heading. One is a trauma to the nervous system, or "shock," due to the nervous susceptibility of the patient and to the urethral instrumentation, without any necessary infection; the other is a trauma to the urethra or other structures, and is due to septic absorption. For the prevention of the first, morphia is well-nigh a specific, while the avoidance of the other calls for careful antiseptic precautions and the employment of skill and great gentleness in the manipulation of the instruments. The septic type of urethral fever occurs in different degrees: by a transitory chill and fever only, when systemic absorp- 
tion thus expresses itself, but is promptly overcome by the defensive forces of the system, or by immediate use of local and internal disinfection. A more pronounced form expresses itself in recurring attacks of chill and fever, when disinfectants have not been properly employed, or where the system is unable successfully to combat the septic invasion. A still more severe form is that which continues in a more or less chronic state, owing to an inherent unhealthy condition, dependent upon bad kidneys, or preëxistent chronic inflammation of the prostate, cystitis, etc. The two types, the nervous and the septic, may be combined in one individual, and the more susceptible the patient, the lesser the degree of local disturbance necessary to produce such reaction. In other words, a nervous and susceptible patient (low antibodies) may sustain a marked reaction from slight urethral damage, and a relatively severe attack of chill and fever from greater trauma, while the opposite type of individual (high antibodies) may be able to sustain a much more pronounced urethral injury without reaction. The source of infection comes from the presence of microörganisms, which normally inhabit the urethra, or from the passage of infected urine over the freshly denuded urethral membrane. Local conditions, such as stricture, prostatic enlargement and residual urine, are apt to favor the occurrence, by retaining septic products in contact with the injured mucous membrane.

\section{EXAMINATION OF URINE (CLINICAL)}

It is often necessary to await the result of the laboratory findings before the clinical diagnosis can be relied upon, and the progress of the disease, as well as the action of treatment, must be constantly determined in the same manner. Therefore the laboratory is an important part of the urological equipment, or, at least, a full knowledge of the essentials of laboratory examination should be possessed, so that none of the significant diagnostic features shall be overlooked.

The Urinary Examination is of first importance, and it is necessary to have a thorough acquaintance with both the normal composition and the pathological variations of the urine in order to become proficient in diagnosis. The diet, regimen and general habits of life are duly weighed in connection with the urinary observation, as not infrequently the presence of albumin is due to diet alone. The same may hold true for transitory glycosuria and other conditions. The quantity and density of the urine are affected in like manner.

Collection of Urine.-For gross examination, or preliminary observation as to the transparency, acidity or decomposition, the urine may be passed into a good sized glass or graduate, when the presence 
or absence of pus, blood, phosphates, etc., may be noted, and ordinary chemical, or even microscopical examination of the centrifuged sediment be made. But for a bacteriological investigation the urine should be collected in a sterile manner, which means either by catheter, especially in the case of a female, or after the external parts have been cleansed and the urethra flushed with sterile water, before the patient is allowed to urinate into a sterile vessel.

To preserve the specimen, it should be kept in a cold place when possible. There is no ideal chemical preservative. Chloroform or thymol are preferable to others. Chloroform is apt to produce confusing sugar reactions and thymol may produce reactions which resemble bile pigment, but otherwise they have no objection-a few drops to each 4 ounces of urine. Formalin interferes seriously with the chemical examination.

Quantity of Urine.-The average quantity of urine voided by a healthy adult has already been stated to be 1500 c.c. (50 ounces) In accordance with the effect of daily regimen, exercise, etc., variations are readily caused in this amount, by surface elimination through perspiration, or by the drinking of generous quantities of liquid. Such factors in the decrease or increase of urine must be taken into account.

Inspection. Color.-The normal color of urine is light amber or straw yellow. Concentration is a dominant factor in determining the depth of color. Almost colorless urine may be present in health, as may fluid of brownish-red shade. In polyuria, especially in nervous individuals, the hue is pale; in oliguria, as in febrile conditions, it is dark. The presence of bile or bile coloring matter causes the urine to have a brownish-yellow color with a greenish cast and the froth of such urine is yellow. The presence of blood or hemoglobin turns urine to a shade varying between deep red and pale pink or a smoky brown. The color of alkaline (phosphatic) urine is cloudy yellow, sometimes flocculent. This cloudiness simulates purulent urine and bacteriuria. Acid urine which does not contain pus or bacteria is clear, except when there is a great excess of oxalates (oxaluria) or urates. Occasionally the appearance of the urine is affected by the elimination therein of certain chemical agents. The blue or greenish-blue tinge following the use of methylene blue is familiar. The internal use of carbolic acid, salol or guaiacol may cause the urine to become smoky black. The ingestion of senna and rhubarb causes a reddish-brown, and santonin a yellowish color.

The presence of pus may be readily recognized by the experienced eye, although it may at times require the addition of a few drops of 
acetic acid to differentiate between it and phosphates. The presence of bacteria alone (bacteriuria) gives the urine a pale smoky hue, and the deposit of an excess of urates has a slightly pinkish cast. The latter are dissolved with heat. The presence of blood is usually self-evident.

Odor.-The odor of normal urine is characteristic. It is not fetid nor offensive, but aromatic in quality. This quality is more or less marked, according to its density. Febrile urine is sharp and volatile in odor on account of its concentration. In chronic cystitis when, owing to obstruction, a portion of the urine is retained in the bladder and undergoes decomposition, the odor may become ammoniacal in character unless it is disinfected by bladder lavage or internal antiseptics. In conditions of excessive suppuration, mainly of the kidneys, where there is a large amount of pus and retained urine, as in pyonephrosis, the urine has a foul, putrid odor. This is also the case where there is necrosis of the tissues, as in ulceration and neoplasm of the bladder. A fecal odor is given to the urine when recto-vesical fistula exists. Diabetic urine has a sweetish odor.

Inspection alone will often determine the presence in the urine of certain abnormal constituents, or of the precipitation of some normal ingredients, of which the principal ones are pus, blood, bacteria, phosphates, urates, oxalates.

Pus in the Urine.-Normal urine may contain mucus in strings or granular masses and filaments of mucus with some leucocytes and epithelial cells from bladder and urethra, known as shreds. It is an extreme view, shared in by many individuals, that such elements appearing in the urine are of necessity a sign of disease. It is true that certain varieties of shreds composed of pus and epithelial masses are symptomatic of different lesions of the urethral canal, to be considered later, but they are accompanied by other physical signs which give evidence of an existing morbid condition. Purulent urine is turbid. The presence of pus changes the consistency as well as the transparency of the urine, and when allowed to stand, settles in a creamy deposit, yellowish or greenish in color. If there is any question as to the nature of such deposit, the addition of acetic acid, as already stated, will promptly decide it, as the precipitate of phosphates, the most likely source of error, is quickly removed thereby, whereas pus is unaffected. The presence of pus may be demonstrated chemically by mixing with the urine liquor potassa in about equal parts, and shaking well, which causes the separation of the contained pus in a ropy string, leaving the rest of the liquid perfectly transparent, or by shaking up with aqua ammonia, about 1 part to 10 or 20 , which causes the entire urine to become of a syrupy consistence. 
Free pus in the urine is evidence of suppuration somewhere along the genito-urinary tract. When it is suspended or deposited in the form of masses or filaments, it occurs as a result of some lesion of the urethral tube or accessory glands. The common cause of pus in the urethra, which is washed out by the urinary current, is acute or chronic gonorrhea, in which case the pus is washed out by the first flow of urine, whether it comes from the anterior or posterior urethra. If the front canal is first washed clean, before the urine is passed, the pus present comes from the posterior urethra or bladder; if from the bladder, it will appear alike in all sections of urine passed. Pus is present in the bladder in all types of cystitis, whether the cause be a stricture of the urethra, gonorrheal invasion, hypertrophy of the prostate, tuberculosis, calculus or neoplasm. See Plate V.

Purulent urine from the bladder may be acid or alkaline. The latter reaction is frequently caused by the presence of ammoniacal decomposition, and is more common in obstructive conditions, where there is residual urine. Pus may come down from the kidneys in the urine and be present in the bladder without there being any symptoms of cystitis, as a result of pyelitis, pyelo-nephritis, pyonephrosis caused by renal calculi or tubercle, etc. Such conditions are recognized and a definite diagnosis made by cystoscopic observation and ureteral eatheterism (q.v., index).

As a rule, pus originating in the kidney settles down in the bottom of a graduate in a compact layer, and does not show itself in thick, ropy masses, as is of ten the case in alkaline decomposition in the bladder during chronic cystitis. The most common confusion is due to the presence of amorphous urates or phosphates. The former will disappear on the application of heat; the latter are dissipated by a few drops of acetic acid.

Bacteriuria.-The presence of bacteria in the urine may give to it a turbid appearance, which, when inspected closely, has a pale, vaporous character. It does not settle down like pus after standing, and when such urine contains both pus and bacteria, in combination, if a portion is centrifuged for several minutes the pus will be precipitated in a solid deposit, and the upper part of the urine will remain cloudy from the bacteria in suspension, although some of the bacteria are carried down with the pus.

A bacteriuria pure and simple is generally produced by the presence in the urine of numerous quantities of bacteria of the colon group, which are rod-shaped bacilli; and while this organism is one of the frequent causes of cystitis, it is, when introduced into a healthy bladder, non-pathogenic (Melchior), which explains the existence of 
this form of bacteriuria without the presence of pus. When, however, the bladder is damaged or diseased, a cystitis is lighted up by these organisms, which have found their way into the urine by contamination, or directly from the intestinal tract. We therefore have turbid urine which is produced by bacteria alone, or by a combination of pus and bacteria. The other forms of bacteria which are found in the urine are the pathogenic varieties, and are mentioned under the head of Bacteriology of the Urine, p. 101.

Phosphates.-Excessively nervous and mentally active individuals are prone to eliminate large quantities of alkaline phosphates in the urine, which give to it a cloudy appearance sometimes mistaken for pus. Unlike the bacterial cloudiness, it settles down to the bottom of the urine, and the addition of a few drops of acetic acid causes its disappearance, which produces no effect on either the bacterial or purulent urine.

Urates in excess will give a cloudiness to the urine which is promptly removed by the application of heat. The sediment caused by urates is more likely to occur in cold weather and in individuals of a gouty or rheumatic tendency, or following a highly nitrogenous diet. It is of a pinkish muddy appearance, and is not necessarily of any significance.

Oxalates.-The presence of oxalates in excess, namely, calcium oxalate crystals, will sometimes be the sole cause of cloudy urine. They occur in neurotic individuals of sedentary habit or with gastrointestinal derangement, due to faulty metabolism. Their presence is recognized by the highly acid condition of the urine, and by microscopic examination, which reveals in abundance the characteristic crystals of oxalate of lime (see Fig. 63, p. 100).

Blood in the Urine.- When fluid blood is present in the urine in large amount, it gives the urine a bright red or brownish-red color. Alkaline urine heightens this color. If the blood is decomposed, the urine assumes a brownish or smoky hue. Blood may also show itself in clots, or the quantity may be so small as to appear only microscopically, red blood cells being discovered; and, as is the case whenever blood is present, by testing, albumin will be found. If hemoglobin alone is present causing a dark brown or black color, the spectroscope will reveal the cause, if any doubt exists.

Blood from the kidney is evenly distributed with the urine. It may be bright red in acute congestion or from active bleeding, as in the case of injury from calculus, etc. It is dark red and smoky when it has been retained in the kidney for some time. The detection of blood casts in the urine is always a positive proof of the source of the blood 
from the kidneys. But the cystoscope in surgical lesions determines this question beyond doubt (see p. 129).

There is nothing characteristic about blood from the ureters. Here, again, the cystoscope and ureteral catheter will help to clear up the diagnosis.

The causes of blood from the upper urinary tract are stone (ureteral and renal), tuberculosis and neoplasm; and hemorrhage may occur as a result of inflammation not dependent upon any of these surgical conditions. Blood comes from the bladder in small amounts, as a result of acute and chronic cystitis, and in copious quantity it is caused by calculus, tuberculosis or neoplasm. When the blood is retained in the bladder, it becomes changed in character and darker in hue. During urination the latter part is brighter than the first, showing fresh bleeding; the reverse is the case when the blood is from the prostate or posterior urethra, the fresh blood appearing first. In sharp inflammation of the front urethra some blood appears with the first urine, and in acute posterior urethritis and urethro-cystitis, blood appears at the end of urination-terminal bleeding. 


\section{CHAPTER IV}

\section{DIAGNOSIS (Continued)}

\section{CHEMICAL AND MICROSCOPICAL URINARY EXAMINATION}

\section{EXAMINATION OF URINE (CHEMICAL)}

The examination of the urine is conducted for the purpose of finding an excess or defect in the quantity of normal constituents or the presence of one or more abnormal ingredients. This information is obtained by chemical and microscopic analysis.

\section{Normal Ingredients Secreted in 24 Hours by Healthy Adult on Mixed Diet Urea, about $30 \mathrm{gm}$., $500 \mathrm{grs}$. Uric acid, 0.5-0.8 gm., 7-12 grs. Phosphoric acid, about $3 \mathrm{gm}$. Chlorides, 12 to $15 \mathrm{gm}$.}

Normal Urine

Quantity in 24 hours, 1500 c.c. (50 ounces).

Color, light amber or straw yellow. Odor, aromatic.

Reaction, acid.

Specific gravity, 1020.

\section{Abrormal Ingredients}

Albumin (serum albumin and globulin).

Sugar (glucose).

Acetone and diacetic acid.
Bile pigment.

Indican.

Blood.

Fat. $\left\{\begin{array}{l}\text { Lipuria. } \\ \text { Chyluria. }\end{array}\right.$

Pus.

Bacteria.

Casts.

\section{Estimation of Normal Ingredients}

Urea.-The elimination of urea in the urine represents the metamorphosis or combustion of the proteid portion of the tissues and alimentary substances in the body; that is, it comprises the largest percentage of the total nitrogen excreted ( 85 to 90 per cent.); the balance of the nitrogen is contained in the free ammonia and purin bodies of the urine (uric acid and purin bases). 
The estimation of the total nitrogen output in the urine is a lengthy and complex proceeding, requiring elaborate apparatus. It is important in measuring accurately the functionating capacity of the kidneys, and is of special significance in connection with the operation of nephrectomy, when any doubt exists as to the possibility of post-operative uremia on account of the deficiency of the opposite kidney. In most instances, however, the estimation of the urea (in special cases also the chlorides), combined with the other available tests for measuring the urinary function (Chap. VI), together with the other laboratory and clinical features, have proved a reliable basis for conclusion on this question. And by means of such guidance, the percentage of mortality following operation has been materially reduced. The elimination of urea is diminished in renal disease, and in all forms of malnutrition. The quantity of urea excreted is increased by the amount of proteid food intake and the wear and tear of the body, whether from exercise or increased animal heat as a result of febrile activity. These facts should be taken into account, and should be weighed in the endeavor to reckon the reserve capacity of a given organ.

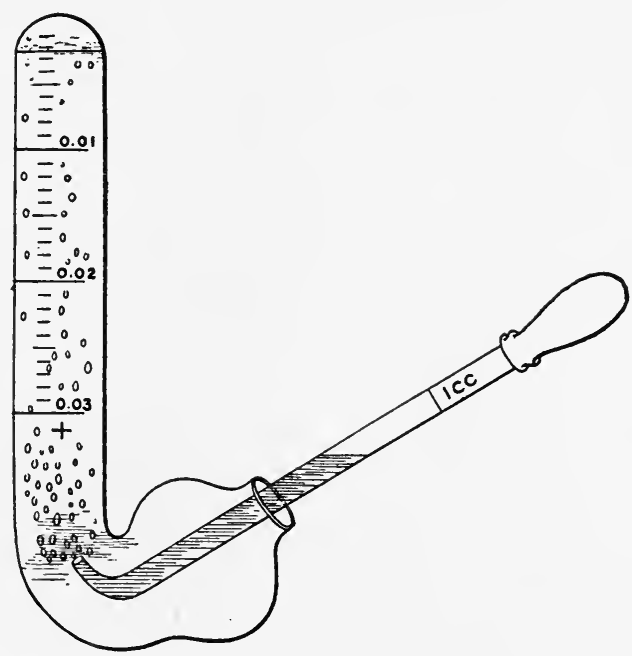

FIG. 51.-Doremus ureometer.

Quantitative Test for Urea.-The apparatus commonly used for this purpose is the Doremus Ureometer (Fig. 51), the test solution, a hydrobromite of soda mixture, prepared by adding to 25 c.c. of sodium hydroxide solution 1 c.c. of pure bromine and mixing thoroughly. The upright arm and bend of the ureometer are filled with the hydrobromite solution, so that there are no air bubbles at the closed top of 
the tube. The pipette is filled up to 1 c.c. with urine, and this is deliberately injected by directing the curved end of the pipette beyond the bend, and thus forcing the urine into the upright arm. The gas which is then generated by contact of the urine with the hydrobromite solution cannot escape into the bulb, but mounts to the top of the tube, displacing the fluid downward. The space occupied in the tube by the gas set free represents the amount of nitrogen liberated, which bears a fixed relation to the amount of urea. The result is read from the scale on the tube, which is marked in large divisions $0.01,0.02$ and 0.03 , and subdivided in tenths. The divisions 1,2 and 3 represent corresponding percentages, or as many centigrams of urea to each gram of urine; (the smaller subdivisions represent milligrams). Thus, at the level of 0.02 we have

$$
0.02 \underset{\text { Urea to urine }}{\mathrm{gm}}: 1.0 \text { c.c. : : } .02 \times 100: 1.0 \times 100 \text { (or } 2: 100 \text { ) }=2 \text { per cent. }
$$

More elaborate and accurate laboratory apparatus are devised, but they are not suited for common usage, nor are they necessary for clinical diagnosis, in view of the accessory means of investigation that are always employed in routine practice (see Chap. VI).

Uric Acid.-The quantitative estimate of uric acid has no important clinical significance apart from the urea estimation. The crystals of uric acid are recognized microscopically in the course of such investigation (see p. 100).

Chlorides.-The chlorides present in the urine as sodium chloride are an important solid ingredient, and they exist in such amount (12-15 gm. in 24 hours) as to represent a significant proportion of the excretory output of the kidneys. It is, therefore, considered important by some urologists, in measuring the total or comparative renal function, to estimate the quantity of the chlorides. It may be stated, however, that while a knowledge of such may furnish interesting data, helpful in grave and obscure cases, yet, in the majority of instances in the diagnosis of surgical lesions, their estimation may be dispensed with.

Phosphoric Acid.-There is likewise no diagnostic value in estimating the total amount of phosphoric acid in the urine. The precipitation of phosphates and their recognition have already been considered under the clinical examination of the urine $(q . v$.$) . The$ crystals of ammonio-magnesium phosphates are recognized by the microscope in the urinary sediment (see p. 100).

Reaction.-The normal urine is acid in reaction. This applies to the mixed twenty-four hour quantity, as there is variation throughout the day in this physical property of the urine. It is affected by the 
ingestion of solids and liquids, physical and mental activity. The first morning urine is usually the most acid of the twenty-four hours. A proteid diet will increase, and a vegetable diet diminish the acidity. Generally speaking, the degree of acidity bears a relation to the specific gravity and quantity of the urine excreted. In nervous individuals the amount of alkaline phosphates eliminated is increased, the total quantity of urine large, and the degree of acidity low; whereas, in phlegmatic persons, with relatively small urinary output, the degree of acidity is high. In certain neuroses combined with intestinal derangements and a defective metabolism, the acidity runs high. In chronic Bright's disease the tendency is toward lowered acidity. In febrile conditions the acidity is strong. Urine which is retained in the bladder and undergoes ammoniacal decomposition has a strongly alkaline reaction.

The common method of testing the reaction with litmus, which is turned red with acid and blue with alkaline urine, is adequate for ordinary usage. To test the reaction for percentage of acidity, more complicated methods are employed, notably the phenolphthalein method, which is seldom necessary. This test is based upon the fact that phenolphthalein is red in an alkaline and is decolorized in an acid medium. A standard solution of sodium hydroxide ( $1 / 10$ normal) is used to neutralize the effect of the acid in a given quantity of urine (10 c.c.) upon a standard solution of phenolphthalein ( 1 per cent.) and the result measured. Thus, if 10 c.c. of urine are mixed with two drops of 1 per cent. phenolphthalein and sodium hydrate solution added, drop by drop, the reddish color produced by the alkaline solution will appear but temporarily until the point of neutralization of the acid in the urine is reached, when the color remains. The amount of soda solution used to this point is noted. Then multiply the number of cubic centimeters of soda solution by 100 and divide by 10 - the amount of urine tested-to obtain the percentage of acidity. Thus, if for example, the amount of soda solution was 30 c.c. : $\frac{30 \times 100}{10}=30$ per cent.

Specific Gravity.-The specific gravity of any urine varies according to the proportion of solids to the total liquid. This necessarily is not a fixed relation, but is affected by various influences, namely: the amount of fluid ingested, character of diet, and surface or skin evaporation. The mean standard of the mixed twenty-four hour urine, however, is placed at 1020 , distilled water at $15.6^{\circ}$ C. $\left(60^{\circ}\right.$ F. $)$ being 1000 . As stated, the variations in specific gravity and acidity are dependent more or less upon the same conditions. When these two properties of 
the urine are relatively high, and remain so for a considerable period, an irritation of the urethra or bladder is occasionally produced, owing to the density and hyperacidity of the urine. This may be mistaken for some inflammatory condition, which will subside promptly following the use of alkaline medication and the copious drinking of mineral water. Beyond this, the specific gravity has no bearing upon the maladies of the lower urinary tract. As to the kidney, however, it is more or less an indicator of the functional activity of the organs, being an index of the total solids excreted. In advanced kidney lesions, such as chronic interstitial nephritis, the function of elimination is badly impaired and the solid constituents of the urine reduced, and, therefore, the specific gravity is low. In diabetes mellitus, on account of the presence of sugar in the urine, the specific gravity runs as high as 1040. For estimating the specific gravity a good urinometer should be employed, which means one that has been properly tested, is not too large, so as to require as small an amount of urine as possible for the test, and the urinometer should not fit the glass too closely.

\section{Abnormal Ingredients in the Urine}

Albumin (Serum Albumin and Serum Globulin).-The great importance given to the presence of albumin in the urine is dependent upon the fact that it is a constant symptom of morbid conditions of the kidneys, and is often the first evidence discovered of such trouble. The stress laid upon this fact by routine physical examination for life insurance, etc., has tended to give to this symptom alone too great significance. It is not inconsistent with health to find a slight trace of albumin in the urine in individuals under a free proteid diet. And it should always be borne in mind that certain albuminous substances present in the urine from local causes, such as blood, pus and prostatic fluid, may, without any constitutional deterioration, be the explanation of an albuminuria of moderate degree, which in reality is a false albuminuria, as distinguished from true. While true albuminuria is a manifestation of organic disease of the kidney, affecting the integrity of the blood-vessels or tubules, it may result from transient causes, such as sudden changes in blood-pressure, due to diet, exercise, nervous shocks and other influences upon the vasomotor system; or from agencies in the blood itself, when such substances as turpentine, carbonates, etc., in excess, or toxins are present.

The first question, then, when albumin is discovered in the urine is, "where does it come from?" Other evidence will not be lacking to clear up this question. It is only necessary that a thorough knowledge 
of the situation should be obtained by appropriate diagnostic measures. An examination of the urine alone will often direct attention to the probable source of trouble by the discovery of such elements as pus, blood, etc., and with the distinguishing features of casts or characteristic epithelia.

Tests for Albumin.-To make a satisfactory test of the urine for albumin, the specimen should be clear and free from decomposition. If necessary, it should be filtered through heavy filter paper, and if, in spite of this, the urine still remains turbid, it should be mixed with French chalk and charcoal or calcined magnesia, and then be filtered.

Heat Test.-Fill the test-tube two-thirds full of clear urine. Boil the upper portion in the Bunsen flame. If a cloudiness appears it is either albumin or earthy phosphates, in which case the addition of one or two drops of nitric or acetic acid will determine the question. The clearing up of the cloud proves that it is phosphates and the reverse that albumin is present. The reason for the addition of acid in this test is to set free any albumin that may be combined in the urine as albuminates which are not precipitated by heat. On the other hand, an excess of acid may form acid albumin, which is also not precipitated by heat. The precaution should always be taken, therefore, to boil the urine first and add the acid carefully, drop by drop. When this test is properly performed, it will detect minute quantities of albumin in the urine.

Heller's Cold Nitric Acid Test.-About 2 c.c. of fuming nitric acid are put in a conical test glass. The urine is then allowed to trickle slowly down the side of the glass from a pipette, while the glass is held on a slant, so that the urine will become imposed upon the surface of the acid. If albumin is present, it will appear in a clear-cut opaque white zone at the juncture of the two fluids. The nitrate of urea is an unlikely source of error; it appears above the albumin zone, and is of brownish hue. Attempt has been made, and it may to a certain extent be useful, to make a quantitative estimate of the albumin with this test by gauging the size and density of the albumin zone. Such an estimation is purely comparative, but by frequent usage and experience the eye becomes expert in this method.

It should be remembered that in patients using such resinous drugs as turpentine, sandalwood and copaiba, the urine will yield a precipitate upon boiling that may be mistaken for albumin, as it does not dissolve upon the addition of acid. If there is any doubt in this respect, the excess of acid will cause this precipitation to disappear.

The above tests fulfill most requirements, and are capable of detecting minute quantities of albumin. Exceptionally, only, are other 
tests required. Generally speaking, their use is a matter of elaboration of technic.

Ferrocyanide of Potash and Acetic Acid Test.-This test involves the use of acetic acid and a 1 per cent. solution of ferrocyanide of potash. About 10 c.c. of urine are acidulated with a few drops of acetic acid (3 to 5 ); to this several drops of the ferrocyanide solution are added. A flocculent precipitate or cloudiness denotes the presence of albumin, and appears in proportion to the amount of the latter present.

Other delicate tests employed for the detection of small traces of albumin are by means of the sulphosalicylic acid reagent and the phenol acetic acid test of Millard.

The Quantitative Estimation of Albumin.-This is accomplished by precipitation with acid and boiling, and then weighing or measuring the amount of deposit. Thus we obtain the estimate by weight or bulk. The Esbach albuminometer (Fig. 52) is a means of approximating the quantitative estimate of albumin. In this method the albumin is coagulated by the addition of a test solution of picric and citric acids (10 and 20 parts per 1000 distilled water) and the result read from a scale on the side of the albuminometer after the liquid has been allowed to settle for twenty-four hours. A line on the tube (U) indicates the point to which the urine should reach, and another (R) for the amount of reagent, after which they are thoroughly shaken. The figures on the scale represent

FIG. 52.Albuminometer. the number of grams per litre, or parts per 1000: thus, the level of the figure 5 indicates 5 parts to the 1000 , and to gain the percentage, divide by 10 , thus:

$$
5: 1000:: 5 / 10: 1000 / 10 \text { or } 0.5: 100,0.5 \text { per cent. }
$$

Tests for Sugar (Glucose).-Glycosuria is a constitutional disorder, and not in any sense an affection of the urinary organs. Its importance to the urinary surgeon rests entirely upon the bearing the particular condition that underlies the glycosuria may have upon the general health of the patient in connection with the treatment of some coexistent malady of the sexual or urinary organs, or perhaps, on the other hand, what causative agency the latter condition may possess in so affecting the metabolism as to be the main contributory factor thereof. Glycosuria is not necessarily of diabetic origin, though commonly so. Alimentary excesses in the carbohydrates, and alimentary disturbances may produce a transient glycosuria. The existence 
of either of these conditions is to be taken into account in the case of prospective operation upon the urinary tract, although not of necessity a serious complication, other conditions being favorable.

Fehling's test is the time-honored method of finding sugar in the urine, and is generally employed. The reagents are (a) solution of copper, and (b) alkaline solution. One centimeter of each of these reagents is mixed with 4 c.c. of water in a test-tube and boiled to see that no precipitate occurs. To this the urine is added drop by drop, and boiled after each addition. If sugar is present a heavy yellowish or orange precipitate will occur of the oxide of copper. If this does not appear after an amount of urine equal to the test solution has been added, and after the mixture has cooled, no sugar is present. The quantity of sugar present is determined by the amount of test solution required to produce the precipitation of the copper. A table giving the amount each minim of test solution represents is printed upon the box containing the reagents, which is a convenient form of keeping them. Compressed tablets for making the copper and alkaline solutions can also be had, and are standardized for quantitative estimation. These are preferable to the solutions, on account of the instability of the latter.

There are many other tests for sugar in the urine, such as Trommer's, Haines', the bismuth tests and the fermentation quantitative method. For ordinary purposes in determining the presence of sugar, and whether the amount is large or small, as well as any marked changes from one time to another, the Fehling test is entirely adequate.

Acetone and diacetic acid need only be mentioned here in their relation to diabetes and as general prognostic signs. Their presence in the urine may or may not be combined. When they appear in the course of diabetes, the outlook is most unfavorable and their discovery in the urine is a grave feature in the clinical history of other constitutional maladies, such as carcinoma, autointoxication and infectious diseases generally.

Indican.-The presence of indican in the urine is of importance only as an evidence of an unsatisfactory condition of the system, being dependent upon intestinal putrefaction, a sluggish state of the liver, or constipation. As it always may be helpful in the treatment of other maladies to eliminate any unhealthy condition elsewhere which may affect the general well being or interfere with the response to therapeutic measures, it is desirable that indicanuria should be recognized, and if possible, corrected.

Heller's Test for Indican.--1. Remove any albumin that may be present by precipitation (heat and nitric acid and filtration). 
2. Add one or two drops of nitric acid to 4 c.c. of hydrochloric acid.

3. Add fifteen drops of urine; mix thoroughly, and allow to stand. In from five to fifteen minutes the color change occurs which indicates the amount of indican present, according to the depth of color and time of appearance. The color produced is amethyst, which will probably be seen in any urine in ten or fifteen minutes. When the color is very marked and appears quickly, or is of a very deep hue, it is an indication that indican is present in excess.

Another indican test: Mix 5 c.c. of urine with equal amount of hydrochloric acid (c.p.), add slowly about the same quantity (5 c.c.) of $1 / 2$ of 1 per cent. solution potassium permanganate and then $21 / 2$ c.c. chloroform and shake thoroughly. Indican in mild amount produces a purplish hue or a deep indigo; still deeper hue, greenish or reddish signifies marked indicanuria with different degrees of autotoxemia.

Bile Pigment and Acid.-In diseases of the liver (congestion, cirrhosis and atrophy) producing jaundice, bile is found in the urine, and will be recognized by the color of the urine, which is a yellowishor greenish-brown, and the froth is a greenish-yellow.

Gmelin's test for bile pigment consists of dropping the urine upon a thin layer of fuming nitric acid on a white plate, which produces a play of colors at the point of contact, beginning with green, then violet, then red, and last, yellow:

Rosenbach's test is the same as the previous one, only the urine is dropped upon filter paper, and the play of colors furnished by contact of the nitric acid in like manner.

Hemoglobinuria. - The presence of blood in the urine in visible amount has already been referred to in the consideration of the clinical examination of the urine (p. 96). The existence of blood in too small a quantity to be seen by the naked eye is recognized by detecting the red blood-corpuscles under the microscope. When hemoglobin or the coloring matter of the blood passes into the urine without the blood cells, as it does in certain diseases which produce their effect upon the kidneys, such as scarlet fever, pernicious forms of malaria and diseases of malnutrition, like scurvy, etc., its presence is established chemically or by the spectroscope. When the blood cells have undergone disintegration in the urine on account of decomposition, hemoglobin will also be found alone.

Heller's Test.-To a given amount of urine in a test-tube, add one-third the volume of sodium hydrate. Upon boiling, the earthy phosphates are precipitated, stained red with the coloring matter of the blood.

Lipuria and Chyluria.-Fat appears on the surface of the urine 
in drops in lipuria, and in the form of an emulsion in chyluria. The latter condition is due to the escape of lymph into the urine, owing to the presence of filaria sanguinis or from fractures of the long bones or from abscess cavities. The presence of fat globules in the urine may also result from foods rich in fatty constituents. The possibility of their being derived from instruments should naturally be taken into consideration.

\section{EXAMINATION OF URINE (MICROSCOPIC)}

The specimen of urine for microscopic examination should always be fresh, as epithelial cells, casts and other elements undergo disintegration as a result of decomposition. The sediment may be obtained by gravity after the urine has been allowed to settle for a number of hours

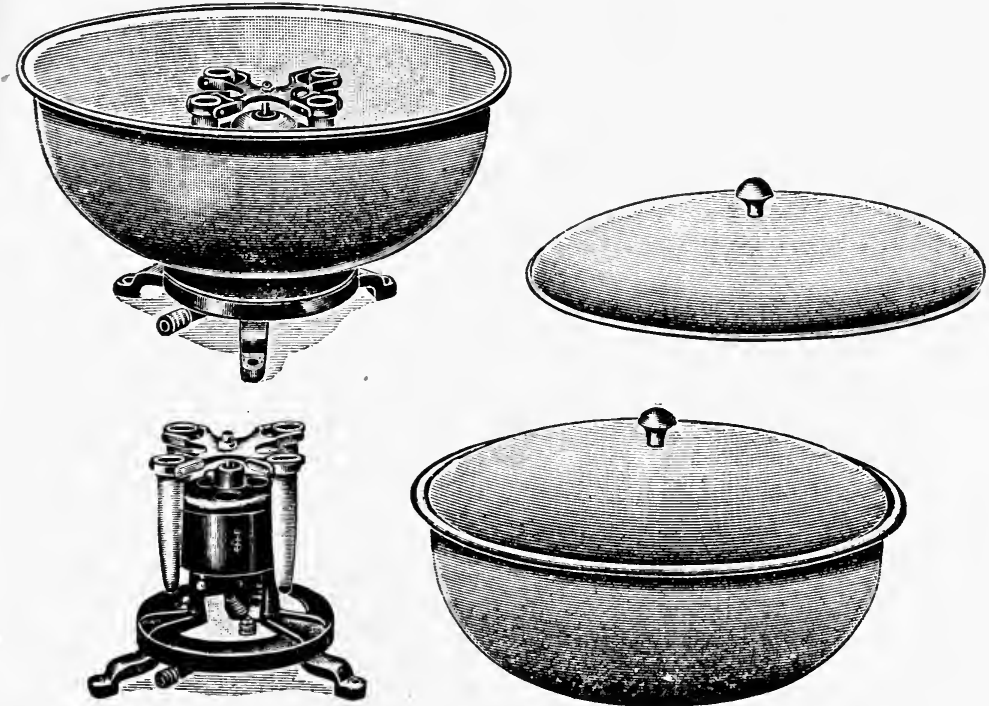

FIG. 53.-Electric centrifuge.

in a conical glass, which is covered and kept in a cool temperature. Preferably, the sediment should be obtained at once by centrifugation. A variety of apparatus are manufactured for this purpose, which are propelled by hand, by water and by electricity. The electrical centrifuge is most satisfactory (Fig. 53), if current is available, on account of the uniformity of the speed thereof, 2000 to 3000 revolutions per minute. Three minutes is a sufficient period to gravitate the sediment with such an apparatus. A drop of the sediment is taken from the 
bottom of the centrifuge tube by means of a fine pipette with a rubber nipple, is placed upon a microscope slide and covered with a coverglass.

A good microscope is equipped with several objectives and eyepieces. For urinary examination, the low and medium power objectives (16 and $4 \mathrm{~mm}$.) are used, and for bacteriological work an oil emulsion lens $1.9 \mathrm{~mm}$. is necessary.

Red Blood Cells. - Red blood corpuscles are seen by the microscope in the urine as a rule unchanged and unaltered in shape. Their normal appearance is that of a biconcave disk of a slightly yellowish hue. They contain no nuclei, but when the periphery is focused, the central portion has the appearance of a round nucleus. When changed by their presence in the urine, their borders become irregular or crenated. They appear in the urinary sediment with other elements, according to the nature and location of the causative factor.

Pus cells are round in shape, and about twice the size of red blood cells, freely granular, and contain one or two nuclei. To clearly differentiate the nuclei of pus cells, add a little acetic acid to the slide, which will produce a clearance of the finely granular background, leaving the clear-cut nuclei in relief. Another test for pus cells, when mistaken for epithelial cells, is to allow a little Gram solution to run under the cover-glass. The epithelial cells turn light yellow, and the leucocytes a dark brown color. The pus cells also undergo changes: in the alkaline urine they swell to a globular shape, or rupture and coalesce in a state of decomposition. Pus cells are seen in association with the characteristic epithelia of the region of the urinary tract affected, and form the greater part of most urinary sediments.

Pus cells from the lower urinary tract appear for the most part as individual bodies while those from the kidney show a tendency to clumping or massing. This fact is made use of in accumulating diagnostic data.

Epithelium.-A few epithelial cells are found in all urine under normal conditions, notably from the urethra, bladder and vagina, but occasionally from various parts of the urinary tract. The recognition of different types of cells is often the means of determining the source of blood or pus in the urine, and many forms may be differentiated beyond question. But there is at times difficulty in stating without some doubt the source of some epithelium, and this is especially true with regard to the round cells of the prostatic urethra and the ureter or the pelvis of the kidney; the last are larger. It is difficult, then, to rely upon the appearance of the 
epithelial .cells alone in determining their source, and only the eye of the most practised microscopist can draw the necessarily fine distinctions in this respect. The different types of epithelial cells are the flat or pavement (urethra, bladder and vagina); round or

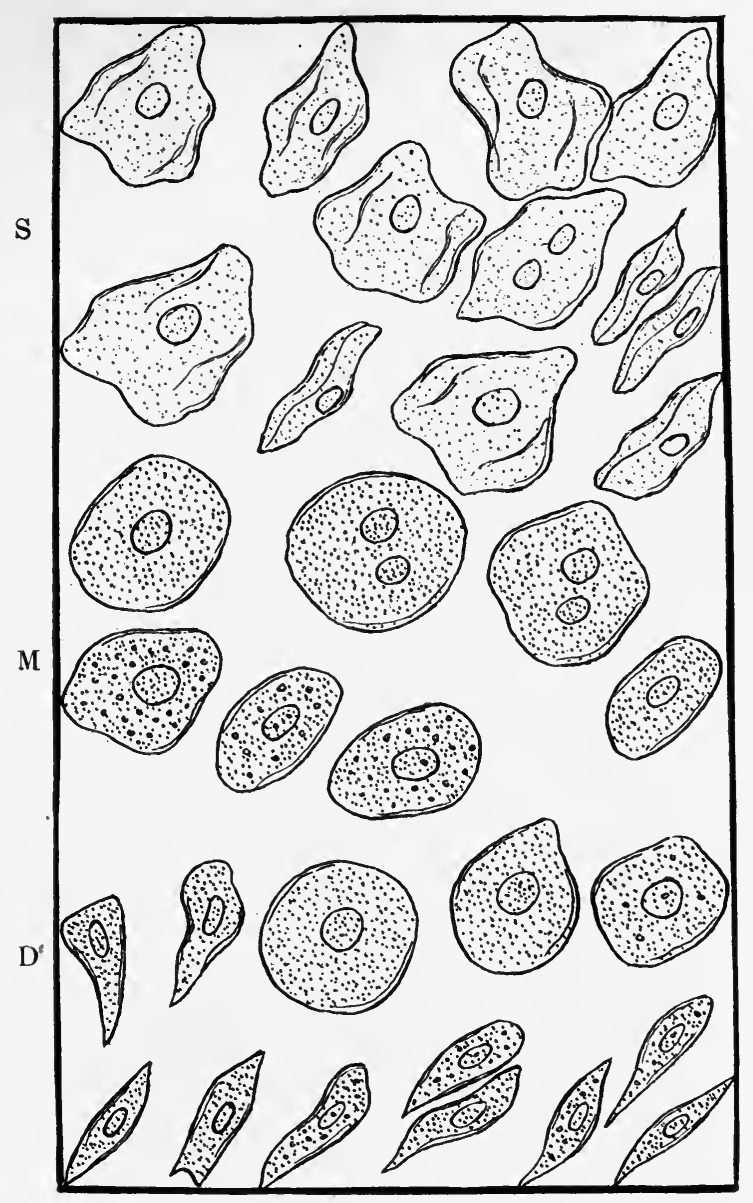

FIg. 54.-Bladder epithelia. S, Superficial layer; M, middle layer; D, deep layer (Heitzmann.)

cuboidal (urethra, prostate, bladder, vagina, ureter and kidney); cylindrical or caudate (urethra, prostate, seminal vesicles, bladder, ureter and kidney). The pus cell serves as a standard of measurement for the comparative sizes of the different epithelial cells. Generally speaking, the large pavement epithelium of the bladder is 
about six or seven pus-cell diameters in width (vaginal epithelium is somewhat larger). The same type of cell from the urethra is about five pus-cell diameters in width, and the urethral columnar cells are about four pus-cell diameters in their widest portion. The cuboidal cells of the bladder are nearly as large as the more superficial flat cells. The round epithelia from the prostate are the same size as those from the ureter, and smaller than those of the pelvis of the kidney, or about two pus-cell diameters. Those from the kidney tubules are much smaller, being one and one-third the diameter of a pus cell. The round cells from the kidney pelvis are about three pus-cell diameters. The caudate or pear-shaped cells from the

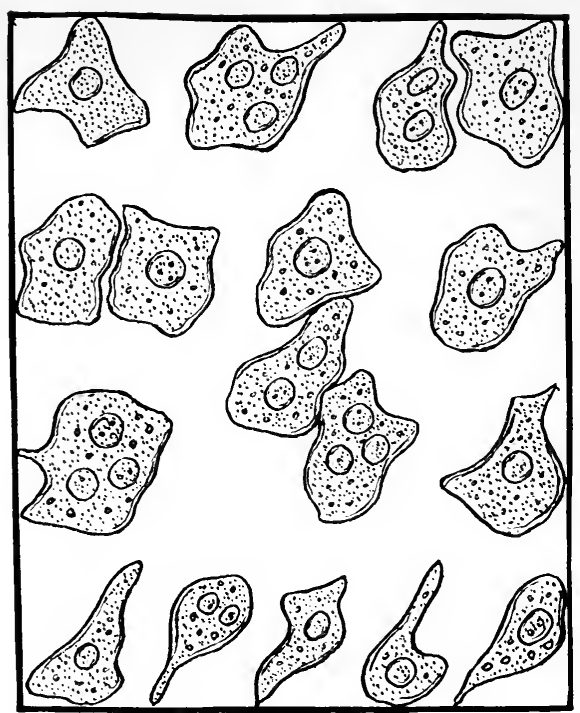

FIG. 55.-Urethral epithelia. (Heitzmann.)

pelvis are about the diameter of a pus cell in width, and about four diameters in length. The same type of cell from the deeper layers of the bladder is much larger.

Bladder Epithelium.-Generally speaking, pavement or flat cells come from the superficial layers (Fig. 54), cuboidal or round from the middle layers (Fig. 54) and caudate cells from the deepest epithelial stratum (Fig. 54). They appear separately and in clusters, and in the case of bladder inflammation are seen in the midst of numerous pus cells. The more intense or prolonged the inflammation of the bladder, the deeper the epithelial exfoliation.

Urethral Epithelium (Fig. 55).-Epithelium from the urethra 
varies according to the portion of the canal it comes from. At both extremities, near the meatus and near the opening into the bladder, it is of the stratified pavement variety; beyond the internal meatus or the fossa navicularis, the epithelium is columnar down to the membranous urethra. In the prostatic portion it gradually fuses into the flat form of the bladder, except from the ducts and glands, where it is columnar and cuboidal. These latter, as stated, resemble closely the cells from the renal pelvis, but there may be other distinguishing features in the sediment to aid in determining their source. The cells from the seminal vesicles and ejaculatory ducts are ciliated columnar epithelium.

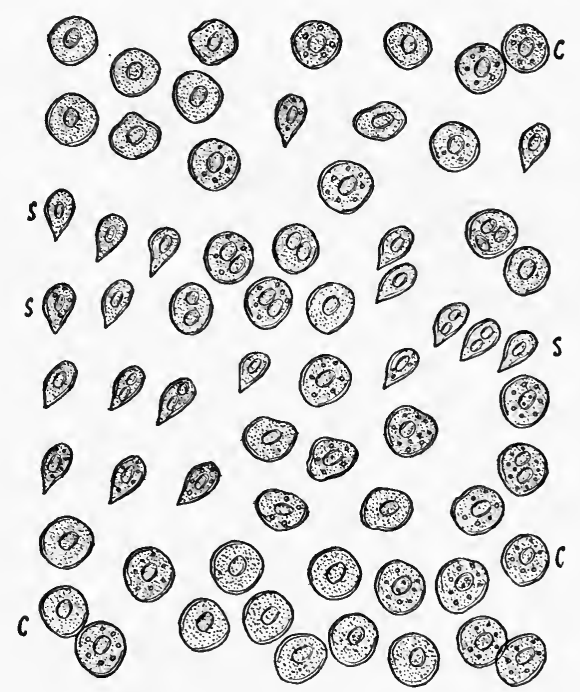

FIG. 56.-Renal epithelia. S, Straight tubes; C, convoluted tubes. (Heitzmann.)

Renal Epithelium.-From the pelvis of the kidney, the epithelial cells are both caudate and round. The latter resemble the prostatic cells, but are larger, and are smaller than those from the bladder. The caudate form is more characteristic (Fig. 56). The renal cells which come from the secretory tubules of the kidney are smaller than those from the pelvis; their nuclei are larger in proportion to their size, and more granular. These cells appear in the urine in all stages of renal inflammation and degeneration, when they also are found in the epithelial casts. These round epithelia come from convoluted tubules; from the straight tubules, the cells are columnar in variety. 
The cells from the uretters are round from the surface, resembling closely the prostatic cells, and caudate from the deeper layers (Fig. 57).

Renal Casts. - It is not within the scope of this treatise to go fully into the consideration of the maladies producing the different kinds of renal casts, the clinical significance of which has more a medical than a surgical bearing. But as in some surgical lesions their presence forms a part of the clinical picture, and as some unilateral lesions of the kidneys exist which are, in their etiology, diagnosis and treatment, of surgical import, a knowledge of the variety and meaning of these products is of interest to the urologist.

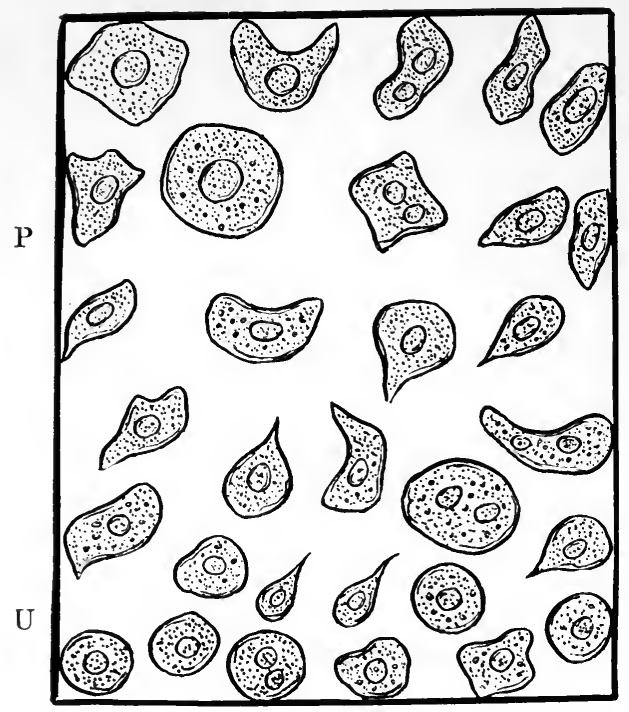

FIG. 57.-Epithelia from the (P) pelvis and (U) ureter. (Heitzmann.)

Generally speaking, the different types of casts are hyaline granular, epithelial and waxy. To these bodies may be added pus cells, blood corpuscles, fat globules, crystals and bacteria, changing the denomination of the casts according to the predominant element of which they are composed. Casts are cylindrical moulds of the renal tubules, formed primarily of albuminous material which on account of blood pressure or morbid tissue-change, finds its way into the renal structure and assumes different characteristic peculiarities, according to the diseased condition of the kidney.

Hyaline Casts.-This type is tubular in contour, usually with rounded extremities of homogenous translucent structure and a variety of sizes (Fig. 58). They are frequently difficult to see, requiring fine 
focusing, or darkening of the field. They may be very finely granular, are usually symmetrical in outline, but sometimes twisted and irregular in shape. Hyaline casts may appear in the urine of normal individuals under certain conditions of the kidneys, when they are subjected to high blood-pressure or transient irritation, but are also present in most acute and chronic diseases of the kidneys in conjunction with other casts, when the presence of albumin is also a feature.

Epithelial Casts.-These casts indicate exfoliation of renal epithelium. The epithelium may be attached or embedded. The whole

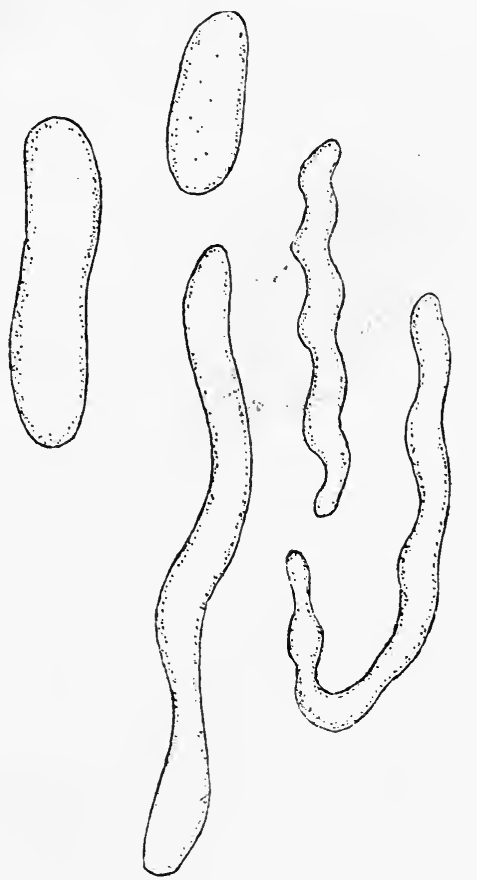

FIG. 58.-Hyaline casts. (Heitzmann.)

or a part of the cast-body may contain epithelial cells (Fig. 59) which are generally vague in outline. They indicate an acute desquamative process of the parenchyma of the kidney. When the epithelial cells undergo disintegration, as a result of a more advanced morbid condition, from the resulting epithelial particles are derived the so-called granular casts. These bodies are of various sizes, irregular in shape, and finely or coarsely granular (Fig. 60), according to the size of the broken-down fragments. They occur in all forms of kidney disease, commonly acute and chronic parenchymatous nephritis. 
Blood casts occur in acute congestion and inflammation of the kidney, accompanied by intrarenal hemorrhage resulting from stone, neoplasm or tuberculosis.

Fatty casts are composed of a hyaline cylinder studded with fat globules of different sizes, and occasionally with the needle crystals of fatty acids. Their appearance is distinguished from the coarsely granular casts in being highly refractive. They are present in chronic nephritis of the parenchymatous type.

Waxy or amyloid casts are really a form of the hyaline variety. They are gelatinous and refractive in appearance, of a tallow-like

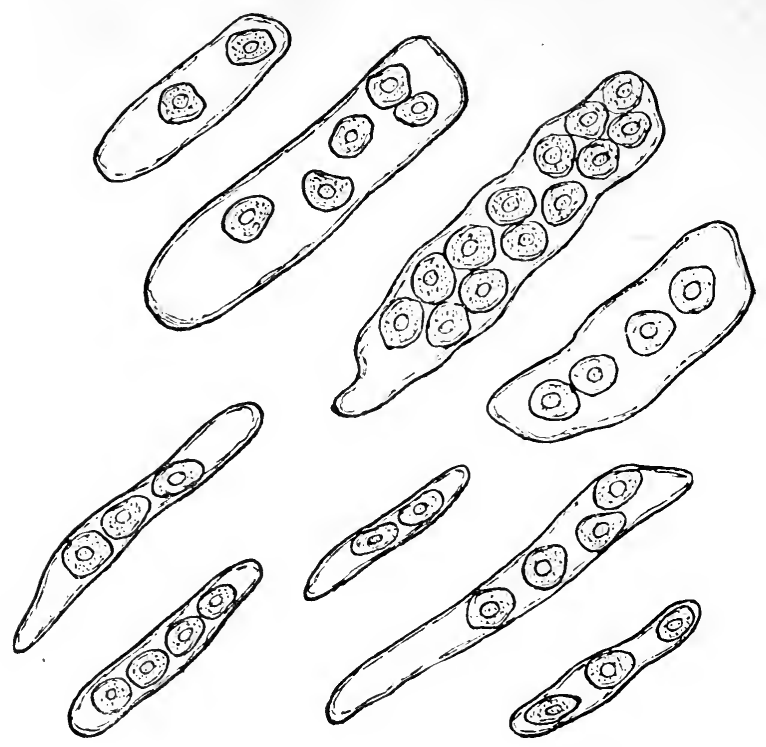

FIG. 59.-Epithelial casts. (Heitzmann.)

character, sometimes broken and indented. They indicate an advanced chronic state of inflammation of prolonged duration and bad prognosis.

Other types of casts, such as pus casts, crystalline casts and bacterial casts are so designated because these particular elements are a component part of or cover the surface of the hyaline cylinders. Pus casts occur in chronic suppuration of the kidney.

False Casts.-These formations are usually shreds of mucus moulded into various lengths and irregular shapes. They are differentiated from true casts by their greater length, tapering ends, not clean cut or round, and their variety of sizes. If they contain 


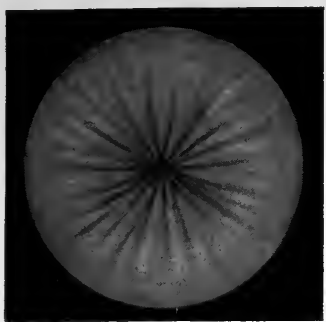

Fıg. 1.

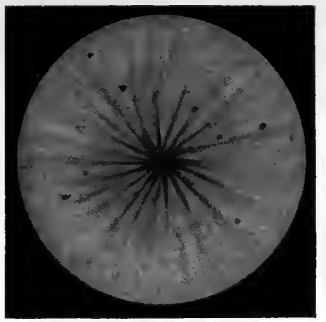

FIG. 2

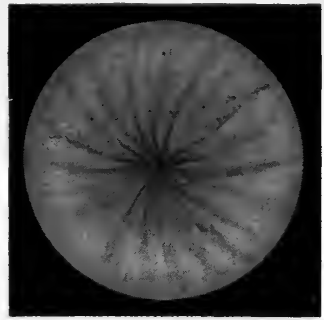

Fig. 3.

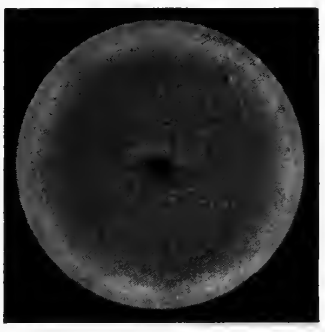

FIG. 4.

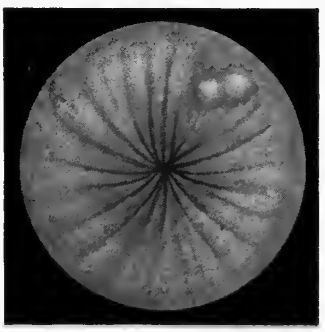

Fig. 6.

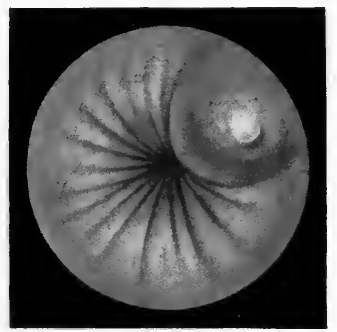

FIG 5.

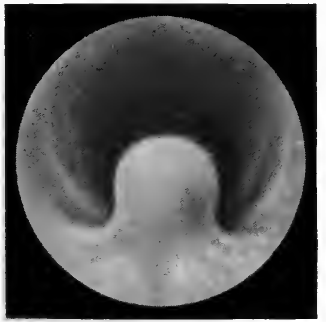

FIg. 7.

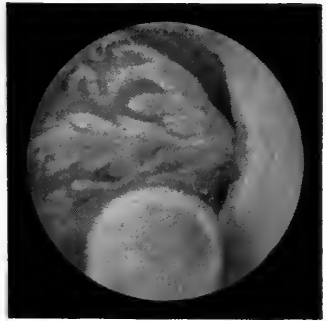

Fig. 8.

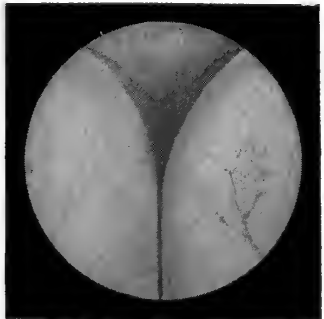

FIG. 9 .

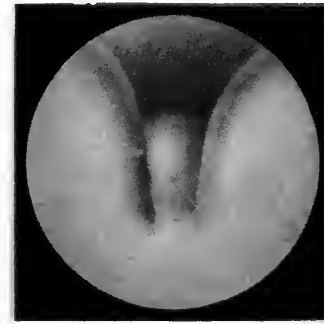

FIG. 10.

Fig. 1.-Normal Urethral Mucosa. FIg. 2.-Chronic Urethritis with Erosions. Fig. 3.-Chronic Urethritis, Vesicular. Fig. 4.-Stricture of the Urethra. Fig. 5.-Follicular Abscess. Fig. 6.-Cysts of the Mucosa. FIg. 7.-Posterior Urethra. The Verumontanum. Fig. 8.-Papilloma of Prostatic Urethra. FIG. 9.-Bi-lateral Prostatic Hypertrophy; Posterior Urethral View. FIG. 10.--Bi-lateral and Median Prostatic Hypertrophy; Posterior Urethral View. 

granules, as they sometimes do, acetic acid quickly dissolves them. These bodies originate both in the kidney and the bladder, and in distinguishing them, it will be found that they do not conform to the characteristics of any of the types of true casts. This fact, together with the absence of any other evidence suggestive of a condition that could account for the presence of true casts, should prevent confusion.

Acid urine may contain a deposit of amorphous urates, urate of soda and potash, uric acid and calcium, oxalate crystals.

Alkaline urine may contain amorphous phosphates (calcium phosphate), triple phosphate or ammonio-magnesium-phosphate crystals, urate of ammonium crystals.

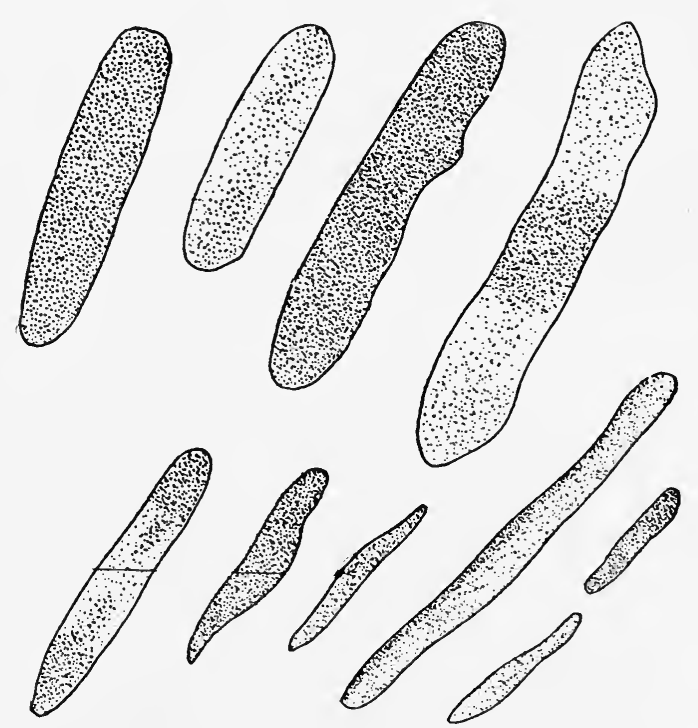

Fig. 60.-Granular casts. (Heitzmann.)

Other substances more rarely found in urinary deposits are crystals of leucin and tyrosin, cystin, bilirubin (crystalline and amorphous) and cholesterin. The first two are the result of distinctive diseases of the liver, cystin of putrefaction in the intestinal tract. Bilirubin is present in urine containing bile, and cholesterin is a product of broken-down tissue or fatty degeneration.

Uric acid crystals occur in the urine as a "reddish" granular deposit, visible to the naked eye. They are the result of a highly nitrogenous diet, and sometimes, but not necessarily, an evidence of renal lithiasis or uric acid diathesis. They appear microscopically in a variety of shapes, such as rhombic, parallelogram, whetstone 
and stellate formations. They are generally of a bright yellow hue, but may be colorless (Fig. 61).

Amorphous urates show in the urine a characteristic brick dust deposit, and under the microscope yellowish or brownish granules.

Ammonium urates are the so-called "hedge hog" crystals, being

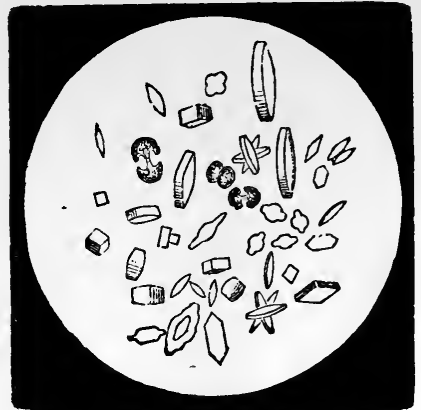

FIG. 61.-Crystals of uric acid. (Kirke's Physiology.)

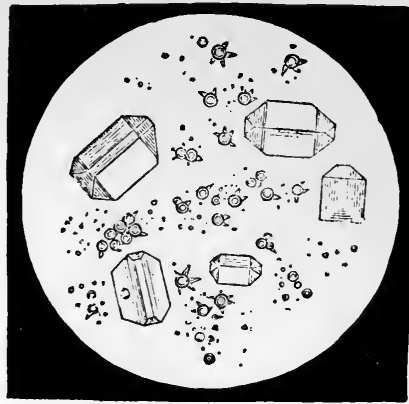

Fig. 62.-Crystals of triple phosphates and urate of ammonium. (Kirke's Physiology.)

spherical bodies presenting fine surface projections, some of them long and irregular, like thorns, and others symmetrical, like chestnut burrs (Fig. 62). They are the same color as the uric acid crystals, but occur in alkaline urine often in conjunction with the ammoniomagnesium crystals.

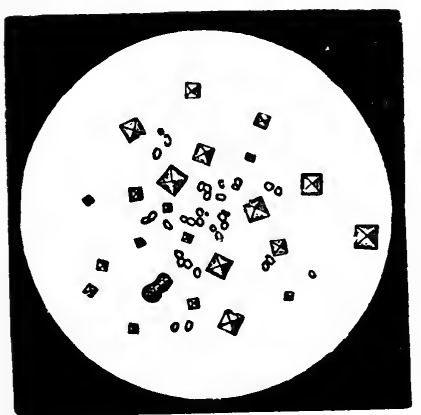

Calcium oxalate crystals occur in two common forms, the octahedral or envelope shape and the dumb-bell or hourglass variety (Fig. 63). They may be present in normal urine following certain articles of diet, notably vegetables and fruits which contain oxalic acid. Their constant presence may be an evidence of neurasthenic condition, an accompaniment of defective metabolism, characterized by dyspepsia, languor, malaise, etc.

Fig. 63.-Crystals of oxalate of When present in large numbers, with a lime. (Kirke's Physiology.) tendency to conglomerate, they may be significant of renal calculus of similar composition; but this by no means follows.

Ammonio-magnesium or Triple Phosphate Crystals (Fig. 62).These crystals are the result of the ammoniacal decomposition of the urine, and usually occur in the bladder, although they may also exist 
in the pelvis of the kidney. They appear microscopically in "coffinlid" or oblong prism form and stellate or fern-like variety. Some of the former are like the stone of a seal ring (Fig. 63). They are present in the bladder in chronic cystitis, and in old chronic infections of the kidney, and under favoring conditions, i.e., ulceration of the mucosa, bacterial activity and mucus formation, may result in the production of renal or vesical calculi.

Amorphous phosphate is the alkaline deposit of calcium phosphate, producing "phosphaturia," which is indicative of a corresponding alkaline diet or of a neurasthenic condition. The appearance in the urine is a flocculent powdery deposit, and microscopically a whitish granular sediment.

\section{Summary of Urinary Sediments}

Hyaline and epithelial casts, together with cells from the tubules of the kidney, indicate inflammation of the kidney of an acute degree; granular casts, the more chronic forms of renal disease, and waxy and fatty casts, a still more advanced stage.

Red blood cells and blood casts suggest hemorrhage in the kidney. Fresh blood cells in numerous quantities, without kidney elements, but with bladder epithelium, point to vesical hemorrhage. Pus cells, with cells from the pelvis or renal tubules, signify suppuration in the kidney, pyelitis or pyelonephritis. Pus cells in abundance, with bladder or urethral epithelium, are evidence of cystitis or urethritis. The addition of triple phosphate crystals denotes chronic cystitis, with partial or complete retention of urine. Added to these elements, we may find in the urine the mixed and special forms of pathogenic bacteria, defining the nature and cause of the inflammatory lesion.

\section{Bacteriology of the Urine}

The normal urine is sterile, and when it passes through the urethra, it becomes contaminated by the microörganisms which are always present in the anterior section. Therefore, for bacteriological examination, when it becomes important to isolate certain organisms which are a factor in existing lesions, the urine should be taken by means of a sterile catheter and received in a sterile vessel. In certain infectious diseases, the bacteria may pass through the genito-urinary tract without causing a lesion thereof.

The microörganisms which are found in the uterine are pathogenic and non-pathogenic. The latter cause the decomposition of urine outside the body, namely: the micrococcus ureæ and the bacterium 
urea, etc.; yeast molds and fungi are found in fermenting and decomposing urine, especially in diabetes. Many varieties of organisms may be found by bacteriological examination of the urine. In inflammation of the urinary system practically all the organisms capable of producing infection have been demonstrated, and in general infections bacteria may be excreted by the kidneys with or without causing lesions. The principal pathogenic bacteria found in the urine are the staphylococcus pyogenes aureus and albus;

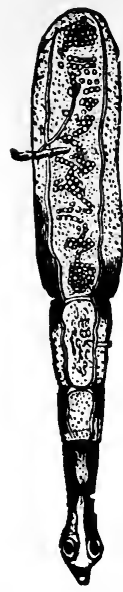

Fig. 64.Tænia echinococcus. streptococcus pyogenes; proteus vulgaris; pneumo coccus; bacterium coli; and the specific organisms, gonococcus and tubercle bacillus, as well as those of typhoid, glanders, anthrax, etc. Frequently there is a mixed or combined infection with these organisms. Typhoid bacilli are found in the urine of one-third to one-half the cases of typhoid fever. The same is true of paratyphoid infections. When present they are usually in considerable numbers and may persist for months. And mention may be made of the animal parasites, which are found in the urine in rare conditions, the filaria sanguinis; the parasite of chyluria; the distoma hematobium indigenous to the tropics, which, when present, are a cause of hematuria, and the echinococcus, the head of the parasite and the hooklets of which are found free in the urine in the event of hydatid cysts of the kidney (Fig. 64).

Bacillus Coli.-The colon bacillus and its numerous kindred varieties known as the colon group are natural inhabitants of the intestinal tract and are found frequently in small and large quantities in the urine. In many of the cases of cystitis and pyelitis reported as due to the colon bacillus, that organism is frequently present by contamination. In the bladder they probably reach there by diapedesis through the lymph channels, and in the kidney in the same manner or by hematogenous route. It should also be remembered that the urine may contain colon bacilli in such numbers as to cause clouding without any lesion being present. This organism is, however, the factor in the great majority of cases of non-tuberculous cystitis. ${ }^{1}$ It is also the principal pyogenic factor in mixed and tuberculous infections. Its importance in mild renal suppuration, while disputed, is becoming generally recognized. ${ }^{1}$ Frequently there is a mixed

\footnotetext{
1 Guyon believed it to be the most frequent etiological factor while Rovsing, on the other hand, held that it was rather innocuous and never produced destructive lesions of the kidney.

Anthony Bassler (Med. Record, July 6, 1912) examined 191 fresh urines and found B, coli present in over 9 per cent. in persons having no genito-urinary symptoms.
} 
infection in combination with the staphylococcus, streptococcus, bacillus pyocyaneus, or tubercle bacillus.

The. Streptococcus and Staphylococcus Pyogenes Aureus (or Albus).- Next to the colon bacillus these are the most common causative microörganisms in infections of the kidney and bladder. They may be conveyed to the bladder from the urethra and are likely to become pathogenic under the circumstances of the rough handling of instruments, or other traumatism, in the presence of a preexistent morbid local condition, or a generally lowered resistance due to constitutional depreciation (tuberculosis, etc.). Hematogenous infection by these organisms also occurs (see Bacterial Infection of Kidney); the streptococcus is usually traceable to a suppurative focus of the mucous membranes; the presence of staphylococcus pyogenes albus in the urine may possibly be due to contamination from a skin lesion.

Gonococcus.-On account of the frequency of gonorrheal urethritis gonococci are common occupants of the urethra and may be carried into the bladder or spread there by cellular infiltration as well as to any part of the urethral adnexa. Invasion of the bladder by the gonococcus is usually limited to the neck. General cystitis following gonorrheal urethritis may be due to other organisms for which the gonococcus has paved the way. Gonococci have been frequently found in the serous exudate of the tunica vaginalis of gonorrheal epididymitis and cases of infections of the pelvis of the kidney in which a pure culture of the gonococcus is obtained are now and again reported. Infections of the prostate are mainly due to this organism. ${ }^{1}$

Tubercle Bacillus.-Tubercle bacilli are discovered in the urine in tuberculous infection of the urogenital system. These are, however, sometimes difficult to find in the urine and the number present gives no evidence of the extent of the process. In early tuberculosis of the renal papillæ the urine may contain large numbers of bacilli, while in old processes involving one pole or practically the entire kidney there may be extraordinarily few, which may be due to the fact that the foci or area involved may be completely devoid of normal secretory substance and closed off from the renal pelvis.

The differentiation of the tubercle from the smegma bacillus, which is found in the preputial and vulval excretions, is important and sometimes difficult. By taking the urine with a catheter after sterilization of the external parts the risk of contamination is practically

\footnotetext{
1 Segond gives the bacteria found in prostatitis in the order of their frequency as follows: Gonococcus, staphylococcus, streptococcus, B. coli, pneumococcus, anaerobes.
} 
avoided. Several methods of differential staining have been devised, depending on the fact that the smegma bacillus is more easily decolorized by alcohol. It is claimed that antiformin dissolves all bacteria except the tubercle bacillus. These methods are useful but cannot be absolutely relied upon. While the morphology suffices in the majority of instances, the final reliance in doubtful cases must be placed in the results of animal inoculation. 


\section{CHAPTER V}

\section{SERODIAGNOSIS AND SEROTHERAPY}

The action of both serodiagnosis and serotherapy depends upon the properties of certain substances contained in the blood of all mammalia, which substances are known as antibodies. These antibodies are governed in their action by a specific law which action is taken advantage of for diagnostic and therapeutic purposes.

Antibodies are of several kinds, and their specific action in the blood serum is demonstrated in the presence of infective organisms. Thus, "agglutinins" possess the power of gathering the infecting organism into clumps; "precipitins" produce a precipitation of the same; "antitoxins" neutralize the product of the bacteria; "bacteriolysins" dissolve them, whereas "bacteriotropins" and "opsonins" so change the bacteria that they are the more readily taken up by the leucocytes. Thus is produced through the agency of these antibodies what is known as immunity. When such immunity occurs spontaneously following recovery from an infectious disease, the body as a result is in a state of complete resistance to that disease or of "active immunity." The same condition may be produced artificially by injecting into the individual or animal the serum of an animal which is already actively immune (passive immunity).

\section{SERODIAGNOSIS}

The specific antibodies used for diagnostic purpose are the agglutinins, the precipitins and lysins. The phenomenon of agglutination is made use of most commonly in the diagnosis of typhoid fever. The precipitation test is little used for clinical diagnosis. We are mostly concerned here with the action of the bacteriolysins and hemolysins.

It was discovered in bacteriological research (Pfeiffer) that the peritoneal exudate of the guinea-pig immunized to cholera vibrio had the power of causing the disintegration and subsequent disappearance of living vibrios (bacteriolysis) and the blood serum of an immunized animal may be utilized in the blood of another to produce its specific action of hemolysis. 
Complement Fixation Tests.-If immune serum containing a lytic substance be kept for several days or be heated for $1 / 2$ hour at $56^{\circ} \mathrm{C}$. it becomes inactive. Its activity may then be restored by the addition of fresh serum from a normal non-immune animal of the same or different species. The reactive substance is known as "complement." Lysis is therefore a process for the production of which two substances are necessary, a lysin (amboceptor) or specific antibody, the product of immunization of tried stability by temperature test under $60^{\circ} \mathrm{C}$., and a complement which is a non-specific component part of normal serum.

The Wassermann Reaction.-The Wassermann reaction is a test for the presence of the syphilitic antibody in a given serum and depends, like all complement fixation tests, on the fact that antigen brought in contact with a specific antibody in a serum forms a combination with that antibody and in so doing, "absorbs complement." The absorption or non-absorption of complement is made evident by the addition of a second (hemolytic) system after incubating the first. The normal constituent of fresh serum (complement) is destroyed in the patient's serum by heating for $1 / 2$ hour at $56^{\circ} \mathrm{C}$. A definite amount of complement is then added in the form of fresh guinea-pig serum. As a hemolytic system in the regular Wassermann technic, a suspension of washed sheep corpuscles is used and the serum of a rabbit which has been immunized to sheep corpuscles. In the latter, the complement is destroyed as in the patient's serum, by heating $1 / 2$ hour at $56^{\circ} \mathrm{C}$. (This is done to eliminate unknown quantities of compliment.) For the rabbit's serum, to produce hemolysis of the sheep corpuscles, complement is necessary. If then, antibody is present in the human serum, complement will be "fixed" by the first system and no hemolysis will result on the addition of hemolytic system. On the other hand, if no antibody is present, complement will remain free and hemolysis of the sheep corpuscles will result. Wassermann, at first, used as an antigen an aqueous extract of the liver of a syphilitic fetus. It was found later that extract of normal organs (heart, liver) would serve as well as those containing the spirochætæ pallida. The nature of the substance in syphilitic blood on which the reaction depends is not known. It is spoken of as antibody because of its development with that of the disease and its disappearance with that of the disease.

The results of the reaction quoted by different workers vary. ${ }^{1}$

\footnotetext{
${ }^{1}$ Boas (Die Wassermansche Reaktion, Berlin, 1911) reports primary syphilis 974 cases, 56.5 per cent. positive; secondary syphilis 3762 cases, 88 per cent. positive; tertiary syphilis 830 cases, 80 per cent. positive; latent syphilis 1111 cases during the first three or four years after infection, 46 per cent. positive; 1452 cases longer standing, 30 per cent, positive. Tabes dorsalis, 360 cases, 70 per cent. positive; dementia paralytica 95 to 100 per cent. positive.
} 
The reaction usually appears about six weeks after the infection and reported results in primary syphilis differ from as low as 18 to as high as 90 per cent. of positive reactions. In secondary syphilis, reports vary from 77 to 100 per cent. positive reaction.

Tertiary syphilis yields from 45 to 100 per cent. positive reaction according to different writers.

In late and obscure cases where the regular Wassermann yields a negative reaction, especially where the clinical history tends to cast doubt upon this result the spinal fluid is obtained by lumbar puncture and submitted to the complement fixation test. In such cases it is often possible to secure the confirmation sought for, the spirochætæ being lodged in the spinal canal in sufficient number to produce a positive reaction. This result has an important bearing upon the future course of the malady and the prognosis thereof.

In congenital syphilis, statistics vary considerably but the percentage of positive reaction, in cases with symptoms, is high. The reaction may be negative at birth and positive a few weeks later.

A positive reaction will usually disappear under treatment with mercury. The disappearance may precede the disappearance of symptoms but more often is coincident with it or does not follow for a considerable interval. The reaction may disappear immediately after the treatment with salvarsan but is more likely to clear up slowly. After a negative reaction has been obtained following treatment with either mercury or salvarsan, a positive reaction may again appear. Rarely, a reaction previously negative will become positive after treatment. In some cases of weakly positive or uncertain reaction a strongly positive reaction will follow the administration of a single or small dose of salvarsan. This is alleged to be due to the continued presence of inactive spirochætæ which become "reactivated" by the salvarsan not being of sufficient potency to totally destroy them. This effect has been relied upon by some as a final test as to the presence of these parasites. It also suggests an explanation of neuro recurrences following the use of the remedy-possibly in too moderate dosage.

Noguchi Modification of Wassermann Reaction. ${ }^{1}$ - The principal difference in this modification from the Wassermann technic lies in the use of a suspension of human corpuscles and an antihuman hemolytic amboceptor, which latter is preserved by saturating filter paper with the serum and drying. Other modifications of the

\footnotetext{
1 Noguchi, Serum Diagnosis of Syphilis, Philadelphia, 1911.
} 
Wassermann technic are those of Bauer, Stern, Wechselmann, and Jacobaeus.

Method of Obtaining the Blood for Complement Fixation Tests.It should be borne in mind that the portion of the blood required for complement fixation test is the serum only and this portion must be carefully separated before the test can be satisfactorily conducted. It is not practical to transmit blood specimens from a distance for this purpose. If the serum is carefully separated by high centrifugation and placed in a sterile, hermetically sealed tube, transportation that does not require over twenty-four hours in transit is feasible. For the Wassermann complement fixation test, from 4 to 5 c.c. of blood are required. For the Noguchi a lesser amount is sufficient or about 1 c.c. For the gonorrheal complement fixation test, about the same amount as the Wassermann is required. The specimen is obtained in the following manner: The arm is cleansed at the bend of the elbow and a rubber tube is placed above the lower end of the humerus, and clamped. The arm is allowed to hang down and the patient is instructed to close the hand tightly. As the veins bulge into prominence a No. 19 gauge needle is thrust into the lumen of the vessel from below upward. The presence of the end of the needle within the vein is evidenced by the beginning flow of blood, which may be received in a test-tube or syringe. A smaller quantity of blood may also be obtained from the finger tip and collected in a Wright tube. In this manner a liberal supply of blood is rapidly obtained for several examinations. The specimen is then placed in a centrifuge tube and the serum is either separated at once by an electric centrifuge or it is corked and sent to the laboratory for the serodiagnostic test.

Complement Fixation Tests for Gonococcus Infections.-The technic is essentially the same as that of other complement fixation tests, but as different strains of gonococcus differ considerably and the serum of the patient infected with one strain will fix complement only in the presence of an antigen of the same or a very closely related strain, it is necessary to use in the test a polyvalent antigen. This antigen should consist of the extracts of from Io to $\mathbf{5}$ different strains of gonococcus. The greater the number the more reliable will be the result.

The phenomenon of complement fixation is not obtained in acute anterior gonorrheal urethritis and not until at least six weeks have elapsed following acute infection. The test seems to be of considerable value in chronic infections due to the gonococcus, such as chronic urethritis, chronic prostatitis, gonorrheal arthritis, etc. In these cases 
the percentage of positive reactions is high. ${ }^{1}$ In cases where the presence of the gonococcus is demonstrated it has been found positive in $\mathbf{1 0 0}$ per cent. and positive also in a high percentage of cases in those where the gonococcus could not be demonstrated but which gave a probable or possible gonorrheal history.

Ecchinococcus Disease.-The complement fixation test has been used with success in the diagnosis of ecchinococcus disease. As antigen the cyst fluid from a human being or sheep is used. In other particulars, the technic is similar to that of the Wassermann reaction.

The complement fixation tests have also been employed for the detection of renal tuberculosis, but as there are theoretical and practical objections to the test, further proof of its value is needed. ${ }^{2}$

The requirements for the Wassermann and other complement fixation tests prohibit their employment by the practitioner. Not only is a well-equipped laboratory necessary, containing a powerful centrifuge, water-baths, incubator, ice-chest, etc., but also the housing, and constant care of the guinea-pigs, whose serum furnishes the complement for the reaction, and rabbits immunized against sheep corpuscles for the amboceptor. Moreover, time, experience and skill are indispensible, and uniformly accurate results are assured only where ample laboratory assistance is possible, and where similar work is constantly being conducted for control purposes.

\section{Diagnosis with Bacterial Extracts and Fluid Cultures}

The Luetin Reaction for Syphilis.-The material for diagnostic injection is prepared by grinding an ascitic agar culture of spirochætæ pallida in a mortar, diluting the paste thus obtained with an ascitic fluid culture of the spirochætæ, heating the mixture at $60^{\circ} \mathrm{C}$. for an hour and adding 0.5 per cent. carbolic acid. For the control, uninoculated media is prepared in the same way and 0.5 per cent. carbolic acid. About 0.05 c.c. of the material is injected intradermically as

1 Schwartz and McNeal, Jour. of Amer. Med. Asso., May, 1911.

Chronic urethritis of gonorrheal origin:

No. of Positives
Cases

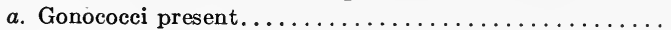

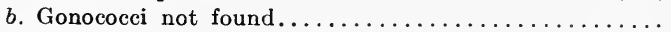

c. Examination for gonococei made............... Chronic prostatitis:

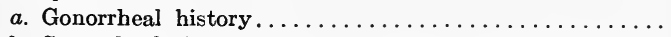

b. Gonorrheal history doubtful................ Joint affections

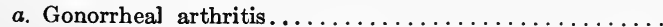

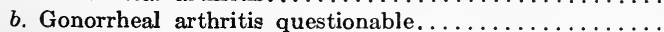

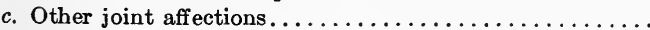

${ }^{2}$ Debre and Paraf; Comptes Rendus d. l. Soc. de Biol., II, 1911.

$\begin{array}{rrr}4 & 4 & 100 \\ 36 & 27 & 80 \\ 8 & 7 & 90 \\ 25 & 17 & 67 \\ 2 & 1 & 50 \\ 14 & 14 & 100 \\ 7 & 4 & 57 \\ 9 & 1 & 11\end{array}$


superficially as possible; the luetin in one arm and the control in the other. In normal individuals a small erythematous area or papule appears at the site of both injections which disappears within fortyeight hours. The positive reaction may be papular or pustular. In the former, the papule appears in twenty-four to forty-eight hours; it is 0.5 to $1 \mathrm{~mm}$. in diameter and is usually surrounded by a diffuse zone of redness. The dimensions and redness increase for the following three or four days and gradually recede. The early course of the pustular form is like that of the papular. About the fourth day, the surface of the papule becomes edematous, softening gradually in the centre, and the resulting pustule ruptures spontaneously, producing a small ulcer with indurated edges which raises a crust and gradually heals. In rare cases, the reaction may be delayed and shows only the slight papular formation seen in normal cases; then after the lapse of ten days or more, there may occur a sudden lighting up at the point of injection which goes on to pustule formation. The reaction is usually absent in primary and secondary stages when it is more apt to occur following treatment with mercury or salvarsan, especially the latter. It is most commonly positive in tertiary and hereditary cases. ${ }^{1}$

Tuberculin Diagnosis of Tuberculosis.-Tuberculin is employed for diagnosis as follows: (a) Koch's subcutaneous method, (b) Von Pirquet's cutaneous reaction (Moro's ointment), (c) Calmette ophthalmic reaction.

Old tuberculin (T. Tuberculin, see p. 113) is used in all cases. This is injected subcutaneously in minute quantity (Koch) or endermically (Von Pirquet); rubbed into the skin in 50 per cent. ointment (Moro) or a freshly prepared 2 per cent. dilution dropped into the eye.

The far advanced cases with cachexia generally favor the reaction to any of the above tests. The Von Pirquet is the most specific in children, but is of no diagnostic value in adults as over 70 per cent. of the latter give positive reactions.

\section{VACCINE AND SERUM THERAPY}

A brief explanation of the condition of immunity has already been given under Serodiagnosis. Closely allied to the process of immunity is that of anaphylaxis. Immunity, however, is a process beneficial to the organism, while anaphylaxis is the opposite, being a state of

1 Noguchi, (Journ. of Experimental Med., 1911) obtained a reaction in 100 per cent. of manifest tertiary cases and 94 per cent. of latent tertiary and 96 per cent. of hereditary cases.: 
increased sensitiveness. If a guinea-pig is injected with a small quantity of horse serum (1 c.c.) and after a period of ten to fourteen days is again injected with a larger amount of horse serum (3 to 5 c.c.), severe symptoms appear within a few minutes, and usually death follows within one hour (Theobald Smith reaction). This condition of exaggerated susceptibility may be produced by other foreign proteids. The subject has been much investigated but is not as yet understood. The practical importance is the danger of producing this condition by improper use of antitoxic sera.

The Opsonic Index.- "Opsonins" is the name given to substances existing in serum which so change bacteria as to make them more readily taken up by phagocytes. Their exact nature is unknown. Opsonins are demonstrated by incubating a mixture of leucocytes, bacteria, and serum, and noting in stained smears the number of bacteria ingested by the leucocytes. The relation between the number of ingested bacteria and the leucocytes is termed the phagocytic count. ${ }^{1}$ The phagocytic index represents the number of bacteria taken up by a leucocyte.

In skilled hands the results of testing the opsonic index seem to be of value. This complicated procedure, however, has been given up to a large extent, and reliance is placed upon the clinical manifestations as a guide in the administration of vaccines.

Immediately after the injection of a vaccine the opsonic index is lowered ("negative phase"). After several days it begins to rise ("positive phase") until it reaches a point considerably above normal, when it again falls. Vaccines should be given during or at the height of the positive phase. Injection during the negative phase results in further depression of the index.

Production of Active Immunity.-Active immunity is produced by the injection of living bacteria, dead bacteria, or bacterial extracts. Living, fully virulent bacteria are seldom used in man. In the cases where living organisms are used a weakly infectious or attenuated form is employed (e.g., small-pox vaccine, antirabic virus).

Immunization with Dead Bacteria.-The best results from vaccine treatment are often obtained when the particular strain of organism is used which is the cause of the patient's infection ("autogenous

1 Wright found that the phagocytic count of infected individuals was low compared with that of normal individuals. The relation between the two he expressed in the form of a fraction. This is known as the "opsonic index." Wright believes that the opsonic index expresses the organism's resistance to infection. He also believes that inasmuch as opsonins are specific it may be used as an aid in diagnosis. For example, the opsonic index of a tuberculous individual is low for the tubercle bacillus, but normal for other bacteria. When an animal is immunized against a certain bacterium, its opsonic index for that bacterium is increased. 
vaccine"). This, however, is not possible in many cases, and a stock vaccine must be resorted to. As the latter are prepared from selected active strains, their potency should be reliable.

The method of preparation is essentially the same in different infections. The organism is grown on large surfaces of a suitable medium. When a good growth is obtained it is removed in a small volume of sterile normal salt solution and shaken with a few glass beads. A convenient method of standardization is that of Wright. Into a capillary pipette, marked a short distance from the tip, are drawn two or three volumes of citrate solution (1 per cent. sodium citrate in 0.85 per cent. salt solution) to prevent clotting of the blood to be added; one volume of blood from the finger tip is then drawn up, separated from the citrate solution by an air bubble, and finally one volume of the bacterial suspension. The whole is then thoroughly mixed by drawing in and out of the pipette on a glass slide, and is finally smeared and stained. From a comparative count of bacteria and cells an estimation of the number of bacteria per volume can easily be made, assuming the red blood corpuscles to number five million per cubic millimetre. The bacteria are then killed at the lowest temperature which will destroy the life of the organism (one hour at $58^{\circ}$ C.). A sterility test should be made by transplants to suitable media. One-half per cent. carbolic acid is then added to the suspension of killed bacteria, and they are sealed in proper doses.

Injections are given subcutaneously in gradually increasing dose at intervals of three or more days. There may be edema and tenderness at the site of injection, and some general malaise, but any marked systemic reaction indicates that the dose is too large. The practice of following the opsonic index during treatment has been largely abandoned. The doses used by different individuals vary somewhat, but the following table gives the usual minimum and maximum limits.

\begin{tabular}{|c|c|c|}
\hline & Minimum & Maximum \\
\hline Sta & million & 2000 millic \\
\hline Str $x_{2}$ & $2 \frac{1}{2} \mathrm{~m}$ & 100 millic \\
\hline Pne & $2 \frac{1}{2} \mathrm{mil}$ & 100 \\
\hline Gon & $2 \frac{1}{2}$ mil & 300 millio \\
\hline 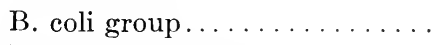 & 5 & 2000 millio \\
\hline eus............... & $\mathrm{mi}$ & $1000 \mathrm{mi}$ \\
\hline Tuberculin (B. E.). & $0.00003 \mathrm{mg}$. & $0.0005 \mathrm{mg}$ \\
\hline
\end{tabular}

When the specific bacterial agency of a given case can be determined, that particular organism is selected for treatment by vaccine, for which purpose the stock vaccine may be tried, and often satisfactorily. On the other hand, an autogenous vaccine may act when a stock fails. 
In the late stages of gonorrheal infection and its complications, the pyogenic organisms, notably the staphylococcus, are often responsible, in the presence of the gonococcus, for the production of such lesions. Therefore, the combined vaccine (stock) of the gonococcus and staphylococcus is used successfully in these cases.

When the exact bacterial character of a morbid lesion is not determined a combined vaccine may be given composed of streptococcus, staphylococcus albus and citreus, bacillus coli and pneumococcus (Van Cott). This may more effectively combat the malady, on account of its complex bacteriology, than the vaccine of a single variety

Bacterial extracts and filtrates are also used to produce active immunity and as a therapeutic measure against infective processes. Of great importance in this category is the treatment of tuberculous lesions.

Tuberculin therapy, however, has not been attended by brilliant success and while there is some difference of opinion, satisfactory results are not sufficiently numerous to earn for it an undisputed claim to popularity. In some instances it is believed that favorable results are obtained by regarding the lesion as that of a mixed infection and bringing in to use the vaccine or filtrate of the pyogenic organisms in combination.

This much may be stated, that no vaccine or bacterial extract is of any service in tuberculosis against the debilitating influence of bad hygiene and improper climatic surrounding or in the case of an active surgical focus, until such lesion has received appropriate surgical treatment. The preparations of tuberculin commonly employed at the present time are the new tuberculin (T. R.) and the bacillary emulsion (B. E.). The dose is arranged as to size and interval to avoid general reaction especially fever, and varies from $1 / 4000$ to $1 / 500$ of a milligram of T. R. which is the mildest form. The emulsion is used in doses from $1 / 100,000$ of a milligram, given from three to seven days apart and continued over a long period.

Bacterial Filtrates.-(Phylacogens-Schaefer). These filtrates are prepared from the various mïcroörganisms in certain combinations and differ from the regular bacterial vaccines in not containing dead bacteria but a derivative of them by filtration.

The mixed infection vaccine is a filtrate composed of the staphylococcus pyogenes aureus, albus and citreus, colon bacillus, typhoid bacillus, pneumococcus, bacillus pyocyaneus, streptococcus pyogenes and the streptococcus erysipelatis. The different organisms are grown in bouillon or agar cultures and are then mixed in known proportions, being standardized in accordance with the usual dosage. 
Its therapeutic indication is in those cases of chronic infective lesions caused by invasion of a variety of bacteria and not dependent upon the specific effect of a single organism although such an organism may have been primarily the causative factor.

Gonorrheal filtrate (phylacogen) is composed of 50 per cent. of the mixed and 50 per cent. of gonorrheal filtrate obtained from a culture of gonococcus and combined in a standardized strength.

The action of the bacterial filtrates (phylacogens) is to promote the production of antibodies like that of dead bacteria and therefore create an active immunity. Their indication is more particularly in subacute and chronic conditions.

The method of using bacterial filtrates is much the same as that of the bacterial vaccines, namely, subcutaneously, and like the vaccines they should never be given during the negative phase, which occurs after the injection when the opsonic index is lowered; but during the positive stage, or after the reaction has subsided. The intervals between injections therefore are from one to three days and the dosage usually employed is from 1 to 5 c.c. of the mixed and 5 to 10 c.c. of the gonorrheal vaccine, beginning with 1 c.c. of the mixed vaccine and 5 c.c. of the gonorrheal and gradually increasing.

There usually follows a local reaction in the form of swelling, redness and soreness around the area of the injection which continues from two to five days. The general reaction may be entirely lacking or may involve a slight chilly sensation, sometimes a distinct chill accompanied by nausea and general malaise. This indisposition usually passes off in from twelve to twenty-four hours. The most scrupulous care should be exercised in the administration of the subcutaneous injection which requires that the syringe and needles should be freshly boiled, the site of the injection cleansed and painted with iodine.

The intravenous injection of phylacogens of the mixed variety has been tried in some instances and the results obtained have been more prompt and strikingly effective. This method, however, is too dangerous and experimental to warrant endorsement at present or to justify its employment except in the most skilled hands and with cautious precision; in fact, it is reported that death has occurred following this method and although such an outcome is an extreme one, it should serve the purpose of instilling great care in its employment. When such method is used the smallest needle attached to a tuberculin syringe is selected; the needle is carefully inserted into the lumen of the vein and a small quantity of blood is drawn to insure the location of the needle. The syringe is then uncoupled and another syringe containing 
the vaccine replaces it. A much smaller dose of the vaccine is employed than subcutaneously, not over 0.25 to 0.50 c.c. and this amount is injected with great deliberation allowing a full minute for each 0.50 c.c. This precaution provides for the slow and gradual diffusion of the preparation in the blood current and minimizes the danger of sudden and severe reaction. The general reaction following this method of introduction is more marked than that accompanying the subcutaneous treatment and the result is proportionately more rapid. Extraordinary care should be adopted in the preparation of the arm and in the technic in general of this operation. The indications for repeating the dose are the same as with the subcutaneous injection.

Passive Immunity.-As already explained (p. 105) in biologic experiments the serum of an animal which is actively immune is injected into another animal to produce passive immunity; in other words, when the system is unable to produce antibodies, this defect is met by the injection of a serum containing these antibodies with a view of actively combating an acute infectious process.

Gonorrheal serum is prepared from the serum of an immunized animal, and is employed more particularly during the acute stage of systemic invasions. The serum is put up in ampules of from 2 to 10 c.c. The dose should be 2 c.c. and repeated two or three days in succession in similar or increased amount but should not be given at long intervals on account of the possibility of producing anaphylaxis (see p. 110).

The serum may be given in combination with the vaccines at the beginning of the treatment for one, two or three injections when the sensitizing influence may prove of value, following which, the treatment may be continued with the vaccine alone.

\section{General Indications for the use of Vaccine and Serum in Lesions of the Genito-urinary Tract}

The use of vaccine, serum or bacterial filtrate has little or no value during the acute stage of urethritis. At this period, the lesion is purely a local one and has not reached the blood current; that is, so far as our present methods of investigation can determine, it is impossible at this stage to distinguish its existence by serodiagnosis. (See Complement Fixation Test for Gonorrhea, p. 108.) Most of the complications of gonorrhea occur after the disease has reached the posterior urethra and it is from this point that systemic invasion usually transpires. The general indications, therefore, for the use of serotherapy in gonorrheal conditions are as follows: 
(1) At the onset of an acute systemic invasion as evidenced by the occurrence of acute toxemia in connection with gonorrheal inflammation.

(2) For the various complications of acute gonorrhea occurring after the inflammation has reached the deeper structures, namely, acute epididymitis, prostatitis, cystitis, pyelitis.

(3) In the metastatic complications of gonorrhea involving the tendons, joints and bursæ, $(a)$ of acute type, $(b)$ of subacute and chronic type.

(4) For chronic gonorrheal complications and sequellæ involving the urethral adnexa, namely, the prostate, seminal vesicles, testicles, bladder, etc.

In the acute stage, the gonococcus and its toxins are the sole causative factors; in the chronic stage, there is apt to be a combination of the staphylococcus, the colon bacillus, etc., which together with the gonococcus are responsible for the production of the complicating lesions. The most brilliant results from the use of vaccine therapy have been encountered in the chronic stage of gonorrheal involvement; and this may be attributed to the production of an active immunity by the action of the vaccine in which respect the system is lacking. In the treatment of the chronic condition, it should always be borne in mind that the more distant the period when the lesion occurs from the initial infection, the more likely it is that the other organisms besides the gonococcus are responsible for the existence of the lesion ; and, therefore, the treatment should be directed with a view of combating the mixed infection by the employment of a mixed vaccine. In any case in which a specific organism can be found predominating it is usually possible to obtain an autogenous vaccine for treatment, but in such cases where this is impossible, a mixed vaccine or bacterial filtrate should be employed.

Acute Gonorrheal Toxemia.-When there occurs during an attack of acute gonorrhea the evidence of acute systemic invasion as manifested by chills, high fever, nausea and general malaise, the indication is that the system is unable to produce sufficient antibodies for the purpose of active immunization, and the production of passive immunity is therefore in order; to this end, serum and not vaccine is indicated. From 2 to 5 c.c. of antigonococcic serum should be administered subcutaneously in the gluteal region and repeated every day in increasing amounts up to 5 or 10 c.c. until three or four such injections have been used. This will usually have the result of reducing the fever but will exercise little influence upon the local condition. Following such treatment, if the systemic conditions subside, local measures may be resumed and further serum treatment is not needed. 
Complications of Acute Gonorrhea.-At the onset of the occurrence of acute epididymitis, prostatitis, cystitis, and pyelitis, there is usually the mildest amount of systemic invasion and as these conditions belong more to the acute than the chronic form of the malady, so they are the least favorably affected by vaccine and serum treatment. In general terms, the more pronounced the systemic invasion the more likely is there to be a favorable response to the vaccine treatment and this is also the case when acute exacerbations occur after prolonged duration of the malady. In such instances, favorable results are obtained by the use of the gonorrheal and combined gonorrheal vaccine and the gonorrheal bacterial filtrate, the gonorrheal and mixed phylacogens. If the gonococcus is abundantly present in the urethral discharge, the gonorrheal vaccine or phylacogen may be used, and if the gonococcus is not demonstrable or in only small number, while the staphylococcus or other microörganisms are present in large numbers, the gonorrheal combined vaccine or mixed phylacogen is preferred. The dose should be 5 c.c. to begin with and repeated every second or third day in increasing quantity up to 10 c.c. for five or six doses. This treatment is supplementary to the usual measures adopted. (See Index.) When suppuration occurs in connection with any of the complicating lesions of gonorrhea, such as prostatic or testicular abscess, vaccines are worse than useless and the moment such a condition is recognized, surgical measures should be resorted to promptly.

Metastatic Complications of Gonorrhea.-In the most acute manifestations of tendon, bursæ and joint lesion which commonly occur during the early acute stage of gonorrhea denoting a mildly acute systemic invasion, it is usually found that the prompt employment of antigonococcic serum in such cases is followed by quick improvement. Five cubic centimeters of antigonococcic serum should be injected subcutaneously every day for three to five days. In favorable cases, the result is obtained at the end of this period and no further treatment is necessary unless the condition passes into a more or less subacute or chronic stage when the treatment of that stage is followed, which involves the adoption of vaccines.

Subacute and chronic tendon and joint involvement, the common form of gonorrheal rheumatism. In these cases, one or more joints are involved and the tendons and bursæ may or may not be included. The condition, beginning in a subacute form, is likely to be chronic and protracted. The treatment requires patience and persistence and the addition of such general and localized measures as are usually employed for the malady in question. (See Gonorrheal Rheumatism.) Some cases are favorably treated by the use of gonorrheal 
vaccine beginning with $20,000,000$ dead bacteria and running up as high as from 100 to $300,000,000$, the repeated injection being given during the negative stage or during the period when the reaction has subsided. Other cases are favorably influenced by the use of the combined vaccine, giving the gonococcus vaccine and a combined mixed culture vaccine (Van Cott) (see p. 113), one cubic centimetre of the latter in combination with the usual amount of the gonorrheal vaccine. In other cases, the combined mixed culture vaccine may prove of value alone without the gonococcus vaccine, which is likely to be the case when there is no longer any gonococcus infection. Again, eases have shown a more ready response to the use of the bacterial filtrate (phylacogens) either the gonorrheal or the mixed, the former where the gonorrheal infection dominates the situation and the latter where invasion with other microörganisms is responsible for a mixed infection. These preparations are given in the usual doses of from 2 to 10 c.c. and at such intervals as the occurrence of reaction and severity thereof suggest ( 2 to 4 days). In old chronic cases the treatment should be kept up over a long period; the greater the duration the longer the interval between injections (once a week to 10 days), the object being first to establish and then maintain active immunity to the infective agent until the latter is overcome.

In the chronic sequellæ of gonorrhea, involving the urethral adnexa, the mode of treatment adopted is much the same as outlined for chronic joint affections. Here in addition to employing the complement fixation test of the blood, the presence of the specific organism as a continued factor in the trouble is further sought out by examination of the inflammatory exudate, be that from the prostate, the seminal vesical or other part of the uro-genital tract. Treatment is then selected upon a specific or mixed infection basis, and the directions given above for chronic joint complications may be followed.

A good rule to be adopted is to examine the urethral exudate and determine its bacterial content, and where possible to employ the complement fixation test for the gonococcus as a means of detecting the present and continued influence of this organism as a systemic factor, and upon the result of such laboratory investigation base the selection of vaccine or bacterin treatment. Where such measures are impossible, it is necessary to adopt the lines already suggested as a means of guidance in the application of treatment. 


\section{CHAPTER VI}

\section{URETHROSCOPIC AND CYSTOSCOPIC DIAGNOSIS AND TECHNIC}

\section{URETHROSCOPY}

Anterior Urethroscopy.-The visual or direct examination of the urethral tube by electric illumination is an important adjunct to diagnostic technic. The instruments employed for this procedure are of two kinds-one that projects the light from without (Fig. 65) and the other that introduces the light into the canal. Good instruments are made of both types. Some urologists prefer one and some the other method. It is well to be equipped with both instruments and become accustomed to the use of either the direct or the reflected illumination. All kinds of urethroscopes use similar straight endoscopic tubes which range in size from 24 to $28 \mathrm{~F}$. Modern instruments designed (Fig. 65) are modifications of the Otis Leiter urethroscope, composed of miniature lamp and condenser in very small and convenient size attached to the outside of the endoscopic tube which projects a strong light into the calibre thereof. A magnifying glass is applied to bring the urethroscopic picture nearer to the eye and intensify the definition.

The other type of urethroscope (Fig. 66) supplies the light within the urethra by means of a minute cold incandescent lamp on a staff or light carrier which is inserted inside the tube; this instrument is manufactured by several instrument makers, foreign and domestic, under different names. The general principle is the same. The electric current for all urethroscopes is obtained either from dry or wet cell battery and, by the use of a rheostat or controller (Fig. 68), the street current, direct or alternating, may be utilized.

The Goldschmidt (Fig. 67) urethroscope is an instrument with an irrigating attachment. While the endoscope is in use the urethra is distended with a current of water, the lamp being so placed as to be beyond the visual field, the lesion being illuminated from behind. The hydraulic pressure by causing dilatation of the canal amplifies the field of vision. The method of distending the urethra by means of air is also employed in the areo-urethroscopes (Fig. 65-B).

Posterior urethroscopy is much more practical than formerly. This is on account of the improvement in instrument manu- 
facture. The instruments employed in posterior urethroscopy, like those for anterior, are provided with direct illumination by means of a small incandescent lamp. The Swinburne instrument has a curved beak for introduction (Fig. 69), and the endoscopic tube contains the lamp which is brought in close contact with the lesion.

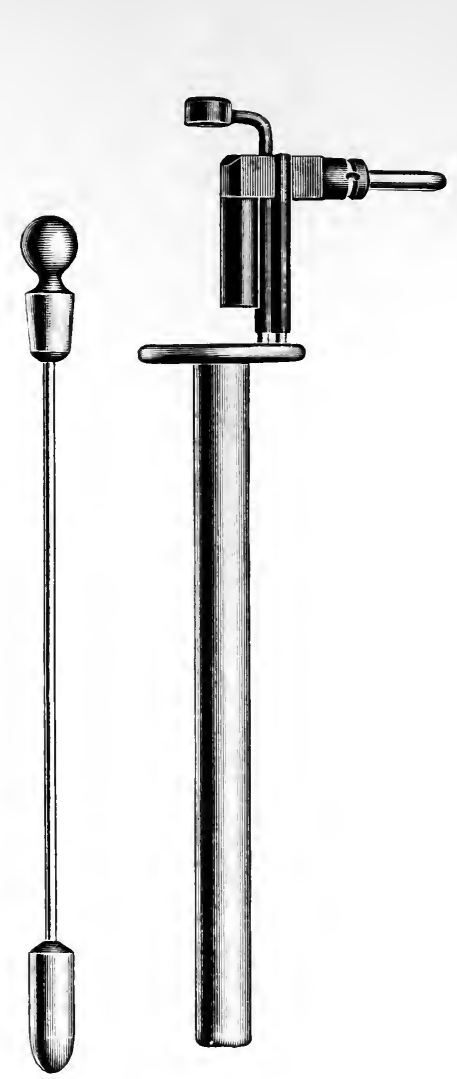

A

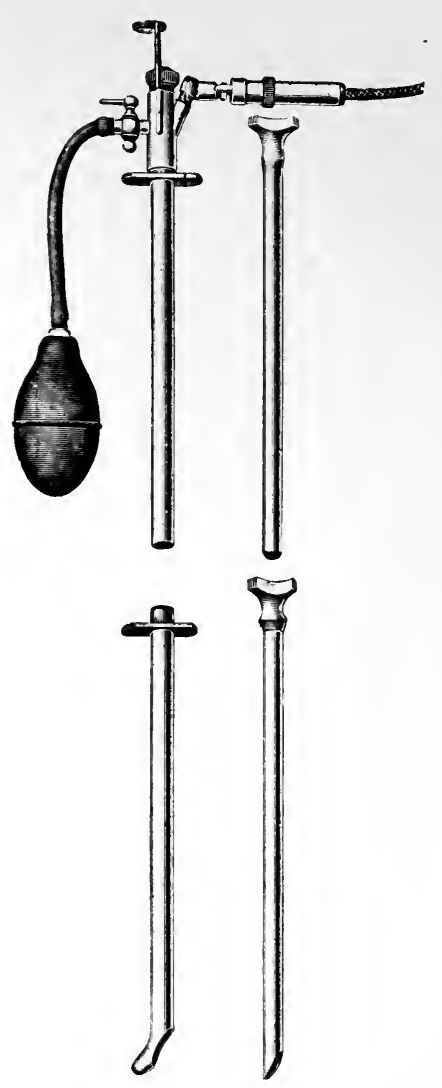

$\mathrm{B}$

FIG. 65.-Urethroscopes using projected light for illumination. (Otis.) A, Squier's modification; B, Hayden's modification.

Through this instrument, a small area of prostatic canal may be viewed, applications may be made to the sinus of the prostate and the veru montanum and it is more suitable for such treatment than for exploration; introduction sometimes causes bleeding which must be continually removed with a cotton applicator to permit satisfactory inspection, and sometimes permits the leakage of urine during the 
examination which must be removed before proceeding. I prefer the instruments which employ distention of the canal by irrigation for examination of the posterior urethra. The Goldschmidt instrument (Fig. 67, A and B) already mentioned may be used posteriorly and the Buerger, a more recent modification, gives an excellent visual field of the posterior canal and the different anatomical characteristics of this region. This instrument is known as the cysto-urethroscope (Fig. 70) and can be used for diagnosis or for the purpose of applying treatment.

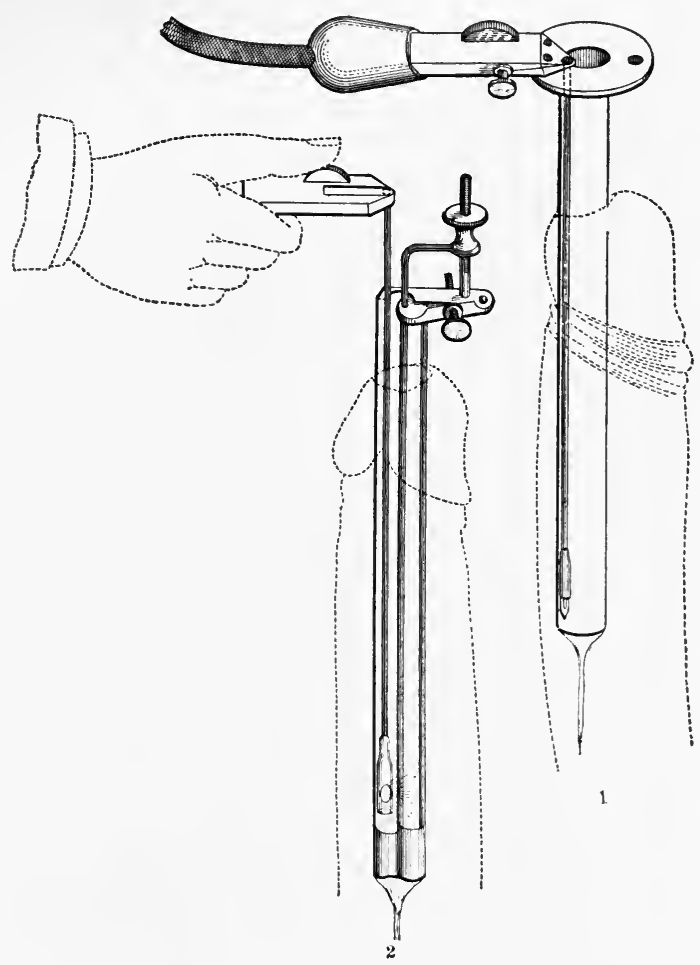

Fig. 66.-Author's urethral endoscope and speculum. Direct illumination.

The author's cystoscope was designed for investigation or treatment of the bladder, under continuous irrigation and may also be used as a cysto-urethroscope (Fig. 78). With the cysto-urethroscope the posterior urethra can be thoroughly inspected and through one of the ureter catheter canals may be passed an electric cautery wire for application of fulgeration or high frequency current, while examination of the neck of the bladder from the anterior aspect to the trigone, 
is accomplished without changing the instrument, as the irrigating device affords continuous vision. The combined features of this instrument give it an extended field of usefulness. For further description see Cystoscopy.

Position of Patient.--The patient may be placed in either a recumbent or semi-recumbent position; the latter is preferable. For this posture, a suitable table is used (the author's modification of the Isaac's table has proved satisfactory) (Fig. 79). The knees are bent, the feet rest upon foot supporters, causing a slight elevation only of the knees

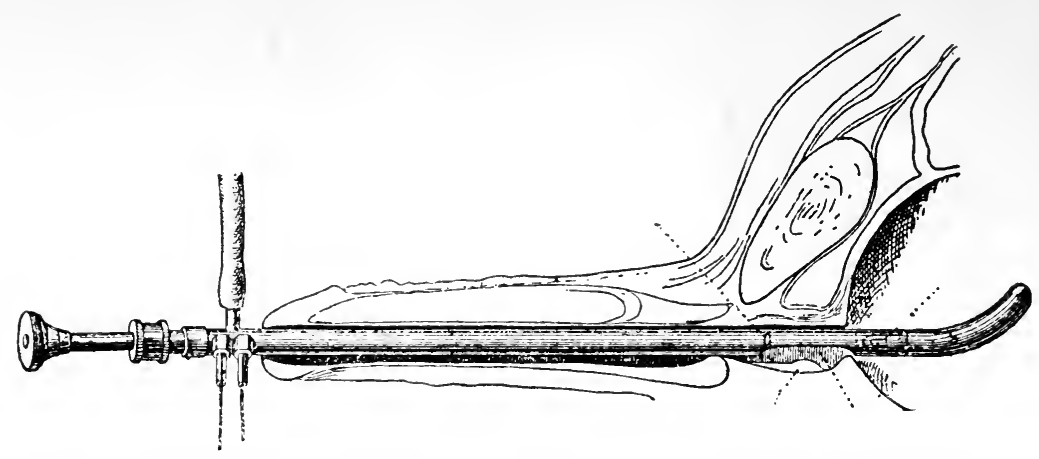

B

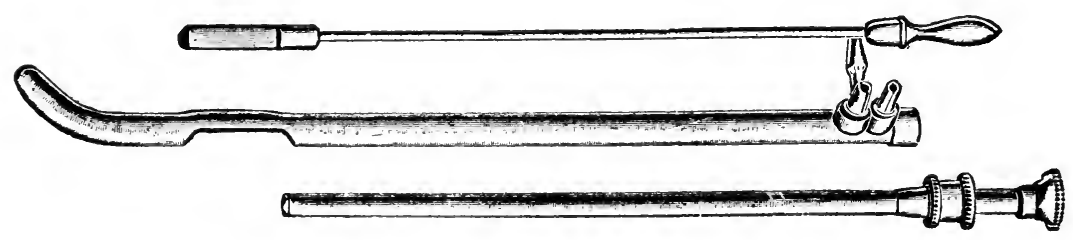

A

FIG. 67.-Goldschmidt's urethroseope (A); instrument introduced (B).

above the table. The surgeon stands or sits between the patient's knees so as to bring the eyes on a level with the opening in the tube.

The Technic of Urethroscopy of the Anterior Canal.-The largest size endoscopic tube that will comfortably enter the meatus is selected. Sometimes on account of the meatus being congenitally tight, it is not possible to introduce the smallest tube. In such cases it is essetinal to enlarge the opening. The larger the tube, the clearer the field of vision but too large a tube for the orifice will interfere with the proper examination causing pain and injury. The urethra may be previously anesthetized with a solution of cocain, novocain, or alypin 
(see p. 46). The tube is well lubricated to allow free manipulation and is then introduced down to the bottom of the anterior canal which brings its terminal end up to the triangular ligament. The obturator is then withdrawn and the electric light attachment introduced in the case of direct illumination or applied to the disk on the end of the tube when reflected light is employed (Figs. 65 and 66).

To make an intelligent endoscopic examination of the anterior canal, a familiarity with the normal anatomical conditions is necessary. The general purpose of urethroscopy is to observe the color and lustre of the mucosa and to recognize any departure from the normal in this respect, to search for the presence of a foreign body or of any morbid lesions, such as granulations upon the surface of the mucosa, dilatations of the follicle mouths, polypoid or papillomatous excrescences, or variations in the contour of the urethral tube as might be produced by stricture formation (Plate II). The normal appearance of the urethroscopic picture is that of a small funnel-shaped pouch beyond the end of the endoscopic tube, the apex of which converges in the centre. The color is slightly yellow with pinkish striations and a normally glistening surface. The openings of the follicles of the urethra, the lacunæ of Morgagni, appear in the form of longitudinal depressions. It should be borne in mind that the pressure of the instrument may mechanically cause anemia of the adjacent area or a hyperemia of the area beyond the tube.

Variations from the normal of this picture are

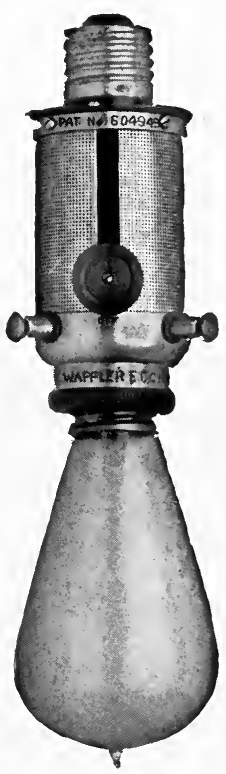

FIG. 68.--Electric controller. encountered (Plate II), represented by differences in color, general or in circumscribed areas; small ulcerations or erosions, as found in chronic urethritis; herpetic vesicles shown in clusters or confluent patches; follicular cyst or abscess swelling; papillomata; thickening of the mucous membrane by fibrous or cellular infiltration showing a loss of softness and distensibility.

The observation is made as the tube is withdrawn. Therefore, the canal is inspected from the triangular ligament forward to the meatus, unless a stricture of the canal of sufficient contraction to prevent the introduction of the tube limits the distance.

Strictures of the Urethra.- The urethroscope permits an inspection of the morbid appearances of the urethra in the region of the stricture, affects the elasticity of the mucous membrane and the uniformity 
of the funnel-shaped cone at the end of the tube. The color may show an abnormally blanched area which does not change in tint with the pressure of the instrument to the same extent as does the normal membrane. It shows also a loss of elasticity giving the

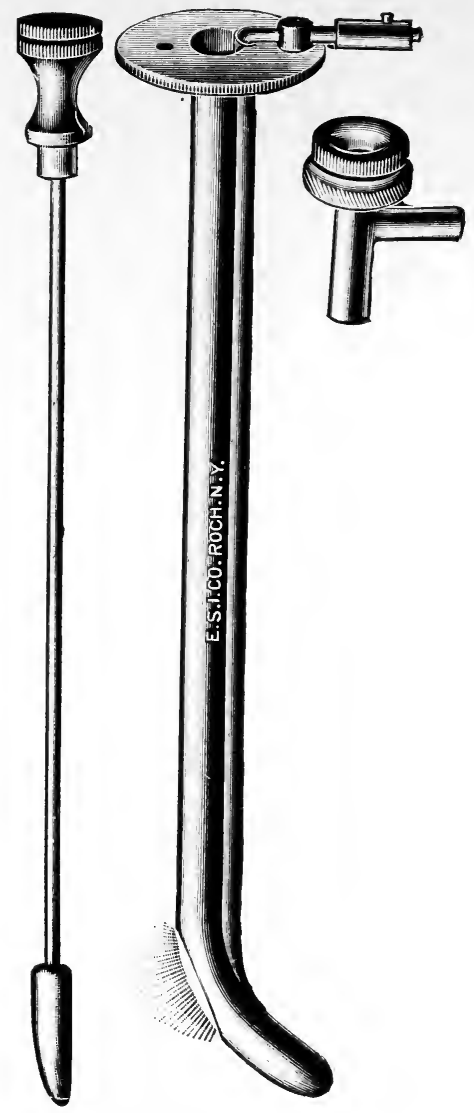

FIG. 69. - Swinburne posterior urethroscope.

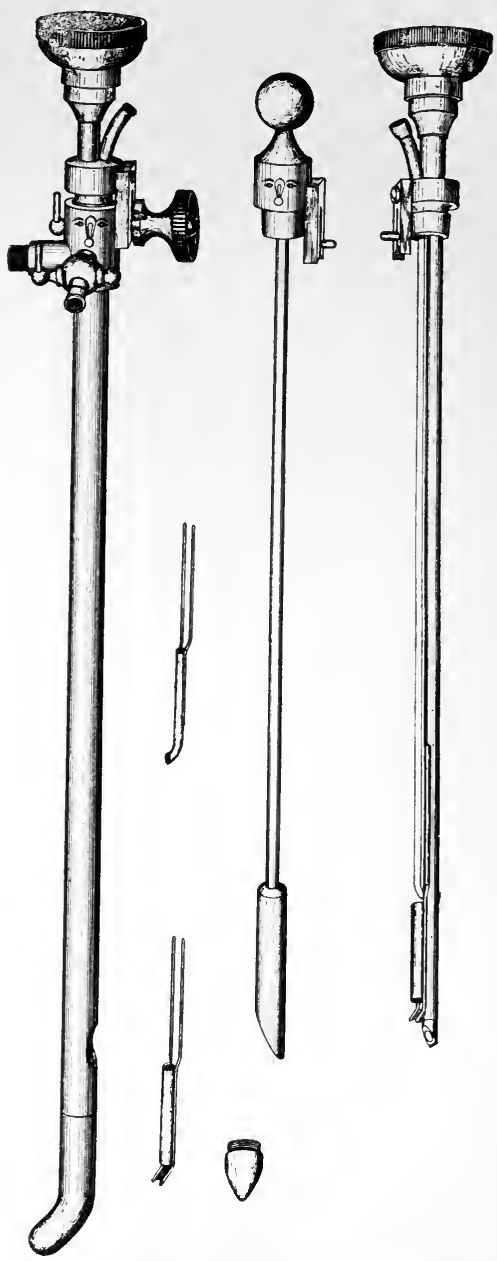

FIG. 70.--Buerger cystourethroscope.

impression of thickening of the tissues to a greater or less extent, according as the stricture may be of a linear or annular variety. The distortion of the conical pouch at the end of the tube is occasioned by the orifice of the stricture which instead of being central, may be anterior, posterior or lateral. In certain strictures when it is difficult to 
introduce even the smallest instrument through the orifice thereof, it is possible to begin dilatation by finding the tiny opening of the stricture with the aid of the urethroscope and then to pass a small bougie through this orifice under direct ocular observation.

Chronic Urethritis.- It is unnecessary to use this method, either for examination or treatment in many cases of simple chronic urethritis and never in acute urethritis. Different types of chronic urethritis are recognized through the urethroscope; in one there will be observed localized areas of congestion, in another granular urethritis, before the stage characteristic of stricture of connective-tissue formation is reached, when is seen a mottled area covered with small elevations reddish and yellowish in hue. This may appear in patches or covering a generalized area. (See Plate II.) Infiltration of the submucous tissue causes concavities of the surface and alterations in the lacunæ mouths. These changes are accompanied by corresponding alterations in appearance, affecting the color and lustre. The surface of the membrane loses its normal pink color and becomes white and lustreless and the infiltration also causes loss of the normal urethral folds in the affected area.

Ulcerations and Erosions.-A spotted appearance of the urethra, as seen through the urethroscope, may be produced by alterations in the epithelium in the form of patches of thickened epithelia which have lost their normal color. This condition has been likened to psoriasis of the skin (Oberlander). Erosions of the surface may be seen in spots denuded of epithelium, which, in chronic urethritis, have an unhealthy appearance, but when produced by the introduction of instruments appear as simple abrasions. Ulcerations are characterized by loss of substance and a depression of the surface. (See Plate II.) They may occur in various sizes and may be the result of simple chronic inflammation or around the meatus may present the special type of ulceration of the soft or hard chancre. The differentiation between these two types is generally in the greater amount of induration and thickening surrounding the latter. Soft chancre is found deeper in the urethra than hard. Tuberculosis of the urethral mucosa may be the cause of ulcerations and nodular granulations and when present increase the difficulty of examination, on account of great sensitiveness of the canal, which is likely to cause increased irritability or to light up acute inflammation from the prolonged use of the instrument.

Glandular Changes.-As already stated, under normal conditions, the opening of the lacunæ of Morgagni are seen as small longitudinal excavations. The glands of Littré cannot be seen in the normal 
urethra. When the latter are affected by chronic inflammation, however, they are seen in the urethroscopic picture as pin-head indentations of a reddish color. The normal appearance of the lacunæ of Morgagni is changed by chronic inflammation causing them to be more gaping and their openings congested from which sometimes may be seen to exudate purulent discharge.. Retention cysts of these glands are sometimes observed. (See Plate II.)

Urethral Polypi and Papillomata.-Papillomata are more common in the posterior than the anterior section of the urethra although they may appear in any portion of the canal. Polyp of the urethra is rare, but when present is easily recognized. Carcinoma of the urethra does occur and is a definitely diffused condition which may be recognized by palpation. Its effect upon the urethral tube is that of generalized stricture.

Technic of Posterior Urethroscopy.-The instruments for this procedure have already been described. The position of the patient is the same as that for cystoscopy (Fig. 81). The instrument which has a curved beak is introduced and the obturator removed. The normal appearance differs from the anterior canal on account of the anatomical construction. This applies more particularly to the complex arrangement of the prostatic eanal well observed with the cysto-urethroscope which provides for hydrostatic dilatation during inspection. Thus may be observed the typical picture of the prostatic urethra; upon the floor is the veru montanum which forms a promintory; the depressions on either side contain the prostatic sinuses in which are seen the orifices of the prostatic ducts. On the summit of the veru montanum is the sinus pocularis or utricle, with the openings of the ejaculatory ducts on its lateral margins. (Fig. 3, p. 4). Beyond this is seen the sphincteric orifice of the neck of the bladder and if the instrument is carried onward into the bladder, the anatomical structure of the trigone or bas-fond may be viewed. (See Plate II.)

In posterior urethroscopy the same deviations from the normal color and elasticity of the mucous membrane are to be observed and changes in the glandular structures are likewise visible. It is possible by pressure of the prostate gland to observe an exudate of purulent material from the glandular mouths. In addition, congestion and hypertrophy of the veru montanum are appreciated and, as stated, the presence of papillomata or other growths are more likely to be encountered here than in the anterior portion. (See Plate II.) Foreign bodies may be discovered in both portions of the canal which may have been introduced from without or by calculus formations. 


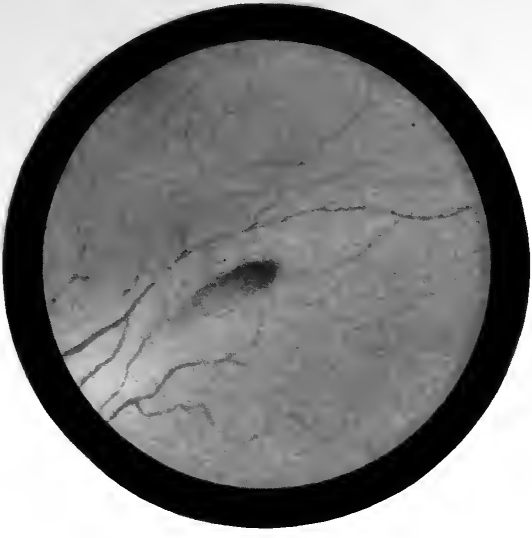

FIG. 1.-Normal Ureteral Orifice.

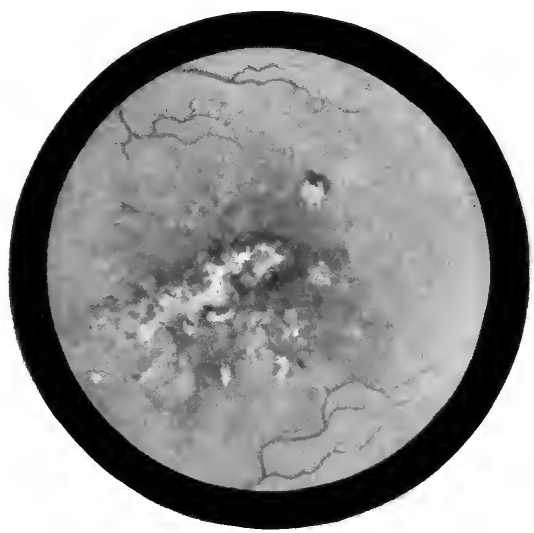

Fig. 3.-Tuberculous Ulceration of the Ureter and Kidney.

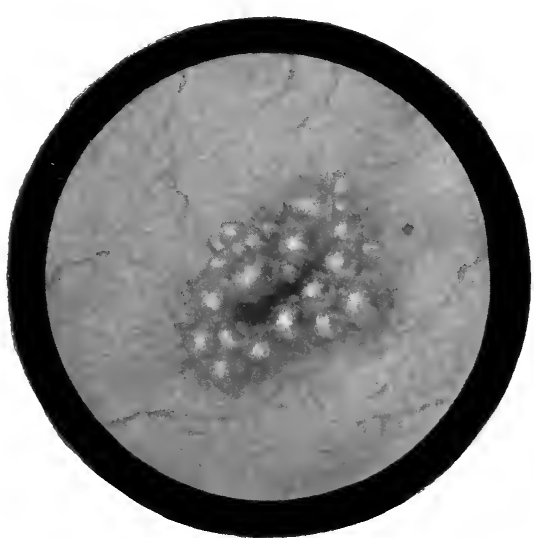

Frg. 5.-Bullous Edema around Ureteral Opening.

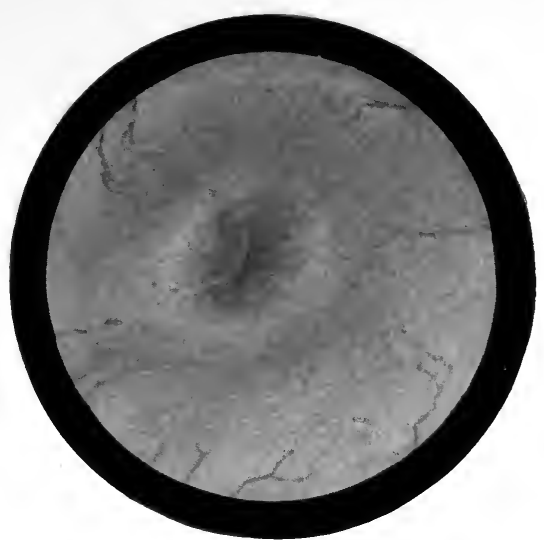

FIG. 2.-Areolar Infection of the Ureter.

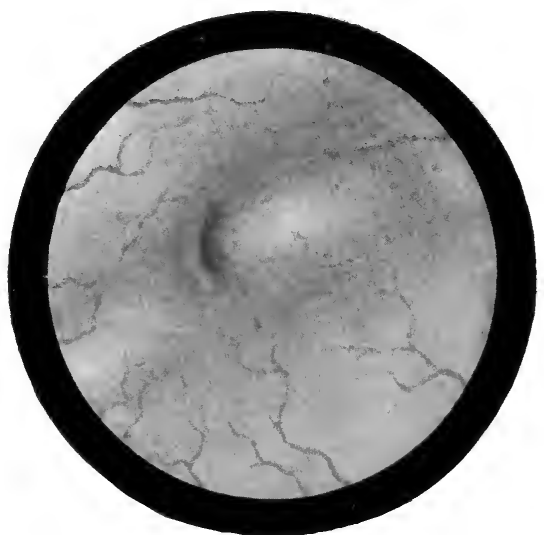

Fig. 4.-Calculus Arrested at Ureteral Orifice.

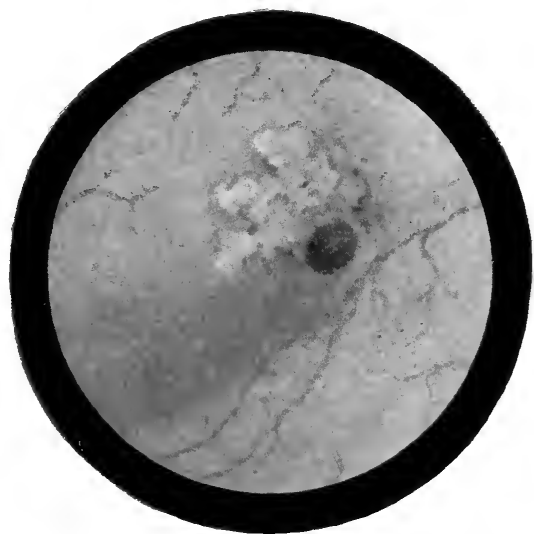

FIG. 6.-Pyelo-nephritis (non-tuberculous) with Destruction of Kidney. 

Treatment Through the Urethroscope. Anterior Urethral Lesions. -These lesions comprise localized patches of congestion, glandular and herpetic areas and ulcerations that do not yield to the ordinary methods employed for the treatment of chronic urethritis. In such cases, when these lesions can be located through the urethroscope, they may be treated by direct applications. The nitrate of silver is a common and effective therapeutic agent which is applied by means of a cotton plug on the end of a wooden or metallic applicator in a strong solution, from 10 to 50 per cent., after first wiping away the secretion from the area to be treated; or, better still, a small portion of solid lunar caustic is fused on the end of the applicator and thus the lesion is effectively cauterized. Small follicular abscesses and sinuses may be incised through the endoscopic tube by means of a slender knife, curetied with the urethral curette and cauterized with lunar caustic or injected with strong solutions of protargol 20 to 30 per cent. (Fig. 71).

Foreign bodies located in the anterior urethra by urethroscopic examination when of the smooth and small variety are sometimes flushed out by retrograde irrigation or rubbed off with an applicator or small tampon. The urethral alligator forceps are used to remove calculi or other foreign bodies lodged in the canal.

Papillomata or other growths may be removed with the urethral snare or curette and their surface cauterized as above or still better, they may be destroyed by the direct application of the high frequency current (Oudin) with an insulated wire through the endoscope. This method has great value in the treatment of intravesical growths (see p.

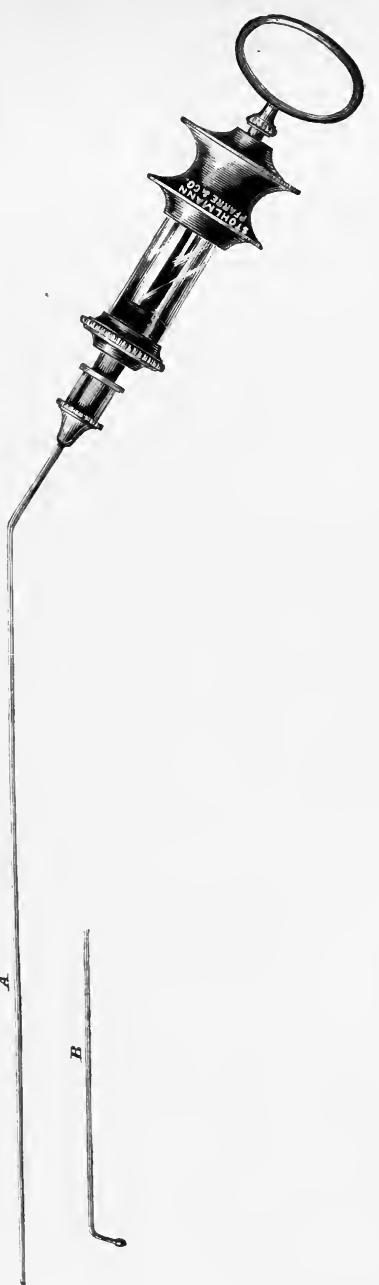

Fig. 71.-Urethral endoscopic syringe. 153). When used in the urethra each treatment therewith should entail only a momentary application, and be repeated as needed. Gonorrheal warts when discovered deep in the canal with the endoscope are treated in like manner or the denuded surfaces, after their re- 
moval with a blunt curette. Subsequent urethroscopy will determine the success of these procedures.

Strictures of the urethra are not treated through the endoscope, as they are more simply and satisfactorily managed by instrumental means without this procedure, except, as already mentioned, it may be possible to enter a stricture otherwise impassable, by finding the orifice through the urethroscope and thus to commence the treatment of dilatation which is carried on afterward in the usual way (see Index).

Posterior Urethral Lesions.-As in anterior urethroscopy, posterior urethroscopy may be employed for direct application to ulcerated areas of the mucosa, etc., of unusual persistence. The greatest opportunity for the use of the prostatic urethroscope is found in treating the veru montanum for chronic inflammation and enlargement, the result of prolonged congestion, a cause for partial impotence and other sexual irregularities (see Index). In such conditions, the direct application of the solid silver nitrate caustic is indicated and may be employed as already suggested-by fusing upon the end of an applicator, or strong solution of silver nitrate (10 to 20 per cent.) upon cotton wound around the applicator. When this treatment is undertaken, the veru montanum alone should receive the application and the excess of the caustic should be wiped away with cotton. If any portion of the solid silver is allowed to remain in the urethra, a severe reaction may be entailed causing tenesmus and painful urination which may last for several days to the great discomfort of the patient. This does not occur when the application is confined to a small area. Treatments should be repeated once in five to ten days according to the reaction produced and the recovery therefrom.

Papillomata and other growths may be removed from the posterior urethra in the same manner described for the anterior portion and here again the employment of the high frequency current will be found especially efficacious. The cysto-urethroscope (see p. 124) by means of distention of the canal, will not only afford an extensive view, but also a satisfactory means of employing this treatment. It furthermore permits complete vision of the orifice of the bladder as well as the posterior urethra and the discovery of lesions that may extend to this point which otherwise might be overlooked with the ordinary posterior urethroscope that has not so extended a field of vision.

Calculi lodged in the posterior urethra are not easily removed with the endoscope but are better pushed back into the bladder with a catheter or other instrument and then removed by a lithotrite or washing tube (see Litholapaxy, p. 492). 


\section{CYSTOSCOPY}

\section{Instruments, Technic, Diagnostic Examination}

Direct examination of the bladder and of the kidney outlets by electric illumination is a means of diagnosis that has gained increasing importance through the development of instruments and greater facility in their use that has been attained by wider experience. It is not necessary to go into the history of the evolution of these instruments, which in the beginning were of crude manufacture and primative design. It is not so long ago that in the very class of cases in which cystoscopic examination now proves of greatest value, attempts to accomplish the end in view were unsuccessful on account of difficulties which the instruments were unable to overcome. This advancement is due to the progressive improvement in the mechanism, the ample means of lighting, and the excellent arrangement of the lens system. The results are that the field of vision has been enlarged, the introduction of the instrument is much less painful, and, by irrigating attachments, a clear field is obtained for a continuous period. Foreign and domestic manufacturers have produced these instruments under the direction and from the designs of those who have been prominent in the field of cystoscopy, whose experience has enabled them to appreciate the requirements and defects. The early instruments of Nitze and Casper are types from which have been developed the improvements suggested by Albarran, Tilden-Brown, and others. The principles of the modern instruments are more or less the same, most of them being refinements of original designs.

Generally speaking, cystoscopes have always been divided into two classes: the direct and the indirect, which applies to the line of vision employed. To these two types of instruments are added attachments which enlarge their usefulness - namely, those for catheterization of the ureters and for affording continuous irrigating during examination. Still more elaborate attachments are provided for operating purposes and the means of making permanent records of cystoscopic pictures by special photographic equipment.

Some instruments are made with the light and the window on the concave surface of the shaft, so that in inspecting the floor of the bladder, it is necessary to rotate the instrument after it has been introduced (Fig. 72). This arrangement is reversed in some of the foreign and domestic instruments, the lamp and window being on the convex side (Fig. 73). The system of lenses in the telescopic portion formerly conveyed the image to the eye of the operator as an inverted object, 
somewhat magnified, but with the improvements in more recent instruments, the image is transposed before it reaches the eye, and therefore is seen in its normal, upright position (Fig. 75). The

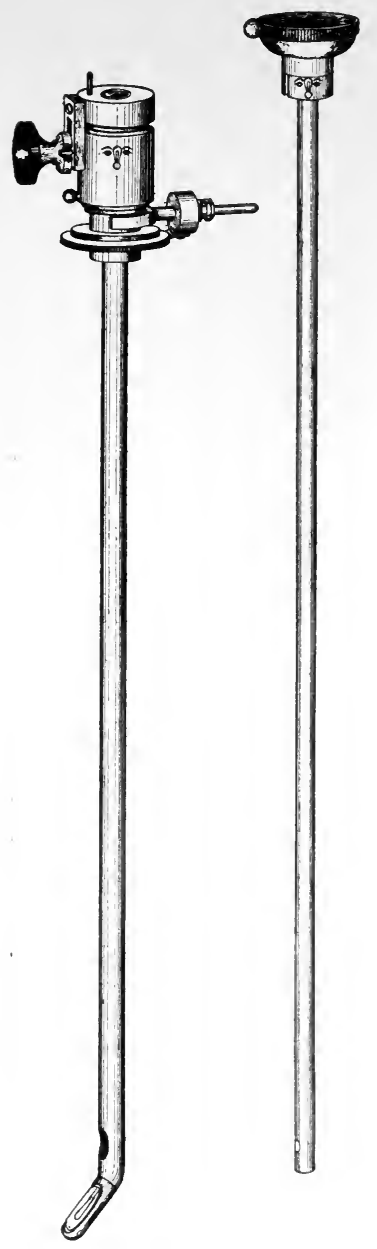

A

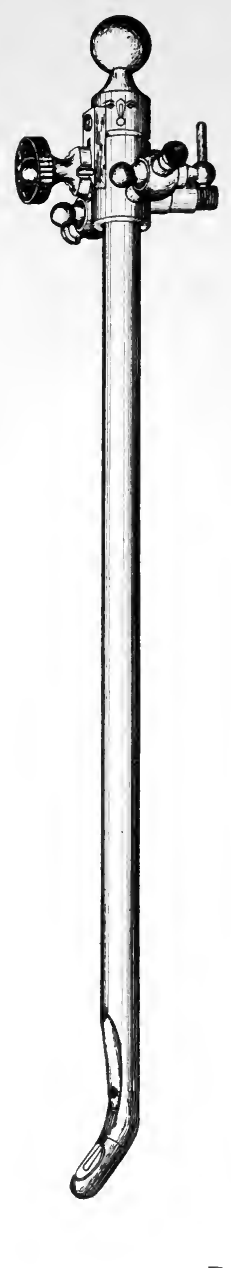

$\mathrm{B}$
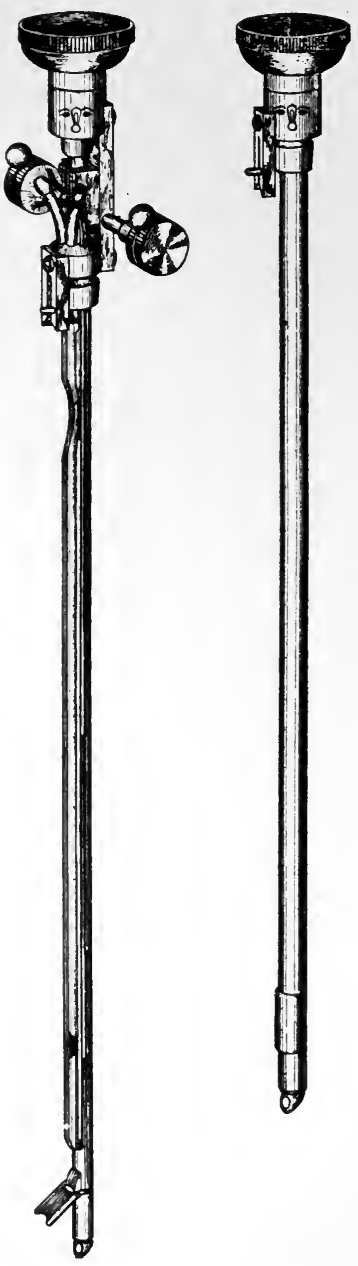

FIG. 72.-Types of cystoscopes; window on concave surface. A, Otis examining cystoscope; B, Brown-Buerger examining and ureteral catheterizing.

direct vision cystoscope presents the object directly in front of the eye of the operator (Fig. 74), while the indirect instrument shows the object from a rectangular point of view (Fig. 75), the object being received upon a prism and reflected through the system of lenses in the telescope. 
This latter instrument, although seemingly more complex, is by all means the most satisfactory, and even though the direct instrument may be preferable in some cases when the object can be placed on a direct line with the eye of the operator, this is not easily accomplished with respect to the floor of the bladder, on account of the elevation of the urethral orifice and the depth of the bas-fond; consequently, it is easier to find the ureteral mouths with the indirect instrument, and
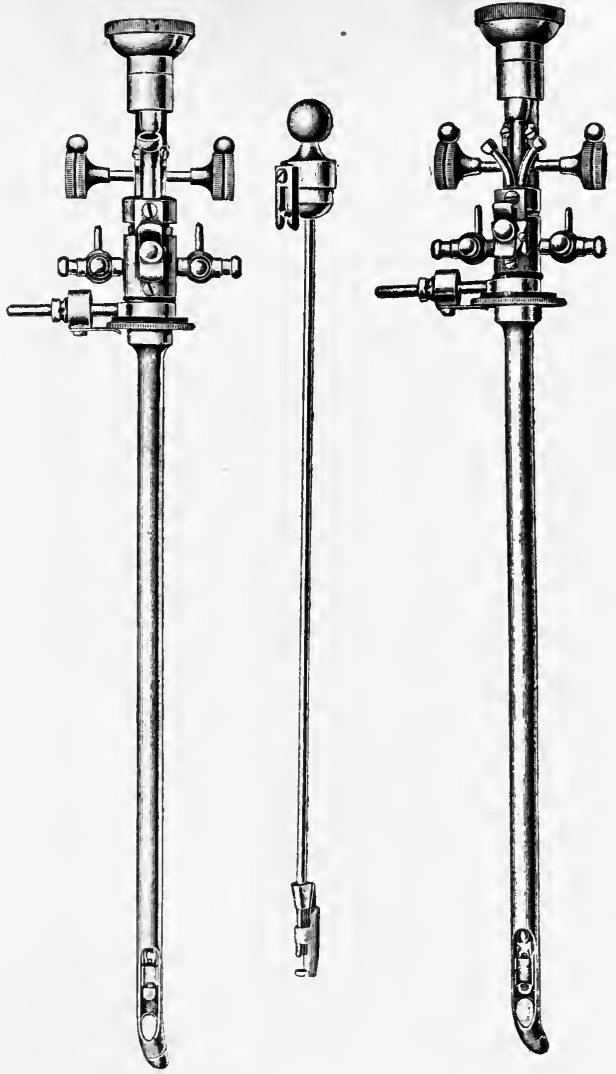

FIG. 73.-Type of cystoscope with window on convex surface of shaft.

this may apply to the female as well as the male bladder, although it is easier to view the ureteral mouths with the direct vision cystoscope in the female than in the male bladder. The particular advantage of the direct vision cystoscope with catheter channels is found when the application of the high frequency (Oudin) electric current is undertaken for the destruction of a growth in close proximity to the vesical orifice, which is sometimes difficult to treat with the indirect instru- 
ment. The region closely adjacent to the neck of the bladder is, therefore, better reached by the direct vision cystoscope or by the cysto-urethroscope of Buerger (see p. 124) or the author's cystoscope (see Fig. 78).

Theoretically, it would seem easier to introduce the ureteral catheter with the direct instrument after the orifice has been located, but by means of the deflecting device (Fig. 72, B) attached to the indirect cystoscope, it is possible to change at will the angle of the catheter,

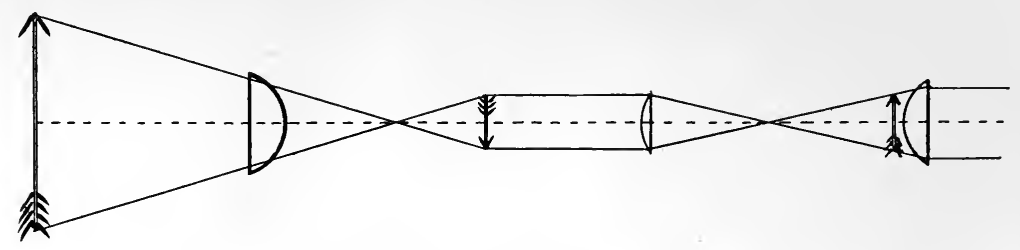

Fig. 74.-Lens system of direct vision cystoscope.

and thus the facility with which the ureters may be catheterized with this instrument has been greatly improved.

The component parts of the cystoscope are the sheath on the terminal end of which is the incandescent lamp, and the telescope (Fig. 78). The lamps commonly used are 4 volts $2 / 10$ ampere, and known as cold lamps, not generating a high degree of heat. The telescope, which is introduced into the sheath, has at its proximal end the ocular, and at its terminal portion, the prism or window. This latter, in the direct instrument, is a simple lens on a direct line with the ocular, and in

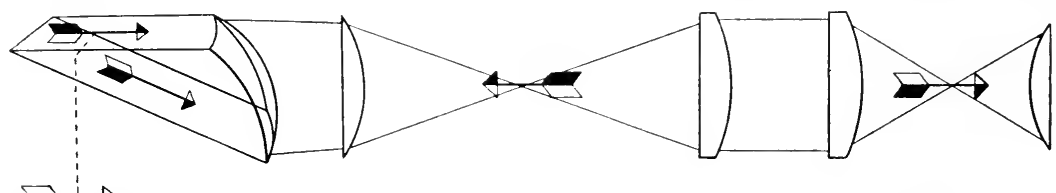

FIG. 75.-Lens system of indirect cystoscope, corrected image.

the indirect instrument is a prismatic lens on the shaft of the telescope, near the end. It is either an ordinary examining telescope for general inspection, or it is provided with one or two catheter canals for catheterizing the ureters. The same sheath may be used for the introduction of the operating devices such as the electro-cautery, probes, knife, snare and traction forceps. The same instrument may also be used for photographic purposes, as demonstrated by the cystoscopic pictures shown (Fig. 76). These pictures were obtained through the author's cystoscope, with a camera attachment, and accomplished 


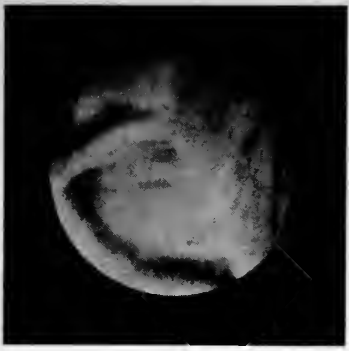

FIG. $76(a)$.- Region of bladder beyond ureter-Nitze photographic cystoscope. (Jacoby Atlas of Cystoscopy.)

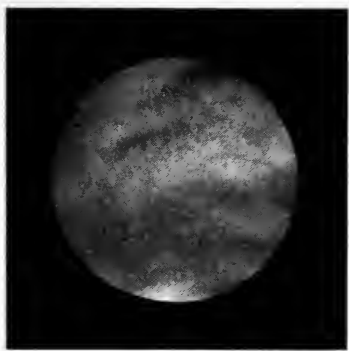

FIG. 76 (b).-View of ureteral orifice and fold. (Jacoby.)

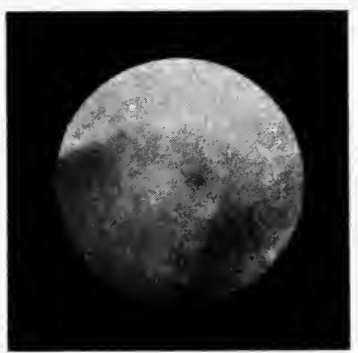

FIG. $76(c)$.-Ureteral opening and eminence. (Jacoby.)

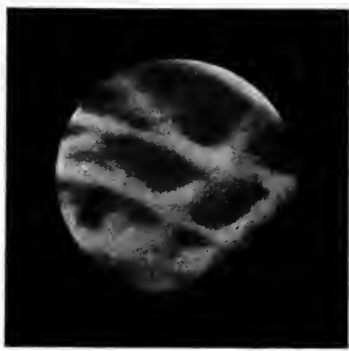

FIG. $76(d)$-Trabecular interspaces. (Jacoby.)

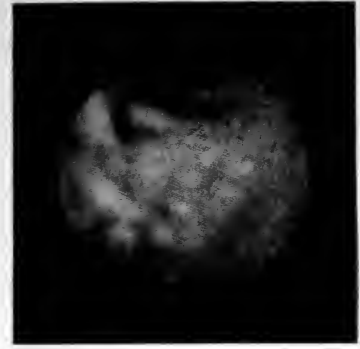

Fig. $76(e)$.- Same as $76(a)$ taken with author's cystoscope and photographic attachment.

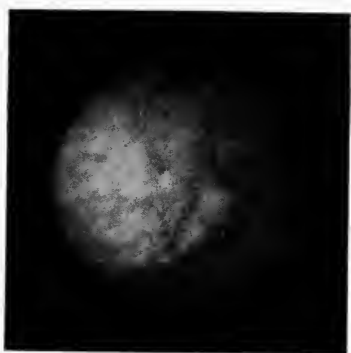

FIG. $76(f)$. - Same as Fig. $76(b)$. (Author's cystoscope.)

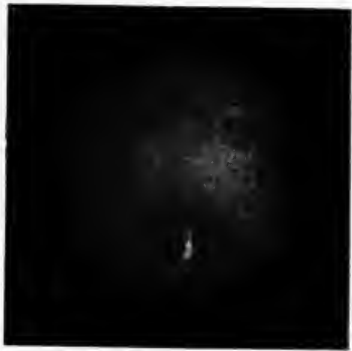

FIG. $76(g)$.- Same as Fig. $76(c)$. (Author's cystoscope.)

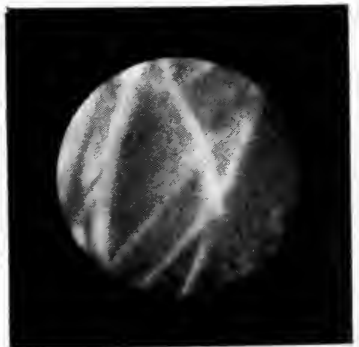

FIG. $76(h)$. - Trabecular bands. (Jacoby.) 
the end in view without the necessity of larger, more elaborate and expensive devices of foreign manufacture. 'The average size of modern instruments, which combine the possibilities of the examining and the ureter-catheterizing cystoscope is about $21 \mathrm{~F}$. The electric current is furnished from a dry or wet battery, or from the street current, and a controller or rheostat (Fig. 68). The current is turned on and off by a switch in the battery or controller or at the instrument. Some instruments are constructed with and some without irrigating attachments, the purpose of which is to obtain, without the removal of the instrument, a clear field in the bladder after it has become clouded by blood or pus. In many instances this irrigating attachment is impractical on account of the time required to empty the bladder through the irrigating canal, an important consideration in cases where the active accumulation of pus or blood produces a very rapid clouding of the field of vision. (Fig. 87.)

In order to obtain the largest possible field of vision, it is necessary that the bladder be distended. Some instruments are constructed to employ air as the distending medium; others employ a clear, aseptic, watery solution, namely, boracic acid, saline or plain sterile water. The watery medium is much more desirable, and the best results are obtained by its use, as the walls of the bladder are fully distended, the lens of the cystoscope is kept clean, and foreign objects in the bladder, especially papillomata and all pedunculated growths are successfully exposed in detail.

Generally speaking, in normal cases, and in those that depart from the normal to a slight degree, almost any good cystoscope can demonstrate its utility. It is in the difficult and trying cases-those in which the bladder contains a large amount of pus, either from an active cystitis or a constant flow from one or both kidneys; those cases in which there is admixture of blood from the kdney or from an intravesical lesion; and those cases in which it is difficult to introduce the instrument on account of obstruction of the urethra or elevation of the prostate, with more or less bleeding as a result-it is under circumstances such as these that the value of the cystoscope is tried, and under which cystoscopy has not proved a success, either through the inefficiency of the instrument or the failure of the operator to properly manipulate it. To meet all difficulties, the best instrument is one that is smoothest and not too long in the beak, to facilitate introduction; one that is not beyond 23 calibre French, and while presenting an ample field of vision, still provides for double ureteral catheterization and preferably a means of cleaning the lens of the visual field by quickly emptying the bladder of its contained fluid, allowing refilling 
during the process of examination. The ordinary inspection cystoscope, which is intended to afford general inspection of the bladder, having none of the special attachments, may be several sizes below 23 French. This instrument, however, has a limited field of usage, and in cases where the admixture of blood or pus occurs at too rapid intervals, the examination is sometimes interfered with. Again, if, after inspecting the bladder, ureteral catheterization is required, it is necessary to remove this instrument and introduce another at a time when it may be found that the patient has stood as much as possible and the neck of the bladder has become irritated. It may be necessary, on this account, to postpone completion of the examination until another time. For simple bladder investigation, however, this instrument has a field of usefulness, and a satisfactory type is the small size Nitze or the Otis cystoscope. Most of the other instruments, however, of somewhat larger calibre (23 French) are provided with simple examining telescopes, so that they may be used interchangeably for inspection and for ureteral catheterization without removing

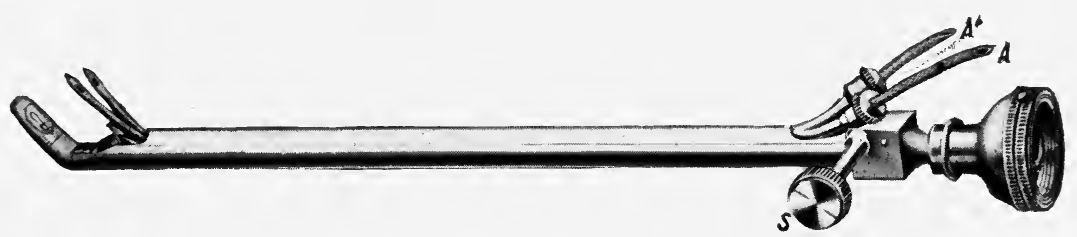

FIG. 77.-Nitze catheterizing cystoscope.

the sheath, and through the same sheath may be introduced operating devices, such as cautery wires for the treatment of vesical growths, etc. The Nitze cystoscope is the original type of instrument much used at the present day. The foreign manufacture is still preferred by some urologists (Fig. 77). The size of the sheath is about 23 French, with the double ureteral catheterization attachment. It has the advantage that the catheter canals are attached to the sheath of the instrument and not to the telescope (as in some American instruments, Fig. 72, B), so that the telescope may be removed to cleanse the bladder, while the catheters remain in situ. This is particularly useful in cases where double catheterization of the ureters is indicated, and one catheter having been introduced, the field of vision becomes obscured by blood or pus, rendering it difficult to locate the second ureter. The telescope can be removed with the unused catheter, the bladder cleansed and the telescope replaced with the second catheter, when it may be possible readily to locate the second ureter and accomplish the purpose of double catheterism. 
The Brown-Buerger Cystoscope (Fig. 72, B).-This instrument of domestic make, developed along the lines of the Nitze, represents improvement in manufacture and design. The sheath is also 21 French, and may be used for simple inspection with an examining telescope, or for double ureteral catheterization by removing the simple telescope and introducing the double ureteral catheterizing attachment, which is supplied with a deflector to change the angle of the catheter, to facilitate its introduction. It is also supplied with two irrigating channels, which, however, on account of the smallness of their size, are generally useful only to fill the bladder, and inadequate when it becomes necessary to empty; therefore, in cases where it is necessary to empty the bladder, both the telescopic portion and the catheters have to be removed. This offers a disadvantage already mentioned in undertaking double catheterization, where one catheter has been introduced and the field has become clouded before the second ureter has been found and entered. This may be of occasional occurrence only, but it is very trying when it happens, as it may necessitate resorting to the less satisfactory means of inserting a canula into the bladder and thus obtaining the urine from one kidney from the catheterized ureter, and from the other through a canula in the bladder mingled with the secretion therefrom.

The author's cystoscope (Fig. 78) was designed to combine the advantages of a cystoscope and cysto-urethroscope. Many prefer the separate instruments which have been continuously improved. This instrument contains no new principles, but assembles important features derived from the use of many different instruments. In brief, these comprise indirect vision from the concave surface of the sheath, thus bringing the floor of the bladder immediately into view without rotation (important when ureteral catheterization is undertaken); an ample field of vision, with new system of lenses, which means a brilliant field and a corrected image; a large examining telescope for inspection of the bladder, bringing from $75^{\circ}$ to $90^{\circ}$ of the field into view; two ureteral catheter channels in the sheath, separate from the telescope, which may be removed without disturbing the catheters, and thus the catheter canals are further away from the eye, giving greater facility to the hand of the operator and less likelihood of leakage reaching the eye of the examiner. There is a small irrigating channel running through the catheter septum, with which to cleanse the lens during operation. There is a large exit irrigating channel attached to the shaft, sufficiently large to permit evacuation of the bladder contents, and, with the other irrigating channel, to accomplish continual irrigation during usage (Fig. 78). Thus, with the 

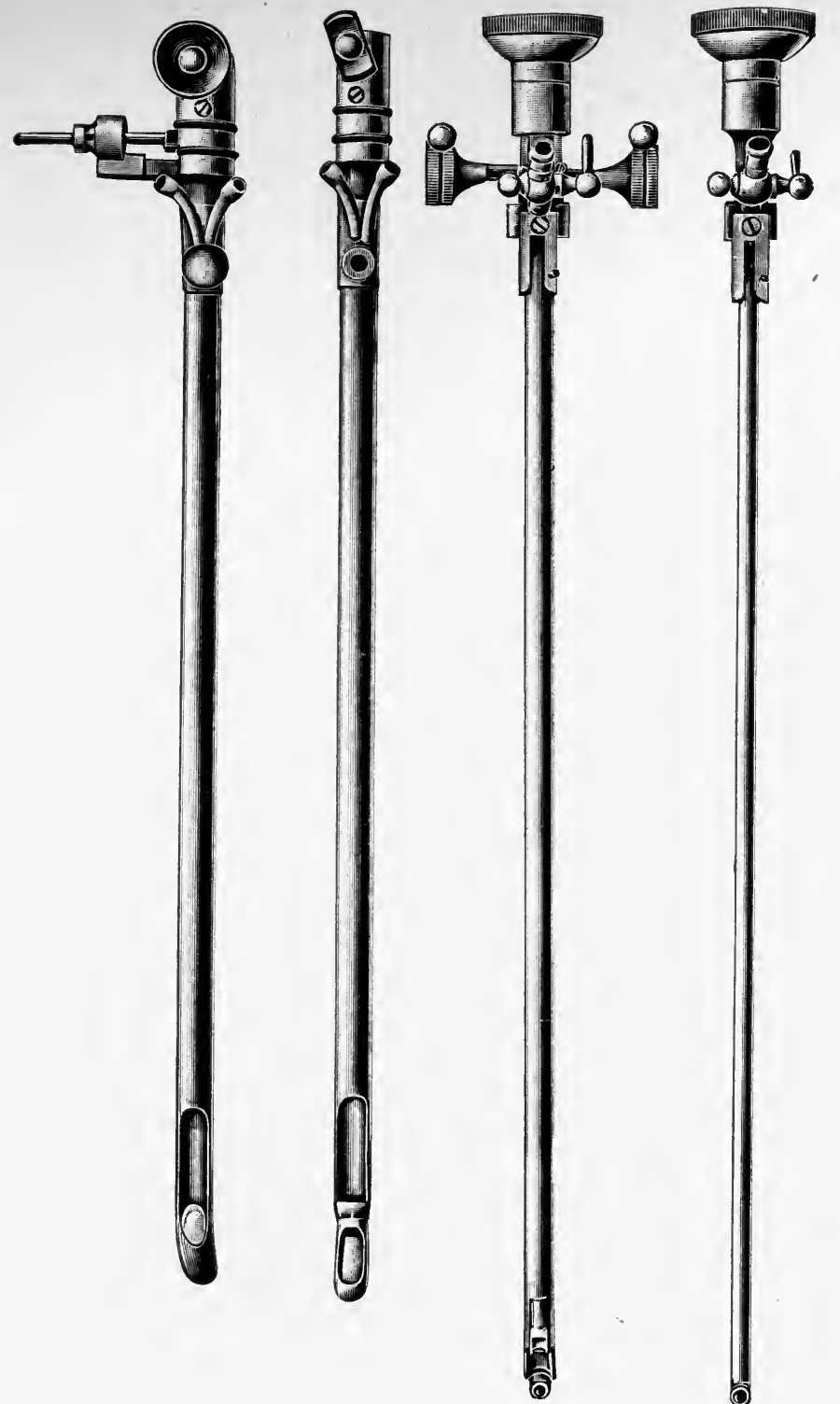

Fig. 78.-The author's double irrigating cystoscope with convex and concave sheaths. 
two ureteral canals, there are four channels connected with the vesical orifice, two of which may be used for irrigating. Where one of the ureteral canals only is in use, as in the case of electric cauterization, the insulated wire occupies the other catheter canal. (See Fig. 91.) Finally, on account of its short beak, the moderate angle between the prism and the light places this instrument between a direct and indirect vision, and it is able to detect and attack lesions adjacent to the neck of the bladder. By withdrawing the instrument into the urethra while the irrigation is continued and the walls distended, ample inspection of the posterial urethral canal may be accomplished, and if need be, cauterization of papillomatous growths in this region with the insulated electric wire and Oudin current.

A second sheath is also designed for this instrument with the window on the convex side of the shaft (Fig. 78), found more satisfactory in some cases for inspection of the fundus of the bladder and also affords more effective lighting for photography.

\section{The Technic of Cystoscopy}

General Requirements.-The cystoscopic room should contain all of the essential features of an aseptic operating room (Fig. 79), with additional provision for affording the means of shutting out the light. An ample-sized instrument table should be provided, upon which are placed the various articles required. These include small glass (urethral) syringes; a large glass syringe (100 c.c.); instillator; urethral. catheters of various sizes; ureteral catheters; sounds, always more than one; cystoscopes; sterile towels and wipes and glass graduates; large glasses and test-tubes. In addition, there are prepared solutions for the hands and cleansing the patient, the irrigator filled with salt or boracic-acid solution, with an ample additional supply on hand; plain, sterilized lubricant and special lubricant for local anesthesia of urethra (see p. 47); a proper operating table, provided with a drip pan and surgeon's stool (Fig. 79).

Position of Patient.-The semi-upright position is found to be the most satisfactory, with the knees flexed a little above the level of the table (the author's modification of the Isaac's cystoscopic table), the feet being placed on properly arranged rests (Fig. 80). This table affords such position, and is equipped with two drip pans, one to be used for cleansing the patient, and after the parts are draped with a sterilized and perforated sheet, it is removed and is replaced by a clean sterilized pan.

The preparations being complete, the operation is carried on in the following steps: 
1. The application to the posterior urethra of an anesthetic lubricant by means of a special instillator ${ }^{1}$ (Fig. 33).

2. Test of cystoscope light before introduction.

3. Introduction of cystoscope detached from battery cord, withdrawal of obturator.

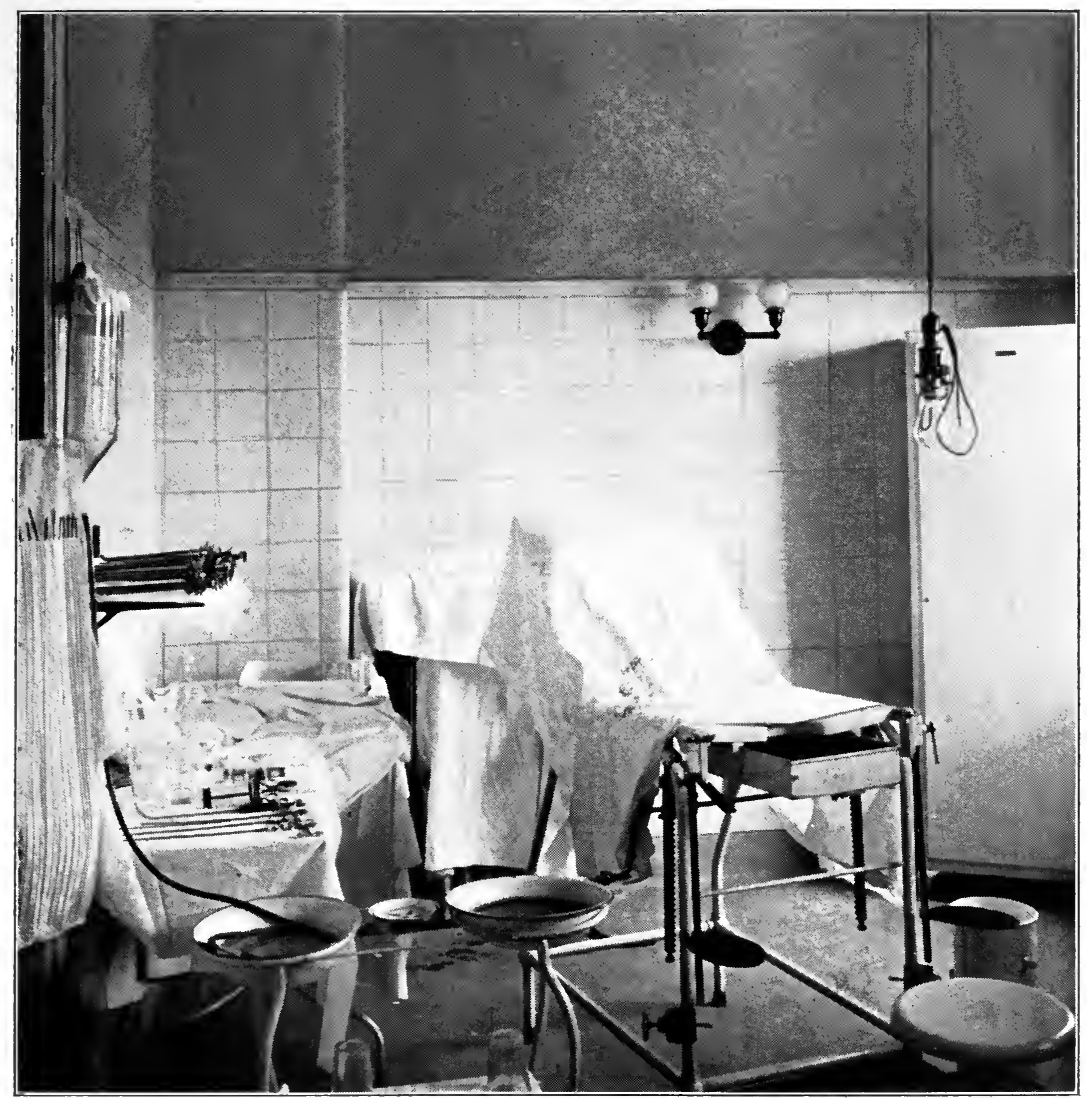

FIG. 79.--Operating room prepared for cystoscopy.

4. Irrigation of bladder through eystoscopic sheath (Fig. 80). This may be done with 100 c.c. syringe with most cystoscopes; with the author's instrument, the irrigating and cleansing of the bladder is conducted by alternately occluding and opening of the outlet of the sheath. During the process of irrigation the return flow is received in a clean glass for inspection of the transparency of the returned

${ }^{1}$ N. B. This is not always necessary. Many cystoscopies can be painlessly conducted without anesthesia. 


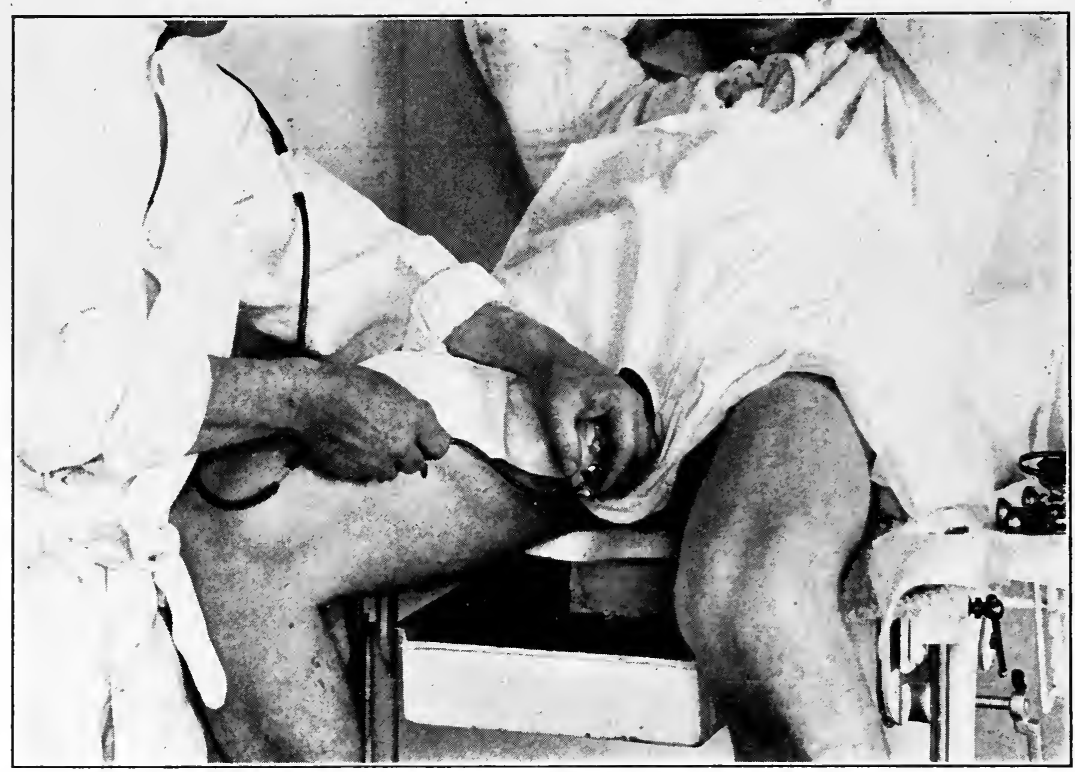

A

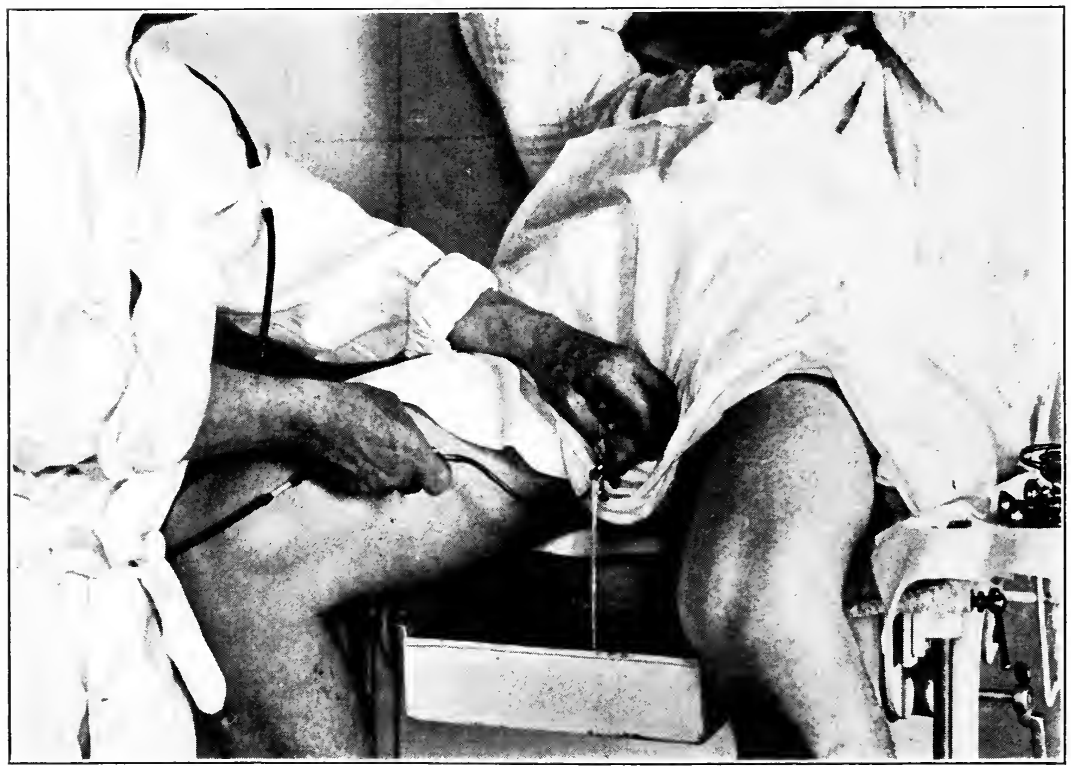

B

FIG. 80.-Cystoscopy. Irrigation of bladder through sheath of cystoscope. A (above), inflow; B (below), outflow. 
fluid, and this washing is continued until the latter is absolutely clean and transparent.

5. The telescopic tube is introduced into the sheath of the cystoscope and the battery cords attached.

6. Filling of bladder through irrigating channel. From 125 to 200 c.c. ( 4 to 6 ounces) of fluid are commonly necessary for bladder inspection. With the irrigating cystoscope, inspection may be commenced forthwith (Fig. 87), and a view of the bladder obtained as distention is accomplished.

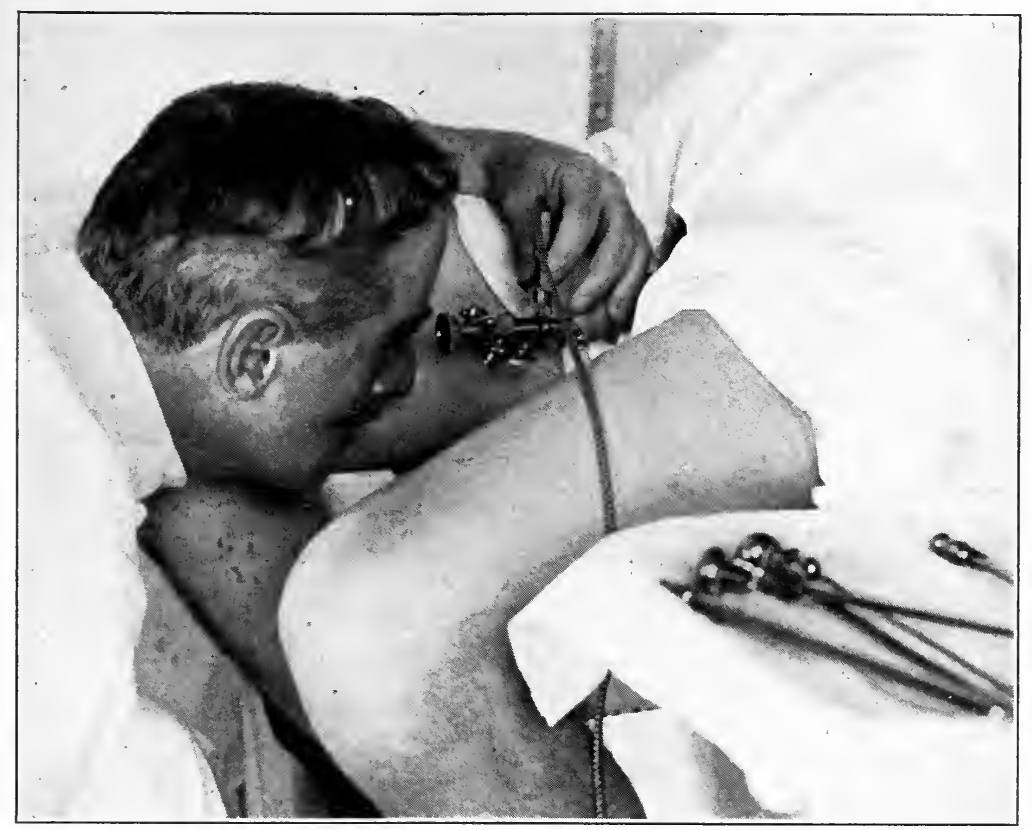

FIG. 81.-Cystoscopy. Inspection of bladder.

To become proficient in cystoscopy it is desirable that the examiner be thoroughly familiar with the appearances of the interior of the bladder under normal conditions.

Generally speaking, the cavity of the bladder is composed of summit, side-walls, trigone and bas-fond. These regions are inspected systematically by following certain normal anatomical landmarks.

In conducting a cystoscopic survey, it is convenient to divide the interior of the bladder by segments corresponding to the dial of a watch, and to have this subdivision (Fig. 82) in mind in its relation to the normal landmarks, for the purpose of identifying the location of any abnormality or pathological lesion thereof. 
The instrument having been introduced with the window upward, it is slowly withdrawn until an air-bubble having the appearance of a drop of oil, comes in view at the beginning of the superior aspect, which, although not always present, when seen at this site, distinguishes the summit of the bladder or the first anatomical landmark. By rotating the instrument from side to side at this point, between the radii of 10 to 12 , and 12 to 2 of the dial, the entire vault is inspected; and when it is further withdrawn within the same radii, a dull red curtain appears, which is the upper margin of the vesical sphincter, and repre-

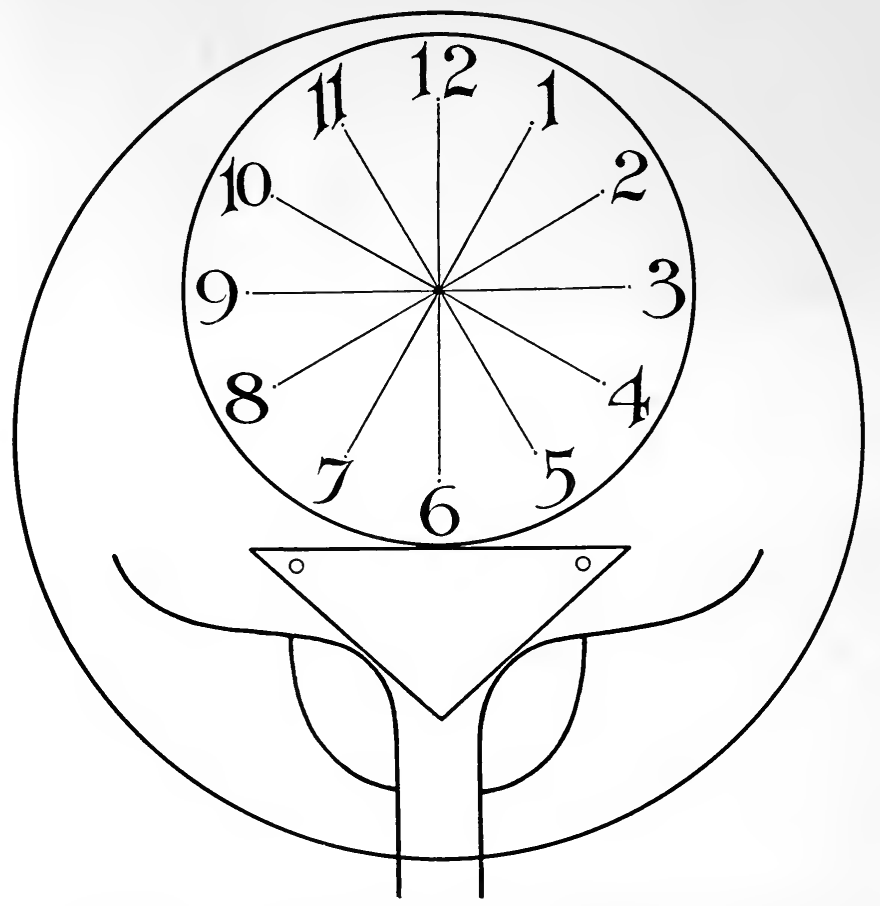

FIG. 82.-Diagram for locating cystoscopic landmarks, and abnormalities.

sents the second landmark. Holding the instrument fixed in this position, and gently rotating it first on one side and then on the other, the lateral margins of the sphincter will be seen until the window is turned directly downward to 6 o'clock, when the outline becomes lost in a hazy field which marks the trigone, and is the third anatomical landmark. The vision is hazy at this point, because the prism of the instrument lies close to the bladder mucosa, and in order to overcome this, it is necessary for the operator to depress the ocular or proximal end of the instrument, until the picture of the trigone becomes clear 
and distinct (Fig. 83). Now, holding the instrument in this position, keeping it in midline, or at 6 o'clock, the operator introduces it slowly until the base of the trigone or interureteric ridge comes into

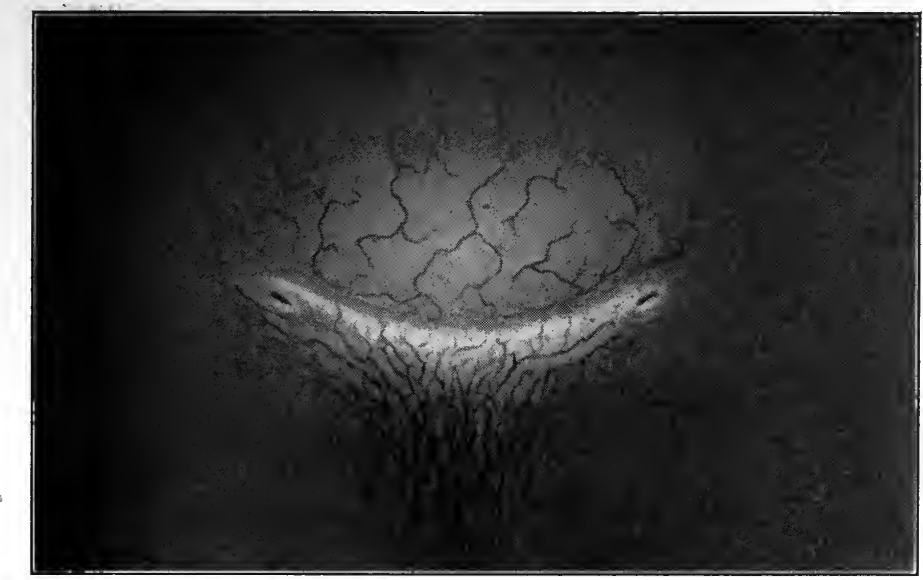

Fig. 83.-Cystoscopic picture of trigone and ureteral openings.

view, which is the fourth landmark. This landmark represents the base of a triangle, the apex of which is the urethral orifice, and the sides

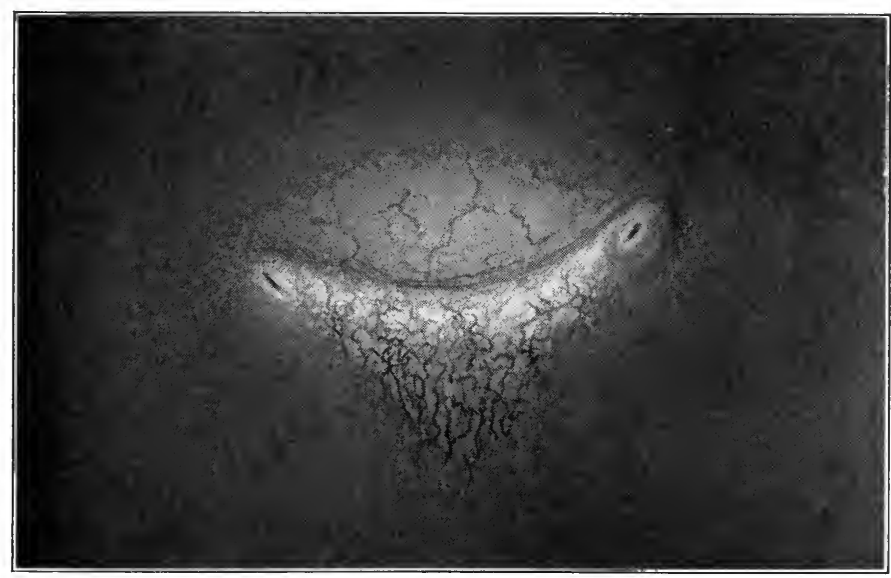

FIG. 84.-Cystoscopic picture of ureteral openings, showing slight deviation from the normal symmetrical relation.

of which are the two lateral lines extending from the urethral orifice to the ureteral openings (Fig. 82). Having located the base of the trigone, by following it deliberately to the left, the left lateral angle of 
the triangle comes into view, at which may be seen at the radius of 7 o'clock, the left ureteral opening. By rotating the instrument to the right, always keeping the base of the trigone in sight, the right lateral

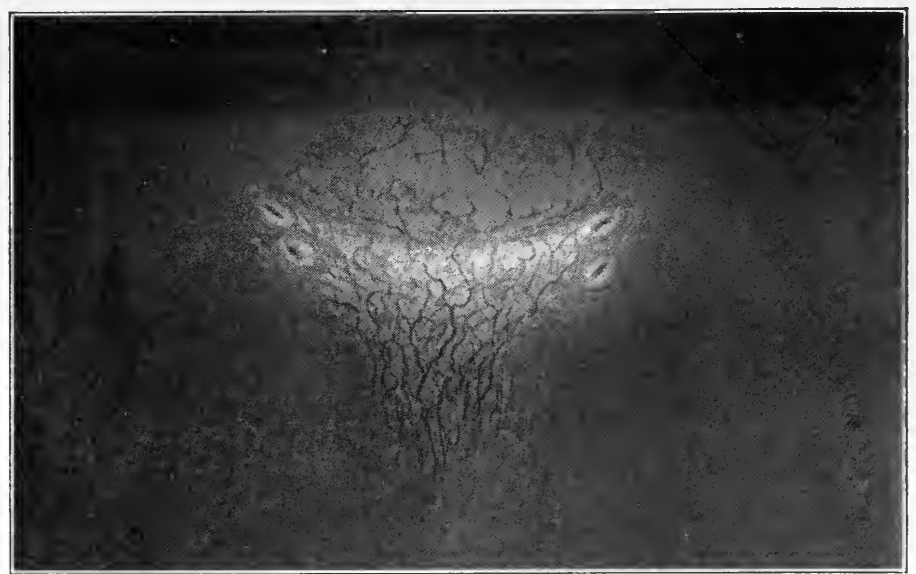

Fig. 85.-Cystoscopic picture showing anomaly of two ureteral mouths on both sides.

angle of the triangle is seen and the right ureteral opening, at the radius of 5 o'clock. These two lateral angles of the triangle are the fifth

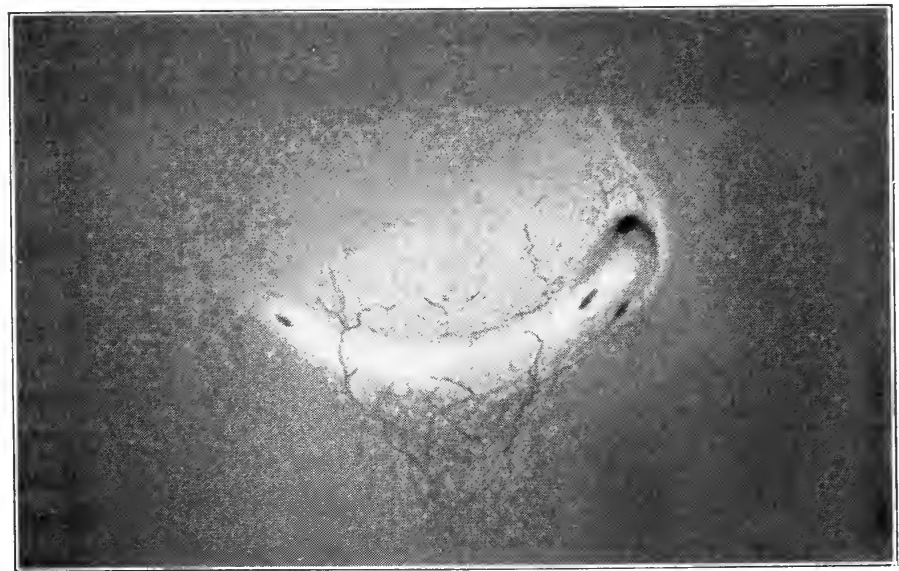

FIG. 86.-Cystoscopic picture showing blind pouch in region of ureteral opening. (L.)

anatomical landmark of the bladder, and are the most important guide to the location of the ureteral openings.

These various landmarks are fixed points, but their relation to each 
other is not similar in all cases. For instance, the difference between the sides and the angles of the imaginary triangle may vary in different individuals, and as a result, the relation of the ureteral openings to each other, and to the urethral orifice will be somewhat asymmetrical. Thus, instead of the location of the ureteral mouths being at 5 and at 7

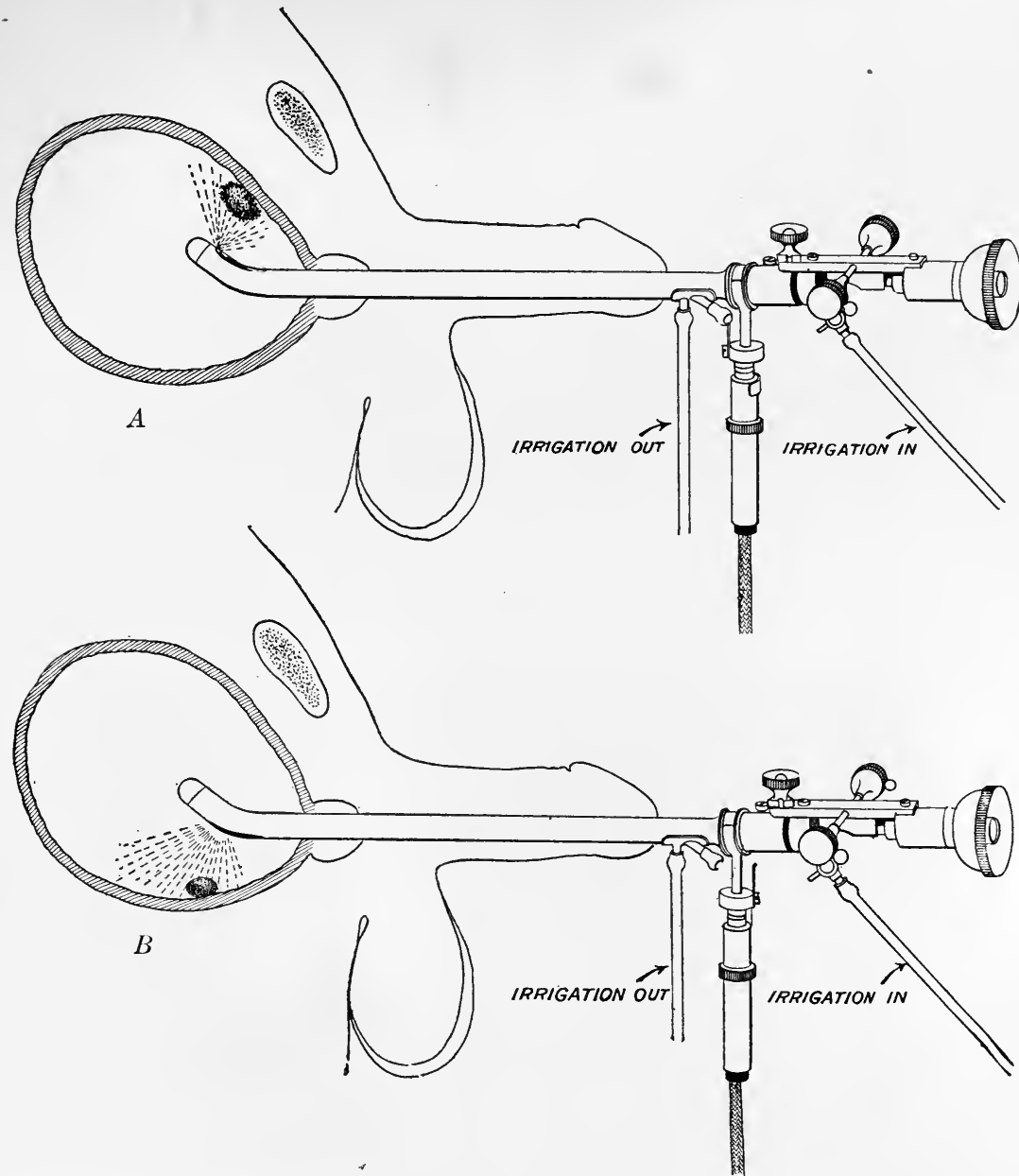

Fig. 87.-Cystoscopy. Inspecting roof of bladder. $A$, Window on concave surface of shaft; $B$, inspecting floor of bladder. Window on convex surface.

of the dial, one of them may be between 4 and 5 (Fig. 84) or both of them may be at a different angle. By becoming familiar, however, with all of the fixed landmarks described, it should be possible to find them with the least amount of difficulty.

After inspecting the ureters, by turning the instrument with the 
window toward the median line of the trigone, and still further introducing it, the bas-fond, or that portion of the base behind the trigone, is inspected (Fig. 83).

Throughout the entire examination, the instrument should be supported and kept in the median line, for it is by the swaying of the shaft from side to side that most distress is caused the patient.

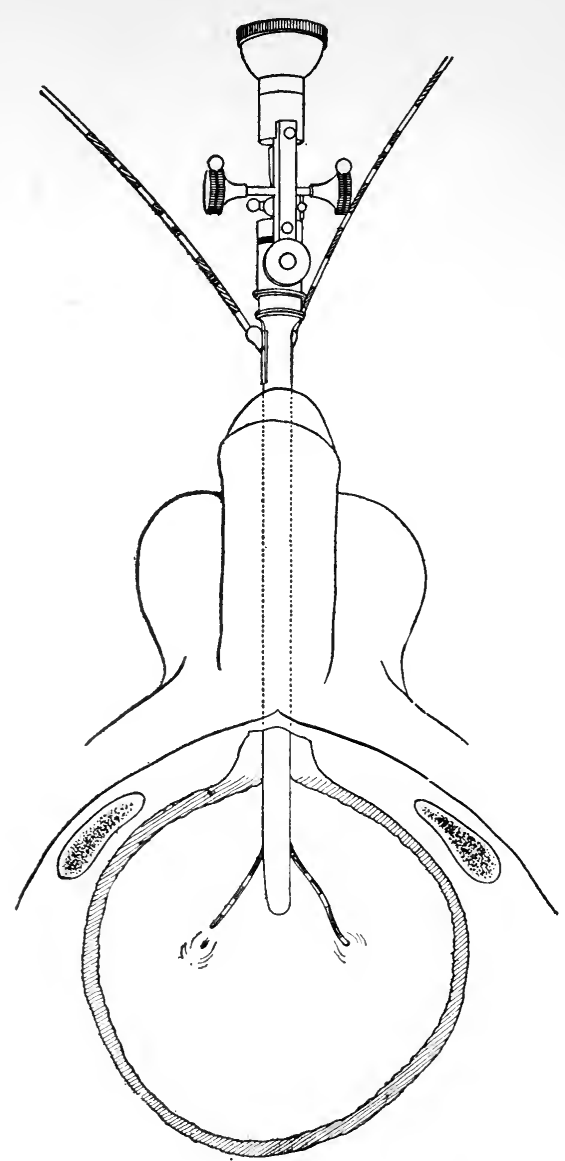

Fig. 88.-Cystoscopy. Ureteral catheterism with indirect instrument.

When the relations at the base of the bladder are distorted by inflammation some difficulty may be experienced in finding one or both ureteral openings; and in exceptional instances the anomaly of more than one ureteral mouth on one or both sides may be discovered (Fig. 85), or a blind pocket in the locality of the ureteral opening may confuse the observer (Fig. 86). With the convex instrument, that 
which has the window upon the side opposite to the beak, the base of the bladder comes first into view and it is not necessary to rotate the instrument completely to find the ureteral orifice (Fig. 87, B.) For immediate catheterization of the ureters this instrument is. most serviceable.

Catheterization of the Ureters (Fig. 88).-The base of the bladder is brought into the field as already described; first one and then the other ureteral orifice is located. The one to be catheterized is plainly viewed, the window of the cystoscope being kept at a slight distance from the ureteral opening, while the catheter is slowly inserted into the field of vision. After it is introduced about $1 \mathrm{~cm}$., the instrument and the catheter are advanced toward the ureteral mouth, which enables the operator to determine the angle of the catheter as compared with the direction of the ureter. The deflector is then turned sufficiently to give the catheter the required pitch, and if possible, instrument and catheter are advanced together to bring the tip of the catheter against and into the ureteral mouth, when it is gently introduced; and if this manœuvre succeeds, it is advanced cautiously until it reaches the pelvis of the kidney, previous to which the deflector is turned to its normal position and the eye of the operator notes the distance that the catheter is entered, which is made possible by the markings provided on the ureteral catheters (Fig. 89), plainly observed through the cystoscope. The same operation is repeated on the opposite side, unless the bladder has become clouded, in which case, with the irrigating cystoscope, a clear field is again obtained, by evacuating the contained fluid and refilling.

The sizes and style of ureteral catheters vary to a certain extent. The best are of foreign manufacture. The terminal end is bougie, olivary or whistle type (Fig. 89). For average use in the adult the sizes from 5 to 7 are customarily employed. It is only in those instances where there is a question of extra catheter flow or leakage along-side and where it is deemed important to collect the exact total amount for functional test, that an extra large size is employed or one of a conical shape (Garceau), that upon being introduced will fit the

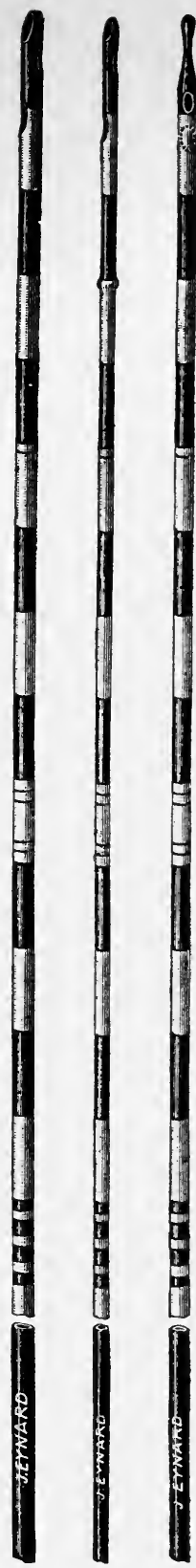

Frg. 89.-

Ureteral catheters. 
orifice tightly. The difficulty about such large catheters is either that two cannot be used at a time for double catheterism or an unusually large cystoscope is rendered necessary.

\section{Cystoscopy in Abnormal Conditions of the Bladder}

Cystoscopic examination of the interior of the bladder is employed to investigate chronic inflammatory conditions, to detect the presence of neoplasm, stone, and other foreign bodies and to inspect the region around the neck of the bladder in the case of prostatic and other obstruction thereof.

Chronic inflammation of the bladder when viewed by cystoscopic observation may be confined to the region of the neck of the bladder or be distributed over the entire cavity. The ordinary evidences of inflammation may be seen, such as vascular exaggeration, ecchymosis, and at times ulcerations. In old chronic conditions, when the mucosa has undergone epithelial transformation and fibrous infiltration, plaques of cicatricial areas and leucoplakia may be seen here and there. When the inflammation is confined to the neck of the bladder in the region of the triangle of the base and sphincter (Fig. 83), a highly congested and reddened area is revealed, spoken of as trigonitis.

In tuberculous inflammation characteristic appearances are observed. Occasionally minute grayish or yellowish tubercles are discovered studded over portions of the mucous membrane. What is more common is an area of intense inflammation containing ecchymoses and granulations with here and there punched out ulcerations. A favorite site is the region of the ureteral orifices from which the inflammation may be seen to radiate, which is especially the case when a tuberculous bladder lesion is secondary to that of one of the upper urinary tract. This may take the form of an areolar infection, of extensive ulceration, or of bullous edema (Plate III, Fig. 2).

Varices of the bladder occur, although they are somewhat rare, and are recognized by cystoscopy. In chronic cystitis about the neck of the bladder behind an orificial stricture or hypertrophied prostate there is usually extensive ecchymosis of the mucosa, which in more extreme conditions may exist in the form of varices of the tortuous veins especially in old subjects with arterial sclerosis. Such a condition may lead to spontaneous hemorrhage, possibly of severe degree. Relief is obtained by bladder lavage with nitrate of silver and measures directed against the existing lesion of stricture or prostatic hypertrophy.

Vesical Calculus. - While stone in the bladder is usually found by intravesical palpation, it is possible by the aid of the cystoscope to 
discover a calculus or calculi that might otherwise be overlooked or be beyond the reach of the searcher. This may be the case when stone is present in a deep bas-fond behind a stricture of the neck or prostatic enlargement. Encysted stone and one hidden in a diverticulum or between the trabecula of old chronic inflammation may also be detected through the cystoscope (Fig. 87).

Tumors of the Bladder.- It is in the discovery of morbid growth of the interior of the bladder that cystoscopy is of greatest value. Many small papillomata have been thus detected and their removal by minor means made possible, before they have attained large dimensions and perhaps undergone malignant transformation. These vesical growths are readily detected by means of cystoscopy in the transparent fluid medium, and whereas formerly it was necessary to effect their removal by open operation, or the unsatisfactory method of piece-meal destruction with the cystoscope, the valuable expedient of destroying these growths by the application of the high-frequency current (Oudin) has practically revolutionized the treatment of bladder neoplasms (see p. 153). These tumors appear as small and large pedunculated excrescences located upon different portions of the vesical mucosa, or they may be sessile. Through the cystoscope they appear like different forms of aquatic plants; their surfaces may be pale pinkish in hue or bright red; or their color may be whitish in portions denoting necrosis or calcareous deposits (see Vesical Neoplasm).

It is difficult to distinguish positively through the cystoscope between benign and malignant growths. It is a well-known fact that papillomata of the bladder may belong to either variety. The age of the patient is taken into consideration, and degenerative changes if apparent through the cystoscopic examination are usually significant of malignancy. When it is possible, a portion of the growth should be obtained for laboratory examination (see p. 453).

Bladder Diverticula.-These congenital and acquired malformations of the bladder are seen upon cystoscopic examination in their respective forms. The acquired diverticula which are due to chronic inflammation and thinning of the bladder wall between the fibrous trabecula, appear through the cystoscope like irregular holes punched out of the bladder wall, usually in a furrow; sometimes the bottom of the sinus can be seen and other times it is invisible (Fig. 174, p. 416). Congenital diverticulum may be seen in the bladder that is not the seat of chronic inflammation and is recognized as a circular opening of different dimensions, from the size that is almost invisible to that large enough to contain a good-sized calculus (Fig. 175, p. 417).

Prostatic enlargement and other abnormalities of the bladder outlet 
are investigated by means of the cystoscope. A retrograde vision cystoscope is not necessary to make this examination on account of the great increase in the visual field of the present indirect cystoscopes being ordinarily from 75 to 90 per cent.; and instruments are being constructed to increase materially this percentage so as virtually to be able, from the interior of the bladder, to take in the entire area around the outlet. Then again the instruments, which are constructed with irrigating device, make it possible to inspect the interior of the vesical orifice on being withdrawn and the cysto-urethroscope furnishes a view from the urethral aspect (Plate II, Figs. 9 and 10).

To inspect the intravesical prostatic growth the indirect instrument is introduced just within the bladder orifice and by rotating at different angles a systematic view of the prostatic projection is obtained (see p. 401). Thus may be seen in segments the bilateral growth, the superior commissure and the middle lobe (Plate II, Fig. 10). A medium isthmus or bar may also be taken account of, and an unusual orificial contraction may often be appreciated, more especially when viewed from the urethral aspect.

Abnormalities of the Ureteral Orifices. - The normal view of the ureteral mouth is a minute slit or aperture superimposed upon the ureteric ridge (Plate III, Fig. 1). Anomalous appearances of this ureteroscopic picture are seen in the displacement or total absence of the ureter from its normal site. In the unusual condition of double or triple ureter as many abnormal orifices will be found in the region of the trigone (Fig. 85).

Alterations from the normal in the contour and condition of the ureter opening are produced by different morbid lesions. In place of a contracting slit there may appear a rigid and rounded aperture which may be the result of a chronic lesion of the kidney-a pyelonephritis or pyelonephrosis (Plate III, Fig. 6). Such a ureter may be surrounded by ecchymosis and inflammatory change and exude a thick and purulent discharge.

Ulcerations involving the mouth of the ureter produce an irregularity of the opening; bullous edema may appear in the adjacent region or hide the orifice from view. These latter appearances are especially significant of tuberculosis of the upper urinary tract (Plate III, Figs. 3 and 5).

Cystic protrusion of the ureter is seen in some instances as a congenital lesion and false pockets may also be present (Fig. 86).

Encapsulated stone in the ureteral mouth produces a rounded swelling and an edematous areola causing a damming of the urinary flow, and interference with the introduction of the catheter (Plate III, Fig. 4). 
In completing the cystoscopic diagnosis, following the visual inspection of the ureteral mouths, the catheters are introduced to obtain the urine separately from both kidneys. The catheterized specimens are then submitted to microscopic and chemical examination and incidentally the capacity of a suspected morbid kidney is com pared with that of the opposite organ by means of the functional urinary tests (see p. 155).

Pyelometry or Measurement of the Renal Pelvis.-This technic is resorted to as a means of further investigating the cause of a kidney lesion when other measures fail to supply adequate diagnostic information, as in the instance of intermittent attacks of renal colic, when the radiograph does not reveal the presence of calculus (see Index).

To accomplish properly this undertaking, it is necessary that the ureter be catheterized, or both ureters, in case a comparative measurement is desired, and that the ureteral catheters satisfactorily occlude the ureteral mouths, in order that there be no return or extra-catheter flow. This may be tested by injecting into the ureteral catheter with a hypodermic syringe about 1 or 2 c.c. of test solution, observing through the cystoscope whether or not leakage occurs, before making the measurement with a solution of argyrol or collargol, the same as used for pyelographic injection (see p. 168). A small graduate glass, containing a known quantity of solution (about 50 or 60 c.c.) is held by the assistant or nurse, and of this amount sufficient is injected slowly through the ureteral catheter until a sharp colicky pain in the kidney region is produced, signifying pelvic distention. This amount indicates the capacity of the pelvis, and any marked excess above 15 c.c. may be considered as caused by abnormal distention. In the examination it is customary to compare the two sides. When evidence of pelvic distention is presented, as a result of this procedure, the condition is further investigated, and the result confirmed by pyelography, or radiographic examination of the pelvis following the injection of an opaque liquid, such as argyrol or other silver solution (see p. 168).

The Luys Urine Separator.-In certain cases in which for one reason or another the ureters cannot be catheterized, yet in which it is important to obtain the urines separately from the two kidneys, it will be found that the Luys separator may prove of considerable value (Fig. $90, \mathrm{~A})$. This is especially the case in females, in whom the instrument has its greatest utility. As a matter of fact with the improved instruments the cases are much fewer in which the cystoscope cannot be employed but there are still some in which even the most skilled may find it difficult. It is in such cases that the separator is helpful. These cases are those in which, on account of great distortion of the 
vesical mucosa as a result of cystitis and secondary changes, it is impossible to locate the ureteral mouths.

The instrument is introduced in the same manner as a ureteral sound with the Béniqué curve. When properly in place the convex surface of the curve rests on the floor of the bladder against the fundus between the ureteral orifices (Fig. 90, B). By revolving the handle, a membrane or diaphragm is then raised so as to completely fill the curve of the instrument; this separates the two sides and directs the flow of

A
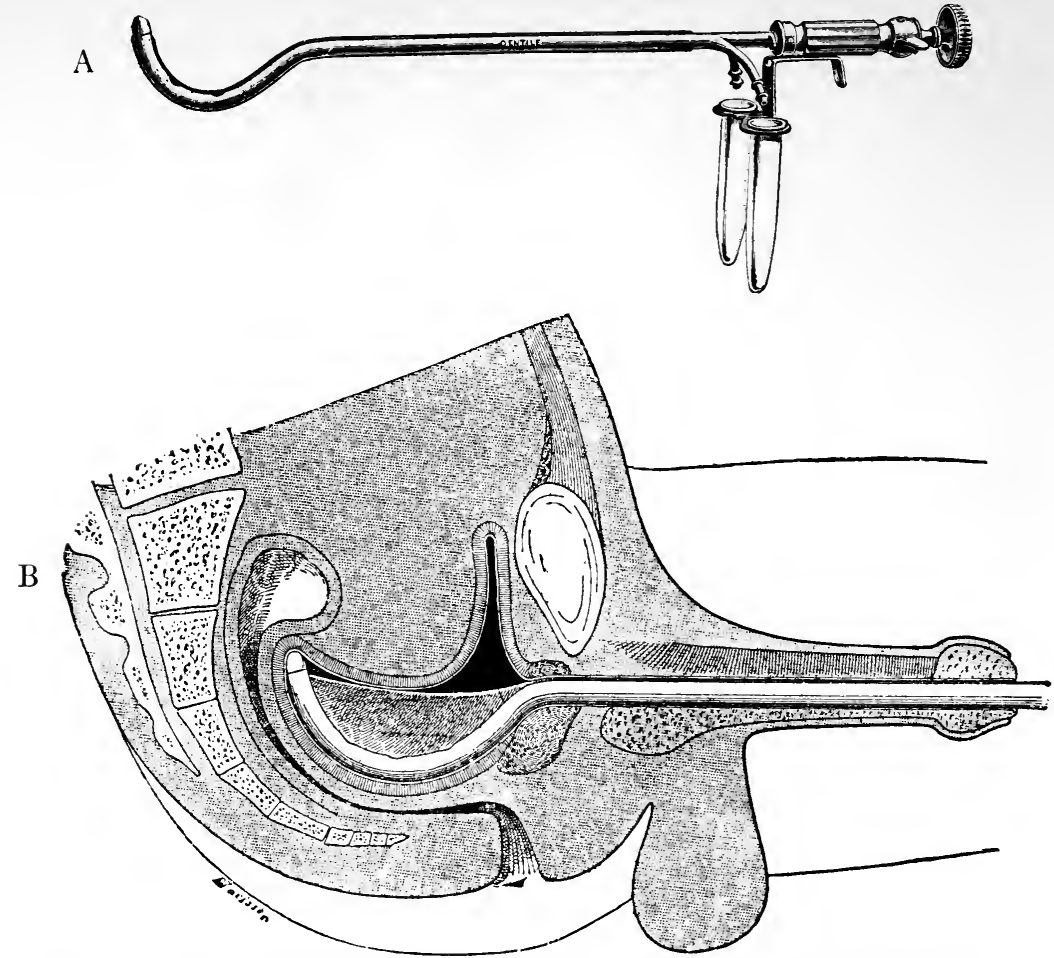

FIG. 90.-Luys urine separator. A, Instrument; B, separator in position.

the ureteral streams into the orifices of the separator on either side of the membrane whence it passes through the canals of the instrument into the receiving tubes.

This instrument has found favor with competent observers and should be included in the urologist's armamentarium. ${ }^{1}$

The specimens obtained are submitted to chemical and microscopical examination and the renal functional tests are employed in the same manner as in connection with ureteral catheterism.

1 The Luys Urine Separator, second report of forty-one cases, B. S. Barringer, Boston Med. Journal, April, 1899. 


\section{Treatment through the Cystoscope}

Destruction of Vesical Growths by High Frequency Electricity.The advantage of this treatment is that it can be employed through any good ureter-cystoscope, direct or indirect, through the catheter canal of which is passed the insulated cable or wire which conducts the high frequency current (Oudin). The tumor is destroyed by immediate necrosis. The high frequency current is obtained from a coil apparatus attached to the street current. There seems to be no limit to the size of benign growths that can be treated in this manner. The method is as follows: the preliminary steps of an ordinary cystoscopy

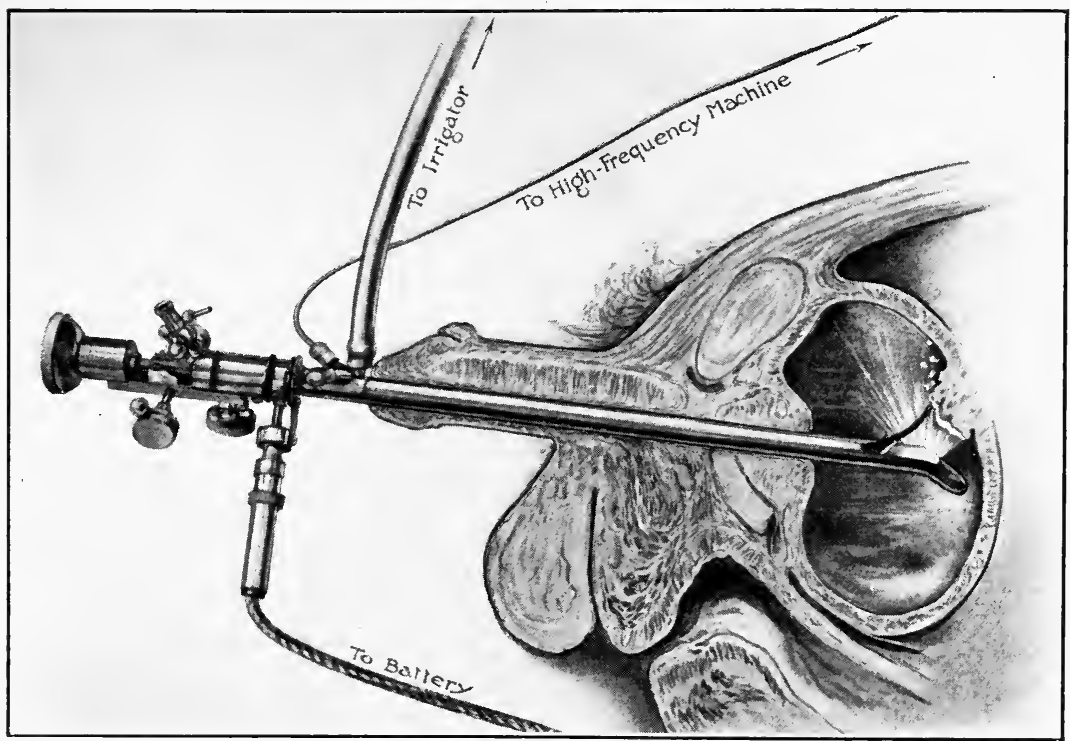

Fig. 91.-Destruction of vesical neoplasm with high frequency current. (Oudin.)

are first carried out (see p. 138). After the bladder is cleaned and a good view of the tumor obtained, the high frequency apparatus is tested, the spark gap being set at from 5 to $7 \mathrm{~mm}$. (about $1 / 4$ in.) according to the potency desired. The wider the gap, the larger the spark and the stronger will be the effect of current exerted upon the neoplasm. The insulated wire is now introduced through the catheter canal and its end, which has been previously denuded of insulation, is made to disappear within the substance of the tumor close to the pedicle. The current is then turned on, with frequent interruptions, for intervals of five to fifteen seconds at a time (Fig. 91). The treatment 
is continued until there is complete blanching of the surface of the tumor and is renewed about once a week or in sensitive and difficult cases at longer periods. Cystoscopic investigation should always be made after the work is discontinued, to make sure that the treatment has been effective. There is no question whatever as to the immediate effects of this operative procedure and even though there persists a tendency to the return of the growth, the method is so simple that frequent repetitions are not a serious objection and it is reasonable to hope that ultimately all benign cases will succumb to its effects and that some malignant ones may be controlled thereby.

Treatment by Means of the Ureteral Catheters.-This expedient may be employed for the treatment of chronic pyelitis, to inject into the pelvis of the kidney mild antiseptic solutions. Such lavage of the kidney has been accompanied by beneficial results in catarrhal conditions which prove rebellious to the ordinary methods of internal treatment by urinary disinfectants. This method is entirely unsuited to cases of advanced infective lesions in which surgical intervention is demanded. It should also be discountenanced as a common practice in cases that give evidence of catarrhal conditions in so slight a manner as the presence of only a few leucocytes in the catheterized specimen.

The solutions employed are 2 to 5 per cent. argyrol, boric acid 2 per cent. and silver nitrate 1-3000. About 5 to 10 c.c. of fluid are injected with a glass hypodermic syringe and allowed to return two or three times, and this is repeated once or twice weekly, care being observed that no reactionary irritation is caused thereby, which should be a signal to discontinue.

Drainage of the kidney from above downward may be resorted to by ureteral catheterization in some cases with decided benefit, and possibly a more severe surgical operation avoided thereby when for one reason or another postponement thereof is considered expedient. Such cases as temporary blocking of the urine on account of a twisted ureter or movable kidney may be so treated; or temporary suppression due to the blocking of the kidneys with thick and purulent secretion, in the case of double pyonephrosis when an open surgical operation is deemed too dangerous. ${ }^{1}$

1 Lilienthal (Am. Jour. Urology, February, 1912) reports four strikingly successful cases. 


\section{CHAPTER VII}

\section{FUNCTIONAL RENAL DIAGNOSIS AND ROENTGENOGRAPHY}

Functional Tests.-The normal physiological function of the kidneys or departure therefrom is studied by various methods for determining the character of the urinary secretion, and the elimination by these organs of certain substances introduced into the system for purposes of such examination. This measurement is made by comparison of the separate secretion of the two kidneys with the established standard under normal conditions; account is also taken of the relative capacities of a suspected abnormal kidney and of the opposite organ.

One means of effecting this observation is to measure the output of the normal ingredients of the urine, notably urea or nitrogen, and chloride of sodium. There is a normal variation in these ingredients in one individual as compared with another, and also in the same individual under different conditions, such as the amount of food of nitrogenous character consumed, and the amount of fluid and exercise taken. Therefore, this variation occurs during different parts of the day, which renders it necessary, for an accurate estimation of the total work performed by the kidneys in a given case, to take account of the amount and kind of food consumed and the extent of body activity. For practical purposes, it is customary to study the apparent activity of these organs, adopting a routine method of examination, combining the result of the functional test with observations made along other lines, namely: the clinical history, the general physical examination, the cystoscopy, and the presence of pathological elements in the urine of one or both kidneys, such as pus, blood, etc. The conclusion thus obtained from the preponderating evidence is usually reliable.

These functional urinary tests are employed in conjunction with other methods of examination, as a means of diagnosis of an existing kidney lesion and as a basis of deciding upon the propriety of operation or perhaps removal of one kidney; at the same time the nature and extent of the lesion in one organ are elicited, with the capacity of the opposite one, thus determining whether the latter is sufficient to carry on the body work alone. 
The various methods of making functional urinary tests that have been employed are cryoscopy or estimation of the freezing-point of the urine and comparison with that of the blood; measurement of the electrical conductivity; colorimetric estimation or measurement of the elimination in the urine of certain analine substances, namely, methylene-blue, indigo-carmine and phenolsulphonephthalein, when injected hypodermically in a given amount; the elimination of sugar in the urine after the injection of phlorizin; a comparative observation of the specific gravity and total solids; the estimation of total nitrogen or urea volume; and the polyuria test. It is not necessary to dwell at length upon the method of employment of all these various tests. Some have much more value than others and it is advisable to select from these for regular usage those in which the greatest reliance can be placed, which are not too difficult to employ, and are within reach of most observers. In other words, a practical working technic that can be depended upon in the majority of cases should be acquired, which, combined with other means of observation, has proved its value by adequate experience.

Of the methods mentioned above, that of cryoscopy is too complicated and difficult for general usage. This also applies to the method of electrical conductivity. Of the colorimetric tests the indigocarmine and phenolsulphonephthalein are employed most often while the latter is by far the more efficient. The phlorizin test gives variable results, and while its use may be of value in conjunction with one of the others, it is usually superfluous. The specific gravity is regularly recorded in most cases; for accuracy it should in reality depend upon the total twenty-four hour specimen. The total urea estimate is also a routine observation and for reliability, the conclusions from it, like the cryoscopic test, should be based upon the amount of urea in the blood at the same time. The polyuria test, or response on the part of an affected kidney to the forced blood pressure produced by the ingestion of copious draughts of water, is an interesting and sometimes telling observation but consumes an unduly long period. The best of all methods, taking into consideration the practicability and results obtained are the phenolsulphonephthalein test and estimation of the urea volume. Taken together with the chemical and pathological examination of the urine and the clinical data obtained from other sources, such a combination will furnish the means of reaching a reliable diagnosis in most instances. It is well to have at command a working knowledge of the other important tests and these are phlorizin and indigo-carmine which should be available for use as an alternative or a means of confirmation. 


\section{Method of Employing the Functional Urinary Tests}

Regular Technic.-As a preliminary measure, it is proper in all cases to collect for laboratory examination a total twenty-four hour specimen, or at least to subject to such examination a gross specimen of morning urine, before the cystoscopic investigation and the functional urinary test.

The usual steps of the cystoscopy are carried out (see p. 138), including catheterization of both ureters. A preliminary gross specimen of urine is usually first collected for separate examination; or, if it is deemed inexpedient to consume the time, separate specimens for chemical and microscopic examination are obtained previous to and independent of that collected for the functional test.

The Colorimetric Test.-This method of exploring the functional activity of the kidneys has long been considered a valuable accessory to the diagnostic investigation of these organs; more recently it has become of exceptional value on account of greater development and the reliability that can be placed upon the quantitative estimation obtained.

The injection of an aniline solution, during the course of the cystoscopic examination, is resorted to, to note the period, following the injection of such material, that the color appears from the ureteral orifices or ureteral catheters. It is also employed in the case of a misplaced ureter, or one that is hidden by circumscribed inflammation and thickening, for the purpose of finding such ureteral outlet, and thus demonstrating the existence of a secreting kidney on that side, when doubt of such has existed on account of a failure to find its outlet. Such a demonstration may have its value, but is of minor significance compared with the greater information elicited by the colorimetric test that is available for a total quantitative estimation; such is the phenolsulphonephthalein test, the best of the colorimetric method.

Phenolsulphonephthalein.- The demonstration by able investigators that this drug is eliminated from the body almost exclusively by the kidneys suggested the possibility of its unusual value for the purpose of measuring the kidney function. Extensive experiments have been made, which have successfully attested this fact. ${ }^{1}$ The preparation commonly employed is a standard alkaline solution of

\footnotetext{
1 Rowntree and Geraghty (Journ. Pharmacology and Experimental Therapeutics, July, 1910, No. 6, "An Experimental and Clinical Study of the Functional Activity of the Kidneys," etc., etc., state among the conclusions reached that: "Phenolsulphonephthalein is better adapted for use as a functional test than any other drug previously employed for the same purpose, on account of ts early appearance in the urine and the rapidity and completeness of elimination by the kidney." (4) "In unilateral and bilateral kidney disease, the absolute amount of work done by each kidney, as well as the relative proportion, can be determined when the urines are obtained separately."
} 
the drug, I c.c. of which is equivalent to $6 \mathrm{mg}$. of phenolsulphonephthalein, the customary amount that is used in conducting the test.

As a preliminary measure, to insure activity of the kidneys, one or two glasses of water are administered about one-half hour before starting the test. The dose of 1 c.c. is then injected intramuscularly, after the ureteral catheters have been introduced. For a satisfactory examination, the urine is collected for a full hour, and sometimes longer, as it has been determined that the average amount eliminated for the first hour, under normal conditions, is from 40 to 60 per cent., and for the second hour, from 20 to 25 per cent. A comparative observation may be made for a period of shorter duration, from onehalf to three-quarters of an hour, but such an abbreviated period is of less value for estimating the actual kidney function, although it may be sufficient in comparing an obviously diseased organ with an apparently normal companion, when other evidences point in the same direction, the main question being thus satisfactorily determined.

The time of the first appearance of the dye in the urine is, under normal conditions, between five and ten minutes, although this fact is not to be relied upon alone, as in some cases in which the total output is well up in the normal limits, the first appearance may be delayed for as long as fifteen minutes. The characteristic color of this test is apparent only in a strongly alkaline medium, and requires, therefore, the addition to the urine of a few drops of a solution of sodium hydroxide, 25 per cent., to bring out the color. This should not be done, however, when the total quantity is being estimated, until the entire amount for the stated period is collected. The accumulated urine may be separated, when being collected, in periods of one-half or one hour.

There are different methods of reading the result obtained in the urine collected for quantitative estimation. For this purpose a colorimeter may be used. The Duboseq apparatus is a large and expensive colorimeter, but affords accurate readings. A simplified substitute for this may be obtained in compact form, with which the percentages are read upon a scale, when the color of the urine specimen is matched with the standard solution. ${ }^{1}$

A useful and easy means of estimating the quantity of the dye has been suggested ${ }^{2}$ which is always available. This consists in preparing a series of test-tubes of equal size with different percentages of the standard solution, in variations of 5 per cent., and from a minimum of 10 to a maximum of 60 per cent. The diluted urinary specimen is then matched with the test-tube of the standard

1 Hellige's Colorimeter.

2 Hugh Cabot, Trans. Am. Ass. Genito-urinary Surgs., vol. vi, 1911. 
solution of similar intensity and color, which gives a result within 5 per cent. These diluted standard solutions should be made fresh, as in the weaker strength of solutions there is a tendency for the color to change. This, however, may be conveniently and rapidly done by simply mixing the solution in as many dilutions as is necessary to match a given diluted specimen of urine.

The standard solution of the dye is prepared for comparison with the urine specimen, by adding $6 \mathrm{mg} .{ }^{1}$ of the dye to 1 liter of distilled water, and rendered alkaline with four or five drops of 25 per cent. caustic soda solution. The specimen of urine obtained is then diluted up to about 200 c.c. with distilled water, having been previously filtered; it is then rendered alkaline by the addition of 20 drops of 25 per cent. caustic soda solution, and further diluted with distilled water up to 1 liter. Both the dye solution and the urine having been equalized in quantity up to 1 liter a given quantity of the diluted dye solution (1 c.c.) is taken and further diluted in different percentages until the similarity of color with that of the urine is obtained which indicates the percentage of dye present.

Table for mixing comparative solutions of standard phenolsulphonephthalein:

$\begin{array}{cccc}\begin{array}{c}\text { * Units of Standard } \\ \text { Dye Solution. }\end{array} & \begin{array}{c}\text { Units of } \\ \text { Water. }\end{array} & & \begin{array}{c}\text { Per } \\ \text { cent. }\end{array} \\ 5 & 95 & = & 5 \\ 10 & 90 & = & 10 \\ 15 & 85 & = & 15 \\ 20 & 80 & = & 20 \\ 25 & 75 & = & 25 \\ 30 & 70 & = & 30 \\ 35 & 65 & = & 35 \\ 40 & 60 & = & 40 \\ 45 & 55 & = & 45 \\ 50 & 50 & = & 50 \\ * 6 \text { mg. in 1 liter of water. } & & \end{array}$

$\begin{array}{cccc}\text { * Units of Standard } & \begin{array}{c}\text { Units of } \\ \text { Dye Solution. } \\ \text { Water. }\end{array} & \begin{array}{c}\text { Per } \\ \text { cent. }\end{array} \\ 55 & 45 & = & 55 \\ 60 & 40 & = & 60 \\ 65 & 35 & = & 65 \\ 70 & 30 & = & 70 \\ 75 & 25 & = & 75 \\ 80 & 20 & = & 80 \\ 85 & 15 & = & 85 \\ 90 & 10 & = & 90 \\ 95 & 5 & = & 95\end{array}$

The units in above table may be represented by minims or cubic centimeters. The per cent. desired is first decided: Thus if it be 10 per cent.,

Minims 10 (Dye sol.) +90 (Water) $=10$ per cent.

$$
10 \text { c.c. (Dye sol.) + } 90 \text { c.c. (Water) }
$$

or

$$
1.0 \text { c.c. }+9.0 \text { c.c. }=10 \text { per cent. }
$$

In other words in the case of cubic centimeters divide the figures by 10

${ }^{1}$ Ampules of 1 c.c. containing $6 \mathrm{mg}$. are obtainable. 
to reduce the bulk employed. Any number of solutions may be rapidly made up in this manner until that corresponding with the color of the tested urine is obtained.

The intravenous injection of the standard amount of phenolsulphonephthalein is employed instead of subcutaneously, as a more rapid means of obtaining the desired result. This has proved very satisfactory, and diminishes materially the time required for collecting the urine from the ureteral catheters. In this manner a practical observation may be made in fifteen to thirty minutes, during which time in normal cases from 15 to 25 per cent. of the dye is obtained.

It is often useful to make an observation of the gross amount of the dye excreted by both kidneys before or following an operation, as, for instance, in the case of prostatic hypertrophy, to determine the reliance that may be placed upon the kidneys, following the strain of operation. In this case the usual amount of the dye is injected subcutaneously or intravenously, the bladder is emptied and then washed by means of the catheter, following which the urine is collected in periods of one-half hour for as long as may be desired, up to one or two hours, and the same means of estimating the amount of elimination are employed.

The amount of dye excreted does not depend upon the amount of urine, and reveals approximately the extent of impairment of the kidney. It is generally considered the most reliable test by means of which the actual functional capacity of the kidney is determined.

Indigo-Carmine.-Before the introduction of the previous method, this test was used much more frequently. At the present time, however, it has given place largely to phenolsulphonephthalein for a number of reasons, the most important of which are: that it is less accurate, and that not more than 25 per cent. of the amount injected is eliminated by way of the kidneys. It is, however, more rapidly excreted, and appears sooner in the urine than methylene blue, and therefore still continues to be used as a rapid means of approximately making a comparative estimate of the function of the kidneys, or of ascertaining the normal presence of two organs.

In using this preparation to estimate the period that elapses between the injection and its appearance through the ureteral catheter, after the introduction of the latter, about 2 c.c. of saturated solution of indigo-carmine are injected intramuscularly into the gluteal region, and the time is taken of its first faint appearance, and again when the color caused by its elimination is a deep hue. Generally speaking, under normal conditions, the blue color is visible in from ten to twelve minutes after injection; this quickly becomes pronounced, later 
becoming a green color, which persists to a lesser degree for about twenty-four hours until the customary 25 per cent. of the whole is eliminated. The longer the delay in discoloration, the greater the disturbance of function of the kidney, and the same is true of the intensity of the discoloration.

Phlorizin.-Following the hypodermic injection of this substance, sugar is discovered in the urine. This fact has been made use of as a functional urinary test. As before stated, it unfortunately is not of invariable accuracy; some normal cases may reveal no sugar at all, and there may be a marked diminution in the amount, with only mild lesions.

It may be employed in conjunction with the other tests, when from 1 to 4 c.c. of a half of 1 per cent. solution are injected, and account is carefully noted of the time of appearance of the sugar in the urinary secretion obtained through the ureteral catheters. On account of the tendency to deterioration, fresh solutions should invariably be used.

The quantitative estimation of the glycosuria is so troublesome as to be impractical for routine usage.

The total nitrogen and urea estimation is complicated by the difficulty of the test and the necessity of having to know the exact diet of the individual; for complete estimation, a twenty-four hour specimen of urine is needed, which it is impractical and inadvisable to obtain, in collecting the urine by ureteral catheters. A simple expedient for routine examination is therefore resorted to, of estimating the urea volume on the basis of the percentage of the quantity present in the amount of urine collected. This is necessarily only relative, but, combined with other data, serves a useful purpose in the investigation. To make this estimation, the percentage of urea present in a given specimen is ascertained, and from this the urea volume is figured. The amount of grams per liter is also reckoned. For example, in a given case 5 c.c. of urine are obtained from one kidney $(\mathrm{R})$; and the urea present equals 1 per cent. From the other kidney (L) 3 c.c. of urine are collected and the urea present is 2 per cent.; thus from one 'side (R) the estimation of the urea volume shows $(5$ c.c. $\times .01=.05) 5 \mathrm{cg}$. in 5 c.c. urine and $10 \mathrm{gm}$. to the liter from the other side $(\mathrm{L})$ the result shows $(3$ c.c. $\times .02=.06) 6 \mathrm{cg}$. urea volume in 3 c.c. or about $20 \mathrm{gm}$. to the liter.

Practical Summary of the Method of Estimating the Relative Functional Capacity of the Two Kidneys.-Both ureters are catheterized, and the urine is collected in separate test-tubes. The specimens obtained are then subjected to the routine examination, including laboratory analysis and functional test. 
In a given case, the result,may be illustrated as follows:

\begin{tabular}{|c|c|c|}
\hline \multicolumn{3}{|c|}{ Laboratory Analysis } \\
\hline & Left Kidney & Right Kidney \\
\hline Quantity obtained. & 3 c.c. & 7 c.c. \\
\hline Time of collection... & 10 minutes. & 10 minutes. \\
\hline Transparency........... & Clear. & Turbid. \\
\hline Reaction............ & Acid. & Acid. \\
\hline Sp. grav... & 1015. & 1005. \\
\hline Albumen............. & Absent. & Present. \\
\hline Urea ............... & 2 per cent. & $1 / 2$ per cent. \\
\hline Total Urea Volume. & $.06 \mathrm{cg}$ & $.035 \mathrm{mg}$ \\
\hline Grams per liter......... & About $20 \mathrm{gm}$. & About $5 \mathrm{gm}$. \\
\hline Microscope: & & \\
\hline Pus.. & None. & Numerous cells. \\
\hline Blood.... & Few cells. & Many cells. \\
\hline Casts.... . . . . & None. & None. \\
\hline Crystals............ & None. & None. \\
\hline Other elements....... & None. & $\begin{array}{l}\text { Miscellaneous bacteria } \\
\text { and a few tubercle } \\
\text { bacilli. }\end{array}$ \\
\hline
\end{tabular}

Functional Test

1 c.c. phenolsulphonephthalein (6 mg.) injected intramuscularly.

Time of Collection 2 Hours.

\begin{tabular}{|c|c|}
\hline opearance. & 15 minutes. \\
\hline $\mathrm{Fil}$ & 40 per cent. \\
\hline ur & 20 per cent. \\
\hline
\end{tabular}

The interpretation of this result would support the diagnosis of a tuberculous lesion of the right kidney and a deficiently functionating organ, as revealed by both the urea volume and functional test, while the organ on the opposite side is normal and capable of carrying on the excretory function alone.

\section{ROENTGENOGRAPHIC DIAGNOSIS OF THE URINARY TRACT}

Roentgenography has its greatest field of usefulness at the present time in connection with the kidney, and especially for the detection of renal and ureteral calculi (Figs. 92, 93). While it has an increasing field of usefulness in investigating obscure and otherwise unexplained conditions of the ureterogenital tract, it may be superfluous as a means of observing conditions that can be more expeditiously and effectively diagnosed by other methods. For instance, a stone in the bladder can readily be detected by vesical palpation with the proper sounding instrument, and may be seen with the cystoscope; in such a case, roent- 
genography is a waste of time. Experimental investigation with the roentgen-ray, however, may be the means of illuminating the diagnosis of conditions that are not explained by cystoscopic observation; in the case of children, and in some instances old people, in which cystoscopy is impractical it has an important use (Fig. 94). The field of roentgen-ray diagnosis has been extended substantially through the

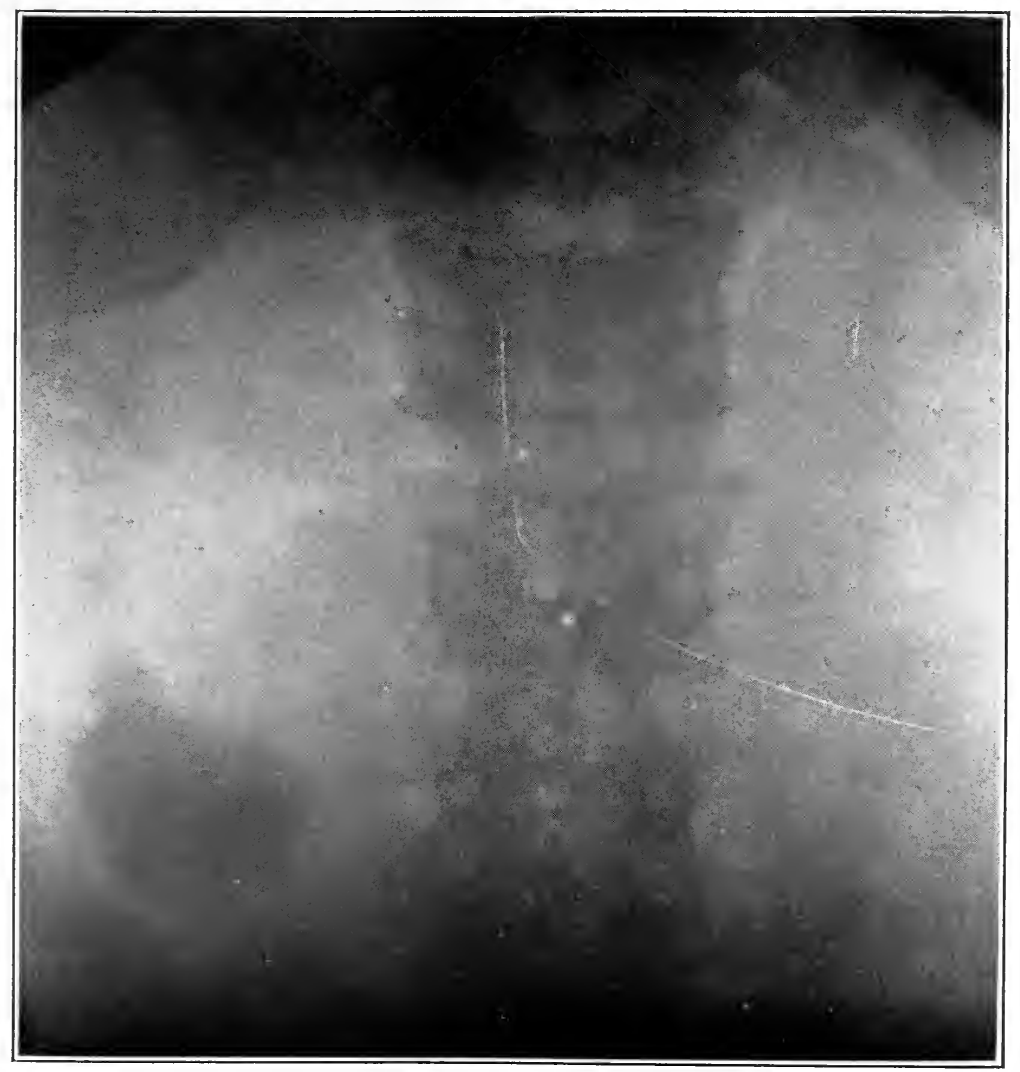

FIG. 92.-Roentgenogram of renal calculus.

aid of the stiletted or opaque catheter and the injection into the bladder, ureters and pelvis of the kidney of the opaque solutions of silver salts or thorium 10 per cent. By this means it is aimed to discover unexplained mechanical obstructions at the neck of the bladder, vesical calculi, ureteral obstructions, and cystic enlargements, displacements, and anomalies of the kidney. It is also possible by means 
of a good roentgenogram to distinguish the outline of an enlarged kidney, expecially when such kidney is the seat of a morbid process, such as the existence of tuberculous foci. In obtaining a roentgenogram of the urinary apparatus, calculi when present cast a shadow which is visible upon the plate (Figs. 92 and 95), because the permeability of the rays is different from that of the soft parts, the muscles and tendons surrounding them, and because permeability differs according to the composition of the stone. Oxalates and phosphates, which are repre-

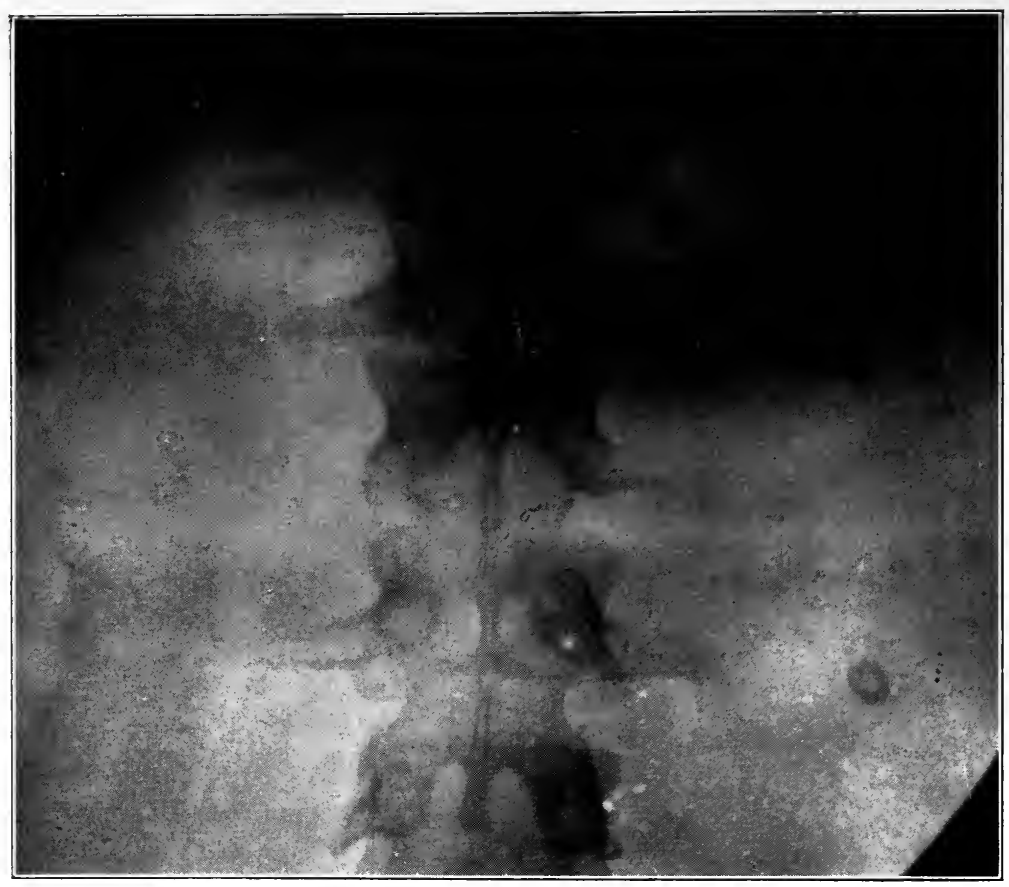

Fig. 93.-Roentgenogram of ureteral stone.

sented in the oxalates of lime and triple phosphate stones, give a distinct image, and those of cystine, which are rare, also cast a faint shadow; whereas, on the contrary, uric acid stones bear a resemblance to the soft parts in their relation to the rays, and in consequence cast an indistinct or no shadow upon the plates when they are of pure uric acid composition. This is the reason why pure uric acid gravel and calculi of small size are not revealed by roentgenographic examination. Uric acid stones, however, are commonly of mixed composition, containing in addition phosphate or oxalates. 
In order to insure a satisfactory roentgen-ray examination, the patient should be prepared by free catharsis, preferably castor oil, 30 to 60 c.c., the evening before examination. If the bowels have not moved in the morning, he should be given a copious enema. It is advisable that the roentgenogram then be taken before the ingestion of any food.

A satisfactory roentgenogram of the kidney and ureter is obtained by placing the patient upon his back. Ordinarily, plates are taken in two directions; one with the tube focused between the umbilicus and

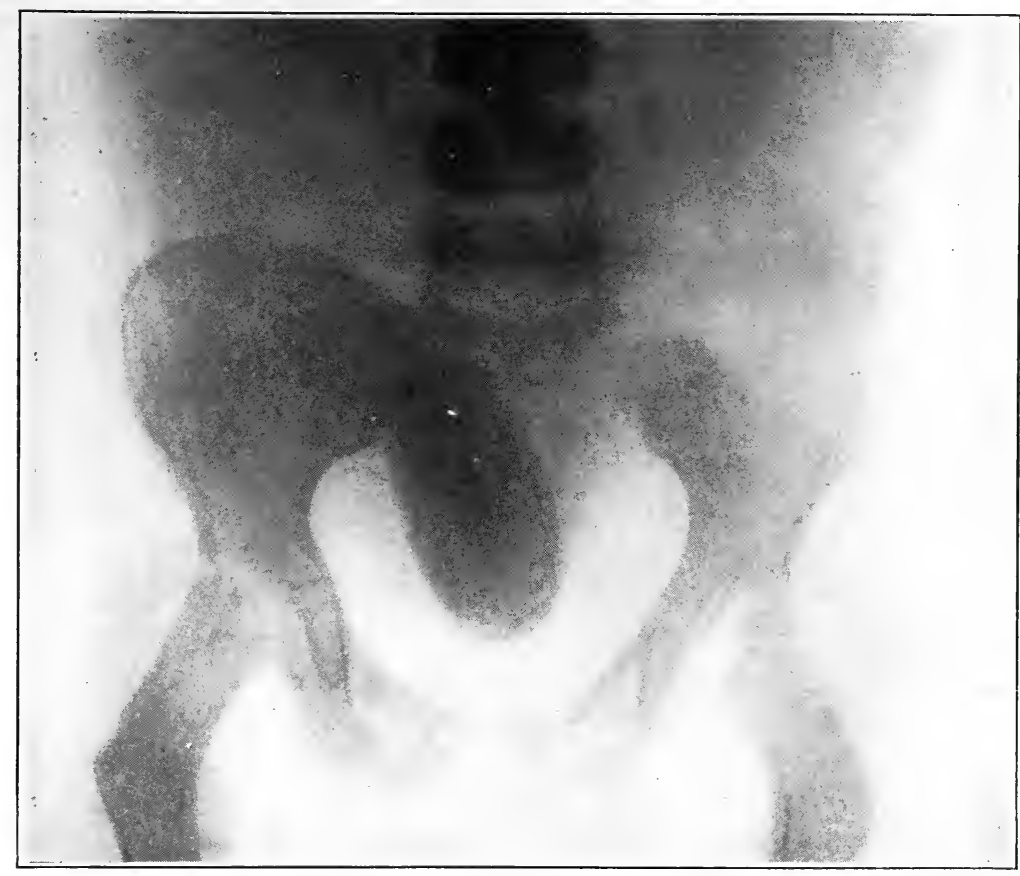

Fig. 94.-Roentgenogram of large vesical calculus. (Child.) (Author's case.)

xiphoid, pointing slightly upward; this takes in the kidney and upper portion of the ureter; the other, by focusing the tube midway between the umbilicus and symphysis pubis, pointing somewhat downward, which will take in the lower ureteral region and ureterovesical outlet. In both instances, the best results are obtained by using firm compression, especially in fat subjects. In all cases of doubtful shadows, confirmatory plates should be taken.

The essential requirements of successful kidney plates are that the transverse processes of the lumbar vertebræ be shown, and that the 
groove of the outer border of the psoas muscle is also clearly revealed. An excellent roentgenogram may outline the entire kidney. The lower pole should at least be distinctly recognizable. Stones in the kidney or pelvis are usually easy to demonstrate by roentgenographic plates; localization may be difficult, unless the kidney itself throws a shadow

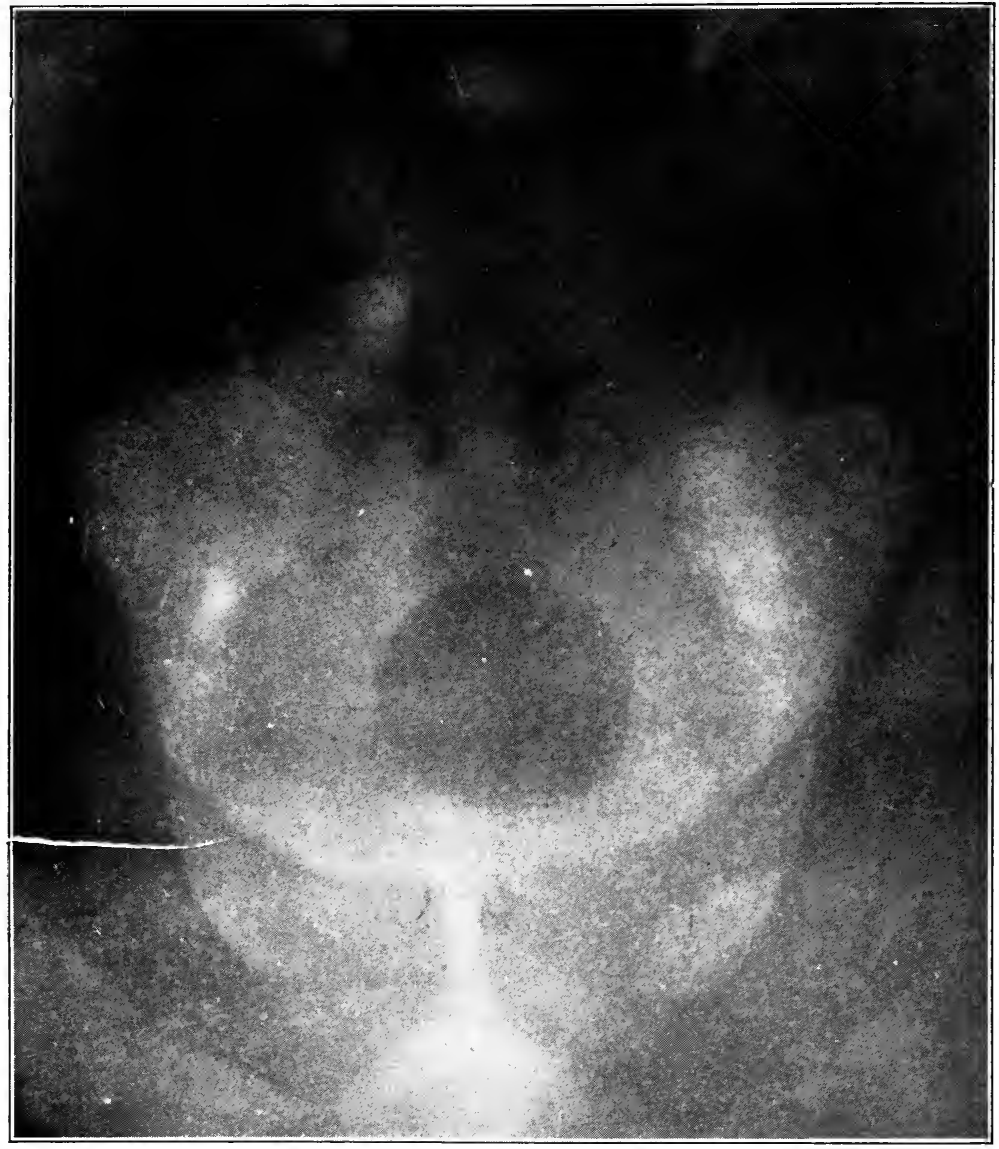

Fig. 95.--Roentgenogram of resical calculus. (Adult.)

or stereoscopic views are taken. Pyelography affords the best means of localization of renal and pelvic stone. A tuberculous kidney may simulate calculus.

Stones in the ureter, notably when they have passed the pelvic brim, offer special difficulty in diagnosis from phleboliths. Errors in this respect may be prevented by the use of a shadow-catheter 
(Fig. 96). Such a catheter may be the ordinary ureteral catheter, stiletted, or a specially manufactured leaded catheter; or by injecting silver solution (collargol or argyrol or thorium) previous to the intro-

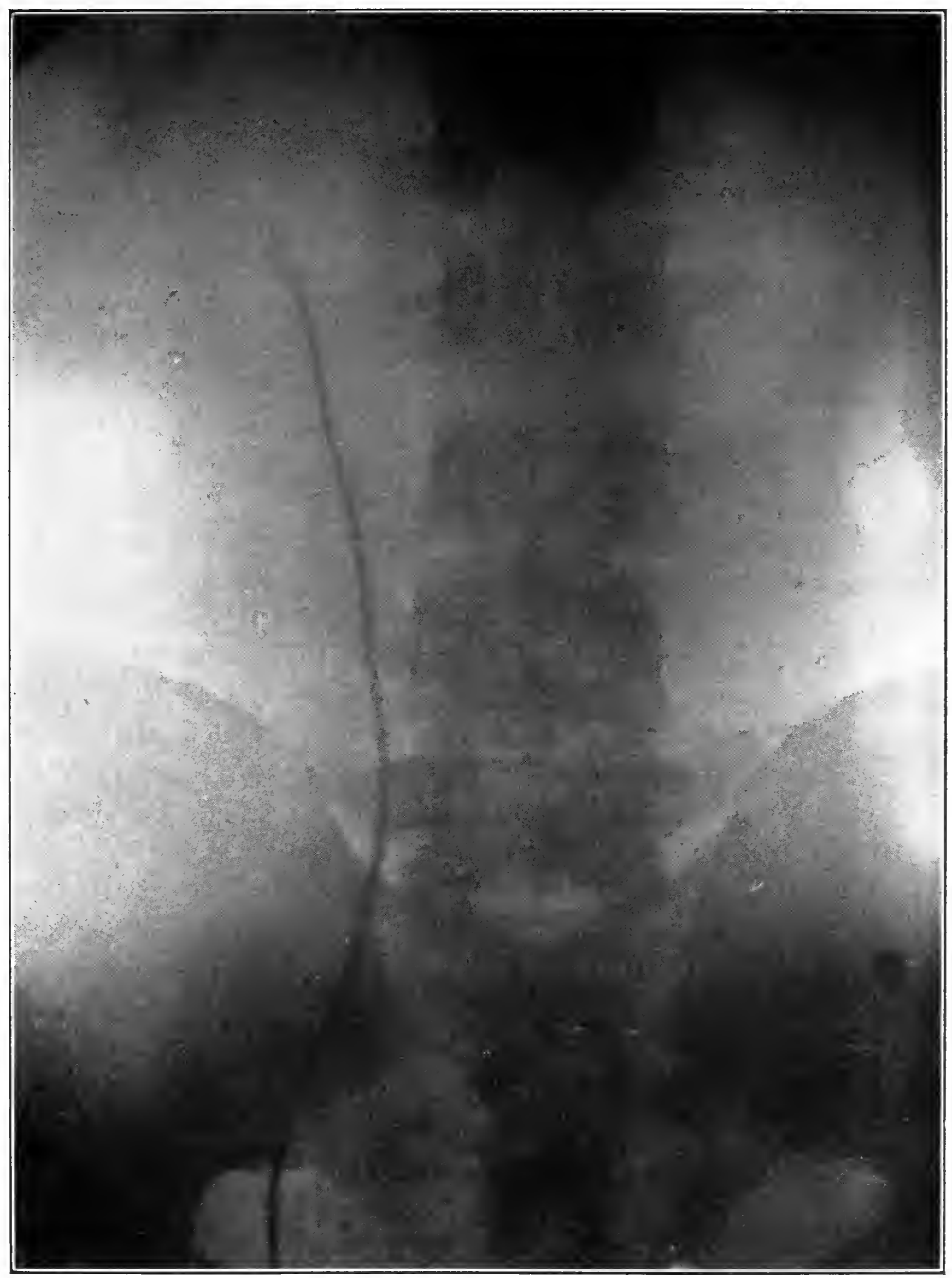

FIG. 96.-Roentgenogram of shadow catheter.

duction thereof, enough of the solution adhering to give a good shadow. If the shadow is more than $1 \mathrm{~cm}$. away from the line of the shadowed catheter, a negative diagnosis of calculus may be made. When the 
shadow is within this area, the result is doubtful, as the shadow may still be a calculus without overlapping the ureteral catheter. Under such conditions, pyelography affords a more accurate method of differentiation. ${ }^{1}$ Stereoscopic examination under these circumstances will clear the diagnosis.

Pyelography and Cystography.-The subnitrate of bismuth was first employed to demonstrate a bladder diverticulum by the roentgenogram. ${ }^{2}$ This method, however, has been entirely replaced by the injection of opaque silver solutions, following which the bladder has been studied under various conditions, satisfactory roentgenograms being taken with 2 per cent. or stronger collargol solutions. ${ }^{3}$ In this manner, bladder diverticula are demonstrated, contraction of the cavity is shown, and variations from the normal contour of the resicoprostatic outline. $^{4}$ Roentgen examination after inflation of the bladder with air often gives valuable information: e.g., intra-vesical growth, etc.

Pyelography has a still greater diagnostic value than cystography, and its employment elicits important evidence in many conditions, among which are dilatation of the pelvis, when it may be employed in connection with pyelometry (see p. 151); hydronephrosis, which may be seen to occupy the kidney substance or the pelvis; cystic kidney; pyonephrosis, which may reveal under this examination the characteristic shadow of the irregular lobulated enlargement (Fig. 97); renal tumors, causing various distortions of the pelvis; and finally, ureteral stone and constrictions with dilated pouch above the point of obstruction may be brought out in graphic detail by this method.

A 10 to 15 per cent. solution of collargol has been used in this work; much stronger solutions of argyrol have been tried and seem to be harmless; 10 per cent. of collargol, however, is of sufficient strength to obtain the desired shadow and a 10 per cent. solution of thorium gives excellent results. It is preferable to insert the catheters only a few centimeters within the ureters, which tends to prevent the occurrence of colic and also renders obstructions and dilatation along the course of the ureter visible upon the roentgenographic plate. The amount of fluid injected must vary in different cases, according to the dimensions of the pelvis, as determined by previous measurement; or action in this particular is guided by the sensation of pain complained

${ }^{1}$ Braasch, The Radiograph Shadow of the Cystoscope, Surg., Gyn. and Obst., April, 1911.

${ }^{2}$ Wulff, Fortschritte für Roentgenstr., VIII, 193.

3 Voelcher u. Lichtenberg, Muenchener med. Woch., 1905, 33. These authors found that the bladder, under moderate distention appears pear shape, with the apex downward. The exterior pressure of prolapsus uteri produced an asymmetry of the bladder contour. Prostatic hypertrophy showed, as a rule, a flattening of the apex.

${ }^{4}$ A radiographic study of the region of the neck of the bladder has been more recently conducted by Barringer and McKee (Trans. Amer. Urol. Assoc., 1912). 
of during the deliberate and careful injection, always by gravity, as forced injection with a syringe is responsible for much of the damage reported in pyelographic work. When the catheter is advanced up into the pelvis of the kidney it is well to use one that allows some leakage to obviate the occurrence of severe pain. Additional and sometimes valuable information is obtained by changing the position

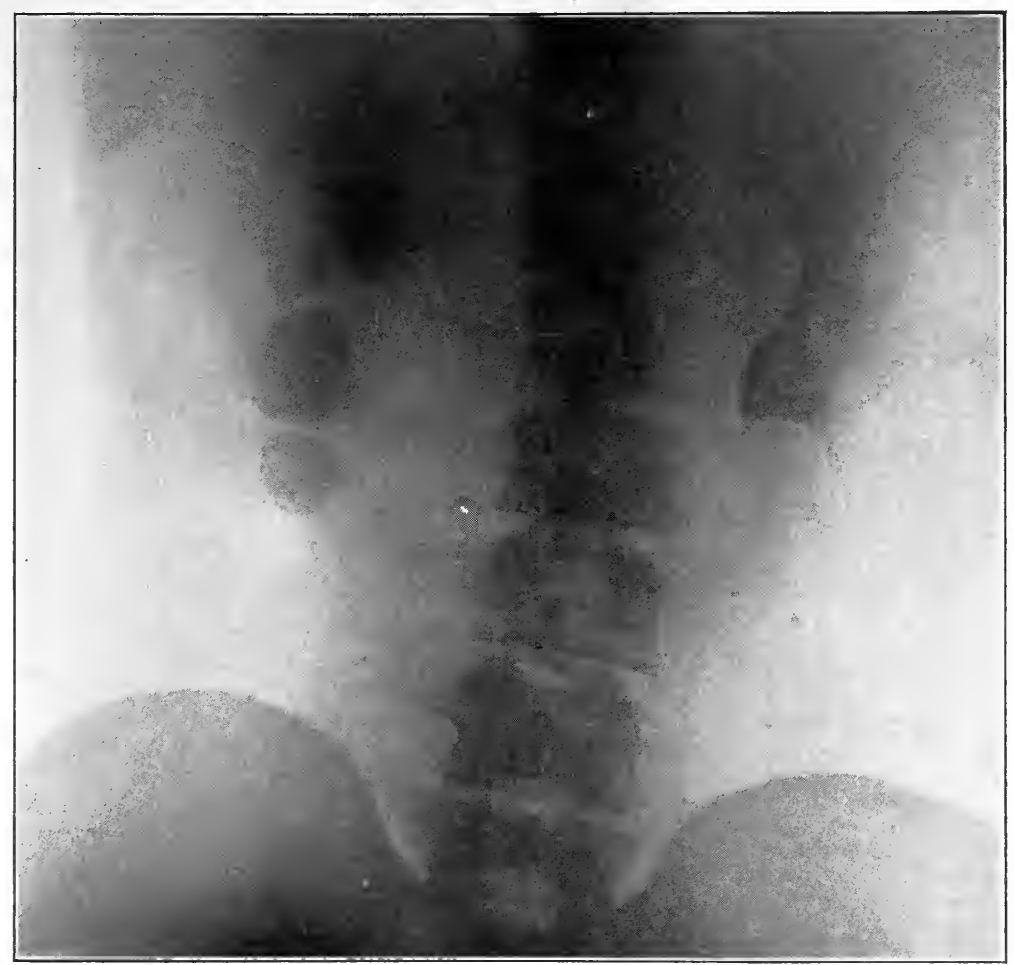

FIG. 97.-Pyelography showing lobulated kidney on one side, and normal pelvis opposite. (Author's case.)

of the patient in taking a series of roentgenograms in connection with pyelography; thus the changing position of a prolapsed and mobile organ may be shown in a picture taken in the upright position, compared with one in the recumbent posture, and explanation thus afforded of painful renal crises caused by pelvic retention (see Hydronephrosis). 


\section{CHAPTER VIII \\ MALADIES OF THE PENIS \\ INJURIES, INFLAMMATION, GROWTHS}

Wounds of the Penis.-Injuries of the penis may occur as contusions, punctures and lacerations.

Contusions.- Such injury may be due to a crushing blow which does not necessarily break or rupture the skin but causes laceration of the subsurface vessels and hemorrhage into the loose, cellular tissue, the outward appearance being that of general swelling and ecchymosis. The greater the injury, the greater the depth thereof, sometimes involving the corpora cavernosa, thus greatly increasing the internal hemorrhage, and the condition may then be a part of an injury involving rupture of the urethra, when the latter condition becomes of prime importance, as extravasation and retention of urine may result (see Index).

The clinical course of contusions of the penis is that of contusions elsewhere and the possible dangers, therefore, are infection, leading to acute inflammation and suppuration, or extreme swelling and gangrene.

Treatment.-In mild cases, rest, wet dressings (composed of aluminum acetate 2 per cent., carbolic acid, 1 per cent., or dilute lead water) with gentle pressure, will generally bring about recovery. In severe cases, when inflammation is intense and suppuration occurs, incision and free drainage is necessary. If rupture of the urethra forms a part of the injury, the treatment is of the latter condition, very likely requiring the introduction of an indwelling catheter (Fig. 41 , p. 53), to prevent constant infiltration with urine into the submucous structures.

Punctured, Lacerated and Incised Wounds.-These injuries may be superficial or deep. They may be a part of a general injury or inflicted by such an implement as a knife or nail, independent of any other involvement. The symptoms of pain, hemorrhage and distortion of the anatomical structure vary according to the character and extent of the injury. In a punctured wound, the superficial injury may be slight, but there may be much subsurface hemorrhage producing swelling and deformity. Lacerated wounds may denude the superficial structure or separate them from the deeper parts causing much hemorrhage and severe reaction. Incised wounds may be longitudinal 170 
or transverse, in either case producing complete or incomplete amputation and resulting in dangerous or even fatal hemorrhage.

The treatment of these injuries is upon proper surgical principles. Thorough cleansing and strict antisepsis of the parts are required and then such suturing of the incised or lacerated area as may appear necessary, hemorrhage having been first controlled either by hemostatic forceps and ligature or by hot sterile water. An excellent means of hemostasis for capillary and venous oozing may be found in the application of pulverized formaldehyd gelatin (glutol-Schleich). Further control is exercised by the application of a splint and a snug bandage. If the urethra is not ruptured, the introduction of a catheter is not required but the dressing may have to be loosened to permit urination. Priapism should be controlled by an opiate, hypodermatically or in a suppository or, better still, by the administration of 1 or 2 grams of bromide of sodium or potassium at night.

Luxation of the Penis.-This accident is usually a part of a more general injury suffered by the patient, as a result of which the organ is forced out of its sheath, downward into the scrotum, outward upon the loin or upward over the pubic bone. The urethra may or may not be ruptured, and there may be accompanying laceration of the superficial' structures. The condition is recognized by the resulting displacement, which is accompanied by extensive subcutaneous tumefaction; retention of urine usually exists, accompanied by extravasation, if the urethra is ruptured.

Treatment.-The penis should be replaced at once and the condition of the urethra investigated. Hemorrhage is checked and the same course followed as for lacerated wounds; in severe cases accompanied by retention and extravasation of urine, external urethrotomy is indicated.

Fracture of the Penis.-Any accident causing forcible bending of the organ may result in rupture of its fibrous sheath. This is accompanied by great pain and extensive extravasation of blood. The urethra may also be ruptured. Rupture of the corpus spongiosum, or what is spoken of as breaking the chordee, is caused by forcibly bending the curvature of chordee during an attack of acute gonorrhea. This is also followed by hemorrhage and swelling and later results in traumatic stricture. The excessive deposit of fibrous connective tissue as a result of fracture of the penis may lead to deformity and functional impairment.

Treatment.-Immediate treatment calls for means to arrest hemorrhage and control swelling. This is best accomplished by the introduction of an indwelling catheter (p. 53) and the application of wet 
dressings of lead water or carbolic solution which should be kept in place by the gentle pressure of a snugly applied bandage.

Inflammatory Conditions of the Penis.-Inflammation of the penis may involve the entire organ as cellulitis, lymphangitis or phlebitis, or it may attack the skin of the prepuce and mucous membrane of the glans (balanitis, balanoposthitis).

Cellulitis.-The occurrence of this inflammation of the organ is either in connection with one of the lacerated or contused wounds already mentioned, as a complication to severe inflammation of the urethra, usually gonorrheal in character, or as an extension of severe inflammation within the prepuce (balanitis, balanoposthitis, q.v.). Furthermore, cellulitis may extend from a follicular inflammation which latter has occurred as a complication of gonorrheal or nonspecific urethritis (q.v., p. 242), when suppuration frequently occurs. Further extension of cellulitis involves the perifollicular tissue and inflammation of the corpora cavernosa (cavernitis), and this latter condition may become chronic, as a sequel of which an infiltration of fibrous tissue may take place in the form of single or multiple fibrotic tumors in the sheath of the corpora cavernosa.

Fibrous plaques of the copora cavernosa are frequently encountered. They were formerly looked upon with some curiosity on account of the small number of cases that had been reported.

To-day, in the large clinics and in private practice, they are not considered a great rarity. The fibrosis occurs in a circumscribed area; sometimes one, sometimes two or more of such are found in the sheath of the corpora cavernosa, anywhere from the corona glandis back to the root of the penis. They feel like cartilaginous plates, are composed primarily of fibrous tissue and later may undergo calcification or ossification. These circumscribed areas of fibrous deposit, which may be secondary to previous injury and inflammation, are said to occur with greater likelihood in those of gouty diathesis. The pathology, therefore, is primarily inflammation and secondarily fibrosis which may ultimately, although exceptionally, undergo calcification and ossification. There may be no symptoms in connection with the development of this condition until it acquires such a size as to call the attention of the patient, who may recognize the hardened area or areas or, when, on account of its obstruction to the free circulation in the cavernous tissues, erection becomes partially interfered with and curvature results. The condition is a chronic one and no palliative measures have been found that have proved successful in removing the hardened area. Such injections as thiosinamin and fibrolysin, the author has tried without success. Removal with the 
knife is justifiable, if the area is not too extensive, and I have performed this operation successfully on more than one occasion with alleged improvement in the erectal power.

The treatment of cellulitis of the penis requires first, a recognition of the cause thereof. If the condition occurs in connection with a local injury, its care is that of such inflammation elsewhere, namely, wet dressings and bandaging; if suppuration ensues, surgical drainage is necessary; and if it is a complication of acute urethritis, its treatment is simply supplemental to the measures adopted for the care of the primary malady.

Lymphangitis is usually a complication of gonorrheal inflammation of the urethra or of an inflammation around the glans and prepuce (q.v., p. 241).

Elephantiasis of the penis occurs not alone but as a part of the same condition affecting the scrotum, when it is due to mechanical interference with the return circulation, dependent upon the extensive deposit of connective tissue after operation upon the abdomen or removal of the inguinal glands. This, in effect, amounts to a chronic form of edema, which may exist in mild or considerable degree, according to the extent of the preëxistent cause.

In tropical countries, the presence of the filaria sanguinis hominis in the lymph vessels may be the underlying reason for this lesion.

\section{Inflammation of the Glans Penis and Prepuce}

Balanitis-Balanoposthitis.-Uncleanliness, the retention of irritating discharges and a congenitally long foreskin are predisposing factors to this condition which is a common complication of gonorrheal inflammation. Balanitis is inflammation of the surface of the glans and posthitis of the mucous lining of the prepuce. The two conditions coëxist.

The clinical symptoms are redness and moisture of the mucous membrane, surrounding the glans and foreskin with a certain amount of creamy secretion of offensive odor, accompanied by constant itching or burning of the parts. In more advanced form, the mucous surface may become covered with excoriations. The whole substance of the prepuce may be swollen sufficiently to occasion inflammatory phimosis. The excoriations result in irregular patches and when the irritation persists, they go on to form ulcerations superficial in character, often simulating the appearance of chancroid (p. 682).

Chronic balanoposthitis occurs in men past middle age with tight and pendulous foreskin. The inflammation is mild in character and as it extends over a long period, produces thickening of the mucous 
surface of the prepuce, which ${ }_{b}$ when retracted, is found to be covered with granular prominences or superficial excoriations. Sometimes retraction is impossible and there is a resulting compression and atrophy of the glans penis.

The complications of balanoposthitis are phimosis, paraphimosis, vegetations, lymphangitis, inguinal adenitis, gangrene.

Phimosis is the direct result of inflammatory swelling. Paraphimosis is due to thickening of the retracted foreskin (p. 180). Lymphangitis is similar to that which occurs with chancroids of the penis (see index). Inguinal adenitis sometimes accompanies lymphangitis as a complication of balanitis, and resembles the same condition occurring in connection with chancroids (p. 690).

Vegetations or papylomata complicate balanoposthitis as a result of the same conditions that cause the latter trouble. Gangrene and sloughing of the constricted prepuce may result from excessive swelling of the parts, entailing ultimate deformity due to cicatricial contraction.

The diagnosis of balanitis is simple in most cases but it may be confounded with herpes, chancroid or gonorrhea. When the inflammation is highly acute and ulcerations exist upon the foreskin, it is difficult to distinguish ordinary balanitis from ulcerated herpes. In the early stage, the outline of herpetic vesicles may be recognized (p. 176).

Chancroid is more apt to be confounded with balanitis, complicated by deep ulcerations. The course of the inflammation is much more severe and rapid in chancroid. Lymphangitis and adenitis are quite common in the latter, while in simple balanoposthitis they are less likely to be present and treatment is apt to be more promptly effective.

In subpreputial chancre or chancroid, when the foreskin will not retract, the diagnosis remains doubtful until the preputial cavity can be inspected or other signs clear up the diagnosis. When there is a copious flow of pus, if there be phimosis, balanitis may be mistaken for acute gonorrhea. This question is settled by examination of the discharge for the gonococcus, and by determining whether or not the flow exudes from the meatus after washing the cavity of the prepuce.

Treatment.-When the prepuce can be retracted, mild balanitis may be speedily relieved by cleanliness and the use of a mild disinfectant or astringent lotion. For this purpose may be used the following:

R. Acid. boric.............. 2 gm. $(3 \mathrm{ss})$

Zinc sulphocarbolate......... 0.065 gm. (gr. i)

Water.................. 125 c.c. (马iv) 
R. Zinc sulphate............. $0.065 \mathrm{gm}$.

Phenol ................... c.

Acid boric $\ldots \ldots \ldots \ldots \ldots \ldots \ldots \ldots 4$ c.c.

Spirits lavander compound ....... 8 c.c.

Water ................. $110 \quad$ c.c

R. Goulard's extract ...........2 c.c.

Water................. 120 c.c.

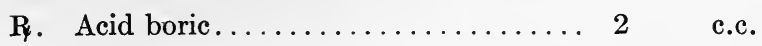

Borax................... 4 c.c.

Zinc chloride $\ldots \ldots \ldots \ldots \ldots \ldots \ldots \ldots . .1 \ldots \ldots$ c.c.

Formalin solution ........... $0.2 \quad$ c.c.

Distilled extract witch hazel...... $25 \quad$ c.c.

Distilled water............. $75 \quad$ c.c.

A strip of thin gauze cut in the centre and moistened in one of the above lotions is laid around the glans and the prepuce pulled forward to its natural position. Dressings should be repeated several times a day and the irritating secretions should always be removed by washing with warm water and plain soap. Sometimes a dry dressing acts better than a moist one, especially when erosions or ulcerations exist. Under these circumstances, the discharge should be frequently removed and the glans and prepuce carefully washed with a mild disinfectant solution (corrosive sublimate 1:6000 or 8000), after which the parts should be dried with absorbent cotton and the surface dusted with bismuth, perhaps containing a little boric acid or calomel, or with iodide of thymol. In chronic cases and when the ulcerations persist in spite of the soothing form of treatment, the application of a solution of nitrate of silver ( 2 to 6 per cent.), or 10 per cent. argyrol by means of a camel's-hair brush may assist in bringing about a more rapid cure. When the prepuce cannot be retracted, its culde-sac must be cleansed thoroughly with hot water and one of the above lotions, by means of a duck-bill syringe. This should be done frequently during the day, as often as the discharge reaccumulates. If the parts are generally swollen, a wet dressing of aluminum acetate or mild sublimate solution should be employed in addition to the above measures. If, by the adoption of such means, improvement is deferred, or if inflammation is so intense that sloughing of the prepuce be threatened, it is better to relieve tension by bilateral incision of the foreskin (see p. 340). If upon exposure of the region behind the glans, chancroid is discovered, its treatment should be conducted in accordance with the instructions laid down for chancroidal phimosis. 
In all chronic cases of balanitis with phimosis and thickening of the mucous membrane, circumcision (q.v.) affords proper means of relief and should be resorted to. Lotions and astringent injection in these cases afford little relief, as relapses are liable to recur continually. For those who are more than ordinarily predisposed to these conditions, the prophylactic measures consist of frequent cleansing of the parts and careful drying thereafter.

Erosive and Gangrenous Balanitis.-A definite and individual infection of the glans penis, which is not an ordinary balano-posthitis, although sometimes confused with it and, on account of its virulence and persistence, with chancroidal ulceration. It has been recognized and described by a number of foreign observers, and primarily in this country by Corbus and Harris, ${ }^{1}$ so that it has been clearly distinguished as a distinct pathological entity. Bacteriologically, it is due to a dual infection by a vibrio and a spirochete. The same bacteriologic findings are discovered in noma and Vincent's angina, which are the etiologic factors of gangrenous stomatitis. The conditions which have been found to favor the growth of these organisms are heat, moisture, uncleanliness and the absence of air, which conditions are peculiarly afforded by the glans penis, with a long and tight foreskin.

Therefore, in cases of sluggish ulceration, of gangrenous character a bacteriological examination should be undertaken, in view of the possibility of the existence of this special type of erosive and gangrenous balanitis. In such case, there will be found, by culture, typical vibrios and spirochetes, which bacteriological combination characterizes this type of ulceration and determines the diagnosis.

The treatment is cleanliness and antiseptic applications, which are promptly effective when the parts are exposed to the air, as the organisms are anaerobic.

\section{Herpes Progenitalis}

Herpetic vesicles, single or multiple, appear upon the mucous membrane covering the glans and prepuce and upon the cutaneous surface as well. They may be located in the sulcus, behind the corona, in the folds of the frenum on either side, around or inside the meatus, or on any portion of the integument. When occurring upon the skin, the vesicles dry down and form scabs, but when situated upon the mucous lining, they run a different course owing to the rupture of the vesicle which takes place early, leaving more or less superficial ulcerations. In the case of a single vesicle, this is irregularly round, but when

${ }^{1}$ Journal A. M. A., 1908, p. 1474. 
several vesicles lie close together, they may become fused into a single patch of irregular outline. The symptoms attending this lesion are burning or itching of the surface and a certain amount of watery secretion, which, when allowed to collect, becomes purulent. Subpreputial herpes, after the vesicles break, may produce balanoposthitis if the secretion be allowed to accumulate. When the ulcerations become unusually deep, as a result of neglect of treatment, and are accompanied by inguinal adenitis, the sores may be confounded with chancroidal ulceration. In reaching a diagnosis, it should be remembered that the herpetic condition starts in vesicles, generally in clusters, which precede the appearance of ulceration, and that bubo is less common than with chancroid. Differential diagnosis is sometimes difficult, but a decision may be reached by evidence of auto-inoculation. The pus of ulcerated herpes will not reproduce the typical ulcer which is seen upon the contiguous. surface as a result of auto-inoculation with chancroidal pus. The duration of herpes when uncomplicated is from three or four days to a fortnight.

The causes of herpes are local irritation and diathetic predisposition, uncleanliness and the decomposition of sebaceous material, especially when the prepuce is long and contracted. Full-blooded, gouty, rheumatic and neurotic individuals seem to possess a predisposition to the affection. Sometimes herpes progenitalis is attended by rather pronounced neuralgic pain-a feature which may be marked in any kind of herpes, reaching its highest expression in herpes zoster.

Treatment.-For cutaneous herpes, prevent the vesicles from breaking by covering the patch with collodion and keep it covered until it is well. If the vesicles break, dust them with bismuth or iodide of thymol. On the mucous surface, dress the vesicles daily with an ointment of zinc and tar and dust with bismuth or iodide of thymol when they break. After excoriations occur, the treatment is generally the same as for balanitis, requiring that the irritating discharge should be removed and the parts cleansed and maintained in this condition by suitable disinfectant lotions. As indicated in the previous section on balanitis, after using the lotion the parts should be thoroughly dried and a mild dusting-powder applied-bismuth, zinc, starch or iodide of thymol. Underlying constitutional conditions should be attended to. In chronic and relapsing herpes, in cases accompanied by sharp neuralgic pains and in those showing a tendency to persistent ulceration, it may be necessary to stimulate the surface; for this purpose may be employed a 10 per cent. solution of nitrate of silver or 10 per cent. argyrol. When a long, tight prepuce is the predisposing cause, circumcision is the natural remedy. 


\section{Venereal Warts-Papillomata}

Vegetations, verrucæ or venereal warts (Fig. 98), as they are commonly called, spring up readily in both sexes about the genitals if acrid and irritating discharges be retained until they have had time to decompose. Pathologically, they consist of highly vascular papillary outgrowths, composed of an increase of the epithelial and connective tissue. They are not necessarily of venereal origin, but are

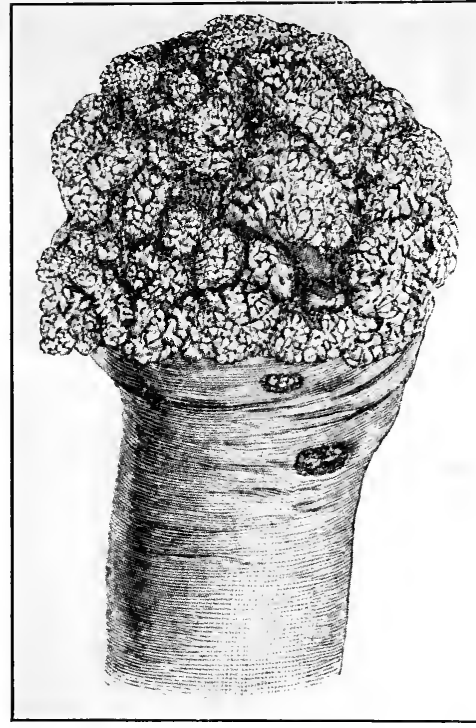

FIG. 98.-Papilloma of penis. common under a tight prepuce in connection with gonorrhea and are often found complicating balanitis when there is no urethral inflammation. Those that occur upon the mucous membrane are soft and yielding in character; upon the integument, they are harder. When dry they resemble ordinary skin warts and are composed of pointed, epithelial prominences which grow up into a raspberry-like mass, varying from the size of a pin head to that of a hickory nut. They may spread out as a dry, velvety growth over a large surface. The soft warts are commonly situated behind the corona glandis on each side of the frenum, but may be encountered on any portion of the mucous suface of the prepuce. They are sometimes found in the mouth of and deep in the urethra and are distributed also over the scrotum and frequently around the anus. They may be scattered in irregular collections in one or more of these sites, or may be gathered so abundantly upon the glans penis as to bury it completely, giving it a cauliflower-like appearance. If the prepuce be long, the extensive outgrowth may emerge through the orifice, or, on account of a tight phimosis and consequent subpreputial pressure, may produce gangrene and perforation of the prepuce.

The inflammation due to these excrescences and their irritating discharge may result in balano-posthitis and inflammatory phimosis.

Paraphimosis may also come on during a retraction of the pre- 
puce on account of the hyperplastic inflammatory condition of the tissues due to the presence of these growths (p. 180).

The contagiousness of the venereal warts has been maintained and is probably well founded.

Diagnosis. - The presence of these growths is self-evident. They should not be confounded with "condylomata lata" which are of specific origin. Such an error can be made only when the vegetations or warts have existed for a long time and have undergone changes which have resulted in their becoming more dense with pronounced flattening of the surface. If there is any doubt, a blood examination should be made. Epithelioma at the beginning of its course may be mistaken for condylomata. The former appears late in life and is accompanied by infiltration of the adjacent tissues and enlargement of the nearest chain of glands. In a case of doubt, in which the suspicion has been raised, microscopic examination will decide.

Treatment.-The prophylaxis against these warty excrescences is cleanliness, especially in the event of balanitis or of gonorrhea. When the warts are encountered in cases in which the foreskin can be retracted they may be removed with scissors and their bases cauterized with nitric acid or other caustic, the surface being anesthetized with $5 \%$ novocaine solution. When the growths cover an extensive area, they may be thoroughly scraped away by means of a sharp curette and the denuded surface cauterized with acid and treated with a dry dusting-powder composed of calomel, oxide of zinc and bismuth, or iodide of thymol.

A most satisfactory way to treat these warts is to apply

P. Acid. salicylic.............. 4 gm. ( $3 \mathrm{i})$

Acid. acetic................. 30 c.c. (年 $\mathrm{i})$

This combination forms a curd-like mixture which generally will remove warts of all sizes upon the foreskin and prepuce, leaving a smooth surface within twelve or twenty-four hours. In using this mixture, a sufficient quantity of the dregs should be deposited upon the growth to cover the entire surface and be allowed to dry. One or more applications are required according to the extent of the growth.

When vegetations occur within a phimosed foreskin, it may be necessary to slit or cut away the latter. Finally, these vegetations can be effectually destroyed by the application of the Oudin high frequency current (see p. 153). 
Phimosis, Paraphimosis.-Phimosis is said to exist when the orifice of the prepuce is too small to permit the glans penis to be uncovered. It may occur congenitally but is also acquired as a result of secondary contraction following attacks of balano-posthitis, either alone or in combination with the presence of herpes, vegetations, chancroid or gonorrhea. Most infants are born with redundant foreskins which persist up to six or seven years, when the preputial opening generally assumes normal dimensions.

Phimosis persists in various degrees as a congenital formation, producing a contracted orifice which may simply prevent retraction of the foreskin or may be so tight as to impede the flow of urine. Such an extreme condition will naturally favor the occurrence of balanitis and interfere with the proper treatment of the morbid conditions which attack the interior of the prepuce.

The radical treatment for phimosis is circumcision (see Op. Surg., p. 342), an operation, which on account of its sanitary and hygienic character, may be unhesitatingly recommended at all ages as a means of permanent relief for unsatisfactory local conditions.

Distressing local and general nervous phenomena in patients with pronounced congenital phimosis, are sometimes benefited by removal of this source of irritation; and especially is the operation resorted to in young children as an attempt to relieve persistent nocturnal enuresis, in which condition it is sometimes but not always successful.

Inflammatory phimosis is a transient trouble due to inflammation of the prepuce. It may leave behind a true phimosis as a result of chronic thickening.

The treatment of inflammatory phimosis consists in treating the cause which underlies it; in addition, evaporating lotions in the form of wet dressings may be applied while the prepuce is kept continually washed with an antiseptic solution composed of carbolic acid, $1: 100$, or corrosive sublimate, 1:5000. It is better not to incise an inflammatory phimosis unless the condition inside the prepuce demands it. When such is the case, the operation should be performed by making two lateral incisions, and completing the circumcision when the inflammatory condition and local lesions have yielded to treatment.

Paraphimosis is that condition which exists when the prepuce gets behind the corona glandis and cannot be turned. Occurring with gonorrhea, this is caused by edema of the retracted foreskin. Such inflammatory paraphimosis may depend also upon balanitis, herpes, chancroid or chancre. When seen early, there may be little inflammation of the glans or prepuce but when the condition continues 
and is unrelieved, inflammation may reach a high degree, edema of the prepuce becomes very extensive, the constricting portion behind the corona appears like a cartilaginous ring, and the glans penis may even become gangrenous from strangulation.

Sometimes, although the foreskin is not long enough to cover the glans, edema may occur, giving the same appearance as in the case of paraphimosis with contracted and long prepuce. Under these circumstances, the deeper parts are not strangulated and there is no occasion to attempt reduction.

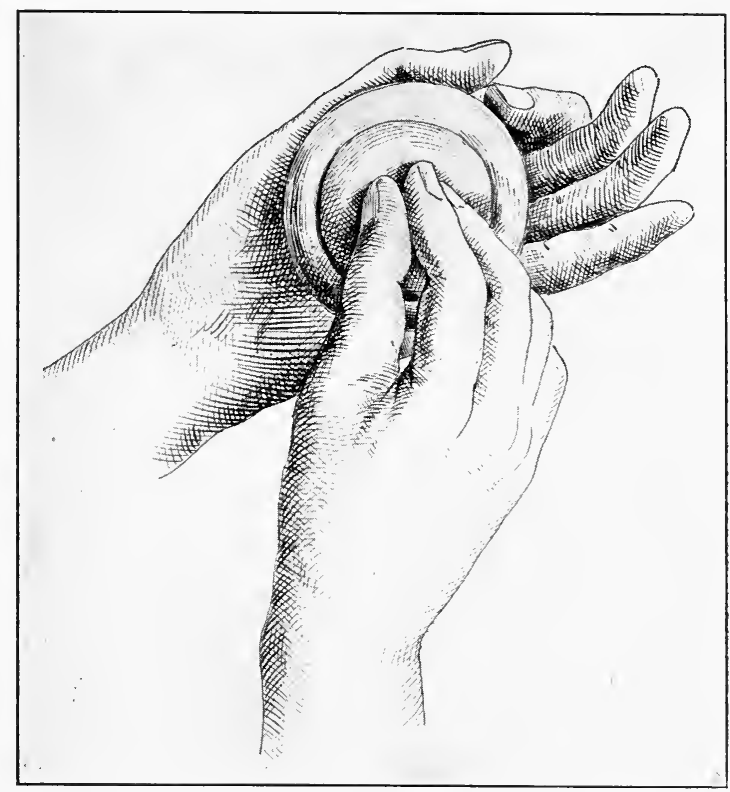

FIG. 99.-Reduction of paraphimosis. First step.

The treatment of paraphimosis calls for return of the prepuce over the glans after careful cleansing in cold, antiseptic lotion. This becomes imperative should positive strangulation of the penis occur, as evidenced by the bluish-black color and tense swelling of the glans, in order to prevent necrosis and sloughing. This may be done in several ways always beginning with thorough lubrication. One way is to encircle the penis with one hand (Fig. 99), or with the thumb and index-finger of one hand, using the other hand to knead and compress the glans and push it back through the constricted ring of the prepuce. Another is to grasp the penis behind the constricted portion between 
the index- and middle fingers of both hands, making pressure with the thumbs on each side of the glans at the same time pulling the strictured prepuce forward, the purpose being to make the glans as small as possible and to draw the constriction over it rather than to push the glans through the stricture (Fig. 100). Still another method is to compress the swollen glans by means of a half inch muslin or rubber bandage, applying it from the apex to base, and then endeavor to retract the prepuce forward with the bandage in place by one of the manipulations or to remove the bandage and immediately attempt reduction before the engorgement has had time to return to the

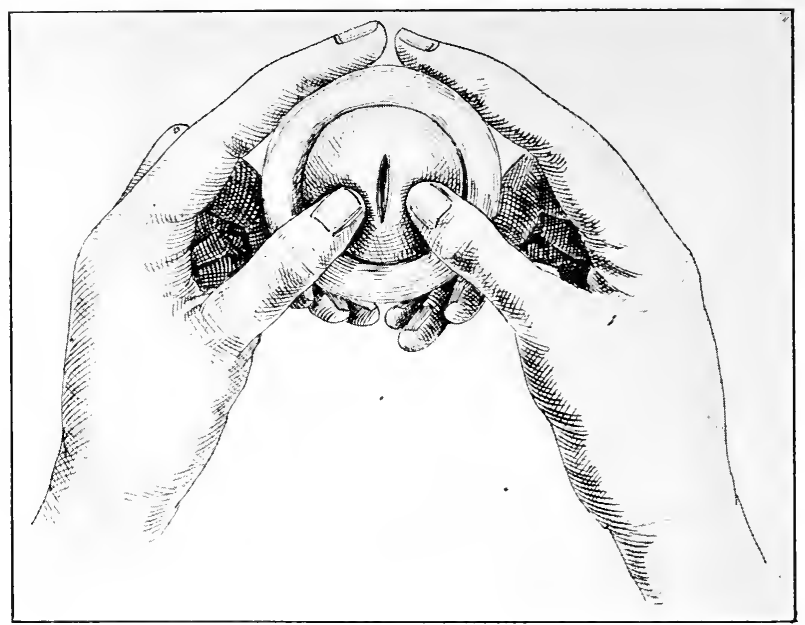

Fig. 100.-Reduction of paraphimosis. Last step.

same extent. When attempts at reduction fail, the constriction must be divided with the knife. To accomplish this, a few drops of $1 / 2$ per cent. novocaine are injected with hypodermic syringe behind the constriction, or thorough local anesthesia is previously obtained (see p. 501); a blunt tenotomy knife is then introduced on its flat surface beneath the stricture and is made to cut outward or the incision may be made directly down upon the sheath of the penis. Sometimes the inflammatory infiltration of the tissue necessitates the division of the stricture at several points.

\section{Tumors of the Penis}

Tumors of the penis are benign and malignant. In the benign group are included cysts and lipoma, fibroma and papilloma, horny 
growths. Cysts and lipoma occur in the areolar tissue of the penis but are rare and require no special consideration. Fibrosis of the penis has already been mentioned (see p. 172). Papillomata are a common form of benign growth, usually spoken of as vegetations (see p. 178). Horny growths and excrescences are derived from the skin and the glans penis and are important on account of the tendency to subsequent development of carcinoma and therefore such excrescences together with the entire base should be removed as soon as discovered.

Malignant tumors of the penis are carcinoma and sarcoma. The latter is rare, while the former is the most common growth of the penis and usually occurs in the form of squamous-celled epithelioma. The relative frequency of occurrence of this form of cancer of the penis compared with its appearance on other parts of the body has been estimated at from 2 to 3 per cent. It seldom occurs as a metastasis. The most usual period of its appearance is in middle age and early old age. I have operated upon one case of extensive cancer of the penis, however, in a patient a little over thirty years of age. The percentage of its occurrence in those of the Hebraic race is strikingly small. Venereal disease is alleged to have some causative influence, but to what extent cannot be stated. It is not possible to ascribe any significance to the contact with a cancerous cervix. Traumatism is usually mentioned as a predisposing factor. In most instances the growth has its origin in the glans, less frequently on the prepuce, the urethra is only exceptionally involved. Glandular involvement is the rule, and even after the most radical removal the tendency to recurrence in from a few months to over ten years is great. In one case in my experience, following complete emasculation, extensive recurrence appeared in seven months and in another, there has been no recurrence after twelve years.

Clinical Course.-The initial appearance is that of a warty excrescence. Ulceration and induration soon follow and as the growth extends, the surrounding parts become edematous, swollen and discolored, the general appearance being that of a raw, fungating, exuberant tumor. The occurrence of pain as a rule is not marked. Micturition becomes painful and difficult as the outlet of the urethra is encroached upon. The tumor emits a sero-sanguinolent discharge which, in individuals whose habits are not cleanly, becomes foul. Constitutional depreciation soon makes its appearance and emaciation of an extreme degree develops, the patient acquiring a sallow and anemic color peculiar to general cachexia. The duration of the disease varies in different individuals who have not submitted to operation from less than a year to over five years. Difficulty of diag- 
nosis is found only in the early stages and notably in individuals in whom the growth is hidden by a phimosis. The appearance of an indolent excrescence upon the glans penis in an elderly individual should always arouse suspicion and prompt removal of a section of tissue for histological examination should be resorted to.

Prognosis. - The most favorable outlook for the patient exists when the growth is discovered early, and is completely removed before involvement of the inguinal glands. Generally speaking, when the inguinal glands have become involved, a fatal outcome in a short period is more likely.

Treatment.-The treatment of this malignant neoplasm is entirely surgical; and best results are obtained by early and radical action, except when the patient is seen after the cancer has made general and hopeless invasion, at which time the treatment must be symptomatic and palliative. If the growth is no more than an ulcerated excrescence of the glans penis, prompt excision and cauterization are demanded; but when malignant neoplasm has extended itself much beyond the limits of the glans penis nothing short of partial or complete amputation (see Op. Surg., p. 360) should be practised and total emasculation, including the inguinal glands, represents the most radical course of complete removal. Striking instances of life prolonged by skillful surgical interference are encountered following both complete and partial amputation. In the latter instance, however, it is important to employ supplementary $x$-ray or radium treatment the moment the slightest suspicion of a return is detected, or even before such appearance, as a prophylactic measure.

Sarcoma of the penis is very rare. It occurs as a nodular growth in the corpora of the penis, generally bilateral, which enlarges rapidly and is accompanied by involvement of the inguinal glands. Metastases are common. The outlook is bad in spite of surgical intervention. It can usually be distinguished from the benign form of neoplasm by its more rapid growth and the pronounced inguinal gland enlargement.

Anomalies of the Penis.-From a surgical aspect, anomalies of the penis are embodied in the consideration of malformations of the urethra. The rare anomaly of "double penis" is referred to in literature but is simply an anatomical curiosity and requires no special consideration. In connection with other congenital anomalies, there is rarely absence of the penis producing pseudo-hermaphroditism.

Chancroid of the Penis is considered in a separate section, Chap. XXXVIII, p. 681. 


\section{MALFORMATIONS AND WOUNDS OF THE URETHRA}

Malformations of the Urethra.-At one time, during the growth of the embryo, the ureter and the urethra are continuous; after birth the urethra may be completely absent or partially obliterated as a result of improper development. A so-called "double urethra" may have an abnormal communication with the ureter or rectum. Finally the urethra may be congenitally sacculated, strictured or fissured. Congenital stricture of the urethra, which occurs in various types, is a common form of urethral malformation (see Stricture of the Urethra, p. 252).

The congenital fissures of the urethra are of great surgical importance and embrace the most conspicuous malformation of the external genitals, known as hypospadias and epispadias.

Absence or complete obliteration of the urethra is exceptional, and, like occlusion of the ureter, usually causes a fatal congenital hydronephrosis. If the child is born alive, urine is discharged through a vicarious passage, such as a perineal or rectal fistula or patent urachus. Associated anomalies of the other urinary organs are usually present. When complete obliteration occurs in the vicinity of the meatus, immediate meatotomy may be performed to relieve the condition. Incomplete obliteration occurs in different portions of the canal and is considered under the title of Congenital Stricture (q.v.).

"Double urethra" is due to congenital periurethral fistula and usually constitutes a blind pouch lined by mucous membrane which may be discovered during endoscopic examination. Smaller diverticula or pouches of the urethra occur which are brought to light by urethroscopic examination or by the introduction of a sound. Folds due to valvular malformation occur in different parts of the canal, and when occurring in the prostatic canal, may give rise to urethral irritability that may furnish an explanation of dribbling or incontinence. When discovered, these folds should be incised through the urethroscope as, during inflammatory conditions, they may be a site for the lodgment of infectious material and a source of annoyance and relapsing inflammation.

Fissures of the Urethra-Hypospadias.-Hypospadias is a congenital defect in development in which the termination of the urethra is not at 
its normal site in the glans but on the under penile surface at some point between the perineum and the glans. It is far more common than epispadias. Varieties.-(1) Balanic hypospadias or hypospadias of the glans, in which the opening is abnormally located in the glans or just behind it; (2) hypospadias of the body of the penis when the fissure is under the shaft of the organ, somewhere between the peno-scrotal junction and the glans; (3) scrotal or perineal hypospadias in which the opening is between the peno-scrotal junction and the perineum involving a cleft of the scrotum as a part of the abnormality.

This anomaly is due to abnormal development and faulty union of the three embryological segments of the urethra. In conjunction with any of the above varieties, there may be found a separate opening in that portion of the urethra anterior to the anomalous fissure, terminating in a blind canal, disunited with its posterior section.

Symptoms. - In cases of mild degree, there is nothing more than the obvious deformity. In the more extreme cases, the consequences of disturbance of urination occur, involving dribbling and excoriation of the parts and interference with normal coitus. Simple inspection is sufficient to recognize the condition. In the extreme types in the infant there may be a question of sex, as undescended testis is sometimes a concomitant anomaly. Rectal or abdominal palpation may give evidence of the existence of the prostate or uterus.

Treatment.-In slight degrees of malformation, interference is not called for unless the patient demands it. In the more advanced cases, on account of the annoying symptoms attending the condition and of the embarrassment likely to ensue later in life from its existence, it is the obvious duty of parents to resort to surgical advice and endeavor to have the deformity corrected in the proper manner, which may take a number of years to accomplish to a satisfactory end. In the mild degrees of this deformity, the operation may be performed when the patient is two or three years old, but in the more marked degrees thereof it is better to wait until the child is seven or eight years of age, although some preliminary steps may be taken earlier in life. The parts are more satisfactorily developed and it is easier to obtain cooperation of the patient when the final steps are not undertaken at too early an age. The factors which have an important bearing upon the outcome of the operation are the descent of the testicles and the size of these organs, the size of the penis, the extent of malformation and the condition of the surrounding skin. As there is usually incurvation of the penis, together with the faulty opening of the urethra, preliminary efforts may be made in earlier years to reduce the general deformity before the final operation of establishing the 
normal continuity of the urethra is undertaken. For description of operation for hypospadias, see p. 277.

Epispadias.-In this deformity, the urethral opening is on the upper side of the penis at some point back of the normal orifice. Incomplete closure of the anterior wall of the bladder (exstrophy), is often associated with epispadias. The latter may exist without exstrophy but the reverse does not occur. Other congenital deformities may coexist with epispadias such as absence of prostate, imperforate anus and fistula of the urachus. Epispadias is less common than hypospadias, far more grave in its consequences and more difficult to correct. The different degrees of epispadias are represented by a simple type in which the opening is above and behind the glans penis, and another when the opening lies between the glans and the symphysis and a third, in which the most marked degree of deformity exists, namely, complete epispadias in which the whole urethra is open as far as the bladder. There are several degrees of this deformity in the female where the opening is either in the clitoris, beneath the symphysis or behind the symphysis.

Symptoms.-As in hypospadias, in mild degrees of this abnormality the symptoms are comparatively slight except the annoyance of the discharge of urine and the difficulty of coitus. The greatest annoyance in advanced cases of epispadias is the continuous incontinence of urine with the consequent excoriation and eczema of the adjacent parts. In such cases, the patient is both sterile and impotent.

Treatment.-As in the allied condition of hypospadias, the treatment is purely surgical. The mild cases should be left alone; in the severe cases, the proceeding is both difficult and tedious, and requires most patient and skillful plastic surgery (see Op. Surg., p. 281).

Wounds of the Urethra.-Injury to the urethra may be a part of an accident involving the penis, in which case the injury is from without; on the other hand, a damage may occur during instrumental manipulation or the passage of a foreign body (calculus, etc.), when it is derived from within. In an accident involving injuries to the penis, the question whether or not the urethra is implicated must always have an important bearing upon the gravity of the injury and the treatment indicated. Such wounds may produce subcutaneous contusion or incomplete laceration of the urethra and, when more severe, complete rupture of the canal may result. An incised wound may be longitudinal or oblique or transverse. Those in the longitudinal axis of the canal naturally heal more easily without subsequent stricture. Gunshot wounds may occur in which case there 
is more or less irregular laceration. In one case that I operated upon, a lad was walking along the street and received a chance bullet in the perineum, causing laceration of the urethra and subsequent fistula, and stricture. These latter conditions comprise the most important consequences of wounds of the urethra. The immediate consideration pertains to the question of hemorrhage which is more or less profuse according to the extent of the injury and the amount of tissue that is lacerated. Transverse wounds bleed with great freedom.

Treatment.-The treatment is for the most part that outlined in the chapter on wounds of the penis. First of all, cleanliness and, where possible, primary suture. This may be carried out satisfactorily in incised wounds but to a lesser extent in contused and lacerated injuries, where there is likely to be a certain amount of sloughing. The subsequent occurrence of stricture and urethral fistula will depend upon the extent of laceration and the success with which primary suture can be applied. The technic of operation in these cases is the same' as that for urethral and urethro-perineal fistula and resection of the urethra (see Op. Surg., p. 293).

Rupture of the urethra occurs as the result of an injury or as the pathological sequence in the case of an impassable stricture.

Traumatic rupture of the urethra occurs at whatever portion of the canal the injury is inflicted, whether it be as the result of a blow from the outside or of instrumental damage upon the inside. The common site of interior urethral damage is the bulbous portion of the urethra where false passages are most frequently inflicted (see Fig. 50, p. 74): and the common site of rupture from outside injury is the perineal or bulbo-membranous section. This generally results from falling astride a bar or barrel. The extent of the rupture may be incomplete, that is, all three of the tunics of the urethra may not be severed or the entire lumen is not involved or there may be complete separation of the urethral tube into two portions.

Symptoms.- In mild cases of incomplete rupture of the urethral cylinder or where only a portion of the circumference has been severed, pain and urethral hemorrhage are the only symptoms; and when a soft rubber catheter can be introduced into the bladder without difficulty, the probability of incomplete rupture is borne out and the treatment becomes simplified. In such cases the catheter may be left in situ and the bladder drained for a period of four or five days to a reek during which time the urethra is kept clean and if no undue swelling occurs as an evidence of infiltration, a successful outcome may be expected. On the other hand, where the symptoms are more severe, where the hemorrhage is not only urethral but there is extravasation of blood 
into the structures of the penis as well, where there is retention of urine and the development of a clearly defined tumor in the region of the laceration, complete rupture is likely.

When the rupture occurs in the posterior urethra, there may be no copious hemorrhage from without, simply a bloody oozing and no evidence of perineal tumor. There is, however, retention of urine, and the catheter, when introduced, is arrested in the perineal region and obtains no urine. If by chance, or because the urethra is not completely severed, the catheter is slipped into the bladder, the urine obtained is freely blood-stained as distinguished from the clear urine which is obtained by catheter in rupture of the anterior urethra.

Complications of rupture of the urethra are extravasation of urine (see p. 266), and urinary septicemia which may develop if the diagnosis is not made early and proper surgical measures adopted. The ultimate sequel is traumatic stricture (see p. 257).

Treatment.-As already stated, the treatment of the mild forms of slight and incomplete rupture of the urethra demands urethral cleanliness and catheterization, and usually the employment of the indwelling catheter for such period as may be necessary to demonstrate that urine may be passed normally without hemorrhage or infiltration. The more severe cases demand prompt surgical measures in order to avoid urinary extravasation and sepsis and to forestall the occurrence of traumatic stricture, viz.: perineal section or retrograde urethrotomy (see p. 288 and p. 473). When rupture of the urethra is the result of neglected stricture, the first evidence may be extravasation of urine or complete retention. In this case, external perineal urethrotomy is indicated as in the other types of stricture.

Urethral Fistulæ.-Fistulæ of the urethra are classified into three groups, (1) penile fistulæ, (2) perineo-urethral fistulæ, (3) urethrorectal fistulæ. The causes of urethral fistulæ are traumatism, inflammatory conditions following traumatism and infection, malignancy and tuberculosis. The penile variety, as a rule, is a sequel of gonorrheal follicular abscess (see p. 242). The perineo-urethro and urethroperineo-scrotal fistulæ occasionally follow operation but generally are the result of periurethral inflammation, prostatic or follicular abscess. In this variety of urethral fistula, differing from that in the anterior urethra, which is small and only slightly indurated, there is much sclerotic periurethral tissue as a result of inflammation.

The urethro-rectal fistula is sometimes the result of careless instrumentation, sometimes follows operation or occurs incidental to it (perineal prostatectomy); it may be the result of a prostatic abscess or of traumatism. 
The presence of perineo-urethral fistula is self-evident. They vary in size and number and in the quantity of urine passed by such route. There may be one opening connected with the urethra and two or three externally in the perineum and scrotum. The surrounding tissues are generally inflamed and thickened and there may be associated one or more purulent foci. In the case of rectal fistula, a part or all the urine is voided by rectum. In occasional instances, liquid feces pass through the urethra. It may be difficult to detect urethrorectal fistulæ when they are very small; in such instances, methylene blue is administered so as to color the urine when recognition is rendered more easy.

Treatment. - The treatment of all urethral fistulæ depends upon the condition of the lesion and the adjacent parts. Such treatment is palliative, local and operative. Some fistulæ are so small and the local condition so favorable that cauterization with the fine point of the galvanocautery, with the high frequency Oudin current or with the injection of "caustic pyrozone" may be sufficient to eause complete closure by destruction of the epithelium lining the fistulous tract. These fistulie of the anterior urethra are sometimes treated endoscopically (see p. 127), or from the outside by one of the above methods; and when such methods have failed I have succeeded in a number of instances in obliterating fistulæ of the pendulous urethra by carefully dissecting down the fistulous tract to its entrance into the urethra when it is surrounded by a silk ligature and tied off, following which the ligature is left long and without cutting off the dissected fistulous tract, the wound is allowed to granulate (see Op. Surg., p. 297).

If stricture is present, it is not reasonable to expect satisfactory closure of a fistula to take place until the normal calibre of the urethra is reëstablished, in fact, in some instances where stricture has been permitted to recontract to small dimensions, dilatation of the stricture alone may be sufficient to bring about closure of the fistula, which, however, will reopen should the stricture be allowed to recontract.

When the fistulæ are more extensive, surrounded by indurated tissue and are connected with several openings which have burrowed through the surrounding tissues in different directions, nothing short of careful, surgical treatment will yield permanent results. In such cases, successful surgical operation entails free opening of all fistulous areas down to the urethra, obliteration by removal of the artificial tracts and careful suturing, layer by layer, of the urethra and superstructures. Special operations have been devised by different operators for perineo- and urethro-rectal fistulæ (see Operative Surgery, p. 297). 


\section{CHAPTER X \\ URETHRITIS-GONORRHEA}

\section{BACTERIOLOGY-PATHOLOGY-CLINICAL COURSE}

Inflammation of the Urethra. - Urethritis is, according to its duration, acute or chronic; according to its location, anterior or posterior; and according to its cause, specific or non-specific.

\section{Acute Urethritis}

(Chronic urethritis is considered in a separate section (p. 227).

\section{Location}

Acute Anterior Urethritis involves that portion of the canal in front of the triangular ligament and posterior urethritis that portion behind it. This division is not merely an arbitrary distinction, as the direction of treatment generally depends upon the existence of one or the coëxistence of both of these conditions.

Acute (primary) gonorrhea always begins at the meatus.

Acute non-specific urethritis may spring from any portion of the canal or involve its entire length at the onset.

Acute posterior urethritis occurs either as an extension backward of inflammation in the front canal or as a result of acute exacerbation of old chronic trouble in the follicles or adjacent anatomical structures of the posterior urethra.

\section{Causation}

Non-specific urethritis, as its name implies, differs from gonorrhea in that it is not referable to a single cause. It is etiologically complex. Its causes arise from without and from within the urethra. Generally speaking, it comprises those cases of urethral inflammation not directly traceable to the gonococcus. Clinically, a diagnosis between it and gonorrhea often cannot be made, which is due to the fact that the symptoms of inflammation of the urethra are alike, whether their cause be specific or non-specific. Bacterio- 
logically, a distinction must always exist between non-specific urethritis and gonorrhea, although practically such a difference oftentimes cannot be demonstrated except by resort to the microscope.

The intensely contagious quality of gonorrheal pus can be demonstrated by direct experiment from urethra to urethra. It is demonstrated upon the victim who has exposed himself to it in sexual intercourse; and the eyes of a patient with gonorrhea may also attest the powerful contagiousness of this disease. By the discovery of the gonococcus of Neisser and its cultivation and reinoculation upon the human urethra, the true etiological factor of gonorrhea was disclosed. A gleet after gonorrhea which is nearly well, may give a gonorrhea to a woman if the gonococcus be still present, as will a mild lurking inflammation in the vagina readily poison the male when its secretion harbors this specific organism.

Simple urethritis may develop following contact with the irritating and germ-laden vaginal discharges, but not unless the urethra has been damaged by previous gonorrhea or unless the individual is debilitated, overstimulated with liquor or suffering coincidentally from very acid urine, or unless he overdoes himself sexually. Each of these causes is capable alone of producing urethritis when the mucous membrane is in a morbid or congested condition, but all are more prone to be predisposing in their tendency, and the means of inducing acute inflammation, through the activity of the various microörganisms which commonly infest the urethra and vagina, and which, although not necessarily pathogenic, may become so under favorable conditions.

When a patient is exposed to true gonorrhea, he is almost certain to become poisoned without the coöperation of any of the adjuvants mentioned above; simple contact is sufficient.

The theoretical distinction, therefore, between gonorrhea and urethritis is clear; the clinical distinction is often equally so. Yet, without doubt, an intense urethritis is one and the same in its symptoms, whether or not its cause be gonorrheal.

From what has been written it may be seen that the cause of true virulent gonorrhea is single-namely, previous contact of the infected person with the gonorrheal pus of another; while the causes of catarrhal inflammation are varied. These causes are predisposing and exciting, and the more potent the exciting cause the less susceptible need be the urethra to succumb to its influence; while on the other hand, the more abnormal the urethral canal happens to be, the more likely is it to light up a catarrhal inflammation from an otherwise slight or inadequate irritation. 
Thus are determined the different forms of acute non-specific inflammation of the urethra.

Traumatic Urethritis.-If the urethra be healthy or unhealthy, it becomes inflamed readily by contact with the gonococcus, and the healthy urethra becomes the seat of inflammation as the result of mechanical violence, such as the rough introduction of surgical instruments, or following chemical irritation, from the injection of strong applications for experimental or medicinal purposes, or with the idea of employing a prophylactic against supposed infection. Under these circumstances the organisms which ordinarily are inert in the urethra exert a more or less virulent action against it as a result of the traumatism. The healthy urethra may also become inflamed by the combined influences of sexual excitement, intense and prolonged, and contact with such fertilizing media as leucorrheal pus, menstrual blood, etc.

An unhealthy urethra is always ripe and ready for inflammation. In diabetic, lithemic and tuberculous subjects the urethra is especially prone to become inflamed and yields to the results of traumatism mentioned above with unusual readiness. Besides these constitutional predisposing causes, a local predisposition may be present, with the existence of such morbid conditions as a granular and hyperemic area, ulceration, stricture, polyp, or some latent lesion seated in any of the follicles or accessory glands.

Therefore, traumatic violence, when sufficiently severe, will produce inflammation of the urethra with little susceptibility on the part of the urethra, while a mild amount of trauma will produce an undue amount of irritation upon a urethra made susceptible by constitutional or local predisposition.

Other causes of traumatic or irritative urethritis are the passage in the urine of such irritating substances as copaiba cubebs, cantharides, turpentine, iodide of potassium and excessive quantities of alcohol. These irritants are not causative factors except when taken in excess, and in the case of alcohol, when combined with other factors, such as excessive sexual indulgence, and when there is already present a mucous membrane damaged from preëxistent disease.

The treatment of all types of urethral inflammation is considered under one section (p. 207).

The important lesson to be derived here is the value of prophylaxis, which involves a constant consideration for the damaged and sensitive urethra, careful handling of all instruments used, on the part of the surgeon, and the avoidance of all excesses and irritating influences on the part of all who would escape the penalty thereof. 
Diathetic Urethritis.-This is in effect a form of traumatic urethritis, being dependent upon the passage of urine made irritating by the presence of large quantities of urates, phosphates or oxalates over a hypersensitive mucous membrane, but is so named on account of the fact that it occurs in those individuals who are victims of diathetic conditions which render all their mucous surfaces specially sensitive. This is the case in gouty or rheumatic conditions in which the urine is overloaded with urates and uric acid and exercises a caustic influence upon the tender canal. It has already been stated that these subjects are susceptible to the other causes of urethral inflammation and hence, bad subjects when they acquire gonorrhea, being more than ordinarily prone to prolongation of their trouble into an obstinate chronic affection. This is especially true in strumous or tuberculous individuals who may develop an unhealthy condition of the mucosa in the form of hyperemic and granular changes, and prove exceedingly resistant to the ordinary methods of treatment.

The appropriate treatment of such cases is more hygienic than local and especial attention is to be given to the underlying diathesis.

Eruptive Urethritis.-A catarrhal discharge from the urethra may accompany an eczematous eruption which involves the external genitals, due to an attendant hyperemia of the mucous membrane and hyperacidity of the urine.

Herpetic urethritis is seen in connection with the extension into the canal of herpetic lesions in or about the orifice. When the clusters of small pin-head vesicles break down and become confluent, they may be mistaken for mucous patches or atypical chancre. The diagnosis is determined by their early disappearance and by negative bacteriologic findings (see section on Chancre).

Chancre of the urethra will cause a urethral discharge when it involves the entrance of the urethra, and in some instances it develops entirely inside the orifice, when its existence is not discovered until a characteristic hardness surrounding its site is recognized or it is revealed by an endoscopic examination. The discharge accompanying a chancre of the urethra is thin and serosanguinolent and there is present a notable amount of edema, which persists for several weeks, or as long as the sore is active. A recently acquired gonorrhea will produce a marked swelling and induration at the site of the infection on either side of the frenum, and may be mistaken for hard chancre, when at the onset, owing to a break in the epithelial lining, it has entered one of the follicles on the floor of the fossa navicularis. The bacteriological examination and the progressive development of the gonorrhea will determine the diagnosis. 


\section{Bacteriology of Urethritis}

The bacterial flora of the human urethra comprises various microörganisms the presence of which are harmless in the quiescent state, but the same organisms assume more virulent properties under certain conditions, as seen in those forms of urethritis which come under the title of non-specific inflammation. These different organisms have been isolated and studied. They comprise many varieties-streptococci, staphylococci and bacilli. In cases of non-specific urethritis or pseudogonorrheal inflammation, examination of the discharge reveals the presence of one or several of these types of bacteria, which, under the adverse conditions, contribute to the causative agency, but by reinoculation they are not productive of a similar inflammation. They are, therefore, properly considered as only one of the factors that enter into the causation of simple urethritis, and there are cases in which the microbic element plays a very minor rôle.

The virulence of the gonococcus, on the other hand, is now undisputed; and it alone can be regarded as capable of transmitting from one individual to another that form of urethral inflammation which is broadly spoken of as gonorrheal. There is a possible exception in the so-called micrococcus catarrhalis (studied by Wohlstein, Berlin and Elser, New York), Gram-negative cocci, and, bacteriologically speaking, cultural methods are required to differentiate them unqualifiedly from gonococci. But the knowledge of this exception is so obscure that its rare occurrence and insignificant virulence serve more to emphasize the rule.

The Gonococcus.- The various localities in which the gonococcus is found most commonly are the urethra and prostate of man, the urethra, Bartholins glands and the cervix uteri of woman. It may be the cause of epididymitis, cystitis and pyelitis, endometritis, salpingitis, oöphoritis, peritonitis, proctitis; also, conjunctivitis, rhinitis, otitis, arthritis. It occurs also as a cause of pleuritis and malignant endocarditis.

Flattened epithelial cells seem to serve as a better protection against urethritis than the columnar epithelia, but as already stated, it may penetrate beyond the epithelia and reach the connective tissue beneath. Immunity does not follow infection.

Attempts to inoculate the lower animals are not successful, and do not produce the true gonococci of Neisser, but may yield a spurious organism somewhat resembling it morphologically, but which can be differentiated from it by means of the Gram staining method, properly conducted. 
The gonococcus is a parasitic organism that thrives best upon mucous or serous surfaces, and hence finds a ready medium in the urethral mucosa. When deposited outside the body, it soon ceases to be virile, but remaining in the urethra after a gonorrhea, may lie dormant for a long period, and may at any time produce gonorrhea in another, or an acute exacerbation in the same person. This organism is easily recognized by means of the microscope in cover-glass preparations but in doubtful cases, cultivation upon a proper medium may be necessary to demonstrate its existence. The advantages of cultures are in those cases in which a few shreds or filaments are present in the urine and nothing more, and in the case of patients who suffer from a very mild catarrhal gleet, and who contemplate matrimony. In these cases the gonococci, if present at all, may be so scarce that the microscope fails to find them until their number is greatly amplified by propagation upon a suitable medium. But when there are present in a given discharge suspicious organisms which can be seen with a microscope to resemble gonococci, the diagnosis may be made satisfactorily, without cultivation, by means of the Gram (q.v.) method of staining. (See Plate IV.)

Laboratory Technic. - The gonococcus was discovered by Neisser in 1879 in gonorrheal pus, and was first cultivated by Bumm in 1885 . These organisms consist morphologically of micrococci, usually joined in pairs (diplococci) or in groups of four. The separate elements are flattened or hemispherical with the flattened surfaces approximated, and in the stained preparation present an unstained interspace. The approximated faces show a slight concavity. Their average size is $1.25 \mu$ in the long diameter. Their multiplication occurs by fission alternately in two planes, as a result of which groups of four are frequently observed. The microscopical appearances are not entirely characteristic of the gonococcus, as various other diplococci cannot be distinguished morphologically from the gonococcus. It readily stains with the basic aniline colors, such as blue, violet, red and brown. It is negative to Gram's stain, that is to say, it is decolorized by alcohol after the use of iodine solution in this method. The characteristic action of the Gram method upon the gonococcus is the best and most reliable means of establishing its identity, and when confounded with other diplococci, the error is due to faulty technic. In perform. ing the Gram test, great pains must be taken in every detail, to make this test conclusive.

Another distinguishing characteristic, but of lesser diagnostic value, is the fact that the diplococcus gonorrhea is found within the protoplasm of the pus cells. It is found also within the epithelial cells. 
Pus cells are observed entirely filled with these organisms in a stained specimen of gonorrheal pus. (See Plate IV.)

Technic with Single Stain.-In a clear clinical case of gonorrhea, the gonococci can be rapidly stained and recognized by the morphological characteristics above described, and being so numerous, many pus cells will present the typical appearance shown in Plate IV. A thin smear of pus is spread upon a microscope slide and dried in the Bunsen flame until the slide is heated almost too hot to hold, but not sufficiently to scorch the smear. It is then treated for two minutes with a standard solution of methylene blue (Loeffler's) or Erhlich's solution of gentian violet. The excess of staining fluid is removed with filter paper until thoroughly dried in this manner, when it is ready for examination, preferably with $1.9 \mathrm{~mm}$. oil-immersion lens.

The single staining method is not to be relied upon in doubtful or chronic cases; in these it is necessary to resort to the differential test by counterstaining and Gram's solution.

Gram Method. - Spread a very fine film of discharge upon a coverglass. Allow this to dry spontaneously or apply gentle heat. "Fix" by passing the cover-glass through the flame of an alcohol or Bunsen burner until thoroughly dired, but not scorched. Stain with erystal violet, $1 / 2$ of 1 per cent., 20 seconds, or methylene blue solution, preferably that made with 1 per cent. carbolated water, for a period of one minute. Remove the surplus solution with filter paper, but do not wash with water. Next immerse in the Gram solution for one minute. Decolorize with alcohol until the specimen is clear to the naked eye. This should take about one minute and a half. Now restain with one per cent. neutral red, 30 seconds, or a solution of Bismarck brown. Wash again in water, dry the cover-glass and examine with $1.9 \mathrm{~mm}$. oil-immersion objective.

Plate IV, Fig. 1, shows all organisms colored by first stain.

Plate IV, Fig. 2, shows the smear after it has been treated by the Gram solution and decolorized with alcohol. The gonococci do not show, having been decolorized (negative to the Gram stain), while other organisms which receive the Gram stain are plainly visible.

Plate IV,Fig. 3, after the application of the second, or contrast stain, shows the ordinary organisms, as before, stained with the Gram, while the gonococci are colored by the Neutral red.

To fix the identity of the gonococcus, there should be diplococci of the right form, size and arrangement; they must be negative to Gram staining, and may be observed within the protoplasm or outside of the pus cells. The cultural method of establishing the identity of 
the gonococcus includes also recourse to the Gram staining as a final means of identification.

The gonococcus does not grow upon the ordinary culture media. It is best grown upon human blood serum (Heiman) and requires thorough experience with this special method, to be successful. It can only be performed, therefore, in a laboratory properly equipped for this purpose. The inoculation of the blood culture plates should be made directly from the urethra if possible, and if it is desired to use the urinary sediment it should be done immediately after such specimen is voided or drawn by catheter.

\section{PATHOLOGY AND CLINICAL COURSE OF URETHRITIS}

The pathology of acute non-specific urethritis is the same as that of catarrhal inflammation elsewhere, and consists of a hyperemia of the blood-vessels resulting from local irritation, chemical, traumatic or bacterial, as the case may be. This hyperemia is accompanied by serous exudation and desquamation of epithelium. Leucocytes occur in variable numbers and purulent urethritis follows according to the activity of the irritation (Fig. 101). This purulent urethritis may follow as a result of infection from a virulent organism, or as a result of severe or continuous mechanical irritation (see Bacteriology). The common cause of virulent urethritis is infection by the gonococcus. The presence of this organism in the mucosa determines the activity of the inflammatory process and marks the difference between the pathology of urethritis of a catarrhal nature and that of a gonorrheal type.

Catarrhal inflammation will often subside spontaneously, without medical treatment, and unlike the gonorrheal inflammation, does not show the same tendency to extend into the deeper structures or to give rise to complications with the same frequency as the more virulent condition. Is a result of infection from gonorrhea, the pavement epithelium of the urethra presents more or less resistance to the attack of the gonococci, and, therefore, unless there happens to be a fissure or break in the lining of the epithelial coating at the meatus, no deeper extension takes place in this locality; but when abrasions have been produced, the inflammation then extends deeper into the submucosa, and causes periurethral involvement and follicular inflammation, sometimes leading to abscess formation which may rupture on the floor of the urethra or on the outside. Such complications may be produced by the use of an improper syringe and by abrasions of the surface in the introduction of an instrument. 
The gonococci, after infection, proliferate upon the pavement epithelium of the fossa navicularis and some reach the columnar epithelia of the pendulous urethra, spreading themselves by rapid growth over the lining of this portion of the canal and penetrating into and between the relaxing cells, thence reaching the deeper regions, such as the pockets or excretory ducts of the urethral glands. Sometimes, early in the disease, from the third to the seventh day, the gonococci may be found extending into the connective tissue at the

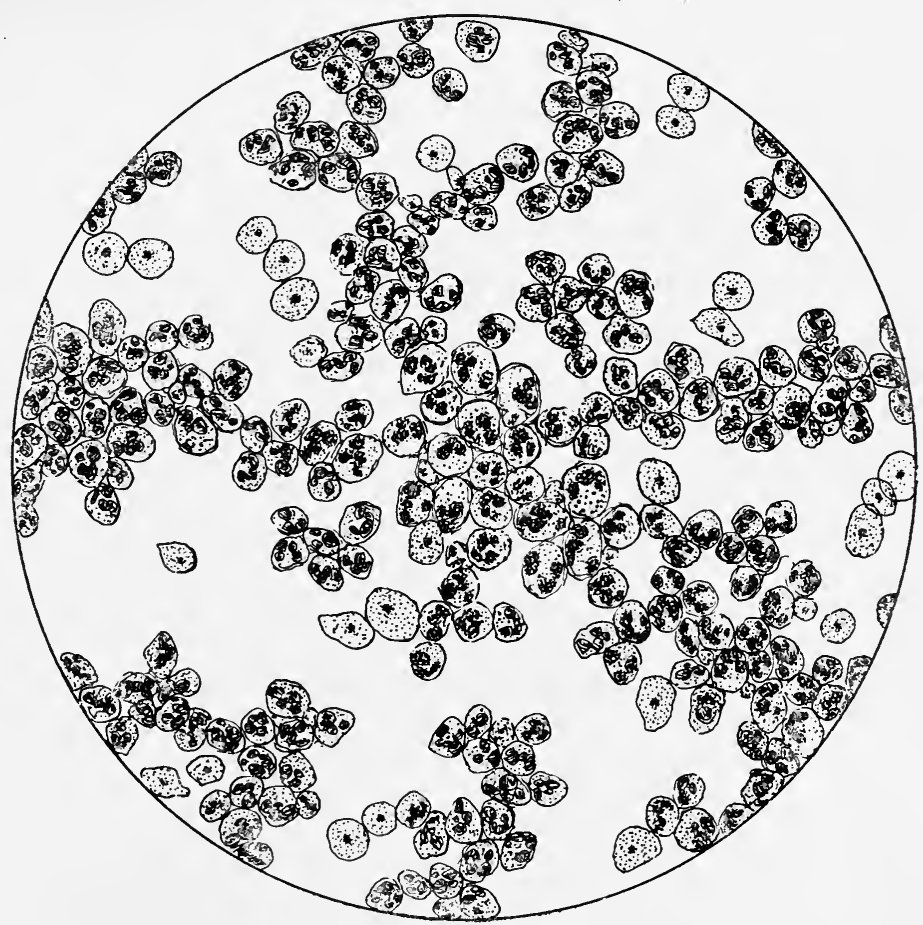

FIG. 101.-Acute urethritis.

point of most marked inflammation. When the gonococci have thus penetrated deeply into the tissues, they may produce further morbid changes as a result of their entrance into the blood current and the production of metastasis in other parts of the body. This serious development is the exception, and is found only where body resistance has become weakened by some constitutional cause or in the event of severe local damage to the urethral structure during the height of the inflammatory process. 
Generally speaking, gonorrheal inflammation is superficial, and largely an epithelial attack. Proliferation in the connective tissues is not the rule. The abundant serous exudate and cellular infiltration which occur as the result of the stimulation of the exciters of inflammation, the gonococci, is a natural effort to remove the latter by bringing them to the surface. The inflammatory exudate in the tissues responds to the stimulation of the poison and the anti-bacterial properties of the serum tends to impede proliferation of the microörganisms and render them inert. Thus, when the disease has not gone beyond the mucous epithelial lining and has terminated with or without treatment, the superficial layers of stratified epithelium are cast off with their contained gonococci. The removal of the gonococci from the pouches and glands of Littre which are involved as part of the deeper structures occurs naturally with greater difficulty. The involvement of these deeper structures is responsible for the greater intensity of the symptoms and the occurrence of such complications as folliculitis, periurethral inflammation, lymphangitis, etc. Active chordee is due to the extension of inflammation into the erectal sinuses of the corpus spongiosum and corpora cavernosa.

Incubation of Acute Gonorrheal Urethritis.-One of the distinctive differences and differential diagnostic features between acute gonorrheal and non-specific urethritis is the period of incubation, which, in the case of the latter, is insignificant, as the onset of the malady follows closely upon the cause for the same or as the culmination of a number of irritating factors. For instance, acute non-specific urethritis follows immediately a sexual strain or as a result of an alcoholic debauch. In the case of gonorrheal urethritis, on the other hand, a period of incubation always exists, which varies from three to seven days, five or six days being the average. In subsequent attacks, the incubation period is usually shorter. The addition of such exciting causes as would produce non-specific urethritis, such as physical strain and alcoholic excess, would tend to shorten proportionately the incubation of gonorrhea. On the other hand, a case of non-specific urethritis may have an apparent incubation period on account of a suspicious exposure at a suitable intervening period, without, however, being the cause of infection.

\section{Symptoms of Urethritis}

Simple or Non-specific Urethritis.-In this form the patient gives himself the disease more often than his partner gives it to him. The evidence of irritation appears almost simultaneously with the cause 
or on the second day following; sometimes it is delayed longer. A damaged mucous membrane with any one of a number of exciting causes is sufficient to kindle the slumbering congestion into an active discharging inflammation with the aid of those microörganisms which constantly inhabit the urethra, and under normal conditions remain there as harmless saprophytes.

In these cases the discharge may originate at a certain distance within the urethra from the very start, or it may commence at the meatus. The patient has intercourse, perhaps, with a woman who has no gonorrhea, who at most has a purulent leucorrhea. In twentyfour to forty-eight hours he presents himself to the physician for inspection, stating that he has an attack of gonorrhea.

When inspection shows that the lips of the meatus urinarius are not swollen, the attack may not have begun at the meatus. When, however, the cause of irritation is sufficiently severe to involve the entire canal, the evidences of commencing irritation appear at the meatus. This discharge may be thick and purulent from its very start, or thin and mucoid. There may be little or no itching or tingling along the course of the urethra. There is some heat and smarting in the urethra during the urinary act, but very little discomfort at other times.

Under conditions of bad hygiene, alcoholic or other excesses, and general neglect, it may assume all the quality of intense gonorrheal urethritis, accompanied by the complications of gonorrhea, and be absolutely indistinguishable from it clinically; or it may subside in a few days, or at most, weeks, under moderate symptomatic treatment, and give very little discomfort. The latter termination is most common. Of course, it should be borne in mind that any case of recurrent urethritis may be truly specific, in that the causative agency is the gonococcus; this organism having remained dormant in the urethra for a long period and becoming attenuated to a harmless degree temporarily, may be rendered active again by irritating causes, and resume all of its former perilous character.

Symptoms of Specific Urethritis.-Primary gonorrheal urethritis always commences at the meatus, inoculation with gonorrheal pus being the cause, and there is always a period of incubation between the moment of exposure and the outbreak of the first symptom. The first symptom in these cases is a redness, edema, and pouting of the meatus. The patient feels a sensation of tickling and itching, either at the orifice, or at a point just within the urethra, upon its under side. These sensations keep the patient's mind fixed upon his genitals and call upon him to empty his bladder rather more frequently than usual. 
The passage of urine over the tender orifice of the urethra causes a hot, stinging pain, an ardor urinæ more or less intense in different patients.

Between the lips of the pouting meatus, perhaps faintly sealed with dried mucus, a drop of watery pus is seen during the first twenty-four hours. During the first week the drop becomes more purulent, then somewhat greenish in hue from admixture with blood, and all the disagreeable sensations increase, while from day to day the discharge becomes more copious and more purulent, except when under the influence of restraining treatment.

If the inflammation is allowed to run unchecked at any period of the disease, it extends from the surface of the mucosa through the minute ducts into the mucous glands of the urethra and may spread thence to the delicate meshes of the spongy tissue of the corpus spongiosum. Erections not only become frequent and painful, but the meshes of tissue, becoming stiffened and agglutinated together by the inflammatory process over a given area, the penis becomes curved, and what is known as chordee is produced.

The intensity of the inflammation, and hence the symptoms produced, vary in different cases according to the susceptibility of the individual and the promptness with which treatment is instituted.

During the second or third week, the inflammation having extended backward, the deep urethra becomes involved and from this site the most common complications of gonorrhea are derived on account of its complex structure and its relation to the adnexa. Among the most common complications which occur after the disease has reached this portion of the canal are swollen testicle, active inflammation of the prostate gland, urethro-cystitis, retention of urine, and even extension upward into the pelvis of the kidney.

Posterior Urethritis.--The distinction between anterior and posterior urethritis is one of location, posterior urethritis involving that portion of the urethra which is beyond the bulb. The involvement of this portion of the canal during acute urethritis is the result of the gradual march of the inflammation backward. It may be precipitated early in the career of the disease by unwise and rough treatment, such as the introduction of instruments or forced irrigation. Generally speaking, it is common for the inflammation to reach this portion of the canal in the declining stage, and in favorable cases this may not be of serious consequence, on account of the fact that the disease has become attenuated and is more or less readily controlled by the measures of treatment adopted. If, on the other hand, inflammation is driven backward into the posterior portion of the urethra early in the 
disease, on account of possessing all of the virulent properties then in activity, it becomes more difficult to handle and more likely to extend still deeper and produce those complications which are commonly derived from that portion of the canal.

Acute posterior urethritis may also light up from a chronic focus of inflammation which is a remnant of earlier trouble and produce all of the symptoms characteristic of the same condition following acute anterior urethritis. The distinction between simple posterior urethritis and posterior gonorrhea is the same as in the anterior urethra, namely, the presence or not of the gonococcus as the causative factor.

The symptoms of posterior urethritis are those of urethro-cystitis, on account of the contiguity of the neck of the bladder and the posterior urethra; and the onset of this condition is generally signalized by urgency and frequency of urination of a degree in accordance with the intensity of the inflammation. The frequency varies from every two to three hours to almost a constant and uncontrollable desire to void, which, in severe cases, is accompanied by tenesmus - severe terminal pain and the passage of blood. The urine under these conditions is freely purulent, and if passed in two separate glasses, will be found to be cloudy in both flows, differing from anterior urethritis, where the second flow of urine is clear. This form of posterior urethritis is, as stated, significant of extension of inflammation to the mucosa of the neck of the bladder. When differing from this type, the extension of trouble in the posterior urethra involves the prostate gland alone, extending into the parenchyma (parenchymatous prostatitis), the symptoms are those peculiar to this condition (q.v., p. 370) and may then be more suggestive of obstruction in the posterior urethra rather than a high degree of irritation of the neck of the bladder.

With the occurrence of posterior urethritis, there may be constitutional disturbances, according to the intensity of the inflammation and the susceptibility of the individual. These constitutional symptoms are significant of toxemia, and may amount simply to a general malaise or an acute febrile attack which may last one or several days, and then disappear, on account of the adjustment of the system of the individual by the development of a constitutional resistance to the invasion.

When in the case of the involvement of the prostate the febrile symptoms are preceded by a chill, such a symptom may indicate the formation of pus in one or more of the follicles of the prostate or the beginning of prostatic abscess. 


\section{Diagnosis of Urethritis}

Before deciding upon the proper course of treatment in a given case of urethritis, the determination of the cause upon which it depends is a necessary preliminary step.

In the light of our present knowledge regarding the causative agency of the gonococcus in all cases of specific urethritis, it is hardly necessary to state that it is always proper to resort to the means already dwelt upon to determine the question of its presence in a purulent discharge from the urethra.

A careful history of each case should be obtained first of all, as by such means it is often possible to form a tentative diagnosis, which may be confirmed or disproved when the examination is completed. A proper history comprises the period of incubation and duration of the present attack, the number of previous attacks and duration of each one, and the proximity of the last previous to the present attack, as well as the intervals between attacks. A number of attacks of urethral inflammation, one following closely upon the other, are likely to be a prolongation of the original infection, and the cause will be found in the persistence of the primary cause or the existence of a structural change in the urethra, or extension of the inflammation to the neighboring parts or adnexa of the urethra. On the other hand, a first attack of acute urethral inflammation following a suspicious coitus, is always strongly suggestive, on its face, of specific urethritis.

The diagnosis of acute urethritis is generally easy, and depends upon the presence of a purulent discharge from the urethra with the attendant symptoms of inflammation. It must be distinguished from a balanoposthitis when the discharge collects between the glans and the prepuce and exudes from a phimosed opening.

The hypersection of the glands of the urethra and the prostate is of a non-purulent character, and thus is recognized. The diagnosis generally lies between specific and non-specific urethritis, and is determined by bacteriologic examination.

Finally, when the gonococcus is sought for, and whether it be found or not, if the case appears to be one of a succession of attacks which have occurred at various intervals, structural changes in the urethra or morbid conditions of the adnexa must be located, when the acute stage subsides under the appropriate treatment. These underlying conditions of acute urethritis are considered in the section on Chronic Urethritis.

The Urine in Acute Urethritis (Plate V).-In the early stages of 
acute urethritis, and when the inflammation is confined to the anterior section of the canal, the total amount of discharge is derived from this area so that if the urine is passed without cleansing the anterior canal by injection, the first flow is cloudy and the second clear. If, however, before voiding, the front urethra is cleansed by irrigation with lukewarm water, the first urine passed is clear. Later on in the malady when the posterior urethra is invaded, if the urine is passed without cleansing the anterior canal, the same result will be obtained as in the earlier stage, namely, the first flow is cloudy, the second clear; and even though the anterior urethra is cleansed before urination, the first flow of urine still will be cloudy, as only that portion of the canal in front of the triangular ligament has been cleansed-the discharge in the posterior portion is carried out with the first urine. The second urine in this case is clear, there being no generalized cystitis; but when there is a general cystitis, as well as urethritis, both the first and the second urine will be cloudy, whether or not the anterior section of the canal is cleansed. If the cystitis be confined to the neck of the bladder, and the urine is passed in three instead of two sections, the first two glasses will be cloudy, and the second clear. While, on the other hand, if the inflammation of the bladder is generalized, or produces a profuse amount of discharge which is mixed with the entire urine, all three sections will then be cloudy and sometimes the third one will contain a greater amount of purulent secretion than the first two on account of deposit of sediment in the bladder. It should be borne in mind that the same cloudiness of the three sections of the urine passed separately is seen with implication of the kidneys, as in that case the purulent secretion comes down from the kidneys (Plate V). The special features of such urine are discussed in the appropriate chapters.

The general appearance of the urine, therefore, with respect to its transparency or otherwise when observed in different sections, signifies the location of the inflammation and also the stage of the same, and often its intensity as well.

As the inflammation of the urethra declines and becomes subacute or is confined to a small section of the canal, the amount of purulent exudate being small, it requires but a small jet of urine to clear the canal of such secretion, and the balance of the urine passed is clear. When the inflammation has practically subsided, this exudate will be composed of one or more shreds, which shreds represent a collection of leucocytes and epithelial desquamation, which may or may not contain microbial elements, specific or otherwise. 


\section{Duration of Urethritis}

The duration of non-specific urethritis, when the exciting cause is derived from alcoholic excess or other means of local irritation, is generally short and readily controlled by the removal of such cause.

When the acute inflammation springs from a urethra already the seat of structural changes, the result of previous trouble, it will of ten subside in a short period under mild treatment, only to blossom out again later, when sufficient cause recurs. Its duration, therefore, will depend upon the character and persistence of the underlying cause.

Urethritis occurring in lithemic, tuberculous and cachectic subjects is apt to assume a severe form, and to extend over a prolonged period. Primary attacks of gonorrhea generally endure longer, other things being equal, than do subsequent recurrences.

True gonorrhea varies in its duration according to the intensity of the inflammation, the treatment employed, and the time at which treatment is commenced. A period of four to six weeks may be considered an average duration, and in severe cases, longer periods are not excessive. In exceptional instances, a cure may be possible in as short a period as one or two weeks, and even less, but such duration must be considered abortive, and difficult to attain.

To obtain the best results, the patient must be seen early in the term of his disease, and the treatment continued in a careful but energetic manner. It is more creditable to the surgeon to conduct a case through the course of the disease free from complications than to shorten its duration by one or two weeks. 


\section{CHAPTER XI \\ TREATMENT OF ACU'TE URE'THRITIS}

\section{Hygienic-Internal-Local}

The treatment of a mild case of non-specific urethritis often resolves itself into a simple removal of the cause underlying it. The treatment of specific urethritis, and of the more intense form of non-specific urethritis, requires resort to active local antiseptic measures, to be hereinafter described. The treatment of urethritis in general comprises the adoption of measures hygienic, internal and local.

Hygienic Measures.-Absolute regularity of life should be enjoined. The patient should rest as much as possible, avoid exercise and fatigue and in severe cases, absolute confinement may shorten the period of the disease and render it more tractable to treatment. The bowels should be watched and kept free and the amount of food taken should be moderate, its quality bland and unstimulating. Milk is an excellent article of diet in all cases, and when it cannot be easily digested, peptonized or skimmed milk may be ordered. The articles of food to be avoided in acute cases are all rich made dishes and indigestible articles, condiments, pickles and acids, strong tea and coffee, and alcoholic stimulants of every description. Asparagus is harmful to some patients.

In fact, were no other measures available, the ideal hygienic treatment of confinement in bed on an exclusive milk diet and freedom from all injurious agencies would prove an effectual means of treating gonorrheal urethritis, and would no doubt, in some fresh and un. complicated cases, lead to recovery. Smoking is not objectionable.

Everything which tends to sexual excitement, whether by thoughts, conversation or actions, should be interdicted.

Special precaution should be observed in handling the genitals; failure to observe this sanitary injunction exposes the eyes of the patient to inadvertent inoculation. The caution of extreme cleanliness should be very forcibly given to each patient, and frequently repeated and insisted upon.

As a final precaution, it is well for the patient to carry his testicles in a suspensory bandage, since the tendency to epididymitis is decidedly lessened thereby. 
All hygienic measures should be employed during the entire course of a urethral discharge, but the foregoing restrictions may be relaxed more or less in mild, non-specific cases.

Internal Treatment.-This comprises the use of:

Alkalies and diuretics, to render the urine bland and unirritating.

Balsams, santal oil, etc., which act favorably upon the mucous membrane of the urethral tract in passing out of the system.

Sedatives and anodynes, to lessen ardor urinæ when intense and control chordee.

Internal antiseptics are sometimes used for their bactericidal action. For this purpose, salol, 0.4 to $0.6 \mathrm{gm}$. (5 to $10 \mathrm{gr}$.) and hexamethylenamin 0.5 to $0.6 \mathrm{gm}$. ( $71 / 2$ to $10 \mathrm{gr}$.) three times a day have been given, but possess no established value.

The internal treatment of urethritis has as its objects, to aid in allaying the inflammation by removal of any irritating factors within its reach; to secure the comfort of the patient by the control of symptoms. The irritating factors of urethritis are the passage of dense and highly acid urine, and the added congestion of the inflamed area caused by sexual excitation.

The predominant symptoms of urethritis, which are a cause of distress to the patient, are burning urination (ardor urinæ) painful erections and chordee.

The purpose, therefore, of treatment, is to neutralize the acidity of the urine and diminish its intensity by alkalies and diluents; to exercise a soothing influence upon the mucous membrane by the use of such internal agents as are known to possess this property during elimination by the urine, and to control as far as possible the tendency to priapism and chordee by sedatives and carminatives.

In posterior urethritis when the train of symptoms of urgency, frequency, precipitancy of urination and tenesmus is in force, anodynes and other special remedies are called for.

In the case of simple non-specific urethritis, internal treatment is often sufficient to effect a cure, and patients may cure themselves in this manner. In more severe cases of non-specific urethritis and notably in gonorrhea, the internal treatment is necessarily subordinate to the local.

Alkalies and Diluents. - To produce the alkaline and diluent action, the ordinary commercial soda mint tablets are serviceable in connection with the liberal use of spring water, or of imported or domestic vichy. The citrate of potash may be given as an alkali, 0.4, 0.6 or $1 \mathrm{gm}$. $(5,10$ or 15 gr. $)$, three times daily, or more preferably by adding it to one of the various combinations in liquid or capsule form. 
Santal and Other Oils.-Of all the internal preparations in vogue, there is nothing more useful than the yellow oil of East India sandal. wood. This may be ordered in capsules, hard or soft, 0.6 to 1 c.c. $(m x-x v)$ three times a day. Some manufacturing chemists make capsules containing the oil of sandalwood, 0.6 c.c. $(\mathrm{mx})$, combined with 0.3 or $0.65 \mathrm{gm}$. ( 5 or $10 \mathrm{gr}$.) of the citrate of potash, which are dispensed under a proprietary name; and other balsamic oils are put up in the same manner.

The oil of sandalwood may also be given in a liquid mixture for those who prefer a liquid to a capsule. The following combination of the oil with an alkali is a good one:

R. Oil of sandalwood............. 30 c.c. $(\tilde{3} \mathrm{j})$

Citrate of potash or liq. potassa... $8 \mathrm{gm}$. (3ij)

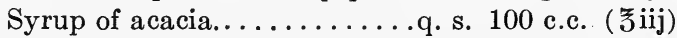

Mix.-Teaspoonful after meals.

Wintergreen may be substituted for sandalwood when the latter disagrees, and the effect is often satisfactory.

Anodynes and Sedatives.-When the symptom of painful urination becomes intensely severe, an anodyne may be added in the form of codeine, $0.032 \mathrm{gm}$. (1/2 gr.) to each 4 c.c. (3i), or the same may be given separately in tablet form, $0.032 \mathrm{gm}$. (1/2 gr.) each. The tincture of hyoscyamus is also used for controlling ardor urinæ, and may be added to the above prescription, 0.3 c.c. $(\mathrm{mv})$, to each 4 c.c. (3i).

For painful erections which occur at night, a larger dose of codeine $0.13 \mathrm{gm}$. ( $2 \mathrm{gr}$.) may be taken before retiring and may prevent the occurrence of chordee. Other remedies which are given for nocturnal priapism and chordee are phenacetine, 0.324 to $0.65 \mathrm{gm}$. (5 to $10 \mathrm{gr}$.); ammonol, 0.324 to $1.3 \mathrm{gm}$. (5 to $20 \mathrm{gr}$.) ; lupulin, 2 to $4 \mathrm{gm}$. (30 to $60 \mathrm{gr}$.), and bromide of potash, 2 to $4 \mathrm{gm}$. (30 to $60 \mathrm{gr}$.). The last preparation is usually the most effective. I commonly order a mixture containing $15 \mathrm{gm}$. ( $3 \mathrm{ss}$ ) bromide of potash and 30 c.c. ( $3 \mathrm{i})$ of water, of which the patient is directed to take from 4 to 8 c.c. (3i-ii) on retiring.

Internal Antiseptics.-Great dependence is not to be placed upon the efficacy of the internal antiseptics in acute urethritis, although they are commended by some writers and are alleged to exercise an inhibitory influence upon the growth of the specific organisms. This may be the case, but I am not able to confirm it by personal experience. Salol, 0.6 to $1 \mathrm{gm}$. (10 to $15 \mathrm{gr}$.), two or three times daily, is used for this purpose. Methylene blue, 0.065 or $0.13 \mathrm{gm}$. ( 1 or $2 \mathrm{gr}$.), three times daily and the various formaldehyd preparations, $0.3,0.65$ or $1 \mathrm{gm}$. (5, 10 or $15 \mathrm{gr}$.$) , t. i. d. I prefer the last of these, if any.$ 
Cubebs and copaiba, both time honored remedies in the treatment of urethral inflammation, are more indicated in the chronic stages and declining forms of the malady. These remedies are given in the wellknown formula, "Lafayette mixture," or in capsules.

Capsules put up by different drug houses contain various combinations of santal oil, copaiba, cubebs, salol and methylene blue, from which may be selected a formula suitable to the requirements of the case in hand. Generally speaking, as stated, the internal treatment of gonorrhea is symptomatic and subordinate to the local measures, which is also the case in most non-specific inflammations in which there is likely to be, within the urethra itself, or in one of the adnexa, a persistent or concomitant lesion of a nore or less chronic character, which calls for recognition and appropriate treatment, either at once or later, when the acute stage has subsided. Of this more is written in the section on Chronic Urethritis, under which heading most cases of non-specific urethritis more properly belong.

To summarize the internal treatment of acute urethritis: in the early stage the oil of sandalwood as a balsamic is a most efficient internal remedy; to this should be added an alkali, and the free use of water recommended to dilute the urine. In the declining stage and in subacute urethritis the addition of copaiba, cubebs or turpentine is sometimes useful, as is also the case in acute exacerbations of chronic urethritis. Internal antiseptics have no specific action, but may prove useful additions to the internal medication. The following combinations are put up in capsule form by different manufacturing chemists:

Oil of sandalwood.

Oil of terpentine.

Citrate of potash.

Salol.

Hexamethylenamine.

Oil of sandalwood.

Resin of kava kava.

Salol. .................. $0.23 \mathrm{gm}$. (gm. iiiss)

Oleoresin cubeb.............. 0.3 c.c.

Pepsin.................... $0.065 \quad$ (gr. i)

Copaiba................... 0.3 c.c. $(\eta v)$

Sandalwood ................. 0.3 c.c. $(\eta \mathrm{v})$

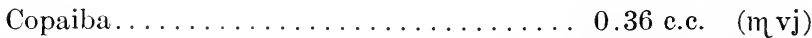

Oleoresin cubeb.............. 0.12 c.c. (mij)

Sandalwood ..................... (mij) 
When that train of symptoms supervenes which indicates the existence of posterior urethritis, namely, urgency, frequency and painful urination with tenesmus, the tincture of hyoscyamus will be found to be a sovereign remedy, and if urination is accompanied by terminal bleeding, the fluid extract of senecio aureus will often effectually control this symptom. In very severe cases, codeine is added.

The following prescription is employed and modified according to indication for it on account of the painful symptoms attending posterior urethritis, and when the irritation is great owing to the intensity of the inflammation in the earlier stage:

R. Codeine.............................

Alcohol . . 0.2 to 0.4 gr. (gr. iii-vi)

Oil of sandalwood or wintergreen.......... 15 c.c. (杼ss.)

Fluid extract senecio aureus................ 30 c.c. ( $(5 \mathrm{i})$

Tincture hyoscyamus................... 60 c.c. ( (气

Liquor potassa..................... 8 c.c. (3ij)

Syrup acacia.............enough to make 100 c.c (节ij)

Mix.-Teaspoonful three or four times daily.

In this mixture the codeine need not be included unless needed. The oil may also be removed and given instead in capsule form.

Finally, some cases of non-specific urethritis, which occur as a result of an oversensitive state of the urethra, accompanying diathetic conditions, anemia and malnutrition, require such internal remedies as will directly benefit the underlying condition. Iron and arsenic as general tonics, cod-liver oil and creosote for some cases, will at times, in addition to the local or internal treatment already instituted, be the means of bringing about a favorable result.

Before proceeding to the consideration of the local treatment of acute urethritis, attention should be called to the fact that there are practised two methods of treating this malady, either one of which may result in a safe and complete cure, but with a decided difference in the period of duration, namely: the expectant method, which avoids the employment of local measures in the early stage, when the acute symptoms run high, and relies upon the free use during this period of alkaline diuretics and diluents, allowing the discharge to flow freely without restriction, and only when the acute stage has materially subsided are local measures adopted. The latter consist of disinfectants and astringents in the form of irrigations or injections. The duration of the disease is materially prolonged by this temporizing method.

The alternative course, and the one strongly recommended, consists in the adoption of local measures at the onset of the inflam- 
mation for the purpose of destroying or restraining the microbic agents and curtailing the duration of the disease.

\section{Prophylaxis}

The prophylaxis of gonorrhea is in reality more important than its treatment, and this is the more keenly appreciated when its extensive propagation and far-reaching injurious influences are brought to mind. The individual who exposes himself to the pernicious effects of gonorrheal poisoning might be expected to pay the penalty without complaining of the consequent suffering, but the innocent ones who, without contributory offense, suffer from participation in the consequences, should, humanely speaking, be protected by every means that sanitary teaching can command. As an evidence of the far-reaching injury wrought by gonorrheal infection we have the percentage of gynecological operations, variously stated as being between 20 and 50 per cent. of the number performed, resulting from infection due to uncured gonorrheal inflammation in the male. The blindness of infants has been stated to be due to specific vaginitis existent in the parturient canal prior to or at the time of birth, approximately 15 per cent.

These reasons alone are sufficient to emphasize the appalling evil of this malady, which is too of ten treated as insignificant; and besides, when we consider the many ills that may be inflicted upon the individual himself, such as stricture, chronic cystitis, inflammation of one or both testicles, and the secondary results, involving impotence and sterility, leading to unfruitful and unhappy wedlock, we are still more impressed with the importance of exercising sanitary measures as far as possible and of teaching prophylaxis. In the first place, when a patient is treated for true virulent gonorrhea, the great contagiousness of his malady should be strongly impressed upon his mind and care should be exercised to determine before he is released from treatment that he is no longer a dangerous factor in this respect. Above all, it should be impressed upon him that this disease, having once ,been contracted, retains its virulence to the very end and that even after the discharge has ceased there may still lurk contagious elements which may propagate the disease to another, and that a laboratory examination is necessary to determine their presence or absence.

This brings up the question so often asked and so important in this connection: when is gonorrheal inflammation cured so far as its contagiousness is concerned, and when is the patient, once the victim of this malady, a proper candidate for matrimony? By referring to the section on the Bacteriology of Gonorrhea, it will be seen that for 
ordinary purposes the specific organism of this disease can be found and recognized by the usual cover-glass examination with the microscope', but that in the more obscure conditions of chronic urethritis and where there is apparently existent only a catarrhal inflammation, yielding but a few shreds in the urine, the ordinary cover-glass examination is not sufficient to give a reliable result as to the existence or non-existence of gonococci, and it becomes necessary to resort to more searching methods, which involve the employment of cultures.

When a patient has been under treatment for a sufficient length of time and, as a result thereof, all discharge has ceased and the urine has become absolutely clear he is apparently clinically well, but should be submitted to proper tests. I generally then have him report, preferably, in the early morning before any urine has been voided, the first part of which he is directed to pass into a clean vessel; and then, after thoroughly massaging the prostate, the seminal vesicles and the entire urethra, the urine is passed into another clean vessel in sufficient quantity to wash the anterior and posterior canal of whatever exudate has been obtained by the previous manipulation. These two specimens are subjected to careful centrifugation until all the sediment is obtained. This sediment is then examined in the usual manner for gonococci. If the result is negative as to gonococci a second or confirmatory examination is made, following a previous irritation of the urethra, which is caused first by the passage of a fullsized metallic instrument through the urethra and later in the day or evening by drinking some beer or other form of alcoholic stimulant, after which the patient returns on the following day for the test, made in the same manner as before. This test should be sufficient in most instances where no clinical symptoms remain following a recent gonorrheal inflammation. If the discharge still persists or when there is free pus present in the urine in sufficient quantity to be seen on ocular observation, it is customary to confirm the negative findings of the microscopic examination by the compliment fixation test (p. 106). In those instances in which pus is present, following irritation of the urethra by the passage of an instrument, it is a good plan to cause retention of the discharge in the urethra by the application of an adhesive strap at the meatus, to be left in place for a period of about two hours, and before the next urination a specimen of this discharge is obtained for cover-glass or culture examination.

It is hardly necessary to state that when the physician is asked to give his sanction to matrimony it is important that every possible investigation be made before final opinion is given. On the other hand, it is possible to assume too extreme an attitude when conditions do not 
warrant it. If the patient has been pronounced cured clinically, if there is no evidence of free pus in the urine, and if the microscopical examination of the urethral exudate obtained from the canal following massage of the prostate and vesicles reveals, after repeated trials, not only no specific organisms, but few pus cells and only a few epithelia, a single cultural test, in confirmation of the negative microscopic findings, may be made in deference to the peace of mind of the individual or as a matter of routine on the part of the physician.

If, on the other hand, there is still remaining in the canal or present in the urine free pus, the additional measures described of irritating the urethra and several subsequent examinations are needed to establish the reliability of the search. I am inclined to believe that a satisfactory conclusion can be arrived at with repeated microscopic examinations, properly made, with the Gram differential test and that the culture tubes will sometimes fail to find the gonococci when present as well as will the cover-glass examinations, for it is possible for the shreds present in the urine at one passage to contain gonococci while those of another time may be entirely free from these organisms. Therefore, if the gonococci are present in only a single isolated area and cannot be found by ordinary microscopic examination, they may also be missed in cultural tests and be found later following an exacerbation of inflammation brought on by mechanical means-the passage of a sound-or when the discharges of the canal are pent up by being retained, thus affording a favorable medium for their growth and propagation.

Examination of the blood by complement fixation test is the last and best means of detecting uncured gonorrhea and infectious lesions that are not revealed in the urethral exudates, as is demonstrated by the satisfactory results obtained in competent hands. The difficulty of obtaining and keeping reliable test sera, confines the use of this method to a specially equipped laboratory. Generally speaking it may be stated that this test, properly conducted, possesses even a greater percentage of reliability than the complement fixation for syphilis (see Serodiagnosis, p. 108).

So much for the prevention of the spread of this virulent malady beyond its original possessor; now, as to the protection of those who have exposed themselves sexually to possible contagion, or personal prophylaxis. When a patient appears and requests a preventative treatment against possible infection following a recent coitus, I usually employ a copious irrigation of the anterior urethra, with a 1-6000 solution of permanganate of potassium, and I have not known any 
contagion to follow this precaution when adopted within twelve hours after exposure. Although no absolute assurance can be given, and the shorter the interval the more reliable the result, yet it is a proper precaution at any time before the development of trouble. In cases that have allowed several days to elapse, and where the fear of contagion rests on more than mere suspicion, a solution of protargol, 1/2 of 1 per cent., or argyrol, 10 per cent., may be injected, following the irrigation of permanganate, and retained several minutes, or a small cotton wick containing a lanoline paste of $1 / 2$ of 1 per cent. protargol may be inserted $1 / 2$ to 1 inch within the meatus and retained there until the next urination.

For the patient's own use a powder containing $0.5 \operatorname{gram}(71 / 2$ grains) of protargol is ordered, which he is told to dissolve in a tumblerful of water. As soon after coitus as possible the penis is immersed in this solution for from five to ten minutes, and if the period elapsed has been over six hours, or in especially suspicious instances, an injection of the same solution or of 10 per cent. argyrol is employed in addition to the immersion.

As an additional precaution against inoculation with syphilis, the penis may be annointed with a paste composed of 10 per cent. calomel.

To encourage a universal custom of prophylaxis such preventative methods should be generally urged, and in discharging a patient cured of gonorrhea, realizing that in most instances he will again be exposed, despite the strong injunctions and warnings that may be given, it is proper to impress upon him the importance of adopting prompt prophylactic measures in the event of an exposure and the proper method should be explained for this object.

\section{LOCAL TREATMENT OF URETHRITIS}

In some cases of non-specific urethritis local measures play an important rôle in the treatment; while in other non-specific cases, notably those which arise from or are prolonged by diathetic and constitutional causes, such means are often unnecessary, and may even aid in prolonging the symptoms. In specific cases generally it is the local treatment, in all stages, that is of prime consideration.

General Considerations. - The rationale of the local treatment of urethritis is expressed in the different properties of the various topical applications, which properties are astringent, stimulating and antiseptic. In the mildest forms of non-specific inflammation a local astringent is often the best means of controlling the discharge and cur- 
tailing the catarrhal inflammation. In the more chronic inflammatory conditions a certain amount of stimulation is demanded; but in this class of cases, as has been stated in the section on Internal Treatment, there is likely to be an underlying morbid condition of the mucous membrane or complicating lesion of the adnexa, the recognition and removal of which are paramount to complete the cure; and, therefore, for a better understanding of the treatment of this type the reader is referred to the section on Chronic Urethritis.

The local treatment of acute urethritis, generally speaking, resolves itself into the local treatment of gonorrhea or gonorrheal urethritis. The local treatment of non-specific urethritis is either the treatment of declining gonorrheal urethritis after the specific element has been abolished or the treatment of catarrhal inflammation which is an expression of some underlying morbid condition of the canal or its adnexa, which, as already stated, will come under the heading of Chronic Urethritis and its Treatment and is fully considered in that chapter.

The Local Treatment of Acute Gonorrhea is of first importance. Beyond question, the majority of cases of acute urethritis not associated with preëxistent lesions of the anterior or posterior urethra or of the adnexa-in other words, not acute exacerbations of chronic urethritis-are instances of true gonorrhea. While there are cases, as already described, of simple urethritis not of gonorrheal origin, their number is so small that their importance is insignificant compared to that of gonorrhea in considering the local treatment.

The different methods of applying treatment to the urethra in acute gonorrhea are irrigations, hand injections and instillations. A description of these methods is given in the chapter on General Technic (q.v.) and the different agents employed are mentioned in a later section. In accordance with the theory of the modern treatment of gonorrhea, the important object in view is the destruction of the gonococcus in all localities where it can be reached; and to this end, such local measures are employed as will accomplish the result with the least, or with no damage to the mucosa or, in other words, with the avoidance of injury of any sort. As the disease is primarily a local infection, the measures adopted to accomplish the end should be essentially antiseptic. It is, therefore, desirable that the mucous membrane be subjected for the longest possible time to the applications of the selected germicidal remedy, or exposed to it as often as is consistent with freedom from damage. This treatment is to be kept up until assurance is had that there no longer remains any poisonous element in the canal or its adnexa. 
Notwithstanding that there are agents with strong gonococcicidal properties, and that the irritating qualities of these agents have been reduced to a minimum when they are put to therapeutic test, it is found that certain conditions exist which limit their action; for while they are capable of destroying gonococci, and while this can be demonstrated by laboratory experiment, yet the gonococci by proliferation propagate themselves into localities which cannot be reached even by those applications to which are imputed an unusually deep action; which fact teaches that if therapeutic measures were relied upon entirely in the treatment of this disease, a cure would not be possible.

In combination with the local treatment, we must, as one writer puts it, form a judgment as to how far we may be able to influence the infectious process with our remedies and study the question as to how we may be able best to support the natural defensive forces of the organism, and how we may interfere least with the same. In line with this purpose of treatment, the most effective method which produces the quickest result and is least apt to be accompanied by complications is the frequent use by hand injections of one of the silver compounds retained in the urethra as long a time as possible, or at least, as long and as often as can be done without producing irritation, that is to say, marked irritation. I qualify this statement, because a mild amount of irritation, when not continuous, instead of being undesirable is even capable of aiding favorable progress.

We know that in response to the action of the poison upon the mucous membrane of the urethra a hyperemia is produced, accompanied by exudate of pus corpuscles and serum. This inflammatory exudate stream occurs in the opposite direction to the proliferation of the gonococci and has the tendency to remove the cocci from the tissues. When they are carried to the surface of the mucosa they are met during the course of treatment by the bactericidal action of the local application and are destroyed. Besides acting directly upon the cocci which are free upon the surface of the mucosa and causing their death, the antiseptic action of the local application tends to render the mucosa a bad habitat or dwelling-place for these organisms and the bactericidal action is extended into the deeper tissues reinforced by the natural protective agency of the organism. As a part of this protective agency, there occur also epithelial alterations of the mucosa involving the production of many-layered pavement epithelial formations, rendering the further penetration of the cocci difficult.

The anatomical changes which occur in the course of acute gonorrheal inflammation are part of the natural defensive forces of the body, and a knowledge of them helps us to realize the limit of the 
action of therapeutic agencies and suggests the conditions that have to be taken into account in directing the treatment. While a knowledge of these facts does not teach or explain the different results obtained, it helps us to theorize upon the different actions of the various agents employed, which actions are not always consistent with the potency of the antibacterial or chemical properties of the remedies.

It has been determined by comparison of the bactericidal properties of the various remedies used in the local treatment of gonorrhea that those which produce or possess the most antiseptic activity are not always the most satisfactory clinically. Different degrees of irritation are also possessed by these various preparations: one-the nitrate of silver-experiment reveals, has a special action upon the epithelial layer, causing transition of the upper layers of the stratified epithelium; while another-protargol-produces no formation of stratified epithelium, but causes abundant round-cell infiltration in the epithelial and connective-tissue layers. The organic silver preparations are alleged to exert a deeper action than silver nitrate. Chemical experiments, however, do not always concur with the clinical results and the latter are much more to be depended upon for practical purposes. But even though chemical demonstration fails, it seems that the organic silver combinations with even slight antiseptic properties still exert favorable action in controlling the inflammatory process.

The practical conclusion is not to confine the treatment of gonorrhea in any one case to the adoption of a single remedy or to any one of the silver preparations, but to change agents according to conditions and the action of treatment during the progress of the disease. This method accords with the clinical results obtained by experience and conforms to the general didactic clinical teaching of beginning treatment by using antiseptics, followed by antiseptic astringents, and terminating with pure astringents.

We therefore institute treatment upon the principle of destroying the gonococcus at all points within reach where its presence may be demonstrated or may be assumed to exist. To this end we first aim to remove all the free gonococci which proliferate upon the surface of the mucosa; we further aim to destroy the gonococci which exist between the epithelium and still exist after the mechanical removal of those upon the surface. These are the organisms which are present in the folds of the mucosa and in the mouths of the glands. If they cannot be destroyed, the purpose of treatment is to interfere as much as possible with their proliferative action, and so to influence the tissues that the latter become a poor nutrient medium for their growth. 
To accomplish this purpose we select a remedy which by bacteriological experiment has demonstrated its bactericidal action so far as the gonococcus is concerned. This action will enable it to destroy the gonococci which remain upon the surface of the mucosa. Next, it must have an action penetrating in quality, to enable it to reach some of the cocci which are beyond the surface-those which are in the folds or in the mouths of the glands. It should possess also a lasting quality in order to produce upon the mucous membrane the effect of making it a poor medium for the growth of the microörganisms; and, finally, the remedy must not antagonize the natural protective action of local hyperemia, but treatment should be directed from time to time toward assisting this agency, for it must be borne in mind that the exudate flow associated with inflammation is an important resource for bringing to the surface the exciters or producers of infection which we cannot possibly reach with our remedies and which are, therefore, inaccessible to therapeutic action. We have, in the organic silver compounds, agents which fulfil all the chemical and therapeutic requirements. Their action is sometimes assisted by alternating from one to another throughout the course of treatment and we certainly have in the use of irrigations, especially with permanganate of potassium, a valuable means of supplementing the treatment and of accomplishing the purposes for which treatment is intended. Many excellent results are obtained by the employment of the irrigations alone, but still better results and more rapid cures are accomplished by the combined use of the silver preparations and the permanganate irrigations.

The efficacy of the irrigations may be due in part to a certain amount of pressure and stretching of the urethra, which being followed afterward by relaxation, causes a mild degree of hyperemia of the mucosa, a serous saturation of the tissues; and the exudation toward the surface of the mucosa is in line with the theoretical purpose of treatment. Following the teachings of Bier, we are learning to take advantage of this hyperemia and its consequences in inflammatory conditions in all parts of the body.

The mechanical distention of the canal by means of irrigation unquestionably produces a local hyperemia and the occurrence of this local hyperemia in response to a permanganate irrigation in a case of simple acute urethritis may explain the prompt and effectual cure that is seen to follow a single treatment of this kind, and may also explain the cessation of a subacute urethritis involving the anterior and posterior urethra when treatment is directed only to the anterior urethra by means of such irrigation with permanganate of potash; the posterior urethra is not included, yet shares in the general cessation of 
the catarrhal inflammation. This is not possible, however, when the inflammation is of gonorrheal origin, for we have to deal with virulent organisms which proliferate in the deeper tissues and cannot be removed at once by the mechanical means of irrigations or injections nor overcome by the increase in the natural protective power of artificial hyperemia. We have resource, therefore, to the most powerful gonococcicidal remedies at our command, and those which exercise the deepest action, as soon as possible after the disease has started; and these special remedies must be kept in contact with the inflamed and infected canal long enough to make the increase and proliferation of the organisms difficult.

Silver Preparations.-Chemical experience has shown that the different organic silver compounds exert a less harmful influence upon the tissues than does the nitrate of silver, and that the former possess a strong bactericidal property. Of these silver compounds, argyrol, collargol, novargan and protargol are the most important, and while others have been introduced and their properties extolled, it is sufficient to mention these four as representatives of the class of remedies to which they belong. They are all related to the nitrate of silver, and have more or less common bactericidal tendencies. The protargol is probably more irritating than the others and possesses some special properties already mentioned. Argyrol and collargol are much alike in action, and are used in the same strength solution. They are in most instances entirely free from irritating properties, when used in solution up to 10 per cent., and of ten even up to 15 and 20 per cent. I usually order a solution of 10 per cent. argyrol for the patient's own use, and he is directed to employ this injection two or three times a day, and oftener if possible, retaining the same in the urethral canal as long as he conveniently can-at least 5 to 10 minutes. When the patient calls at the office for periodical inspection and examination, if he has been unable to retain the preparation for more than a short time, he is treated with a very much longer injection than usual. To attain this end, I have adopted a special urethral clamp, constructed of hard rubber and made so that the pressure is directed upon the terminus of the urethra alone, and does not constrict the surrounding tissues; in this way the clamp can be left in place for 15 minutes, or even longer, and thus the action of the remedy is maintained for a long period (Fig. 31, p. 44). Many patients find no difficulty whatever in retaining the injections in the urethra as long as is desirable by compressing with the ends of the fingers. This treatment is commenced at the very onset of the disease, or as soon as the patient reports for treatment; and if he has reported early enough, before 
inflammatory edema and swelling have set in, the prompt action of the gonococcicide is likely to ward off the occurrence of severe inflammatory conditions.

The Abortive Treatment of Gonorrhea, to be successful, must be resorted to within a few hours after the first appearance of the urethra] discharge. While many of the local antiseptics commonly employed at the present time have the effect of shortening the course of a gonorrhea, they cannot be considered as abortive measures. A treatment which is deserving of this title must quash the disease at its onset and prevent its evolution. For this purpose the permanganate of potassium has been employed in strong solution $(1: 500$ and $1: 1000)$ by copious irrigations of the anterior urethra a few hours after the discharge appears; also the nitrate of silver (2-4 per cent. solution) and argyrol or collargol (20 to 30 per cent.), which are injected with a small urethral syringe or pipette of a capacity of about 1 to 2 c.c., just inside the meatus. The pain and distress following the use of silver nitrate may be intense, but when successful, the sero-sanguinolent discharge which subsequently appears subsides of its own accord in a day or two. This treatment is uncertain, and not employed for this reason, unless the patient is seen early enough. Its success depends upon the destruction of the gonococci while they still lurk upon the pavement epithelium of the fossa navicularis, which, unless broken, prevents their penetration, and before they have spread to the deeper portions of the canal.

Astringent injections of the urethra are capable of rendering considerable service in urethritis, but when improperly used, they may' occasion much mischief.

In gonorrheal urethritis, if they are used at the beginning of the disease, there is danger of carrying the inflammation backward and of lighting up posterior urethral complications, especially when the agent employed possesses astringent properties only. Therefore, if the local treatment is not directly antagonistic to the life of the gonococcus, such as may be obtained by the application of the silver preparations or by copious irrigations of the urethra with permanganate of potassium, it is far better to postpone all local measures until the later stage, when the acute symptoms have subsided; but even at this stage, if it is found that the gonococcus is still existent in the urethral discharge, it is necessary to employ the same remedies that are known to be destructive to its existence, rather than astringent applications which possess no such agency.

Frequently in subacute urethritis and in non-specific cases generally, one of the astringent injections may be employed with good effect. 
The discharge which is checked by the use of an injection of ten promptly returns, however, when such injection is discontinued. This relapsing urethritis is generally due to morbid changes in the urethra and to underlying constitutional conditions. Such cases of relapsing urethritis which persistently recur properly come under the head of chronic urethritis.

A final precaution concerning the use of injections is that they occasionally produce and keep up a discharge on account of being inappropriately strong. This is quite likely to occur with those patients who frighten themselves into the belief that they have gonorrhea when such does not exist, and who commence a fierce onslaught on the urethra with injections, a treatment which promptly excites a flow of pus and confirms their fears. On the other hand, at the end of a gonorrhea, when an injection of undue strength has been employed to arrest the discharge, an oozing of gleety mucus may be kept up by the persistent use of a strong injection.

In either one of this class of cases rapid improvement follows a cessation of the injection.

In injecting the urethra, a proper syringe should be used (see description of Urethral Instruments and Technic).

The following formulæ of zinc sulphate, sulphocarbolate and permanganate, alum, subacetate of lead, bismuth and fluid extract of hydrastis represent the different combinations of these agents as they are used for astringent injections.

1. R. Zinc sulphate Dilute lead water

Mix. To be filtered.

2. R. Zinc sulphate

Bismuth.

Powdered acacia.

Water.

Mix. To be well shaken.
$0.2-0.4 \mathrm{gm}$.

125 c.c.

$0.26 \mathrm{gm}$.

$8 \mathrm{gm}$.

$30 \mathrm{gm}$.

100 c.c. (gr. iij-vi)

(亏)iv)

(gr. iv)

(5ij)

(รi)

(รiii)

3. R. Zine permanganate ...... $0.065-0.26 \mathrm{gm}$. (gr. i-iv)

Water............... 125 c.c.

4. R. Zinc sulpho-carbolate..... 0.13-0.26 gm. Water .............. 125 c.c.

(gr. ii-iv)

(争iv)

5 R. Zine sulphate.

$0.13-0.26 \mathrm{gm}$.

Fluid extract hydrastis

4-16 c.c.

Water.............. 125 c.c.

(gr. ij-iv)

(5i-iv)

(争iv)

6. R.

$0.26-0.52 \mathrm{gm}$.

(gr. iv-viij)

Zine sulphate......... $0.13-0.26$ c.c.

Water.............. 125 c.c.

(gr. ij-iv)

( 亏iv) 
The frequency with which the above astringent preparations should be used must be determined according to the quantity of the discharge and the controlling influence exerted upon it by the injection. A proper rule to follow is to use the injection only sufficiently often to keep the discharge in check. When, in spite of its usage for a reasonable length of time, the discharge persists, or when relapses occur as often as the injection is discontinued, it may be taken as an indication that the agent employed is acting as a source of irritation rather than a means of relief, or that there is trouble deeper down the canal than is reached by the injection (posterior urethritis) or perhaps deeper in the tissues themselves.

Urethral and Intravesical Irrigations.-Irrigations of the urethra by means of copious quantities of permanganate of potassium have long been a favorite method of treating simple and gonorrheal urethritis. More recently this method has been partially superseded by the practice of using the organic silver compounds, on account of the more satisfactory results accompanying the use of the latter preparations in specific urethritis. But irrigation of the urethra with permanganate of potassium has still an important place in genitourinary technic, as many cases of simple urethritis are best treated in this way, and it must also still be considered an important agent in combination with the use of the silver compounds in the treatment of gonorrhea. I would reiterate here what has been stated previously, that the best local treatment of gonorrhea consists in the regular use of the silver preparations already described.

Irrigation of the urethra is by no means restricted to the treatment of urethral inflammation. It is desirable at times before and after the passage of instruments, and may be the means of preventing subsequent inflammation and of avoiding urethral chill. For this purpose may be employed normal salt solution, 1 per cent., boracic acid or permanganate of potash, 1 to 8000 and 1 to 4000 . Bichloride of mercury is also used to irrigate the urethra, and was formerly a favorite method of treating gonorrhea, but has fallen into disuse on account of the more satisfactory results attending the use of other agents. When used as a disinfecting irrigation, it is employed in the anterior urethra in a strength of 1 to 10,000 and 1 to 20,000 .

Janet, of Paris, popularized the method which bears his name, of treating gonorrhea with the irrigations of permanganate of potash. In employing these irrigations he followed a rigidly systematic routine, irrigating daily both the posterior and anterior urethra during the course of the treatment, whether or not the posterior section was involved. 
This method of carrying out the treatment I have not found desirable. (Description of Anterior and Posterior Urethral Irrigation is given on p. 37.) Irrigation of the posterior urethra without a catheter is likely to be productive of undue irritation and to lead to complications in acute inflammations of the urethra and should not, therefore, be carried on as a routine practice. While in certain individuals, not oversensitive in their tissues, and in old, chronic inflammations this plan is useful and is not so likely to produce harmful results, yet I prefer in most instances to rely upon the catheter in irrigating the posterior urethra and bladder, and always in acute urethritis.

For a number of years I have been in the habit of employing in private and hospital practice an apparatus designed for use for either anterior or intravesical irrigation (see p. 36). For irrigation of the anterior urethra, about one liter (qt.) of fluid is used and for the posterior urethra, 500 c.c. or less, according to the capacity of the bladder.

In supplementing the treatment of gonorrhea by means of the silver compound injections, irrigations of permanganate of potassium are employed every two or three days, and sometimes every day, the strength of the solution being from $0.2-0.32 \mathrm{gm}$. to the liter (3 to 5 grs. to qt.). For the treatment of simple non-specific urethritis, the irrigation of potassium permanganate is employed in the same strength. In this latter class of cases sometimes a single wash is sufficient to control the discharge, and in more active or severe cases, a daily irrigation is given for four, five or seven days, after which time, the acute activity of the malady having subsided, it often becomes necessary to determine whether or not there is any underlying morbid condition in the urethra or the prostate gland calling for further treatment.

Treatment of Posterior Urethritis.-The importance of treatment of posterior urethritis is due not only to the intensity of the symptoms, but more to the fact that it is from the posterior urethra that the disease usually takes its unfavorable course when such occurs; that is to say, extension from this point leads to the further complication of acute cystitis or invasion of the bladder, acute epididymitis from extension down the rasa differentia, acute prostatitis or seminal vesiculitis from implication of these organs, on account of their contiguity and direct contact with the posterior canal; and, finally, it is likely that this point is more frequently the port of entry of the disease into the general circulation, resulting in metastases and more distant complications, namely, involvement of the joints, tendons and other synovial membranes. It does not necessarily follow, when the 
posterior urethra is invaded, that any of these complications will occur. Other things being equal, the earlier the stage of the inflammation (namely, when the virulence of the process is greatest), the greater the danger. The consequent aim of treatment, therefore, should be to reduce this factor, so that when the posterior urethra is reached, if at all, the type of inflammation is as nearly catarrhal as possible. When posterior urethritis occurs during an acute attack, as evidenced by the characteristic symptoms, the question arises, whether to treat the existing condition by local or internal measures, or both.

This question must often be decided by the conditions attending the particular case. For instance, if local treatment has already been vigorous and posterior urethritis supervenes, such complication may be in part the result of too aggressive an attack, being a mixed infection composed of the gonorrheal germs and the ordinary pathogenic organisms, which latter may in this instance predominate. In such a case the best results are obtained by desisting from local measures temporarily and insisting upon body rest until the acute stage of inflammation subsides, at which point local treatment may be resumed. In this case the internal treatment would consist of balsamics, alkalies and sedatives (see p. 208). On the other hand, when, during the course of an acute urethritis the existence of posterior urethritis is established by the examination of the urine (see p. 202) rather than by a sudden acuteness of the symptoms, local measures promptly applied to the posterior section of the canal may have an immediate and restraining influence thereupon. If in doubt as to the advisability of adopting local measures, it is proper to make an experimental investigation of such with great gentleness, and to continue the same only so long as there appears to be no resulting irritation (see Local Treatment). When there coexists with the posterior urethritis any of the other complications named, the appropriate treatment must be adopted (see Complications of Urethritis).

The dressings of the penis in urethritis, when the discharge is abundant, become a matter of importance, and may prevent the occurrence of balanitis and posthitis, and of vegetations around the foreskin, all of which conditions are frequently due to the collection of irritating discharges. When the discharge from the penis is slight and of a nonpurulent character, a plug of cotton, dry or moistened with mild sublimate solution, placed over the meatus and retained by the prepuce, may be a sufficient dressing; but when copious, it is necessary to provide a means of catching the flow and protecting the clothing. Gonorrhea bags, made of rubber or muslin, are sold by druggists for this 
purpose. The bag is suspended from the waist by means of tapes, and a piece of absorbent cotton, dry or moistened with an antiseptic solution, is placed inside. The cotton should be changed frequently. Another dressing may be worn, in connection with an ordinary suspensory bandage, consisting of a piece of absorbent gauze folded in the shape of a triangle. The base of this is placed underneath the penis, and the three corners are folded over and pinned together to the band of the suspensory; or a square of gauze, with a smaller square of absorbent cotton, may be applied to the end of the penis and held in place by a loose elastic band. Such a dressing must be changed frequently, according to the amount of discharge. In all cases, general cleanliness should be strictly observed. The patient should be instructed to keep the hands clean, as well as the local parts, and should be duly cautioned regarding the dangers of contagion, particularly with respect to his own eyes. The discharge should not be allowed to accumulate behind the foreskin. A mild antiseptic wash should be employed daily, and if the foreskin cannot be retracted, a duckbill or ordinary syringe should be used to wash the cavity of the prepuce. For purposes of general cleanliness, as well as when balanitis or posthitis exists, a solution of corrosive sublimate, 1:6000 or 8000 , or permanganate of potash, 1 to 4000 , may be used. 


\section{CHAPTER XII}

\section{CHRONIC URETHRITIS}

Causation.-Chronic, like acute urethritis, may be specific or non-specific, anterior or posterior, or both. An acute urethritis becomes chronic when the gonococci, having located in the submucous tissue or follicles, remain there for a prolonged period, in spite of treatment. This form of chronic urethritis is directly traceable to the continued lodgement of the gonococci in the urethra, and is due to misdirected or unwise treatment, neglect or carelessness on the part of the patient, or, in some cases, to an inherent condition, such as a lithemic or tuberculous constitution, which renders the tissues more vulnerable and resistant to treatment.

There is no portion of the canal which may not be the seat of chronic trouble, and locations of special frequency are the areas behind the points of natural contraction in the anterior and posterior portions. These are about $71 / 2$ centimeters ( 3 in.) from the meatus, the sinus of the bulb generally running into the adjacent membranous portion (antero-posterior urethritis) and the sinus of the prostate. In the prostate the lesion may be follicular, as in the anterior canal, or extend deeper into the substance of the gland (parenchymatous prostatitis); and finally, on account of the direct proximity of the opening of the bladder to the posterior urethra, this part is often implicated in the chronic condition (urethro-cystitis).

A cure of urethritis is likely to follow the effectual removal from the urethra of the gonorrheal organisms, although in many cases a catarrhal discharge will continue in spite of such removal, on account of structural changes (ulcerations, granulations, stricture) and other morbid conditions beyond the urethral calibre in the follicles, Cowper's gland, prostate, seminal vesicles, the testicles and their ducts, all of which require special treatment for their relief.

The causes that tend toward the chronicity of urethritis are direct and indirect. The indirect are constitutional: bad hygiene, gout, rheumatism, tuberculosis; and local: congenital and acquired narrowing of the canal. The direct causes are those which prolong the stay of the gonococci in the urethra, or that tend to damage the 
already inflamed tissues, namely: neglect of treatment, excessive treatment, sexual and alcoholic excesses, and the occurrence of complications during the early course of the disease which apparently disappear during the acute stage, yet lie dormant until lighted up afresh by any of the usual causes of urethral irritation. Such forms of chronic, relapsing urethritis are found located in the urethral follicles, in the prostate and seminal vesicles, and in the testicles.

Chronic urethritis is usually traceable, directly or indirectly, to gonorrhea, although individuals of a gouty or lithemic constitution may have a more or less chronic urethral catarrh, never having had gonorrheal infection, which catarrh is due to the congestive tendency of their mucous membranes in general and to the irritating qualities of the urine.

Other forms of chronic urethritis, not of gonorrheal origin, such as may be caused by the presence of papillomata, polypi, encysted calculi and foreign bodies, are considered under the division on Diagnosis and Endoscopic Examination of the Urethra.

Clinically speaking, we recognize several forms of chronic urethritis:

First, one which is a direct continuation of recent acute inflammation, in which the symptoms are pronounced and the discharge is of a variable character, but of ten rather free. This may last any time from three to six weeks-even longer.

In the second form the discharge is decidedly scanty, and has been present for a much longer period, perhaps for years.

A third form shows itself as a continual tendency to recurring attacks of acute or subacute urethritis, between which there may be practically no sign of trouble.

These several forms are dependent upon various causes. The first may be due to the continued stay of gonococci in the urethra, to constitutional debility, to the occurrence of complications or to badly directed treatment, and sometimes to long-continued or too energetic local measures. The second and third forms are generally dependent upon stricture or other morbid changes in the urethra or adjacent structures, or due to catarrhal and mildly inflammatory conditions in which the gonococcus may be present alone or in combination with other pathogenic bacteria excited into activity by various causesintercourse, alcohol, fatigue, etc., or are fostered by unfavorable diathetic conditions, gout, scrofula, tubercle, etc.

The most important question to be considered with respect to chronic urethritis concerns the presence of the gonococcus. Many cases of chronic urethral catarrh may continue for years with no discoverable evidence of the primary etiological factor, while some cases 
are found which retain this virulent element for a suprisingly long time.

Pathology.-The morbid changes of chronic urethritis are dependent upon small cell infiltration superficially, causing alterations in the color and transparency of the mucous membrane, and in the deeperlying tissue producing structural changes of the epithelia, alterations in the follicles and glands, which become distended with retained inflammatory products and obliterated as a result of the formation of new tissue. Finally, this process in parts of the canal may become the starting-point of stricture, of which it is the transitional stage.

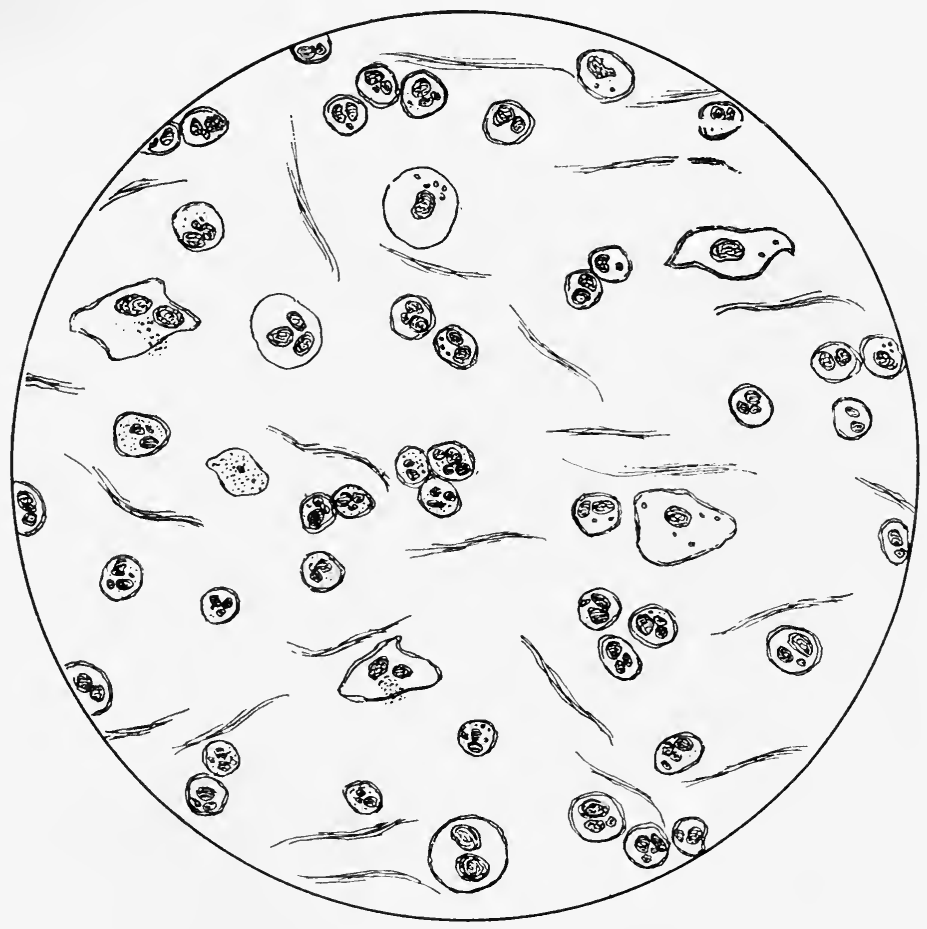

FIG. 102.-Chronic urethritis.

Chronic infiltrative changes are not necessarily the beginning of stricture in the urethra, the formation of narrowing of the canal being the determining feature of the latter condition. These infiltrative changes have a predilection for the glandular elements of the urethra, and commonly involve the glands of Littré and the lacunæ Morgagni with the surrounding tissues. While the process may go on to the formation of stricture, this is not necessarily the result; but it may 
continue for an indefinite period as a localized chronic inflammation, producing a discharge of leucocytes and desquamated epithelial tissue (Fig. 102).

In the posterior urethra the changes are more or less analogous, but owing to the structures here subject to involvement, the condition becomes more serious and the symptoms more complex. The mucous membrane is swollen and congested, the verumontanum elevated and softened. The glands and follicles present the same changes as in the anterior urethra, and one or both of the ejaculatory ducts may become catarrhal or even occluded. The inflammation in the prostatic canal may result in engorgement of the neck of the bladder and eventually in contracture of the sphincter (see Chronic Prostatitis, p. 375 and Prostatic Hypertrophy, p. 385).

Clinical Course.-The discharge in chronic urethritis is represented by a more or less continuous flow of pus, sometimes by the so-called "morning drop," or merely by urinary shreds.

In chronic anterior urethritis the discharge is continuous. It may be slight, and the quantity may be greater on arising in the morning, but this is due to overnight accumulation. It may be so scanty as to cause a slight gluing together of the meatus, or, on the other hand, it may be continuously active. In the latter instance, the existence of anterior urethritis is self-evident, although there may often coexist posterior urethral inflammation. The discharge is practically the only symptom present in chronic anterior urethritis, as there is commonly no pain or other discomfort, although the patient sometimes complains of some one particular sensitive area. By obtaining the urine in two separate flows, it will be seen that the second flow is perfectly clear; or, having previously washed out the anterior canal, the urine voided will be found entirely free from pus (Plate V, Figs. 5 and 6 ).

In chronic posterior urethritis, unless accompanied by anterior inflammation, the discharge is intermittent. When slight, it appears only in the passage of the urine, but when more abundant, collects at the meatus in the form of a morning drop, or the flow may be provoked by the act of straining at stool. There is not uncommonly a certain amount of frequency of urination; the desire is urgent, but not imperative, and accompanied by more or less burning at the end of the penis. When the pain and the desire to urinate persist after the urine is voided, such is an indication that the neck of the bladder is actively involved in the chronic lesion, and when there is pain in the perineum in the intervals between urination, there exists a prostatitis in conjunction with the urethritis.

Diagnosis.-In reaching a diagnosis regarding the location of 
chronic urethritis, it should be remembered that the discharge is liable to be continuous in anterior, and intermittent in posterior urethritis; that the symptoms of urgent, frequent and painful passage of the urine are absent in anterior and may or may not be present in posterior urethritis. Unless there is coexistent a certain amount of cystitis, these latter symptoms are not marked.

If a conical bulbous bougie is passed into the anterior urethra (a bulb as large as the meatus will admit) down to, but not into the membranous portion, when the urethritis is confined to the anterior canal a tender spot may be detected somewhere along the urethra during the introduction of the instrument; and when it is withdrawn, a small amount of pus may be found upon the shoulder of the bulb. The damaged area in this manner may be located in the bulb, in the fossa navicularis, or in some part of the urethra between these points. In the case of stricture, the site of the inflammatory area will be found behind the points of contraction. In exploring the urethra with a blunt steel or bulbous instrument, after previously washing the anterior canal, when the posterior canal alone is involved, it will be noted that no pus is expressed from the anterior region; but as soon as the posterior urethra is reached, a sharp pain is produced, and the withdrawal of the instrument may be accompanied by a discharge of pus.

In chronic urethritis the urine may contain free pus, which produces a certain amount of turbidity, or it may be clear, except for the presence of filaments or shreds, which cause a moderate amount of cloudiness only when the urine has stood some time after passage (Plate V). If the anterior urethra is cleansed by irrigation or injection before urination, chronic posterior urethritis will be evidenced by the clouded or shreddy appearance of urine passed after such operation, whereas if the lesion is confined to the anterior portion, the shreds or filaments will be removed by the lavage, and the subsequent urine passed will be clear. It is quite common that both the anterior and the posterior urethra are involved in the chronic inflammation.

For a more careful location of the lesion of chronic urethritis the 4 glass test is employed. In the first glass is contained the urine after washing the anterior urethra, by injection or irrigation with plain sterile water and is the first jet of urine, which carries the washing of the posterior section of urethra. The second glass contains the washing of the neck of bladder (urethro-vesical). The third glass contains the urine as it exists in the bladder, after the passage of which digital massage of the prostate and prostatic urethra is instituted, following which the urine is received in the fourth glass (Plate V, Fig. 11). In chronic anterior urethritis, the first glass contains 
all of the evidence. In posterior urethritis, the second and fourth glasses give evidence of this condition, the fourth glass more particularly when the body of the prostate is implicated. When the bladder shares in the existence of the chronic lesions the third, as well as the fourth glass, gives evidence of this fact.

There is also a 6 glass test for locating the source of purulent secretions in the urine, but for all practical purposes that already described is sufficient, as it is preferable to gain more positive and accurate information regarding the nature and locality of the lesion by instrumental examination, and especially by means of anterior and posterior urethroscopy, which yield an exact knowledge of the condition of the urethral mucosa and the presence of any lesion upon its surface. For a satisfactory examination, therefore, of chronic lesions of the anterior and posterior urethra, it is an important part of urological training to be skilled in the urethral examination by means of the urethroscope and cysto-urethroscope, and to become familiar with the latest types of instruments (see section on Urethroscopy).

When the symptoms of urethritis point toward inflammation of the prostate gland, digital examination by rectal touch will determine the condition of this organ as well as that of the seminal vesicles. The existence of cystitis in conjunction with urethritis will be recognized by the symptoms and by the examination of the urine. These complications are to be considered in a later chapter.

The Infectiousness of Chronic Gonorrhea.-Deserving of careful consideration, and a most important class of cases, are the patients who consult the physician with regard to the advisability of matrimony. The paramount question for investigation concerns the presence of gonococci in any portion of the urethral tract. If they are shown to be present in the discharge, infection is possible, and the patient must submit to treatment; but there are still other cases where the gonococci are not demonstrable in the visible discharge, and yet may be present in the follicles, in the prostate or elsewhere in the urethral tract. These infectious microörganisms may exist in the tissues underlying the surface of the mucosa, where they may live for months, or even years, and now and then by chance give evidence of their presence through a temporary outbreak.

The urologist must pursue a course of wisdom and discretion, which means that he must never overlook the danger involved in the transmission of this malady to another, who may be an unconscious and innocent victim. This does not mean, however, that he must accept a narrow-minded view expressed by some, that once the victim 
of gonorrhea, the hope of cure is lost; but better this than the more dangerous and casual treatment of this subject by those who feel safe when the disease is manifested by no more than a morning drop or shreds in the urine. It is an unquestionable fact that many who have once contracted this disease never really get rid of the urinary filaments, and that many of these are freed of the infectious element, and proper subjects for matrimony so far as the question of contagion is concerned. Yet, on the other hand, there are cases that show no gonococei in the free discharge, and yet, upon more careful examination, the virulent element is found to be still present. The composition of the discharge has little to do with the question of virulence, that is to say, whether it consists only of pus cells or a combination of pus with epithelia. The proper method of deciding the question of contagion is given in a previous chapter (see p. 195). The presence of gonorrheal organisms determines that the patient is still infectious; but if they cannot be demonstrated, even though the urine contains filaments, after repeated trials, centrifugation of the precipitate and active irritation of the urethra, when such investigation has been conducted by a careful observer and with proper laboratory technic, it has been customary to sanction matrimony. But in doubtful cases resort should be had to the Complement Fixation Test of the Blood, and better still as a regular routine practice see p. 108. In a personal experience of many years, I have had to decide this question in numerous cases, and know of not one in which the decision proved to be unwise or unauthorized. Therefore, the extreme statement made that "once gonorrheic, always gonorrheic," is absurd and unwarranted.

Treatment.-Generally speaking, the treatment of chronic urethritis should be varied according to the nature of the case. It is important that a definite diagnosis be made and the location of the lesion be determined before beginning treatment. When the case appears to be one which is a direct continuation of recent acute urethritis, and resembles in its activity the acute stage, all the restrictions enjoined upon the patient during that stage are enforced; in fact, the prolongation to a chronic state is often due to a failure to observe such precautions, and as the condition may be due to the continued presence of the gonococcus in the urethra, then its removal becomes the most important part of the treatment. The occurrence of one of the complications during the acute stage may be the determining cause of a chronic lesion and suggest the direction of treatment, as a chronic, localized urethritis may depend upon a morbid lesion in one of the accessory glands or follicles of the urethra, which occurred primarily as a complication of the early stage, and the cure of such a lesion will effect the removal of 
the dependent chronic condition. If constitutional debility is the cause alone for the continuance of chronic inflammation of the urethra, a knowledge of this fact is important in the application of treatment; and, finally, it is sometimes the case that nothing more than long-continued local treatment may be a sole cause of the prolonged trouble and, therefore, its cessation along may lead to the desired result. The longer the discharge has existed-in other words, the more chronic the case - the more difficult it is to treat.

The treatment of these cases, therefore, entails discovery of the underlying cause and its treatment by the proper measures. Those cases which have had a long existence and appear in frequent relapses of active inflammation from causes which should not affect the healthy urethra, are more frequently the subjects of lesions in the prostate and seminal vesicles. Patients of a tubercular, gouty or rheumatic diathesis are much more prone to develop chronic lesions than more healthy individuals. To summarize, therefore, the following important questions must be investigated before instituting treatment in all cases of chronic urethritis:

1. The continued presence of the gonococcus.

2. The probability of too long continued irritating local treatment.

3. The existence of structural change in the urethra (stricture) and the extension of inflammation into the neighboring tissues and organs (folliculitis, prostatitis, seminal vesiculitis and cystitis).

A preliminary investigation of each case should determine whether one or more of the above factors possess any causative agency.

When the gonococcus is discovered in the discharge from a recent chronic urethritis, the local measures which are adopted for the acute stage of the malady may be sufficient to accomplish recovery (see p. 215). This will not be the case, however, when there exist localized morbid lesions in the adjacent structures, which must be sought out by proper diagnostic technic and treated according to the indications. If no definite cause for a continuation of the discharge is discovered when rigorous local measures have been pursued for a long period, examination may discover that the persistence of symptoms is due to irritation, and that by instituting a more simple regime, a favorable result will be obtained. This may be brought about by the cessation of local measures and the use of internal medication alone. Concomitant constitutional disorders should, as far as possible, be corrected by rational measures of treatment.

Internal Treatment.-The nearer the condition of chronic urethritis approaches the acute stage, the more appropriate are those internal 
remedies referred to in the treatment of the latter (p. 208). When the discharge is more or less free, the use of oil of sandalwood or of gaultheria is indicated. The oleoresin of cubebs and copaiba are more suitable for the chronic than for the acute stage. Combinations of these remedies in capsule form are put up by manufacturing pharmacists. When the posterior urethra or the adjacent portion of the bladder is the seat of the trouble, causing symptoms of irritability, such as painful urination, tenesmus or frequency, tincture of hyoscyamus and the fluid extract of cornsilk are useful remedies: 0.30 to 0.60 c.c. $(m \vee$ to $x)$ of the former, and 2 to 4 c.c. ( 3 ss to i) of the latter may be given after each meal in combination with other remedies. When the urine is highly concentrated and overacid, it should be diluted by diuretics and diluent mineral waters. A selection may be made from the formulæ given in the Treatment of Acute Urethritis, to suit the individual case (p. 208). Finally, emulsion of cod liver oil and hypophosphites, when the general health demands such medication, may prove extremely helpful.

Local Treatment.-The statement already made with respect to internal medication applies with equal force to the local treatment of chronic urethritis, viz.: that as the character of the symptoms approaches those of the acute stage, such local measures as are commonly used in the treatment of the latter should be adopted. When the discharge is free and purulent, the employment of one of the organic silver compounds, by means of hand injections, or the use of injections of permanganate of potassium is indicated. If the gonococcus is found to be still present in the discharge of the urethra, the injection of the silver preparations may be especially relied upon; but after the activity of the inflammation has subsided to a certain degree, such treatment having counteracted its virulence, or on account of the removal of the irritating factor of a too active treatment, it becomes necessary to investigate the character and location of the chronic urethral lesion, for upon such investigation must depend the further direction of treatment.

The local medication of the urethral canal is effected by means of irrigations, instillations, injections, soluble bougies. The proper manner of applying these various methods of technic is described in the section on General Technic. The selection of one or the other of these agencies depends upon the lesion to be treated. Many of the chronic urethral lesions are in reality the result of complications which have occurred during the course of the acute stage, and their treatment is described under the section on Complications of Urethritis (see p. 241). The proper analysis of each case, therefore, is essential to the selection 
of treatment. Many cases of chronic urethritis are cured as the result of well directed local treatment; others get well when local treatment which has been too long continued is stopped. Some patients who are undermined in general health, or subjects of tuberculosis or other diatheses, improve or recover entirely when transplanted to a proper climate; while other patients, in whom the urethritis is due to structural alterations in the canal (stricture, etc.), necessarily do not improve unless treatment is directed against the particular lesion present.

The use of astringent injections by the patient is sometimes useful in correcting a simple catarrhal condition, and may control the discharge from the anterior urethra. They may also be used to supplement the local treatment conducted by the surgeon, and when enforced absence of the patient temporarily precludes the adoption of other measures. For this purpose, one of the injections given on page 222 may be ordered. Urethral bougies and cylinders of cocoa butter containing some medicinal astringents, antiseptics, etc., that are retained in the urethra and, when melted, liberate the therapeutic agent, are composed of a mild combination of nitrate of silver or of one of the organic silver compounds, sulphate of zinc, salicylic acid and ichthyol, according as it is desired to produce disinfectant, counterirritant or desquamative action.

Irrigations are employed both for the anterior and the posterior urethra (p. 223). The permanganate of potassium has a wide range of utility, and is employed in strength of from 1 to 8000 or 1 to 4000 . The favorable effect of this agent is often promptly exhibited. It is especially useful in anterior urethritis and in mild chronic inflammation of the superficial area of the prostatic urethra and bladder mucous membrane. When inflammation is chronic, and as a result has invaded more deeply the mucous lining of the urethral canal, it may be found that one of the stronger local disinfectants, such as nitrate of silver, or the organic silver compounds, will prove more beneficial. The nitrate of silver irrigation is more effectual in its use for conditions of the posterior urethra and the bladder, as are also the other silver compounds. Nitrate of silver is employed in solutions of from 0.065 to $0.19 \mathrm{gm}$. to 500 c.c. ( 1 to $3 \mathrm{gr}$. to a pint), and protargol, 0.65 to $2 \mathrm{gm}$. to 500 c.c. (10 to $30 \mathrm{gr}$. to a pint); argyrol and collargol from 10 per cent. to 25 per cent. These irrigations are usually made at intervals of from one to three days, according to the amount of pus secreted as evidenced by the anterior flow, the appearance of the urine, and the control exercised by the treatment, which is continued until the urine becomes clear, or until it is determined that the treatment is a source of irritation, or is producing no apparent benefit. These irri- 
gations should be conducted in the manner described (p. 37). In some cases, no other treatment may be necessary; in others, a milder degree of trouble may persist, following the use of one of these applications, and remain stationary. This condition may then be benefited to a greater degree by the substitution of one of the other agents, perhaps of more astringent action, or by the same application in more concentrated form. This is accomplished by changing the method of treatment from irrigation to that of instillation of the urethra.

Instillations are employed for both the anterior and posterior urethra. The method of their usage and the proper instruments to employ are described in the chapter on Technic (p. 33). By this means, a small quantity of solutions possessing astringent, antiseptic and mildly caustic properties is deposited in different portions of the canal.

When the application is made to the anterior urethra, the liquid will naturally escape from the meatus, but should be retained by compression of the lips for a period of one or more minutes, as in the case of an injection. When these applications are made to the membranous or prostatic portions of the canal, the fluid does not escape upon withdrawal of the instrument, being retained by the compressor-urethræ muscle. From 2 to 5 c.c. and of some agents 20 or 30 c.c. are deposited in this manner with one of the instillating syringes. The stronger the solution, the smaller the amount employed. The bladder should be emptied prior to the application, to insure as long an interval as possible before its removal by urination.

Of the various topical applications employed as instillations, the following have proven to be of value: sulphate of thallin, sulphate of copper, nitrate of silver, protargol, novargan, argyrol.

Sulphate of thallin is mildly counterirritating and astringent and its ultimate action is distinctly sedative. It is not suitable for use when the gonococci are known to be still located in the canal, but is especially employed in mild catarrhal conditions of the urethra, attended by neuralgic symptoms, when the latter are disproportionately severe as compared with the extent of the lesion. It is usually employed in a solution of from 3 to 12 per cent. except in very hypersensitive conditions of the canal, when it is more desirable to begin with milder solutions of from 2 to 4 per cent. When it is found during treatment of a mild catarrhal posterior urethritis, without any infectious element remaining, accompanied by mucous discharge, that the deep urethra sustains instrumental interference poorly, the sulphate of thallin instillation is a satisfactory preliminary application, and will sometimes produce an effect sufficiently beneficial to justify 
the discontinuance of treatment thereafter, or being one of the mildest forms of deep urethral application, it may be used to prepare the way for and lead up to the stronger ones, which may not at first be well tolerated. The preparation is generally kept on hand in a 12 per cent. solution, which is the full strength, in a dark colored bottle. From 2 to 4 c.c. $(m \times x x$ to $\mathrm{lx})$ of the solution are injected at each application. It is not likely to be irritating, except for a short period, and the tendency to expel it by urination which occurs in some instances will quickly disappear.

Sulphate of copper is much more astringent than the agent previously described, and is somewhat more irritating, although in weak solutions it has but little effect. It is also more suited to the posterior than the anterior urethra. It is essentially an astringent, and its particular effect is to harden the surface mucosa when it is hyperesthetic following an antecedent prolonged or acute inflammation. In such instances it is best preceded by the sulphate of thallin, which is milder in its influence and produces less reaction. It may be kept conveniently in a 10 per cent. glycerine solution, which will not precipitate. The desired strength is obtained by the addition of water to get a solution of from $1 / 5$ to 1 per cent. or even 2 per cent. strength.

Nitrate of Silver.-This agent is probably more often used for urethral application than any other, and is of great value. It is stronger than either of the previous applications, and exercises a mild, caustic influence which is desirable in granular conditions of the mucous membrane. The indications for the use of thallin and sulphate of copper in sensitive conditions of the urethral canal apply also to the nitrate of silver, which, however, is capable of exercising a much higher degree of counterirritation and possesses a much greater disinfectant property. It is also employed with good effect as an instillation to the anterior urethra, on account of its bactericidal action. In that type of chronic urethritis which is a prolongation of more acute trouble, it may be employed as a substitute for the permanganate of potassium irrigation when it has been tried and found ineffective or only partially beneficial; also in certain cases of chronic urethritis in which there has occurred a sudden lighting up of more active trouble, involving the posterior urethra. One application may sometimes exercise a prompt controlling influence. In cases where it is well tolerated, it should be used in progressively increasing strength. It is generally customary to begin with a very mild solution, to test the urethra, say $1 / 10$ of 1 per cent., and work up gradually to as high as 2 per cent. A few drops only of this solution are deposited in the posterior canal, and 
where it is desired to include the mucosa of the vesical sphincter, a larger quantity is injected, 1 to 2 c.c., ( $m \times x$ to $\mathrm{xxx}$ ).

Protargol.-This substance has already been referred to in the consideration of acute urethritis. Its special value as an instillation is in gonorrheal urethritis of the posterior urethra. It is suitable to the more chronic forms of posterior urethritis. Its beneficial effects are obtained in chronic urethritis involving the mucosa, and particularly in cases which, on account of their chronicity, have extended somewhat deeply into the tissues. Successful results are more likely to be obtained when the process is a generalized one than when it is due to a more deep-seated malady, covering a smaller area. Its best effect is obtained from strong solutions in chronic conditions; the average strength is from 5 to 10 per cent. in the posterior urethra, and sometimes it is used as strong as 20 to 25 per cent. These solutions are much stronger than are commonly used in the anterior canal. In chronic posterior urethritis, following closely on gonorrhea, the use of protargol is often attended by markedly good results, either in combination with the permanganate of potassium irrigation or after the latter remedy has been discontinued. In using the stronger solutions, 5 to 10 per cent., care should be exercised not to increase the strength too rapidly or abruptly, as it is accompanied sometimes by marked symptoms of irritation.

Novargan is one of the organic silver compounds which is not so irritating as protargol, nor is it as effective in the same type of cases. There are some cases, however, in which it seems to be of value as an instillation, and more effective than either protargol or the nitrate of silver. This effect may be negative and owing to its lack of irritating quality, but there are some cases that seem to do well under its usage. I am accustomed, therefore, to employ this remedy interchangeably with the other silver compounds, and especially during the course of a treatment when it is deemed advisable to avoid irritation and still continue some form of local treatment. The following is the solution commonly used:

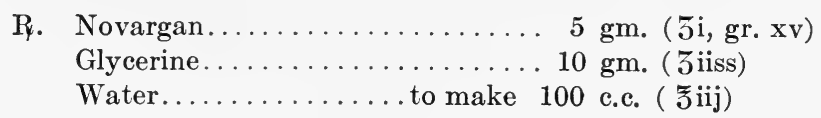

As in the case of other instillations, 2 to 4 c.c. ( $m x x x$ to $l x)$ of the solution are deposited in the posterior urethra. It may be used also in the anterior portion, but is not as effective here as either permanganate or protargol in the majority of cases.

Argyrol is used as an instillation in both the anterior and posterior 
urethra for chronic inflammation, especially when such inflammation still harbors the presence of the gonococci, and even when this is not the case. It may be employed as an instillation in solutions of from 10 to 25 per cent. in the usual manner. It is also used in chronic conditions and in the declining stage of posterior urethritis involving an area around the neck of the bladder, when a larger amount (20 to 30 c.c.) of a milder solution ( 5 to 10 per cent.) is injected by means of a catheter into the posterior urethra and retained (see pages 35 and 40).

Such treatments are conducted two or three times per week according to the effect thereof. 


\section{CHAPTER XIII}

\section{COMPLICATIONS OF ACUTE AND CHRONIC URETHRITIS}

\section{INFLAMMATION OF URETHRAL FOLLICLES-PERIURETHRAL ABSCESS - COWPERITIS}

The common complications of urethritis consist of adenitis and lymphangitis, inflammation of the glands of the urethra, folliculitis, periurethritis, cystitis, prostatitis, seminal vesiculitis and epididymitis, which inflammatory conditions occur in connection with the chronic as well as with the acute stages of the disease, especially when the gonococci are still present in the urethra, but also after these microbial elements are no longer found. Their consideration, therefore, applies to both the specific and non-specific forms of inflammation. These complications result from extension of the inflammatory process into the follicles and glands and the lymphatic vessels adjacent to the urethra, and by further extension through the blood current more distant parts of the body are implicated.

Adenitis and Lymphangitis.-In some cases of highly acute inflammation, especially gonorrheal, the lymphatics of the penis and the inguinal glands become infected. This may involve the lymphatics around the prepuce or extend along the dorsum, producing a redness of the skin, edema and swelling of the subcutaneous tissues. The glands in the groin may become enlarged and brawny, but seldom suppurate. The former condition is usually due to lack of cleanliness of the parts, or to a continually tight meatus interfering with the proper escape of the contained discharge. Involvement of the glands may be induced by external injury or violent exertion.

The treatment of lymphangitis and adenitis is the same here as elsewhere, namely, removal of the cause by proper cleanliness, and application to the inflamed organ of wet dressings, which should be frequently changed; for such dressings dilute acetate of lead and 1 per cent. phenol or creolin solutions are used. For the enlarged glands, the application of the tincture of iodine or a belladonna ointment is desirable, and in severe cases, complete rest in bed. Should the gland undergo suppuration, incision and drainage will be required. 
Inflammation of the Urethral Follicles.-The follicles of the urethra always participate more or less in acute inflammation of the canal. In chordee the follicles at the affected spot are implicated and may be the route by which the inflammation reaches the deeper tissues. These mild inflammations get well spontaneously, as a rule, when the surface congestion goes down.

Follicular inflammation of the urethra is due to some form of local irritation when added to the already congested canal, or during an attack of urethritis, by irritating injections, frequent erections, nocturnal emissions, etc. When a single follicle or a group of follicles is attacked by an extension of inflammation, each one may be felt under the skin, generally on the floor of the urethra, like a small

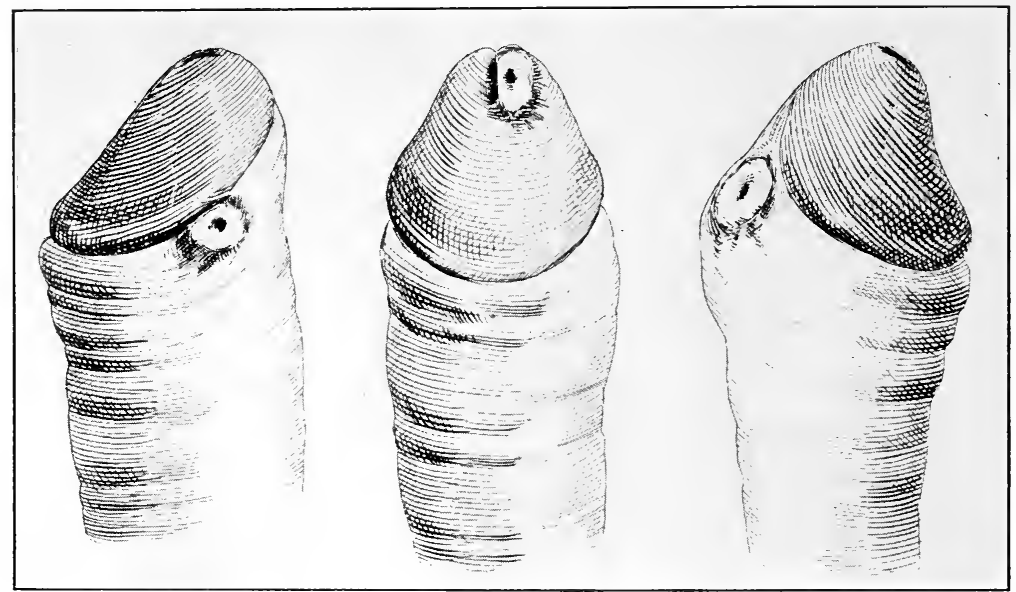

FIG. 103.-Folliculitis.

shot or pea. When the follicular mouth becomes closed during the course of such an inflammation, an abscess is formed in the dilated sac of the follicle, which eventually discharges itself externally through the skin, or internally through the natural duct of the gland. This condition is prone to become chronic, and when the gonococcus is present in the urethral follicles, it offers an ever-present pocket of infection (Fig. 103). When an external opening occurs, urethral fistula may be the ultimate result. A favorite site of these follicular abscesses is behind the meatus in the fossa navicularis on one or both sides of the frenum. The inflammation, if unabated, may extend into the cellular tissue, periurethral inflammation (Fig. 104). When a cellular inflammation occurs, the outline of the follicle is generally 
lost and a phlegmon is formed which is not likely to undergo resolution, but discharges itself either externally or internally, or both. In the latter instance, as in the case of a follicular abscess, a urethral fistula may result. The inflammation may extend into the corpora cavernosa, causing an induration which, during the course of the acute disease, produces the symptoms of chordee. This usually undergoes resolution, unless the exudate, instead of being absorbed, remains, when permanent curvature and deformity will result.

Treatment.-At the onset the treatment of follicular and periurethral inflammation should be palliative-rest, freedom from local irritation, and mild urethral applications, if any be adopted, together with fomentations or wet dressings of 1 per cent. phenol or weak corrosive sublimate to the swollen organ. Irritating injections should be positively avoided. If irrigations of permanganate of potassium are used, they may be continued under light pressure in mild strength $(1: 6000)$.

When suppuration occurs, and the abscess points externally, an incision through the skin should be made to evacuate the pus, except when the follicular abscess points near the meatus, and can be reached from the interior. In this instance an internal incision should be made, and the abscess treated

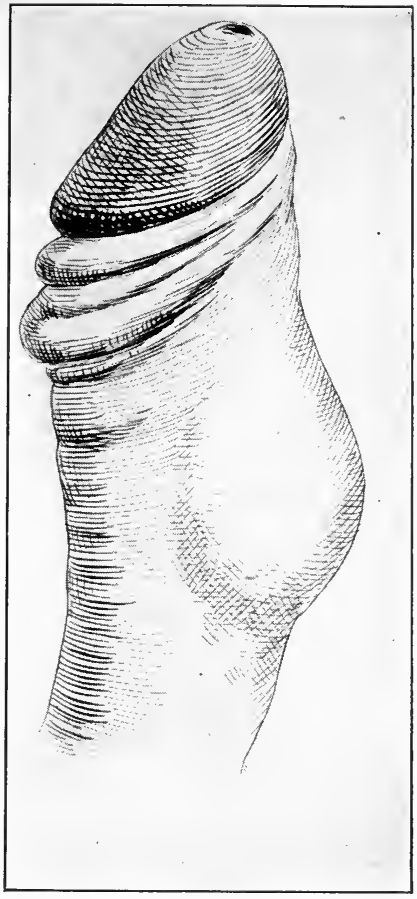

Fig. 104.-Periurethral abscess. on the inside, thereby possibly preventing the formation of complete urethral fistula.

A method of treating these abscesses after they have discharged, whether externally or internally, is by the injection into the cavity of a small amount of peroxide of hydrogen (see also Urethroscopy, p. 119). By this method contraction and closure of the sac sometimes may be produced, and the danger of fistula be avoided. These solutions are applied by means of a urethral pipette.

Other methods of treating these abscesses are based upon general surgical principles of antisepsis and drainage, which require that after the abscess is opened it shall be injected at regular intervals with 
antiseptic solution, and made to'heal from the bottom by packing with gauze.

The high frequency fulgurating current by means of a small insulated wire through an endoscopic tube internally, or to the vent of the sinus externally, is also employed to cauterize these fistulæ and sinuses, and may effect their closure when the opening is not too large.

Inflammation of Cowper's Glands.-Another form of follicular inflammation of the urethra occurs in the glands of the bulbous portion known as Cowper's glands-cow-

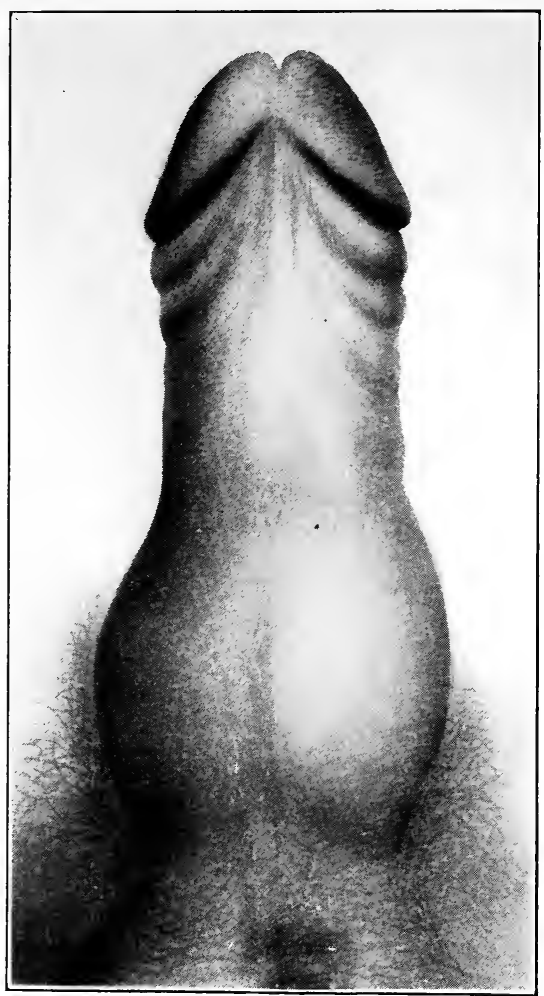

Firi. 105.-Abscess of Cowper's glands and pericowperitis. peritis and pericowperitis. The causes are much the same as in follicular inflammation in other portions of the urethra, some kind of direct local irritation or traumatism during an attack of urethritis, generally gonorrheal.

The primary symptom of this complication is pain in the perineal region, which when occurring during the acute stage of urethritis should always suggest the possibility of inflammation in one of these glands. Examination reveals a painful and indurated sweiling in the perineum, corresponding to the locality of Cowper's glands, generally on one side only. It is important to recognize this condition at its onset, as in the majority of instances, when discovered early enough, resolution may be brought about by rest and hot local applications. When this latter course is not taken, suppuration occurs, characterized by the enlargement of the swelling in a more or less oval shape, somewhat pointed toward the anal region. The swelling later diffuses itself into the surrounding tissues (pericowperitis), and extends forward in front of the scrotum (Fig. 105), when the surface of the skin reddens, the tumor softens, and in some cases becomes the size of a hen's egg. 
When suppuration occurs, the abscess may open both into the urethra and through the perineum. More often, however, the tumor resolves without suppuration, especially if recognized early and submitted to rest and careful treatment.

Certain cases that have suppurated and discharged in one or both directions, notably if not well treated or if the general health be undermined, terminate in chronic cowperitis, with internal or external fistula, or both, a very obstinate and annoying malady. If there be complete urethro-perineal fistual, it may permit leakage during urination, or the size of the fistula may be too small to permit the passage of a visible amount of urine and be demonstrable only by injection.

The symptoms peculiar to cowperitis are those of pain, swelling, induration and suppuration corresponding to the anatomical situation of Cowper's glands, occurring during the course of acute urethritis. Therefore, the diagnosis based upon these symptoms is not difficult; it should not be confounded with perineal inflammation or urinary infiltration. When the glands are swollen they may be palpated with the index-finger in the rectum and the thumb on the outside, by compressing the tissues between the apex of the prostate and the perineum (see Fig. 47, p. 67). When there is a general pericowperitis, its recognition is more difficult, and a distinction between it and urinary infiltration from the urethra may be troublesome, but the latter is usually a result of tight stricture following old chronic inflammation of long standing.

While malignant disease and tubercle do occur in this region, they have been very seldom observed. In distinguishing either one of these conditions it should be borne in mind that the course and duration of acute cowperitis are rapid and short, usually not lasting over five or six days, while both tubercle and cancer are essentially chronic in character. Generally speaking, a chronic cowperitis which persistently resists treatment should lead to the supposition of the existence of underlying tubercle, and, therefore, calls for careful bacteriological examination of the pus and fistulous scrapings.

Treatment.-It is most important to recognize cowperitis at its inception. Absolute rest should be enjoined, all direct urethral applications discontinued during the acute stage, and external applications, hot or cold, substituted, in the one case, poultices or the electric heater, in the other the ice bag. Gentle massage of the swelling may also be employed. As soon as suppuration becomes evident it is necessary to evacuate the pus, and for this purpose a free incision should be made over the most prominent point of the swelling. The abscess should be washed out with an antiseptic solution and the 
cavity packed lightly with gauze, for the purpose of encouraging contraction and healing from the bottom toward the surface. As soon as cicatrization commences, and even before the cavity has contracted completely, the method suggested for the treatment of follicular abscess of the urethra is to be recommended.

Acute Prostatitis is a common complication of gonorrhea and chronic prostatitis is a frequent sequel. A description of these conditions will be found in the section on Maladies of the Prostate. Other common complications such as gonorrheal inflammation of the seminal vesicles, gonorrheal inflammation of the bladder (urethrocystitis) and swollen testicle (epididymo-orchitis) are all considered in the sections upon the maladies of these different organs.

\section{Extragenital Complications of Gonorrhea}

Some of these complications, like the more frequent varieties, are derived from direct contact with the gonorrheal virus: for example, ophthalmia and proctitis; others are transmitted through the blood and lymph currents. Of the latter, inflammation of the joints, bursæ, tendons and periosteum are common, while implications of the membranous lining of the blood-vessels and serous cavities are more rare occurrences.

Gonorrheal proctitis is rare. It may arise from unnatural practice, or from careless habits, especially when there preëxist abrasions of the rectal mucosa. When this condition occurs, it is prone to become more or less chronic. Examination reveals an excoriated membrane, accompanied by tenesmus, a tormenting burning and painful bowel action. A purulent foul discharge, stained with blood, will contain the gonorrheal organisms. Localized ulcers are likely to develop, and prolapse may occur.

The treatment calls for the most careful cleanliness, which requires frequent ablutions of the affected membrane, together with hot sitzbaths, permanganate of potassium solutions and saturated solutions of boric acid. The pain and tenesmus may be relieved by suppositories of opium. The local application of an analgesic in the form of a powder (anesthesine) or of a lubricant, the same as is used for anesthesia of the urethra (see p. 46), containing alypin 5 per cent., may prove useful. Finally, solutions of nitrate of silver are used to disinfect the mucous membrane and to cauterize the fissures and ulcers.

Gonorrheal Inflammation of the Nasal and Buccal Mucous Membranes although very uncommon, does occur, in which case the presence of gonococci has been revealed in the secretions. The disease 
may have been contracted through the medium of dressings, towels or pocket handkerchiefs, or by direct contact.

Gonorrheal Ophthalmia. - This disease occurs in the adult as a result of direct transmission from the urethra to the eye, generally by the hands of the patient. By virtue of its location and the importance of the treatment, this complication of gonorrhea belongs to the department of ophthalmology. It is important, however, to appreciate the danger of infecting the eye during the course of gonorrhea and the proper means of preventing such a serious complication. If there were any need of confirmation as to the contagiousness of gonorrhea, the instances of its transmission to the eye during its course furnish striking examples. In the newly born, this infection occurs at the time of birth, from direct contact of the eye with the diseased secretion of the vagina. The period of incubation in the adult is from twelve to forty-eight hours, and in the infantile condition, it occurs on the third or fourth day after inoculation. It is important, therefore, that the surgeon shall keep constantly in mind the danger of transmission, and that patients should always be instructed regarding proper means of protecting themselves against this dangerous complication.

The means of prevention involve scrupulous cleanliness on the part of the patient. Frequent cleansing of the hands is an important measure which should never be omitted after the necessary handling of the infected parts. An additional measure which is not amiss is to use an antiseptic hand solution in the form of bichloride of mercury, 1 to 3000 or 4000 . Wrappings and wearing apparel which have become soiled with the discharge should be carefully washed in boiling water and not allowed to come in contact with other articles.

Symptoms.-Great redness and swelling of the conjunctiva will be noticed, accompanied by a serous secretion characteristic of catarrhal inflammation from another cause, but the intensity of the inflammation rapidly increases, marked by extensive swelling and edema of the lids, together with sharp burning pain and exudation. Gonococci are found in the pus cells and in the epithelium, and later migrate into the lymph spaces. In severe cases, an ulceration of the cornea occurs as a result of the intraocular exudate, and this ulceration may lead to perforation with prolapse of the iris and permanent cicatricial alteration of the symmetry of the eye-ball.

Diagnosis.-The determining feature of the malady is the presence of numerous gonococci in the secretion. The great intensity of the symptoms is a diagnostic evidence of its existence. Inflammation of the eye, occurring during the course of gonorrhea, always leads to the suspicion of direct infection. 
Prognosis.-The tendency ${ }^{*}$ of gonorrheal ophthalmia in the adult is destructive, and unless discovery is sufficiently early, and unless the condition succumbs promptly to active remedial measures, partial or total destruction of the eye-sight is the result.

Non-virulent Ophthalmia.- This occurs in individuals with chronic urethral lesions, and is produced by toxic transmission through the circulation, and not by direct infection from the urethra. This is not, properly speaking, gonorrheal ophthalmia, and gonococci, if present at all in the urethra or elsewhere, have not any etiological significance. This form of ophthalmia must be differentiated from the virulent type. It occurs, as stated, as a systemic infection, and is sometimes spoken of as rheumatic conjunctivitis. The conjunctiva alone may be the seat of trouble, marked by redness and swelling, pain or uneasiness, and scanty muco-purulent exudation. Again, the cornea sometimes shows a cloudiness and becomes more prominent, from overdistention with fluid. The iris may present the main seat of trouble, though not showing much change of color, while the pupil may be somewhat dilated and irregular. In all cases of iritis or conjunctivitis of uncertain causation, the etiology rests upon syphilis, gout or urethral inflammation. It is well, therefore, to make a practice of seeking out old urethral lesions, as a possible explanation. These non-specific affections of the eye get well without compromising the structure of the eye or its function, but are liable to relapse during subsequent attacks of urethral inflammation. In the infant, conjunctivitis may be caused by any mechanical irritant, in which case the gonococci will not be present. The bacteriologic urethral examination and the complement fixation tests of the blood for both gonorrhea and syphilis are diagnostic resources to be relied upon.

Treatment of Ophthalmia.-In the newly born, the treatment consists of immediate applications to the eye of a 2 per cent. solution of nitrate of silver, as suggested by Credé. In cases of doubt regarding the infectiousness of the malady and of the bacteriological character, it is well to follow the course of treatment as a means of precaution, for the irritation following will subside spontaneously. The treatment of gonorrheal ophthalmia in the adult belongs, properly, to the province of the eye specialist. Treatment consists of frequent ablutions of the eye with a mild antiseptic solution of boric acid to remove the accumulated secretion, frequent application of one of the silver compounds, preferably argyrol or collargolum, 25 per cent., four or five times a day, and in the intervals the application of cold compresses, which are kept upon ice, and cast aside whenever removed. Solutions of atropine are used to reduce intraocular pressure. 
In cases of unilateral infection, the opposite eye should be protected by preventative measures, which consist of dropping into the conjunctiva daily a 10 per cent. solution of argyrol. During the course of treatment, the eyes are screened from the light by confining the patient in a dark room.

In the pseudo-gonorrheal or rheumatic form of ophthalmia, the coexistent urethral lesion is sought out and treated with appropriate measures (see Chronic Urethritis, p. 227) and the eye treated symptomatically.

\section{GONORRHEAL ARTHRITIS}

Causation.-Gonorrheal rheumatism is a most important complication of gonorrhea in both sexes. It is more common in men than in women, and occurs at all ages, children being very prone to it. Its frequency is stated to be between 1 and 2 per cent. of the cases of gonorrhea. The different joints are affected in the following order of frequency: most commonly the knee, and next the elbow-joints, then the ankle, the joints of the hand, and the shoulder. Arthritis more frequently appears after the second or third week of urethral inflammation, when the latter has invaded the posterior canal, from which point metastases usually occur, although in severe cases, when the inflammation has been driven backward, it may occur much earlier in the course of urethritis, and has been observed within a few days after the onset. This metastasis of urethral gonorrhea is caused by the entrance of the gonococcus into the circulation, whence invasion of the particular joints occurs. As a demonstration of the causative agency of the gonococci, cultures of the latter have been grown from joint effusions, and although it is not always possible to make this demonstration, the absence of the gonococcus does not disprove the gonorrheal origin of arthritis. The presence of these organisms in the circulation may lead to the entrance simultaneously of pyogenic microörganisms and their invasion of the joint; thus, a mixed infection occurs, which at the time of examination reveals the presence of pyogenic bacteria only.

Pathology.-The pathological lesion in gonorrheal arthritis is ordinarily that of a synovitis with excessive plastic exudation. When the articular ends of the bones are involved, the lesion is that of an osteoarthritis; beginning in this situation the periosteum and cartilages of the joint become implicated, and extension into the synovial capsule eventually occurs, the hydrarthrosis then being secondary to the osteoarthritis. This latter form of gonorrheal arthritis is prone to leave permanent changes and impairment of function. 
Symptoms.-The inflammation of the joints may be acute from the start, or on the other hand, may be subacute or chronic in its type. Acute invasions are characterized by local pain, swelling and redness of the joint, accompanied by febrile symptoms. These symptoms attack one or more joints, generally predominating in one location. Movement of the affected joint is impaired, and sometimes abolished Examination will reveal fluctuation and tenderness. A few days after the onset, in mild cases, the affection tends to become subacute in character, when, if it does not follow the favorable course of resolution and disappear, it assumes the stage of chronicity. In severe cases the acute stage may be prolonged, sometimes over several weeks. Even after protracted duration, the joint may be restored to normal movement by absorption of the exudate, or if this does not occur, and in the event of intraarticular changes, such as thickening of the cartilages and ramification of the epiphyses, the joint may be permanently deformed, or even ankylosed. Suppuration sometimes occurs, which renders the attack more serious and active, locally and constitutionally.

Diagnosis. - There should be no difficulty in recognizing the existence of gonorrheal rheumatism, as it always occurs in connection with acute urethral inflammation or an exacerbation of chronic urethral lesions. Care must be exercised to differentiate it from ordinary rheumatism. When arthritis occurs during the course of urethritis, it naturally leads to the suspicion of gonorrheal rheumatism. The local symptoms are usually less severe and the constitutional disturbance of milder degree than in acute inflammatory rheumatism. Ordinary rheumatism may respond promptly to the action of salicylates and to local applications and immobilization of the joints, to which rheumatism of the gonorrheal type shows little response, but may subside concurrently with the decline of an acute exacerbation of chronic urethritis.

Prognosis.-There is sometimes a prompt abatement of gonorrheal arthritis after short duration, and especially as a result of appropriate treatment, although the tendency is strong toward chronicity and to relapses in connection with subsequent urethral outbreaks. The later functional condition of the joints, as already stated, depends upon the nature of the lesion at the onset. It is likely to be little impaired when the attack has been a hydrarthrosis pure and simple, and more damaged when the lesion has started in the joint surfaces rather than in the synovial sac.

Under the division of gonorrheal arthritides there also occurs inflammation of the tendons, bursæ and muscles, of the periosteum 
and of the bones. These other gonorrheal lesions, tendosynovitis, bursitis, myositis, periostitis and osteomyelitis, generally occur in association with joint affections, and may assume an acute, subacute or chronic course.

Gonorrheal tendosynovitis may appear during the first few weeks of gonorrheal inflammation as an independent lesion. There are generally some febrile symptoms, accompanied by pain and swelling in the region of one or more tendons, with redness of the skin and edema surrounding the area involved. The extensor tendons on the dorsal surface of the hand and forearm are more often involved. If the tendency is not toward reabsorption of the exudate and resolution thereof, relapses occur and a chronic course results.

Gonorrheal Bursitis usually accompanies joint invasions. The common locations are those of the tendo Achilles, the patella, the olecranon or the os calcis. Gonorrheal myositis usually accompanies involvement of an adjacent joint, but sometimes occurs by itself. The local symptoms are those of a localized inflammation, redness, swelling and tenderness in the region of the muscle affected.

Gonorrheal Periostitis and Osteomyelitis attack the epiphyses of the long bones and generally form part of the lesion of osteoarthritic type of gonorrheal metastasic rheumatism, which invades the ends of the long bones, and generally goes on to further involvement of the joint in the development of hydrarthrosis.

Treatment.-The treatment of all gonorrheal arthrides calls for immobilization of the parts affected during the acute stage, and the application of an ice-bag to the swollen and tender joints. Proper attention must be given to the urethral lesion, in accordance with the usual measures employed. Internal medication is of little value. The solutions and tablets of iodine preparations have been tried and found ineffective. Of greatest importance is the employment of specific serums and vaccines, which afford more definite curative results in this particular form of metastases than in any other of the conditions in which the serums or vaccines are recommended. For more detailed description and directions, see chapter on Vaccines and Serums, p. 110.

Other gonorrheal metastases involve the nerve centres and ganglia and nerve sheaths, more particularly the sciatic and crural nerves of the leg.

Heart lesions occur in a fair percentage of cases of gonorrheal arthritis notably gonorrheal endocarditis. The treatment is that of the systemic malady supplemented by serum or vaccine therapy (see p. 110). 


\section{CHAPTER XIV \\ STRIC'TURE OF THE URETHRA}

Stricture of the Urethra appears under various denominations of more or less common usage, i.e., organic or true stricture; spasmodic, muscular and inflammatory stricture. Etiologically, stricture is spoken of as being congenital or acquired.

The congenital form is due to a fetal defect in development the exact cause of which is not known.

Acquired or Organic Stricture may be gonorrheal or traumatic. Acquired stricture is a cicatricial narrowing of the canal, due to submucous inflammatory exudation, with the formation of fibrous tissue, and is a common result of gonorrhea in the male, but may be due to traumatic violence, mechanical or chemical. It also represents what is known as organic stricture in contradistinction from "inflammatory" and spasmodic stricture, in that it is a permanent lesion, while the term "inflammatory stricture" has been used to denote a constriction produced by soft inflammatory cellular infiltration, which may be absorbed, or be retained and later become a fibrotic stenosis. It has also been used to signify simple inflammatory, edematous and congestive swelling sufficient to diminish the calibre of the canal, but not properly called stricture in accordance with what is generally understood by this title.

\section{Spasmodic Stricture}

Spasmodic Stricture may depend upon a multitude of causes, general as well as local; moral as well as physical. Moreover, it may complicate the other forms of stricture of the canal, and add to. their significance in consequence. Spasmodic action of the muscles of the deep urethra produces an obstruction which may result in retention of urine, and, therefore, has been called "stricture"; but as the cause may exist outside of the urethra, being reflex, and as this condition sometimes coexists with true stricture, it is more properly designated urethral spasm.

Urethral Spasm or "Spasmodic Stricture" is generally susceptible of very easy demonstration. 
A patient is operated upon for hemorrhoids or hernia, for example; the reflex irritation causes a spasm of the deep urethral muscles and he is unable to void his urine. The passage of a soft rubber catheter is accomplished without much difficulty, if there be no organic obstruction, and voluntary urination returns sometimes after the catheter has been employed only once, and sometimes after several days' usage.

A patient having had several attacks of complete retention of urine called upon the author. He had reached the age of prostatic hypertrophy, and had been told that such was the cause of his trouble. Indeed, he had just departed from a hospital, because orchidectomy had been proposed and rejected by him. Examination revealed that there was no undue frequency of urination by day, nor even at night; that the bladder emptied itself completely; that every attack of retention had been relieved instantaneously by the single passage of a catheter, and was separated by a long interval from each previous attack. Exploration of the urethra demonstrated the existence of a true organic stricture, but one which was quite permeable and of large calibre. The prostate was practically normal. The retention of urine had been caused by spasmodic contraction of the deep urethral muscles, urethral spasm complicating true organic stricture.

The writer remembers the case of a youth with a highly nervous temperament who had an organic stricture of the membranous urethra, which he was wont to treat in the most casual manner, resorting to relief only when compelled by necessity to do so. One time he was caught away from civilization, up in the mountains, with an attack of complete retention of urine, which occurred suddenly and in spite of the fact that he had been able previously to urinate, although with some difficulty. He boarded a train, and was tortured for twenty-four hours with an overdistended bladder, which fortunately did not rupture, but dribbled continuously en route. A small-sized catheter was passed, and 2000 c.c. of limpid fluid withdrawn. At that time voluntary urination did not return, and this fact was used as a means of inducing the patient to accept operation. For three days he continued in a state of retention, being relieved by catheter; at the end of this period he had yielded and consented to operation with much trepidation. But upon the author's arrival, prepared to operate, and at the sight of the instrument table laden with surgeon's tools, he rushed horror stricken into his bath-room, and then and there did he urinate, much to his satisfaction. This was another case of spasm in conjunction with organic stricture; the stricture was not impassable, although of small calibre.

When urethral spasm occurs independent of true stricture it must 
be remembered that a diagnosis of tight stricture may be made, because a filiform bougie is passed with difficulty and grasped in the attempt to withdraw it, and there may be nothing more in the case than spasmodic contraction of the deep urethra, as proved by the fact that a large blunt steel sound, held gently against the face of the obstacle, will after short delay slip of its own weight into the bladder.

Regarding the location of urethral spasm, it is probably found only in the membranous portion; and, although some authors have claimed its existence in the anterior canal, it has not been satisfactorily demonstrated that spasmodic contraction of this region occurs. In some cases it is quite difficult to distinguish between urethral spasm and a stricture situated at the bulbo-membranous junction. The resistance of the latter is firm, tense, unyielding, as contrasted with the muscular contraction, which is softer, more yielding and elastic.

If a blunt instrument be held firmly against the face of a "spasmodic stricture," with gentle, but steady pressure, the contraction often yields suddenly, and the instrument enters, causing a certain amount of pain; but in the case of true stricture, the resistance is firm and tight, and is not suddenly overcome by the gentle force of an instrument larger than the calibre of the stricture.

The attendant symptoms will render additional assistance in distinguishing between these two conditions. In the case of urethral spasm, the evidence of urinary obstruction is of irregular character. Sometimes the difficulty lies at the commencement of urination, while the continuation of the act is normal. Again, during the entire act an effort is required to pass the water, or the stream may be abruptly interrupted before the bladder is emptied; while in other cases, partial or complete retention may occur. The presence of urethral spasm is clearly demonstrated when the functional difficulty is not constant but variable, and an attempt to pass a small bougie is unsuccessful. An organic obstacle, if small enough to obstruct the bougie, will yield constant symptoms. When even the smallest instrument will not pass, in the case of spasm, a very large blunt steel sound will sometimes enter without hesitation or delay.

The causes of urethral spasm are direct and indirect. The action of the different emotions in a nervously constructed individual is alone sufficient at times to prevent him from voiding urine. Thus, under the influence of shame, nervous excitement or fear, a perfectly healthy person may be unable to pass water for a long time. Deep urethral spasm is the cause of such retention.

Some people cannot pass water at all under certain conditions. For instance, a patient, when asked to urinate in the presence of the 
physician, will try and persistently fail, but will promptly respond when left to himself. Some individuals are never able to pass water on a railroad train, and are thus prevented from travelling long distances.

Of the various local causes of "spasmodic stricture" may be mentioned posterior urethritis and urethro-cystitis, irritation due to abnormal condition of the urine or to certain urethral medications, stone in the bladder, irritation of the posterior urethra from the rectum, and deep urethral irritation from other causes, such as sexual overindulgence, withdrawal, etc., and acute inflammation in the anterior urethra, organic stricture and contracted meatus.

The variable condition in which a deep organic stricture is disclosed, namely, sometimes permitting a reasonably free stream of urine, again so tightly closed up that only a few drops can be painfully voided, is undoubtedly often due to spasm rather than to any purely inflammatory change in the stricture itself. That form of partial or complete retention sometimes seen in connection with slight stricture of large calibre is certainly due to spasm of the membranous sphincter, as proved by the ease with which many of these cases permit the passage of a large-sized steel instrument without the employment of force.

Treatment of Spasm.-To handle intelligently this class of cases, it is important to separate the cause from the effect. The various causative factors have been mentioned above, and upon their removal may depend the successful treatment. The disposition to this abnormal tendency of deep urethral spasm is associated with other organic lesions, such as true stricture, when the latter must be reckoned with according to appropriate indications.

The result of spasmodic stricture, namely, complete or incomplete stoppage of the urine, is what calls attention to the existence of such spasm. Sometimes the single passage of a catheter, followed by the gentle introduction of a steel sound, will bring about instant relief of the immediate condition. It is well to remember that an overdistended bladder is especially susceptible to infection, consequently, the greatest care must be observed in regard to catheterization. A hot sitz-bath is often employed with success. But it is necessary to follow up the underlying cause. The occurrence of deep urethral spasm is in itself an indication of deep urethral irritability. Therefore, besides working on the exterior cause, such as nervous excitability, etc., any source of reflex local irritation, such as the coexistence of true stricture, prostatic congestion caused by sexual overindulgence or withdrawal during coitus, irritation of the region adjacent to the urinary organs, the rectum, the pubis and the groins must be taken into consideration. It may be necessary to treat such deep urethral irritation 
by direct topical applications, and for this purpose are recommended instillations of mild solutions of nitrate of silver in increasing strength by means of the deep urethral syringe, commencing with a solution of $1 / 8$ of 1 per cent., and increasing same at intervals of two or three days, up to three times the original strength. Protargol, 1 to 5 per cent., may also be used and sulphate of thallin, 2 to 12 per cent. solution, is an excellent application for very sensitive individuals. (See p. 237.)

\section{ORGANIC OR TRUE STRICTURE}

While true stricture is considered a narrowing of the canal, it is in reality a loss of distensibility of the affected part (Sir Charles Bell).

Congenital Stricture.- There are four different types of congenital stricture: the cylindrical or annular, the diaphragmatic, the valvular, and bands or spurs. One stricture only is present commonly; but two or more may coexist. The annular form is more common at the meatus, but may occur anywhere throughout the urethra, or involve its entire length.

The diaphragmatic form consists of a fold of mucous membrane which contains an opening centrally or peripherally located. This form is found at the opening of the meatus, or behind the lacuna magna, or at the bulb.

The valvular type is formed by mucous folds, and is more common to the prostatic urethra, but may occur elsewhere.

Bridles, spurs or bands, do not occupy the whole circumference of the canal; they are small irregularities-projections of the mucosa, - which may occur anywhere.

Associated with congenital stricture, there may be other lesions, such as phimosis, hypospadias, and diverticule. As in the case of acquired organic stricture, secondary lesions may occur, such as urethritis, anterior and posterior, prostatitis, hypertrophy of the bladder, dilatation of ureters and pelves, etc.

Causation.-The condition of congenital stricture is not uncommon and syphilis in the antecedents has been stated to stand in some pathological relationship, but such a claim cannot be substantiated. The pathogenic explanation can only be found in the arrest of development during the embryonic stage, as it is known that the urethra consists of three distinct structures during its embryonic development, namely, the posterior urethra, the spongy and balanic portions, each of which has different embryological origin (see Embryology, p. 27); and these component parts are separated from each other by other 
structures, which eventually disappear and leave the continuous canal. Hence, if the disappearance is incomplete, occlusion of the canal may result at this point. This explains the occurrence of strictures at the juncture of the balanic and spongy urethras, and at the juncture of the spongy and prostatic sections. In other portions, the explanations must be found in anomalies of epithelial development.

The symptoms of congenital stricture vary with the location and the degree of occlusion. In very wide strictures there may be no symptoms, or only a nocturnal incontinence. In the more severe types there may be a prodromal period of prolonged urination and fine stream, followed by frequency, which may eventually end in retention. The obstruction is readily recognized with a bulbous bougie, and further exploration and investigation may be made by means of the urethroscope. The severe types, when untreated, may grow progressively worse, and the condition become serious on account of the development of secondary lesions and infections. In those cases, when not discovered until the development of a gonorrhea, the result is the aggravation of the acquired condition. It is possible that some instances of severe stricture, following soon after the acquisition of gonorrhea, are cases of preëxistent congenital stricture. In concluding the diagnosis, differentiation must be made from spasm, traumatic lesions and foreign bodies. Prognosis varies with the severity of the occlusion and existence and nature of complications.

The treatment does not differ from that of the acquired form of stricture. Dilatation may sometimes suffice, but where dilatation cannot be employed, or produces only a temporary benefit, internal urethrotomy must be resorted to (see p. 283), when the obstruction is in the spongy portion, and external urethrotomy (p. 287) when the obstruction is complete and exists in the bulbous or prostatic urethra.

\section{ACQUIRED ORGANIC STRICTURE}

Causation.-Most cases of organic stricture owe their existence to gonorrhea. Injury to the urethra, resulting in complete rupture of the canal, or lacerations and abrasions of a lesser degree, blows or falls upon the perineum, forcible bending of the penis during erection, all may be followed by the production of traumatic organic stricture. Trauma or ulceration causes loss of urethral tissue, that is replaced by contracting connective tissue. Inflammatory stricture is often the first stage of organic stricture. The cause and effect are more closely associated in traumatic than in gonorrheal stricture, and the more severe the injury, the more rapid the occurrence of stricture. 
When stricture of the urethra results from gonorrhea it is due rather to a long-continued inflammatory process than to an inflammation intense in character but short in duration. The development is generally slow. It is seldom encountered before the second year following gonorrhea, and may not appear for a much longer time, ten or twenty years after the primary attack. Such strictures are very likely to invade the deeper urethral structures, while strictures following simple urethritis are not apt to extend beyond the superficial tissues.

From observations gleaned from various sources, it appears that in a majority of instances stricture is detected between two and ten years after the original inflammation. The more quickly cure of the urethritis is effected the less the danger of stricture formation.

Stricture may occur at any age, but is most common between the ages of thirty and forty. Stricture following gonorrhea is said to occur more often in white men than in negroes. Women may suffer from this affection. About 60 per cent. of cases are located between the bulbo-membranous junction and the peno-scrotal angle; the remaining cases are about equally distributed throughout the other portions of the anterior canal.

Stricture of the prostatic urethra anterior to the vesical orifice does not occur as a result of gonorrheal inflammation. But cicatricial contraction or fibrotic stenosis of the neck of the bladder is not an uncommon result of gonorrheal inflammation of the deep urethra or of the interior of the bladder, a condition which should be thoroughly recognized and appreciated. On account of its near association with the prostatic urethra, and its symptoms, which are closely allied to those of prostatic hypertrophy, this malady is described, under the title of Contracture of the Neck of the Bladder, in connection with other maladies of the bladder (p. 435).

Stricture following gonorrhea occurs most commonly near the bulb, and may be continued into the membranous urethra, or this portion of the canal may be dilated, as well as the prostatic portion behind an organic stricture involving the section of the urethra anterior to it.

Stricture due to trauma may develop anywhere in the urethra, the commonest locations being the bulb, the membranous or prostatic parts, one or all of them, according to the nature and extent of the injury.

Cicatricial contracton due to chancre or chancroid is generally found near the meatus.

Strictures vary in length, although generally not exceeding one- 
half centimeter (about $1 / 5$ in.). In the case of an apparently long stricture, it will be found that the condition is due to several individual adjacent strictures. As many as fourteen strictures (points of contraction) in one urethra have been found, all of which may belong to one or two strictured areas; and the number varies from this to a single constriction.

Morbid Changes.-The pathological beginning of stricture is inflammation; the resulting sequence is cicatricial or scar formation.

At first there is round-cell infiltration in the submucous connective tissue. This cell proliferation is cellular activity and leucocytosis. The extent of this process determines the future development and extent of the lesion. Spindle cell transformation next ensues. Extensive capillary increase in the mucosa forms granulation tissue, which is the soft or inflammatory stricture, the transitional stage toward cicatricial or true stricture. The infiltration of the lacunæ and follicles produces surface elevations of these structures in the beginning, and later the extensive connective tissue formation results in their atrophy. Leucocytes invade not only the subepithelial mucosa, but parts of the periglandular tissue as well. The intercellular substance increases and at the same time becomes broken up into fibrillæ, and thus is formed new connective tissue, the character of which is hard or soft, according to its density on the one hand or vascularity on the other. The more chronic, the more callous it becomes. Thickening of the urethral epithelium, with a constant tendency toward the conversion of the cylindrical cells to those of the pavement form and separation of the various layers by serous exudate favors exfoliation. The attendant urethral discharge is thus composed of a mixture of upper necrotic layers of epithelium with the secretion of the mucous glands and more or less leucocytes. The characteristic contraction of newlydeveloped connective tissue compresses the vessels of the part, after causing their obliteration, which explains the change of color of the morbid area, as seen through the endoscope, to that of a dull grey with tendinous striæ.

The practical importance of these pathological changes is their effect upon the calibre of the urethra. The pathologic changes which begin in the submucosa of the spongiosum may be confined here and form a narrow constricting band-the linear stricture (Fig. 106) - or a wide flattened area of irregular distribution-the annular variety of stricture (Fig. 107); on the other hand, the deeper parts-the corpora cavernosa, the muscular and erectile tissue, may become so involved as to be transformed into scar tissue; the walls of the urethra, thickened and cartilaginous, thus lose their distensibility, and the lumen is 
more or less completely obstructed. Thus is formed the tortuous or callous species of stricture, the distortion of the lumen sometimes amounting to occlusion (Fig. 108). If the narrowing due to the pathological changes be slight, there is present a stricture of wide calibre. If the growth of connective tissue is directed into the urethral

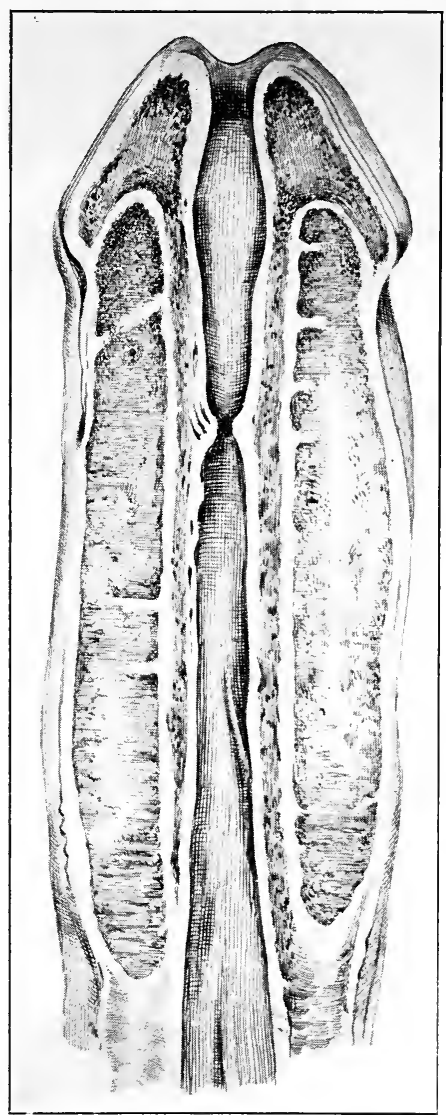

FIG. 106.-Stricture of urethra. Linear variety.

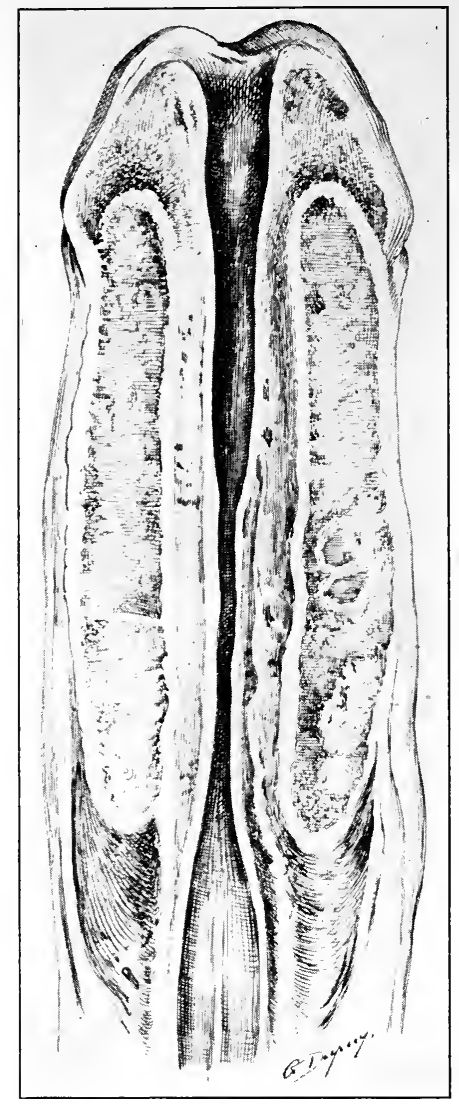

FIG. 107.-Stricture of urethra. Annular variety.

lumen there is valvular formation; while extensive deposit of cicatricial tissue may produce a twisting of the urethra and a small tortuous passage.

The characteristic formation of gonorrheal stricture is more complex and irregular than that of trauma, which is more compact and circumscribed. 
The portion of the urethra behind a stricture is dilated, sometimes forming a pouch, which is the seat of inflammatory exudate, causing erosion of the thinned mucous membrane.

In the light of our knowledge of the pathological formation of stricture tissue, it can be understood how, in exploring the urethra, the impression might be derived, that multiple stricture existed from the fact that an extensive area infiltrated

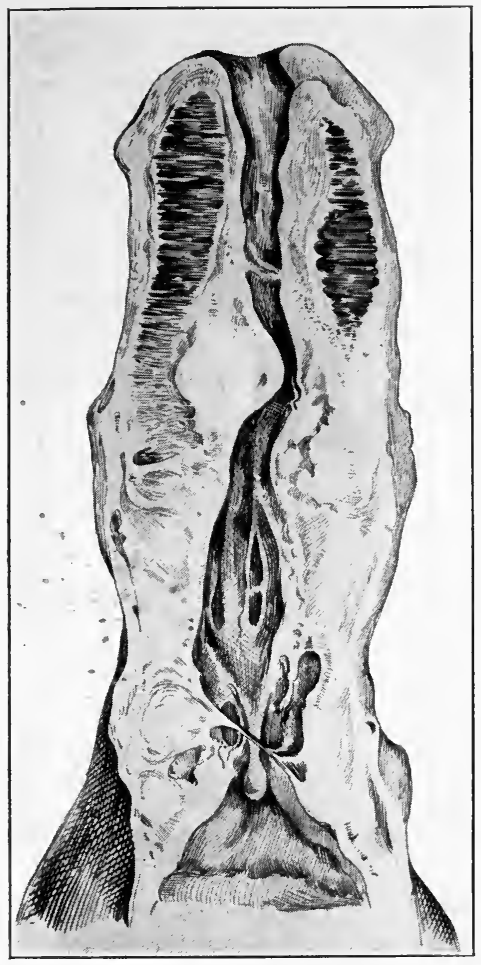

FIG. 108.-Tortuous stricture. with fibrous tissue must vary at different points in the degree of stenosis (Fig. 108). In other words, a stricture commencing in any part of the spongy urethra may reach the maximum degree of contraction at the bulbo-membranous junction; or,

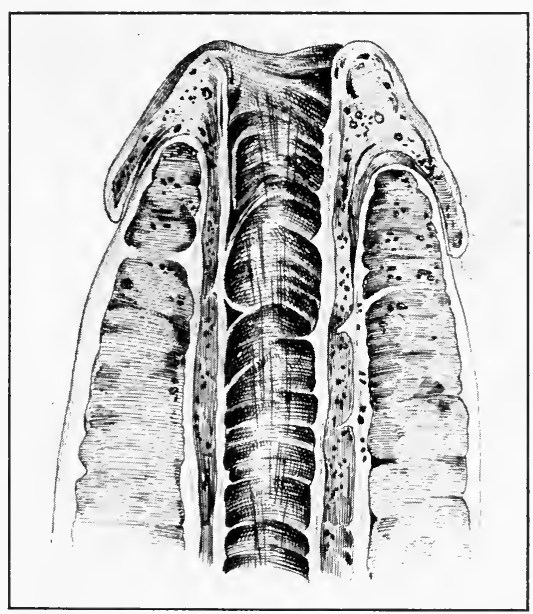

FIG. 109.-Section of urethra showing anatomical bands.

on the other hand, at one or more points anterior to the bulb, on account of an increase of deposit of cicatricial tissue at these points, in which case the exploring instrument would detect what seemed to be more than one stricture.

The classification of strictures into those of large and small calibre is, of course, simply an arbitrary one and is governed by no established standard. It is generally understood that a stricture which will permit the introduction of an instrument $5 \mathrm{~mm}$. in diameter (15 
French) or larger may be considered one of large calibre, while anything below this is regarded as of small calibration.

In order to determine whether or not the urethra is the seat of a narrowing such as should properly come under the title of stricture it is customary to have in mind a recognized standard for the normal urethra to compare with. Any pronounced variation below the natural calibre must be considered a "stricture of the urethra." It is important, however, in determining the natural calibre of the urethra to recognize that anatomical points of narrowing (Fig. 109) exist normally, and that between these anatomical points the urethra may be dilated beyond what should be regarded as its normal calibre.

The objection to the division of strictures into those of small and large calibre is that unless the standard be placed at a reasonable size strictures of large calibre will undoubtedly oftentimes be discovered at one of the points of physiological narrowing; and another fact to be remembered is that there exists no definite relation between the circumference of the penis and the calibre of the urethra, hence, the fallacy that may arise from the use of the urethrometer, an instrument devised for exploratory purposes, having a hinged extremity which can be expanded to a bulbous shape between sizes 20 and 45 French. It is hardly possible that a urethra explored with this instrument, anywhere near the maximum size or beyond an average of 30 French, will be found free from some variation in its calibre, and, therefore, being considered as the seat of stricture, must lead to most indiscriminate and unnecessary interference either by cutting or by overdistention. A conservative estimate of the average size of the canal in the majority of cases places the normal calibre at from 28 to 30 French, and while exceptionally it may be somewhat above or below these sizes, in the larger number of cases it is safe to follow this standard both for exploring the urethra and in the treatment of stricture. It is sometimes useful to treat by overdistention with the urethral dilator, much beyond the sizes given, strictures of wide dimensions, but this does not alter the established standard for the urethral calibre.

The meatus externally and inside the orifice is the most commonly constricted point and is subject to considerable variation. This smallness, which is a congenital deformity and not a pathological condition, never calls for interference unless it be the probable cause of symptoms such as reflex spasm of the membranous sphincter, or unless it interferes with the proper treatment with instruments of morbid conditions more deeply seated. About the middle of the spongy portion, from 2 to 3 inches from the meatus, there exists 
another point of physiological narrowing which is sometimes thought to be a stricture of large calibre by those who examine the urethra with the idea that it should possess a calibre uniform throughout its whole course, and at the triangular ligament is another point of normal anatomical narrowing which should not be mistaken for stricture (see Anatomy): Behind the meatus is a dilated area known as the fossa navicularis. Another dilated cul-de-sac exists in the sinus of the bulb, in front of the triangular ligament, the location of which is readily recognized by the introduction and withdrawal of an instrument, especially a bulbous bougie. Such points simply represent areas of uneven dilatability and will exist whether such an individual possesses a healthy urethra or whether it is the seat of real stricture.

Clinical symptoms.- Strictures of wide calibre, when they are sensitive, or have become inflamed, as by instrumentation, irritating urine, etc., may give rise to spasmodic and irritable troubles in the deep urethra. Sometimes, on the other hand, such strictures in a quiescent state, especially near the meatus, may have nothing whatever to do with troubles deeper in the canal. It is possible that such strictures may produce no symptoms until they have contracted down to a marked degree.

The most common symptom of strictures of large calibre in the pendulous or the deep urethra is a more or less purulent discharge. If such a discharge persists more than six months after the initial attack of gonorrhea, the possible development of stricture should always be considered.

As stated above, stricture of large calibre may go a long time undiscovered; being of gonorrheal origin, its formation is usually slow. As the strictured area contracts, the patient becomes accustomed to the slight effort involved during the act of urination and may fail to recognize any alteration from the normal state until a period is reached when he becomes conscious of a pronounced modification, either in the size or force of the urinary stream. This may be twisted, or may be uniformly small or flattened or it may be expelled in two separate jets. Such changes in the volume and form of the stream are not invariably significant of the existence of true stricture, as they may be noted in acute and chronic urethritis, in prostatitis, and in urethral spasm, in which instances the actual calibre of the canal is determined by exploration and found to be normal.

Of much greater importance is the projectile force of the stream during the act of urination as an index of the tone of the bladder muscle; but even when there exists a marked contraction of the urethra due to stricture, the bladder, having become reinforced by compen- 
satory muscular hypertrophy, may eject the stream from the urethra with reasonable force. Soon, however, the bladder muscle is unable to compensate for the obstruction in the canal, the stream is not only much diminished in size, but ceases to be projected and either falls perpendicularly from the end of the penis in a small stream or is voided by drops with considerable effort. In such a case the ejaculation of semen also loses its force. The fluid is slowly passed from the urethra or regurgitates into the bladder. So-called incontinence of urine, due to an overflow from the overdistended bladder now of ten exists. These symptoms denote tight organic stricture. During the progress of this condition, as a result of catching cold or from the effect of instrumental examination, congestion of the surface of the stricture or spasm of the compressor urethræ muscle is produced, from either of which causes the passage becomes occluded and urinary retention occurs. This may be sudden and complete.

Retention of urine is more likely to accompany stricture involving the deeper regions; it may occur, however, as a result of reflex spasm in connection with stricture in the anterior portion. Some patients are more liable to be attacked by this symptom than others, especially those of a neurotic temperament, although anything which is likely to cause hyperemia of the mucous membrane overlying the compressor urethræ muscle, such as cold, alcoholic excess, or the passage of instruments, may be the means of provoking this complication.

The urine of a patient suffering with stricture is generally not clear. An inflamed condition behind the constricted area of the urethra, due to irritation caused by the impact of the urinary stream as it strikes the narrowed walls results in increased secretion, which shows itself in the form of shreds or filaments. If, from extension of the process, cystitis develops, the urine becomes turbid.

Urethral discharge, one of the earliest symptoms, more or less muco-purulent in character, is generally found in all cases of stricture of small calibre, but may be absent. The quantity of the secretion is generally scant, sometimes rather free. It is commonly a morning drop, but may also occur during the day. A free discharge is the exception rather than the rule, and the pus which is formed at the inflamed and congested area in connection with any stricture may simply appear in the urine in the form of shreds and masses.

A certain amount of uneasiness and smarting during urination may exist in connection with strictures, but such subjective symptoms may be entirely absent.

Increased frequency and urgency of urination may be due to posterior urethritis or urethro-cystitis. 
Stricture in any part of the urethra may be attended by pain felt either at the point where the stricture is located or referred to the meatus or to some other portion of the canal, or there may be no pain whatever. Frequency of urination is much more marked when the deeper regions are involved, such urinary frequency being relatively greater by day than by night.

Diagnosis.-In a majority of cases, a careful study of the foregoing symptoms will afford the surgeon sufficient facts upon which to base the diagnosis, although it must be borne in mind that an abnormal stream, slow micturition, or increased frequency, incontinence, retention, gleet and loss of sexual power may all be significant of other maladies producing urinary obstruction or retention, such as hypertrophy of the prostate and its allied condition, contraction of the vesical neck, or paralysis of the bladder. In establishing the diagnosis, the physical examination is the means upon which to depend for definite data as to the location, size and texture of a suspected case of stricture. This physical examination of the urethra is conducted by means of bougies of various kinds and solid metal steel sounds. These instruments form an important part of the urologist's armamentarium and their full description belongs to that section (see Chapter II).

Finally, an important part of the diagnostic procedure involves the recognition and careful investigation of the various complications and sequelæ that may result from the presence of stricture.

\section{Complications and Sequelæ of Stricture}

Urethritis in some degree is a common accompaniment of stricture. If from one of the possible sources of irritation, it is aggravated to a severe extent, an increased closure of the canal follows, and incomplete or complete retention of urine results. This, as already stated, may come on suddenly and may be the occasion that first induces the patient to seek relief.

Epididymitis, sometimes acute and sometimes mild and relapsing, occurs in connection with strictures, notably those which are irritable, inflamed, or of small calibre and so situated as to affect the deeper portion of the urethra and the vesical neck.

The inflamed area behind the urethral narrowing has a tendency to produce an erosion of the adjacent tissues. This may amount to nothing more than ulceration and thickening, but if it extends beyond the mucosa, whether from the collection of decomposing urine or purulent discharge, infiltration occurs and extension into the follicles may lead to follicular abscess or periurethral infiltration. Partial 
rupture of the urethra from progressive ulceration or instrumental damage is the cause of urinary extravasation into the tissues.

Extravasation more commonly is the result of a violent or continuous straining effort to overcome the resistance of a tight stricture. The urine is forced into the surrounding tissues, being limited in its course, according to the site at which rupture occurs, by the anatomical attachment of the fasciæ enveloping the different regions. Thus, when rupture occurs in the pendulous urethra it is followed by swelling of the penis, which may be limited within the fascia surrounding the corpus spongiosum, or, overcoming the resistance of this membrane, extend into the connective tissues of the penis and scrotum. This form of extravasation is rare.

Extravasation in front of the triangular ligament through the bulbous portion of the urethra inundates the perineum, and being limited by the attachments of this fascia, cannot reach the pelvic cavity or the tissues of the lower extremity, but is directed upward over the hypogastrium.

Rupture of the urethra in the membranous portion is limited by both layers of the triangular ligament, and at first meets the strong resistance offered by the fibrous tissues; suppuration and later ulceration with a consequent fistulous tract through one or the other layers of the ligament into the pelvis or toward the perineum then result.

Finally, rupture of the urethra behind the triangular ligament may lead to extravasation around the rectum in the periprostatic region, or going above the prostate, may extend into the cellular tissue of the pelvis.

Extravasation is self-evident when it occurs in front of or behind the triangular ligament, provided the quantity of infiltrated fluid be sufficient to attract notice; otherwise, it may pass undetected until other symptoms occur. Sometimes, however, the patient complains of having felt something give way during a straining effort, followed by a certain sense of relief, which is unaccompanied by the normal passage of urine. Extravasation between the layers of the triangular ligament may not be recognized until further extension of the process occurs or until the onset of constitutional symptoms. These symptoms consist of general depression and weakness, pyrexia, dry tongue, sometimes ushered in by a chill; and, if the condition continues unrelieved, the pyrexia increases, and severe urinary septicemia supervenes, which may end fatally. The local symptoms consist of swelling in the region to which the extravasation has extended; the scrotum may be much enlarged, the tissues over the hypogastrium and perineum may be tense and red, or bluish and dusky and edematous. Actual destruction of 
the tissues may occur as a result of sloughing and gangrene. The scrotum sometimes sloughs entirely, leaving the testicles bare. The more septic the urine in cases of extravasation the more rapid is the occurrence of the systemic symptoms and the more profound the resulting septicemia.

Urinary Fistula.-Small quantities of urine sometimes escape from the urethra, resulting in urinary abscess and the formation of fistula. Suppuration may originate in the glandular structures closely related to the urethra. After penetrating through the lumen, the pus may find its way to the exterior, thus forming a channel or fistulous tract. An abscess cavity communicating with the urethra may persist for a long time. When the urethral outlet thereof becomes closed, a fistulous opening is formed in one or more places, through the perineum, backward into the rectum, or in front of the scrotum.

The ultimate result of long-continued tight stricture is generally chronic inflammation of the bladder attended by thickening of the vesical wall with contracture of the neck, contraction of its cavity, or sometimes dilatation and atony, resulting perhaps in constant dribbling of urine due to overflow of the distended organ and loss of power of the vesical and membranous sphineters.

Finally, as the result of tight stricture extends itself, dilatation of the ureter and pelvis of the kidney and pyelonephritis may develop, which in extreme cases lead to a fatal issue from acute uremia; or, after long duration, to a slowly progressive condition of kidney insufficiency.

Prognosis.-As far as the immediate result is concerned, the prognosis of stricture is favorable although the character of the constriction and the location thereof are modifying factors. Simple contractions of the urethra, wherever located, are easily overcome, except cicatricial narrowing of the meatus, which is rebellious.

When a stricture is resilient or tortuous, it offers more difficulties of treatment than it does without these features. Stricture due to injury is far more resistent to treatment than that of gonorrheal origin, although here again, the extent of the trauma has a potent influence on the outcome. Relapse in the form of recontraction and sequelæ of serious nature are almost invariably the result of neglect. Neglected stricture produces serious complicated conditions, involving first the bladder and later the kidneys, which may become irremediable. The haphazard statement made that "once a stricture, always a stricture" is derived from the fact that many cases exist that recur after continual dilatation and even after cutting, but inasmuch as the object of treatment is the removal of that which mainly gives to stricture its 
significance, namely, the narrowing of the urethral tube and the consequent intesference with the urinary function, it may be stated, that the cure of stricture is satisfactorily effected for a prolonged period, and often permanently, when the proper means of treatment are employed and when careful observance of the rules laid down is adhered to.

Treatment.- The general treatment of stricture is subordinate to the surgical management of the local condition which is more or less that of the attendant urethritis or complications thereof (see Index), and the care of the general health.

The surgical treatment of stricture is of first importance. The principal measures employed are dilatation (progressive or rapid), urethrotomy (internal and external), resection, electrolysis. Not any one of these methods is applicable to the treatment of all cases.

Electrolysis. - With respect to electrolysis, time has not changed the conviction obtained from early education and experience that extravagant claims made in its favor have not been established. The most that can be conceded to this method of treatment, so far as the action of the electrolytic current is concerned in whatever form it may be administered, whether it be low or high milliamperage or high frequency, is that it may exert a certain degree of paralytic or antispasmodic influence affecting the unstriped muscular tissue, on account of which the spasmodic element of the stricture may relax. Upon organic stricture it has no effect whatsoever. The field of its usefulness, therefore, is so excessively limited that it is hardly worth consideration with other measures at hand so much more practical and reliable.

It is unreasonable to state that all strictures should be cut, a doctrine that was at one time too largely followed on the theory that the urethra was a canal with uniform calibre throughout and that it was necessary to remove by incision any encroachment thereupon. It is equally unreasonable, on the other hand, to say that all strictures must be dilated. The choice between dilatation and cutting should, if possible, be made in favor of the former when its employment is consistent with the attainment of the principal object of treatment, namely, the re-establishment of the normal calibre of the canal at the seat of the stricture and maintenance of the same for as long a period as is possible. The size of the stricture is not necessarily a guide to the course of treatment. Some very tight strictures can be easily dilated, whereas some strictures of large calibre, although yielding readily, to the dilating instrument, yet, on account of elasticity and resiliency recontract so rapidly that they are a source of constant irritation, 
and nothing approaching a cure is possible until cutting is resorted to. From a textural standpoint, strictures are divided into soft, hard and nodular formation. Soft stricture is an early inflammatory stage and, whether of the anterior or posterior urethra, should respond to treatment by dilatation.

Hard strictures are fibrous in character and, therefore, have passed through the stage of chronic inflammation with its attendant cicatricial formation. Here again, dilatation should be attempted first and when it is found that the contraction is intractable beyond a certain point, urethrotomy should be resorted to. Generally speaking, the measure of success is the extent to which the contraction can be distended and maintained and the effect produced by such result upon the coexisting symptoms. Therefore, strictures of either small or large calibre which, although easily dilated, promptly recontract, rendering it necessary to reintroduce the dilating instrument at too frequent intervals, necessarily demand the more radical method of cutting in an attempt to elongate the period before recontraction occurs. Strictures of the anterior urethra are usually more unyielding than those of the membranous portion. Strictures of the posterior urethra accompanied by chronic or severe urethro-cystitis, outside of other considerations, often are treated more suitably by external urethrotomy on account of the advantages to the bladder and posterior urethra of efficient and continuous drainage afforded by operation. Those strictures peculiar to the bulbous and bulbo-membranous urethra in the composition of which there is an unusually large and irregular deposit of fibrous tissue, should be treated by external urethrotomy, and the mass of indurated material removed by the operation of resection and suturing of the urethra. Irritable strictures or those which occur in a urethra unusually susceptible to instrumental interference, as evidenced by sharp reaction (chills, etc.) and local irritation, in spite of gentleness of manipulation and careful antisepsis, should be dilated at long intervals only, in the attempt to overcome such undue susceptibility, which certainly cannot be accomplished by too persistent attacks at short intervals. Strictures of the anterior urethra requiring cutting should be treated by internal urethrotomy (see p. 283) and those of the bulbo-membranous junction or behind it should be treated by external urethrotomy (see p. 287).

The Treatment of Stricture by Progressive Dilatation.--Before instrumental introduction, it is well to flush out the anterior canal with a mild, non-astringent wash, either plain sterile water or mild boric solution preferred. Anything in the nature of an irritant will render the examination of the canal more difficult (see p. 68). The steel- 
nickeled instruments, conical in shape and double-tapered (see p. 40) are used with satisfaction in dilating the canal either in the pendulous or deep portion provided an instrument as large as a No. 20 F. can be introduced. Should the stricture be smaller than this size, it is well to commence with a soft and flexible instrument. The instrument, of a size that the urethra can readily receive, is gently introduced and immediately withdrawn, after which, the progression in number and size of instruments reintroduced is governed by the irritability of the urethra, the resistance of the constriction and the hemorrhage produced. It is far better to desist following the introduction of one or two instruments until more thoroughly acquainted with the peculiarities of a given case, rather than persist at the expense of a sharp local or general reaction, much to the discomfort and dissatisfaction of the patient. Such reliable acquaintance with the features of a given case is obtained only after careful observation following the results of several examinations and treatments; and patience in this respect is well rewarded. After treatment by dilatation, the urethra should be washed with a warm, mild disinfectant solution, either 1 per cent. boric acid or permanganate of potassium 1 to 6000 (see Irrigation of the Urethra, p. 37). The discharge from the urethra often visibly increases the following day but this subsides spontaneously at the expiration of a few days if the treatment has not been too severe.

All evidence of mechanical irritation should subside before resumption of treatment and the result of the second instrumentation will be less irritating than the first unless the interval between them is made too short. The most effective treatment, after a large-sized instrument has been reached, is that which permits an interval of one week or more between the introductions thereof. When the full size of the urethra has been reached, attempts should be made to discontinue the introduction of the instrument for as long a period as possible without recontraction. This period can only be determined by extending the intervals between each visit until, as a result of observation, a period suitable to the individual case is determined. Sometimes treatment is discontinued for an indefinite period, either from necessity or choice, in which case the patient should be warned that recurring symptoms (irritability, discharge, etc.) imply recontraction and dilatation must be resumed and furthermore, that no dependence can be placed upon the permanent distention of the canal when once a stricture has been established, exceptions to the contrary notwithstanding. It is, therefore, better to forestall trouble by submitting to inspection at appropriate intervals.

In those cases in which the symptoms yield, but do not entirely 
disappear, and those in which the required intervals for dilatation are unduly short, it may be necessary to resort to urethrotomy.

Impassable stricture is one that produces complete retention of urine or is impassable to the introduction of an instrument of any size, or both. Some strictures cause complete retention of urine and yet it is possible to pass an instrument of sufficient size to empty the bladder. Such strictures occur at the bulbo-membranous urethra and are due to the combination of spasmodic with organic stricture. Stricture which is impassable both to the urine and the introduction of an instrument of sufficient size to draw the urine, requires immediate action by dilatation if possible, which if unsuccessful, must be followed either by suprapubic tapping (see p. 471) or by external urethrotomy (see p. 287). Some strictures which at first appear to be impassable to the introduction of any instrument, may yield to a patient and careful trial with the diminutive urethral bougies, filiforms or stricture finders described in instrumental technic (see p. 44), one of which may be left in place. The patient may then successfully urinate alongside of the bougie and gradual dilatation may be accomplished in increasing sizes, by means of the tunnel sound or metal instrument of Guyon (see p. 43, Fig. 29).

Rapid and prolonged dilatation is accomplished by means of the Kollmann urethral dilator fashioned on the principle of the Otis urethrometer but of different patterns, on the lines of the straight sound for the anterior urethra, the single curve for the posterior urethra and the double or Béniqué curve (see description of instruments, p. 46). These instruments, when closed, are introduced through the stricture and then expanded for the purpose of producing distention as high as may be desired or as the contraction will permit without laceration. The dilatator may be removed almost immediately or may be left in place from three to ten minutes. These instruments have no particular advantage over the smooth steel sound when the meatus will permit the introduction of the latter; they are more particularly useful for the more distensible portion of the posterior urethra in strictures of wide calibre where it is impossible to introduce a sound without splitting the meatus. They are used also for distending contracture of the neck of the bladder with mild degree of stenosis and for the relief of reflex neuralgic symptoms dependent upon deep urethral spasm. Great care should be observed in the employment of these powerful dilators as severe traumatic urethritis and swollen testicle may result from their unwise and careless employment.

For further description of instrumental technic see Chapter II. The Treatment of Stricture by Internal Urethrotomy.-This opera- 
tion is generally applicable to the pendulous urethra only. The dangers of internal urethrotomy of the posterior urethra are severe hemorrhage, extravasation of urine, retention and urinary sepsis, any of which conditions may demand external urethrotomy later as a matter of necessity. Internal urethrotomy is indicated in the first place when dilatation is required at unreasonably short intervals. In this particular, however, it must be stated that even after cutting there is no assurance that the stricture will remain permanently distended to the full size, although this may occur. In any event, the result should be much elongation of the intervals between the instrumental dilatations. Stricture of large calibre in the pendulous urethra may be cut by internal urethrotomy and its symptoms forever cease without the necessity of further use of instruments. Such occurrence, however, can only be determined by the lapse of time. Resilient stricture or one of elastic calibre is often incurable except by the knife. Small, tight strictures in the pendulous urethra are probably best managed by internal urethrotomy, being easily reached in this manner and the results on the whole warrant it; and this is true of all strictures of small calibre in the pendulous urethra when they are fibrous and resistant, as they are quite certain in the long run to require cutting. If they prove too narrow to receive the urethrotome or cutting instrument, the calibre may be increased to the required size by dilatation and urethrotomy may be performed thereafter. (For a description of Internal Urethrotomy, see p. 283.)

It may be difficult to determine how deeply to cut a given stricture in the pendulous urethra. This cannot be decided on any arbitrary scale. It may be stated as a general proposition that the strictured area that will not yield to dilatation should be cut so as to be somewhat larger than the normal urethra at that point and the best results are obtained by a single cut just beyond the diseased into the healthy tissue. This result is probably most often obtained by accepting the size No. 30 French, 20 American, as the normal average standard, recognizing that some individuals may have a smaller and others a larger calibre than this size.

Should the meatus be strictured congenitally or by disease, it is necessary to perform a preliminary meatotomy.

The bleeding after internal urethrotomy is variable; in some cases, little or no blood escapes at the time of operation but later, at the next urination or during erection, hemorrhage may become quite profuse. The same means will usually suffice to arrest it as are given in connection with the operation (p. 285).

The after-treatment consists in the employment of a conical steel 
sound at appropriate intervals, until the cut surface of the urethra shall have healed over. The first interval may be forty-eight hours, the second four days and the third six, after which one or two passages of the sound at weekly intervals may terminate the case temporarily until observation can be made as to the tendency of recontraction based upon which must be the decision as to future instrumentation. It should be taken into account that symptoms which sometimes persist after stricture has been cut and after the attendant irritation has had time to subside, may be due to inflammation deep in the canal (posterior urethritis), which will require appropriate treatment.

The complications attending urethrotomy are those of acute urethritis, which may be avoided by strict cleanliness, by keeping the patient quiet upon his back for four to seven days upon a mild diet and the free use of bland diluents. One complication that has been noted is the formation of new fibrous tissue in the corpus spongiosum resulting in painful erection and chordee persisting for a long period and resulting in curvature of the penis. The cause of this condition is that the cut has been made too far beyond the strictured area and confines of the urethra. This should not occur if the reasonable standard of measurement given is adhered to.

External Perineal Urethrotomy.-This procedure may be adopted either as a method of choice or as an emergent necessity. It is resorted to as a matter of expediency for deep urethral stricture whether of small or wide calibre, which, on account of the element of resiliency, shows a too rapid tendency to relapse following dilatation and in strictures of the anterior or posterior canal accompanied by acute or chronic posterior inflammation of the urethra and bladder, when it is deemed advisable to afford the benefits of continuous drainage, other means having failed. It is employed as an operation of necessity in such conditions as extravasation of urine, prostatic or perineal abscess, urethro-perineal fistula and complete retention of urine, and when the stricture is impermeable to an instrument passed through the meatus. This last contingency does not necessarily demand external urethrotomy. It is also possible in such a case to aspirate the bladder above the pubic bone (see p. 471) and if need be, to repeat this procedure on one or two occasions; or to introduce through the aspirating canula a small sized catheter which is left in situ, after the removal of the canula, as a means of continuous drainage; and the day following this method of aspiration or drainage another effort is made to pass the stricture by means of a whalebone bougie which, owing to the relieved tension, is very likely to be successful. From such a beginning, progressive and successful dilatation may be instituted. 
Retrograde catheterism is resorted to by opening the bladder suprapubically, in order to reveal the urethral opening during a perineal section without a guide, when prolonged efforts to locate the urethra through the perineum have been unsuccessful. A very resourceful substitute is that of retrograde cystourethrotomy through a suprapubic canula. (See Operative Surgery, p. 472.)

Stone in the bladder as a complication of stricture naturally calls for external urethrotomy in order that the two conditions may be reached by a single operation.

The primary consideration in external urethrotomy operations, whether done as a matter of expediency or necessity, is the question of the permeability of the urethra which determines whether or not the operation can be performed with or without a guide in the canal (see Operative Surgery, p. 287). This is an important distinction as perineal section or external urethrotomy with a guide is a simple operation, whereas that without a guide is much more difficult and may prove exceedingly tedious on account of distortion and deflection of the urethra. It is, therefore, important to exercise patience and skill in obtaining an entrance to the bladder by way of the anterior urethra before operating without a guide. This may be accomplished under a general anesthetic when it has been unsuccessful without such means.

The after-treatment following the operation of external urethrotomy depends upon certain conditions. At the time of operation, a drainage tube is introduced through the perineum into the bladder. In simple and uncomplicated cases, this tube can be removed in twentyfour or forty-eight hours along with the packing that is introduced to control hemorrhage. But in severe cases of prolonged operation involving a large denuded area and requiring much packing to control hemorrhage thoroughly, the after-treatment is more protracted, it being impossible to remove all the packing for several days and it is necessary to keep the perineal tube in place meanwhile. Furthermore, in cases of chronic cystitis of long standing, it may be deemed advisable to institute drainage for a longer period, under which circumstances, the tube may be left in situ for a week's time, during which period daily lavage of the bladker is conducted with boric acid 2 per cent. and nitrate of silver 1 to 16,000 .

Following the removal of the tube, a sound is passed into the bladder, starting, perhaps, with one or two sizes below the full size; and this dilatation is kept up weekly or semi-weekly until complete closure of the wound has taken place, after which the intervals are extended according to the peculiarities of each individual case. For 
the first twenty-four or forty-eight hours after the perineal tube is removed, all of the urine passes by way of the perineal wound, following which it is divided in varying quantities between the perineum and the natural route. As the wound heals, which it usually does without assistance, the urine gradually passes entirely by way of the urethra, often permanently at the end of the first week. Finally, it is of greatest importance to impress upon the patient the necessity of establishing a regular practice of having recourse to the sound at required intervals. This may be once a month or once in six months as the case may be, but many relapses occur either because the surgeon has not called attention to this necessity or because the patient has neglected to follow his injunction. Recontraction, however, is not invariable after external urethrotomy; it is possible, with the regular and systematic use of sounds following this operation, to gradually lengthen the interval and finally discontinue them entirely without recontraction; but it is a wise precaution in all cases to acquaint the patient with the continuous possibility of a return, and to urge him, as a precaution, to report at long intervals for exploratory examination. 


\section{CHAPTER XV}

\section{OPERATIVE SURGERY OF THE URETHRA}

\section{OPERATIONS FOR HYPOSPADIAS ; URETHROTOMY, INTERNAL AND EXTERNAL; SUTURE AND RESECTION}

The operations performed upon the urethral tube are demanded to remove congenital defects and morbid growths, to repair injuries and lacerations, and to correct structural changes and other pathological conditions which have occurred as the result of infective inflammation or traumatism.

The principal difficulty to be contended with is the necessity of keeping open the urethral canal to allow the passage of the urine at regular intervals which necessarily exercises an influence upon the healing process. As a matter of fact, however, surgical wounds of the urethra heal kindly provided suitable drainage is established.

Generally speaking, the operations are either internally or externally performed, the former to remove morbid growths, foreign bodies, etc., to enlarge the calibre of the canal (internal urethrotomy) and to incise glandular abscesses. The external operations are for the correction of congenital deformity, the removal of fistulous tracts and the enlargement and excision of portions of the canal (external urethrotomy).

Both local and general anesthesia are employed.

Local anesthesia is obtained by inflation of the canal with a 2 per cent. cocaine or kindred solution (novocain, beta-eucaine, alypin) or by the introduction of an anesthetic lubricant (see p. 46). This method is applicable to internal urethrotomy and operations through the endoscope (q.v., pp. 127 and 500).

Local anesthesia for external operations is acquired by anesthesia of the urethra as above and by infiltration around the urethral tube in the particular area to be operated upon with a weak cocaine solution or other local anesthetic (see special section on local anesthesia p. 501).

Preparation for Operations upon the Urethra.-Before internal operations, the external parts are properly cleansed with soap and water and wash of mercuric bichloride. Shaving of the pubis is not usually practised. The urethra is irrigated with a mild solution of permanganate of potassium 1:8000 of a 2 per cent. boric solution, in sufficient quantity to remove retained secretion and discharge, the urine having been passed preliminary thereto. 
The preparation for external operations upon the urethra is of greater moment. The parts should be carefully shaved and the surface prepared as before any surgical operation with green soap, bichloride of mercury solution, etc. Sometimes the surface is prepared by the application of equal parts alcohol and tincture of iodine, as a further precaution. When general anesthesia is employed, the usual preparation as to thorough evacuation of the bowels and fasting is included.

\section{Hypospadias}

It is generally desirable to precede any attempt at correcting this deformity by a perineal urethrotomy (see p. 287), with a view of carrying off the urine through a perineal tube and thus effectively protect the operative wound; as when a catheter is tied in the anterior urethra, instead of making a perineal opening, unsuccessful

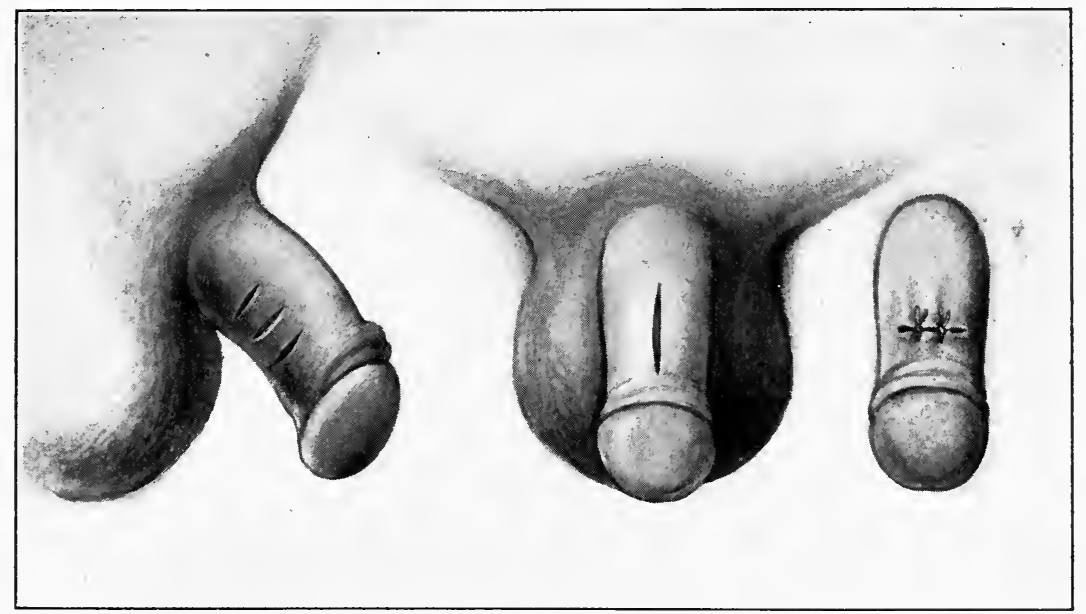

Fig. 110.-Hypospadias. Preliminary method of correcting curvature.

attempts have resulted chiefly on account of leakage of the urine around the sutured urethra and infection of the field of operation. This operation should not be performed until the patient has reached an age when he can be reasoned with and proper coöperation obtained. This may be from four to six years, according to temperament.

There are three important features embraced in the technic (Fig. 110): (a) the correction of deformity or downward curvature of the penis, which exists to a greater or less extent in different cases; (b) providing a new canal from the fistula or false opening to the natural 
terminus; and finally (c) the establishment of a continuity between the newly made and the old canal. This last is the most difficult step in the procedure.

The correction of the downward curvature of the corpora cavernosa is accomplished by transverse incisions of the fibrous sheath of the corpora cavernosa laterally; and by longitudinal incision on the dorsum, reuniting the latter wound by means of a suture in the opposite direction (Fig. 110). This should be done as a preliminary step and on one or more occasions, as may be required, during the earlier development of the patient; it may embrace a period of from two months to a year previous to the final operation.

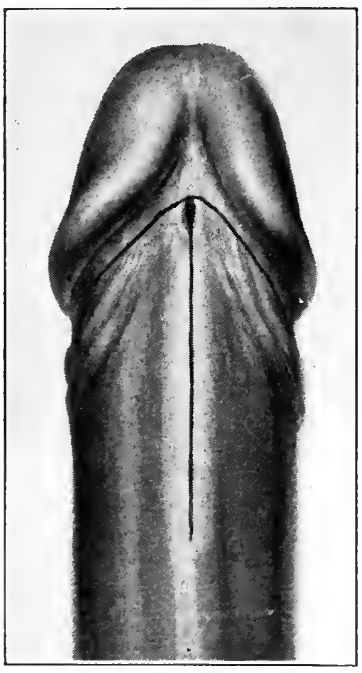

Fig. 111.-Beck's operation for balanic hypospadias. Incision.

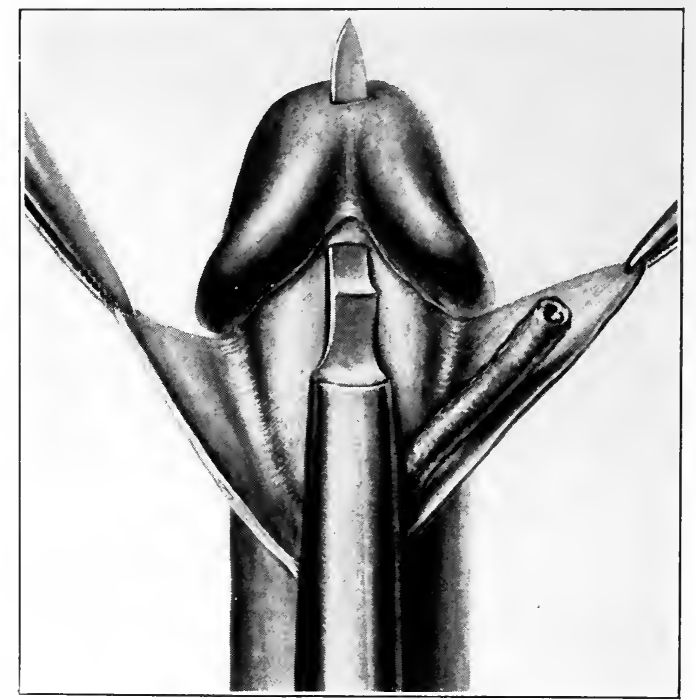

FIG. 112.--Beck's operation for hypospadias. Tumelling of glans after separation of urethra.

In the technic known as "Beck's operation," the formation of a canal from the site of the hypospadias to the end of the glans penis is accomplished by sliding forward the urethra and suturing it at that point. The urethra is first freed from its attachments to the corpora cavernosa by a single longitudinal incision or by two parallel incisionsone on either side of the tube-beginning at the abnormal opening and ending somewhere between that and the peno-scrotal angle (Fig. 111). The corpus spongiosum ensheathing the urethra is then carefully dissected with a small, sharp knife or ophthalmic scissors. When this is completed, a tunnel is made with a bistoury through the glans penis 
from the site of the normal meatus to that of the hypospadias aperture (Fig. 112). Hemorrhage in the glans is controlled by the application of hot water and pressure. The anterior portion of the urethra is freed from its covering of skin to the extent of the length required to allow the urethra to pass through the tunnelled passage in the glans. A pair of thumb forceps passed through the new meatus and the glans pulls the released urethra through the tunnel (Fig. 113); it is then sutured in correct position with fine catgut or silk. The flap of skin dissected from the urethra is sutured to the under surface of the penis and the free corpus spongiosum is also sutured in its new position. The opera-

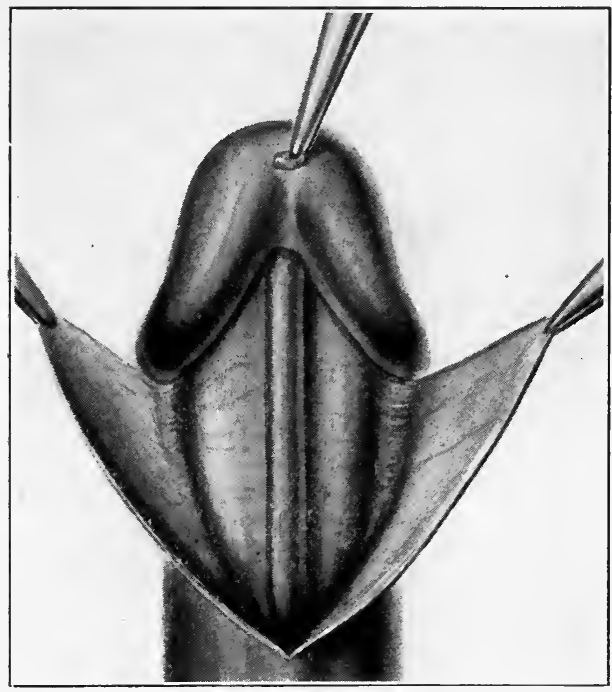

FIG. 113.-Beck's operation for hypospadias. Drawing urethra through tunnel in glans.

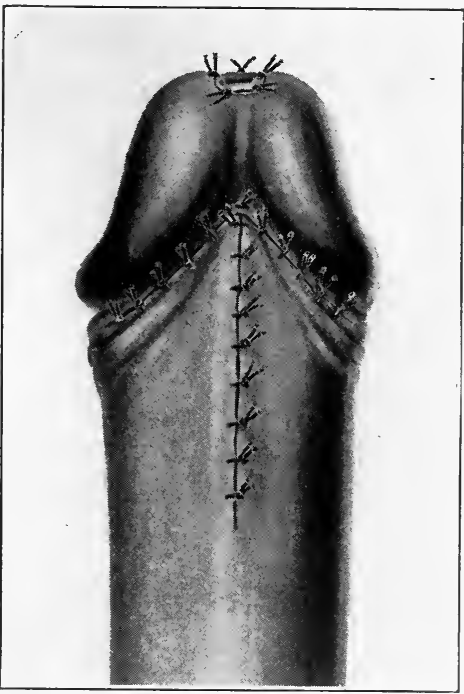

FIG. 114.-Beck's operation for penile hypospadias completed.

tion is completed with additional sutures between the skin surfaces (Fig. 114). While this operation is satisfactory for balanic hypospadias, in more pronounced cases, where the distance between the tip of the glans and the abnormal opening is much greater, the foreshortened urethra unduly exaggerates the downward curvature of the penis and the correction of this part of the deformity is not effected. In such cases the following is preferable:

The Technic of Nové-Josserand.-A Thiersch graft is obtained from the thigh, somewhat longer than the length of the new canal requires and about $3 \mathrm{~cm}$. wide. It is rolled with the raw surface outward around a short gum-elastic bougie or glass rod and tied at both ends (Figs. 115, 116). This is introduced through the canal dug through 
the glans and left there for ten days at the end of which time the bougie is removed and the redundant end trimmed off. The new

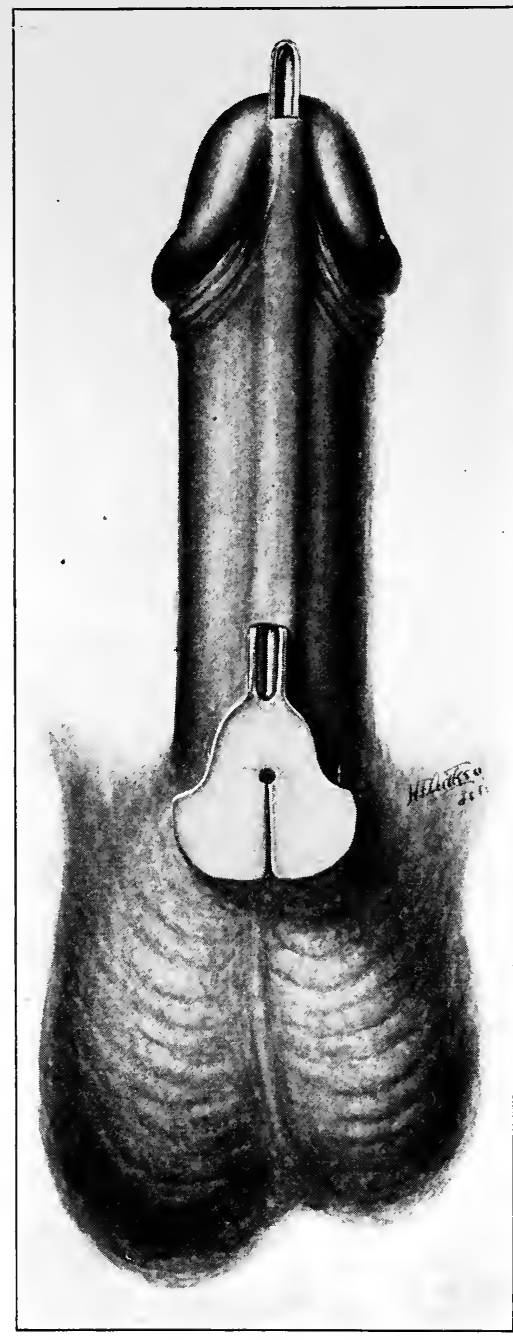

Fig. 115.-Nové-Josserand technic for hypospadias. Tunnelling passage for new canal. canal thus made is shaped by the continued introduction of bougies until a satisfactory result is obtained. Finally, suture together of the ends of the two canals is necessary to complete the undertaking and may require more than one attempt.

A venous or ureteral transplantation has been tried in preference to the thigh graft and a flap turned back from the scrotum has also been used (Rochet) instead of a graft (Figs. 117 and 118).

Epispadias.-The simple forms of this malformation, that is, those involving the penile portion, may be let alone on account of the dissatisfaction attending most operative procedures for relief therefrom; or the technic of NovéJosserand as performed in cases of hypospadias may be employed here. For the more severe forms of epispadias, where the sphincter of the bladder is involved, in the less extreme type, attempt may be made to restore the sphincter by incision and suture with a view of bringing the separated muscular ends together, and then follow with the Nové-Josserand technic. In the most extreme variety of this deformity, the operation is that of exstrophy of the bladder. (See p. 472.)

\section{Requirements for Hypospadias and Epispadias Operations}

General anesthesia required.

(Preliminary perineal section, see p. 2 7.) 
Scalpels, straight blunt-pointed bistoury and scissors.

Grooved director. 2 pairs of thumb forceps.

Silver female catheter. Artery clamps.

Needles and needle holder.

Tube of lubricating jelly.

Perineal tube. Soft rubber and silk-woven catheters.

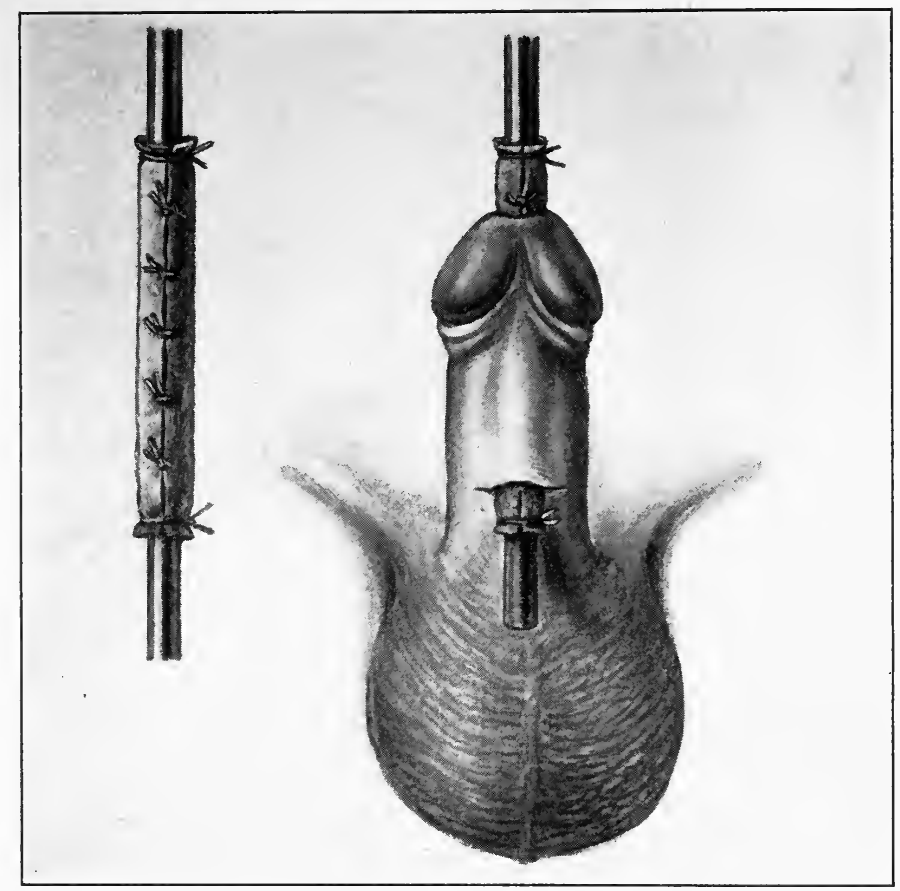

FIG. 116.-Nové-Josserand technic. Preparation of graft and introduction through new canal.

00 and No. 1 chromic and plain catgut.

Glutol.

Gauze packing,

Bladder syringe, 100 c.c.; boric and alum solutions.

\section{Meatotomy}

With a hypodermic syringe and needle, a few drops of one-fifth of 1 per cent. cocaine, or one-half of 1 per cent. novocaine solution are injected into the region to be incised. This should cause a small bleb to rise and very little delay is necessary before proceeding. 
A straight, blunt-pointed knife is now introduced into the urethra and the meatus enlarged by a vertical incision directed toward the floor of the urethra.

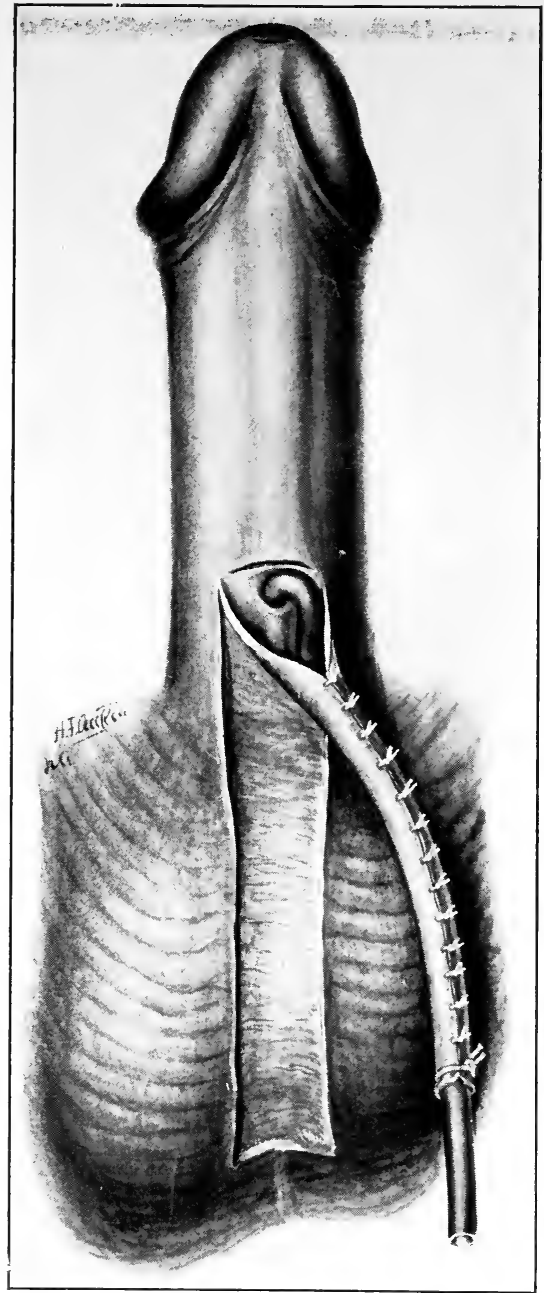

Fig. 117.-Rochet technic. Flap turned back from scrotum.

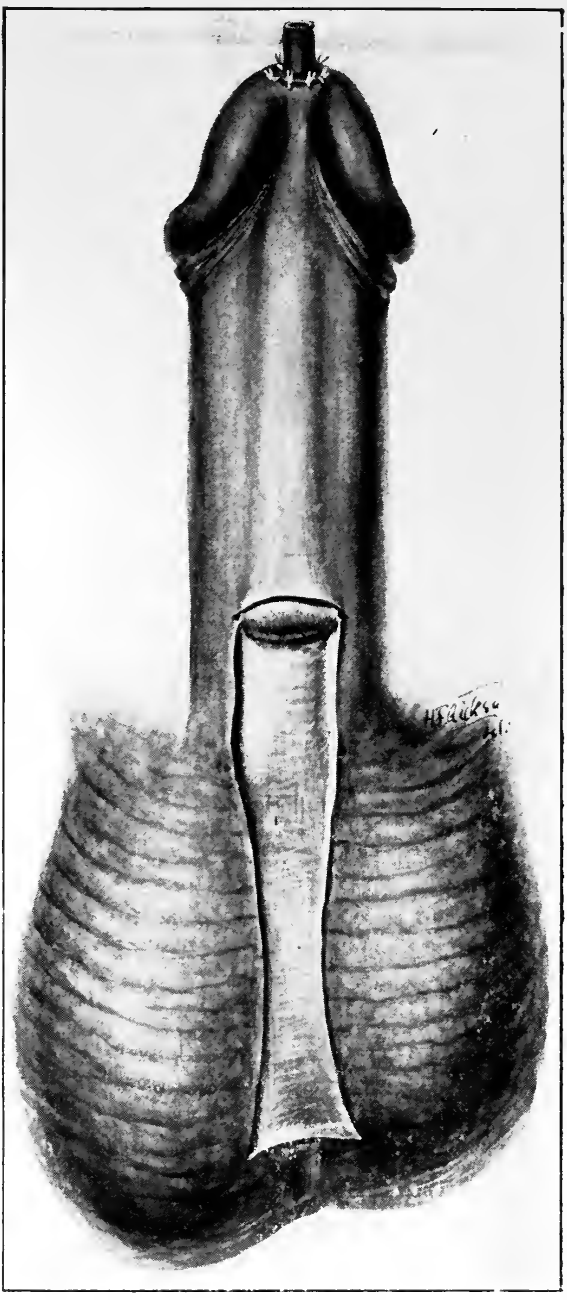

Fig. 118.- Same as Fig. 117. Completed.

The extent of the incision should be in keeping with the size of the penis. In the average case, about 30 French.

Frequently, after enlarging the external meatus, on introducing a large calibre bougie or sound, a second obstruction is found $2 \mathrm{~cm}$. 
( $3 / 4$ in.) behind the first one and corresponding to the posterior limit of the navicular fossa. This, of course, must be also divided on the floor of the urethra so as to admit of a No. 30 French. No ligatures are required; compression with a snug bandage is always sufficient to control the oozing even if abundant at first. Every other day, after the operation, a straight, short, thick conical sound must be passed through the meatus to prevent reunion of the cut surfaces.

\section{Requirements for Operation}

Hypodermic syringe and needle.

Cocaine solution one-fifth of 1 per cent., or novocain $5 / 10$ per cent. Straight probe-pointed bistoury.

Lubricating jelly.

Bulbous bougies and sounds Nos. 26 to $32 \mathrm{~F}$.

Absorbent cotton and gauze.

\section{INTERNAL URETHROTOMY}

This operation or internal incision of the urethra is performed from behind forward when the calibre of the stricture will allow the passage of the dilating urethrotome (Otis) which is about 16 French (Fig. 119) scale; and from before backward when it is only possible to get by the contraction with a much smaller instrument in which case, the Maisonneuve urethrotome is employed (Fig. 120).

After the regular preparation and local anesthesia, for which purpose anesthetic jelly is injected and retained ten minutes (see p. 46, also p. 500), bulbous or olivary bougies well lubricated are passed into the urethra and the calibre of the stricture and its distance from the meatus measured. The distance of the stricture from the meatus is now measured off on the Otis urethrotome and the latter introduced to a point about $12 \mathrm{~mm}$. ( $\frac{1}{2} \mathrm{in}$.) behind the strictured area and held in such a position that the sharp edge of the knife is exactly in the dorsal median line against the roof of the urethra, corresponding to the fibrous septum that exists between the corpora cavernosa.

The penis being held moderately on the stretch, the urethrotome is dilated up to the required size and the knife drawn from behind forward, completely through the strictured area and then returned to its former position in its sheath (Fig. 121).

This double action is effected in a few seconds and should result in a complete division of the scar tissue. The urethrotome is then closed and withdrawn and a No. $30 \mathrm{~F}$. steel sound or bulbous 
bougie is introduced into the urethra to ascertain whether the reintroduction of the urethrotome is necessary or not. If not, two splints

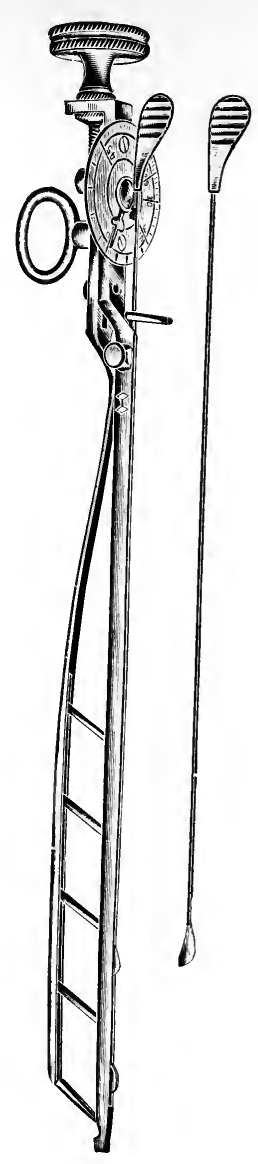

FIG. 119.-Otis dilating urethrotome.

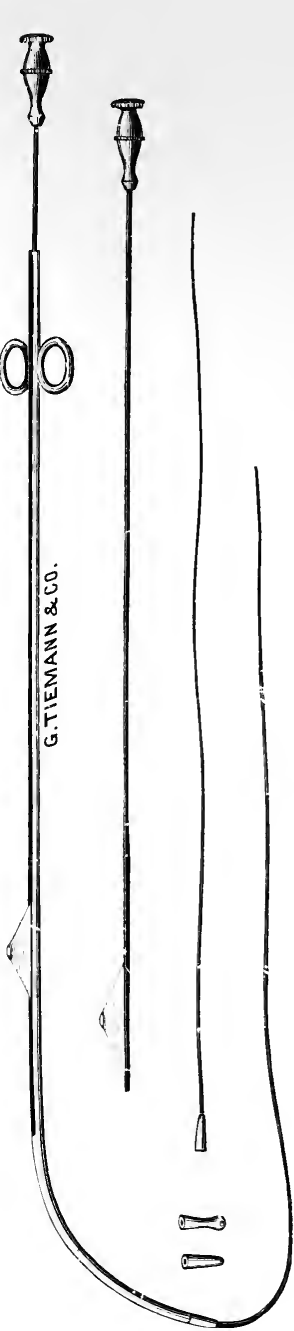

A

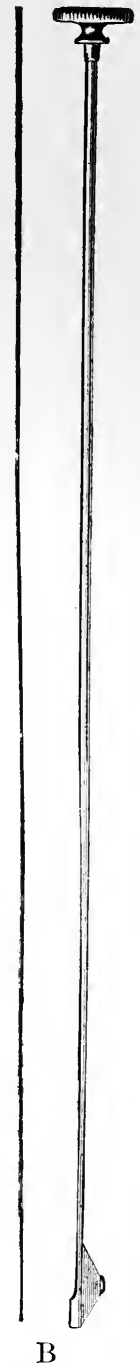

Fig. 120.-A. Urethrotome, B. Urethrotome blade with evelet and bougie. (Maissonneuve.)

improvised from ordinary cardboard, padded with a small layer of cotton and bound by a gauze bandage are applied. One splint is placed on the dorsal and the other on the ventral surface of the penis 
extending anteriorly to within $12 \mathrm{~mm}$. ( $\frac{1}{2} \mathrm{in}$.) of the tip of the penis and posteriorly to the root of the scrotum, fastened in place by strips of adhesive plaster. The amount of compression used should be sufficient to stop hemorrhage while not enough to interfere with urination.

The splints may be removed on the second day and within the week a sound is introduced to insure the continued patency of the canal. Some urinary antiseptic (hexamethylenamine or salol) is to be taken

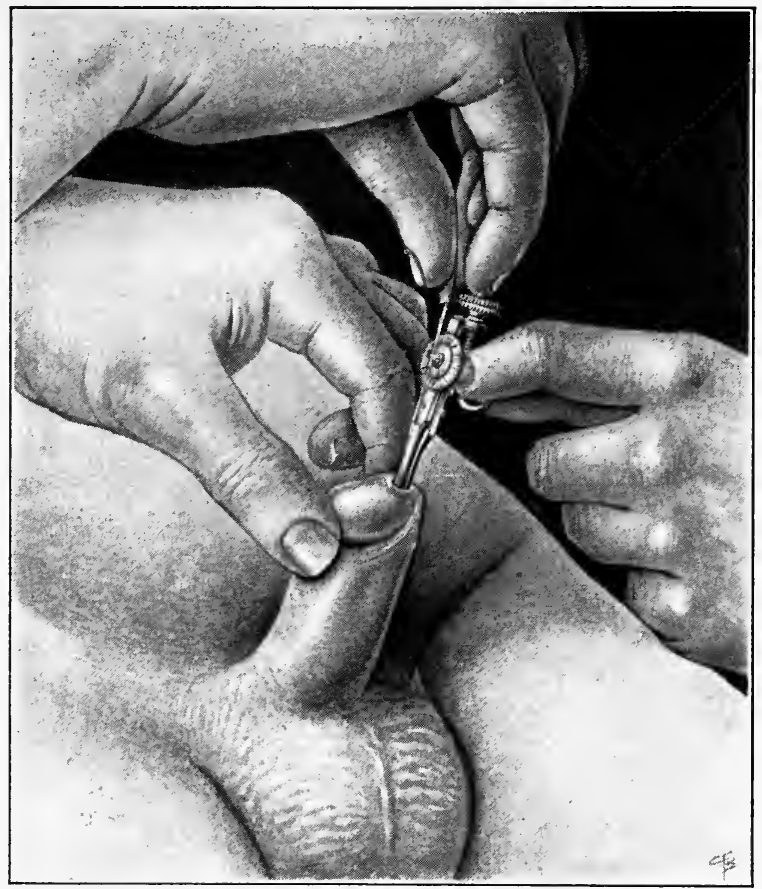

Fig. 121.-Internal urethrotomy. Incision from behind forward.

by the patient until healing has progressed satisfactorily. The use of cocaine with each introduction of the sound is to be condemned on account of possible post-operative bleeding and free absorption. The patient should be made to understand that cutting the stricture is not of necesssity a cure of the condition as recontraction is almost bound to occur, unless steps are taken at regular intervals to prevent it.

Strictures tighter than No. 15 French cannot be cut with the Otis instrument unless they are first dilated, as this urethrotome is about 
that calibre. In such cases, the Maissonneuve Urethrotome (Fig. 120,A) which has a calibre of No. 8 French is resorted to. This instrument is provided with a gum-elastic bougie of No. 3 French which carries a small metal end that may be screwed to the tip of the instrument and acts as a guide. The guide is first introduced, then the metal

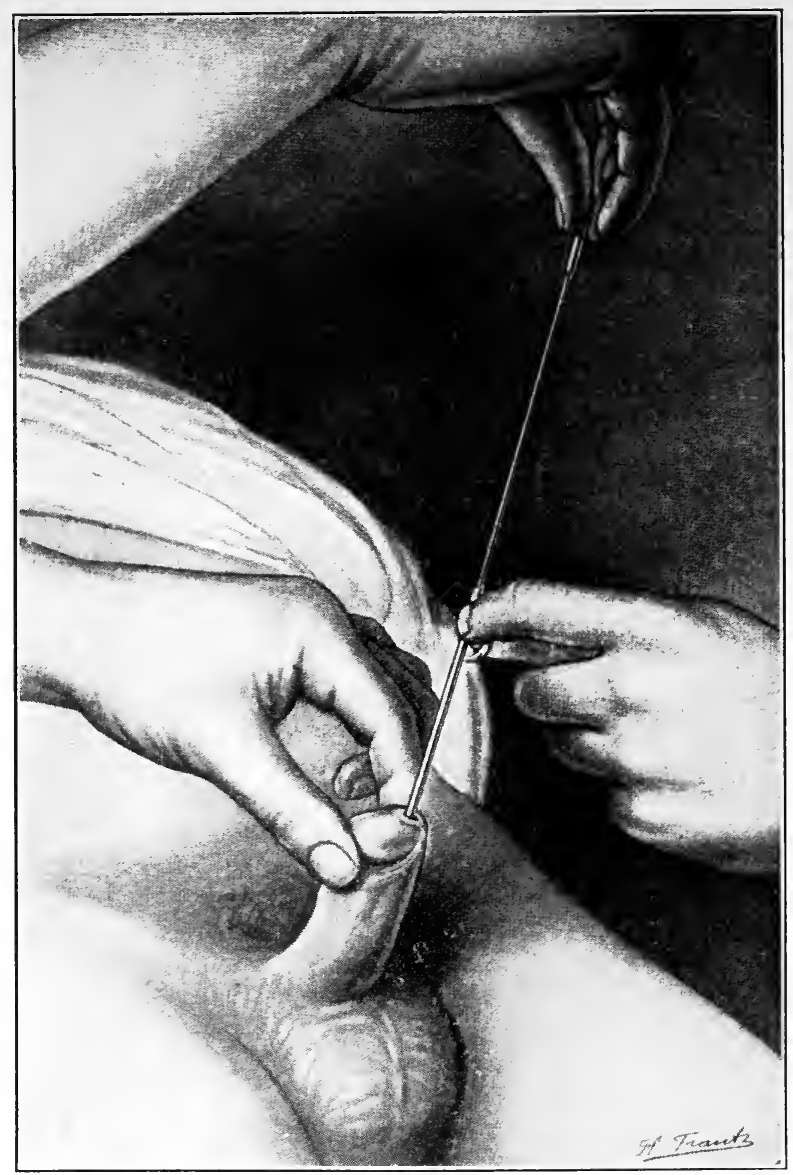

Fig. 122.-Internal urethrotomy. Incision from before backward. (After

grooved director is serewed on and passed through the stricture. The knife is now fitted into the groove toward the dorsum and pushed through the contracted area thus dividing the stricture from before backwards (Fig. 122). The knife and guide are now removed in the order named. 
Subsequent steps are the same as described after using the Otis instrument.

After the incision has been made, it is inadvisable to introduce instruments beyond the cut-off muscle without carefully cleansing the urethra with antiseptic irrigation to provide against the occurrence of urethral chill. For this purpose may be used permanganate of potassium 1 to 6000 , or silver nitrate, $0.065 \mathrm{gm}$. to $500 \mathrm{c.c}$. It is a common practice to install an indwelling catheter for twenty-four hours following the operation to drain the urine (Fig. 41, p. 52); and this may prove a helpful expedient to avoid pain and hemorrhage. In this case the splints are dispensed with.

In cases where the stricture is even too tight to admit a Maisonneuve, it is necessary to dilate the contraction with filiform bougies to the required calibre. Should this fail, external urethrotomy must be done, or retrograde cysto-urethrotomy (p. 187 and p. 473).

\section{Requirements for Operation}

Blunt pointed glass urethral syringe.

5 per cent. Alypin lubricating jelly for anesthesia. Bulbous bougies and sounds, No. 26 to 32 French.

Tape measure or ruler.

Otis dilating urethrotome.

Maissonneuve urethrotome.

Lubricating jelly.

Pasteboard splints for the control of hemorrhage.

Adhesive plaster strips.

Absorbent cotton.

Gauze finger bandage.

\section{EXTERNAL URETHROTOMY}

The technic of external urethrotomy is demanded in a number of surgical conditions and up to a certain point is the same for most of them; variation being required according to the indication for operation in each instance, which will be described under their respective headings.

The urethra is opened externally as a means of reaching the bladder for drainage (perineal cystotomy). The operation is the same whether it is necessitated on account of the requirement for drainage in the bladder itself or of the urethra. It is performed for stricture of the urethra behind the bulbous portion, for the complications and sequelæ of stricture and chronic inflammation of the urethra, namely, fistulæ, 
perineal abscess, and extravasation of urine, and maybe required in case of injury to or complete rupture of the canal.

The operation may be done under local anesthesia, but general

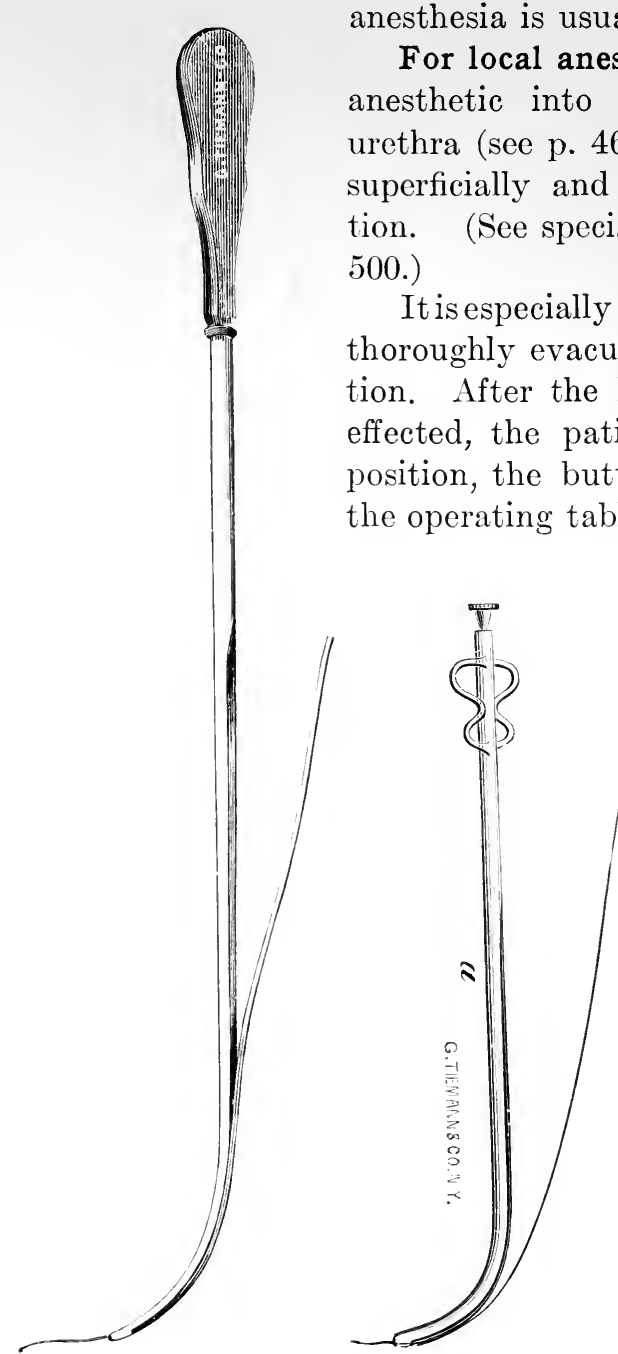

FIG. 123.-Grooved sound and catheter with filiform guide.

A staff or tunnelled sound is well lubricated and introduced into the bladder, or, if this is not possible, as in the case of tight stricture, a filiform bougie is introduced as a director and a grooved catheter or sound passed over it (Fig. 123). The guide, whichever it may be, is held steadily in the median line by an assistant, together with the penis and the scrotum. With a sharp scalpel, an incision $4 \mathrm{~cm}$. (1 1/2 inches) long is made in the perineal midline ending 12 $\mathrm{mm}$. (1/2 inch) in front of the anus. This incision divides the skin, subcutaneous tissue and fat. The point of the knife next finds the groove in the guide which is accomplished by directing it through the muscles with a steady thrust, while the assistant deflects the handle of the guide toward the patient's abdomen and makes the tissues bulge slightly in the operative field. 
After feeling the guide, which is easily recognized by the grating of the knife, the opening of the urethra is enlarged by cutting somewhat further along the groove of the guide; the straight, bluntpointed bistoury (Fig. 124) is now passed alongside of the scalpel until it touches the staff; the scalpel being removed, the operator holds the bistoury with one hand, and with the other grasps the handle of the guide which he introduces further into the bladder, following it with the bistoury for about $2 \mathrm{~cm}$. (3/4 in.). A grooved director or gorget canula (Fig. 125) is now passed alongside of the bistoury into the posterior urethra or bladder, and the bistoury removed together with the guide. A female catheter is guided alongside the grooved director into the bladder, the escape of urine from this or from gorget canula demonstrates satisfactorily that the bladder is entered. The catheter is now removed and the index finger is introduced to explore the posterior urethra and the bladder outlet.

Stricture of the urethra anterior to the incision may be cut either by the blunt pointed bistoury guided by a grooved director, or the staff brought out through the incision and reversed so that its groove

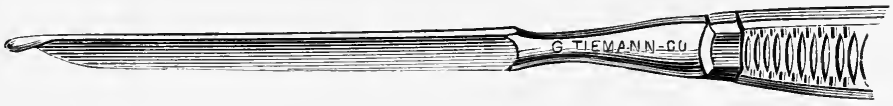

FIG. 124.-Blizzard knife for perineal section.

looks toward the pubic arch, or with the urethrotome as already described, making a combined external and internal urethrotomy (see p. 283); this division of the stricture should be on the roof of the urethra.

The next step when operating for stricture is to pass a full-sized sound, making sure that the urethra is continuous and that no false passage exists; if any be found, it is necessary to incise it on a level with the urethra, leaving as smooth a canal as possible. Any bleeding vessels must be ligated, using small plain catgut for such purpose, although it is seldom necessary to tie any vessels in a simple urethrotomy: the oozing from the small vessels and the cavernous tissue being controlled by compression with gauze pads placed around the perineal tube held in place by a " $\mathrm{T}$ " bandage. The perineal tube which is used for drainage of the bladder after perineal section, should have a No. 30 French calibre, although a smaller size may be employed where there is not much hemorrhage or oozing. It is important to place the tube properly in the bladder so that fluid injected returns freely. A good perineal tube should have an opening at the end and also a large lateral eye (Fig. 126). 
The perineal tube of solid metal (Alexander) or that with an irrigating attachment (Hagner) may be preferred in some cases where bleeding is free to make reinsertion more easy in case it has to be removed on account of blood clots.

It is important to place the perineal tube so that it will drain to the best advantage by measuring the distance to the bladder with the finger through the perineal wound.

When satisfactorily placed it is securely fastened by a stitch through the lips of the wound of silkworm gut which is made to encircle the tube.

Bleeding from the perineal wound is controlled by packing with gauze impregnated with formaldehyde gelatin (Glutol) mostly toward the bulb of the urethra as most of the hemorrhage comes from that point. The gelatin though usually dispensed with, is occasionally very useful.

Bleeding behind the bulb is also controlled by packing (Fig. 192) aided by injections into the bladder of alum solution, one dram of which is added to a pint of hot, sterile water. ${ }^{1}$ Pads of gauze slit to their centre are slipped over the tube and held against the perineum by a snug " $\mathrm{T}$ " bandage. After the patient is returned to bed a large glass coupler is fitted to the perineal tube and connected with a rubber tube of $32 \mathrm{~F}$. calibre sufficiently long to reach a large bottle placed alongside the bed to eatch the drainage. The connecting tube should be supported by a sling of tape or adhesive plaster so that it does not pull on the perineal tube.

The after care of the patient is important. Besides the ordinary discomforts following all surgical operations, there often exists an intense desire to Frg. $125 .-$
Probe-pointed grooved catheter sufficiently from the anesthetic to understand, he must staff (Tilden be acquainted with the conditions of drainage from the
Brown.)

bladder and the fact that the tube acts as a foreign body, causing his desire to urinate, but that yielding to this is useless and simply favors bleeding. This explanation always has a good effect, and gains the confidence and coöperation of the patient. Morphine sulphate, $.016 \mathrm{gm}$. (1/4 gr.) hypodermatcially should be given

I Squibb's Compound Alum Powder is the preparation used for this purpose. 
and repeated if pain and tenesmus persist. As a rule the tube is well tolerated after twelve to twenty-four hours.

An experienced nurse will appreciate the importance of watching for hemorrhage, for insufficient drainage from the tube, and excessive. pain. A staining of the dressings around the site of operation is of no consequence and is to be expected; but if the oozing becomes abundant, enough to wet them through thoroughly, extending upon the draw sheet, especially if it is of a bright red color, there may be active hemorrhage of which the surgeon should be notified at once, and the cause ascertained and corrected. It may be necessary to reopen the wound and repack, although this is seldom the case. Fresh bleeding is commonly due to retention of urine and damming back of blood into the bladder, causing the patient to strain continuously. Thus a vicious cycle sets in; the blood clogging the eye of the tube causes retention of urine; the patient's straining to expel the latter causes more bleeding and so the symptoms become more and more urgent until an alarming condition develops. It is necessary to give clear and explicit instructions to the nurse to observe the quantity of drain-

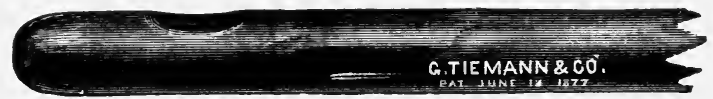

Fig. 126.-Perineal tube.

age (half an ounce to an ounce an hour for the first twenty-four hours being normal), and to order sufficient morphine to dull the painful sensations kept up by the presence of the tube in the bladder. Perhaps the straining to urinate may be due to a faulty position of the tube which has worked out of position; it may be simply clogged by blood; or there may be a kink in the perineal tube or in that attached to the glass coupler. To investigate the cause of improper drainage the glass coupler and connecting tube should be disconnected from the perineal tube and cleaned; then with a hand syringe, warm boric solution is injected in small amounts, half an ounce to an o unce at a time, with a series of short, quick jerky motions of the pl unger, in order to displace any clots that may be in the eye of the tube; the fluid returned through the tube should be measured and note taken of the amount, compared with th at injected; if many clots are obtained, they are commonly the cause of trou ble. If only part or none at all of the fluid returns, there may be a mass of clotted blood in the bladder or the tube is either in too far, or what is more likely out too far, the eye being in the posterior urethra. Uncontrollable straining, with 
or without hemorrhage, indicates marked intolerance of the bladder and in such cases it is wise to take away the tube at once. Twentyfour hours after the operation, the packing, if any has been used, is removed; it is well to have it moistened by injecting boric solution, or 25 per cent. peroxide of hydrogen in water, every ten minutes for an hour before removing. As it is impossible to know whether or not the removal of the packing will start up fresh bleeding, it is slowly and carefully withdrawn while watching for signs of free bleeding. If none occurs, it should be all removed at once; but if there is much bleeding the removal is temporarily stopped and a pad of gauze held firmly against the perineum for a minute or so to decide this question. If no further bleeding is seen, and this is usually the case, a little more packing, or all of it is taken away; if however, bleeding continues, it may be necessary to wait twelve or twenty-four hours before making another attempt at removal. Daily irrigations of boric solution or silver nitrate ( 1 to 16,000 ) should be given. The tube is left in the bladder for forty-eight hours in simple cases and longer in other cases according to conditions. This is governed by the advantage to be derived from bladder drainage. It is well at this juncture to advise the patient of what will follow. The urine, all of which may pass through the perineal wound at first will come gradually more and more by way of the urethra. The change is sometimes quite sudden, sometimes slow. There may be temporary relapses of perineal urination before complete healing which occurs in two or three weeks. This explanation saves much disappointment to the patient.

Balsam of peru and castor oil, equal parts, is an excellent application to promote granulation and hasten healing.

\section{Requirements for Operation}

Tunnelled sounds and filiform bougies. Soft rubber and silk woven catheters.

Double tapered sounds No. 26 to No. 32 French.

Scalpel and blunt pointed straight bistoury.

Tube of lubricant.

Grooved director;

Female silver catheter $\}$ or gorget canula (Fig. 125).

Artery clamps.

Two pair of thumb forceps.

Perineal tube (rubber or solid).

Needles and needle holder.

1 tube No. 1 plain and chromic catgut. 1 tube of silkworm gut. 
Gauze packing; gauze pads for perineal tube; "T" bandage.

Bladder syringe, 100 e.c.

Boric and alum solutions.

\section{SUTURE OF THE URETHRA}

As already stated, wounds of the urethra usually heal satisfactorily; partial loss of structure is supplied by the process of granulation; and, as there is a tendency to subsequent narrowing of the lumen following suture of the urethra, it is proper to keep this fact in mind. Longitudinal wounds should be sutured transversely and thus provide a widening of the lumen (Fig. 127).

Complete circular suture of a transverse wound is undesirable. In such cases, it is better to establish the continuity of the canal by partial suture only upon the roof leaving the sides and the floor to reunite by granulation (Fig. 127, II and III).

Operation for Traumatic Rupture of the Urethra.-A blunt instrument is passed through the anterior urethra until it can be felt in the perineum. The usual longitudinal incision for external urethrotomy is made or in severe cases with a great deal of contusion of the parts requiring more room, the reversed $\mathrm{Y}$ or $\mathrm{V}$-shaped incision for prostatectomy is employed (Fig. 209, p. 481). All extravasated blood is washed away from the tissues to bring them as nearly as possible into their normal relation. The anterior section of the urethra is found by means of the guide. As much of this portion of the urethral tube as may be necessary is exposed even to the extent of cutting completely through the bulb. This should be done as nearly as possible in the middle line (Fig. 127). If there is incomplete rupture, it is easier to follow backward the unretracted posterior portion by means of a probe or grooved director which will lead into the prostatic canal and thence to the bladder. If there is complete rupture, however, this is much more difficult and a prolonged search is required to find the proximal end. Failing in this, it is necessary to open the bladder above the pubis and perform retrograde catheterism, or retro-vesical urethrotomy through a supra-pubic cannula (see p. 473). When the two ends of the urethra are found, it s necessary to suture the lacerated portion so that the roof and sides are reunited but the floor is left open (Fig. 127, II). If the bulb has been bisected it should be reunited as carefully as possible to help to control the bleeding. An indwelling catheter is inserted through the urethra and the perineal wound is only partially closed so as to provide adequate drainage to the sutured urethra and relieve it of any undue tension. 
Resection of the Urethra.-In that type of stricture which is styled tortuous or innodular and especially in traumatic stricture it may be necessary to resect a portion of the canal in order to establish a satisfactory calibre that can be maintained. The urethra is exposed by perineal incision down to the strictured portion (Fig. 127). The

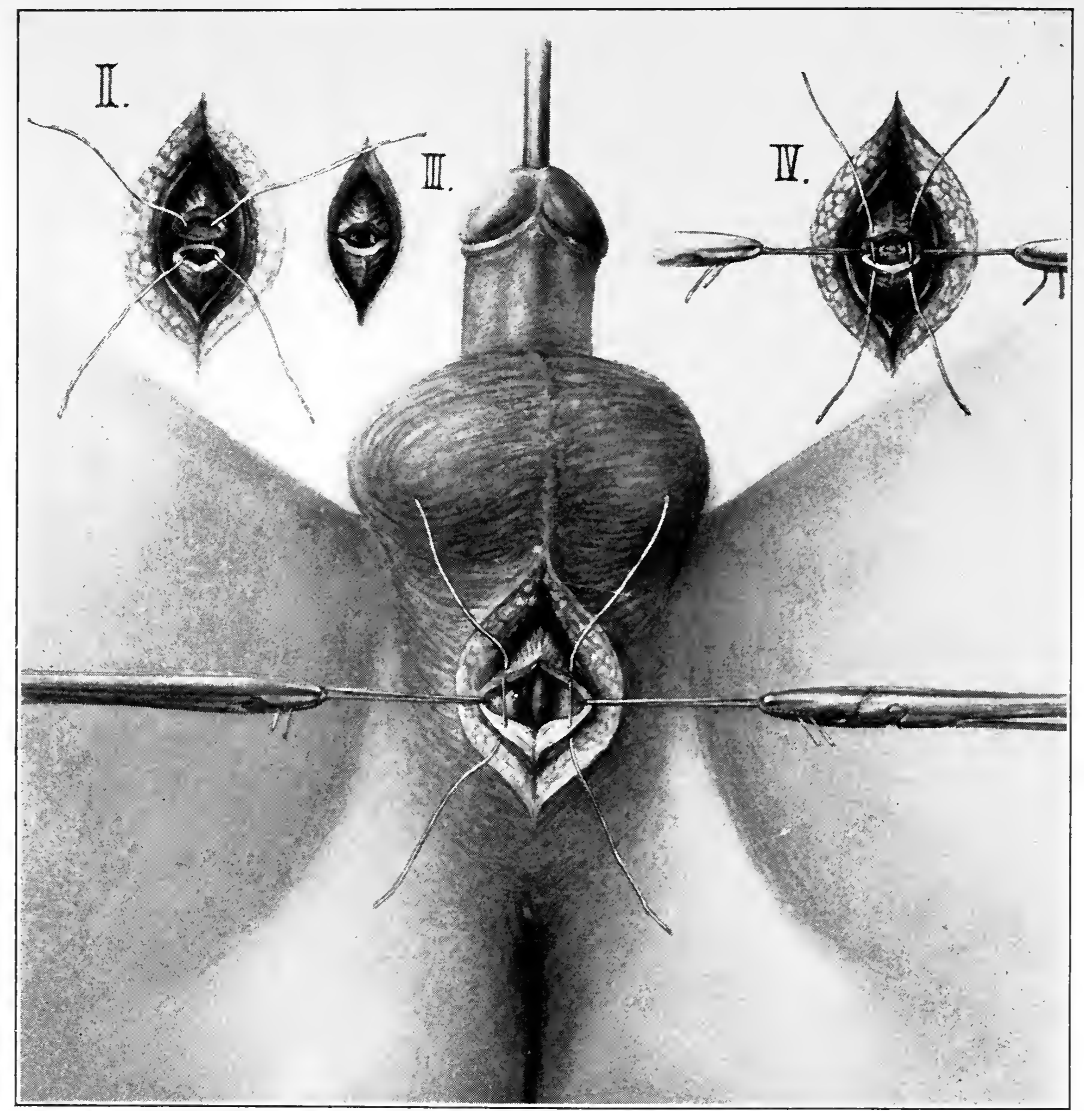

Fig. 127.-Resection of urethra. Suture of longitudinal slit to prevent contraction. II and III, End to end union leaving vent to be filled in. IV, End to end union complete suture.

cicatricial area is dissected away as well as any fistulous tracts connected with it. The two ends of the urethra are then approximated by suture as in the case of rupture of the urethra (Figs. 127, II and III). This is performed without difficulty if the gap is short and if not, the anterior portion of the urethra can be freed from its bearings with little difficulty and reunion obtained without undue traction. To avoid ten- 
sion, it is important to freely mobilize the urethra. The amount of dissection required for this purpose varies in different cases (Fig. 127, IV).

The avoidance of infection and infiltration in the region of the sutured urethra is best attained by adequate drainage down to the point of union and the prevention of undue tension upon the newlymade watershed. This is assisted by keeping the urethra clean

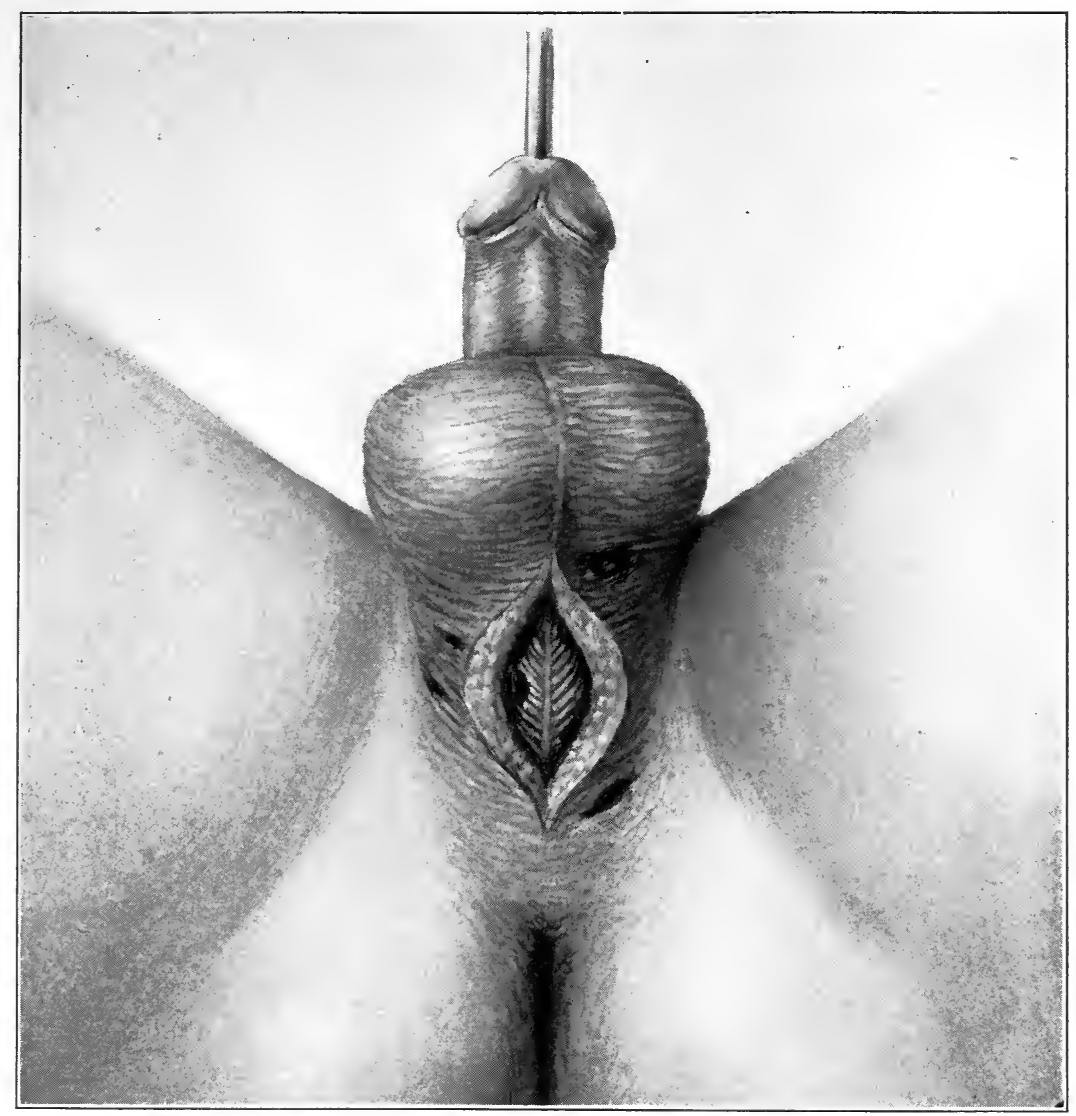

FIG. 128.-External urethrotomy for perineourethral abscess and fistulæ.

and free of accumulated discharge. The indwelling catheter is used in some cases; in other instances the urethra is tapped behind the suture line and a drainage tube inserted into the bladder at that point. $^{1}$ I have obtained excellent results following complete suture and the insertion of an indwelling catheter. The important point

1 Hugh Cabot (Boston Med. and Surg. Jour., Vol. CLXI, Dec. 9, 1909) prefers this expedient and has developed a special technic as a result of a combination of different methods. 
in such cases is the provision of drainage from the outside of the sutured urethra through the external wound.

Periurethral Abscesses.-Those of the penile portion resulting from the extension of a folliculitis demand only a longitudinal incision over the most prominent point followed by evacuation of the pus, swabbing of the cavity with hydrogen peroxide and packing. Perineal periurethral abscesses, which are localized urinary infiltrations, are opened by a long, median incision, down to the urethra, even

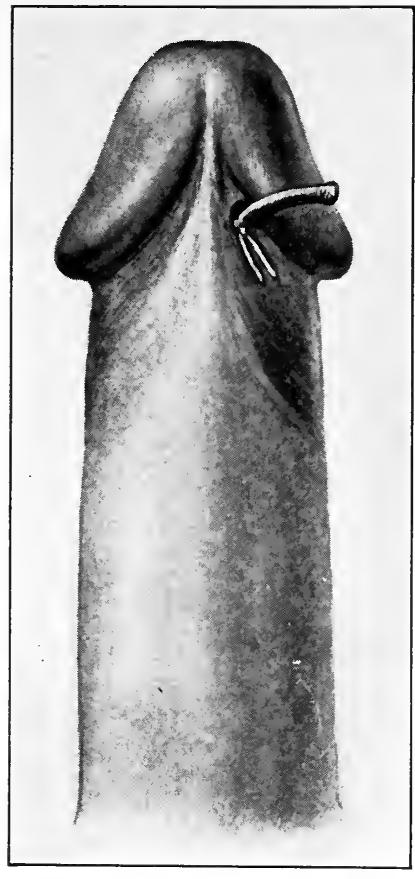

FIG. 129.-Excision and ligation of fistula near meatus. though the principal point of swelling is lateral (Fig. 128). All recesses are incised or broken open by the finger and a drain introduced up to the highest point. Diffuse urinary extravasation demands, besides the median incision, free incisions elsewhere wherever there is evidence of tumefaction. The unhealthy tissues in such instances must be freely disinfected with 1 to 20 carbolic acid followed with pure alcohol or with equal parts of tincture of iodine; and necrotic tissues are removed with scissors or by a sharp currette. As a sequel to these severe conditions urethral fistulæ are common occurrences, hardly to be aroided. These are treated at a later date by proper surgical measures.

Operation for Urethral and Perineal Fistulæ.-Fistulæ of the penile urethra may or may not require preliminary perineal drainage to ensure complete closure. Fistula of the fossa navicularis which is a more or less common sequel of follicular abscess in this locality has proved often an obstinate condition. Some small apertures at times may be closed by cauterization with the high frequency current (see p. 153). Other cases are successfully mastered by a simple operative technic, as follows:

After cocainization of the immediate surrounding area, the exterior orifice is encircled by an incision through the skin. The fistulous tract, which is usually irregular, is then dissected throughout its entire course down to but not through the urethra. This isolated, tubular tract is then ligated close to the urethra, the ligature as well as the 
fistulous tract being left long, and the wound is permitted to remain entirely open to granulate (see Fig. 129). In a few days, the ligature comes away with the sloughed tract leaving a granulated surface which usually closes up the urethral fistulous opening.

Fistula of the pendulous urethra lower down may be slight or extensive. Operation involves excision of the tract and surrounding fibrous tissue. The method of applying sutures will then depend upon the

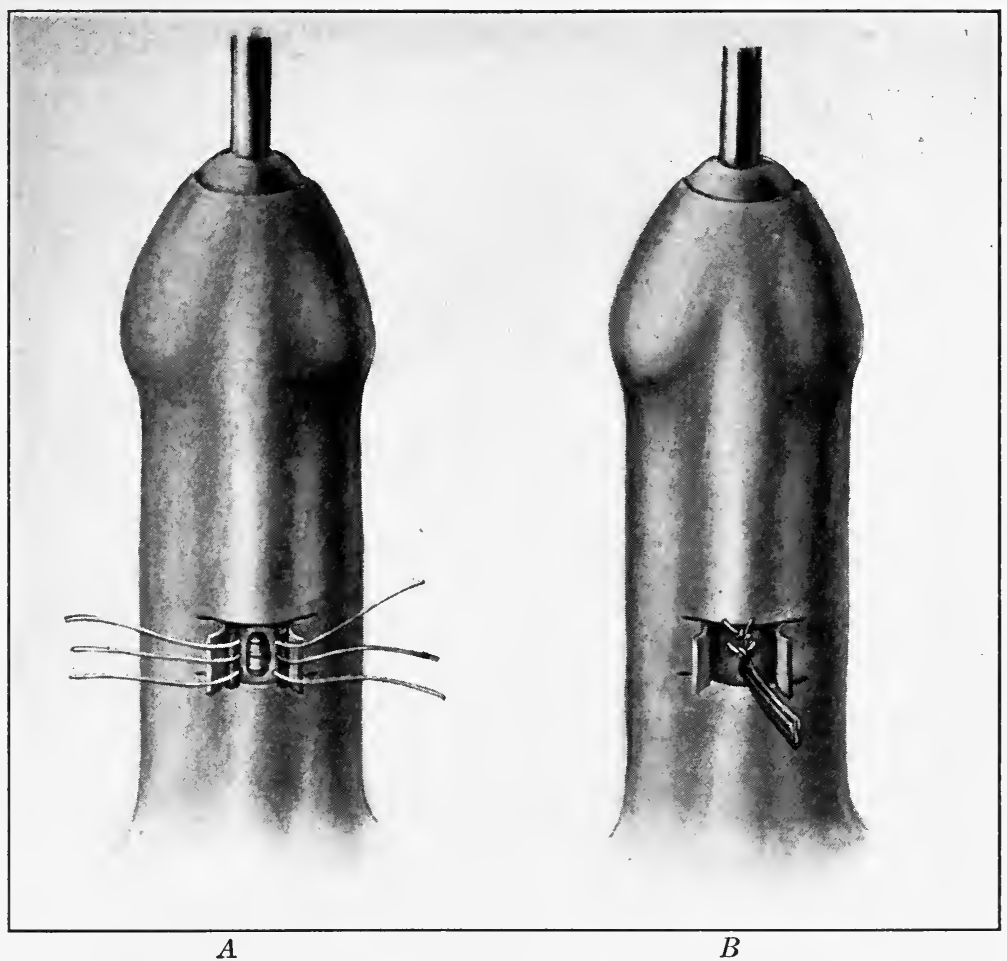

Fig. 130.-Operation for anterior urethral fistula. $A$, Suture of mucosa and submucosa. $B$, Suture of muscularis.

most available tissues for the purpose (see Suture of Urethra). The principle involved is the same in all cases and that is careful union, layer by layer of the mucosa, submucosa and muscularis and particular observance of the rule to carry a drain down to the sutured urethral cylinder (Fig. 130). I have operated successfully on many cases by this method. If perineal drainage is not arranged for, the indwelling catheter is employed (Figs. 41 and 42, p. 52). Drainage in either instance is maintained for about a week. 
The instruments, etc., required are about the same as are used in External Urethrotomy (q. v. p. 187).

Operation for Perineo-urethral Fistulæ. -The patient is placed in the lithotomy position, a tunnelled sound or guide introduced into the bladder and a perineal section performed with exposure of as much of the urethra as may be necessary in each individual case. When the fistula is located, the tract and infiltrated tissue are then excised as in resection of the urethra (see also Peri-urethral abscess).

The skin and muscular tissue are separated by dissection in their respective layers and their freshened edges united by interrupted sutures of chromic catgut, silkworm gut being used for the skin. A small cigarette drain is inserted through the skin and the deeper layers of muscular tissue down to the sutured urethra and emerges through the most dependent portion of the wound near the anus. An indwelling catheter is introduced or an opening is made into the urethra at another point behind the sutured portion and a tube is inserted therein for drainage. The drain is removed in twenty-four hours. The catheter is left in place six or seven days during which time the bladder is irrigated regularly twice a day with boric acid solution.

(For other operations upon the urethra see section on Bladder and Prostate.) 


\section{CHAPTER XVI}

\section{MALADIES OF THE TESTICLE, SPERMATIC CORD AND COVERING}

The Scrotum.-A deformity of the scrotum forms a part of that congenital malformation called hypospadias (q.v.). Other malformation occurs only in connection with abnormalities of the testicle (see p. 302).

Injury and Wounds of the Scrotum.-The scrotum, like other portions of the body, may be subjected to wounds of different kinds, contusions, lacerations and puncture. These may occur without injury to the testicle.

Contusion of the scrotum, not involving the testicle, causes no such excruciating pain as is the case when the latter organ is injured. On account of the great vascularity, there is likely to be much extravasation of blood which sometimes distends the sac to considerable size. If the distension is extreme, gangrene may supervene, but usually, under suitable applications of cold lotions, lead and opium wash, aluminum acetate or the ice-bag, together with suspension upon an adhesive band drawn across the thighs, the blood is absorbed without suppuration or inflammation. If abscess formation occurs, incision and drainage are demanded.

Lacerated or punctured wounds of the scrotum are generally accompanied by free hemorrhage. They are treated on general antiseptic principles and usually heal with surprising promptness.

Infected wounds should be treated as elsewhere with appropriate drainage and wet antiseptic applications.

Cellulitis of the scrotum occurs as a result of injury, and suppuration may take place. When there is great distension and bogginess of the tissues, it must be distinguished from rupture of the urethra anterior to the triangular ligament when the extravasated urine finds its way from the cellular tissues into the scrotum. The coexistent stricture and the more profound systemic involvement in the latter condition should prevent any mistake in diagnosis. Edema of the scrotum also occurs as a result of the systemic expression of heart and kidney disease.

Emphysema or pneumoscrotum occurs in consequence of the direct entrance of air into the tissues of the scrotum through a wound from 
the outside or from a cavity left by an extraperitoneal abdominal wound, when it finds its way around the tissues of the cord; or as a result of general emphysema due to the escape of gas from one of the hollow viscera. It is more likely to be the result of inflammation due to infection with certain gas-forming microörganisms, in which case it is likely to be the accompaniment of emphysematous gangrene.

The treatment is on the general surgical principles of free incision and drainage and removal of the necrotic tissue.

Elephantiasis of the Scrotum.-Lymph-scrotum. Beside the forms of edema already mentioned, the tissues of the scrotum may assume extraordinary dimensions from interference with the lymph circulation. This disturbance is due to the extensive deposit of connective tissue after an operation upon, or wound of, the scrotum following the removal of the inguinal lymphatic glands. In rare instances, it is due to the presence of filaria sanguinis hominis, which, being present in the lymph channels, acts as the obstructing cause of the impeded circulation. This malady is endemic in tropical regions but is rare in this part of the country.

The treatment of the mechanical type of lymph-scrotum consists in the application of wet, evaporating lotions, of alcohol and glycerine or aluminum acetate, and if these mild measures fail, surgical intervention, involving amputation of the redundant tissues, is demanded.

In the parasitic form of this malady, operation is less hopeful on account of the difficulty in reaching the parent filariæ. ${ }^{1}$

Cutaneous Affections of the Scrotum.-The scrotum is subject to various cutaneous lesions which may be part of a generalized eruption or may be localized.

Eczema.-Eczema frequently attacks this portion of the body on account of the susceptible texture of the skin and owing to the irritation to which it is subjected by the exudation of perspiration in warm weather. Persons of gouty and rheumatic diathesis and those who tend to obesity are especially susceptible. The appearance is that of one or the other type of cutaneous eruption. Commonly, it is papular or squamous and the symptoms are those of itching and burning.

The treatment demands cleanliness of the parts and the aroidance of friction, which is aided by the use of a suspensory bandage and the application of an appropriate dusting powder such as stearate of zinc, Lassar's zinc oxide paste or plain zinc oxide ointment. In some

\footnotetext{
1 Cunningham of Boston, Annuls of Surgery, October, 1906, quoted in Watson and Cunningham's treatise, reports the successful result obtained by operation in one case although the parent worm was not found.
} 
instances, the addition of a small quantity of tar (picis liquida) in the latter ointment (1 part to 10 or 12) is useful.

The therapeutic action of the X-ray by means of the Coolidge tube has proven in some instances a sovereign remedy.

Erythema intertrigo is a superficial irritation of the scrotum, occurring mostly in children who wet themselves and patients who suffer from urinary dribbling, especially when the urine is rendered more irritating on account of a gouty or rheumatic diathesis. The treatment requires extreme cleanliness and protection of the parts, and the application of a mild dusting powder, such as oleate or stearate of zine which, if applied frequently, will protect the skin from moisture. Again the Coolidge X-ray tube is a valuable expedient.

Pruritus is an exceedingly annoying condition when it attacks the scrotum and adjacent tissues. It occurs more particularly in neurotic, diabetic and rheumatic individuals. The tendency to irritate the parts by friction is often so irresistible that thickening of the skin and dermatitis are likely to result. The treatment, when effective, is a source of great comfort to the individual. It consists in the exercise of cleanliness, the wearing of a suspensory bandage to keep the parts separated, and the use of a local sedative together with a dusting powder. Stearate of zinc, talcum, calamine lotion (pink wash) are generally mentioned but not always effective. X-ray treatment will often succeed where other measures fail. In all cases of pruritus, careful search for the presence of pediculi should be made.

Pediculi Pubis.-These parasites infest the genitalia including the scrotum. They may be propagated from one person to another and from intermediate source (public toilets). The parasites attach themselves to the skin and the ova to the hairs. The common treatment is the application of the blue or mercurial ointment. One or two applications of this usually effect a cure. It is not always necessary to shave the parts. The tincture of delphinium or larkspur is also employed with success. Other applications favorably spoken of, are powdered calomel or calomel ointment (calomelol). The latter is much more agreeable than the dirty blue ointment.

Scabies, when found on the scrotum, is a part of the general invasion of this parasitic malady and calls for the most painstaking. attention to the directions laid down in the ordinary method of treatment, consisting of the disinfection of all clothing and the application, on several successive occasions, of strong sulphur ointment.

Tumors of the Scrotum.-Benign growths attack the scrotum as 
elsewhere. The skin of the scrofum is replete with sebaceous glands and hence is the seat of small cysts of this type. They are harmless and need not be removed unless in deference to the wishes of the subject or unless they attain undue dimensions.

Molluscum contagiosum attacks the scrotum of children, and consists of small, waxy, endermic cysts which become umbilicated. They may disappear spontaneously but are contagious and should be treated separately by evacuation of the sac contents and complete cauterization with phenol or preferably creatin.

Carcinoma of the Scrotum.--Epithelioma or chimney-sweep's cancer is the most common type of malignant growth that attacks the scrotum. It makes its appearance at first as a watery nodule of slowly progressive growth; following the formation of a scab, excoriation occurs, exposing a deep ulceration with uneven, granulating surface and undermined, indurated margin. If not checked, this malignant growth extends more rapidly and may involve the entire scrotum or extend inward and include the testicle. Death may occur from progressive, constitutional depreciation, preceded, perhaps, by severe hemorrhage. Treatment should be commenced as early as possible. If discovered before ulceration has taken place, extirpation of the nodule and free cauterization may cause a subsidence; later, free excision of all the parts involved, including the inguinal glands, becomes necessary.

Other tumors of the scrotum are lipomata, enchondromata, osteomata and sarcomata. These are not frequent and require no special description; their treatment is surgical.

Gumma of the scrotum occurs but its consideration belongs essentially to the disease underlying it (see Chapter XL).

\section{ANOMALIES OF THE TESTICLE}

\section{Anomalies of the Testicle occur in}

$a$. Number; $b$. size; $c$. location.

Anomalies of Number.-Complete absence of the testicles and their adnexa is exceedingly rare (anorchism). Absence is usually unilateral or there is a fusion of the two glands which may be intra-abdominal; on the other hand, the testicle may be present and the epididymis or vas absent. If both testicles are absent, there is an associated rudimentary condition of the other genitals. The alleged existence of a supernumerary testis has, in most instances, been explained by some pathological condition such as a separated epididymis, a fibromatous growth or an encysted hydrocele. 
Anomalies of Size. ${ }^{1-A t r o p h y}$ of the testicle usually occurs in the undescended gland. In some instances, the position of the atrophied organ is normal. The size may remain stationary or increase. The nature of this condition is a congenital defect, the cause of which is unexplained.

Enlargement of one testicle may exist and usually occurs as a result of a deficiency in size of the opposite organ or its total absence.

Anomalies of Location.-The testicle may be arrested at any point from its primordial abdominal location to its destination in the scrotum (cryptorchism) or it may wander from its normal location to some distant site (ectopia). Retained testicle may be unilateral or bilateral. It may be retained in the abdomen, in the inguinal canal or in the external abdominal ring.

A shortening of the vas deferens has been mentioned as a cause of retained testicle and in operating, this difficulty has been taken into account. It has been satisfactorily and frequently proven, however, that such is not the case- that the vas deferens is not shortened when the testicle is retained but of ample length, and indeed, that it sometimes extends downward into the scrotum and back again to the site of the retained gland. On the other hand, it has been equally well demonstrated that the spermatic arteries are shortened and it is suggested as very likely that this anatomical defect is responsible for the condition of undescended testes. At all events, successful results in operating have been attained by taking this fact into account and in returning the testicle to its normal location, after ligating these arteries, preserving with great care the artery of the vas deferens as the source of blood-supply. ${ }^{2}$

Atrophy usually results on account of the abnormal location of the undescended testicle and when placed in the inguinal canal, it is subject to injury and prone to inflammation and even malignancy

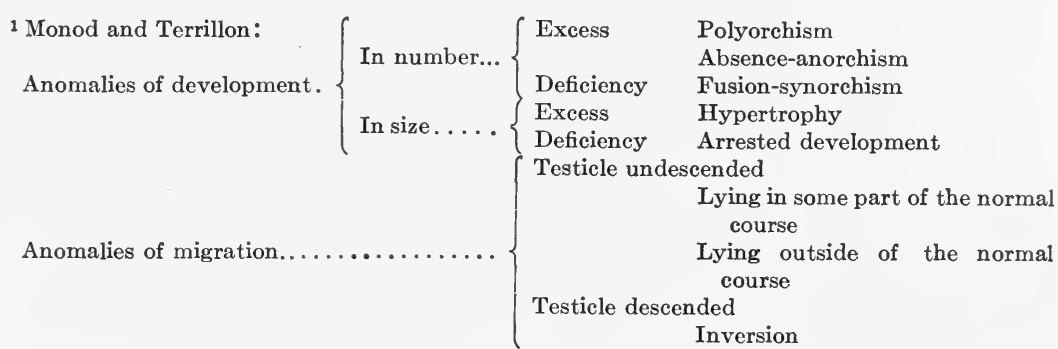

z Moscheowitz, "Annals of Surgery," December, 1910, calls attention to Bevan's operation for retained testicle and emphasizes the soundness of his claim with regard to the length of the vas deferens and shortness of the spermatic arteries in these cases. The writer, following in the footsteps of Bevan, has reported a striking array of cases, all cases without exception being attended by good results. 
A common complication of retained testicle is that of hernia, on account of incomplete closure of the peritoneal sac accompanying it.

The testicle may be undescended at birth and descend to its normal position later on. While this may occur after the expiration of several months, it is less likely after the lapse of one or two years. On the other hand, a descended testicle may. from injury or strain, be dislocated backward into the abdominal ring and remain there.

The coverings of the testicle may be anomalous in that the scrotum may consist of two separate chambers, the testicles therein being normal otherwise - a defect in fetal development. The testicle may be partly or completely inverted in the scrotum when it is more likely to be in the upper and anterior portion, an important fact in connection with the introduction of the needle for aspiration of hydrocele.

Ectopia implies the migration of the testicle outside of the abdominal cavity to some point distant from its normal location. This may be in the region of the abdomen, in the perineum or beneath the skin of the thigh.

The treatment of misplaced or undescended testicle is surgical and entails transferring the organ from the abnormal to the normal position. This has been accomplished when the organ is outside the abdomen in the inguinal canal or elsewhere but is not so easy when it is retained in the abdominal cavity. The alternative operative course is orchidectomy. If operative interference has been postponed until after puberty, the organ, so far as its functional activity is concerned, may be useless, therefore, operation should be undertaken in early youth.

If the retained organ is a source of continuous pain or the seat of recurring inflammation, operation is imperative and it should be removed rather than left in its abnormal position if it is deemed impossible to accomplish successfully the operation of placing it where it belongs as atrophy always occurs and malignancy is imminent.

I operated upon a case in which the body of the testicle was in the internal abdominal ring and the globus major end of the epididymis was in the external ring. The latter had undergone purulent inflammation as a result of injury and was the source of continuous pain. In this instance, removal was the natural means of relief (Fig. 131). (For description of the operation for Undescended Testis, see p. 359.)

Anomalies of the Spermatic Cord.-In the normal anatomical arrangement, the vas deferens connects the testicle and the seminal vesicle and the prostate. The vasa deferentia open separately in the utricle on the floor of the prostatic urethra or, exceptionally, they 
may fuse at that site. Under anomalous conditions, the cord may be absent in part or entirely, which may occur in spite of a normally descended testicle. Other anomalies comprise a fusion of the two cords into one or a double cord on one side. During fetal life, there is a communication between the ureter and the vas and this may persist after birth.

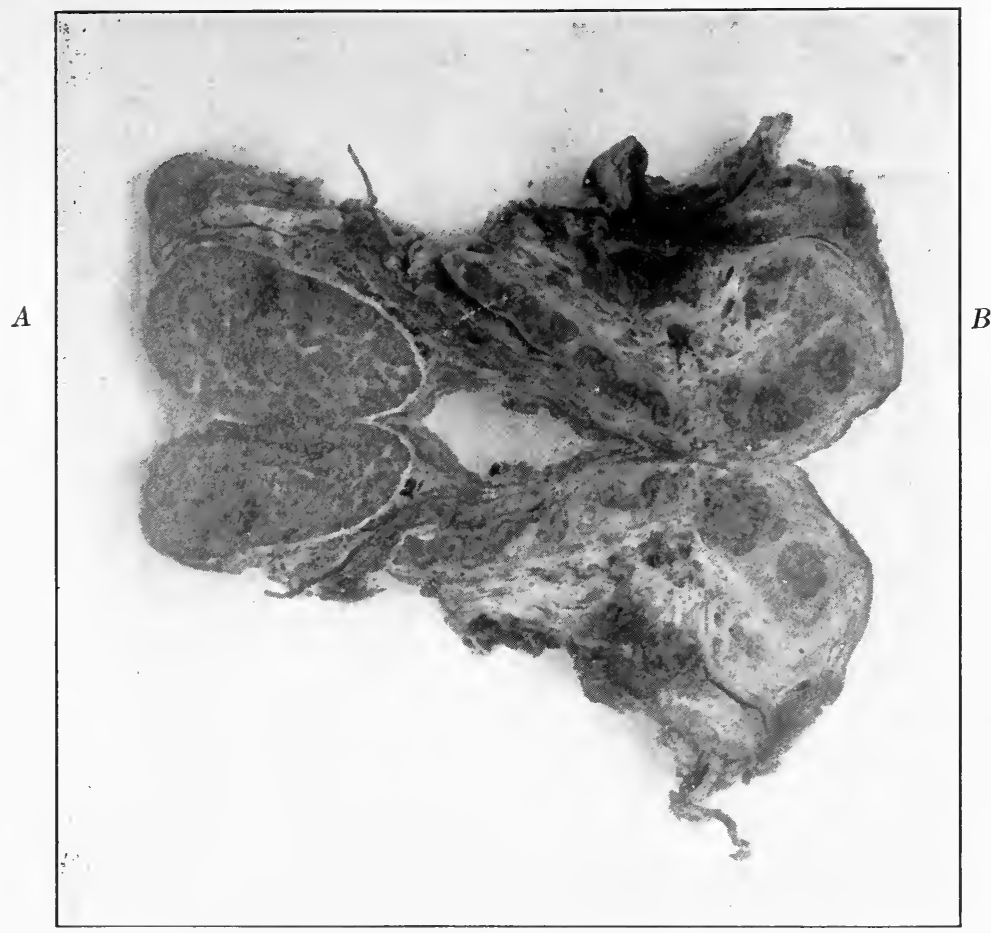

FIG. 131.- Specimen of retained testicle. (Author's case.) A, Abdominal testicle. $B$, Aberrant epididymis.

\section{WOUNDS AND INJURIES OF THE TESTICLE}

On account of the protected situation of the testes in the sac of the scrotum and of their free mobility, injury of these organs is rendered less likely. When injury of the testicle is associated with a wound of the scrotum, this fact is made known by the excruciating pain which ensues which is likely to cause the subject thereof to faint. The cause may be a direct blow or fall astride some oblong object or in connection with riding a bicycle or horse. If the testicle is already the seat of 
previous disease, especially if it be tuberculous, it becomes susceptible to slighter injuries on that account.

Contusion of the testicle produces swelling and effusion, and if this be the extent of the condition, rest, suspension and the application of an ice-bag or cold lotion are sufficient to afford relief. If inflammation ensues, which is evidenced by increase in swelling and pain, the treatment is that of epididymitis or orchitis (see p. 307). Atrophy sometimes follows contusion of the testicle but this is not commonly the result.

Dislocation or luxation of the testicle may be caused by direct injury or severe strain, when the gland is forced into the inguinal canal over the pubis or into the thigh. I have seen a case which occurred while the patient, a butcher, was lifting a large hind of beef. The testicle, an undeveloped gland, was retracted into the inguinal canal and remained there.

The testicle is likely to become acutely inflamed and later undergo atrophy as a result of this accident. If attempts to replace it are unsuccessful, its removal may be necessary as in the case of the congenitally retained organ.

\section{Punctured and Lacerated Wounds of the Testicle}

Such wounds may occur in connection with injury to the scrotum. If swelling and infection do not take place, healing generally progresses without untoward event. When the tunica albuginea or covering of the testicle is opened as the result of a lacerated wound, protrusion of the tubular structure of the interior may occur in which case, immediate suture, under careful antiseptic precautions, should be practised, otherwise the treatment of such wounds is upon general antiseptic principles. Puncture of the testicle may be the result of the careless introduction of the trocar in which case, however, if the instrument is clean, no evil result ensues.

Gangrene of the testicle may occur as a result of extensive laceration of the gland and surrounding tissues causing impairment of the blood supply, but this is more likely to follow laceration or torsion of the spermatic cord.

Torsion of the Cord.-Gangrene is the ultimate outcome of this condition unless it is recognized and corrected early (Fig. 132). Simple strain is the usual direct causative factor and malposition of the organ favors the occurrence. ${ }^{1}$

\footnotetext{
1 Scudder, Annals of Surgery, 1901, Vol. XXXIV, reports thirty-two cases in fifteen of whioh the organ was misplaced.
} 
The symptom-complex of torsion is not unlike that of strangulated hernia, without the evidence of intestinal obstruction. There are, however, pain, swelling and tenderness along the inguinal canal and the constitutional disturbance of vomiting and pyrexia. The general condition is that of shock. It is differentiated from strangulated hernia in most instances by the extreme acuteness of the local symptoms, and less profound constitutional disturbance. The diagnosis may sometimes not be made until the nature of the condition is exposed by operation. The outcome may be a spontaneous untwisting of the cord and a relief of the testicle, or it may proceed to gangrene when extirpa-

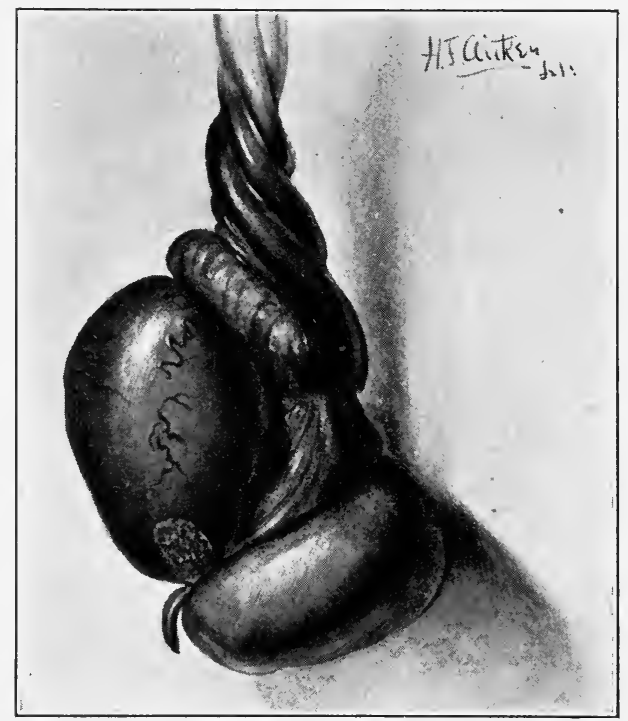

Fig. 132.-Torsion of the spermatic cord. (Am. Practice of Surgery.)

tion becomes necessary. If the condition is encountered early enough untwisting of the cord may be attempted and prove successful eitherby manipulation or through an open incision. In the majority of instances, operation is called for and removal of the testicle frequently becomes necessary.

\section{ORCHITIS-EPIDIDYMITIS}

Orchitis.-Inflammation of the body of the testicle may be acute or chronic. Tuberculosis is a common cause and is discussed in another section. The etiological factors of primary orchitis are traumatism, 
extension of inflammation from a neighboring infected focus and infection by hematogenous transmission. Primary orchitis is much less common than epididymitis. Secondary orchitis has its origin in epididymitis and the two conditions often coexist (epididymo-orchitis). Traumatism is likely to involve both the testicle and epididymis and attention has already been called to the frequency of injuries to the undescended testicles.

Inflammation of the testicle may result from infection from the posterior urethra, bladder or seminal vesicles, but in such case the inflammatory process usually involves the epididymis as well. The transmission of infection to the testicle through the blood stream during the course of an acute infectious disease (scarlatina, variola, typhoid, pyemia, etc.) does occur but is not common, with the notable exception of the metastasis which sometimes occurs as a complication of mumps. This type of orchitis may be unilateral or bilateral. It usually develops after puberty and involves the body of the testicle alone. Atrophy sometimes succeeds this inflammation.

The pathological changes which occur with orchitis consist of serous infiltration with marked hyperemia causing great tension within the tunica albuginea and when the tension is excessive and the infection severe, suppuration is likely to result. In cases of severe traumatism, gangrene with complete destruction of the gland may exist. In other cases of high tension without rupture of the tunic the pressure may bring about fatty degeneration and atrophy of the gland. If resolution takes place, the tissues return to their normal condition and there are not left the characteristic nodules that occur after epididymitis. In the case of suppuration of the gland, this may appear in single or multiple foci. Adhesions form between the tunica albuginea and the tunica vaginalis in which case a fluctuating focus is recognized beneath the skin or it may rupture and leave a fistulous tract connected with the testicle. If the suppurative condition is not relieved by proper surgical drainage, extensive destruction of the testicle may result and a large indurated and infiltrated area surrounding the gland may cause pressure to be exerted upon its substance and ultimate atrophy.

Symptoms. - In the case of systemic invasion the symptoms are general and are such as might be expected with the fever of a transient toxemia. The local symptoms are pain and swelling of the affected gland and pain at the groin. The pain is the more severe on account of the unyielding character of the tunica albuginea. Palpation of the organ will elicit tenderness and hardness; sometimes there are soft foci indicative of suppuration; in non-tuberculous or non- 
specific cases it is not nodular. In reaching a diagnosis the distinction must be made between a simple, and a specific or tuberculous inflammation, from epididymitis, hydrocele and neoplasm. So far as syphilis and tuberculosis are concerned (see Index) the condition is more likely to be chronic, although tubercular inflammation sometimes comes on in acute form but is usually a part of an associated epididymitis. As a matter of fact, orchitis 'and epididymitis are commonly associated, and the presence or absence of tuberculosis as a causative factor must be determined by investigating the etiology, by the more active systemic disturbance when tubercular, by the tuberculin reaction and by the existence of other constitutional evidence.

Palpation and transillumination will serve to distinguish orchitis from hydrocele. (See Hydrocele, p. 317.) Suppuration cannot always be recognized as palpation of the testicle is sometimes deceptive and the pus focus may be deep. Protracted inflammation that is not tubercular and does not resolve will usually reveal upon operation a deep focus of suppuration.

Treatment.-The palliative measures which are first indicated consist in elevating the inflamed gland upon a proper support (see p. 314) and in the application of hot fomentations in the form of poultices, wet dressings ( 1 per cent. phenol solution or 2 per cent. aluminum acetate) or the use of the electric heater pad (Fig. 136) for such length of time as may be necessary to determine whether or not the inflammation tends to subside under these measures. If such is not the case, it is likely to be

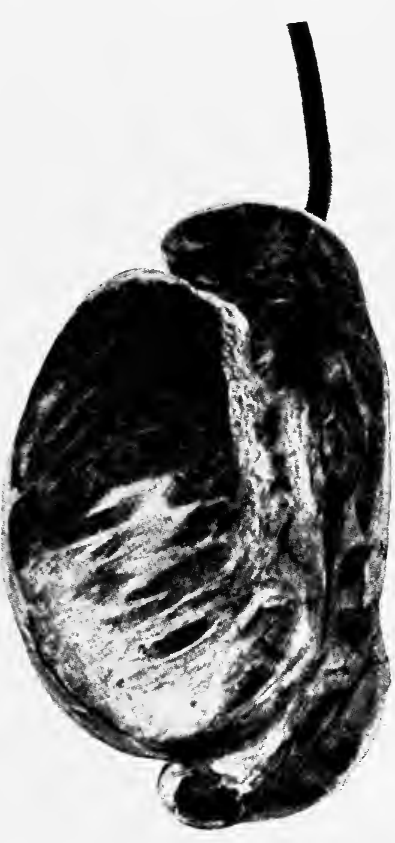

FIg. 133.-Extensive fibrous infiltration of the testicle. due, as stated, to the existence of interior suppuration and incision is required. This entails exposure of the testicle through an incision in the skin and tunica vaginalis, and drainage of the organ upon general surgical principles. (See Operative Surgery.)

Chronic orchitis is not a common sequence of acute inflammation of the testicle and is usually due to tuberculosis or syphilis. (See Index.) If resolution of the inflammation does not occur and interior tension has been great, fibrous interstitial changes result in the production of new connective tissue at the expense of the normal struc- 
ture and atrophy ensues (Fig. 133). This is accompanied by no symptoms, simply a gradual shrinkage of the gland for which there is no particular remedy.

Epididymitis.-Epididymitis, like orchitis, occurs as a result of traumatism and infection; a combination of these influences is generally responsible for both conditions. Hematogenous infection occurs in such diseases as syphilis, tuberculosis and other systemic affections. Inflammation of the posterior urethra due to gonorrheal inflammation, stricture, etc., is the most common cause-in a word, epididymitis is one of the most common complications of acute and chronic gonorrhea. Like gonorrheal cystitis, it may come on as a result of extension of inflammation from the posterior urethra in which case it involves the mouth of the ejaculatory duct and thence through the vas deferens, the testicle. Its most common stage of occurrence in the course of gonorrhea is at the end of two or three weeks. The date of appearance of epididymitis, however, is by no means fixed. It may come on during the first few days and may be encountered at any later period.

Chronic posterior urethritis is a constant predisposing factor and any of the well-recognized sources of urethral irritation is the immediate exciting cause. Among these causes may be mentioned too strong urethral injection, introduction of a sound or other instrument of exploratory character without due gentleness, sexual irritation of any sort, excessive drinking, violent exercise, any of which is capable of damaging the testicle mechanically and, as it were, calling down the inflammation from the urethra. The testicle once attacked by epididymitis, is distinctly more prone to recurrence. There is a predisposition to the same complication in the event of future occurrences of gonorrheal inflammation and operations involving the posterior urethra in those who have once been a victim of testicular inflammation are often followed by post-operative complication of the same condition.

Morbid Changes.- The tail or the head of the epididymis is usually the primary site of the inflammatory process and later the entire structure may become involved; the body of the testicle is usually spared. As a natural consequence of the inflammation, the epithelial linings of the tubules become infiltrated with serous exudate. Pus cells are mingled with the seminal fluid and undue tension is the result. Resolution is the common termination, although suppuration may result in the formation of small pus foci or in the development of diffuse abscess. When the inflammation is intense, there may be a consequent effusion in the tunica vaginalis or hydrocele. Examina- 
tion of the fluid in the tunica vaginalis frequently reveals the presence of the gonococcus.

A common sequel to the active form of inflammation here is the deposit of hard nodules caused by proliferation of connective tissue, which are sometimes permanent but which usually soften to considerable extent during the process of resolution. If the latter does not occur, there results a barrier to the passage of spermatozoa on that side.

Symptoms.-Acute inflammation of the epididymis is ushered in by pain and heaviness in the region between the testicle and the groin. Accompanying these physical signs, there may be some constitutional disturbance in the form of marked elevation of temperature and general malaise. When these preliminary symptoms are not severe and the individual immediately yields to the natural demand for rest, perhaps under the influence of some antiphlogistic or counter-irritant treatment, the attack may prove to be abortive and disappear without further progress. This, however, is not usually the case. The discomfort increases in intensity until the pain becomes exceedingly severe, especially with the movements of the body; the affected portion is acutely sensitive to pressure. In the early stage, the different divisions of the epididymis and its separation from the testicle can be readily distinguished; as the enlargement continues the whole testicle apparently becomes swollen, tender and red with a maximum amount of tenderness in the globus major or minor. Inflammation and swelling of the cord may occur simultaneously with that of the epididymis or later on. When there has been a preëxistent flow of pus from the urethra, the discharge is diminished or stops entirely but this disappearance is only temporary and it usually returns when the inflammation in the testicle subsides. As the testicle increases in all its dimensions, fluid collects in the tunica vaginalis, making the enlargement oval in shape. The scrotum becomes red and hot and is sometimes the seat of edematous effusion. The intensity of the symptoms and extent to which the inflammation reaches vary in different cases. In some, there may be nothing more than an involvement of the head or the tail of the epididymis about the size of a hickory nut which lasts only a few days and promptly subsides under palliative measures. Again, the swelling may be so great and the suffering so intense that there is great difficulty in obtaining relief by the ordinary measures at hand. In these more severe cases, there is probably pus formation beneath the resistant fascia of the epididymis and an excessive amount of serous effusion in the tunica vaginalis. When the cord is involved, the swelling, tenderness and pain are associated also with the region of the groin. The first attack of epididymitis accompanying gonorrhea, 
like the urethral inflammation itself, is prone to be more formidable than subsequent exacerbation of the same malady.

The non-specific forms of epididymitis, those which occur as the result of injury or strain, may be comparatively insignificant, but when occurring as a complication of chronic prostatitis, or during the course of treatment of stricture of the urethra, assume all the active properties of the most acute form of this malady.

In acute cases, it usually takes from two to seven days for the swelling to reach its height, after which there may be a stationary period of another seven days, and then a declining period of about a week.

The duration of the attack is controlled by the proper adoption of treatment and especially where the operative procedure is resorted to. (See Operative Surgery, p. 356.)

The diagnosis of epididymitis is self evident when it occurs in the usual clinical sequence. (See Tuberculosis of Testicle.)

Sequels and Chronic Course of Epididymitis.-The immediate outlook of acute inflammation of the testicle is good. Subsidence of the inflammation generally occurs with or without treatment in two or three weeks. There are certain ultimate results, however, that give to this malady an unusual importance.

Patients who have once suffered an attack of epididymitis are prone to recurrences, if chronic inflammation in the prostate or posterior urethritis is allowed to remain uncured. The testicle which is or has been the seat of inflammation offers a favorable soil for tuberculous infection and the latter involvement is not an uncommon occurrence as the first active sign of this constitutional malady in individuals who have otherwise been free from evidence thereof beyond a certain degree of general physical debility.

Abscess of the testicle, when it occurs to the extent of appearing on the outside of the scrotum, may be the cause of the loss of considerable structure and consequent functional impairment.

Neuralgic pains of a more or less paroxysmal character are complained of as an after occurrence in some cases of epididymitis, especially by patients of neurotic temperament.

Atrophy of the testicle occurs as a sequel of chronic inflammation more often in those cases in which the inflammation has been exceedingly intense and has lasted over a long period.

Chronic infiltration of the tubules causing a blocking of the seminal ducts exists in a number of cases and chronic inflammation of an extensive area may persist with a coexistent tendency to relapsing attacks of acute inflammation. In such cases of persistent inflam- 
mation, chronic hydrocele also occurs as a sequence of swollen testicle.

Azoöspermia is produced by localized, nodular deposits in the tail or the head of the epididymis, following bilateral epididymitis, with a resulting sterility. This is more likely to be the case when infiltration exists in the globus minor or tail on account of the greater number of the tubules in the globus major whereas the testicular duct is narrowed down to a single canal in the tail of the testicle. When there is complete blocking of both seminal canals, the seminal fluid will contain no spermatozoa whatever and the victim thereof is perfectly sterile. (See Azoöspermia.) Where there is incomplete blocking of the canal, either in the testicle or at some other point in the seminal duct, the spermatozoa may be partially but not entirely absent with diminished procreative capacity (oligo-spermia). Both of these conditions may also occur as the result of seminal vesiculitis. It is customary to conclude that occlusion always exists after the occurrence of double epididymitis, yet as a matter of fact, a certain percentage of cases of inflammation of the epididymis resolve completely and the only possible means of determining the question of sterility is by microscopic examination of the seminal discharge when the presence or absence of spermatozoa will be recognized.

For the treatment of azoöspermia (vesicular) see Vesiculitis, p. 337, and testicular, see Operative Surgery, p. 357.

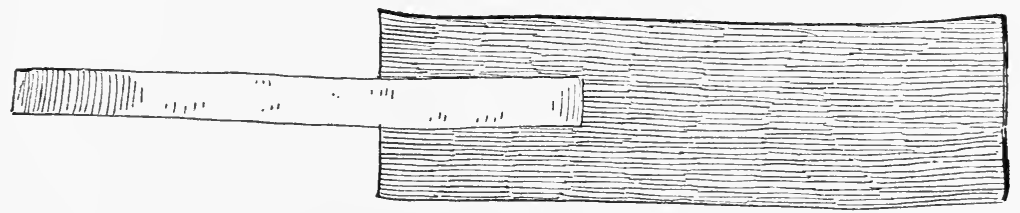

FIG. 134.-Rubber bandage for compression of testicle.

Treatment of Epididymitis.-Prophylactic measures are, as always, of prime importance. In conditions of acute inflammation of the urethra, precaution should be taken to protect the testicles by wearing a properly fitted suspensory bandage, and by avoidance of all physical and sexual strain. Strong injections, overzealous treatment and the unwise passage of a sound during the course of specific or during the active stage of any inflammation are likely to precipitate the condition.

The aim of treatment therefore during the acute stage is complete rest and the avoidance of all irritating factors, which factors are enu- 
merated as the exciting causes of this malady. If the attack is caught in the initial stage, it is sometimes possible by immediate rest and the application of a sharp counter-irritation over the tender area of the beginning inflammation, in the form of a strong solution of guaiacol 25 to 50 per cent. in glycerine, to cause a quick subsidence of the attack. This application should be made with a camel's hair brush over the site of the swelling in sufficient amount to produce some irritation and redness but not enough to blister. If a favorable

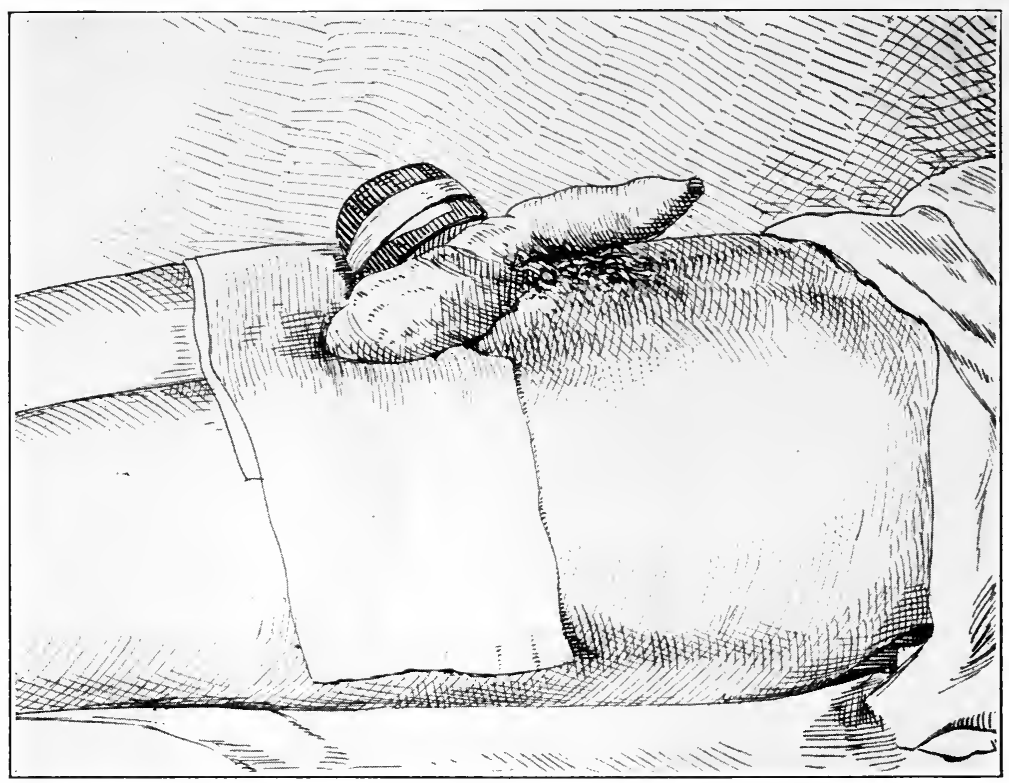

FIG. 135.- Rubber bandage and adhesive strap applied to swollen testicle.

effect is obtained, the result is prompt and occurs within twenty-four hours. Failing in this, it becomes necessary to resort to other measures of treatment, palliative, sero-therapy, or operative. The patient is therefore put to bed, the scrotum is suspended upon an adhesive plaster band drawn across the thighs (see Fig. 135) and one of three courses may be selected.

1. The Palliative Method of Treatment.-This involves the constant application of heat and moisture in the form of flaxseed poultices, hot fomentations or the electric heater (Fig. 136) so long as the acute stage of inflammation continues. This generally lasts a week or ten days. At the end of this period when the acute tenderness has subsided 
to a sufficient degree to allow gentle pressure without undue pain, the condition calls for the additional expedient of continuous gentle pressure. This is best obtained by the use of a rubber elastic band (the same as is used for bandaging the extremities) a section of which about $7.6 \mathrm{~cm}$. ( 3 inches) wide and 15 to $18 \mathrm{~cm}$. (6 or 7 inches) long is employed. To the end of this section of rubber bandage is attached a strip of adhesive plaster (see Fig. 134). The swollen gland is isolated from the opposite organ and bound with the rubber bandage with as much pressure as the patient can comfortably stand, fastening the bandage in place by means of the rubber adhesive strap. This means to constant elastic pressure when not too tight exercises a prompt and favorable influence upon the declining stage of the inflammation and

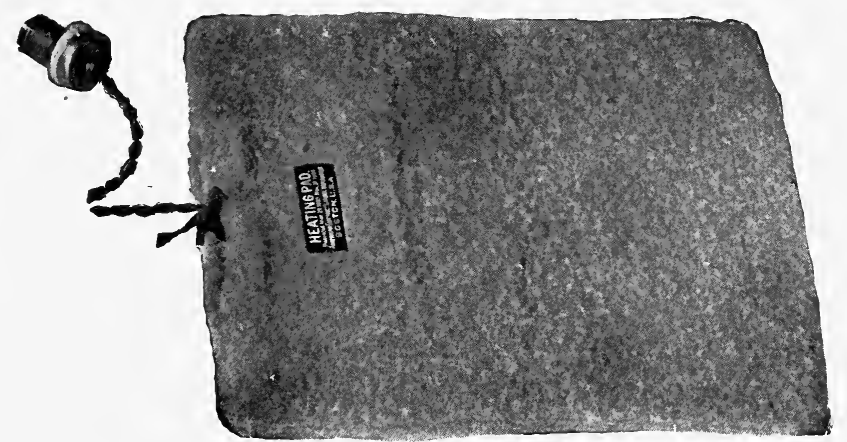

Fig. 136.-Electric heater pad.

ends to shorten the confinement in bed. As soon as it is evident that the swelling is reducing under this means of treatment, the patient is allowed to be up and about with the testicle supported in a proper suspensory bandage.

The ice-bag is a common resource to the extent of being almost a routine method in some quarters but while this means of treatment exercises a controlling influence upon the pain accompanying the malady, it is in no sense a curative agent and tends more to retard than assist recovery. Heat and moisture are usually to be preferred. There are no special internal remedies of value; anodynes and other therapeutic agents have their proper indications.

2. The Use of Vaccines and Serums.-Acute epididymitis, especially the gonorrheal type, offers a favorite indication for the use of sero-therapy and some cases of prompt cessation of this complication have occurred under such treatment; other cases have pursued their usual course in spite of such measures. It is proper, however, to have 
in mind that this modern method of combating infective organisms is not likely to act favorably in the presence of a local focus of pus, in which case reliance can be placed only upon the usual surgical measures. (See section on Serums and Vaccines, p. 110.)

3. Operative Treatment of Epididymitis.-When abscess of the testicle involves the tunica vaginalis and whenever surface fluctuation is evident, surgical drainage is called for as in the case of any abscess elsewhere. This may follow acute or chronic epididymitis; and when there is extensive destruction and necrosis, may call for removal of the epididymis (epididymectomy) (see Operative Surgery, p. 358).

The operative technic for acute inflammation of the testicle (Hagner's Operation) demands especial consideration, and is that which is resorted to in extra intense inflammation and swelling with a view of producing prompt subsidence of the acute symptoms, a shortening of the duration of the malady and less likelihood of secondary infiltration from nodular swelling in the affected gland. The technic is simple and, inasmuch as pus foci probably form in a fair proportion of cases, when there is any question of the existence of such in a given case, the operation should be considered. (See Operative Surgery, p. 356.) In all cases of epididymitis, there is more or less effusion in the cavity of the tunica vaginalis making an acute hydrocele. The size of this is sometimes considerable and relief may be afforded the patient by puncturing the tunica vaginalis alone.

Chronic relapsing epididymitis may be treated by vasectomy (ligation of the vas deferens) on the affected side. (See Operative Surgery, p. 355.)

As already stated, sterility due to azoöspermia results from nodular obstruction of the seminal ducts as a sequence of inflammation in the epididymis. This condition has been successfully operated upon by anastomosis of the epididymis with the vas deferens. (Martin's operation.) (See Operative Surgery, p. 357.) 


\section{CHAPTER XVII}

\section{HYDROCELE-VARICOCELE-GROWTHS OF THE TESTICLE}

Hydrocele, or what is known as the common variety of this malady, consists of a serous effusion in the sac of the tunica vaginalis, Fig. 137. Other varieties of hydrocele possess different relations to the sac of the tunica vaginalis; thus, hydrocele of the cord surrounds this structure and lies above the tunica vaginalis. It may be encysted hydrocele

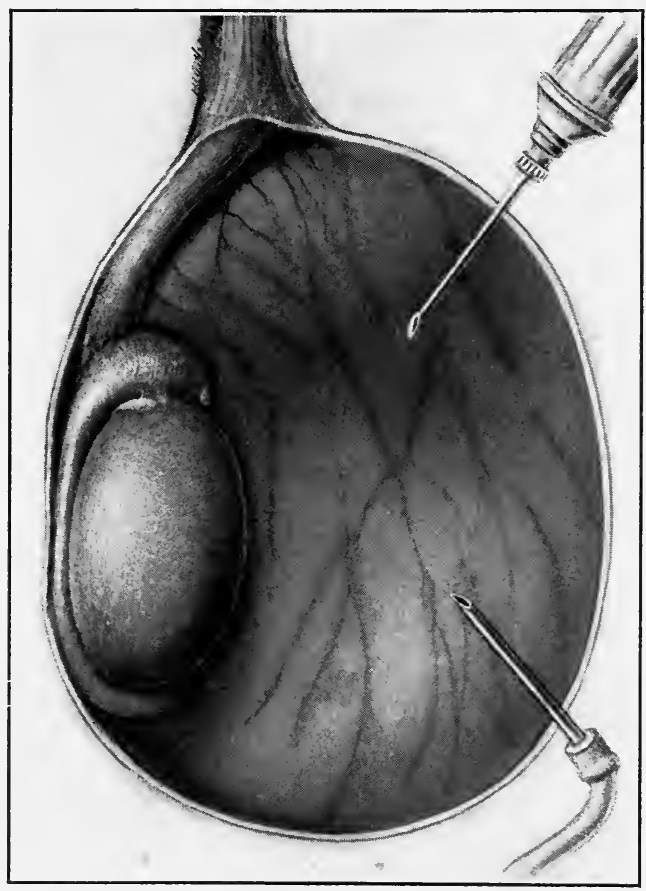

FIg. 137.-Hydrocele.

of the cord or have an opening connecting with the peritoneal cavity, like a congenital hydrocele but not connected with the tunica vaginalis, whereas congenital hydrocele proper, like congenital hernia, is due to a connection between both the peritoneal cavity and the cavity of the tunica vaginalis. 
Hydrocele may be bilocular or multilocular, being divided into two or more compartments by membranous extensions. Hydrocele is either an independent malady or it occurs as a symptom of disease of the epididymis or testicle, tuberculous, syphilitic, neoplastic or simple inflammatory. When simple and uninfected, the content of the sac is a clear, serous liquid; when infected and bacterial, it is cloudy. It may contain blood (hematocele) or spermatic elements (spermatocele).

Acute Hydrocele. - This term is applied to inflammation with effusion in the tunica vaginalis propria, when the fluid may be serous alone, or combined with plastic exudation, or purulent. It may be the result of a direct trauma to the scrotum or testicle, or a symptom of an acute epididymitis, especially when the latter occurs as a complication of gonorrhea.

Clinical History.-The symptoms depend upon the intensity of the cause. It is, in effect, a localized, acute inflammation with serous exudate. The pain is sometimes slight and at other times severe. Fibrinous exudation is the result of an intensified activity due to severe injury or microbial invasion, and may lead to obliteration of the cavity of the tunica. If suppuration occurs and is not relieved by incision, an external outlet is likely to be formed. In simple uncomplicated hydrocele, when the fluid is clear, this fact may be demonstrated by the translucency test, which consists of transilluminating the hydrocele sac with a bright light, and looking through a tube placed against the opposite side of the scrotum.

Treatment.-The treatment is ordinarily palliative and consists of rest, elevation of the part and the application of suitable evaporating lotions, such as aluminum acetate, saturated solution of sulphate of magnesia or the ice-bag; in cases of great distention and sharp pain, puncture may be performed to relieve the tension; otherwise the treatment is of the underlying condition of the testicle of which it is a symptom. If, after the acute symptoms have subsided, the fluid in the sac remains and persists for a period of six weeks or more, the hydrocele becomes chronic and should be treated as such.

If there is pus formation, as evidenced by systemic reaction, edema and redness of the parts, incision and drainage are required.

Chronic Hydrocele.-When the fluid exudate accumulates slowly over a prolonged period or persists indefinitely after an acute inflammation, the condition is that of a chronic effusion and represents what is more commonly understood by the term hydrocele. With respect to the etiology of this condition, the persistence of an acute hydrocele has already been mentioned. Traumatism, gonorrheal and other affections of the testicle may lead to its development; there are besides, however, 
certain cases which, appearing to have no special etiological factor, are described as idiopathic, but there is undoubtedly some underlying irritating cause or disturbance in the local circulation that, if discovered, would explain the cause thereof. ${ }^{1}$ There is no discrimination as to age or as to which side is affected. The predisposition is a local condition of the parts, congenital or acquired, structural or inflammatory.

Pathology.-The pathology of hydrocele is that of the sac, its fluid contents and the condition of the testicles. The tunica vaginalis is almost invariably thickened which is more likely in old cases; especially when the sac has been subjected to tapping on one or more occasions followed by an irritating injection as a means of treatment, the sac wall may become fibrous. The fluid is usually serous and of the appearance of clear, amber urine, unless it has been changed as a result of treatment or unless it becomes discolored from the admixture of decomposed blood or bacteria, when it loses its transparency and becomes brown or turbid.

It usually contains epithelial cells and cholesterin crystals in moderation; when the latter are present in considerable quantity, they give to the fluid an opaque, saponaceous appearance. The presence of

${ }^{1}$ Jacobson's Classification of Chronic Hydrocele:

$\left\{\begin{array}{l}\text { Hydrocele of The Tu- } \\ \text { a. Hica Vaginalis. }-\mathrm{T} \mathrm{h} \mathrm{e} \\ \text { nica } \\ \text { fluid is in a sac connected } \\ \text { with that of the tunica } \\ \text { vaginalis. }\end{array}\right.$

b. Encysted Hydrocele.The fluid is in a sac distinct from that of the tunica vaginalis.
1. Ordinary Hydrocele.-The fluid distends the closed sac of the tunica vaginalis.

2. Congenital Hydrocele.-A communication exists between the cavity of the tunica vaginalis and that of the peritoneum.

3. Infantile Hydrocele.-The tunica vaginalis and the funicular process are distended with fluid, but these are shut off from the peritoneal cavity by an obliteration placed usually at the external ring.

4. Inguinal Hydrocele.-Hydrocele in relation with a retained testis.

1. Encysted Hydrocele of the Epididymis.-The fluid is encysted in the neighborhood of the epididymis.

2. Encysted Hydrocele of the Testis.-The fluid is encysted between the tunica albuginea and the inner surface of the tunica vaginalis.

a. Diffused.-The fluid forms a serous collection of the nature of edema in the cellular tissue of the cord.

b. Encysted. - The fluid is contained in a distinct sac originating usually (1) in some unobliterated part of the processus funiculo-vaginalis; (2) in a cyst formed independently of this process, e.g., by dilatation of persistent tubules of the organ of Giraldes.

a. With other Coexisting Hydroceles:-e.g., (1) hydrocele of the tunica vaginalis with encysted hydrocele of the testis; (2) hydrocele of the tunica vaginalis with encysted hydrocele of the cord; (3) hydrocele of the tunica vaginalis with diffused hydrocele of the cord.

b. With Hernia:-e.g., (1) hydrocele of the tunica vaginalis with inguinal hernia; (2) hydrocele of the cord with inguinal hernia.
}

IV. Hydrocele of the sac of a hernia. 
spermatozoa, changing the character of the cyst to a spermatocele, renders the appearance of the fluid contents whitish in hue. There are sometimes present in the mass of fluid, free fibrinous bodies composed of a combination of earthy salts and fibrin.

The cavity of the sac is usually monolocular but may be subdivided by adhesions or septa, under which conditions, the division may be such that one sac is in the abdomen and the other in the scrotum or the subdivision may be in the scrotum.

The testicle may be unaffected and in its normal locality which is behind the hydrocele accumulation; it may, however, be the seat of disease involving either the epididymis or body of the organ and there may be a secondary atrophy of the gland.

Very exceptionally, the testicle is in front instead of behind the fluid. This is due to congenital misplacement.

Clinical History.-The most apparent symptom of hydrocele is that of the pear-shaped enlargement of the scrotum on the affected side, with the tapered or conical end of the enlargement pointing toward the inguinal region. The size varies widely from a size so small that the patient is unaware of its presence to that of enormous dimensions, so large as to contain an inconceivable quantity of fluid. Over 2000 c.c. of fluid have often been obtained by many operators and some report cases that have yielded far in excess of this amount. As the sac enlarges with the accumulation of the fluid, it causes a corresponding amount of discomfort to the patient owing to its weight and pressure. As this increases, there occurs retraction of the prepuce which occasions difficulty of urination and the parts are likely to become excoriated owing to the impossibility of keeping the skin from becoming wet with the urine. Manual examination discovers a fluctuating tumor of tense and elastic nature; percussion elicits a flatness; there is no pain or other evidence of inflammation and in distinction from hernia, there is no impulse felt upon coughing. The most important diagnostic sign of simple hydrocele is the translucency of the fluid contents which is observed by transillumination and inspection through a tube. It is important not to confuse hydrocele with a scrotal hernia. In the latter condition, the weight of the tumor is not so great and the characteristic features are impulse upon coughing, a resonant percussion note, and extension into the inguinal canal with the history of gradual enlargement from above downward, instead of from below upward, as in the case of hydrocele. Tumors of the testicle sometimes offer difficulties in diagnosis as they may be accompanied by a certain amount of fluid contents. The special difficulty is experienced with the soft tumors of the testicle. Enlargement of the inguinal glands is 
to be looked for. in the latter instance; and in any case where a fair size aspirating needle of clear lumen has been introduced with the purpose of withdrawing fluid from a suspected hydrocele which fails to flow freely and yields little more than a small quantity of bloody fluid, yet the end of the needle seems to be free within the scrotum, there is strong reason for suspicion of a malignant neoplasm, and incision may be necessary to decide the question.

Treatment.-Spontaneous cure sometimes takes place in childhood and in some cases of small size following the irritation induced by a single tapping. In most instances, however, radical cure requires the adoption of one of two resources-(1) aspiration and injection or (2) radical removal of the hydrocele by open operation following the technic of one of the different methods employed for this purpose (see p. 350).

Aspiration is only a temporary mode of relief and must be performed as often as the hydrocele sac refills. This occurs at variable periods in different individuals. Following aspiration, the injection into the sac of an irritating agent such as iodine, carbolic acid, ether chloroform, alcohol, has been adopted with effective results. Carbolic acid is by far the most preferable as the percentage of successful results following its use is much greater than with any of the other agents. I prefer the open, operative method of treating hydrocele as under the advanced facilities of present-day practice, the operation is devoid of any risk and sure of success. But there are certain select cases of simple hydrocele of small size, especially in those who. strongly object to cutting, that may be very effectively handled and brilliantly cured, without the necessity of laying up for more than twentyfour or forty-eight hours, by means of aspiration and injection with pure carbolic acid. For a description, see p. 352.

Hematocele.-When the sac content of any form of hydrocele is composed of blood, the same is designated a hematocele. This alteration in the fluid of hydrocele is usually the result of traumatism. When of recent origin as from an external injury or following the introduction of a canula for aspiration, the color is that of fresh blood. After the lapse of time, the blood undergoes disintegration and the content of the sac assumes in consequence a dirty brown color and the admixture of shreds of fibrinous material gives to it the character of coffee grounds. When the contents of the tunica vaginalis are composed entirely of fresh blood, as, for instance, following a surgical operation or when the flow of blood occurs in the substance of the testicle, it is more properly spoken of as a hematoma. Finally a spontaneous hemorrhage may occur in the sac of a hydrocele as a 
result of arterial degeneration in chronic debilitated individuals or in scorbutus. The symptoms of hematocele differ insignificantly from those of hydrocele, except with respect to the fluid contents of the sac. In the acute form, there are local tenderness and pain due to the sudden access of distention. That form which is not acute but occurs slowly may present no symptoms and be unrevealed until the character of the fluid is discovered. If the hemorrhage is slow but sufficiently extensive to fill the entire sac, the result may be a confusion in diagnosis and the resulting gelatinous mass may press upon and cause atrophy of the testicle and give the appearance of a neoplasm.

Treatment.- In the acute type, the treatment is the same as for acute hydrocele - namely, rest in bed, suspension and the application of an ice-bag.

If the distention by such means is not controlled and if aspiration with a good sized needle cannot be accomplished, it may be necessary to open the sac, to evacuate the contents and control the hemorrhage as a means of saving the testicle. Suppuration demands incision and drainage. In the old and more chronic cases, the treatment calls for the same operative procedure as for chronic hydrocele. If operation is deemed inadvisable on account of the existence of systemic conditions, the latter should receive appropriate treatment and the local condition should be managed with proper palliative measures.

Congenital Hydrocele. - To appreciate the nature of this form of hydrocele, it must be remembered that, during the process of generation, the testicle descends to its destination, through the inguinal canal, carrying along with it a pouch of peritoneum which eventually becomes the tunica vaginalis and which is normally obliterated from the peritoneal cavity. When this does not occur, there remains a communication with the tunica raginalis which favors the existence of congenital hydrocele or congenital hernia or both. Occurrence may take place during infancy, generally the result of traumatism, or during active muscular strain. The presence of hydrocele is recognized by its translucency; the presence of hernia or its coexistence is distinguished by its tympanitic percussion and gurgling on manipulation.

Treatment.-The hydrocele alone or in combination with hernia may disappear spontaneously or may be reduced by application of an ice-bag and elevation of the pelvis, following which, it may remain in situ. Otherwise, open operation is called for.

Infantile hydrocele is located in the tunica vaginalis above the testicle from which it is walled off. It extends upward toward the inguinal ring into the funicular process, but, differing from congenital 
hydrocele, is shut off from the peritoneal cavity and is therefore irreducible. It is usually accompanied by hernia, in which case its treatment demands open operation, otherwise aspiration and injection. Congenital hydrocele has been found in adults.

Hydrocele of the Spermatic Cord.-Encysted hydrocele of the cord results from a failure of a portion of the vaginal process to become obliterated, hence a serous exudate accumulates in the cavity. Another variety of this same condition may exist as a supernumerary cyst inside of a hernial sac or an old hernia. In the case of an old hernial sac, the neck has become obliterated and encysted hydrocele may remain. There are no peculiar symptoms that characterize this condition; there is nothing more than the existence of a cyst lying along the cord between the testis and the external ring. It should be differentiated from congenital inguinal hernia, in which case the characteristic signs of hernia are to be taken into account and should prevent any confusion; namely, a reducible protrusion yielding a resonant percussion note and giving an impulse on coughing. Even though the congenital hernia is irreducible, the earlier history of the case should aid in determining

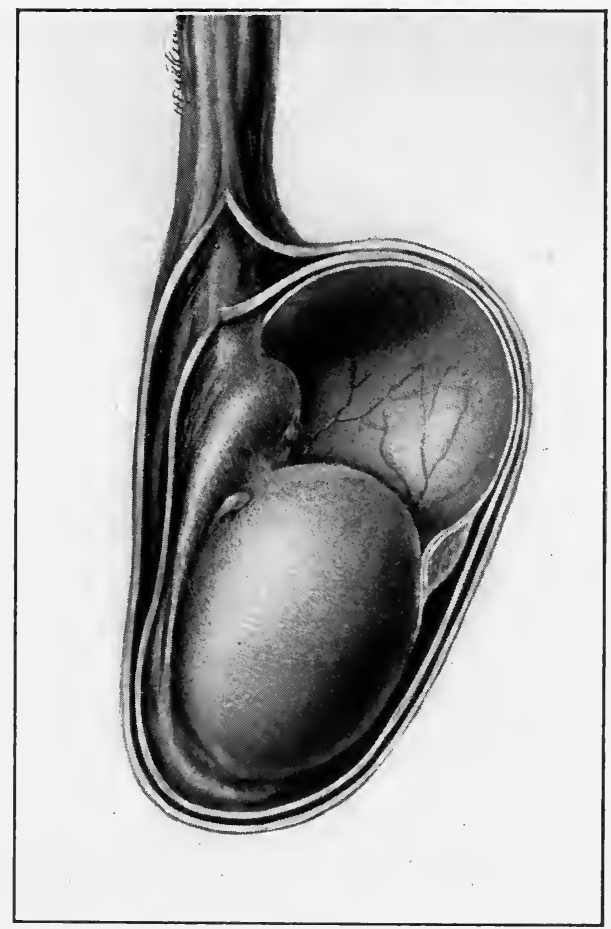

FIG. 138.-Spermatocele. (Am. Practice of Surgery.) its nature. The treatment is the same as that of hydrocele elsewhere: tapping alone, in children and in small, thin-walled sacs, generally will yield permanent results. The most reliable method, however, in straightforward cases, is the aspiration and carbolic acid method (see p. 350). Should the encysted tumor become a hematocele, on account of the occurrence of internal hemorrhage, it should be treated surgically through an open incision and its complete or partial removal accomplished. 
Spermatocele.-The distinguishing feature of spermatocele is the presence of numerous spermatozoa in the hydrocele sac, the cause of which is said to be the open communication of a seminal duct with the spermatic cyst. These cysts are adjacent to but independent of the tunica vaginalis (Fig. 138). They may be small, single or multiple, and properly speaking, are epididymal cysts, developing in or around the epididymis and closely resembling hydrocele of the cord. When the spermatic elements are found in the fluid of a hydrocele of the tunica vaginalis, it is due no doubt to a rupture of one of these cysts into the tunica. The symptoms of spermatocele are inconspicuous. The diagnosis is based upon the location of the cyst or cysts which is at the junction of the epididymis and the testicle. They may be small when first discovered but may grow to considerable size upward along the cord or downward, extra- or intravaginally. In differentiating from hydrocele of the cord, aspiration and disclosure of the nature of the fluid may be the only means of determining the diagnosis. The treatment consists of aspiration which affords temporary relief; radical cure is secured by the injection of a small amount of carbolic acid or excision of the cyst wall (see p. 352).

Galactocele or Chylocele.- - This affection is caused by an exudation of chyle from the lymphatics of the cord into the cavity of the tunica vaginalis. The condition is very rare and occurs mostly in the tropics. It is due to the presence of the filaria sanguinus hominis in the lymph channels, which cause an engorgement and vicarious leakage of the lymphatic fluid into the vicinity of the tunica from the adjacent vessels. The symptoms are those of ordinary hydrocele, there being no translucency to the fluid, and in addition the manifestation of filariasis. Tapping as a means of treatment is of no value; very extensive effort should be made to remove the parasite with the affected lymphatics of the spermatic cord, including excision of the sac.

\section{VARICOCELE}

The network of veins located in the scrotum and surrounding the spermatic cord and testicle is known as the pampiniform plexus. The term varicocele is applied to the varicose condition of these veins that very frequently exists.

Causation.-The condition commonly occurs in the young and persists from youth to old age except when in the latter, the occurrence of varicosity in the veins of the lower extremities diverts the tension from the pampiniform plexus, and results in the disappearance of preexistent varicocele. It is more frequent on the left side although both 
sides may be involved. The reasons given for its greater prevalence on the left side are the fact that the left testicle hangs lower than the right, that the left spermatic vein enters the left renal vein at a right angle and is subject to the pressure of the rectum, while the corresponding vein on the opposite side enters the vena cava at an acute angle. On account of the tortuous and loose arrangement of these vessels, their susceptibility to engorgement is not surprising. It is evident, in view of the existent anatomical arrangement, that varicocele is predisposed by the dependent weight of the blood contained in the great plexus of veins together with the column of blood that extends from this plexus up to the point of anastomosis with the abdominal circulation. It commonly begins at the period of adolescence and is more frequent in the inactive than the active; sexual excesses and irregularities encourage its development and increase its growth. It is not uncommon to find a moderate varicocele visible in a young man, kept up by a series of strained conditions, that will disappear under the more quiescent sexual relations of marital existence. The occurrence of varicocele caused by intraabdominal back-pressure of a large morbid growth is rare.

Pathology.-Primarily, there is elongation of the veins, stretching of the valves and thickening of the vessel walls; later, fatty degeneration may occur at the expense of their normal elasticity. Occasionally, phleboliths or venous concretions may be felt in the lumen of the vessels.

Clinical History.-There may be no symptoms whatever associated with varicocele even though of considerable size. Indeed, such symptoms as are sometimes complained of, namely: dragging pain and heaviness in the region of the groin and neuralgic sensations in the testicles are as likely to be present with a varicocele of small dimensions as they are to be absent in one of larger size. When such symptoms are in existence, they are more in evidence after physical exertion and are relieved by rest and support of the veins with a suspensory bandage, (see Sexual Neuroses p. 507).

Sexual neurasthenia is a common accompaniment of varicocele in an individual of nervous temperament who is fanciful and hypochondriacal. These neurasthenic manifestations are headache, depression and other neurotic symptoms of indefinite and obscure character. The condition of varicocele may vary but little in years and there is no serious danger of any kind associated with it unless the growth is of such considerable size as to cause direct pressure upon the testicle and consequent atrophy. This is very unusual, but is used with other extreme statements by unprincipled practitioners and quacks as a 
means of obtaining patients and of inducing them to submit to operation or some special form of treatment.

The objective symptoms are swelling and relaxation of the scrotum on the side affected, while palpation detects the mass of dilated veins which is usually likened to a handful of earthworms. These are much more marked and readily felt in the upright position as when the patient assumes a recumbent posture, the varicocele largely disappears. This is the distinctive feature in diagnosis but there should be no difficulty in this respect unless there is a hydrocele present which adds somewhat to the difficulty of recognition. Hernia is distinguished by the presence of characteristic signs such as reducibility, gurgling upon manipulation and tympanitic percussion as well as the previous history prior to the gradual development of this latter condition.

Treatment.-No treatment whatever may be required in many cases, even those of pronounced degree, or at most, the local use of a cold, astringent wash, such as witch-hazel, night and morning, and the employment of a well-fitting suspensory bandage, together with regulation of the bowels, and an avoidance of such aggravating circumstances as excessive sexual indulgences of any character.

The indications for radical surgical treatment exist when the testicle begins to atrophy, when the size of the growth becomes uncomfortably large, when there is complaint of localized pain of a continuous or annoying degree. In those cases that developed reflex neurotic symptoms, involving sexual neurasthenia or neuralgic crises, the psychic effect alone of a successful radical operation may be a sufficient justification for its adoption; but, better still, in mild cases, which are more or less self-inflicted, in wayward and fractious youths, is the wise admonition concerning the detrimental effect of the exciting sexual agencies and the assurance of abatement of symptoms under favorable hygienic conditions.

The surgical procedures which are advocated for the removal of varicocele are subcutaneous ligation, which is being practised less at the present day than formerly but may still be preferred in some instances of moderate degree, and excision of the vein by open operation with or without partial ablation of the scrotum (see p. 347).

Occasionally, following operation unhappy sequelæ occur, such as atrophy of the testicle; in other cases, the reflex symptoms, instead of being relieved by operation, are intensified thereby. In some instances traumatic hydrocele develops. Atrophy of the testicle, however, is very exceptional and, even though the vas deferens is ligated or incised, it need not follow. In the majority of cases, the results attending operation are extremely satisfactory. 


\section{TUMORS OF THE TESTICLE AND EPIDIDYMIS}

Tumors of the testicle and epididymis appear in the various prevalent forms of neoplasm and are often of mixed variety. They are more commonly malignant than not. Carcinoma and sarcoma represent the malignant types while those of the non-malignant variety are the usual types of benign growths occurring elsewhere such as fibroma, osteoma, myoma and cystoma. It is difficult, in classification, to draw the line of demarcation between the benign and the malignant growths of the testicle or, by examination, to distinguish between them, for it is quite common for a tumor to start as a benign and later become a malignant neoplasm.

Carcinoma of the Testicle.-There is some difference of opinion as to the relative frequency of carcinoma and sarcoma. In my own cases, sarcoma is the more frequent and this seems to be the belief with the majority of observers. Carcinoma occurs as a solid, compact growth of the scirrhus variety or as a soft, medullary tumor which is more common than the former and is more rapid in growth. There is little definite knowledge concerning the etiology but traumatism seems to be one of the agencies that enter into the causation of all tumors of the testicle. The undescended testicle is particularly prone to malignant involvement on account of its greater exposure to injury. Other predisposing factors are such inflammatory conditions as complicate gonorrhea and chronic urethritis, epididymitis and epididymoorchitis. Youth and age are both susceptible to malignant tumors of the testicle but the common period is between the ages of thirty and fifty.

The contour of the tumor is ovoid or irregular; the latter, when the growth has forced its way through the tunica albuginea and distends the tunica vaginalis and scrotal sac until adhesion to the latter occurs; then it shows a tendency to break through the walls and extend outwardly as an exuberant growth.

The prognosis is bad, metastasis and recurrence being frequent.

Sarcoma.-Sarcoma occurs in middle age and childhood and in advanced years. ${ }^{1}$

The clinical course of the development of sarcoma is similar to that of carcinoma and, therefore, differential diagnosis can be made only by microscopic examination of a section of the growth. Almost all known types of sarcoma are found in the testicles. In general, they

\footnotetext{
${ }^{1}$ Kobler, American Journal of Medical Science, 1899, CXVII, 535, reports a series of 114 cases. Of these. 71 per cent. were between the ages of twenty-one and fifty. Of seventy-five of these cases examined histologically, 65 per cent, were round or spindle-celled varieties mixed; of the remainder, there was a scattering of cystic, alveolar and spindle-celled variety.
} 
may be either soft or hard in texture according to the nature of their structure. They extend from the body of the testicle to the epididymis and thence up the spermatic cord (Fig. 139). They possess a marked tendency to metastasis in other organs.

One case upon which I operated proved to be a mixed roundcell sarcoma. Two years after complete extirpation of the testicle and the cord, the patient was taken ill with symptoms of pleurisy and with what seemed to be a broncho-pneumonia, from which he grew rapidly worse and died. On the autopsy table was revealed the most extensive spread of secondary sarcomatous growth imaginable, sarcoma being found in the lungs, in the mediastinum and pleural cavities, in the pericardium and in the liver and spleen as well.

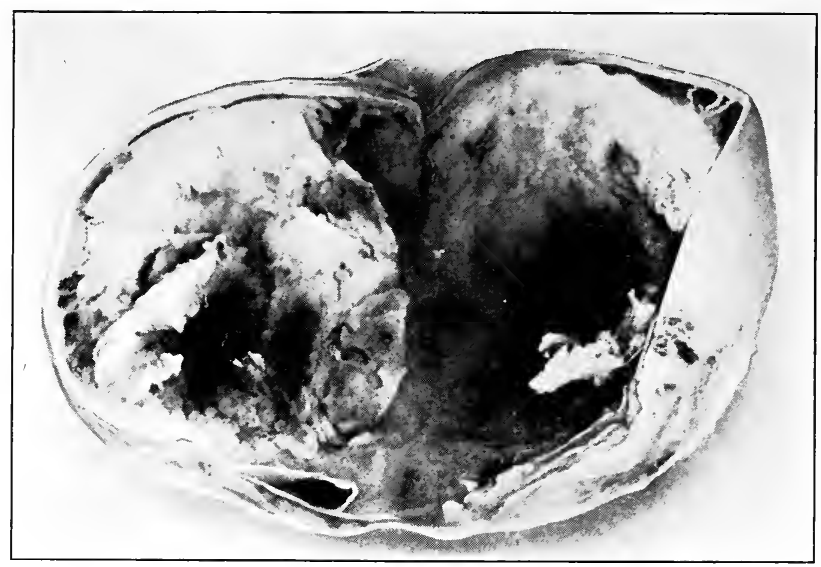

Fig. 139.-Sarcoma of the testicle.

Traumatism is revealed in the history of a large percentage of the cases.

The prognosis of sarcoma is bad; prompt and radical operative measures for this malignant growth, as well as for carcinoma, are demanded, and the earlier in their existence operation is performed, the better the outlook for the patient.

Cystoma or Cystic Testicle.-Cysts of the testicle occur as simple retention cysts, the contents of which may be mucoid, clear or opaque. They may be single or multiple and may occur in the epididymis or in the body of the gland. When they contain spermatozoa, they are spermatic cysts or spermatocele (see p. 324). Cystic degeneration may take place in both the benign and malignant growths of the testicle. Among the benign growths which undergo eystic changes are 
the cystic fibroma and cyst-adenoma. It occurs in the malignant tumors of either carcinomatous or sarcomatous varieties.

Teratomata.-Teratomata, like dermoid cysts, are congenital growths, the dermoid cyst containing embryonal material in the form of hair, bone, teeth, etc., while the teratomata are a mixed variety of tumors which contain the congenital remnant of blastodermic tissue in the form of chorio-epithelium which is their distinguishing characteristic; otherwise, they present the features of carcinoma or sarcoma or of a mixed variety of growths and are exceedingly malignant which is the practical significance concerning them and suggests their early recognition and removal. They assume a large size in some instances and often present a conglomerate pathology as they may contain the elements of more than one type of neoplasm, benign and malignant.

Tumors of the Cord.-Malignant growths of the testicle and their recurrences may extend upward into the inguinal canal and involve the spermatic cord. Independent and primary involvement of the cord by the different types of neoplasm is rare.

Lipoma occurs in the region of the inguinal canal and may involve the tissues surrounding the spermatic cord. Such a fatty tumor is sometimes confused with an omental hernia, although differentiation should not be difficult as the growth in question is irreducible and painless, lobulated to the touch, and yields no impulse on coughing. 


\section{CHAPTER XVIII}

\section{MALADIES OF THE SEMINAL VESICLES}

The seminal vesicles, in addition to acting as temporary reservoirs for the seminal secretion, also contribute to it a constituent of diluent character which has an important function in preserving the vitality of the spermatic elements.

On account of the anatomical arrangement between the vas deferens and the tubular interior of the vesicle, it is possible to inject the vas through an opening made for this purpose with a view of treating locally the vesicular canal. Such injection will pass through the vas, thence through the seminal vesicle and finally reach the posterior urethra. The vas deferens on its route from the testicle to the prostatic urethra meets the apex of the vesicle and by a union with it forms the ejaculatory duct. A valvular arrangement at the point of union provides for the testicular secretion to pass into the vesicle. It is possible to palpate the seminal vesicles through the rectum, but not to reach their entire length.

Abnormalities of the Seminal Vesicles.-Abnormalities of the vesicles include the absence of one or both of them. The ejaculatory ducts may be absent entirely or in part. Malformations of these organs include fusion of the two glands or of the ejaculatory ducts, persistence of the fetal communication of the latter with the ureter, as well as an anomalous opening of these ducts in the termination of the urethra instead of in the prostatic portion. These malformations usually are associated with defects in the development of other portions of the urogenital apparatus.

Such malformations have little clinical significance excepting in so far as they may affect the viability of the fluid and thereby occasion the sterility of the individual.

Injuries of the Seminal Vesicle.-The vesicles are seldom injured by exterior wounds and only when associated with severe injury involving the surrounding parts. ${ }^{1}$ During operations upon the structures around the neck of the bladder including cystotomy, prostatec-

\footnotetext{
${ }_{1}$ Watson and Cunningham (Genito-Urinary Diseases) mention an instance of accidental injury of the vesicles in the case of a sailor who fell from the yardarm of a vessel astride an upright belaying-pin; the blunt end of the pin projected into the perineum, passed behind the bladder, lacerating the vesicles, among other parts seriously damaged.
} 
tomy and lithotomy, the vesicles may be injured directly by laceration and indirectly as a result of inflammation, in which case, the vasa deferentia or both ejaculatory ducts may become severed or occluded and sterility result.

The treatment of injuries of the vesicles will depend generally upon the immediate surgical requirements. When there is evidence of occlusion of the excretory ducts and consequent interference with the exit of the seminal elements, attempt may be made to pass a small filiform probe through the lumen of the canal by opening the vasa deferentia through a wound in the scrotum or by the injection of an antiseptic fluid such as 5 or 10 per cent. argyrol solution through the same opening (see p. 355), according to the method of treating chronic seminal vesiculitis.

Cysts of the Seminal Vesicles.-As a result of obstruction of the ejaculatory duct due to inflammatory process or pressure from without, distention of the vesicle may be caused and a retention cyst involving the entire vesicle or one or more of its diverticulæ may be formed ${ }^{1}$ and as one or more of the alveoli become cut off and occluded, there are produced small unilocular and multilocular cysts attached to the parent vesicle by a pedicle.

It is probable that some morbid condition within the cavity of the vesicles exists in addition to the obstruction to the ejaculatory ducts as a cause for the cystic condition.

There may be no symptoms until the cyst reaches sufficient dimensions to exercise pressure. Rectal palpation will recognize the cystic swelling or it may be seen through a proctoscope.

Treatment.- The proper treatment of cysts of the seminal vesicle is aspiration of the sac contents. In the case reported below (Jacobson), the condition was cured following two tappings.

If, after repetition, this method fails to effect a cure, open drainage of the seminal vesicles may be instituted through a perineal incision according to the method of Fuller, for chronic vesiculitis (see Operative Surgery, p. 491).

Concretions.-Calculi occur in the seminal vesicles but usually are not of sufficient clinical significance to be discovered during life. They consist of a number of small, millet-seed concretions occupying the diverticula of the vesicle or they may become considerably larger and obstruct the main excretory duct. They are composed of the inspissated seminal secretion combined with earthy salts and are more common in the aged. While it is unusual for them to cause any

\footnotetext{
1 Jacobson (Diseases of the Male Organs of Generation) quotes a case in which ten pints of a brownish serous fluid were drawn off from a vesicle.
} 
special symptom, when such oecurs, it is in the nature of a spermatic colic. The pain is spasmodic and intense, caused by the impaction of the concretion obstructing the passage of the ejaculatory duct. It may occur during the night, associated with an abortive seminal emission. A dull rectal pain may occur independently, on account of the presence of these concretions and such pain may be increased by micturition and defecation. It is not improbable that some of the obscure neuralgic pains directed toward this region may be accounted for in this manner. Calcification of the seminal vesicles occurs in connection with a generalized chronic inflammation of these organs and of the vasa deferentia. ${ }^{1}$

Diagnosis.-Concretions of the vesicles may be recognized by rectal palpation or their presence suspected on account of the existent symptoms.

Treatment.-No treatment is necessary unless the symptoms are of sufficient intensity, in which case pain is met by hot sitz baths, hot rectal injections and suppositories. In cases in which the conditions warrant it, vesiculotomy (drainage of the vesicles), or even vesiculectomy may be performed (see Operative Surgery, p. 491). Free vesicular drainage is adequate in most cases.

Tumors of the seminal vesicles are mostly extensions from the prostate and bladder or rectum; especially does cancer, involving the floor of the bladder, extend into these organs. It is alleged that both carcinoma and sarcoma may have their primary seat in the vesicles but certainly it is not of frequent occurrence or is not discovered until other parts are involved, when it is difficult to state from which direction the growth has originally spread. ${ }^{2}$

Tuberculosis of the Seminal Vesicles (see Urogenital Tuberculosis).

\section{Acute Seminal Vesiculitis}

\section{Spermato-cystitis}

Seminal Vesiculitis may occur as a separate and distinct affection as the result of the invasion of the bacillus coli communis and the common pyogenic microörganisms into the vesicles in combination with or without the gonococcus. Any inflammatory condi-

\footnotetext{
1 The Author operated upon one case suspected to be tuberculosis and discovered calcareous degeneration involving both the vesicles and the prostate. The case was one of very old, protracted inflammation involving the prostate, the seminal vesicles and the adjacent tissues.

2 Casper ("Genito-Urinary Diseases," p. 461) refers to a case which he observed in which a nodular tumor above the prostate and separated from it by a furrow, was palpated. This tumor destroyed the seminal vesicle and broke through into the rectum. There is some doubt, however, whether or not this growth was not originally a vesical cancer.
} 
tion existing in the prostatic sinus may extend by continuity into the vesicular reservoirs, but gonorrheal urethritis is more liable than any other inflammation to take this course. It is believed that this extension of inflammation from the prostate may be by way of the blood-vessels or lymph channels as well as by direct propagation. While gonorrhea is the etiological factor in most instances, it is possible that traumatism, great physical strain, especially in the presence of mild catarrhal inflammation, may light up an acute exacerbation.

This is more possible in individuals of a neurotic temperament and those whose organs are made more susceptible by the tuberculous diathesis or excessive malnutrition. When the invasion occurs during the height of a virulent gonorrheal infection, the process gradually involves the entire vesicle and extends to the adjacent tissues as well, in which case there is a perivesiculitis or a plastic exudate surrounding the affected organ and a circumscribed inflammation of the neighboring wall of the bladder. Complete resolution may occur under favorable conditions, but there is a tendency for the acute condition to pass into the chronic form or suppuration and localized abscess may develop; otherwise the pus forces an entrance through the excretory duct into the urethra.

Symptoms.-The symptoms are those accompanying prostatitis or urethro-cystitis; consequently, it is difficult to make a sharp differentiation between these conditions. Naturally, the finger of the experienced diagnostician is more skilled in determining this question and in well-marked cases, the exploring finger will readily detect an edematous, hot and sensitive area in the region of the affected vesicle which will be more easily recognized if unilaterally than if bilaterally involved, as in the latter case, the swelling is between the two organs and it obliterates the landmarks between them and the base of the bladder.

In very virulent infections, both the prostate and vesicles are involved on one or both sides. Rectal examination causes great pain and marked infiltration is detected in the regions of both organs. There is apt to be, at the height of this condition, marked febrile reaction.

The general symptoms are a sense of tension, vesical pain, tenesmus, painful erections and sometimes spermatozoa in the urethral discharges and purulent or bloody seminal emissions. The pain may radiate toward the abdomen and raise the suspicion of appendicitis or renal colic. Defecation may be painful and dysuria is marked in severe cases and in others is according to the degree of the urethrocystitis accompanying this condition. 
As the symptoms are not.entirely distinctive of this disorder, the diagnosis must depend upon rectal examination.

Prognosis.-In favorable cases, spontaneous resolution occurs but the course of the inflammation takes on great severity in exceptional instances; this is more likely where the bacterial invasion has been unusually severe, where the patient's resistance is weak or where, during the course of acute gonorrheal inflammation, unfavorable conditions have been invited by excessive indulgence in alcohol or otherwise. When abscess forms, as is sometimes the case, it may discharge itself forward into the urethra or backward into the rectum. Such abscesses have been known to empty into the abdominal cavity causing fatal peritonitis, or require surgical intervention to direct the course of the pus externally. The common termination of seminal vesiculitis after several weeks' duration is in the chronic form of the malady.

Treatment.-The treatment of acute vesiculitis is the same as that of acute prostatitis, namely, rest in bed upon a light diet devoid of highly seasoned stimulating food, freedom from all sexual excitement, the internal use of balsamics, alkalies and diuretics, as the symptoms may call for (see p. 208), and the local application of heat to the perineum. Massage and rectal douches are not warranted in the acute stage, being liable to cause irritation and do harm. At the inception of the acute invasion of gonorrheal infection, the use of autogenous or stock vaccines of the gonorrheal or combined variety are indicated (see section on Serums and Vaccines). If an abscess forms in spite of these measures, its contents should be evacuated by a rectal incision as soon as fluctuation can be distinctly felt. When the abscess has discharged itself spontaneously or by incision and the acute symptoms have subsided, treatment is the same as for the chronic condition.

\section{Chronic Vesiculitis}

Chronic seminal vesiculitis is a protraction of the acute trouble which is as stated, more than likely to occur. The symptoms peculiar to the chronic stage are less marked than in the acute disorder. They may be summed up as those that relate to the vesicle itself and to an accompanying chronic prostatitis, and those that depend upon a coexistent chronic urethritis. The last-named condition produces a certain amount of urethral discharge or gleet. Chronic vesiculitis, therefore, is responsible for many of the cases of chronic urethritis that have to be dealt with, the cause of which, as mentioned in the section on chronic urethritis, must be sought for in a morbid condition of the structures outside of the urethral canal. Frequent relapses of chronic urethritis are likely to be caused by latent lesions that exist in these 
organs or in the prostate. If the inflammation includes the area surrounding the neck of the bladder, there will be present symptoms associated with urination involving a certain amount of urgency and perhaps pain at the end of the urinary act. The discharge from the urethra is purulent and may contain spermatozoa and other elements of the seminal secretion, due to a leakage of the ejaculatory ducts (Fig. 140) as a result of thickening, and loss of contractility of these outlets. There may be also a discharge of prostatic secretion caused

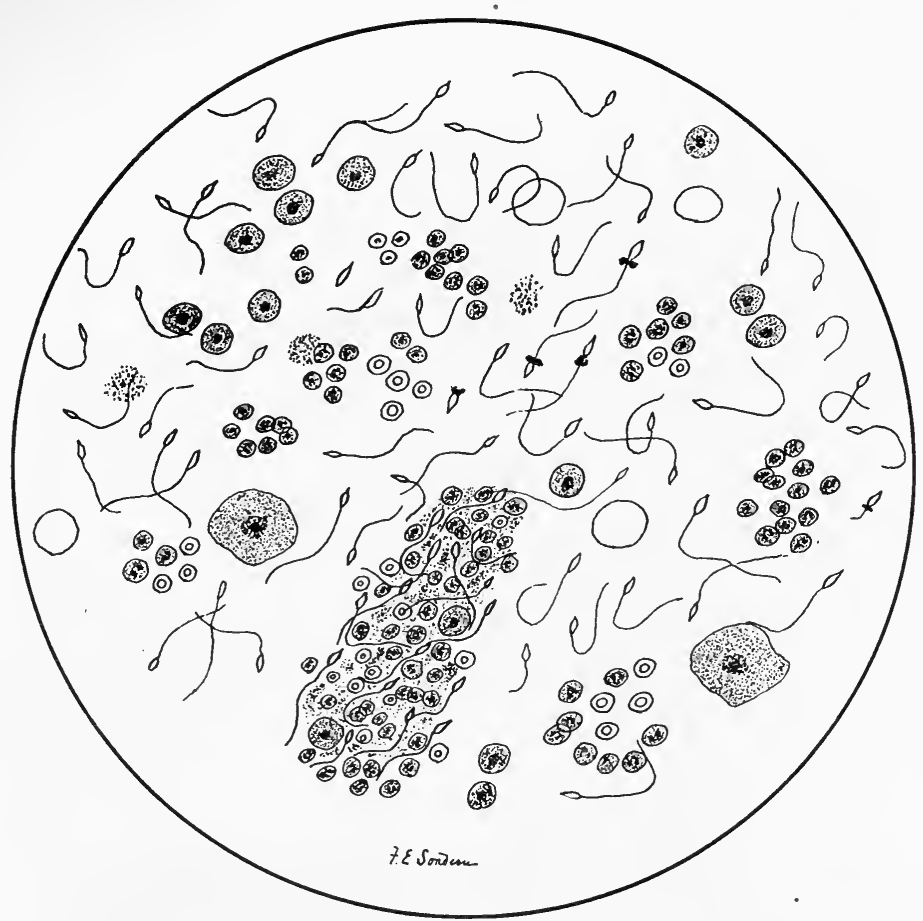

FIG. 140.- Spermatocystitis and spermatorrhea.

by chronic prostatitis; this is produced more particularly by pressure of massage (see p. 378). The symptoms referable to the vesicles and prostate are: first, those elicited from the patient and second, those obtained from rectal palpation. The subjective symptom is a dull pain referable to the region of the rectum and perineum and felt more particularly when pressure is exerted in one manner or another. Such pain, in mild cases, is not always present. Nervous symptoms which are mentioned as characterizing chronic prostatitis, are very likely to be associated with the chronic stage of this malady. They represent a 
form of sexual neurasthenia which causes an excessive degree of depression and hyperchondria. Sexual symptoms may also be present with consequent modification of the seminal secretion-oligospermia, necro-spermia, etc. (Fig. 141) involving partial or complete sterility (see p. 515).

Rectal Examination.-There may not be discovered by digital exploration a circumscribed enlargement of the vesicle in all cases; in fact, as a result of the acute condition, there may be found a general

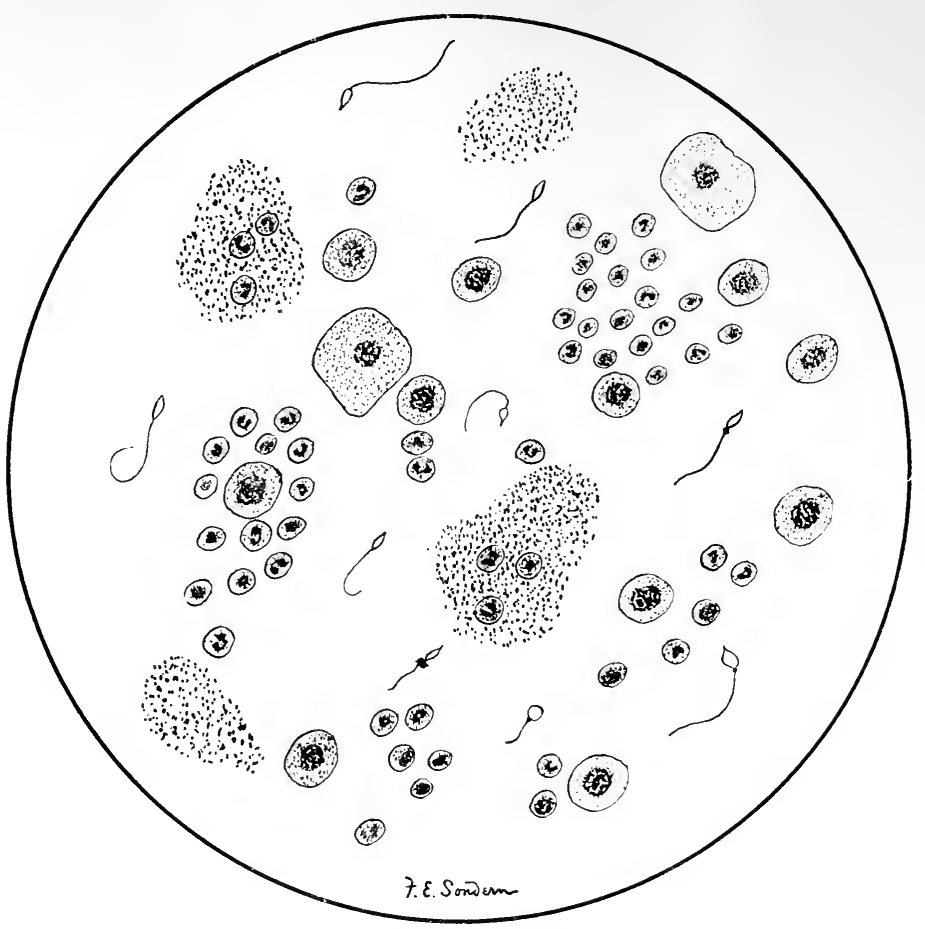

FIG. 141.-Chronic gonorrheal prostatitis and vesiculitis. Urine after stripping vesicles.

thickening of the area in the region of one or both of the vesicles at the base of the bladder above the prostate; or there may be recognized a greater amount of swelling in one of the vesicles than in the other and there may also be distinguished the characteristic conditions of chronic prostatitis with symmetrical enlargement of this gland. Expression of the contents of the vesicles with the finger or with a hard rubber instrument and massage of the prostate will obtain a certain amount of the pent-up secretions of these organs along with the inflammatory 
products. This material will be made to exude into the urethra and may be seen at the meatus at the time of the examination or be washed out by urination or lavage. An examination of the contents of the inflamed organs thus obtained will reveal the presence of spermatozoa generally dead, some distorted and broken up; also epithelia from the prostate and vesicles as well as pus and granular bodies in varying quantities (Fig. 141). Various microörganisms may also be discovered in stained smears.

Prognosis.-There is no danger in the existence of chronic vesiculitis; the only unfavorable feature is the tendency of some cases to be protracted and obstinate in spite of treatment. It may be stated, however, that under the present day methods of diagnosis and direct treatment, the number of such cases is relatively small.

Treatment.-The object of treatment is primarily to free the vesicles from the lodgment of bacterial poison, to evacuate the pent-up secretions in which the former may be contained and to endeavor to establish and maintain good drainage of the excretory duct. To accomplish the first two of these requirements, massage and stripping of the vesicles is an important measure of treatment. This manœuvre should be conducted by the surgeon with skilful but gentle manipulation, otherwise the end is not successfully attained and harm, rather than good, is wrought by the lighting up of more acute inflammation or by extending the inflammation from the vesicles into the testicle. Therefore, judgment should be exercised as to the amount of pressure employed and the intervals between the treatments. The patient is placed in a bending-over position on his feet or preferably in a kneechest position on the operating table or couch. After insertion of the finger, the prostate is felt above the internal sphincter, and above the prostate on either side laterally are felt the apex and as much of the body of the vesicle as can be reached by the examining finger. The treatment is performed by a downward stripping with the end of the finger which is applied to both of the organs, the amount of pressure being governed more or less by the degree of pain inflicted. Assistance may sometimes be had by counter-pressure with the opposite hand against the lower abdomen, force being exerted toward the bottom of the pelvis. After the vesicles have been attacked in this manner, the prostate should also be massaged by a firm, rotary and lateral movement of the finger over its entire circumference. At a subsequent time, when it has been determined that the patient sustains the treatment well, the upper pole of the vesicle may also be reached and treated by means of a hard rubber masseur (see Fig. 142) which is always introduced after the finger has completed the massage of that 
portion within its reach and the operator has been akle to take careful note of the lay of the parts for his guidance in the introduction of the masseur. This treatment should be repeated from two to four times a week, sometimes only once a week at the beginning, according to the response of the patient thereto and the presence or absence of irritation thereafter. This treatment may be supplemented and good drainage of the vesicles encouraged by the adoption of hydropathic methods of treatment as obtained by the use of hot rectal irrigations of saline solution similar to the method recommended in chronic prostatitis, q.v. p. 382 .

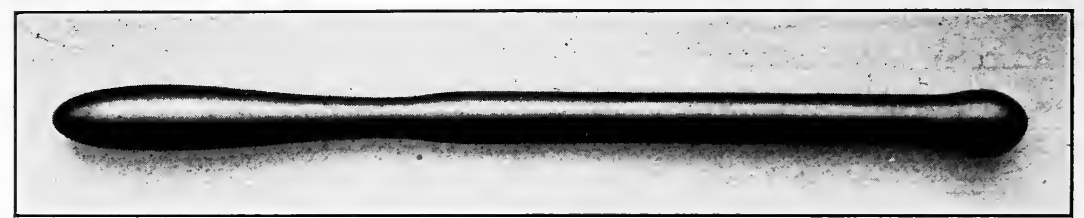

FIG. 142.-Prostatic masseur.

While the mechanical measures above described may succeed in accomplishing one of the requisites of successful treatment laid down, namely, that of evacuating the stored-up inflammatory contents of the diseased organs, and thereby aid in eliminating quantities of organisms, many of them perhaps inert and others that may not be so harmless - yet to antagonize more directly the microbial element of the malady which exists to a greater or less extent in different cases, recourse to the vaccine and the serum treatments appropriately combined, will in some cases produce prompt and favorable results.

In cases that are obstinately resistant to prolonged treatment, other chronic conditions may have a bearing upon the prospect of recovery and must be taken into account in the direction of treatment. Thus, an infection of great virulence propagated from the deep urethra would be likely to involve the base of the bladder as well as the prostate and vesicles, resulting in a fibrotic contracture (stricture) of the neck of the bladder which calls for surgical intervention for the latter condition (see p. 435). Tuberculous involvement of the seminal vesicles and prostate should always be suspected in chronic and indolent cases (see Chapter XXXVII).

The method of making direct applications to the interior of the vesicles by injecting through the tubular extensions of these organs, the vasa deferentia, solutions of one of the silver salts is recommended in more chronic and obstinate cases when other measures have failed. 
It has been demonstrated that by tapping the vas deferens in the scrotum, such solutions injected through the proximal end of this duct will reach the seminal vesicle, pass through its entire interior and then by way of the ampullæ and ejaculatory ducts pass into the posterior urethra. This expedient may be the means of reaching portions of the vas that are otherwise unaffected by treatment and of opening up the excretory duct occluded by old retained secretions. ${ }^{1}$ The vas deferens may be stitched to the skin with fine silk passed through the cut edges, thus enabling the treatment to be repeated as often as desired. I have tested this treatment in a number of cases with favorable results but believe it should be supplemented with the bacterial vaccine treatment as well (p. 354).

1 Belfield of Chicago was first active in this method of treatment and designated the condition of the vesicles demanding it, "pus tubes in the male." 


\section{CHAPTER XIX}

\section{THE OPERATIVE SURGERY OF THE EXTERNAL GEN- ITALS, THE PENIS, SCROTUM, TESTICLE AND SPERMATIC CORD}

The operations upon the external genitals are performed under both local and general anesthesia. They comprise the operations upon the foreskin, incomplete and complete circumcision; incision and removal of diseased inguinal glands (bubo); operations upon the scrotum and its contents, the testicles and spermatic cord; partial and complete removal of the external genital organs.

The preparation for operation is similar to that of all surgical operations including the proper cleansing and disinfection of the parts before and at the time, care being exercised in this particular not to excoriate the parts by excessive friction or too strong antiseptics as the skin of this region is especially sensitive. When mercuric chloride solutions are used from 1 to 3000 to 1 to 6000 is sufficient and the weaker strength always when a wet dressing is left on. For rapid sterilization and extra disinfection the parts may be covered with a solution of equal parts of alcohol and iodine which is only warranted on these sensitive organs when a virulent infection is present as this solution will cause sharp smarting and excoriation in this region. On most occasions it is better to employ cleansing with green soap and water and sponging with a 1 to 5000 bichloride of mercury solution in such procedures as the closed operations of the scrotum (aspiration of hydrocele and subcutaneous ligation of varicocele) and those upon the foreskin, when the interior of the prepuce should also be thoroughly cleansed by retracting it or by plentiful injections if this is impossible. For open operations, such as those upon the interior of the scrotum (hydrocele, varicocele, ligation of vas deferens, etc.), it is necessary to follow the usual custom of a double preparation several hours before and at the time of the operation.

Operations for Phimosis. Bilateral Incision of the Prepuce. (Fig. 143.) - The cocaine solution (1/5 of 1 per cent.) or novocain (1/2 of 1 per cent.) is injected subcutaneously on both sides of the foreskin from the preputial opening backward to about the site of the corona glandis. The mucous membrane lining of the preputial cavity is anesthetized by 
alypin lubricant or an effort made to introduce the needle inside the prepuce and inject the mucous surface in the same area as the skin.

The prepuce is drawn down with a pair of artery clamps above and below and the incisions made bilaterally with blunt pointed scissors from the end of the prepuce back to the coronal sulcus. This gives an upper and lower flap which will allow free inspection of the entire glans and mucous surface.

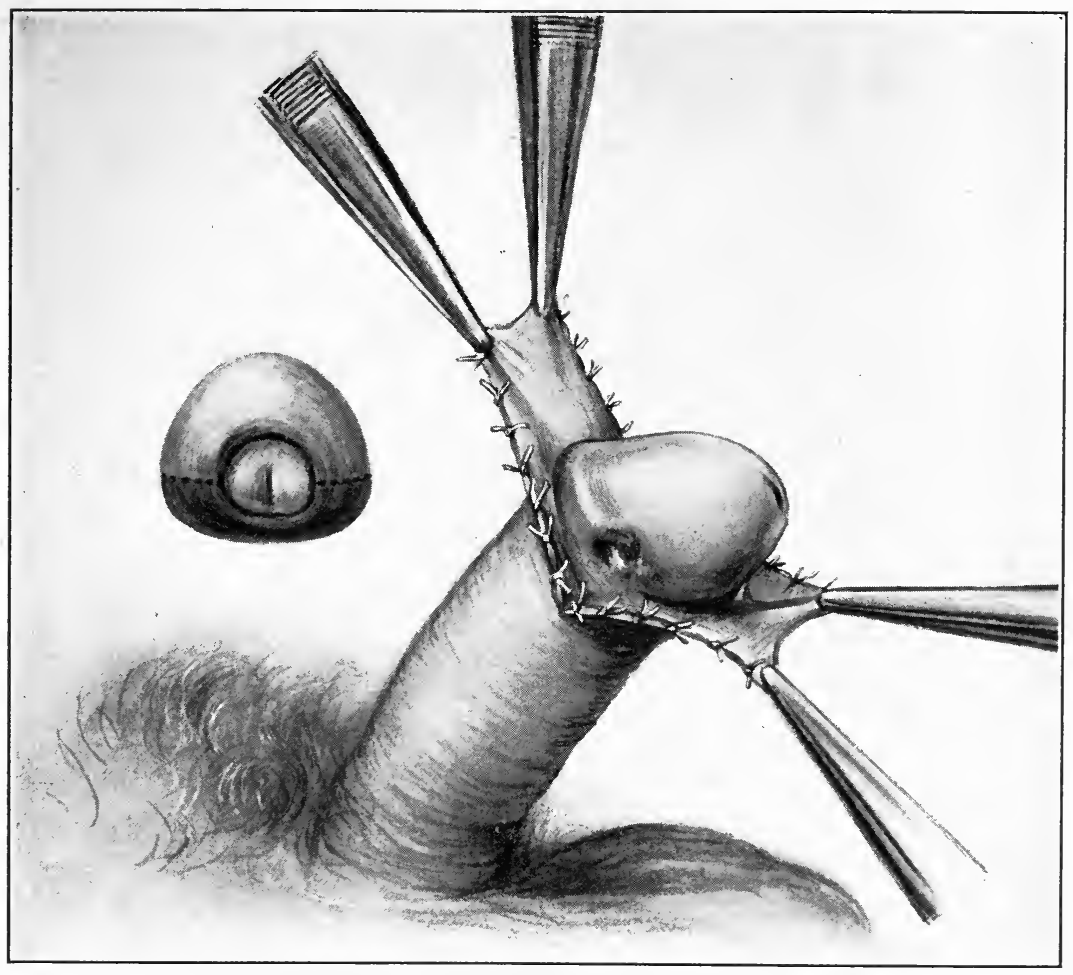

Fig. 143.-Bilateral incision of prepuce for exposure of glans.

Bleeding vessels are caught and tied with plain catgut or interrupted sutures are inserted through the two layers of the foreskin on both sides in sufficient number to control the hemorrhage.

A wet phenol (1 to 50) or bichloride (1-5000) solution dressing is applied and renewed as often as the local condition requires. The subsequent treatment depends upon the underlying lesion (see balanitis or chancroid). 


\section{Requirements for Operation}

Hypodermic syringe and needle.

Cocaine solution $1 / 5$ of 1 per cent. or novocain $1 / 2$ of 1 per cent.

Alypin lubricant 5 per cent.

$1 / 2$-ounce glass urethral syringe.

1 pair of straight, blunt pointed scissors.

6 artery clamps; needle holder and needles.

2 pairs of thumb forceps; grooved director; No. 1 plain catgut.

Gauze bandages and bichloride of mercury solution, black wash or 1 to 50 phenol solution.

Division of the Frenum. - A few drops of $1 / 5$ of 1 per cent. cocaine solution are injected along the line of attachment of the frenum. A needle with plain catgut is passed beneath the frenum where it joins the mucous layer of the foreskin and another close to its insertion into the glans. Both stitches take in the whole thickness and are tied securely, after which the intervening part is incised with scissors. Dust with iodide of thymol and dress with boric or carbolic solution.

\section{Requirements for Operation}

No. 1 plain catgut. Cocaine solution $1 / 5$ of 1 per cent.

Needle holder and needle. Hypodermic syringe and needle. 1 pair of scissors.

\section{CIRCUMCISION}

In children under twelve years of age, with very few exceptions, a general anesthetic is necessary. In adults local anesthesia is always satisfactory and preferable.

Peripheral anesthesia of the foreskin is obtained by infiltrating the prepuce subcutaneously and the sub-mucous area in the line of the incision with a $5 / 10$ per cent. solution of novocain. The line of the incision may be preliminarily marked off on the skin with iodine (Fig. 144). The foreskin is then punctured with the syringe and the solution freely injected subcutaneously above and below this line. The needle is then made to penetrate further until it reaches the submucous area adjacent to the coronary sulcus and the solution is infiltrated all the way around. One or two punctures should be enough to encircle the entire prepuce. If retraction of the foreskin is not possible, after the cavity has been cleansed with an antiseptic solution an injection of Alypin lubricant 5 per cent. is introduced and retained five minutes. This method of anesthesia is most commonly used but 
complete distal anesthesia of the foreskin may be obtained by infiltrating the base of the penis (see special section p. 501).

The incision should be oblique and not circular (see Fig. 144); the amount of tissue removed being greater on the dorsal than on the ventral surface. A circular cut shortens the foreskin but enlarges the

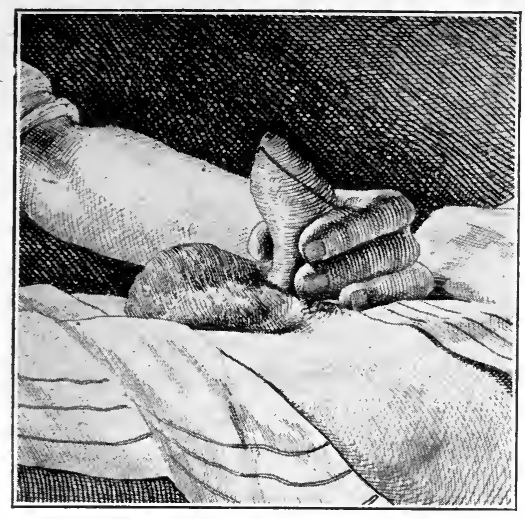

FIg. 144.-Oblique line of incision.

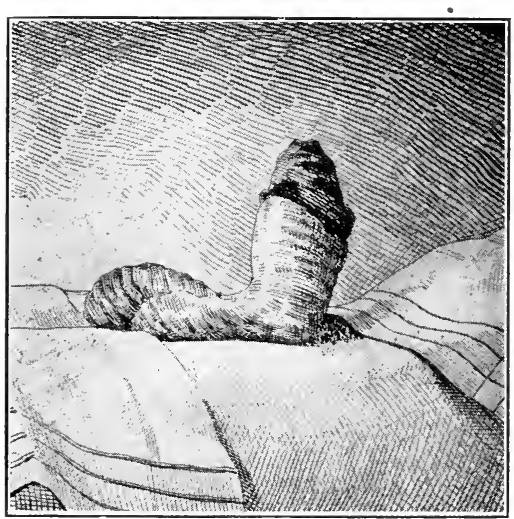

FIG. 146.-Mucosa covering glans after removal of forceps.

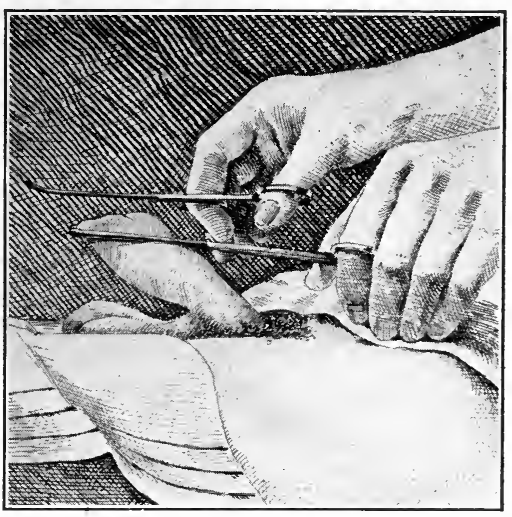

FIG. 145.-Forceps applied to foreskin.

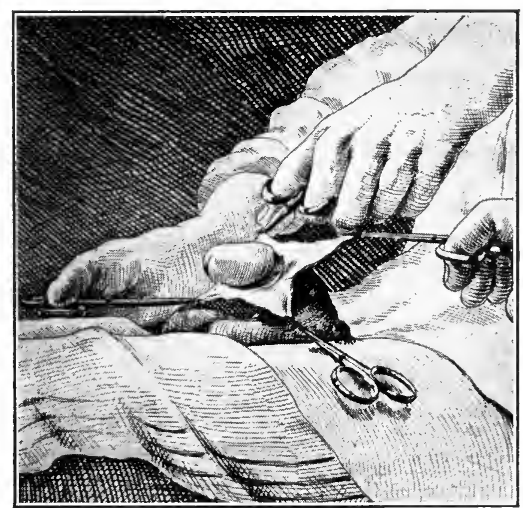

FIG. 147.-Glans exposed, trimming of mucosa.

aperture insufficiently so that an unsatisfactory result may be produced amounting to a paraphymosis. With a pair of circumcision forceps (Fig. 145) the foreskin is clamped obliquely care being taken to avoid the glans penis. The prepuce beyond the forceps is now excised and the latter removed when it will be observed that the skin has retracted but that the mucosa still covers the glans penis 
(Fig. 146). This is now slit up the dorsal aspect to within $6 \mathrm{~mm} .(1 / 4$ inch), of its attachment in adults and to within $3 \mathrm{~mm}$. (1/8 in.) in infants (Fig. 147) and is then trimmed away evenly on both sides to the frenum.

The field of operation has been kept comparatively bloodless by constricting the penis near the pubis with a soft rubber catheter as a

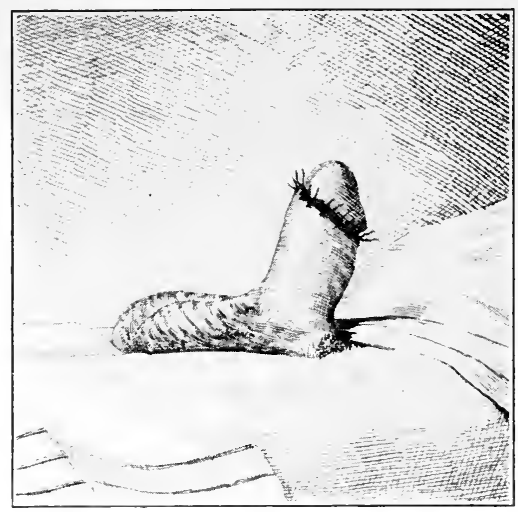

FIG. 148.-Operation completed.

tourniquet. This is now released, the bleeding vessels caught with artery forceps and tied. The artery of the frenum and the dorsal veins sometimes bleed freely.

The skin and mucous layer are next sewed together as evenly as possible. For this purpose No. 0 plain catgut is used in the case of infants and for older children up to twelve years No. 1 plain catgut.

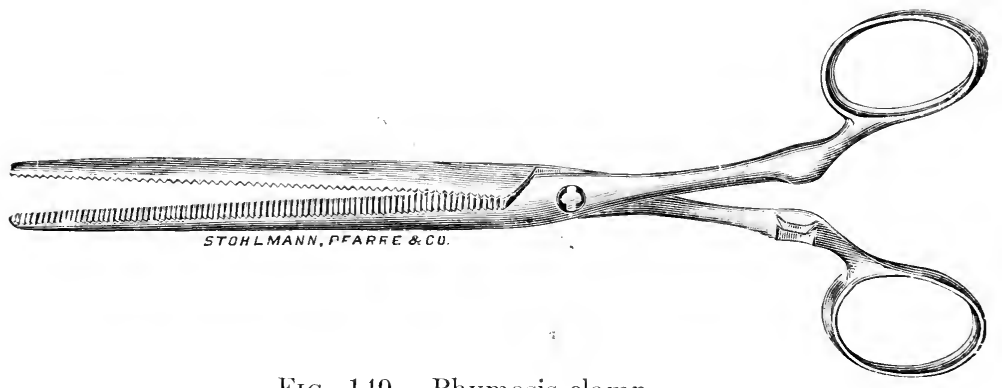

FIG. 149.-Phymosis clamp.

For adults, horsehair is the best suture material. These sutures are placed about $5 \mathrm{~mm}$. (1/5 inch) apart (Fig. 148).

No dressing is used for infants and children; the parts are protected from rubbing against the clothing with a gauze pad; a daily bathing with lukewarm boracic acid solution cleanses the parts and lessens 
the discomfort. In adults, the ends of the tied horsehair sutures are left long, and between them, a narrow strip of plain or iodoform gauze, lubricated with sterile petrolatum, about $13 \mathrm{~mm}$. wide folded on itself is tied along the line of suture (see Fig. 150), beginning and ending on the dorsum so as to encircle the penis completely.

Instruction is given to keep the dressing free from urine contamination, by urinating in the upright posture and drying the parts thereafter.

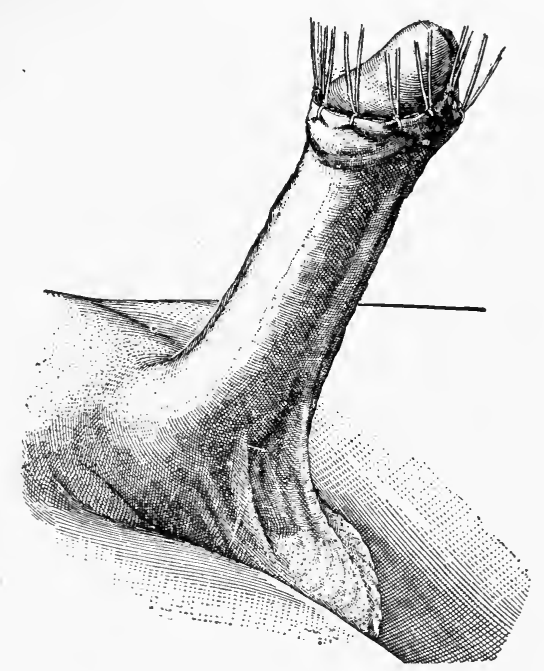

FIG. 150.-Circumcision. Showing method of tying gauze strip around incision.

The stitches should be removed on the fifth to the seventh day and complete healing occurs in ten to fourteen days.

The after-treatment consists in keeping the parts clean and dry and dusting with thymol iodide or other antiseptic powder.

\section{Requirements for Operation}

Rubber catheter for tourniquet.

Hypodermic syringe and needle.

Cocaine solution $1 / 5$ of 1 per cent., or novocain $1 / 2$ of 1 per cent. Phymosis forceps (Fig. 149).

1 scalpel.

1 pair of scissors, curved on the flat.

2 pairs of thumb forceps.

6 artery clamps.

Needle holder and needles.

No. 00 and No. 0 plain catgut, one tube each; 1 tube of horsehair. 


\section{Incision and Excision of Inguinal Glands}

Simple incision of the inguinal glands is required to evacuate the contents of suppurating bubo. This may be done under local anesthesia (preferably by freezing with ethyl chloride) and when the abscess is near the surface and complete drainage is accomplished resolution may result. But in indolent and especially tuberculous conditions or where there exist multiple suppurative foci in the structure of one or more glands complete extirpation becomes necessary.

Inguinal Adenectomy. - This operation is performed for broken down and indolent glands in the groin, such as chronic bubo. It is also a part of the operation of amputation of the penis for cancer and complete emasculation.

A free oblique incision 10 to 12 centimeters ( 4 to 5 in.) long is made in the groin, and in the case of bubo the free pus expressed and wiped away with gauze or by irrigation with bichloride of mercury 1 to 5000 .

By blunt dissection, the nodes are freed and removed, care being taken to avoid wounding the large inguinal vessels if the dissection has to be carried down to the deep abdominal fascia.

The entire cavity is then swabbed with bichloride of mercury solution 1 to 2000 or tincture of iodine and alcohol equal parts; bleeding points are ligated with catgut and the cavity is packed with iodoform gauze.

The dressing is changed every day and a mixture of equal parts castor oil and Peru balsam is used as a stimulant to hasten granulation. If the skin is not too much destroyed by the suppurative process, the edges may be approximated by silkworm gut sutures and the entire cavity filled up with 10 per cent. iodoform and petrolatum. Lymph stasis is apt to follow extensive removal, causing more or less edema of the lower extremity but this subsides when a collateral lymph circulation has been established.

\section{Requirements for Inguinal Adenectomy}

General anesthesia.

1 scalpel.

1 dozen artery clamps.

1 pair blunt pointed scissors.

Needle holder and needles.

1 sharp curette; 1 dull curette. 
Plain catgut, silkworm gut.

Iodoform gauze packing.

Iodoform ointment 10 per cent.

\section{OPERATION FOR VARICOCELE}

Subcutaneous Ligation.-This operation is suitable for small varicoceles when one or two large trunks can be isolated and when the open method is objected to by the patient.

The patient stands up to make the veins distend. The subcutaneous ligation is effected by isolating a venous trunk from the rest of the veins and the vas deferens. The scrotum is transfixed with a silk ligature on one side of the vein, and is then passed on the other side of the vein so as to enter and exit through the same punctures in the dartos, the silk being picked up, drawn around the vein and out again through the point of entrance. One ligature is placed below and one above on each venous trunk to be tied. Special needles (Keyes modification of Reverdin needle) are used, the eye of which opens from the handle. With the thumb and index finger of one hand the vas and all other veins excepting the one to be tied are held on one side by pinching together the front and rear walls of the scrotum. The needle threaded is then thrust from front to rear completely through the scrotum and the ligature is removed from the eye. One end of the ligature now hangs in front and the other in the back. The needle is now withdrawn as far as the entrance puncture, but not entirely out; it is then reinserted on the outside of the vein and is made to exit out of the same hole posteriorly. The end of the silk behind is picked up in the eye of the needle and it is then pulled to the front and outside thus completely encircling the vein. This process is repeated in as many points as necessary to place two ligatures on each varicose trunk, one above and one below.

The patient now lies down on the operating table and the ligatures are tied tightly, beginning below and ending above. The subcutaneous tissues caught in the ligatures are forcibly separated by pulling apart the anterior and posterior scrotal walls and thus the knots after the ligatures are cut close are made to disappear. A little chloroform inhalation is necessary for the last part of the operation.

Collodion and absorbent cotton are applied to the punctures and a suspensory bandage is worn. Confinement in bed for three to six days is generally advisable. The swelling gradually subsides. 


\section{Requirements for Väicocele, Subcutaneous Method}

2 Keyes needles. 1 hypodermic syringe and needle. Cocaine, $1 / 5$ per cent. or novocain $1 / 2$ of 1 per cent.

1 tube of heavy twisted silk.

Gas or chloroform when tying.

Open Operation for Varicocele (Fig. 151).-This operation is generally preferred to the closed method since it has become possible

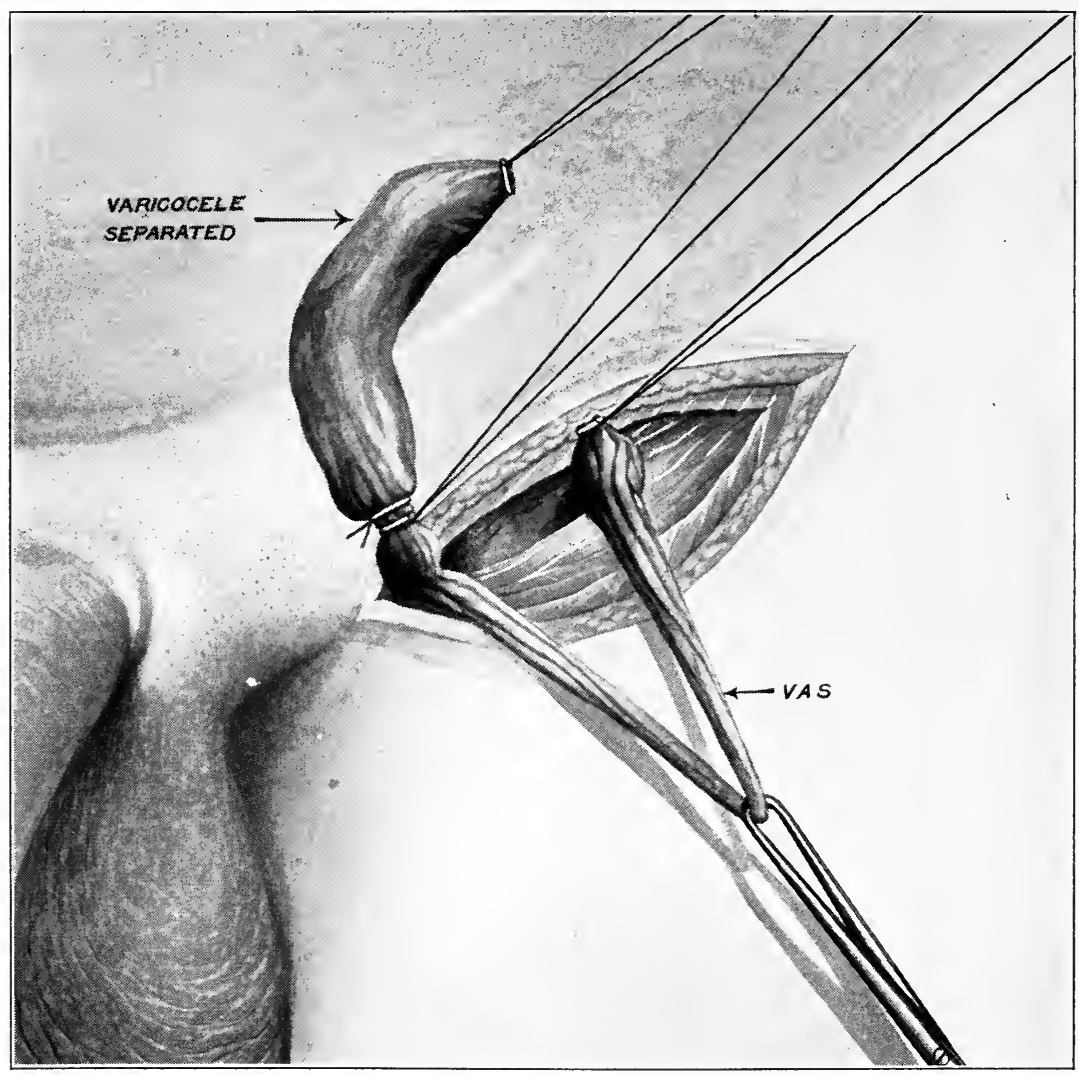

FIG. 151.- Open operation for varicocele.

in most instances to employ local anesthesia and it is considered more radical, probably because the veins are not only ligated but a considerable portion of them removed as well. While many cases can be satisfactorily handled under local anesthesia, the neurotic temperament of some patients who seek relief from varicocele is 
likely to embarrass the operator; therefore in such cases, general anesthesia is preferred. (See Local Anesthesia, p. 501.)

An oblique incision is made 5 to 8 centimeters ( 2 or 3 in.) long over the external ring and a little above the spine of the pubis extending downward toward the scrotum. This cut into the adipose tissue is met by some active hemorrhage from the superficial veins which are ligated forthwith, and the tissues are pushed aside and retracted downward to the fascia overlying the spermatic cord. The cord is easily detected and after the fascia is slit with a pair of scissors it is elevated upon a grooved director or dressing forceps and held in the incision. The operator and his assistant now lift the thin, delicate coverings of the cord with thumb forceps and by putting them on the stretch crosswise, it becomes possible to divide them without injury to the vessels which are readily seen through the translucent tissues when thus elevated. The veins of the pampiniform plexus are united into three or four large and tortuous trunks before entering the abdominal ring; two or three of these trunks are liberated carefully from the vas deferens and tied above with two ligatures (Fig. 151) between which the isolated portion of the varicocele is severed with a pair of scissors. The lower segment now contains that portion of the varicocele, between the division and testicle, which portion is to be excised. By careful dissection and transillumination of the tissues, with the aid of an assistant, this lower segment is followed down to within $2 \mathrm{~cm}$. (3/4 inch) of the epididymis, great care being exercised to avoid the vas. When satisfactory separation is accomplished, it will be found that whereas the venous plexus was composed of a few trunks above, below it has become a network of veins bound together by connective tissue. The centre of this mass is transfixed by double ligature and tied externally and internally and the ends of these ligatures are again tied to one another. Another ligature is put upon this segment of veins above the lower one and by cutting between them the entire varicocele thus excised is removed. On account of the importance of securing the ligatures around the cut ends of the veins against possible slipping, it is advisable to stitch the long end of the ligature through the remaining trunk of veins and then tie the two divided ends together. It is very important to make sure that no bleeding points are left untied before the wound is sutured. If the tunica vaginalis has been opened and the testicle exposed, it should be closed with one or two stitches of plain catgut.

The skin and superficial tissues are brought together by interrupted sutures, preferably horsehair or silkworm gut, the ends of which are left long and used to tie a narrow strip of plain or iodoform gauze 
over the incision and upon this is placed a pad of plain gauze held by adhesive strips. The scrotum is supported upon an adhesive plaster band carried across the thighs or by means of a suspensory bandage. The patient is confined to bed for about one week, following which he is able to be up and around and there persists for several weeks a certain amount of swelling at the site of the ligated veins. Sometimes a traumatic hydrocele is produced which may be absorbed spontaneously or may require tapping. It is of little consequence The testicle sometimes swells but healthy resolution usually occurs.

\section{Requirements for Operation}

Hypodermic syringe and needle.

Scalpel.

Pair of blunt pointed scissors.

1 dozen pair of artery forceps.

Grooved director.

Needle holder, and needles.

2 pairs of thumb forceps.

Tube of No. 1 chromic catgut.

2 tubes of No. 1 plain catgut.

Tube of silkworm gut.

Suspensory bandage.

Closed Operation for Hydrocele.-Aspiration of the sac of simple hydrocele accompanied by the injection of pure undiluted carbolic acid results in satisfactory and permanent removal of this condition in a certain number of instances. To be successful, it is necessary that the case be one of simple hydrocele, uncomplicated by disease of the testicle or constitutional disorder (tuberculosis or syphilis), and that the local conditions be entirely favorable, that is to say that the fluid is perfectly clear, uninfected and uncontaminated with blood, bacteria or pus. To determine the indication for this technic in a given case, the sac of the hydrocele is transilluminated and the location and contour of the testicle are outlined. A specimen of the fluid for inspection may also be obtained by hypodermic syringe. When this particular technic is decided upon, the following steps are carried out, the surface first being prepared by the application of iodine and alcohol.

A small hypodermic needle is introduced at the top of the sac (Fig. 152). A good-sized aspirating needle is then inserted at the lower portion and the two needles are brought together to make sure that their ends are free in the same cavity and not in separate sacs. 
The aspirating needle is now attached to the connecting tube of the aspirating bottle from which the air has been exhausted and the fluid in the sac is completely and thoroughly evacuated. When this has been satisfactorily accomplished, the aspirating needle is withdrawn, the smaller needle having been kept carefully in situ. The hypodermic syringe filled with pure deliquesced carbolic acid is now firmly attached to its needle and the required amount of acid is slowly injected. The needle is then quickly withdrawn being careful not

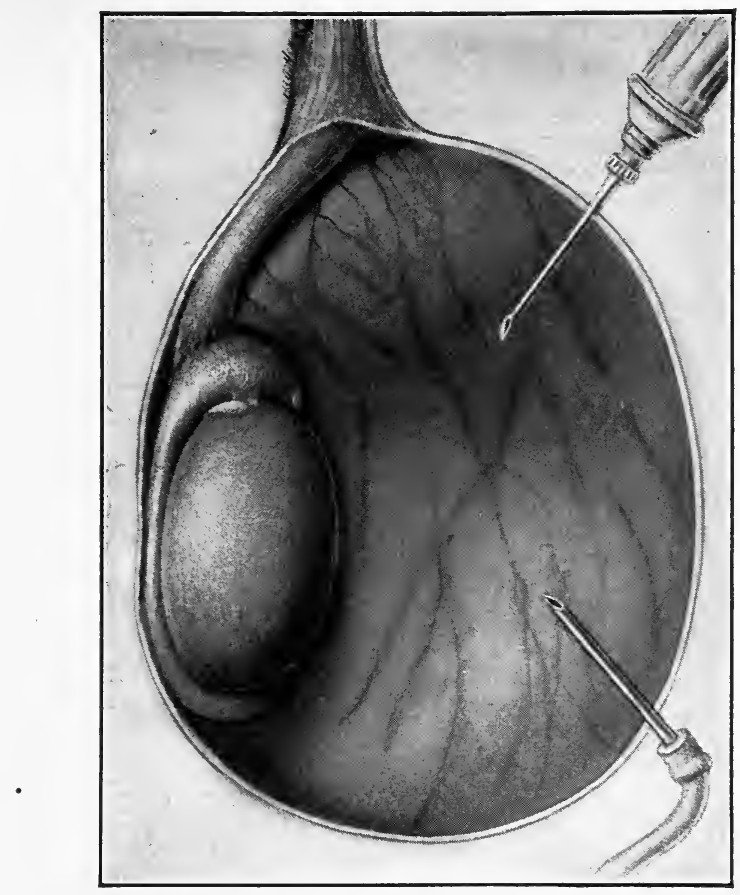

FIG. 152.-Radical operation for hydrocele. Injection method.

to spill any of the acid on the outside tissues. The scrotum is then kneaded together to distribute the acid thoroughly within the sac. The punctures are then sealed with collodion and the scrotum placed in a suspensory bandage.

For small hydrocele sacs (that is from 60 to 125 c.c.) from one to two cubic centimeters of the carbolic acid are used and for those of larger dimensions about 1 c.c. for each additional 60 c.c. of fluid. In case any of the acid is spilled upon the tissues at the time of operation immersion with alcohol immediately will counteract the escharotic effect of the acid. 
Following this injection treatment, the hydrocele sac is liable partially to refill. This is due to the traumatism exercised by the carbolic acid and when this fluid is again aspirated, at the end of one or two weeks, successful cases will not refill. When the latter occurs, however, in spite of the injection, it then becomes necessary to resort to the open operation for the relief of this condition.

Requirements for Tapping and Carbolic Acid Injection of Hydrocele

Sixty minim hypodermic syringe and needle.

Pure deliquesced carbolic acid.

Alcohol. Iodine.

Aspirating needle.

Aspirator and bottle.

Collodion; suspensory bandage.

Spermatocele.-This form of cystic growth of the testicle may be treated in the same manner as simple hydrocele, namely, by aspiration and injection with pure carbolic acid. A single small needle only is used for both aspiration and injection. For the latter $1 / 2$ to 1 c.c. of carbolic acid are usually sufficient. In obstinate recurring cases it may be necessary to lay open the scrotum overlying the cyst and after exposing the same it is incised, the contents evacuated, and the interior treated with pure carbolic acid and then alcohol or the cyst wall is removed with scissors, hemorrhage controlled and the wound sutured with a small drain.

Open Operation for Hydrocele.-Local anesthesia may be sufficient for this operation but a general anesthetic is sometimes insisted upon. (See Local Anesthesia, p. 501.)

The parts having been properly prepared, a longitudinal incision is made over the most prominent portion of the sac 8 to 10 centimeters (3 or $4 \mathrm{in.}$ ) in length down to the tunica vaginalis. A small aperture is then made in the sac through which the fluid is allowed to escape until it is emptied. The aperture is then seized on either side by a pair of artery clamps and is slit completely open. In cases of moderate size, this sac is simply everted and sewed backward, leaving the testicle on the outside, and the cavity of the scrotum is then closed by suture. When the hydrocele is large, the sac wall is partially excised with scissors or only a fringe is left 1 to $2 \mathrm{~cm}$. (1/2 to $3 / 4$ inch) deep on either side of the epididymis. These cut edges are sewn hindwise by continuous catgut stitch, thus closing in the epididymis but leaving the testicle uncovered (Fig. 153). Great care should be taken in locating all bleeding points. The wound is closed with silkworm gut and sutured without drainage unless there is much 
inflammation of the testicle or infection of the hydrocele sac in which case a cigarette drain may be inserted and left in place until conditions warrant its removal at subsequent dressing.

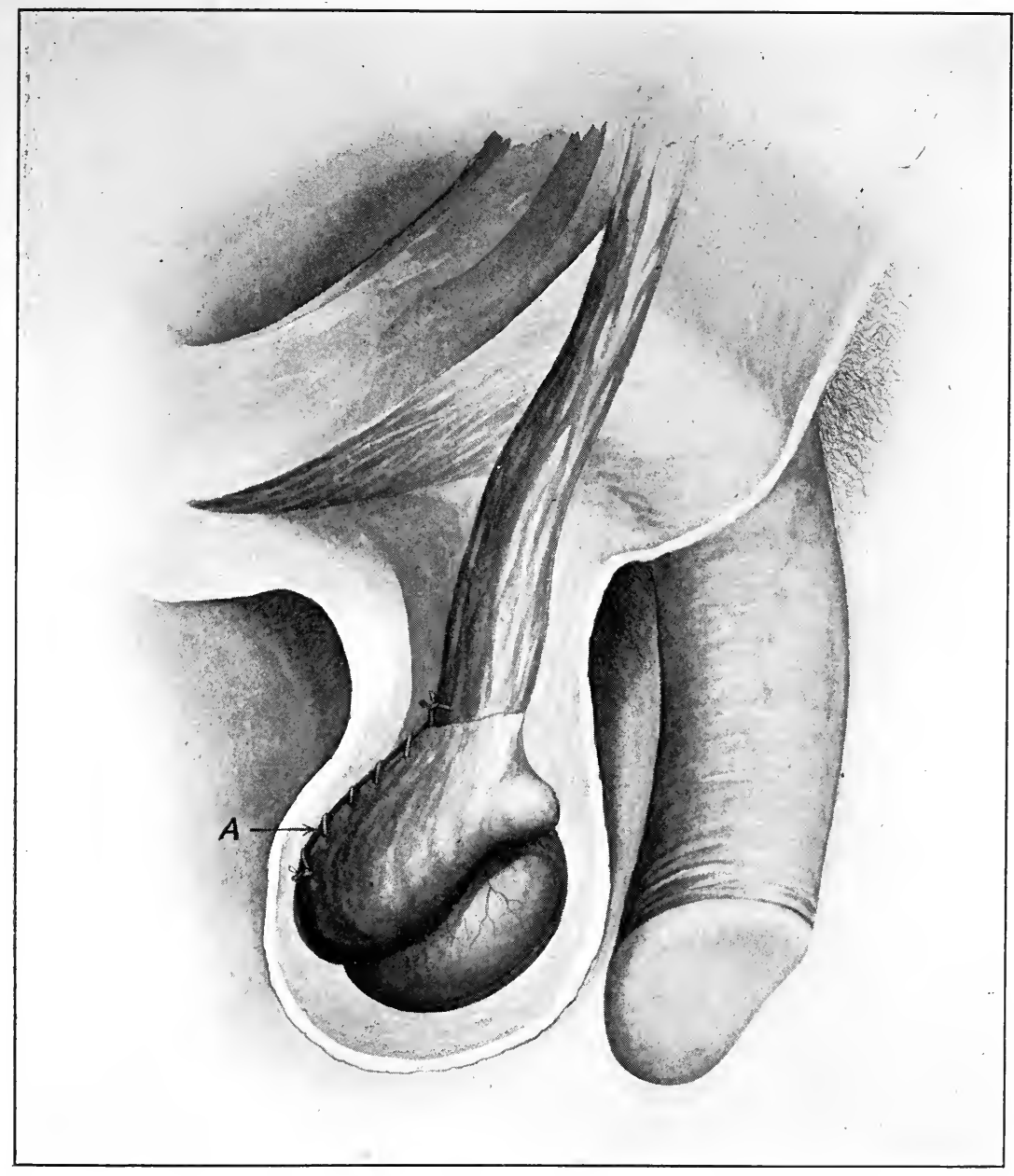

FIG. 153.--Open operation for hydrocele.

\section{Requirements for Operation}

Local or general anesthesia.

Scalpel. Scissors. Grooved director.

Needle holder and needles.

No. 1 plain catgut, horse hair or

Silkworm gut, chromic catgut.

Cigarette gutta percha drain.
Suspensory bandage.

Dozen artery clamps. 
Vasotomy.-The vas deferens may be exposed in the region of the inguinal ring or in the scrotum (Fig. 154). The latter region is usually more accessible. By careful manipulation, the vas is isolated from the venous plexus as in the subcutaneous operation for varicocele.

Having been isolated, it is brought immediately beneath the surface of the dartos in a fold of this sac and this isolated area is anesthetized by the injection of a small quantity of $1 / 5$ of 1 per cent.

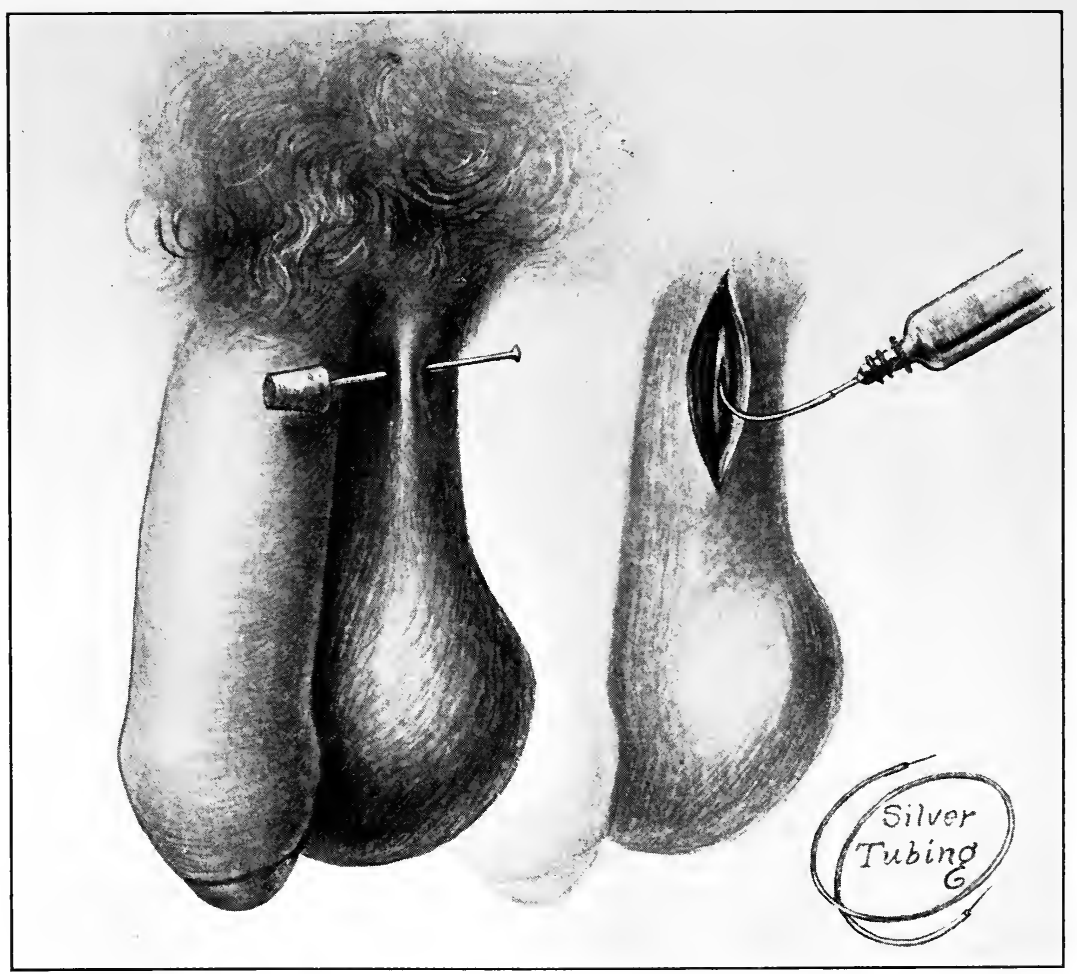

Fig. 154.-Scrotal vasotomy. A, Transfixation of vas. B, Incision and injection of vas.

cocain solution or 5/10 per cent. novocain and a good sized hair-lip or bank pin is made to transfix the two walls of the scrotum thus locking the vas in this isolated space.

With a small sharp knife, the fold of the scrotum is incised and the vas is readily exposed. A longitudinal slit is then made in the exposed vas 1 centimeter (about $1 / 2$ in.) in length (Fig. 154). It is now possible to introduce a small hypodermic needle into the lumen of the duct 
and inject therein a solution of one of the silver compounds, namely, argyrol, collargol, etc., and to demonstrate whether or not the seminal vesicle or ampulla of the ejaculatory duct is patent by introducing a catheter through the urethra into the bladder when the injected fluid will be obtained, unless some obstruction to the flow exists. In cases of chronic seminal vesiculitis in which it is desired to treat the interior of the vesicles or of any portion of the seminal canal by periodic injections, a fine piece of silver tubing may be tied in the lumen of the vas with a catgut ligature and left in situ for several days (Fig. 154). Through this tube the treatment may be administered, consisting of the injection of from 1 to 2 c.c. of argyrol, collargol 5 to 10 per cent., or protargol 1 to 2 per cent. This medicament, on account of the anatomical arrangement of the seminal vesicles, must pass through the entire tubular canal of the latter before reaching the sinus of the prostate and it is possible in this manner to open up a closure of the seminal canal caused by inflammatory exudate existing in some portion above the opening in the vas deferens. In cases, however, where the closure is below this point in the vas or at its juncture with the epididymis, the operation of anastomosis of the vas with the epididymis (Martin's operation) may be resorted to. After the treatment is discontinued, the wound is kept clean and allowed to granulate.

\section{Requirements for Operation}

Hypodermic syringe and needle.

Cocain solution $2 / 10$ per cent. or $5 / 10$ per cent. novocain.

Large bank pin.

Small, sharp scalpel.

2 pairs of small thumb forceps.

1 pair of small scissors.

1 or 2 artery clamps.

Small size chromic and plain catgut.

Blunt pointed hypodermic needle.

Needles and needle holder.

Finest sized silver tubing obtainable.

Bladder catheter.

Vasectomy.-This technic is employed for ligation and resection of the vas deferens on account of relapsing inflammation of the testicles, due to frequent descent of inflammation from the posterior urethra, especially when repeated instrumentation is necessary, as in the case of regular catheterism. The operation is performed under local anesthesia. 
A straight or "V"-shaped incision is made over the spine of the pubis and the spermatic cord is exposed and elevated, as in the case of the open operation for varicocele, by delicate dissection and separation of the coverings of the cord. This is accomplished by first infiltrating with cocain or novocain solution and then carefully snipping the elevated structures until the duct is exposed. A section about half an inch is severed between two ligatures and removed. The cord is then returned and the skin sutured without drainage, any bleeding points having been previously secured.

The operation may be conducted, if preferred, by the same technic as described for vasotomy, the section of the vas deferens being removed in the scrotal region instead of as above.

Requirements for operation are the same as for vasotomy.

Epididymotomy.-The operation of exposure and incision of the sheath of the epididymis is resorted to in cases of intense inflammation of the epididymis occurring as a complication of gonorrhea, as a result of which expedient, it is found in many instances that prompt relief of the painful symptoms ensues with speedy resolution and less likelihood of blocking of the epididymal canal with nodular infiltration. ${ }^{1}$

On account of the acute inflammatory condition of the organ, general anesthesia is often but not always necessary. The testicle is seized in one hand and an incision is made over the most prominent portion of the inflamed epididymis. The tunica vaginalis is opened sufficiently to permit the testicle to be delivered. The epididymis is then examined carefully and several punctures are made through its tense capsule with a small knife, areas being selected where the inflammatory swelling, edema and hardness are felt. When pus escapes from any of the punctures, the aperture is enlarged, thoroughly evacuated and cleansed with a solution of 1 to 1000 of bichloride of mercury, followed by normal salt solution. All other pus cavities are treated in like manner. Sometimes no pus is obtained, but notwithstanding this marked diminution of the tension within the epididymis is produced by multiple punctures. The serous fluid usually found within the tunica vaginalis is evacuated when the sac is opened and this cavity is now cleansed with 1 to 1000 bichloride of mercury followed by normal salt solution. The wound is then completely closed except for a cigarette drain inserted at the lower angle, which is removed at the end of twenty-four hours.

\footnotetext{
${ }^{1}$ Hagner of Washington is responsible for successfully demonstrating the value of this operation and has reported extensive experience to prove its claims. Annals of Surgery, Dec., 1908.

The auther has found pus in 80 per cent. of the cases but has found that it makes little difference in the result obtained whether or not pus is present.
} 


\section{Requirements for Operation}

Sharp scalpel.

2 pairs of thumb forceps.

6 artery clamps.

1 pair of scissors.

Chromic and plain catgut.

Silkworm gut.

Cigarette drain.

Suspensory bandage.

Needles and needle holder.

Epididymo-Vasotomy. ${ }^{1}$ - The object of the operation is to cure sterility and aims to establish an epididymo-deferential anastomosis with a view of gaining a free passage of the spermatozoa from the epididymis into the testicular duct, when such is prevented by an obstruction located in the tail of the epididymis on both sides, owing to the persistence of gonorrheal infiltration.

An opening is made through the scrotum and the testicle is delivered and placed in a warm antiseptic towel. A small incision is made through the sheath over the head of the epididymis and if the spermatic ducts or portion of the epididymis exposed thereby are opened on the testicle side of the obstruction, there will occur a flow of milky fluid which contains many freely motile spermatozoa. The object then is to anastomose this portion of the testicle with a segment of the vas deferens. To this end, a longitudinal slit, a trifle over $5 \mathrm{~mm}$. $(1 / 4$ inch) in length is made into the lumen of the duct. It is desirable that a preliminary investigation be made, of the patency of the seminal vesical and ampulla on that side, by injecting a mild solution of argyrol (3 per cent.) as described in the operation for vasotomy (see p. 354). The tunica albuginea of the testicle, which has already been slit, is then sutured to the cut edge of the vas deferens, on each side thereof, by four very fine silk or silver wire sutures and the wound is carefully closed.

The percentage of success is hard to state; the procedure, however, is without any definite risk. This operation is only suitable for cases in which the obstruction is in the epididymis.

\section{Requirements for Operation}

Scalpel.

6 artery clamps.

Small retractors.

1 Martin of Philadelphia was the first to describe and operate successfully with this technic. 
Very small scissors.

Thumb forceps.

Ophthalmic knife.

Catgut, fine silk.

Silkworm gut.

Fine and coarse needles and holder.

Hypodermic syringe.

Argyrol solution.

Blunt hypodermic needle.

Epididymectomy.-General anesthesia is usual.

After the preliminary preparation, the scrotum is steadied with the left hand of the operator, and with a scalpel a free incision is made over the most prominent part of the epididymis. The tunica vaginalis overlying this portion is incised and separated and the entire epididymis and testicle are exposed. When found to be the site of extensive degeneration and removal is considered necessary, the whole epididymis including all visible area of diseased tissue is dissected away from its attachment to the body of the testicle by means of a pair of blunt scissors and thumb forceps. The cut end of the vas deferens is ligated and the curette used freely where necessary. After carefully ligating all the bleeding points, the wound is sewed with silkworm gut, leaving a cigarette drain extending from the most dependent portion of the incision. After the application of the outer dressings the scrotum is supported in a suspensory bandage.

\section{Requirements for Operation}

Scalpel.

Pair of scissors curved on the flat.

6 artery clamps.

1 curette.

Plain catgut, silkworm gut.

Needle holder and needles.

Suspensory bandage.

Orchidectomy (Castration).--Most cases require general anesthesia, although in some where there is no great amount of swelling or inflammation local anesthesia may suffice. (See special section, p. 501.)

A longitudinal incision 7 to $9 \mathrm{~cm}$. long ( $23 / 4$ to $31 / 2$ inches) is made extending downward from the spine of the pubis, exposing the testicle and cord which are promptly delivered through the wound. The vas deferens, which is recognized in the lateral posterior portion of the cord, is isolated and tied near the external abdominal ring. 
After severing the vas, the remainder of the cord exclusive thereof, is then secured by two separate ligatures of heavy chromic gut and is divided between them. After the removal of the testicle and cord, the remaining section of the latter is secured in one of two ways, i.e.: by transfixing with a double ligature or the individual vessels may be tied at the exposed end. The stump of the vas deferens is then touched with pure carbolic acid and immersed in alcohol. The end of the ligature fastened to the cord is secured at the upper corner of the incision as a matter of precaution in case it is necessary to find it if by any chance hemorrhage should occur after the operation. There is no reason, however, why such complication should ensue when the technic has been carefully followed. Most conditions requiring removal of the testicle demand drainage, in which case a cigarette drain is inserted, otherwise the wound is closed with silkworm sutures. In castration for neoplasm, it is not necessary to remove the inguinal glands as they do not communicate directly with the lymphatics of the testicle.

\section{Requirements for Operation}

General or local anesthesia.

Scalpel.

1 pair of scissors.

1 doz. artery clamps.

Needle holder and needles.
Ligature carrier.

Retractors.

Chromic and plain catgut.

Silkworm gut.

Carbolic acid and alcohol.

\section{Operation for Undescended Testis}

The most satisfactory technic for this operation is what is known as Bevan's Technic, ${ }^{1}$ which is based upon the belief that one of the principal difficulties in this condition is the rudimentary development and undue shortness of the spermatic vessels and therefore involves division of the spermatic cord but not the vas deferens.

The incision of the skin is the same as that for inguinal hernia, parallel with Poupart's ligament including all the structures down to the external oblique aponeurosis which is split. The membranous coverings of the cord are separated and the peritoneal cavity closed by ligating at the neck what would correspond to a hernial sac, having previously withdrawn the testicle if the latter happens to be intraabdominal. The portion of the sac remaining adjacent to the tunica vaginalis is cut off and sutured. Careful separation of the cord is completed and gentle traction upon the testicle will determine whether

\footnotetext{
1 Longinbeck's Archiv., LXXII, p. 1035; also Moschowitz, Annals of Surgery, December, 1910.
} 
it can or cannot be deposited in the scrotal sac. When this cannot be accomplished the technic of the present operation demands that the spermatic vessels be divided and ligated, as a result of which no difficulty should be encountered in bringing the testicle to its normal site in the scrotum. During the proceeding it is important to protect and save the vas deferens and to free it from attachments throughout its course.

No retention sutures are required to anchor the testicle.

The canal between the tunica vaginalis and the groin is reenforced by a buried suture to prevent retraction of the testicle and the wound is then closed by suturing the corresponding tissues together without drainage.

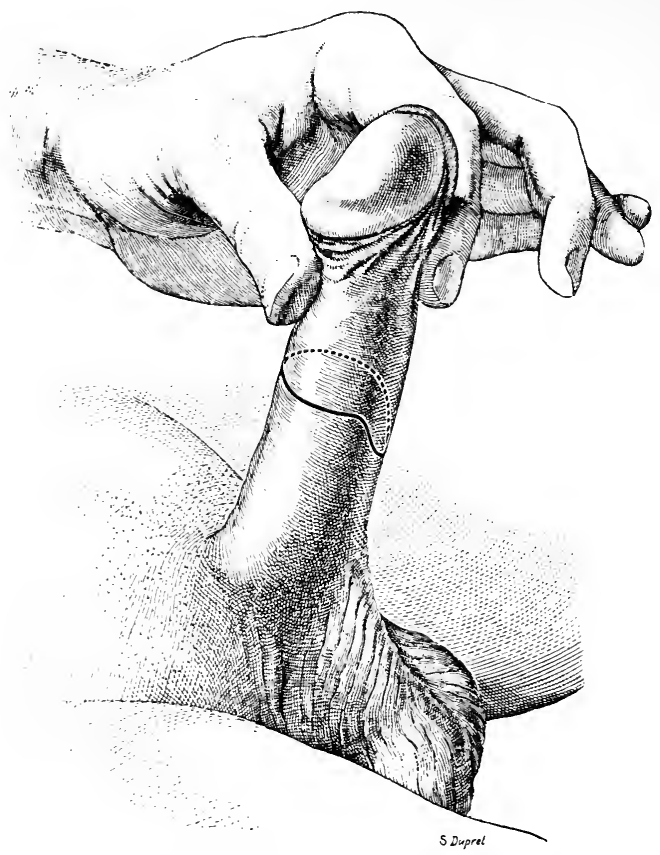

FIG. 155.-Conservative amputation of penis. Incision. (After Legueu.)

Amputation of the Penis (Conservative).-General anesthesia is not necessary unless as a preliminary measure, it is desired to remove the inguinal glands. To this end, incision is made on either side from the root of the penis extending " $V$ " shaped on either side for about $9 \mathrm{~cm}$. (3 $1 / 2$ in.). After removal of the glands the wounds are stitched up with silk or silkworm gut. In other cases, after local anesthesia has been obtained (see special section, p. 499) a rubber ligature or soft rub- 
ber catheter is made to encircle the penis close to the pubis and is fastened with a clamp. The skin of the penis is now cut through by circular incision down to the erectile bodies and urethra. This should be well behind the diseased area. The corpora cavernosa are severed at the point of retraction of the skin flap and the corpus spongiosum is incised about $3 \mathrm{~mm}$. in front of them. All accessible bleeding points are caught and tied after the tourniquet is removed. Further hemor-

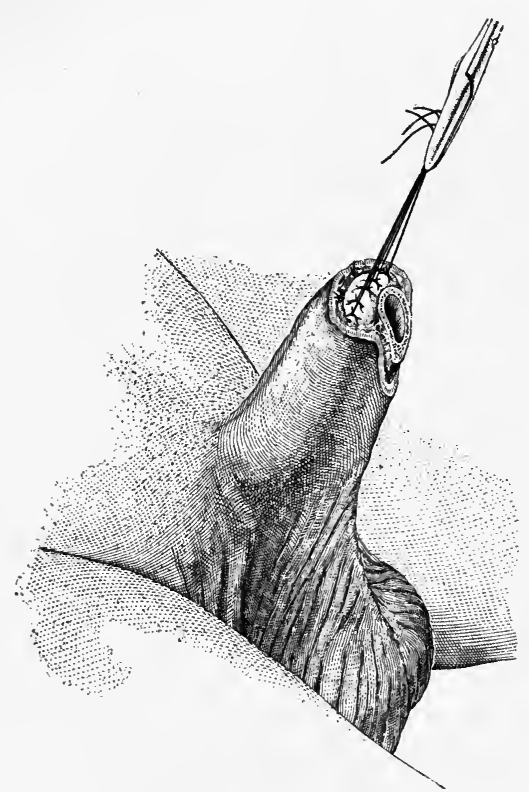

FIG. 156.- Same as Fig. 155. Suture of corpora cavernosa. (After Legueu.)

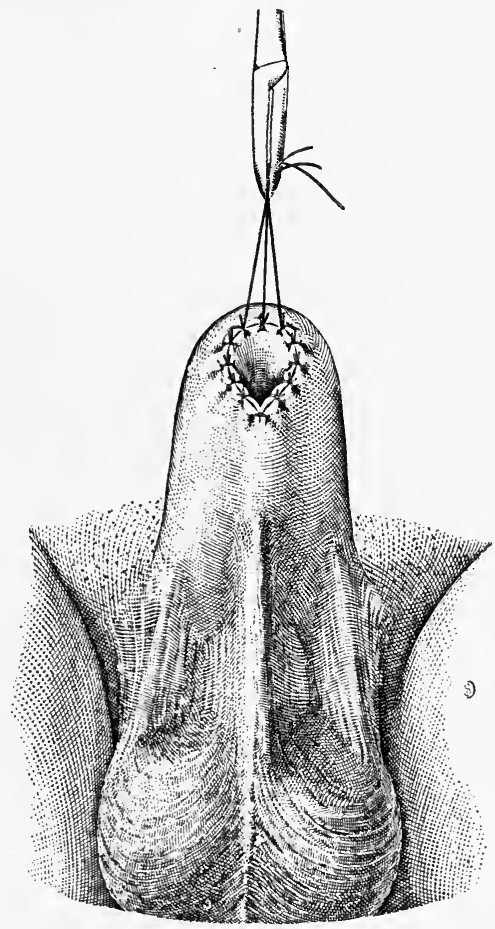

Fig. 157.-Same as Fig. 155. Completed. (After Legueu.)

rhage is lessened by hot solutions and finally by the suturing of the fibrous sheaths of the corpora cavernosa over the exposed ends (Fig. 156). The urethra being split along the bottom is then sutured to the skin and the latter is sutured over the exposed corpora (Fig. 157). A retained catheter is placed in the bladder for continuous drainage.

This operation may prove to be ineffectual and recurrence of the disease may necessitate more radical removal or complete emasculation. 


\section{Requirements for Operation}

Rubber ligature or soft rubber catheter for tourniquet.

Rubber catheter for drainage.

Scalpel.

1 pair of scissors.

2 pairs of thumb forceps.

1 doz. artery clamps.

Groove director.

Needle holder and needles.

Plain, chromic gut and silk.

Silkworm gut.

Complete Emasculation.-This operation embraces the technic of two other operative procedures already described, namely, inguinal adenectomy and castration.

As in the case of conservative amputation, two oblique incisions are made starting from the base of the penis and extending outward on either side above Poupart's ligament for about 9 or $10 \mathrm{~cm}$. (3 $1 / 2$ to 4

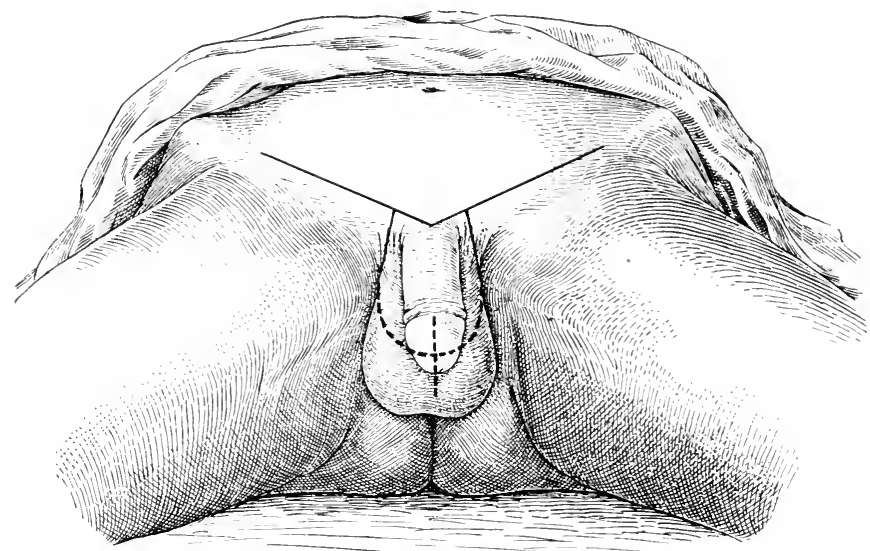

FIG. 158.-Line of incisions for complete emasculation.

inches) (Fig. 158). All of the inguinal glands, superficial and deep, are removed by careful dissection with as little handling and bruising as possible. The bleeding vessels are ligated and the wound closed with interrupted silkworm gut sutures. This step is performed with the patient on his back, and when completed, the patient is changed to the lithotomy position. A sound or guide is then introduced into the bladder; upon this guide, an incision is made in the perineum for the purpose of exposing the urethra, much the same as in external 
urethrotomy (p. 287) when resection or reunion of the urethra is undertaken. The urethra is therefore exposed and dissected laterally from the bulbous portion back to the triangular ligament when it is opened transversely and incised completely about $4 \mathrm{~cm}$. (I I/2 inches) anterior to the triangular ligament. The guide is then withdrawn. The proximal end of the urethra is dissected free from its attachments, split longitudinally and anchored to the skin of the perineum. A large curved incision is now made beginning on one side near the base of the penis, sweeping around the penis, beneath the penoscrotal junction and meeting a similar incision from the opposite side in the median raphe of the perineum at the point where the perineal incision has already been made (Fig. 158). Beginning above, the spermatic

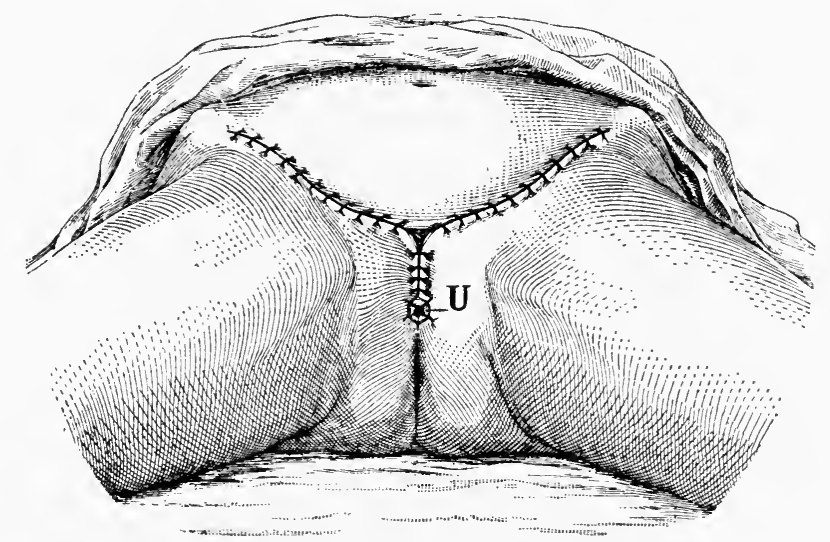

FIg. 159.-Line of union following complete emasculation. U, Urethra. (After Lеguеu).

cord is drawn upon and the testicle delivered, the cord being ligated at the external abdominal ring as already described for the operation of Castration (see p. 358). The same step is repeated on the opposite side. While traction is being made upon the penis wrapped in moist antiseptic dressings, the suspensory ligament which is felt at its attachment to the front of the symphysis pubis is divided on either side. The crura or prolongations of the cavernous bodies are separated by periosteal elevator from their attachment to the rami of the pubis and ischium. At this stage the greatest difficulty is encountered; severe hemorrhage is likely to occur from the arteries of the crura and may be controlled by passing ligatures on each side between the crura and the suspensory ligament by means of an aneurysm needle. 
When total extirpation is completed the superfluous scrotal tissues are to be trimmed away, and the flaps neatly joined together with silkworm gut suture, especially in the region of the urethra (Fig. 159). The urethra is finally carefully sutured to the skin in the perineal region and a soft rubber catheter cut about half the usual length is introduced and stitched in situ. The operation is completed by the application of a snug " $\mathrm{T}$ " bandage and the continued oozing from small vessels controlled by the addition of sufficient loose gauze to exercise firm pressure beneath the bandage.

\section{Requirements for Operation}

Soft rubber catheter.

Scalpels.

100 c.c. syringe.

$11 / 2$ dozen artery clamps.

Thumb forceps.

Scissors.

Periosteal elevator.

Needles and needle holder.

Cautery.

6 tubes each of plain, small and large catgut.

6 tubes each of chromic, small and large catgut.

3 tubes of silkworm gut. 


\section{CHAPTER XX}

\section{MALADIES OF THE PROSTATE}

\section{ATROPHY, IN JURIES, NEOPLASMS, CALCULI, ACUTE AND CHRONIC PROSTATITIS}

Atrophy of the Prostate.-This condition may develop during life and is occasionally congenital. In fact, total absence of the prostate has been noted in connection with other anomalies of the sexual organs, but is very uncommon. Arrested development of the prostate may exist as a result of injury to or destruction of the testicles before puberty. The general debilitating effect of chronic constitutional disease during early life may exercise an arresting influence upon the development of the sexual organs and incidentally the prostate. A secondary condition of atrophy may occur as a result of abscess of the gland and as an after condition of hypertrophy in advanced age.

The symptoms associated with prostatic atrophy are sexual and urinary. There is naturally a concomitant sexual decline and sometimes a development of urinary incontinence, especially at night. The diagnosis is determined by the existence of the associated symptoms and by rectal examination. There is little to be done in the matter of treatment that holds out any reasonable expectation of improvement. Urethral sounds, electricity and such other routine measures have been employed without any notable success.

Cysts of the Prostate.-Prostatic cysts are occasionaliy encountered in urethroscopic and rectal examinations. It is well to mention the hydatid variety as a possibility although most observers do not have the opportunity to meet such a rarity. Conclusive evidence of hydatid cysts here as elsewhere is the discovery of the characteristic hooklets of the echinococcus. (See p. 102, Fig. 64.)

Retention cysts of the prostate may result because of the occlusion of the natural opening of one of the follicles. These occasionally occur posteriorly when they may be felt per rectum; or anteriorly in the sinus pocularis or prostatic utricle where they may exercise a certain degree of interference with the urinary function and may be revealed by posterior urethroscopic examination (see Chap. VI). 
Large cysts of the prostate are usually the result of congenital formation and require differentiation from a vesical neoplasm or diverticulum of the bladder. The diagnosis in such cases rests upon the result of cystoscopic or urethroscopic investigation and rectal examination. The treatment of cystic formations depends upon the extent of the growth. Evacuation of the cyst contents may be necessary to relieve urinary obstruction. This may be accomplished by a simple puncture or incision through the endoscopic tube. Refilling of the sac may require more surgical intervention which may involve perineal or suprapubic section (see Operative Surgery).

Injuries of the Prostate.-Traumatic damage of the prostate often occurs as a result of careless or unwise introduction of instruments (False Passages, p. 73). The prostate gland may be otherwise injured from the outside by direct blows, by kicks or contusions. The evidence of such injury is signalized by the symptoms of congestion and inflammation. (See p. 370.) More active symptoms arise from extension of the injury to the urethra, producing complete or incomplete tears and resulting in hemorrhage, extravasation of urine and general infection. An ordinary wound of the prostate, not involving the urethra, is generally transitory in its effect but when the urethra is involved, the result may be that of a copious hemorrhage or complete retention of the urine may occur, or urinary extravasation, in which case the treatment is necessarily not that of congestion or inflammation of the gland but of the secondary condition, which may require surgical intervention, as in the case of extravasation of urine (see Extravasation of Urine, p. 266); or the temporary arrangement for suitable drainage by the use of an indwelling catheter (see p. 52), as in the case of injury or incomplete rupture of the urethra.

Neoplasms of the Prostate.-Benign growths of the prostate are not common except in connection with the ordinary condition of prostatic hypertrophy (see p. 385). Thus, myomata may be discovered as a component part of the enlargement of prostatic hypertrophy surrounded by other types of pathologic formation. Fibromata may also form a portion of the growth of prostatic enlargement but are usually the result of protracted chronic inflammation.

The malignant tumors of the prostate, carcinoma and sarcoma which are the usual types, are composed of glandular, epithelial and connective-tissue elements.

Primary carcinoma of the prostate is more common than sarcoma. It usually develops late in life. Its etiology is of uncertain character. There need be no history of preëxistant gonorrhea or heredity as the causative agency. This neoplastic growth occurs either in a 
circumscribed and symmetrical nodule or as a diffuse infiltration. A beginning benign prostatic hypertrophy may be eventually complicated with malignant involvement. It has been demonstrated that primary cancer of the prostate always commences in the posterior lobe or commissure (Young) and never in the lateral lobes, but may later extend into the lateral lobes; while just the reverse is the case with benign prostatic hypertrophy. Be this as it may, from clinical observation cancer is encountered in two classes: one where it is confined within the capsule and may or may not be recognized by rectal palpation; the other where it has broken through the capsule and extends upward to the seminal vesicle and base of the bladder. There are two types of prostatic carcinoma - the soft or medullary and the hard or scirrhus. A prostatic carcinoma may unquestionably be the point from which a general extension of malignancy is derived, especially extension into the bladder; circumscribed carcinoma of the prostate may remain as such and be undiscovered until complete enucleation of the gland for prostatic hypertrophy with obstruction when the malignant character is discovered during pathological examination. Diffuse carcinoma of the prostate resembles a similar condition in the mammary gland of the female. The consistence of the carcinomatous gland when examined per rectum is found to present an extreme degree of hardness, much greater than that of prostatic hypertrophy.

Duration.-While a fatal issue is the usual outcome of cancer of the prostate, it is surprising how in some instances patients may continue to live on for a long period before the fatal ending occurs. In one instance observed by me, the patient revealed a cancer as large as the fist which caused obstruction of the urinary outlet but only moderate pain and which persisted without marked symptoms after my first observation for two years and until a rectovesical fistula had formed; following this, but not much before, there was progressive depreciation in general health.

Symptoms.-The early growth of prostatic cancer is insidious. The first symptom may be pain in the perineal and rectal regions sometimes extending down the thighs, which is due to the pressure of the growth upon the sacral nerves and ganglia. If sufficiently large, partial or complete stoppage of the urine may be caused. Slight hematuria may be a feature, and pain in the perineum may be constant or intermittent. Such pain is sharp and cutting in character, usually attends urination and evacuation of the bowels. When infection of the bladder occurs, the symptoms all become aggravated. Anuria may signalize the extensive growth of the tumor supposed to produce pressure upon the ureters and in the same manner urethral 
obstruction may result. Hematuria as an accompanying symptom of pain occurs at the beginning of urination instead of at the end as in the case of intravesical causes of bleeding. During the course of the progressive development of the growth, rectal symptoms may occur in the form of tenesmus, hemorrhoids and the discharge of mucus. The deep-seated glands eventually become involved but not usually the more superficial chains. Constitutional disturbances supervene-emaciation, cachexia - which mark the closing scene of the malady. The diagnosis is made by the great hardness of the gland as revealed by rectal examination, the contour of which may be irregular or symmetrical; the cysto-urethroscopic observation; the obstructive symptoms which, however, are also common in benign prostatic enlargement; the rather significant painful symptoms and finally the general depreciation. It is not always possible to make a diagnosis of a prostatic cancer by palpation.

Treatment.-The only hope of partial cure and prolongation of life by surgical intervention lies in the early discovery of the neoplasm which, when the growth is confined within the capsule, sometimes occurs at the time of the removal of the gland for supposed benign hypertrophy; an event that may result most fortunately for the patient in prolonging life. In case the capsule of the prostate happens to be involved or in the event of the neoplasm having extended outside the capsule, the operation may exercise little more than a palliative influence in some cases, while in others the cancer will proceed to extend with even more rapid growth.

Those cases in which a clinical diagnosis is possible before too great extension and glandular involvement have occurred may be offered the choice of a radical perineal excision (Young) in the hope of prolonging life thereby, or the equal uncertainty of allowing nature to take its course. The end results following radical operation are not sufficiently impressive to make the selection an easy one. The number of instances of unmistakably malignant prostates not operated upon that have lived longer than is customary to expect following radical operation, is sufficiently large to command attention. If these cases are called exceptions, so are those that live beyond a period of three years after operation, not to mention the dangers and difficulties of the operation itself.

If the choice lies between complete removal and no operation in the early period of cancer, in the later stage the course lies between partial removal and awaiting the time when total occlusion of the bladder outlet necessitates an operation for the purpose of affording an opening to permit the escape of urine. The operation of partial 
removal of the cancerous growth has little value. In one case of mine in which there was complete closure of the prostatic urethra and extension of the growth upward, the patient lived four years following the establishment of permanent suprapubic drainage and was able to attend to his business the greater part of this period. Some patients are singularly free from pain while others seem to suffer excessively. In the latter instances, the free use of opium is demanded either by suppository or internally.

Sarcoma.-Sarcoma of the prostate, although a pelvic tumor rarely seen, ${ }^{1}$ occurs in children as well as in those of mature and advanced years, but most cases have occurred in the young.

The etiology of these growths has not been determined.

In children, the principal indication is sudden retention of urine without apparent cause. In the adult, pain on urination and defecation or constant pain in the lower abdomen is experienced, accompanied sometimes, but not always, by hematuria. Frequency of urination probably due to retention is likely to occur. - Bimanual palpation per rectum detects a tumor in the region occupied by the prostate, sometimes filling the entire pelvis. In adults, the symptoms of sarcoma and carcinoma are similar.

The neoplasm is large, irregular and ovoid, firm and elastic. ${ }^{2}$ The bladder is more often encroached upon than involved by the growth; the urethra becomes distorted and the seminal vesicles are almost annihilated.

The neoplasm is of rapid growth and metastasis usually takes place. Without operative treatment, the termination is invariably fatal within two months to two years.

Treatment.-If the lesion is discovered at an early stage and is not too extelisive, surgical removal by way of the perineum may prolong life for several years; but when discovered in children and advanced adult cases, palliative treatment is usually the only recourse.

Prostatic Calculi.-Calculi formed in the bladder may migrate into the prostatic urethra or may become arrested in this region and lie partially within the urethra and partially within the bladder, where their size is increased by incrustation of urinary salts (Fig. 181, p. 442). Phosphatic calcareous deposits may incrustate upon the mucous membrane of the prostatic urethra extending into the follicles favored by morbid conditions of the mucosa and ammoniacal

${ }^{1}$ Gibson (Trans. Am. Ass. of G.-U. Surgeons 1909) tabulates thirty-seven authenticated cases, two of which are his own.

${ }^{2}$ Legueu. " Histologically, prostatic sarcoma is globocellular, fusocellular or has polymorphous cells. Certain tumors are composed of a variety of tissues, myosarcomata, angiosarcomata, chondrosarcomata and rhabdomyomata." 
deposits of the urine, but true prostatic calculi are developed primarily in the follicles of the gland and are derived from the precipitation of the salts of the prostatic secretion. These calculi are usually small and sometimes very numerous. I have operated upon a case in which there were found several hundred millet-seed calculi stored away in a pouch of the prostate gland. While these calculi may remain dormant for years in situ, they sometimes burrow their way through the sheath of the gland and form urethral and external fistulæ.

The symptoms they cause are those of prostatitis and urinary obstruction, mainly pain and dysuria sometimes accompanied by hematuria and purulent urine. The presence of these formations may be discovered during the course of an operation or be revealed by rectal palpation with the stone searcher or by endoscopic exploration.

Treatment.-As stated, patients may live on for years without knowledge of the existence of prostatic calculi and hence no treatment may be applied. When symptoms arrive of sufficient activity to call for measures of relief, surgical intervention is required which involves external perineal urethrotomy (see p. 288) and removal of the calculus or calculi through an incision of sufficient size to evacuate the contained stones. If a calculus is in the prostatic urethra, whether primarily so or having been derived from the bladder, it may be removed through a prostatic endoscope or by litholapaxy (q.v.)

\section{CONGESTION AND INFLAMMATION OF 'THE PROSTATE ACUTE AND CHRONIC PROSTATITIS}

Congestion of the prostate is the condition that commonly precedes inflammation of the gland. Inflammation is the result of microbic invasion; it is predisposed by preliminary congestion which occurs without the assistance of microbic invasion, being more or less mechanical in character. The prostate is rendered susceptible to the occurrence of congestion by any direct active stimulation, such as instrumentation and irritating urine, or by indirect nervous excitation, especially sexual. Relief of congestion of the prostate entails removal of the cause thereof, be that local irritation or excessive physiological excitation. If this is not accomplished, the condition will assume a more advanced stage in the development of actual inflammation.

Prostatitis.-Inflammation of the prostate is the result of infection of the gland by microörganisms in the presence of a favorable soilusually congestion. Gonorrhea is a common cause; traumatism 
also may be followed by pyogenic infection of the prostate, or be the cause of preëxisting congestion that leads the way to inflammation. Acute inflammation may also occur as a result of hematogenous or lymphogenous invasion, the microbial element being the staphylococcus, streptococcus or colon bacillus. The inflammatory course and symptoms in this case are similar to such inflammation occurring after urethral invasion, and the possibility of such occurrence should be appreciated.

Prostatitis occurs as a sequel or complication of non-gonorrhœal urethritis, notably when stricture is present, and it may also occur in connection with sexual strain especially where there is preëxistent prostatic disease, tuberculosis, cancer, etc.

Follicular Prostatitis.-Mild prostatitis is a frequent form of posterior urethritis. Confined mostly to the surface and to the follicles of this portion of the urethra, it is not, when promptly treated, or when the virulence is slight, of long duration.

Following invasion, the prostatic urethra becomes congested and swollen and the superficial follicles become engorged and their mouths dilated with contained pus which, exuding backwards, materially increases the purulent flow and adds to the cloudiness of the urine.

The symptoms consist of somewhat urgent and frequent desire to urinate, signifying congestion of the adjacent mucosa of the bladder orifice and this varies in degree according to the intensity of the inflammation. The desire to urinate may persist after the bladder has been emptied, and there is likely to be pain at the end of the urinary act as the coexistence of a certain amount of urethro-cystitis or inflammation of the neck of the bladder is more than likely. In fact the symptoms are those of the latter disorder. There is usually a pain or soreness in the perineum that calls attention to the prostate as the seat of trouble. In mild cases, this amounts only to a deep urethral tickling.

Besides the existence of a certain amount of urethral discharge, the urine is purulent and the presence of pus from the posterior urethra may be demonstrated by washing out the forward canal before the urine is voided (see Plate V). Sometimes a few drops of blood trickle away after the urinary act.

Parenchymatous Prostatitis.-A further extension of the malady involves the parenchyma of the organ and may or may not result in prostatic abscess. When prostatitis attacks the body of the gland, the latter becomes increased to several times its normal size; its consistence is firm and resistant; the tissues are engorged with blood and distended with an exudation which is at first serous and later of a 
purulent character. Sometimes one-half of the gland is more enlarged than the other, and sometimes lumps and nodes are felt upon the surface on account of irregular distribution of the inflammatory process.

This glandular invasion is either a direct urethral extension or a lymphatic transmission, more of ten the former. The lack of normal resistance on the part of the individual, both general and local, has an important bearing upon the susceptibility thereto. Therefore, systemic depreciation, temporary or inherent, and excessive prostatic congestion, are determining factors.

Symptoms.-Acute prostatitis, involving at once the whole gland, is sharp in its onset, sometimes preceded by a chill and followed by marked pyrexia. Attention is immediately attracted to the prostate by the pain in the perineal region, accompanied by a dragging or bearing-down sensation. These symptoms often supervene after the unwise or careless use of instruments in the posterior urethra during an acute anterior urethritis. Urination becomes painful and difficult. A few drops of urine retained in the prostatic canal cause a distressing spasmodic sensation. Between such paroxysms, the calls to urinate are not as frequent and imperative as in the case of cystitis. There is more likely to be difficulty in voiding the urine, owing to swelling of the prostate. This may lead to incomplete or even complete retention of urine. Pain in the rectum is also complained of; defecation intensifies it and provokes vesical and rectal spasm. If the prostate be examined by the rectum, it is found to be hot, swollen and intensely painful. The enlargement may be symmetrical or confined to one lateral half. When the symptoms are unusually intense, the possibility of prostatic abscess should be borne in mind.

Abscess of the prostate occurs as the result of the formation of multiple pus foci which are distributed throughout the glandular structure and later unite into one pus sac of variable size.

In the early stage, these small pus collections are separated by thick and resisting walls, generally seated beneath the urethral mucous membrane and sometimes more deeply. Later, the dividing walls give way and the small sacs communicate with one another. The ejaculatory ducts may become thickened and filled with pus and the seminal vesicles may become involved-acute vesiculitis.

Suppuration occurring in the substance of the prostate gland during an attack of acute prostatitis is marked by a chill, fever and general malaise. The local symptoms become aggravated, the sensitiveness being intensified by movements of the body. Defecation is rendered most painful. All intense symptoms cease almost instantly after the evacuation of the pus, when the malady takes this 
favorable turn, which is often the case, or when the abscess is treated surgically (see p. 474). Sometimes, however, when operation is resisted, sepsis increases, the temperature mounts and the indications point toward general systemic infection. This unfavorable course is of grave import and menaces life unless relieved by proper surgical measures. When prostatic abscess breaks toward the urethra, rupture usually occurs during straining at stool or the spasmodic efforts attendant upon urination. When the evacuation of pus is complete, recovery is progressive, but when evacuation is incomplete the gland becomes the site of a chronic prostatitis of long duration, or when all the foci are not deeply drained, complications are to be feared and exacerbation may occur. Not infrequently, the pus burrows toward the rectum and either discharges directly into the bowel or more often diffuses itself into the cellular tissue between the prostate and the rectum -periprostatic abscess.

Periprostatic Abscess.-When this complication ensues, rectal examination detects the general diffusion of the inflammatory thickening, blotting out the limits of the prostate gland. Laterally the tissues surrounding the prostate effectually limit the course of the pus, but below the resistance is less, and there the pus diffuses itself and may open into the urethra, the rectum, the ischiorectal fossa or the perineum. When opening into the urethra occurs, the result is urethro-rectal fistula.

Periprostatic abscess may arise from other causes, wounds or laceration of the rectum, from inflammation of the bladder or seminal vesicles or from extension through the venous and lymphatic vessels, but more commonly it is propagated from the prostate gland.

Diagnosis of Acute Prostatitis.-Follicular prostatitis occurring during an attack of gonorrhea is signalized by increased urgency and frequency of urination, the occurrence of pus in both flows of the urine, and often by a coincident decrease of purulent discharge from the meatus. These symptoms, however, also occur in cystitis, and the two conditions are likely to coexist-hence the term urethrocystitis (p. 422). The subjective symptoms peculiar to the prostate, already outlined, are likely to direct attention to this organ as factor in the existing condition.

When inflammation attacks the body of the gland, most of the subjective phenomena are intensified, and when suppuration occurs, marked constitutional symptoms, i.e., chills, rigors, pyrexia, etc., supervene.

The nature of the trouble is easily recognized by the swelling, tension and tenderness of the enlarged gland, and it is furthermore 
characterized by a feeling of soreness and enlargement in the perineal region which may be particularly noticeable when pressure is brought to bear upon this region, as in sitting down. Rectal examination will detect a hard, painful tumor of unequal proportions and sometimes a fluctuating point.

Prostatitis involving the body of the gland and cystitis are often confounded. The differential features are the greater frequency, urgency and vesical tenesmus in the case of cystitis and the characteristic difficulty of urination and sometimes retention of urine in prostatitis. Rectal examination will reveal decisively the existence of one or the coexistence of both of these troubles.

Cowperitis is distinguished by recognizing the seat of the inflammation to be the anatomical region of Cowper's gland in front of the deep urethra, and by excluding prostatitis by rectal examination. A seminal vesiculitis may be present with the same symptomatology as that of prostatitis and should be sought for at the onset by rectal examination.

When periprostatitis occurs, the swelling diffuses itself in the surrounding tissues, including the seminal vesicles and the recognition of the original trouble may then be much more difficult.

Treatment.--The treatment of acute follicular prostatitis is that of posterior urethritis (see p. 203).

The aim of treatment in acute parenchymatous prostatitis should be to prevent the occurrence of suppuration, which calls for palliative measures. Hot sitz-baths or hot applications to the perineum are generally a source of comfort to the patient, in which case they should be used freely. This may be accomplished by the hot water bag or by the electrical heating device (p. 315). Anodynes may be given to allay pain, which is sometimes exceedingly acute and often remains in spite of the use of such remedies, pushed almost to the point of narcotism. Morphine hypodermically and suppositories of opium, belladonna and cocaine may be employed. Confinement in bed is essential. No other medicinal treatment has any particular value. Bacterins and antigonococcic serums have proved successful in some cases and should be considered (see p. 110). In all acute complications of gonorrhea, the question of using serotherapy is likely to be considered. The diet should be plain and the bowels relaxed by appropriate means. The urine, if highly charged and acid, should be diluted by alkaline diuretics, and if retention occurs, the catheter must be used regularly and with the utmost gentleness. It is generally the case in acute parenchymatous prostatitis that moderation of the acute pain does not occur until the tension in the gland is relieved by the 
subsidence of the inflammation, or, when suppuration occurs, until the evacuation of the pus. When it becomes evident that pus has formed, drainage is called for at once. As already stated, the abscess may point toward the urethra, the rectum or the perineum. When it points toward the urethra, it often leads to urinary retention and the pus escapes either spontaneously or during the use of the catheter. Superficial fluctuation, detected by rectal touch, calls for puncture through the wall of the gut, or better through the perineum behind the prostatic capsule (see p. 475).

If the pus be deeply seated, a more surgical and better drainage is attained by the perineal incision, dissection being carefully made upward until pus is reached. The advantage of this method is that urinary fistula is quite certainly averted. Sometimes pointing of the abscess takes this direction, in which instance, a superficial incision is all that is required. The cavity is then washed out with hot normal salt solution and if the hemorrhage be great, it is packed with absorbent gauze. The gauze may be removed in twenty-four or forty-eight hours and replaced or not, as seems best. The aftertreatment consists in keeping the external opening pervious until the cavity contracts down, which should be encouraged, if necessary, by stimulation. This, however, is generally not required, as when proper drainage is afforded the cavity rapidly contracts.

\section{CHRONIC PROSTATITIS}

Chronic prostatitis is the natural sequel of the acute form, commonly the outcome of gonorrheal or non-specific inflammation. It may occur, however, as a slow and insidious development, the result of chronic congestion from more or less continuous excitation, sexual or mechanical (instrumental, urinary, etc.) and microbial. In the last instance, the particular microbial element may be found in the urine or the purulent exudation from the urethra. In cases of socalled aseptic or amicrobic forms of prostatitis, the possibility of anaerobic bacteria (fungi) should be considered as an explanation of its existence. These may be found only by culture method and obtained from the prostatic secretion through the urethroscopic tube.

Chronic prostatitis is of frequent occurrence and often exists for a prolonged period unrecognized. As an independent malady it is therefore of greater frequency than acute prostatitis, which is generally more closely associated with gonorrheal or non-specific urethritis. Many cases yield symptoms of little note and are only detected by examination of the prostatic secretion obtained by massage of the 
prostate after thorough cleansing of the urethra, or there may be noted a slight, sticky discharge of whitish hue which causes a glueing together of the meatus in the morning or a crust upon the outer surface. At other times, the discharge is more copious.

Prostatorrhea is a disorder which resembles chronic prostatitis, in that it is a mild and chronic condition accompanied by few or no symptoms other than a small amount of urethral discharge appearing at varying intervals. The discharge of prostatorrhea is not muco-

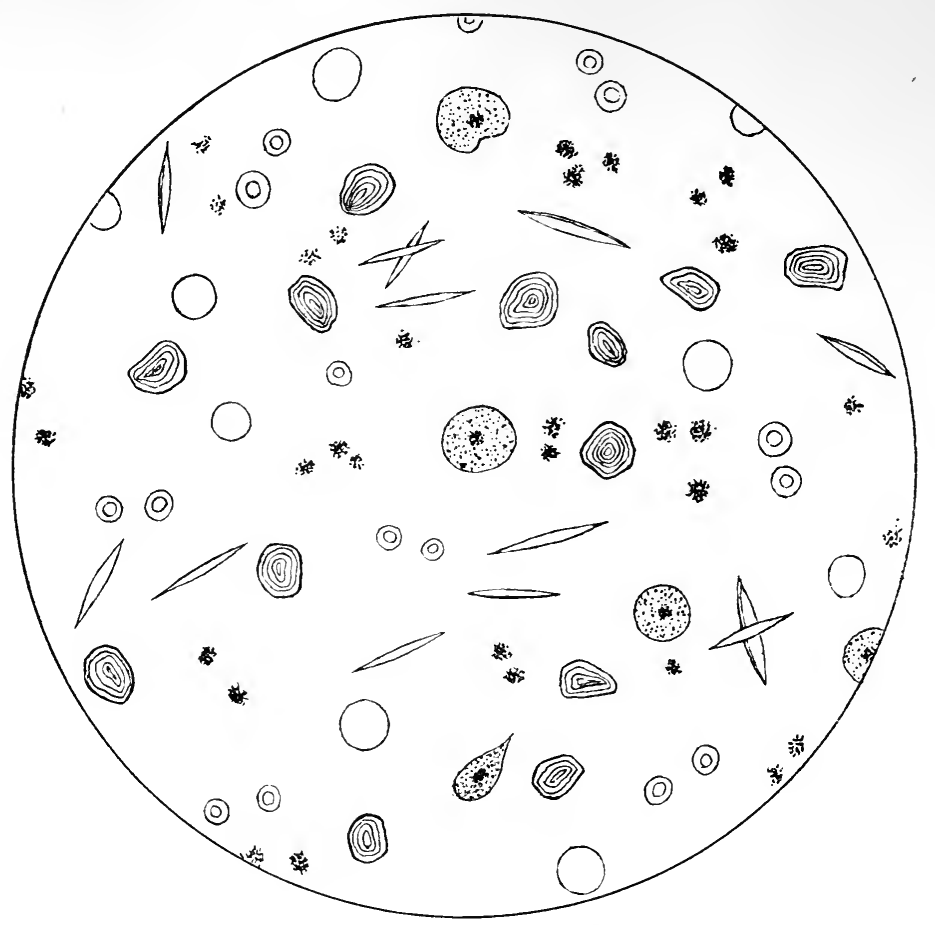

FIG. 160.-Prostatorrhea.

purulent, but simply an excess of the normal prostatic secretion (Fig. $160)$; and the malady has not necessarily any venereal origin. These two troubles are often confounded and the terms used interchangeably.

Chronic prostatitis is a latent inflammation, a remnant of preëxistent acute trouble; whereas prostatorrhea consists in a leakage of the prostatic secretion due to the relaxation of the mouths of the prostatic ducts, and may be caused by masturbation, excessive sexual indulgence, etc. Both of these maladies are liable to be attended by various reflex nervous phenomena, psychical disturbances 
and sexual debility and neurasthenia. The significance of the discharge is overestimated by the patient, who often, particularly in prostatorrhea, imagines that the flow is a seminal loss, and he therefore aggravates all his symptoms by mental introspection.

Besides the mild type of prostatitis which approaches so nearly to prostatorrhea, there is another in which the suppuration in the prostatic sinus is more profuse, the dilated sinus containing many drops of pus, as may be demonstrated by washing out the pouch. One or more of the follicles of the prostate may be distended with secretion, which exudes from their dilated mouths into the prostatic sinus. There may exist an interstitial prostatic pus cavity, the remnant of an old prostatic abscess, which has only partially contracted down. Examination per rectum in chronic parenchymatous prostatitis will detect prostatic enlargement, generally unsymmetrical, sometimes nodular, and pressure upon the gland will express a quantity of its fluid contents which will consist of pus cells and prostatic secretion, differing from that which is obtained by pressure in a case of prostatorrhea, which consists of a prostatic secretion pure and simple and is devoid of the products of inflammation (Fig. 160).

There is an interstitial form of chronic prostatitis involving cellular infiltration of the gland and the obliteration of its glandular elements by the formation of new fibrous tissue. Such a condition may eventually lead to atrophy, contraction and diminution in the size of the organ. There is no distinct line of demarcation between the follicular, the parenchymatous and the interstitial forms of prostatitis, any one of which may occur alone. They are likely to coexist, and the classification is more or less arbitrary.

Symptoms.-Chronic prostatitis often exists without any symptoms of sufficient moment to attract the attention of the patient. A chronic follicular prostatitis involving the ejaculatory ducts and the prostatic sinus may be attended with pain during the sexual act. The principal symptom is a certain amount of purulent discharge, more or less continuous and increased by the muscular exertions of the body (stool, etc.). A much higher grade of trouble, involving interstitial changes and parenchymatous inflammation, may exist without a corresponding increase in the intensity of the symptoms. Heat and bearing down in the perineum or rectum are sometimes complained of. Neuralgic pains radiating down the legs and toward the fundus of the bladder are also a source of annoyance. Reflex nervous symptoms and mental depression are often present, but belong equally to the clinical history of all chronic disturbances of the genital apparatus and therefore are not especially significant. Func- 
tional impotence or premature ejaculation due to hyperesthesia of the ejaculatory ducts sometimes occur (see p. 515). Sterility is possible from azoöspermia, caused by obstruction of the seminal ducts in some part of their course.

Excessive flow of the prostatic secretion (prostatorrhea) may be present, due to dilatation of the excretory ducts of the prostate, in which case, however, inflammatory products are found mingled with the prostatic discharge. This discharge, as in prostatorrhea simplex, may be increased by movements of the body and by defecation. True spermatorrhea, as shown by the presence of spermatozoa in the discharge, the result of inflammatory catarrhal dilatation of the seminal ducts, often complicates chronic prostatitis. The prostate is always sensitive when examined per rectum, a feature more or less marked in different individuals. The nature of the discharge obtained by pressure upon the prostate varies, being sometimes a free gush of normal prostatic secretion with the admixture of a small amount of purulent matter, at others a large quantity of pus combined with only a few of the elements of the normal prostatic fluid, demonstrating that while these two conditions of prostatorrhea and prostatitis may be differentiated theoretically, yet, clinically, they are less easily separated and are liable to coexist. Strictly speaking, prostatorrhea is a symptom, not a disease-a symptom which enters more or less into the clinical history of prostatitis.

Diagnosis.-The diagnosis of chronic prostatitis is made by rectal examination and the character of the prostatic secretion squeezed out by digital pressure. The subjective symptoms are not to be relied upon. Microscopical examination of the discharge is important, to differentiate prostatitis, prostatorrhea simplex, spermatorrhea, urethritis and urethrorrhea. In making such investigation, it is important to examine the discharge: first, that which may be lying in the anterior urethra; next, that which is found in the urine; and finally that which is found in the last urine, after the prostate has been massaged through the rectum. The physical character of the urethral discharge is known to vary in the different conditions named. A purulent discharge signifies an inflammation in the anterior or posterior portion of the canal. Prostatorrhea simplex, as already stated, yields an excess of prostatic secretion which escapes from the urethra spontaneously and during muscular effort. Such a discharge is neutral or mildly acid in reaction. It has the seminal odor. It is white in appearance and is smooth and slippery. A microscopical examination reveals the accompanying picture which contains amyloid, hyaline and lecithin bodies, epithelium and Boettcher's crystals 
(Fig. 160), the latter being seen only in secretions from the prostate and may be produced by the addition of a 1 per cent. solution of phosphate of ammonia. This secretion, as will be seen, possesses none of the elements of inflammation. Its presence would indicate the coexistence of prostatorrhea with prostatitis, from which, theoretically at least, prostatorrhea simplex must be distinguished.

Urethrorrhea ex libidine consists of a hypersecretion from the glands of the urethra. The discharge is thin and watery, sticky and tenacious, and the patient may notice that upon placing his finger over the meatus, the discharge may be drawn out for some distance, resembling in this particular a bit of gum or sap. It has no seminal odor and it consists microscopically of a collection of flat epithelial cells and some free mucus, but of none of the elements which are found in the secretion of a prostatorrhea. This discharge is also, in an uncomplicated case, entirely free from the elements of inflammation.

Spermatorrhea produces a discharge which is gelatinous in character, mildly alkaline in reaction and possesses the seminal odor. Microscopically, we find the unmistakable evidence of the character of this discharge in the presence of spermatozoa (Fig. 140, p. 335). We have also the elements of the prostatic secretion, and in inflammatory conditions we find the products of inflammation; but the presence of spermatozoa in a secretion from the urethra at any time except following an emission is the one sign necessary to determine the existence of spermatorrhea, which condition may exist as a result of an atonic state with dilatation of the seminal ducts, or as a symptom of prostatitis or vesiculitis.

The discharge of prostatitis may be scanty or abundant. It oozes spontaneously from the urethra, but in order to establish its origin it is better first to wash out the urethra with warm saline or boric-acid solution and then obtain the discharge for examination, from the first flow of urine, or examine that which is expressed from the gland by pressure through the rectum. The gross appearance of this discharge is more or less purulent, differing from that of prostatorrhea and that of urethrorrhea, which do not possess this characteristic. It has nothing whatever in its gross appearance to distinguish it from the inflammatory discharge of other portions of the canal, and it is therefore important that it be obtained for examination after the anterior urethra shall have been cleansed: The exudation from a chronic prostatitis will present a microscopical picture as shown in Fig. 161, containing granular phosphates, leucocytes and characteristic epithelia from the prostatic urethra (see p. 95), and in addition, 
the elements of the normal prostatic secretion as seen in prostatorrhea (Fig. 160) may be found.

The gross appearance of the urine in chronic prostatitis may show only the presence of urethral filaments or of a small or large quantity of free pus. There is sometimes a solitary shred of a definite tadpole shape, as though it had been expelled from the mouth of one of the ejaculatory ducts. Microscopically, these shreds are composed of pus cells and mucus and also may contain any of the other elements of the prostatic or seminal secretion.

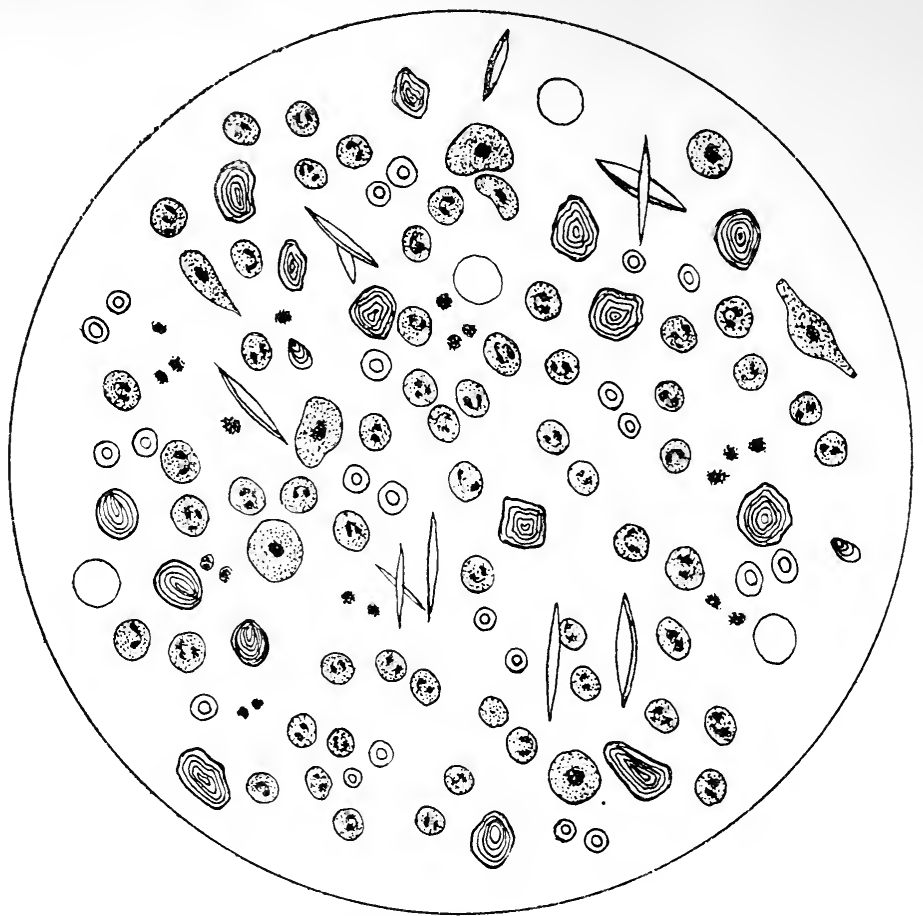

FIG. 161.-Chronic prostatitis.

The examination per rectum will distinguish any alteration in the size and sensibility of the prostate and implication of the seminal vesicles which, if distended by catarrhal secretion, may be felt beyond the prostate on each side of the base of the bladder, varying in size from a peanut to that of a small egg. Cystitis of the neck of the bladder is readily identified by the frequency and pain of urination, notably at and after the expulsion of the last portion of urine, and of ten by the existence in the last flow of a greater amount of pus than in the beginning. Cystitis of ten coexists with chronic prostatitis. 
Treatment.-In the adoption of local treatment for chronic prostatitis by way of the urethra, measures which are a source of irritation rather than a means of relief, should be avoided. Such are the unnecessary passage of dilators and other instruments intended to overdistend the canal, and the use of medicated bougies. Some cases of chronic prostatitis require about the same treatment and management as that recommended for chronic urethritis (see p. 233), namely, mild irrigations of permanganate of potassium, nitrate of silver, protargol, argyrol, etc. The method of irrigating the prostatic urethra has been already described (p. 39). Instillations are also often found useful in this type of prostatitis. The nitrate of silver heads the list. It should be tested gently for it may disagree. In solutions of from 0.003 to $0.6 \mathrm{gm}$. (gr. ss to $\mathrm{x}$ ) to the ounce, and sometimes stronger, it is used probably more often than the other preparations because it is quite generally effective, often brilliantly so. A few drops only of the strong solutions are needed, the quantity being reduced as the strength of the solution is increased. Thallin and the other drugs already referred to (see p. 237) will be found to suit some of the cases. The use of the endoscope in the posterior urethra may be employed in suitable cases, notably those that are of the superficial follicular variety of long standing, for the purpose of making direct application of a caustic agent, nitrate of silver, for example, to the veru montanum. If the parenchyma of the prostate is involved as well as the superficial area, these surface applications, if used alone, will not yield satisfactory results.

To reach deep-seated chronic inflammatory alterations of the prostate, such measures must be instituted as will stimulate favorably the circulation of the organ. With this object, digital massage of the prostate through the rectum is universally practised. By this manœuvre it is intended to force out the purulent and pent-up secretion in the swollen and turgid follicles, and by inducing a responsive activity of the circulation, cause resorption of inflammatory exudate. This manipulation is often quite useful in chronic cases. It should be practised at regular intervals, once or twice a week, but never with a vigor or frequency sufficient to cause undue irritation. There is no doubt that practitioners who have developed an increasing fervor for active treatment of chronic urethral lesions are wont in some instances to protract rather than relieve this particular condition by too frequent or too vigorous rubbing of the gland. Like all other local treatment, it should be employed with judgment and watchfulness as to the immediate and lasting effect produced. Another excellent means of treating chronic parenchymatous inflammation of the prostate, which may be 
used alone or as a supplementary measure to massage, is the application of moist heat to the region of the prostate by rectal irrigation. The rectum will tolerate a high degree of temperature when employed in this manner and strikingly beneficial results are obtained in old chronic cases of prostatitis which have resisted long continued efforts directed toward the urethral surface of the gland alone. A recurrent tube devised for this purpose (Fig. 162) is attached to a fountain syringe; the hot water passes into one arm of the tube and enters the rectum through two small apertures near the distal end. A large opening at the extremity connecting with an inside tube allows

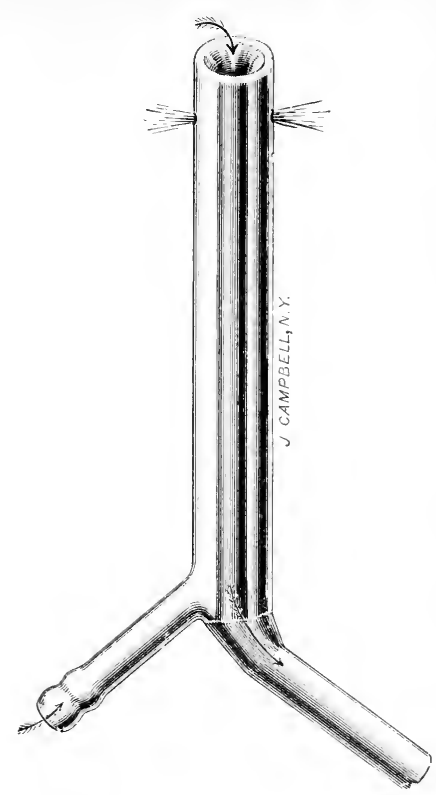

Fig. 162.-Author's double current rectal tube. of the immediate return of the hot water from the rectum and thus a continuous circulation of moist heat around the prostate is kept up so long as the operation is continued, which generally should be from ten to fifteen minutes. The end of the tube needs a little manipulation to insure continuous outflow of the fluid. Instead of the sitting posture which is assumed in the accompanying figure (Fig. 163) the knee-chest position may be substituted, or when conducted by an attendant the recumbent posture on a douche pan is used. The temperature of the water should be from $43^{\circ}$ to $54^{\circ} \mathrm{C}$. $\left(110^{\circ}\right.$ to $130^{\circ}$ F.). One tablespoonful of common salt is added to each liter of water, or the same amount of citrate of potash, four liters of water being used each time. Sometimes ichthyol, 4 cc. to the liter, are added. It is commonly ordered once a night at first, then every second night, and finally once or twice a week. Prompt improvement is often noted under this measure without other local treatment, as shown by the change in the character of the urine and the reduction in the size of the gland; but it is sometimes necessary to urge the continued use of this method for a long period before satisfactory results are obtained.

It is claimed that there is an actinic effect produced by the use of the high frequency current which is useful in some cases. The virtue of such treatment has decided limitations and there is too great a tendency to overstate the benefits derived therefrom. It is quite easy to understand that such pyrotechnic display is liked by the 
patient and profitable to the physician, but it should not be resorted to in empiric fashion. The high frequency current is obtained from a coil or plate machine and glass vacuum electrodes are used. The direct application of heat and oscillatory stimulation of the parts are thus obtained. The action is not a specific one. But the heat and stimulation do help some neuralgic cases and may lower congestion. In old chronic inflammation it may be tried, but is of doubtful value. For the enlargement of true prostate hypertrophy it is of no use.

The technic of using this apparatus consists in having the patient in the recumbent position; one electrode, a metal plate, is fastened over

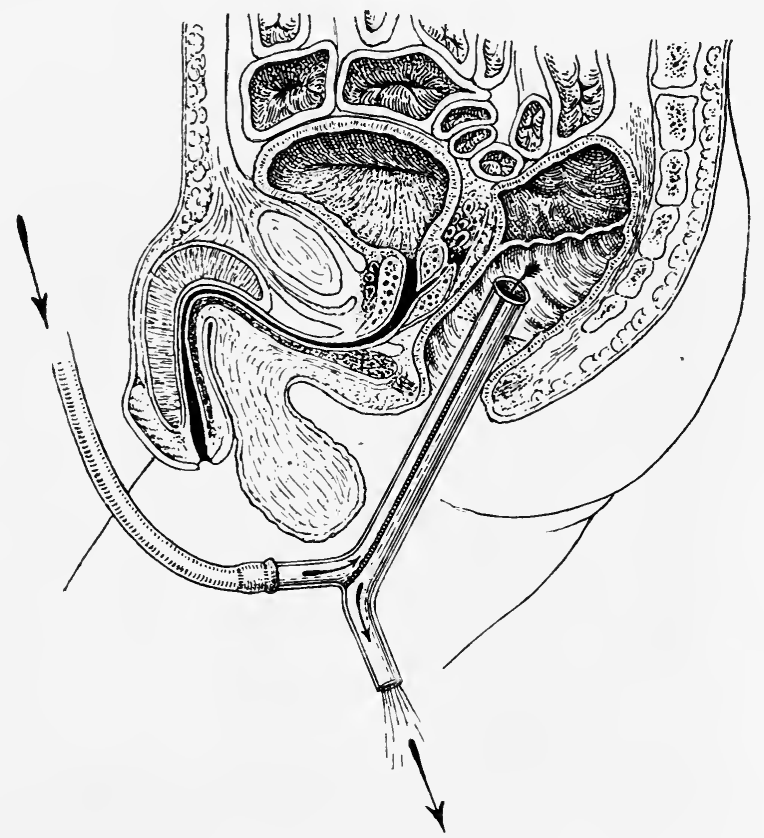

FIG. 163.- Recurrent rectal irrigation of prostate and vesicles.

the lumbar region, the other a glass or metal prostatic electrode, (Fig. 164) is introduced into the rectum. The application is kept up ten or fifteen minutes with a current of from 200 to 700 milliamperes. The same apparatus is used for obtaining the current employed for the trans-urethral treatment of vesical neoplasms (see p. 153).

The general condition of the patient should be investigated and any disturbance of nutrition properly treated. Iron, arsenic and hypophosphites have their indications as tonics and cod-liver oil seems to possess a special value in debilitated conditions. Pros- 
tatitis may be tuberculous in character or occur in individuals who have the tuberculous diathesis. Indeed, posterior urethritis is not infrequently the exciting cause of local tuberculous deposit. Such cases naturally do not get well under instillations, irrigations or the rectal douche. Sulphate of thallin sometimes comforts the irritability and hot rectal douche may do the same, but transportation to a favorable climate and the usual internal constitutional remedies are more to the point.

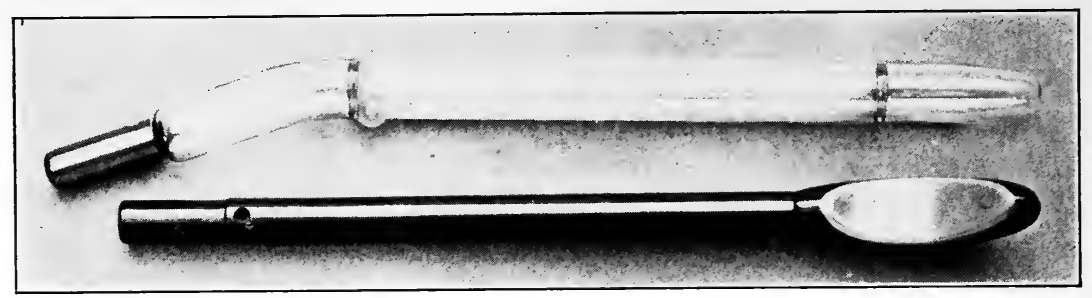

FIG. 164.-Metal and glass prostatic electrodes.

Vaccine therapy is of value in some cases of chronic infections of the prostate and by its use it is possible to control some otherwise rebellious conditions; other cases fail entirely to show any responsive action. Where it is possible to demonstrate a predominating organism, an autogenous vaccine may be prepared or a mixed stock vaccine of selected strains is sometimes used. The dosage and intervals between injections must be governed by effect produced and reaction following. (See Chapter V, p. 110.)

Finally, some old chronic cases of interstitial prostatitis are not cured short of surgical measures, entailing in cases perineal section and cureting and drainage of an old abscess cavity (see p. 474), or galvano-cauterization of the contracted orifice (see p. 476) or the total removal of the gland (p. 479). 


\section{CHAPTER XXI}

\section{PROSTATIC HYPERTROPHY}

\section{CAUSATION-PATHOLOGY_CLINICAL COURSE}

On account of the situation of the prostate at the confluence of the seminal and urinary canals, enlargement of this gland produces detrimental effects upon the sexual and urinary functions and becomes a predisposing factor to disease of both the bladder and kidneys.

Prostatic hypertrophy, therefore, not only exercises considerable influence upon the health and comfort of the individual but is among the important agencies that combine to make up the percentage of human mortality. It is generally regarded as a malady of advanced years, nevertheless its occurrence in individuals of middle age or even the young is recognized, and while in many cases the condition is not brought to notice until advanced age, it is evident that it has commenced years before.

The size of the enlargement is in no sense indicated by the activity of the symptoms which is owing to the fact that the urethral passage is not necessarily seriously affected even though the enlargement be considerable and many instances are found in postmortem examinations in which enlargement of the gland is marked and yet there has been no symptom thereof during life. A very moderate enlargement, on the other hand, which extends into the prostatic urethra or becomes elevated behind the bladder outlet, may be sufficient to cause serious trouble.

Causation.-As already stated, prostatic hypertrophy is commonly discovered in old men, that is to say, discovered when its effects are most likely to make themselves felt and when the symptoms it produces are in greatest activity. This is usually after the age of sixty, but it is often encountered and first revealed between the ages of fifty and sixty. Its frequency of occurrence is generally stated to be about 33 per cent. of all normal men and of these, in the neighborhood of 40 per cent. present symptoms. ${ }^{1}$

Many and varied have been the theories concerning the cause of

1 Sir Henry Thompson gives 34 per cent. of men reaching the age of sixty as having enlarged prostate and 15 to 16 per cent. of the total having symptoms. Johnson, Internat. Jour. of Surg., 1909 , XII, found hypertrophy in 79 per cent. of 360 prostates examined with only 16 per cent. showing symptoms. 
prostatic hypertrophy and yet, it is still not satisfactorily explained. To sum up briefly as far as is known of the causative agency resulting in hypertrophy of the prostate, is to state that it consists of factors which are active principally during and after middle age which tend to bring about overgrowth of one or both of the two glandular elements, the epithelial and connective tissue. Great activity of the sexual functions may be one of these factors.

The theory of arterioclerosis, suggested in earlier years, is entirely unacceptable and has been refuted by different observers. ${ }^{1}$ Arteriosclerosis of the bladder may exist as a part of a general circulatory sclerosis but is not an explanation of the symptoms of prostatism when they exist.

Strong arguments have been advanced and diligent efforts have been made to prove that gonorrhea bears an important relation to the etiology of prostatic hypertrophy. This theory has its exponents and has been ably supported but such a teaching as the theory of the inflammatory nature of this malady, in spite of the arguments of its adherents, cannot be proven by pathological findings or the credible clinical histories of many individuals who are subjects of this trouble. ${ }^{2}$

Pathology.-The chief pathological changes that take place during the evolution of a hypertrophied prostate (microscopic changes) are overgrowth of glandular and of connective tissue, one or the other of these predominating. It is stated that in a few cases, there is some increase in the muscular elements of the glands and in others that new ascini develop, this condition representing true adenoma. ${ }^{3}$

This is the type of growth that has given rise to the neoplastic theory of the affection. ${ }^{4}$ The adenomatous appearance is explained by many observers who have closely studied the subject as being due to the choking up and consequent dilatation of the ducts of original ascini by proliferated epithelial cells, ${ }^{5}$ as well as mucus, amyloid bodies, serum and various products of cellular overactivity. ${ }^{6}$

\footnotetext{
${ }^{1}$ Casper, Genito-urinary Diseases, states regarding the association of prostatic hypertrophy and arteriosclerosis, "Both are affections of old age and therefore are often associated but they bear no causal relation to one another . . . . In cases in which complete retention of urine has existed for years, the contractile power of the bladder remains unweakened. This would be impossible were the wall of the bladder much affected by the sclerotic degeneration."

${ }^{2}$ Ciechanowski, Ann. des Mal. des Org. Gen.-Urin., 1901, XIX, undertakes to show that the changes found in prostatic hypertrophy, no matter what may be the distinctive course in an individual case, are due to the effects of early gonorrheal inflammation. Watson and Cunningham, Genito-urinary Diseases, Keyes, Diseases of the Genito-urinary Organs, Casper, Genito-urinary Diseases, and Legueu, Traite Surgical d'Urologie, all disagree with this theory.

${ }^{3}$ Watson and Cunningham.

${ }^{4}$ Greene and Brooks.

${ }^{5}$ Watson and Cunningham.

6 Greene and Brooks.
} 
Gross Appearances.-It is well to note here that the prostate in which the glandular elements are chiefly effective becomes large and soft whereas those where the connective-tissue elements bear the brunt of the changes, are firm and small. ${ }^{1}$ The condition, therefore, is not in any sense a hypertrophy but a hyperplasia, a degenerative, rather than a constructive formation. Other observations regarding the gross appearances of the gland relate to its contour and separate portions of it that are involved. The condition may predominate in one or both lateral lobes or the morbid enlargement may be almost entirely in the isthmus between these lobes forming what is known as a third or middle lobe of various dimensions. ${ }^{2}$

The resulting growth, as it occurs in a portion or involves the entire body of the gland produces a symmetrical or irregular outline. The volume of a hypertrophied prostate varies; the average weight being from 60 to 100 grams; whereas the enlarged gland often attains the weight of from 100 to 200 grams. In exceptional cases, the weight is still greater and markedly in contrast to that of the normal gland which averages 20 grams. Symmetrical enlargement of the entire gland produces a spherical body with equal lateral lobes, a mass which presses backward into the rectum. Frequently in the large lobes there are isolated masses of dense structure analogous to the lobules found in a fibromatous uterus. The groove normally separating the lateral lobes disappears and thus it loses its chestnut shape. In asymmetrical enlargement, one lobe is excessively developed. Overdevelopment of the median portion forming the so-named "third"

1 Watson and Cunningham.

${ }^{2}$ Tabulation, showing the frequency of the different varieties of prostatic hypertrophy, taken from Keyes, Diseases of the Genito-urinary Organs with observations of Moullin added from Watson and Cunningham.

\begin{tabular}{|c|c|c|c|c|c|c|c|}
\hline & Thompson & Predal & Desnos & Motz & Watson & Moullin & Total \\
\hline 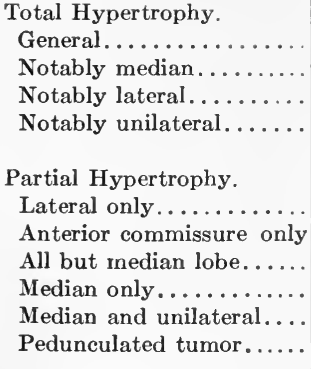 & 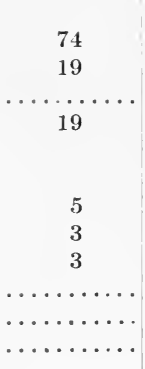 & 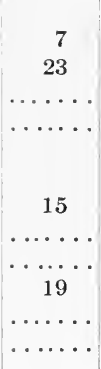 & 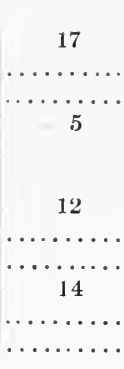 & $\begin{array}{c}10 \\
\ldots \ldots \\
\ldots \ldots \\
\ldots \ldots \\
1 \\
\ldots \ldots\end{array}$ & $\begin{array}{c}14 \\
\ldots \ldots \ldots \\
\ldots \ldots \ldots \\
\ldots \ldots \ldots \\
\\
\\
\\
4 \\
\cdots \ldots \ldots \\
\cdots \ldots \\
9 \\
2 \\
1\end{array}$ & 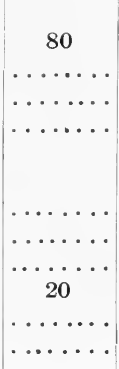 & $\begin{array}{r}46 \\
3 \\
3 \\
62 \\
3 \\
1\end{array}$ \\
\hline & 123 & 64 & 48 & 27 & 30 & 100 & 392 \\
\hline
\end{tabular}


lobe or Home's lobe may reach a very large size as compared with the rest of the gland. It is sometimes sessile and other times pedunculated. This is the most rapid growth of prostatic hypertrophy and involves the glandular tissue belonging to the posterior commissure or isthmus. Thus, it will be found that the extension of the prostatic growth, when symmetrical, may be mostly anterior to the bladder with a moderate bulging into this viscus, producing its obstruction largely

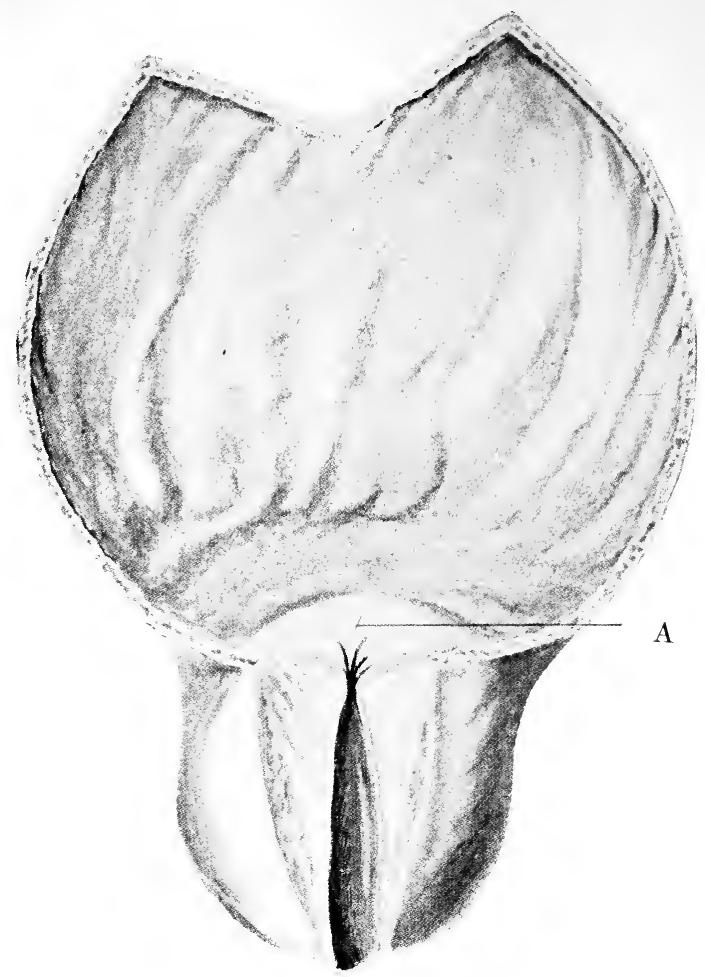

FIG. 165.-A, Bilateral prostatic hypertrophy causing elevation of median isthmus. (International Clinics-Chetwood.)

by compression of the urethra and elevation of the posterior commissure (Fig. 165); in other cases, there is a much greater, intravesical growth and the site of the obstruction, be it lateral or median, lies behind the vesical outlet (Fig. 166). This is an important consideration in connection with the choice of operation for prostate hypertrophy. ${ }^{1}$

1 Legueu gives a compilation of the statistics of various observers as to the frequency of involvement of different parts of the prostate. Hypertrophy, localized in the anterior commissure 3; hypertrophy limited to the lateral lobes 103; hypertrophy limited to or predominating in the median lobe (posterior commissure) 117; general hypertrophy 206. 
Alterations in the fibrous sheath often bring about conditions of great importance to the surgeon.

Thickening of this structure without adhesion to the surface of the gland makes enucleation an easy matter whereas adhesion to the prostate offers one of the difficulties during operation making it impossible to obtain a good line of cleavage. ${ }^{1}$ Finally, the interposition of fibrous tissue as a result of old, chronic prostatitis, a supplemental condition to prostatic hypertrophy, alters the character of the prostatic growth and interferes with the ease of removal; and

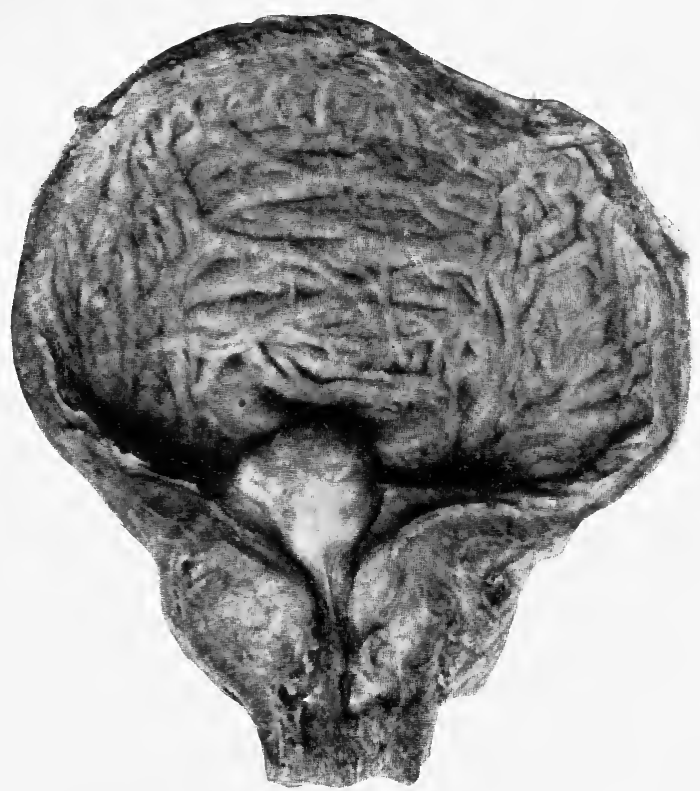

FIG. 166.-Prostatic hypertrophy. Obstruction intravesical (bilateral and middle lobe.) (Aschoff's Pathology.)

when these changes are superimposed upon the neck of the bladder as a result of chronic cystitis or prostatitis, contracture or stricture of the orifice as an added condition (Fig. 179) exaggerates the obstruction to the bladder outlet, greatly interferes with urination and has to be reckoned with at the time of operation (see Contracture of the Neck of the Bladder, p. 435).

Changes in the Urethra.-Of greater importance than the size of the prostate is the change that it works upon the urethral outlet of the bladder. Under normal conditions, the vesical orifice is on the

${ }^{1}$ Watson and Cunningham. 
same plane as the floor of the prostatic canal (Fig. 180, p. 438), the latter being capacious and distensible. Under the changes wrought by prostatic enlargement, the entrance to the urethra is elevated, and contracted by the encroachment of a concentric growth (Fig. 170) and it is partially or entirely closed by the valvular obstruction of a small or large, intravesical middle lobe (Fig. 166). The prostatic urethra is elongated by antero-posterior enlargement of the prostate; its calibre is compressed transversely by an extensive bilateral enlargement (Fig. 165) and its direction is changed by elevation of its entire length or by irregular protrusions of growth upon the floor or roof (Fig. 167); its course may be twisted by enlargement of one lateral

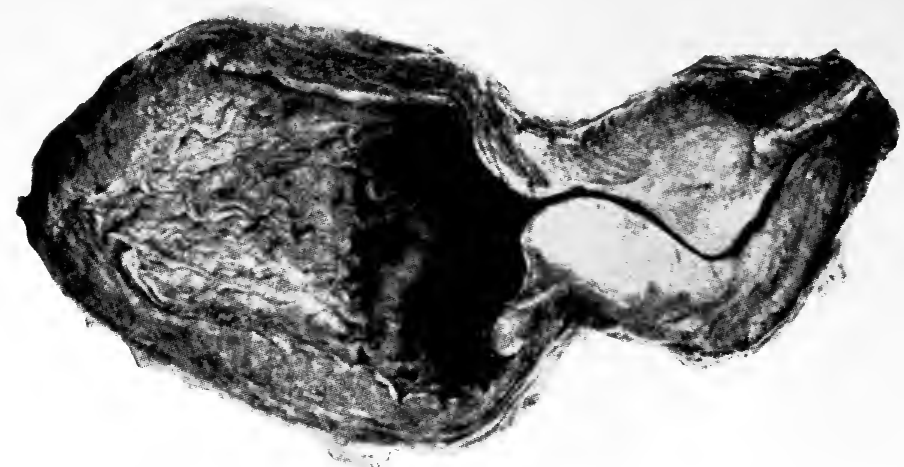

FIG. 167.-Prostatic hypertrophy intraurethral obstruction. (Aschoff's Pathology.)

lobe which forces the urethra to one side or the tube may become practically bifurcated by the overgrowth of the prostate in the median line. ${ }^{1}$ These various distortions of the urethra render catheterism exceedingly difficult (Figs. 168 and 169).

Secondary Changes Due to Prostatic Hypertrophy.-These changes comprise alterations in the bladder itself with attendant functional impairment and its effect upon the ureters and kidneys. The causes of such secondary conditions are partly mechanical and partly the result of microbial invasion. The bladder in some cases becomes hypertrophied and in other instances atrophied. The initial and constant lesion is hypertrophy, followed later by atrophy. Infection and inflammation aggravate the vesical lesion and favor the development of sclerosis. ${ }^{2}$ Before infection has occurred, there is a tendency for the bladder to undergo great distention: This may occur simul-

1 Casper.

${ }^{2}$ Legueu. 
taneously with hypertrophy ${ }^{1}$ and as the bladder undergoes atrophy, the patient is hardly conscious of the gradual distention until it reaches such a point that there is an almost constant urgency of urination; complete retention is then established, accompanied by the incontinence of overflow.

The greater the obstruction and the longer it has existed, the more advanced are these secondary conditions. They are caused by futile effort on the part of the bladder to carry out its normal function of complete evacuation, whereupon the muscular mechanism working against continuous obstruction in a sense receives back again the force it expends and becomes hypertrophied. This hypertrophy of the vesical musculature on account of its reticulated construction, offers lines of greater resistance with intermediate spaces that are more yielding so that irregular trabeculæ are formed on the internal aspect of the bladder. The inter-trabecular spaces, under the continued back pressure, tend to form diverticulæ or pouches which are sometimes of considerable dimensions. In advanced cases, when infection of the bladder usually has occurred, urine is apt to be retained in these saccules and secondary stone may be formed from the deposit of sediment. The trigone of the bladder, or that portion directly behind the urethral orifice and in front of the inter-ureteric ridge, on account of the marked hypertrophy of this elevation, is a point where sacculation is most pronounced. In this pouch the retained urine precipitates its alkaline salts which, together with the products of irritation and inflammation, mucus, bacteria, etc., combine to make up the composition of secondary calculi. As an extension of this process in the bladder, the ureters and kidneys share in the changes wrought by mechanical obstruction and vesical distention. Dilatation of the ureters, the renal pelvis and of the kidneys themselves may occur (hydronephrosis) as a part of the general, vesico-renal distention (see Hydronephrosis, page 575), but this condition is liable to be reached at a period when infection has already occurred and is therefore likely to be pyonephrosis rather than hydronephrosis. Such general distention of the urinary tract from below upward, which necessarily involves both sides, occurs only in extreme cases and is exceptional. More frequent are conditions derived by infection from the bladder, such as ureteritis, pyelitis and pyelonephritis, which may implicate one and not both kidneys or the infection may occur on both sides following the constant retention of a large amount of infected urine which may possibly infect these organs by hematogenous transmission without dilatation and upward distention of the ureters and

1 Casper. 
kidneys. Then is produced that condition, which is often observed in late cases, of mild chronic urinary toxemia, the principal evidence of which is a functional renal insufficiency-polyuria, especially nocturnal-the urine being pale, bacterial and of a very low specific gravity.

In the prostate itself, there may occur complications due to inflammation setting up, which may form an abscess; and on account of occlusion of the ascini, may be a cause for the formation and collection of prostatic calculi, ${ }^{1}$ and this same engorgement may extend. through the ejaculatory ducts to the seminal vesicles.

Other important complications incident to hypertrophy of the prostate are the changes in the blood-vessels. The vessels of the prostatic plexus not infrequently become permanently distended and this varicose condition extends to the floor of the bladder. This circulatory engorgement render's the individual more prone to frequent transitory attacks of retention before the stage of permanent retention has been reached. On account of these conditions, hemorrhage may occur from the varicose vessels of the mucosa, of the prostate and of the trigone. ${ }^{2}$

Clinical Symptoms.- The symptoms of enlargement of the prostate are principally those that relate to the function of the bladder rather than that of the prostate, being characterized by disturbances of micturition; these are changes in the frequency of urination which are due to incomplete emptying of the bladder and to cystitis; changes in the stream due to obstruction of the orifice of the bladder and of the prostatic urethra and dribbling of the urine due to overflow (distention) and spasmodic incontinence (irritability).

Frequent Urination.-The frequency of urination, peculiar to prostatic hypertrophy, is particularly noticeable at night time; in fact, one of the first evidences with approaching years of beginning prostatic obstruction is the necessity of rising once or twice a night to urinate. Ordinarily, an individual under normal conditions will pass the night through without the necessity of rising. This may mean eight or ten hours interval, whereas the necessity to rise twice during this period, means an interval of three or four hours and a diminished bladder capacity or incomplete evacuation thereof. There is a relative frequency also by day, but it is not so noticeable. The explanation of nocturnal frequency is partially the residual urine,

\footnotetext{
1 I operated upon a case of complete retention of urine in an aged patient with prostatic hypertrophy and removed from the interior of the prostate innumerable millet-seed calculi (C. H. C.).

${ }^{2}$ I have seen cases of vesico-prostatic retention which have had severe attacks of hemorrhage from no other cause than that of an erosion of a varicose vessel (C. H. C.). Casper, Genito-urinary Diseases, refers to these conditions and states that "an injury may result in profuse and of ten uncontrollable hemorrhage" and he states "I have frequently seen such occurrences and indeed have had two patients bleed to death from this very form of hemorrhage."
} 
which is usually greater at night, and the engorgement of the circulation of the prostate and adjacent bladder region, also probably greater at night. These two contributing factors limit the distensibility of the sac and hence make the call to urinate more pressing, causing the patient to be aroused from his sleep. When inflammation has set in, these conditions are intensified, the irritability of the bladder being greater, the calls to urinate are still more frequent day and night. This is also the result of accumulating retention, leaving but a small space between the mean and extreme capacity of the bladder which finally reaches the point of more or less continuous dribbling or overflow. This particular feature occurs especially at night when the patient, on account of the somnolent condition is not aroused by the almost continual necessity to relieve the overdistended bladder: whereas, in the daytime, the slight remaining capacity to urinate prevents the occurrence of overflow until the bladder shuts down entirely and complete distention results, when the overflow may occur by day as well as at night. Increase in the quantity of urine secreted (polyuria) aggravates the symptom of frequency of urination (polakiuria). The former is an expression of the kidney activity and the latter of the irritability of the bladder. In the early stages, damming back upon the kidneys and their consequent congestion diminishes rather than increases the flow of urine, whereas in the later stages of kidney implication, as heretofore stated, the destruction of the kidney substance leads to a diminution in their functional activity with respect to the elimination of solid constituents and there is a relative increase of fluid secreted producing a larger amount of urine of low specific gravity. Polakiuria or frequency, on the other hand bears a direct relation to the irritability of the parts which is more or less variable, being increased by the declining hours of the night and intensified in the daytime as well as at night when infection of the bladder and inflammation set in.

Dysuria.-This symptom is referable to the difficulty of urination and the occurrence of pain; the former depends upon the mechanical obstruction to the flow and the latter on this as well as the existence of irritability or inflammation in the urethro-vesical space. The difficulty of urination, caused in the first place by the obstruction to the flow, is evidenced in the difficulty of starting the stream. Later, the continued efforts and straining against the obstruction result in a lack of force and the stream instead of being projected, falls in a perpendicular manner with diminished impetus. This is at first overcome by bringing into action the abdominal muscles but later, even this extra effort loses effect. The impelling force of the final muscular contraction at the end of urination also loses its tone, this terminal impulse of 
the cut-off muscle is wanting and the end, instead of being a jet is a dribbling. Pain bears a relation to the existence of cystitis $(q . v$.$) ,$ abrasion or ulceration and may be present at the end or during urination. In the presence of a vesical calculus the pain is characteristic of this condition (see p. 445), caused by the contraction of the congested bladder membrane against the foreign body.

Hematuria.-There is usually no blood at the end of urination unless the enlargement of the prostate is complicated by the existence of stone, of ulceration, or unless the mucous membrane has been injured by the passage of the catheter or other instrumentation.

The symptoms due to renal changes are characteristic of the nature and extent of these changes and may consist of renal insufficiency or the consequences of renal infection, general urinary septicemia.

Progress and Course of Prostatic Hypertrophy.-It is well to consider separately the successive stages that usually occur. The earliest stage is premonitory; following this is the stage with retention and no distention of the bladder, and the third stage is that of retention associated with distention. ${ }^{1}$

The symptoms of the first stage are, as a rule, insignificant and often taken little account of by the individual, it being generally accepted as foreordained that males, after having passed the age of fifty, should rise once or twice during the night to urinate. This may be all that is noticeable over a long period, but looking backward, it is generally possible to perceive the increase in this tendency, and the necessity to rise becomes more insistent and frequent. Along with the symptom of urinary frequency occurs the slowly developing dysuria, first noticeable in the form of hesitancy in the beginning of the flow. This is more apparent in the morning or following any cause that may lead to congestion of the prostate, such as excesses of eating or drinking, physical fatigue or exposure to cold. One of the peculiar manifestations of this symptom is described by the patient as occurring in the morning on rising when, after passing a small quantity of urine, there is a stoppage and in a short time, another call when the flow comes more copiously and freer than before. Loss of projectile power is another feature of this stage and develops as slowly and progressively as the other indications which may extend over months or years.

The second stage of prostatic hypertrophy differs from the first only in degree of symptoms produced and there may not be a distinguishing period of separation. The difference is that in the first stage, the obstructing prostate acts as an impediment to the passage of urine, as evidenced by the beginning symptoms of dysuria, but

${ }^{1}$ Casper. 
at this early stage, there is compensatory muscular action exerted by the bladder which aids in overriding the abnormal resistance of an elevated urethra or one that is partially obstructed by median or lateral intrusion.

When the second stage arrives, this compensation which is in reality a hypertrophy, loses its power and incomplete retention commences. Thus in the first stage it is possible to find by introduction of the catheter very little or no residual urine. In the second stage, however, the obstruction having increased and the compensatory action having failed, residual urine is a prominent symptom which varies commonly from 50 to 200 c.c. and up to complete retention of the third and last stage.

As the second stage progresses, there may be intermediate attacks of complete retention which are relieved by the catheter or subside spontaneously. Furthermore, the symptom of dysuria increases and frequency of urination becomes greater as the retention increases. Infection may occur and bring matters to a climax, intensifying the symptoms of urgency, frequency and precipitancy of urination. On the other hand the second, like the first stage, may be continued for a considerable period without danger to the patient, especially if the distention of the bladder is gradual and infection does not occur. One of two courses generally ensues. Infection is produced by the introduction of instruments in the course of examination but the painful and distressing symptoms resulting therefrom are relieved by appropriate treatment and the progress of the malady proceeds as before; or the continuance of infection initiates the beginning of a distressing existence and impairment of general health, that usually influences the patient's state of mind to accept a surgical operation. On the other hand, if the situation has not reached a climax as above described, complete retention eventually ensues which attack of retention may be the first, or one of several occurrences, and the third stage is ushered in. At this time, catheterization has no lasting effect upon the relief of the retention-the condition is that of complete stoppage; voluntary urination is entirely out of the question and pain is excessive if the bladder is allowed to become distended. The situation has reached an impass where the patient must accept one of two alternatives - that of habitual and exclusive use of the catheter at required intervals or the operation of removal of the hypertrophied gland to relieve the obstructive condition (prostatectomy). The condition of the urine during the first stage is, as a rule, clear; during the second stage, it may be clear at first and later become clouded with the bacteria and pus owing to infection. This may occur 
early or late in the second stage and even up to the third and last stage the urine may remain clear and continue so during this period until late infection ensues. Infection usually occurs from instrumentation, but in cases of chronic prostatism with persistent posterior urethritis, it may occur spontaneously.

The constitutional symptoms of prostatic hypertrophy are those that occur as a result of general depreciation of health from nervous debility and interference with sleep and, when septic infection occurs, from systemic absorption. The latter symptoms may begin with a chill and fever and a general malaise, or the onset may be less pronounced and the systemic condition show itself in digestive disturbances and malnutrition, resembling in a measure a cachexia which may lean to the suspicion that the condition is one of malignancy rather that benign prostatic hypertrophy, but it is in no sense as profound a state as characterizes the latter condition. 


\section{CHAPTER XXII \\ PROSTATIC HYPERTROPHY (Continued) \\ DIAGNOSIS AND TREATMENT}

Diagnosis.-The diagnostic investigation of a patient presenting the symptoms already described aims not only to recognize the presence of benign prostatic hypertrophy and differentiate the same from other morbid conditions but to determine the type of enlargement in a given case and the particular stage thereof. There are certain cardinal symptoms and associated conditions which, taken together, make a presumptive diagnosis. These are the symptoms which collectively are known of as "prostatism," occurring in an individual past the age of fifty or fifty-five years. In addition to the urinary disturbance, there may be also evidence of mild systemic disorder such as digestive impairment, constitutional debility, but not cachexia or emaciation, significant of malignancy.

The physical examination embraces:

(1) Rectal palpation.

(2) The introduction of a catheter:

(a) To take account of the permeability and course of the prostatic urethra.

(b) To determine the presence and quantity of retained or residual urine.

(c) To take account of the muscular tone of the bladder as evidenced by its projectile force.

(d) To measure the urethral distance.

(e) To determine the bladder capacity.

(3) Intravesical palpation to detect the presence of a foreign body (stone) and to recognize the intravesical projection of the prostate.

(4) Cystoscopy.

1. Rectal Examination.-This exploration (see p. 65) should be conducted before the bladder is emptied and again after its contents have been evacuated. It is intended to ascertain the general outline and size of the gland, its consistency, elasticity and irregularity of surface.

2. Catheterization.-The introduction of the catheter should be undertaken with every precaution and care to avoid irritation and 
infection. This is especially important in patients with uninfected and dilated bladders. Such individuals are peculiarly prone to infection and the occurrence thereof may prove disastrous in those of poor resistance and impaired kidneys. The detailed instructions set forth in the section on general technic (see p. 53) with regard to the handling of instruments and the preparation of the patient are carried out. The patient is directed to void as much of the urine as is possible. A catheter, preferably of soft rubber, is then gently introduced. It may be that the soft rubber catheter will not pass beyond the prostatic urethra, on account of the distortion of the urethra to one side or the other, or elevation of the floor by the growth of the gland or on account of the existence of an intravesical median lobe. In one case it may be necessary to employ a more rigid catheter of the olivary type (see Fig. 168); in another an elbow catheter of the
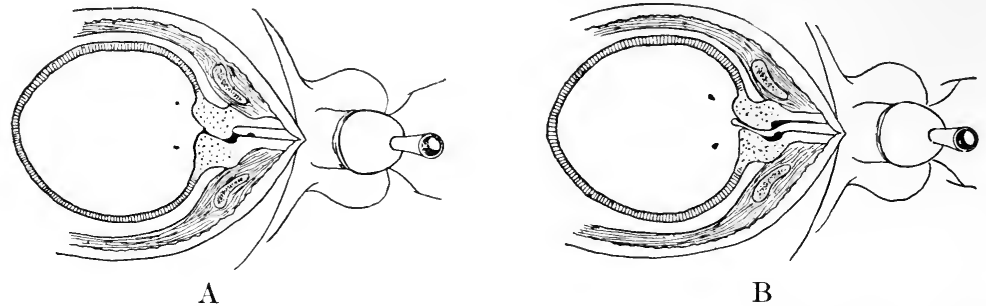

FIG. 168.-Catheterism in prostatic obstruction. A, Soft rubber catheter obstructed by tortuous prostatic canal. B, Obstruction overcome by olivary catheter.

single or bi-coudé variety (see Fig. 169, and A, B, C, D), in still another the full curved prostatic catheter (Fig. 38, p. 50) or the Guyon director with a soft rubber instrument. In any case, the avoidance of force or undue haste is of great importance. After the catheter has been fully introduced, the amount of urine obtained is taken account of, which represents the residual or retained urine, provided the patient has been able to urinate before the examination. The result, however, is not necessarily final unless confirmed by more than one examination, as conditions affecting the congestion of the gland which have an influence upon the amount of retained urine may change from time to time. During the emptying of the bladder, observation should be made of the force of the flow whether it is projected slightly, if at all, from the end of the catheter or whether it is necessary to use manual pressure above the pubis to evacuate a small or large amount of the contained urine.

The bladder capacity is tested by injecting as much of a mild anti- 
septic solution as can be comfortably retained and taking note thereof. This observation is not necessary except in cases where there is evident a marked decrease below the normal capacity (400 to 500 c.c.).
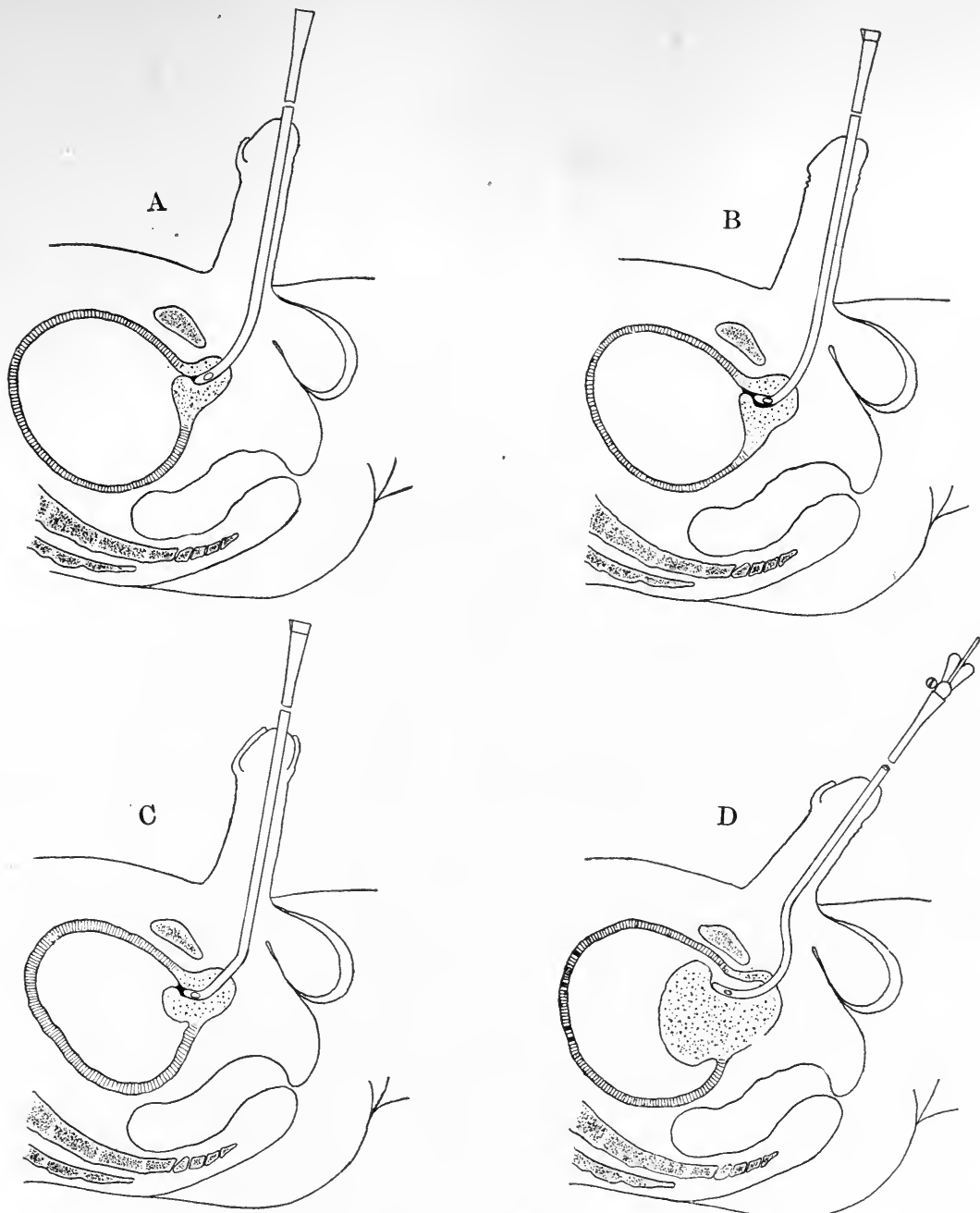

FIg. 169.-Catheterism in obstructive conditions of the prostate. A, Soft rubber catheter intercepted in prostatic urethra. B, Single elbowed catheter used to surmount obstacle in prostatic urethra. C, Double elbowed catheter used to surmount obstacle in bladder. D, Introduction of soft rubber catheter on director (Béniqué curve.)

The measurement of the urethral length is obtained by moving the catheter backward and forward until a point where the flow begins is satisfactorily determined, from which point the urethral distance is 
measured on the catheter when it is withdrawn; "with a catheter that is marked with gradations, the urethral length may be determined, then and there. The normal urethral length is between 18 and 20 centimeters ( 7 and 8 in.) and any marked elongation signifies that much lengthening of the prostatic portion.

3. Palpation of the Interior of the Bladder.-This is accomplish as already described (p. 75) by means of the short beak sound (Thompson searcher). The introduction of this instrument in experienced hands will recognize the general direction and length of the prostatic urethra. After it reaches the bladder, by rotation from side to side the unusual projection of the prostatic growth will be appreciated. The elevation of a middle lobe upon the floor will interfere materially with the introduction of the instrument and by turning the beak of the latter downward, the middle lobe is engaged and this, as well as the hypertrophied bar, is recognized. By palpation the prostatic growth may be roughly measured but when the parts are sensitive and congested, great caution should be exercised with this instrument, as otherwise damage may be inflicted. It is more particularly relied upon in cases of moderate prostatic hypertrophy, to distinguish between that and stricture of the neck of the bladder and to determine the presence of vesical calculus when, on account of the existence of hematuria and other characteristic symptoms, such a complication is suspected.

Generally speaking, the diagnosis of prostatic hypertrophy by means of the measures above described is not a difficult matter. Of greater importance is the appreciation of the attendant conditionsthe size of the growth and its general direction, inflammation of the bladder, due to infection, overdistention, atony and the presence of such complications as stone in the bladder and marked renal involvement.

The size of the growth is appreciated by rectal examination and intravesical palpation as already described. This question may have a bearing upon the choice of operation (see p. 410). Infection and inflammation of the bladder are recognized by the symptoms and by the urinary examination.

Overdistention and atony when revealed by exploration with the catheter signify the existence of back pressure of prolonged duration; the opposite condition of contraction and limited bladder capacity is the result of unusual irritability caused possibly by a growth or foreign body (stone) or by deep inflammation and pericystitis, in which case it has a bearing upon the prognosis or the degree of relief that may be expected from operative intervention.

The presence of stone in the bladder has also a bearing upon the 
choice of operation; whereas renal implication is an all important factor affecting the selection of operation, the prognosis thereof and the future health of the patient.

When these ordinary measures of examination do not suffice to give the desired information with respect to the important questions involved, cystoscopic exploration must be resorted to.

Cystoscopy.-With the present improvement in instruments and technic it is practically possible to make a cystoscopic examination in almost all cases and if carefully and skillfully undertaken, no damage need be inflicted thereby. In exceptional cases it should not be practised on account of great irritability and large median lobe projection. When successfully accomplished it is possible to outline the intravesical projection of the lateral lobes and the superior commissure, to inspect a pedunculated middle lobe on either side and on withdrawing the cystoscope with the dilatation of continuous irrigation, it is possible to view this lobe from the urethral side or if not present to see the middle commissure between the lateral lobes. (See Cystoscopy.)

The examination will also detect changes in the vesical mucosa due to cystitis which is usually centralized around the neck. Characteristic diverticula and trabeculæ caused by vesical distention and muscular overexertion are recognized. In some cases, calculi that could not be touched by vesical palpation on account of the depth of the bas-fond may be discovered. The condition of the kidneys is also investigated when demanded

Profuse hematuria attending prostatic hypertrophy usually suggests the existence of some other condition such as vesical neoplasm or stone. In such cases, cystoscopic examination is of great importance.

Hypertrophied prostate must be differentiated from tumors of the bladder, malignant growth of the prostate and retention of urine due to other causes than that of the hypertrophied gland. In the case of vesical tumors and calculus the cystoscope will recognize the particular lesion and it is in such conditions that the skill of the operator and the value of the instrument will be tested.

In the case of malignant neoplasm of the prostate, rectal palpation is able to distinguish the texture of a malignant growth when it is of an extremely hard variety or extensively diffused in character but border-line cases and those which have no predominating feature of this sort are difficult to distinguish from dense, fibrotic thickening of the prostate and malignant scirrhous growths. The cystoscope is not to be relied upon to detect malignant growths confined to the prostate but will reveal any metastatic extension within the bladder. The age 
of the patient has some bearing, although malignancy occurs in the young. Cachexia and marked physical depreciation are important features to be taken account of, but in the case of a slowly progressive and long standing neoplasm, the occurrence thereof is insidious and difficult to detect until its presence becomes markedly in evidence.

The important types of prostatic enlargement commonly met with are as follows: (1) Generalized and symmetrical prostatic hypertrophy involving alike the median and lateral lobes which form a

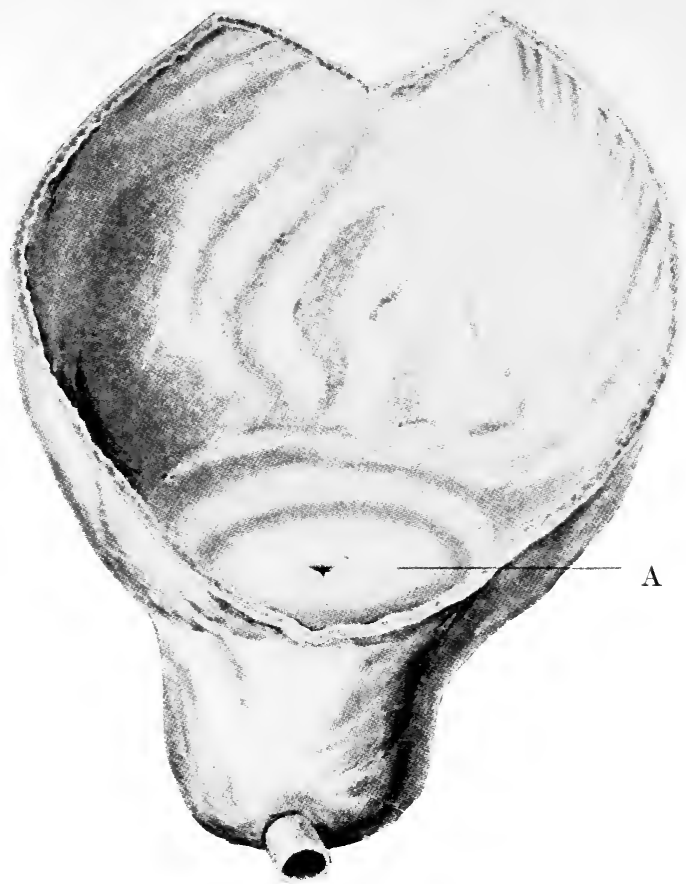

FIG. 170.-Concentric sphincteric. A, Prostatic hypertrophy. (Chetwood, International Clinics.)

single mass, encircling and compressing the prostatic urethra and bulging more or less upon the floor of the bladder. This form of prostatic hypertrophy is felt as a globular tumor by rectal palpation and, if examined with the cystoscope, is seen as a concentric protrusion surrounding the bladder outlet (Fig. 170).

(2) Bilateral prostatic hypertrophy without median lobe but with elevation of the posterior fold of the urethral outlet by the lateral enlargements which extend into the urethra and backward into the bladder. This form is felt by rectal palpation as a bifid enlargement 
of the gland without rectal elevation posteriorly from the floor of the bladder, at the site of the posterior commissure (Fig. 165). Inspection with the cystoscope may be difficult on account of elevation of the urethral fold; but with care it is successfully accomplished and it is possible to recognize the bilateral abuttment of the two lobes of the prostate, also to view the deepened bas-fond behind the elevated orifice.

(3) Bilateral prostatic hypertrophy with enlargement as well as elevation of the posterior fold of the bladder outlet caused by hypertrophy of the posterior commissure in the form of a middle lobe (see Fig. 166). In this form, the enlargement, per rectum, is more symmetrical, there is generalized lateral enlargement and the growth

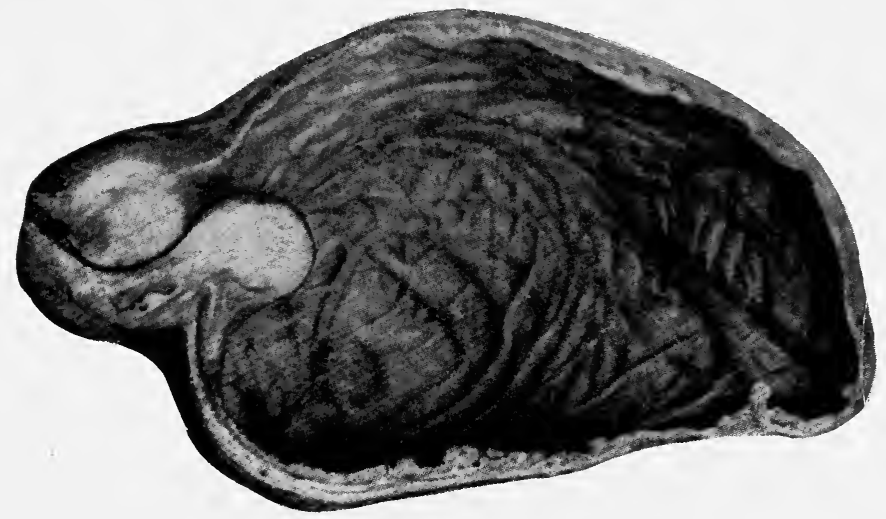

FIG. 171.-Prostatic hypertrophy obstruction intravesical. (Middle lobe.) (Aschoff's Pathology.)

of the posterior commissure is distinguished posteriorly and above at the site of the floor of the bladder. Cystoscopy is still more difficult to accomplish than in the previous type and, if effected, the presence of the middle lobe is recognized with a still deeper basfond behind it.

(4) In this class, median enlargement alone is the main feature of the growth and may occur as a pedunculated, lobular mass extending from the posterior isthmus (Fig. 171) or as a broad, transverse bar (Fig. 172) or as a tongue-like projection from the median isthmus, an exaggeration of the latter type of median lobe which covers the orifice of the bladder much like the glottis in the pharynx (Fig. 173). Very little rectal protrusion is distinguished in this class of prostatic enlargement although there is always some and it is principally felt in the region of the floor of the bladder. Cystoscopic 
examination is sometimes difficult and causes sharp reaction in these cases except where the bar is slight or there is a small pedunculated lobe which may readily be seen and outlined.

Neuralgic symptoms are more likely to occur with malignant growths of the prostate than with benign hypertrophy.

Retention of urine brought about by other causes than prostatic enlargement may be due to contracture of the neck of the bladder,

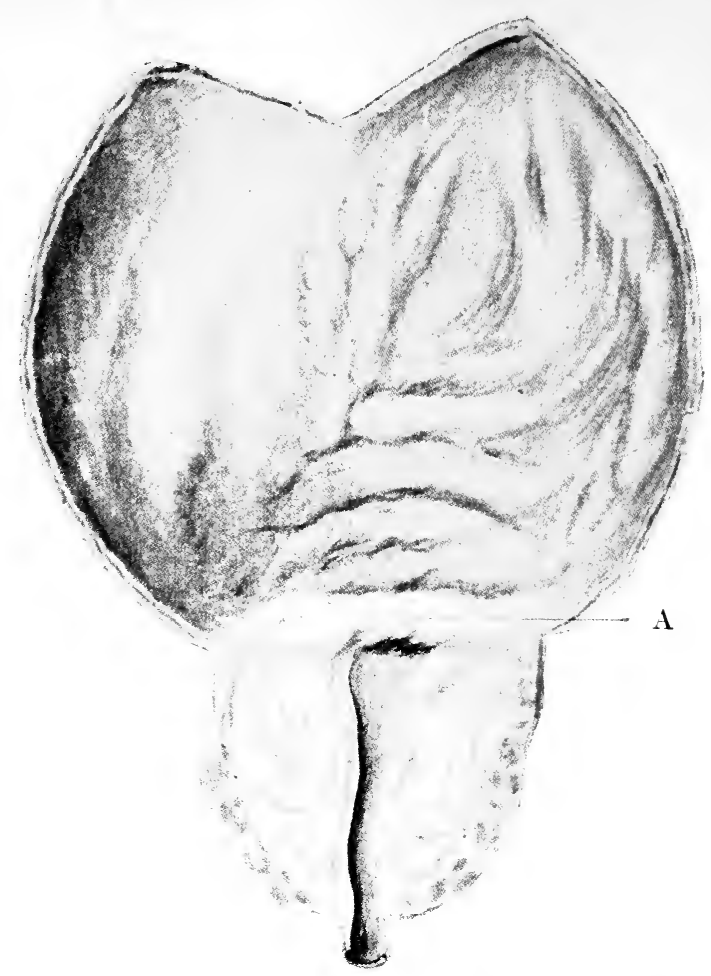

FIG. 172.-Prostatic hypert rophy with (A) bar formation. (Chetwood, International Clinics.)

stricture of the urethra, to central nerve lesions, and, it is alleged, to other causes as well, which are not fully explained. (For description of Stricture and Contracture of the Neck of the Bladder, see pp. 252 and 435.) In such obstructive conditions, as well as with atony due to paralysis from central nerve lesions, the clinical history, the short urethra, the absence of prostatic enlargement, as determined by rectal and vesical palpation, should be the means of determining the diagnosis. 
When tabes develops late in life in combination with hypertrophy, it may be difficult to measure the extent of the one or the other of these causes of the vesical retention and a careful study of the case is necessitated.

Prognosis.-As already stated, about 35 per cent. of men past the age of fifty have more or less hypertrophy of the prostate but only 15 per cent. of these present symptoms that require treatment. Of these cases, the number is divided into various groups and in formulating an opinion or venturing a prognosis, much depends upon the particular stage or class in which the individual happens to belong. Those factors which have the most important bearing upon the out-

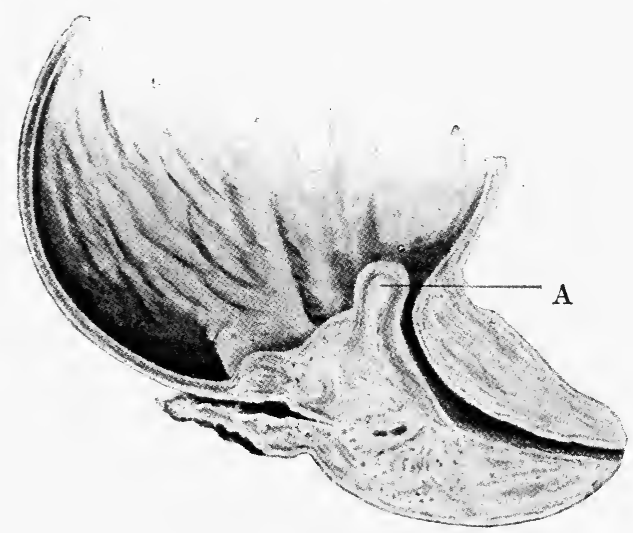

FIg. 173.- Side section of (A) middle enlargement of prostate. (Chetwood, International Clinics.)

look of a given case are whether or not it is benign or uncomplicated, how far the kidneys may have become implicated and whether or not surgical intervention is demanded.

Simple benign prostatic hypertrophy, as such, is without danger, but the malady is a progressive one and in many instances entails serious and sometimes fatal consequences. Before this period arrives, however, the condition may remain stationary over a long time. Many prostatics, under favorable conditions, live in comfort and security indefinitely, but nevertheless they are always exposed to the danger of a variety of complications. The occurrence of infection has an important bearing upon the course of the malady and also the prognosis, for when infection occurs early, the inflammation entailed thereby leads to serious disturbance of micturition and a tendency to the formation of a small and contracted bladder. In those cases in which the bladder undergoes pronounced dilatation, before the 
period of infection is ushered in, the outlook is more favorable. Moreover, the grave complication of general urinary sepsis is more likely to occur with a small and unyielding bladder than one which gradually accommodates itself by compensatory dilatation. Generally speaking then, the prognosis in a given case depends upon whether such a case is encountered during the early stage-the stage of development (before retention and infection have occurred). During this period, under palliative measures, the prospect is the most favorable. If the question of operation is considered at this time, the percentage of risk, so far as the operation is concerned, is the minimum. At this period, however, few patients are prepared to entertain the question of operative intervention.

During the next stage, when dilatation and retention have occurred, the prognosis depends on whether palliative or operative measures are adopted. In both instances the result bears a relation to the occurrence of infection and the possibility of its removal. Finally, during the last stage, when dilatation, complete retention and infection have developed, the prognosis depends entirely upon the attendant complications that may coexist, which refers more particularly to the upper urinary tract, and the depth to which inflammation may have extended in the lower urinary tract. Where these conditions have not advanced to a considerable degree, the outcome following operation may prove very satisfactory, whereas, when the upper urinary tract is seriously involved, on account of dilatation and infection (pyonephrosis), and where the bladder inflammation has extended so deeply into its structures that a thickened and contracted cavity has been produced, little more than temporary relief can be expected from either operative or palliative measures and the outlook is toward a progressive course, leading to a fatal termination.

A further consideration of the prognosis of prostatic obstruction, which is largely mechanical and necessarily surgical, must embrace a fair exposition of the end-result following operative intervention, and this must consequently be comparative; for what would seem a good result in one case might be a decidedly bad result in another. Thus a patient, both of whose kidneys are badly damaged who is compelled to rely upon the use of a catheter on account of complete or incomplete retention of urine, may have to submit to operation because of the inability to introduce the catheter; the outcome of such an operation will afford only partial relief to the patient as compared with what might be expected from an operation earlier in the course of his malady.

The development of complications, after operation, has a certain 
bearing upon the result of operation and the prognosis in that particular case. The unexpected existence of cancer may be a determining cause of death in some prostatic operations. The occurrence of septic thrombosis and pulmonary embolism, in spite of all necessary precautions and skill, may abruptly carry off an otherwise promising case. The condition of the kidneys, as already stated, has an important bearing upon the chances of the patient. Taking it all in all, the mortality following prostatectomy, whether perineal or suprapubic, cannot be considered high, has been decidedly lowered by experience and skill and at the present time may be considered as somewhere between 4 and 10 per cent., according to the stage in which the operation is performed, and certain other factors that have an important bearing, such as the age of the patient, the local conditions and the existence of complications in the upper urinary tract.

Treatment. - The methods of treatment are generally considered under two headings-palliative and operative; and inasmuch as the discussion of operation is likely to come up in nearly every case, this question resolves itself into a consideration of when to operate and when not to operate, the answer to which will demand a knowledge of what may be expected without operation in a given case and what the operation will yield in the same case if it be submitted to.

Palliative Measures. - In the first place, the patient to obtain the best from a palliative course must lead an existence that is most conducive to the success of such measures. This entails a regulation of life with the omission of all excesses, a moderate routine of eating, drinking and physical exercise. The measures adopted for the conduct of treatment under this course may be divided into hygienic, medicinal and local treatment.

Hygienic Measures. - The keynote of the hygiene of prostatic hypertrophy is the avoidance of all factors favoring congestion of the gland. This embraces excesses of all kinds, exposure to cold and wet and overtaxing of the bladder by retention of urine for a prolonged period. Some prostatics are prone to congestion of the gland as a result of chilling of the body, especially of the feet, and the first attack of retention that occurs in many individuals often follows the excessive indulgence in alcohol. When congestion occurs, rest in bed and warm hip baths with a bland diet may be the means of tiding over that period. When cystitis supervenes, the general measures suggested for this trouble are in order, including a restricted dietary preferably a milk diet, with such special medication as the activity of the symptoms calls for.

Medical Measures.-There are no remedies that have a specific 
value in hypertrophy of the prostate so far as effecting a reduction in the size of the hypertrophied gland. Symptomatic medication is called for according to the individual requirements, namely, laxatives, tonics and diuretics. The main indication during the first stage is the avoidance and reduction of congestion. To this end, ergot has been employed with moderate success. The following formula may be tried:

R. Fluid extract Ergot........... 4 c.c. (3j)

Strontium bromide........... 2 c.c. (3ss)

Peppermint water-enough to make. 100 c.c. ( (̋iij)

S. One teaspoonful three or four times a day in water.

When infection has occurred, the medicinal treatment is aimed to control the resulting cystitis. To this end, urinary antiseptics are in order, the most common of which are the preparations of hexamethylenamine, salol, benzoate of soda, and biborate of soda. A combination that suits admirably some cases as a urinary disinfectant is salol with biborate of soda, $0.32 \mathrm{gm}$. ( $5 \mathrm{gr}$.) each, three times daily. In the case of alkaline urine, benzoate of soda, $0.65 \mathrm{gm}$. or boric acid 0.2 to $0.32 \mathrm{gm}$. three times daily may prove useful, but the most effective remedy to prevent ammoniacal decomposition and to reduce the amount of pus in the urine is one of the formaldehyd preparations (urotropin, cystogen, helmitol, formine) 0.32 to $0.65 \mathrm{gm}$. (5 to 10 gr.) three or four times in twenty-four hours.

Practically speaking, it is advisable to make use of all of these preparations by alternating one with the other at different periods. In those cases in which there is an overconcentrated and acid condition of the urine, the citrate and acetate of potash will be found useful, together with the ingestion of alkaline mineral water and a reduction of the amount of sugar in the diet.

Local Measures. Catheterization.-Generally speaking, it is customary to give any patient who so desires it, the benefit of the so-called catheter life. Some patients will improve under this course while others will find that the method is a source of irritation rather than a means of relief and must be abandoned. (For description and directions as to the care of catheters, see p. 56.)

The indications for the use of catheters are to relieve the bladder of its retained or residual urine, as a means of introducing local disinfectants and for general cleansing of the bladder. For the first indication, the catheter is employed as often as may be necessary to avoid overfrequent urination and especially before retiring to obviate the necessity of rising during the night. On the whole, the use of the 
catheter is to be considered an objection to be resorted to only as the choice of evils. Vesical sepsis, which is one of the complications likely to arise during the course of prostatic hypertrophy and which has a marked bearing upon the patient's condition, the progress of his case and the direction of treatment, usually first occurs in connection with the introduction of the catheter. Therefore, the greatest possible caution should be observed when the use of the catheter is unavoidable. It is demanded for the relief of the bladder in one of the following conditions:

(1) Complete retention of urine with or without cystitis.

(2) Incomplete retention of urine with and without cystitis.

Complete Retention of Urine.-This condition occurs as a sudden development during the course of the first or second stage of prostatic hypertrophy and as a result of one of the various causes of congestion of the gland. In this case it may be relieved by the introduction of the catheter continued for several days, following which the former condition may be resumed and voluntary urination performed at the customary interval. On the other hand, a second attack of urinary stoppage may mark the beginning of chronic vesical retention. In either case, it becomes necessary to determine whether palliative or operative measures should be resorted to. If palliative measures are decided upon, the catheter must be employed at such intervals as are necessary to produce adequate comfort and eliminate the necessity of too frequent urination.

Infection and cystitis, in conjunction with complete retention of urine, exist either as an antecedent condition to the retention or come on as a result of the latter, during the course thereof, in connection with the necessary introduction of instruments. When cystitis antedates the occurrence of retention, it may be due to an old chronic prostatitis or urethro-cystitis, of early origin; and it may be due to instrumental examination or treatment. In either case, the treatment is that of chronic cystitis from any other cause (see p. 427). When infection occurs in connection with the instrumental treatment of complete retention, prompt measures of antisepsis must be conducted with a view of controlling the septic infection. These measures of treatment embrace those already described of regular catheterism followed each time with bladder disinfection with boric acid 2 per cent. and nitrate of silver one-eighth of 1 per cent. alternately, and a careful observation of all the rules of antisepsis, combined with the use of internal disinfectants (see medicinal treatment). If infection is not controlled a serious result may ensue from the extension upward to the kidney or prolonged chronic cystitis, which will have an important 
bearing upon the progress and outcome of the case, and which usually determines the decision in favor of operation.

Incomplete Retention of Urine.-During the early stages of prostatic hypertrophy the amount of residual urine is insignificant. This increases with the development of the case over a short or long period. At first it may be entirely disregarded but when the quantity reaches an amount that limits the bladder capacity and shortens the intervals between urination to an inconvenient extent, some measure of relief becomes necessary, which involves either the habitual use of the catheter or the acceptance of a surgical operation for total removal of the gland.

When infection has occurred and cystitis is active, treatment becomes a necessity, whether the residuum is small or large and then again the same question is entertained with reference to the adoption of conservative or operative measures.

If palliative measures are decided upon, the treatment of cystitis is demanded with the aim of ensuring the comfort of the patient by the relief of pain, limiting the undue frequency of urination or a too frequent demand for the catheter. In deciding the proper course to pursue in this situation, certain reasonable rules may serve for guidance:

Palliative measures should not be persisted in when they fail, after a sufficient trial, to produce and maintain an abatement of symptoms.

First infection of the bladder is not alone sufficient reason for operation unless palliative measures fail to subdue the resulting inflammatory conditions.

Recurring infection of the bladder demands operative intervention; in the case of ascending infection of the kidneys the attendant risk may be less with than without such operative course.

The selection of operation is to be discussed hereafter. Generally speaking, the results following operation are on the whole satisfactory and operative intervention is justified in a large percentage of cases; nevertheless, there are a certain number that should be left alone, notably some aged patients whose conditions do not warrant the risk of surgical interference with the peaceful lot of their few remaining years except in emergent conditions.

Operative Treatment of Prostatic Hypertrophy.-The operations commonly performed for prostatic hypertrophy and its attendant conditions may be divided into

(1) Palliative.

(a) Operations for drainage.

(b) Galvano-cautery operation (Bottini and Chetwood). 
(2) Radical.

(a) Total perineal prostatectomy.

(b) Total suprapubic prostatectomy.

Cystotomy as a palliative procedure is performed as a preliminary measure to complete prostatectomy, and is spoken of as the first stage of the two-step operation, which is the conservative operation with minimum hazard to be undertaken in cases that are classified as bad risks; cases in which the local conditions are exceedingly unfavorable, in which the advantage of preliminary drainage is incontestable. A patient whose general condition is so poor that there is grave question whether an operation of magnitude can be sustained is afforded by the simple operation of preliminary drainage, a test of his vitality and an opportunity to rally before submitting to the final and more hazardous procedure. The preliminary operation has also its local influence upon the parts to be operated upon. In the case of a highly congested gland there is likely to be produced, as a result of the preliminary drainage, a subsidence of this condition, hence hemorrhage is less liable to occur; and the duration of the second operation is minimized, but whether short or long, the freedom from shock is favorably influenced. This after-condition, which is a source of serious concern and a menace to the success of many cases, is materially lessened by the preliminary period of drainage; and the second operation is done with very much less likelihood of its occurrence, in from five to seven days after drainage has been instituted.

Therefore, in all cases of doubtful risk, where the conditions with respect to disinfection and antiseptic surroundings are not of the most satisfactory nature, where there is a question as to the patient's ability to sustain the ordeal of operation, a rapid preliminary opening, perineal or suprapubic, will serve to make matters much more favorable for both the operator and the patient for a subsequent perineal or suprapubic prostatectomy, as the case may be. In extremely severe cases, it is possible to do the preliminary procedure of drainage under local anesthesia. (See External Urethrotomy and Suprapubic Drainage, pp. 287, 460 and 501.)

Galvano-cautery Operations.--The operations of galvano-cautery prostatotomy (see pp. 477 and 498) are limited in their usefulness in prostatic hypertrophy entirely to that type which closely approximates the character of contracture or stricture of the neck of the bladder; in other words, the circular or concentric variety of prostatic hypertrophy comprising an hypertrophy of the glands of the sphincteric portion of the prostate, together with fibrous infiltration surrounding them. As already stated in another section, the 
author is strongly opposed to the Bottini technic (see p. 477) for prostatic or other obstructive conditions around the neck of the bladder. In such cases as are suitable for the galvano-cautery operation, it is always advised to follow the method of galvano-cautery prostatotomy through a perineal opening.

Other palliative operations which have had a very limited and passing vogue such as castration, vasectomy and ligation of the internal iliac arteries, are entirely abandoned.

Prostatectomy.-The indications for prostatectomy or complete removal of the gland have already been outlined in the preliminary consideration of treatment. When operation has been determined upon, and accepted by the patient, the question as to the method of operating must be decided thereupon, and the selection made between intraurethral perineal prostatectomy, extraurethral perineal prostatectomy and suprapubic prostatectomy.

There was a time when there seemed to be a tendency on the part of surgeons in general to take sides on the subject of operating upon the prostate, some believing that perineal prostatectomy was invariably the operation of choice, others that the suprapubic operation was equally to be preferred. At the present there is a truce between these disputing factions, and it is generally accepted that some cases of prostatic growth, mainly anterior to the bladder, are best approached by the low or perineal operation. Other cases of extensive intravesical projection may be reached with greater facility through the suprapubic opening, while still other intermediate types of cases may be handled as well by either method of operation as happens to suit the inclination of the surgeon. The willingness to operate by whichever route seems best suited to the existent condition is the proper state of mind for the surgeon to acquire, and to discourage too much operative fervor in favor of one method to the exclusion of another. The perineal operation is appropriate when the prostate is small and fibrous and where removal of that portion which involves the floor of the prostatic urethra will lower the vesical orifice sufficiently to make a clear right of way, and where there is not much likelihood of the remaining portion, the superior commissure, subsequently enlarging and causing renewed obstruction.

The aim of either operation is primarily toward the relief of the prostatic obstruction and incidentally, the removal of the resulting conditions of cystitis, retained urine and urinary sepsis. When these unfavorable conditions are remedied, the operation is a complete success, yet necessarily, in all cases the result after operation must be compared with the previous condition of the patient and in those 
cases in which the bladder has been clean before operation, when the kidneys are in fairiy satisfactory condition, the result naturally has been very much better than in cases with extensive and deep bladder inflammation and damaged kidneys.

On account of the diversity and different degrees of səverity it is not possible to state a fair estimate of the percentage of mortality or the end results in a miscellaneous collection of cases without a proper analysis of each one. So far as the residual urine, and voluntary urination are concerned in the large majority of cases, when the prostatic obstruction is removed completely, whether it be by the perineal or suprapubic route, the functionating power of the bladder is returned. Inflammation, however, may remain for an indefinite period in longstanding cases, and when there has occurred permanent contracture of the bladder, a continued frequency of urination and sometimes a dysuria will persist, which is controlled to a certain extent by bladder lavage and after-treatment.

Incontinence of urine, urinary fistula and recto-urethral fistula, stone in the bladder and swollen testicle are among the complications that occur in a certain percentage of cases (in about 2 to 4 per cent. of them). Fistula and urinary dribbling are much more likely to follow the perineal than suprapubic operation, as they seldom if ever follow the suprapubic technic. Postoperative stone in the bladder and swollen testicle occur in about the same percentage after both operations, the latter almost always in those patients who have given a history of previous testicular inflammation and a chronic urethral lesion. On this account, it has been my invariable custom to perform a previous or simultaneous resection of the vas deferens in such cases. The serious objection raised to suprapubic prostatectomy has been its relatively higher mortality than the perineal operation but improvement in technic tending to shorten the duration of the operation and minimize the danger of hemorrhage has brought this operation into more favor.

The choice between intraurethral and extraurethral perineal prostatectomy is one of individual preference to a certain extent. Some prostates can be rapidly and effectually removed by a median urethrotomy, notably those which tend to encroach in that direction. Very large prostates and those with median projection which are removed perineally are perhaps better attacked by the extraurethral method as both the bladder sphincters are less liable to be invaded and the chance of incontinence is diminished thereby. (For description of Technics, see p. 479.)

The care of the patient following operation is an important factor 
in insuring a successful result. In perineal cases, it involves the same details embraced in all perineal operations. In the first twenty-four or forty-eight hours, it is a matter of properly attending to the drainage arrangements to guard against the formation and retention of blood clots and hindrance of the flow thereby. This is accomplished by continuous irrigation during the period of preliminary hemorrhage by means of a double current tube. When this difficulty has ceased, continuous irrigation is stopped and the further arrangements for drainage will depend upon the condition of the bladder. Generally speaking, the tube may be removed in three days, after which the bladder is kept clean by the insertion of an indwelling catheter and by daily washing, or by the regular reinsertion of a tube through the perineal wound or the urethra for the latter purpose.

In suprapubic cases, the requirements of after-treatment are the same as in the perineal but the problem of drainage is a more difficult one. For the first twenty-four or forty-eight hours, there is the same demand for continuous irrigation to avoid the accumulation of blood and clot formation. This is accomplished by a special drainage tube (Fig. 218, p. 488). After twenty-four or forty-eight hours, when the packing is removed, an excellent method of keeping the suprapubic wound dry and thus insuring a placid healing, is by means of an electric pump which may be attached to any outlet and accomplishes the purpose, with ordinary care (Fig. 221, p. 489). This apparatus by the action of gentle suction, through the agency of a vacuum bottle, constantly clears the bladder of the accumulated urine. With the end of the first week it is generally possible to remove the suprapubic drainage tube and insert an indwelling catheter, getting the patient out of bed as early as possible. 


\section{CHAPTER XXIII}

\section{MALADIES OF THE BLADDER}

\section{MALFORMATIONS, DIVERTICULA, INJURIES, INFLAMMA- TION, ATONY}

Congenital malformation of the bladder may occur in a partial absence of its walls or a fusion of the walls with other tissues or organs, which constitutes what is known as exstrophy of the bladder, and which may occur alone or be associated with deformities of the adjacent organs.

Exstrophy of the bladder involves the absence of the anterior wall of this viscus with a protrusion of the posterior wall through a cleft in the abdominal parietes. Thus is presented below the umbilicus and above the pubis an appearance of a turgid, rose-colored mucous membrane, not unlike a prolapsed rectum. This deformity exists in various degrees. It may be accompanied by separation of the pubic arch, there being no symphysis, and is usually associated with epispadias (see p. 281). The testicles may be ectopic or atrophied. Where there is absence of the bladder wall posteriorly, the intestine or anus may communicate with the interior of the cavity.

The cause of this malformation is arrested development during fetal life, the lateral portions of the urogenital flap, after completion, having failed to unite.

The danger in exstrophy is renal infection; the exterior parts are continually bathed in exuding urine which undergoes decomposition, and the ureteral mouths are exposed. The only means of relief are surgical, which are difficult and tedious and often require many attempts before a satisfactory result is accomplished (see Operative Surgery p. 187.) The palliative treatment of exstrophy consists in the wearing of a properly fitted urinal to protect the parts from excoriation. This has not been satisfactorily accomplished.

Total absence of the bladder may occur, usually as part of an extensive general fetal deformity resulting in the death of the infant.

So-called "multiple" bladder may be caused by a large sacculation where a misplaced ureter opens into a dilated pouch (see Diverticulum) or by the existence of a septum of tissue dividing the bladder completely or incompletely into two portions. 
Deformity of the Bladder.-The bladder may be greatly hypertrophied or atrophied. These two conditions are acquired: hypertrophy, as a result of protracted inflammation and excessive functional activity behind a mechanical obstruction, and is therefore an accompaniment of those maladies which are the cause of such obstructive conditions (see Stricture, Prostatic Hypertrophy, etc., p. 252 and 385). Atrophy of the bladder is also a secondary condition due to lesions of the central nervous system or to overdistention of the organ, when it may be secondary to hypertrophy.

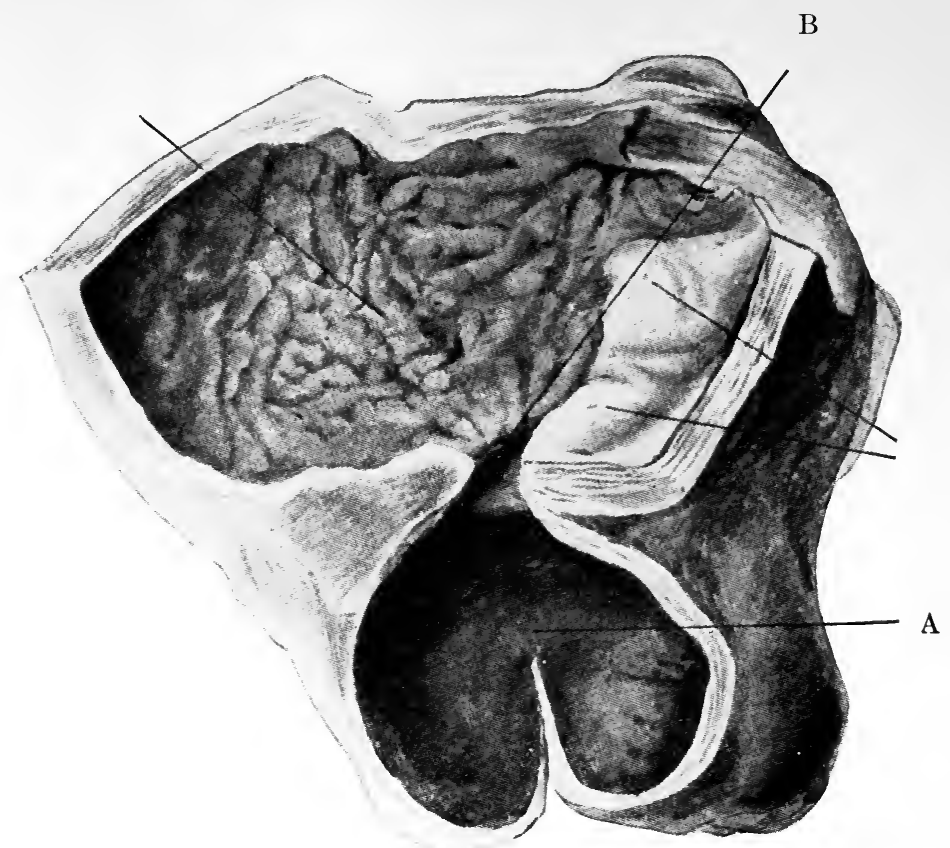

FIG. 174.-Diverticulum of the bladder. A, Cavity. B, Orifice of same. (Rumpel.)

Diverticulum of the Bladder.-Diverticula of the bladder are of two kinds-congenital and acquired. The acquired form is more common. It occurs as a result of overdistention of the inflamed organ on account of obstruction to its outlet (prostatic hypertrophy or stricture) and consists in the bulging of the wall of this viscus in the inter-trabecular spaces of any portion thereof. (See p. 134, Fig. 76, $d$ and $h$.)

The congenital form of diverticula also may be due to obstruction of the urinary flow during embryological development, and may or may not contain the ureteral orifice. If the ureter opens into a con- 
genital diverticulum of the bladder, it may be due to an embryonal displacement of the ureter connected with a dilated pouch or the ureteral opening may be included in the diverticulum by the gradual distention and enlargement thereof. All diverticula contain a mucous lining. In the acquired form, the walls are thinned by distention and the muscular covering usually absent. The congenital form is composed of the same coatings as that of the bladder cavity proper. The size of diverticula are variable. In the acquired form, there may be a number of small or one or two large ones, varying from the size of a pea to that of a lemon. The congenital kind are usually single and may be small cyst-like cavities at the ureteral outlet or be located upon the lateral or posterior walls of the bladder, where their size is without limit and imay fill the entire pelvic cavity (Figs. 174 and 175). : Any

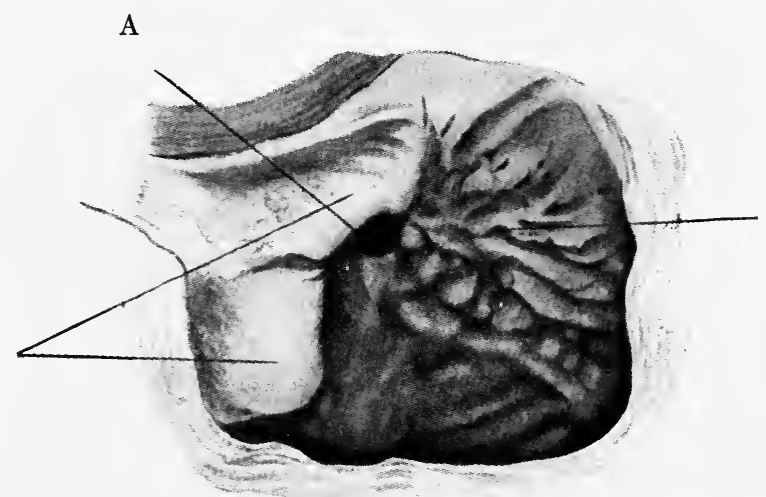

FIG. 175.-Same as Fig. 174. Showing orifice A. (Behind trigone.)

obstruction to urination naturally acts as a cause of increasing the size of these pouches, whether congenital or acquired. They are therefore more commonly found in men than in women.

Symptoms. - The symptoms of diverticulum of the bladder are those of an accompanying cystitis where infection has occurred, but such are not characteristic of the existing condition. Diverticulum may be suspected where, after a free evacuation of the bladder, there occurs in a surprisingly short period, a subsequent call to urinate and a second and almost equal evacuation of urine. With the introduction of the catheter to empty the bladder, it may be possible after apparent complete evacuation to pass the catheter into another cavity and obtain a large supply of urine. The most effective and satisfactory means of diagnosis, however, is cystoscopic examination of the interior of the bladder. 
Complete or incomplete retention of urine is likely to be an accompanying condition of diverticulum.

The treatment involves regular vesical lavage and catheterism according to the requirement for such measures. Some cases are quiescent and require no treatment whatever. Other cases, accompanied by infection, inflammation, calculi and obstruction to the bladder outlet, demand surgical intervention involving incision of the partition between the sac and the bladder, stretching of the orifice of the diverticulum, excision of the sac and suture of the orifice with or without transplantation of the ureter (see Operative Surgery, p. 469).

A small cyst or saccule of the lower end of the ureter may be treated by incision through the cystoscope; large cysts must be treated as above.

The patent urachus may lead to a cyst or diverticulum in this region at the apex of the bladder; the resulting cavity may remain as a cystic tumor or, by infection, form an abscess. In the case of a cystic tumor, it may be recognized by cystoscopy and where abscess forms, it may appear at the umbilicus.

Cystocele or hernia of the bladder signifies a protrusion of the bladder into the hernial sac. This generally occurs in the inguinal region and is not an uncommon accompaniment of inguinal hernia. It should always be suspected when an unexplained extra sac is discovered in the hernial wound at the time of operation. The bladder has been unwittingly opened in such a case and the nature of the trouble recognized by the exit of urine. In any case of doubt, it is better to introduce a steel sound through the urethra into the bladder and settle the matter so as to avoid the mistake of opening the bladder wall in the operating wound.

Hernia of the bladder may occur in any part of the abdominal wall, especially following extensive suppuration in the prevesical space. I have seen a case of this character where extensive suppuration followed an infected varicocele operation, the pus having burrowed through the prevesical space to the opposite side of the abdomen; and subsequent to the occurrence of a double inguinal hernia, a cystocele developed on one side of the abdominal wall.

Treatment.-Partial relief from this condition may be effected by a properly fitted abdominal bandage or truss. Radical cure is accomplished only by surgical procedure, as in the case of hernial protrusion elsewhere, which entails replacement and careful suturing of the overlying structures.

Injuries of the Bladder.-An injury to the bladder may be intra- or 
extraperitoneal. Such a wound may be part of an extensive injury involving other organs or may occur alone in the form of a punctured or gunshot wound, a laceration or rupture.

Symptoms.-The implication of the bladder, when a part of a general accident, is discovered during the examination by the passage of bloody urine from the urethra or the escape of urine from the exterior wound and by the location of the pain. If the bladder injury is not discovered at the time of the accident, it may be revealed later by the symptoms of cystitis or, in the case of intraperitoneal injury, by the development of peritonitis. The outcome depends upon the extent of the damage and is much more grave when the injury is intraperitoneal. Extensive suppuration caused by the conveyance of foreign material into the bladder or in the surrounding structures at the time of the injury produces a more serious constitutional condition on account of secondary inflammation and resulting fistulæ. The important symptom of bladder injury which leads to the suspicion thereof and upon which the diagnosis depends, is the appearance of blood in the urine and, when there is an exterior wound, the outflow of urine at that point is conclusive evidence of this complication. In gunshot wounds of the abdomen followed by severe shock, laparotomy is performed to trace the course of the bullet and to determine the character of the injury. The question of the bladder being injured in such a case should always be considered, and the catheter should be passed to determine the continuity of the bladder and to reveal the presence of blood.

The treatment of injuries to the bladder depends upon the character of the wound. Lacerations and punctures intra- or extraperitoneal require suture and drainage (see Operative Surgery, p. 461) much the same as rupture.

Rupture of the Bladder.- The most common form of bladder injury is that of rupture. This may be caused in connection with fracture of the bony pelvis, or by a direct blow when the organ is distended, and, in old and inflamed conditions, when the walls have become thinned by continuous straining, during an attempt at evacuation or as a result of sudden or spasmodic movement of the body. I have operated upon a case of subcutaneous rupture of the bladder in an old man with enlarged prostate and complete retention of urine which followed a suprapubic aspiration to relieve retention after a futile attempt to pass the catheter. In this case, the tear in the bladder wall was an extension of the puncture made in drawing off the urine and was due to the impaired condition of the tissues as a result of prolonged inflammation. As in the case of other injuries to the 
bladder, the important consideration in connection with rupture is the involvement of the peritoneum.

Rupture of the bladder may occur in the posterior wall, when it may be either extra- or intraperitoneal, and it may also occur at the base of the bladder behind or in front when a rupture of the urethra may also be included.

Symptoms.-There may be no noteworthy symptoms immediately after a severe injury to direct attention to the existence of rupture of the bladder unless the bladder injury is extensive; but the occurrence of blood in the urine, which is the important symptom, should raise the suspicion of its existence. Shock is always present in greater or less degree according to the gravity of the injury. Straining and ineffectual efforts to pass the water may be caused by the accumulation of blood clots in the bladder or the coexistence of rupture of the urethra. The diagnosis is confirmed by the passage of a catheter and the discovery of a large quantity of bloody urine in the bladder following a severe injury. Further exploration is made by rectal examination and abdominal palpation, which may reveal the location in one or the other of these regions of a swelling caused by blood or extravasated urine outside of the bladder.

Treatment.-Prompt surgical intervention may be the means of avoiding severe complications and of diminishing the gravity of the situation. In the case of extraperitoneal injuries or rupture, by suprapubic section and proper drainage the same condition may be instituted as exists after a suprapubic cystotomy before infection has been allowed to occur. If the case is one of intraperitoneal rupture, the tear must be located and properly sutured, after which drainage of the bladder through a suprapubic wound is provided. (The technic of operations for rupture of the bladder is described in section on Operative Surgery, see p. 471).

\section{ACUTE AND CHRONIC CYSTITIS.}

Causation.-The essential cause of cystitis is bacterial contamination but such bacterial invasion, to produce cystitis, must be in the presence of a contributing factor which renders the organ readily susceptible to microbial infection. In the commonest form of cystitis, the susceptibility thereto and the bacterial infection are derived simultaneously; and this refers to that type of cystitis which comes as a result of extension of inflammation, be that from the posterior urethra, which is the commonest of all, from the ureter or from a neighboring focus of suppuration outside of the bladder. The less 
common method of invasion is by way of the blood current or lymph stream. In this case, the predisposing and infecting agencies are derived separately. The predisposing factors in general are embraced in an enumeration of all causes of vesical irritation or congestion, namely:

Highly irritating urine due to an excess of acid or alkaline constituents; the presence in the urine of certain chemical substances in excess, such as turpentine, copaiba, cubeb, cantharides; overindulgence in alcoholic beverages, etc.

Physical strain, and anything that may produce traumatism directly or indirectly, such as violent exercise, falls and other injuries; and direct wounds, such as the unwise and careless introduction of instruments.

The microörganisms that are responsible for the production of cystitis are the gonococcus, staphylococcus, streptococcus, pneumococcus, typhoid bacillus and tubercle bacillus. (See Bacteriology of Urine, p. 101.) As already stated, the presence of the organisms must be accompanied by a favorable soil before infection occurs but the more virulent the organism and the more numerous they are, the less a coexisting predisposition is necessary, as the organisms themselves may afford the source of irritation. In the case of extension of inflammation the morbid lesion thereof follows the route of the bacteria from one area to the other; such as occurs when posterior urethritis extends from the posterior urethra into the adjoining portion of the neck of the bladder. This, morever, may be precipitated by any of the predisposing causes already mentioned.

Cystitis is divided first of all, into acute and chronic forms and is subdivided according to its specific bacterial character, namely gonorrheal, tuberculous, mixed infection.

Tuberculous cystitis is considered in the section on Tuberculosis of the Urogenital tract.

Finally, cystitis is again subdivided according to the type of inflammation manifested, acid cystitis, alkaline cystitis, membranous or fibrinous cystitis, etc.

The most common of all forms of cystitis is that which accompanies or follows inflammation of the urethra which is therefore generally directly or indirectly of gonorrheal origin and the gonorrheal organisms themselves may be present or the inflammation of the bladder may be due to a mixed infection, being an invasion, by other pathogenic organisms.

The next most common form of cystitis is that which accompanies the presence of foreign bodies in the bladder, neoplasm and 
stone, and of retained urine as the result of urethral obstruction, prostatic hypertrophy or spinal disease. Cystitis may also be caused by the presence of certain animal parasites. (See Examination of Urine.)

\section{Acute Cystitis}

Pathology of Acute Cystitis.-Preliminary to cystitis, there is congestion; then follows cellular infiltration with round cells and leucocytes which occurs beneath the epithelial lining of the mucosa and unites with epithelial proliferation, composing the inflammatory exudate. These irritative changes are due to the presence of microörganisms, the exciters of inflammation, and an effort on the part of the tissues in conjunction with an excess of serous exudation, to expel them. As the inflammation becomes more intense and spreads deeper, there is proliferation of the capillary vessels which causes distention and edema of the tissues; sometimes rupture of the small vessels produces surface ecchymoses, and ultimately necrosis and ulceration. The inflammation usually confines itself to the mucous membrane and immediate submucosa but in the more intense forms, extension into the other coats of the bladder may result, involving infiltration of the muscular layers and the deposit therein of fibrous tissue, leading, at first to a loss of distensibility of the bladder and later to secondary contracture. Finally, the inflammation in the most severe form may result in a complete exfoliation of the whole mucosa (diphtheritic cystitis); or in an extension beyond the bladder following the involvement of its entire structure, and thus is produced a pericystitis. Resolution of the inflammation, when it is confined to the mucous membrane is usually complete, and may leave no secondary effects; but when the deeper structures are involved, the repair is relatively slower and the secondary condition, like that following inflammation elsewhere in the urinary tract, namely, the formation of interstitial tissue and contraction. This contraction, in severe and extensive cases may involve the whole bladder cavity but more frequently is centred at the bladder orifice, in the neighborhood of the sphincter, in the form of stricture or contracture of the bladder outlet.

The lesions of acute gonorrheal cystitis are generally confined to the neck of the bladder and are likely to be combined with similar lesions of the posterior urethra. The term "urethro-cystitis" has been applied to this condition signifying inflammation commencing in the posterior urethra and extending into the bladder. The mucous membrane in the vicinity of the neck of the bladder becomes swollen and hyperemic in continuation with that in the prostatic urethra. Ecchymoses appear here and there and the general mucosa becomes 

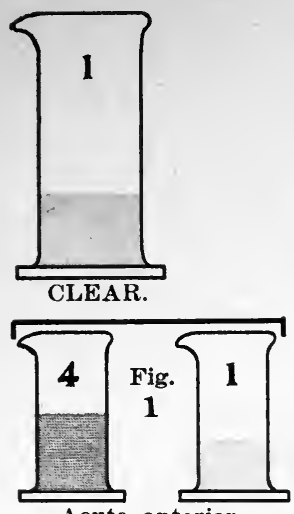

Acute anterior urethritis.

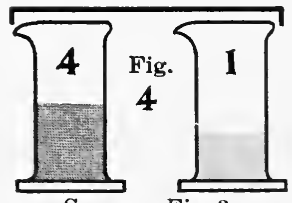

Same as Fig. 3.

After cleansing an-

terior urethra.

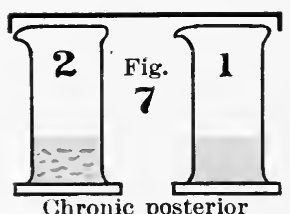

urethritis.
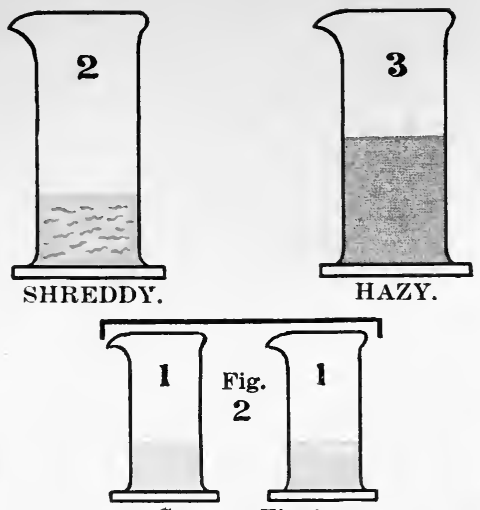

Same as Fig. 1. After cleansing anterior urethra.

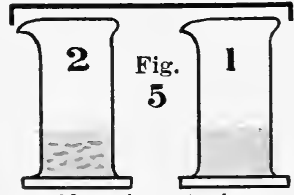

Chronic anterior urethritis.

\section{CLINICAL EXAMINATION OF URINE}
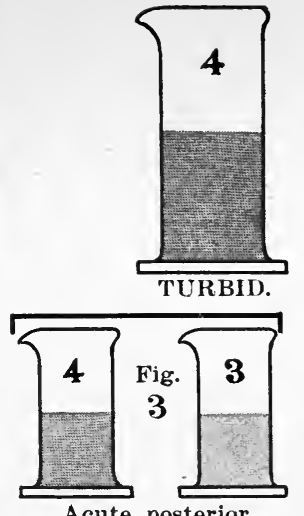

Acute posterior urethritis.

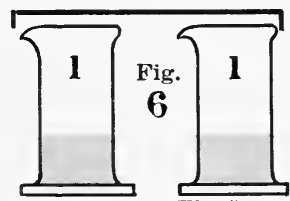

Same as Fig. 5. After cleansing anterior urethra.

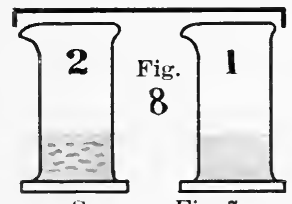

Same as Fig. 7 .

After cleansing anterior urethra.

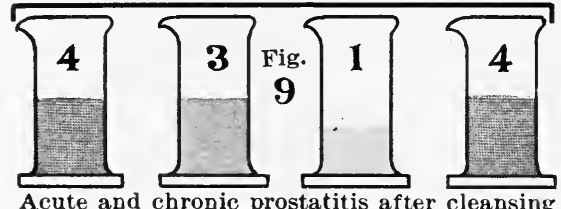

Acute and chronic prostatitis after cleansing anterior urethra. 1. (4) Posterior urethral. 2. (3) Urethro-vesical. 3. (1) Bladder. 4. (4) After prostatic massage.

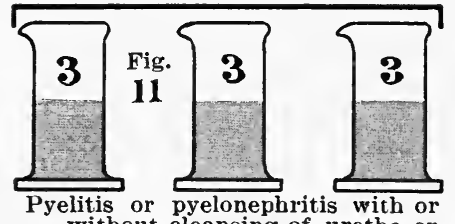

without cleansing of uretha or bladder. First. second and third flows.

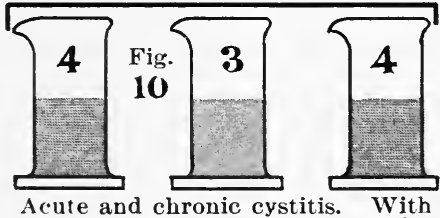
or without cleansing of anterior urethra. First, second and third flows.

Different Combinations of Urines In Test Glasses, Before and After Cleansing the Anterior Urethra. 

infiltrated with serum, the epithelium swollen and desquamated in areas, forming a portion of the purulent detritus in the urine. The gonococcus is found in the purulent sediment in some cases; in others, its presence cannot be demonstrated while many other microörganisms are found in large numbers.

Symptoms of Acute Cystitis.-The symptoms of acute cystitis are the same whether inflammation be caused by invasion with the gonococcus or other microörganisms, the difference being in the degree of intensity of the inflammation. In gonorrheal inflammation, these symptoms come on during the course of acute inflammation of the urethra, generally after the latter has been active for two or three weeks, but, like acute epididymitis, they may appear earlier as a result of any of the exciting causes which tend to carry the inflammation into the posterior urethra and the neck of the bladder. They may also occur abruptly as a result of the same causes during the course of chronic urethritis.

The characteristic train of symptoms of acute cystitis is represented by frequent and urgent desire to urinate accompanied with sharp pain and tenesmus (terminal pain) and the passage of turbid, purulent and sometimes bloody urine.

In all forms of cystitis, there exists a certain amount of urinary urgency; this becomes intense and imperative combined with pain and hematuria in the more acute condition. The blood may appear as a discoloration of the last drops of urine or be of sufficient quantity to redden the entire flow. Involuntary or spasmodic urination occurs in the very acute cases when, on account of the imperative call to urinate, the patient is unable to restrain the bladder contraction.

Complete or incomplete retention is sometimes a symptom of acute cystitis as a result of urethral spasm or swelling and edema of the tissues around the neck of the bladder (see Spasmodic Stricture).

The urine in acute cystitis is generally acid, loaded with pus and contains epithelia from the bladder and urethra and sometimes blood.

When the urine is passed in two glasses (whether or not the urethra be previously washed), both flows are turbid, the second one being as cloudy as the first and sometimes more so; when the urine is passed in three glasses, the third flow will of ten be cloudy from the presence of pus, sometimes more so (on account of settlement in the bottom of the bladder) and sometimes less (when inflammation is confined to the neck of the bladder); but it will always be turbid, which is not the case when the posterior urethra alone is involved (see Plate V-Fig. 10).

At the onset of the invasion of acute cystitis, constitutional symp- 
toms may occur in the form of febrile derangement, general depression, anorexia, etc. These symptoms generally clear up after the inflammation has continued a few days and as a result of the sero activity of the blood. The diagnosis of cystitis is made without difficulty especially accompanying the characteristic clinical history. Sharp vesical irritability due to hyperacidity of the urine is sometimes confused with it; the distinguishing feature in this case is the presence of a large amount of uric acid or urates and the absence of pus.

Uncomplicated acute cystitis is generally amenable to treatment and gets well in a period varying from a week in ordinary cases to several weeks in severe ones. The prolonged cases are usually due to gonorrhea following which chronic cystitis often persists with more or less fibrotic thickening of the urethral orifice.

Treatment of Acute Cystitis.- The treatment is general, internal and local.

General treatment involves confinement to bed upon a restricted and unstimulating diet, the application of heat to the suprapubic and perineal regions and careful regulation of the bowels. Such articles of food and drink which are enumerated in the dietetic section on the treatment of gonorrhea (see p. 207) are equally to be avoided in this condition. An exclusively milk diet has special merit.

The medicinal treatment comprises the use of anodynes, and alkaline diluents of all kinds. Anodynes hold the first rank; the frequency of urination must be controlled and vesical spasm overcome. The following combination affords relief in mild cases:

R. Tincture of hyoscyamus.......... 25 c.c

Liquor potassa or potassium citrate...... 10 c.c.

Syrup acacia enough to make.......... 100 c.c.

M. Teaspoonful every 4 to 6 hours.

To this may be added, or taken alone in capsule form, one of the internal antiseptic oils, namely: the oil of sandalwood or the oil of gaultheria, 1 c.c. (mxv) t.i.d.; or one of the sandalwood compounds or derivatives, furnished in proprietary form, may be substituted. When such sedative measures fail to control urinary frequency, a positive anodyne must be employed, in which case 3 to $6 \mathrm{cg}$. (about $1 / 2$ to $1 \mathrm{gr}$.) of the extract of opium in suppository form, with 2 to $3 \mathrm{cg}$. (1/3 to $1 / 2$ gr.) of belladonna extract may be used, and repeated as often as necessary to modify the frequency of urination.

Codein internally in tablet triturate $3 \mathrm{cg}$. (1/2 gr.) every three to four hours may be used for the same purpose.

For a liquid preparation: R. Tincture opium (McMunn), 25, 
cascara aromatic 30 to 50, Elixir lactopeptin, enough to make 100 c.c. -1 teaspoonful three or four times a day.

By persistence in such means, especially under the favorable conditions of body rest, the pain, tenesmus and frequency of urination should subside, and as this occurs, in urethral cases, a discharge begins to reappear at the meatus, upon the return of which the addition of the time honored remedy of copaiba is indicated, on account of the beneficial influence this drug sometimes exercises upon the bladder in postgonorrheal inflammation. It may be given in $1 / 2$ to 1 c.c. ( $m$ vii-xv) doses t.i.d.

In all cases of cystitis, alkalies and diluent mineral waters are indicated when the urine in strongly acid and of high specific gravity. Hyperalkalinity is to be avoided as well as hyperacidity.

Internal disinfectants are of assistance, notably in alkaline conditions. Salol is given alone or in combination with the balsamics in the declining stage, 3 to $6 \mathrm{gm}$. (gr. $\mathrm{v}-\mathrm{x}$ ) three times daily. See formulæ, p. 208.

Methylene blue is inferior as a disinfectant and has the disadvantage of coloring the urine. It seems to suit some cases, however, and is given in pill or capsule form .06 to $.13 \mathrm{gm}$. ( 1 to $2 \mathrm{gr}$.) three times a day. Special formulæ combined with balsamics are also to be had.

Hexamethylenamine, the ammonia salt of formaldehyd (urotropin, cystogen, helmitol, formin, etc.), is a most valuable urinary disinfectant. It is especially effective in alkaline and strongly bacterial urine. It is therefore more often used in the chronic form of cystitis: 2 to $4 \mathrm{gm}$. (30 to $60 \mathrm{gr}$.) in 24 hours in divided doses.

Vaccines.-The method of reaching microbial invasions by the use of vaccines is applied to this, as it is to almost every portion of the urogenital tract. Specific (gonorrheal), autogenous and polyvalent vaccines are used. (See Chapter on Serotherapy).

Local Treatment.-During the acute stage of eystitis, it is usually better to depend upon the palliative measures and the internal treatment already detailed. Local measures are generally to be interdicted during this period as they are liable to increase the irritation rather than to allay it. Exception may be made in some cases of gonorrheal cystitis right at the inception thereof, in which instances the local treatment is similar to that employed in posterior urethritis $(q . v$.) the prompt application of which may be of value, but when unwisely or carelessly adopted may prove a distinct irritant. In other cases, the local treatment is reserved until the subacute or chronic stage supervenes, for a description of which see Local Treatment of Chronic Urethritis. 


\section{Chronic Cystitis}

When cystitis has reached a stationary period and is prolonged thereafter, it may be regarded as in the chronic stage. This stage usually follows a preëxisting acute phase, and is therefore accompanied by the continued stay in the bladder of pyogenic microörganisms.

Pathology of Chronic Cystitis.-The pathological changes more frequently involve the deeper structures than in the acute stage. The same characteristics are presented as are found in the acute condition, namely, cellular infiltration, submucous engorgement and vascular proliferation, with the addition of an increase of connective tissue in circumscribed areas or in more diffused formation. Ulcerations occur upon the surface as a result of necrotic changes, upon which may be seen phosphatic incrustations, and cicatricial areas denoting exfoliation and repair, with a secondary deposit of new fibrous tissue. Here and there may be viewed leukoplakia, or irregular patches upon the surface of the mucosa, in which epithelial degeneration and transformation into that of the thick pavement variety has occurred, giving the appearance of a bleached surface, irregularly outlined by pinkish margins of the unaltered portions. In-advanced cases, on account of the reticulated arrangement of the muscular coats of the bladder, the irregular thickening of the muscular coat and the thinning of the mucous membrane between the bands of connective tissue, forms what is known as trabeculation, a series of bridges with intervening cavities. These may occur uniformly over the entire bladder or, where inflammation has been circumscribed at one point, a large cavity may form in that particular region. These cavities are in the nature of diverticula. They may harbor infected urine and favor the deposit of calcareous material and, by such retention, develop secondary stone of various sizes and number.

In long-standing cases the wall of the bladder may eventually become enormously thick and perivesical proliferation may also be evident.

Symptoms of Chronic Cystitis.-The symptoms are the same as in the acute form but of much milder degree, and, as time goes on, they tend gradually to diminish in activity. When the structural changes already described produce a permanent alteration of the musculature there exists more or less retained urine or a permanently contracted bladder, according as these changes involve the neck of the bladder alone or the entire cavity. The retained urine is purulent and bacterial; it may be acid (Fig. 176) or, when ammoniacal decom- 
position sets in, it is strongly alkaline, and contains the crystals of ammonio magnesium phosphate (Fig. 177). Cases of chronic cystitis are always subject to acute exacerbation. The systemic symptoms are very much less in evidence in chronic than in acute cystitis, save in cases with chronic retention of ammoniacal and decomposed urine when there may exist a low grade of urinary toxemia.

Treatment of Chronic Cystitis.-While the treatment of acute cystitis is largely general and medicinal, that of chronic inflammation

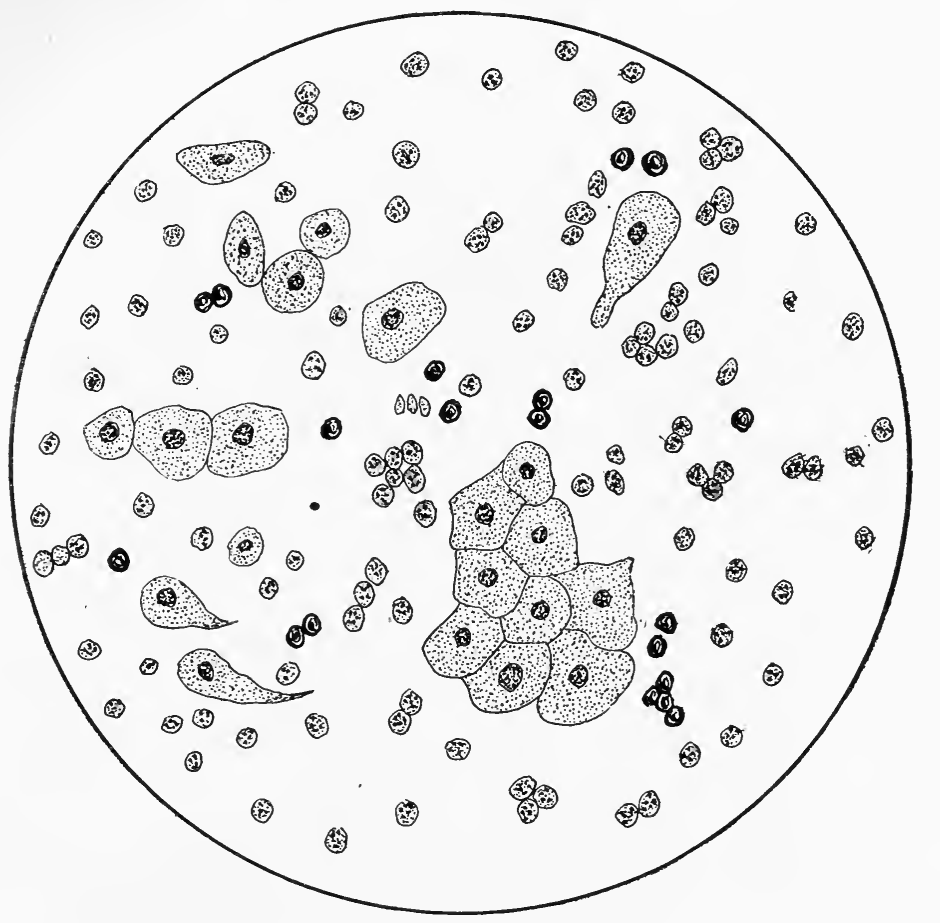

FIG. 176.-Chronic cystitis. (Acid.)

is for the most part local. There is not the same necessity of enforced rest and restricted diet except with respect to the use of alcoholic beverages and undue physical exertion.

As far as medicinal measures are concerned, anodynes are not usually required. The balsamic oils may be given in the form of sandalwood or wintergreen as suggested in acute cystitis and posterior urethritis, when symptoms of irritation exist. Copaiba and cubebs while formerly given in protracted forms of inflammation are seldom employed at present. 
Of greater importance in chronic cystitis is the use of the internal disinfectants, notably salol or one of the formaldehyd preparations (urotropin, cystogen, helmitol, formin). The former may be given in 0.3 or $0.6 \mathrm{gm}$. doses three times daily, alone or in combination with the balsamics (capsule salol compound, capsule salol and santal compound) or it may be combined with an equal amount of biborate of soda in powder form or with the benzoate of soda. One or the other of these combinations may be used interchangeably according to the

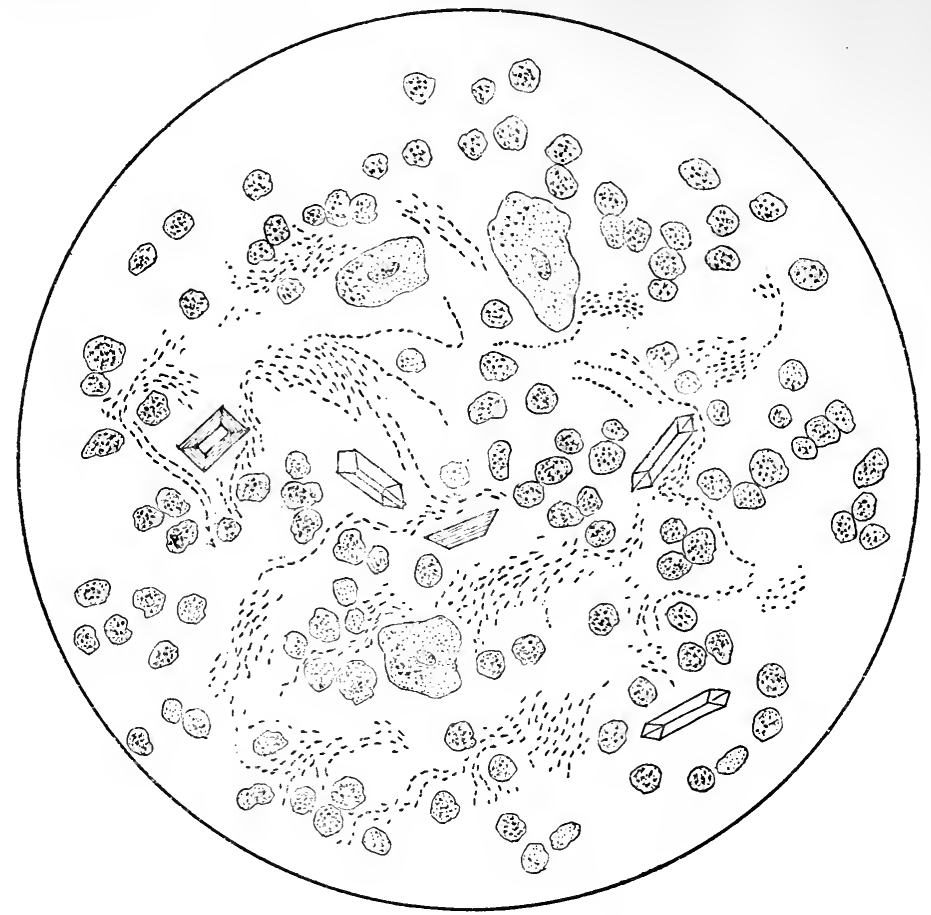

FIG. 177.-Chronic eystitis. (Alkaline.)

effect produced. The salol and biborate of soda seems to suit well some of the old chronic cases with retention but inasmuch as the stomach may be sooner or later affected, it is well to substitute one of the preparations named above of hexamethylenamine. This last internal disinfectant is especially useful in the alkaline forms of chronic cystitis and may be given in doses of from $0.3 \mathrm{gm}$. ( $5 \mathrm{gr}$.) up. An average dose is $0.5 \mathrm{gm}$. (7 $1 / 2 \mathrm{gr}$.) three times daily taken with one-half tumblerful of water. In some cases this remedy has been pushed to the full point of tolerance: 3.0 to $6.0 \mathrm{gm}$. $(45$ to $60 \mathrm{gr}$.) 
daily, with reputed beneficial results but generally speaking, the average dose is employed.

Local Treatment.-The local treatment of chronic cystitis is of prime importance, and generally speaking the medicinal agent and the quantity used should be fitted to the individual peculiarity of different cases. Some cases of mild degree seem to be irritated by strong local applications, but are benefited by irrigation with plain hot normal salt solution or 2 or 3 per cent. boracic acid. Other cases are favorably treated with irrigations of permanganate of potassium employed in the same manner as in antero-posterior urethritis (see p. 40). The favorable effect of permanganate of potassium when it occurs is usually prompt in action and if such is not the case, it should be discontinued for some other preparation. Solutions of nitrate of silver often prove more efficacious than any other remedial agent in the treatment of chronic cystitis. This applies especially to the alkaline forms. The strength of the solution may be varied from 0.06 to $0.25 \mathrm{gm}$. to a litre (about 1 to 16,000 to 1 to 4000 ) according to the tolerance of the bladder thereto. This should be used in the same manner as permanganate solutions being injected into the bladder through the posterior urethra, in order to reach the latter region with the treatment (see p. 40). If there exists a certain amount of anterior discharge, it is proper to irrigate the anterior urethra before attempting posterior irrigation but if nitrate of silver is employed, some other solution than this agent should be used for the anterior urethra on account of its strongly astringent effect which will interfere with the later introduction of the catheter.

The organic silver compounds, argyrol, protargol, etc., will be found to be valuable topical agents for direct application to the bladder in cases of chronic cystitis. The selection of one or the other of these preparations depends to a certain extent upon the chronicity of the condition. Argyrol is less irritating and generally used in the less chronic form of inflammation in which the cystitis is confined more to the region around the neck of the bladder. In such instances, it is not necessary to use so copious a quantity of the solution and it may be introduced by means of the 100 c.c. syringe with a soft rubber or woven catheter, following a preliminary cleansing with a mild boric or saline solution of the cavity, so as to free it of its purulent contents. The catheter is then slightly withdrawn into the posterior urethra and from 25 to 100 c.c. of 5 to 10 per cent. argyrol solution are introduced, and allowed to remain until it is necessary to urinate. This treatment is conducted daily or every second day according to the absence of 
irritation therefrom, and the resulting benefit. It is usually very effective and especially in cases of postgonorrheal cystitis.

Protargol is somewhat more irritating than the previous preparation and is found useful in the more chronic forms of cystitis. Solutions of from .75 to $2.0 \mathrm{gm}$. (12 to $30 \mathrm{gr}$.) to 500 c.c. are useful, the total capacity of the bladder being injected and allowed to remain for a few minutes, or a portion of the amount is allowed to remain until the next urination. This solution is useful in both the gonorrheal and non-gonorrheal forms of cystitis.

Bichloride of mercury is sometimes found helpful as an irrigation in the chronic forms of cystitis in solutions of 1 to 20,000 to 1 to 16,000 . The method of Guyon of using this agent as an instillation in tuberculous cystitis is also valuable in other forms of indolent, chroniccystitis. The usual manner of giving such instillations (see p. 669) consists of injecting into the neck of the bladder about 2 c.c. ( $\mathrm{mxxx}$ ) of the solution commencing with 1 to 16,000 and working up to about 1 to 4000, at intervals of two to four days, according to the effect produced and the absence of reaction.

When after a certain period, the cystitis has yielded to treatment and examination of the test glasses shows that the condition is concentrated in the posterior urethra, bladder irrigations may be discontinued, and treatment by instillation adopted as described under the treatment of chronic urethritis.

The bisulphate of quinine is regarded by some as a useful agent in the treatment of chronic posterior urethritis and cystitis. Its action is certainly not unfailing; it may, however, be employed with some benefit alternately with one of the silver preparations where the added astringent effect may be needed. In such cases, it is employed in the proportion of 1 to 6000 to 1 to 3000 as an irrigation.

In all cases of chronic cystitis, the antecedent cause is to be determined, inasmuch as the persistence of such cause may be accountable for the bladder inflammation, which applies to the existence of stricture chronic prostatitis, chronic seminal vesiculitis and, in non-gonorrheal cases, prostatic hypertrophy, vesical neoplasm or stone. Such concomitant conditions must be sought out and appropriately treated, as suggested under their respective headings. When, after continued local treatment, chronic cystitis persists, perineal cystotomy for drainage may become necessary, in which case the bladder is opened by central perineal incision, as is performed for stricture (see p. 287). Before. such operative measure is adopted, a careful cystoscopic examination should be made and the ureters catheterized, as in all cases of prolonged chronic cystitis, more important than the 
cystitis itself may be the coexistence of pyelitis, due to ascending infection.

If it is found by cystoscopic examination that there exist ulcerations or velvety granulations of the mucosa, a curettage, followed by the introduction of a drainage tube, may produce beneficial result. If a contracture and fibrosis of the vesical sphincter is discovered, relief of this condition is sought by special operative technic either through the endoscopic tube (Goldschmidt), by means of the urethra! punch (Young) or galvanocautery incision through a perineal opening (Chetwood), which may result in the removal not only of the obstructive condition but of the chronic cystitis depending upon it (see p. 477).

Prevesical Abscess.-Suppuration in the space of Retzius, or prevesical abscess, implies an infection of this anatomical area by pathogenic organisms, which may be derived from a direct injury, or from extravasation of urine following a ruptured bladder or urethra, or a suprapubic cystotomy, or an infection through the lymphatic system or blood-current. I operated upon such a case, in which there was a very large phlegmon of the space of Retzius, and in which it was discovered by perineal section that there was no connection from the urethral or vesical membranes as a source of contamination. The pus was drained suprapubically, and the urine obtained from the bladder by catheter was transparent and uninfected. The organism in this case was the bacillus pyocyaneus.

The symptoms of suppuration in the space of Retzius are generally local. The general symptoms are in inverse proportion to the resistance to the toxic absorption, and are those of general septicemia; great malaise, anorexia; and severe constitutional disturbance may be ushered in by chill. The local symptoms are those of pain and swelling and disturbance of the bladder from pressure, and perhaps partial obstruction of the urethra. If this condition occurs following a suprapubic cystotomy, prevesical abscess is then suspected, on account of the systemic disturbances, before the local symptoms are much in evidence. If the focus is unrelieved, the pus continues to accumulate, and burrows in the direction of the bladder or rectum, and possibly the peritoneum.

The diagnosis is not difficult when the condition has advanced to the extent of producing a tumor in the prevesical region, and in the absence of any bladder indications. But if there is present coexistent bladder distention, the extent of such is established by the disappearance of the swelling, after the bladder has been drained with a catheter. Following an operation upon the bladder, and before swelling has 
developed, if systemic disturbance raises the question of some purulent focus, a grooved director, passed into the prevesical space, will bring to light the nature of the trouble by the discharge of a quantity of pus.

Treatment.-The treatment is based upon recognized surgical principles, and requires the opening of the cavity, free cleansing and drainage. Sometimes it will be desirable to make a counter-opening through the perineum, to obtain dependent drainage. Any concomitant condition that exists, such as a cystitis, calls for appropriate treatment (see p. 427). For severe constitutional disturbance, due to systemic absorption, the pyogenic bacterial vaccines are indicated.

\section{Atony of the Bladder.}

Vesical atony signifies a loss of muscular tone. The causes for this condition are divided into two sets, namely: central and peripheral, both of which exercise their influence upon the functional action of the organ by impairment of its nerve energy. The central causes more properly belong to the department of neurology but it is necessary that the urologist should be aware of the nature and character of such central lesions as the cause of vesical atony in order to avoid diagnostic error. The neurological causes, generally speaking, are sclerosis of the posterior columns of the spinal cord (tabes) meningomyelitis and injuries to the spinal cord involving fracture, dislocation, or distortion of the vertebral column. Other neuropathic agencies are assigned to disease of the bladder nerves and ganglia.

The local causes of atony are varied. Generally speaking, they comprise any mechanical interference with the normal muscular contractility of the vesical cavity. These mechanical causes are obstructive and inflammatory.

Obstructive Causes of Atony.--In this class belong all obstructions to the bladder outflow, namely: malignant or benign growths of the prostate, stricture of the urethra and obstructing tumors. In consequence of the futile attempt of the bladder muscle to fulfill its function of emptying the cavity, being compelled to work against a firm resistance and to suffer continuous overdistention, it eventually loses tone and contractility. Previous to this state, there may be an intermediate one of hypertrophy and the sclerosis which ultimately results may be muscular, arterial or general. Be that as it may, experience teaches that atony of the bladder supervenes upon prolonged mechanical obstruction of its outlet. 
The inflammatory cause of atony is of infectious origin and is a protracted local inflammation of the bladder of sufficient depth to involve the submucous and muscular structures which leads to the deposit of fibrous tissue encroaching upon the muscular coat. This condition, like that produced by obstruction of the bladder outlet, is a sclerosis and also mechanical in its interference with the contraction of the muscular coat. Its action, however, is direct, while that of obstruction is indirect.

There are a number of cases reported of atony of the bladder assigned to other causes than that of obstruction or organic nervous diseases. Such causes have been variously mentioned as arteriosclerosis, disturbance in the circulation of the vesico-prostatic plexus, etc. It is possible that a more exact examination of the functional mechanism of the vesico-prostatic outlet than was possible by cystoscopic observation in some of these cases might reveal some slight valvular abnormality to account for the condition while others may belong to the category of diseases of the central nervous system in spite of the inability to obtain evidence to support such diagnosis.

Those cases that are unmistakably outside of either of these divisions are not as yet fully explained.

Diagnosis.-Atony of the bladder is easily recognized, and selfevident, when, upon the introduction of the catheter to relieve complete or incomplete retention of urine, it is observed that the flow of urine, instead of coming with its normal force, is slow, and the outflow is not projected from the end of the catheter, but drops in a rectangu. lar stream. If any urine is passed voluntarily, the quantity is small, and the same lack of force in the stream is noticed as when the catheter is passed. The absence of normal muscular contractility is further noted from the necessity of compressing the lower abdominal wall to evacuate the bladder contents, by forced muscular contraction of the abdominal muscles in the case of voluntary urination, and by pressure of the hand of the operator, when the urine is removed by catheter. Of greater difficulty, and more importance, is the discovery of the cause of the existing atony, before attempting any means of relief, as such means often involve a surgical procedure, and it is important to know beforehand whether the cause of the trouble is of central (neuropathic) or peripheral (local) origin.

The presence of a central cause for atony is recognized by the attendant conditions that accompany it; in other words, those evidences that leave no doubt as to the existence of a spinal lesion, and those signs which suggest the probability of such. In this connection it should be remembered that bladder manifestations may be the 
very first signs of tabes dorsalis, and are often confounded, when they occur in individuals of mature age, with prostatism. Bladder atony occurring with other neuropathic conditions would naturally be ascribed to the nervous lesion. The possibility of nerve conditions coming on insidiously should be kept in mind, and, therefore, in reaching a diagnosis they should be eliminated by testing the general and pupillary reflexes, the intraocular conditions and the peripheral sensory reaction. Examination of the blood and spinal fluid by sero-diagnosis is essential; a test course of mercury and potash in unexplained cases is sometimes employed but is an indirect and unscientific mode of procedure.

The existence of mechanical obstruction of the neck of the bladder, in the form of a tumor, enlargement of the prostate, or stricture, furnishes ample explanation of atony. The absence of such obstructive lesion necessitates the discovery of some other local cause, which, as already stated, might be chronic interstitial inflammation.

Treatment.-Adoption of treatment for atony naturally depends upon the diagnosis and the conditions that exist in the bladder. In the case of chronic spinal lesions, requiring the continuous use of the catheter, the treatment may be that of an attendant cystitis, which involves the use of internal disinfectants and bladder washing (see p. 427). In obstructive conditions, the treatment is that of the obstruction itself; and, usually speaking, with rare exceptions, a complete removal of this obstruction leads to the ultimate relief of the atony. (See Stricture, p. 268; Contracture of the Neck of the Bladder, p. 435.) Where there coexists one of the other causes of atony, namely: a concomitant spinal lesion or an interstitial hyperplastic inflammation of the bladder, with arterial, muscular or nerve sclerosis, in spite of such coexistence, it may be deemed expedient to remove the obstructive cause as a sine qua non to successful treatment, even though such success, comparatively speaking, is of a much lesser degree than is the case in uncomplicated obstructions.

The interstitial form of atony-that which follows prolonged and deep inflammation-may yield to a certain extent to treatment, by bladder distention, lavage, and abdominal massage, but such local measures are limited in their effect when the chronic inflammation of the bladder is of very long duration. If there still exists in the situation an active bacterial agency, specific or mixed in character, resort to bacterial vaccines is in order (see Chapter V, Vaccines and Serums, p. 110). The advisability of surgical drainage in old cases of chronic inflammation would naturally be considered in some of the cases of this character (see Chronic Cystitis, p. 430). 


\section{Contracture of the Neck of the Bladder}

Contracture of the neck of the bladder is a common sequel of chronic cystitis and is a stenosis of the sphincteric orifice caused by inflammatory infiltration and contraction. This same malady has been described under different denominations by various writers who, while not entirely in accord, refer to the same condition or to different phases of it. ${ }^{1}$

Causation.-The cause of this pathological condition is found in long standing chronic inflammation centered in the region of the

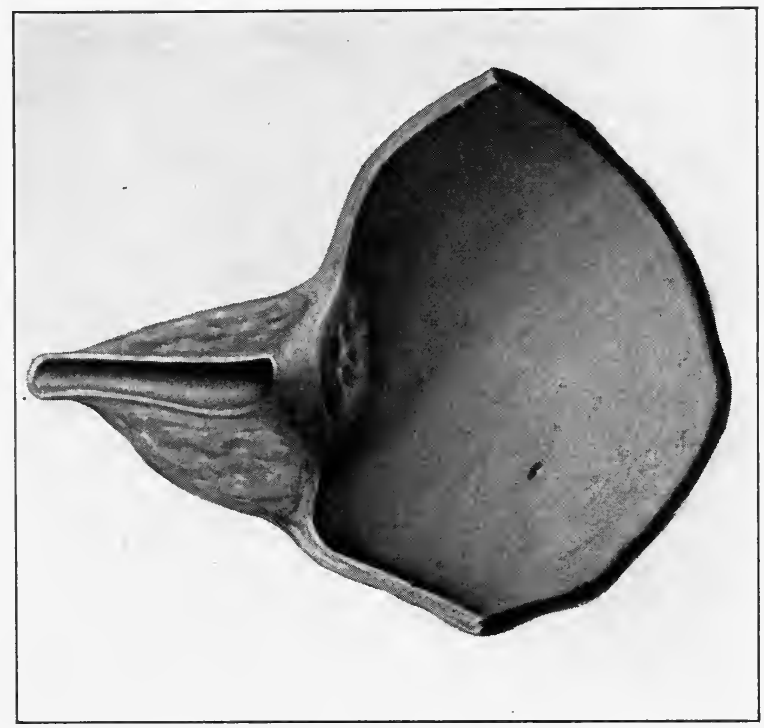

FIG. 178.-Contracture and elevation of vesical outlet.

trigone (behind) or in the prostatic urethra (in front). It is commonly but not necessarily of gonorrheal origin. A prostatic abscess, stone in the bladder, traumatism or any of the conditions involving

\footnotetext{
${ }^{1}$ Sokell (De la Contracture douleureuse du col de la Vessie) in 1874, described a condition which bears a close resemblance to the malady in question.

Civiale, (Traite practique, Vol. II, p. 244) refers to a simple urethro-vesical fold of the mucous membrane in some cases and in others a dense barrier containing fibrous or muscular tissue.

Guthrie (Diseases of the Bladder and Urethra, 1836) mentions chronic thickening of the neck of the bladder.

Mercier (Researches du le traitement des maladies des organs urinaires, Paris, 1856) speaks of the existence of the urethro-vesical barrier produced by spasm from prolonged irritation.

Von Frisch (Die Krankheiten der Prostata, 1899) referred to long standing cases of spasm of the sphincter resulting in inability to empty the bladder and residual urine amounting to several hundred cubic centimeters.

Sir Henry Thompson (Diseases of the Prostate) recognizes in his writings the existence of organic obstruction of the bladder outlet apart from that caused by prostatic hypertrophy.
} 
prolonged suppuration in the region of the neck of the bladder or prostatic urethra may lead to the development of this lesion; and it is of great importance to remember that it may coexist with prostatic hypertrophy so that it becomes one of the elements producing the obstruction in the latter malady (Fig. 179).

Pathology.-The pathology of this condition, as stated under its causation, is chronic inflammation, with secondary fibrotic contraction, producing sometimes almost total occlusion with elevation of the opening above the floor of the prostatic urethra (Figs. 178 and 179). The inflammation involves the periurethral glands and is accompanied by round-celled infiltration into the tissues between the glands and invades the sphincter muscles. The mucosa becomes hypertrophied and polypoid. Infiltration may also extend into the stroma of the prostate gland.

Symptoms.-The symptoms of contracture of the neck of the bladder are analogous to those of stone in the bladder and prostatic hypertrophy, hence contracture is frequently a cause of that condition which has been designated "prostatism without enlargement of the prostate," and it is therefore one of the mechanical causes of atony of the bladder (q.v.). The predominant symptom is frequency of urination. The calls to urinate vary from half hourly to every two hours day and night. The desire is usually imperative and if not responded to promptly is accompanied by marked pain in the bladder region and spasmodic urination. Difficulty in starting the flow is noted in some cases and attacks of complete retention of urine supervene in many instances. In long-standing cases, the force of the stream is distinctly diminished while residual urine exists in varying quantities in most of the cases. In the early stages, however, when the bladder muscle undergoes compensatory hypertrophy, to overcome the resistance, the residual urine may be inappreciable but this state of affairs cannot continue as the bladder soon loses its tone and partial retention is the result. As in the case of prostatic hypertrophy, in uninfected cases, the urine may be perfectly clear. When infection occurs, it contains all the elements of chronic inflammation (see Chronic Cystitis). Examination per rectum reveals nothing characteristic of this condition although, as stated, prostatic hypertrophy may coexist. Examination by way of the urethra with a short beaked sound or searcher meets an obstruction beyond the prostatic sinus and is feit to override this barrier upon entering the bladder, after which the same obstruction may be recognized, when the beak of the instrument is turned downward toward the floor of the bladder by making traction out- 
ward. In cases of simple contracture, by rotating this instrument from side to side, an absence of prostatic enlargement can be detected. The urethral length is not elongated unless there be a coexistent median or lateral prostatic growth. With the cystourethroscope (Buerger) or the author's irrigating cystoscope, the parts around the neck of the bladder can be inspected, in which case it will be found that. there is general congestion on both the prostatic and vesical face of the orifice; there may be some swelling and congestion recognizable around the entire orifice and the elevation of the plane of the vesical outlet above that of the prostatic urethra may be visible. Inflammation is seen to extend backward around the trigone or forward

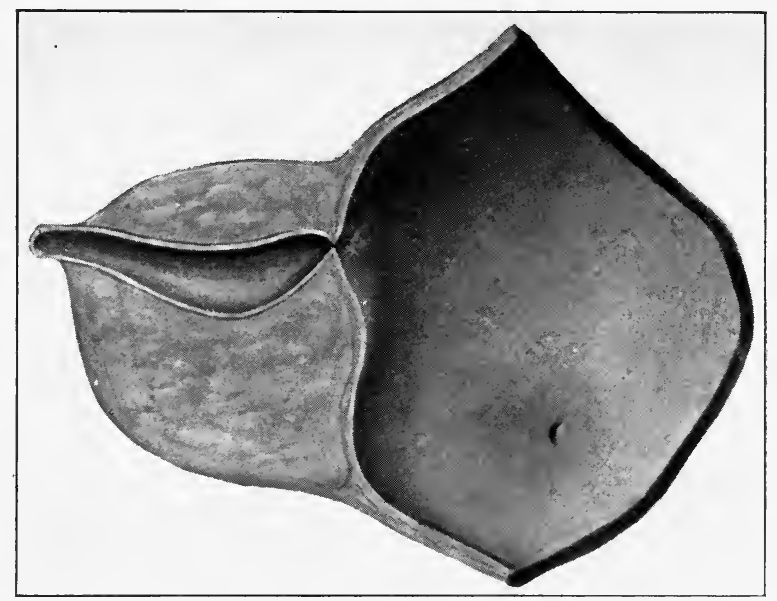

FIG. 179.-Prostatic enlargement. Obstruction caused by contracture of orifice.

into the prostatic sinus. By taking account of these clinical features, the diagnosis between this malady in individuals of advanced years and prostatic hypertrophy should be made without difficulty. It should be borne in mind that the two conditions may coexist, an important consideration in the event of operation aimed at the removal of the obstruction (Fig. 179).

Diagnosis.-Generally speaking, it may be stated tht contracture of the neck of the bladder is a frequent cause of complete retention of urine in a patient presenting a normal urethral length and a prostate of normal dimensions in the absence of tabes or other central lesions or stricture of the urethra. The Thompson Searcher (Fig. 187), may enter without difficulty or may meet obstruction at the vesical orifice and as stated above, its orificial contraction may be recognized by 
turning the beak of the instrument toward the bas-fond. A full-sized sound may enter the bladder without marked difficulty as this is the most distensible part of the canal and moderate contraction may not be detected by the passage of a sound. The absence of prostatic intravesical growth to account for the symptoms will be noted. The cystoscope will confirm the diagnosis and eliminate the question of stone or prostatic intravesical enlargement. Finally the diagnosis will be ratified, following a perineal incision, when the finger passed through the membranous and prostatic urethra reaches at the neck of the bladder an impassable orifice so tight as to barely admit the end of the finger or completely to obstruct its entrance.

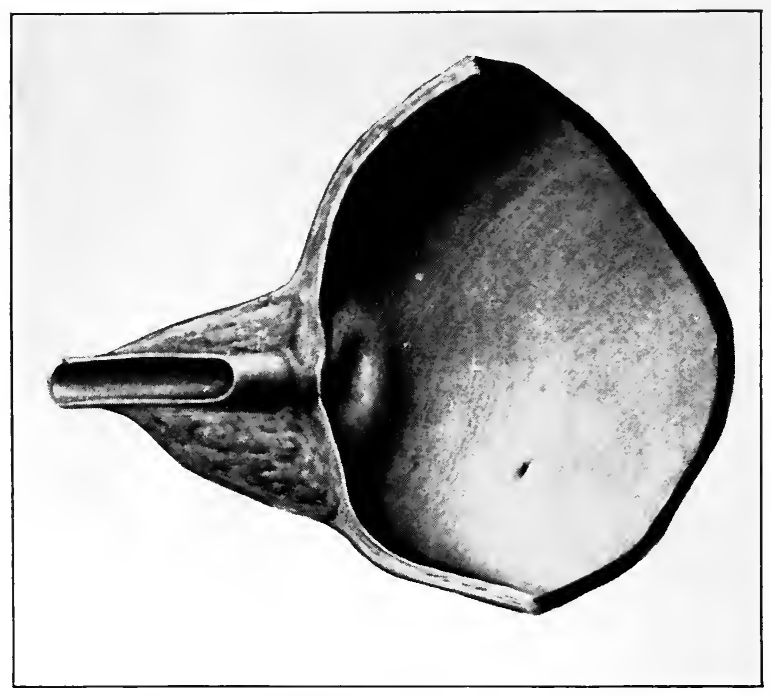

FIG. 180.-Normal bladder outlet.

In the normal subject, the vesical orifice (Fig. 180) is of sufficient dimensions to admit freely the entrance of the finger with nothing more than an elastic resistance. When, however, contracture is present the orifice may be so stenosed as to prevent the introduction of the finger entirely or so rigid as to tear if a forced entrance is made (Fig. 178).

Treatment.-The treatment of this condition, if intended to be radical, must aim at the removal of the existing obstruction. Such methods as forced dilatation and chemical cauterization have been tried and yielded at best only temporary relief. They are therefore not to be recommended upon any other basis. The only satis- 
factory surgical means involves free incision of the obstructing area down to the plane of the floor of the urethra. ${ }^{1}$

The Bottini operation is a modification of the earlier Mercier operation in which, instead of using an ordinary knife blade, the cut is performed by the galvano-caustic incision. The Bottini technic which was introduced over thirty-five years ago was intended to lessen the danger of hemorrhage attendant upon transurethral prostatotomy. That an incision made by a galvano-cautery knife is less likely to be followed by hemorrhage and more likely to leave a permanent channel is beyond question but when such an operation is conducted through the urethra it is accompanied by all the other disadvantages attending the same operation without cauterization, among which are its lack of precision, and difficulty of performance even in the hands of expert operators who have had considerable experience and good results following its employment are relatively few. In spite of this, the Bottini technic has its adherents. I believe, however, that the more satisfactory surgical means of attacking this obstacle is by a modified Bottini technic involving a perineal opening to the neck of the bladder. The application of the galvano-cautery knife is made through this opening which is also employed for examination of the obstructing area before and after incision and as a means of postoperative drainage. For description of the Galvano-Cautery Prostatotomy (see p. 477).

Following the operation of galvano-cautery prostatotomy the treatment is practically the same as that after perineal cystotomy for other conditions, such as stone in the bladder, stricture, etc. A drainage tube is kept in situ for twenty-four or forty-eight hours through which the bladder is washed twice daily with mild nitrate of silver and boric acid solution. After removal of the perineal tube, the washing of the bladder is continued by the introduction of the catheter daily, at first through the perineal wound and later through the urethra.

The following cases illustrate the malady of contracture of the neck of the bladder in its different phases and show the resemblance in the clinical history to that of vesical calculus and prostatic hypertrophy.

Patient, E. H. B., aged 39 complains of symptoms suggestive of chronic cystitis. Urination of variable frequency from every five minutes when on his feet to every three hours when quiet. He urinates at night from five to eight times. There is pain during the act of urination which is variable, being intensified by exercise. He has at times

\footnotetext{
1 Internal urethrotomy was performed long years ago under the inspiration of Mercier who was one of the first to conceive the idea of cutting down the neck of the bladder through the uretbral channel by means of a specially devised instrument.
} 
voided blood with the urine, the greatest amount having followed some unusual exertion. The trouble has been most marked in the last two months and the patient has been compelled to rise two or three times during the night for the past seven or eight years. Venereal history denied. No tuberculous family history.

The prostate is inflamed and enlarged; not very tender. The urine is turbid, purulent and slightly blood stained. Search for stone is negative. A rigid bar is felt with the Thompson Searcher at the neck of the bladder behind a long prostatic sinus. A gush of fresh blood follows the withdrawal of the searcher. The capacity of the bladder is 150 c.c., urethral length $20 \mathrm{~cm}$. (8 in.); residual urine 45 c.c.

Operation. Perineal Galvano-Prostatomy.-A marked contracture barely admitting the tip of the index-finger is felt and is incised with the galvano-cautery knife after which a perineal tube is inserted for drainage. No reaction follows and the usual after-treatment is instituted. Patient returns home at the end of three weeks. ${ }^{1}$

Patient, E. R., aged 45, has had bladder symptoms for about ten years. He urinates hourly day and night. At times he has been eompelled to rise as often as fifteen times during a night. He denies venereal history. After 300 c.c. have been passed 60 c.c. of residuum are drawn which is perfectly clear and free of inflammatory elements. The prostate is negative. No stone is found by palpation with the Thompson Searcher but an exceedingly rigid ring or collar is detected at the bladder orifice. Operation: perineal galvano-prostatotomy. Through the perineal cut a dense fibrotic ring at the neck of the bladder is felt with the index-finger which is found to be as thick as an ordinary lead-pencil. This is incised with the galvano-cautery incisor to the size of $1 \mathrm{~cm}$. A perineal tube is inserted for drainage. The patient leaves the hospital much improved, urinating from three to five times during the day and not at all during the night. No residual urine. ${ }^{2}$

\footnotetext{
${ }^{1}$ This patient is seen at the end of ten years following operation; he has remained perfectly well; the urine is clear and free of inflammatory elements and there is no pain or difficulty in urination.

2 This patient was seen over five years after the operation; the satisfactory condition following operation continued to exist.
} 


\section{CHAPTER XXIV}

\section{MALADIES OF THE BLADDER (Continued)}

\section{VESICAL CALCULUS AND NEOPLASM}

Stone in the bladder may be primary or secondary. Primary stone is formed within the bladder, whereas the nucleus of secondary stone is derived from the kidney and becomes augmented in the bladder by continuous deposit of salts from the urine. Such a nucleus, when small, may pass into the urethra and be voided during urination. Secondary stone, therefore, is primarily a renal calculus. It may occur at any age of life with a greater tendency in men than women, on account of the shortness of the urethra in the latter, which is more favorable to the expulsion of a small stone derived from the kidney. The formation and growth of stone in the bladder are favored by obstruction to the outlet such as occurs with stricture or enlargement of the prostate; by cystitis and decomposition of urine and by such predisposing chemical conditions of the urine as an excess of phosphates, uric acid or oxalate of lime salts.

Primary calculi in the bladder are essentially the result of the decomposition of urine or other cause for the concentration and precipitation of urinary salts. Therefore, the habitual usage of drinking water with large precentage of lime salts, peculiar to certain locations, may be cited as a predisposing but not an essential causative factor of primary vesical as well as renal calculi. Chronic cystitis is a constant cause of primary stone and may be a sequel of secondary calculus after the latter has been present for a sufficient period to produce irritation. Retention of urine, a damaged condition of the vesical mucosa, and bacteriuria are all strong factors in the formation of primary or secondary vesical calculus, while defective metabolism, faulty oxidation and gouty tendencies, go to make up the predisposing causes thereof.

The nucleus of secondary calculus is generally composed of oxalate of lime, or uric acid and, after remaining in the bladder a certain period, becomes progressively enlarged by the further deposit of the same salts, when the system tends to produce these elements to an excessive degree; or in obstructive conditions of the bladder, by the deposit of the salts obtained from the decomposition of the urine, the ammonio-magnesium phosphates and carbonate of lime. 
The nucleus of a primary bladder stone is likely to be formed of the products of inflammation, such as mucus, blood and bacteria. A foreign body may also act as a nucleus in primary stone. Such a foreign body may have reached the bladder from the hands of the patient or medical attendant during an attempt to dilate a stricture or with the introduction of the catheter. Calculous formations have been deposited around bits of twig, hair pins, broken ends of catheters, etc. A case of the author's, illustrating this (Fig. 181, A) shows the terminal end of the catheter forming the nucleus of a bladder stone. The medical attendant had attempted to introduce an ancient and friable rubber catheter which broke off in the deep urethra and failing in the effort to extract

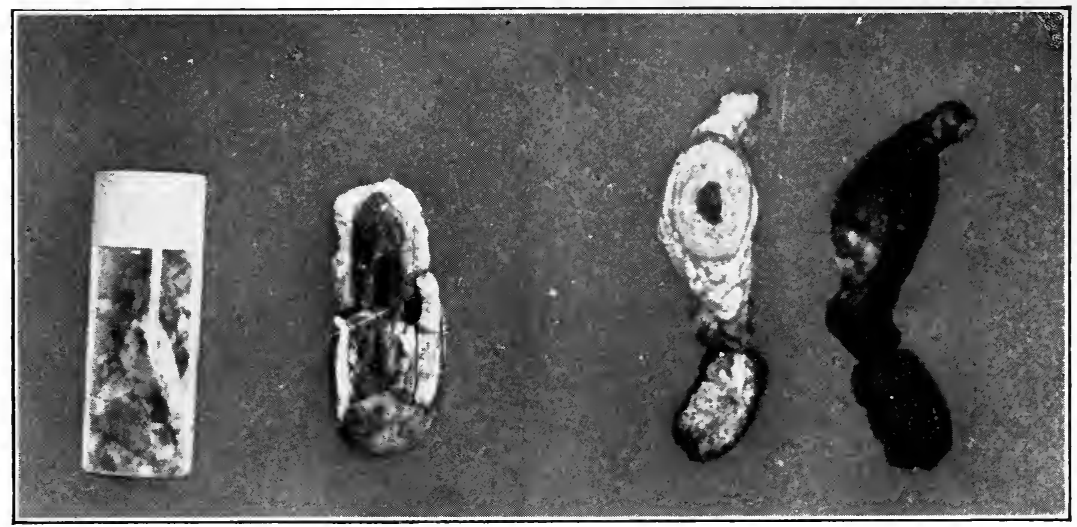

A

$\mathrm{B}$

FIG. 181.-1, specimen of vesical calculus. End of catheter as nucleus. B, Urethrovesical calculus from child.

the broken portion, it was decided to force it back into the bladder as one would treat the cork of a bottle. Three years later the stone was removed by suprapubic section.

When the nucleus of a secondary stone in the bladder is derived from the kidney and is composed of the salts of uric acid, urate of soda, a compound or mixed variety of stone is formed by the encrustation of the ammonio-magnesium phosphates. This mixed variety forms a large number of the vesical stones removed (Fig. 183).

Climate and locality seem to exercise a predisposing influence to the formation of stone, as well as custom and habits; the imbibition of acid wines which tend to promote intestinal fermentation, favors the production of the oxalate formations, and the habitual drinking of hard water which contains a large amount of lime contributes to the 
formation of calcareous deposit. In this country, stone is especially prevalent in the Southern States, and in foreign countries, England, India and China furnish frequent cases.

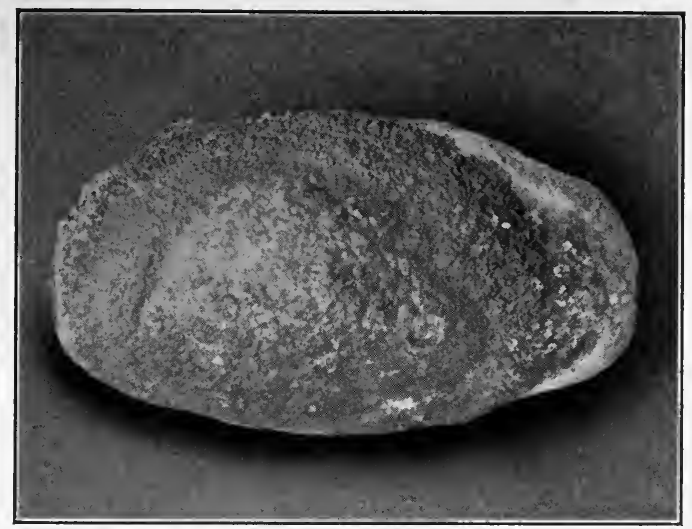

FIG. 182.-Large uric acid calculus from bladder of child, weight 360 grains. (Author's case.)

Volume.-The size may vary from that of a bit of gravel to one of considerable volume-several ounces. They may be single or multiple. Several hundred have been found in one bladder. Usually when large

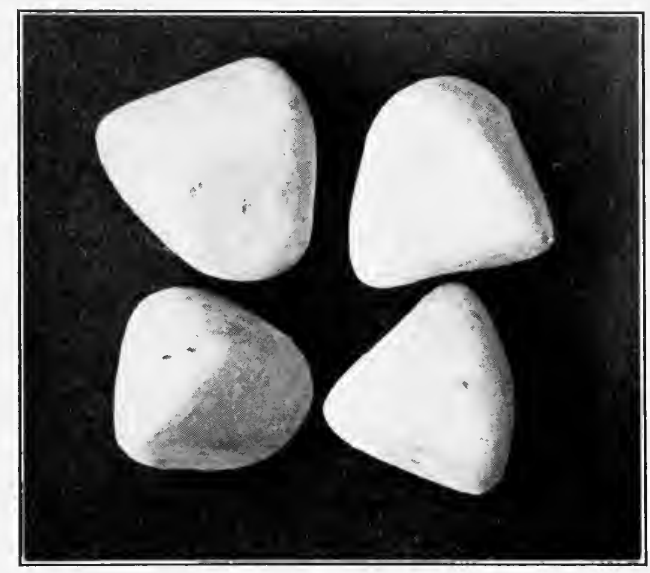

FIG. 183.- Phosphatic calculi.

in number, they are small in size. The accompanying figure illustrates an unusually large-sized stone in a youthful patient (Fig. 182). (See Roentgenogram, Fig. 94, p. 164.) 
Form and Consistence.-The uric acid stone is round, oblong, or oval, smooth and hard. The color is yellowish or red (Fig. 184). The oxalate stones are irregular and mulberry-like on the surface and exceedingly hard. The color is grayish-black (Fig. 185). The phos-

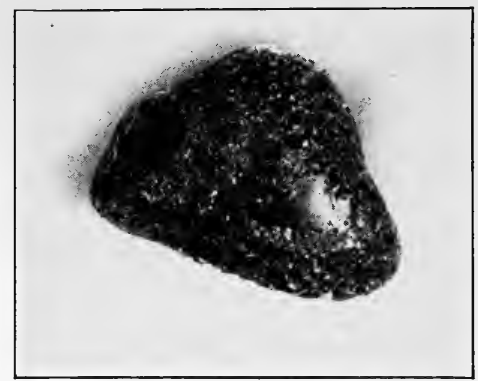

FIG. 184.-Uric acid stone.

phatic calculi are softer in consistence, sometimes flat or round and when multiple have several surfaces or facets marking the points of contact with each other (Fig. 183). When pure phosphatic, they

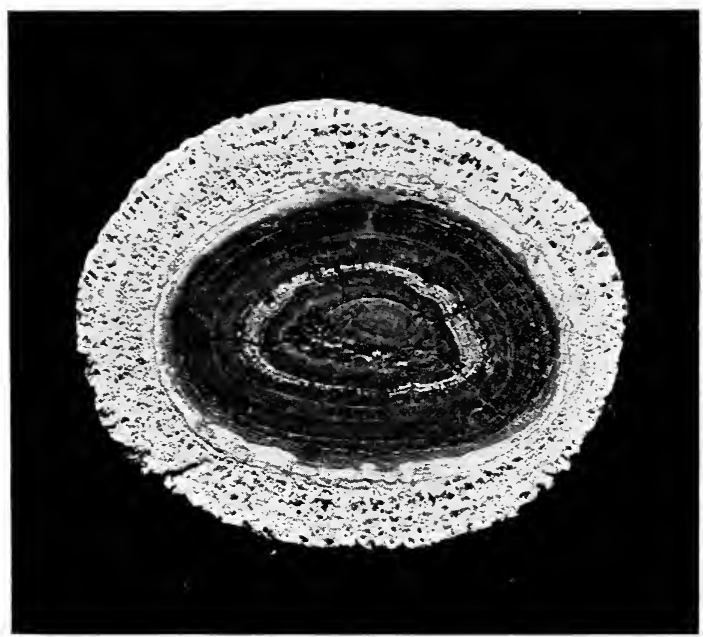

Fig. 185.-Vesical calculus. Centre calcium oxalate. Circumference, phosphates (Delafield and Prudden.)

are whitish in appearance, or whitish and reddish when mixed with urates. Cystine calculi are rare. Their surface is slightly roughened, the color is greenish and they are translucent in appearance.

Site.-Stone in the bladder is usually free and movable although it 
may be attached to the bladder wall or encysted in a pouch of the bladder. The calculus may be urethro-vesical, partly in the urethra and partly in the bladder (Fig. 181, B) or partly in the ureter and bladder. The common location, however, is in the most dependent portion of the bas-fond where it lies from gravitation and grows from continuous deposit. It may, however, rest in a diverticulum where its growth is favored by the stagnation of urine in this anomalous sacculus. Encapsulation of the stone occurs from its retention in some portion of the bladder by means of the formation of trabecular bands which fix its position at one point where it becomes attached.

Symptoms. - It is possible for the bladder to accommodate itself to the presence of stones so favorably that no marked symptoms are derived. I have seen the case of a stone in the bladder of an old man as large as a good-sized peach which did not give rise to symptoms until very late in life. This, however, is the exception, as symptoms of cystitis usually give evidence of the presence of stone early in the existence thereof. Cystitis is the natural outcome of stone in the bladder and furnishes the symptoms usually associated therewith, the symptoms of pain and hematuria predominating. Hematuria is especially significant, and is usually of the terminal variety occurring at the end of urination. The inflamed bladder wall contracts down upon the foreign body, producing the emission of blood and with it pain and tenesmus. In the quiescent stage of stone, before the symptoms of inflammation supervene, there may be only microscopic blood in the urine and a moderate amount of pain which, together with the presence in the urine of an excessive crystalline deposit, lead to the suspicion. of stone as the probable cause. When the activity of the condition increases with the accession of marked cystitis, there occur the disturbances of micturition: painful and frequent urination, arrest of the stream during passage, and sometimes retention of urine.

Painful urination is caused by pressure upon the inflamed mucosa, arrest of the stream by lodgment of the calculus in the bladder outlet during the passage of urine, and retention by the complete obstruction of the outlet. The symptoms of pain and frequency of urination are naturally increased by the movements of the body and thus, one of the diagnostic features of stone in the bladder is that these symptoms are more aggravated by body activity and somewhat relieved by rest in the horizontal position. Spontaneous arrest of the stream during the passage of urine is often a diagnostic symptom of stone, but not exclusively so, as this same symptom occurs in other mechanical obstructions of the bladder outlet and in neuropathic conditions. Partial retention of urine occurs with calculus in the bladder but 
complete retention is generally due to the coexistence of other obstructive conditions of the neck of the bladder, namely: of stricture or of prostatic obstruction.

Seat of Pain.-The pain of stone in the bladder is located at the extremity of the urethra at the end of urination and is excited inde-

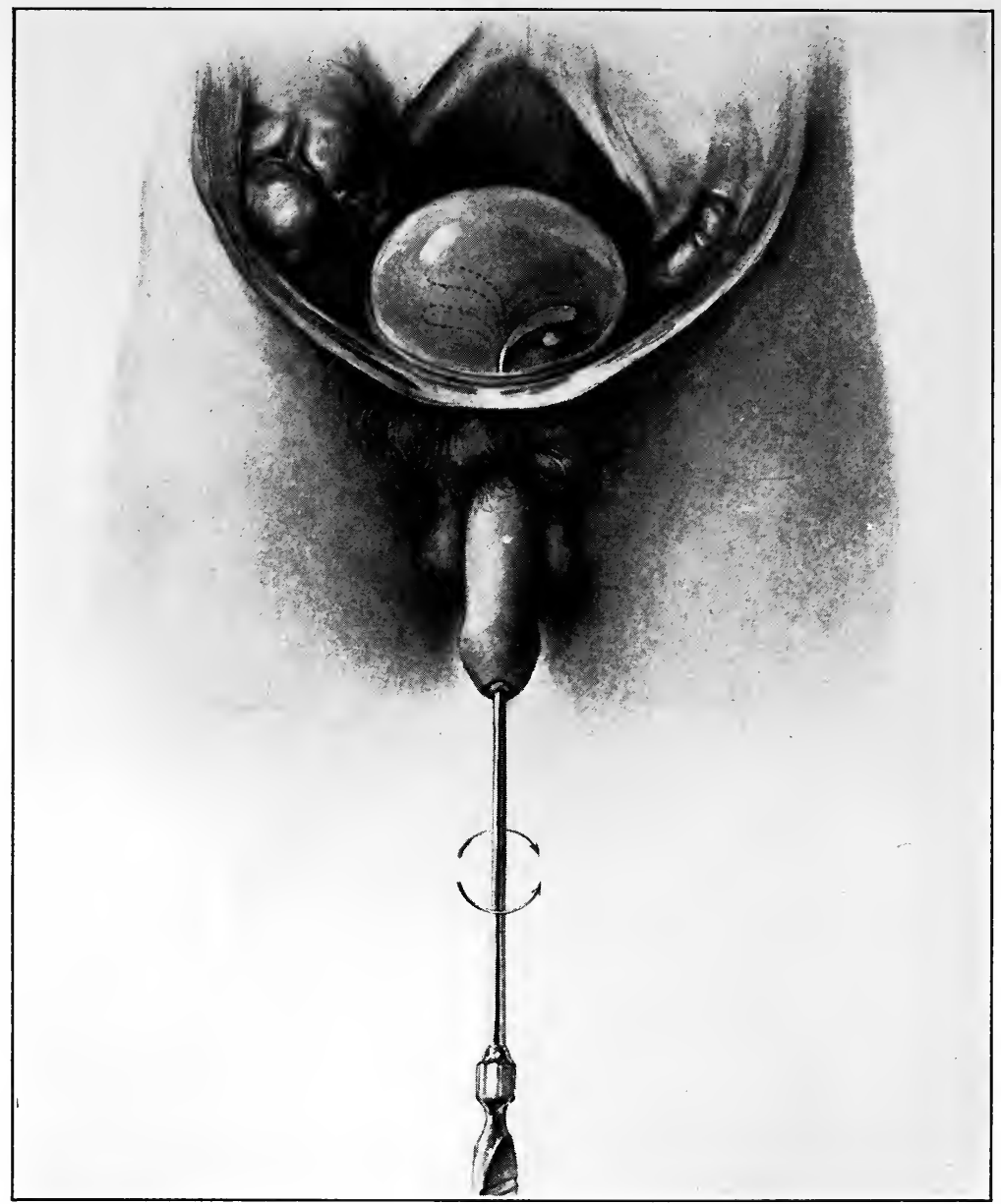

Fig. 186.-Palpation of bladder with Thompson's searcher.

pendently of urination by the movements of the body. When the stone has reached a sufficient size to produce pressure on account of its weight, there is a sense of fullness and pain in the rectum and groin. Diagnosis. - The diagnosis of stone cannot be positively made from the symptoms, although it may be strongly suspected in the case 
of cystitis with pain which is increased by movements of the body and characterized by the presence of blood in the urine, especially at the end of urination. The symptoms of weight in the rectum and perineum and pain transmitted toward the end of the urethra give greater weight to this suspicion. Instrumental examination, namely: palpation of the stone by the use of a searcher (Fig. 187) and cystoscopic examination furnish prompt and reliable means of diagnosis. The exploring instrument is the simplest and most rapid means of determining the presence of stone in the bladder and its use obviates the necessity of the more painful and prolonged measure of cystoscopy. A satisfactory exploration of the bladder with the palpating instrument requires that the bladder be filled with fluid. While this may be accomplished with the bladder full of urine, it is better and more surgical first to empty the bladder of its contents and after thoroughly washing it, to fill it with an antiseptic fluid such as boracic acid and then introduce the searcher, which, when rotated lightly from side to side (Fig. 186), will usually detect the presence of stone unless it is in a sacculus, between trabeculæ or hidden behind an enlarged prostate. It is possible by such palpation to gain a knowledge as to whether the calculus is single or multiple and also some idea as to its size and consistence. When there is any question of the diagnosis, it is necessary to resort to the cystoscope. Cystoscopy in children is difficult and Roentgenography may be more satisfactory. Cystoscopy may detect the existence of impacted calculi in the adult where palpation might fail; it is essential for the recognition of diverticula, and when it is necessary to differentiate between stone and neoplasm, as the symptoms of frequency of urination, hematuria and pain are common to both conditions. In prostatic hypertrophy in the aged, there are pain, frequency of urination, cystitis and sometimes hematuria, but the aggravation of these symptoms by movement of the body is not a feature as in stone. Again, these symptoms of prostatism are likely to be as active during the night as in the day and the coexistence of stone with enlargement of the prostate will in all probability produce an intensity of the symptoms that leads to the suspicion of its presence. The appearance of an excessive amount of mucus in the urine with cystitis always suggests the possibility of stone in the bladder and in the case of prostatic enlargement, the 
presence of gelatinous sanguinolent secretion in spite of the daily catheter and bladder washing, is strong evidence of this complication.

The prognosis of stone in the bladder is usually favorable when its removal can be successfully accomplished.

Treatment.-The treatment of stone in the bladder depends upon its size and the attendant local and general conditions.

Litholapaxy is the method of choice in competent hands for simple stone and when there does not exist some special contra-indication to its use. (See Operative Surgery, pages 492 to 499.) It may be difficult to accomplish with stricture of the urethra or in obstructive conditions of the prostate and more desirable to resort to such surgical procedure as will reach the obstructive lesion, which means to perform an external urethrotomy or prostatectomy, as may be indicated (see p. 479) at the same time. In cases of multiple stones or stones of large size, it may be considered the simplest method of procedure to open the bladder and remove the calculi rather than to attempt to crush them by a litholapaxy. (See Suprapubic and Perineal Lithotomy, Operative Surgery.) It may also be more desirable to remove the stone by opening the bladder in cases of chronic inflammation when the drainage resulting from such an operation affords more favorable conditions for the relief of the secondary cystitis which, on the other hand, may be aggravated by the manipulation of instruments during a litholapaxy. In cases of encapsulated stone or diverticula, litholapaxy is not practical and the removal by cystotomy is essential. A stone may be removed by means of the median or lateral perineal section (see Operative Surgery, p. 458), but on account of the limited space at the neck of the bladder and the possibility of damage to the structures in this region, suprapubic section is much more desirable, except in the case of small stones and in cases of stricture and prostatic enlargement operated upon by perineal route when it is natural to remove the stone through the perineal incision. Special caution should be exercised to explore the cavity of the bladder in cases of chronic cystitis with enlargement of the prostate or stricture, as not infrequently the presence of stone in the bladder has been overlooked. The choice of operation in the case of stone will depend upon the condition of the stone itself, the size of the urethra and the condition of the prostate and of the bladder. The stone may be too hard or too large to be crushed, and it should be free within the bladder to be favorable for litholapaxy. In children, lithotomy is preferable; the youthful bladder heals readily. Enlargement of the prostate always presents a difficulty to litholapaxy and is a reason for lithotomy. When litholapaxy has been performed and chronic cystitis does not 
improve, it may be necessary to perform a secondary cystotomy as a means of drainage. (For description of operation, see p. 492.)

\section{TUMORS OF THE BLADDER}

Since the advent of cystoscopy, and more especially since complete bladder exploration has been made possible in a large range of cases by the great improvement in the instruments manufactured, the frequency of neoplasms of the bladder has become more apparent. This frequency bears a certain relation to that with which hematuria occurs and while some hematuria is of renal and some of bladder origin, there is little question that many of those cases which occurred in early days without satisfactory explanation of the cause and were therefore designated essential hematuria and idiopathic hematuria, may have been due to small papillary growths of the bladder which were not recognized in the absence of the cystoscope or on account of the difficulty of making a satisfactory cystoscopy with the older instruments, without irrigating attachments. A common site of neoplasms of the bladder is around the ureteral orifices, but they invade other portions as well; they occur more of ten in the adult than in the young, and are more common in males than females. These tumors may be either benign or malignant, being the latter in fully half of the cases. Both types may exist in different parts of the bladder at the same time, especially in recurrences following operation.

\section{Varieties of Bladder Tumors ${ }^{1}$}

Papilloma is the common type of benign and carcinoma of the malignant variety of tumor. Other less common forms of non-malignant tumors are fibroma, myoma and cysts. Of the malignant growths sarcoma is much more rare than carcinoma.

Neoplasms vary also in number and form. They may be single

1 Kuster and Davis, Division of Vesical Tumors.

1. Epithelial group.

(a) Papilloma.

(b) Carcinoma.

(c) Adenoma.

(d) Cysts.

2. Connective tissue group.

(a) Sarcoma.
(b) Myxoma.
(c) Fibroma.

(d) Angioma.

3. Muscular group.

(a) Myoma. 
or multiple, sessile or pedunculated. The most striking feature that the urologist becomes aware of in connection with the observation of vesical tumors is the persistency with which they recur, and their strong tendency to malignancy.

Papillomata. ${ }^{1-}$-Papilloma is a villous growth; and here it must be clearly appreciated that while the terms villous growth and papilloma have been used generally to designate a benign growth of the bladder, the same type of growth may be malignant. A malignant papilloma may exist as such from its inception, or it may become so later, by malignant invasion, ${ }^{2}$ or, in the case of a recurrence, following surgical removal. A papillary growth may be pediculated or sessile.

The pediculated growth which is more likely to be benign occurs as a separate tumor, consisting of a fimbriated villous mass, attached to the bladder by a pedicle (Fig. 188, A). This pedicle may be of a size that is easily visible or it may be so short as to be hidden by the overlying growth. These pedunculated tumors vary in size and contour from the size and appearance of an unripe mulberry to that of a good-sized tomato. They are racemosed growths of connective tissue covered with epithelia. The sessile papilioma springs directly from the bladder wall in separate villi, single, multiple or in a fimbriated mass, covering a small or extensive area from the size of the end of a tooth pick to that of the entire bladder (Fig. 188, C).

Other Benign Tumors. - Of the other, more rare varieties of benign growths, fibroma occurs as a submucous infiltrate of dense fibrous tissue.

Myoma is rare, has its origin in the muscular structure, and may either extend in the direction of the vesical cavity, as a submucous growth, or extravesically, and be recognized as a tumor outside of the bladder.

Cysts of the vesical mucous membrane contain a viscid fluid; they may be few or multiple, and are probably glandular protrusions.

Dermoid cyst of the bladder has been reported.

Myxomata have also been found but their infrequency is much greater than the transitional forms of malignant growths which they outwardly resemble. They are composed of mucous and fibrous tissue.

Carcinomata.-Carcinoma of the bladder may appear, as already

${ }_{1}$ Bremerman (Trans. Am. Urolog. Assoc. 1911) gives papilloma as about 3 to 5 per cent. of all genito-urinary conditions. More common in adult life; more frequent in males-3 to 1 . Recurrence is usually outside the site of the previous operative field. Reports twelve cases and concludes that all papilloma should be regarded as malignant. Papilloma should be suspected in long-standing cases of cystitis (persisting in spite of treatment).

${ }^{2}$ Von Frisch (Wein. klin. Wochenschrift, 1907, pp. 1203-7) found beginning carcinoma infiltration in 107 of 201 apparent benign papillomata examined. 
stated, as a villous growth, pedunculated or sessile, this fact making it more difficult to make a diagnosis by cystoscopic appearances. Carcinoma also occurs as an infiltrating growth of the walls of the bladder, when it may extend out into the cavity as a sessile tumor or may be confined entirely within the intramural structure: then it is

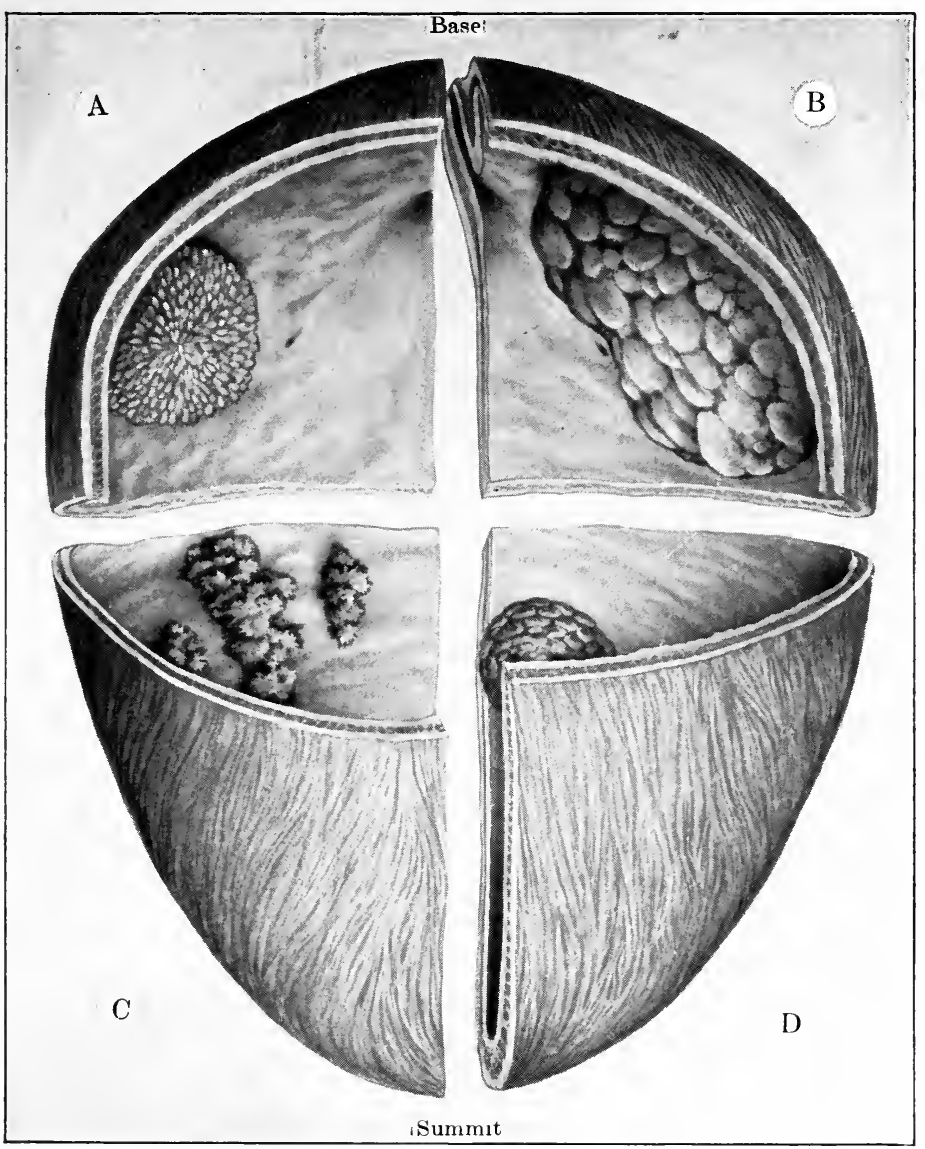

FIG. 188.-Different types of vesical neoplasm. A, Papilloma of base pedunculated. B, Lobulated sessile growth of base. C, Sessile papilloma. D, Lobulated and pediculated carcinoma of summit.

essentially an infiltrated mass growing backward. Carcinoma may also occur as a pediculated lobular, instead of a papillary growth (Fig. 188, B and D). It is usually fungous on the surface, while infiltration of the base of any vesical neoplasm is a recognized feature of malignancy. 
Sarcoma is much rarer than'carcinoma, and assumes different types, round or spindle celled, in pedunculated, sessile and infiltrated varieties. It may be soft or hard in structure, and may extend from the bladder into the surrounding tissues. Like carcinoma, it develops varying degrees of malignancy, as shown by the extensive nature of its growth, inside or beyond the bladder, and its rapidity of recurrence. Differentiation between carcinoma and sarcoma is only possible by microscopic examination. In fact, no positive diagnosis can be made as to the nature of vesical neoplasm until the laboratory findings have been reported upon.

Etiology of Vesical Neoplasm.-The causative agency of bladder tumors is, at the present time, beyond the scope of scientific knowledge. The parasitic theory has been advanced, but not proven. Occupation as a predisposing cause has been advocated, in that there has been noted a certain prevalence among dye workers, but this is not generally accepted. Injury and inflammation may be considered exciting causes, and metastases from other organs and glands, notably the adjacent prostate gland, may be the primary source; but the percentage of tumors of the bladder arising from a preëxistent prostatic focus is not great. Malignancy frequently invades an originally benign type, and recurrences following the latter are prone to appear in malignant form. Propagation, therefore, may be stated to be by direct contact, being grafts produced by implantation of the original tumor upon the previously infected surface of the mucosa. It also occurs by extension to the contiguous tissues, and from more distant sources, through metastatic propagation.

Pathology.-The pathological anatomy of bladder tumors differs, according to the variety of the growth. Benign papillomata occur as a soft mass of villous tissue, pedunculated or sessile in character, and extremely friable. The pedicle at its base is an offshoot of the normal mucous membrane. This pedicle is soft or hard, according to its density, produced by a greater or lesser amount of fibrous tissue. The sessile tumors extend more deeply into the mucosa, and frequently contain offshoots that extend into the submucosa, in which case they are more likely to be malignant. This invasion of the submucous structure may be microscopic, or apparent to a visual examination. The structure of the benign bladder tumor contains cellular and fibrous tissue, with no definite orderly arrangement. The structure of the other benign tumors is according to the type in which they appearfibrous, muscular or cystic.

The pathological anatomy of malignant neoplasms has the same characteristics as distinguish them when they occur in other localities. 
The carcinomata, generally speaking, are epithelial degenerations or epitheliomatous vegetations, pedunculated or diffusely implanted over the bladder wall (Fig. 188, B).

Infiltrated growths invade the mural structure of the bladder at the expense of the normal tissue, interstitially within the separate coats, and between them; and extend extravesically, as well as intravesically, sometimes appearing beneath the abdominal walls, where they may be felt by palpation. These tumors present the most varying structure, which may be lobulated, reticulated, or irregularly infiltrated, upon the mucous surface of which may develop irregular ulcerations, resulting from the distention of the mucous membrane and necrosis of the neoplastic covering.

Symptoms.-The symptoms of bladder neoplasm are the same, whether it be benign or malignant; and the most common symptom is hematuria, which, generally speaking, is greater in the non-malignant than in the malignant varieties, and may, in the former, be the only outward sign of the existence of the growth. Any tumor, however, that is located near enough to the bladder outlet to cause obstruction may produce as a result difficulty of urination and partial retention of urine. If cystitis coexists, there are the usual symptoms of this condition in the form of painful and frequent urination. Malignant growths, in addition to the symptoms of hematuria and obstruction of urine, are also likely to be the seat of little or much pain according to their location and the size. When ulceration and necrosis occur, there is produced a consequent inflammatory condition, with proportionately active symptoms of pain and tenesmus, decomposition of urine, and, finally, profound systemic sepsis. When the growth extends outside of the bladder, it may be palpated in the abdominal wall. The more definite clinical character and extent of all growths are best observed by cystoscopic examination (see p. 129).

Diagnosis.-The diagnosis of a vesical growth is accomplished with the cystoscope. Free bleeding is sometimes a difficulty attending this operation, but the end is achieved by quick action and free irrigation. Large neoplastic growths and those that have extended beyond the interior of the bladder may be recognized by their severe symptoms and by abdominal and rectal palpation. It is possible to recognize the probable nature of such growths by cystoscopic observation, although it is unsafe to rely conclusively upon such observation without the aid of the microscope. The peculiar features that have been given in the description of the different varieties of neoplasm may be taken into account in forming an opinion and in the case of the passage of fragments of the growth or when such can be obtained by operative 
manipulation, a positive diagnosis may be reached prior to recommending mild or severe measures of treatment.

It has been demonstrated by careful study of extensive material (Buerger, Mandlebaum) that certain peculiarities in the shape and arrangement of the cells indicate either primary carcinoma or carcinomatous transformation, and more especially with the added indication of infiltration of the stroma. ${ }^{1}$

Treatment.-In considering the question of treatment of vesical tumors, their strong tendency to recurrence must always be kept in mind. Generally speaking, treatment may be palliative or operative. The operative measures that have been adopted are as follows:

I. Transurethral (Cystoscopic).

(a) Morcellation and removal of the growth by means of the Operative Cystoscope. ${ }^{1}$

(b) Cauterization through the cystoscope.

(a) With the galvanocautery.

(b) By high frequency current (Oudin).

II. Open Operation-Suprapubic section.

(a) For excision of tumor.

(b) For drainage.

III. Transperitoneal operation for complete removal of bladder and neoplasm including transplantation of the ureters.

The obstinate recurrence of tumors that on the face have appeared non-malignant, in spite of their total removal, emphasizes the necessity of complete and far-reaching extirpation if a radical course is decided upon.

Transurethral Methods.-The operating cystoscope is employed to remove intravesical growths by means of a snare and cautery. ${ }^{2}$ This necessarily entails a number of sittings which vary according to the size of the tumor and the success with which it is attacked. This work is confined to the expert cystoscopist and one whose outfit includes this rather complex apparatus. The method is naturally unsuited to the treatment of malignant neoplasms and the successful cases reported are practically all benign ones.

The work has therefore been confined to a few observers only, and it has been difficult to obtain end reports based upon thorough cystoscopic observation at long intervals.

\footnotetext{
1 Department of Surgical Pathology, Mt. Sinai Hosp.

2 Young (Trans. Amer. Genito-Urinary Surg., 1909), introduced a new operating cystoscope refers to Nietze's report of cases.
} 
Intravesical Destruction of Vesical Tumors by the High Frequency Current. ${ }^{1}$-This somewhat modern technic has yielded results that have deservedly won for it strong approval and unquestionably is superior to the previous eystoscopic method so far as facility, prompt result and the amount of pain produced are concerned.

Moreover it is likely in view of the reports that have accumulated that the end results will equal any other operative measure employed to destroy benign growths. It may be possible to produce radical destruction and cessation of some growths in which malignancy is suspected, provided such growths are attacked when they are of small size and before marked infiltration of the submucous structure has ensued. Generally speaking, for extensively malignant and largely diffused growths, it can hardly be considered an effective means of treatment although palliation has seemed to occur in connection with its use even in some cases where such a result could barely be expected. For description of technic, see p. 153.

Excision of Growths by Open Operation.-This operative procedure is conducted through a supra-pubic incision and the neoplasm is removed with a section of the bladder wall. In benign growths it is possible to make a clean cut operation and by such a method to eliminate the likelihood of recurrence. But the previously mentioned high frequency treatment is preferable when cystoscopic observation and section obtained for pathological examination give evidence of nonmalignancy. This operation of open excision may also be performed in combination with the free application of actual cauterization, when preliminary attacks with the high frequency current upon the more dense benign tumors have failed; and even in the case of malignant growths, when they are circumscribed in area and apparently have not extended beyond the bladder wall. There is a possibility in such instances of a long period ensuing without recurrence; yet there is always the grave danger that instead of such hopeful outcome a rapid return will occur, even though at the time of operation conditions appeared especially favorable for complete removal.

Complete Removal of the Bladder.-In malignant growths, when the submucous structure is infiltrated, whether or not they extend over a large area of the bladder, nothing short of obliteration of this viscus or complete excision may offer the greatest reliance of forestalling

\footnotetext{
1 To Edwin Beer (New York) belongs the credit of developing this important method of successfully destroying bladder tumor and of insisting upon its virtue in the face of opposition. See Removal of Neoplasms of the Urinary Bladder, Genito-Urinary Association, 1910; Annals of Surgery, August, 1911. Also E. L. Keyes, Jr., Transactions of American Genito-Urinary Surgeons, 1910, Report of Treatment of Bladder Tumors by High Frequency Current.

B. A. Thomas (Surgery, Gynecology and Obstetrics, April, 1912). The Destruction of Tumors of the Urinary Bladder by a High Frequency Current, etc.
} 
recurrence. ${ }^{1}$ This entails transplantation of the ureters in the intestine or in the lumbar region. Such an operation can be performed in two steps by first side-tracking the urine, which may result in a prompt relief of the painful symptoms, and later the complete removal of the growth including the bladder is effected under more favorable conditions. There is naturally a large element of risk of infection of the kidney when the ureters are united to the intestine or exposed externally in the loin, yet such a complication under existing conditions would seem imminent without transplantation unless life is forfeited before this has had time to occur.

The operation of complete removal of the bladder and transplantation of the ureters at the same sitting involves such a serious risk that it is much preferable to do the work in two steps.

As between transplantation of the ureters into the intestine $o . x$ rectum and double nephrostomy, the latter operation is believed to be followed less often by ascending infection of the kidneys. There is beyond doubt always danger of pyelonephritis, but the serious menace to life from the onwara march of the malignant growth, not to mention the possibility of renal infection in any case, renders the risk following double nephrostomy the lesser of these evils. ${ }^{2}$

(For description of Operative Technic, see page 639.)

The danger of metastases is ever present as in all malignant growths, ${ }^{3}$ therefore it may be a question to be decided by the patient himself whether or not he would prefer to live out his time with palliative treatment. It is to be hoped, however, that future progress will make the indication for operation more definite. Some surgeons are prone to feel that it is better to allow such patients to die by the knife than to live out a life of progressive deterioration, but no fixed law can be laid down to settle so serious a question; the circumstances of each case must govern the action.

Palliative Treatment.-Any operative proceeding short of complete removal of the growth aims to be palliative. Thus, in cases where it is deemed inexpedient or impossible to accomplish entire removal of the tumor, its partial excision is undertaken with the intention of prolonging life or of enabling the patient to exercise the bladder function with less difficulty. Generally speaking, such partial operations are not satisfactory save in exceptional instances in which

\footnotetext{
1 Rovsing of Copenhagen believes that there is a far better chance of recovery following total than partial removal of bladder.

2 Rovsing believes that the danger following operation has been diminished by this expedient and Watson of Boston has spoken strongly in favor of this procedure backed up by successful experience. Hugh Cabot of Boston is also favorably disposed toward this technic and reports a case (Trans. Am. Assoc. Genito-Urinary Surg., 1909).

${ }^{3}$ Rovsing remarks: "The future fate of patients who survive the dangers of operation is still a gloomy one."
} 
there is a surprising tolerance both on the part of the neoplasm and the patient to surgical "interference" and there is always the question whether or not life would not have been as endurable and enduring without such an attempt to improve matters. If palliative operation is deemed advisable in place of complete removal of the disease, it would seem wiser to resort to external ureterostomy alone as a means of obtaining the comfort attendant upon keeping the urine away from the diseased bladder, in the same manner as when excision is contemplated; or a suprapubic cystostomy for continuous bladder drainage. Some patients have lived on comfortably for years following this latter operation, wearing a suitable portable urinal (see Fig. 189) to receive the urine and such a course is demanded when complete occlusion of the bladder outlet renders urination impossible. It has the disadvantage, however, of permitting the diseased parts to be continually bathed in the accumulated urine and there is, as a result, more or less bladder inflammation and attendant pain. This may be somewhat controlled by palliative measures of lavage and anodynes. (For description of different operative technics, see pages 464 to 470 .)

Symptomatic palliative treatment of bladder growths consists in treating the attendant symptoms of bladder tumor which are, as stated, pain and hematuria associated with more or less cystitis. The pain must be met with the judicious use of anodynes either internally or by rectal suppository. In the end stages, the frequent use of the

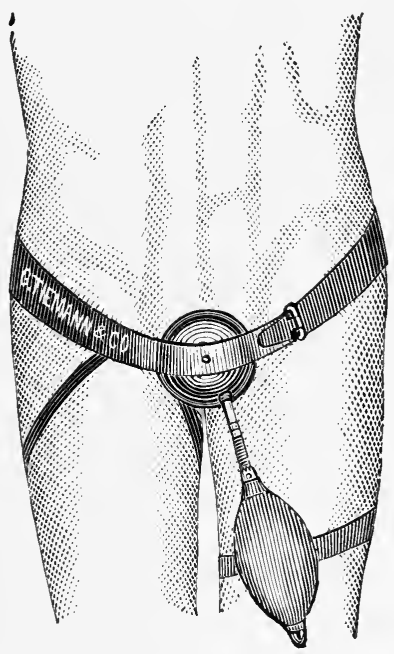

FIG. 189.-Urinal for suprapubic drainage. hypodermic syringe becomes necessary.

Hematuria may be controlled to a certain extent by bladder lavage. It frequently disappears at once in benign growths under the influence of the high frequency current already described and is controlled to a lesser degree by the same method in some malignant growths. Cystitis is to be treated according to its extent and the coexisting conditions. Some patients tolerate local measures very poorly and are made to suffer increasingly by such means. On the other hand, the disinfection of the bladder by gentle lavage with such applications as nitrate of silver $(1-16,000)$ or 2 per cent. boracic acid may be the means of lessening irritation. Internally no treatment is of any value beyond the symptomatic use of anodyne and internal disinfectants. (Hexamethylenamine, etc., see Treatment of Cystitis.) 


\section{CHAPTER XXV.}

\section{OPERA'TIVE SURGERY OF THE BLADDER, PROSTATE AND SEMINAL VESICLES}

The Bladder.-The bladder may be approached through the perineum or through a suprapubic opening. The operations upon the bladder proper are performed for the purpose of drainage, for the removal of foreign bodies (calculi) and neoplasms, for correcting deformity (diverticulum) and for the partial or complete extirpation of the organ on account of cancerous growth. In addition to the

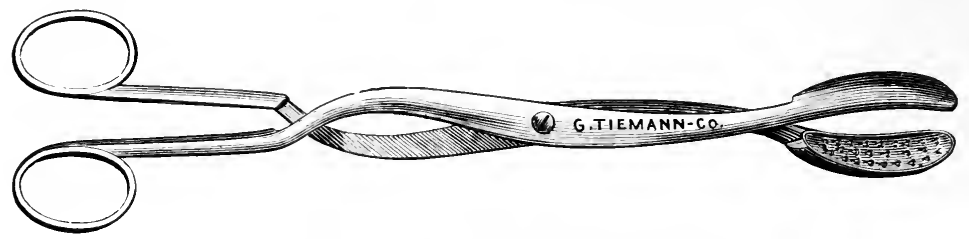

FIG. 190.-Straight lithotomy forceps.

operations upon the bladder itself, this organ is entered during other operations that are required for surgical conditions involving the adjacent regions.

External urethrotomy has already been described (see p. 287) and while this technic is essentially for morbid conditions involving the urethra, the bladder is invariably reached during the operation,

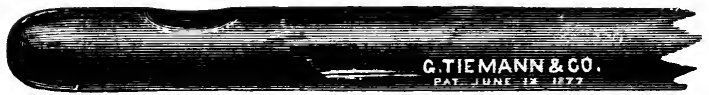

Fig. 191.-Perineal drainage tube.

not only as a landmark, but also to afford drainage, either on account of the lesion of the urethra itself or because of the coexistence of chronic inflammation within the bladder.

Perineal cystotomy, therefore, when performed for drainage, is the same as external urethrotomy. When this operation is done for the removal of a foreign body, namely, a calculus, the stone forceps (Fig. 
190) is passed through the perineal wound, through the prostatic urethra and thence to the bladder, and the stone if not too large, being grasped by the instrument, is removed through the normal neck of the bladder. Such an operation, properly speaking, is median perineal lithotomy. Should the stone prove to be too large for re-

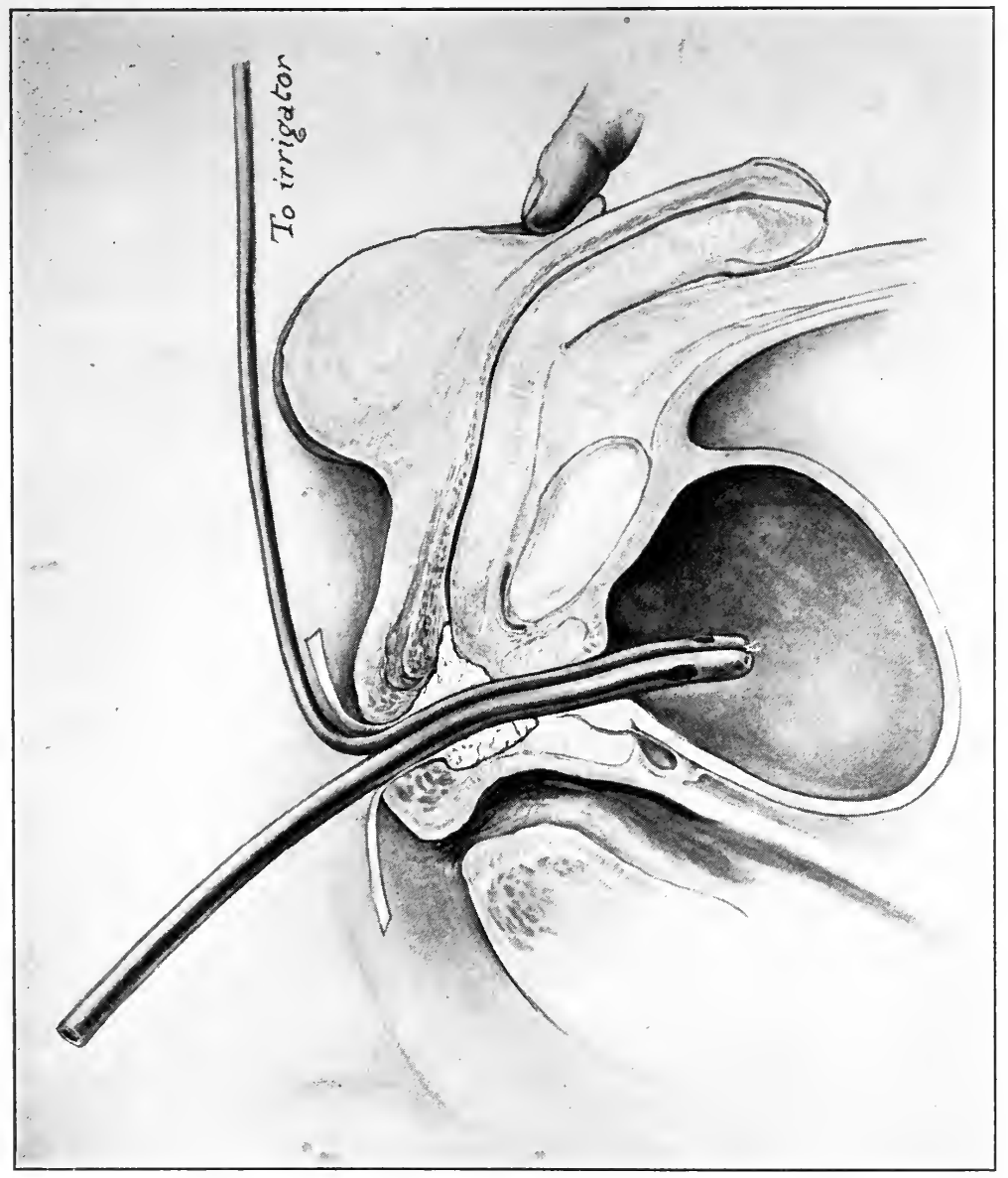

Frg. 192.-Continuous irrigation of bladder and packing after perineal operations.

moval in this manner, it must be either broken or crushed and washed out through the perineal wound through a straight litholapaxy tube (Fig. 227, p. 496), or the opening in the bladder must be made larger by lateral incision. In this case hemorrhage from the neck of the bladder is likely to be very free, which must be taken into account at the 
time of operation and in the arrangement for drainage afterward (see over). Incision of the neck of the bladder is not advisable on account of the amount of hemorrhage that ensues and the difficulty of controlling it. One of the other methods of removing stones, namely, litholapaxy or suprapubic section, is more desirable.

Drainage of the bladder through a perineal opening is accomplished by inserting into the bladder through the external urethrotomy wound a good-sized perineal tube (see Fig. 191) which is tied or sutured in situ. Drainage is then continued according to the requirements of the case. In connection with operations accompanied by much hemorrhage, a narrow gauze tape is packed around the perineal tube, forward toward the bulb and backward toward the bladder on either side. This is sometimes spread with powdered formaldehyd gelatin (glutol) when the hemorrhage is outside of the bladder, but when the hemorrhage occurs inside the bladder, either from its neck or general mucous surface, it is very important to see to it that the blood is not allowed to remain within the cavity to form blood clots which offer a double menace to the successful progress of the case. First, they plug up the drainage tube and interfere with drainage and second, they act as foreign bodies, causing continual straining and additional bleeding, which sometimes leads to a dangerous situation. To avoid this, it is well to provide for continuous irrigation of the bladder for a few days so as to remove the fluid blood as quickly as it flows until the bleeding has ceased (Fig. 192). This may be accomplished by a double rubber tube, one for inflow and the other for the outflow, which may be made by suturing a small eathether to the ordinary perineal tube. Double rubber drainage tubes for this purpose are manufactured or a Hagner metal double current drainage tube may be used. When it is satisfactorily determined that bleeding has ceased it is not necessary to continue the irrigation.

\section{Instruments Required.-Same as for external perineal urethrotomy.}

\section{SUPRAPUBIC CYSTOTOMY}

The bladder is approached through the suprapubic opening for the purpose of removing foreign bodies (calculi) for diagnostic investigation and for reaching the adjacent structures, namely, the lower end of the ureter and the prostate (suprapubic prostatectomy $q . v$.). The interior of the bladder may be examined through the suprapubic incision, the urethral orifice explored and the ureteral openings inspected and examined by bougie or catheter. By means of 
electric illumination the entire surface of the interior of the bladder may be examined (Fig. 194).

The approach to the bladder suprapubically may be intra- or extra-peritoneal. The latter is performed when the interior of the cavity alone is to be reached and the former when a larger field of operation is required on account of hemorrhage from lacerations of the bladder, the removal of large growths and the complete extirpation of the viscus.

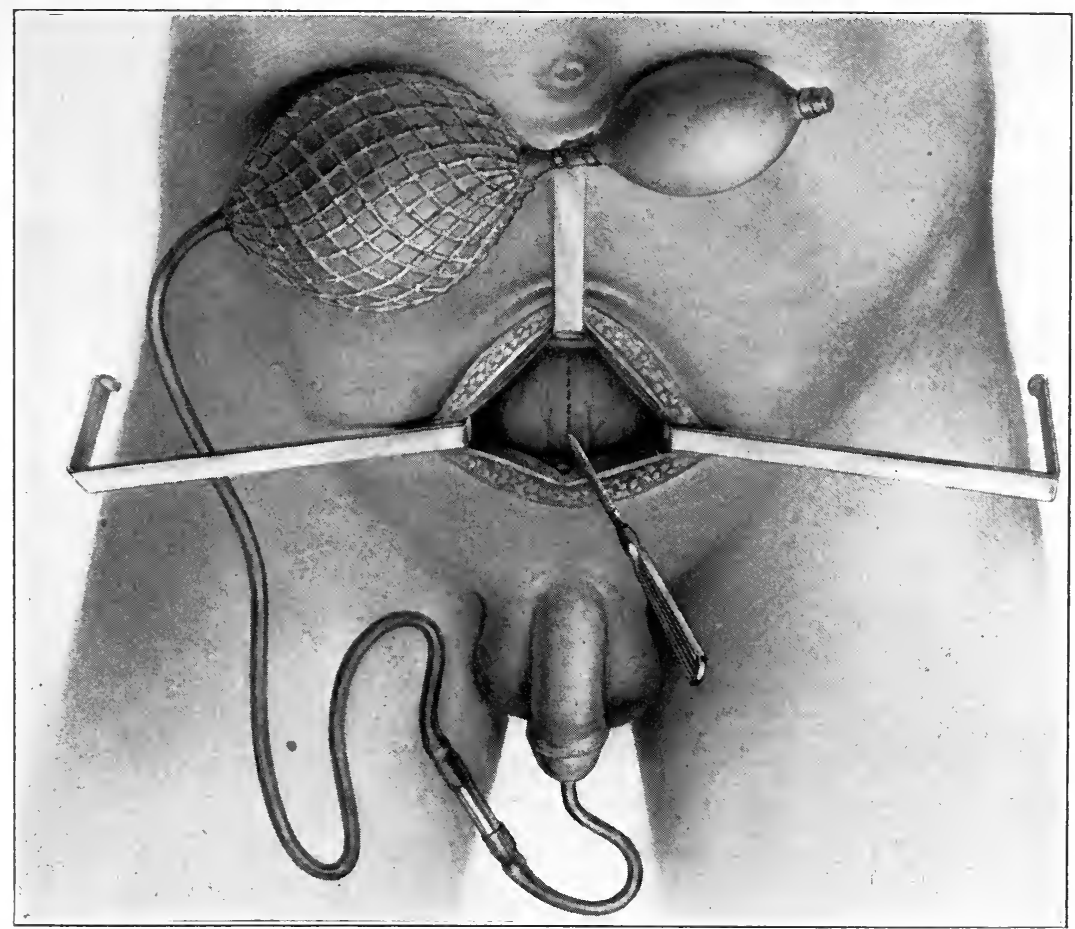

FIG. 193.-Suprapubic incision of the bladder; air inflation.

Extraperitoneal Suprapubic Cystotomy.-The patient lies in the ordinary dorsal position under general anesthesia. In some cases, where desirable, when a rapid entrance to the bladder is necessitated, local anesthesia may be employed, in which case, the tissues overlying the suprapubic region are infiltrated with five-tenths per cent. novocain. The patient may be given one-half hour previous to operation an injection of $.016 \mathrm{gm}$. (1/4 gr.) of morphine and $.0004 \mathrm{gm} .(1 / 150$ 
gr.) of atrophine sulphate. Previous to the operation, the catheter is introduced and the bladder is carefully cleansed with boric acid solution. The catheter is left in situ, or is reintroduced just previous to making the incision. A transverse incision is now made just above the pubic bone about 15 centimeters ( 6 in.) in length through the skin and superficial fascia down to the deep fascia. The deep fascia is now slit in the same direction and separated from its attachment to the rectus muscles. The rectus muscles are then separated in the median

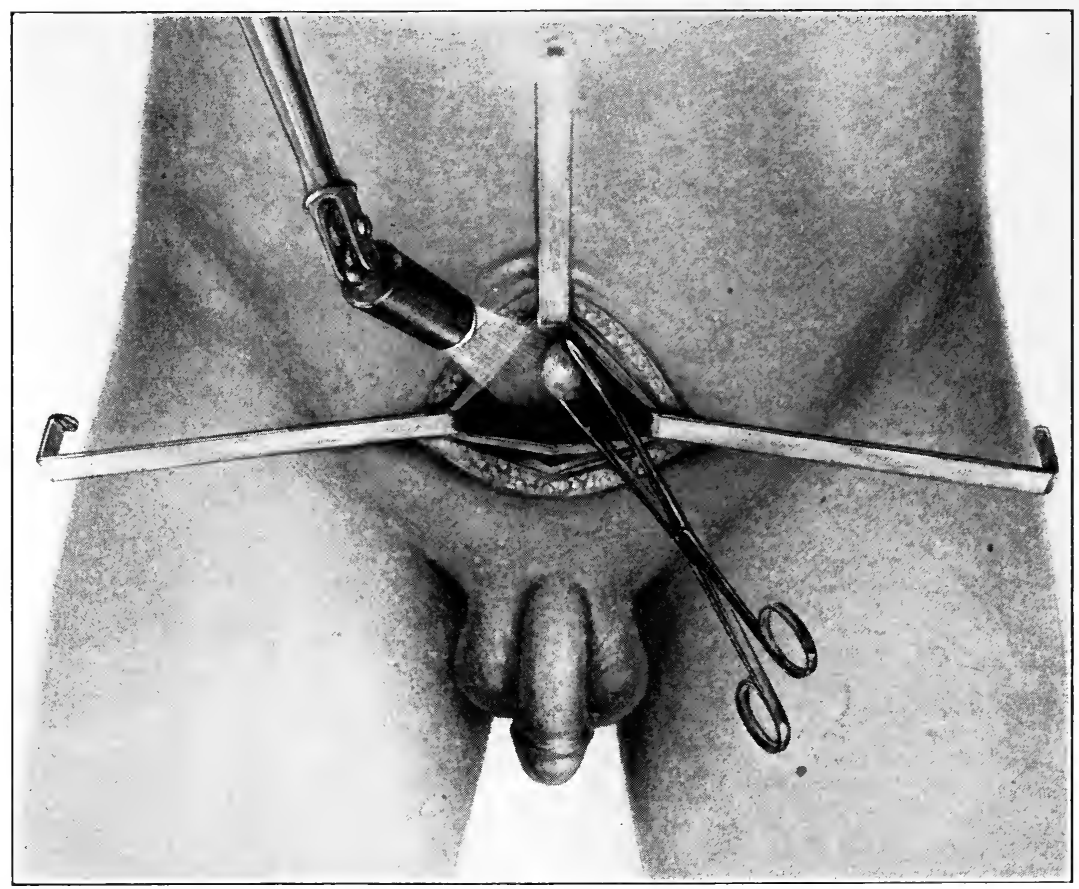

FIG. 194.-Suprapubic section of the bladder. Illumination and extraction of stone.

line and held on each side by lateral retractors. The peritoneal cul-desac, which naturally falls down over the bladder when empty, is pushed upward with the overlying adipose tissue and held there by means of a retractor over a small gauze pad.

The longitudinal incision is often used by preference. In this case, incision is made in the median line above the symphysis 12 to 15 centimeters (5 or 6 in.) in length, down to the deep fascia, which is then split in the median line in the same direction, and the rectus muscles 
separated and held aside as already described. At this point, whether the longitudinal or transverse incision is made, a double inflator bulb is attached to the catheter in the urethra and an assistant slowly distends the bladder by allowing the air to escape from the bulb through the catheter (Fig. 193). Meanwhile, the operator feels the distended bladder bulging upward into the wound, and with a scalpel, a longitudinal incision one or two inches in length is made through the bladder wall, allowing the air to escape. Before the scalpel is removed from the

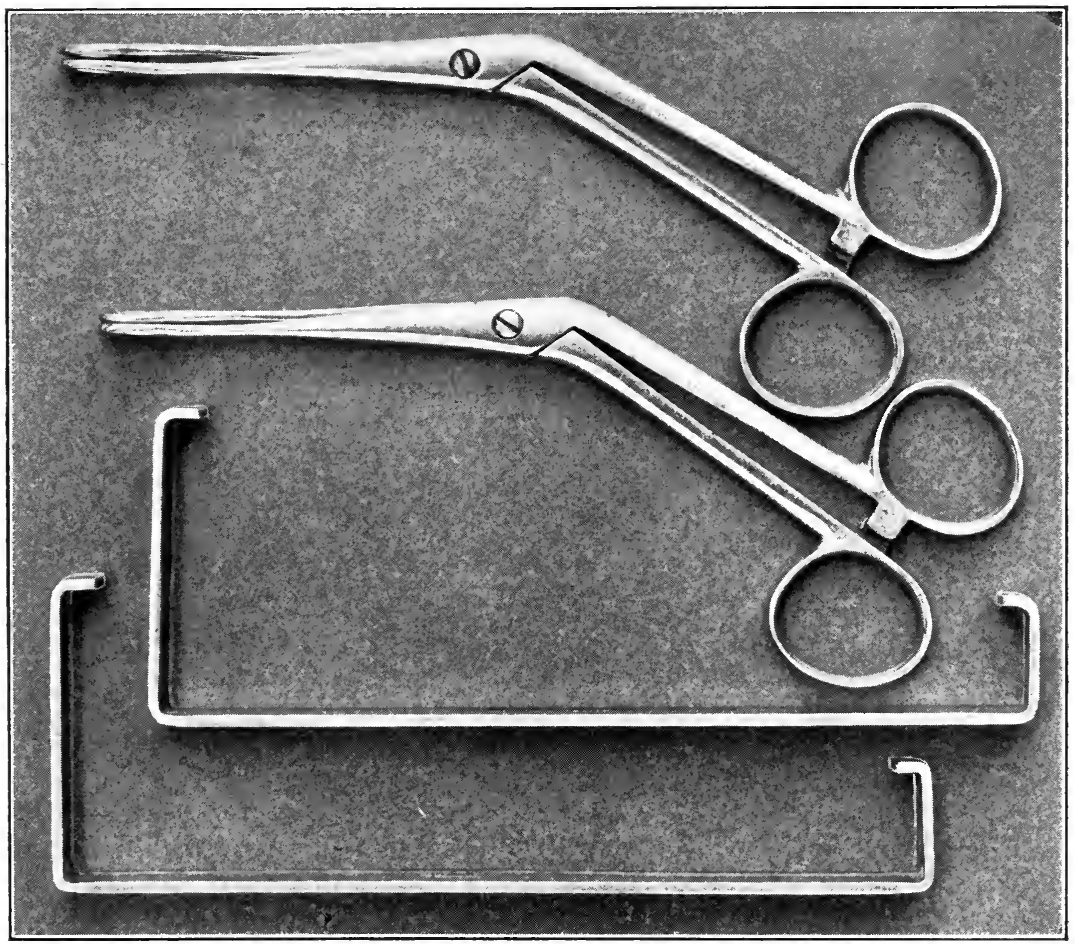

FIG. 195.-Bladder holders and retractors.

cavity, a retractor (see Fig. 195) is introduced and the wound is held open, permitting the introduction of the finger for exploration of the interior of the cavity. Further steps depend upon the existing condition, namely: stone, prostatic hypertrophy (see Suprapubic Prostatectomy), extirpation of the bladder for neoplasm, etc.

Suprapubic lithotomy is performed when the stone is too large or too hard to be crushed by litholapaxy, when suprapubic drainage is desirable or when suprapubic prostatectomy is required. For removal of vesical calculi, the stone forceps (Fig. 196) are inserted alongside 
the finger as a guide and as many as are present removed in such manner (Fig. 194). A careful search of the bladder is then made with the finger or more thorough examination of the interior by means of electric illumination is conducted, intravesical retractors being inserted on both sides and above. When the interior of the bladder has been properly dealt with, operation is completed by suture and the provision for drainage. A good-sized rubber drainage tube is fitted into the opening in the bladder, the end of which is kept from pressing upon its floor, and, when the bladder incision is properly closed by sutures, the drainage tube is held in place thereby. These sutures are passed so as to invert the edges of the bladder and include all the layers but the mucosa. Two rows of sutures are usually employed; one of these stitches below is left long and tied to the fascia so as to close the prevesical space (Fig. 197, A) and one is tied to the fascia above to exclude the peritoneal pouch (Fig. 197, B).

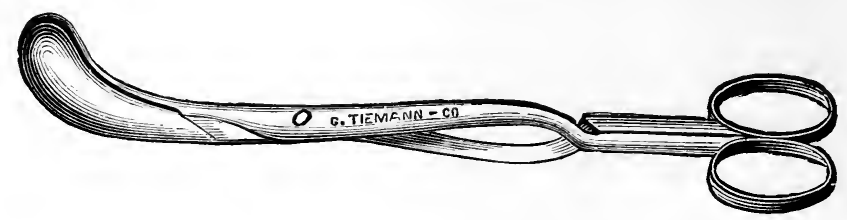

FIG. 196.-Curved lithotomy forceps.

One of the sutures is also tied around the drainage tube to keep it in proper position. The skin is then sutured with silkworm gut. As in perineal cystotomy, if there is any tendency to intravesical bleeding, it is important to institute continuous irrigation of the bladder for one or more days until this tendency has subsided. For this purpose, a special drainage tube is employed (see Fig. 218) which permits the introduction of a mild antiseptic or styptic fluid through a small inlet tube and free exit through the larger opening of the drainage tube. Alum 4 or 6 grams to 500 c.c. of hot sterile water will be found useful to check the bladder oozing and may be used also in the continuous irrigation alternately with boric acid solution when obstinate bleeding demands it.

Intravesical Removal of Tumors.-After opening the bladder according to the above description, further action is governed by the indication in each individual case. When vesical neoplasm is exposed, diagnosis may have been made by previous cystoscopic examination q. v.) and it may be determined malignant character precludes the possibility of intravesical removal. In such cases one of several courses is open:- simple bladder drainage (cystostomy), complete 
extirpation of the bladder at once or at a subsequent date, following perhaps the preliminary operation of double ureterostomy to divert the urine. (See Treatment of Bladder Tumors, p. 454.)

If preliminary cystoscopic examination and observation at the time of operation has decided the operator to perform intravesical removal, the bladder is carefully laid open as above described; if the tumor be of the sessile type, broadly implanted, it must be either totally destroyed by application of the cautery or partial resection of the bladder is called for. This procedure is simplest in the case of

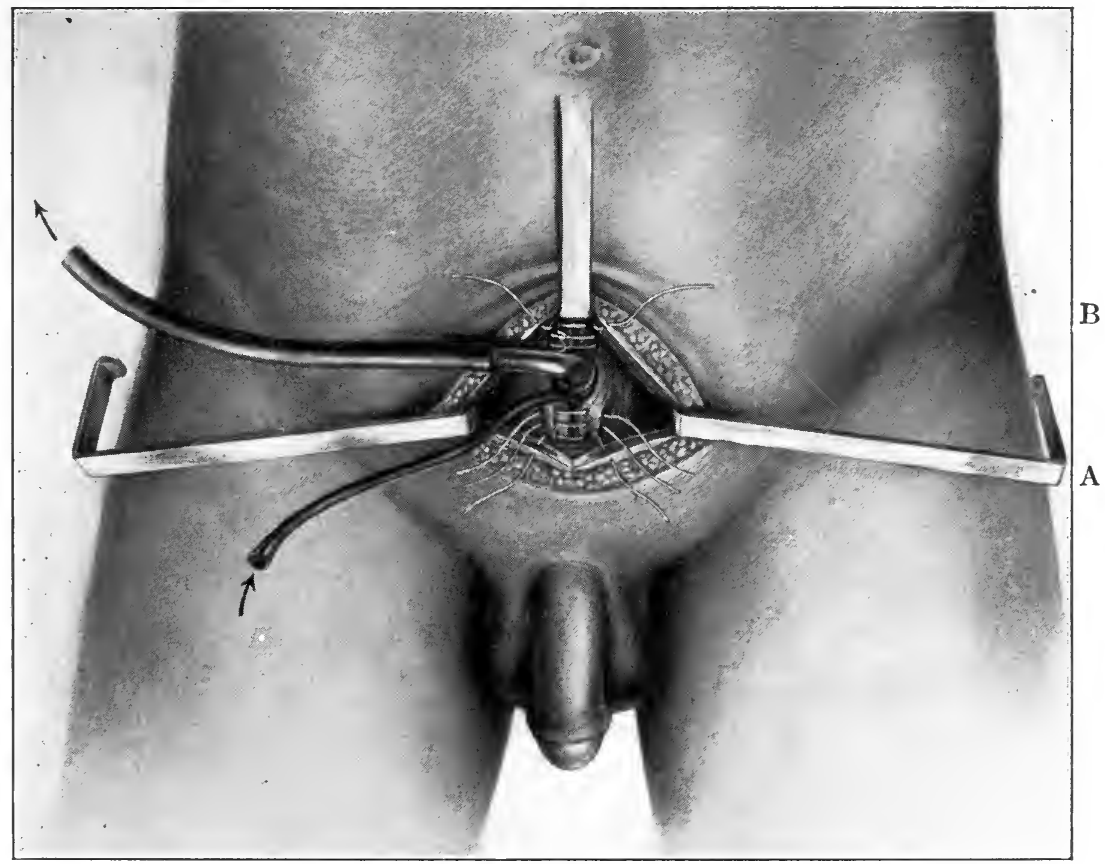

FIG. 197.- Suture and drainage of bladder after suprapubic cystotomy.

tumors of the fundus of the bladder; but when the bladder tumor involves the region of the base and is in the vicinity of the ureter, not infrequently, the latter duct must be severed during the course of the operation and reimplanted elsewhere (Fig. 199). Under such conditions the transperitoneal operation is performed.

Where the tumor is easily accessible in the vault of the bladder, an incision is made half an inch beyond the outline of the growth, through the mucosa and deepened into the tissues of the entire thickness of the bladder. The whole excised area of the vesical wall is then raised 
and removed with utmost gentleness by means of scissors and forceps. In spite of the clean cut removal of the tumor in this manner, it is better to treat the denuded surfaces freely with the Paquelin cautery on account of the danger of malignancy in so many cases (Fig. 198). The bladder is then approximated with interrupted

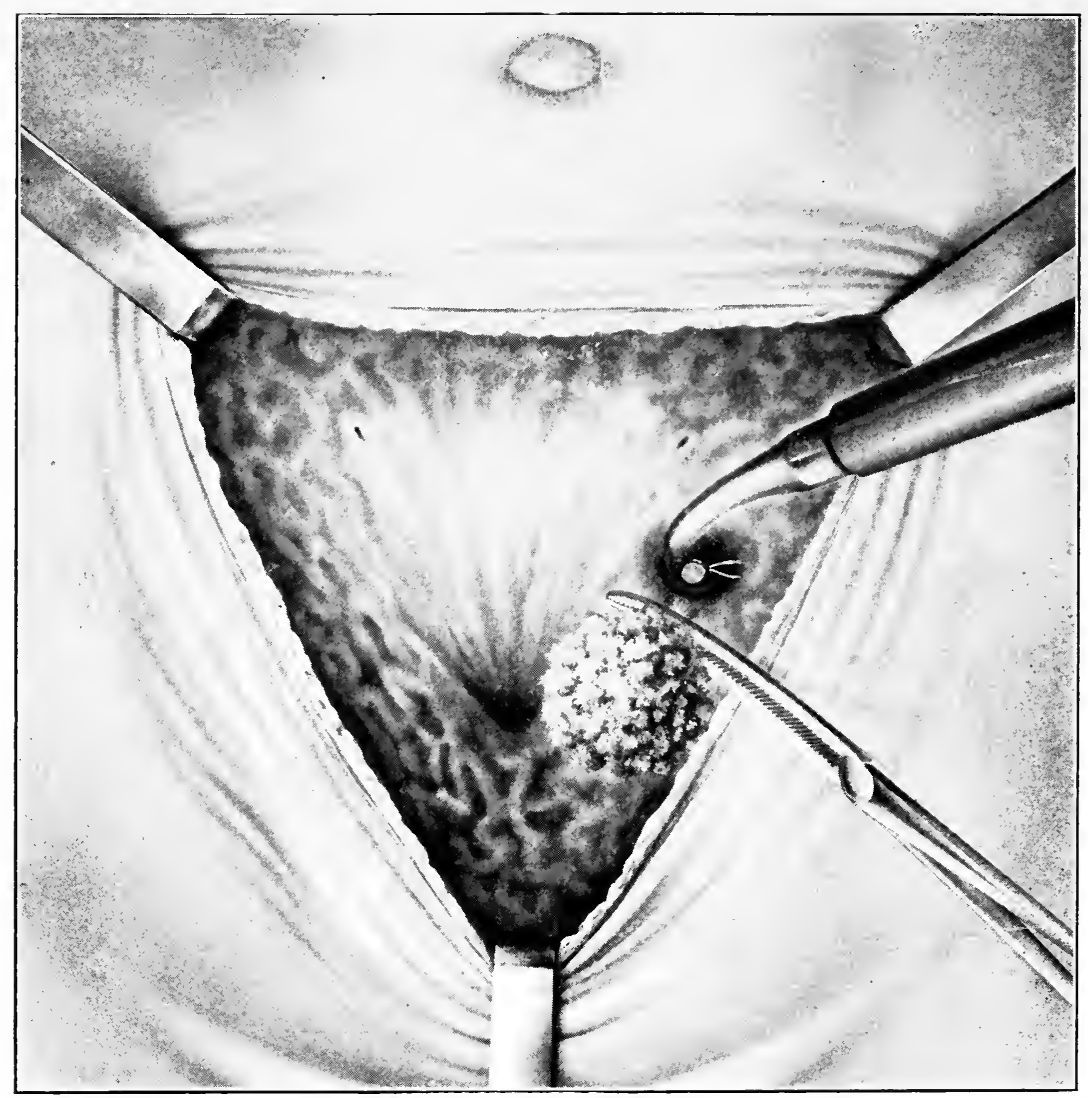

FIG. 198.-Intravesical removal of neoplasm. Cauterizing base. (After Mayo.)

sutures, and drainage is instituted (see Suprapubic Cystotomy, p. 461) and packing is inserted between the bladder and the peritoneal sac.

If the tumor is pediculated, the base of the pedicle is exposed at the site of its implantation and is transfixed close to the surface of the bladder with a double ligature; the two halves are tied separately and then the entire pedicle is ligated. The tumor is then snipped off with a pair of scissors and the base thoroughly cauterized with the 
Paquelin cautery (Fig. 198). If the bladder wall be infiltrated or if the pedicle extend beyond the mucosa, the area in question is dissected away by an incision $1 / 2$ inch beyond its extension and the Paquelin cautery applied. Sutures are then inserted from the outside, when"the

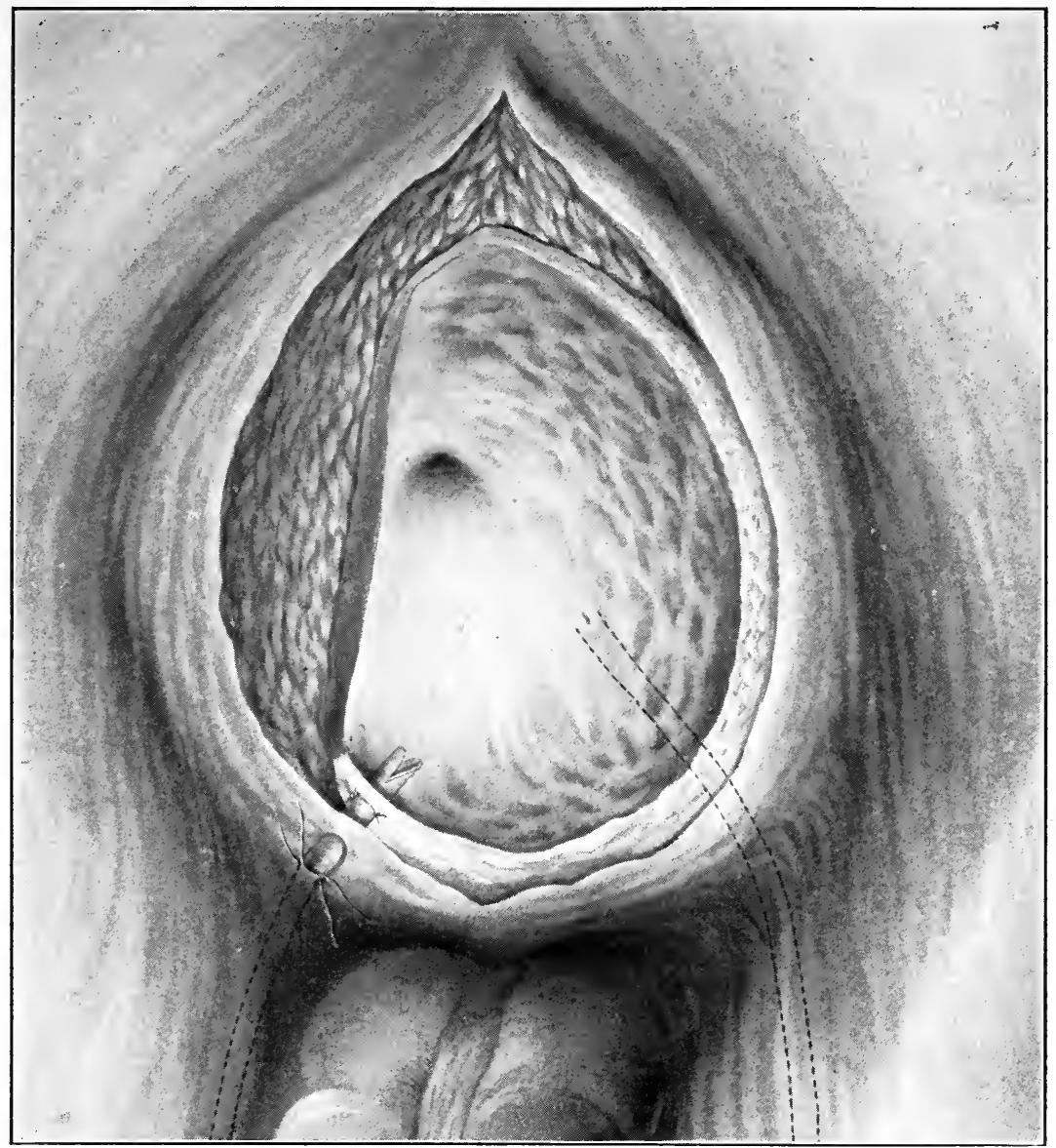

FIG. 199.-Partial intraperitoneal excision of the bladder and reimplantation of ureter. (After Mayo.)

entire thickness of bladder wall has been excised, so as to turn the charred area inward.

Transperitoneal Cystotomy.-In this instance, the longitudinal incision is made longer than in the extraperitoneal operation and extends sometimes from the symphysis to the naval or beyond, the patient being placed in the Trendelenberg position. After the peri- 
toneal cavity is opened and retractors are inserted, the abdominal cavity is walled off by large gauze compresses before opening the bladder cavity. A longitudinal incision is then made in the median line of the bladder and the interior of its cavity exposed. Pediculated growths are removed in the manner already described and those

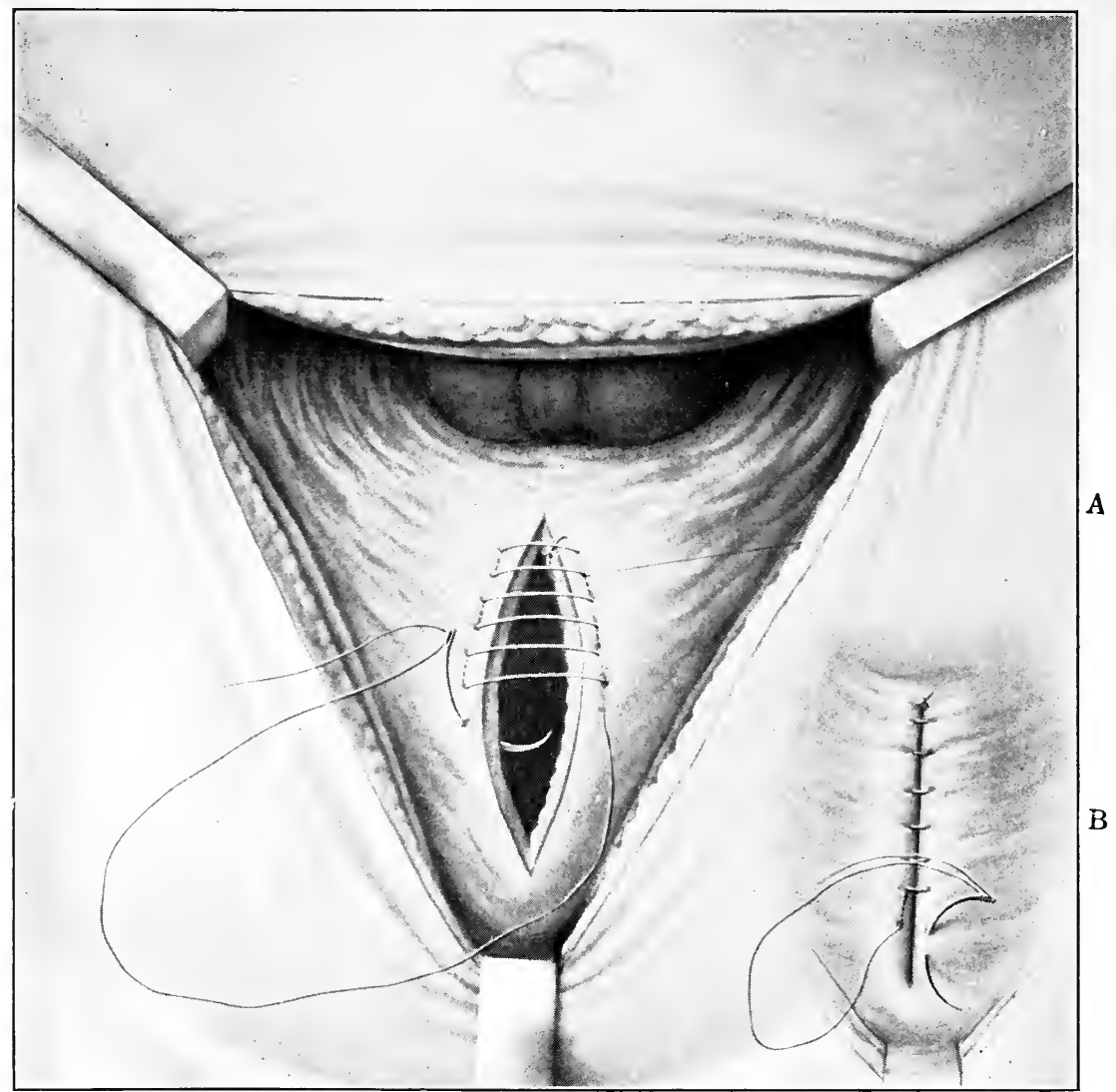

FIG. 200.- Suture of the bladder wall following intraperitoneal operation. A, First row. B, second row. (After Mayo.)

growths involving a large area of the bladder wall are excised with a portion of the healthy surrounding structure as well (Fig. 199). Where the vicinity of the ureter is involved by a malignant growth, the ureter must be tied with one ligature and severed outside the bladder and reimplanted at another site in the remaining portion of the viscus, having been drawn through an opening in the peritoneum 
for this purpose (Fig. 199). After removal of the required area of bladder structure, as well as any lymphatic nodes that may be found, the opening in the bladder is closed by a double row of sutures.

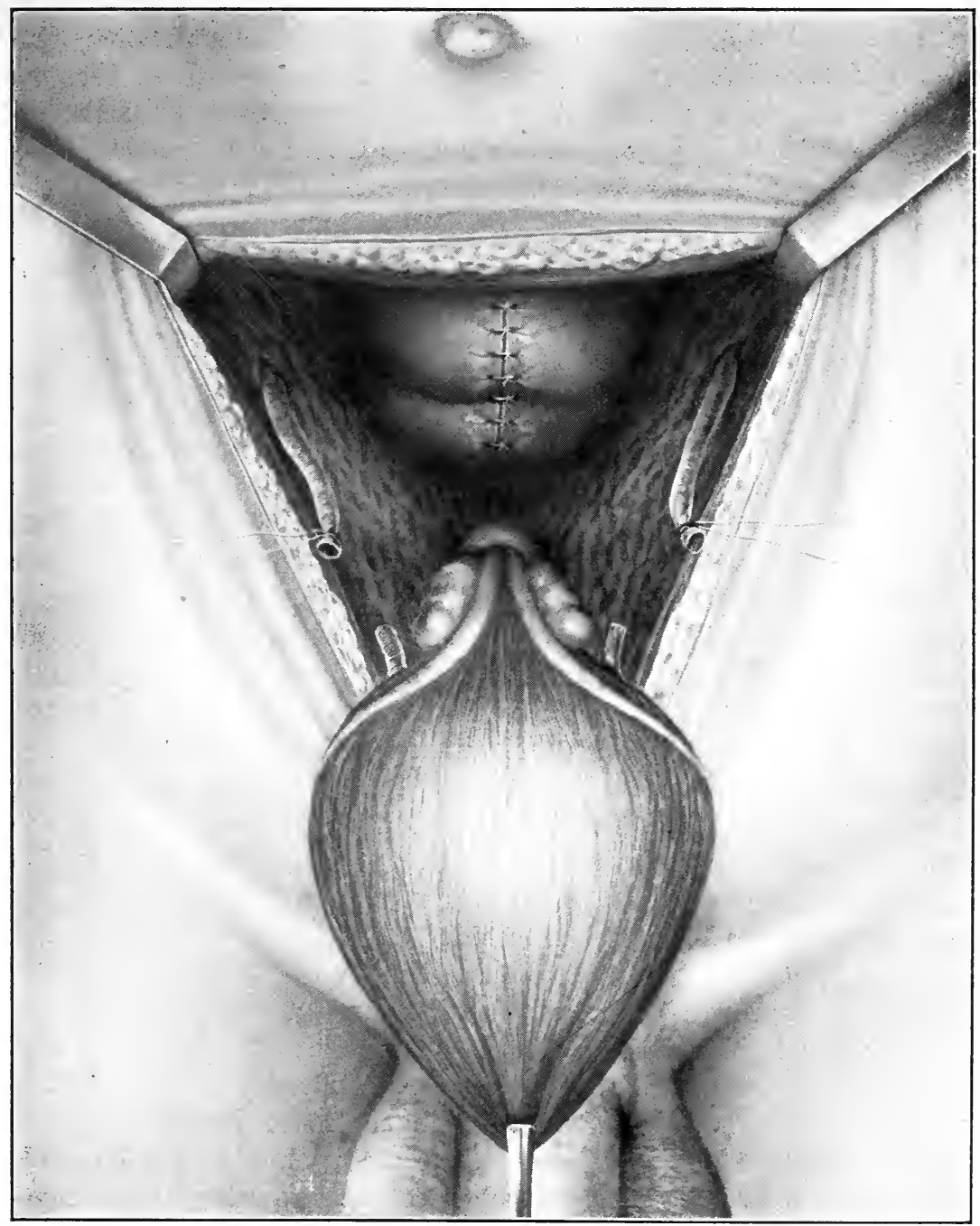

FIG. 201.-Complete extirpation of the bladder. (After Legueu.)

The first row, a mattress suture, includes all the coats and the second row of silk or linen forms a protecting line of peritoneal suture over the first line of closure ${ }^{1}$ (Fig. 200). The bladder is closed without 1 Mayo, Annals of Surg., July, 1908. 
drainage. The peritoneal cavity may or may not be drained, according to choice.

Diverticulum of the bladder is handled surgically according to the nature and extent thereof which is determined by previous cystoscopic investigation and roentgenography (p. 129). In some instances extraperitoneal cystotomy is performed and the partition divided between the sacculus and the main bladder cavity accompanied by the removal of contained calculi. In other cases where the diverticulum is attached to the bladder by a prolongation or neck or includes the opening of one of the ureters, its complete removal demands the same technic as for vesical neoplasm, namely, transperitoneal cystotomy and possibly ureteral implantation.

Total Removal of the Bladder.-This operation involves transperitoneal exposure of the bladder much the same as that already described for partial removal. In this instance, however, preliminary provision must be made for the diversion of the urinary secretion, and most authorities favor the external nephrostomy which provides for the reception of the urine through the lumbar region rather than transplantation of the ureters by intestinal anastomosis. When this important requirement has been accomplished in a previous step (see p. 639), the removal of the bladder is simplified thereby. The median incision having been made, as already described, the peritoneum is freely separated from the surface of the bladder and is then closed by careful suture to avoid contamination. The remainder of the operation is carried on by blunt dissection, the vesical cavity being excised from its urethral attachment including the prostate or at the beginning of the prostatic urethra (Fig. 201). After control of the hemorrhage, the wound is closed with adequate drainage.

Rupture of the Bladder.-In case of extraperitoneal rupture, the operation is simple and the technic is similar to that of suprapubic cystotomy. Through the suprapubic incision, the rent in the bladder is found; this is closed by a double suture line (see Fig. 197) and drainage accomplished through a separate suprapubic opening if the urine is infected, or by an indwelling catheter in the urethra if the urine is clean and uninfected. The pelvis should then be carefully washed out with a mild antiseptic solution and dried with gauze wipes (see Suprapubic Cystotomy, p. 460).

Intraperitoneal Rupture.- In this condition, the bladder is exposed by transperitoneal incision, the same as that for reaching the bladder for partial removal. The abdominal cavity is carefully cleansed and mopped free of urine. The closure of the tear is effected in the same manner as set forth in suture of the bladder following partial removal 
(Fig. 200), the sutured area always being protected by a peritoneal covering. In case of extensive rupture low down on the posterior surface, involving the Douglas cul-de-sac, extra- as well as intraperitoneal, after repair by suture of the intraperitoneal laceration it is well to drain the prevesical space (see p. 467).

Exstrophy of the Bladder.-Many operations for this condition are difficult of performance and unsatisfactory in the result. The exceptions are Maydl's operation and Peter's modification of the same.

Maydl's Operation.-This technic involves the implantation of the whole trigonal segment, including the ureteral orifices, into the structure of the sigmoid, segregating a loop of that portion of the intestine for this purpose, and combining with a lateral anastomosis of the same so as to divert the current of feces away from the area of the implanted ureters.

Ureteral catheters are first passed up into both of the ureters. The exposed bladder is dissected free extraperitoneally, and totally excised with the exception of a quadrilateral section embracing the two ureteral openings which is left intact with the attached ureters.

The abdominal cavity is then opened by transperitoneal incision. A loop of the sigmoid flexure of the colon is drawn down and sutured together as for lateral anastomosis, which anastomosis is made at the base of the loop; the bowel is then returned to the abdomen with the exception of the rounded top, at which point, the trigonal implantation is effected by a double row of sutures similar to those used in gastroenterostomy, the mucous membrane and muscular coats being sutured separately. The sigmoid flexure is anchored to the abdominal wall and the wound is closed. In spite of the risk of peritoneal infection in this operation, the results recorded are good.

Peter's Operation.-This procedure is entirely extraperitoneal. Each ureter is dissected with its sphincteric opening and is implanted through a separate slit made in the rectum, the vesical end of the ureter being left free in the rectal lumen. The rectal slit is closed by a double row of sutures the outer coat being attached to the circumference of the ureter.

Suprapubic Puncture and Drainage.-In certain cases of complete retention of the urine, when the urethra is found to be entirely impassable, as in cases of prostatic hypertrophy and stricture of the urethra, suprapubic puncture and drainage may prove the means of temporarily relieving an otherwise painful and serious situation and justify the delay of operation when local or general conditions do not favor such an undertaking. The bladder is generally distended above the pubic bone. The parts are first thoroughly shaved and 
cleansed. A small area about 2 centimeters above the pubic bone is infiltrated with five-tenths per cent. novocain solution. A goodsized cannula and trocar is prepared and a small-sized catheter is selected that may be readily introduced through the lumen of the cannula. The catheter should be of a size about No. 9 F. scale and the cannula proportionately larger. With the trocar in place and the parts anesthetized, the bladder is punctured by a careful thrust, the trocar is then withdrawn and, while the urine is flowing, the catheter is passed through the cannula and enough of its length introduced to reach the floor of the bladder (Fig. 202). The cannula is

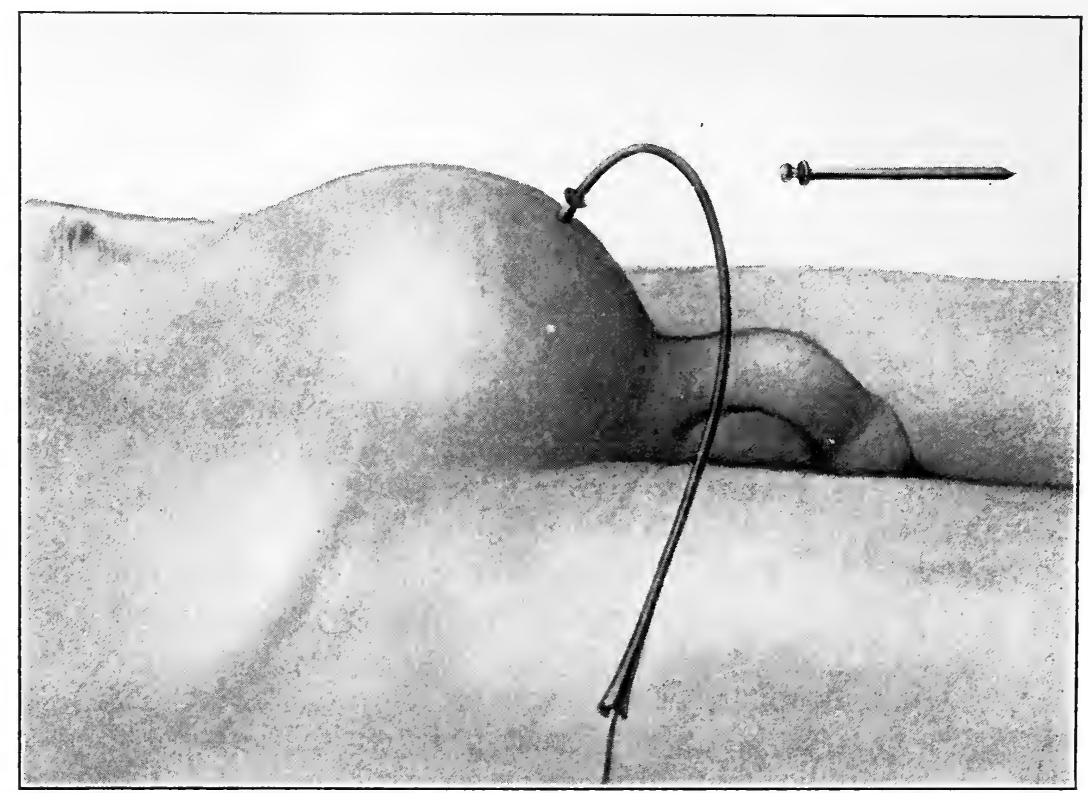

FIG. 202.-Suprapubic puncture and drainage for complete retention of urine.

then carefully withdrawn without disturbing the catheter. After the bladder is emptied the catheter is attached, by means of a glass union and rubber tube, to a drainage bottle or urinal.

Retrograde Cysto-urethrotomy through Suprapubic Cannula.-The preceding expedient (Fig. 202) is utilized also for solving the difficulty arising out of impassable urethral stricture, to save the unnecessary time involved in performing a perineal urethrotomy without a guide. The technic (Sinclair ${ }^{1}$ ) is a combination of suprapubic puncture and cystoscopy through the suprapubic cannula. The steps are as follows:

${ }^{1}$ N. Y. Med. Journ. April 4, 1914. 
The cannula and trochar are introduced as described above for drainage (Fig. 203, 1). The bladder is then thoroughly cleansed, by way of the cannula, as for cystoscopy, until the returning fluid is perfectly clear. Six or eight ounces of fluid are retained in the bladder, and an indirect cystoscopic telescope, which fits the cannula exactly, is intro-

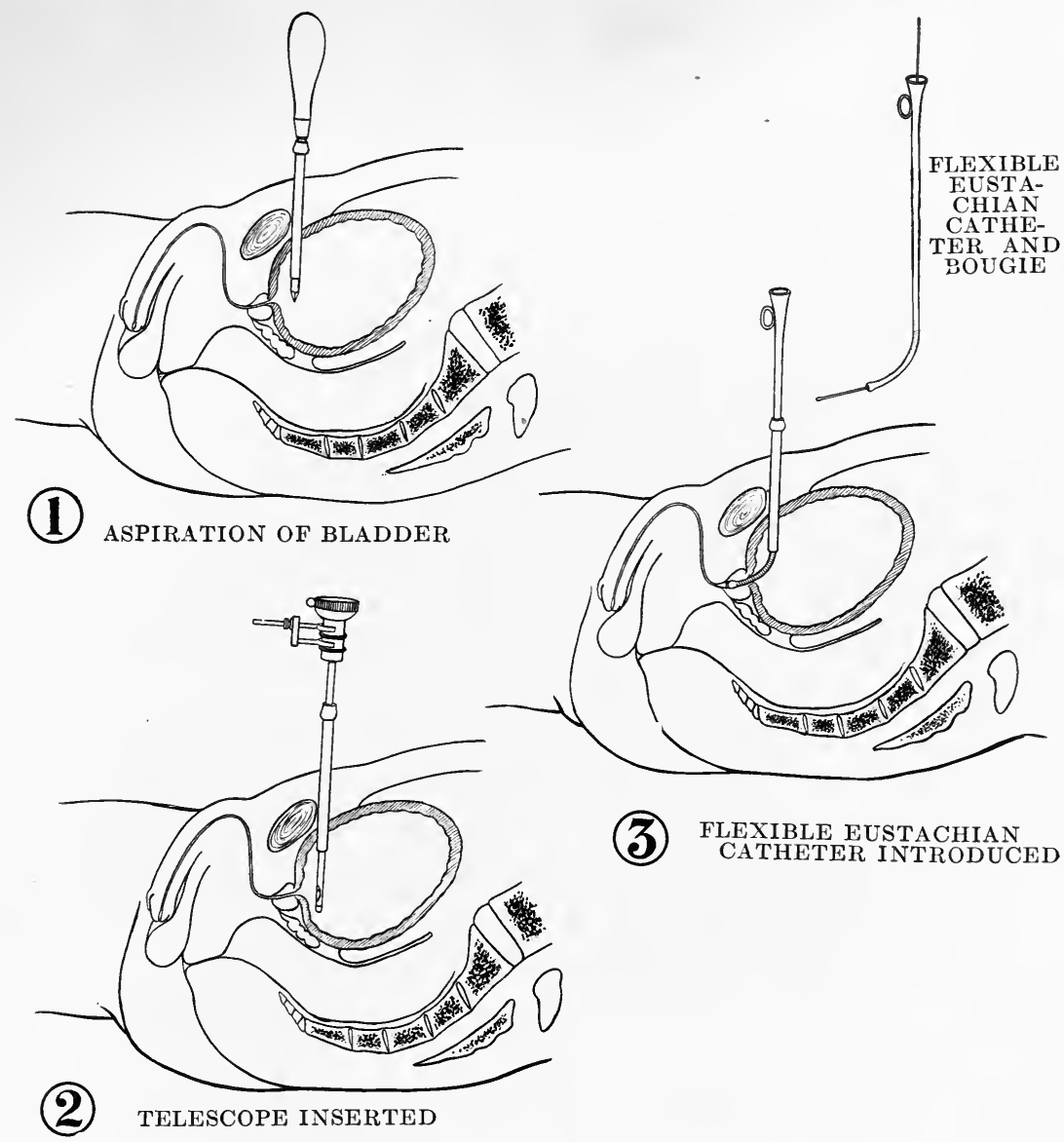

FIG. 203.-Retrograde Cysto-urethrotomy through Cannula.

duced (Fig. 203, 2) and through it the internal urethral orifice is viewed with absolute clearness. The distal end of the cannula being directly applied to the bladder outlet, the telescope is removed and is replaced by a flexible Eustachian catheter, which, when its obturator is removed, it will be found, will readily enter the urethral orifice (Fig. $203,3)$ and pass into the posterior urethra. A gloved finger intro- 
duced into the rectum may now determine the satisfactory accomplishment of this proceeding. In some instances, the Eustachian catheter may be advanced into the membranous urethra. In other cases, an extra long filiform bougie may be passed through the cannula into the anterior urethra and out of the meatus. In the latter instance, an internal urethrotomy may be performed, by passing over the filiform as a guide, a urethrotome blade (Fig. 120, p. 384) having an eyelet (see internal urethrotomy, page 283) or, if the bougie is not passable as above, the patient is placed in the lithotomy position, and upon the Eustachian catheter, as a guide in the posterior urethra, the operation of external urethrotomy is performed (see p. 287) the posterior urethra being easily reached with the cannula as a guide, thus simplifying the operation, and avoiding the prolonged procedure of perineal section without a guide.

Cystostomy.-This procedure is required for permanent suprapubic drainage. A small longitudinal suprapubic incision is made, the bladder having been previously washed and distended with saline or normal salt solution. The overlying adipose tissues are pushed upward and two large silk sutures are inserted about 2 centimeters apart laterally. The bladder is then laid open by a longitudinal incision about 3 centimeters in length; while the bladder is held up with the two silk sutures, the mucous membrane is stitched by a continuous circular ineision into the skin, the silk sutures being then withdrawn, and a drainage tube is inserted.

\section{OPERATIONS UPON THE PROSTATE}

Prostatotomy.-The prostate may be reached by way of the urethra (external perineal urethrotomy, p. 287) when the incision is identically the same as that of external urethrotomy for exposing the urethra or for drainage of the bladder.

Intraurethral prostatotomy is performed for prostatic abscess, for chronic prostatitis, for the removal of calculi. In the case of chronic prostatitis, it may be resorted to simply as a matter of drainage. In the case of prostatic abscess, the urethra is opened when it is evident that the abscess is pointing in that direction on account of the interference with urination. Often, the abscess will be evacuated at the moment of incision of the urethra upon the staff or is ruptured by the introduction of the examining finger. When the pus flows through the opening into the urethra, evacuation is aided by passing the index-finger into the abscess cavity and gently separating the septa between the purulent foci, following which, the abscess cavity is 
cleansed with copious irrigation with boric acid or 1 per cent. protargol solution: a perineal drainage tube is then introduced, as already described (p. 287). The drainage tube is kept in place for three or four days. Although thoroughness is required in the performance of

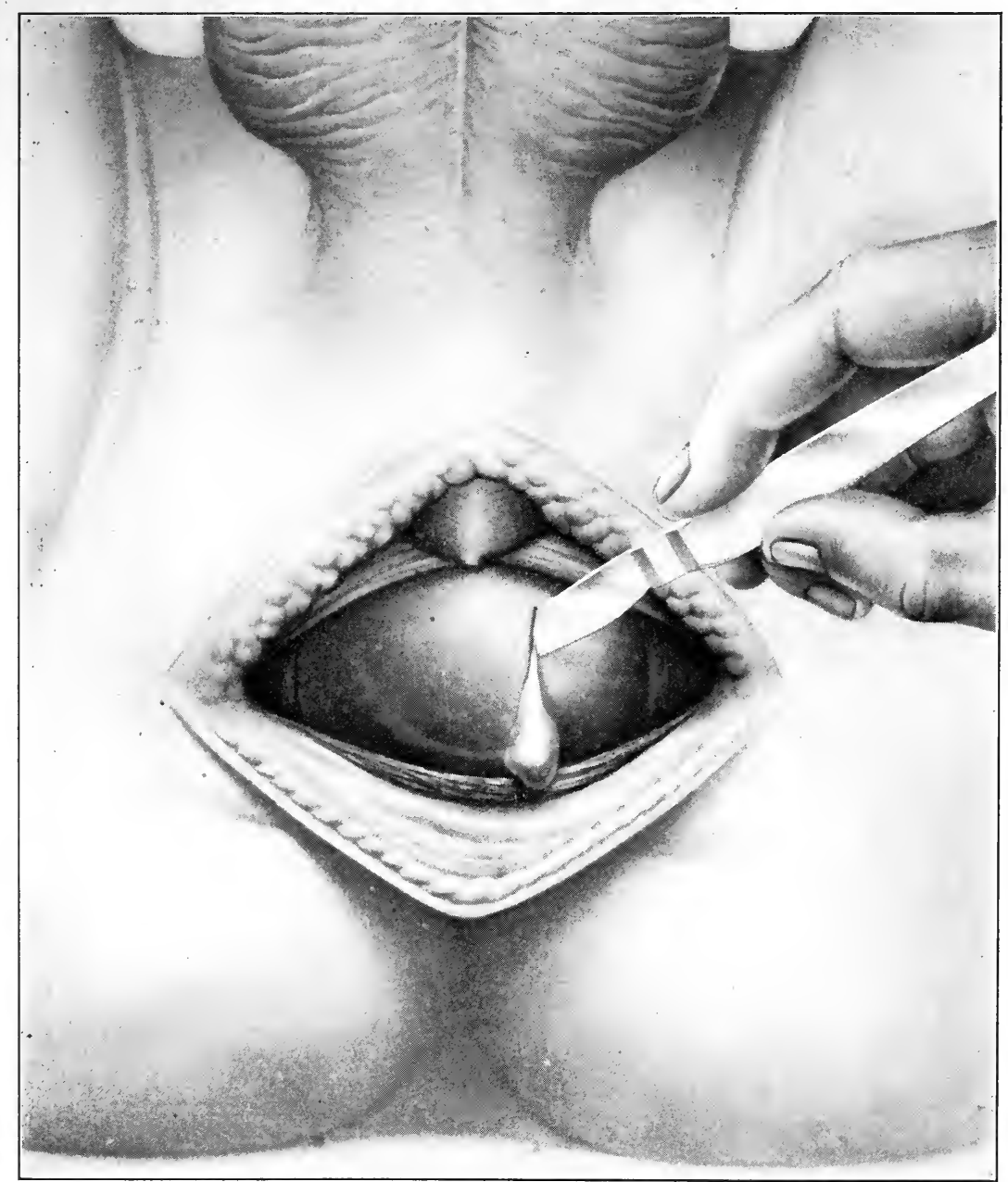

FIg. 204.-Incision of prostatic abscess through capsule. (After Legueu.)

this technic, great gentleness is necessary on account of the special tendency to epididymitis in these cases.

Complete enucleation of the prostate surrounding the abscess cavity may be accomplished in the same manner as an intraurethral prostatectomy $(q . v$.) but is seldom necessary. 
Prostatotomy for prostatic calculi is still the same operation and simply involves removal of the prostatic stones by a blunt or sharp scoop in case they are small and multiple, or extraction with a stone forceps when of larger dimensions.

Extraurethral prostatotomy or opening the prostate through the posterior surface of the gland is resorted to in the case of prostatic abscess which is pointing toward the posterior aspect of the prostate. The incision is the same as that for perineal prostatectomy when the gland is enucleated from behind and not through the urethra; Kocher's

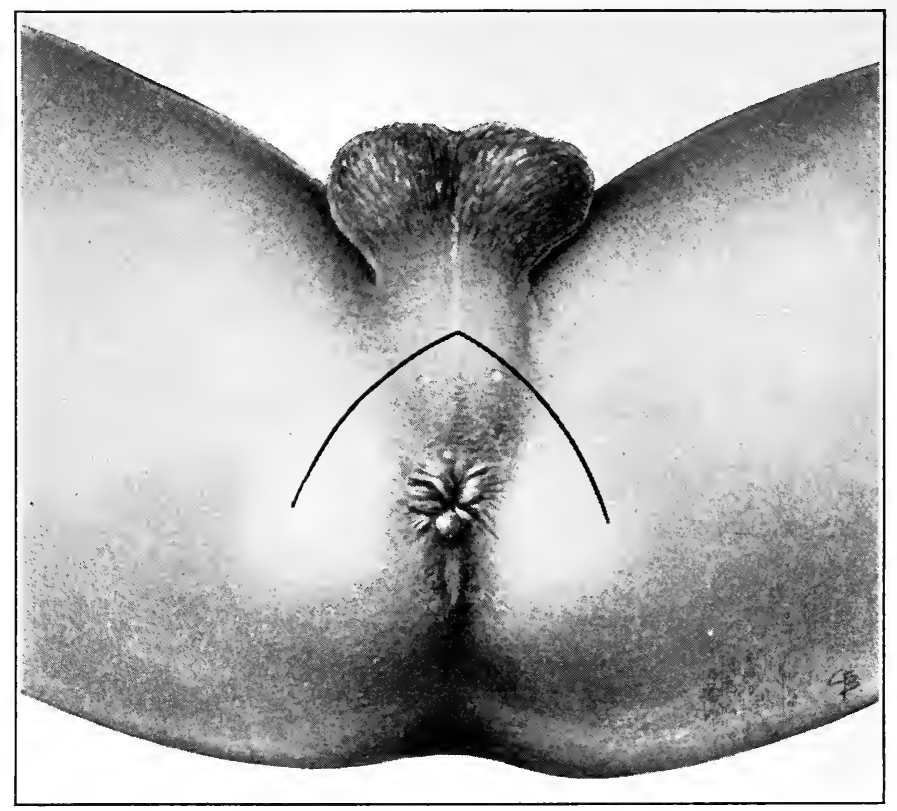

FIg. 205.-Outline of Krocher's incision.

incision or the reversed "V" may be used (see Figs. 205 and 209). The skin and superficial fascia are opened, a sound in the urethra gives the location of the bulb; by blunt dissection, the tissues are separated downward and backward until the bulging prostate is exposed behind the membranous urethra. The most prominent point is selected and incised with a knife or broken through with a blunt instrument (Fig. 204). The abscess cavity is emptied and thoroughly cleansed. Unhealthy prostatic tissue may be removed when it is apparent that it is broken down by multiple abscess foci. It is to be remembered, however, that here, as in the case of simple abscess 
elsewhere, satisfactory resolution is likely to follow complete and adequate surgical drainage.

Perineal Galvano-Prostatotomy.-This operation, which is the author's technic, may be spoken of as a modified Bottini operation, the latter being a galvano-prostatotomy within the urethra without a perineal opening (see p. 477). The indication for this operation has already been spoken of in the section on Contracture of the Neck of the Bladder, see p. 435.

The instrument is composed of a handle and sheath and several sizes of cautery blades (Fig. 206). The handle of the instrument is graduated so that the dimensions of the cut may be determined. The sliding of the knife is effected by traction instead of the rotation of a wheel, so that the operator readily appreciates the progress

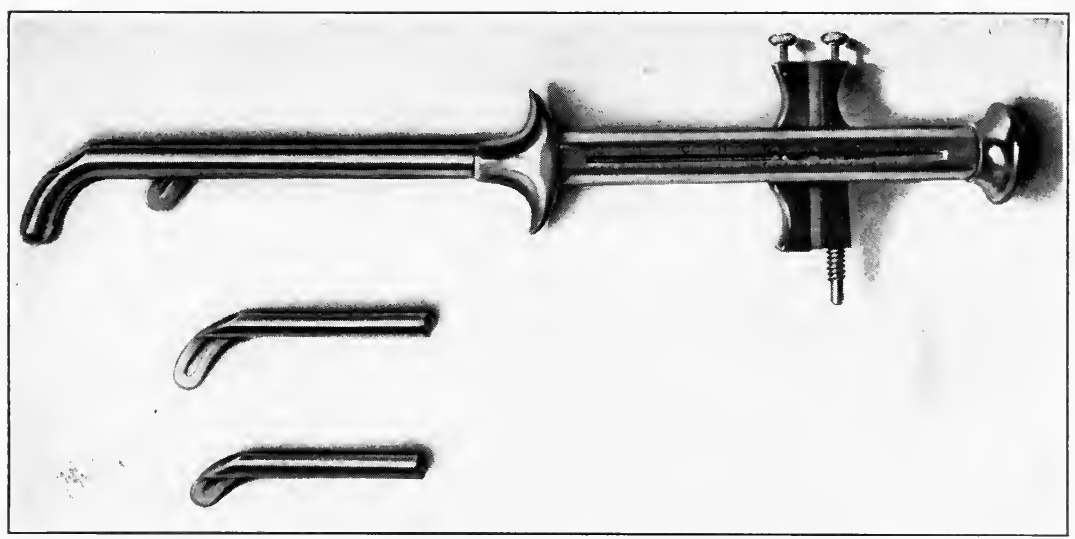

FIg. 206.-Author's galvanocautery prostatic incisor.

of the blade and the density of the tissues during cauterization. One hand is required to operate the instrument and the index-finger of the other hand is free to be introduced into the rectum. No cooling device is attached to the instrument. The circulation during the operation of a cold, sterilized solution through the urethra and out through the perineal wound is required to keep the handle of the instrument cool without affecting the blade (Fig. 207). The current is supplied by a storage battery or preferably from the street current with the aid of a motor transformer and rheostat. About 50 amperes are required to heat the knife to a white heat which is cooled to a certain extent as it passes through the tissues.

The patient is placed in the lithotomy position, the bladder having been previously washed with boric acid solution. The preliminary 
step of external perineal uretbrotomy is performed (see p. 287). The bladder having been reached, the staff is withdrawn and the finger introduced through the perineal opening. In cases of marked contracture, whether or not accompanied by hypertrophy, the ves-

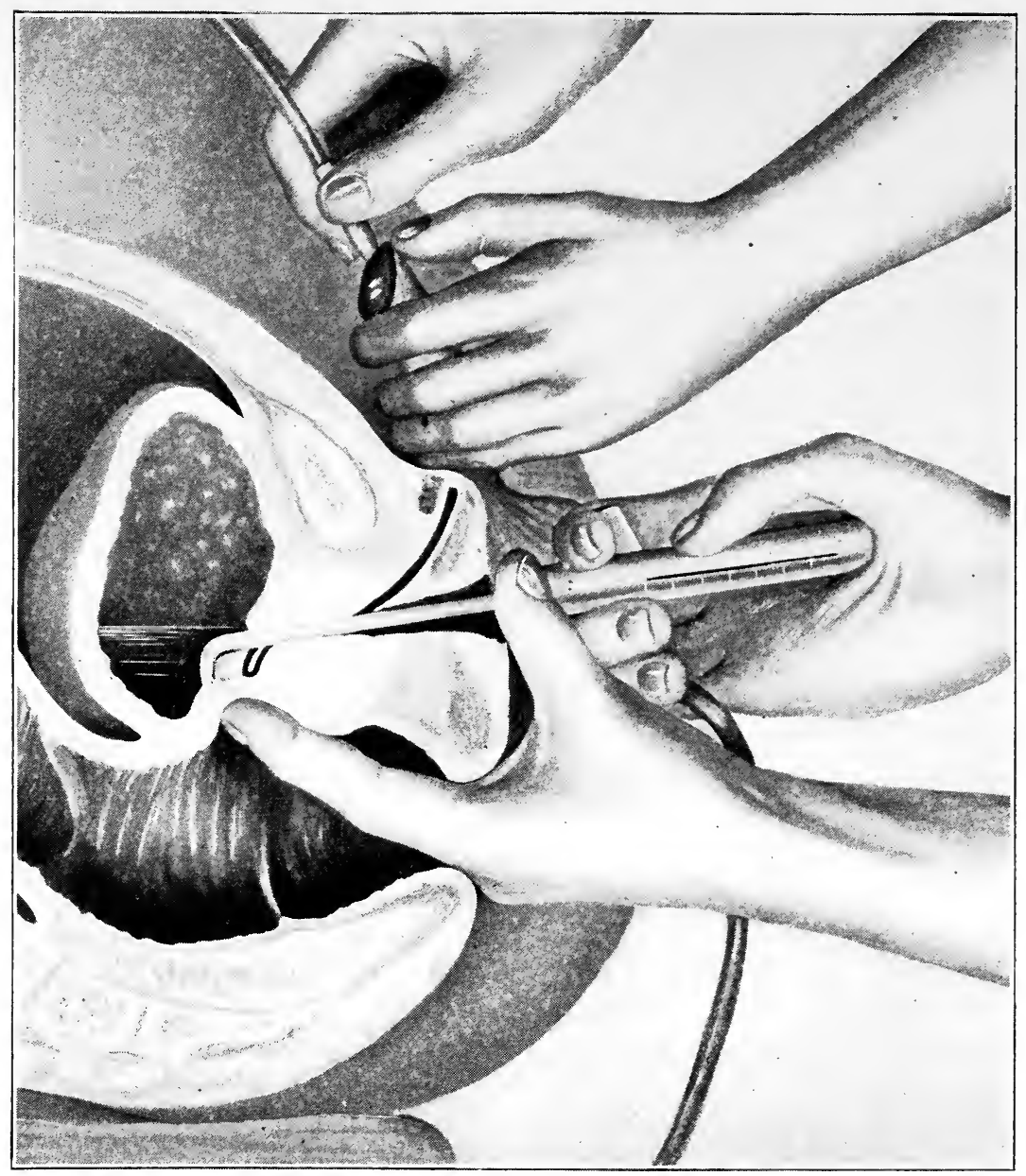

FIG. 207.-Galvanoprostatotomy. (Author's technic.)

ical orifice may be too tight to admit the examining finger. This opening is not forced and torn by the finger but is enlarged by the first incision of the cautery knife. The instrument, having been previously tested, is introduced through the perineal opening; the index-finger of the left hand feels the beak through the rectum; and irrigation through 
the urethra is commenced. The operator now gives the signal to turn on the current; ten seconds are allowed to heat the knife after which it is slowly unsheathed by drawing outward (Fig. 207). From onehalf to three-quarters of a minute's time is generally allowed to complete an incision of moderate length $(1-2 \mathrm{cms}$.) and return the knife to the sheath. A longer period is required for incision of greater length. After returning the knife to the sheath, the irrigation is forced through the perineal opening before withdrawal of the heated instrument. The finger of the operator is now gently introduced into the bladder and a careful exploration is made. In cases of simple contracture, a single incision of 1 centimeter is generally sufficient to complete the operation. In cases of wide hypertrophied collar formation, a double incision may be necessary, one on either side, or a second incision to deepen the first, the aim being to render the neck of the bladder readily accessible to the examining finger. Care should be taken not to draw the knife too far outward into the prostatic urethra or too deeply through the contracted tissue completely severing the internal sphincter beyond.

Following the galvano-cautery incision, a perineal drainage tube is introduced and the after-treatment is that following external perineal urethrotomy, the perineal tube being usually left in place one or two days according to the condition of the bladder. Daily washing of the bladder is required through the perineal tube, and, after its removal, by means of a catheter introduced through the perineal opening and later through the urethra (see External Urethrotomy p. 287).

\section{PROSTATECTOMY}

Prostatectomy, or removal of the prostate gland, may be effected through the perineum or suprapubically. For a discussion of the relative merits and indications of these two operative procedures, see p. 410.

Perineal prostatectomy is subdivided into intraurethral or median prostatectomy and extraurethral prostatectomy.

Intraurethral Perineal Prostatectomy.-The patient is placed in the lithotomy position under general anesthesia and the first step of the operation is that of an ordinary external urethrotomy, effort being made to enter the urethra as far behind the bulb as possible by drawing the latter forward with a retractor, and incising the recto-urethralis muscle surrounding the membranous urethra. The finger is then slipped into the neck of the bladder through the membranous urethra, 
the staff having been removed (p. 287). The general contour of the prostate is carefully palpated with the examining finger. If the enlarged prostate is bilateral and symmetrical in type, a point is selected on the lateral wall of the prostatic urethra. A sharp-pointed bistoury is

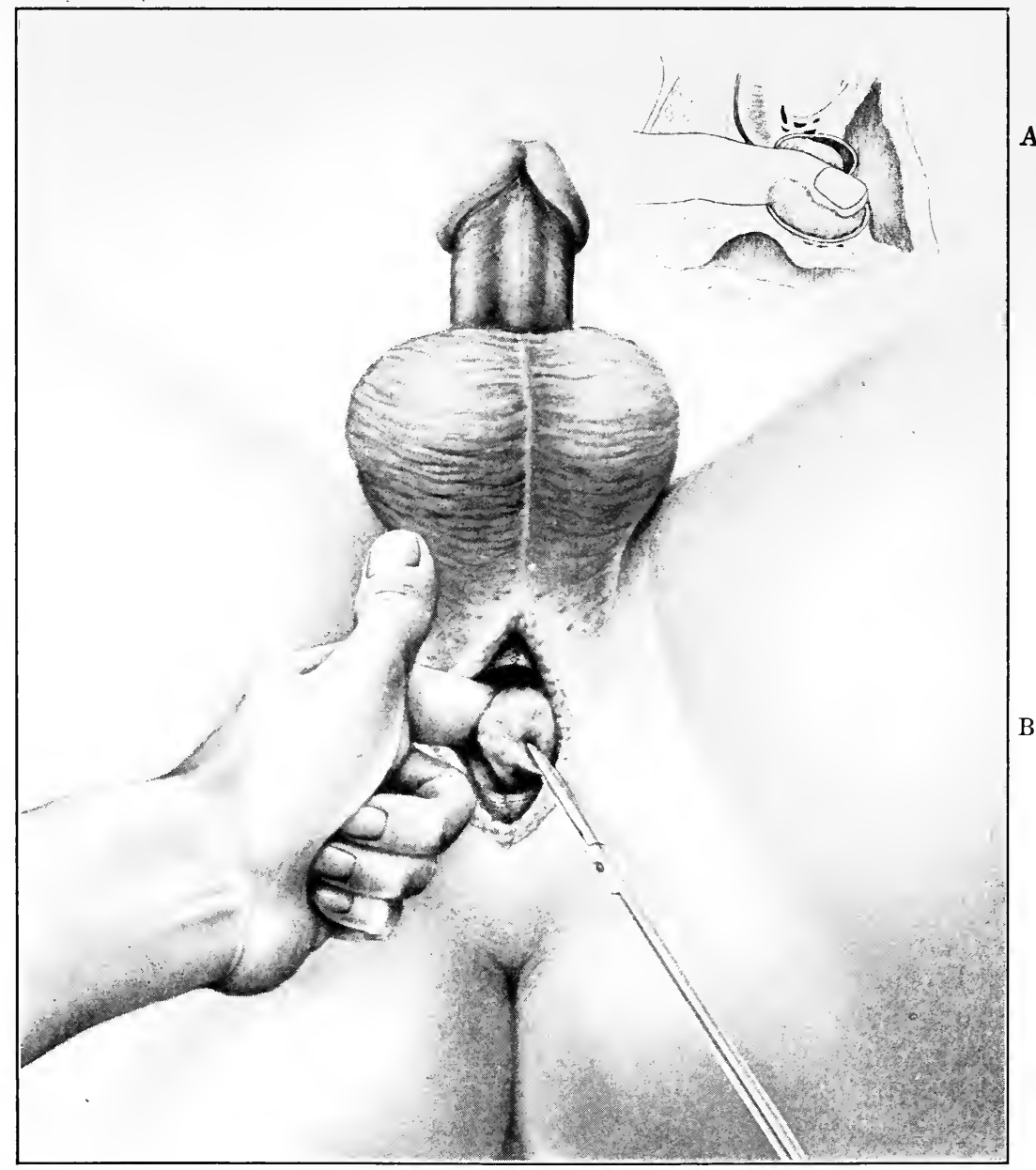

FIG. 208.-Perineal prostatectomy (intraurethral). A, Enucleation of prostate from capsule. B, Removal of lobe through perineal wound.

introduced into the prostatic canal and a longitudinal incision is made laterally at the point selected. This incision must be made of sufficient depth to reach the glandular tissue. The forefinger is then thrust through this opening and feels for the line of cleavage between the glandular nodes and the capsule which should not be difficult to 
find (Fig. 208, A). Enucleation is continued in this manner and when a portion of the gland can be seized with the volsellum forceps, assistance is obtained by traction (Fig. 208, B). The urethral mucous membrane is separated from the liberated lobe when possible but usually it is more or less torn during the process of removal. The same procedure is repeated on the opposite side and when a middle lobe is also present, it is enucleated by passing the finger back into the bladder; or, when the lobe is pedunculated, it may be drawn down into the perineal wound by a tenaculum or tractor and removed.

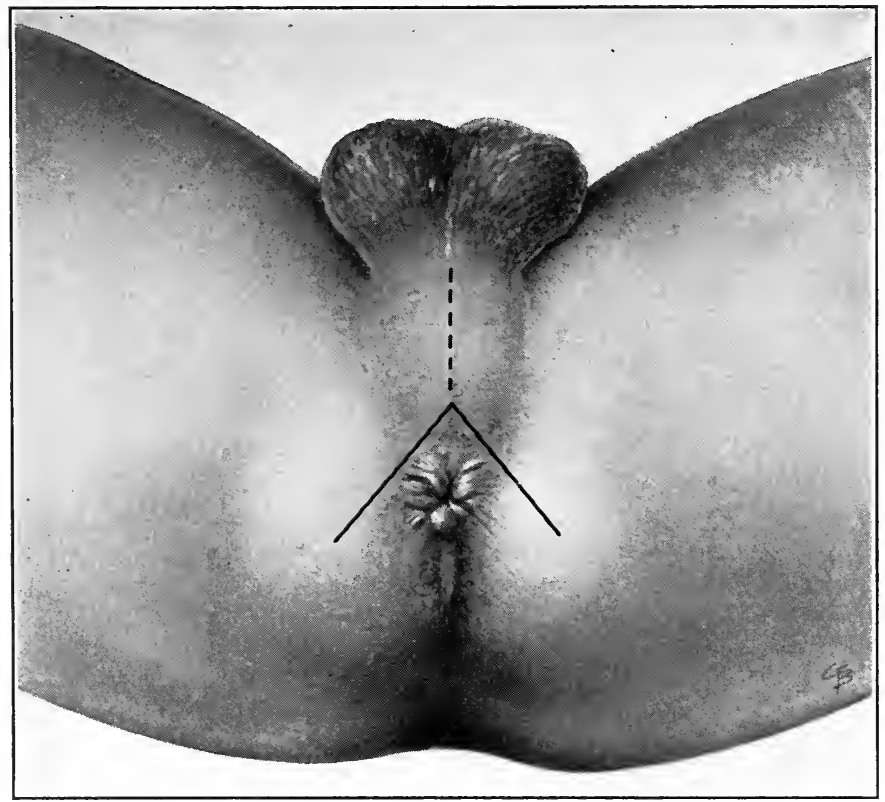

FIG. 209.-Outline of perineal incisions, median and inverted V.

When the prostatic growth, however, is found before operation to be largely intravesical, the suprapubic method of operating affords a more direct route of reaching this type of enlargement (see p. 485). Following removal of the prostate, the cavity is carefully packed with gauze wicks $3 \mathrm{~cm}$. wide, spread with formaldehyd gelatin (glutol), a drainage tube is inserted and fastened in the bladder, and the directions given for perineal drainage are followed (see p.458).

Extraurethral Perineal Prostatectomy.-The patient is placed in an extreme lithotomy position so that the perineum is raised on a horizontal plane. A sound is introduced into the urethra. A prerectal 
incision is made in an inverted" "V" (Fig. 209) shape from about 5 centimeters ( 2 in.) in front of the anus backward to a point midway between the anus and the tuberosity of the ischium on either side. ${ }^{1}$

This incision is carried through the superficial structures and is

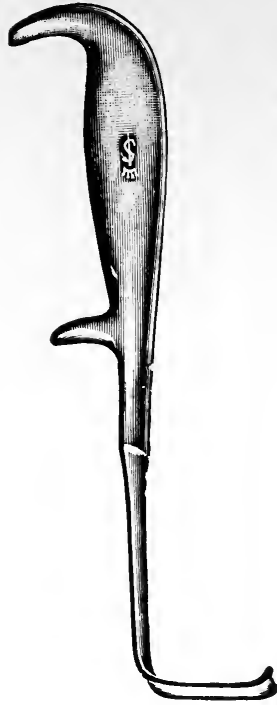

A

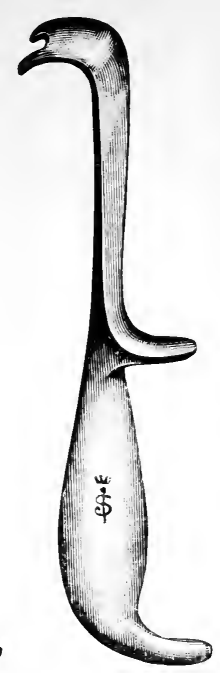

$\mathrm{B}$

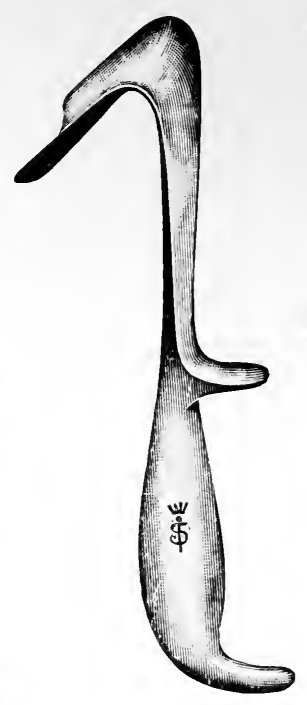

C

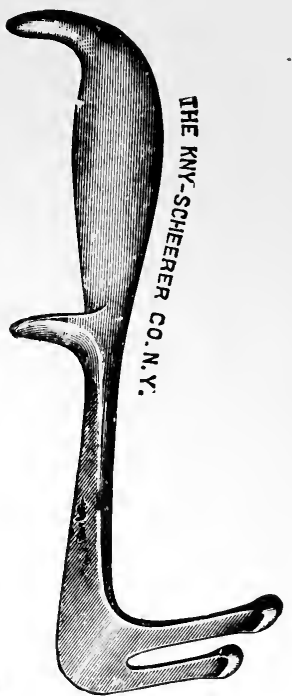

D

FIG. 210.-Retractors for extraurethral perineal prostatectomy. (Young.)

deepened by blunt dissection with the handle of the knife. A bifid retractor is then inserted (Fig. 210, D) to draw the tissues backward and stretch the central perineal tendon, which is incised just behind the bulb and as far from the anus as possible. This is important to

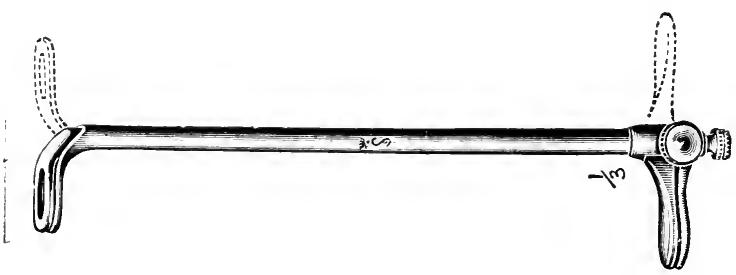

FIG. 211.-Prostatic tractor. (Young.)

avoid wounding the rectum, but care should also be taken not to injure the vascular bulb of the urethra. The Young bifid retractor (Fig. 210,B) is then used to pull forward the bulb and expose the membranous urethra covered by the fibres of the recto-urethralis

1 Technic of Young. 
muscle, which latter are divided with scissors as near the urethra as possible. This muscle marks the point where lies the plane of cleavage between the prostate and rectum, as after severing the muscle, the rectum is readily separated and pushed away from the prostatic capsule without great bleeding. The posterior bifid retractor is then withdrawn and a broader flat retractor and two lateral retractors in-

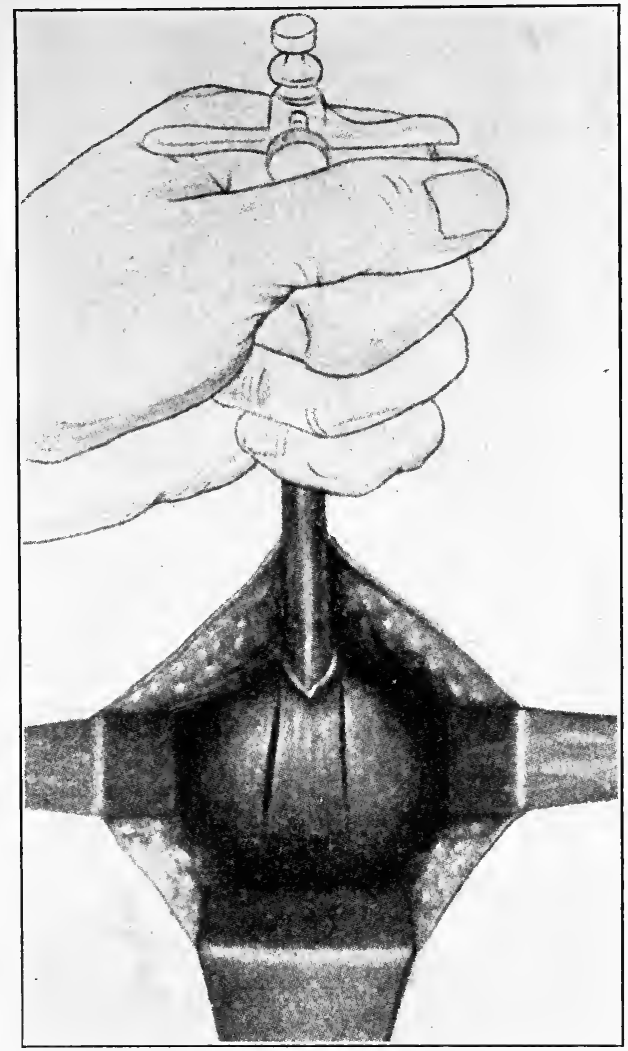

FIG. 212.-Perineal prostatectomy-extraurethral. Traction downward on prostate. (Young.)

serted (Fig. 210, a and c); the membranous urethra is opened upon a sound at the apex of the prostate. This opening may be held apart by means of a suture passed through each lip of the incision. The sound is then removed and Young's prostatic retractor (Fig. 211) is inserted into the bladder and opened. Traction is then exerted outward (see Figs. 212 and 213) which brings the prostate bulging into the perineal wound. Two vertical incisions are now made in the capsule of the 
prostate, one on either side, to ${ }^{*}$ cover the entire length of the lobe of the prostate about $1.5 \mathrm{~cm}$. deep on either side of the median line,

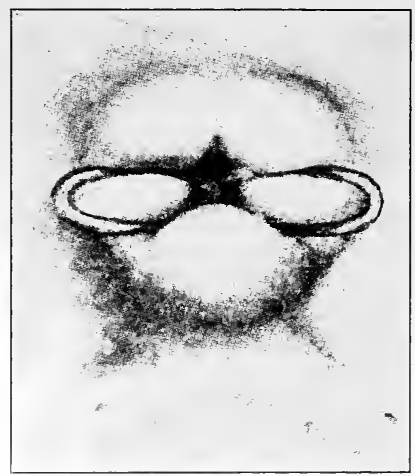

Fig. 213.- Same as Fig. 212. Vesical view.

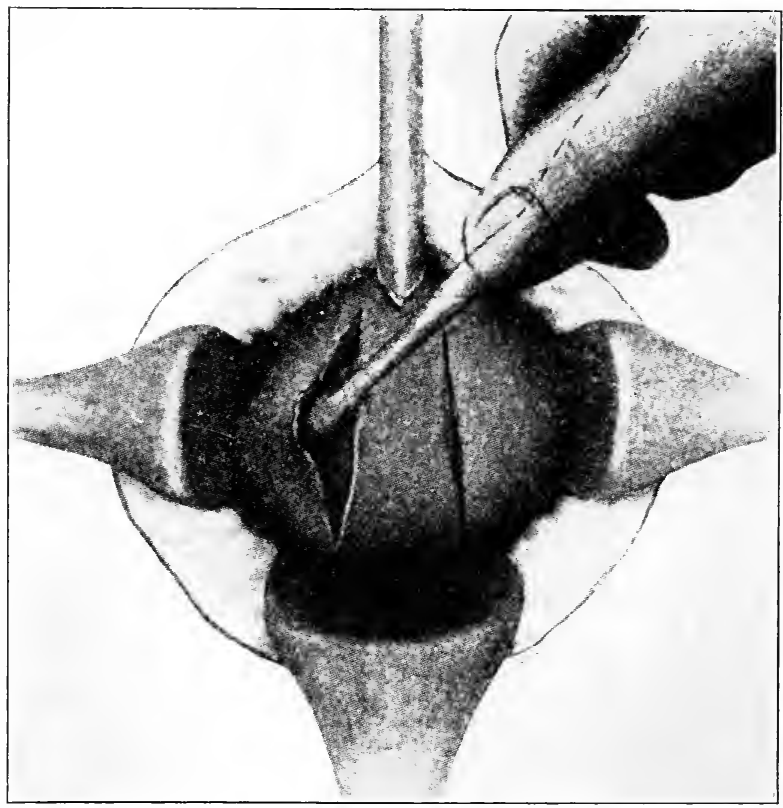

Fig. 214.-Perineal prostatectomy. Extraurethral enucleation of lateral lobe. (Young.)

about $1.5 \mathrm{~cm}$. apart in front and $1.8 \mathrm{~cm}$. behind. The finger is then introduced through the incisions and finds the proper line of cleavage between the smooth capsule and the lobulated hypertrophied 
tissue. The plane of cleavage is followed around as far as possible, gentle traction being exerted upon the prostatic tissue by means of a volsellum or prostatic forceps. A blunt instrument like a periosteal elevator may be employed to assist the separation (Fig. 214) of the lobes until the point is reached where it adheres to the urethra, when a sharper instrument may be required, either scissors or small bistoury. This manœuvre is repeated on the opposite side. When a middle or intravesical lobe also exists, the tractor is turned upon this portion and it is drawn into the cavity left by the already enucleated lateral lobe. The bladder is irrigated copiously and the prostatic cavity loosely packed with gauze. The edges of the levator ani muscle are then brought together by a single chromic gut stitch in the midline to protect the rectum. A double current drainage tube is inserted through the urethra into the bladder, after ligating all vessels, care being observed that undue pressure is not exerted by the packing upon the rectal wall. Continuous irrigation is kept up until there is no bleeding; the perineal tube is removed after forty-eight hours and, as in all cases of perineal prostatectomy, the patient is gotten out of bed as early as possible.

\section{Instruments Required for Perineal Prostatectomy}

Scalpel.

Sound (medium-sized).

Urethral staff.

Hemostatic forceps.

Thumb forceps.

Scissors.

Blunt and sharp periosteal elevator.

Bifid bulb retractor (Young).

Bifid rectal retractor (Young).

Broad posterior retractor (Young). Extraurethral operation.

Lateral retractors (Young).

Prostatic tractor (Young).

Prostatic forceps.

Needles and needle holder.

Double current drainage tube.

Suprapubic Prostatectomy.-This operation is performed for the removal of the prostate in one stage under advantageous conditions, that is to say, when the patient's general condition warrants it and when the local bladder conditions are not unusually septic. The operation is more properly performed in two steps when the general 


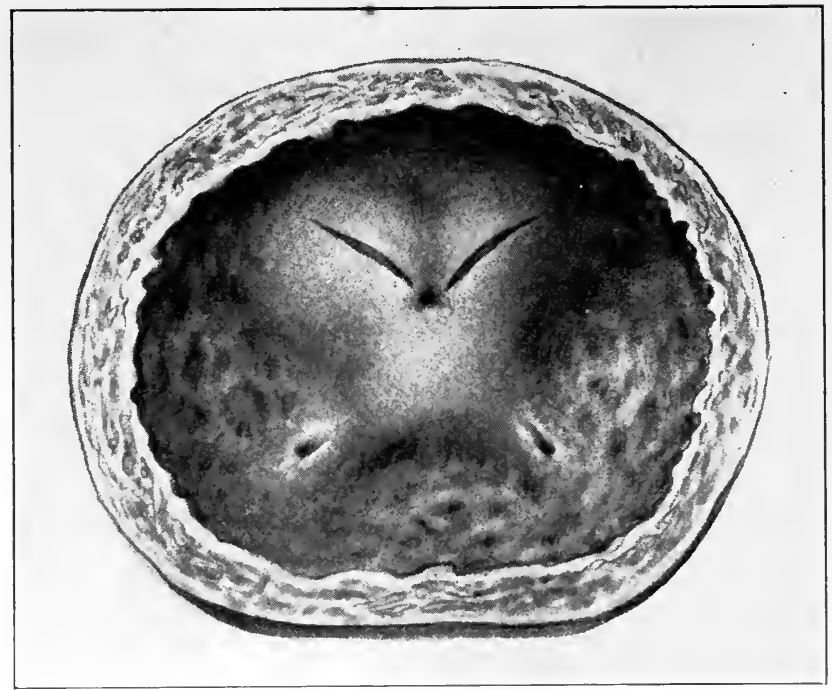

Fig. 215.-Suprapubic prostatectomy. Incision of capsule.

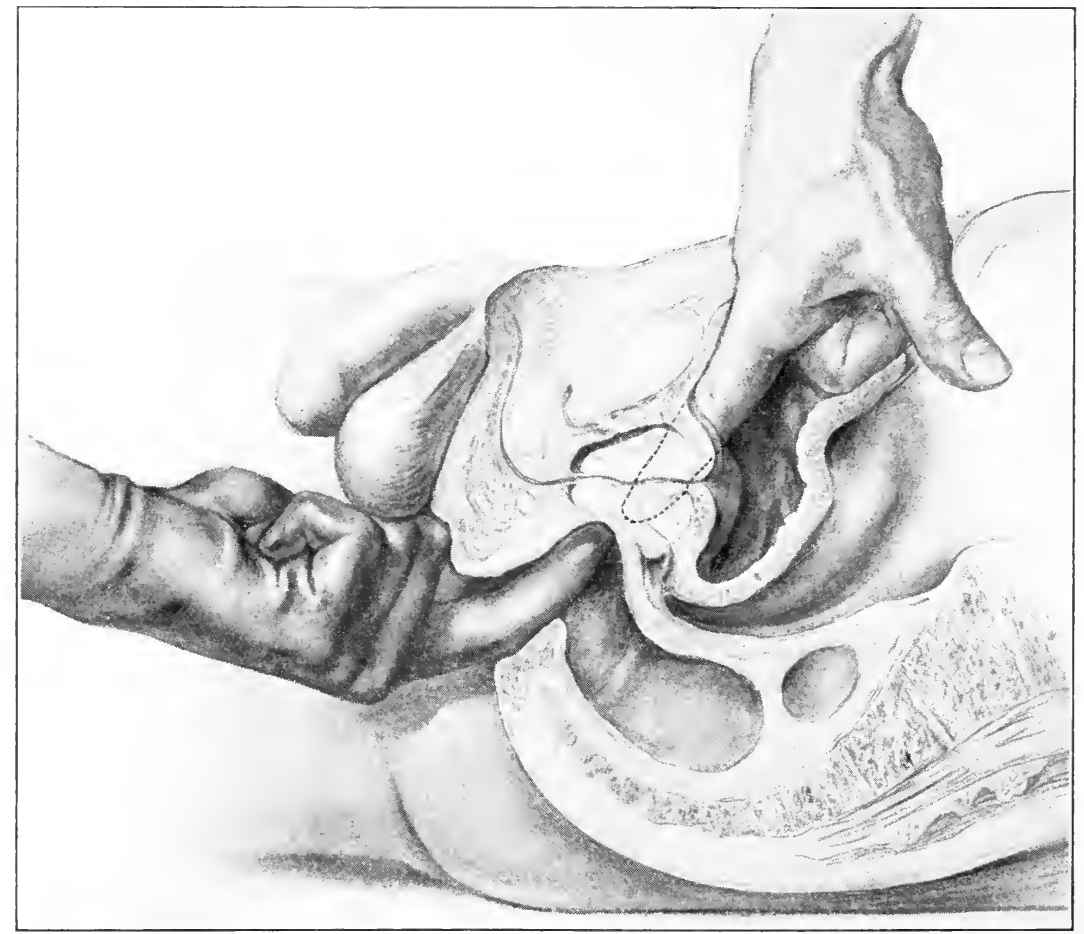

FIG. 216.-Suprapubic prostatectomy. Finger in rectum assisting enucleation. 
condition of the patient is poor on account of great debility, urinary sepsis and kidney insufficiency. Under these conditions, the preliminary step of opening the bladder for drainage, namely, suprapubic cystotomy (see p. 460), will cause an improvement in the local conditions and tend to fortify the patient for the subsequent operation. The preliminary step may be performed under local anesthesia or under a short general anesthesia. When calculus is present,

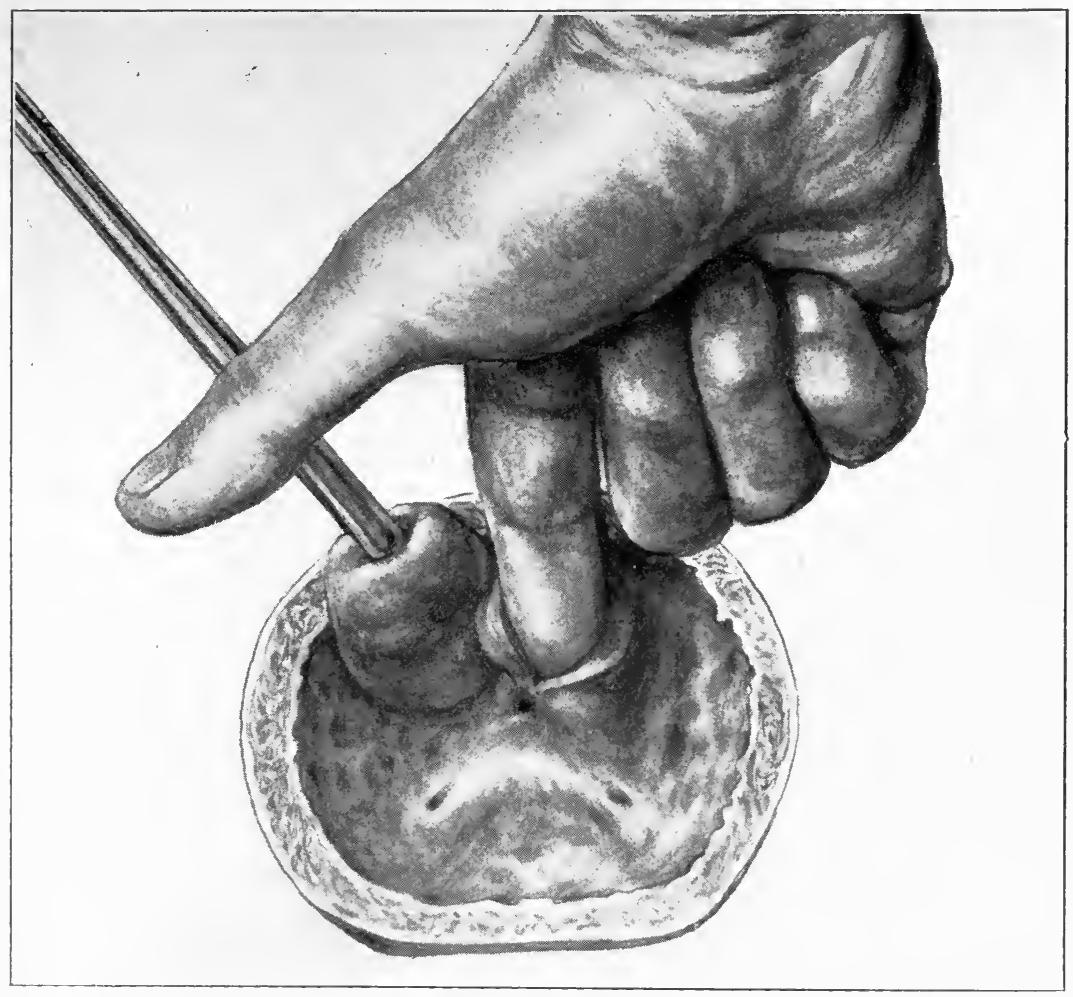

Frg. 217.-Suprapubic prostatectomy. Removal of one lobe and digital separation of the other.

removal of the latter is accomplished and the beneficial effects of preliminary drainage insured before the final removal of the hypertrophied glands several days later.

The bladder having been reached by suprapubic cystotomy (see p. 460) special retractors are introduced into the bladder (see Fig. 195), the patient is placed in a moderate Trendelenburg position so that the parts around the neck of the bladder may be inspected. An 
incision is then made through the mucosa upon the most prominent portion of the prostate on one side of the median line or within the prostatic urethra (Fig. 215). The end of the finger next seeks the proper plane of cleavage, which, when found, aids materially in

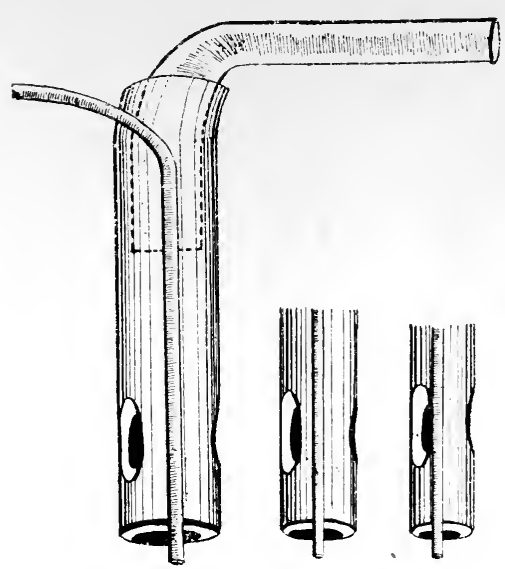

FIG. 218.-Suprapubic drainage tube with irrigating attachment. the rapid progress of enucleation. Following this line of cleavage, the forefinger separates the lateral lobes on all sides down to the apex of the gland. Assistance is had by introducing two fingers of the opposite hand into the rectum (Fig. 216). When one lobe or the entire gland is freed, it is grasped by a volsellum forceps and extracted through the vesical incision (Fig. 217). When such enucleation can be accomplished rapidly without much tearing of the mucosa, the amount of bleeding is inconsiderable, but usually the urethra is torn and hemorrhage results which may become very copious. The ease with which the prostatic tissue is removed varies with every individual case. When the capsule is not adherent on account of chronic inflammation, and when the amount of fibrous tissue within the prostatic structure is not great, the operation is relatively easy and the reverse conditions render the operation so much the more difficult. If there is little bleeding after enucleation, on account of the smoothness with which it has been accomplished, no fear need be felt as to subsequent hemorrhage, but when the bleed-

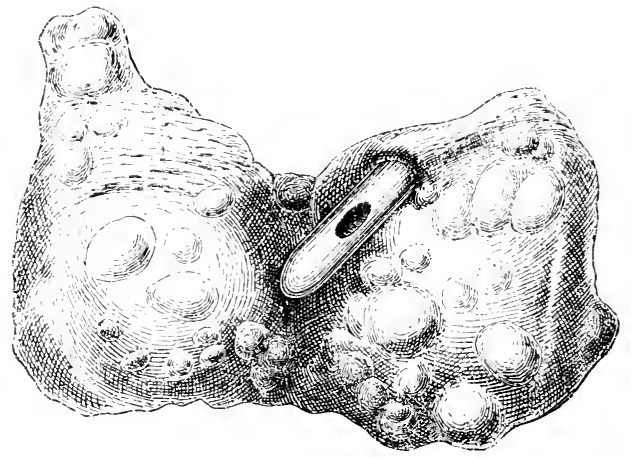

Fig. 219.-Carcinoma of prostate removed by suprapubic section. ing is copious and there has been much laceration of the tissues, it is safer to have recourse to some means of packing the cavity to render excessive post-operative hemorrhage impossible. For this purpose, I have used with success a long piece of narrow tape wound up in the form of a rosette, attached? at both ends to a piece of silk, one end of 
which passes through the urethra and the other out through the suprapubic wound so that by pulling the urethral end of this packing it makes firm pressure on the walls of the prostatic cavity, while pulling on the other end will unfold the tape and permit its easy removal. This special tampon or rosette is thoroughly immersed in balsam of Peru before introduction which further adds to the facility of its removal. A very practical and effective substitute for packing as a means of controlling hemor-

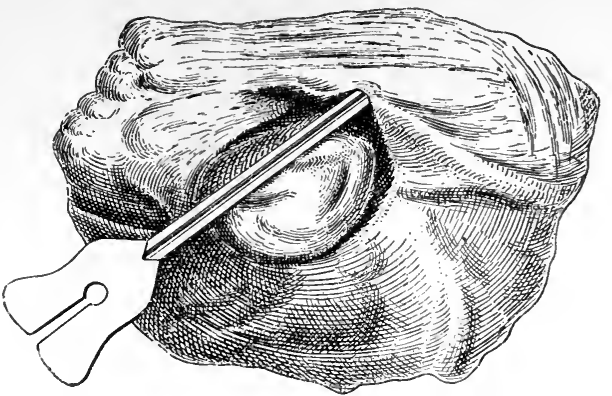

FIG. 220.-Saecimen of prostate removed by suprapubic section.

rhage is the rubber tampon bag suggested by Hagner. This device, when inflated fills the cavity left by the removed prostate. It is

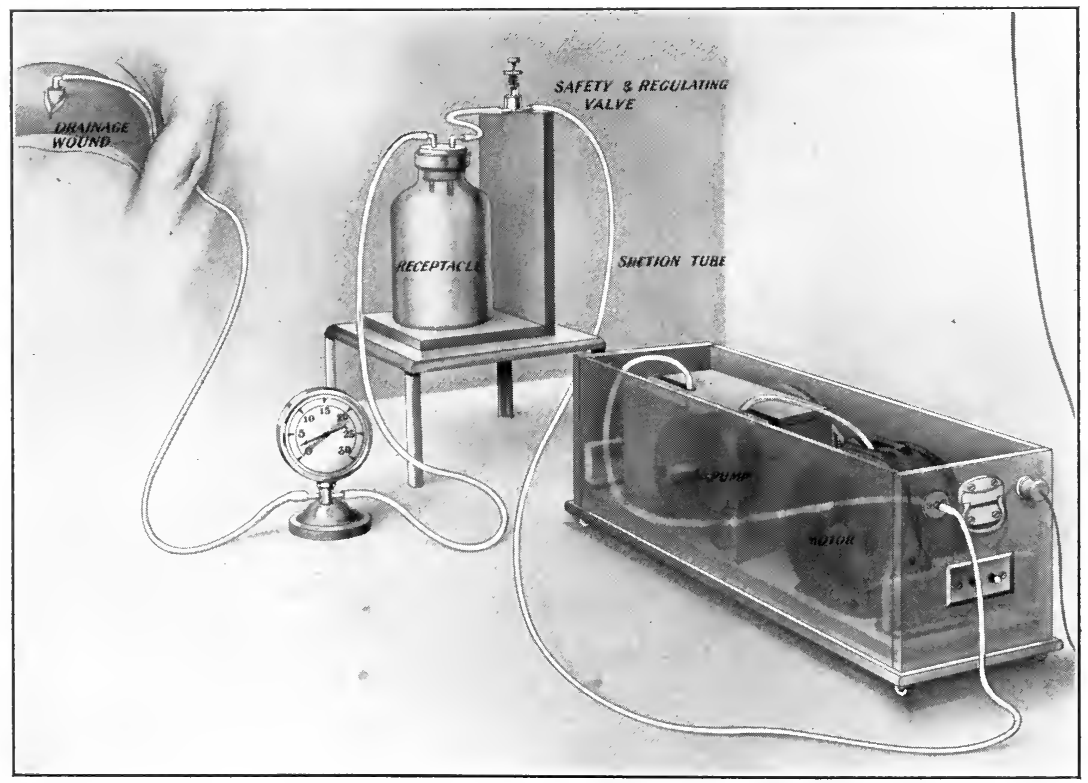

FIG. 221.-Electric suction apparatus for draining bladder.

taken out through the suprapubic wound twenty-four or forty-eight hours after operation.

Drainage is? established as already described in suprapubic cystot- 
omy by means of the author's"special suprapubic drainage tube which is a large tube, of the Freyer type attached to which is a small catheter tube for irrigation (Fig. 218).

As already advised, irrigation is continued until there is no evidence of bleeding so as to avoid the formation of blood clots in the bladder (Fig. 197). The packing can generally be removed at the end of twenty-four hours. The suprapubic drainage tube is left in place for about a week at the end of which an indwelling catheter is introduced into the urethra and the suprapubic wound is allowed to heal. Previous to reaching this stage drainage may be satisfactorily provided and the wound kept unusually dry, in spite of the urinary secretion, by adopting the plan of attaching a suction and hydraulic vacuum apparatus to the bladder drainage tube; or an electric vacuum pump may be employed in place of the hydraulic suction when current is available (Fig. 221). During the post-operative period, the patient is kept in the semi-upright position and effort is made to get him out of bed as early as possible.

\section{Requirements for Suprapubic Prostatectomy}

Scalpel.

Hemostatic clamps.

Thumb forceps.

Bladder retractors.

Bladder forceps.

Scissors.
Prostatic forceps.

Needles and needle holder.

Suture material.

Tampon.

Special drainage tube.

Bladder inflator.

Radical Removal of the Prostate for Carcinoma.-The prostate may be removed by enucleation when examination may reveal the nature of the growth to be carcinomatous. Such an operation is the same as ordinary prostatectomy (Fig. 479). The question of resorting to a more radical operation for complete removal of the prostate with the capsule and a portion of the neck of the bladder, the seminal vesicals and a segment of the rasa deferentia must be deliberately decided and all conditions weighed in each case. The technic (Young) is briefly as follows:

The posterior surface of the prostate is exposed as already described in extraurethral perineal prostatectomy, but the tissues are separated further back between the rectum and the prostate so as to bare also the seminal vesicles. The urethra is opened and the tractor introduced. The pubo-prostatic ligaments being put upon the stretch are cut on each side with scissors. Strong downward traction is exerted upon the mass; the ureteral mouths are sought for and the base of the bladder incised in circular fashion between the ureteral 
mouths and the urethral outlet. As much of the vas deferens as can be brought down on each side is cut away. The retracted bladder is seized with forceps and the urethra is approximated and sutured to the bladder. A drainage tube is inserted and the after-treatment is the same as that following perineal prostatectomy.

Instruments required for operation are the same as required for perineal prostatectomy.

Vesiculotomy and Vesiculectomy.-Exposure of the seminal vesicles is effected in the same manner as above described for radical

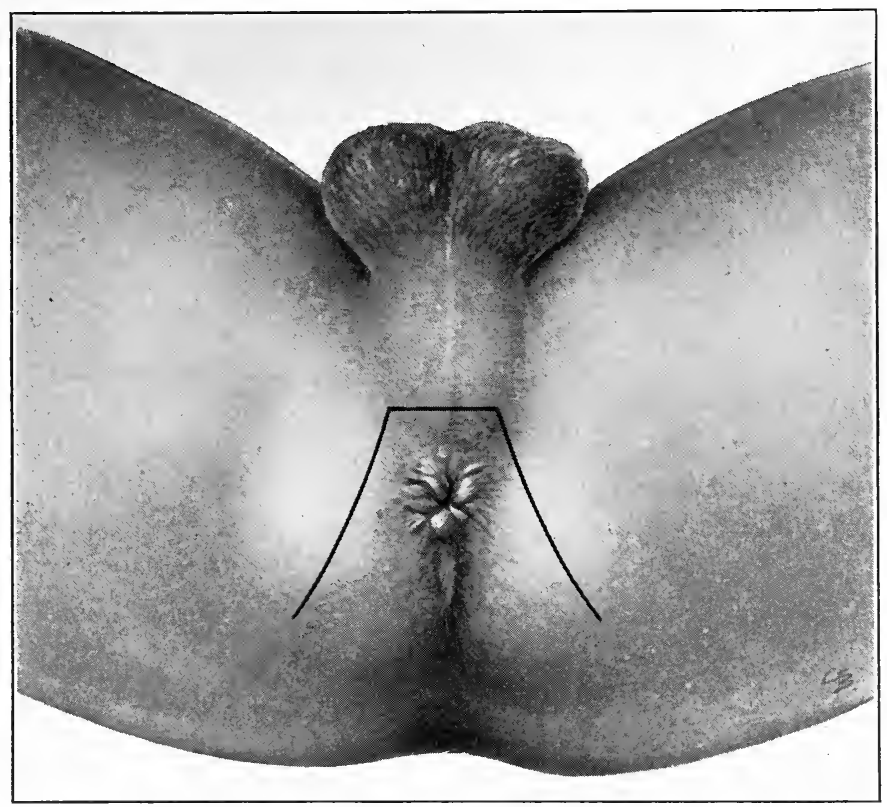

Frg. 222.-Outline of incision for reaching seminal vesicles. (Fuller.)

removal of prostatic carcinoma. Vesiculotomy is also performed by the technic of Fuller for drainage of these organs. The perineal opening consists of two long lateral incisions on either side of the anus joined by a transverse incision closely in front of it (Fig. 222). This type of incision allows plenty of room and permits the operator to avoid wounding the rectum which is aided by keeping forward in front of this viscus and separating the parts above the perineum by blunt dissection until the prostate is reached and the vesicles are exposed on either side of it. They then may be incised and curetted and drained. This operation has been frequently performed for 
chronic vesiculitis and systemic gonorrheal invasion such as gonorrheal arthritis. Complete removal of the vesicles is less of ten required, is very difficult and of doubtful expediency. When such removal is indicated, tuberculous or cancerous invasion is the reason therefor.

\section{TRANSURETHRAL OPERATIONS UPON THE BLADDER AND PROSTATE}

The operations performed by way of the urethra are those which are conducted through the endoscopic tube for removal of foreign bodies and for the treatment of lesions in the anterior and posterior urethra (for which see section on Urethroscopy). Those operations which are performed in the bladder cavity are for the removal of foreign bodies and vesical calculus, the destruction of vesical neoplasm by high frequency current (see section on Cystoscopy); operations upon the bladder orifice for the treatment of stricture or contracture thereof and certain prostatic obstructions of limited size and central projection.

Litholapaxy.-This operation entails the intravesical crushing of calculi too large to be removed whole or to be passed through the urethra, followed by immediate evacuation of the broken up calculus through a washing tube by means of suction. Preliminary to this operation, careful examination is made to determine probable location of the stone, whether it is movable or attached and whether an individual case is best suited to this method of operation (see p. 448). In suitable indications, the results are most farorable and the operation exceedingly satisfactory. Most cases of stone in the bladder are accompanied by cystitis. The cystitis is often caused by the presence of stone and is relieved by its removal; when, however, the stone is due to the cystitis and not the cystitis to the stone, there is likely to be a condition behind it, that will not be relieved by removal of the stone and, therefore, litholapaxy may be unsuitable or only palliative. The size of the urethra must be sufficiently large to admit readily the litholapaxy instruments, and the more capacious, its calibre, the better. In cases of stricture, it is necessary to dilate the canal to at least $26 \mathrm{~F}$. or to perform a preliminary urethrotomy; otherwise an open operation with the purpose of reaching both the conditions at the same time is preferable. While the existence of prostatic hypertrophy offers a difficulty to the performance of litholapaxy, there may be some cases in which it is deemed advisable for one reason or another, to remove the bladder stone, primarily, in spite of the necessity of resorting to prostatectomy at a later date.

The instruments are the essential feature of this operation and 
will therefore be described before the operation itself. They consist of lithotrites, evacuating tubes, evacuator or washer.

Lithotrites.-There are several models of these instruments, the most commonly used of which are the Bigelow, the Keyes, the Reliquè
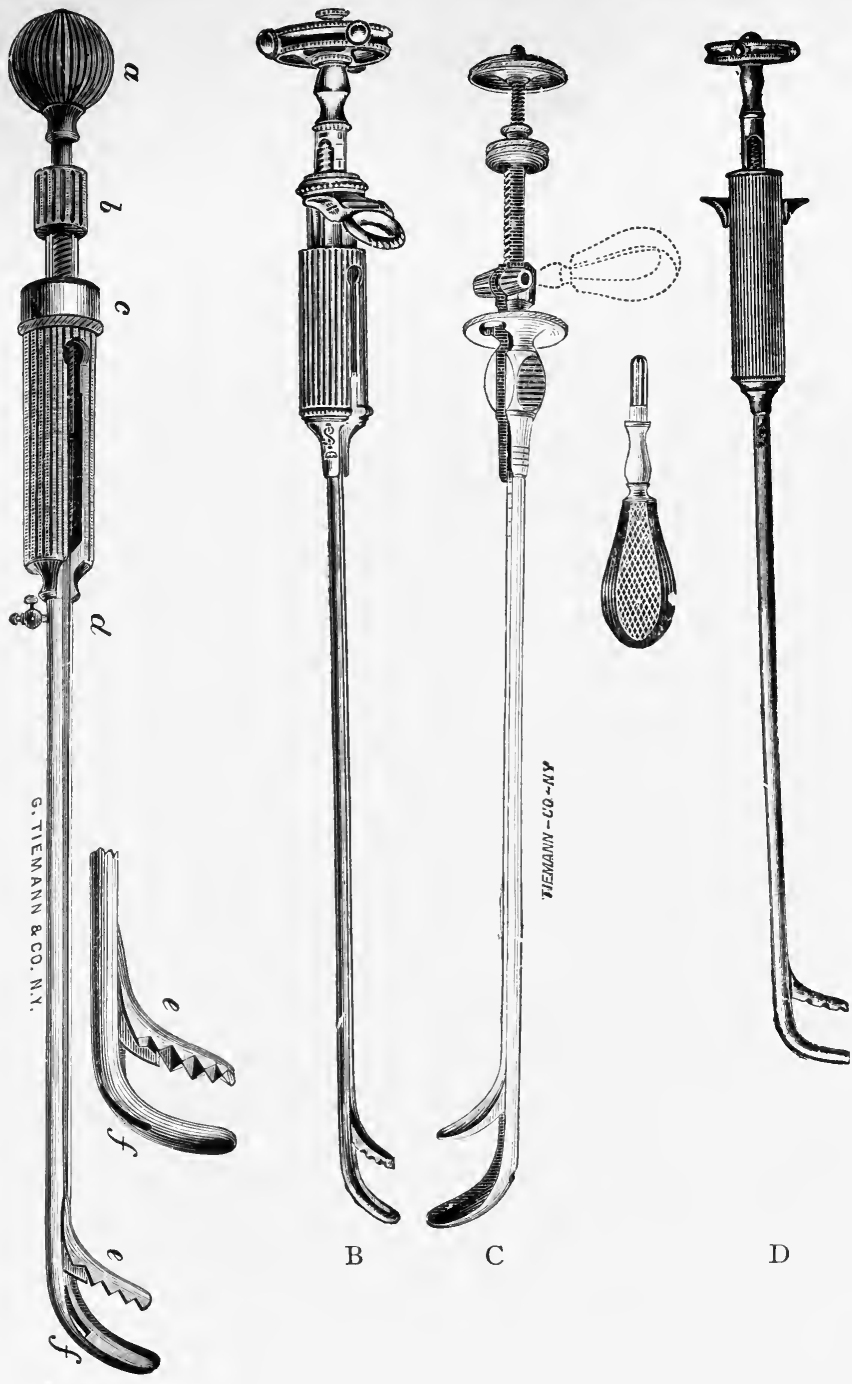

A

Frg. 223.-Lithotrites. A, Bigelow pattern. B, Reliquet. C, Spoon bill. D, Keyes.

(Fig. 223). These lithotrites are made with different curves and lengths of beak. The longer beak is suitable for use where there is a 
deep bas-fond as in the case of prostatic enlargement. The lithotrite is divided into a male and female blade. The male blade fits into the female and the latter is either fenestrated or closed (Fig. 224). The fenestrated blade is used to crush hard fragments; the non-fenestrated to crush soft prostatic concretions likely to clog the fenestrated instrument and the closed or spoon-shaped instrument picks up small fragments much easier than the fenestrated, while the latter is more powerful. We, therefore, begin with the fenestrated instrument when the stone is large and finish with the closed or spoon bill (Fig. 223-C).

Evacuating tubes are also of different size and pattern. The sizes run from 26 to $31 \mathrm{~F}$. for use in adults and smaller sizes for children.

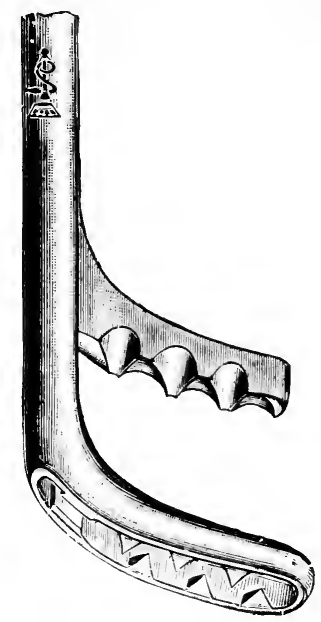

A

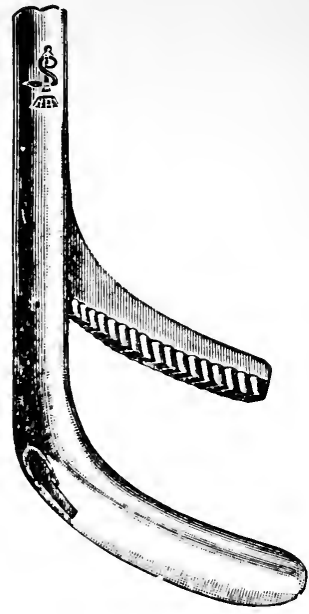

$B$

FIg. 224.- Lithotrite blades. A, Fenestrated and B, non-fenestrated.

They may be straight or curved; the curved tubes are mostly used in male adult operations and the straight tube for the female and perineal litholapaxy. Other variations in the tubes consist in the location of the eye of same which may be on the concave surface of the curve (Fig. 225), at the terminal end of the instrument (Bigelow) (Fig. $227-\mathrm{C})$ or on both sides.

The washer (Fig. 227, A) is the aspirating attachment to the tube which produces evacuation by the action of suction. The instrument commonly used in this country is the Bigelow washer. The Chismore instrument is a model that combines a lithotrite and evacuating tube so that the crushing of the stone and evacuation thereof may be completed without exchanging the instruments. The same idea is represented in the instrument of Young (Fig. 226) which includes a cystoscopic attachment for use at the same sitting, but is 
seldom employed. The other instruments required are several sizes of curved sounds, catheters of various kinds, elbowed and straight, 100 c.c. syringe, boric acid and nitrate of silver solutions.

Litholapaxy may be undertaken under local anesthesia in some cases. It is performed in the office of the operator but this is only appropriate for small stones and in simple cases. Local anesthesia is employed, by the same method as used for cystoscopy, in simple cases; (see p. 46) for large stones more complete local or light general anesthesia is necessary (p. 500). The bladder is first

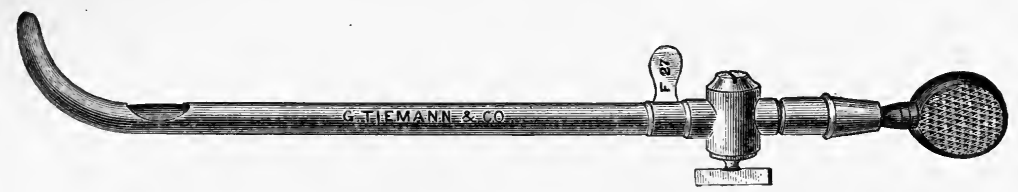

Fig. 225.-Keyes evacuating tube.

thoroughly washed out and cleansed with mild boric acid solution, 1 or 2 per cent. It is very important never to use strongly astringent preparations such as nitrate of silver or bichoride of mercury which interfere with the introduction of the instrument and the ease of manipulation. The well-lubricated lithotrite of good size and fenestrated type is introduced into the bladder, 250 c.c. of lukewarm boric acid solution having been previously injected. The lithotrite is rotated from side to side until the calculus is palpated with the beak thereof. The blades are then opened and the stone grasped

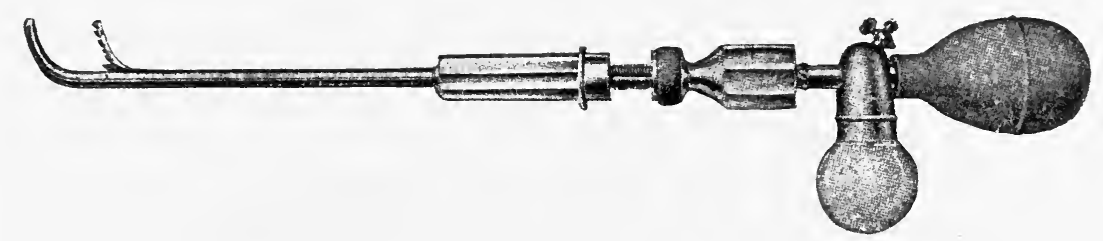

FIG. 226.-Young lithotrite with evacuator attached.

(Fig. 228), the lock of the instrument having been opened so that the sliding action is possible. The catch is then turned so as to lock the blades, the beak is rotated to the middle line of the vesical cavity and if this movement is perfectly free, it is evident that the mucosa has not been pinched by the blades and the operation of crushing the stone can be carried on (Figs. 228 and 229). This is accomplished by turning the ratchet wheel at the end of the lithotrite until the blades are completely approximated. If the stone has been held within the grasp of the instrument the fracture of the fragments will be felt as well as the softness or hardness of the texture thereof. 
On the other hand, if the mucosa is caught in the grasp of the instrument in engaging the stone the lithotrite will not turn freely, therefore, the grip should be at once released and the lithotrite turned for another trial in the same manner.

In the case of a very hard stone (oxylate of lime or uric acid) the calculus will break into several large fragments first and then each one of the subdivisions must be caught and crushed in like manner until all the pieces are sufficiently small to pass through the washing tube. This is only determined by crushing all particles that can be caught and which, as indicated on the handle of the lithotrite, are of sufficient size to require crushing, after which the washing tube is

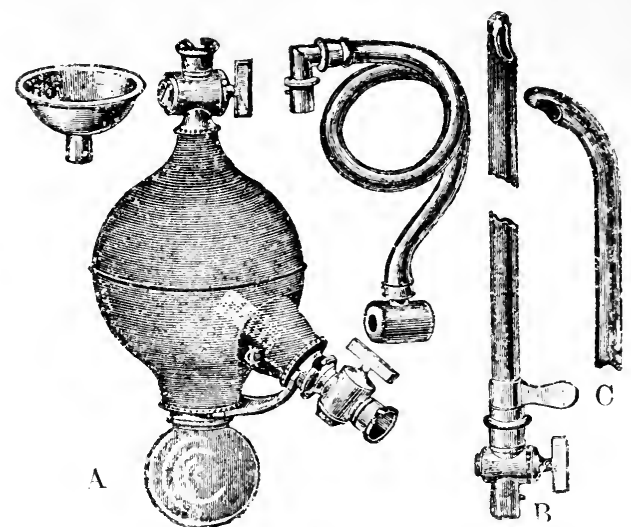

FIG. 227.-Bigelow washer. Straight and curved evacuating tubes. introduced and as much débris as possible is washed out (Fig. 230). The suction of the water through the tube reveals the presence of other fragments by the metallic click that is heard or felt and is caused by the knock against the instrument of the remaining pieces too large to enter the lumen of the tube. The position of the eye of the evacuating tube is changed from time to time during the washing so as to find any particles or fragments that may lie between the trabeculæ of a chronically inflamed bladder. The operation of crushing and flushing is kept up until it can be satisfactorily demonstrated by the washing in and out of the fluid through the evacuator and by palpation with the searcher, that no more fragments remain.

When the operation is satisfactorily completed, the bladder is left as clean as may be by irrigation with boric acid 2 per cent., followed by nitrate of silver solution one-eighth of 1 per cent. A retained catheter is left in place for two or three days to obviate the necessity of urination over the irritated and bruised canal, at the end of which period, when the catheter is removed, the patient is enabled to urinate with greater comfort. While the indwelling catheter is in place, the bladder should be washed daily with boric acid and silver solutions and the patient should be given an internal disinfectant in the form of hexamethylenamine $(0.5 \mathrm{gm}$. three times a day) or salol (0.4 gm. three or four times a day). Other treatment is 
similar to that of cystitis, namely, rest in bed on a bland diet, the administration of diluent drinks and in the early period after the operation, a moderate amount of anodyne if necessary.

After the expiration of several weeks following the removal of bladder stone by litholapaxy a careful cystoscopic examination should be made to determine whether complete evacuation has been accomplished. In case it is found that a fragment remains it may be possible to remove the same by means of the spoon bill lithotrite (Fig. 223) under local anesthesia.

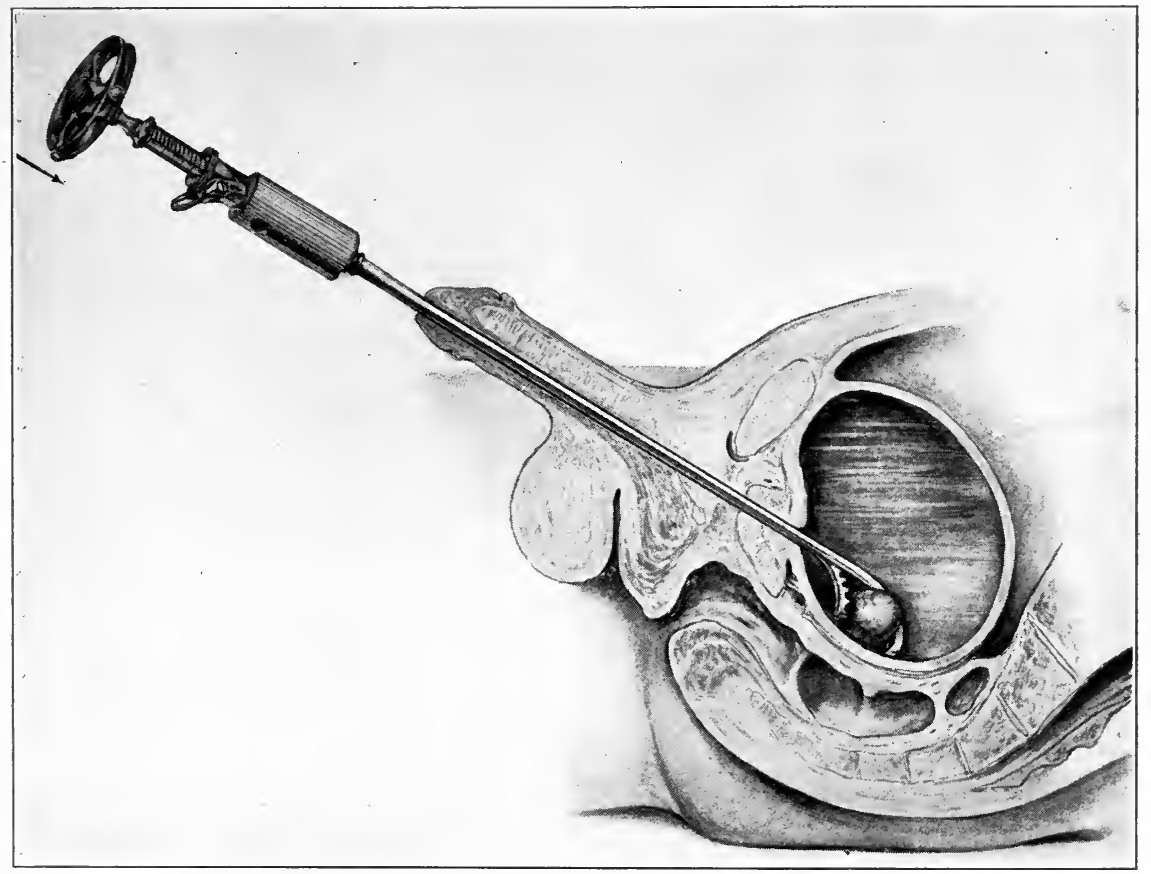

FIG. 228.-Litholapaxy. Grasping of stone on floor of bladder.

Transurethral Operations upon the Bladder Outlet.-These operations comprise the galvano-cautery incision or Bottini technic, galvanocauterization through the endoscope (Goldschmidt) and the Young "punch" operation. As has been already stated in a previous section, the method of operating upon the vesical orifice practised and preferred by the author involving incision or removal of a portion of the vesicoprostate ring, is that which provides a perineal drainage opening. The opportunity afforded to make a careful examination (p. 477) and the advantage of surgical drainage would seem to outweigh auy 
objection thereto. Yet clever-operators practise and advocate the other methods with good results. Therefore, in such hands they are successful. It would seem that their employment is suited to special cases in experienced hands and not for general adoption.

The Bottini operation is done in the dark without the guidance of ocular inspection or tactile control. Notwithstanding this the operation has its adherents, the more conservative of whom give it a restricted field of usefulness with a tendency to become increasingly more restricted. The instrument resembles a lithotrite in shape.

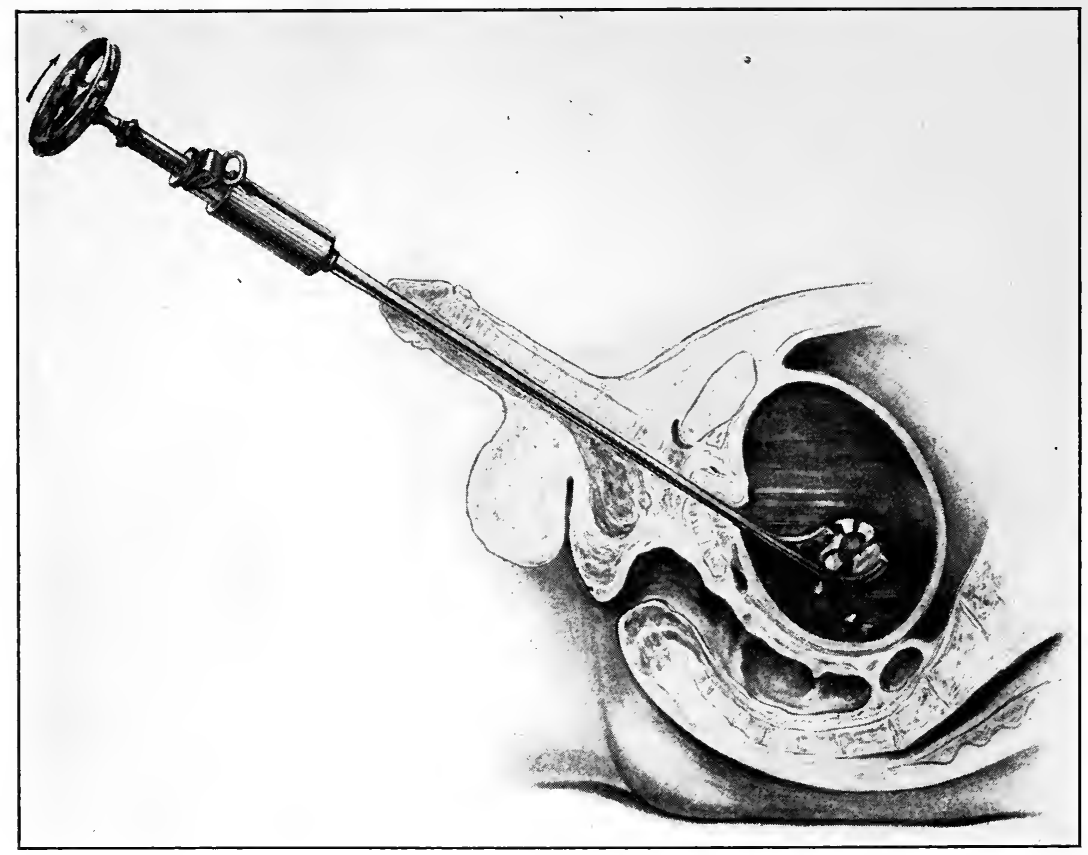

FIG. 229.-Litholapaxy. Crushing stone away from floor of bladder.

The outside sheath contains a platinum blade controlled by a wheel on the handle (Fig. 231). After introduction the beak is turned down and drawn against the vesical constriction; the blade when heated to about 50 amperes is then made to emerge slowly by turning the wheel. The number and size of the cuts must be predetermined by cystoscopic examinations. My personal belief is that operation should only be mentioned to be condemned.

Removal of section of the vesical orifice by "punch" operation (Young) (Fig. 232).-This technic is designed to correct obtrusions 
of collar and polypoid formation by excision through the urethra with a cleverly devised instrument, the author of which makes a good showing of cases, better probably than is possible in less expert hands. An important feature of the treatment is the immediate provision for satisfactory urethral drainage to guard against the accumulation of free blood and clotting in the bladder.

The Goldschmidt urethroscope is contrived to reach the same condition as that attacked by the Bottini incision and perineal gal-

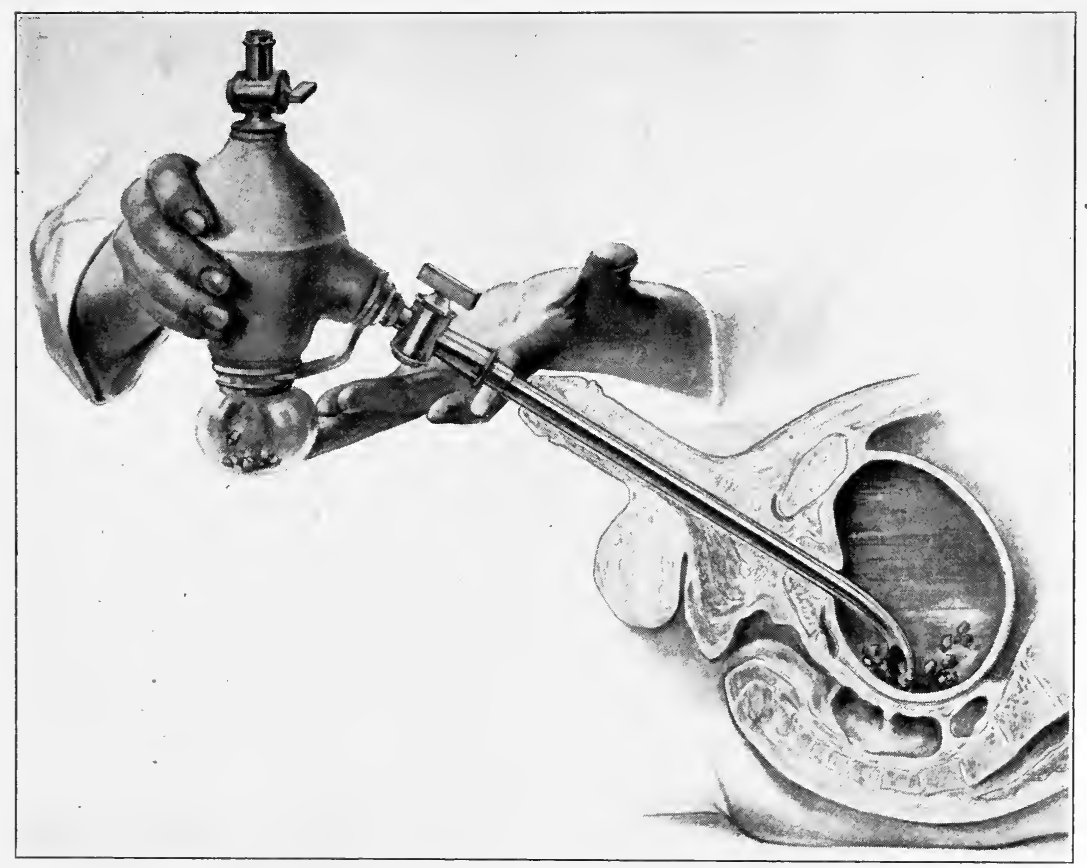

FIg. 230.- Removal of fragments with Bigelow washer after crushing.

vano-cauterization, a cautery being applied by means of the endoscopic tube after visual inspection through its lumen. Report upon this procedure ${ }^{1}$ gives good results.

\section{LOCAL ANESTHESIA FOR OPERATIONS UPON THE LOWER URINARY TRACT AND EXTERNAL GENITALS}

The mucous membrane of the anterior and posterior urethra may be satisfactorily anesthetized by the application thereto of a 5/10 per

1 Journ. Am. Med. Ass., 1912. 
cent. solution of novocain, or a 5 per cent. alypin in lubricating jelly which should be retained for five to ten minutes to obtain the full effect (see p. 46). This method is suitable for urethroscopic examination,

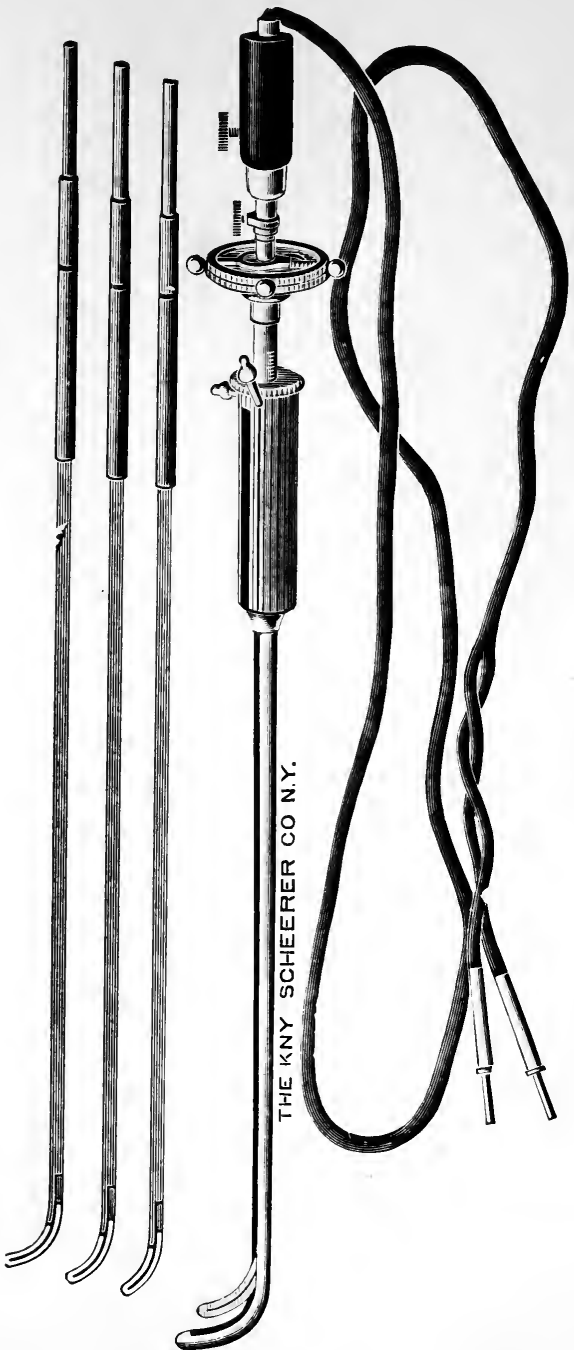

FIG. 231.-Freudendberg Bottini incisor. (Young modification.)

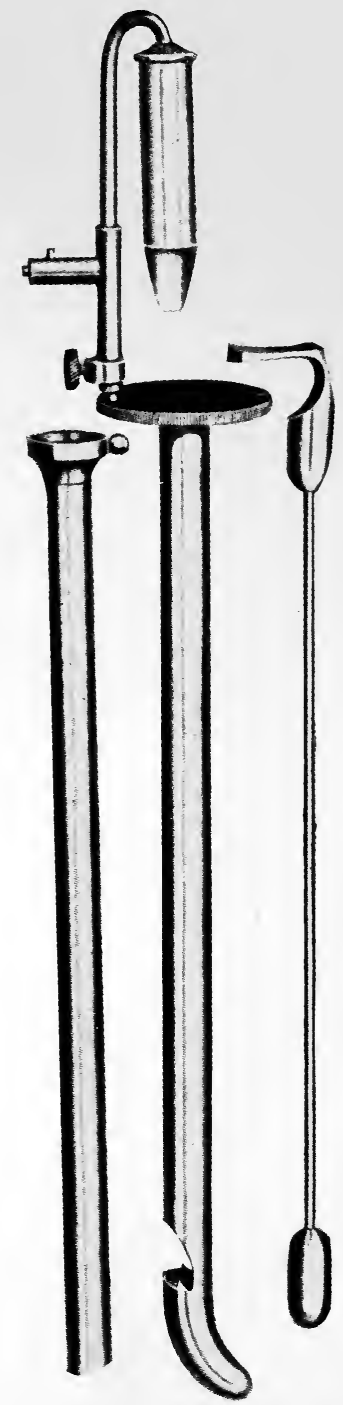

FIG. 232.-Young prostatic

internal urethrotomy, and minor operations upon the urethral tube through an endoscope. It is also sufficient for examinations of the interior of the bladder. For intravesical operations of the trans- 


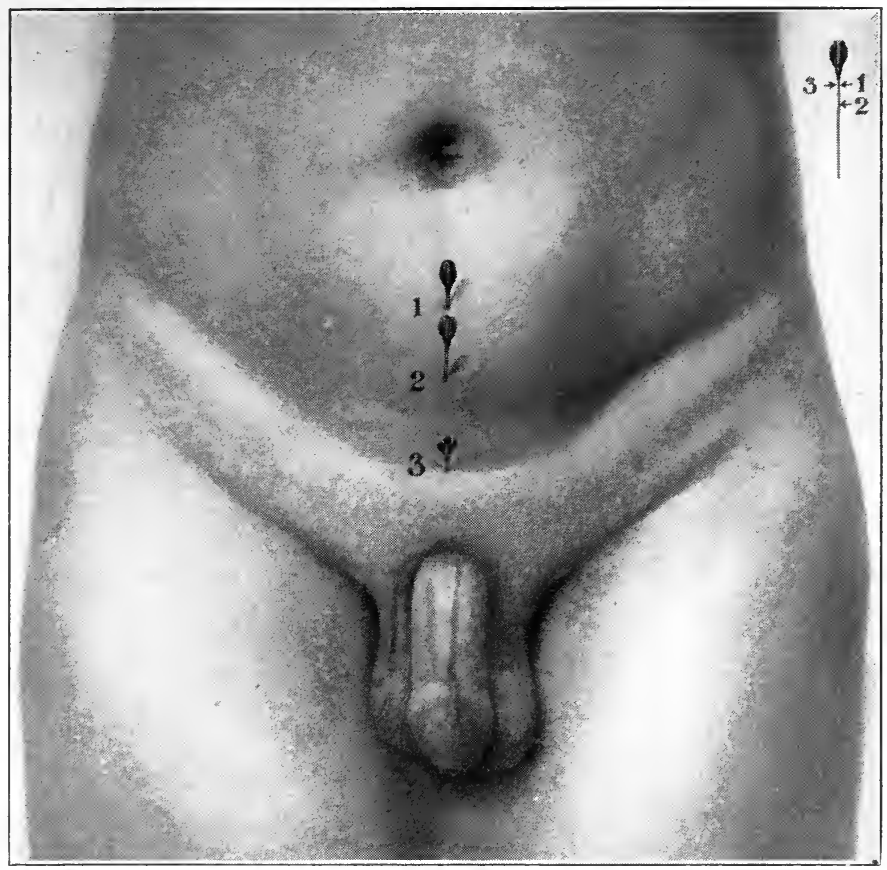

FIG. 1.-Infiltration of suprapubic region for local anæsthesia.

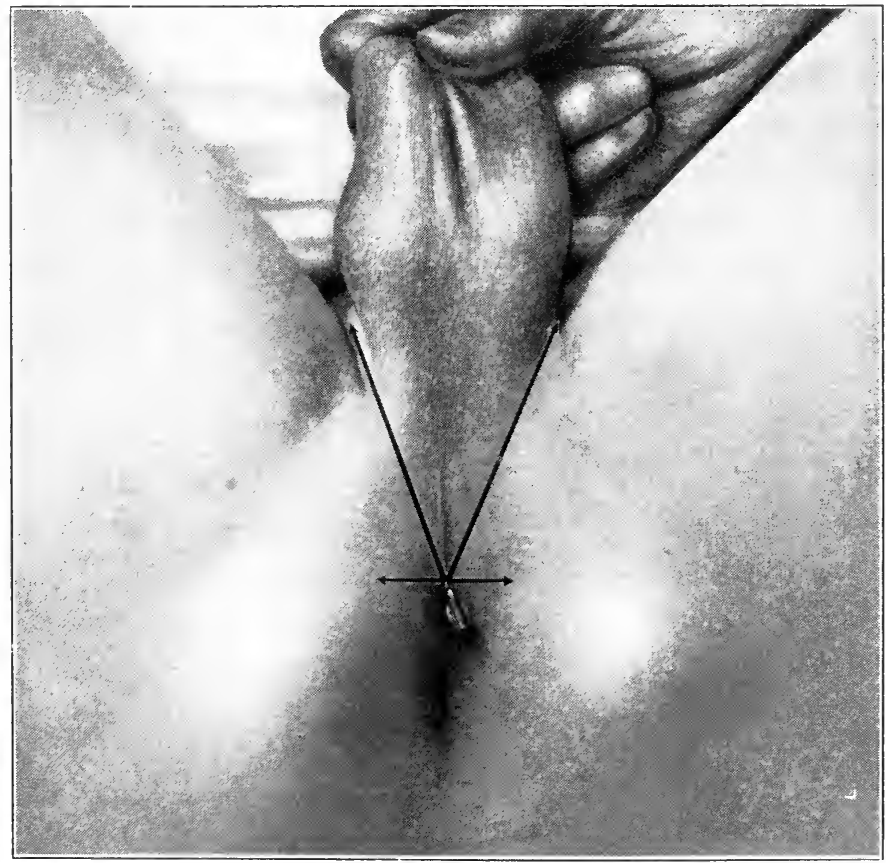

FIG. 2.-Local anæsthesia of perineum. 



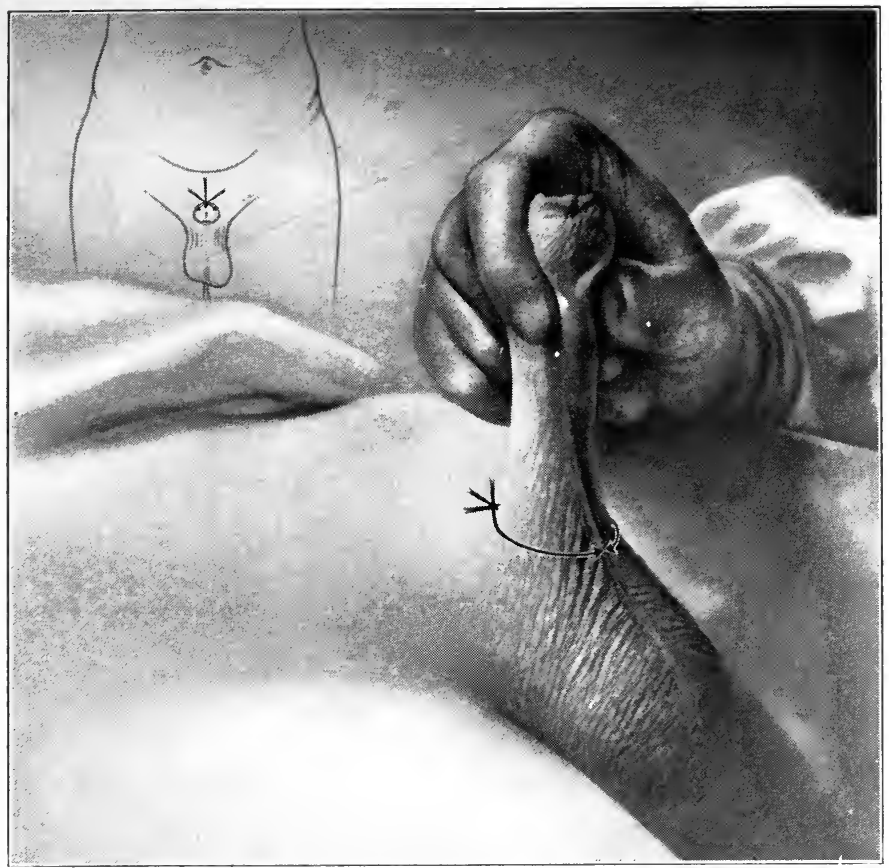

FIG. 1.-Circular infiltration at root of penis for distal andesthesia.

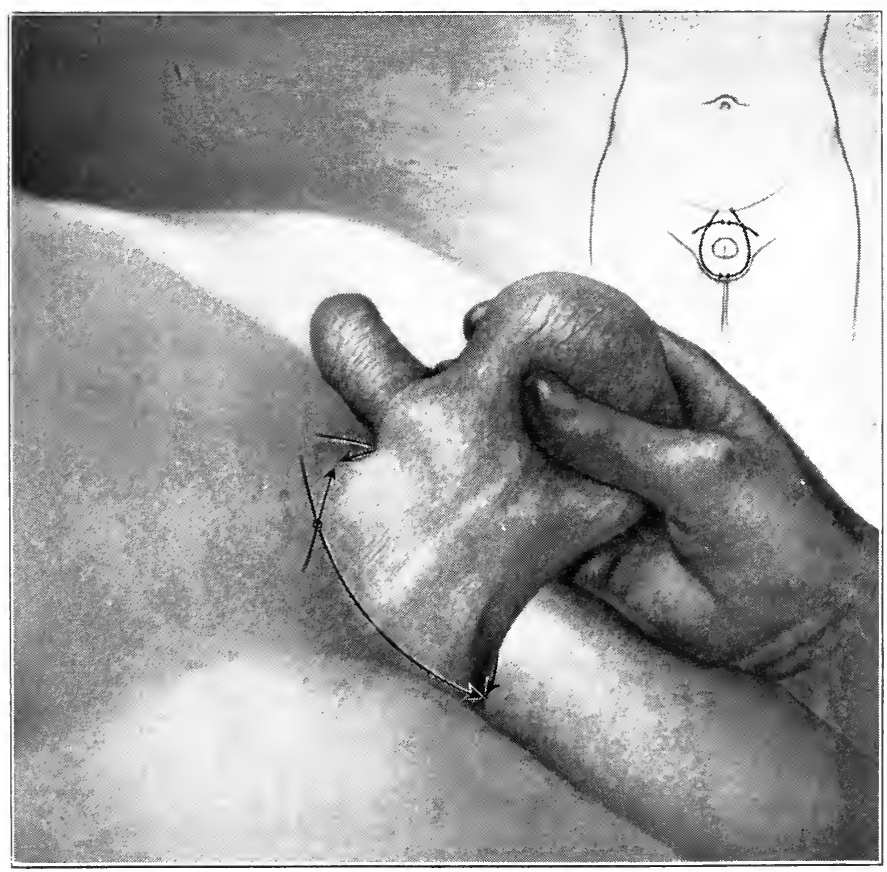

FIG. 2.-Local anæsthesia of external genitals. 

urethral variety (p. 492), it is necessary to render insensible the posterior urethra and neck of the bladder as described (p. 46) and in addition, to distend the cavity of the bladder, after previously washing with a $5 / 10$ per cent. alypin, or a 2/10 per cent. novocain, by preference with a combination of one to eight thousand adrenalin, which solution should be retained for from ten to fifteen minutes.

This method, besides serving to anesthetize the interior of the bladder for transurethral operations may, be used for exploratory open operations as well, when the organ is approached by either the suprapubic or perineal route, in both of which instances, however, it is necessary to supplement the urethral and intravesical injection by hypodermic infiltration in the particular region to be incised, for the purpose of blocking the nerves thereof.

Suprapubic Cystotomy.-The interior of the bladder is prepared as above. An area of about 3 inches is marked with iodine by a perpendicular line (Plate VI, Fig. 1) from a point just above the symphysis. In the area indicated, from 30 to 50 c.c. of $5 / 10$ per cent. novocain solution are injected superficially from the top to the bottom of the line, and then through the deep fascia to the prevesical space. The steps of the operation are then carried out (p. 460) and when the bladder wall is exposed, before it is opened, the line of incision may be further infiltrated, but this is usually not necessary.

External Urethrotomy.-After preparing the bladder and urethra as above, an area is 'marked off in the perineum with iodine, by horizontal and oblique lines (Plate VI, Fig. 2) the former midway between the anus and the perineo-scrotal junction, and the latter at the scrotal junction with the thighs. This area is infiltrated superficially and deeply with from 40 to 50 c.c. of $5 / 10$ per cent. of novocain, for which purpose the needle is carried forward in the direction of the lines between the bulbus urethra and the rectum, and between the prostate and the rectum bilaterally. In the transverse line, the injection is carried superficially, and is then made to penetrate deeply in the ischiorectal fossa on both sides.

Operations Upon the External Genitals.-In making the dorsal or bilateral incision of the prepuce, it is only necessary to infiltrate the area to be incised with a 5/10 per cent. novocain solution (see p. 341).

For circumcision, the method usually employed is that of infiltrating the foreskin, and anesthetizing the interior of the prepuce (p. 342). Complete distal anesthesia of the entire coverings of the penis may be accomplished by a circular infiltration of a 5/10 per cent. novocain solution, around the root of the penis (Plate VII, Fig. 1). The needle is passed as deep as the sheath of the corporal cavernosa, that region 
where these bodies are attached to the symphysis on both sides being encircled by the injection, and the overlying structures from this point, on either side, outward to the root of the penis dorsally, and to the penoscrotal angle anteriorly, is further infiltrated with the same solution, employing in all from 40 to 50 c.c. thereof.

Operations on the Scrotum and Testicle.-For producing local anesthesia on practically the entire external genitalia, it is necessary to block the nerve supply of the spermatic cord, as well as the overlying structures. This nerve supply includes the ilio-inguinal nerve and the genital branch of the genito-crural. The area is first marked off as above (Plate VII, Fig. 2) beginning, on either side, at the point where the spermatic cord emerges from the external ring and around, carrying it beneath the scrotum, to the same point on the opposite side; these two points are then joined together by a line over and above the symphysis. This area is infiltrated superficially with 30 to 40 c.c. of $5 / 10$ per cent. novocain, and then with a like quantity beneath the deep fascia in the same region, special care being taken to thrust the point of the needle on both sides into the fascial coverings of the spermatic cords, and to inject here a liberal amount of the solution. This injection is intended to block the spermatic nerves and the genito-crural as they emerge from the inguinal canal. This local anesthesia embraces the entire contents of the scrotum, and the shaft of the penis, and is suitable for the complete amputation of these organs. By limiting the injected area to one side or the other, the parts included therein may be prepared for a unilateral hydrocele, varicocele, or removal of the testis. In the case of orchidectomy or varicocele operation, it is only necessary to carry the injection through an area represented by onefourth of the distance outlined (Plate VII, Fig. 2) upon the side to be operated upon, which embraces the line of incision, with its central point over the external ring (see pp. 348 and 358). 


\section{CHAPTER XXVI}

\section{FUNCTIONAL DISORDERS OF THE URINARY AND SEXUAL ORGANS-THE NEUROSES}

\section{URINARY NEUROSES}

A strict classification of the urinary disorders that are of nervous origin should include only those conditions that present no active lesion in the urinary organs. Yet it is not possible to draw so sharp a line of distinction, and what is really meant embraces all cases of general neurasthenia that express themselves in functional urinary disturbance without the existence of any important anatomical lesion; and includes those cases in which, while there may exist some sort of lesion along the urinary tract, the clinical manifestations accompanying such lesion are of a severity out of all proportion to the extent thereof. These abnormal conditions include polyuria or an excessive increase of the volume of urine, pollakiuria or increased urinary frequency, anuria, oliguria, vesical neuralgia, irritability and spasm; vesical atony and enuresis.

These conditions, therefore, affect the functions of both the kidney and the bladder.

Polyuria, or increase in the volume of urine, may come from any nervous cause, although it does occur as a symptom of advanced kidney disease, when it will be recognized as such by the urinary examination and general condition of the patient. It is physiological when it occurs as the result of increased consumption of fluid and it is not abnormal when it appears under circumstances of undue nervous excitability. Diabetes insipidus is of purely nervous origin and its particular manifestation is a great increase in the outflow of urine. It is, therefore, more common in nervous subjects than those who are not of that temperament. Certain agents taken internally have a tendency to produce the condition, such as alcohol, hops, turpentine, etc.

The sole symptom of nervous polyuria is increased frequency of urination with a secretion of large quantities of limpid urine of low specific gravity. There need be no pain whatever and irritability, if any exists, may be caused by the undue frequency of urination or be due to a small area of congestion in the neck of the bladder or posterior urethra, aggravated by the increased activity of the organs. 
The treatment of this condition is to allay the nervous cause underlying it by appropriate measures, to reduce the volume of fluids consumed and to eliminate such articles from the diet as tend to stimulate renal activity, such as malt liquors, acid wines, etc. Tobacco and other sources of irritation to the nervous system should also be excluded or the use thereof reduced considerably.

Anuria, or complete cessation of urinary secretion, is a serious symptom of organic kidney disease. It also occurs as a so-called reno-renal reflex (see p. 573), in the kidney opposite to one with an active lesion. Suppression of urine, or incomplete stoppage (oliguria), may develop merely as a nervous symptom in hysterical subjects, especially women, when it has continued for several days. The recognition of the condition when of purely nervous origin must depend upon the satisfactory exclusion of any organic renal lesion such as nephritis, calculus, neoplasm or nephroptosis, and the absence of characteristic symptoms peculiar to any of these maladies. In the purely nervous condition there is a significant lack of great constitutional disturbance in the nature of chills, pyrexia or septicemia. The previous history and examination should be the means of determining the presence of preëxistent disease, and in recognizing an abnormally nervous temperament. The organic condition becomes graver each day and leads to a dangerous situation unless relieved by surgical intervention. The neurotic condition, on the other hand, ceases spontaneously or under the influence of sedative medication-valerian and bromides-or the hypodermic administration of a full dose of morphine.

Pollakiuria.-Increased frequency of the urinary act may be simply a consequence of the increased volume of urine (polyuria); it may be due to an irritability of the neck of the bladder or posterior urethra of sufficient degree to account for its occurrence; or it may be the expression of great nervous excitability that spends its influence through the cerebrospinal or vasomotor system, the bladder centres being for some reason or other unduly sensitive. It may, on the other hand, be due to the condition of the urine itself which on account of unusual concentration or hyperacidity acts as an irritant.

The symptom of urinary frequency under neurotic conditions varies considerably. It may be as frequent as every five to fifteen minutes or from every hour to two hours. It is usually unaffected by the muscular activity of the body and is much less frequent during the night, when the patient is able to sleep, differing in this respect from the same symptom occurring with inflammatory condition of the bladder which causes the patient to rise from his sleep to 
relieve the inflamed organ. The only direct influence that intensifies this condition is the increased nervous excitability of the individual and an irritating state of the urine. The treatment demands an appreciation of the underlying cause, very likely to be found in digestive disturbances and other evidences of defective metabolism, which produce an effect upon the composition of the urine as well as the nervous excitability. The urine therefore should be carefully examined and account taken of high specific gravity or hyperacidity; any defect in this particular must be counteracted by dietary restriction, alkalies and sedatives. An excess of indican will direct attention to the intestinal tract as a possible source of disturbance and of the production of irritating by-products which gain access to the urine. In fact it will be observed in cases with an organic cause for frequent urination, such as prostatic hypertrophy, that an aggravation of this symptom occurs with defective intestinal metabolism, and partial improvement results from giving this proper consideration in directing the treatment.

Oxaluria, which occurs in crystalline form in great excess in the urine (see p. 100) as a result of intestinal indigestion, is a potent cause of polyuria and the correction of this tendency is the proper means of treating such cases. This is accomplished by dietary regulation, excluding such articles as asparagus, rhubarb, cauliflower, by insistence upon outdoor physical exercise and avoidance of undue mental strain. Some of the mineral waters are reputed to have helpful properties in combating this condition, and Wildungen Spring water, a German Spa, is recommended for this purpose.

Vesical Neuralgia.-Under this category are included all painful conditions of the bladder that are not explained by the existence of an anatomical lesion thereof. Among these is included the so-called irritable bladder embraced under the description just given of pollakiuria. Such vesical neuralgia is designated as being either symptomatic or idiopathic, the latter class denoting that no assignable cause in the urinary apparatus is discoverable and therefore the case is one of nervous polyuria or pollakiuria already alluded to.

The improved means of investigation and diagnosis afforded by the cystoscope and the cysto-urethroscope renders it possible to discover otherwise obscure lesions of the vesico-prostatic region which may possibly afford an explanation of some of the cases of vesical neuralgic symptoms; such a lesion may be an area of congestion, a small polyp, or a contracted urethral orifice.

Symptomatic vesical neuralgia may be due to abnormal conditions outside of the bladder in the neighboring organs, such as a hernia, in 
which the bladder may be a part of the contents of the sac or may be dragged upon on account of adhesions; and in women uterine displacement may be the cause of such bladder disturbance; finally disease of the central nervous system, notably locomotor ataxia, may produce vesical neuralgia as a part of visceral crises peculiar thereto. The diagnosis is reached by careful investigation with the means at command, cystoscopy, urethroscopy and general physical examination, as a result of which most of the cases will be explained by the existence of some minor local lesion or upon purely nervous grounds.

The treatment therefore depends upon the result of the examination. In the case of urethral or vesical causation the cystoscope and urethroscope are employed for treatment as well as for examination. In some cases without any other lesion than marked hyperesthesia of the posterior urethra, the application thereto of mild cauterization by deep urethral instillation or through the endoscope may prove of marked benefit. An excellent remedy to commence such counterirritation with is the solution of sulphate of thallin ( 2 to 12 per cent., 1 to 2 c.c.) the effect of which, while in the beginning somewhat irritating, exercises a secondary soothing influence. This may be followed by nitrate of silver solutions (1-1000 to 1-250) as a counterirritant (see p. 237). A further resource that has been employed in neuralgic conditions, in which the nervous element predominates and the local lesion is slight, is the application of heat to the vesico-prostatic region, either through the urethra or rectum. This is accomplished by hot water irrigation, vesical or rectal lavage, or better still by means of the high frequency electric current (D'Arsonval) and vacuum tube electrode. This last method of treatment is given undue importance and value by some, although it may be useful in purely neuralgic conditions and its benefit explained by the counter-irritating influence of the heat and the psychic effect upon the patient. For this purpose the prostatic electrode is used and 500 or more milliamperes given for sessions of from five to ten minutes two and three times a week. (See p. 383.)

Vesical spasm accompanied by retention of urine is in reality a spasm of the posterior urethra and is fully described under spasmodic stricture.

Atony or paresis of the bladder is a condition that simulates stricture or hypertrophy of the prostate, and this condition is also described in a previous section (see p. 432). Some of these cases, however, are not fully understood, and while it is probable that in most of them a mechanical cause exists, be it ever so small, producing spasm and obstruction of the bladder outlet and secondary atony as a result, it is 
believed that there are other causes in some cases not accounted for on mechanical grounds, which act through the nervous supply of the detruser or sphincter muscles, and which are not as yet sufficiently explained (see also Contracture, p. 435).

Enuresis.-This condition of incontinence of urine is almost entirely confined to children under the age of ten years, although the trouble continues after that age is passed and even occurs in adults. It is more likely to be nocturnal. During the day, when the urinary function is under the control of the will, there may exist an imperative demand to urinate at call, or spasmodic incontinence when the call is not responded to.

Various suggestions have been offered to account for this trouble in the absence of any organic lesion of the urinary apparatus. A possible origin of the difficulty, but without an explanation, is that there exists a disparity between the detrusor and sphincter muscles, the action of the former being much stronger than that of the latter; and when the exercise of the will is lost, during sleep, the urine is forced involuntarily from the bladder. The cause of the persistence of this malady after childhood is not apparent, although it usually occurs in those of a neurasthenic type. Certain abnormalities, local and general, may have a bearing upon its presence; as for example, anemia and general debility will aggravate an otherwise nervous temperament and increase the difficulty. Such local conditions as phimosis, anal fissure, deep urethral irritability and congestion around the vesical trigone, all tend to augment the condition.

Treatment.- In the light of the possible contributing factors the treatment is both local and general. Constitutional treatment involves improvement in the general condition by tonics and hygiene; iron, arsenic, hypophosphites and the glycerophosphate of lime and soda are all useful remedies. Any local contributing cause that is discovered should be removed. Preputial adhesions are to be broken up and general cleanliness observed. A narrow meatus should be enlarged to reduce irritation of the urine and phymosis corrected by circumcision. The daily life of the child should be supervised by the regulation of diet, which should be substantial and free of an excess of sweets and acids; plenty of sleep should be insisted upon and life in the outdoor air encouraged. Late in the day the consumption of fluid should be restricted. Of the special means of medication, belladonna has been used more often than any other agent. It is given in full doses which are increased progressively in proportion to the tolerance, and continued over a long period. Effort should be made to appeal to the patient's sense of pride, but encouragement should always be given 
and moral suasion exerted in a gentle and tactful manner; a resort to fright or chastisement is not to be tolerated. In some cases local treatment is useful in the form of mild counter-irritation to the posterior urethra and neck of bladder such as the instillation of a few drops of sulphate of thallin, 3 to 12 per cent., or the nitrate of silver 1-1000 to 1-2000, slowly increased up to 1-500 and stronger if undue irritation is not produced.

Finally, in an attempt to break what seems in some cases to be simply a habit neurosis, the application of the author's urethral clamp may prove successful (see p. 44). This is intended to obstruct the canal without exercising undue pressure or constriction. It is applied just beyond the corona glandis. If the end in view is accomplished for a number of successive nights, the habit may be effectually broken.

\section{SEXUAL NEUROSES}

As in the case of urinary neuroses, the allied conditions of the sexual apparatus are encountered as functional disturbances without organic local lesion, or as a local manifestation of a general neurasthenic condition. Again, there may be a minor lesion of some portion of the sexual apparatus, which produces apparent symptoms out of proportion to the extent thereof. These conditions, generally speaking, affect the physiological function of the sexual apparatus, namely, the flow of semen and the performance of the sexual act. Under this classification will be embraced excessive nocturnal emissions, spermatorrhea or involuntary seminal leakage, and the different types of impotence.

Nocturnal emissions are physiological when they occur within certain limitations, that is to say, when they do not exceed in frequency once a week or once in two weeks; when they happen more persistently, as often as two or three times a week, there is generally the explanation of an unusual excitability in the region of the prostatic sinus, the verumontanum, which is caused by congestion aggravated by prolonged and ungratified sexual excitement. This may be induced by voluntary acts, mental and physical, on the part of the patient, such as indulgence in sensual thoughts, voluptuous pictures, and licentious reading. It is further encouraged by contact with women of immodest and suggestive sexual temperament.

The result of too frequent nocturnal emissions is a pronounced physical and nervous depression; this is partly physical and partly psychic. 
The proper management of such cases involves the exhibition of good judgment and common sense on the part of both physician and patient. The latter should be informed that the condition is not serious, but that it is important to avoid all unnatural and harmful sources of sexual excitation that are possible causative factors. The internal administration of general and nerve tonics is indicated, and such hygienic measures as open-air exercise, cold sponging and general massage.

When it is determined that the area of the posterior urethra is the seat of an excessive degree of hyperesthesia, counter-irritation may be applied by the successive use of instillations, beginning with the sulphate of thallin, and working up gradually to stronger solutions of nitrate of silver as already described (see p. 237); or the treatment may be employed through the endoscope $(q . v$.). The passage of a cold sound at regular intervals, about once a week, is sometimes helpful.

Spermatorrhea.-Leakage of semen without ejaculation is due to a relaxation of the orifices of the ejaculatory ducts. Like involuntary nocturnal pollutions, the most potent cause for this condition is prolonged sexual excitation, either from overindulgence in the sexual act, by unnatural practices such as masturbation, or any other vicious conduct that tends to keep up an abnormal sexual stimulation.

It is possible that the same condition may be produced by an hereditary abnormality of the ejaculatory ducts or by some neuropathic condition without any contributory action on the part of the patient.

The only local symptom of the malady is the involuntary leakage and discharge of spermatozoa independent of the act of coitus. The spermatozoa may be discovered in small or great numbers in the urine, or they may be discharged from the urethra in connection with the exudation of prostatic fluid. Their presence is the essential feature of the condition which should not be confused with prostatorrhea, in which there is an exudation of an excess of prostatic fluid without the presence of spermatozoa (see p. 376). The resulting effect of spermatorrhea is much exaggerated by unscrupulous medical practitioners and charlatans; the feelings of the patient are easily played upon for revenue on account of the nature of the trouble and the depressing influence it produces upon his susceptible nerves. It is difficult for the subject to think otherwise than that the loss of semen means a drain upon the vital forces which if not stopped will lead to total loss of manhood or mentality.

The treatment of these cases requires the exercise of a firm but 
friendly control of the patient's mental aberration, and while it is improper to minimize the importance of this functional derangement, the likelihood of irremedial damage being caused thereby can be discredited. Excesses of all kinds should be positively discouraged and the causative action of such evils emphasized. Unnatural sexual practices with the extreme degree of sexual excitement engendered thereby are among the prevalent causes of this condition, and their continuation precludes any possibility of improvement.

Masturbation is a common form of youthful vice, which if persisted in may lay the way for excessive excitability of the delicate posterior urethra in the region of the ejaculatory ducts, and later a relaxation and leakage of these ducts may ensue. The consciousness of the possible damage wrought by the secret practice of his early youth tends to work upon the mind of the patient and adds to the morbid state thereof. It is proper to encourage such an individual in the belief that damage is not irremedial and that with proper care and conduct a satisfactory result may be obtained. The possibility of such result depends upon the coöperation of the patient, the extent to which the neurotic element in his case can be controlled, and the duration and amount of the localized condition. Many cases may be successfully handled. The treatment involves general systemic and nerve supporting measures in the form of tonies, especially the glycerophosphates, internally or hypodermatically; the latter method is a direct means of administering this medication which has the additional advantage of stimulating the patient's confidence and of keeping him under control. Ampules of the glycerophosphates with and without strychnine may be obtained, and may be used daily or every second day, as the judgment dictates. Where such regular and close attendance is not possible the internal use of lecithin in powder or liquid form or glycerophosphate of lime and soda in liquid or capsule form may be employed. As a means of local stimulation cold douching to the spine under high pressure is useful, and cold may be applied more directly. to the sexual apparatus by the psychrophore (urethral or rectal). Local stimulation may be further obtained by the application of the electrical current, the static and galvanic, by means of a urethral or prostatic electrode. The galvanic current with very low amperage, two or three milliamperes, is alleged to exert a decongesting influence, whereas the static electricity is stimulating and produces contraction. The definite actions of these expedients are not entirely understood, nor as potent as some electrical therapists are prone to believe; their moderate use, however, is justified in suitable cases, and while the patient is inclined to accept favorably the rationale of such treatment, it is not 
proper that the physician should be carried away out of all reason by its possibilities.

\section{IMPOTENCE}

This malady is represented by a weakness or loss of power on the part of the male to properly perform the sexual function. The condition is divided into various types. The important sub-division thereof is impotentia cœundi, or inability to perform, and impotentia generandi, inability to produce (sterility).

In order to perform properly the physiological sexual act the genitals must be normal anatomically, and the power of erection must also be satisfactory. A departure from the normal is caused by a variety of factors which determine the type of impotence in individual cases. Organic impotence is due to an anatomical defect in the construction of the genital organs or to an organic lesion of the portion of central nervous system presiding over the sexual function (Paralytic Impotence). The other forms of impotence which are more commonly met are embraced under the title of functional impotence, which is further sub-divided into psychic impotence, relative impotence and atonic impotence.

Impotentia cœundi manifests itself in several phases which has led to other classification by some writers. Such variation, however, represents more a difference in the clinical history than it does a radical change in the type of malady. Thus impotentia voluptas is a form of impotence exhibited by a loss of sexual desire, this physiological sense having previously existed to a normal extent, that is, normal to the individual in question. Its causation, prognosis and general line of treatment are much the same as other examples of impotence that are not organic. Furthermore, the difference between organic and functional impotence of some types is a matter of degree of severity. In one instance is produced a case of impotence which is temporary and yields to treatment; it is therefore regarded as of a functional order. In another individual, on account of underlying inherent susceptibilities, the same causes produce the same variety of impotence of a more severe character which remains permanent, and is regarded as organic. Thus impotentia libido signifies the possibility of performing the sexual function but a loss of the normal sensual feeling, the same having previously existed to a normal extent. When this condition is born with the individual it is an inherited defect and is therefore organic; when it is acquired as a result of the usual causes it may be either functional or organic according to 
the receptivity of the subject, "that is to say the malady itself is functional but the individual peculiarity may afford for it an organic basis.

The centres of erection are cerebral and spinal. Those in the cerebrum are more or less affected by consciousness and the will, that is, they may be stimulated or suppressed by acts of the mind; whereas the spinal centres are entirely reflex and are governed by vasomotor conditions, local irritation, and reflex stimulation, which may be derived indirectly from the cerebral centre as well as peripherally from the genital organs.

Organic impotence signifies a complete absence of power or of will to perform the sexual function. It may be due to serious structural defects, (a) in the genital organs themselves, (b) in the central nervous system.

The most frequent malformations of the sexual organs interfering with the sexual act are hypospadias, epispadias, exstrophy, extreme smallness of the penis, excessive deviation due to fibrosis, laceration and elephantiasis; congenital defects and acquired conditions of the testicles such as absence of, atrophy and fibrosis or neoplasm. These defects, as may be judged, are both inherited and acquired. Some of the inherited conditions such as hypospadias and deviation, when not of extreme degree, may be corrected by surgical operation and the condition removed, as it is quite likely that outside of the structural defect the individual is otherwise normal. In certain extreme conditions the deformity may be beyond help.

Organic impotence, the cause of which is located in the cerebrospinal region, is beyond the reach of medical aid. Hereditary weakness is the cause of many of these cases, and the form in which they are manifested may be impotentia voluptas or lack of desire, impotensia libido or absence of sensation and impotentia cœundi, absence of erection. Some of these cases of a neurasthenic character are amenable to treatment and partial or marked improvement may result, but usually the condition when hereditary is an inherent one and permanent. Other forms of organic impotence of central nervous origin are caused by organic disease of the brain or spine and are equally out of reach of medical relief. These may be produced by tabes dorsalis, general paralysis and traumatism.

Certain general diseases also produce the condition (symptomatic impotence) directly or indirectly through the vasomotor apparatus, such as diabetes, anemia, extreme obesity, chronic alcoholism, and acute mineral poisoning by such poisons as arsenic, lead, mercury, etc. The outlook in this last type of central nervous impotence may be more favorable than the other forms mentioned and depends upon the 
success with which the intrinsic or general condition can be treated and removed.

Functional impotence like the foregoing organic form may assume the same types, that is to say, the condition may be one of a lack of desire, of sensation, or of erection, which may be atonic-physical, psychic or mental.

Etiology.-The causes of functional impotence are usually the voluntary acts of the individual and lie in sexual excesses of all kinds. Contributing factors are alcoholic abuse, great physical and mental fatigue, worry and care, etc. Chronic inflammation of the posterior urethra, of the prostate gland and seminal vesicles are also common contributory factors of this affection. The causes therefore are both local (mechanical) and general (neurasthenic). Every case of functional impotence is governed to a greater or less degree by these causative agencies and each case has its own peculiar individuality according to the temperament of the subject and the previous clinical history with respect to gonorrheal inflammation and its complications.

The symptoms of functional impotence express themselves in varying degree and character, which together with the contributing factors determine the particular kind of functional impotence that exists. In one case it is a strongly neurasthenic individual, who has practised all manner of sexual excess, who sooner or later becomes aware of the fact that he has developed one or the other forms of sexual incompetency. He may never have had gonorrhea and being neurasthenic, his condition is magnified in his own mind and becomes the more difficult to master. Another type of similar character is seen in an individual of perhaps only mild neurasthenic temperament, but in this instance there has been a prolonged or severe inflammation of the urethra which has left a chronic prostatitis, vesiculitis, or posterior urethritis. In either case there is likely to be, with a normal sexual desire, a lacking sensation, a weak or absent erection or a precipitate and incomplete ejaculation. Different combinations and grades of these two examples are found which decide whether the condition is largely or only partially a neurosis.

The Prognosis is governed by many factors. In the first case it depends upon the extent of the neurasthenic temperament of the individual and the ability of the physician to control it or to remove from the patient's mind what may be in effect an actual phobia. It depends upon the restoration of the general health of the patient and improvement in the nerve tone, the complete removal and separation from the various causative agencies that are present in his case, such as sexual 
overindulgence, abnormal practices or alcoholic excess. The strong contributing mental factor in some cases is a sudden bereavement or financial misfortune, and the effect of such a cause must have time to lose its influence. Finally the outlook depends upon the character and extent of the localized lesion in the sexual apparatus and its partial or complete removal by appropriate treatment.

Treatment.-The treatment of functional impotence, like the prognosis, depends upon the individual conditions in each case. It is important and very essential to begin with that the physician should gain the full confidence of the patient in order to exercise as much control as possible over whatever psychic element may exist. To this end the patient should be encouraged as much as possible, but warned of the pernicious effects of inordinate sexual excess. It should be explained that an overtired and worn-out organ naturally reveals itself by inactivity and failure to respond to the usual stimulation, but that with care and appropriate management a restoration of normal function may be hoped for. This end is aimed at by means of such rational measures as tend to improve and tone up the general nervous system, namely, strychnine and glycerophosphate internally; hydropathy-spinal douche, cold psychrophore, massage and physical exercise. In atonic cases electricity may be employed, galvanic or static, to the spine for general effect or by prostatic electrode to the genital region. It is well to counsel, during the early weeks of treatment at least, a total abstinence from sexual performance, and until such time that it is determined that the general nervous system has been benefited by the treatment employed and that the patient is in a condition of mind to return to the moderate practice of the sexual function without resorting to excess. At first some patients are not prone to be satisfied with what the treatment may have accomplished in comparison with their previous excesses, but those with proper sense of proportion soon learn to appreciate that the condition at hand is more nearly the normal one, whereas their previous wild career was an extravagant excess beyond the normal limits. It is furthermore necessary for a successful outcome to treat satisfactorily the local lesion in the genital apparatus, whatsoever that may be, and careful examination in each case must determine the extent of this to insure its effective removal (see special sections on different urethral maladies, also general diagnosis). Such urethral lesion may amount to an extreme congestion of the prostatic sinus or enlargement of the verumontanum, in which case by the passage of a steel instrument marked hyperesthesia of the deep urethra is recognized, or by endoscopic examination the hypertrophied and engorged verumontanum 
can be detected. Treatment involves counter-irritation of this section of the urethra by systematic use of deep urethral instillations (see p. 237): beginning with the application of the sulphate of thallin 1 or 2 c.c. of a 2 to 12 per cent. solution twice weekly and following later with a nitrate of silver solution at the same intervals, very mild at first (1 to 1000) and gradually working up to the full strength of tolerance, 2 to 5 per cent., of which solution a few drops only are deposited in the prostatic sinus. These measures should be combined with the general course suggested and are often effective. In other cases where there is discovered an enlargement of the verumontanum treatment may be applied thereto through the posterior urethroscope, with solid nitrate of silver fused upon an applicator for two or three such treatments (see p. 127). Excellent results are obtained in some cases by this means. Other lesions of the sexual apparatus such as vesiculitis and prostatitis must receive their appropriate treatment.

When it is found that the local condition, in place of being one of hyperesthesia and congestion, is that of diminished sensibility of the atonic variety such means are employed locally as will tend to improve the circulation and stimulate the parts. To this end is used the cold psychrophore, rectal and urethral, with ice water, for a period of five or ten minutes each night. Mild stimulation to the posterior urethra is also in order, and prostatic massage once or twice a week is often adopted with the same purpose in view. Cold douching under high pressure several times weekly to the lower spinal regions is resorted to in these atonic cases with some success. Finally, it must be repeated that each case is a law unto itself and must be worked out along the proper lines by careful study in conjunction with the patient's coöperation.

Sterility-Impotentia Generandi.-This condition may be due to and entirely dependent upon the existence of impotentia cœundi, but the reverse state of affairs does not follow. There are various degrees of impotentia generandi associated with different conditions of the semen, which may vary from a total absence thereof (aspermia), or complete lack of spermatozoa (azoöspermia), to a diminution in the quantity of the semen (oligospermia) or of the fertilizing elements thereof. Other forms of defective spermatism are found in inflammatory conditions-catarrhal, producing hydrospermia or a thinning thereof and a rapid formation of sperm crystals (Boettchers); purulent and hemorrhagic, a condition of pyospermia or hemospermia in which there are present pus and blood respectively, due to chronic inflammation along the urinary and seminal passages. 
Sterility is therefore relative or complete.-In the latter case it is due to aspermatism or azoöspermia.

Aspermatism may be due to the fact that the semen does not pass through the genital canal, although duly formed. The failure of emission may be permanent or partial. It may be congenital or acquired.

In absolute aspermatism of congenital origin the ejaculation of semen has never taken place, on account of some constitutional defect. When the condition is acquired the loss of ejaculation may be tem-

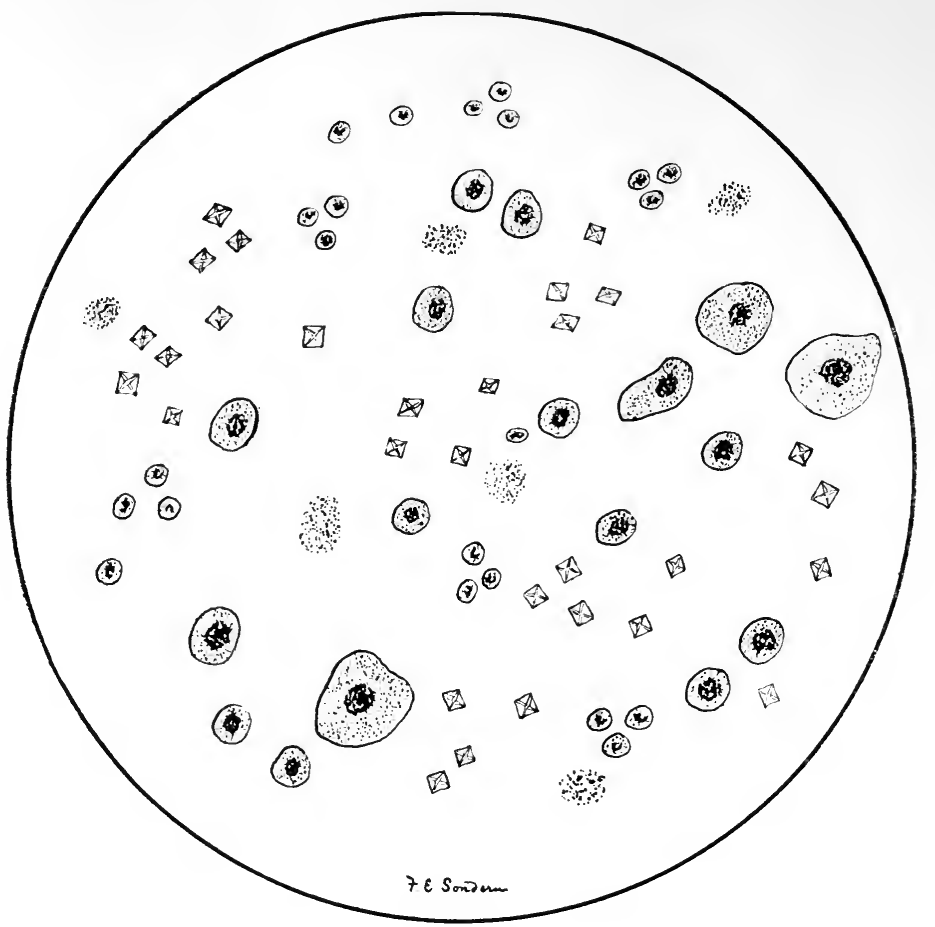

FIG. 233.-Azöospermia and impotentia cœundi.

porary or partial, complete and permanent. In any of these conditions the causes are general and local. The local causes are malformation and distortion of that portion of the genital which unites with the urinary tract, namely, the posterior urethra. This may be due to the traumatism of an external injury or a surgical operation, as a result of which the semen may be projected backward into the bladder or into a cul-de-sac, instead of coming out through the anterior urethra. This fact is determined by endoscopic and cystoscopic investigation and by examination of the urine following an ejaculation. In some 
cases where it is retained in a pouch or cul-de-sac of the urethra it oozes outward some time afterward instead of at the time of ejaculation. Other causes for temporary and partial aspermia are general physical depreciation, and the same factors that produce the nervous forms of impotence.

The treatment of aspermatism and the result therefrom depend upon the existing causative agencies. In organic physical defects, there may be nothing to do. In acquired physical derangement it

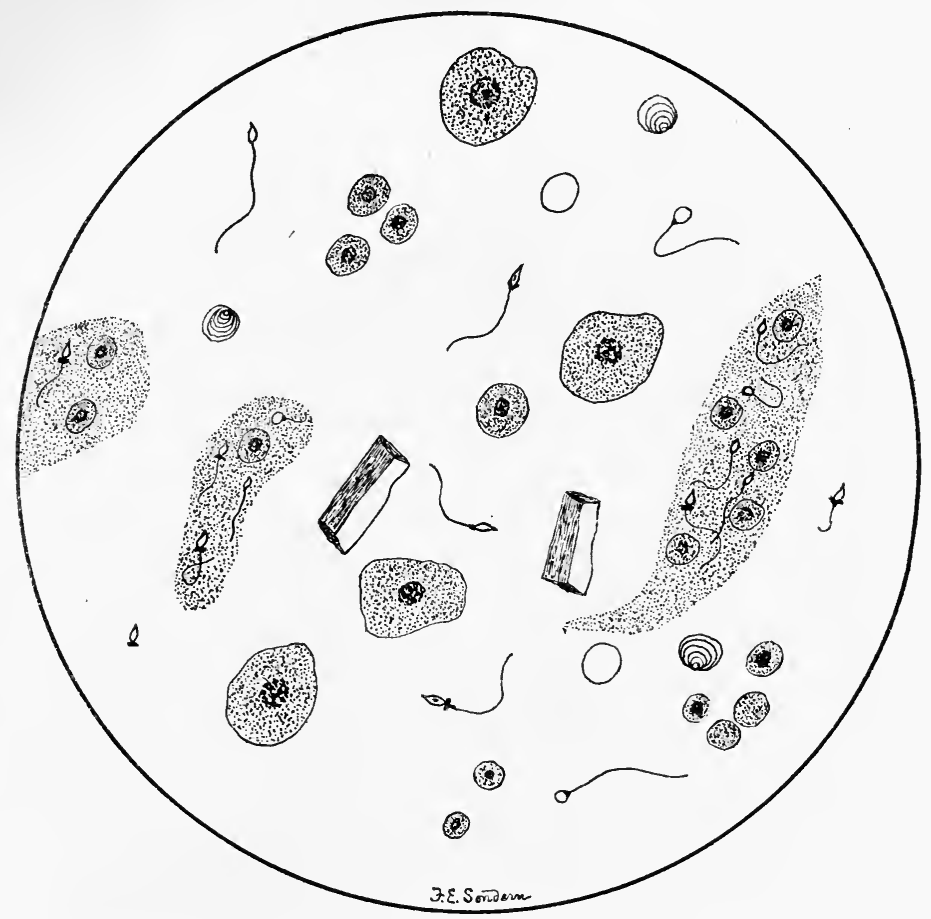

Frg. 234.-Oligospermia.

may be possible to restore the natural flow of the semen outward by plastic operation entailing the dissection and excision of a pouch or removal of an obstruction. The more generalized conditions upon which the acquired forms of aspermatism depend require the same treatment as is adopted for the kindred conditions upon which impotentia cœuendi depends.

Oligospermia, partial absence of the spermatozoa, and azoöspermia (Fig. 233), complete absence of the latter, may be temporary or permanent. In both of these conditions the seminal flow may or may not be 
normal in quantity, and the fertilizing power thereof is partially or totally absent.

Various causes are responsible for the existence of these conditions: there may be an organic azoöspermia on account of congenital physical defects such as anorchism, cryptorchidism, absence of the epididymis and inflammatory disease of the testicles, the most important of which is occlusion of the spermatic duct in some portion of its course from the epididymis to the ampulla of the vas deferens. This last condi-

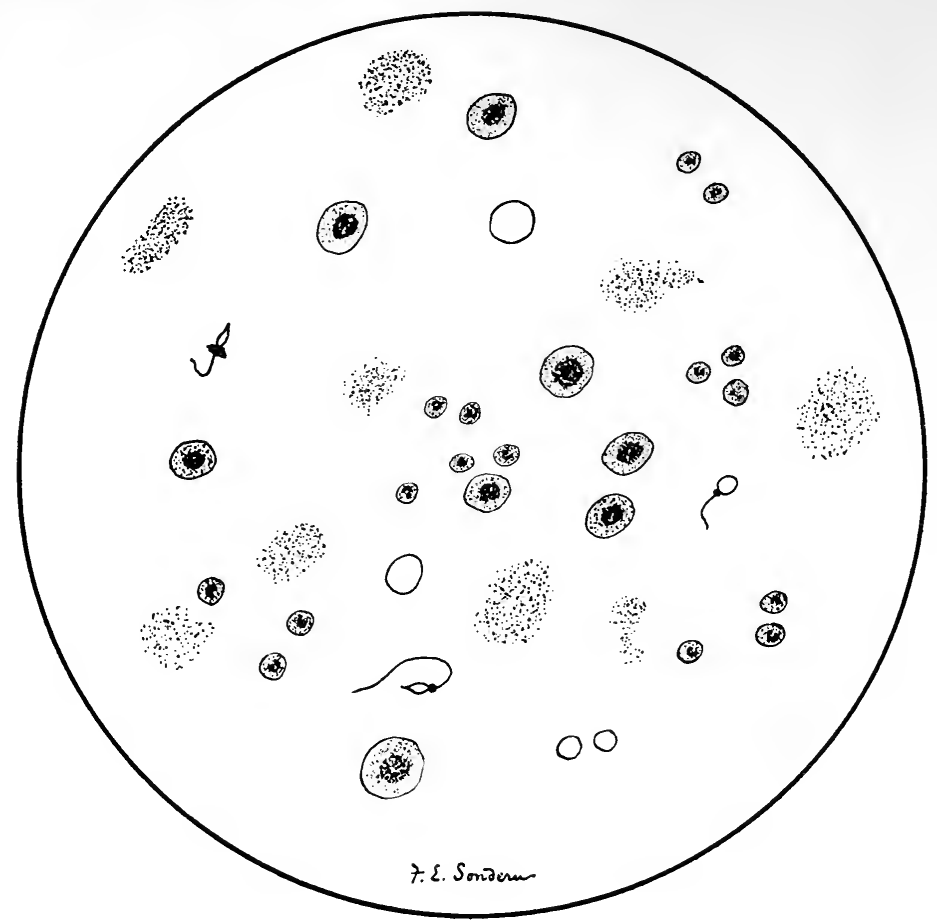

FIG. 235.-Necrospermia and impotentia cœundi.

tion may affect one or both sides. Physiological or temporary azoöand oligospermia may be produced by too frequent sexual practice. Other causes are both general and local; constitutional maladies, such as syphilis, tuberculosis, chronic alcoholism, and pronounced anemia; chronic localized inflammation of the seminal vesicles, of the prostate and of the posterior urethra (Fig. 234).

The diagnosis is made by microscopic examination In azoöspermia there are found no spermatozoa whatever and their place is taken by the other elements of the vesicular and prostatic secretions, 
the spermatic crystals, hyaline bodies and starch corpuscles as well as in oligospermia (Fig. 234) when there are present an abnormally deficient number of spermatozoa, some of which may be non-motile and variously deformed (necrospermia) (Fig. 235). Some of these spermatic bodies may be headless, others may have rounded instead of pear-shaped heads, and some have rigid and abbreviated tails. In other instances there may be present some normal motile spermatozoa mixed with the abnormal varieties named and the other elements of the prostatic secretion.

The prognosis depends upon the cause and the nature of the local condition. In the neurasthenic type it is similar to that of nervous impotence, and varies in each individual case. In those cases resulting from a mechanical physical defect it depends upon the removal of the same.

Treatment.-The treatment varies with the pathological conditions. In nervous cases it is the same as that of impotentia $(q . v$.$) .$ In inflammatory conditions it entails removal of the localized lesion, be that chronic seminal vesiculitis or prostatitis $(q . v$.) or occlusion of the vas deferens somewhere along its course or in the epididymis. The patulous condition of the vas is determined by injection from below upward with argyrol or other silver solution and when the vas is open this is obtained by catheter passed into the urethra (see p. 354). When the duct is found open and there is recognized a hardness in the epididymis, between the latter and the testicle proper, the operation of vaso-epidymal anastomosis may be performed and successful results are reported (see Operative Surgery, p. 357). 


\section{CHAPTER XXVII}

\section{THE UPPER URINARY TRACT}

\section{CONGENITAL ANOMALIES AND WOUNDS OF THE KIDNEY AND URETER}

The embryology of the kidneys (see Chapter I, p. 23) is the same as that of other organs; that is to say, their development in the process of generation proceeds conjointly with that of the blood-vessels. Congenital anomalies originate in the embryonic renal bud and, as has generally been taught, primarily in the vascularization of the kidneys, although this is disputed by some authorities. ${ }^{1}$

There is a wide variation in the character of renal anomalies which appear from a slight departure from the normal to defects so serious that death must result soon after birth.

The increasing importance of the surgery of the kidney and the many operations that are performed, render each one of these defects of practical significance.

These anomalies may embrace:

1. Form.

2. Size.

3. Number.

4. Position.

5. Attachment, and

6. Mobility of the kidney.

Frequency.-In investigating the experience of different observers, it will be found that tabulations by some give different impressions than those of others in regard to the frequency of occurrence of abnormalities. $^{2} \mathrm{My}$ personal experience in kidney surgery is that the abnormalities are rare.

Anomalies of Form.-One or both kidneys may be irregular in form. The variation may be so slight as to have little clinical or surgical significance or the deformity may be so pronounced that it

1 W. A. Newman Dorland, Surgery, Gynecology and Obstetrics, Vol. XIII, 1911, pp. 303-319.

2 In 11,168 autopsies, Morris, Surgical Diseases of the Kidney and Ureter, London, 1901, discovered 53 anomalies of various types or about 1 in 211. Of the total number, 10 were misplaced kidneys, while Brewer finds in 151 post-mortem examinations that 14 were displaced or 9.2 per cent. of this abnormality alone. 
becomes of serious importance to bear in mind that the associated anomalies of the blood-vessels likely to be encountered during operation are a source of danger. The lobulated kidney is a striking example of malformation which applies not alone to its irregular shape (bi-lobular or tri-lobular), but to its accompanying vascular arrangement.

In cases of marked malformation, there is usually displacement as well. The term "fetal kidney" is applied to an organ that shows these characteristic lobules in adult life. The persistence of the lobules is due to arrested development.

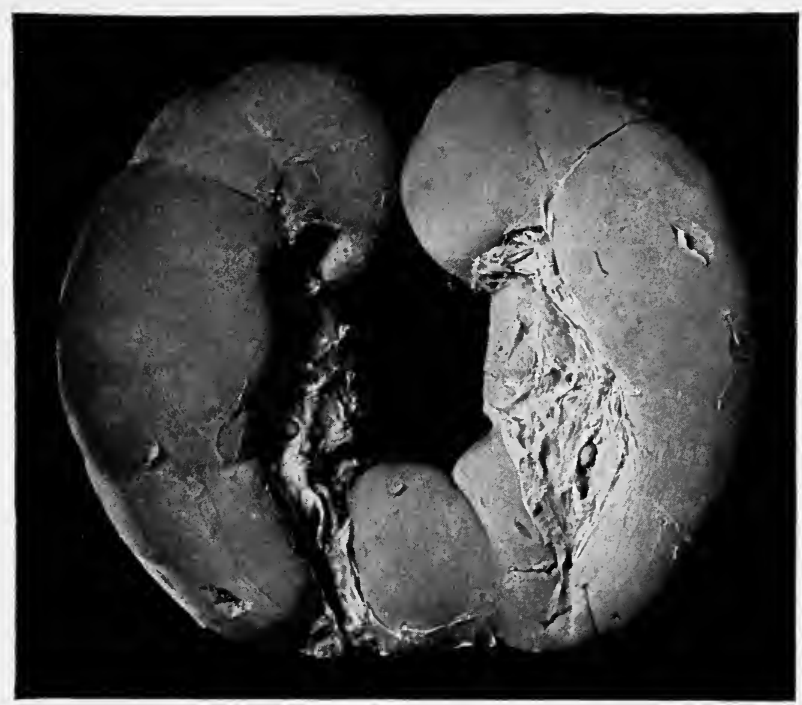

FIg. 237.-Horseshoe kidney. (Delafield and Prudden.)

Malformation may consist in the fusion of the two kidneys, resulting in the "horseshoe" kidney and other irregular forms (Fig. 237). In the fused kidney, the lower poles are united more often than the upper. In the latter condition, the concavity faces downward. The kidney may lie transversely across the vertebral column or its position may be more lateral. As a rule, the malformed kidney is deeply placed and the large vessels invariably lie behind the median part of the organ, while the ureters are usually in front. The connecting bridge of the fused kidney consists of renal tissue or of a band or cord of connective tissue. Commonly the pelvis and ureters are separate and normal although irregularities of the ureters and vessels are not infrequent. 
There may exist two renal pelves and two or more ûreters connected with the same kidney (Fig. 239). These anomalous ureters may unite at one point or another or may have separate entrances through the bladder wall.

The diagnosis by palpation is so difficult that it is not often made except under very favorable conditions for examination, such as a thin subject and flaccid abdominal walls. A good $x$-ray picture may outline malformations, more particularly with the aid of pyelography or ureteral bougie (see chapter on Radiography). The presence of calculi may also disclose the irregular shape of the kidneys upon an $x$-ray plate.

As a rule, anomalies in shape are actual growth perversions, but they may be due to encroachment or abnormality of adjacent organs or tissues.

Anomalies of Size.- Variation in size from the normal is seen in the rudimentary or atrophied kidney. In some cases, the atrophied organ is a mass of connective tissue; again the kidney tissue is replaced by a cyst, both forms being useless for excretory purposes.

The remaining or functionating kidney may be congenitally enlarged, the enlargement being compensatory; and on the other hand instead of an atrophied kidney opposite to one that is hypertrophied, the other organ may be entirely absent. Happily, the single, enlarged kidney seems perfectly capable of doing the work for two, as is evidenced by many post-mortem examinations of those who, having this abnormality, have lived to advanced age and have died from other causes.

While one kidney may be atrophied or even entirely absent, with no trace of other anomaly, other malformations affecting the genitalia, such as atrophy of the testicle, are frequently associated.

Anomalies of Number.-Congenital absence of one kidney is very rare-about one in 2400 individuals (Morris). The great importance of this condition when it does exist, is paramount when nephrectomy is under consideration and it is unlikely to be overlooked with the routine practice of cystoscopic diagnosis.

Supernumerary Kidneys. - Supernumerary kidney is such a rarity that its existence has been doubted. Authentic cases, however, are recorded (Morris) of the discovery of a third kidney with separate ureter attached and independent blood supply.

Anomalies of Position.-The term "dystopia" is used to designate congenital displacement of the kidney. Although an otherwise normal kidney may be displaced, this anomaly is commonly associated with some malformation of the organ (Fig. 238). Displace- 
ment of the kidney occurs most frequently on the left side. Such displacement may vary in extent from a slight distance out of the normal position to that of being lodged in the canal of Nuck; or the two organs may be on the same side of the body. The kidney may lie upon the lower lumbar vertebræ, the so-called "pelvic kidney," and has been found on the sacrum (Casper). It may change its position so that the

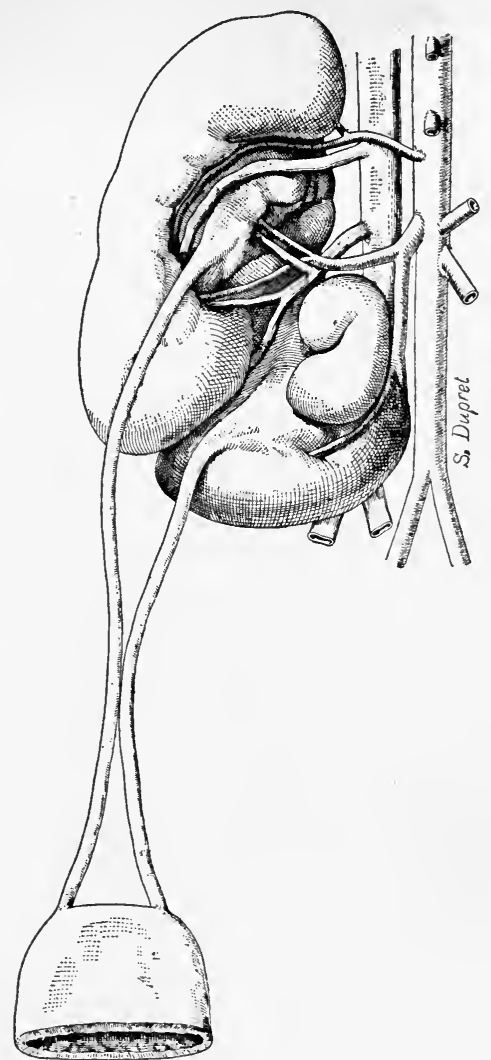

Fig. 238.-Anomalous misplacement of the kidney. (After Legueu.)

hilum may face in any direction other than that of the normal toward the opposite kidney.

The pelvis of the kidney may be displaced to the anterior surface instead of at the mesial border, while otherwise the organ is normal in position.

Misplaced kidney is not a synonymous term for movable or floating kidney. The two conditions may coexist, however. The term "misplaced kidney" refers to a congenital anomaly of position which 
is a fundamental defect of development. Movable kidneys undergo changes of position which are usually temporary in character, although a movable kidney may finally become fixed after it has shifted from its normal position. (See Movable Kidney, p. 588.)

The practical importance of a misplaced kidney consists in (a) its source of danger in parturition, (b) the danger of mistaking it for an intra-abdominal tumor, (c) the untoward effects of pressure upon the organ itself as well as upon adjacent organs and tissues. In the case of a misplaced kidney, the suprarenal body not infrequently remains in its normal position.

Anomalies of Attachment.-When the position of the kidney is abnormal, its attachment is necessarily faulty.

\section{Anomalies of the Ureter}

The significance of abnormalities of the ureters, is the effect they produce upon the kidney as their existence may be the cause of structural changes and functional impairment in this organ, namely, hydronephrosis and atrophy.

Anomalies of the ureter occur in number, in position and in structure. (See Fig. 85 p. 144.)

Anomalies in Number.-Three ureters have been found in a number of instances, and specimens with five and six have been recorded. The accompanying illustration is a demonstration of this anomaly, two ureters being connected with one kidney (Fig. 239).

The possible existence of a supernumerary kidney, with separate ureter is obscure, although such an anomaly is reported.

In the case of an absent kidney, absence of the corresponding ureter may be expected as a rule. In a few isolated instances, however, the inferior end of the ureter in a rudimentary condition appearing as a cord-like structure with no canal, or normal vesical opening, has been encountered. In the case of rudimentary kidney, the ureter sometimes terminates in a blind sac.

It is commonly observed that a horseshoe kidney possesses two pelves and two ureters.

The supernumerary ureters frequently coalesce in the upper part of their course, although this fusion may take place in the lower part or at any other point; or the ureters may remain separate and instead of being fused may enter the bladder at different points, the vesical termination of one being further from the bladder orifice than the other. The duplication may occur on one or both sides.

This abnormality is especially important in diagnosis, as the kidney 
drained by a double ureter may be partially bifurcated, one portion yielding normal urine and the other urine corresponding to the diseased condition of the organ, which may be pyonephrosis or tuberculosis. In such a case there would be difficulty in reconciling the clinical and cystoscopic findings following ureteral catheterization, if the catheter were passsed through the ureter connected with the healthy portion of the kidney.

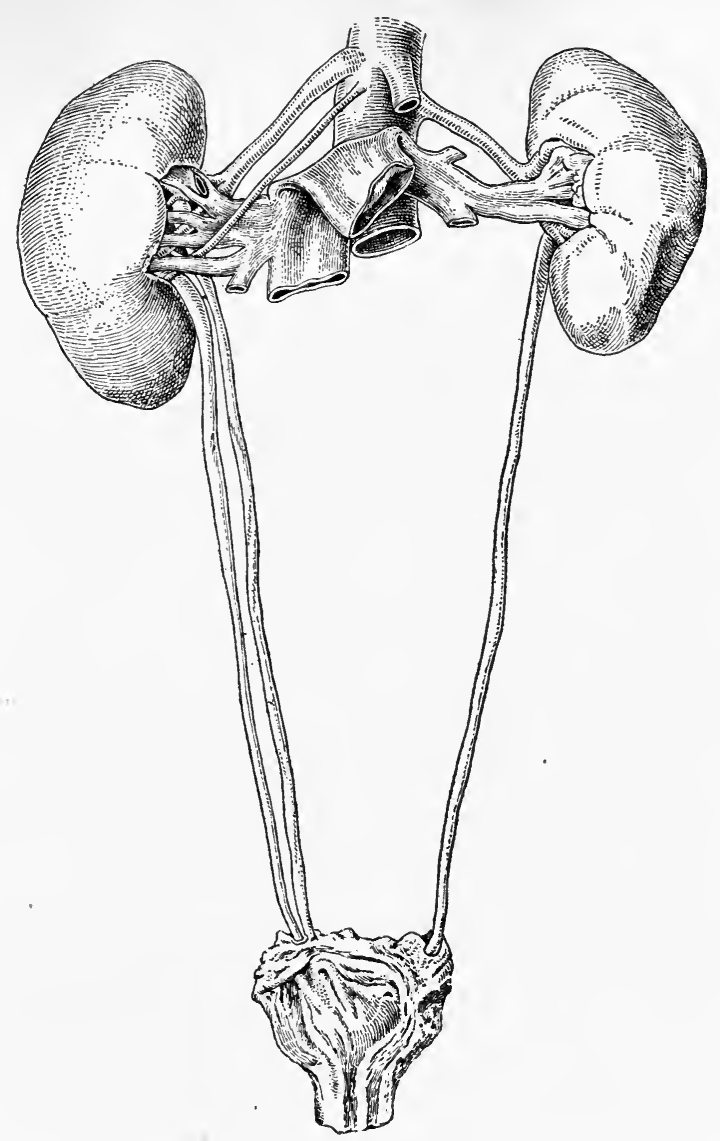

FIg. 239.-Three ureters with separate vesical orifices. (After Legueu.)

Anomalies of Position.-The ureter of one side may open into the bladder on the opposite side. Such a malposition might readily lead to a very serious error in determining the presence of a pathologic condition. The use of the $x$-ray with roentgenographic ureteral catheter has disclosed the existence of such an anomaly (see Roentgenography). Other malpositions of theu reter are found where this canal, instead of 
opening into the bladder is furnished with an anomalous outlet into the vagina. The ureter may terminate high up in the bladder instead of at its base. It has been known to open into the prostatic urethra or into the seminal vesical, and its origin, instead of extending from the dependent portion of the pelvis of the kidney, may follow a course along the lateral wall of the renal pelvis.

Malformation of the Ureter.-The ureter may be imperforate, when a congenitally atrophied kidney results, unless an anastomosis occurs, providing an outlet in some other location, in which case it may open into a diverticulum connected with the bladder. The calibre may be congenitally constricted to such an extent as to cause hydronephrosis. Valvules existing along the course of the ureteral canal may give rise to this latter condition, as may also the obstruction caused by fibrous or vascular bands. (See Hydronephrosis, p. 575.) Stricture of the lumen of the ureter may occur in practically any portion of its course. It is commonly found at its vesical orifice.

Diagnosis.-It is generally the case that these anomalies of the ureter are discovered on the autopsy table or by chance in experimental cystoscopic investigations with the $x$-ray, although, on account of the present knowledge of their occurrence it is quite common to look for such anomalies in cystoscopic diagnosis where there seems to be a confusion between the clinical symptoms and the cystoscopic findings. The advent of the roentgenogram catheter and pyelography in connection with $x$-ray diagnosis has made it possible to discover misplaced and multiple ureters, whereas with the cysto-urethroscope it is possible to detect the outlet of an anomalous ureter even when it is as low down as the urethral canal, affording an explanation in such an instance of urinary incontinence.

Treatment.-Surgical intervention is called for where anomaly of the ureter results in serious morbid condition of the kidney or hampered function, when it may be necessary to transplant the distal extremity of a misplaced ureter into a convenient portion of the bladder or in case of resulting hydronephrosis, plastic operation may be required to relieve this condition. (See Operative Surgery, p. 646.)

Kinks of the Ureter.-In addition to congenital defects in position and direction, the ureters are frequently the seat of irregularities, of course, on account of undue motility of the kidney and their attachment by adhesion, resulting in kinks. This abnormal condition is responsible for the production of renal symptoms, being the means of producing intermittent and permanent hydronephrosis; and the attendant symptoms often simulate so closely other lesions of the kidney that careful cystoscopic examination and radiography are 
required to clear up the diagnosis between such irregularities and renal tuberculosis or lithiasis. (See Cystoscopy, p. 129, and Roentgenography, p. 162.)

Treatment.-When a diagnosis has been made successfully and in some instances where this has not been possible, lumbar nephrotomy is performed, to afford complete exposure of the kidney and the relation to it of the ureter, and to allow such plastic operation as may be required upon the latter and finally a nephropexy, if the kidney is prolapsed or abnormally mobile.

\section{INJURIES OF THE KIDNEY AND URETER}

Injuries of the kidney may occur from a mild contusion to that of complete rupture and division in two separate halves (Fig. 240). Such injuries occur at all ages but necessarily more often in adults than in children and are more common in men than in women, whereas movable kidney is more common in women. Injuries to the kidney may result from direct or indirect violence whether such injury amounts to simple contusion or complete rupture. Inasmuch as it is believed that interior hydraulic pressure is the important factor in the production of rupture of the kidney, the greater the distention of the organ at the time of injury, the more likely is this severe condition to ensue. I have seen and operated upon several cases of rupture resulting from direct injury such as the impact of a falling wall and the pressure of a heavy cart wheel and cases are reported where such injury has occurred from muscular exertion alone (see Plate VIII, Figs. 1, 2).

The injury to the kidney may amount only to a subcapsular hemorrhage and hematuria; there may be a slight laceration of the capsule with extrarenal hemorrhage into the perirenal sac or complete separation of the kidney into two distinct segments.

The pathological condition accompanying injury, varies in different degrees from a limited area of ecchymosis and edema to extensive infiltration with polynuclear cells and degeneration of the epithelium of the tubules with purulent infiltration amounting to acute diffuse suppurative nephritis (Fig. 241).

Symptoms.-In case of slight injury to the kidney there may be nothing more than hematuria and the external evidence of contusion. The symptoms of ruptured kidney are those of localized pain and shock, in proportion to the extent of the injury, and hematuria. Immediately following injury, if severe, the patient is apt to be seized with nausea and pain in the lumbar region. There is generally rigidity of the rectus muscle on the affected side and a 
relatively increased resistance below the costal arch. There is usually marked tenderness in the same region, notably upon deep pressure and if the injury has ruptured the capsule and hemorrhage has been extensive, a rounded mass is felt by bimanual palpation. There is usually
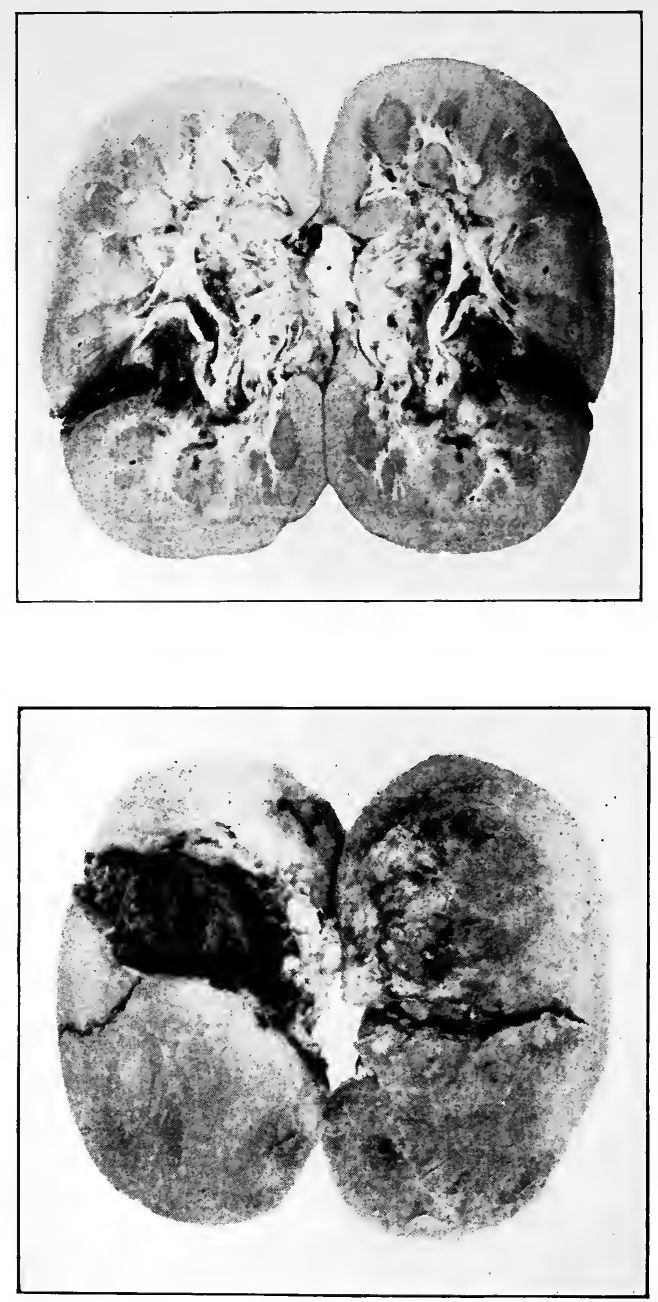

FIG. 240.-Traumatic rupture of kidney. (Author's case.)

an abundance of blood in the urine which may continue for an extended period even when the injury is slight. Sometimes the general condition shows marked evidence of collapse due to the extensive hemorrhage, and at the expiration of several days the temperature may 
become elevated, leucocytosis marked and there may be evidence of toxemia pointing toward acute suppurative inflammation of the kidney.
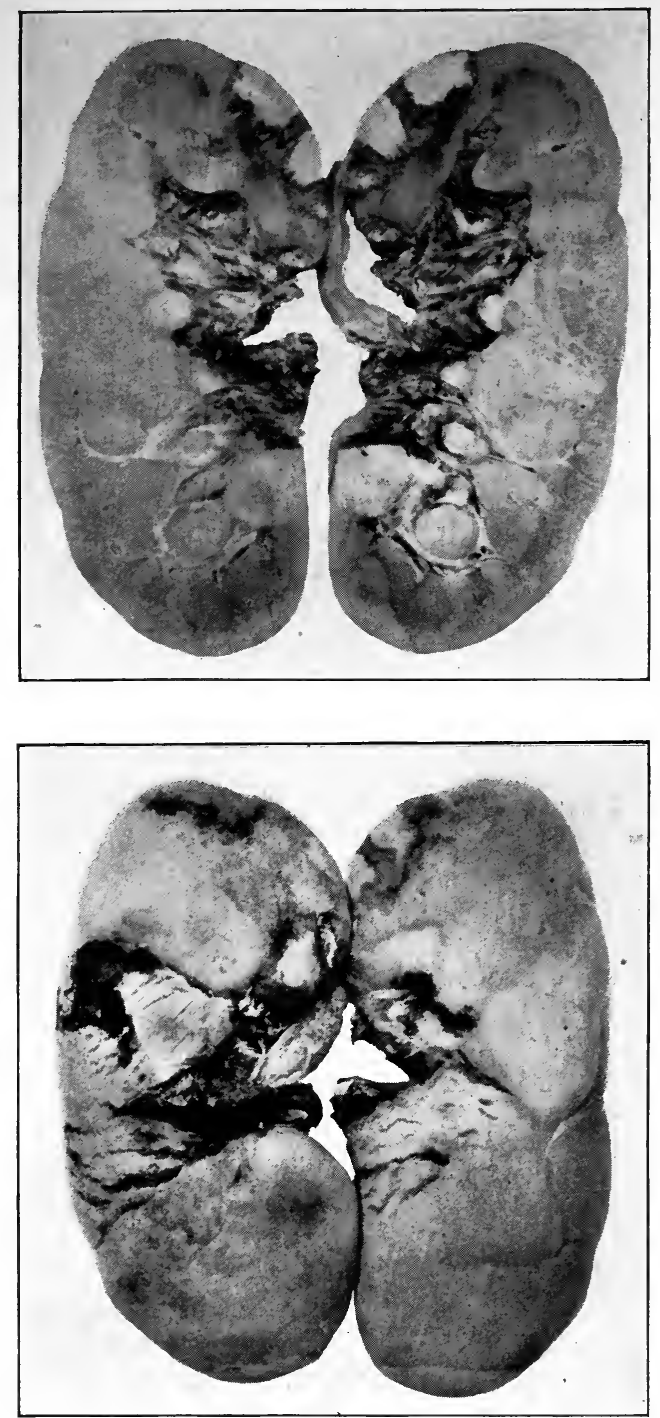

FIG. 241.-Traumatic rupture of kidney. (Author's case.)

The diagnosis must be made on the occurrence of a severe injury of the kidney region, the presence of blood in the urine as an indication of some kind of a wound of the kidney itself, and additional evidence 
of a palpable body with great collapse suggests the likelihood of rupture.

Treatment.-In formulating a rule of treatment for injuries of the kidney, one or the other of two courses is open: first, sympiomatic treatment, and second, exploratory operation. In mild and moderately severe cases of subcutaneous injury to the kidney, purely symptomatic treatment is indicated, namely: rest in bed, the application of an icebag, etc., and with such measures spontaneous recovery frequently occurs. Simple contusions of the kidney without laceration always end in recovery. When it is evident that the capsule of the kidney is lacerated, producing extrarenal hemorrhage and tumor; and when there is persistence of such hemorrhage with marked physical depreciation, exploratory operation is demanded and further action is determined upon after the kidney is exposed and examined. Where the rupture is incomplete and the general condition warrants it, suture and tamponade may be relied upon. Where the large vessels or the ureter

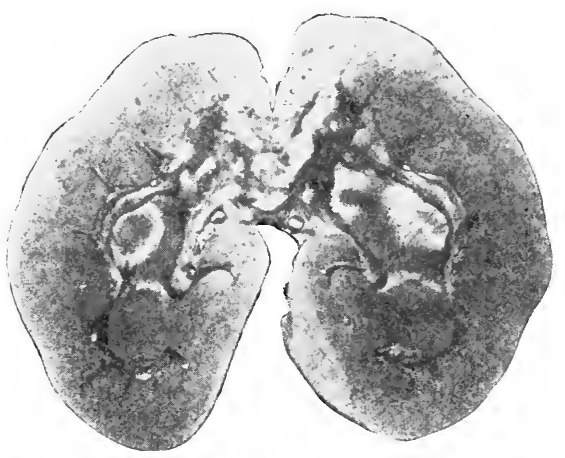

FIG. 242.-Rupture of kidney in child. Complete separation of ureter. Urinary fistula. (Author's case.)

have been lacerated and where the degree of hemorrhage is so great as to threaten total collapse, prompt removal of the kidney is called for. Again, when, after the expiration of several days, there is evidence of suppuration in the kidney on account of the systemic disturbance, leucocytosis and perhaps the urine obtained by ureteral catheterism, it may then become necessary to explore the kidney and proceed according to the conditions found, as in other cases of suppurative involvement of the organ. (See hematogenous infections of the kidney. $\left.{ }^{1}\right)$

1 In two cases operated upon by the author, radical operation was performed in one (Fig. 240) on account of extensive laceration of the organ and extreme hemorrhage and in the other because of beginning suppuration accompanied by marked physical deterioration (Fig. 241). A secondary nephrectomy was performed in another case, that of a child, on account of complete separation between the pelvis and the ureter and the formation of a fistula, (Fig. 242). 
For description of operation of nephrotomy and nephrectomy (see Operative Surgery, p. 632).

The Injuries of the Ureter occur for the most part during surgical operations upon other organs, occasionally during parturition, and in rare instances they are the result of traumatism from gunshot or stab wound. Such gynecological operations as entail removal of the tubes and ovaries accompanied by extensive adhesions are the occasion of injury to the ureter which may involve complete or incomplete division thereof. Ligation of the ureter may also occur during the removal of an extensive growth, and is followed by hydronephrosis or atrophy of the kidney (see Hydronephrosis).

In the case of incomplete injury to the ureter, there may be only slight extravasation of the urine, and cicatrization of the wound may follow without further untoward symptoms, unless a stricture at the site of injury is produced, when secondary hydronephrosis occurs and is recognized subsequently. Complete rupture or division of the ureter results in urinary extravasation, leading to cellulitis or septicemia, which, if not recognized and relieved promptly, becomes a very grave situation.

If injury to the ureter is suspected, diagnostic investigation is made by rectal palpation in the male and vaginal examination in the female, and if necessary, resort to ureteral catheterization. Exploratory operation or reopening of a surgical wound may be necessary.

The surgical means of relieving the condition resulting from severe injury to the ureter involves the operation of ureteral end to end, side to side and end to side anastomosis and vesical implantation. Partial injury, demanding no immediate surgical intervention may subsequently require plastic surgery, as in cases of partial occlusion of the ureter from cicatrization, accompanied by hydronephrosis; or secondary nephrectomy may be necessary on account of accidental ligation of the ureter. In cases of rupture of the ureter due to injury and division during a surgical operation, the point of injury is sought out through the original wound or a special abdominal incision; or the site of the injury is located by retro-peritoneal section. A description of the various operations on the ureter is given in the section on Operative Surgery, p. 632.

Fistula of the Ureter.-Fistula occurs as an ultimate result of an accident during surgical operation, or following an external injury, and more especially following the passage of a calculus down the ureter and the resulting damage inflicted by a stone too large to be delivered through the natural channel. Fistulæ may be congenital in the case of an anomalous position of the ureter. When acquired as a result of 
injury, they occur more often in the female than in the male, in the course of extensive gynecological surgery. Such fistulæ may be ureterouterine, uretero-vesical and uretero-vaginal. Other forms are ureterointestinal and uretero-abdominal. In all of these cases, the existence of such condition is demonstrated by the passage of urine through the fistulous opening, and if there be any doubt, diagnosis is established by giving the patient internally or hypodermatically methylene blue or indigo carmine to color the urine as it passes through the fistulous tract.

Treatment of fistulous ureter depends upon the condition of the kidney and the location of the fistula. Spontaneous closure of the fistula may occur. The cure depends upon the result of such spontaneous disappearance or of successful surgical intervention involving reëstablishment of the continuity of the urinary canal. Preliminary investigation of the integrity of the associated kidney should be undertaken in order to determine whether it is not better to remove the kidney, rather than establish the ureteral canal, in the case of a much disabled organ.

Stricture of the Ureter.-Stricture of the ureter may be congenital or acquired. As in the urethral canal, the ureter is prone to congenital narrowing at the point of normal anatomical constriction, one of the most common of which is the orifice of the ureter in the bladder. The usual cause of acquired ureteral stricture is the presence or passage of a calculus, which, on account of its size or the irregularity of its surface, causes inflammation. The secondary conditions following stricture of the ureter are of prime importance. In complete stricture, atrophy of the kidney may result, and in incomplete stricture, hydronephrosis and secondary infection are the natural sequence.

In reaching a diagnosis of stricture of the ureter, cystoscopic investigation, by means of ureteral catheterism, recognizes an obstruction on the affected side. This is usually accompanied with symptoms of pain on the same side and there is also experienced a certain amount of relief at the introduction of the ureteral catheter through the stricture, as the accompanying hydronephrosis or cystic dilatation of the ureter is temporarily relieved thereby.

The treatment of stricture of the ureter consists in an attempt to dilate it with the ureteral catheter and the conical ureteral bougie. Plastic surgery may be undertaken when the stricture is near the upper or renal portion of the ureter, and a meatotomy may be performed when the stricture is at its bladder outlet.

Uretitis.-Inflammation of the ureteral lumen is continuous, with a similar inflammation in the kidney or the bladder. It may be due 
to direct infection carried by the introduction of instruments, as a result of which abscess of the ureter has occurred. It may be due to tuberculosis $(q . v$.). Inflammation of the ureter behind stricture may be accompanied by small cystic distentions of the ureteral follicles or dilatations of the ureteral lumen. A diagnosis of inflammation of the ureter, with dilatation or abscess, may be elicited by palpation along the course of the ureter, cystoscopic observation of the ureteral mouth and the introduction of the ureteral catheter, which is arrested in the case of obstruction. The possibility of an obstruction being caused by external pressure, as in the case of a tumor, should be taken into account, and when present, may be detected by abdominal palpation. 


\section{CHAPTER XXVIII}

\section{CIRCULATORY CHANGES IN THE KIDNEY. NON-SUP- PURATIVE INFLAMMATION. HEMORRHAGIC IN- FARCTION (NON-SUPPURATIVE). ESSENTIAL RENAL HEMATURIA}

Changes in the circulation of the kidney may be either transient or permanent, that is, they may be part of a generalized morbid condition or a preliminary stage to disease of the kidney, infective or non-infective in type. The infective type is of greatest interest to the surgeon, yet the non-infective may at times demand his attention and the proper distinction must be appreciated to avoid confusion in diagnosis.

Anemia and Hyperemia.-These functional changes in the circulation of the kidney are dependent upon its relation to the general cardiovascular apparatus.

Anemia of the kidneys occurs when the blood volume is markedly diminished (severe diarrhea, cholera, persistent vomiting, fevers, etc.). The urine is then very concentrated. The circulation through the kidneys is diminished so as to conserve the fluids in the lymph spaces. In shock and hemorrhage, the anemia of the kidney is a striking feature. In the one case, the blood is diverted from the kidneys into the splanchnic vessels; in the other it escapes from the body.

Anemia of the kidneys also occurs in marked cachexia and in essential blood diseases (secondary and pernicious anemia, leukemia, etc.).

Hyperemia of the kidneys is a condition of greater import, inasmuch as it is more likely to be the forerunner of, or associated with conditions of severe pathological change in the renal parenchyma. It may be active or passive.

Active hyperemia may be caused by any renal irritant among which are to be found drugs like corrosive sublimate, potassium chlorate, potassium iodide, cantharides, cubebs, copaiba, turpentine, etc. It also occurs in acute febrile conditions when it may be more or less transient and in this condition there is associated some albuminous degeneration of the parenchyma. 
It is also likely to occur after severe exposure to cold. Such cases give a history of having fallen into cold water or of even such apparently moderate indiscretion as sitting upon a cold stone slab. This condition may pass imperceptibly into acute nephritis.

Active hyperemia occurs as the first stage of all acute nephrites whether medical or surgical, and whether non-bacterial or bacterial.

The diagnosis of active hyperemia may be made clinically, especially in those cases due to exposure to cold and to ingestion of drugs. It may vary from a condition presenting slight pain in the back, usually higher up than that complained of in other conditions, coupled with a trace of albumin in the urine and an occasional hyaline cast, to a more severe condition shown by exaggeration of these symptoms with diminution of the amount of urine. More grave findings lead to the diagnosis of acute degeneration, the first stage of many types of Bright's disease. It should be held clearly in mind that the diagnosis of hyperemia is to be made with a certain amount of reservation and the patient should be under suspicion of a graver situation until subsequent physical and clinical findings have cleared him of it. This condition is of importance in urology inasmuch as many of the drugs used in therapy appear among the etiological agents. Renal stone should not be forgotten in making a differential diagnosis.

Passive Hyperemia. Congestion.-This condition of venous stasis may be caused by advanced cardiac disease where the renal circulation suffers obstruction, in which case it is bilateral. It may, however, be caused by unilateral obstruction of one of the renal veins by tumors, etc., or may follow upon more or less advanced degree of movable kidney. The condition may also follow thrombosis of the vena cava or renal vein. Though primarily a systemic condition, it is of importance surgically inasmuch as it is an etiological factor in many subsequent changes that occur, and may eventually result in a relative degree of renal insufficiency. In addition, such a kidney is more susceptible to infection, being subnormal in its resistance and when thus infected, it may be found that there is a surgical focus requiring operative intervention and also an excretory apparatus which is, as a whole, deficient in capacity.

\section{DIFFUSE HEMATOGENOUS NON-SUPPURATIVE RENAL INFLAMMATION (BRIGHT'S DISEASE)}

The condition of albuminuria and dropsy described by Richard Bright in 1827 as showing morbid changes in the kidney has been shown since his time to be only a small part of the clinical pathology of 
diffuse nephritis. By diffuse is meant an involvement of all the renal elements, glomeruli, tubules and interstitial tissues.

The classification of morbid changes and the intimate relations between them and the clinical features, has never been satisfactory. The exciting cause - the toxic material in the blood stream-is unknown. A given provoking cause may result in a most diverse morbid change and clinical picture. Again, there seems to be no fixed relation between morbid change and clinical features.

The explanation of the condition lies in the meaning of renal insufficiency (uremia) and the causation of cardiovascular changes in the organism.

There seems to be no basis for the idea that in one form of nephritis, the epithelium is involved and in another, the interstitial tissue. Whether acute or chronic, the condition is always somewhat diffuse and having originated in one element, the others are finally more or less involved. That the pathology of the kidney should be looked at quantitatively is beyond question. But the gross and histological picture of the kidney does not explain matters as we are without the explanation of the actual cause of renal insufficiency.

It seems best to classify renal disease according to the nature of the process.

\section{Acute Diffuse Nephritis}

Causation.-A toxic substance circulating in the blood naturally causes bilateral, but not necessarily equal change. Likewise, one portion of the kidney may be affected more than another.

The toxic substance may be a chemical poison, as alcohol, corrosive sublimate, cantharides, mineral acids, lead, arsenic, phosphorus, salicylic acid, phenol, anilin preparation absorbed after application to the skin, chloroform, ether, iodoform in wounds, etc. It may be produced during the course of acute infections as in scarlet fever, diphtheria, variola, more rarely in tonsillitis, erysipelas, septic diseases, typhoid, cholera, pneumonia.

It may occur during the course of chronic conditions as tuberculosis, diabetes, carcinoma, syphilis, icterus, etc. Sudden exposure to cold has already been recognized as a cause for renal congestion which may develop into the severest forms of nephritis. Acute nephritis following severe burns is very common. Pregnancy is a factor of great import.

Morbid Changes.-The kidney varies in gross appearance. In milder degrees of acute nephritis there are no apparent changes. The 
organ may not even be enlarged. In many cases, however, the kidney is enlarged, flabby and friable. The color varies from light to dark red. It may be pale and mottled. Hemorrhages show themselves as dots or streaks of intenser shade. The cortex is swollen. The glomeruli are, in some cases, prominent, being enlarged and congested; again, they are pale. In certain instances, the capsule may be easily stripped off.

Microscopically, the tubular and glomerular epithelium is cloudy and swollen. The connective tissue is infiltrated with small round cells. The capillaries may be choked with cells and thrombi. The kidneys as a whole are involved but the morbid changes may not occur uniformly throughout the organs, the changes being more marked in certain parts.

The special location of the morbid process gives rise to a variety of terms designating the region in which the involvement predominates. Thus, we have tubular or parenchymatous nephritis, glomerular nephritis and interstitial nephritis, according as the tubules, the glomeruli or the interstitial tissues are most involved.

Clinical Features.-In some cases of acute nephritis, especially those which follow poisonings and suffer most marked degeneration of the kidneys, the only symptom may be certain urinary changes. This urinary evidence may range from a mere trace of albumin and an occasional red cell to the characteristic urine of acute nephritis. Such a urine shows a marked diminution to about one-third the usual amount-at times, complete anuria. The urine is very concentrated, usually turbid, due to the formed elements including casts, sero-hemorrhagic ("smoky") or bloody in hue and acid. The percentage of albumin is very high becoming at times .5 to 1 per cent. so that the urine boils practically solid. The microscope shows a profusion of red and white cells and casts, hyaline, granular, and blood casts; epithelium and hemoglobin.

Some cases of acute nephritis may start somewhat insidiously with only edema of eyelids and ankles calling attention to the process, but as a rule the occurrence is quite abrupt, following a well-known causative factor. The onset may be ushered in with some fever at times, or a chill and temperature $39.5^{\circ}$ to $41^{\circ} \mathrm{C}$. (103 to $106^{\circ} \mathrm{F}$.); there are pain or tenderness in the loins and costo-vertebral regions, frequency of micturition and nausea. Other symptoms are characteristic pallor of the face and edema which, beginning in the lower lids, spreads rapidly over the entire face. The pulse is usually slightly increased in frequency and there is distinct hypertension.

Gastro-intestinal symptoms such as nausea, vomiting or diarrhea, 
and nervous symptoms such as headache, delirium, coma and convulsions, are not uncommon. In severe cases, the edema involves the skin of the entire body, notably infiltration of the scrotum or labia majora. The serous cavities (pleura, pericardium and peritoneum) may become involved and the edema may involve even the lungs and glottis with fatal results.

After some weeks, retinal changes such as occur in chronic nephritis and also hypertrophy of the heart occur. The blood shows marked secondary anemia after some time has elapsed (increase in the fluid content, decrease in the hemoglobin and red cells).

The prognosis is good as to recovery from the acute attack. Acute nephritis rarely ends fatally. Children, especially, show a marked recuperative power and as a rule recover in a few weeks. The chances, however, are that even after recovery, the patient may retain changes in the kidney that will lead to one of the types of chronic nephritis, either soon afterward or after a lapse of years. Cases prolonged for several months are apt to pass directly into the chronic type of nephritis.

Treatment.- The known irritating causes of nephritis should be avoided, among which are the use of drugs, alcohol, etc., already enumerated. In cases of acute infectious disease particularly apt to be followed by nephritis, the patient should be carefully watched and protected from exhaustion and cold; confinement in bed, a bland diet of such articles as milk, buttermilk, gruels, and the free use of diuretic water should be enforced.

In cases that are associated with marked edema, a salt-free diet is worth trying as sometimes it acts in a remarkable manner. In order to give this procedure a fair trial, even minute amounts of salt should be scrupulously avoided.

Symptoms requiring special treatment must be met with as they arise.

Edema may become so tense as to cause cracking of the skin with large areas liable to infection. In such cases where fluid nourishment has been forced without comparative diuretic effect, it may be advisable to institute a "dry" diet meeting the intense thirst with cracked ice, fruit juices, etc.

Impending uremia may be averted by hot wet packs or hot-air baths. In uremic coma, phlebotomy may be of marked advantage: a removal of 250 to 500 c.c. (8 to 16 oz.) of blood and substitution of normal saline in similar quantity may bring the patient completely out of coma. Other symptoms (convulsions, etc.), must be treated accordingly. 
Chronic Nephritis (Chronic Diffuse Nephritis, Chronic Parenchymatous Nephritis-Large White Kidney; Chronic Desquamative Nephritis-Second Stage of Bright's Disease).-In certain cases of renal affection the onset of the disease is insidious, the course is prolonged and the symptoms are persistent. Such a state of affairs constitutes chronic nephritis. The acute and chronic types overlap each other to a certain extent. It is not, therefore, uncommon to hear such terms as subacute and subchronic applied to transition types of nephritis. Very often, the onset of chronic nephritis is so insidious that the physician is unable to obtain any definite history of the acute stage. This is true of most forms of chronic nephritis.

Causation.-The etiological factors of chronic nephritis, if their influence is sufficiently prolonged, are identical with those of the acute form. This toxic agent may be a drug, food or poison, or it may be developed in the body during the course of some disease, possibly as a sequel of disturbed metabolism. Lead and alcohol are the most common extraneous substances that injure the renal structure.

Alcohol in itself is irritating; it also has the effect of decreasing the resistance of the cells. When alcohol acts directly on the vessels, causing arteriosclerosis, renal disease is a logical sequence.

Lead has similar effects.

Chronic nephritis occurs as a sequel of the diseases involving disturbances of the metabolism, such as gout and diabetes mellitus. It is not uncommon to find an association between nephritis and hepatic diseases. Alcohol may be the etiological factor in both maladies.

A special congenital tendency to disease of the kidneys exists in some individuals.

Morbid Changes.-The most striking gross changes are the increase in size and the color. The kidney is swollen and rather soft and the capsule peels off easily. The surface is smooth, the color is red when the blood-vessels are generally congested, but grayish-yellow if the epithelium is fatty. A variegated appearance is caused by the occurrence of infarcts. When there is a considerable degree of hemorrhage into the renal tissues, the condition is termed chronic hemorrhagic nephritis. When the fatty changes are marked, there results the so-called large white fatty tumors and when the infarcts are prominent, the surface becomes irregular.

It has already been pointed out that the morbid process is never limited to the epithelial elements. The interstitial tissue is always involved to a certain extent. Therefore, there can never be a pure type of chronic parenchymatous nephritis. The interstitial connect- 
ive tissue continues to increase, until finally, the kidney that was at first enlarged, slowly becomes contracted while its surface becomes granular (the secondary contracted kidney).

There are cases in which the parenchymatous changes are not sufficiently marked to enlarge the kidney in the first stages. Therefore, the contraction proceeds slowly until the genuine contracted kidney with granular surface results. This kidney may be red from hyperemia or white from anemia.

As has already been indicated, it seems least confusing to classify nephritis according to its morbid changes in the kidney. Variations in nephritis from the anatomical as well as from the clinical standpoint, are the result of the intensity of the morbid process, its rate of development, and the extent of the tissues involved.

This quantitative view of renal disease is after all the only Iogical one. Histologically, one can often find all the types of disease in one kidney.

Clinical Features.-As in many cases, the invasion of chronic Bright's disease is insidious. Dropsy is of ten the first symptom noticed. The urinary findings are most important. Among these are albumin and casts. The presence of albumin in the urine gives evidence of epithelial depreciation severe enough to allow leakage of serum. One theory considers the excretion of albumen an inflammatory exudation. ${ }^{1}$

Renal epithelium and red and white cells are also found in the urine.

The renal cells lose their power of excretion, consequently toxic material accumulates in the blood and the general condition of the patient suffers. A very characteristic and constant symptom of chronic Bright's disease is general physical weakness and gradual failure of health.

Anemia with its associated phenomena-cardiac palpation and breathlessness on exertion-is a common symptom of chronic renal disease.

In order to make up for lack of power in the kidneys, the heart is overworked, raising the arterial blood-pressure (increasing the pulse tension) and bringing about hypertrophy of the left ventricle and at times, subsequent right-sided hypertrophy also. Precordial apprehension and palpitation often develop. In consequence of the cardiac change, the area of cardiac dullness is increased, especially toward the left, and the aortic second sound is accentuated.

\footnotetext{
I Casper: "The casts are either exudation products, being formed by coagulation of the albumin exuded from the blood-vessels or they develop from the union, fusion and degeneration. of epithelial cells cast off from the uriniferous tubules."
} 
Uremia may occur in a variety of forms. In certain cases the nervous system bears the brunt of the disturbance, in others the gastrointestinal tract suffers most, while again, the respiratory system shows the most marked effect of the circulating toxins. Albuminuric retinitis may become well marked.

Eye symptoms, headache especially, sudden vertigo, dyspnea without apparent cause and indigestion are all symptoms pointing to nephritis.

The skin and hair are subject to certain disturbances with renal insufficiency. The hair becomes dry and breaks easily and the skin becomes eczematous. Pains in the back are often significant of renal disturbance, having long been associated with chronic nephritis as a symptom.

The pain of chronic renal disease is situated low down, is usually bilateral in the region of the fourth or fifth lumbar vertebra, and resembles a typical lumbago.

Transient hematuria may appear now and then, but rarely.

Edema of the face (eyelids) and dropsical effusions (thorax and abdomen) are characteristic. This is quite different from dropsy of cardiac disease which appears first in the dependent regions of the body.

The belief that diagnosis is easily made from the chemical and microscopical examination of the urine is practically untrue. This statement does not mean that the various tests and methods of examination may' not give useful information, but in many cases the exact diagnosis of the definite types of renal inflammation cannot be made from urinary analysis.

If the total amount of solids in the twenty-four hours remains below 70 grams, the deficiency, accounted for in no other way by exercise or diet, may well suggest the existence of interstitial nephritis. On the other hand, if the solids rise considerably above this amount, the indications are renal overwork.

Even if albumin and casts are not detected in the urine, a high blood-pressure without other demonstrable cause will suffice for the establishment of diagnosis of Bright's disease.

A relative renal insufficiency may also be demonstrated by catheterization of each ureter.

The prognosis in chronic nephritis depends largely upon the extent of the morbid process.

The disease may not reach a fatal termination for years. This is especially true when the manner of living can be favorably regulated. On the other hand, the patient may die after a year or two. 
The physician must not allow himself to be deceived, for although the patient's condition may be undergoing apparent improvement, the malady may be making sure progress and the kidney be slowly contracting.

Four types of contracted kidney are accepted: 1. Secondary contracted kidney, a sequel of the large white kidney. 2. Primary contracted kidney, originating in acute nephritis and not going through the large white kidney stage. 3. Arteriosclerotic kidney, due to sclerosis of the renal vessels and so degenerative rather than inflammatory. 4. Senile. ${ }^{1}$

Primary interstitial nephritis bears a close anatomical resemblance to the other types of chronic interstitial kidney, but is recognized as a "separate entity" for clinical reasons. The onset is so insidious that no noticeable symptoms occur until the kidney is already contracted. The etiology is commonly obscure. As a rule, the kidney is smaller than the secondary contracted organ. The color is dull red and the capsule adherent. In the pelvis are masses of fat and the kidney as a whole is surrounded by fat. The branches of the renal artery are markedly sclerosed. As a rule the patient is gouty.

The "genuine contracted kidney," "gouty kidney" "or red granular kidney" represents that form of Bright's disease called primary chronic interstitial nephritis.

Treatment of Chronic Nephritis.-The judicious treatment of Bright's disease puts to proof the physician's skill. Each patient requires individual care and there is usually a demand for an entire change of regimen. The conditions of other organs must be carefully determined.

Prophylaxis is very important and has already been considered under acute nephritis.

Alcohol and condiments are excluded. Certain cases do particularly well on an exclusively milk diet; however, this is not true of all instances.

Individual treatment is most important and the special idiosyncrasies of the patient must be studied and respected. The increase of albumin, blood and casts after certain foods, warns against such articles of diet.

The question of the advisability of nitrogenous food must be answered in every individual case according to the especial effects in each case. And the variations are great. The ease with which combustion in the body is accomplished will be the deciding factor. Broths are contraindicated by some authorities on account of their purin content.

1 Adami and Nichols, “Principles of Pathology," Vol. II. 
As to water drinking, the amount ordered depends upon different conditions. Especially in cases of interstitial nephritis, the quantity should be restricted than otherwise.

But when the patient is gouty or rheumatic, a more generous supply should be allowed. Indeed, in nearly all cases, a large quantity of water is of benefit if not pushed to excess. Clothing should be light but warm. Chilling must be avoided.

As to the most favorable climate for nephritics, rules are somewhat flexible. However, it is well to choose a climate where extremes of temperature and where sudden changes do not occur. The drier climates are also more favorable. The condition of myocarditis and arteriosclerosis is unfavorable to a high altitude.

Exercise, especially walking, if the patient is able to be about, is advisable. Massage is a good substitute for exercise for sedentary or bed-ridden cases.

Warm baths are helpful therapeutic measures; the extent of the reaction must be watched, catching cold and undue fatigue being avoided. In favorable instances, warm or hot baths keep the skin in its best functionating condition and are beneficial. Turkish baths must be taken with caution.

The various symptoms may be met by appropriate medication as they arise.

\section{Non-suppurative Infarction of the Kidney}

\section{Synonyms.-Bland infarction, hemorrhagic infarct, etc.}

Renal Infarction is a morbid process due to obstruction of the circulation in one or more portions of the kidney, and as the renal afferent circulation branches rapidly into a fan-shaped structure of terminal arteries, that is, arteries supplying areas which have very little collateral circulation, the kidney lends itself readily to such circulatory change.

Causation.-The pathogeny of the non-suppurating infarcted kidney (see also hematogenous infection, p. 611) originates from that portion of the circulation that lies between the left side of the heart and the bifurcation of the renal artery. The infarcts are found, as a rule, in left-sided valvular disease, which is usually described as rheumatic in type. That type of infarct associated with malignant (usually streptococcus) endocarditis does not necessarily break down into purulent foci. During the last decade it has been noted that these infarcts, non-suppurative in character as they are, may be and are found typically associated with chronic bacterial endocarditis. In these cases, which may run long courses of even a year or more, the 
blood stream will yield on careful culture the small streptococcus. ${ }^{1}$ This organism does not cause suppuration, and will form the typical infarctions described above, not only in the kidney, but in other parts of the body (spleen, etc.).

In addition to the heart valves as an etiological factor, the atheromatous aorta must not be overlooked. There are many cases where, with careful dissection and examination of the renal artery, no thrombus of macroscopic size can be discovered, and where a fairly recent infarct yields no explanation other than arterial spasm. Of course, in some cases fragments of tumor or a large clot have been found plugging the lumen of the artery, but, as a rule, at the autopsy table thrombi are entirely organized and undiscoverable.

Morbid Changes.-The infarct, as a rule, consists of a pyramidal area of kidney tissue having an apex toward the hilus of the kidney and a subcapsular base; it may vary in size from that of a pea to even half the entire substance of the organ. The area may be extremely irregular, so that it is difficult to make out its quadrilateral shape; the non-suppurative infarcted area rapidly becomes grayish or glary, due to the anemia of the tissue. Soon it softens, and at this stage it is surrounded by a hyperemic zone of compensatory circulation, which may measure several millimeters in diameter, when coagulation necrosis ensues.

Microscopically, the infarcted tissue shows coagulation necrosis; the cells are distended and granular, the nuclei are pale and in all the stages of lysis down to complete disintegration. The basophilic granules are scattered through the cells, giving the tissue a bluish tint when stained with hematoxylin. The hemorrhagic zone shows dilatation and engorgement of the capillaries, minute extravasation of blood, and areas of polynuclear leucocytes. Later on, the infarct may soften to a yellow semi-fluid consistency, not due, however, to the presence of suppuration, as smears or scrapings will show. On section, the tissue is often impregnated with microscopic, glistening, somewhat stellate crystals, which may be some hemoglobin derivative. The cellular outlines are completely lost. These infarets occasionally break down completely or become adherent to other organs (e.g., spleen, colon); they rarely become infected.

Later, the infarct gradually organizes with retraction of its periphery and later still of its entire area. It may become almost fibromatous. The kidney now becomes indented and distorted irregularly by these infarcted areas, which, when extensive, give to it a lobulated contour. Meanwhile, it has apparently suffered no functional inconvenience,

1 Vide, Lenhartz, Die Septischen Erkrankungen, Chap. Endocarditis. 
even in cases where it has become deformed to the most advanced degree. The embolic contracted kidney of Bright's disease is to be considered a Bright's disease kidney with infarcts, that is, a kidney representing two distinct conditions, in which the nephritic symptoms are due entirely to the former.

Clinical Features.-Clinically, the diagnosis of renal infarct may be made in typical cases. The onset may occur with vomiting, pain in the loin of more or less dull character, but sometimes radiating. Urinary symptoms may be present, namely, suppression of urine with later appearance of polyuria. After careful centrifugation, at times a few scattered red blood cells may be found in the urine.

The diagnosis is, of course, only to be surmised in the presence of a cardiac condition. The urinary findings are to be judged only after careful weighing with previous urinary reports, as the possibility of a passive hyperemia of the kidney should be taken into consideration. The condition is more likely to be diagnosed when the characteristic conditions are coupled with the presence of the small streptococcus in the blood stream. Although renal infarction is to be considered as a medical rather than a surgical condition, it may become a pitfall of diagnosis, of great importance to the surgeon. In the first place, when the condition occurs on the right side, it can be mistaken easily for cholecystitis or appendicitis. In the absence of the occasional red blood cells in the urine, and in the presence of a low leucocytosis, the exhibition of astute judgment is called for. Again, the condition must not be confused with unilateral septic renal infarcts $(q . v$.). A carefully made blood culture will often be necessary to differentiate this condition, as well as to prevent the removal of a kidney in the case of this essentially medical disease. Such an error has been committed, and the diagnosis of malignant endocarditis made by the pathologist following the skillful removal of a kidney, the surgeon suspecting septic infarct. Operation is likely to be disastrous on account of the systemic condition of the patient, who is suffering from a general constitutional malady.

Its distinction is of peculiar importance as operation upon such a patient, even though immediately recovered from, may be the factor which engenders fairly rapid cardiac deterioration.

Bland infarction of the left kidney must be differentiated from that of the spleen. This is a difficult matter, as the latter is so rarely diagnosticated. Clinically, however, an attack of left-sided pain, in the presence of a spleen enlarged to percussion and palpation in conditions such as leukemia, polycythemia (Osler's disease), pseudoleukemia, etc., should suggest the presence of a splenic involvement. 
Renal infarctions not uncommonly occur rather painlessly, and are only suspected when the etiological factors are found after a complete examination. There is rarely macroscopic hematuria, as is occasionally seen in early tuberculous nephritis, which by its sudden onset may simulate bland infarction.

Renal tumors and perinephric abscess and movable kidney with crises, must be held in mind (see Index).

Treatment.-Surgically, renal infarcts demand no treatment. Their importance lies in the fact that they are often at the bottom of obscure conditions of abdominal pain, and help to complicate the clinical picture and cloud the diagnosis.

\section{Essential Renal Hematuria}

Renal hematuria is one of the most constant and important symptoms of morbid lesions of the kidney. It is especially significant of renal calculus, neoplasm, etc. The question is, does it ever occur independent of any morbid lesions of the kidney as would be inferred from such designations as essential renal hematuria-idiopathic renal hematuria? Many instances are reported of cases of unilateral hematuria in which none of the common causes of hematuria existed and furthermore in which it was impossible to detect even slight evidence of gross anatomical changes as a cause of the condition. It is hard to believe that it is possible for hemorrhage of the kidney to occur without the existence of some morbid lesion, be that lesion ever so slight, notwithstanding the views held by some writers. ${ }^{1}$ It is more reasonable to conclude that an undiscovered lesion has existed in such cases of hematuria, which has disappeared following palliative operation or some other expedient. To discover such cause, it is necessary that more than a casual examination of the kidney be made and as there are few autopsies reported, a careful study has been lacking in most instances. ${ }^{2}$

The view that active hematuria from the kidney can occur without

1 Hale White, Essential Renal Hematuria, Quar. Journ. of Med., July, 1911.

2 H. A. Fowler, Wash. Trans. Am. Urological Assoc. 1911, reports a case of profuse renal hemorrhage cured by nephrectomy. The kidney on the operation table showed no gross signs of disease outside of extreme anemia and there was no abnormality apparent in the pelvis or in the papillæ. Pathological examination with a lens failed to discover varices in the papillæ as an explanation of the bleeding. A studied histological examination of many sections of this kidney, while presenting various abnormalities, according to the author, failed to explain the cause of the hemorrhage.

I operated upon a similar case in which the hemorrhage was also so profuse as to produce extreme anemia. In this case, however, nephrotomy and decapsulation were performed and the hemorrhage promptly ceased. There was no adequate explanation of the cause of the hemorrhage from the gross observation of the kidney. A section of the cortex removed from an area, suggestive of an old infarct, revealed the evidence of very mild parenchymatous inflammation. 
there being any evidence of abnormality after careful investigation has been held and advocated by different writers in earlier times. ${ }^{1}$ On the other hand, others have held that there is really no such thing as bleeding from a pathologically normal kidney; that if such kidneys be carefully examined, some slight lesion will be discovered. The point of divergence between these opposite views seems to be that while it is recognized that cases of unilateral hematuria occur without obvious explanation, that in most, if not in all of these cases, there does exist some slight morbid condition of the kidney, but there is a question raised whether the anatomical findings are sufficient to explain satisfactorily the occurrence of active hematuria. The following are some of the pathological conditions which may be found in different cases:

Nephritis; even slight lesions are believed to be responsible for hemorrhage from the infected organ. ${ }^{2}$ Again it is pointed out that the very first symptom of nephritis, is hemorrhage. ${ }^{3}$ In some instances, there have been found glomerular nephritis with connective tissue formation encircling the glomeruli. ${ }^{4}$

Again following hematuria unilateral nephritis has been found at autopsy. ${ }^{5}$

Colon bacilli have been found in the kidneys with and without malposition of the organ. ${ }^{6}$

Capillary nevus or angioma of the renal papillæ have been discovered as the cause. ${ }^{7}$

Many cases of so-called essential renal hematuria may be explained on this ground and be undiscovered inasmuch as renal decapsulation and fixation have been demonstrated to be an effective means of curing it. ${ }^{8}$

${ }^{1}$ Legueu, Anal. des mals des. org. genito-urinaire, 1891; Klemperer, Deut. med. Woch., 1897; Spencer, Trans. London Clin. Assoc., 1904; Illyes, Deut. med. Woch., 1906.

${ }^{2}$ Kapsammer, Wien. klin. Woch, Jan. 20, 1906.

${ }^{3}$ Katzenberg, Zeitschrift fr. Urologie, 1908, 125.

${ }^{4}$ In Fowler's case (loc. cit.) a satisfactory knowledge of the condition of the glomeruli was not obtainable although the hemorrhage appeared to have occurred by way of the glomeruli and tubules. Albrecht, Wien. klin. Woch., Jan. 20, 1906.

${ }^{5}$ Rayer; Trait des Maladies des Reins. Author's statement: A recent case of unilateral renal hematuria demonstrated by ureteral catheterization a one-sided nephritis with deficient functionating capacity upon the affected side, by urea estimation and phenosulphonephthalein tests. The hematuria totally disappeared in this case following renal decapsulation.

${ }^{6}$ Rovsing, Centralblat für die Krankheiten der Harn und Sexual, etc., 1898, Author's note: I have operated upon a similiar case of renal hematuria in which, before operation, nothing could be discovered by ureteral catheterism and $x$-ray and when the kidney was exposed by nephrotomy nothing more than a movable and misplaced organ to all appearances normal was revealed. In this instance hemorrhage ceased entirely following partial decapsulation and nephropexy.

${ }^{7}$ Hugh. Cabot, Amer. Jour. Med. Sci., 1909.

${ }^{8}$ P. M. Pilcher, Bkln. Trans. Am. Urol. Assoc. 1901, Renal Varix. The writer reports four such cases and calls attention to the fact that a gross examination will not discover the cause which is revealed by microscopic examination of the renal papillæ which shows a typical angiomatous disease. 
Small and hidden areas of tuberculosis may exist as a cause for the symptom of hematuria but such cases do not come under the designation applied to the condition under description.

Clinical Symptoms.- The occurrence of hematuria, is, as a rule, not, painful, but there may be the accompaniment of pain radiating down the ureteral region, especially when, in addition to the fluid blood, clots are formed and passed. The bleeding may be so severe as to cause practically exsanguination and necessitate nephrectomy. ${ }^{1}$ In women, it may be associated with amenorrhea and may cause its increase and lead to the suggestion of a vicarious menstruation. ${ }^{2}$

Diagnosis.-The term essential hematuria should not be used too loosely, simply because the cause thereof cannot be demonstrated. A small papillary growth or polyp might cause such hematuria, and yet be impossible to demonstrate without exploratory nephrotomy. Tuberculosis should be considered and sought for in all instances as well as the presence of small calculi by radiographic examination. It might also prove useful to study the comparative functional activity of the two kidneys by colorimetric or other functional tests. The prognosis is good in all operated cases. In untreated cases, it is possible to understand that the patient might succumb to the hemorrhage.

Treatment.-Various measures of treatment have been recommended and all, strangely enough, have had more or less good effect; namely, simple exposure of the kidney without further interference or accompanied by acupuncture, decapsulation, nephrotomy and tamponing, nephrotomy and cauterization of the renal papillæ. Finally cases have been reported in which simple ureteral catheterism and nothing more has been followed by cessation of hemorrhage for a long period. ${ }^{3}$ When, after exhaustive diagnostic study, the common causes of renal hemorrhage have been excluded, namely: neoplasm, stone, tuberculosis and demonstrable nephritis, the most advisable course in such cases is resort to palliative measures-rest and the internal administration of therapeutic agents that have demonstrated some efficacy by previous experience, as the oil of turpentine, tincture of the chloride of iron and ergot, the fluid extract of senecio aureus, followed by ureteral catheterism and possibly pelvic lavage. When such palliative measures fail the blood serum may be administered by subcutaneous injection as successfully tried in hemophilia of the newborn and suggested by the investigator (Welch) ${ }^{4}$ in other conditions.

\footnotetext{
1 Fowler (loc. cit.).

${ }^{2}$ Schüller, Wien. klin. Woch., 1904, 477.

3 F. R. Hagner, Annals of Surg., 1908.

4 J. E. Welch, Normal Human Blood Serum as a Curative Agent in Hemophilia Neonatorum, Am. J. Med. Sci., June, 1910. 2nd Ref. Barringer, Amer. Assoc. G.-U. Surgs., 1912.
} 
If these measures fail and an operative course is decided upon, nephrotomy may be performed without opening the kidney, the capsule stripped and the kidney fixed in normal position or the pelvis may be opened and the calices inspected. In extreme cases, nephrectomy may be called for. (For description of operations see Operative Surgery, p. 632.) 


\section{CHAPTER XXIX \\ TUMORS OF THE KIDNEY AND URETER}

Causation.-Practically nothing definite is known of the causation of new growths of the kidney, although such factors as irritation and prolonged infection are usually mentioned. As in some other parts of the body, cases are recorded where renal neoplasm was associated with tuberculosis, calculus, pyelonephritis and pyonephrosis. ${ }^{1}$ Whether these have had any causative connection is difficult to say. Nevertheless, there may be nothing of the sort in many cases. The occurrence seems to be somewhat more frequent in males than females. ${ }^{2}$ The right side is affected slightly more often than the left, but this is of little importance. Bilateral tumors are found relatively more of ten in children. In adults, bilateral tumors are rare. ${ }^{3}$

Various classifications ${ }^{4}$ of renal tumors may be made. A convenient one is the following:

${ }^{1}$ P. Albrecht, Klinich und Pathologische Anatomie der Hypernephrome 34 Congress der Deutschen Gesellschaft für Chir., Berlin, 1905; Battle, Tumor of the Kidney with Calculi, Brit. Med. Jour., 1895; Habershon, A Case of Pyelitis, Med. Times and Gazette-Lancet, 1880.

No. cases Males Females

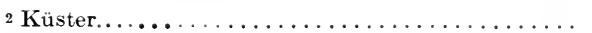

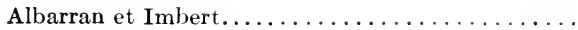

601

402

348

68

227

45

3 Thirteen times in 606 cases (Küster).

${ }^{4}$ Classification of renal tumors, modified from Garceau (Tumors of the Kidney, 1909).

$A$. Solid tumors of the renal parenchyma. B. Embryonic tumors.

Malignant.

1. Hypernephroma.

2. Carcinoma.

3. Sarcoma.

Round cell.

Spindle cell.

Fibrosarcoma.

Alveolar sarcoma.

Liposarcoma angiosarcoma. Benign.

4. Adenoma (malignant).
1. Adenoma.
2. Angioma.
3. Lipoma.
4. Fibroma.

1. Dermoid (teratoma).

2 Rhabdomyoma.

3. Mixed tumors.

$C$. Tumors of renal pelvis.

1. Epithelial growths.

a. Papilloma.

b. Papillary epithelioma.

c. Non-papillary epithelioma.

2. Mesodermal growths.

$a$. Alveolar and round cell sarcoma.

b. Angiosarcoma.

$D$. Tumors of the capsule.

$E$. Pararenal tumors.

$F$. Cysts and cystic kidneys. 
I. Tumors derived from tissue, of renal histogenesis-sarcoma, carcinoma and adenoma.

II. Tumors derived from tissue, not of renal histogenesis-hypernephroma and mixed tumors.

The latter group is of far more importance clinically, as it makes up the great majority of all growths which the surgeon is called upon to treat.

Another classification places them in either benign or malignant category.

\section{HYPERNEPHROMA}

Causation.-The histogenetic development of this growth is entirely of adrenal tissue. ${ }^{1}$ It commonly occurs in adults, and in mature years, but it has been found also in extreme youth and at advanced old age. It is more frequently found in males, and upon the right side. Bilateral cases occur, and may be due to secondary deposit, as the tumor most of ten travels by venous route.

Morbid Changes.-Although the kidney is the most frequent site, remnants of the adrenal may be found in various other organs, namely, the ovary, the testicle, the epididymis, the spermatic cord, along the spermatic vessels, in the liver and the pancreas. These remnants, however, are not as frequently found, even in the kidney, as the malignant neoplasm itself. ${ }^{2}$ It should be understood that they are non-malignant. They vary in size from a split pea to a walnut, and may be found under the capsule, but usually in the region of Bertin's columns. ${ }^{3}$

These original tumors are of the structure of adrenal cortex. They are deep yellow, and separated from the kidney tissue by a thin capsule, and may remain benign until found at postmortem, but on account of their rarity at autopsy, it is probable that they usually develop into malignant tumors.

\footnotetext{
1 On account of its fatty appearance, it was once thought to be a lipoma, but this idea was corrected by Grawitz, who was one of the first to insist upon its adrenal origin. Virchow Arch., Bd. XCIII, 1883; Berl. klin. Woch., 1884.

Being of epithelial origin, it has been called "Epithelioma à cellules claires," Albarran et Imbert, Les Tumeurs des riens, Paris, 1903.

The term hypernephroma was first used by Lubarsch, Virch. Arch., 1894, Vol. CXXXV, p. 195.

They were long thought to be derived from epithelium of the convoluted tubules, from endothelium of blood or lymph vessels, but are undoubtedly of adrenal origin. Recently in investigating their adrenal origin, they have been placed with the mesotheliomata, as being due to Wolffian elements, Wilson and Willis, Jour. Med. Res., 1910, XXIV; also Wilson, Ann. Surg., Feb., 1912. 1906.

2 Neuhäuser Das Hypernephroide Carcinom und Sarkom, Arch. für klin. Chir., Bd. LXXIX,

Such remnants occurred but once in 2000 postmortems, Hall, Hypernephroma, Arch. Internal Medicine, Nov. 15, 1908.

${ }^{3}$ Lubarsch, Virch. Arch., Bd. CXXXV, 1894.
} 
Having attained development, the tumors form masses which may be larger than an adult's head. It is very common to find a large tumor with a portion of renal tissue attached to it. This renal tissue may replace half or two-thirds of the kidney, where the tumor develops from one or the other pole, or a tumor centrally located may have a rim of renal tissue at its upper or lower pole. The gross changes therein are very slight.

The tumors may distort the pelvis by traction, or they may grow into the pelvis, but as a rule, they show a greater tendency to grow into the veins, forming soft friable masses, which may extend into the vena cava, and even up to the right auricle. ${ }^{1}$

On gross section, the tumor is yellow, except in parts where hemorrhage and degenerative changes have occurred. The secondary formation of cysts is exceptional.

There is a great deal of confusion about the histology of hypernephroma, because it may assume several shapes. The unit cell is a large, somewhat polygonal cell, which is said to have a fat lecithin and cholesterin content, different from that of other tumors, ${ }^{2}$ but corresponding closely with the relative and actual amount in the adrenal. The tumors present a rather close mesh-work of capillaries, flanked by rows of these typical cells. ${ }^{3}$ The arrangement is of the narrow tubular type, sometimes with an expansion of these tubules into alveoli, filled with clear or finely granular material; rarely, the cell columns may be hollowed out and be in drrect connection with capillaries, giving a picture similar to angiosarcoma.

A common form is that in which the tubular and alveolar arrangement is further modified into a broad-cell columnar type, interspersed with capillaries (similar to that found in perithelioma). Giant cells may be found, and various types of degeneration, even to complete cellular necrosis. The tumors in such cases present grossly a most variegated appearance.

The histological diagnosis is thus seen to be very difficult. The yellow color and the tendency to degeneration are both of great diagnostic importance. ${ }^{4}$

The kidney in direct contact with the tumor shows a reactive zone of interstitial alteration. The opposite kidney may show signs due to toxic change (albuminuria, etc.), which may vanish after nephrectomy

\footnotetext{
${ }^{1}$ Wyler, Centralblatt für die Krankheiten der Harn und Sexual Organe, 1898.

2 The fats and lipoids of malignant renal hypernephromas, Internat. Clin., 1908, No. 2.

${ }^{3}$ These cells have a striking resemblance to some of the cells seen in the decidua.

${ }^{4}$ Crofton has found that the watery extract of the tumor will reduce uncooked starch to dextrose and maltose. Boiled starch solution, colored with iodin will be decolorized in a few seconds or minutes by a few drops of this extract. It does not occur with other tumors. Crofton, Chemical Diagnosis of Hypernephromas, Jour. Amer. Med. Assoc., 1903, XL, 91.
} 
of the morbid organ. It may also show marked compensatory hypertrophy, so much as to be mistaken for a tumor growth.

Hypernephroma may spread by direct extension into adjacent tissue, or form neighboring nodes. This may be the case in local recurrence after removal, with protrusion through the scar. Distant metastasis occurs early, the venous route being the most usual, in the lungs, liver, bones, etc. Rarely the brain and opposite kidney are affected. Lymphogenous metastasis to distant places (e.g., left supra-clavicular regions) has occurred. Urogenous metastasis (implantation hypernephroma) in the pelvis and lower ureter is rare if it occurs at all. ${ }^{1}$

Bone metastases are of great importance; ${ }^{2}$ they are usually centrally located in the marrow, and act like medullary sarcomata, causing pathological fracture. The skull, femur, humerus and clavicle are the favorite sites. These tumors may be so soft and vascular as to pulsate or fluctuate. They may be the sole metastasis, and may occur without any renal symptoms whatever. In some of these cases, even a thorough kidney examination has failed to reveal a palpable kidney.

\section{CARCINOMA OF THE KIDNEY}

Carcinoma of the kidney is not of fiequent occurrence. ${ }^{3}$ These tumors are considered by some ${ }^{4}$ as developed from renal tubules, and by others ${ }^{5}$ as a later stage of renal adenoma. The cells of the carcinomata are usually massed together, with no regular order, and invade the renal tissue widely. The tumor does not usually become very large, or distort the kidney much before it terminates fatally. Metastasis usually occurs only to neighboring glands, but the lungs or liver also may be affected. Growth into the renal pelvis or ureter is common. ${ }^{6}$

\section{SARCOMA OF THE KIDNEY}

This neoplasm usually originates from the capsule or perirenal tissue. It may be small or large round celled, the former being more malignant. Other classified forms also occur (see Table, p. 550). They are usually soft nodular growths, grayish in color, and showing

\footnotetext{
1 Clairmont, Beiträge zur Nierenchirurgie, Archiv f. klin. Chir., Bd. LXXIX, 1906.

2 Scudder, Ann. Surg., Dec., 1906, XLIV, 851.

3 Newhäuser (loc. cit.).

4 Albarran and Imbert (loc. cit).

${ }^{5}$ Weichselbaum und Greenisch, Das Adenom der Niere, Wiener med. Jahrbücher, 1883.

${ }^{6}$ Newhäuser (loc. cit.), carcinoma occurred seven times in 102 renal tumors.
} 
degenerated areas. They occur also in children, but such growths on careful examination are more likely to be mixed tumors. ${ }^{1}$

Adenoma.-The benign form occurs in small cortical masses, generally smaller than walnut size. They are usually papillomatous in formation, but may be tubular or alveolar. They may be multiple, and there seems to be some relation between them and the compensatory hypertrophy of chronic nephritis; hence, they occur only in later life. $^{2}$

This tumor may have the same histology, and yet be malignant. It may prove just as fatal as carcinoma, but is not so likely to give rise to metastases.

Angioma.- A rare condition, but of extreme interest clinically, inasmuch as it occurs in the renal pelvis, and at the tips of the pyraniids (see Chapter on Essential Renal Hematuria).

Lipoma.-Fat infiltration and replacement of tissue in various conditions of non-suppurative and suppurative renal inflammation, are very common, but lipomata are rare, and are usually very small. Occasional cases have been reported of tumors as large as a child's head. ${ }^{3}$

Fibroma.-As a rule, it is insignificant in importance and small. A fibroma 20 pounds in weight has been reported. ${ }^{4}$

Embryonic Tumors.-Dermoids are very rare. They contain actual fetal inclusions and reproductions of heterogeneous tissue, and even fetal parts. ${ }^{5}$

Rhabdomyoma is also a very exceptional tumor, aithough found widely scattered throughout the body. ${ }^{6}$

Mixed tumors are scarce, and occur in infants. ${ }^{7}$

These tumors have been variously described as sarcoma, adenosarcoma, fibrosarcoma, etc., when more extensive study of various parts of a given tumor would show it to be not such a specific form but a mixed tumor. ${ }^{8}$ The latter and the sarcomata make up most of the tumors in children.

\section{Tumors of the Renal Pelvis and Ureter}

The epithelial growths need very little explanation. The papilloma is the most common, and is similar to the type found in the bladder.

1 Eight were found in 102 cases, Newhäuser (loc. cit.).

${ }^{2}$ Ludek, Virch. Arch., Bd. CXXXIII, 1893.

${ }^{3}$ Kelnach, Renal Growths, London. Mitchell, N. Y. Med. Jour., Nov., 1908.

${ }^{4}$ Bruntzall, Berl. klin. Woch., 1882, Vol. XIX, p. 745.

${ }^{5}$ Hoechel, Berl. klin. Woch., 1902, Vol. XXXIX, p. 964.

${ }^{6}$ Brock, Zeit. für Ges. d. Arzti in Wien, 1894, Vol. V, p. 331.

${ }^{7}$ Wihns, Die Niesch geschw ülste der Niere, 1899.

${ }^{8}$ Walker, Ann. Surq., 1897, Vol. XXVI, p. 586. 
It is very delicate and fragile. It shows a tendency to malignancy ${ }^{1}$ and is then called papillary epithelioma. When malignant, it propagates itself by direct extension down the ureter $^{2}$ and by metastasis.

Squamous celled carcinoma (epithelioma) occurs also in the pelvis. It is very rare.

The coincidence of hemorrhagic nephritis ${ }^{3}$ with pelvic tumor is noted. There may also occur hydronephrosis ${ }^{4}$ with intermittent hematonephrosis. ${ }^{5}$

Tumors of the Ureter occur ${ }^{6}$ primarily, but as a 1 ule, these tumors are metastatic by direct extension or implantation, more often as an extension from the renal pelvis. These tumors partake of the same characteristics as those of renal growth. Some form of malignant neoplasm is more common than any of the benign type. Papillary growth is the common variety which may start in benign form and become transitional and then malignant. A diagnosis of these growths may be difficult to make, the only symptoms being hemorrhage and pain which may be ascribed to renal calculus. All available information by cystoscopy and ureteral catheterism should be obtained, including the functional capacity of the kidney (see p. 155) in spite of which, however, an exploratory operation may be required to confirm or clear up the diagnosis. The existence of a papillary growth of the ureter, extending from the kidney, will call for an extirpation of the organ, on account of the chances of malignancy, and probable return if growth alone is removed.

Mesodermal tumors occur in the pelvis and ureter, but are very rare. $^{7}$

Pararenal Tumors.-Such growths depend for their origin upon misplaced adrenal elements or remains of the Wolffian body, which later may persist throughout the course of the ureter, even as far as the epididymis. They may be of the same histology as tumors of the capsule, and differ only in that they are not so intimately connected anatomically. Among them are found the hypernephromata, which arise from the adrenal itself ${ }^{8}$ or those arising from adrenal elements in the retro-peritoneal tissues. ${ }^{9}$ 1906.

${ }_{1}$ Graemve et Wanthy, Epithelioma papillome, etc., Jour. de Chir. Ann. de la Soc. Velge de Chir.,

2 Kohlhardt, Virch. Arch., Bd. CXLVIII, 1897.

${ }^{3}$ Walter, Monats berichte für Urologie, 1902.

${ }^{4}$ Frankl, Centralblatt für Chirurgie, 1901.

E Luffier, Hematonephrose intermittente, etc., Soc., de Chir., Paris, 1894.

${ }^{6} \mathrm{~L}$. Adler, Bietrag zur Kenntniss der primären Tumoren des Ureters, Monats Berichte für Urologie, 1905.

${ }^{7}$ Frisch, Primäres angiosarkom des Nierrenbeckens, Gesellschaft der ärtze Wien, 1892. Martin, Tumeur du rein chez. un Enfant de cinq mois., Soc. des Sciences Med., de Lyon, 1896.

8 Israel, Zur diagnose der Uebernieren geschwülste, Deut. med. Woch., 1905. 1884 .

9 Chiari, Zur Kenntnis der accessorischen Uebennieren des Menschen, Zeitsch. für Heilkunde, 
These tumors are among the largest abdominal tumors. They may weigh 18 to 22.5 kilo. They occur most commonly in women past middle life. Their size allows them to cause symptoms chiefly by pressure. ${ }^{1}$

\section{Clinical Features of Renal Tumors}

The chief clinical signs of neoplasm of the kidney are hematuria, pain and tumor, but these symptoms likewise occur in other conditions of the kidney, and must be qualified. In addition, there are various other signs which point to renal tumor, to be taken into account.

Hematuria.- When painless and profuse, it is very characteristic. It is usually spontaneous, and although likely to be profuse, slight tinging of the urine and microscopical hematuria also occur. Hematuria, while a frequent symptom in adults, is less so in children. ${ }^{2}$ This rarity of hematuria in the cases in children is the consensus of opinion. Generally speaking, hematuria in some amount is more likely to be observed when cases are carefully studied. ${ }^{3}$ Clots may accumulate in the ureter and be expelled as ureteral casts; renal colic may be associated with this process. ${ }^{4}$

Hemorrhage is usually from the tumor itself, and hence is very common in pelvic growths or pelvic invasions, but may be absent ${ }^{5}$ and in cases where only the kidney proper is involved, it is favored by the existing stasis. ${ }^{6}$

Pain.--In the early stages, pain occurs in about 20 per cent. ${ }^{7}$ When present, it is usually dull, and confined to the loin-rarely has any radiation in the beginning. It is due in some cases to tension upon the capsule, and to hemorrhages in the growth itself. Later in the disease, the involvement of the nerve trunks may give typical neuralgia radiating from the lumbar region to the front and toward the groin.

Lumbar Mass.-In children, as a rule, the enlargement is the first sign that calls attention to the condition. In adults, the loin tumor is palpable, but the chief complaint may be either pain or hematuria. However, cases are not uncommon in which the kidney is only palpable at its lower pole, or is not palpable at all.

140 to 50 pounds.

${ }^{2}$ Küster (loc. cit.) reports hematuria in 52 per cent. of 379 tumors in adults, whereas he found only 16 per cent. in 133 tumors occurring in children.

${ }^{3}$ Kapsammer found it in nineteen of twenty-one cases.

${ }^{4}$ Schede, Neue Erfahrungen ueber, Nieren Extirpation, Jahrbücher der Hamburger Statts Anstattungen, 1889.

${ }^{5} \mathrm{P}$. Albrecht, loc. cit., supra.

${ }^{6}$ Israel, Chir. klin. der Nierenkrankheiten.

7 Albarran et Imbert, Les Tumeurs de Riens. 
Very small nodules in the kidney may give rise to extensive metastasis. In some cases, attention is only called to the tumor when a metastasis has occurred.

The tumors may have any size, even to filling almost the entire abdomen, as is often the case in children, when at times they may be easily perceived by inspection. The tumor is usually firm, somewhat irregular and when large, may move noticeably with respiration.

Symptoms associated with urination are not common, but may occur from the passage of blood coagula, and reflex disturbances, such as retention of urine, may result.

The urine may contain traces of albumin or sugar, aside from the hematuria which is the usual finding. Bits of tumor may be found in the urine in some cases, but not much reliance can be put in the diagnosis of single cancer cells.

Varicocele is not an uncommon symptom, and occurs on the same side as the tumor. It is a sign in favor of malignancy, as the pressure is due to glandular involvement as a rule. Differing from the ordinary variety, it does not vanish when the patient lies down. ${ }^{1}$

Varicosities of the lower extremities and abdominal walls may occur, as well as secondary thrombosis of the vena cava, due to tumor, the latter being more common in children.

Other rarer symptoms are:

Gastric and intestinal symptoms due to pressure, and confusion with the diagnosis of carcinoma of the stomach may result. Intestinal symptoms (alternating constipation and diarrhea) occur with the huge tumors found in children. Jaundice has been seen, and may be due either to pressure on the right side or to absorption of extravasated blood in the tumor. The sallowness which is a mild icterus is very common, and is characteristic of the cachexia of malignancy.

Diagnosis.-Clinically, one should consider any mass in the lumbar region as a possible renal growth. There has been a tendency on the part of medical writers to define too definitely and to be too dogmatic about the symptomatology of the various types of renal growths. Certain definite clinical pictures have been described as belonging to tumors of the kidney, of the pelvis, etc. While it is very easy to reason from a pathological standpoint in this manner, as a matter of fact it is often not possible to separate clinically tumor of kidney, pelvis, capsule and pararenal region. In the case of a mass in the

\footnotetext{
1 Hochenegg, Ueber Diagnose und Klinischen Bedeutung Varikocele bei Malignen Nierentumoren, Zeitsch. f. klin. Med., Bd. LXII, 1907.
} 
lumbar and abdominal regions, other possible causes for such regional enlargement must be held in mind, pancreatic cysts, retroperitoneal sarcoma, Hodgkins' disease, movable kidney, tumor of hepatic flexure, or even of stomach, hydronephrosis, etc. (see Chapter on Pyonephrosis).

The different methods of investigation and various data derived from complete physical examination (e.g., general glandular enlargement, enlarged liver or spleen, complete blood count, etc.) may lead to a working diagnosis. The cystoscope, under such circumstances, may give valuable diagnostic evidence. In the absence of hematuria there may be a marked diminution in the amount of urine on the side upon which the mass is felt, and such urine may present low specific gravity, diminished urea, and show a deficient functional test (see p. 155). In case absolutely no urine is secreted, a fairly accurate diagnosis of renal neoplasm may be made.

However, in many cases, pararenal growths of large size may give a similar picture. These, however, are more likely to cause marked pressure signs (dyspnea, rena caval pressure, etc.). A normal pelvis determined by measurement or pyelography might lead to error by diverting attention to some non-renal condition. In such instances, the condition of the urine renders assistance in many ways: first, as regards the presence of such abnormal elements as blood, pus, etc., and, second, as to the relative quantity present of normal constituents, namely, urea, chlorides, etc. (see p. 83). The finding of abundant suppurative elements points toward an inflammatory lesion. The ureteral catheter may demonstrate the existence of a collection of retained fluid suggestive of hydronephrosis, and pelvic measurement, coupled with pyelography, may verify it.

Clots forming casts of part of the ureter are more likely to occur in tumors of the pelvis or renal tumors which have invaded the pelvis of the kidney; but examination of the lower urinary tract with satisfactory roentgenograms is necessary to exclude the possibility of ureteral calculus.

The diagnosis of pelvic or renal tumor should not be made from isolated cells found in the urine, as they cannot be satisfactorily judged; even fragments of tumor should be considered very carefully, as pelvic and vesical papillomata may have a very similar histology, hence, careful cystoscopy should be made before a diagnosis of pelvic papilloma is to be ventured. ${ }^{1}$

A stream of bloody urine coming from one ureteral orifice, a nega-

\footnotetext{
1 Fenwick has noticed, in growth in the renal pelvis, a peculiar cystoscopic picture. The meatus of the affected ureter shows a dull-colored swelling and elongation, like a swollen meatus of the male urethra; or the orifice, normal in size, may show roughened edges and a dull red-colored interior. This may occur after the passage of clots, but is not a necessary result.
} 
tive radiograph for calculus, and diminished functional capacity on the part of the same kidney, are significant of renal tumor. But one must not forget that there are other causes of hematuria, such as early tuberculosis, nephritis (q.v.), and, therefore, detailed examination should be made in each instance. If the pelvis is gently injected, it is possible to provoke a dull pain similar to the pain complained of by the patient. If not, the chances are strongly in favor of a non-renal mass. Pyelography should be of value in working up the diagnosis (see Chapter on Roentgenography).

Broadly speaking, the tumor of the kidney which occurs most often in adults, is, in the majority of instances a hypernephroma and gives as a rule the cardinal symptoms, pain, mass and hematuria, followed by cachexia and metastasis. ${ }^{1}$

The tumor of the kidney which occurs in children, in the first five years. is usually very huge, causing marked asymmetry of the abdomen, is more likely to be associated with pressure signs or signs of abdominal distention than with hematuria, and pathologically is usually the sarcoma or mixed tumor.

Prognosis.-It is usually very poor, even after operation; however, no other treatment is of any avail. Serum therapy (Coley) in the case of inoperable sarcomata may be tried. Recurrence usually takes place within a year.

Treatment.-The only proper treatment is nephrectomy in all growths where there is any suspicion of malignancy. Exploration may be made and frozen section to justify partial nephrectomy or enucleation in cases of benign histological neoplasms. This should, however, not be allowed in adenoma and in small tumors of adrenal histology, as they are prone to be very malignant. Should the kidney show nothing grossly, the pelvis must be exposed for the source of the hematuria.

In angioma of the pelvis results have been obtained by cauterization with Paquelin cautery. (See Essential Hematuria.)

Papilloma of the pelvis demands nephrectomy, as it may be benign or malignant.

In operating upon hypernephromata, it is well to use a large transverse lumbar incision, and having opened the peritoneum purposely, the renal vein should be ligated close to the cava so as not to sweep fragile fragments into the circulation. The tumor is then removed in a retrograde manner, the artery also having been ligated at the same time.

1 Of thirty-two cases from the Mayo clinic, twenty-nine showed pain in the renal region; twentyeight had macroscopic hematuria, and thirty had a palpable mass. 


\section{Cysts and Cystic Kidneys}

Morbid Changes.-The multiple retention cysts of the kidneys are of no surgical importance. They are usually from the size of a pin head to that of a pea, multiple, bilateral, and due to blocking of the tubules by contraction of connective tissue in the course of chronic interstitial nephritis. Large sized single cysts occur. They may communicate with the pelvis, are usually unilateral, and may hold as much as 10 litres. $^{1}$

\section{Polycystic Kidneys}

They are usually bilateral. ${ }^{2}$ Grossly, they may be small and not clinically demonstrable, but may be the size of a man's head. One may be larger than the other. They are formed of a mass of cysts which contain colloid-like material of various colors. The capsule is thick and hard, and the entire organ will collapse on section, unless previously hardened. The fluid may vary from urinous fluid to dark brown, and at times even show purulent change, and the chance of secondary infection is great, as these patients, like nephritics, are prone to die of secondary septic conditions.

The tissue on section may show grossly no renal tissue at all, and even microscopically it may be almost impossible to find. Nevertheless, kidneys of such histology may be sufficient to carry on their function until late in life.

Theories of pathogenesis are :

(a) The product of a proliferative process.

(b) The product of a chronic interstitial nephritis.

(c) Congenital defects.

This last theory is the one most in favor at present. They occur in families, and very often are associated with other congenital malformations and cyst formation in other organs.

Clinical Features.- They occur in two types of cases: An infant at birth presents severe dystocia, and an examination of the prominent abdomen reveals palpable tumors on both sides. Death follows very soon, due to renal insufficiency. The adult form presents an insidious onset, usually in women between the ages of forty to fifty years, having remained latent until then. The symptoms are then the gradual march or the rapid onset of renal insufficiency. Hematuria and pains in the loins are not uncommon, and very often a tumor is felt unilaterally only. The urine shows changes of granular kidney.

${ }^{1}$ Rendu, Gros Cyste du rein droit., Ann. de Gynæk., 1898.

${ }^{2}$ Lejars, Du gros rein polycystic de l'adulte, Thèse de Paris, 1888 (60 cases). 
Diagnosis.-Cystoscopy and ureteral catheterization show a marked diminution of renal excretory function, but differentiation cannot be made from nephritis. They are also lixely to have the cardiac hypertrophy, hypertension, etc., of nephritis. The presence of a palpable tumor is of most value in differentiation.

Treatment.- See General Treatment of Renal Tumors. 


\section{CHAPTER XXX}

\section{RENAL AND URETERAL CALCULI. CALCULUS ANURIA}

Stone may occur in any part of the upper urinary tract but is most common in the kidney. Many stones located in the ureter and bladder originate in the kidney and increase in size after acquiring their new location. They may be unilateral or bilateral and occur at all ages. ${ }^{1}$ Large stones are usually single; small stones may be multiple or single. They vary in size from fine gravel to that of extensive coral-like masses branching into the calices and filling the pelvis. The geographical localities in which renal lithiasis is said to be common are the regions of eastern Europe, India, Egypt, Southern Russia

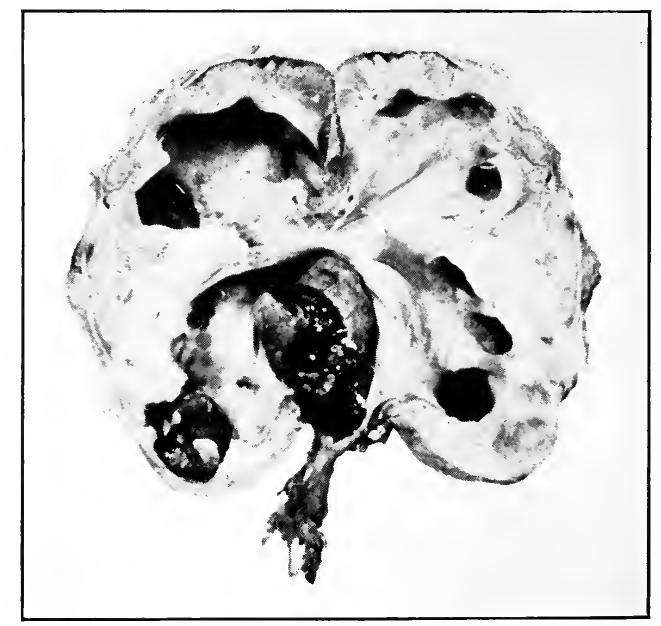

FIG. 243.-Kidney containing two uric acid stones. (Author's case )

and in the northern part of the British Isles, notably Scotland. The commonest forms are urates, phosphates and oxylates.

Uric acid or urate stones are reddish in color, smooth and round in contour and hard (Fig. 243). Phosphatic stones are white, generally not smooth on the surface and soft or friable in consistency (Fig. 244).

1 Kapsammer, (Monats. Berichter für Urologie, 1906) found at autopsy twenty-two out of seventy-three cases bilateral. 
Oxalate stones are exceedingly hard, irregular on the surface with small protuberances (mulberry calculi), grayish-brown in color (Fig. 245). Rarely a stone is encountered which is composed of cystine which is oft and a yellowish-green color. Many stones are of mixed composition. The nucleus of primary stone is as a rule in infancy and youth the urate of ammonia or uric acid, and in later life calcium oxalate.

Causation.-The causation of renal calculus depends in the first place upon whether the calculus is primary or secondary. A primary calculus may be described as one that forms in a kidney in which there is no morbid process. A secondary calculus, on the other hand, is one which is formed under the influence of some preëxistent patho-

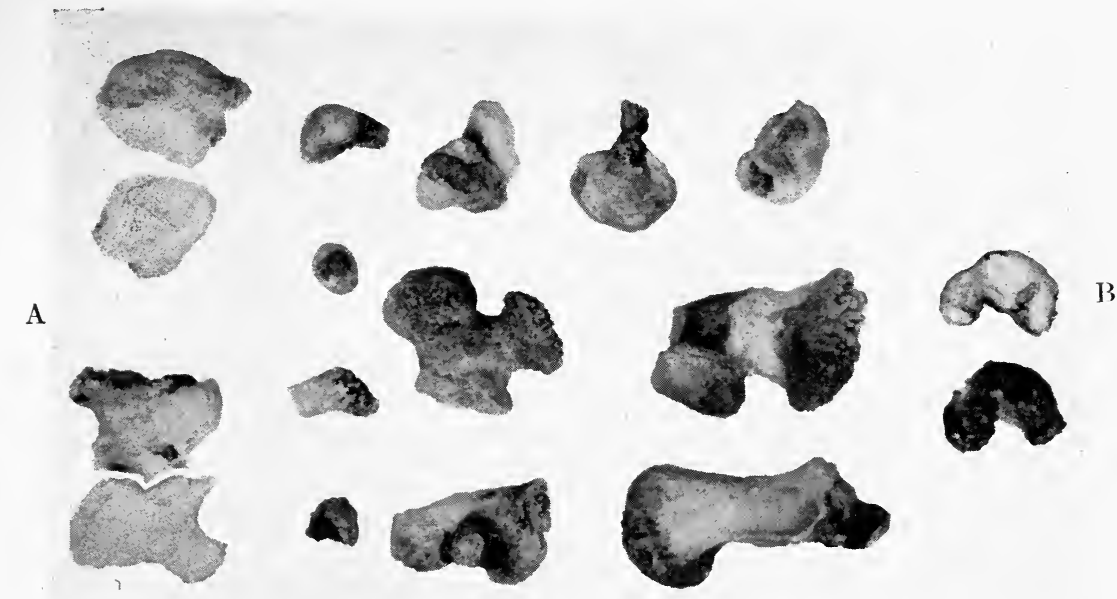

FIG. 244.- Phosphatic renal calculi (one-half actual size). A, Calculi from pelvis and calices of kidney. B, Calculus from cellular tissue, outside of pelvis and ureter. (Author's case.)

logical condition in the kidney, namely pyelitis, pyelonephritis or pyonephrosis. Thus, urate, oxylate and cystine stones are nearly always primary, no matter what may occur later, in the way of infection and inflammation as a result of their presence; they are deposited in aseptic kidneys on account of a combination of causes most of which are not essentially renal (Fig. 245). Phosphatic and carbonate stones are usually deposited in connection with infection (Fig. 244). There are certain influences which play an important rôle in the production of renal calculus; these are diet, diminution of the fluid intake, excessive drinking of acid wines, consumption of nitrogenous food in excess; disturbances of metabolism-gout, rheumatism, etc.; heredi- 
tary predisposition. Of all the causative agencies mentioned, it requires a combination of several or an overwhelming influence of one to determine the formation of stone. The most important cause that exercises in any sense a specific influence is situated in the kidney itself and is therefore local. Such cause involves the presence of a foreign body of some sort, which acts as the primary focus or nucleus of stone. For instance, this may be parasitic, such as the echinococcus hooklet or the ova of Bilharzia, but these are exceedingly rare and the rôle of a nucleus may be as well supplied by the presence in the kidney of microörganisms which, while not pathogenic, may in combination with colloid material (mucus, fibrin, etc.), become the primary focus

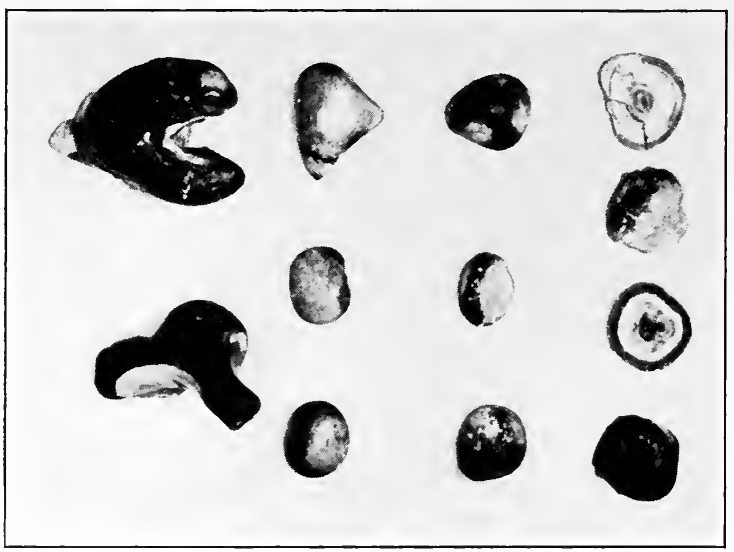

FIG. 245.-Primary renal calculi (aseptic) (uric acid and calcium oxalate).

(Author's case.)

of crystalline deposit, which eventually forms a small or large renal calculus. ${ }^{1}$

Morbid Changes in the Kidney.-In cases of aseptic stone in the kidney, there may be evident very slight morbid changes. Stones may thus form in the pelvis and pass on into the ureter and bladder without any visible deterioration of the kidney. When hematuria occurs, there is caused some local traumatism, but this may not be demonstrable short of a pathological examination of the kidney structure upon the autopsy table. On the other hand, the changes in the kidney associated with the presence of calculus may be those of the gouty kidney or chronic interstitial nephritis; there may occur localized damage due to pressure causing atrophy of the pyramids and connec-

${ }^{1}$ Israel, Chir. klin. der Nieren krankheiten, 1901. 
tive tissue proliferation, and obstructive consequences, namely, circumscribed or general hydronephrosis. These consequences demonstrate the importance of surgical intervention in renal calculus even before infection occurs; and when this latter condition ensues in the pelvis or parenchyma of the kidney, all the accompanying morbid changes from mild pyelitis to severe pyonephrosis are encountered (see Bacterial Infection of kidney, p. 599).

In combination with renal tuberculosis or neoplasm, the presence of stone is of secondary importance to the pathological changes peculiar to these conditions.

The opposite kidney often shows histological changes of chronic nephritis, which are alleged to be due to the elimination of toxic substances or to the existence of a generally defective metabolism (the gouty or uric acid diathesis). ${ }^{1}$

Clinical Symptoms.-The important clincial evidence of the existence of renal stone is comprised in the occurrence of :

(1) Pain.

(a) Chronic (obscure).

(b) Acute (renal colic).

(2) Urinary symptoms.

It has frequently been demonstrated that stones may lie dormant in the pelvis or parenchyma of the kidney without causing any symptoms for years, ${ }^{2}$ being finally brought to light as the result of a thorough examination (radiography) following the appearance of pain. On the other hand, deep-seated stones may give rise to irregular lumbar pain of a varying degree of severity and duration, usually brought to notice by muscular activity such as is associated with stepping from a vehicle or being jolted about. In this instance, the patient may reveal nothing more than slight costo-vertebral tenderness on examination or not even this, whereas examination of the urine may show only microscopic evidence of blood and a trace of albumin. The additional presence in the urine of uric acid or oxalate of lime crystals should suggest the advisability of radiographic examination and thus determine the diagnosis. Other examples of dormant cases of renal calculus are first. brought to light by the occurrence of marked hematuria, with or without the presence of pain.

The typical picture of renal colic is a classical one on account of its

${ }^{1}$ Leclerq (Lyon Medicale, 1909, N. 2) has called attention to cases of leucemia in which there were renal stones and a high uric acid content of the blood therein.

The increased saline constituent in the blood in cases of primary stone has been proven as mentioned by Rosenbach (Mittheilungen aus den Grenzgebieten der Medizin u, Chirurgie, Bd. XXII.

2 The 15 kidney stones shown in fig. 244 gave no subjective symptoms whatever directed to the kidney. 
striking features, which are easily recognized. The pain is intense and usually sudden of onset. It generally radiates from the kidney region down to the groin and testicle. Nausea and vomiting are frequent accompaniments; during the paroxysm, there is of ten strangury and frequent micturition of small quantities of bloody or blood-tinged urine. The attack usually stops spontaneously, similar to its onset, at the expiration of one or more hours with the patient often well-nigh exhausted. Such severe pain is usually associated with the engorgement of a small calculus in the ureter, and while it is naturally on the side upon which the calculus is located, it is sometimes bilateral and may occur in the opposite kidney, thus demonstrating the unreliability of following this symptom alone ${ }^{1}$ (see Reflex Anuria, p. 573). The sudden cessation of pain signifies the return of the stone to the kidney pelvis or its passage downward into the bladder, following which, vesical irritation may indicate its new location or it may be passed through the urethra during urination. Following an attack of kidney colic, there is commonly a persistent soreness in the loin for some time afterward, due to hypercongestion or possibly hydronephrosis.

Urinary Symptoms.-During the attack the urine is voided in small quantities of high specific gravity and is accompanied by pain during the passage. It contains albumen and blood and sometimes crystalline deposit. In the case of ureteral stone low down, blood casts of the ureteral lumen may be passed, which are more or less characteristic. Following the attack, there is likely to occur a polyuria when pale urine of low specific gravity is voided. Scanty and high colored urine is a common urinary symptom of renal calculus. Anuria sometimes occurs and is conclusive evidence (see p. 573).

The course of kidney calculus is chronic. Stone rarely perforates the kidney causing perinephritic abscess. A small stone will pass into the bladder and will remain there as a nucleus for secondary bladder stone or, as stated, may be voided through the urethra. Hydronephrosis or atrophy may result from the prolonged presence of ureteropelvic stone. The more likely ultimate result, however, is infection. The accompanying condition of the kidney therefore in which stone is usually discovered, is that of pyelitis, pyelonephritis, or pyonephrosis.

Diagnosis.-The pain of renal calculus may be simulated by hydronephrosis (q. v., Chapter XXXI), by nephralgia, occurring in the course of chronic nephritis, early tuberculosis and tumor. Severe colic may follow an appendicial exudate and in some cases red blood cells

\footnotetext{
1 Albarran, Malad. du Rein, etc. Trait de Chirurgie P. Newman, Reno-renal Reflex, Glasgow Medical Journ., 1904.
} 
have been found in the urine. Cases of renal colic have occurred with stone at the ureteropelvic junction, causing pain in the right iliac region, accompanied by a rise of temperature, rendering the diagnosis more obscure. The leucocyte test in renal calculus, however, is usually negative.

Hematuria is the most obscure of all symptoms although it is of great value. It may occur without pain in cases of stone and with pain in cases of tumor, hydronephrosis, tuberculosis, etc. It is seldom as constant or abundant in calculus as in the cases of neoplasm of the pelvis, and it is typically associated with pain and increased by exertion or jarring with renal calculus. It may, however, occur in an apparently normal kidney or one slightly diseased (see Essential Renal Hematuria).

The final diagnosis of stone rests upon the roentgenographic evidence thereof. Reliable plates can be obtained in most instances when carefully made and should be repeated to verify the finding. Some individuals are poor subjects and the results obtained unsatisfactory. In a few cases of pure uric acid stone, the $x$-ray picture will fail to give a shadow. In such cases of uncertainty, reliance must be placed upon the other diagnostic measures and conclusions drawn from the net result. The clinical history will prove of value, but painful hematuria alone has no more than relative significance. Cystoscopy is of utmost value and should be a routine practice, not only as an adjunct to the radiographic investigation but also to test the capacity of the opposite kidney, especially in cases of pyonephrotic stone in which the question of nephrectomy may be considered.

Cystoscopic Examination.-As a rule, in uncomplicated cases of calculus of the upper urinary tract, the bladder is normal with only a slight hyperemia around the ureteral orifice, from which may or may not be observed a jet of bloody urine.

The ureteral catheter may furnish information by detecting a blocking of the canal at any point and the waxed tipped ureteral bougie may give the characteristic marks upon the tip when withdrawn. ${ }^{1}$ Examination of the catheterized specimen of urine may yield further information, viz: the presence of blood and crystals. Estimation of the urea excretion may show a slight comparative diminution upon the affected side. A very marked discrepancy between the two sides, namely, great polyuria with low specific gravity from the affected organ, would raise a suspicion of hydronephrosis. The colorimetric functional urinary test may show slight difference between the two sides in uncomplicated cases, whereas in infected lithiasis, the char-

1 Kelly, Amer. Journ. Ob., Vol. XLIII, 1901. 
acteristic evidence of pyelitis and pyelonephritis with defective renal capacity will be recognized. (See Cystoscopic Diagnosis and Functional Urinary Tests.)

Treatment.- The treatment may be divided into palliative and operative measures. As a preliminary consideration, mention may be made of the methods to be adopted to discourage the formation of renal calculus in those who have inherited a predisposition or have revealed a favorable diathesis by the presence of an excess of crystalline deposit of one or the other common ingredients of calculous formation (urates, oxalates). Under such conditions, hygienic methods should be adopted that will tend to aid the active elimination of the urinary salts through the other channels which aid in their excretion. Thus, the action of the skin should be encouraged by regular bathing and exercise; and deep and systematic breathing exercise encouraged.

The diet should be composed of simple and plain articles of food, eliminating such substances as are rich in purins or contain a high degree of acidity. Thus are excluded foods containing a large percentage of nuclein: brains, sweet breads, kidney, etc. Fresh meat of all kinds should be consumed in moderation as well as raw fruits, especially berries and canned articles of food, to combat the uric acid tendency. The acid vegetables and fruits and acid wines and condiments encourage the production of oxalate of lime crystals and are, therefore, to be excluded in individuals presenting a tendency in this direction. As a further measure of prophylaxis, the free use of distilled or alkaline water should be encouraged. The bowels should be kept open and any irregularities of digestion corrected by such rational measures as moderate eating, careful mastication, dietary restriction and exercise.

There is no remedy that will dissolve calculi, notwithstanding the groundless and unwarranted claims to the contrary. Piperazine taken with copious draughts of mineral water in doses of $1 / 2$ to 1 gram three times a day will aid in keeping in solution uric acid salts or gravel and in the case of very small stones may, to a certain extent, dissolve the surface thereof so as to aid in their dislodgment and passage; but the mechanical influence of the free diuresis caused by copious libations of water may fullfil the same requirements. It is not amiss, however, to administer piperazine as a prophylactic or preliminary means of treatment so long as the impression is not given that calculous formations of any size will be thereby dissolved.

During the attack of renal colic, the patient may be benefited by hot bath, hot fomentations, suppositories of belladonna and morphine 
and hypodermatic injections of morphine according to the intensity and duration of the pain. Sometimes chloroform inhalations cause relief. Glycerine has been given in doses of from 50 to 100 c.c. with alleged benefit (Hermann). Atropine by hypodermic injection is also said to relieve the pain and relax the spasm during passage of the stone down the ureter. Generally speaking, however, morphine hypodermically and the application of heat by means of a hot bath or the electric heater pad afford the most substantial means of assistance and other measures are not so much to be relied upon. Finally, in some cases, where the presence of stone from the kidney is recognized by roentgenographic diagnosis, its downward passage may be assisted by injection through the ureteral catheter of sterilized olive oil. This may be done at intervals between paroxysms.

Operative Treatment of Renal Calculus. - The operations upon the upper urinary tract for the removal of stone are nephrolithotomy, nephrotomy, nephrectomy and pyelotomy, anterior and posterior.

In renal calculus without infection, nephrolithotomy is the operation of choice; it should be accompanied by exploration of the pelvis and the passage of a bougie from the pelvis to the ureter. In the case of pelvic stone, pyelotomy may be preferable if the stone is not too large. Posterior incision is preferable for this operation (see Operative Surgery) as the anterior surface of the pelvis is more vascular and may be crossed by aberrant arteries. Nephrectomy is not performed unless there is extensive destruction of the renal tissue or marked infection which in the judgment of the operator is too great to be relieved by drainage; or unless there is uncontrollable hemorrhage or a ureteral obstruction not amenable to treatment or operation. It should not be attempted without sufficient knowledge of the functionating capacity of the opposite kidney.

For operations on the kidneys see p. 632.

\section{Ureteral Calculi}

Calculi of various composition located in the ureter are usually small, but occasionally surprisingly large stones have been deposited in the lower end of the ureter, and have been removed by operation (Figs. 246 and 247). The common variety of ureteral calculus is round or oblong and irregular, and of a size that is capable of forcing its way along the ureteral channel. Such a calculus when dislodged gives rise to the usual symptoms of renal colic, which occur during the passage of the calculus or its arrest in some portion of the ureteral tube. This may be near the pelvic end of the ureter, or in the lower end, where it often becomes lodged in the mural or that portion of the 
ureter which enters the bladder wall. When the stone is not movable, and does not completely occlude the ureteral canal, no symptoms may occur, and it sometimes remains impacted for years, unrecognized.

The symptoms of stone in the ureter resemble those of renal calculi, the difference being in the location of the pain. When the calculus is located high up, near the pelvis, the pain is in the lumbar and sub-

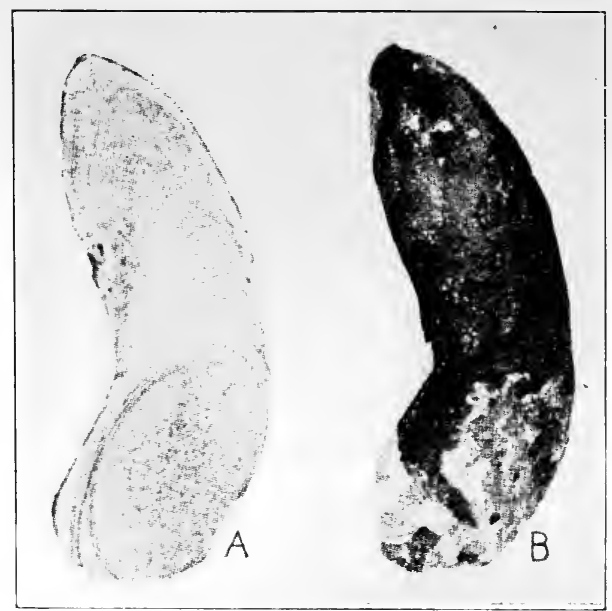

Fig. 246.--Stone removed from ureter. (Gibson.)

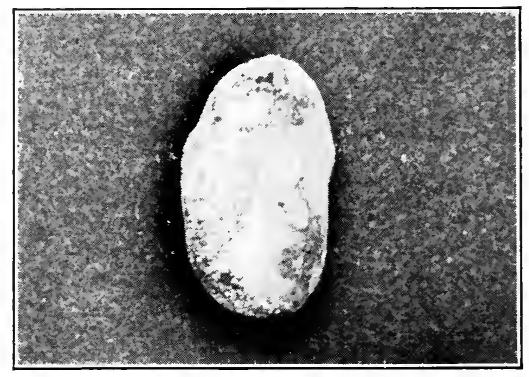

FIG. 247.-Stone from lower end of ureter. (Author's case.)

costal regions, radiating down toward the groin and into the penis or testicles. The pain is often so intense as to cause nausea and vomiting. It sometimes comes on suddenly and disappears suddenly, due to the fact that the stone is forced back again into the pelvis; at other times the pain is continuously severe, and only partially relieved by large doses of morphine. There is usually a continuous 
soreness with exacerbations of pain so long as the calculus remains in the ureter. If the stone migrates down the ureteral canal, the pain radiates toward the abdomen, groin and testicles, and when it reaches the ureteral orifice in the bladder, it is felt in the lower pelvic region and may be accompanied by vesical tenesmus and urinary frequency, which frequency, however, is often the accompaniment of renal colic. On the other hand, with complete occlusion of the ureter, there may be anuria.

Diagnosis.-Renal colic and anuria are unmistakable symptoms of calculus with complete occlusion of the ureter. Without anuria, ureteral renal colic on the right side is sometimes confounded with appendicitis. The diagnosis is confirmed by the $x$-ray and in most cases with the ureteral bougie (see Roentgenography, p. 162).

Cystoscopic Examination.-The meatus, in the case of a stone in the lower ureter near the bladder, may show a pouting or swelling (Plate III, Fig. 4); and a urinary stasis may be evident by the irregular flow of urine from the obstructed ureter. A stone may be seen protruding from the ureteral mouth or unilateral hematuria may be observed.

Treatment.-When the existence of a ureteral calculus is established, the question of its disposal must be considered. This may be accomplished in three ways: it may be passed spontaneously; it may be assisted in passage by dilatation of the mouth of the ureter and the injection of sterile olive oil; or it may be removed by surgical intervention. In some cases, by the ingestion of copious draughts of water, the patient may assist the passage of the calculus down the ureter into the bladder, when it will be voided with the urinary passage. In view of the possibility of accomplishing this end, the patient should be instructed to pass the urine always through a piece of surgical gauze or cheese cloth, in order to catch the calculus, if passed. The gradual descent of the calculus under such conditions may sometimes be observed by $x$-ray photography. The roentgenogram (Fig. 248) illustrates a case in point, the patient successfully passed the calculus spontaneously. Where the calculus does not pass spontaneously, but reaches the lower extremity of the ureter, it may be assisted by dilatations with a conical bougie and its passage lubricated by the injection of oil through the ureteral catheter.

Operation for Ureteral Calculi.-Ureteral stones in the middle portion may be reached during a lumbar operation by a further extension of the incision downward and forward, keeping the dissection extraperitoneal. (See Operative Surgery, p. 646.) For a calculus in the lower section of the ureter, uretero-lithotomy may be performed 
through an anterior, oblique inguinal incision also entirely extraperitoneal. (See Operative Surgery, p. 647.) In women, stone in the lowest part of the ureter may be reached through the vagina, or may be removed by suprapubic trans-cystic route (see Operative Surgery, p. 648).

Finally, a small stone lodged at the uretero-vesical orifice may be

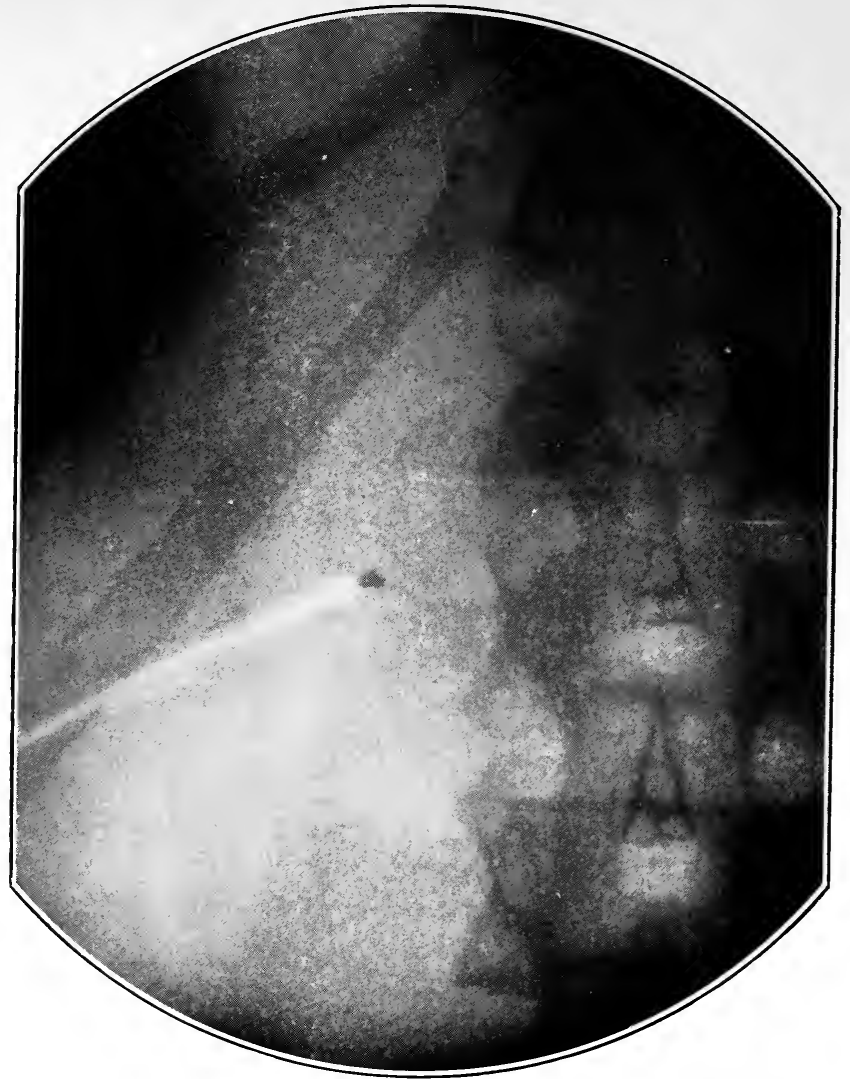

FIG. 248.-Radiograph of small ureteral stone in passage.

delivered by an incision of the meatus through the cystoscope or through a suprapubic opening.

\section{Calculus Anuria}

The condition of calculus anuria is signalized by sudden stoppage of urine in the course of calculus disease of the kidney or ureter.

Causation.-A variety of causes may be responsible for the development of this complication, namely: 
(a) Sudden and complete obstruction of one kidney, caused by the presence of stone.

(b) Simultaneous obstruction of both ureters.

(c) Congenital anomalies such as a fused kidney with one patent ureter which becomes obstructed; or an anastomosed ureter common to both kidneys.

(d) Obstruction of one ureter and suppression of urine in the other kidney by what is called reno-renal reflex.

Calculous anuria being an acute and grave condition, no time is allowed to pursue the investigation of the functional value of the two sides. That reno-renal reflex does exist has been proven by the fact that both kidneys can recover punctually after unilateral nephrotomy. It has also been demonstrated experimentally and while there may exist in the unobstructed kidney structural changes, as a predisposing factor to the ocsurrence of simultaneous suppression yet cases have been reported in which such anuria occurred without the coexistence of any appreciable morbid condition. ${ }^{1}$

Morbid Changes. - The condition of anuria of a kidney which is the seat of stone is not that of retention in the renal pelvis as is evidenced by the fact that during nephrotomy no appreciable amount of fluid is found in the pelvis.

The effects upon the kidney of sudden obstruction are, increase in the intrarenal pressure, renal congestion and edema with consequent enlargement; impaired urinary secretion, from a very small amount to complete anuria. This condition in a calculus obstructed kidney may be accompanied by degenerative and inflammatory changes in the opposite kidney which may or may not be the seat of a calculus or of such morbid condition as may be insignificant and inappreciable.

Clinical Symptoms.-There is a striking resemblance to uremia in cases of anuria due to the retention of the renal internal secretion. The tolerance to anuria may last as long as twenty days. ${ }^{2}$ The general condition of the patient may, in the beginning, continue surprisingly good and such tolerance of the urinary suppression may lead to unnecessary and unwise delay in action. When symptoms significant of uremia manifest themselves, the condition is almost hopeless. The cardinal symptom is the almost or complete absence of any urinary secretion. The condition follows an operation upon one kidney or is associated with renal colic. Any case of sudden anuria in an adult in whom there has been no evidence of advanced nephritis, should

\footnotetext{
1 Watsın, Trans. Am. Ass. G.-U. Surg., 1909.

${ }^{2}$ Russel, Necropsy in case of Anuria.
} 
arouse suspicion of calculous anuria, as should also uremia lasting a few days accompanied by anuria. Chronic nephritis of advanced degree may yield a transient suppression of urine but usually a small amount is voided and examination of such specimen will reveal the presence of various types of renal casts. Roentgenographic investigation will clarify the diagnosis in cases of uncertainty.

Treatment.-Pending the radiographic examination, active measures of treatment should be directed to combat the uremia: hot packs, warm baths and cupping of the loins; the administration of liberal fluids and saline diuretics. Morphine is contraindicated on account of a possible inhibitory action upon the kidney. Double ureteral catheterization and pelvic irrigation with warm saline or with oil on the obstructed side have been resorted to with beneficial result. ${ }^{1}$

Operative Treatment.-If the patient is already uremic, bilateral nephrotomy and partial or complete decapsulation may be performed provided the clinical history points to a diagnosis of calculus. If the patient is not uremic, delay of twenty-four to forty-eight hours is warranted before operating. When unilateral nephrotomy has been performed, the more incapable that kidney is regarded to be, based on its probable sole functioning capacity, the more necessary is it that bilateral exploration be carried out, to the end that the patient may be given the best chance of recovery, by investigation of the other organ. ${ }^{2}$

1 Andre, Anals des mal. des org. genito-urinaire, Paris, 1911, I, p. 132.

${ }^{2}$ Watson, loc. cit. 


\section{CHAPTER XXXI}

\section{HYDRONEPHROSIS}

Definition.-An accumulation of uninfected urine in the pelvis of the kidney. The fluid content of this hydronephrotic sac does not necessarily correspond to the composition of normal urine, as eventually it is converted in character, becoming a saline liquid of low specific gravity, with a small quantity of urea; it may also lose its transparency and contain some blood and detritus.

Other forms of renal distention, to be spoken of later, are pyonephrosis and hematonephrosis, which are distinguished by the presence of pus and blood respectively, instead of uninfected urine.

Hydronephrosis is in reality a symptom, not a malady-a consequence, not a cause; but as it is the essential feature of its underlying cause, this term fittingly describes the malady in question. The general term hydronephrosis embraces different varieties and degrees of the condition.

Causation.-To appreciate the rationale of hydronephrosis, it must be borne in mind that the pelvis of the kidney is not like the urinary bladder, intended as a reservoir for the urine, but is a funnelshaped expansion of the upper end of the ureter, in which to receive the excretion of urine from the various renal pyramids and direct the course of these different urinary streams into the excretory duct from the kidney to the bladder. Under normal conditions, this flow is unimpeded, and even when there is a certain amount of resistance, the kidney is still able to overcome the back pressure without a damming of the flow; but when, from any cause within or outside of the urinary channel, this resistance becomes excessive, there occurs a gradual distention from the point of obstruction upward, and this distention continues so long as the obstacle exists. The result is gradual accumulation of retained urine, which is continuous in the case of a permanent obstruction or may from time to time be emptied out if the obstacle is of an intermittent character. On the other hand, if the obstruction is complete and permanent, as in the case of a ligature of the ureter during a surgical operation, or of congenital occlu- 
sion of the ureter, the result is not a hydronephrosis, but a gradual destruction of the secretory functions of the organ, and its final atrophy.

The causes of hydronephrosis, therefore, may be within or without the kidney and ureter. Inside of the kidney, they may implicate only a portion of its substance, to the extent of one or more calices. ${ }^{1}$ Within the ureter, the obstruction may exist at any point throughout its course, in the form of a structural defect, or the presence of an arrested calculus, blood clot or tumor. Outside of the ureter, an obstruction may result from the pressure of a tumor or inflammatory deposit, and, finally, the obstruction may exist remotely distant from the kidney and ureter, in the lower urinary apparatus, such as stricture of the urethra and prostatic hypertrophy. In these latter instances, the hydronephrosis is in reality only a part of a condition, the real site of which is not located in the kidney, but is, more correctly speaking, a general vesico-renal distention, affecting alike both ureters and both kidneys; and which, furthermore, is much more likely to be a pyonephrosis from the start, or to become an infected hydronephrosis on account of the antecedent inflammatory feature, except in the first stage of prostatic obstruction, before infection of the bladder has taken place.

As a matter of fact, hydronephrosis considered surgically is a unilateral renal expansion, caused by some obstruction to the ureteropelvic junction or the region just below it-in other words, obstruction in the upper urinary tract. Such lesions are encountered, however, as obstructive prostatic hypertrophy, organic stricture, congenital malformation, etc., which conditions cause a bilateral dilatation from below upward, but rarely produce more than a slight or very moderate degree of pelvic distention. In such cases the bladder itself bears the brunt of the distention, or the onset of infection very soon complicates the picture and alters its character.

Again, tumors of the bladder (papillomata, carcinomata, etc.) may obstruct the vesical outlet or block the ureteral opening. Finally, the types of hydronephrosis which result from pressure outside the ureter by pelvic or abdominal neoplasms or inflammatory masses, have been termed by some cysto-uretero-pelvic dilatation, and are really not to be considered as true hydronephroses in the surgical sense. $^{2}$ They are found with cancer of the uterus, of the prostate, of

\footnotetext{
${ }^{1}$ Fugel (Morris, Surgical Diseases of the Kidney and Ureter) had a case in which only one caly $\mathrm{x}$ was distended, but there was a resulting abdominal tumor.

${ }^{2}$ Gardner, Etiology of Hydronephrosis, A nnals of Surgery, 1908, p. 575.
} 
the rectum; with fibroids, procidentia uteri, ovarian cysts, peritoneal exudates, tuberculous glands, etc. ${ }^{1}$

Congenital Hydronephrosis.- Hydronephrosis has been divided arbitrarily into the congenital and acquired forms. To the surgeon, the congenital variety of causation is of vastly greater importance. At one time the acquired hydronephrosis had the larger number of cases in its list, but at the present time the proportion is in favor of the congenital type, many cases having been found to be due partially or even completely to some congenital defect. This is a very important observation, as a very large number of the whole group are now recognized as having congenital elements in their causation. The chief productive defect is the congenital remains of the valves which exist normally in fetal life just at or below the ureteropelvic junction. These valves, according to exhaustive study, ${ }^{2}$ are often of very large proportions; they are formed of mucous membrane, or, in some instances, of both muscularis and mucous membrane. $^{3}$

Besides this congenital malformation, the next in importance is the movable kidney. Most of these are congenital, as is shown by the point at which their arterial supply comes from the aorta. But too much stress may be laid upon the movable kidney as a causative factor alone. The relation between it and a twist in the pedicle, resulting in intermittent hydronephrosis, has been accepted as self-evident. There is reason to doubt this and rather to hold that the intermittence lies in the emptying and re-filling of the pelvis during long uneventful periods of time, whereas the actual kinking of the ureter caused by any change in the position of the kidney results in slow renal crises of pain and oliguria. Such a view is made tenable by the fact that

1 A very excellent table of Etiology of Hydronephrosis, is that of Poufick, Zeigler's Beiträge Zur Pathologische Anatomie, 1911, Vol. L, pages 1-70.

A. With manifest cause:

I. Primary diseases within the uropoietic apparatus Nephrolithiasis........17

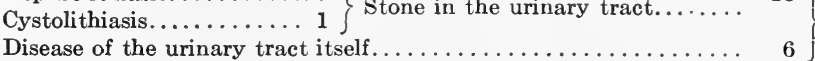

econdary disease within the uropoietic apparatus

Cancer of the uterus.......18

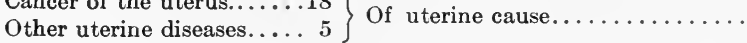

Disease, especially tumor formation, of other abdominal organs... 6

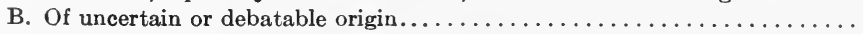

The latter group $B$ contains those which form the hydronephroses of most interest and import to the surgeon.

${ }^{2}$ English, Deut. Zeit. für Chir., 1879, Vol. II.

${ }^{3}$ Wölffler found that in the new-born, 20 to 100 cases still showed these valves in the pelvis or ureter, and every case in which they were present showed transverse folds at the uretero-pelvic junction. 
chronic distention is painless-that many of the largest hydronephroses give no history of renal crises at all, that during the crises of some cases, catheterization of the ureters has shown only a very small amount of urine (e.g., 30 c.c.) in the sac, whereas the same kidney showed polyuria after the attack was over, quite often vastly out of proportion to the size of the sac.

Congenital malformation in the form of stricture of the ureter may be a cause of hydronephrosis, as well as anomalous anastomosis of the ureter, which is likely to be accompanied by a narrowed orifice or an angular deviation of the tube. As already stated in the section on ureteral anomalies, this anastomosis may occur in women in the urethra, between the urethra and the vagina, and in the male, in the prostatic urethra.

The ureter, instead of being derived from the most dependent portion of the pelvis, may extend from its upper portion, resulting in a sacculation which is not afforded the required drainage, or the ureter may depend from the pelvis at such an acute angle as to offer an undue obstruction to the outlet. Finally, cases have been seen in which the cause of the obstruction was found to be an anomalous blood-vessel passing over the upper part of the ureteral tube, causing a compression or distortion of its lumen. ${ }^{1}$

Recently, the existence of anomalous renal arteries has been greatly advocated as a cause of hydronephrosis. ${ }^{2}$ Some French writers are prone to doubt this causative relationship to a great degree, claiming that only arteries that pass behind the pelvis can really kink the ureter. In the light of operative findings, however, we must concede such causative relationship. ${ }^{3}$

Acquired hydronephrosis is of much less interest numerically to the surgeon. The number has, of course, been vastly lessened by throwing into the previous class cases in which there was even a small element of congenital defect.

The most important factors inside the urinary tract are tumors, stones and strictures of the ureter (see note, p. 577). Occasionally, a

1 An interesting case reported by Roberts (Morris, Surgical Disease of the Kidney and Ureter) is that of a double hydronephrosis in a young man, the cause of the lesion on the left side being a contracted and too oblique ureter, while on the right, a branch of the renal artery exerted pressure on the ureter. Another unusual case is reported by La Grave, who found that hydronephrosis had been caused from compression of the ureter by menses retained in one-half of a bifid uterus.

${ }^{2}$ McDonald, Anomalous Arteries and Hydronephrosis (an Anatomical Study), Ann. Surg., 1910. Ekehorn, Die anomalen nierengefässe, etc., Arch. f. klin. Chir., 1907, LXXXII, 955.

Merhel, Die Hydronephrose und ihre Beziehung, etc., Virch. arch., 1908, CXCI, 534.

${ }^{3}$ Mayo (Mayo-Braasch-Maccarty, Relation of Anomalous Renal Blood-vessels to Hydronephrosis, Jour. Am. Med. Assoc., May 1, 1909) found that of twenty-seven hydronephroses operated upon, twenty were associated with such blood-vessels. In order to prove the causative relationship, in thirteen of the twenty cases, he merely severed the vessels and obtained a cure of the condition. 
bladder tumor, or a tumor of the pelvis of the kidney, may create such a condition. Extraneous tumors pressing upon the urinary tract have a numerical importance in this relation. ${ }^{1}$

Impacted stone as a cause of hydronephrosis is now falling into disfavor. The large number of impacted stones without hydronephrosis, and the rise of the congenital view, based on more careful study of the specimens, are responsible for this.

Strictures of the ureter following previous inflammation, due to periureteral adhesions, and in many cases due to the passage of stones -wounds of the ureter-are responsible for a very small number of cases.

High insertions of the ureter into the pelvis are often very deceptive. They seem congenital in many cases, but are really due to kinks of the ureter, with adhesions forming a secondary valve-like process or spur. ${ }^{2}$

Acquired hydronephrosis therefore may be caused by external compression of the ureter, or by causes inside of it. Compression from without may arise from cancer of any abdominal or pelvic organ and other neoplastic growths, copious effusions and extreme ureter displacements, also, hyperextension of the bladder.

Of the conditions within the ureter given as a factor of acquired hydronephrosis, that of displacement of the kidney and consequent bending and kinking of the ureteral tube is one of the commonly ascribed causes. It must be admitted, however, that the bending alone of the tube may be insufficient to produce the obstruction, whereas the additional existence within of a constriction or valvule, or the occurrence externally of periureteritis with adhesions, may furnish the contributing causative agent. There has been considerable discussion on this point among medical authorities, and while the consensus of opinion seems to be that renal prolapse is a frequent reason for the production of hydronephrosis, it is justly questioned as to whether or not, in many cases, the prolapse of the kidney may not be a result, rather than a cause, of the existent condition, and whether in many instances, there has not preëxisted one of those congenital defects of the lumen of the tube in the retention of fetal folds or valvules of mucosa, which, after birth, has acted as a slow, but progressive, cause of incomplete renal drainage and consequent retention, and which has gradually induced an undue mobility of the organ, finally culminating in distention and

\footnotetext{
1 Israel, in a series of forty cases operated upon, found only one which was due to extra-renal tumor pressure, whereas Morris found at autopsy in 381 cases of hydronephrosis almost 90 per cent. of them due to the extraneous pressure of abdominal tumors. On account of their extensive growth and pelvic situation, 274 of these hydronephroses were bilateral.

${ }^{2}$ Morris, Surg. Diseases of the Kidney and Ureter, 1901.
} 
hydronephrosis. However, undue mobility of the kidney must be accepted as one of the causes of acquired hydronephrosis when it is demonstrated that an operation for the fixation of the kidney, following the existence of such a condition, has resulted in complete relief. Yet, on the other hand, it is an important caution to bear in mind that in all cases operated upon for movable kidney in which hydronephrosis has been a symptom, the point of departure of the ureter from the renal pelvis and the calibre of the ureteral canal should always be investigated; otherwise, the operation may fail of complete relief. There is, of course, such a thing as acquired movable kidney, but the proportion of these which are congenital has been mentioned above.

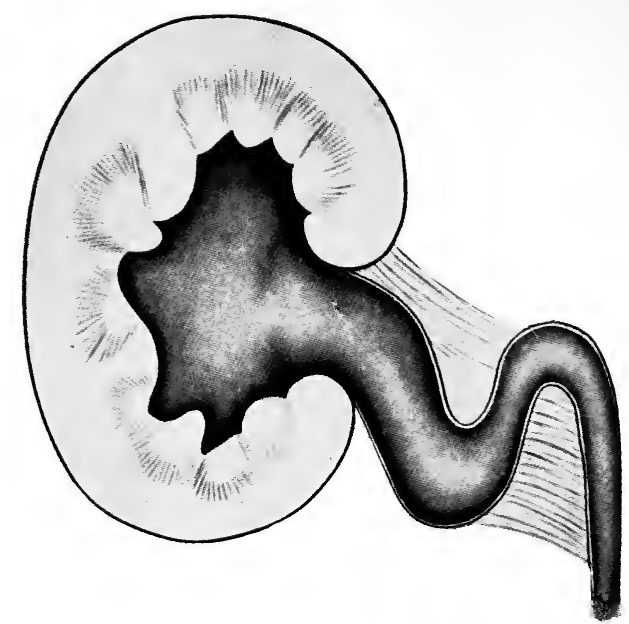

FIG. 249.-Adhesions of ureter causing intermittent hydronephrosis. (Author's case.)

Finally, it should be said that in many cases the factors are intermingled so that classification is very difficult, unless all cases that have even the slightest congenital stigma are put into the congenital class, which is the present tendency. Many acquired conditions can well aggravate an already defective congenital basis.

This leads naturally to the consideration of the so-called "intermittent hydronephrosis," a term that has been objected to by some authors, and perhaps with foundation, as it suggests that the underlying condition is of a transitory character, whereas it is distinctly progressive; yet, as already stated, the cause of its production may be a continuous incomplete occlusion of the canal and an incomplete temporary occlusion. In certain cases where, owing to the mobility 
of the kidney or other changing mechanical conditions, the extent of the obstruction varies in degree, there is, as a result, an ebb and flow in the hydronephrosis and the symptoms resulting therefrom necessarily occur at intermittent intervals.

An incomplete obstruction of the ureter, together with a movable kidney, may become completely obstructed temporarily by movement of that organ, and entirely overcome the intrarenal tension, and may right itself again by changing position. A pronounced bend of the ureter, fixed by adhesions, may exercise a permanent resistance to renal drainage, and by a valvular formation, the degree of closure may from time to time become greater or less, according to the amount of retention within the kidney ${ }^{1}$ (Fig. 249).

Traumatic hydronephrosis is a type of the acquired form. True hydronephrosis may come on several years after an accident to the ureter producing laceration and stricture. Hydronephrosis has been reported as occurring immediately after an accident, but such cases are, in all likelihood, either cases of pseudo-hydronephrosis, or, in other words, a urinary and hematic effusion outside of the kidney, ${ }^{2}$ in the perirenal space, or preëxistent true hydronephrosis, which by chance is revealed at that period. ${ }^{3}$

Calculous Hydronephrosis. - A calculus may act as the cause of hydronephrosis in two ways: (1) by obstructing the uretero-pelvic outlet; or, (2) by being arrested in its downward course in the ureter, and by causing a ureteritis with inflammatory swelling, or by

1 The author operated upon such a case, and in this instance the patient suffered from a paroxysmal pain in the kidney region, which came on slowly and gradually increased to a high degree of intensity, which persisted for a varying period, and then gradually subsided. There was no evidence of a calculus to explain the paroxysm, but it was discovered at operation that the ureter, after leaving the pelvis of the kidney, made a sharp turn upward, and again downward, and that this intervening loop was fastened together by adhesion, and the afferent ureter was adherent to the distended pouch of the pelvis. It seemed that under ordinary circumstances, the resistance to the outflow was not enough to occlude the passage of the urine, but at certain times, accumulation, either within the pelvis or in the loop of the ureter, would exercise pressure, producing a valvular obstruction, which would remain in force until the distention became sufficiently great to force a small leakage through the lumen of the loop and thus produce sufficient alteration in the valvular compression to permit a gradual drainage of the accumulated secretion and relief of the symptoms until another period, when the same conditions recurred with a repetition of the painful symptoms. By releasing the adhesions of the ureter, and by replacement of the kidney in its normal position, complete relief was obtained (Fig. 249).

${ }^{2}$ An example of this description occurred in the author's experience. A lad, while at play, fell down-stairs, landing upon his side. He was taken to the hospital, and while there, developed a swelling in the costo-iliac space a few days after the accident. An abdominal incision was made and the peritoneal cavity exposed. It was then discovered that there was a retroperitoneal protrusion of a large fluctuating mass. The peritoneum was closed, and a lumbar incision made down to the mass in question, which proved to be an accumulation of urine. This was called a traumatic hydronephrosis, but was in reality, as discovered at a secondary operation for urinary fistula, an extravasation of urine into the retroperitoneal space around the kidney (see Fig. 242, p. 530).

${ }^{3}$ Coenen, Deutsch med. Woch., XXXVI, 2411. 
extending its damage deeper into the structure of the ureter, resulting in the formation of a stricture. A calculus, on the other hand, may be secondary to a preëxistent hydronephrosis from one of the other causes already mentioned, and, therefore, it should be remembered that the coexistence of hydronephrosis and renal or ureteral calculus does not necessarily establish the calculus as the cause of the former condition, and in operating for the removal of the calculus, investigation of the lumen and the course of the ureter should be made systematically. ${ }^{1}$

Ureteritis from other causes may bring about a hydronephrosis, and tuberculous ureteritis may also act as one of the causative agencies responsible for that condition.

Morbid Changes.- The retention in the pelvis of the kidney of a collection of urine, the outflow of which is hindered by some obstruction, causes a gradual dilatation and later a sclerosis of the walls of the sac, the distending body encroaches upon the substance of the kidney and the adjacent blood-vessels, causing anemia and gradual atrophy. When the obstruction is permanent and complete, after having existed as an incomplete obstruction for a varying period, it is known as closed hydronephrosis. In this instance the kidney substance becomes totally destroyed and leaves a closed cyst, sometimes of huge dimensions. ${ }^{2}$

The lesser grades of dilatation may be bilateral, and caused by pressure below the pelvic brim. In their early stages, many of the unilateral dilatations must be small, ${ }^{3}$ and the number of these cases recorded is increasing since preoperative ureteral catheterization and pelvic measurement are being more often performed. ${ }^{4}$

The character of the pathological changes which attend the existence of hydronephrosis varies according to the length of time which the condition has existed. Coincident with the dilatation of the pelvis, the calices become widened, hence the intermediate medullary substance is subjected to increasing pressure, and thus follows the atrophy. If the capsule of the kidney becomes stretched and the parenchymatous

${ }^{1}$ Legueu (Traite Chirurgicald'Urologie, 1912) believes that a calculus or gravel may be considered only an obstruction in hydronephrosis, on account of the disturbance it causes in the ureteral circulation.

2 The author operated upon such a case, in which there were between two and three gallons of liquid, which had been taken for a large ovarian cyst, there having been no other symptom than that of the enlargement and discomfort naturally attending such a growth.

${ }^{3}$ Morris, Surgical Diseases of the Kidney and Ureter, makes the following classification: "(1) Simple hydronephrosis with atrophy without expansion; they are the small, flaceid, shriveled kidneys.

(2) Simple hydronephrosis with atrophy and expansion. These often enlarge into huge cysts.

(3) Hydronephrosis with atrophy of the pyramids and thickening and sclerosis of the medullary substance. These kidneys have generally been inflamed, and are prone to suppurate."

${ }^{4}$ For clinical purposes, any pelvis holding 50 c.c. or more should be considered hydronephrotic. 
substance yields to pressure, the connective-tissue septa, being more resistant, gives to the resulting cavity a lobulated contour. ${ }^{1}$

On section, the kidneys present different degrees of morbid changes. There is usually an apparent thinning of the renal parenchyma. The pyramids suffer most, their points becoming flattened against the cortex, which, as a rule, is thinned to a lesser extent. The multilocular cavities communicate freely, their septa being formed by the columns of Bertin. These finally atrophy, so that the sac may be merely represented on its renal side by a thinned layer of renal tissue with traces of septa which formerly existed. The pelvis is generally more or less thickened in its texture, and the outlet may have become much distorted; remains of the valves may be present. The ureter is usually open, although it may be closed. Its entrance may be guarded by a distinct fold, which may be the original congenital fold, or may be formed by the shifting of the organ after the dilatation is well under way.

To summarize the histological changes: ${ }^{2}$ Fibrotic thickening of the true renal capsule; dilatation of the convoluted tubules, giving at times a bulbous appearance; marked arteriosclerosis of the pyramidal vessels. The glomeruli often show slight dilatation, with hyaline material in the space between the vascular mesh and Bowman's membrane, pushing the tuft to the wall. In other places, complete atrophy of the glomeruli and connective-tissue replacement are found. The capsule of the kidney may show large spaces filled with hyaline material.

There is a great deal of new and old connective tissue in the columns of Bertin wherever these can be identified. Collapsed and atrophied collecting tubules and Henle loops are found.

The opposite kidney usually shows proportionate compensatory hypertrophy in cases of unilateral hydronephrosis.

Clinical Features.-Hydronephrosis may exist for years without giving rise to symptoms. As the condition progresses, the presence

\footnotetext{
${ }^{1}$ Anatomical Classification of Hydronephrosis: 1. Hydronephrosis in which the pelvis chiefly is involved.

In these cases there may be only a moderate amount of renal thinning, and the size may be due chiefly to the dilated pelvis (hydronephrose pyelique).

2. Hydronephrosis in which the kidney is about normal in size, but the calices have suffered markedly, and only upon gross section would one suspect such a marked lesion (hydronephrose calicielle).

3. Hydronephrosis in which the kidney dilates to great size with very little or practically no pelvic enlargement. The kidney tissue is, of course, greatly thinned (hydronephrose parenchymateuse).

4. Hydronephrosis in which both elements take part equally (hydronephrose totale).

5. Hydronephrosis in which only one calyx or one portion of the kidney is dilated. This may assume enormous proportions, leaving a good deal of the kidney in quite normal condition. It has been observed with double ureter (hydronephrose polaire).

${ }^{2}$ Vide Poufieck, loc. cit., supra.
} 
of a tumor in the loin becomes evident. This may arise from the existence of pain due to pressure, although, on the other hand, cases are seen without a palpable tumor, in which the distention of the pelvis causes pain in the abdomen and back, radiating downward.

When the swelling is the first sign to attract attention, it may be distinguished by bimanual palpation, which detects an enlargement of varying size occupying the space in which the kidney is normally located. A movable body, suggestive of an enlarged kidney, may be felt with characteristic ballottement. Sometimes fluctuation can be felt, and percussion detects a dulness in large growths, while in those of smaller dimensions, which rise behind the colon, a tympanitic note is heard. The contour of the tumor may be rounded and smooth or lobulated. Pain may be constant and intense in large growths and in smaller enlargements may be entirely absent or it may take the form of colic in intermittent painful crises. This intermittent characteristic is likely to be present when the kidney is movable and when the obstruction is of a variable character so that the distention of the kidney undergoes marked variation, becoming almost complete at one time, and partially subsiding at another. In other words, the pain may fluctuate with the swelling. It is characteristic of these painful crises that during the intermission there is an increased flow of urine, which is considered one of the significant signs of hydronephrosis. Such urine is usually pale and limpid, and of low specific gravity. It may contain mucus, and a small amount of blood. ${ }^{1}$

The symptoms in such cases resemble those of nephrolithiasis, which is important to be remembered in differential diagnosis.

Diagnosis.-The existence of a large palpable tumor in the kidney region, of an elastic and fluctuating character, always suggests the possibility of hydronephrosis. Such a tumor must be differentiated from a pyonephrosis. The latter condition generally gives rise to systemic symptoms, and is usually accompanied by evidence of suppuration, namely, free pus in the urine; nevertheless, this may be absent in a closed pyonephrosis. A large hydronephrotic tumor may be confounded with ovarian cysts, especially when the growth occupies a large portion of the space between the kidney region and the pelvic cavity.

Aspiration of the tumor will not always determine the diagnosis, as the fluid obtained from a large hydronephrotic sac may contain a very small percentage of urea, which may also be present in the fluid of an ovarian cyst. The association of paroxysmal pain and a palpable and

1 Legueu (Traite d'Urologie) speaks of the polyuria at the end of these crises as of neurotic origin, which is more than likely, as the amount of urine passed is out of proportion to the contents of the hydronephrotic sac. 
large kidney, directs attention to this organ as the seat of trouble, but the exact nature of the underlying condition is not so easily determined. Much assistance may be obtained in the differential diagnosis by cystoscopic investigations and functional urinary tests (see p. 155). By this means, it may be determined whether the hydronephrosis is of open or closed type by inspecting the mouth of the ureters, and by catheterization of the ureters. The bladder is usually normal, the ureteral orifice elevated and more or less patulous; the ureteral jet is either indistinct or inconstant.

The catheter passes in easily, but may be somewhat arrested at the uretero-pelvic junction $(20-25 \mathrm{~cm}$.) ; however, it usually slips by, into the pelvis. Whereas little or no urine has flowed before, at this point there will be a steady drip. The comparison with that collected from the other side is an important observation. The fact that the urine runs with a steady drip, instead of the rhythmical contraction of an undistended pelvis, is of decided diagnostic value in the diagnosis of hydronephrosis. There may be an ounce, or vastly more.

The dilated pelvis will admit 50 or more centimetres of catheter, the normal only 30 to $40 \mathrm{~cm}$. The urine from the affected side will be pale, and more or less turbid from the presence of leucocytes or phosphates. It may be slightly alkaline or neutral (in comparison with an opposite normal urine).

Measurement of the pelvis is of value in reaching a diagnosis. A pelvis which will hold 50 c.c. or more before the onset of renal pain is probably hydronephrotic. The distending fluid should be colored blue, and the ureteral orifice watched for marked reflux during the injection. Some cases will hold as much as 200 c.c., more than 150 c.c. denoting very little secreting substance remaining in the kidney. ${ }^{1}$

The composition of the fluid from the affected side should be determined. The quantity of urine secreted is usually less than normal and the character thereof depreciated, but if the obstruction is passed by the ureteral catheter, the quantity of urine may be considerably greater. Complete diagnosis may be accomplished by applying the different functional urinary tests (see p. 155) and by Roentgenography and possibly pyelography. Simple Roentgenography will rarely show anything of diagnostic value except vague outlines of the kidney and the concomitant occurrence of stones. Pyelography is of great practical importance $^{2}$ (see Chapter VII).

\footnotetext{
1 According to Albarran and Braasch, a peculiarity of hydronephrotic kidneys is the absence of sugar elimination from both sides after an hour, as shown by the phloridzin test. Legueu merely claims that the phloridzin and methylen blue tests are retarded on the hydronephrotic side.

${ }^{2}$ Braasch: Trans. Am. Urol. Assoc., 1911, Vol. IV, p. 195; Annals of Surg., 1910, Vol. XV, p. 534; Annals of Surg., Nov., 1910; Surg., Gyn. and Obst., April, 1911.

Uhle-Pfahler, etc., Annals of Surg., 1910, Vol. LI, p. 546.

Hoch and Porges, Prager med. Woch., 1911, XXX VI, 137.
} 
Differential Diagnosis.-Cases that require any fine differentiation cannot be diagnosed without the cystoscopic findings. Without the confirmation of this test, the practitioner will of ten be forced to consider the following:

Gall-stones.-This is one of the most difficult conditions to differentiate, as the patient may present only the history of attacks, with no fever and no jaundice. The presence of atypical gall-bladder pain may be extremely confusing. In the event of the least doubt, cystoscopy should be done, if only for its negative diagnostic value, especially if a catheterized specimen of bladder urine shows a few white cells.

Nephrolithiasis.-Nephrolithiasis may simulate hydronephrosis, and in fact be associated with it. In atypical cases, these conditions cannot be differentiated without the cystoscope and roentgenogram.

Appendicitis must always be considered, as is shown by the number of patients from whom the appendix was removed for indefinite abdominal pain, and who were afterward proven to have hydronephrosis. ${ }^{1}$

In large hydronephrotic sacs, the diagnosis of pancreatic and ovarian cysts, hydrops of the gall-bladder and retroperitoneal neoplasm must be considered (see Pyonephrosis).

Prognosis.-In bilateral hydronephrosis, the prognosis is bad, as in this instance both kidneys are diseased. In uncomplicated unilateral hydronephrosis, the prognosis is favorable. Complications which tend to increase the gravity of the prognosis are: infection causing a pyonephrosis; complete occlusion of the ureter by arrest of a calculus during its passage, with the possibility of anuria and uremia, or extreme distention of the sac and pressure upon other organs, with the danger of intraperitoneal rupture.

Treatment.-The treatment of hydronephrosis must depend upon the cause and the extent of the lesion accompanying the given case. Any measures short of surgical intervention involving exposure of the sac have little value, although in some instances aspiration has been practised with beneficial results; but it cannot be depended upon, and is, therefore, merely an experimental effort, following which open operation may be required. In calculous hydronephrosis, dislodgment of the calculus may afford relief from the symptoms, which is only temporary in the case of a movable kidney, or when there exists any of the other causes for the production of hydronephrosis.

Nephrotomy should first be performed (see p. 632). The distended sac should then be aspirated and its contents removed. The

1 Twenty-five per cent. of Braasch's patients had this experience (loc. cit.). 
lumen and course of the ureter should be carefully explored and the exact cause and nature of the obstruction should, if possible, be determined. If the patient's condition does not warrant further operative measures, a temporary nephrotomy may be done for the purpose of drainage, and a more complete operation performed at a later date. This may involve a plastic operation for removing the obstruction, be it a calculus of the ureter, an obstructing bend or stricture, or ligature of an anomalous blood-vessel. When there is an undue mobility of the kidney, or a flexion of the ureter, replacement and fixation of the organ by nephropexy are required. This, however, should always be done in connection with an examination of the ureter for other causes of obstruction. Quilting, or resection of the hydronephrotic sac, may be deemed advisable, although a considerable degree of shrinkage naturally takes place as a result of the relief, following the removal of the cause of hydronephrosis. In a word, the kidney should not be sacrificed by removal if it is found that there remains a fair degree of secretory substance, and it is most surprising how small an amount of kidney tissue can continue to perform service. When, however, the entire organ is obviously destroyed and it is deemed advisable to remove it in order to preserve the strength and the depreciated health of the patient, immediate nephrectomy may be performed. The more complex operations involve opening up of the uretero-renal canal, uretero-pyelo-anastomosis, and uretero-cystoanastomosis.

Pyeloplasty (Fenger's operation).-This consists in splitting the stricture and suturing it in the direction at right angles to the incision after the manner of Heinke-Miculicz pyloroplasty. ${ }^{1}$

Division of Ureter and Reimplantation.-This is a more difficult procedure, and should be done only in cases of necessity. The most dependent point of the sac is, of course, selected for reimplantation.

In those cases where the hydronephrosis is secondary to obstruction in the lower urinary tract, such as constriction of the urethra and prostatic enlargement, it is futile to remove the secondary result of such obstruction without attacking the cause thereof. Abnormal conditions of all other organs, such as retroflexions of the uterus, etc., that may cause obstruction to the ureteral canal, must be remedied by appropriate operation.

\footnotetext{
1 Mayo is accustomed to do this whenever possible, and to cover the suture line with a fatty fascial flap which he first dissects off.
} 


\section{CHAPTER XXXII}

\section{NEPHROP'TOSIS}

\section{PROLAPSE OF THE KIDNEY, MOVABLE KIDNEY}

Anatomical Considerations.-The kidney is more or less out of the natural position, temporarily or permanently, on account of some traumatic or congenital defect. Normally, it is held quite firmly in the loin by its various anatomical relations (see Chapter on Anatomy, p. 1) pertaining to which it is necessary to bear in mind certain important facts:

The kidney is located deeply in the loin, surrounded by a thick layer of perirenal fat enclosed in a fibrous capsule. This capsule, or perinephric fascia, is adherent to the adjacent structures, and forms the main support of the kidney. An upper prolongation of this fascia on either side attaches it to the diaphragm. The colon passes across the kidneys at both flexures and is attached to the anterior layer of the perinephric fascia, but on the right side the colon does not in any way support the kidney. On the left side the kidney has the advantage that the splenic flexure of the colon is the one point of the colon which practically never varies in position, being held by a strong layer of fascia, the costo-colic ligament, while on the right side the hepatic flexure varies greatly, always retaining, however, its attachment to the kidney. The kidney on the left side is also held up to a great extent by the lieno-renal ligament, a fold of peritoneum which is usually very well developed. The costo-vertebral recess or renal fossæ in women are wide; in men they are long and narrow.

Causation of Renal Mobility.-Although held quite firmly in welldeveloped individuals, the kidney has a certain degree of respiratory descent, amounting to probably $2.5 \mathrm{~cm}$. (1 in.) in the recumbent posture. This is only slightly due to the descent of the liver, and would be greater, were it not that the liver, in its passage downward, rotates upon a horizontal axis, that is, its lower sharp edge performs most of the descent. ${ }^{1}$

The position of the kidney in the erect attitude is affected by the force of gravity, which is more or less constantly in action, and together with a loss of the perinephritic fat, forms a strong combination

1 Addison, Topographical Anatomy of the Abdominal Viscera in Man, Edinburgh, 1901. 
in the causative agency of nephroptosis. In addition to this, the attachment of the colon on the right side is an important factor. It has been found to descend gradually in a large proportion of individuals during their lifetime ${ }^{1}$ and in acquiring a new position naturally tends to draw the kidney with it, which tendency is increased by the existence of habitual constipation. The production of renal prolapse by traumatism has been unquestionably established. It is caused by rupture of the reno-diaphragmatic attachment, and may be produced by apparently moderate trauma, properly directed, in which case the prolapse usually comes on weeks or months after the occurrence of renal trauma.

Congenital defects as causative factors may be summed up as those which cause hydronephrosis and thus secondarily renal mobility. In addition to this, there are quite a large number of congenitally socalled movable kidneys which have acquired their faulty position during fetal life. ${ }^{2}$

In exceptional cases the kidney is totally surrounded by a mesentery, a double layer of peritoneum called "mesonephron," which condition allows of greater mobility, and in time, under the usual causative conditions, may become increased by being stretched.

Many other contributory causes exist, which combine with the foregoing factors in the production of nephroptosis. Thus, pregnancy may bear an important relation, in great measure due to stretching and loss of tone of the abdominal walls. The same conditions may exist after the change of intra-abdominal pressure subsequent upon removal of an abdominal tumor (ovarian cyst, etc.).

Tumors of the kidney, extrinsic tumors (adrenal, etc.), are responsible for some cases.

All the various degrees of splanchnoptosis ${ }^{3}$ involve the kidney, along with all the other abdominal organs. Tight lacing has been held responsible. It is usually found in young people, between twenty-five and thirty-five years of age, and is more commonly found in the female than in the male sex. ${ }^{4}$

As to frequency in general, nephroptosis is not of common occurrence in normally healthy individuals. It has been found to exist

1 Lane, Clinical Journal, June 5, 1901.

2 These kidneys thus alluded to have an arterial supply which comes off from the aorta at a point which is lower than is normal. Bissell, The Pelvic Kidney, Amer. Journ. Obstet., Vol. LXIV, Nov. 4, 1911, draws attention to a more accurate nomenclature of renal malposition, reserving "displacement" for acquired conditions and "misplacement" or dystopia for congenital conditions. Such marked degrees of malposition as are seen in pelvic kidneys may be either of congenital or acquired causation. The arterial supply decides this feature.

${ }^{3}$ Glenard, Les Ptoses Viscerales, 1899.

${ }^{4}$ Wilson and Howell, Movable Kidney, 1908, London, eighty-nine per cent. in a series of 100 cases occurred in women; seventy-four of these were right-sided. 
in from 30 to 40 per cent. of those complaining of neurasthenic symptoms and functional disorders of the digestive system.

Heredity is a factor so far as there may be a tendency to the reproduction of predisposing constitutional defects by inheritance.

Morbid Changes.- The pathological condition in the kidney itself, or in the adjacent region, must be dealt with under the proper headings, such as tumor of the kidney, adrenal, etc., congenital cystic disease of the kidneys, non-inflammatory enlargements of the spleen and liver, and spinal curvature, any of which may be associated directly with movable kidney in a somewhat causative relation.

The kidney usually moves in three ways:

1. Its lower pole passes downward and rotates inward, swinging upon the pedicle as an axis.

2. Its upper pole falls forward, and there is thus a rotation upon a transverse axis.

3. A combination of both 1 and 2 .

The first process is more common, and results in various minor symptoms. The second results in renal crises. Nephroptosis cannot occur to any appreciable degree without at least inward rotation taking place.

As a rule the mobility of the kidney persists (see Clinical Features) but at times the organ becomes adherent to the surrounding tissues in its acquired position. In extreme instances, such an organ may be found adherent to the under surface of the liver, with the renal hilus pointing directly downward to the gall-bladder or to the colon. It has been found almost straddling the vertebral column. It may even be found on the other side of the vertebral column. ${ }^{1}$

Morbid Changes in the Kidney Itself.-Most of these are seen at operation. The mobility of the kidney is then very apparent, as the kidney sometimes jumps out upon the loin, being delivered with a far greater facility than is normal. It is usually diminished in size, flabby and soft in consistency, and pale. Its pedicle is quite elongated. The perirenal fat is usually markedly atrophic, which adds to the facility of locating the kidney. The acute cases in which the surgeon has operated during or just after a renal crisis may show, instead of pallor, a purplish tinge due to venous engorgement from twist of the pedicle. Such a kidney may be enlarged beyond the normal. The capsule is thicker and more opaque than normal, but strips easily, the kidney being rarely the seat of chronic interstitial nephritis.

As a rule the parenchyma removed at operation shows no micro-

1 Cases have been reported (Bramwell) in which the kidney had moved downward and inward, and had compressed the duodenum, causing jaundice or suggesting acute dilatation of the stomach. 
scopical changes of interest. There is occasionally some fatty infiltration of the epithelium. ${ }^{1}$

Movable kidney, the author believes, is wrongly stated to be uncommonly complicated by hydronephrosis, ${ }^{2}$ for statistics in this respect are misleading, as it is impossible to tell the degree of hydronephrosis present by any other means than pelvic measurement either before operation or after removal of the kidney, and by pyelography.

Clinical Features.-There is a wide range in the character of the symptoms presented by the patients suffering from movable kidney. In the first place, the patient may suffer no inconvenience from the condition at all, and it may be found only on routine examination. It is useless to mention such conditions to a patient, unless fairly sure, after repeated examinations, that the mobility is responsible for symptoms, as such ill-advised revelation is responsible for many of the severest cases of hypochondriasis. On the other hand, there are many cases in which the symptoms are really referable to undue renal mobility, and these occur in chronic and acute types.

Malposition of the kidney, with or without occasional twists in its pedicle, causes a certain amount of renal insufficiency and toxic absorption, as it is practically demonstrated from urinary examination and clinical evidence that the movable kidney is often incapable of eliminating the waste products of metabolism, and from this a certain type of chronic renal toxemia results.

A typical clinical syndrome is that of a type of neurasthenia accompanied by a mild degree of general toxemia. Such a type is often seen in young women. The patient usually presents herself with vague abdominal pains. She is poorly nourished, as shown by a complexion of muddy hue. She complains of indigestion, marked constipation, and often has been advised that she has appendicitis.

On physical examination are found a coated tongue, a bad breath, flabby tissues, at times anesthesia of the cornea; the thorax is long and thin, and may show marked mobility of the tenth rib. A slightly protuberant abdomen, unusual in a young girl, may be seen while the patient is in the standing position. The liver may be felt extending three fingers below the free border of the ribs. The kidney is easily palpable, especially on the right side, and can be held down. The

\footnotetext{
1 Rose Bradford, Trans. Path. Soc., 1897, imitated the effects of Dietl crises in dogs, and after some weeks of hydronephrosis thus produced, allowed the kidney to recover by ureterostomy. The organ did recover, becoming a macroscopically perfect kidney, but with microscopic atrophy which he regarded as due to diminution of size of renal cells, disappearance and crowding of tubules.

${ }^{2}$ Wilson and Howell (p. 37) state that hydronephrosis occurred only four times in sixty-two cases of movable kidney operated upon.
} 
abdomen may show marked separation of the recti, such as would follow previous pregnancies.

Some patients undoubtedly suffer from visceral ptosis of a degree far more severe than would be suspected from inspection, coupled with a marked degree of intoxication resulting therefrom, to which the kidney is contributing its share. Severe degrees of neurasthenia and hysteria finally result. These cases are really the bane of the surgeon, as it is impossible, in the light of our present investigations, to say what share the kidney contributes. The gastrointestinal tract is no doubt a strong causative factor.

Another clinical picture is one which closely resembles the symptom-complex complained of in gynecological conditions, in which the chief symptom is pain, located in the back, low down, usually bilateral. This pain is generally dull aching, increased at the menstrual period, and is worse at the end of the day, after hard work. There is a sense of weight and dragging in the lower abdomen. The patient has not lost weight, is frequently stout, but is generally miserable and very neurasthenic. She may give a history of some sort of an abdominal painful crisis, which has happened several times.

On examination, the abdomen is fat, poorly muscled, with very short distance between the lower border of the thorax and the iliac crest. The kidney is easily held down after being grasped, and may be extremely mobile. When some perineal relaxation is present also, it is difficult to decide which region is chiefly at fault. Perineal relaxation is not a part of the general ptosis, but is often associated therewith. These patients may have severe renal crises in their clinical history.

The traumatic movable kidney presents a clinical picture of acute character. $^{1}$ It is likely to occur in active men, and always following some exertion (lifting, tennis, football) or a blow in the side, with disability resulting therefrom for from a few days to a week. At the time of the injury the patient may have hematuria, but this is not essential. This condition is followed, after months or more, by occasional renal crises, when there may occur hematuria of varying degree. The diagnosis may be easy where a palpable kidney is present, as in these eases there is likely to be marked displacement. Otherwise, routine laboratory methods, including pyelography, may be required to reach a satisfactory conclusion.

Renal or Dietl's Crises Associated with Movable Kidney.--The acute renal crisis is one of the most severe pain-crises encountered. They occur usually without direct antecedent cause, such as severe exertion,

\footnotetext{
${ }^{2}$ Newman, Trans. of the Clinical Soc., 1897. Bruce Clarke, Medico-Chir. Trans., 1893.
} 
on the part of the patient who is subject to such attacks. They are usually ushered in with nausea or vomiting, a certain amount of shock, and cold perspiration. The pain is in the abdomen, and usually remains epigastric, but may have renal radiation. The urine is usually very much diminished at the time, and may contain red blood cells. Anuria may occur. The crisis usually continues for several hours, and finally lets up with marked polyuria, consisting of fluid of low gravity. The patients are left exhausted, and continue to have more or less dull loin pain for days. In rare cases there may be renal insufficiency for a day or so (headache, dizziness, spots before the eyes, etc.).

Such crises occur from time to time in patients with movable kidneys, notably the type characterized by a forward prolapse of the upper pole. They may, however, never occur with the most movable of kidneys, and are evidently due to twist of the pedicle, causing strangulation of the vessels and occlusion of the ureter. ${ }^{1}$

In considering the symptoms from a diagnostic standpoint, it is usually impossible to differentiate an acute renal crisis of hydronephrosis from that of nephroptosis. In fact, the conditions are of ten concurrent. Even ordinary renal colic from stone can at times simulate this condition whenever the pain does not radiate typically; or be simulated by it when the pain of renal ptosis does radiate to the groin and genitals. The vomiting, which is reflex, may simulate intestinal obstruction. A supra-pelvic twisted ovarian cyst with a somewhat similar picture, might offer great difficulty in differentiating from a very low-lying twisted kidney. The earlier the patient is seen and examined with respect to the occurrence of the painful crises, the more helpful to the diagnosis. Several hours after the attack the physician may be extremely puzzled, as at this time a general distention with some rigidity and diminution of respiratory motion, and rise of temperature may suggest the existence of traumatic or inflammatory rupture of a portion of the gastrointestinal tract, especially a perforating duodenal ulcer, although in this latter condition the existence of a more profound state of collapse is likely to be apparent. Any vesical symptoms that can be noted (frequent micturition, strangury, urinary findings) should be carefully sought out, and will be of utmost service.

As to the differentiation of nephroptosis, independent of the painful

${ }^{1}$ It is claimed that in some cases there is only vascular torsion, and that these cases can be differentiated by the fact that there is less likely to be suppression; that the urine is more likely to contain albumen, blood, blood casts and hyaline and granular casts, and that on palpation the kidney merely feels enlarged, but there is no loin tumor of great size. These distinctions are rather fine, and will be found impracticable by most practitioners, as other diagnoses will be suggested by their presence. 
clinical crisis above described, the kidney, to be considered surgically a movable kidney, should descend enough so that its upper pole can be held between the thumb and forefinger, and the kidney thus captured remain down during expiration. Such a test should be made in the standing position, or in the dorsal prone position, with the upper half of the examining table raised about 30 degrees. The patient's confidence should be obtained during repeated light palpation through a half dozen or more respiratory phases before the capture is attempted. Such a criterion should be adhered to in stamping the kidney as the cause of the more subacute types of neurasthenia so often seen with movable kidney.

The type which inclines forward upon a transverse axis is par excellence a movable kidney. Such kidneys are apt to become more prominent upon expiration than upon inspiration, in fact, at times may be seen under the abdominal parietes.

As a rule, the anatomical diagnosis of movable kidney in the quiescent stage presents no difficulties. A tumor, somewhat rounded, is found in the loin. It is quite movable, and can be returned to the loin with ease; on pressure a peculiar sickening sensation is felt, similar to that which occurs on testicular pressure. This sensation is felt in the epigastrium, and makes the patient uneasy or even nauseated. At times, however, any movable tumors of the right upper quadrant afford difficulty in diagnosis, such as Riedel lobe of liver, ${ }^{1}$ hydrops or malignancy of the gall-bladder, or a massive gall-stone, tumors of the colon or impacted feces, pyloric tumors, renal neoplasms, any of which may cause some confusion. Light may be shed upon the differential diagnosis by the use of various procedures e.g., bismuth fluoroscopy, Roentgenography of gastrointestinal tract, pyelography, etc.

Riedel's lobes rarely have the amount of sensation on pressure which kidneys have, unless they are coupled with a congestion of cardiac disease, when they may be as tender as an acutely inflamed gall-bladder.

Malignant tumors of the gall-bladder are sooner or later associated with a certain amount of icterus, more or less progressive in character. Hydrops of the gall-bladder and stone very often may have the same hardness, but in their lack of sensitiveness to pressure they differ markedly from that of the kidney. The entire gastrointestinal tract, when under suspicion, should be covered by the diagnostic investigation by repeated stool examinations and Roentgenography.

The recognition of tumors of the kidney, whether hydronephrotic

1 A protuberance or process of the liver which sometimes projects over the gall-bladder in cases of cholelithiasis. 
or neoplastic, must ultimately depend upon the data elicited by ureteral catheterization, assisted by pyelography. This latter, after all, is the most valuable means of disclosing the position of the kidneys and their relations to any abdominal mass which presents itself for diagnosis.

Very movable kidneys in the lower abdomen, and kidneys which have become secondarily attached after their acquired position has been reached, or which are found in the lower abdomen or pelvis from congenital causes, offer great difficulties in diagnosis. They may be confused with ileo-cecal tumors (carcinoma, sarcoma, tuberculosis), with very chronic appendiceal abscesses, many of which are to a certain degree mobile, with foreign body perforation of the intestine, with tumors of the mesentery (lymphosarcoma, fibroma), with ovarian cysts, etc.

Careful rectal and gynecological examinations are of the greatest importance in diagnosis of such conditions.

Neurasthenia and Movable Kidney.-A problem which frequently confronts the surgeon is the differentiation of the "ailment of fancy from the ailment of reality." Unquestionably, certain neurasthenic symptoms are due to nephroptosis. Again, there are cases of real hypochondriasis that ensue as soon as the patient realizes that he has a movable kidney. In deciding the relationship of these two factors, the surgeon must consider:

1. Which condition was primary.

2. The position of the kidney.

3. The effects of replacement of the organ.

4. The clinical history and symptoms.

5. The urinary conditions after crises or so-called crises, and after replacement of the organ.

Evidence has been adduced to place movable kidney among other causes of insanity. ${ }^{1}$

Treatment.-Nephroptosis without pain often may be safely let alone. Too frequently it is found at autopsy that most movable kidneys show nothing of importance-in fact, it is possible that a freely movable kidney is less likely to suffer from constriction of its pedicle.

Regarding the movable kidneys with crises, with very subacute and chronic clinical pictures, it is probable that many of these have been unwisely subjected to operative interference, especially those strongly neurasthenic individuals whose general condition becomes worse as a

1 Suchling, Movable Kidney, London, 1905, gives the following list of mental disorders as partic ularly associated with nephroptosis: loss of memory, mental confusion and mental depression, melancholia and suicidal tendencies, morbid fears, homicidal impulses, emotional disturbances. 
result of the trauma inflicted upon their nervous system by the operation. Some surgeons never operate, except in cases showing acute renal crises. ${ }^{1}$ No such absolute law can be laid down, as certain cases of movable kidney with only subacute paroxysms of pain, etc., can be cured by operation, palliative treatment having been tried unsuccessfully for a certain length of time. Unquestionably, recurring severe acute crises of pain demand surgical intervention as delay may involve distention of the pelvis, diminution of secretory substance, and in time renal insufficiency.

Three classes of treatment are to be considered:

I. Hygienic and Preventative Treatment.-When a patient with movable kidney is undernourished, the general condition should be improved by rest, good food, outdoor exercise, and special exercises for the development of the abdominal muscles. Some cases have been cured by the rapid development of fat alone. Such a result has been derived from forced feeding, and Weir Mitchel rest cure.

In women, the pernicious practice of tight lacing should be prevented. After pregnancy, the use of a binder should be advised until the uterus is well involuted, and puerperal women should not be allowed to get out of bed too soon.

II. Mechanical Treatment.- The mechanical treatment is palliative, and consists chiefly in the employment of an abdominal belt. One of the varieties which can be recommended is that which provides a special pad or truss exerting pressure backward, and from below upward in the costo-iliac space toward the spinal column, for counterresistance. It should be applied by the patient in the recumbent or preferably in the Trendelenburg position. ${ }^{2}$ This position can be obtained by placing an ordinary chair in bed, with its back upward. The pad is objectionable if improperly fitted, and will cause trouble if misdirected pressure is exerted upon the kidney, or when the latter is very morable, as it is likely to slip out from under the pad, and some muscular atrophy is likely to result from prolonged pressure. The snugly fitting belt, without pad, has been found by some to be the best apparatus for ordinary use. It is claimed that by the use of this belt, pressure upon the abdominal contents is made from below upward, without the pad, which is likely to force the kidney out of place. As to this question, circumstances vary in different cases, and proper judgment must be exercised. If the pad suits an individual patient, it should be adopted, with the knowledge that too prolonged usage engenders a dependence upon it and a tendency to muscular

1 Treves, Practitioner, 1905.

2 Treves (loc. cit.) reports good results in chronic cases. 
weakness. Therefore, such mechanical treatment should be supplemented with the appropriate measures for increasing body weight and for physical development. Good exercise for strengthening the abdominal and lumbar muscles is to have the patient lie flat upon the back upon a hard couch or upon the floor, and raise the legs to the vertical position, keeping the knees stiff; also the reverse of raising the upper part of the body to the upright from the recumbent position, without assistance with the hands. These movements should be practised with increasing duration, avoiding undue fatigue.

During the painful crises, hot applications to the abdomen may be of service, but usually morphine is the only immediate means of relief. Great caution must be observed to prevent addiction to the morphine habit. Following the attack, intestinal distention and alimentary derangement demand cathartics and enemata.

Relief may ensue immediately, when it is possible to replace the displaced organ, the procedure being done in the Trendelenburg position. An anesthetic may be required in certain cases. This failing, after five or six hours, or more, the surgeon may have to consider the necessity of operative measures, involving lumbar exploration and kidney fixation and replacement.

III. Operative Treatment.-The operative treatment resolves itself into one procedure, nephropexy. In general, it may be said that complete nephropexy demands a good deal of denudation of the renal capsule, as the capsule itself will not become effectively adherent unless this is done. Sutures are passed through the substance of the kidney as near as possible to the cortical border and thus cause less damage to the parenchyma, and it has been experimentally proven that real adhesion occurs only at the suture points. There is no clinical evidence that any deleterious effects (chronic nephritis, etc.) follow such partial decapsulation. The kidney should hence be suspended by interrupted sutures through its capsule, which has been stripped to the reno-pelvic junction, and these sutures should be passed through the internal oblique and transversalis muscles, and tied. Sutures should not be passed around the last rib. The wound should be sutured without drainage. These are the principles: any refinements may be added, according to the desires of the various surgeons (see p. 637).

The kidney should be delivered, at operation, upon the loin. It should be thoroughly examined for hydronephrosis, and if present, the cause thereof, such as ureteral stone, abnormal ureteral attachment, etc., should be determined. Any abnormal arteries at the lower pole, which may give trouble, should be ligated. It should be placed 
in good position with efficient attachment, and with the least traumatism, and should be placed so that the uretero-pelvic junction is the most dependent point (see Hydronephrosis, p. 580).

The danger of operation is slight, being about 1 per cent. ${ }^{1}$

The results following operation necessarily depend upon the nature of the case and the care with which the procedure is carried out. So far as the general condition is concerned, some neurasthenic patients are improved, and others are not benefited by operation. Judgment should be exercised in the selection of cases in this regard. The kidney is held in place as a result of fixation in a large percentage of cases, and some obtain good results even when the organ does not stay replaced. ${ }^{2}$ The more chronic the condition, the more difficult it is to obtain a satisfactory result (see Operative Surgery, p. 637).

1 Watson and Cunningham, Genito-Urinary Diseases, 1908. In 1000 cases operated by sixteen different surgeons, 1.3 per cent.

2 Watson and Cunningham collected 253 cases. In 158 cases followed one to fifteen years, 83.5 per cent. of absolute cures were obtained.

Wilson and Howell personally studied and followed forty-one cases one to seven years after operation:

No. of cases

Acute cases:

24

Chronic cases:
Cured Greatly improved

Improved

In statu quo

\section{8}

11

1

3

\section{9}

5 


\section{BACTERIAL INFLAMMATION OF THE KIDNEY. PYE- LITIS, PYELONEPHRITIS, SUPPURATIVE NEPHRITIS}

Pyelitis implicates the renal pelvis, while in pyelonephritis the medulla and even the cortex of the kidney are involved. Should suppurative miliary foci ensue, the condition is referred to as suppurative nephritis. When these foci merge into one common sac, the term renal abscess is employed.

These conditions have much in common inasmuch as they are due to changes produced in the organ by bacterial invasion. The process, which may begin in the mildest form (catarrhal pyelitis), may develop progressively and terminate finally in an advanced state of diffuse renal destruction (pyonephrosis).

Causation and Method of Invasion.-The most frequent cause of renal infections is the bacillus coli communis. Next in importance are the staphylococcus, usually aureus (but at times albus) and streptococcus. The other organisms such as bacillus typhosus, bacillus proteus, gonococcus, pneumococcus, bacillus mucosus capsulatus, bacillus pyocyaneus, etc., are infrequent invaders. Mixed cultures are not uncommonly found in advanced lesions where there is extensive destruction of the kidney. Infections with the tubercle bacillus are discussed in a separate chapter (see p. 654).

Infections of the kidney may be

1. Urogenous (or ascending).

2. Metastatic.

a. Unilateral septic infarct, solitary renal abscess, perinephric abscess, etc.

b. In general septicemia.

\section{By direct extension}

a. From neighboring organs (colon, etc.).

b. From without (wounds, etc.).

Although authorities agree upon the existence of such routes, yet in a given case discussion is often raised as to the source of infection and there is also a divergence of opinion regarding the relative frequency of the different methods of invasion.

The urogenous route has long been accepted. Following prolonged cystitis, the direct invasion of one or both ureters and the pelves has been regarded as a natural consequence. This no doubt 
does occur and clinically it seems to be the most natural route. Such an origin of pyelitis has been established by experimental work and yet there are competent observers who will explain the same pyelitis as a result of an invasion of these very organisms by way of the blood stream.

The advent of this view of pelvic infection brings to mind certain well established clinical facts: the rarity of metastatic lesions in advanced infected cases of cystitis with stricture, enlarged prostate, etc., or after perineal section, cystotomy or prostatic enucleation, when there is ample denuded or raw surface from which a bacteremia could readily be acquired. On the other hand, there are cases with an absence of cystitis which develop pyelitis, such as seen in children, of an acute onset with abdominal pain, etc., bacteriuria and very few cellular elements in the urine which would seem to indicate an infection by diapedesis from the colon. These cases have their counterpart in the acute pyelitis of pregnancy, which may not necessarily have a concomitant cystitis.

Many bacteria filter through the kidney with practically no damage to the parenchyma, but such is not always the case; in fact, the meaning of points of low resistance in renal infection has been well shown experimentally. ${ }^{1}$

With infection of the pelvis of the kidney involving severe inflammation of its mucosa, a certain amount of retention and consequent distention, the infection of the parenchyma in an ascending manner would seem a certainty. It would appear natural that by the uriniferous tubules or the endolymphatic apparatus of the kidney, the infection would extend upward toward the cortex, forming bacterial streaks and cortical miliary abscesses; and the latter is seen often at the operating table. Some observers ${ }^{2}$ insist that many of the abscesses in the cortex in these conditions are deposited by a hematogenous route in a kidney of little resistance.

The possibility of a transient bacteremia during the course of a pyelitis cannot be denied, yet the severest forms of pyelitis associated with chills, sweats, etc., do not yield the infecting organism in the blood, and in the same cases, the organism, bacillus coli, bacillus pyocyaneus, etc., are easily recoverable from the urine. Bacteremia can only be demonstrated culturally in the most advanced cases of pyelonephritis. $^{3}$

The likelihood of a lymphatic route of kidney infection is mentioned

1 Brewer.

2 Brewer.

${ }^{3}$ The many observations on bacteremia in general reported by Lenhartz have not yielded other views. 
by some authors and should be given due consideration, but is very doubtful.

That the kidney may be infected by a purely metastatic route in certain cases is not to be denied; the solitary renal or perinephic abscess occurring after abrasions, furuncles, carbuncles, osteomyelitis of the finger or even paronychia apparently bears this out. Here the staphylococcus aureus is the offender. The bacteremia must be a very transient affair as the patients develop renal symptoms only after weeks or months have elapsed, so that the history of the original infection is, as a rule, forgotten.

Cases of severe septicemia, such as occur with acute osteomyelitis, puerperal sepsis, etc., with any pyogenic organism (streptococcus, staphylococcus aureus, etc.) may be associated with miliary cortical abscesses which latter are not the essential surgical lesion but a part of a generalized condition. Here the bacteremia is usually easily demonstrable by blood culture.

Infection of the kidney from without occurs after penetrating wounds or occasionally after nephrotomy for aseptic condition, such as stone.

Pyelitis and Pyelonephritis.-Bacterial inflammation of the pelvis alone is spoken of but it is usually associated with more or less involvement of the kidney. Obstruction to the free passage of urine, resulting in a bacterial infection of this fluid and subsequent decomposition, are a sequence of events associated with its occurrence. Among the causative agencies may be mentioned urethral stricture and prostatic hypertrophy. The vesical distention which occurs with these conditions has an important bearing upon the ascent of many of the infections as the bladder, sooner or later, becomes the seat of cystitis either through catheterization or instrumentation and a source of bacterial contamination.

Again, the vesical atony and retention occurring in hemiplegia, paraplegia, cerebrospinal syphilis and other neurological conditions should be considered as possible factors. A number of cases occur during the course of pregnancy. ${ }^{1}$ Pyelitis in children is not uncommon and is usually associated with cystitis.

Pyelitis may develop in connection with bladder distention with no implication of the ureters whatsoever. The organisms may develop in the bladder without causing an actual cystitis-that is, no involvement of the vesical mucous membrane with exudation of

1 E. Ward (Quart. Jour. Med., Oct., 1908) was able to study 187 cases. He considers the pressure of the uterus, during the latter months, upon the ureters as they cross the pelvic brim as the predisposing cause of pyelitis by the production of stasis and trauma. He considers the ascending route as the route of election. 
leucocytes and secretion of mucus. Such cases are more likely to be called bacteriuria than cystitis, as the urine is only cloudy as a result of the bacterial content, usually colon bacilli (see p. 102). Finally, we look to the systemic infections and intoxications as etiological factors of pyelitis. Under this category come pyemia, typhoid, variola and diphtheria; also the direct irritating effect of such drugs as cantharides, copaiba, etc., as well as other irritating diuretics, and renal calculi, which by their continual irritation sooner or later bring about an infection. Thus, circulatory disturbances of the kidney, notably congestion, furnish a predisposing cause to the development of pyelitis.

Parasites, such as bilharzia, are found in rare cases. Exceptionally, perirenal inflammation may set up pyelitis. The infectious agent may be introduced from without as a result of traumatism. This occurs in wounds, after operation, etc.

Neighboring organs may be the cause of pyelitis-the organisms migrate to the kidney from a diseased intestine, liver or psoas muscle.

Pelvic cellulitis, prolapsed uterus, diseased adnexa, pelvic neoplasms such as fibroid uterine carcinoma, ovarian tumors, etc., may be predisposing causes to pyelitis by pressure stasis in one or both ureters. In this category should be mentioned ureteral stone causing both irritation and tendency toward moderate or severe grades of intermittent hydronephrosis.

The predisposition of all anomalous kidneys to infections should not be forgotten, such as valve formation of the uretero-pelvic junction due to faulty implantation, congenital or acquired hydronephresis and marked misplacement.

The influence of sex has a bearing. The more common occurrence of pyogenic renal disease in men is due to the greater frequency of gonorrheal inflammation of the bladder. In women, the same disease more often attacks the utero-ovarian organs. Again, in men the association of arteriosclerosis, interstitial nephritis and hypertrophied prostate offers conditions favorable for the development of renal infection.

Unilateral pyelitis is not uncommon and occurs more often on the right side. ${ }^{1}$

But, except for the milder types and those having a localized cause (e.g., stone), pyelitis is more apt to be a bilateral affection, especially

\footnotetext{
${ }^{1}$ In 187 cases of pyelitis in pregnant women, Ward found 55 per cent. affected the right kidney, 10 per cent. the left kidney and 35 per cent. both kidneys.

Opitz (Zeitschr. f. Geburts. u. Gyne., Stuttgart, 1905) in a series of sixty-three cases found about the same percentage with however 66 per cent. of right-sided lesions.
} 
when it is encountered in the graver types of the so-called "surgical kidney."

Morbid Changes in Pyelitis and Pyelonephritis.-The renal pelvis presents, grossly, when infected, a variety of appearances which are designated as catarrhal, hemorrhagic, suppurative, membranous and gangrenous.

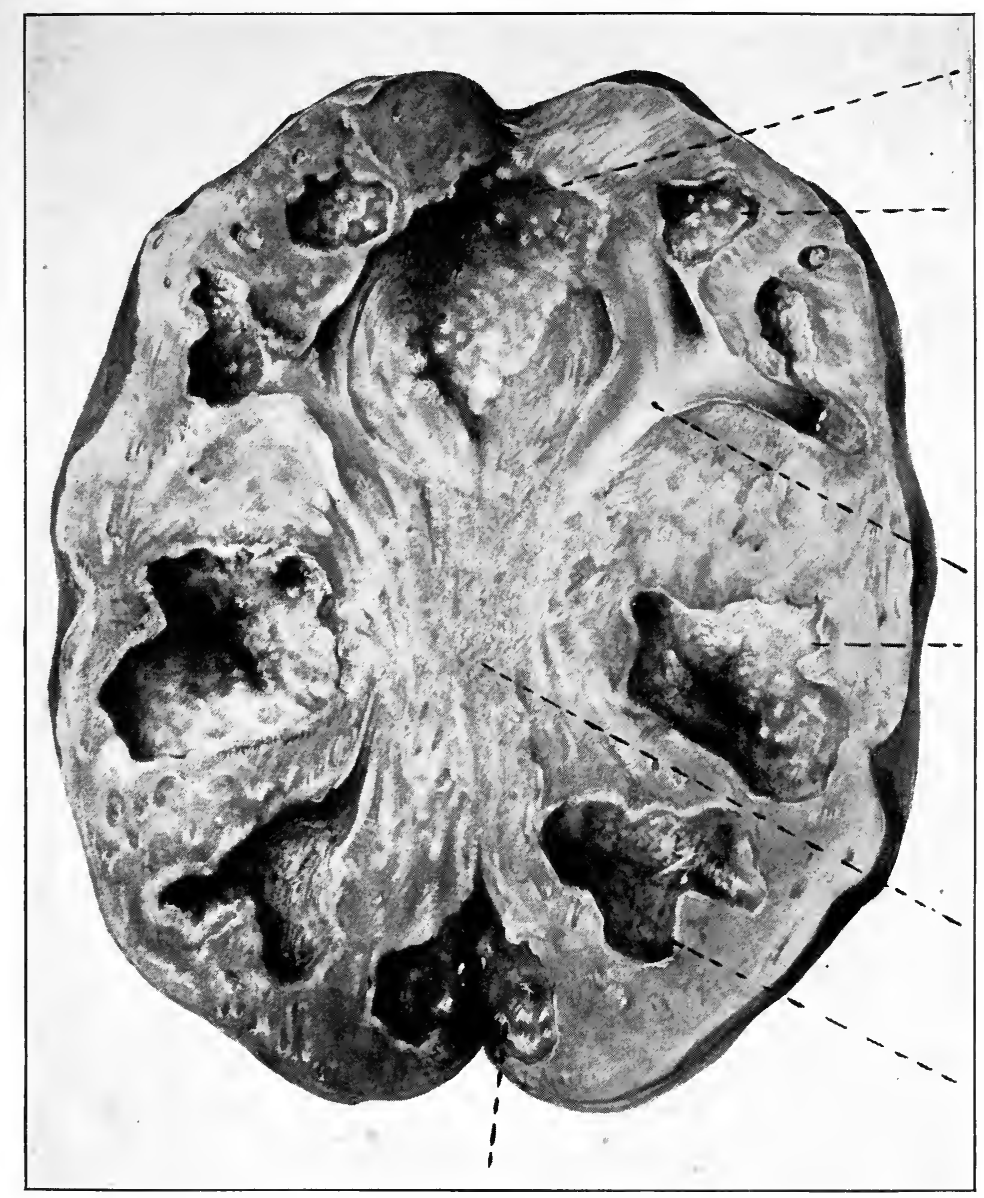

FIG. 250.-Pyelonephritis. Obliteration of ureteropelvic opening. (Author's case.)

In the catarrhal or subacute form, the mucous membrane becomes congested and velvety and covered with a thin layer of mucus or mucopus. Its color varies from grayish-white to reddish. When the inflammation becomes more severe and ecchymotic areas are seen, 
character, the term hemorrhagic is used. In suppurative pyelitis, the pelvis may contain free pus; it becomes, as a rule, dilated by this purulent material, the swelling of the mucous membrane causing a certain amount of encroachment upon the calibre of the ureteropelvic junction and sometimes complete obliteration thereof (Fig. 250).

In membranous, ${ }^{1}$ croupous or fibrinous pyelitis, the pelvis is coated with diphtheritic membrane composed of pus cells and bacteria. The pelvic mucous membrane may show areas of ulceration or gangrene. This is found most commonly with pyelonephritis and with stone in the pelvis. In the former cases the condition may be general, whereas in the cases with calculi, the condition is patchy and is due to pressure ulceration.

The ureter varies in its degree of involvement. It may, in some cases, show no changes at all. In others there may be a few petechial spots, pin-point to pin-head in size, scattered throughout its length. Such ureters, apparently little involved, may have shown changes at their vesical entrance (see Cystoscopy, p. 129). Again, the ureter may be dilated and hypertrophied ranging from its normal size to the thickness of a finger. These cases usually show marked involvement of the mucous membrane, hypertrophy with papillary or valvelike formation and all the types of inflammation of the pelvis that have been described. At times, the entire ureter and pelvis are coated with a fine sandy deposit which may be reddish or grayish, and in consequence the ureter becomes somewhat stiffened.

The moderate grades of infection of the ureter and pelvis may pass into the chronic state with more or less hypertrophy of the mucous membrane, some distortion of the canal or partial stricture formation. In advanced conditions, there is a thickening of the outer coat of the pelvis and ureter with adhesion to the surrounding tissues.

In the more acute conditions, a greater area of the kidney parenchyma is likely to be involved. In some cesses of pyelonephritis, where distention of the pelvis is the main feature, the kidney may only show signs of pressure consisting of blunting of the tips of the calices and dilatation of the pelvis. (See Hydronephrosis and Pyonephrosis).

The kidney itself is more susceptible to infection than to inflammatory pressure, when it is likely to berome enlarged as a result of parenchymatous degeneration and edema. In the moderately advanced stages of nephritic involvement, there may be seen extending in a radiating manner in the columns between the malpighian pyramids,

\footnotetext{
1 "Pseudo-membranous" has been used by Rovsing to designate that form of pyelitis in which shreds made up of fibrin and bacteria are voided in the urine.
} 


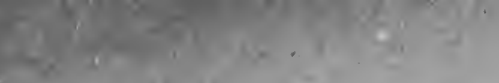

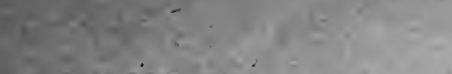

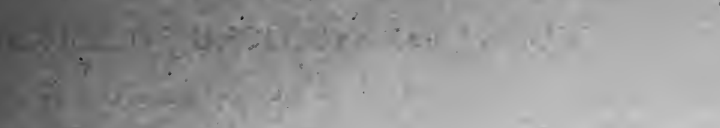

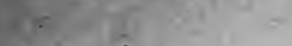

$+\frac{1}{2+\infty} \div$

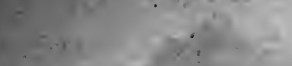

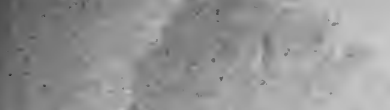

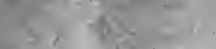
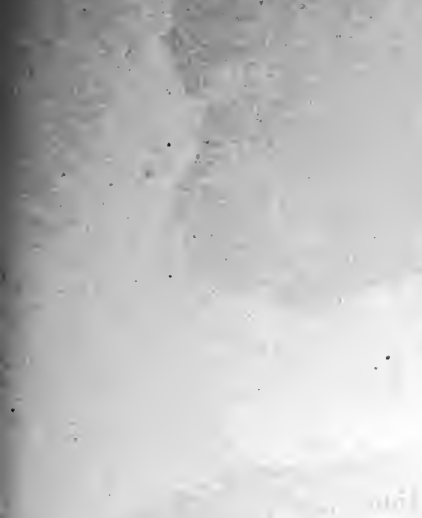

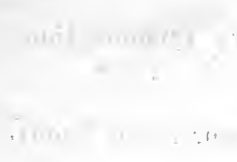

$y^{3}=$

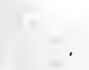

$\div$

-

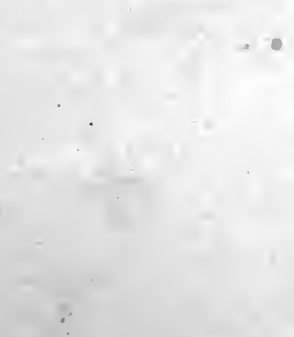




\section{explanation OF PLATE VIII.}

FIG. 1. Rupture of Kidney.

Fia. 2. Ruptured Kidney with Secondary Inflammation.

Fia. 3. Pyelonephrotic Kidney Sac.

FIG. 4. Non-suppurative Nephritis. Hemorrhagic Pyelitis.

Fia. 5. Acute and Chronic Pyelonephritis.

Fia. 6. Old Chronic Nephritis with Calcareous Degeneration.

Practically Non-functionating. 

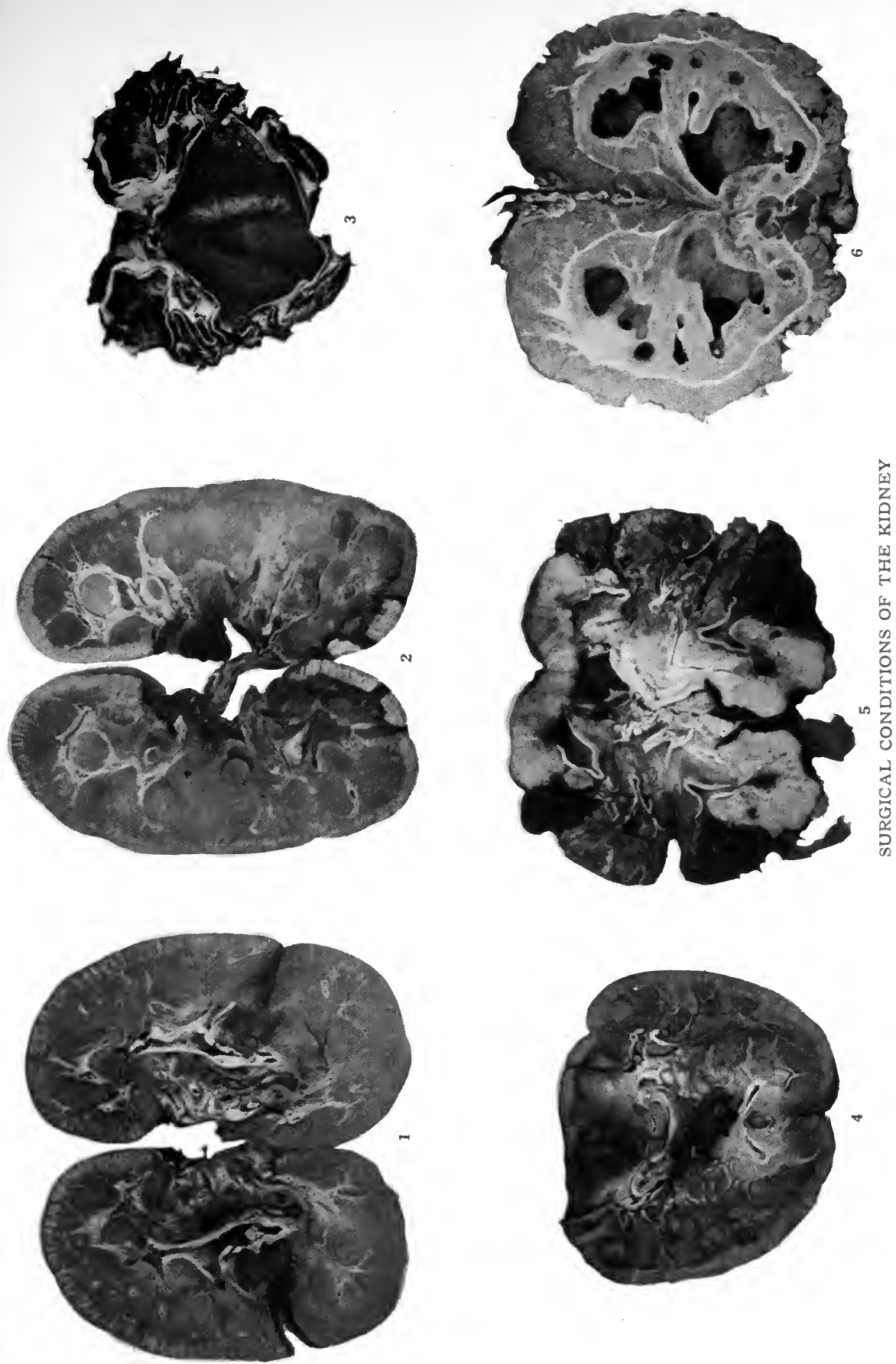

grayish or reddish streaks which represent microscopically stages of intense polynuclear and hemorrhagic involvement (Plate VIII, Fig. 2). The pyramidal tips may be swollen and edematous. The entire kidney may show a more or less advancing type of inflammation, namely: hemorrhagic areas, small grayish pin-head points which represent the early stages of abscess formation, and finally true abscesses, becoming even as large as a hazelnut (Plate VIII, Figs. 5 and 6). These abscesses are as a rule cortical, whether they are due to an ascending pyelonephritis or seem to be of metastatic origin. They may vary in number from one to so many that the entire kidney collapses in a flabby mass on being incised. On the surface of the kidney they may be represented by knob-like prominences.

In the milder forms of renal involvement, where spontaneous resolution takes place, there is advanced degenerative change in the kidney with subsequent fibrosis. The kidney may resemble somewhat closely the kidney of chronic interstitial nephritis and the patient may show signs of gradual renal insufficiency. The perinephric tissue shows considerable change, the fatty or false capsule becomes much thickened and marked fibrotic proliferation occurs, causing compression of the kidney, extending up into the renal fossa following the course of the blood-vessels. It does not, as a rule, develop to the extreme point, such as is seen in pyonephrosis, but it may replace considerable renal tissue near the hilus.

The complete involvement of the uretero-pelvis and kidney is most apt to occur from an ascending infection in association with vesical retention, hence it is usually bilateral, although the two sides may vary considerably in degree (Plate VIII, Figs. 5 and 6). The urogenous or ascending type of infection is not, as a rule, associated with perinephric abscess as it usually ends fatally before rupture of the true capsule occurs.

Clinical Course of Pyelonephritis. - A knowledge of the pathology of pyelitis and pyelonephritis suggests the many different phases in which these maladies may appear. Individual cases are not always regarded from the proper clinical aspect and therefore give rise to confusion in the mind of the clinician or surgeon.

There are certain types of cases represented by different clinical histories that are descriptive of the varieties of pyelitis and pyelonephritis. These varieties are:

Catarrhal or subacute pyelitis, pyelonephritis, acute and chronic.

Suppurative pyelonephritis, acute or fulminating and chronic (surgical kidney).

The Catarrhal or Subacute Variety of Pyelonephritis.- This type of infection is the mildest form that occurs and is likely, on this account, 
to pass undetected. It is usually due to infection by the bacillus coli communis but may also occur during or after an attack of typhoid fever, when the typhoid bacillus is the causative agent. Pyelitis of this type is more apt to occur in women, especially during or after pregnancy. It is quite likely to follow a chronic form, although it may assume a more active acute condition when from any reason the kidney becomes more involved by the infecting organism, the bacillus coli communis, either from a urogenous source or by hematogenous invasion. When the infection is derived from the bladder, it may come as a result of a preëxistent chronic cystitis or, as already stated, the bacilli may be present in the bladder urine without apparently causing any inflammatory disturbance there. When occurring during the course of typhoid fever, it is due to an infection by the organisms during the process of elimination.

The symptoms consist of a septic temperature, of ten accompanied by night sweats, loss of appetite, nausea and languor, and the passage of purulent and bacterial urine. The physical examination reveals slight tenderness in the kidney region by bimanual pressure.

The diagnosis is reached by cystoscopic examination which reveals the fact that pus and bacteria are being discharged from the upper urinary tract. Laboratory examination of the urine will show the presence of pus and of the bacteria of the colon bacillus group.

These cases, if they do not yield to therapeutic measures or subside spontaneously, become more or less chronic or may assume one of the more severe types of renal infection, namely, suppurative pyelonephritis.

Chronic pyelitis is a continuation of the above subacute form and, therefore, due to the same causes. There is a general malaise, with perhaps a loss of weight and mild anemia. The temperature, which is usually not elevated, may from time to time have exacerbations of one or two degrees. There are not usually the local signs of tenderness or pain in the kidney region. There are constant bacteriuria and pyuria, the former being in marked predominance unless there is a coexistent cystitis. The diagnosis is made upon the presence of the bacteriuria with the mild constitutional disturbance and is confirmed by cystoscopy. These cases, if not cured, may continue for a protracted and indefinite period, the mild ones without any particular constitutional depreciation, especially under the controlling influence of treatment, whereas the more severe ones, in the absence of treatment, may develop a gradual renal insufficiency or on account of septic invasion of the parenchyma, a suppurative nephritis or multiple purulent foci.

Treatment of Mild (Catarrhal) Pyelitis.-The treatment is symptomatic and should be adapted to each individual case, some require 
confinement and rest, while others may be allowed to be about. A bland and unstimulating diet should be ordered with avoidance of physical fatigue. The favorite internal remedy is the hexamethylenamine in the form of urotropin or helmitol. This may be given in doses of $1 / 2 \operatorname{gram}(71 / 2 \mathrm{gr}$.) three times a day with or without an equal amount of benzoate of soda with a copious draught of water. Favorable cases of the mild type may yield to this treatment, but it may be necessary to continue it over a long period. Some advise increasing the hexamethylenamine preparation to the full point of tolerance, giving as much as 1 or 2 grams (15 to 30 grs.) at a dose. Generally speaking, smaller doses will yield all the benefit that may be expected from such treatment. Finally, the employment of vaccine therapy should be considered and some good results have been obtained thereby. The vaccine may be a stock vaccine of the infecting organism or of an autogenous vaccine of the same (see p. 110).

\section{SUPPURATIVE PYELONEPHRITIS}

This more severe type of kidney infection occurs in conjunction with cystitis following frequent catheterization or after ureteral catheterization or as a result of the presence of renal calculus. In such cases, the patient may be taken suddenly with a severe chill, the temperature rising to $40^{\circ}$ or $40.5^{\circ} \mathrm{C}$. $\left(104^{\circ}\right.$ or $105^{\circ} \mathrm{F}$.). The patient looks ill and may have an attack of nausea and vomiting at the onset. The pulse and respiration, as a rule, do not correspond to the temperature elevation. On examination there is found tenderness at one or the other costovertebral angle, and on deep pressure in the hypochondrium. The temperature now runs a septic course, ranging from normal to $41^{\circ} \mathrm{C}$. $\left(106^{\circ} \mathrm{F}\right.$.) with chills and sweats and a characteristic blood count (increasing leucocytosis and polynuclear percentage). The kidneys may gradually enlarge and become palpable. In severe fulminating cases, with sharp onset, the course may be less than a week, the patient passing into a typhoid state with muttering delirium, dry tongue and cracked lips, dying within a few days of acute intoxication. Such cases may be difficult to differentiate from typhoid when the history is unobtainable, or they may be confused with pneumonia or pulmonary abscess. In some, however, a bacteriuria is present and a careful culture may yield the bacillus coli, the bacillus pyocyaneus or the bacillus proteus vulgaris. Cystoscopy and ureteral catheterism are at times difficult on account of the severity of the illness but if performed, may yield conclusive information. The same condition may occur during the course of a pregnancy or after re- 
peated pregnancies whenit may be an exacerbation of chronic pyelitis.

The fulminating and rapid course above described may moderate, the septic temperature gradually lower and resolution take place at the end of one to three weeks, or the condition may gradually pass into that of chronic pyelitis or pyonephrosis (p. 620). There may develop a solitary renal abscess, multiple foci or a perinephric abscess. The fulminating form of pyelitis is, as a rule, characterized by the most severe type of morbid changes of suppurative and gangrenous pyelitis described in the previous section.

Chronic Suppurative Pyelonephritis.-This form of pelvic and renal inflammation is insidious of onset and slow in progress. It is apt to develop in connection with infections of the lower urinary tract common to men, such as enlarged prostate with cystitis, stricture of the urethra, chronic gonorrheal cystitis, also vesical tumors and atony of the bladder due to disease of the central nervous system. It represents particularly that which is classically known as "surgical kidney." In the early stage of development, this condition may be unrecognized and it is often difficult to say when its onset occurs. The morbid changes have been described (p. 603).

The symptoms of chronic pyelonephritis are a combination of urinary sepsis (toxemia) and uremia. A terminal stage is signalized by the development of renal insufficiency. In the beginning the temperature may not rise above normal, but as the malady advances it impresses itself upon the general condition of the patient, which is due to absorption of toxins, when gradual urinary toxemia becomes evident. When this state is reached the skin is dry, the tongue dirty with a tendency to dryness on the end, the patient feels prostrated and slowly emaciates. This stage may continue for a more or less indefinite period. Later on there may occur occasional attacks of temperature elevation, possibly preceded by chill. When the terminal stage presents itself, it develops into a state of progressive chronic uremia. The patient emits a peculiar urinous odor, the tongue is prone to be dry, furred or cracked, he becomes drowsy and is aroused with difficulty, hiccoughs occur and finally passes into a comatous condition and dies. This terminal state often is not reached until after a long preliminary period of slowly developing urinary toxemia, during which time the course of the malady is very insidious, and this final condition does not seem to ensue in the case of some individuals until there is almost complete destruction of the renal substance and extensive dilatation of the entire vesico-uretero-renal tract. The urine, throughout, is purulent; it may be of acid reaction in the begin- 
ning, but later on, due to retention and decomposition, it becomes alkaline, yielding a large phosphatic deposit and sometimes phosphatic calculi are formed. Some patients may develop unilateral or bilateral pyonephrosis, at which time the dilated pelvis and kidney may be palpated in the loin (see Pyonephrosis, p. 620).

The diagnosis of chronic suppurative pyelonephritis is made first by the clinical history, namely: a chronic inflammation of the lower urinary tract followed by symptoms of urinary toxemia, but more particularly by laboratory and cystoscopic examination. The gross appearance of the urine is the same as that of cystitis-purulent and bacterial. If the urine is passed in two or three glasses all are equally cloudy (Plate V, Fig. 11). The sediment when it has deposited has often a characteristic waxy appearance. Microscopic examination may find cells from the pelvis and renal tubules (see p. 95). There is usually much more albumin present than the pus would account for. Cystoscopy will determine whether one or both kidneys are the seat of trouble (see p. 129), and functional urinary tests will reveal the probable degree of destruction of the kidneys and their comparative functional capacity (see p. 155).

Treatment of Suppurative Pyelonephritis. - The acute variety of this type of pyelitis may be unilateral and due to instrumentation, in which case palliative measures should be effective. The patient should be treated as in any febrile condition-sponging for high temperature, and symptomatic measures for headache, etc. The most important indication is the free use of fluids; a glassful of water every one or two hours should be taken and hexamethylenamine $\frac{1}{2}$ gram doses three or four times a day. In the acute toxemic cases, great improvement may follow the use of autogenous vaccines of the bacillus coli or bacillus pyocyaneus or other pyogenic organisms or the mixed stock vaccines of these varieties.

In the acutely septic, suppurative, types, especially those of bilateral kidney disease, the general condition of the patient must be the deciding factor as to whether unilateral or bilateral drainage of the pelvis should be done. The recognition by palpation of the development of a renal abscess will leave no question as to the indication for operation unless it is decided to attempt to drain the kidney from below, by ureteral catheterism, as a preliminary measure (see p. 143). In the very toxic unilateral type, the possibility of the existence of multiple hematogenous septic infarcts of the kidney should be borne in mind, and if there be any doubt, exploration should be performed provided the case has been carefully worked up and studied along differential diagnostic lines (Cystoscopy and Roent- 
genography). If exposure of the kidney shows no miliary or gross abscess foci, the nephrotomy or a drainage of the pelvis (pyelotomy) completes the operative procedure (see Operative Surgery, p. 634).

The principles of treatment for chronic suppurative nephritis are practically the same, namely: the free use of diluent mineral waters to wash the kidneys from within outward, urinary disinfection with hexamethylenamine in doses of $\frac{1}{2}$ or 1 gram three times a day, which remedy should not be kept up continuously but omitted from time to time for reasonable periods, and by way of substitution salol and biborate of soda in 0.3 to $0.6 \mathrm{grm}$. (5-10 gr.) doses each may be given. In addition to the above, the patient's general condition should be studied and treated accordingly and such accompanying condition as chronic cystitis and retention of urine should be treated by catheterism and vesical lavage. (See Chronic Cystitis $\mathrm{p}$ 426.)

The question of adopting surgical measures in cases of chronic pyelonephritis must be decided upon by the peculiar features in each individual case, which are determined by cystoscopic examination of the bladder and kidneys, measuring the functional capacity of the latter (see p. 155) and a careful study of the general and local conditions. The terminal state is generally reached when the subject thereof is old and debilitated and the entire urinary tract is diseased. If there is a coexisting obstruction at the bladder outlet, and drainage is deemed expedient, cystotomy may be performed under local anesthesia and a prolonged drainage of the urinary tract accomplished in this manner. Bilateral nephrotomy in these cases after extended duration is not, as a rule, desirable, on account of the poor state of the kidneys which have long been the site of chronic suppuration; and the attendant conditions of toxemia-emaciation and amyloid degeneration-mean that death will soon follow even if the patient should chance to survive the operation. 


\section{CHAPTER XXXIV}

\section{METASTATIC INFECTION OF THE KIDNEY}

\section{UNILATERAL SEPTIC INFARCTS}

A preliminary consideration of hematogenous involvement of the kidney in general is necessary before proceeding to the discussion of the different varieties of this condition. Some of these belong essentially to the domain of surgery, but most of them take a medical classification, are usually of grave import, and should be as clearly differentiated as is possible with our present knowledge.

Metastatic septic infection is an acute pyogenic disease of the kidney, characterized by the formation of single or multiple infarcts deposited by the blood stream. The subject of hematogenous infection of the kidney has of late assumed considerable importance. It is of interest from the standpoint of the internist, as well as from that of the surgeon. Inasmuch as a proportion of the cases show but one actively septic process, namely, a unilateral lesion, whether treated from a medicat or a surgical aspect, it is a borderline subject.

The blood stream of apparently normal individuals at times contain organisms which enter through lesions of the skin or mucous membranes, and are either disposed of by its bactericidal power, or being lodged in one of the organs, produce a morbid lesion. If these organisms are pyogenic, an acute local inflammation usually results. with necrosis and pus formation. The pathogenic microörganisms generally found in such cases are the staphylococcus, the streptococcus. or the bacillus coli. The organism most likely to be associated with the type of transient bacteriuria referred to, localizing in a single focus, is the staphylococcus pyogenes aureus (often albus). ${ }^{1}$

Such a process may occur, with very mild symptoms, associated with a single purulent focus in the kidney and the formation of a perinephric abscess. At times, however, after intervals of weeks, other

\footnotetext{
1 The distinction between the staphylococcus pyogenes aureus and staphylococcus pyogenes albus is of ten very difficult to make. Frequently, the yellow pigment will appear in albus cultures only after seven to nine days' growth on potato. The staphylococcus albus is at times non-pathogenic. For example, the staphylococcus epidermidis albus is a most frequent inhabitant of the hair follicles, and very often found as a contamination in blood cultures. However, the staphylococcus albus is at times pathogenic, and has been found in malignant endocarditis (Lenhartz). It is also found in persistent pustular acne, and in perinephritic abscesses. The staphylococcus pyogenes aureus is practically always pathogenic.
} 
pus foci develop insidiously, a prostatic abscess, an orchitis, a muscular abscess, all being caused by the staphylococcus aureus. As a rule, such cases present no demonstrable bacteremia. They begin insidiously and end in a like manner, in some instances lasting for months or years. When an osteomyelitis occurs during their course, the symptoms usually become fulminating, and staphylococci are easily demonstrable in the blood. During the course of such a severe bacteremia there occur grave visceral lesions, among which are to be found septic infarcts besides those of the kidney, of the lungs, spleen, heart, and muscles, acute endocarditis and purulent pericarditis and pyarthroses of any of the large joints.

On the other hand, the kidney alone may be the seat of such an infection, with profound toxemia, and, being the only focus, may offer ample indication for surgical intervention.

The origin of infection is often very obscure. Some of the staphylococcic infections are undoubtedly associated with furunculosis, carbuncle, paronychia, osteomyelitis of distal phalanges, etc.; others remain unexplained, following acute infections like grippe, scarlet fever, tonsillitis, etc. Entrance through the tonsils is to be considered, as the staphylococcus aureus can be found in the throat, and is discovered in cultures from many cases of acute tonsillitis, either as the predominating organism, or associated with the streptococcus.

In like manner, septic conditions may occur with the streptococcus pyogenes. The staphylococcus aureus is most likely to invade the blood current through the skin, the streptococcus pyogenes as a rule enters by way of the mucous membranes of the body. On account of the fact that the mucous membranes are usually hidden from view, many of these infections resulting in pyemic manifestations, with or without a demonstrable bacteremia, remain obscure. Many, however, have a well-known etiology, such as otitic (otitis media, lateral sinus thrombosis) uterine (after curettage or labor), post-tonsillitic and postpharyngitic, resulting from other suppurative conditions in the nasopharynx (after furuncles of nose, operations in the nose, etc.); others are more obscure, such as bacteremia following secondary infection of new growth or ulceration of the gastrointestinal tract.

Colon bacillus invasions of the blood are very rare. These germs are, however, sometimes found in the blood during life, in suppurative conditions of the genito-urinary tract, such as ascending pyelonephritis. Such conditions are prone to be fatal, and at postmortem examination, show marked involvement of the pelvis, as well as the kidney parenchyma, with abscesses throughout the cortex. These cases may show 
metastatic lesions throughout the viscera, septic endocarditis, abscesses of spleen and kidneys. ${ }^{1}$

They are cases of sharp bacteremia, which are demonstrable by blood culture during life, or, where this is neglected, the metastatic lesions show the presence of the organisms. That a transitory preëxistent bacteremia with the colon bacillus does exist cannot be doubted: witness the cases in which foci apparently have occurred in a single organ, like the kidney $;^{2}$ but the bacteremia in the milder cases is not always demonstrable.

The clinical and anatomical evidence of such cases must all be carefully weighed. Cortical abscesses of the kidney, occurring in the absence of pyelitis, would of course be unquestionable evidence. ${ }^{3}$

\section{Metastatic septic kidneys are divided into}

(a) Unilateral septic infarctions, including some types of perinephric abscess.

(b) Septic infarctions occurring in the course of a general bacteremia.

Unilateral Hematogenous Infarction of the Kidney, including some types of perinephric abscess. - The fact that a kidney shows hematogenous septic infarcts does not necessarily bring it into the domain of surgery, but in assuming this title, which has been used previously by other writers, it is intended to describe a condition which is essentially surgical. ${ }^{4}$

Causation.-The infecting organisms are staphylococcus pyogenes aureus (sometimes albus); streptococcus pyogenes and bacillus coli communis. It is often impossible to designate definitely the point of entry. Some of the invading organisms enter through lesions of the skin, such as pustular acne, sycosis, furuncles and carbuncles. At times parocychia and subacute suppurating processes in the bones or terminal phalanges are responsible for the organisms. Some of the cases have followed acute infections, such as scarlet fever, tonsillitis, pharyngitis; and cases of so-called "grippe" have preceded this condition, while many have shown no distinct preëxisting causation.

Morbid Changes of Unilateral Septic Infarcts.-The perinephric tissue is edematous. At times a very small collection of perineph-

${ }^{1}$ Littman and Barlow, Deut. Arch.f. klin. Med., Bd. LII.

Hetschmann, Wien. klin. Woch., 1896, nr. 18.

2 Brewer, Surg., Gyne., Obstet., 1906, Vol. II.

${ }^{3}$ Posner's experiments (Posner, Berliner Klinik, 1895, have demonstrated the existence of blood invasions by colon bacilli after ligation of the gut.

1 This condition has long been known among the Germans. Israel (Chirurg. Klinich der Nieren Krankheiten) was one of the first to call attention to it. Brewer has contributed notably to bringing the matter forward in this country, principally by calling attention to the early symptoms and showing the advantage of prompt diagnosis and operation. 
ric pus is encountered. The kidney itself is swollen and edematous; its surface is the site of multiple reddish infarcts, which are usually smaller than lentil-size and are at times grouped en masse, raised slightly above the surface. Such a mass may show separate infarcts, reddish in color and having central grayish points, or occasionally may represent a fused fluctuating abscess.

On incision, the infarcts show only a reddish spot, exuding serum, or they may contain a drop or more of pure pus. On examination the serum or pus will yield the organisms already mentioned.

The infarcted areas are usually surrounded by zones of fairly normal-looking or slightly edematous renal parenchyma. The pelvis is normal, or shows a few pin-head sized petechiæ.

The kidney is generally larger than normal.

On microscopic section, the infarcted areas show distinct evidences of abscess formation, with masses of polynuclear leucocytes and various stages of necrosis of renal epithelium. Bacteria are usually so numerous as to need no special stains for their differentiation. The rest of the kidney, which grossly appears normal, may show many multiple bacterial emboli, and usually shows diffuse involvement of the parenchyma (edema, hemorrhages, congestion, albuminous degeneration of the renal epithelium).

In the typical fulminating unilateral case, the kidney is apparently the only organ that is diseased, as the patients usually recover completely following operation, provided there is not too severe a toxemia to sustain the operative procedure.

The subacute type shows nothing more than the morbid changes seen in cases of perinephric abscess (p. 627).

Clinical Course.-There are apparently two distinct classes of clinical onset: acute and subacute in type.

The acute type generally starts with a chill and temperature of $40^{\circ}$ to $40.5^{\circ} \mathrm{C}$. $\left(104^{\circ}\right.$ to $105^{\circ} \mathrm{F}$.). The patient shows the usual signs of an acute septic condition: the face is flushed, the eyes are bright, while the patient answers questions well, there may be some clouding of the intellect, and at times delirium. The pulse and respiration are increased, but not in proportion to the apparent degree of toxemia in comparison with the temperature.

The history may bring to light some of the factors described under causation. The physical examination may show nothing more than slight tenderness in one costovertebral angle. The bladder urine, in typical cases, shows only a trace of albumen, an occasional red cell and a few leucocytes. It may be somewhat scanty and highly colored, as in other febrile conditions. 
Catheterization of the ureters may yield on the affected side a small amount of urine, with a few leucocytes. The normal side may show a larger comparative amount, and an occasional leucocyte.

Careful physical examination, in addition to eliciting costovertebral tenderness, under ordinary circumstances, will show no other clinical evidences, unless the particular symptoms of one of the causative conditions already described; are found in some degree of activity.

The course of the condition may become rapidly fatal within one to three days, with marked febrile activity, or the latter may show signs of abatement, and the general condition improve, the temperature gradually becoming normal, the tenderness disappearing, and the patient be discharged well, with normal urine. Such cases should be held under suspicion for several months, as they may develop perinephric abscess and its sequel.

The milder, or subacute type of metastatic infection follows the same causative factors as the acute, but more properly the condition belongs to the domain of perinephric abscess (see p. 625).

The patient presents the appearance of being sick, but not acutely so; the complexion is sallow. There usually is a complaint of dull pain situated in the costovertebral region or in one or the other of the upper quadrants of the abdomen, and there may be the characteristic attitude of renal tenderness, that is, slightly bent forward, with thorax tilted in the direction to relax the muscles of the affected side.

On examination, distinct unilateral costovertebral or hypochondrial tenderness is elicited on deep firm pressure. The temperature may be $38.5^{\circ}$ to $39.5^{\circ} \mathrm{C}$. $\left(101^{\circ}\right.$ to $103^{\circ} \mathrm{F}$.).

The urine is usually normal.

Careful general examination may reveal one of the causative factors only, the scars of such lesions (carbuncle of the neck, etc.), or there may be a history of an attack of fever associated with an acute infectious disease or a previous fulminating attack, such as already described, and followed by partial resolution.

Diagnosis. - The term unilateral hematogenous septic infarcts of the kidney is restricted here to those cases in which the lesion is located only in the kidney (not the pelvis) and is deposited by way of the blood stream. The rest of the body is entirely free from septic foci other than those (external furuncles, etc.) which are to be considered as the points of absorption. This clinical distinction must be recognized; otherwise the condition cannot be considered as essentially a surgical one, and other varieties of hematogenously infected kidney will cloud the issue and render the differentiation, difficult as it is, still more so. 
The condition is different pathologically from unilateral pyelitis and pyelonephritis occurring without cystitis or ureteritis, which can result also in abscess formations in the cortex, and may necessitate surgical intervention.

In the first place, the diagnosis rests on the clinical pictures already described. In the fulminating variety, where the surgeon is confronted with a condition of the kidney which seems to demand immediate interference, great stress should be laid on the history, which may furnish a causative agency. In the presence of factors which might result in a unilateral urogenous pyelitis (such as cystitis, septic catheterization, etc.), a high temperature should not necessarily convey the idea of abscess or infarct formation. Many cases of acute pyelitis, with chills, fever ranging with septic curve from subnormal to $41^{\circ} \mathrm{C}$. $\left(106^{\circ} \mathrm{F}\right.$.), often clear up in a striking manner under medical treatment. The septic infarct cases are more likely to have continued febrile activity, following a single initial chill, but these facts must be taken into consideration in connection with other features. Patients with septic infarct are also more likely to be profoundly prostrated, whereas those with acute pyelitis do not have the most extreme prostration, even at the time of the height of the temperature.

The urinary findings are of great importance.

In cases of septic infarcts, the urine is almost negative, but a trace of albumen and a few pus cells are found; ureteral catheterization shows but a slight difference in the two sides. This is due to the lack of pelvic involvement. An occasional instance may occur in which rupture of an abscess into the pelvis of the kidney would make this differentiation worthless. As a rule, however, the cases of acute pyelitis show a distinct difference in the urines of the two sides, one of them showing the turbid urine, due to the presence of bacteria, or pus cells, or both, and the cultures of the urine in such instances will show the presence of the infecting organism. ${ }^{1}$

The existence of cystitis as an etiological factor is in favor of pyelitis, and the possible presence of pelvic, and especially of ureteral calculus, should be remembered. Roentgenographic examination will afford the desired information on this question.

Existing pregnancy and a history of many pregnancies would be more likely to favor the diagnosis of pyelitis.

In addition to this type of pyelitis, one should bear in mind that certain types of bilateral urogenous pyelonephritis are likely to give physical signs upon one side only. This is of importance, as such conditions may arise with either ordinary ascending infections or in

1 Brown, J. H. H. Rep., Vol. X. 
advanced conditions of urogenital tuberculosis, with or without the association of renal or pelvic calculi. Such conditions may in some instances warrant the intervention of the surgeon, but it should be with the full knowledge that they are bilateral to some degree. The addition of a streptococcus to a previous infection of pelvis and kidney, adds the element of a mixed infection which brings on the febrile reaction, suggesting unilateral septic infarct. Such kidneys may contain cortical abscesses, or may be in advanced stages of pyonephrosis, and it is difficult to say, on account of the involvement of the pelvis, whether the added infection is urogenous or hematogenous.

In differentiating unilateral septic infarction, the most important series of cases to be separated therefrom are the diseases associated with invasions of the blood stream, easily demonstrable by blood culture and therefore a part of a generalized systemic condition:

Acute septic endocarditis as a rule shows the acute onset, the profound prostration, the chills, fever, sweats, and various metastatic lesions. If these should locate in the kidney, as is usually the case, the formation of septic infarcts and later of abscesses is certain. This essentially medical condition must be clearly differentiated. The cardiac murmur, if present; the retinal hemorrhages, the petechiæ on the skin and in the conjunctival sac, and at times the presence of an etiological factor-history of a former cardiac lesion ${ }^{1}$ - furnish a basis for diagnosis.

Usually the disease progresses without clinical manifestation of any renal involvement, other than an occasional red cell in the urine.

The infecting organism can be cultivated from the blood stream, and at times from the urine. The organisms usually found are the streptococcus pyogenes, the staphylococcus aureus, the pneumococcus, the gonococcus, etc.

Chronic infective endocarditis may at times offer signs of renal embolism.

The diagnosis is usually not so difficult unless seen at the moment of chill and pyrexia, and the blood culture will furnish needed information.

The organisms usually found are the small streptococcus (SchottMüller) and the influenza bacillus. The other organisms (gonococcus) are rarely found. ${ }^{2}$

Non-suppurative unilateral infarcts (see Differential Diagnosis, p. 545) in typical cases present practically no difficulty in diagnosis.

1 Lenhartz, Septischen Krankheiten, 1904. Chapter on Endocarditis.

${ }^{2}$ Litten, Zeitschrift f. klin. Med., Bd. II, 1881. Lenhartz, Münch. med. Woch., 1901, Nos. 28 and 29. Harder, Quarterly Journ. Medicine, 1909, No. 2, p. 289. Libman, J.H. H. Bull., Vol. XVII, No. 184, July, 1906. Schott-Müeller, Münch. med. Woch., Bd. I, No. 20, May, 1903. 
For example, a case of acute rheumatic endocarditis develops slight hematuria and slight deep-seated pain in the loin. If, likewise, the patient is running a high temperature, with an occasional chill, and the pain is very acute, the condition may be obscure. The presence of a cardiac murmur and hematuria are suggestive. The blood examination is important, as leucocytosis and polynucleosis are more likely to be present in a septic condition.

Other septic conditions usually associated with demonstrable bacteremia, particularly acute osteomyelitis, otitic sepsis with sinus thrombosis, and postpartum sepsis, when typical, can form no stumbling block, unless the original focus is somewhat quiescent, and the metastatic lesion overshadows the entire situation. It must be emphasized that whenever such possible portal of entry is found after physical examination, the diagnostician should think twice before considering a metastatic lesion as the sole lesion.

It might be well to call attention to the fact that unilateral septic kidney may be confounded with acute hemorrhagic post-scarlatinal nephritis. Occasionally such a nephritis may start with a chill and temperature of $39.5^{\circ}$ to $40^{\circ} \mathrm{C}$. $\left(103^{\circ}\right.$ to $104^{\circ} \mathrm{F}$.), and very septic symptoms, offering great difficulty in diagnosis, because of the fact that scarlet fever is at times complicated by metastatic abscesses, due to streptococcus pyogenes. Such a nephritis is likely to be associated with smoky urine and facial edema, and such a kidney at the operating table may show hemorrhagic areas which resemble infarcts. No pus will be found, and the spreads from the lesion will show no bacteria. The condition on section will show only the lesions of a very acute nephritis. The kidney may be removed and the patient, being young, may recover in spite of the mistake in diagnosis.

Blood cultures should be resorted to as a last diagnostic aid, and one which is of great importance. This subject is of much interest; space will not allow further consideration here. The reader is referred to the standard text-books and special articles. ${ }^{1}$

The conditions of the blood in unilateral septic infarcts should be studied systematically. However, knowledge pertaining to other conditions of bacteremia will be of service in excluding them, or in directing attention to their presence.

Prognosis.-This depends on many factors, clinical, pathological and therapeutic. In the first place, the patient may succumb to the marked toxemia before even a diagnosis can be made. If operative intervention follows upon a correct diagnosis, the prognosis is very

\footnotetext{
${ }^{1}$ Canon, Lenhartz and E. Libman (loc. cit.), Die Bakteriologie des Blutes bei Infektion Krankheiten, 1902.
} 
good. Certain cases go on to rapid abscess formation, whereupon there is a discharge of pus into the pelvis of the kidney, or into the perinephric space. The former condition is unusual, as the infarcts are very close upon the true capsule of the kidney, or almost entirely within its cortex. Due to the latter, the perinephric involvement more often follows, giving rise to the more subacute type, and consequently a better prognosis. The prognosis under such conditions is that of subphrenic (perinephric) abscess, with its sequelæ, with or without operative intervention ( $q . v$.).

Treatment.-This depends chiefly upon the diagnosis. As the condition is at best obscure, the surgeon should, when in doubt, do an exploratory incision in the loin, exposing the suspected kidney. Should multiple small abscesses be encountered, two courses are open: drainage or nephrectomy. ${ }^{1}$ The advantages of removal of the kidney are great, because, if the diagnosis is correct, the recovery of the patient is practically assured, provided the operation is well stood. Nevertheless the preservation of the organ is worth considering, and the chance that the other kidney is involved should not be lost sight of. Again, incision of the kidney allows the operator to determine more about the exact nature of the infarcted areas. If pus be found, no doubt remains; but if not, spreads should be made, and the presence or absence of polynuclears and bacteria should be demonstrated then and there. The arguments in favor of the removal of tuberculous kidney with multiple foci does not hold here, because of the nature of the infection, which may result favorably, provided drainage is established.

Finally, it should be remembered that there are many cases of general bacteremia which develop metastatic lesions. Should these locate in the kidney, drainage should be instituted, as in any other abscess, subcutaneous or elsewhere. This condition, however, gives a much graver prognosis, and being associated as a rule with foci in other organs, always contraindicates nephrectomy.

\footnotetext{
1 Brewer (loc. cit.) prefers nephrectomy. In one case he reported, however, the patient recovered, following nephrotomy with multiple incisions of the kidney and drainage of the pelvis.
} 


\section{CHAPTER XXXV \\ PYONEPHROSIS AND PERINEPHRITIS}

Pyonephrosis is an end result of bacterial invasion of the kidney. This process may start as a hydronephrosis and, in consequence of a superadded infection, develop uropyonephrosis (infected hydronephrosis). It may begin also as a focal suppuration, single or multiple, and end in the formation of a general abscess cavity in which there is retention of pus and purulent urine with little normal kidney structure remaining. When the process begins in the kidney substance, there is marked dissolution of its parenchyma but only moderate dilatation of the pelvis.

Causation.-The method of bacterial invasion has already been discussed (see p. 599). Generally speaking, some cases of infection have occurred primarily in the kidney and are descending in type (e.g., infected hydronephrosis) while in others, the infection is ascending in character (chronic suppurative pyelonephritis-surgical kidney).

The predisposing factors of pyonephrosis are:

(1) Hydronephrosis.

(2) Previous morbid lesions of the pelvis-pyelitis; pelvic stone.

(3) Previous morbid lesions of the kidney - focal abscess, single or multiple; tuberculosis.

These various maladies are considered under their respective headings. Pyonephrosis, therefore, is a culmination of a number of morbid conditions of different types. Some of these conditions may have given symptoms for some time. In other cases, the first symptom may be that which brings to light the existence of pyonephrosis. ${ }^{1}$

The infecting agents in pyonephrosis are (exclusive of the tubercle bacillus) the ordinary pyogenic organisms. Most cases follow pyelitis. The usual cause is the bacillus coli. ${ }^{2}$

The streptococci and staphylococci are also found and suspicion

\footnotetext{
1 In the Vienna General Hospital, in more than two-thirds of the 750 cases of pyelitis upon which autopsies were performed, a diagnosis of pyelitis had not been made. This does not include the cases which left the hospital undiagnosticated. Kapsammer, Wie diagnost. der Chirurgichen. Nieren, etc., etc. Monats berichte für Úrologie, 1906.

2 Lenhartz found this organism in pure culture in 50 of 60 cases studied. "Artze"-Stuttgart, 1906.
} 
should be directed toward a hematogenous infection when these organisms are present (see p. 615).

Gonococcus infection is occasionally present, and while this organism has been considered a rather infrequent invader of the kidney pelvis, many reports of authentic cases would tend to show a somewhat greater number than has been suspected. ${ }^{1}$

The typhoid and pyocyaneus bacilli have also been found. Mixed cultures are not uncommon.

Morbid Changes.-As already stated, pyonephrosis is the terminal stage of renal suppuration, the pus having become encapsulated in a thick-walled cavity, composed as a rule of a number of chambers which may or may not communicate. The distention of the pelvis varies in degree from a moderate enlargement to that of an enormous sac (Plate VIII, Fig. 3). The former occurs when suppuration begins in the kidney substance, the latter when the outcome is that of an infected hydronephrosis. The mucous membrane is usually completely replaced by granulation tissue. The exterior often presents a lobulated appearance and in some cases it is closely adherent to the perinephric fascia which has become hypertrophied on account of prolonged inflammation. This fatty capsule may be fibrous and firmly adherent to the sac, when it extends upward into the sinus of the kidney and is found in the kidney tissue itself. On section, the organ may completely collapse, showing a large thin-walled cavity. Not infrequently interstitial partitions exist between the abscess compartments which are reinforced by chronic inflammatory thickening. These partitions may contain a certain amount of renal parenchyma, sometimes of fairly normal appearance, especially in the less advanced stages when the infection has been hematogenous. The pus is usually thick and creamy and may be very foul, especially when the infection is due to the bacillus proteus or certain strains of the bacillus coli. The association of renal calculus is very common. Pelvic stones are primary, having been present before infection occurred. They may fill the entire pelvis by prolongation up into the calices. In addition to this, there are secondary stones deposited in the kidney which may be numerous and the presence of these calculi may represent so many abscesses of the malpighian pyramids. Tuberculous pyonephrosis may present the same picture. The walls of the abscesses, however, are lined with numerous tubercles. (See Renal Tuberculosis, p. 654.)

The ureter in pyonephrosis may present a fairly normal appearance except in those cases which have followed a urogenous infection when

\footnotetext{
1 Francis R. Hagner, Transactions of the Amer. Ass. Genito-urinary Surgeons, 1910, collects the reports of nine cases and reports one personal case.
} 


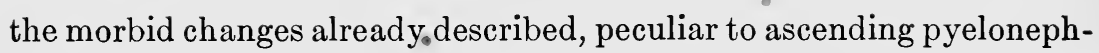
ritis, are evident.

Perinephric abscess may be associated with pyonephrosis and it is usually due to direct extension of the infection through the capsule.

Pyonephroses are referred to as "open" or "closed" according to the patency or closure of the lumen of the uretero-pelvic outlet. The opposite kidney may be normal or the pyonephrotic lesion may be bilateral, in which case there is a double urogenous ascending infection, or the lesion of the opposite kidney may be due to transmission of toxic agents by a hematogenous route from the primarily diseased organ.

Clinical Course. Previous History.-If the patient's previous condition has been carefully reviewed, there is usually a precedent urinary history, which may be a prolonged cystitis, of gonorrheal origin, or possibly not, which has varied in intensity from time to time. Some cases in women have had a protracted cystitis following labor. Any case of pyelitis or cystitis with vague abdominal or lumbar pain should be under suspicion.

Pain. - The pain is usually dull in character and located in either one or the other hypochondrium or loin. There may be only a trace of tenderness on deep pressure. A mass may be felt by bi-manual pressure, rounded and sometimes irregular, or the condition may exist without any palpable kidney.

The urine on gross examination shows nothing more than is found in cystitis-namely, a general turbidity due to the presence of pus and bacteria. As a clinical observation, it is well to note that the presence of a profuse pyuria such as is seen equally distributed when the urine is passed in two or three flows, without marked vesical symptoms (suprapubic pain, tenesmus, frequency of urination, etc.) is suspicious of pyonephrosis or pyelonephritis.

The general constitutional symptoms are slight; the temperature may not be elevated, or at times may rise one or two degrees above the normal; the blood count may show slight or marked leucocytosis.

Such is a general outline of the clinical features in an open or draining pyonephrosis. In cases of closed pyonephrosis, the urinary symptoms are lacking and some of these may exist with only a tumor as their chief symptom; constitutional symptoms in such cases occur in proportion to the acuteness of the morbid inflammatory process; when the condition has become chronic they subside. A veritable connective tissue sac containing no kidney tissue, with nothing more than pus and perhaps some calculi, may give neither urinary evidence nor constitutional symptoms of importance. In the case of a sudden closure, however, ensuing upon an open pyonephrosis such an event 
may be signalized by the occurrence of sharp, constitutional symptoms - chills, fever, nausea and at times, uremic manifestation. At the same time, the urinary evidences may disappear and the bladder urine represent entirely that which is derived from the unaffected kidney.

Diagnosis.-Attention is directed toward the urinary tract, by the history of the case or by examination of the bladder urine. When there is no palpable tumor in the loin, the diagnosis must rest largely upon cystoscopy and ureteral catheterization. Where there is a tumor present the diagnosis is easier. Such an enlargement must be differentiated on the right side from a growth of the liver or distended gall-bladder, a neoplasm of the kidney, retroperitoneal growths and tumor of the colon; and with the same conditions on the left side, except for enlargement of the spleen in place of that of the liver. Perinephric or subphrenic abscess, whether of renal or other origin, must be borne in mind.

Cystoscopy is the main aid to diagnosis in all cases. The bladder, even after a long existing pyuria, may show little or no change. The ureteric orifices may be both slightly inflamed, but usually the ureter of the affected side shows gaping and edema and a plug of pus is not uncommonly found exuding its way out of the opening. Such a picture is characteristic, but it is possible that the ureteroscopic picture may appear far from abnormal even in advanced cases. ${ }^{1}$ Catheterization of the ureters, as a rule, generally obtains from the infected side, turbid, whey-like urine or pure purulent fluid, usually of low specific gravity. The colorimetric functional urinary tests (see p. 155) will reveal delayed excretion away below the normal amount, as compared with the opposite side.

Renal Roentgenography for calculus is of importance; pyelo-Roentgenography may be of service at times. In difficult cases Roentgenography of the colon after the administration of bismuth may serve to differentiate tumors of the stomach and colon.

The prognosis depends upon the condition of the opposite kidney; therefore its capacity should be carefully measured in the course of the cystoscopic investigation. Where the opposite kidney is also involved, the prognosis is poor on account of the danger of renal insufficiency or total anuria before or after an operative procedure. The purely unilateral cases have a good prognosis following appropriate operative intervention. Perinephric abscess, which is not an infre-

1 In a personal case in which both kidneys were involved, on one side the kidney was contracted to the size of a small oyster as the result of a morbid lesion or congenital defect; on this side the ureteral mouth was dilated. The opposite kidney, in which existed the active morbid process presented a huge, pyonephrotic sac and while the ureter was dilated to the size of the small intestine, the urateral mouth on the bladder side was contracted and perfectly normal in appearance. 
quent complication, does not necessarily add to the gravity of the situation. The coexistence of tuberculosis naturally renders the ultimate outcome much less hopeful (see Renal Tuberculosis, p. 654) and secondary infection of pyelonephritis or calculus pyelonephritis with the bacillus tuberculosis is not an uncommon occurrence.

Treatment.-After the existence of pyonephrosis has been established by careful diagnostic means, recourse to surgical measures is unavoidable and even in doubtful cases, an exploratory nephrotomy should be performed, when the pathological condition present will suggest the further operative procedure demanded. The choice of operation to be considered in most cases, lies between nephrectomy and nephrotomy.

Nephrotomy should always be performed when there is doubt as to the functional value of the other kidney, and this may occur when there is great urgency on account of septic or uremic symptoms which do not allow time for careful study of the case. In such instances, a secondary nephrectomy may be performed later on, when the capacity of the other kidney has been established and when improvement in the general condition follows preliminary drainage of the diseased organ.

Nephrotomy is indicated where there is bilateral renal disease and double nephrotomy may be demanded, chiefly in cases in which there exists bilateral renal calculus. Nephrotomy is an undesirable operation in cases of advanced pyonephrosis on account of the possibility of renal fistulæ which are likely to occur and with the remaining diseased tissue, to act as a drain upon the vitality of the patient; and because secondary nephrectomy is a formidable operation.

Nephrectomy.-Primary nephrectomy is always the operation of choice for pyonephrotic kidney if the functional test and laboratory examination show the other kidney to be in good condition. The advantages of this procedure are rapid recovery following the operation of total removal of the diseased organ and elimination of the chance of renal fistula, the occurrence of which after nephrotomy may necessitate the performance of a nephrectomy secondarily. In cases with tuberculous infection, there is no choice; nephrectomy is the operation of necessity. (See Operative Surgery.)

\section{PERINEPHRITIS}

Perinephritis is an acute or chronic inflammatory process in the region of the kidney.

Such a definition gives a wide scope, inasmuch as it embraces any 


$$
\therefore=16=7 y
$$

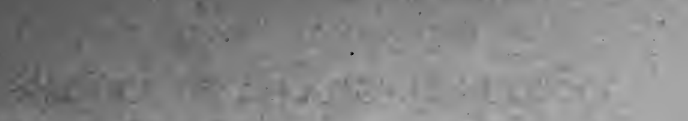

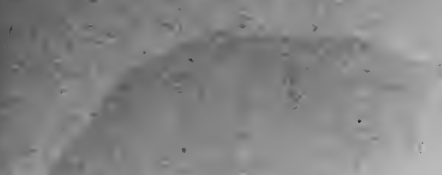

8) $i^{2}=$

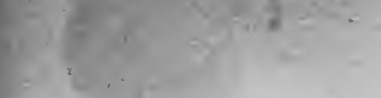

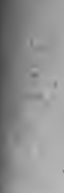

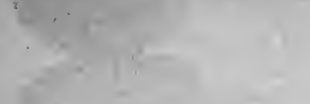

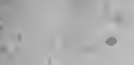

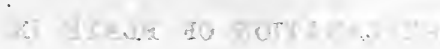

\section{1}

Q. 16,1

$+\cdots, \cdots, \cdots, \cdots$

$+1$. 


\section{EXPLANATION OF PLATE IX.}

Fig. 1. Pyonephrotic Stone Kidney. (Adult.)

Fia. 2. Pyonephrotic Stone Kidney. (Child.)

FIG. 3. Same as Fig. 2. Outer Surface.

Fig. 4. Tuberculous Pyelonephritis.

Fig. 5. Same as Fig. 4. Rupture of abscess posteriorly.

FIg. 6. Tuberculous Kidney.-Cavernous variety. 

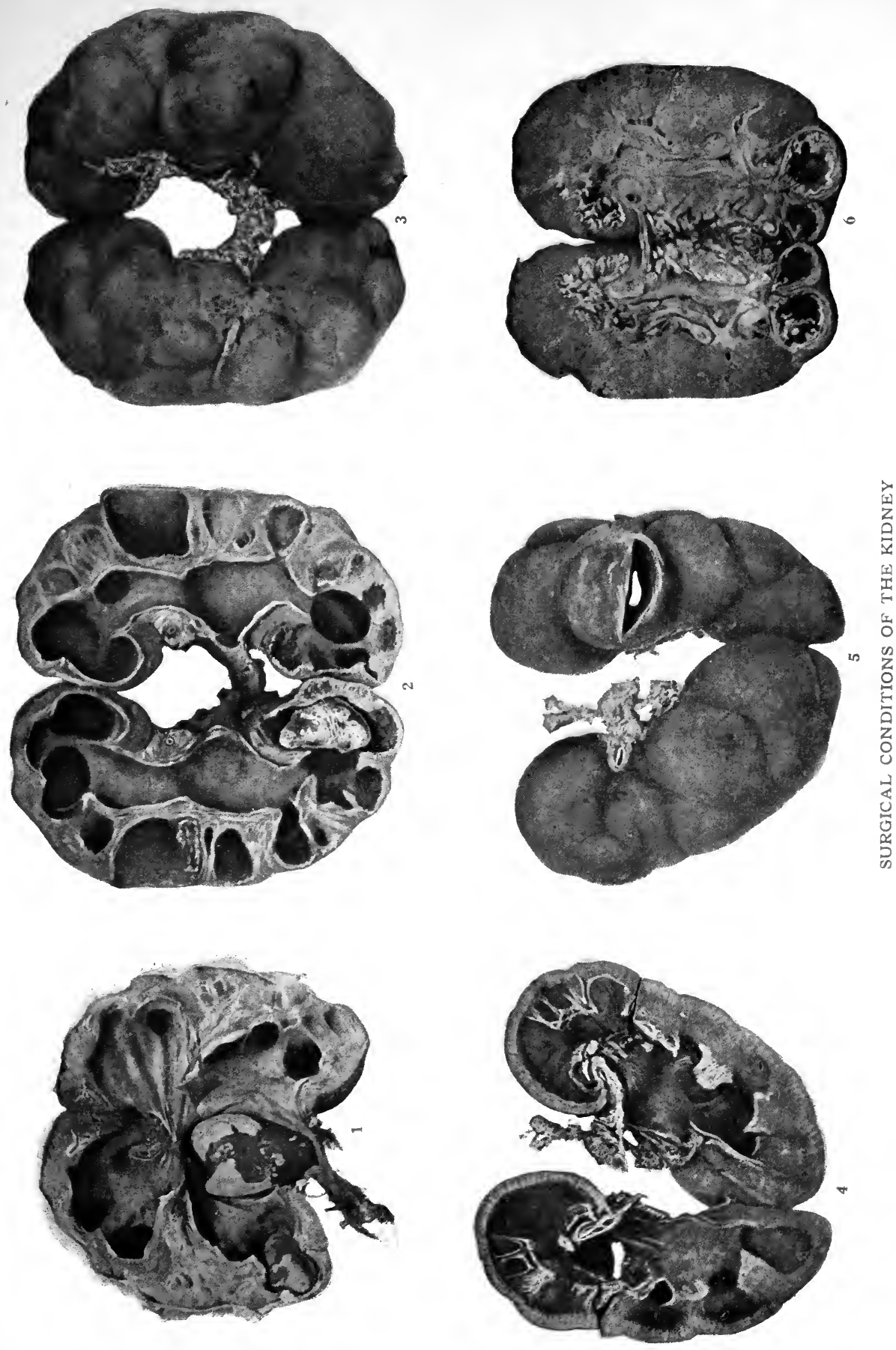

type of inflammation in this location, of any causation. From a surgical standpoint, the importance lies in the fact that the inflammations are retroperitoneal. The etiology is often unknown before, and even after operation, and the question of the point of differentiation between perinephric and subphrenic inflammation may be an academic one. The importance of early diagnosis and indication for operation are the same.

Causation.-Inflammation in the perinephric space may proceed from any sources (see Table ${ }^{1}$ ).

Most of the important causes may be summed up as follows:

a. Of renal origin, ${ }^{2}$ such as inflammations and foreign bodies, which sooner or later become predisposing causes to infection.

$b$. Of gastro-intestinal origin, ${ }^{3}$ inflammation of, operations upon and perforations of the hollow viscera of the gastro-intestinal tract and its appendages.

1 The following table gives an analysis of 230 cases of inflammation in the perirenal or subphrenio region collected by Küster, from an exhaustive study of the literature, Chirurgische Krankheiten der Nieren, Deutsche Chirurgie, 1897-1902 (57 B).

GroUP I, 1-4.

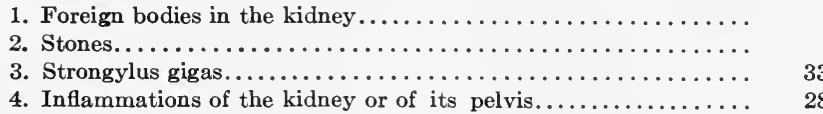

GrodP II, 5-12.

5. Appendicitis............................. 3

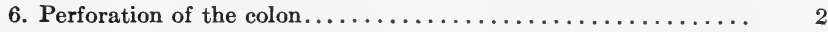

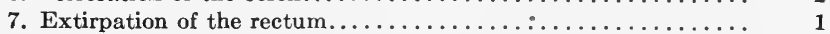

8. Gastric and intestinal inflammation................... 2

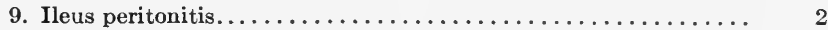

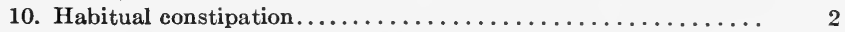

11. Inflammation of the liver and gall-bladder............. 3

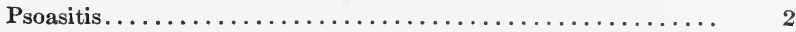

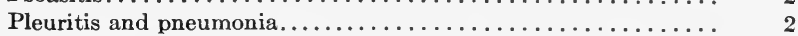

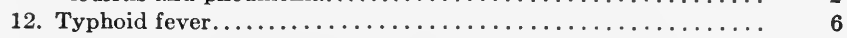

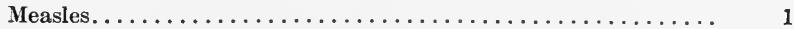

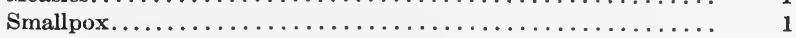

Suppuration in distant points of the body............. 3

GrouP III, 13-15.

13. Contusions and traumatism of the loin $\ldots \ldots \ldots \ldots \ldots \ldots \ldots \ldots \ldots$

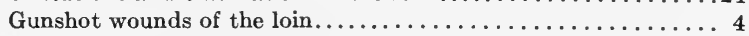

14. Muscular violence............................. 7

15. Strain........................ 42

GrodP IV, 16-17.

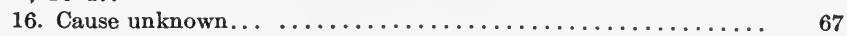

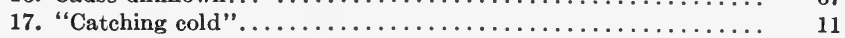

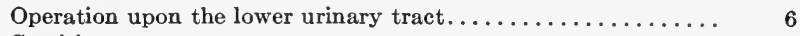

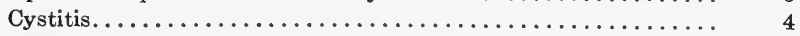

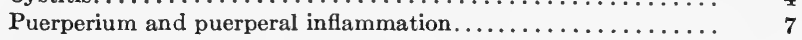

Injuries to and operations upon the male genital organs........ 2

${ }^{2}$ Group I, 1-4.

${ }^{3}$ Group II, 5-12. 
c. Originating after traumatism to the loin, subcutaneous injuries to the kidney. ${ }^{1}$

$d$. Of unknown origin, including catching cold. ${ }^{2}$

Some authors lay stress upon the large proportion due to carious disease of the vertebræ. ${ }^{3}$ This cause may be overlooked in cases where the diagnosis is not made until the mass has gravitated past the loin.

Most of the cases in which the cause thereof is of traumatic origin, or in which the cause is given as unknown, are in all probability of metastatic origin possibly from some far distant focus, either in an active condition, or completely healed. The metastatic deposit may occur during an acute infection (influenza, variola, measles, pleuritis, pneumonia) and the symptoms occur at the time of the infection, or the infecting agents may lie dormant, coming to light weeks or months thereafter, for no apparent reason, ${ }^{4}$ or after certain traumatism to the loin, resulting possibly in either rupture of the kidney or some lowering of its resistance. ${ }^{5}$ (See Chapter XXXIV, Unilateral Septic Infection of the Kidney.)

The bacteriology depends on the group in which the inflammation is placed.

In Group I, of renal origin, the most frequent organism is the colon bacillus; streptococcus, staphylococcus, gonococcus, pyocyaneus bacillus, and mixed cultures are also found. The tubercle bacillus is a very important factor in this group, and pyogenic invasions imposed upon a tuberculous infection often bring about the perirenal inflammatory process.

In Group II, of gastrointestinal origin, any of the bacteria of this tract may be present, especially the colon bacilli, aerobic and anaerobic bacilli and cocci.

In Groups III and IV, the most frequent organism is the staphylococcus pyogenes aureus (rarely albus). Next in frequency are streptococci and bacillus coli.

${ }^{1}$ Group III, 13-15.

2 Group IV, 16-17.

${ }^{3}$ See table, Psoasitis.

"See table "of unknown origin."

5 Such a view would imply that the kidney is involved of tener than is believed to be the case, and such is the opinion of Albrecht, who has reported cases.

W. Albrecht, Beitr. Zur klin. Chir., Vol. L, p. 147 (1906). This author is a strong believer in the view of latent retention of organisms in the kidney advanced by Schnitzler (Beitr. Zur Kenntniss der latentin microörganismen Verhandlungen d. deutschen Gesellschaft für Chir., 1899), who injected three rabbits intravenously with a sublethal dose of staphylococcus aureus, and after three, four and five weeks had elapsed, he bruised the right loin of the first, second and third rabbit, respectively. Staphylococcus aureus abscesses of the right kidney and perinephric space of each of the rabbits occurred regularly, and the left kidneys of two of the rabbits showed signs of healing infections of long duration. This view is in accord with the clinical facts which the author has observed. 
Further than this, the bacteriology cannot be grouped. Occasional cases, occurring after gun-shot wounds, etc., may be infected with any organism present on the integument, or derived from any viscus. Cases which have dissected their way upward by direct.or lymphatic extension from inflammation in the pelvis, will, of course, have the bacteriology determined thereby. Occasional cases which have proceeded from supradiaphragmatic lesions after empyema (usually tuberculous) or after thoracic spondylitis, may give collections of sterile pus, in which the tubercle bacillus is demonstrable with difficulty by ordinary methods.

Morbid Changes.-Perinephritis may be divided into: fibrolipomatous, sclerotic and suppurative cases. The first two varieties depend upon subacute and chronic inflammations in which the lipomatous tissue between the true capsule of the kidney and the outer fibrous capsule is hypertrophied. On cross-section it may show a more or less edematous condition; in the later stages, the fat becomes quite infiltrated with new-formed connective tissue, and finally the entire mass may be changed into one of dense cicatricial tissue which surrounds the kidney, compressing it to a greater or less extent. Such conditions result from the resolution of subacute types of pyogenic infection, usually of the kidney itself, or from tuberculous disease of the bones. They are of importance from a surgical aspect in connection with operation for pyonephrosis.

Suppurative perinephritis may result in minute abscess formation, or in abscesses forming huge abdominal tumors, containing more than a liter of pus. They are surrounded by a zone of inflammatory tissue which may be inches thick in some places, and in others so thin that perforation may occur spontaneously.

Perinephric inflammation may be suprarenal (or subphrenic), infrarenal, prerenal or retrorenal (most common) and may follow certain well recognized routes, either by dissecting its way along planes of fascia or by perforating into some vital organ or space.

The retrorenal abscess being the most common, posterior perforation is the rule. The abscess here is formed in the space between the kidney and the fascia that goes to form the lumbar fascia; gravitating slightly, it points in the direction of the lower and outer portion of the origin of the latissimus dorsi. This is the most usual site of the perforation of abscesses of renal and visceral origin, and these are usually due to acute pyogenic inflammations. Some of these abscesses may also travel upward, under the ligamentum arcuatum internum and enter the thoracic region. The type arising behind the fascia covering the prevertebral muscles may gravitate to the iliac fossæ or into the 
groin and thigh. This is common in those arising from inflammations of bone, and due mostly to tuberculous disease (cold abscesses).

Abscesses not located behind the kidney are likely to perforate into hollow viscera of the abdomen or into the peritoneal cavity itself. ${ }^{1}$

The morbid changes following such spontaneous evacuation may be colonic, pulmonic or external fistulæ, which may result in mixed infections, with progressive phlegmonous processes or in persistent fistulæ from inadequate drainage. Malignant disease may lie at the bottom of such fistulæ, or may result from their prolonged presence; also tuberculous disease of bones or viscera, or sequestra may be present.

Clinical Course.-The diversity of the types of causation and of their resultant complications is very great. ${ }^{2}$

The patients are as a rule ambulant. They complain of abdominal or loin pain, which has occurred rather suddenly. At its onset it is rather severe and knife-like, and later it may have abated somewhat. At times there is pain in the costovertebral region, but rarely has it typical renal radiation. The patient may be observed walking with slight stoop forward, and bent toward the affected side, somewhat suggestive of hip disease. Thus the abnormal side is favored in standing, and the hand may rest on the ilium of the diseased side. The gait is usually very careful.

In the recumbent posture there is a tendency to hold the thigh of the affected side slightly flexed and unilateral costo-vertebral tenderness upon pressure. Some fullness may be visible, diminishing the normal subcostal curve of one loin. There may be tenderness to deep pressure in the hypochondrium of this side. There is likely to be temperature elevation $39^{\circ}$ to $39.5^{\circ} \mathrm{C}$. $\left(102^{\circ}\right.$ to $103^{\circ} \mathrm{F}$.), and pulse and respiration may be increased in proportion. There is either a febrile flush upon the facies, or the skin has a dull, colorless appearance.

As most of these cases are due to cortical involvement of the kidney or extrarenal processes, the urine in such cases shows no changes, or only a trace of albumen (see p. 625). The cases which follow advanced conditions of renal and pelvic involvement may show marked urinary changes, such as pus, albumen, bacteria, etc.

1 Of thirty-four cases of rupture reported by Küster (loc. cit.) perforation occurred as follows:

Pleura and bronchi............................... 18

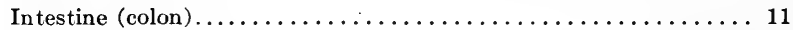

Peritoneal cavity.............................. 2

Bladder or bladder and vagina..................... 3

${ }^{2}$ Küster (loc.. cit.). Herzshy, Centralblat für die Grenzgebiete der Med. und Chir., 1903. Vol. VI, gives a full bibliography and exhaustive study of the important features of renal abscess and perinephritis. 
The blood examination generally gives an increased leucocytosis and polynucleosis.

Variations from the mild or subacute type may occur; the fever may be exaggerated, rising to $40^{\circ}$ to $40.5^{\circ} \mathrm{C}$. $\left(104^{\circ}\right.$ to $105^{\circ} \mathrm{F}$.), together with exaggeration of the constitutional symptoms. The fever is usually continuous; chills are rare. The tenderness in the loin also may be exaggerated, and as the abscess nears the surface there may be severe muscular spasm, and finally edema of the skin. Palpation may reveal a mass in the loin, should the abscess be located on the anterior surface of the kidney; such evidence may be misleading and suggest a neoplasm of the kidney.

There is actually no typical course. Cases may linger on for weeks or months, until rupture occurs into some important organ or elsewhere. The result may be spontaneous recovery, if the organ is the colon, but the development of empyema, pulmonary abscess or fistulæ is usually of very grave import. The expectoration of pus in such cases is an ominous sign. Perforation into other organs, like the bladder, vagina, pelvis of kidney, ureter, rectum, urethra, or sciatic foramen, femoral canal, may result in continuous fistulæ, but sometimes permanent cure follows. Amyloid degeneration and severe anemia are dangers to be expected from prolonged suppuration.

Fistula in the loin is avoided by early diagnosis and operation, and fatal outcome is rarely due to the suppuration itself, but usually ensues upon some complication, which is avoided when cases receive proper surgical treatment.

Diagnosis.-While in many cases diagnosis is easy, in others it is impossible without exploratory operation or until the advent of one of the complications brings to light the actual condition. Again, there are certain cases that give a typical etiology, history and course, which seem to undergo spontaneous resolution.

The etiology, history of the onset of pain in one loin or hypochondrium, to which attention is particularly called, the fever, the loin tenderness or mass, the blood findings, all warrant the suspicion of perinephritis. Exploratory aspiration should always be done with a large needle in the perinephric space. Pus and serum should be submitted to bacteriological examination. Cystoscopy should be resorted to and a careful study made of the urines, combined and separate, both chemically and bacteriologically. If perinephric abscess is suspected, and a unilateral disease of the kidney is discovered by cystoscopic methods, exploration of the loin is justifiable, as in such cases the kidney is generally in an advanced condition of morbid change. (See Chapter on Pyonephrosis.) 
The Roentgen ray should enot be neglected, as its use will throw light upon orthopedic conditions, as well as calculous disease. Roentgenographic pictures of the vertebræ from the mid-dorsal to the lumbosacral articulations should be included.

Osteoarthritis of the hip is one of the difficult varieties for differentiation. In perinephritis the motions of the hip are free in all directions, except that occasionally the patient feels some pain on extension, whereas in hip disease, the favored position of external rotation, abduction and flexion is noted, and attempt to change this produces muscular spasm.

Acute osteomyelitis of the laminæ or of the transverse processes of the vertebral column may be very puzzling, as it may be due to the same etiology (abrasion, furuncle, etc.) as perinephric abscess. It is, however, usually a very fulminating septic type and is more likely to suggest unilateral septic infarct of the kidney. It is of great advantage if the process can be localized as retroperitoneal, as in such a case lumbar incision instead of laparotomy is employed. In suppurating or breaking-down renal or intestinal tumors, the diagnosis may be made only after operation.

Treatment.-In urgent septic conditions, the patient should be given the benefit of an exploratory lumbar incision, even though no pus can be aspirated. Even in non-acute conditions delay cannot be instituted with impunity, on account of the danger of complications in untreated cases.

When possible, it is worth while to have bacteriological examination of aspirated pus made. In cases where there is a doubt as to the orthopedic character, it is advisable to postpone operation until a clear diagnosis can be made by cystoscopy. When pus containing septic microörganisms is obtained, there is no occasion to delay.

Lumbar incision (see Chapter on Operative Surgery) is always preferable. In a few rare cases laparotomy may be indicated, on account of the location of the mass, but even in such instances the incision may be carried forward as far as may be necessary with or without opening the peritoneum.

On the operating table the relation of the abscess to the kidney is learned, and also morbid conditions in the kidney itself, pyonephrotic, tuberculous, malignant, etc., are detected which may suggest an indication for nephrectomy. ${ }^{1}$

The type of the pus encountered may be suggestive of the etiology.

\footnotetext{
I In a personal case of perinephritic abscess operated upon, pus having been aspirated from the loin and also found in the urine, the perinephritic inflammation was discovered to be associated with a malignant neoplasm of the kidney which was removed
} 
Streptococcus or staphylococcus pus is odorless, whereas the pus of bacillus coli has some odor, and the foul odor of the anaerobes and mixed infections is characteristic. In such cases, malignant origin is suspected.

The outcome of the surgically treated cases is usually good. A few result in fistulæ, which usually heal spontaneously or may require extensive secondary operation (nephrectomy, etc.). Orthopedio cases due to tuberculous bone or glandular disease, ${ }^{1}$ which have been incised, usually result in protracted fistulæ and should be submitted to very little or no further surgical interference. More reliance can be placed in general hygienic treatment and proper climatic surroundings, but the prognosis is not good in such cases.

1 See Painter, Report of 6th International Congress on Tuberculosis, Vol. II, p. 61, Washington, D. C., 1908. An excellent discussion and report of some of the cases on the borderline between surgery and orthopedics. 


\section{CHAPTER XXXVI}

\section{OPERATIVE SURGERY OF THE KIDNEY AND URETER}

\section{NEPHROTOMY}

Exposure of the kidney is the first step of all operations upon this organ and this may be effected anteriorly through the peritoneum (transperitoneal) and posteriorly through the lumbar region (retroperitoneal). The retroperitoneal is the more direct route and more frequently adopted. The space between the costal arch above and the vertebral pelvic angle seems to offer a more restricted area than through the peritoneum but, by extending the incision as far as may be necessary toward the median line (subperitoneal), this route affords ample room and has the additional advantage of lessening the risk of peritoneal contamination in the case of suppurative lesions.

The transperitoneal route, therefore, is reserved for the removal of extra large neoplasms and when the diagnosis is doubtful, which instances are exceptional.

Lumbar Nephrotomy.-Exposure of the kidney is required without opening the parenchyma of the organ for the purpose of diagnosis, for nephropexy and for plastic operations upon the pelvis.

The first requirement is to posture the patient properly so as to obtain as wide separation as possible in the space between the costal angle and the iliac crest. The patient is turned on the sound side, resting upon an oblong sandbag or inflated air cushion, with the thigh and knee of the same side flexed, while that of the side to be operated upon is extended.

A variety of incisions are in use (Fig. 251). The vertical incision begins at the eleventh rib and follows downward the margin of the sacrospinal muscles 10 to $13 \mathrm{~cm}$. (4-5 ins.) (a.a.). The oblique incision begins at the centre of the twelfth rib and runs obliquely forward to a point midway between the umbilicus and anterior-superior iliac spine (c.c.). A transverse incision, beginning at the sacro-spinalis muscle runs parallel with the eleventh rib (b.b.). Finally any of these incisions may be combined to gain greater space either at the beginning of an operation or during the course thereof. In other words, the operation may be commenced with a vertical or angular incision and the field enlarged by a transverse cut joining the other incision at 
any point desired. This combination of vertical and transverse incisions is contained in the rectangular incision of Koenig which begins as a vertical incision for 8 to $10 \mathrm{~cm}$. (3-4 ins.) then curves horizontally forward, parallel with the fibres of the abdominal muscles as far as may be necessary, at times almost reaching the umbilicus (d.d.). It is the incision that allows the greatest exposure of both the kidney and the ureter. I commonly employ an oblique or a crescentic incision for exploration of the kidney (Plate I), for pyelotomy, and nephropexy and find that it is the most satisfactory in the largest perecntage of cases (Fig. 252). Such incision divides the skin, subcutaneous tissues and superficial fascia, avoiding the last intercostal, iliohypogastric and ilioinguinal nerves. After proceeding thus far, the bleeding vessels are caught and ligated; the muscular layer is then reached; splitting of the

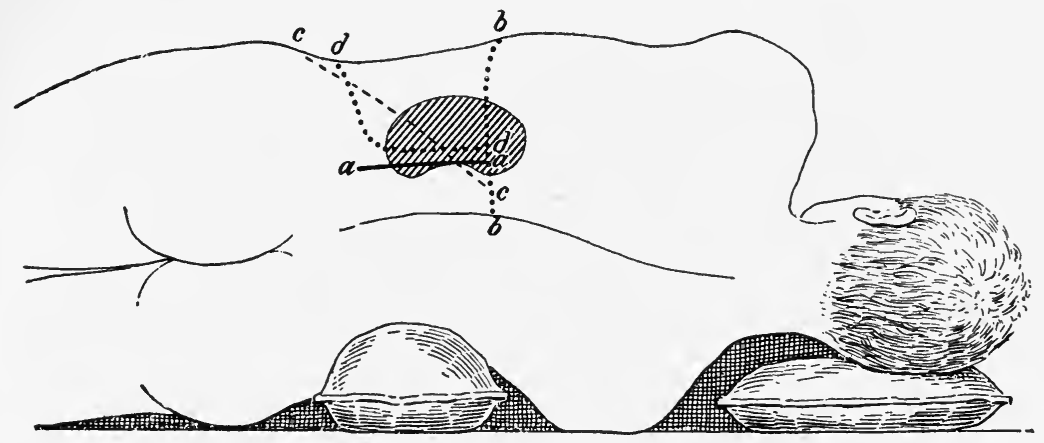

FIG. 251.-Outline of different incisions employed in operative surgery upon the kidney.

muscles gives very little room and is used only when simple exploration is required. Therefore the muscular layer is usually incised. The lower fibres of the latissimus dorsi are seen running upward and outward as well as the posterior fibres of the abdominal oblique muscles in the lower part of an angular or vertical incision. These fibres are divided in the direction of the incision as are those of the serratus posticus inferior which is seen in the upper portion of the incision beneath the latissimus dorsi. The rounded edge of the erectus spinæ muscle running parallel to the spine is now brought into view which muscle is spared, and beyond this are the oblique fibres of the quadratus lumborum. Beneath the latter muscle lies the transversalis fascia and under it the retroperitoneal fat; after pressing these aside the perirenal fascia is revealed. This is incised or torn open with the index-finger and the true capsule of the kidney exposed. The next 
step entails the liberation of the kidney through the opening of its fatty capsule (Plate I). In some cases this is readily accomplished and in others it is difficult, depending upon the preëxistent lesion. When the operation is performed for a movable kidney, this step is accomplished with little trouble as is also the case when the lesion sought is within the kidney substance and has not extended to the cortex, causing perirenal extension and adhesion. Further action depends upon the indication and findings at the time of operation.

Nephrotomy Involving Incision of the Kidney.-This operation is performed for renal calculus (nephrolithotomy) for exploratory pur-

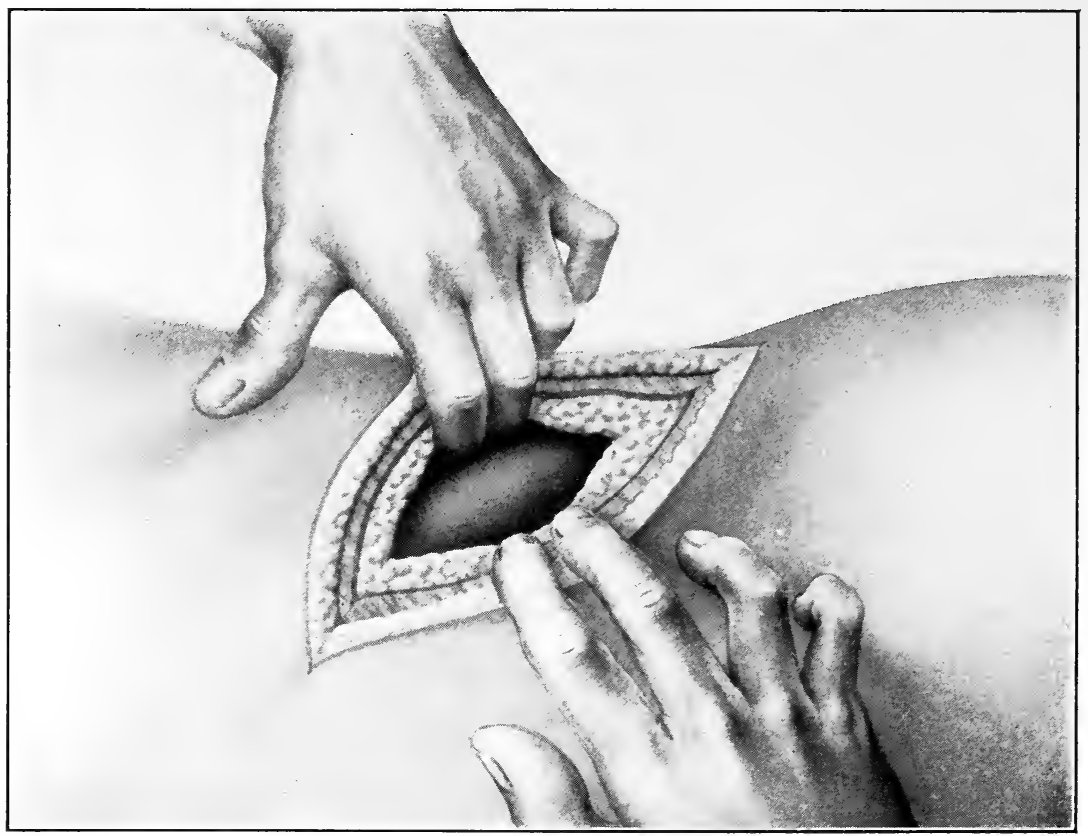

FIG. 252.--Exposure of kidney through loin.

poses, for drainage of the kidney (nephrostomy) and to reach the pelvis either for exploration or for the removal of calculus through this route.

After the kidney has been liberated from its surroundings and is delivered into the wound, one of two courses may be pursued: an assistant slips the hand beneath the organ so as to grasp the blood supply in the pedicle of the organ, or better, a specially devised clamp with rubber tubing over the ends is applied temporarily to the pedicle (Fig. 254), one end being passed between the ureter below and the renal vessels above and the other end above the blood-vessels. The kidney 
is now split along its cortical border through the capsule only from pole to pole in case of decapsulation or for about $5 \mathrm{~cm}$. (2 in.) when exploration of the interior is desired. This incision should run about $6 \mathrm{~mm}$. ( $\mathrm{r} / 4$ in.) behind the convex border of the organ, and should be deepened into the pelvis and enlarged in either direction according to requirement. Through such incision, a finger is slipped into the pelvis and stone, if present, is detected and removed with the forceps (Fig. 254). If difficulty is experienced in obtaining small stones in the pelvis, assistance may be obtained by inflating the latter with a mild

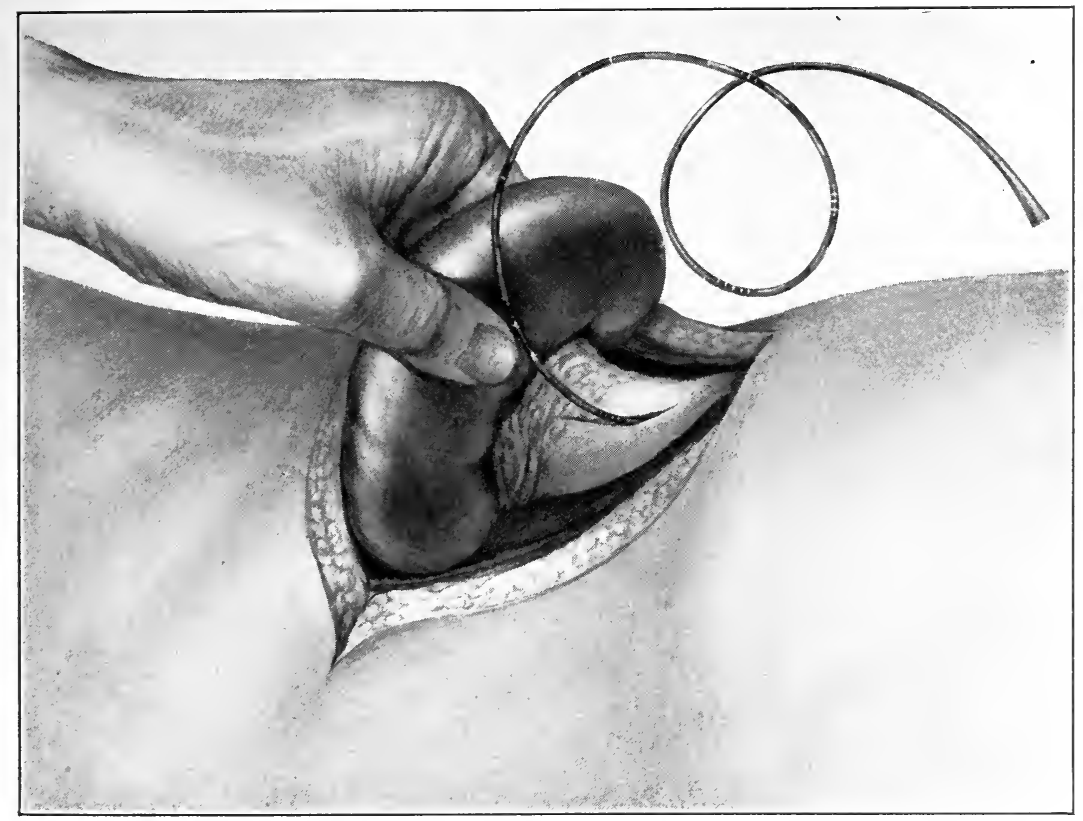

FIG. 253.-Examination of kidney through loin. Pyelotomy and passage of ureteral catheter downwards.

solution after the removal of which the patency of the ureter is determined by passing a ureteral catheter from above downward. Some operations of complete nephrotomy have been accompanied by severe hemorrhage, and to meet this difficulty the method has been devised (Cullen) of incising the kidney from within outward from pole to pole by means of a heavy steel wire. This is carried with a full curve needle through the medulla near the pelvis and with a gentle sawing motion, the organ is laid open.

When the substance of the kidney is not involved and the purpose 
of the operation may be attained thereby, pyelotomy should be performed avoiding the more serious procedure of opening the kidney parenchyma; through a posterior incision of the pelvis, a stone may be removed and the ureter catheterized from above downward (Fig. 253) (also Plate I).

The risk of urinary fistula following incision of the pelvis is less than is usually supposed and complete suture of the same is not demanded, as, with proper drainage from the ureter downward and from the wound outward, the opening heals with few exceptions,

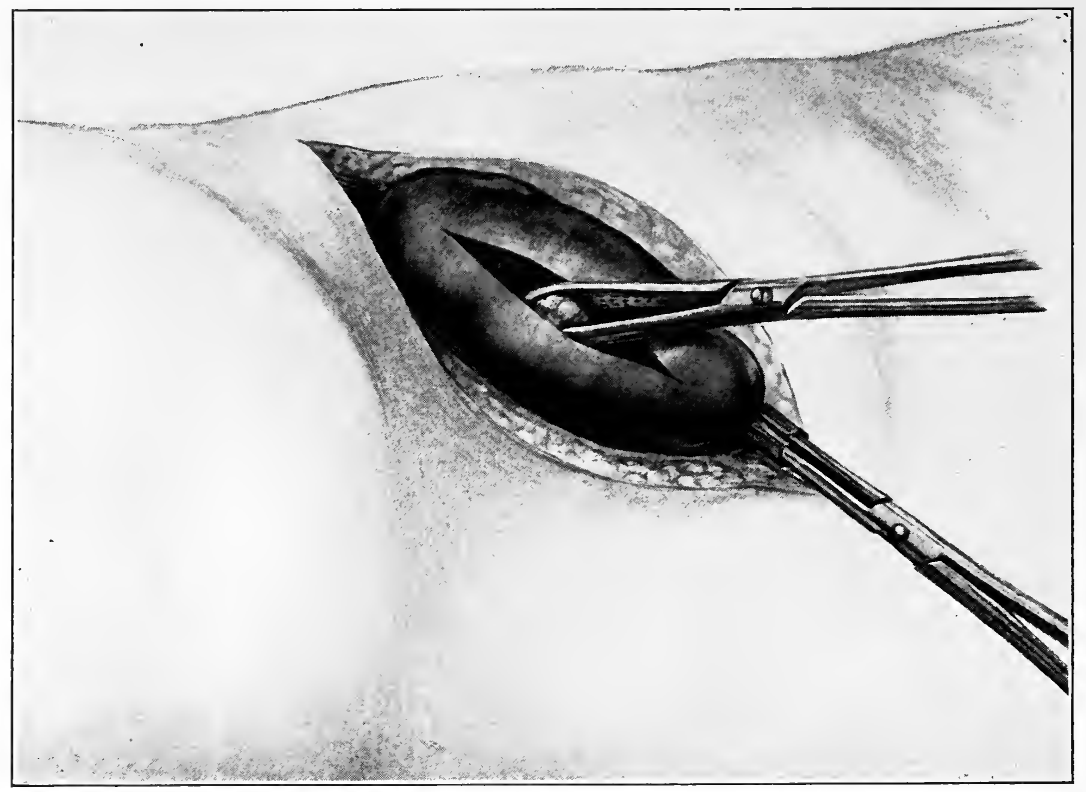

FIG. 254.-Nephrolithotomy.

leakage continuing for about two weeks after the operation. The incision in the pelvis may be enlarged from the ureteral outlet to the middle calix, either for the purpose of removing a large calculus, or to permit inspection of the renal papillæ in the case of suspected renal varix or calcareous incrustations (see Renal Hematuria, p. 546). Following such large incisions the cavity is restored as well as possible with suture and a drain is carried to the outer wound.

Suture of the Kidney.-Incision of the kidney requires suturing first, to encourage satisfactory union and second, to control immediate hemorrhage. The insertion of such sutures necessarily causes a deposit of more or less fibrous tissue in the region controlled by the 
constricting loop; therefore they should be applied with discrimination and in sufficient number only to bring the parts together under satisfactory control. There is a tendency to swelling and congestion of the organ following suture and sometimes post-operative anuria of temporary duration. ${ }^{1}$ This tendency is lessened by intrarenal drainage. Employment of drainage, however, must depend upon the condition that exists in a given case and the activity of the hemorrhage. Stout catgut is used and a large round full curved needle. The sutures are placed mattress fashion about $1 \mathrm{~cm}$. or less apart, the loops thereof being about the same length as the distance between them. These stitches should always be passed through the fibrous capsule, otherwise the kidney substance will be torn through. Following the application of these sutures, the kidney is restored to its normal location, which tends to relieve the congestion and diminishes the bleeding.

The cavity is then washed out with hot water, a drain is inserted, the muscular incisions already described are properly brought together by chromic sutures and the skin closed by silkworm gut.

Nephropexy.-Many special procedures and different methods have been proposed and tried for exposing a movable or misplaced kidney, and for retaining the organ in its anatomical position without undue mobility. The aim and purpose are the same in all instances. In order to obtain the most satisfactory and permanent results, it is desirable to allow the kidney a moderate amount of motion and to secure a position that does not cause twisting of the ureter or a tilting of the upper pole. One of the important requirements in effecting this object, besides the application of sutures, is the careful stripping away of all perirenal fat so that the convex border of the kidney may come in contact with the lumbar muscles and thus favor the development of strong adhesions between them.

The operation is performed through a vertical or angular incision. The kidney is exposed and delivered in the wound as already described. The fat is carefully stripped off the posterior surface. The finger of the operator is then passed into the wound and measure is taken of the height of the renal fossa. This distance is measured on the outer surface of the kidney and marks the point for the introduction of one or two sutures. These sutures are composed of heavy chromic catgut and are passed through the capsule after stripping, or through the capsule and the cortex on either side. They are then tied to the adjacent portion of the deep lumbar muscles. It is not possible to outline definitely the procedure for all cases as cases differ, and if a definite technic is carried out rather than suiting the requirements en-

1 (Cunningham.) 
countered in individual instances, the result is likely to be that the kidney is anchored too far below its normal position. The sutures may be applied through the cortical border above and below (Watson), or through the posterior surface of the capsule of the kidney (Morris), and the selection of one or the other of these methods is of minor importance, the object being to place the sutures in that portion of the kidney which seems most likely to hold the organ up in its normal position without encroaching too much upon the renal structure, or through the denuded capsule alone.

Another satisfactory method of fixation is to pass the sutures through the denuded capsule, after the same is twisted into a pedicle, thus not encroaching upon the kidney substance.

The question of decortication or decapsulation of the kidney at the same time is also determined in each case. Some operators always decapsulate (Edebohls); others partially decorticate (Watson). The object of obtaining strong adhesion is naturally accomplished with greater certainty and in cases of chronic congestion or inflammation, whatever benefit is obtained by such attendant removal of tension, is gained by partial decapsulation, and should be resorted to in appropriate cases.

The lumbar wound is completely closed; union should be obtained by primary intention.

Decapsulation of the Kidney.-This operation has been performed to a great extent for the relief of chronic nephritis (Edebohls), it being thought that abnormal tension was relieved thereby and that through the development of an anastomotic circulation, kidney congestion was further relieved. The operation is less frequently resorted to at the present day for acute and chronic Bright's disease; it may form a part of the technic of fixation of the kidney; it is adopted in some cases of unilateral kidney inflammation (see Renal Hematuria) and decortication or partial decapsulation is performed for acute anuria accompanying renal calculus. The same preliminary steps are followed as already described (see Nephrotomy), the capsule is split along the cortical border and a grooved director or flat instrument is used to elevate the capsule from pole to pole anteriorly and posteriorly (Fig. 255). The stripped capsule is then folded upon itself and allowed to remain upon the anterior and posterior aspect or is snipped away. If fixation sutures are then applied, enough of the capsule must be left to give support to these sutures (see Suture of Kidney). The remainder of the operation is the same as that following nephrotomy and nephropexy. 
Nephrostomy.-Nephrostomy for drainage is accomplished by exposing the kidney as already described; stripping the fatty capsule away from the organ, incising the parenchyma in its dependent portion, introducing the finger into the incision until the sac is reached, containing purulent or hydronephrotic fluid as the case may be, and then inserting a drain and stitching each lip of the renal wound to the lumbar incision. When in certain conditions the operation is required as a means of immediate relief, it is not desirable to expend the time necessary to search for the ureteral outlet; as in such cases at a

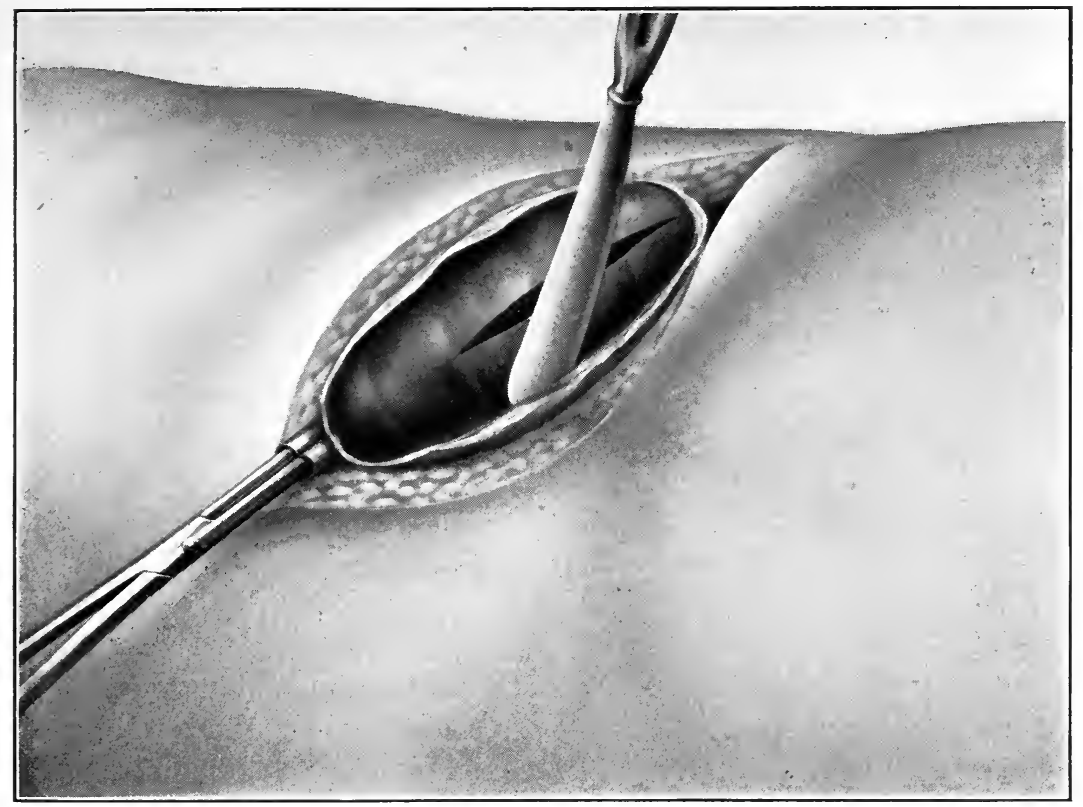

FIG. 255.-Nephrotomy and decapsulation of kidney.

subsequent time when the patient's condition recuperates, a more deliberate operation may be undertaken, making this conservative procedure a preliminary step.

Double nephrostomy is resorted to and highly commended as a preliminary stage to total cystectomy or removal of the bladder for neoplasm (Rovsing and Watson). It is more desirable than ureterostomy as the latter expedient may be followed by kinking of the ureter.

In performing this operation, the kidney is exposed as already described and after opening the kidney through the cortex as is usually done for exploration of the pelvis (see p. 635) the margins of the incision 
on either side are approximated to the fascia and muscle of the lumbar incision by means of carefully applied sutures, preferably of the mattress variety. The kidney is then drained by a special contrivance, the wound being closed around the inserted drainage tube. ${ }^{1}$

\section{NEPHRECTOMY}

As in the case of nephrotomy, nephrectomy, or removal of the kidney is effected in most instances through the loin. The amount of space that is necessary to expose the kidney is afforded by one of the incisions already described, following which, one of two procedures is carried out, namely: intra- or extracapsular removal of the organ. The latter method is more satisfactory and usually accomplished wherever possible. Intracapsular nephrectomy is made necessary by the existence of dense and extensive adhesions which render extracapsular separation of the organ a very difficult and prolonged undertaking.

Complete Nephrectomy.-Exposure of the kidney is followed by its liberation from the surrounding structure. The ease with which this can be accomplished varies considerably and depends upon the amount of extra-capsular adhesion. Sometimes one and sometimes the other pole is more difficult to release. The organ is freed on all sides until the pedicle is reached. The pelvis and ureter are then separated from the blood-vessels (Fig. 253). The ureter is divided between two ligatures as low as possible (Plate I). If the remainder of the ureter is not to be excised, the distal end is cauterized with the Paquelin or pure carbolic acid. A long ligature is left upon the ureter in case it is desired to remove it or introduce a catheter from above downward later in the operation. Having divided the ureter, it becomes much easier to reach the balance of the renal pedicle. The adherent structures (generally made up of adipose tissue) and sometimes the colon are gently separated with the fingers or by means of a small gauze pad. Having accomplished this step satisfactorily, which at times requires great deliberation and care, a good-sized pedicle clamp is placed upon the pedicle close to the kidney but not including the pelvis (Fig. 256). If possible, the veins and arteries should be dissociated and tied separately with stout catgut ligatures, the same being inserted by means

\footnotetext{
1 Watson (Watson and Cunningham, Genito-Urinary Diseases) has embodied the essential features required for permanent renal drainage in a specially devised apparatus which accomplishes the purpose of securing the tube in the fistulous tract so as to give free exit to the urine, thereby keeping the patient dry. This apparatus consists of a rubber cup or shield, perforated in the centre for the drainage tube which enters the kidney and at the dependent portion of its margin to catch the leakage which occurs alongside of the drainage tube. Both of these tubes are connected with a metal receptacle, and cup and receptacle are fastened to the patient's body by means of belt and buckle. The metal receptacle is supplied with an outlet and tube through which it can be cleansed.
} 
of an aneurysm needle. Then the entire pedicle is surrounded by ligature en masse. The vessels are then incised between the clamp and the ligature, and the kidney and clamp are removed together. This method is carried out only when the pedicle is sufficiently free to allow easy separation. In some instances it is necessary to clamp all the blood-vessels at one time then to tie the ligature beneath the clamp, opening the latter only after the kidney has been removed, and another clamp is placed in readiness to catch the pedicle in case the ligature does not control the vessels while the first clamp is being

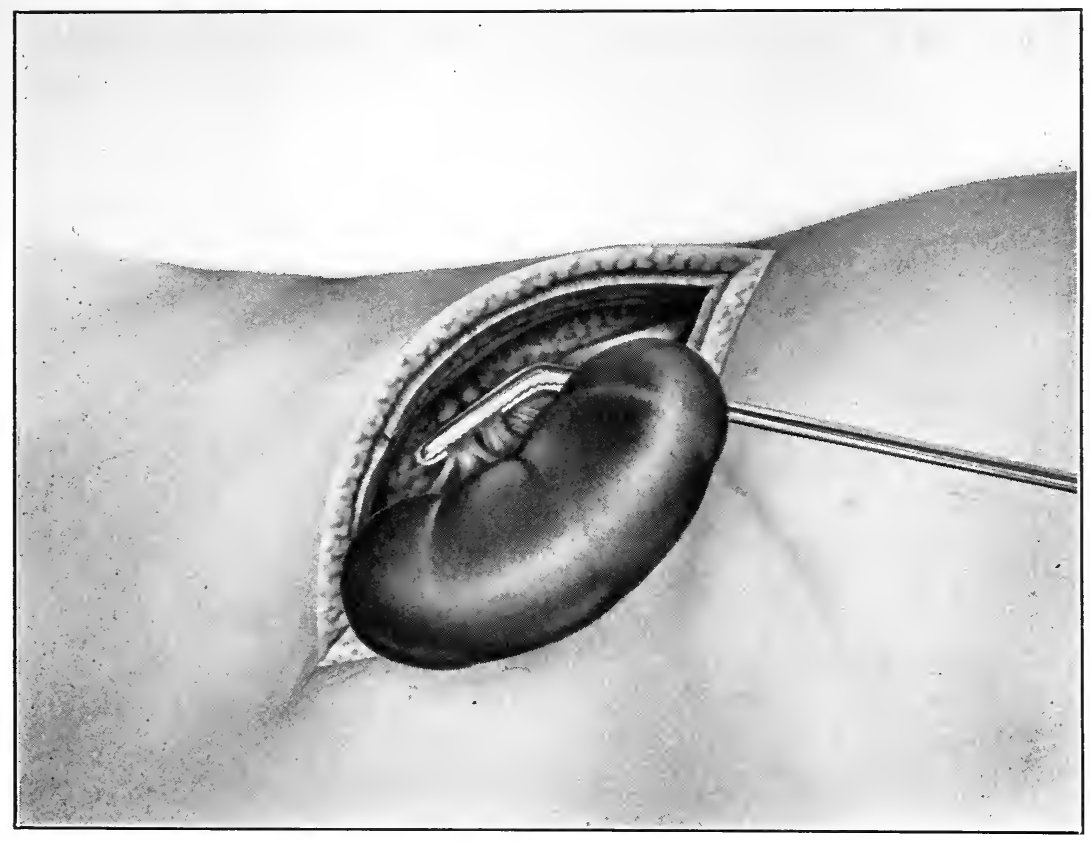

Fig. 256.-Lumbar nephrectomy. Clamping of vessels.

gently released. If it is impossible to ligate the blood-vessels satisfactorily the clamp must be left in situ as a matter of safeguard and is removed three days later.

Any bleeding points that are left after the kidney has been removed are ligated, the cavity is loosely packed with gauze and all of the incised structures, including the muscles, are brought together by chromic gut suture leaving a drainage opening of small or large size according to the character of the wound (Plate I).

Intra- or Subscapular Nephrectomy.-Complete nephrectomy can be accomplished in most cases but may require a great deal of patience 
and time following old chronic pyonephrosis with perinephritis and sometimes after a previous nephrotomy. The time required in some instances may be deemed greater than the patient is able to withstand; therefore a more conservative course must be decided upon. In this case, after splitting the capsule of the kidney, a plane of cleavage is found and the organ is separated from its capsule in all directions. The pedicle is then reached, surrounded by capsule enmeshed in ad-

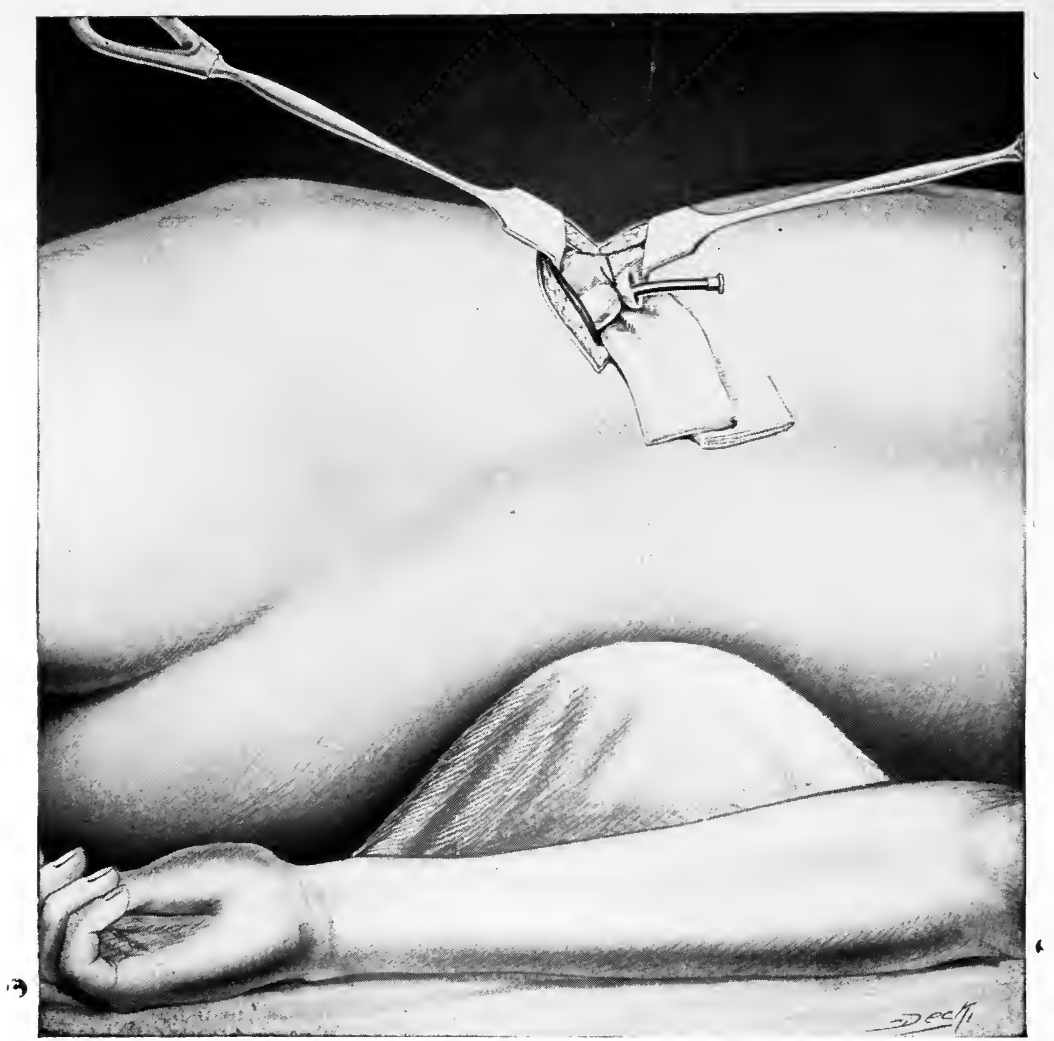

Fig. 257.-Method of removing ureter through abdominal wound after lumbar nephrectomy. Bougie being used as guide. (Lilienthal.)

hesions. It is usually possible to separate the ureter from this mass and ligate it independently, and after its division, a clamp may be applied to the remainder of the pedicle. The kidney is then excised and the pedicle is treated as may be practical in each individual case; sometimes it is possible to separate the vessels from each other and ligate them, as in complete nephrectomy, at other times they must be 
ligated en masse; and in some instances, it is necessary to leave the clamp in place. Such a course is always more safe and sure than to rely upon a ligature that has been doubtfully applied and of the integrity of which there is any question.

Intracapsular nephrectomy fulfills the requirements in pyonephrotic stone-kidney and even in some cases of tubercular intrarenal involvement, but in the case of malignant neoplasm, when it is necessary to remove the adjacent adipose tissue, together with the lumbar aortic glands, it is not sufficiently radical and the lumbar route may not afford sufficient room to accomplish complete removal or the space to reach

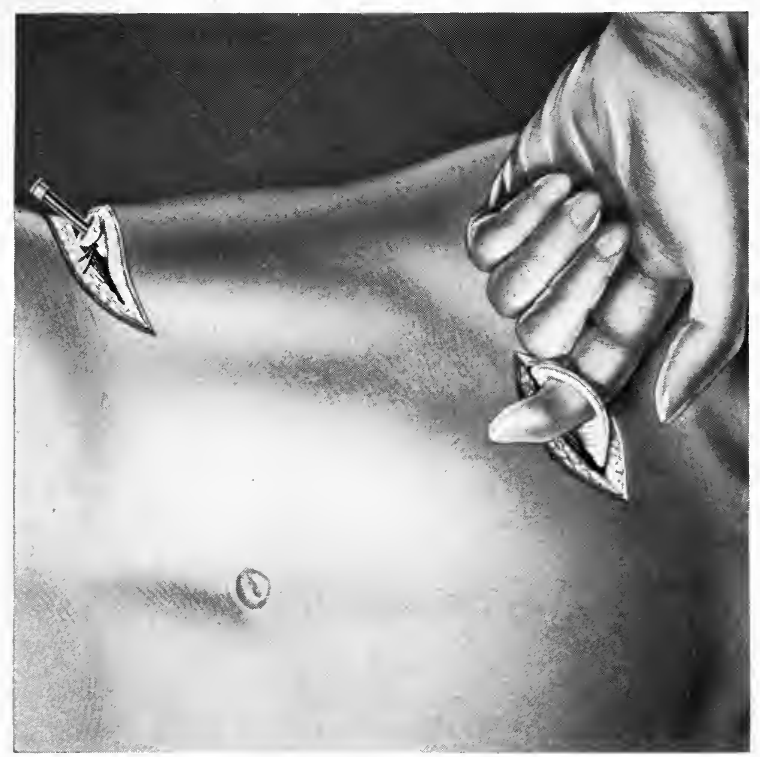

Frg. 258.--Ureter lifted from abdominal incision. Bougie in situ. (Lilienthal.)

the glandular nodes. 'Transperitoneal nephrectomy is therefore undertaken in such instances.

Removal of the ureter in connection with nephrectomy may be an important part of the operation. The oblique incision is lengthened downward and forward, when the ureter is easily followed to the brim of the pelvis and may be ligated there if such a distance is considered sufficient. After the ureter has entered the pelvis, it is more difficult to follow. A practical expedient has been utilized which greatly facilitates this step, ${ }^{1}$ and which requires a less extensive incision (Lilienthal).

1 Howard Lilienthal, Annals of Surgery, April 1, 1911. 
A fair-sized gum-elastic bougie as large as can be easily introduced into the ureter, is pushed through it down to the bladder, and tied above by ligature (Fig. 257). The lumbar wound may now be closed with allowance for drainage. "An oblique incision $11 / 2$ to 3 inches about an inch interior to the anterior-iliac spine is made and carried through the muscles to the peritoneum." The ureter is easily detected by means of the contained bougie and lifted into the wound (Fig. 258). An assistant withdraws the bougie from above and tightens the ureteral ligature. The upper portion of the ureter is then easily drawn into

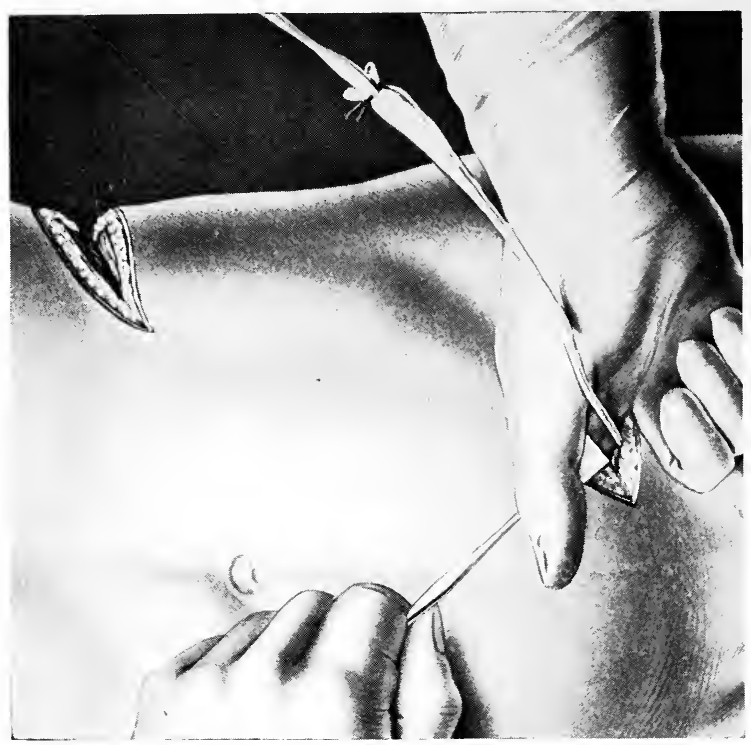

FIG. 259.- Same as Figs. 257 and 258. Ureter drawn through abdominal incision. Bougie removed. (Lilienthal.)

the lower wound (Fig. 259), and can be followed downward and ligated close to the bladder, after which the lower wound is sutured with allowance for a small drain.

Transperitoneal Nephrectomy.-A liberal vertical incision is made at the outside of the rectus muscle and if sufficient space is not afforded thereby may be supplemented by transverse incision later. The peritoneum is opened; the intestines deflected by gauze pads. The posterior peritoneum is then incised externally to the ascending or descending colon, whereby the blood-supply of the intestine is not implicated. A large opening is afforded in this manner, especially if a 
transverse incision is made. The subsequent removal of the kidney and ureter are carried on as already described, after which the peritoneum and abdominal wound are sutured and, if drainage is required, a puncture is made through the lumbar region for this purpose.

\section{Difficulties and Dangers attending Nephrectomy}

The operation is a delicate one and requires adequate skill.

Delivery of the kidney when it is bound by extensive adhesions, should not be accomplished by rough separation but by slow and deliberate dissection with thumb forceps and scissors, gently feeling the way, sometimes with a gauze wipe and at other times with ligature and clamp. As already stated, it is much more satisfactory to remove the kidney complete if the patient can stand the prolonged operation rather than resort to the intracapsular technic.

The satisfactory separation and ligation of the pedicle is the difficult step of all operations. If possible, the component parts should be ligated separately. If this cannot be accomplished, it is often wiser to leave the pedicle clamp in situ to be removed several days later. In reaching the pedicle, the possibility of anomalous blood-vessels should always be borne in mind. An aberrant artery sometimes reaches the lower pole. Such accessory artery may arise from a branch of the aorta, the common or external iliac. Another type is that of two separate renal arteries arising from the aorta. ${ }^{1}$ Three renal arteries are also found. In view of the possibility of this abnormality, it is important to be aware of the danger of hemorrhage as a result and to examine carefully for the presence of abnormal arteries in all cases of nephrectomy or extensive nephrotomy.

In case of extensive adhesion from perinephritis or in the removal of a large neoplasm, there is always the risk of opening the peritoneal cavity. This does not necessarily cause any trouble but the rent should be protected by gauze pads during operation and carefully sutured at the termination thereof. The pleural cavity may also be opened especially when there is an anomalous insertion of the pleura low down. This accident is indicated by a hissing noise with each inspiration. The tear, if sutured at once, should leave no untoward effect.

Injury to the colon is a more serious accident and should be carefully avoided. It is more likely with extensive adhesions and when

\footnotetext{
${ }^{1}$ Eisendrath and Kraus, Am. Journ. of Med. Sci., Vol. IV, No. 16. The Surgical Importance of Accessory Renal Arteries. The authors give a careful review of the various types of abnormal renal arterial supply with statistics upon the subject.
} 
the mesonephron is so short that the intestine is directly adherent to the kidney. A fecal fistula may be caused as the result of sloughing of the gut and in most cases when this appears subsequent to the operation, the surrounding part having become walled off by adhesions, closure of the fistula usually occurs by granulation.

Severe hemorrhage, besides that which occurs from the renal vessels, from slipping of the ligature of the pedicle, may occur from a tear of the large blood-vessels, particularly the vena cava. In such an emergency, which is fraught with great danger, packing of the cavities is the usual means adopted, unless the wound is large enough to reach the bleeding-point satisfactorily to ligate or suture the vena cava and this has been done.

Rupture of the Kidney.-This accident to the kidney is treated surgically according to the extent of the damage. When exploratory nephrotomy is deemed advisable, the kidney is treated by suture and tamponade if the laceration is not too extensive, in which case, the capsule should always be included in the ligature as already described in connection with nephrotomy and nephropexy. In cases where the pedicle has been lacerated and fatal hemorrhage is imminent, complete or partial nephrectomy must be performed (see p. 527).

Partial Nephrectomy.-Resection of the kidney is sometimes performed as above stated, in the event of rupture. Otherwise, it is resorted to in the case of benign lesion such as non-malignant neoplasm but it is never justifiable in tuberculosis. The technic involves incision around that part of the kidney to be removed and suture of the cut surfaces through the capsule thereof which are brought together in close relation.

\section{OPERATIONS UPON THE URETER AND RENAL PELVIS}

Exposure of the Ureter.-The upper portion of the ureter is exposed by the oblique or curved lumbar incision as described in the operation of nephrectomy, q. $v$. The pelvis and iliac portion are exposed either through a vertical anterior abdominal incision along the outer border of the rectus, or semilunar incision above and to the inner side of Poupart's ligament. Inasmuch as an important purpose of such incision is to reach the ureter for the removal of an arrested calculus too large to find its way further down the canal or to be aided in its passage by cystoscopic manœuvre, a liberal exposure of the region in question is necessary. This principal fact has been taken into consideration and is effectually covered by a valuable operative 
technic (Gibson $\left.{ }^{1}\right)$. The incision is begun in the median line above the pubis, extending outward about a finger's breadth above Poupart's ligament until about its middle, when it curves rather abruptly upward and outward to a point on a level with the anterior-superior spine of the ilium (Fig. 260). The aponeurosis of the external and internal oblique are then severed in the line of the skin incision; sometimes some of the fibres of the internal oblique are included. The flap *which is thus outlined is separated upward; at the border of the rectus

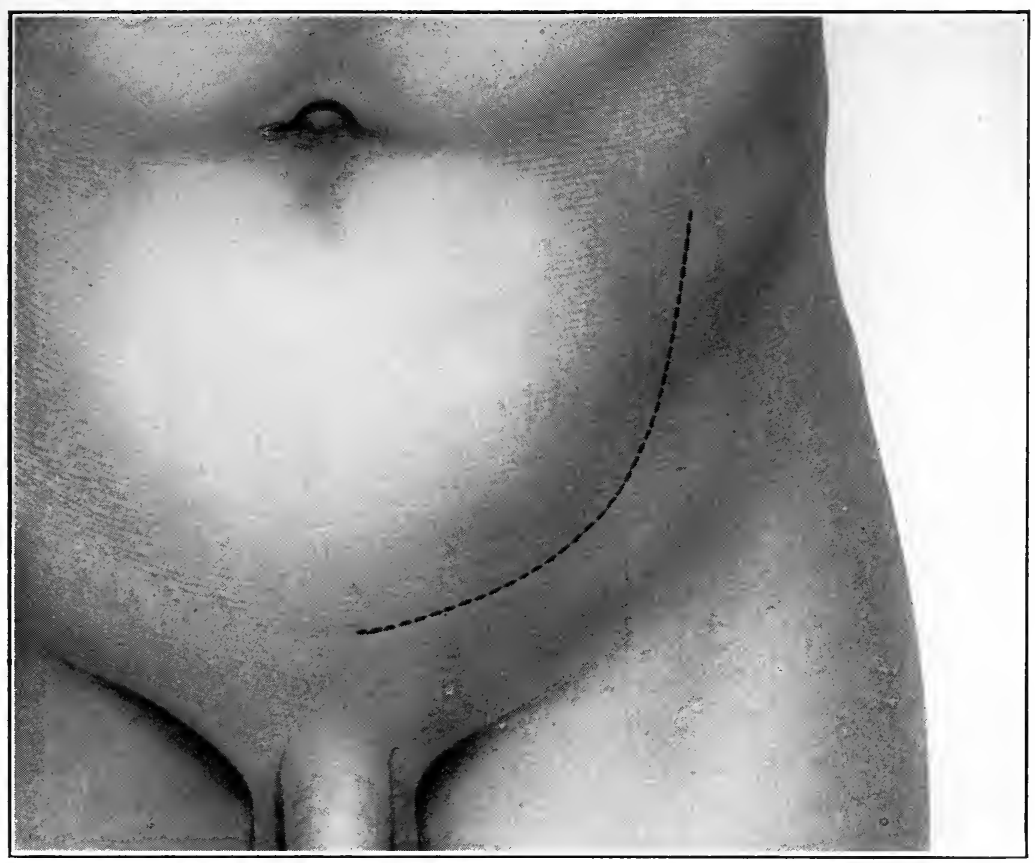

FIG. 260.- Route of approach for the lower portion of the ureter. Line of incision. (Gibson.)

muscle (Fig. 262) the fascia of the transversalis (Fig. 261) is opened by a vertical incision; retractors having been introduced, the peritoneum is separated with the patient in the Trendelenberg position, so that by retracting the rectus muscle inward and the transversalis outward, (Fig. 263) the lower end of the ureter and base of the bladder are exposed in an ample space. Following removal of a stone from this

1 C. L. Gibson, Am. Journ. Med. Sci., January, 1910. The Technic of Operations on the Lower Portion of the Ureter. The author describes at length an admirable method of approaching the lower end of the ureter through the capacious semilunar incision described above and he illustrates the effectiveness of this method by the report of two successful cases of ureteral stone. 
portion of the ureter, it is closed by a double row of stitches, the first of which passes completely through all its coats, while the second row reinforces and buries the first.

Other methods are adopted to reach special portions of the ureter, namely, the vaginal route (Cabot) for the lower portion of the duct in the female and the transvesical route to reach the intravesical orifice for the extraction of stones and incision of cysts.

When ureteral stone is discovered in the lowest portion of the

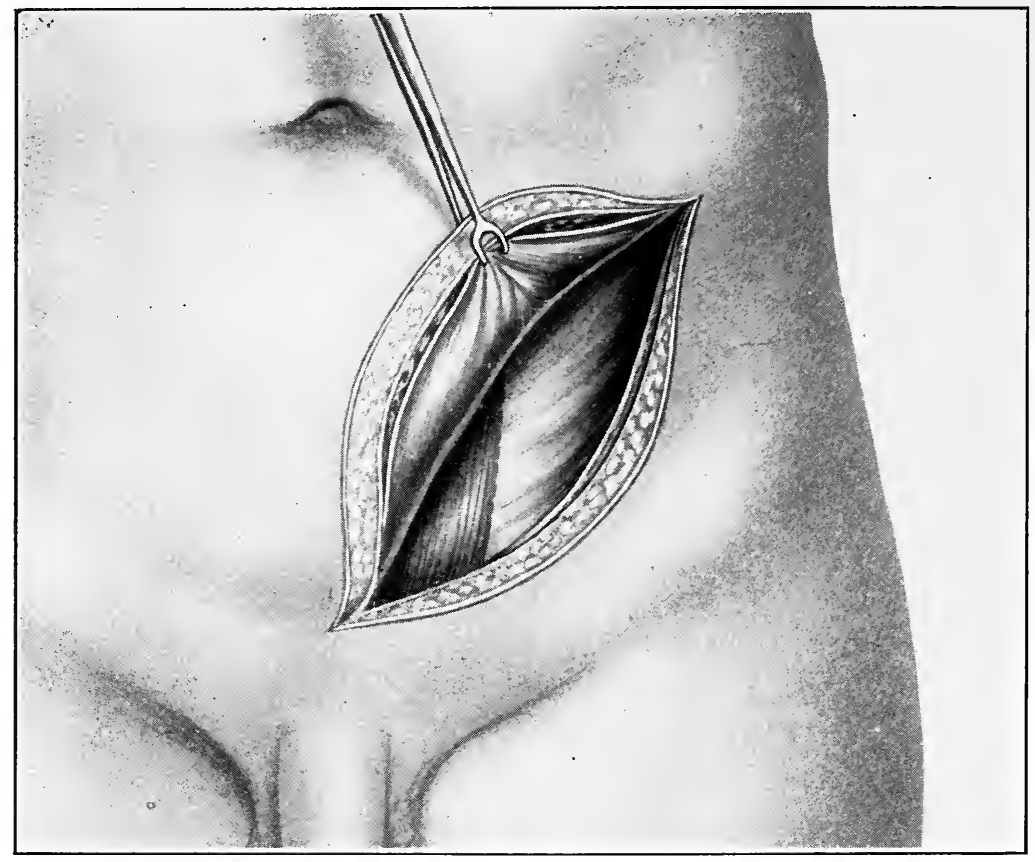

FIG. 261.-Route of approach for the lower portion of the ureter. Exposure of rectus muscle. (Gibson.)

ureter, within $2 \mathrm{~cm}$. (3/4 inch) of the outlet, it may be removed by one of two methods according to its size:-When it has traveled down the ureteral tube and the interval following arrest is not of long duration, it may be successfully aided in its passage by injection through the ureteral catheter of sterile olive oil.

If such an attempt be unsuccessful, or if the stone has been lodged at this site for a long period entailing substantial increase in size, it is reached by the transvesical route: after the bladder is opened by suprapubic incision one finger is introduced in the space of Retzius and 
compression is made toward the bladder to effect delivery of the stone. Stones of large dimensions have been lodged in this vicinity and removed by this method (Fig. 247, p. 570). In some cases, it may be necessary to incise the ureteral orifice to accomplish this feat successfully.

Following exposure of the ureter, further action depends upon the requirements of the case, which may involve incision for the removal of stone; or plastic repair, resection, total extirpation or anastomosis

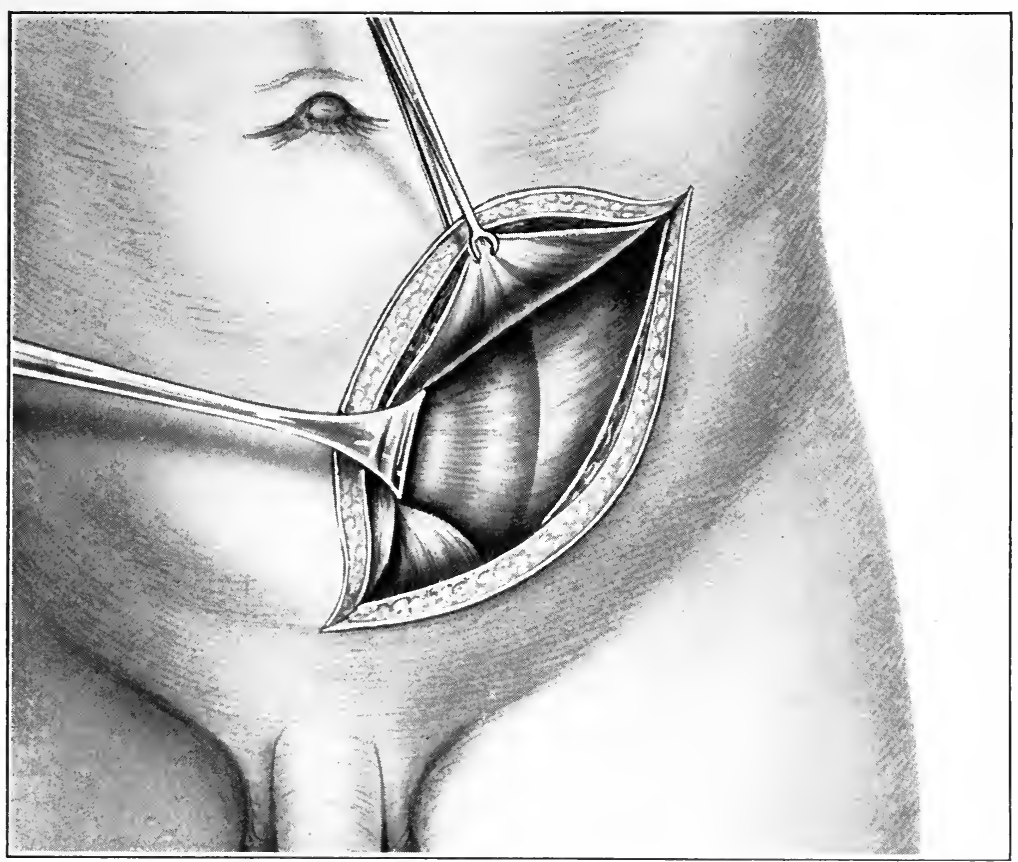

FIG. 262.-Same as Figs. 260 and 261. Exposure of transversalis fascia. (Gibson).

with neighboring organs. Total extirpation has already been described.

As a preliminary step toward ureteral operations, it is desirable by means of cystoscopy to introduce a ureteral catheter as high as possible.

Incision of the ureter in its longitudinal axis is necessary for the removal of an impacted stone, for the purpose of catheterizing the tube from above downward during an operation upon the kidney and as a part of a plastic procedure. Suture of a simple incision of the ureter, may or may not be performed. As a rule, in all non-septic 
cases of simple incision of the upper end of the ureter, healing occurs without such a procedure, although the precaution may be taken; but in septic cases it is desirable to leave the wound open with a drain to the site of operation and it is better generally to dispense with suture than to have it be the means of laceration during the insertion thereof. When no sutures are used a drain should be inserted to the opening in the ureter, otherwise the latter should be earefully united with a double row of sutures which makes it possible to close the abdominal wound

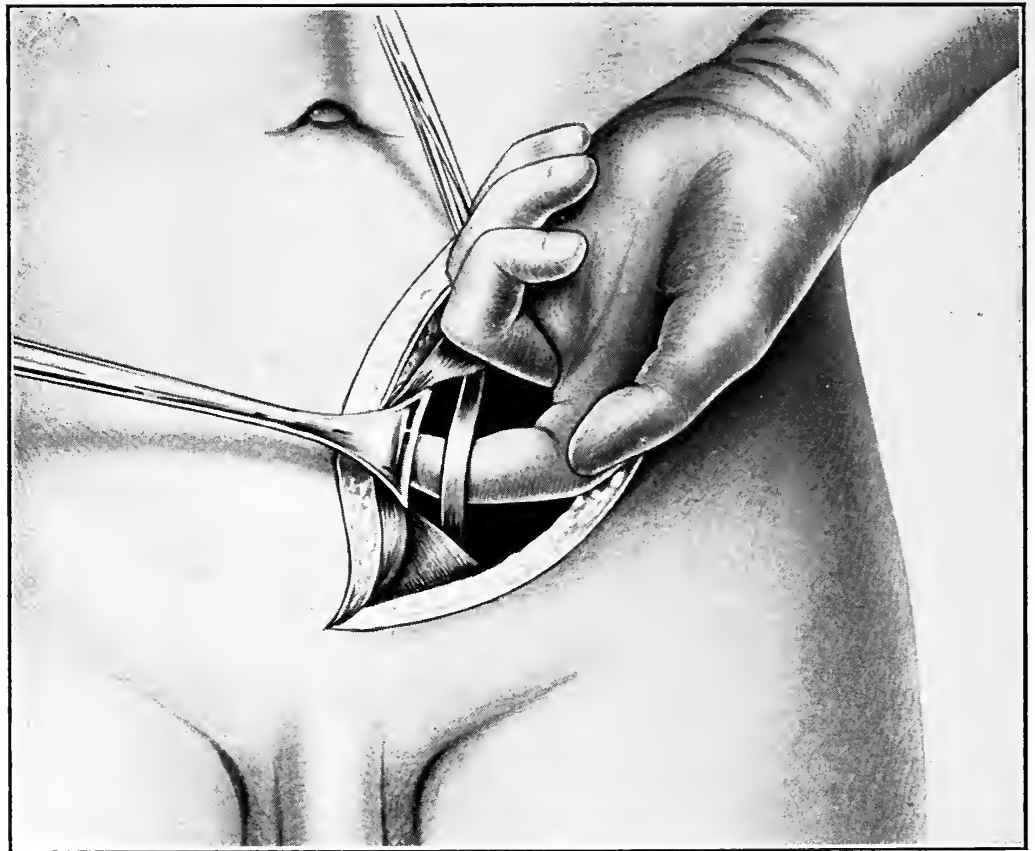

Fig. 263.- Route of approach for the lower portion of the ureter. Elevation of ureter. (Gibson.)

without drainage. In septic cases, however, it is advisable to insert a drain to the ureteral incision which may be removed in twenty-four or forty-eight hours. Besides the repair of surgical wounds, suture of the ureter is required for correcting deformity, traumatic division and stricture. As in suturing the urethral tube, longitudinal wounds of the ureter preferably should be sutured transversely to avoid resulting stricture (Fig. 264).

Resection of the ureter is required for impassable stricture, for tuberculosis and involvement by neoplasm. It is also demanded following accidental division during operation. If the gap between 
the divided ends is not too long, the restoration may be accomplished in one of the following ways: by end to end suture, by end to side approximation, by suture and invagination of the upper into the lower end, lateral-end invagination or side to side anastomosis (Fig. 265). If the upper end reaches as far down as the fundus of the bladder, vesical implantation may be accomplished which is the most successful procedure (Fig. 266). If the gap between the divided ends is too great for any of the above methods of approximation without tension, nephrectomy must be resorted to or retroperitoneal implantation into the opposite ureter.

End to end suture of the ureter is of uncertain outcome. If attempted, the lower end should be split obliquely and dilated before suture to permit invagination and to lessen the likelihood of constriction thereafter (Fig. 265, A). End to side anastomosis is usually employed when one ureter is implanted into the other. Invagination is more reliable in result, although not always successful. In the above technic the vesical end is split on one side for the

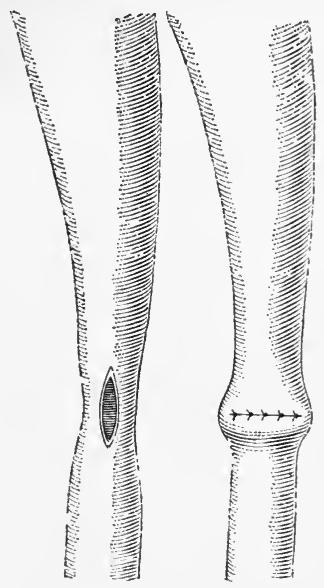

FIG. 264.-Longitudinal incision of ureter sutured transversely. length of about $6 \mathrm{~mm}$. (1/4 inch). Two traction loops, of catgut, one on each side, are passed from without inward through the vesical

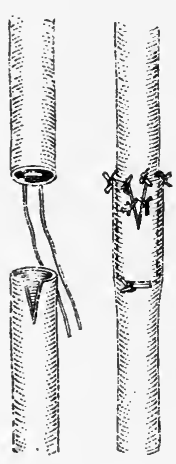

A

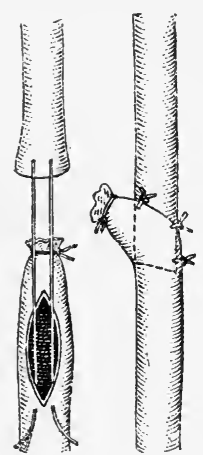

B
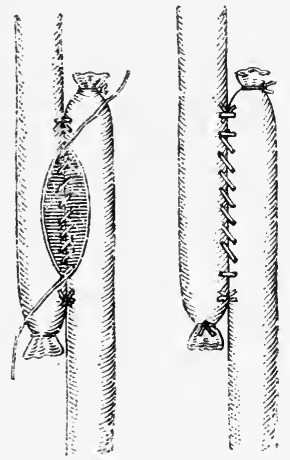

C

Fig. 265.-Ureteral anastomosis. A, End to end. B, End to side. C, Side to side.

end and from within outward through the renal end; again from within outward through the vesical end. Pulling up on these ends telescopes the renal through the vesical portion and the reunion is 
completed by other sutures applied transversely to the split in the vesical end (Pozzi ${ }^{1}$ ).

The end to side invagination is favorably considered by most operators (VanHook-Emmett). In this instance the vesical end is ligated and the renal portion is invaginated through the side of the latter (Fig. 265, B).

Side to side anastomosis is much the same as lateral-entero anastomosis. The ends of both renal and vesical portions are ligated

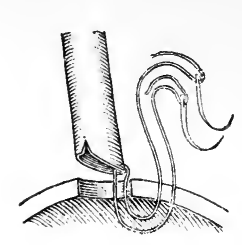
(Fig. 265, C). A longitudinal incision is made about $6 \mathrm{~mm}$. (1/4; inch) in the long axis of both sections about the same distance from the ends. They are united by a row of through and through sutures protected by a second line of Lembert sutures.

Uretero-vesical Anastomosis.-This operation may

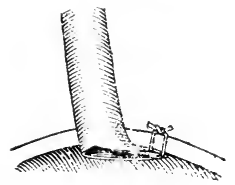

Fig. 266.

Ureterovesical anastomosis.

be performed extra- or intraperitoneally. In all cases, it is an advantage to obtain the peritoneal covering over the suture line. The bladder wall is raised on the tip of the sound and incision is made thereupon (Fig. 266). The ureter is first split lengthwise for .75 to 1 . $\mathrm{cm}$. and is drawn through the bladder hole by means of a traction stitch. Other stitches unite the muscular and peritoneal coats of the bladder to the inserted end and it is finally covered over by peritoneum.

Intestinal anastomosis of the ureter is accomplished in much the same manner as with the bladder. On account of the risk of ascending infection, however, this operation is seldom done except when the sphincteric rings surrounding the orifice can be included in the transplantation of the entire interureteric bas-fond, as in the case of Peter's or Maydl's operation (see p. 471).

1 Trans. Congress de Chirurgie, Paris, 1906. 


\section{CHAPTER XXXVII}

\section{TUBERCULOSIS OF THE UROGENITAL TRACT}

Introduction.-In the present state of knowledge of this subject, as a result of careful observation and study of many cases, it has become established that of ten patients with genito-urinary tuberculosis present symptoms that seem to be referable to one area who are not unlikely to show the essential and active lesion elsewhere. This fact has been amply attested by systematic physical examination involving cystoscopic and laboratory investigation.

While the different varieties of genital and urinary tuberculosis are more or less bound together and often occur in sequence, it is more convenient to separate these varieties in the consideration of them. As a matter of fact, urogenital tuberculosis is never primary; there is usually a preëxistent focus, though ever so small, in the pulmonary tract or in the lymphatic system, entrance having occurred by way of the pulmonary or gastro-intestinal regions. The common types of invasion-namely, acute general miliary tuberculosis, localized pulmonary and gastro-intestinal tuberculosis-to begin with are medical conditions, but may spread to other parts of the body remote from the point of entry, when they become surgical.

That condition in which the urologist is particularly interested is where the tubercle bacilli have spread to the genito-urinary tract and have produced the lesions embraced in the different divisions of urogenital tuberculosis.

\section{VARIETIES OF UROGENITAL TUBERCULOSIS}

(1) Primary Renal Tuberculosis.-The origin of tuberculosis is, in some instances, apparently so quiescent a focus before it reaches the kidney as to lead to the designation of "primary renal tuberculosis." What is really meant is primary as far as the urinary tract is concerned. In this condition, the bacilli have by hematogeonus route become localized in the kidney ${ }^{1}$ where they may remain, the kidney being the sole urogenital focus and clinically, the only focus, the small focus of origin being demonstrable at postmortem only; or the lesion in the kidney may extend to the lower urinary tract.

1 Laroche, De la Touberculeuse primative du rein, Ann. des mal. des org. g.-u., 1896. 
(2) Secondary renal tuberculosis or ascending infection from a tuberculous cystitis or genital tuberculosis. This variety is less common than the previous one.

(3) Tuberculosis of the genital tract, including the testicle, vas and seminal vesicle which may also invade the urinary bladder.

\section{RENAL TUBERCULOSIS}

Causation.-Although many cases of renal tuberculosis are encountered by cystoscopic examination and operated upon in the surgical clinics, from the standpoint of relative frequency as determined by postmortem examination in the general hospital, the number is not great, that is, when active general tuberculosis is excluded. ${ }^{1}$ Whereas in postmortem examinations upon tuberculous patients in general, the kidneys are affected in 10 per cent. ${ }^{2}$ As compared with all surgical lesions of the kidney, its occurrence is considered to be almost onethird in point of frequency. ${ }^{3}$ Touth is a farorite period for the development of renal tuberculosis. The arerage age may be stated as between twenty-five and thirty years.

There is almost an even distribution betreen males and females; some clinicians report more in one and others in the opposite sex. The large majority of cases are undoubtedly of hematogenous origin although instances of ascending infection from the bladder are recorded. These cases are more common in men than in women because of the existence of testicular lesions which may set up trigonal congestion and eventually derelop cystitis, but even under such conditions, ascending infection is not easy, requiring in all probability an insufficiency of the ureteral valves, which is more likely after prolonged and chronic inflammation of the bladder, when reflux may be favored by resical spasm and alteration in the ureteral mouth.

Morbid Changes. (1) Tuberculous Nephritis.-This condition is one of glomerular and tubular nephritis with tubercle bacilli in the urine running a chronic course. ${ }^{5}$

This type of renal tuberculosis must be differentiated from the nephritis of tuberculous subjects which is constantly found upon

${ }^{1}$ In 20,770 autopsies, records of the Vienna General Hospital, Kapsammer, Neirenchirurgie, not counting general tuberculous infections, only 191 cases (1 per cent.) showed renal tuberculosis,

${ }^{2}$ Küster, Deut. Chir., 1902.

${ }^{3}$ Israel, Chir. Klin. Nieren Krankheiten, 1901.

- Watson and Cunningham in a compilation of 373 , the average was 27.66 years.

Braasch found in 203 operations in the Mayo clinic, Journ. Amer. Med. As8oc., Feb., 1912, 29 per cent. above forty years of age.

${ }^{5}$ Pechere, Rein Tuberculeuse, etc., Bull. de la Soc. Royale de Sci. Med., etc., Bruxelles, 1905.

Benard, Les affections tuberculeuses des reins, Ann. des mals des orgs. g.-u., 1905. 
autopsy and is similar to the toxic nephritis of the opposite kidney in cases of actual tuberculous involvement of one organ, which condition often vanishes after nephrectomy.

(2) The Infiltrating Form of Tuberculosis.-Such lesion consists of a diffuse invasion of the kidney substance with tubercles which are studded throughout the parenchyma and are later accompanied by the formation of new connective tissue. The appearance is not unlike that produced by sarcoma. The tendency is not to caseate. ${ }^{1}$ Such kidneys are also associated with parenchymatous proliferation of fibro-lipomatous tissue resembling healed tuberculous deposits. $^{2}$

(3) Papillary Tuberculosis.-This variety is rare but should be held in mind as it may afford an explanation, in the absence of other causative lesions, of hematuria with the presence of tubercle bacilli in the urine. The lesion consists of small ulcerations of the papilli with submucous tubercles.

(4) The Cavernous Form of Renal Tuberculosis.-This form, which is the most common, begins with a deposit of tubercles in the vascular zone at the junction of the renal medulla and cortex. Some of these deposits become caseous and form small or large cavities imbedded in granulation tissue. There may be associated numerous minute subcapsular foci of tubercles surrounded by congested zones. These represent active isolated tubercles and portend grave central extension of the lesion. They resemble the tiny pyogenic infarcts but if examined histologically will be found to contain none of the ordinary bacteria. As a rule, as the lesion progresses, the caseous areas reach a size that may be palpated through the cortex (Fig. 267). They may rupture into the pelvis and produce pyonephrosis without distention or they may form cystic tuberculous areas in the renal parenchyma $\mathrm{m}^{3}$ and rupture externally (Fig. 268).

(5) Tuberculous Pyonephrosis.-As in the case of other bacterial infections of the kidney, the terminal stage of tuberculous inflammation is that of pyonephrosis. The organ becomes a lobulated tumor filled with pus, and upon postmortem section collapses, there being little proliferation of connective tissue between the walls of the cavities, which are lined with translucent tubercles. This lesion usually starts from medullary or papillary lesions which eventually develop pyonephrosis with distention (see Pyonephrosis), becomes secondarily infected

1 Albarran and Imbert, Des Tumeurs des reins.

2 Malgaigne et VanVerts, Tuberculose renale guerie par transformation fibreuse totale, Ann. des mals. des org. g.- u., 1898.

${ }^{3}$ Petit et Lorenzo, Tuberculose kystique du rein sans pyonephrose sac. Anat., 23, XI, 1901. 
with the pyogenic organisms, ${ }^{1}$ or may contain pelvic calculi. Perinephritis is always a marked feature.

The lower urinary tract is nearly always affected to a certain extent in renal tuberculosis even in comparatively early cases. What is very common is a scattered patch here and there in the course of the ureter which shows small elevated, pin-head nodules representing submucous tubercles, and, when conglomerated, forming a thickened

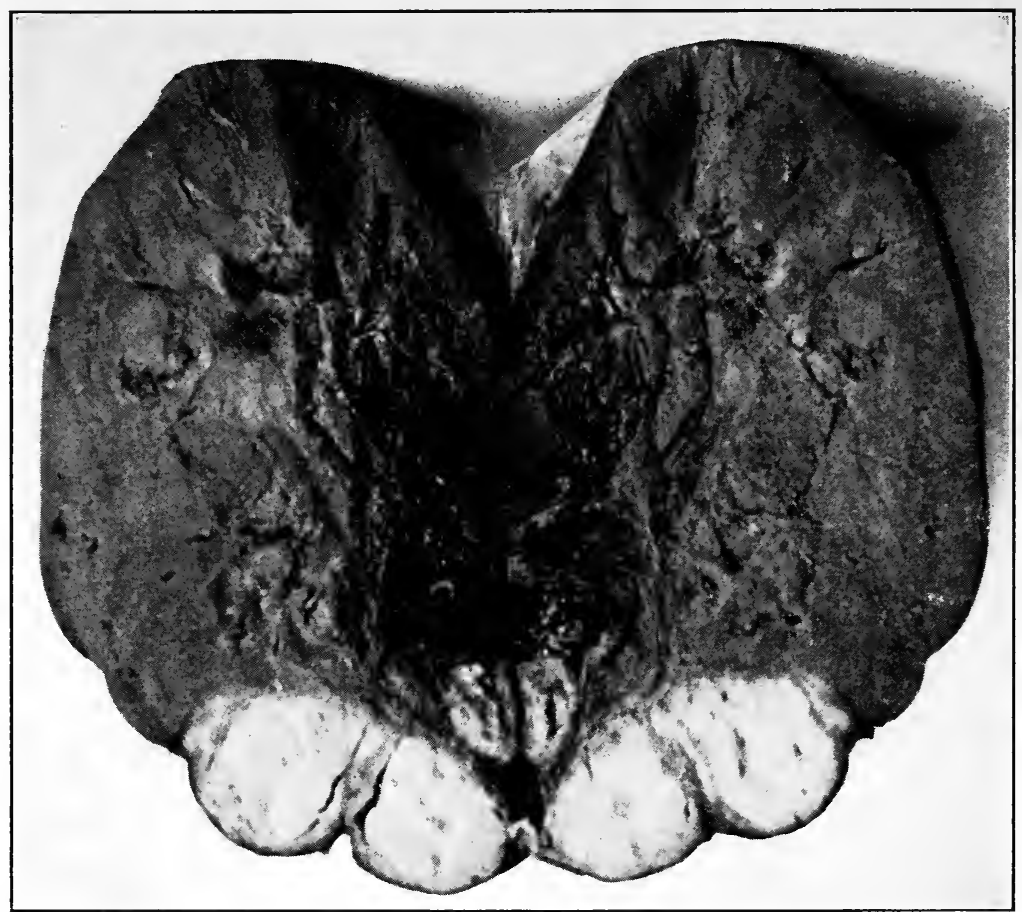

Fig. 267.-Tuberculous kidney. Caseous foci in lower pole.

patch. These are not infrequently found in the portions of the ureter removed during operation or are seen by cystoscopy around or in the mouth of the ureter. A favorite site is just above the pelvic brim. They may occur in cases of early tuberculosis here and there with large spaces of intervening healthy mucous membrane and without any vesical lesion. In advanced cases which present marked cystitis, the ureter is likely to be very much thickened and the mucous membrane over its entire course is replaced by granulomatous tissue accompanied

1 Rafin, Session de l'assoc. Franc. d'urologie, 1906. 
by periureteritis of variable degree in different sections, producing points of stenosis.

The infection of the ureter occurs by contiguity, and by urinary transmission, or it is possible in some cases by a lymphogenous route

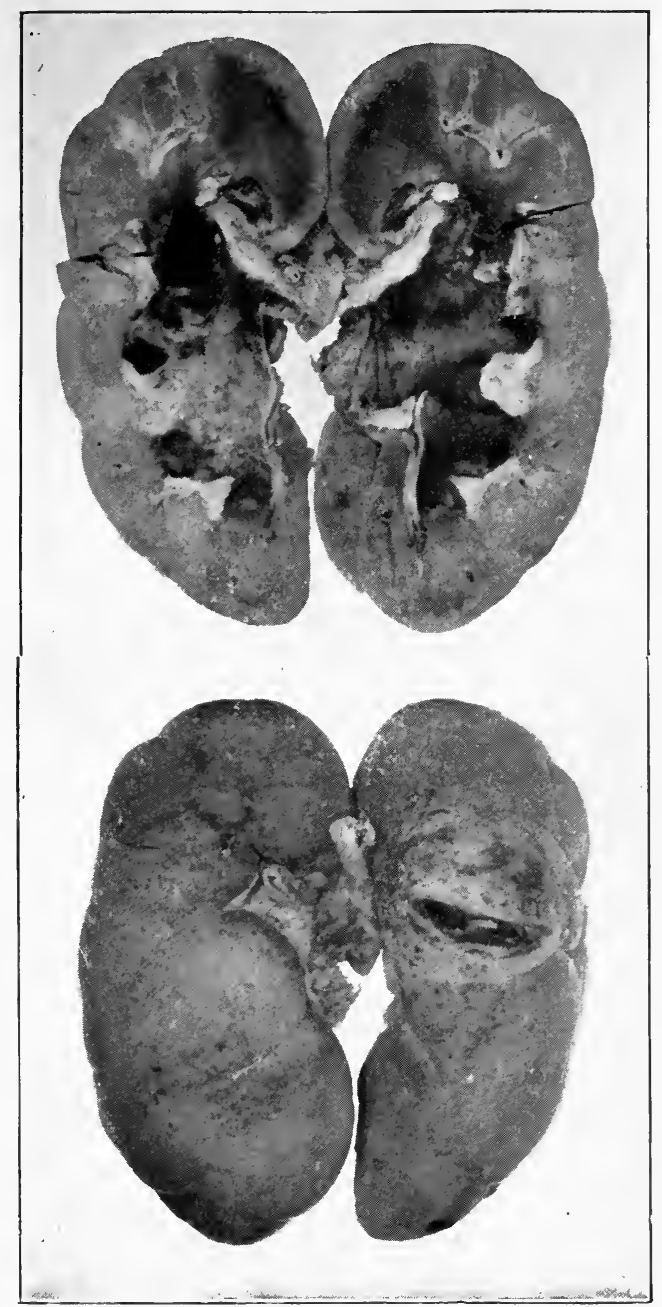

FIG. 268.-Cavernous tuberculous nephritis. Rupture through cortex.

producing the condition of periureteritis without involvement of the mucous membrane. ${ }^{1}$

Concerning the infection of the opposite kidney in the advanced stage of the disease, it is probable that most infections are produced

1 Pousson, Contribution a l'etude de la tuberculose renale, etc., An. des mal. des orgs. g.-u., 1905. 
by hematogenous transmission through the general circulation although it is claimed that a transvesical ascending infection may occur. ${ }^{1}$

Clinical History.-Tuberculous nephritis is not necessarily associated with generalized symptoms. When such symptoms do occur, the kidney is likely to be in an advanced stage of destruction. On the other hand, the most advanced condition of tuberculous pyonephrosis may be encountered with a normal temperature and relatively slight constitutional disturbance, although in such instances a careful investigation of the preliminary history is likely to reveal at some previous time symptoms suggestive of active inflammation such as febrile disturbance, night sweats, loss of flesh, nervous excitability, etc. The onset of secondary infection ${ }^{2}$ as a rule is attended by an exacerbation of general symptoms (fever, sweat, etc.), especially during the closure of a pyonephrosis and interference with the ureteral drainage. Otherwise the condition, as stated, may exist for years without marked general symptoms.

Localized Symptoms of Renal Tuberculosis.-Pain is an important and may be the first symptom of tuberculous renal involvement. It is usually localized in the loin although it may radiate through the shoulder and down the course of the ureter. It is of a dull aching character.

On the other hand, subjective pain may be totally absent.

Tenderness in the loin or at the costovertebral angle is of common occurrence often on account of perinephric involvement. In the event of perinephric abscess, the pain is likely to be severe.

Pain of a radiating character simulating renal colic may be present, even in the absence of ureteral obstruction by caseous emboli and may be regarded as ureteral colic due to local irritation.

Renal Tumor.-The kidney may not be palpable ${ }^{3}$ in the early stage of tuberculosis, whereas at such period the lower pole may be distinguished in thin subjects when it may be sensitive and irregular to the touch. In advanced pyonephrosis, a large irregular mass of more or less lobulated contour may be felt producing a characteristic balottement. It should be remembered that dependence cannot always be placed upon the localized symptoms in renal tuberculosis as pain in the opposite side is sometimes misleading and the compensatory hypertrophy may call attention to the normal kidney. The tuberculous

\footnotetext{
1 Tufier, Etude anat. pathologique et clinique sur la Tuberculeuse renale, Arch. Gen. de Med., 1892. Albarran et Cathelin, D'un mode d'infection de l'autre rein dans la tuberculose renale (Assoc. d'urologie, 1904), suggest a way of transmission from one kidney to the other through a collection of veins which pass toward the diaphragm.

2 Pousson, loc. cit.

${ }^{3}$ Tumor was felt in only 20 per cent. of Braasch's cases loc. cit.
} 
kidney is likely to be less movable than that of neoplasm because of the presence of more or less perinephritis. ${ }^{1}$

Bladder and Urinary Symptoms.-Bladder irritability is one of the most common symptoms encountered in renal tuberculosis. ${ }^{2}$ The bladder may be irritable in the early stages even though it fails to reveal an abnormal condition by cystoscopic examination. The urinary frequency at this time may be due to actual polyuria, and although it may mark the existence of chronic nephritis of the phthisical subject, its presence should lead to the suspicion of renal involvement. In advanced lesions, in such cases as are often beyond surgical intervention, the bladder irritability becomes a most marked feature at which time urination of small amounts may be imperative every few minutes accompanied by great straining of purulent and bloody fluid cotainning tubercle bacilli. In such cases, the thickening of the bladder may be felt by rectum in the male possibly with an induration and thickening of the prostate and seminal vesicles and sometimes the lower end of the ureter as well. The latter in the female may be felt per vaginam.

Hematuria may be microscopic in character or of sufficient quantity to appear in a clinical observation. It is ordinarily a very common, early manifestation. ${ }^{3}$ The appearance of hematuria in tuberculous cases, whether of small or large quantity, always leads to the suspicion of urinary tuberculosis as a likely explanation. The blood in the urine is not as a rule influenced by exercise or rest. Exceptionally, it may exist in a dangerously large amount possibly requiring immediate nephrectomy. Albuminuria is a common symptom which should be given proper consideration as there is an albuminuria of tuberculous subjects which is bilateral, ${ }^{4}$ while the albumen incident to the clinical history of localized renal tuberculosis may be due to the presence of blood or to a tuberculous nephritis, when it is unilateral.

Pyuria.- The presence of pus in the urine is a common accompaniment of renal tuberculosis and may exist in small or large amount, from a few white blood cells to that of a copious quantity. This may be constant or variable from time to time. When a pronounced pyuria is reached it may be due to a pyonephrosis or to extensive vesical involvement. When the pyuria is entirely renal, it may be due to a rupture of a cavity of the parenchyma into the pelvis, and transitory in its appearance; whereas when the pelvis itself is involved (pyelone-

\footnotetext{
${ }^{1}$ Hottinger, Zur Diagnose der Nierentuberculose, Centralblatt für die Krankheiten den Hahn, etc., 1906.

${ }^{2}$ Present in 86 per cent. of Braasch's cases, loc. cit.

${ }^{3}$ Newman, Lancet, 1890 . Hematuria occurred in 60 per cent. of Braasch's case, loc. cit.

'Kahlden, Pathologie and path. anat., Bd. II, 1892.
} 
phritis) the pyuria is usually constant and an apparent cessation occurs only when a constriction at the uretero-pelvic junction is established producing the condition of closed pyonephrosis.

The urine of renal tuberculosis is as a rule acid and the pus, when present, is of a dull grayish tinge and usually settles to the bottom rapidly. Examination should reveal the presence of the tubercle bacilli when careful search is made of the twenty-four-hour accumulation. A hanging-drop specimen of the freshly drawn urine does not contain the ordinary pyogenic organisms and a similar specimen subjected to culture will usually yield a sterile result. On the other hand, the occurrence of a secondary or mixed infection will render this distinction unreliable as a diagnostic guide; but the urine under these conditions, though foul smelling, is generally acid, which is not the case with the fetid urine of pyuria from other causes.

Diagnosis.-The diagnosis of renal tuberculosis is for the most part established by cystoscopic examination and bacteriologic findings. The clinical history will often furnish a well-founded suspicion and in such instances the case should be carefully observed and worked up until diagnosis is confirmed or disproved. No patient under suspicion of tuberculosis of the upper urinary tract should be treated for cystitis without proper investigation.

Cystoscopy.-There are conditions of acute cystitis accompanied by great vesical spasm when it may be practically impossible to conduct a satisfactory cystoscopy but with the proper type of instrument and irrigating attachment requiring a very moderate amount of vesical distention, it is possible at the present time to accomplish this end even in difficult cases in which it was formerly impractical. (See Cystoscopy.)

Examination should be made as short as is consistent with obtaining the desired information. The bladder is usually irritable and participates to a greater or lesser degree in the tuberculous condition. There may be tuberculous erstitis, more especially around the trigone, presenting an intensely inflamed and relvet-like appearance, studded here and there with minute tubercles; and there may be also ecchymoses and ulcerations. (See Tuberculous Cystitis.)

That which is more typical of tuberculous involvement of the kidney is the ureteroscopic picture. The ureter mouth may show simple swelling and redness of the areola (Plate III, Fig. 2). Bullous edema may be seen in the region of the ureter sometimes obscuring the orifice (Plate III, Fig. 2). In more advanced cases, there is a crater-like formation of the ureteral opening and an absence of normal contractility, showing instead an enlarged outlet exuding a thin 
or thick purulent secretion, continuous in flow. This ureteroscopic appearance is more or less typical but at times the cystoscopist is deceived by a similar picture on the opposite side or an appearance approximating this in connection with a non-tuberculous lesion of the kidney (Plate III, Fig. 6). It then becomes a matter of diagnostic necessity to perform double ureteral catheterism. This expedient is frowned upon by some but the evidence it provides is often indispensable. The operation should be done with great care and the ureteral catheters introduced gently and slowly. As an extra precaution, the ureters may be washed out with a small amount of boric solution before removing the catheters. The catheterized urine from both sides should be carefully examined for the presence of blood, pus and tubercle bacilli. As already stated, the pyogenic organisms are not found in abundance except in cases of mixed infection. Tubercle bacilli are sometimes difficult to be found ${ }^{1}$ but careful search should be made by the most approved methods. ${ }^{2}$

The kidneys furthermore should be carefully investigated by the appropriate tests with regard to their relative and combined functional activity. (See Chapter VII, p. 155.) This information is obtained by observing the urea volume and by the colorimetric estimation. A diminished total urea volume is important as indicating a comparative destruction of the secreting renal substance and is to be compared with the work of the opposite kidney. Deductions should be made with a certain degree of caution in cases in which the result is not emphatic inasmuch as the so-called reno-renal reflex from a diseased kidney may exercise a partial inhibitory influence upon the opposite organ, as is sometimes shown by the steady improvement in the urea output after nephrectomy. ${ }^{3}$

Roentgenography is of value in differential diagnosis for the purpose of excluding the presence of stone but it should be remembered that tuberculous foci also throw slight shadows especially when they contain lime salts. With the improved methods of $x$-ray photography and instrument manufacture, the value of this means is gaining increasing importance. Pyelography will reveal dilatation of the ureterabove a constriction and pelvic distention, and sometimes cavities: in the kidney itself. The guinea-pig inoculation test should be resorted to in all suspicious cases of doubtful character: in all cases

1 Rovsing, 6th International Congress of Tuberculosis, Vol. II, p. 269.

2 The Pappenheim method is recommended on account of decolorizing the smegma bacillus. Pappenheim (Berl. klin. Woch., 1898) found the bacilli in 80.7 per cent. of 162 cases of genitourinary tuberculosis.

3 Rovsing. 
of acid bacterial cystitis, where tuberculosis is suspected and not proven. This method of investigation usually requires three weeks' time unless the animal dies of advanced tuberculosis within this period. ${ }^{1}$

In addition to these special means of examination, it is of importance in reaching a diagnosis to seek out any corroborative signs of tuberculosis by means of a general physical examination. The lungs are not necessarily in a sufficiently advanced condition to reveal the fact; the testicles, epididymis, vas deferens and prostate are likely to be involved sooner or later in a certain proportion of cases, and in some, bone or joint lesions are present in an active or quiescent state. The results obtained by tuberculin tests are often confusing and it is desirable, if possible, to reach a diagnosis without this means. The different methods employed are described in the chapter on Sero Diagnosis, p. 105.

The prognosis of a malady of this insidious and far-reaching character must necessarily be conservative, and although it is eventually unfavorable in the majority of cases, it is remarkable how many of the subjects will improve in general condition and body weight after the active lesion has been removed surgically. It is a noteworthy fact how well the remaining kidney, when not the seat of an actual tuberculous focus, will care for its portion of the body economy after nephrectomy of the diseased organ.

The question of spontaneous healing of a tuberculous focus is an interesting one. It is stated on good authority that this does occur (Delafield), which does not refer to that condition, generally recognized, of complete destruction and functional cessation of a previously secreting tuberculous kidney. Even admitting, however, the occurrence of spontaneous healing, it must be considered, in the present state of our surgical knowledge, a curiosity and should not govern the rule of action. The intermittent or remittent course of some cases with the attendant improvement of the urine may be significant of the closure of a pyonephrosis, some of which cases develop perinephritic inflammation and cold lumbar abscess. Secondary infection of tuberculous pyelonephritis renders the prognosis more grave and demands immediate surgical intervention. ${ }^{2}$

1 A. Bloch, Berlin. klin. Woch., 1907, suggests bruising the glands of the groin and then injecting the animal, to stimulate a more rapid glandular involvement as a means of hastening this method of investigation.

${ }^{2}$ Author's case (Plate I), was one that had resisted the offer of operative treatment until active symptoms occurred of an alarming character. The operation revealed a secondary infection which produced a pyogenic abscess of the cortex of the kidney which had almost ruptured into the perinephritic tissues. Nephrectomy was performed, resulting in complete recovery and marked improvement in general health. 
That which has the most important bearing upon the production of an unfavorable prognosis is the occurrence of bilateral involvement and other lesions throughout the body. Perinephritic involvement is always a serious development rendering the prognosis more grave.

The final fatal issue may occur as a result of anemia, septicemia, anuria or uremic poisoning. Death follows operation in a certain proportion of cases. The liability to such occurrence is more or less dependent upon the extent of the localized lesion and that of the generalized condition as evidenced by the presence of other lesions. On the whole, the percentage of mortality is not high for the operation of nephrectomy as reported by competent operators. ${ }^{1}$

The duration of life after nephrectomy has an important bearing upon the prognosis. This, as variously stated, necessarily depends upon whether a case is operated upon early or late; and in a comparison of the duration of life in unoperated cases it must be taken into account whether such cases are unilateral or bilateral. This much may be stated, that the tendency of tuberculosis is to be progressive and the majority of cases of renal tuberculosis, although unilateral for a certain period, if left long enough, tend eventually to become bilateral. ${ }^{2}$

The assembled facts that the tendency of tuberculosis is to be progressive, and while as a rule in the beginning only one kidney is involved, that there is a strong tendency later on to implicate the opposite organ ; that the duration of life following operation ranges from several months to what would seem to be almost a permanent cure; and that the operative mortality is low, compose a strong argument in favor of early operation when tuberculosis of one kidney is established. ${ }^{3} \mathrm{~A}$ conservative estimate of the frequency of permanent cures in early and moderately advanced cases is given as between 60 and 70 per cent.

The percentage of duration of life under medical treatment cannot be considered as promising as that following surgical intervention. Those cases which seem to do well under favorable conditions, and there are some beyond question, are exceptions and should not govern

\footnotetext{
1 Binney (Prognosis of Renal Tuberculosis, Trans. Mass. Med. Soc., June, 1911) states that in the hands of some operators, the mortality in a series of 50 or more cases for unilateral disease is as low as 3 per cent.

Kummel (Arch. klinische Chir., Vol. Ixxxi), gives an operative mortality between 7 and 10 per cent. in a collection of 300 cases.

Watson, Boston Med.Journ., 1907, Vol. clvi.

${ }^{2}$ Chute, Trans. Mass. Med. Soc., June, 1911, calls attention to the observation that 70 per cent. of cases living are found to be unilateral whereas in 250 cases examined at autopsy, only 50 per cent. of unilateral is found.

${ }^{3}$ Binney, loc. cit., reports Israel's results of 75 per cent. living out of a series of 97 nephrectomies, the period of observation being from two months to fifteen years. The bacilli disappeared from the urine in 93 per cent. after nephrectomy.
} 
the rule of action. In the vast majority of instances the tendency is, as already stated, toward progressive advance of the disease, and statistics bear out this assertion. ${ }^{1}$

Treatment.-The treatment of renal tuberculosis is either expectant (including tuberculin medication) or operative. The expectant treatment comprises hygienic measures, careful living and climate regulation. Reliance upon this course should be confined either to the very mild cases which can be under careful observation and in favorable climatic surroundings, to the very advanced cases which are beyond assistance by operative intervention and to those cases which are subjected to a preliminary tuberculin course. (See Serotherapy, p. 110.) In regard to the latter procedure, judgment as to its value is still under suspension. There is little convincing testimony as to its favorable influence. With respect to the propriety of subjecting patients with very slight tuberculous lesion to a preliminary expectant treatment in place of operation, there is decided difference of opinion with the majority in favor of early operation. In favor of the contrary course, however, it may be stated that reliable observers claim that spontaneous cures have occurred but as these cases are so few and are the exceptions, such an alternative course can only be justified with a full knowledge of the uncertainty involved and only under most promising conditions of climatic surrounding and careful management. In the very advanced cases, there is no alternative. In the moderately advanced cases the patient is given the best chance by the operative course.

Operative Treatment.-Total nephrectomy is the operation commonly performed. The ureter should also be removed when involved, although surprisingly good results have been obtained following nephrectomy when the ureter, although diseased, was ligated and left behind. Pronounced involvement of the bladder does not contraindicate nephrectomy; on the contrary, if the involvement is not extremely advanced, marked improvement often follows the operation of nephrectomy. When the testicle is also implicated, orchidectomy should be performed. Partial removal of the kidney is of no value on account of the uncertainty of the diagnosis with respect to the remainder of the organ. Nephrotomy is reserved as a palliative operation in cases of bilateral lesions when required as a matter of immediate necessity for an exigent surgical condition. (Description of operations upon the kidney, see Operative Surgery, p. 632.)

1 Horace Binney, loc cit., quotes Blum and the deduction derived from the latter's cases under medical treatment for six months or longer, thirty-two cases in all, a mortality of 75 per cent., an average duration of life, two years and three months. 


\section{VESICAL TUBERCULOSIS}

The occurrence of tuberculous cystitis primarily is rare as it is usually secondary to renal or genital tuberculosis. Any cystitis of prolonged or doubtful origin should raise a question as to its possible tuberculous character. The mere presence of tubercle bacilli in the urine is not sufficient evidence nor is the contact of such urine necessarily productive of infection. The transmission of tuberculosis during coitus has not been unquestionably established although cases of strange and impressive coincident in this particular are mentioned. Vesical tuberculosis should generally be regarded as a part of and not the sole extent of a urogenital tuberculous lesion, and the percentage of cases in which it forms a part of such lesion is high. ${ }^{1}$

It is less common as an ascending lesion from the genitals. ${ }^{2}$

Morbid Changes.-As a rule, morbid changes characteristic of vesical tuberculosis are located in the region of the ureter or the urethra and seem to centralize at one or the other of these points according as the affection is ascending from the genital tract or descending from the upper urinary region. At first, there are seen dispersed upon the surface of the mucosa, solitary or clustered tubercles. These begin as subepithelial deposits giving the appearance of grayish, transparent granulations. Later, degeneration occurs and the grayish color yields to a yellowish hue. The centre of the nodules becomes excavated with the resulting development of a tuberculous ulcer. When these small ulcerations coalesce, the surface of the bladder becomes more generally involved, presenting marked alterations of the mucous membrane as a result of infiltration and epithelial transformation; or on account of involvement of the muscularis, by hypertrophic changes and secondary contraction, there is impairment of the normal expansion thereof. If the ulceration penetrates deeper, fibrous inflammation of the perivesical tissues is the outcome and adhesions with other organs occur producing fistula and cold abscess. In extreme cases, the contraction of the bladder is so extensive as to allow of the retention of practically no urine whatever.

An examination of the urine will reveal the presence of tubercle bacilli, which are usually readily found.

Symptoms.-Tuberculous cystitis presents all the symptoms of cystitis from other bacterial origin, but with a greater degree of intensity, a decided tendency to chronicity and a constant suscepti-

\footnotetext{
${ }^{1}$ In 86 per cent. of Braasch's cases, loc. cit., the bladder irritability is the primary symptom,
} In some cases this is only a reflex. Judging clinically, it seemed to be present in about twothirds of the cases of tuberculous lesions of the kidney.

2 In thirty-seven cases of Braasch in which the testicles, epididymis or prostate were affected, there were only five in which the bladder showed ulcerative tuberculous lesions. 
bility to irritation. In the beginning there is likely to be only slightly increased urinary frequency with microscopic hematuria. The symptom of hematuria is one of the earliest phases of the trouble, appearing at times as a slight terminal bleeding without apparent preëxisting cause but found upon careful examination to be accompanied by the presence of tubercle bacilli in the urine.

Frequency of urination begins to be noted both day and night, and increases with the development of the disease, according to its progress, slowly or rapidly. In advanced cases the desire to urinate becomes almost constant during the daytime and during the night there may be spasmodic incontinence.

Pain in the beginning is not great and amounts more to an irritability and discomfort, which is experienced when the bladder has reached its capacity and sometimes for a short time after it is emptied. During the active stages of the disease the pain becomes much more intense. It is experienced when attempt is made to hold the urine overtime, or it begins with the action of urination and increases in severity as the last drops are voided. Between urination there is sometimes a burning sensation felt in the suprapubic region or in the perineum, the latter when the prostate is also involved. In the late stage of the malady the pain in connection with urination is almost continuous, on account of the frequency of the latter, and this in spite of the free administration of anodynes.

The urine contains the elements which are commonly found in all inflammatory conditions of the bladder to a greater or less degree, and are detected by both clinical observation and laboratory examination.

The blood in the urine is, as stated, an early manifestation. It may be microscopic but soon becomes evident clinically as a few drops of blood pass with the last urine, or mingled more freely with the entire amount. It occurs in the beginning as a result of congestion around the neck of the bladder as this is the most common point of infection whether derived from a previous genital or renal tuberculosis. Later in the disease the blood becomes more abundant, due to the occurrence of ulcerations in the bladder.

Pus in the urine makes its appearance early in the course of the malady when there is a mixed infection, such as is likely to be the case when there has been an old chronic posterior urethral or bladder lesion. Otherwise the pyuria occurs as a symptom of tuberculous cystitis at the time when ulcerations of the bladder mucosa have developed. It should be borne in mind that the pus in the urine is not necessarily due to bladder inflammation as it may be derived from one or both kidneys (see Renal Tuberculosis, p. 659), and in all cases 
of supposed chronic cystitis, especially when tubercle bacilli are present, this question should be carefully investigated, especially by means of cystoscopy (see p. 129). A characteristic of vesical tuberculosis is that the purulence is constant, while in the case of pyonephrosis it is quite frequently intermittent.

The quantity of urine is usually increased (pollakiuria) due to renal changes. The reaction is always acid except in the event of contamination by the ordinary pyogenic organisms when decomposition sets in and an alkaline urine may be the result.

Incomplete retention is likely to be an accompaniment in some cases due to swelling and edema around the neck of the bladder or to secondary contracture of the vesicle orifice as a result of protracted inflammation. (See Contracture of the Neck of the Bladder, p. 435.)

Sudden attacks of complete retention of urine may occur as is the case with other forms of cystitis, as a result of irritation or injury to the urethral orifice of the bladder, an intensely painful condition which if not relieved is attended with the incontinence of overflow.

Spasmodic incontinence occurs in the later stages when the bladder is contracted on account of deep inflammation and ulceration. Should the neck of the bladder be destroyed by ulcerative invasion a true incontinence may occur.

Diagnosis.-The symptoms of cystitis in general characterize this malady with the distinguishing feature of an insidious and unexplained onset. Recognition of the cystitis is not difficult. The important information to be obtained is whether or not tubercle bacilli are present as an explanation of the cause and what part the cystitis plays in a possible more general urogenital tuberculosis. The aim therefore is to determine whether or not with the vesical inflammation there is coexistent involvement of the upper urinary tract or a genital tuberculosis.

Cystoscopic examination will reveal the lesion in the interior of the bladder, its characteristic appearance and limitation. Swelling, redness, and ulceration of the mucosa are significant. Minute tubercles are occasionally seen distributed over portions of the surface. The swelling may be smooth and velvet-like or present here and there bullous edema. The redness is produced by intense congestion or subsurface hemorrhages in spots or diffused streaks. Ulcerations appear as punched out portions of the mucosa, irregular in outline and a reddish or yellowish surface which bleeds readily. When the disease has extended from the upper urinary tract downward the cystoscopic observation will recognize its localization about the orifice of the ureter from whence it will appear to radiate (Plate III). In 
the case of its extension from genital tuberculosis the process is more likely to be seen toward the fundus of the bladder around the urethral sphincter.

Bimanual examination and palpation may discover a firm and contracted organ which is tender to pressure and not unlike the uterus in feeling (p. 65). At times nodular involvement of the seminal vesicles and prostate is encountered. When the disease has extended from the kidney and ureter the latter may be felt through the rectum on the floor of the bladder above and to the outer side of the seminal vesicle (see p. 66). In the female, ureteral induration is more readily distinguished by vaginal palpation.

Prognosis.-In general the course of vesical tuberculosis is progressive and extension to the adjacent organs is likely to occur. There is a decided variation, however, in the progress of the disease in different individuals, and also according to the type of involvement to which the particular case belongs. In other words, the prognosis of vesical tuberculosis is good if the lesions have originated in the kidney, if the process has not advanced too far, and if nephrectomy is performed $;^{1}$ and the prognosis may be good in a few cases in which there is a mild grade of infection without the tendency to a gradual increasing intensity when such mild condition continues for years. This satisfactory state of affairs is only relatively so and it is only in rare cases that the various factors exerting a favorable influence apparently cause a cessation of the process, either spontaneously or as a result of therapeutic measures. This is less likely to be the case when there is genital involvement, but may follow conservative surgical intervention in the case of the removal or drainage of an active focus in the prostate or testicles. Generally speaking, however, the prognosis of advanced cases, especially those which follow ascending infection from the genital organs, is exceedingly unfarorable, and the outlook is toward progressive advancement with impairment of the bladder function and general constitutional depletion.

Treatment.-The ordinary treatment for cystitis is not only useless but sometimes even harmful in the case of tuberculous involvement. In deciding upon the course of treatment to be pursued it must be determined whether the vesical condition is a secondary infection of renal or genital origin. The selection of treatment will then be controlled by the location of the principal lesion, and later action governed by the indirect influence wrought upon the vesical condition by removal of the primary focus. This applies more particularly to a primary renal

1 Eighty-two per cent. of Braasch's cases (loc. cit.) were alive three years after nephrectomy, and presented either improvement or recovery from the bladder symptoms. 
focus and its removal by nephrectomy. It also applies to active focal lesions in the testicle and epididymis although the removal of such is not so likely to be followed by marked improvement in the vesical condition on account of the likelihood of there being also prostatic or vesicular involvement in connection with that of the testicle.

The treatment of genital tuberculosis is considered under its appropriate heading and should be given due consideration when it is found that there is genital involvement along with the vesical condition.

The treatment of vesical tuberculosis pure and simple embraces general measures including in ternal treatment, local trea tment, operative treatment.

The general treatment is that which is recognized as appropiate for all tuberculous conditions, such as abundance of fresh air, suitable climatic surroundings, good hygienic conditions, etc. In fact, instances of marked improvement are seen in cases with only mild tuberculous involvement of the bladder and genital regions, as a result of transplantation to a proper climate, such as a change from a cold to a warm climate, or to a high and dry altitude.

General tonic medication is accomplished by the various means at command including the internal use of the hypophosphites, iron, quinine and arsenic, cod-liver oil and mixed fats. Cacodylate of soda is also valued for its constitutional effect and is used hypodermically daily or three times a week in doses of 5-10 centigrams each. To this end lecithin has also been used internally as a general tonic and tissue builder and ichthyol in capsules of 0.5 to $1 \mathrm{gm}$. ( $71 / 2$ to $15 \mathrm{gr}$.) three times a day. When the pain becomes severe sedatives and anodynes are demanded; morphine is the most useful of these and can be given hypodermically or in suppositories. Codein is sometimes substituted as a sedative. The extent to which these anodynes can be used must be governed by each individual case.

Local Treatment.-Various local applications have been resorted to in the effort to obtain relief from the distressing symptoms of tuberculous cystitis, and at the same time to exercise some control upon the inflammatory condition.

Irrigations are on the whole of little value, and distention of the bladder is not desirable. The employment of instillations of smaller quantities of solutions is less objectionable and more beneficial. The bichloride of mercury has on the whole proved more effective than any other local application. It is given by means of a deep urethral syringe (see p. 35) in solutions of 1 to 16,000 to 1 to 8000 , from 1 to 2 c.c. $(m x v$ to $\mathrm{xxx})$ at each application, and repeated two or three times a week. In some mild cases daily applications are service- 
able. The strength of the solutions and the frequency of the applications should be governed by the freedom from resulting irritation. It is not desirable to increase the strength or repeat the treatment when irritation is produced. Gomenol seems to possess some advantages as a local application in this condition and is used interchangeably with the sublimate instillations or independent of them. It is employed in a 10 per cent. mixture of sweet oil of almonds and injected in the same manner as the sublimate about 1 to 2 c.c. $(m \times v$ to $\mathrm{xxx})$ at a time and left in contact with the inflamed tissues of the bladder. Of the other local applications that have been used the emulsion of iodoform has met with some favor. The following mixture is prepared:

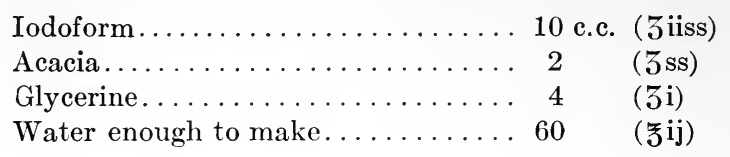

Of this mixture 4 c.c. ( $3 \mathrm{i}$ ), after shaking well, are added to 2 ounces of 2 per cent. boric solution and as much of this solution as can be comfortably retained is injected into the bladder through a small softrubber catheter. After the iodoform has been given time to deposit the greater part of the solution is allowed to return. This treatment is repeated every day. None of the other topical applications has demonstrated any particular value, and all local treatment should be employed with the greatest caution, otherwise more harm than good may result.

Operative Treatment.-Early surgical intervention in tuberculosis of the bladder entailing drainage and curettage has no value and has been abandoned. In the late stages operation is only warranted as a last resort, on account of free hemorrhage and suppuration which are not controlled by local treatment and in the attempt to relieve the bladder from incessant spasm by drainage, when pain becomes intolerable. Under these conditions, in the male, a suprapubic cystostomy is performed as a palliative measure, and in women, a vaginal eystostomy with the same purpose.

The various serums containing tuberculin which have been employed therapeutically in urogenital tuberculosis have not satisfactorily demonstrated their value.

Inasmuch as a number of cases of supposed tuberculous cystitis are due to mixed infection and therefore dependent upon the action of the pyogenic organisms it may be found that some of these cases 
are markedly controlled by the employment of mixed vaccines and bacterines. (See Vaccine Therapy, p. 105.)

\section{GENITAL TUBERCULOSIS}

Tuberculosis of the Prostate.-Genital tuberculosis is more often associated with tuberculosis of the urinary organs, although in a certain number of cases involvement of the prostate or of the testicle or both is an associate condition of phthisis, in which case it may be regarded as a primary involvement of the genital organs, especially when the pulmonary focus is a mild one. There is little doubt that the bacilli may be transmitted to the prostate by way of the blood-vessels or lymph channels, and from the kidney the disease finds easy access to the prostate by way of the bladder, by direct extension and through the urinary stream. Genital tuberculosis having commenced in one of the other organs, the seminal vesicle or testicle, is very likely to implicate the prostate secondarily as it is well recognized that this gland is readily susceptible to any kind of an infection. The youthful subject, being especially prone to prostatic congestion and infection, is a favorable victim for tuberculous involvement.

Heredity seems to exercise a marked predisposition. Even though there be no definite transmission of the disease it is a well observed fact that individuals who give a family history of tuberculosis are especially susceptible to trauma of the prostate, and in such cases injury may be one of the determining causes in the clinical history of a tuberculous prostatitis. Again subjects of this class who develop gonorrhea are particularly liable to protracted cases, and have a special predisposition to prostate gland involvement.

The morbid changes of a tuberculosis of the prostate are similar to those of tuberculosis of other parts. Miliary tuberculosis has no surgical significance, being a part of a generalized process affecting the entire organism.

That type of tuberculosis of the prostate which possesses surgical interest is a chronic condition embracing several stages and different lesions, namely, the deposit of tubercles, the caseation thereof and the formation of abscess. The tubercles appear first as isolated grayish nodules beneath the epithelial lining of the ascini and excretory ducts. They become confluent owing to caseous degeneration, thus forming a caseous nodule surrounded by an infiltrated area of inflammation. Suppuration commonly occurs ending in an abscess of varying dimensions. These abscess formations may involve a good part of the gland in a single sac or there may be several large foci. Like abscess of the 
prostate from other causes there is a tendency to rupture, if not relieved surgically, in which case evacuation may occur externally, or into the urethra, bladder or rectum, resulting in the production of fistulous tracts, having a marked tendency to chronicity.

Symptoms.-When the process commences in the centre of the prostate development may be slow and the symptoms insignificant until the surface of the gland is reached or the urethra and bladder invaded. When the urethro-resical mucosa becomes involved the symptoms are those of urethro-cystitis, including pain and disturbances of urination with often a mucopurulent or bloody discharge. Palpation per rectum will detect the enlarged prostate gland with hard and uneven surface, and sometimes a softened area denoting suppuration. The diagnosis is confirmed by the presence of tubercle bacilli in the discharge expressed from the prostate, and the presence of other foci of genital tuberculosis in the testicle or seminal resicle will have an important bearing thereupon.

The tuberculous prostate is extremely sensitive to pressure. The recognition of the boundary of the prostate may be disturbed by simultaneous involvement of the seminal vesicle producing a diffused and irregular mass covering the area of the base of the bladder felt by rectal palpation.

The personal and family history are also to be taken into account. The prognosis is grave, although in some cases the progress is slow. The most unfarorable aspect is the likelihood of bladder involvement, and the outbreak of a more generalized condition.

Treatment. - Generally speaking the treatment is palliative as there are no radical measures of value. Such hygienic precautions as are advised in all tuberculous conditions are appropriate here and if permanent location in a suitable climate is possible such a course should be urged. Chronic gonorrheal lesions, if present, should be carefully treated having always in mind the great gentleness necessary in connection with instrumentation. Local applications are of little value so far as the prostate itself is concerned. When the bladder becomes involved the treatment is that of vesical tuberculosis (q.v.). Various operations have been practised including complete extirpation, but on account of the coexistence of periprostatic and perirectal infiltration as well as associated infection of the seminal resicles, complete operation is well-nigh impossible and if performed the second state of the patient may be worse than the first. This is notably the case when the operation is performed in unfarorable climatic surroundings where the opening-up of uninvaded tissues tends to invite fresh infection. When abscess of the prostate occurs a palliative operation 
for evacuation of the pus and drainage must be resorted to, on which occasion a careful enucleation of tuberculous tissue may be included in the operation without attempting complete radical removal.

The Seminal Vesicles.-Tuberculosis of these organs is rarely discovered as a primary infection. Although rectal palpation may detect nodules in the vesicles without obvious involvement of the prostate the condition is usually associated with lesions of some other part of the genitourinary tract, very commonly the testicle on account of the possibility of its conveyance by means of the semen. The prostate, vesicle and epididymis may all be implicated at the same time. Adults are more commonly affected than children, owing to the activity of these organs and the possibility of trauma and gonorrheal inflammation, which tend to furnish a predisposition to tuberculous involvement in any of the genital organs.

The morbid changes resemble those attacking the prostate. Tubercles are deposited beneath the surface of the mucosa producing confluent and caseous patches. Nodular tuberculosis is characterized by the formation of larger tubercles which may be felt from the outside of the vesicle by rectal palpation in circumscribed areas; or there may occur diffused infiltration of the entire gland incident to the tuberculous invasion which upon rectal palpation is felt as a hard and irregular mass in the vesicular region beyond the prostate. If both vesicles are affected infiltration of the intervening space occurs so that their outline on the floor of the bladder cannot be distinguished.

Symptoms.-There may be no subjective symptoms, or the patient may complain of a sense of heaviness in the perineum and pain may be complained of during and after urination. With the involvement of the adjacent organs, notably the bladder, the symptoms are in keeping with the coexisting conditions. With the progress of the malady, changes in the quality of the semen occur, oligo and azoöspermia are to be expected and finally the sexual function gradually becomes obliterated. Diagnosis depends almost entirely upon rectal palpation and the discovery of tubercle bacilli in the urine and semen. Vesicular tuberculosis may develop during the course of gonorrheal infection of the deep urethra, when the usual signs of seminal vesiculitis are observed, and after the subsidence of the acute stage, tuberculous nodules are formed in the vesicles which may be detected by rectal palpation. These should not be confused with ordinary inflammatory infiltration which is likely to follow any acute involvement of the vesicle.

Treatment.-The palliative method of treatment is preferable in most cases and such a course of treatment embraces the usual hygienic 
measures and restrictions re̊garding physical strain and physiological exertion, together with the administration of cod-liver oil, general tonic medication and the other therapeutic agents employed in tuberculous cystitis (p. 638). If the disease tends to progress in spite of such care and precautionary measures, removal to appropriate climatic surroundings should, if possible, be accomplished. Surgical intervention should only be resorted to in extreme cases and notably in those in which the lesion is localized in the vesicle and can be effectually reached by perineal incision. (See Operative Surgery, p. 491.) Even in such cases it is a question whether vesiculotomy and drainage are not preferable to an attempt at complete removal.

There is no local treatment of especial value for tuberculous vesiculitis. Therapeutic measures generally are governed by symptomatic indications.

With regard to the possibilities of vaccine treatment the same may be said here as in the case of tuberculous cystitis or prostatitis, that the combined invasion by pyogenic organisms may yield a favorable result from the treatment with mixed vaccine or bacterines. (See p. 113.)

The Testicle, Epididymis and Vas Deferens. - A most common site of tuberculosis of the external genitals is the epididymis. The diseased process is generally limited to this structure for some time before attacking the body of the testicle. The tail of the epididymis is its most favorite location (Fig. 271), although the head is sometimes independently or jointly involved (Fig. 270). From the epididymis, the disease extends by way of the mediastinum to the body of the testicle (Fig. 269). The development of tuberculosis of the testicle may be primary so far as the genito-urinary tract is concerned, by transmission through the blood or lymph channels from the pulmonary or gastro-intestinal region, but is much less common than a primary focus in the kidney. Infection may also occur as a result of extension from the neighboring organs, the bladder, prostate and seminal vesicles, or possibly from one testicle to the other. The disease may begin as a renal tuberculosis and clescend through the urinary canal to the epididymis and testicle. When the disease attacks the body of the testicle it may occur as a primary focus, but is usually derived by extension from the epididymis.

Hereditary predisposition may furnish a partial explanation of some cases, but such an element is not at all essential, while traumatism and previous inflammation of a protracted nature are important exciting factors in determining the deposit of tuberculous infection in this region. There may or may not be discovered an active tuber- 
culous focus in another part of the body. It is only necessary that the tubercle bacilli be present in the blood for them to find a point of weak resistance, and this the testicle furnishes when rendered receptive by injury or prolonged inflammation.

Tuberculosis of the testicle is most commonly met in individuals in the prime of life; its occurrence in extreme youth and old age is rare. The process is as a rule in the beginning unilateral and may extend to the prostate and thence to the bladder or the opposite testicle.

Morbid Changes.-The tubercles are deposited in the walls of the tubular ducts of the epididymis, causing active cellular proliferation and production of characteristic tuberculous tissue. This has the

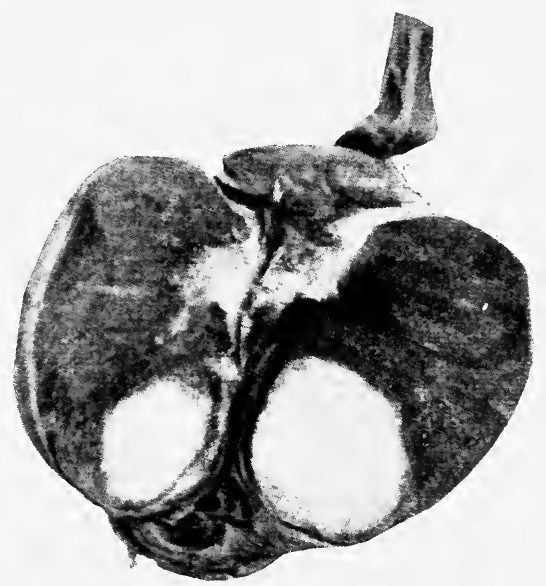

FIG. 269.-Tuberculosis of the body of the testicle.

tendency to form nodules and undergo caseous softening which in some instances ultimately become cavities (Fig. 269). The testicle on section is seen to present the same characteristic lesions in the seminiferous tubules which become caseous nodules. Granulation tissue is also produced at the expense of the normal structure of the testicle, or the entire testicle may be converted into a cheesy mass. The tunica vaginalis, while not at first involved, becomes adherent to the testicle and as a result of inflammatory irritation serous effusion or hydrocele is produced. Later there are perforations of the tunica albuginea and of the tunica vaginalis with resulting external fistulæ.

Clinical History.-Invasion of the testicle may be an acute and rapid development in which case there is pain and swelling and all of the accompaniments of an acute epididymitis, without, however, 
the history of a preëxistent acute urethritis. Such invasions are known to occur not alone in individuals in whom a tuberculous diathesis can be recognized but also in those who have appeared to be strong and well.

Generally, however, the development of the disease is slow and there is in the beginning little local or constitutional evidence of its presence. Sooner or later palpation will detect a swelling in the epididymis, usually the tail, or in the body of the testicle. This swelling may consist of a small nodule the size of a pea or chestnut, or the entire epididymis may be indurated and enlarged with nodular projections in the region of the tail or the head or both (Figs. 270

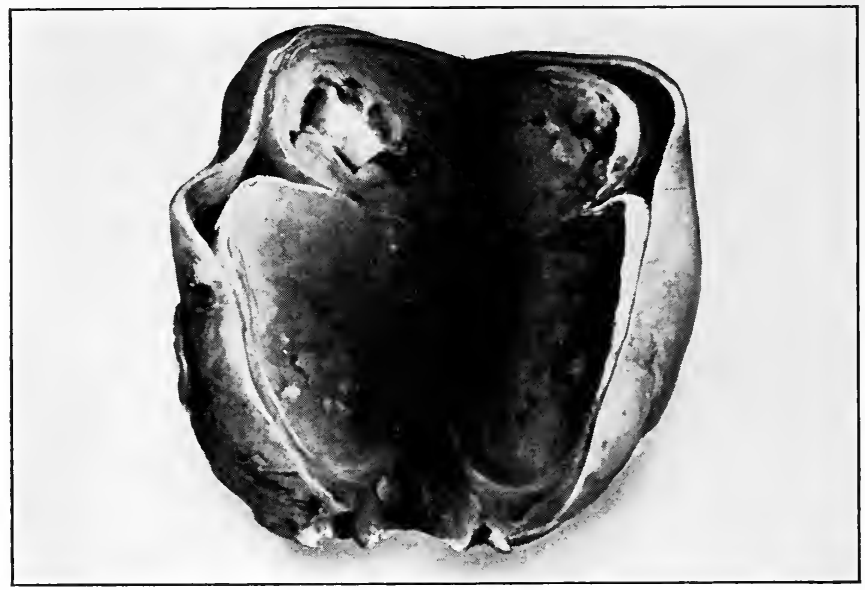

FIG. 270.-Tuberculosis of the head of the epididymis.

and 271). The vas deferens may not be involved, when it will feel perfectly normal on examination. At other times the hardening and thickening of the vas from the point of its attachment of the testicle to where it disappears in the groin may be distinguished by palpation. In this case it is increased double in size and is very much less flexible. When the process extends toward the surface and one or more foci find an external outlet, as many fistulous tracts are observed, and these are surrounded by areas of inflammatory edema leading down to the epididymis or testicle.

Diagnosis.-The suspicion of tuberculous involvement of the testicle is aroused following an acute swelling when the clinical history does not furnish sufficient explanation of such an outbreak in the presence of a dormant deep urethral lesion. Constitutional impair- 
ment under such conditions, with resistance to the ordinary palliative means of relief, showing a tendency to become chronic, would strengthen the suspicion, and later developments will serve to settle the question. When the genital focus is a local manifestation which has occurred along with a generalized tuberculous condition, the diagnosis is not, as a rule, doubtful. In the slow and insidious form of the malady this very feature alone is enough to raise suspicion. This is sustained by the local evidences of nodular formation, the tendency to adhesion to the tunics and outside covering, and the formation of fistulous tracts which will sometimes emit a discharge containing tubercle bacilli.

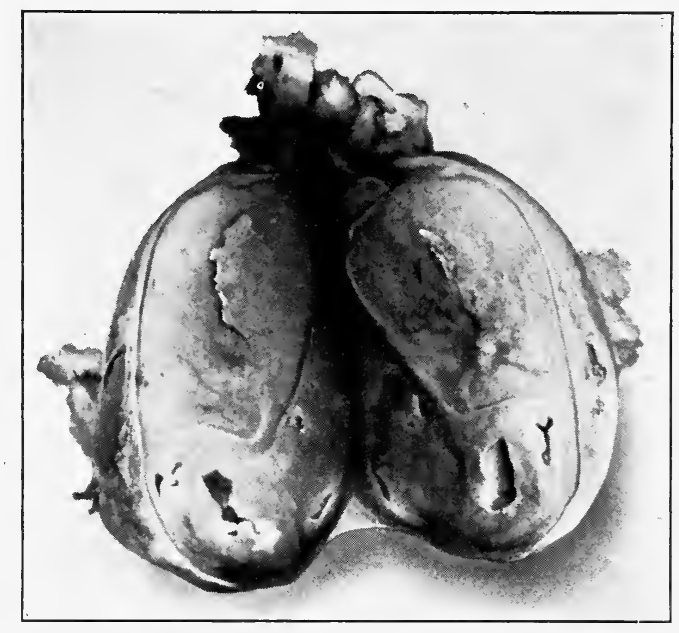

FIG. 271.-Tuberculosis of the tail of the epididymis.

Prognosis.-So far as the patient's general health is concerned the prognosis is governed by the general systemic condition, that is, the extent of such process and the body resistance thereto. So far as the testicle itself is concerned and its effect upon the general system, these depend largely upon the dissemination of the disease. If it is limited to one testicle, the epididymis or the entire gland may be removed which may bring about an apparent end of trouble. Secondary recurrence in such instances will depend upon the condition of the adjacent organs; and, if they are affected, whether or not they are secondary to the testicular lesion, in such a case it is possible that the removal of the testicle be followed by subsidence of an active condition in the prostate or seminal vesicle. When, on the other hand, involve- 
ment of the testicle is the result of an extension from the adjacent organs, such as the bladder or prostate, the prognosis must be regarded as much less favorable and secondary recurrence in the other testicle is likely.

Treatment.-The indications vary according to the phase in which the involvement of the testicle is presented. In the acute form the treatment is entirely palliative and the same as that for acute epididymitis from other cause. When the acute stage has subsided further action will depend upon the general and local conditions. The various constitutional measures that are of recognized value should be resorted to, such as iron, arsenic and cod-liver oil internally and the other remedies recommended for vesical tuberculosis, including nutritious food, and out-door life. In mild lesions under proper climatic surroundings it is possible to obtain a spontaneous cure. In the more indolent and chronic variety of the malady, when the disease becomes a definite surgical focus, operative intervention of some kind is called for, and the different operations resorted to are epididymectomy, castration, complete removal of the testicle and genital canal, including the seminal vesicle. (See p. 358.)

Epididymectomy.-When the disease is confined to the epididymis, the body of the testicle not being involved, removal of that portion of the organ may afford a satisfactory result and be accompanied by great improvement in general health. Naturally following this, as well as all operations for tuberculosis of the testicle, attention should be directed to the care of the general health and the adoption of such measures as will tend to fortify resistance against the activity of the disease by further invasion.

Castration.- - This operation becomes necessary where the body of the testicle as well as the epididymis is involved and in this case as much of the vas deferens as possible should be removed at the same time. In some cases where there is found to be a diseased kidney and testicle on the same side, removal of both these organs may have the effect of exercising a decidedly beneficial influence upon the general health and be the means of prolonging life.

Bilateral Castration.-Double castration will naturally be objected to and the demand therefor must be weighed carefully. If both testicles are extensively and irretrievably damaged there is no choice. There are many cases, however, in which a unilateral castration accompanied by an epididymectomy or partial removal of the opposite testicle, followed by appropriate constitutional treatment and hygienic measures, might be the means of preserving the general health, as well as affording the patient whatever benefit is to be derived from the 
retention of a small portion of the organ, be that physiological (internal secretion) or psychological (mental satisfaction).

Complete removal of the genital canal, including the seminal vesicles is not usually followed by satisfactory results unless it be in that part of the country where favorable climatic surroundings tend to aid the tuberculous patient in resisting his inherent malady during the postoperative stage. Except under such favorable conditions it is wiser to effect a removal of the active surgical focus, the testicle and as much of the cord as possible and then to resort to appropriate treatment along constitutional and hygienic lines. If the patient can be transferred to an appropriate climate so much the better. (For Operations upon the Testicle and Epididymis, see Chapter XIX.)

Tuberculosis of the Urethra.-Involvement of the urethra by tuberculosis is not frequent. The infection is usually by direct transmission and is therefore more common in the posterior region where it is an extension from the bladder or prostate. Periurethral tuberculous lesions are derived through the lymphatic system from a near-by tuberculous source. The morbid appearances in the anterior or posterior urethra are granulations and ulcerations. When the lesion is periurethral the resulting lesion is an infiltration over a circumscribed or diffused area which causes more or less stenosis of the urethral calibre.

The symptoms of this type of urethral involvement depend upon the character and extent of the same. In general the symptoms are those of urethritis or disturbances of urination. If the condition is in the anterior urethra there will be a mild urethritis accompanied by the usual symptoms without satisfactory explanation. If it is in the posterior urethra the symptoms are those of urethro-cystitis and similar to vesical tuberculosis. When the submucous or periurethral structures are involved palpation will reveal induration and infiltration and catheterization is interfered with. In case the usual explanation for the occurrence of urethritis and resulting stricture cannot be found, urethroscopic examination reveals changes in the mucosa and ulcerations suggestive of tuberculosis; and when there is present indolent inguinal adenitis, and perhaps other tuberculous lesions are discovered elsewhere throughout the body the diagnosis of this condition may be established. It is possible, however, to be confused by a persistent and indolent urethritis of chronic granular type in a tuberculous subject.

Treatment.-The treatment is more or less symptomatic. In subjects of a pronounced tuberculous constitution such measures as are generally approved and already mentioned should be resorted to. 
The local treatment in mild cases of uncertain character should be palliative and the same measures as those adopted for urethritis of an indolent type in sensitive individuals whose general condition suggests malnutrition and inability to throw off the localized condition. In such cases it is likely that improvement in general health will favorably affect the urethral condition. When the condition is unquestionably tuberculous the most effective treatment is the instillation or injection of bichloride of mercury from 1-16,000 to $1-10,000$ as recommended for tuberculous cystitis. The use of iodoform emulsion and boric solution is also recommended when the lesions are painful (p. 670). In case of stricture the gentlest kind of manipulation is called for and as it is possible that dilation may prove ineffective internal urethrotomy may be demanded and drainage instituted. It is not to be expected, however, in such cases, as in all others of a tuberculous nature that surgical measures will avail against unfavorable constitutional and hygienic conditions. 


\section{CHAPTER XXXVIII}

\section{CHANCROID}

\section{CAUSATION AND CLINICAL HISTORY-COMPLICATIONS- TREATMENT}

Chancroid is a local and contagious ulcer exuding a virulent pus, commonly venereal in origin, but freely inoculable from its own secretions from one person to another or upon the individual already a victim to it. These features give to the chancroidal ulcer a definite individuality and distinguish it emphatically from the typical chancre or primary lesion of syphilis, which, while a local ulcer, is the initial manifestation of a general constitutional disease. This difference has been recognized from earliest times, but the identity of the syphilitic sore and this purely local lesion was only settled during the present century. In noting this distinction, it is well always to bear in mind the existence of a so-called mixed chancre, a lesion that may result from infection with both of these maladies.

The name of chancroid is established by usage and generally employed in this country. It has otherwise been spoken of as "soft" chancre to distinguish it from "hard" chancre which is a term usually applied to the primary syphilitic lesion. Such a qualifying title has a disadvantage for general usage as it implies invariable characteristics which do not exist always but, on the contrary, may be reversed. This peculiarity of "hardness" or induration, however, is so thoroughly typical of the syphilitic chancre that it is commonly identified with it and properly so, whereas the condition of hardness simulating it in chancroid is exceptional.

To acquire the chancroidal ulcer it is necessary that there be an abrasion of the surface but it is possible that the virus may remain in contact with an unabraded surface and by irritation and erosion gain entrance after a few days' time. Conditions of uncleanliness naturally conduce to infection, but it is only necessary that the virus should be implanted upon a denuded or wounded surface to become inoculated.

Causation. Site and Number.-Chancroid is a venereal ulcer and therefore commonly makes its appearance on the genitals, although it may appear in any region that is infected by the virus of chancroid.

In the male, the ulcer commonly appears upon the glans and the 
prepuce, notably the sulcus near the frenum and the preputial margin (Fig. 272). The common site in the female is the fourchette, the labia and the region of the urethral orifice. It also occurs in both sexes around the anus and rectum.

Infection occurs by direct contact which is the usual method but contagion may be transferred indirectly through some intermediate object such as soiled linen dressings or the hands. It is possible, but

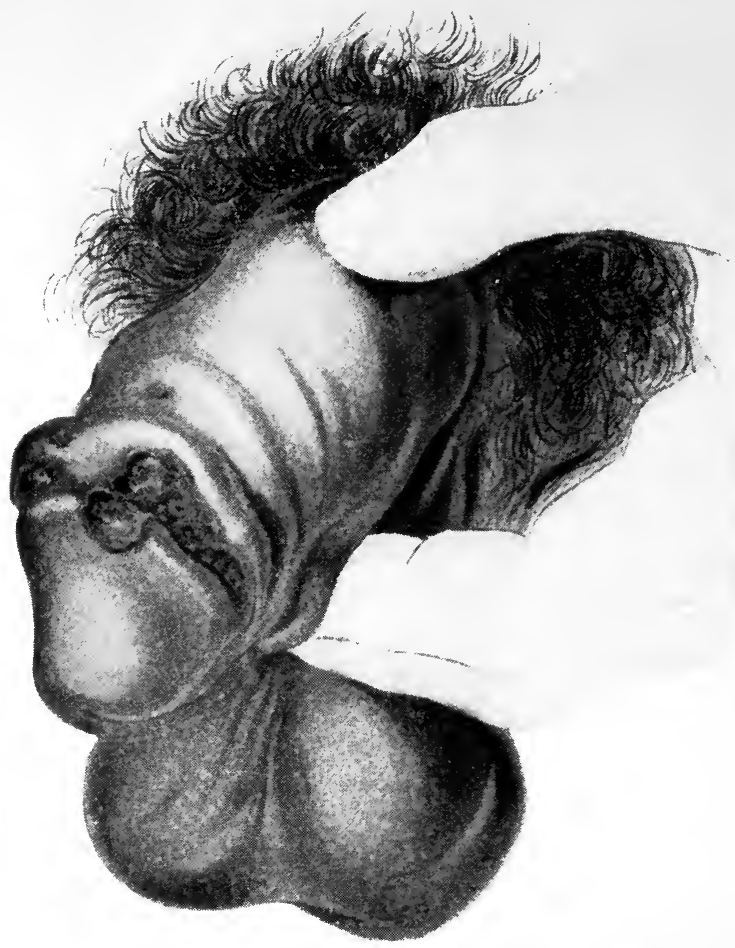

FIG. 2-2.-Chancroids of prepuce and glans. (Johnston's Atlas.)

not likely, that the ragina of the female or the prepuce of the male may harbor the virus of chancroid and be an intermediate object of transmission without becoming infected.

Inoculability of Chancroid.-It is possible to reproduce typical ulcers by reinoculation in various parts of the body of the patient. There is a difference in the susceptibility of different regions to this transmission. Sores produced on the face and upper parts of the body are less obstinate than those upon the abdomen and thighs. Temporary immunity is developed in an area subjected to first inoculations. 
In view of the characteristic inoculability of chancroid, it will be recognized that it usually occurs as a multiple lesion. These multiple lesions may represent separate points of original infection or some of the sores may be auto-infections, sometimes on the surface of the prepuce contiguous to an original sore or at some other point where an abrasion has existed and the virus of the sore has contaminated it.

Frequency.-The frequency of chancroid is seen more often in hospital and dispensary patients than among the better classes on account of the partial protection afforded by cleanliness. This also applies to the reinoculation and spread of this malady upon individuals of the dispensary class who, in reality, present the severe types of chancroid that are seen.

Pathology.- - The area involved by chancroid is an erosive inflammation surrounded by extensive round cell infiltration including the papillæ and the walls of the blood vessels. The latter are increased in number as a part of the inflammation and erosion is at times so rapid that severe hemorrhage occurs; or, on the other hand, infiltration is so extensive that gangrene and sloughing result with much loss of structure. The varieties of chancroid are classified in accordance with the different morbid features which distinguish the local conditions. The common variety of chancroid appears, as already described, as an erosive ulcer (Fig. 272). When this ulcer becomes unduly inflamed and irritated, it acquires an elevated appearance and some degree of hardness (inflamed chancroid). This form of chancroid is sometimes confused with the syphilitic chancre. Inflammation of the surrounding parts, due to retention of secretion and uncleanliness, affects the adjacent tissues and leads to phimosis and balanoposthitis, another condition of inflamed chancroid. When the virus has been implanted in the mouth of a follicle, the so-called follicular chancroid is developed, in which case the inoculation is beneath the surface and eventually appears as a large edematous pustule which ruptures externally. When the inflammation of chancroid is of a still greater degree of intensity, due to some inherent morbid condition of the individual, it assumes a sloughing, indolent character and is called phagedenic. This phagedena may become more or less chronic and when the area covered by the sore is irregular and shows a tendency to heal in one part and extend itself into another, it is called serpiginous chancroid.

Bacteriology.-There has been difference of opinion as to whether or not the pathogenic organisms of different varieties combine to produce chancroid; but it is without doubt due to a specific organism. The DuCrey-Unna bacillus is believed to be the specific organism of 
the ulcer but has not been grown in pure culture in an artificial medium. This organism is a strepto-bacillus appearing in parallel chains occurring in and without the cells (Fig. 273).

Symptoms.- The period of incubation is brief, usually between three and five days after exposure. Cases of more protracted incubation are due to the retention of the virulent pus in contact with an unabraded surface which later becomes inoculated. A follicular chancroid is produced by admission of the chancroidal virus into the mouth of a follicle in which case the incubation is slow as the ulcer develops
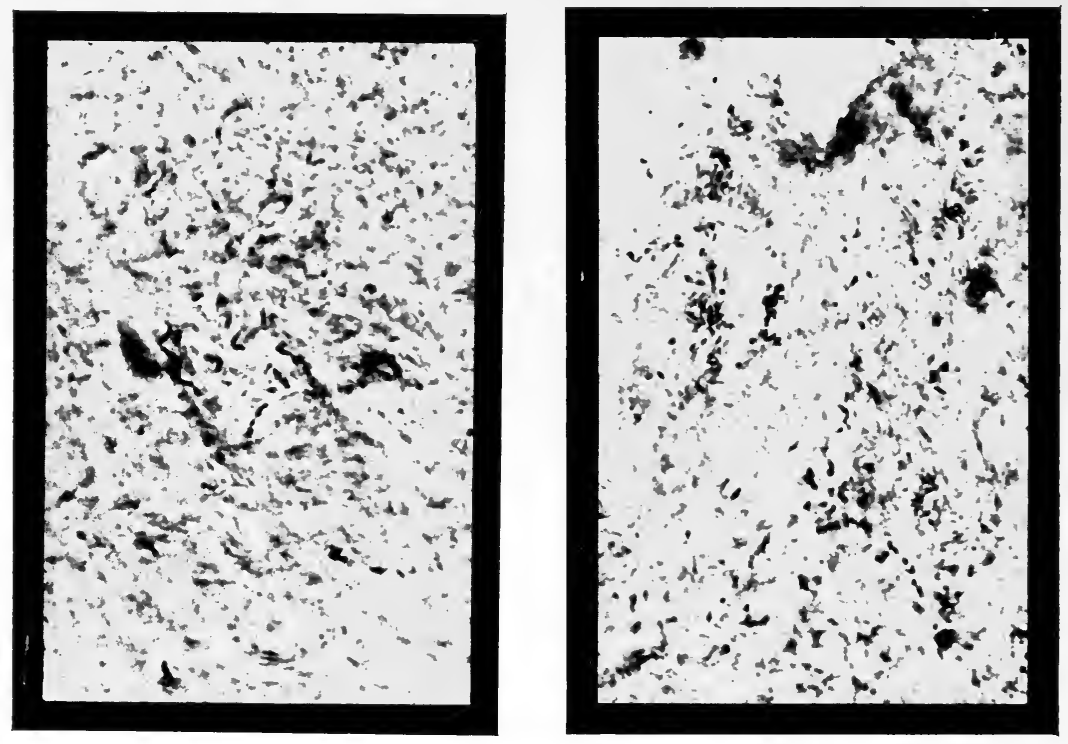

FIG. 273.-Unna-Ducrey streptobacillus of chancroid. (Johnston's Atlas.)

within the follicular sac and later appears on the outside. In the ordinary run of cases, within two or three days after infection, there is a reddened area which very soon develops a papule that in turn becomes a pustule; upon rupture of the latter, which occurs in rapid succession, there results a small, round or oval ulcer with overhanging, undermined and irregular edges. The surface of the ulcer is also irregular and covered with grayish, necrotic tissue and exudes a thick, purulent secretion. In some cases, there is a marked amount of inflammatory edema surrounding the ulcer, which gives to it a characteristic hardness that leads to confusion with the syphilitic chancre. An inflamed chancroid due to uncleanliness and phimosis with retention of secretion, is accompanied by marked balanoposthitis that 
causes much swelling and tension of the parts, sometimes rendering it impossible to retract the foreskin, or resulting paraphymosis and a tendency to frequent auto-inoculations. Such auto-inoculation usually occurs on the surface of the parts that come in contact with the already existing ulceration (Fig. 274).

Duration.-The course of chancroid is naturally controlled by treatment, which may be abortive. Uncleanliness tends to prolong its existence and encourage its spread. When no complications occur

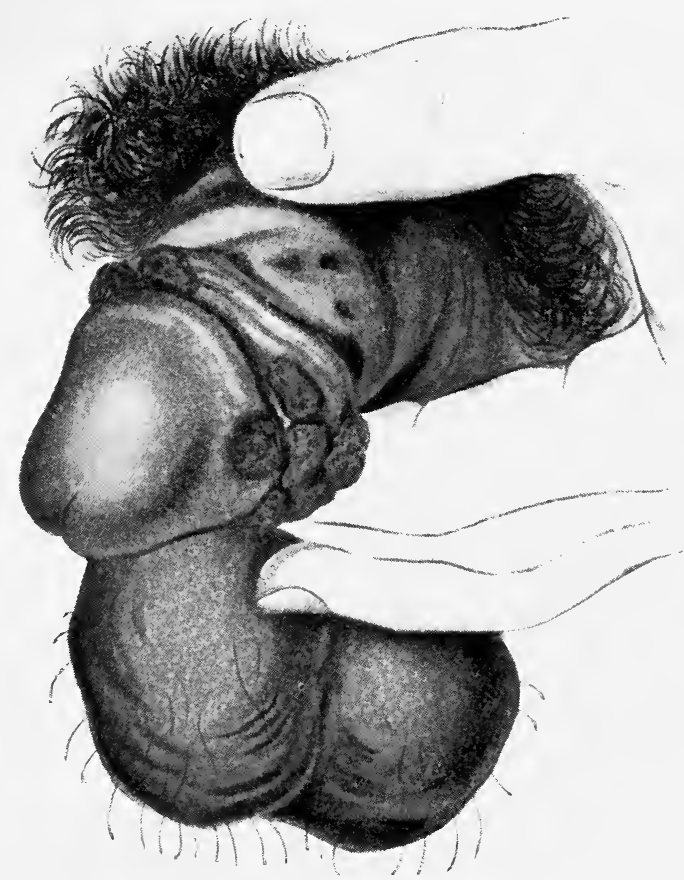

FIG. 274.-Multiple inflamed chancroids. (Johnston's Atlas.)

and without treatment, the ulcer increases in size for from one to two weeks, it then becomes stationary where it remains for a similar period and then begins to heal by granulation. The virulence of the sore gradually declines when healthy granulation occurs following local, antiseptic or caustic measures.

Diagnosis.-Chancroidal ulceration is recognized by its characteristic appearance. It is important to make a differential diagnosis between the syphilitic chancre and ulcerated herpes which latter may sometimes offer great difficulties and in some cases it is impossible to decide the question from the appearance alone. The possibility of 
a mixed sore should always be borne in mind. Chancroid must also be distinguished from mucous patches, gummatous ulcerations, ordinary ulcerated erosions, tuberculous ulcers and epithelioma. The autoinoculability of chancroid is its most distinctive characteristic. Prolonged observation is often necessary to a correct diagnosis. There is always the possibility that a beginning chancroid may later develop the characteristic appearance of a syphilitic chancre, having been acquired simultaneously with it and appearing after a preliminary incubation period. The recognition of spirochaetæ in smears and possibly a positive Wassermann blood examination, may be the means of settling the diagnosis, as will certainly the appearance of secondary manifestations.

The syphilitic chancre is fully described in a later section (q. v.). In connection with the question of differential diagnosis it is well to remember that while commonly the chancroid is an unindurated sore and chancre usually displays a peculiar hardness, that this is not invariably the rule and that a chronic and indolent chancroid may present a certain degree of hardness. Generally speaking, the surface of the chancroid, as well as the circumference, is irregular and uneven as compared with the more symmetrical and smooth outlines of the chancre. The chancroid is flat and undermined whereas the characteristic of elevation belongs more to the primary syphilitic lesion.

The exudate and secretion from the chancroid is regularly purulent or sanguino-purulent; that of the chancre is thin and viscid. There is glandular involvement present with both of these lesions: in chancroid, generally a glandular and periglandular infiltration; in chancre, solitary, distinct glandular induration or polyglandular swelling, easily distinguished and freely movable under the skin. The diagnosis between chancroid and the secondary syphilitic ulcerations is difficult only when the latter have undergone some inflammatory irritation. The presence of lesions of syphilis in other parts of the body is taken account of, also the laboratory finding as a result of the blood examination.

The ulcerated gumma may appear under the corona glandis near the frenum and resemble chancroid. It starts beneath the surface in an indurated pustule and extends inwardly, as well as outwardly. There is a characteristic indolence about it that is peculiar to gumma. The previous history of the patient and the blood examination, as well as the disappearance of the growth under specific treatment are means of reaching a positive diagnosis.

Ulcerated herpes are more likely to be confused with chancroid when it occurs as a solitary ulcer. It is not so deep as chancroidal 
ulceration nor so distinctive. A few days' observation should clear up the diagnosis on account of the usual prompt response of herpes to appropriate treatment; simple ulceration of balanitis, abrasions, mechanical fissures, etc., are distinguished in the same way by the absence of the characteristic appearance of chancroid and the lesser tendency to destructive extension. The more debilitated the subject upon whom such ulcerations occur, the more sluggish are they likely to be and consequently the more difficult is the diagnosis.

Tuberculous ulcers are rarely confused with chancroid as the occurrence of the former is uncommon. They are likely to be associated with tuberculous lesions elsewhere. If uncertainty exists, resort is had to the laboratory expedients such as examination of smears and scrapings, tuberculin and inoculation of the guinea-pig.

Epithelioma occurs as a papillomatous growth or exuberant neoplasm and is distinctively a destructive ulceration. It more often occurs late in life although I have operated upon one case in a male thirty-five years old. Microscopic examination of a section of the tissue leaves no question as to the diagnosis.

Complications of Chancroid.-The most important complication of chancroid is that which imparts to it the character of "mixed" sore owing to the coexistence of the primary lesion of syphilis. This mixed sore is one of the varieties of chancroid which is difficult to diagnose on account of its complex nature. Sooner or later, however, the existence of the mixed infection is established by the development of syphilitic lesions.

Inflammation of the chancroid and the adjacent parts is a complication which produces another variety of this venereal sore-the inflamed chancroid. Ordinarily, the chancroid possesses the local characteristics already described, confined to the area of the sore, but under the adverse conditions of neglect and the irritation of uncleanliness or on account of a physical depreciation, the tissue surrounding the sore are affected and there results an edema of the underlying structure of the ulcer, swelling and edema of the tissues beyond it. In the case of a chancroid situated upon the glans and within the prepuce, phimosis with inability to retract the prepuce is produced and, as a result of erosions and abrasions, numerous reinfections within the prepuce and around the preputial border occur (Fig. 275). This complicating condition makes it impossible to reach the chancroid and prolongs its duration. Sometimes, this inflammatory swelling becomes so extensive behind the tightened prepuce that gangrene and sloughing develop with protrusion of the glans through the resulting opening. 
Not an uncommon complicating occurrence in connection with the location of chancroid in the sulcus on either side of the frenum is perforation of the frenum. This happens as a result of extension and may produce a permanent hole or complete destruction of the attachment of the frenum to the glans (Fig. 276). The occurrence, sometimes, is accompanied by hemorrhage from the eroded artery of the frenum.

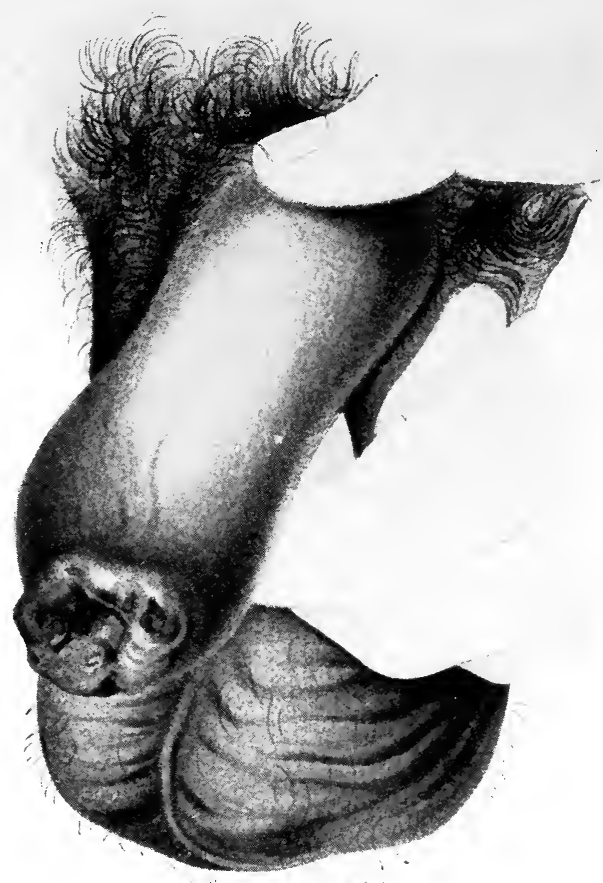

FIG. 275.-Chancroids of margin of prepuce. (Johnston's Atlas.)

Inflammatory paraphimosis complicates chancroid when the prepuce is short or when retracted for the purpose of examining or treating the ulcer, at which time, increased swelling prevents reduction. This complication, however, is not serious when the circulation of the glands is not endangered by the constriction and when reduction can be accomplished after the ulcer has been reached for treatment.

Phagedena.-This is looked upon as being the most formidable local complication of chancroid and its more or less frequent occurrence formerly, has established the name of phagedenic chancroid. On 
account of the successful measures of local treatment, this type of chancroidal ulcer is seldom seen at the present day although every now and then, a striking case is seen in dispensary practice, or is reported. The primary cause of such a complication is neglect or unfavorable local conditions in addition to the existence of constitutional debility. Phagedena has been known to take the form of gangrene and sloughing to such an extent that no tissues are spared, and a large portion of

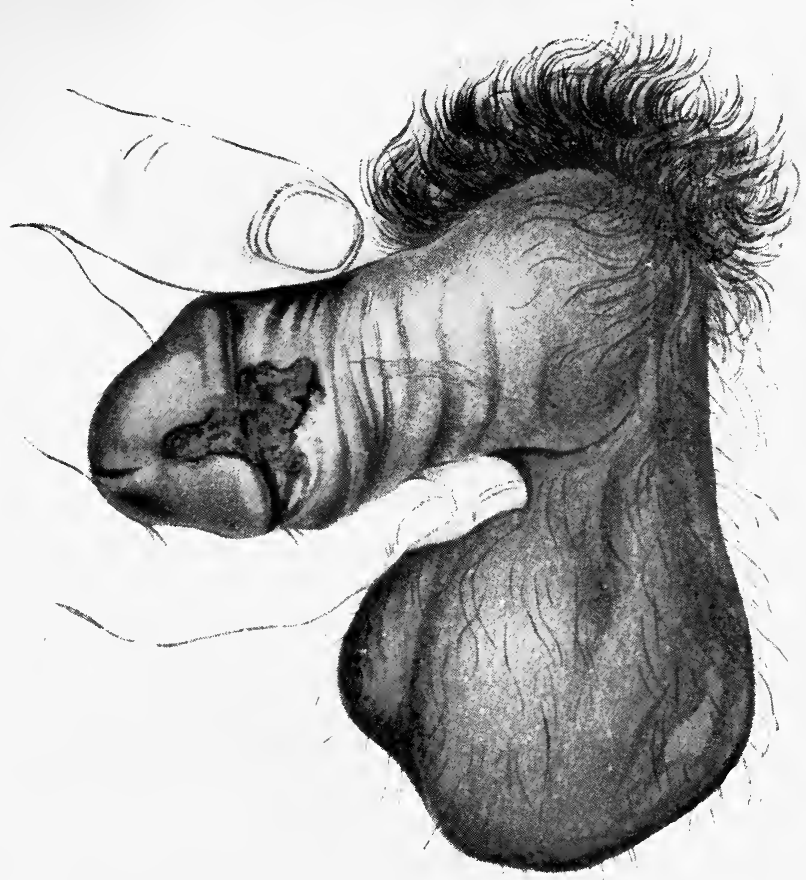

FIG. 276.-Chancroids in region of frenum. (Johnston's Atlas.)

the glans is destroyed; or it assumes a more chronic condition in the form of what has been called serpiginous phagedena, in which form the ulcer advances progressively over a large area, healing in one location as it advances in another. This type is probably more likely to be dependent upon a coexistent constitutional defect (diabetes, anemia, malnutrition, etc.).

Lymphangitis.-Inflammation of one or more of the lymphatic vessels upon the dorsum or sides of the organ is not of frequent occurrence and is rarely seen except in connection with lymphadenitis and bubo. 
Inguinal Adenitis.-Chancroidal bubo is the most common complication of chancroid and is present in a large percentage of cases in slight or extensive degree. It usually occurs in the groin corresponding to the side upon which the ulcer is located but may appear on the opposite side owing to abnormal lymphatic supply. Double bubo may occur in the instance of a single sore when the latter is located on the median line of the frenum. Implication of the inguinal glands may accompany any infective lesion along the line of the lymphatic system of the penis, such as acute gonorrheal inflammation, and any other type of ulceration.

Varieties of Bubo.-When the bubo is represented by a simple inflammatory adenitis, the glands in the groin involved present a similar condition to adenitis from any other cause in the same location or elsewhere amounting to swelling and tenderness of the individual glands which are freely recognizable or movable under the skin. When the inflammation is more virulent, as is usually the case with chancroidal ulcer, there is a coexistent periadenitis as the inflammatory process extends to the periglandular tissue. Under these conditions, palpation fails to detect the individual glands but recognizes an irregular painful swelling consisting of the inflamed glands and intervening structure matted together, adherent to the skin and underlying tissues. This condition usually tends to suppuration and opens spontaneously by rupture unless incised, following which, under favorable conditions, there is a slow but progressive tendency toward resolution. But when individual glands contain purulent foci which have not reached the surface progress is delayed by slow absorption or by suppuration toward the surface.

General constitutional depreciation, especially tuberculous, may impart to the local glandular condition a character of indolence which materially affects the progress and healing thereof and may require more radical surgical intervention, to the extent of complete extirpation of the glands. (See p. 346.)

Virulent Bubo. - The bacteriological character of chancroidal bubo is that of any glandular inflammation, namely, the pyogenic microörganisms. A certain number of cases, however, after suppuration and opening, assume a form which has been designated virulent bubo because it has acquired the characteristic of the original chancroidal ulcer in general appearance and in the property of auto-inoculability. There has been some question as to the origin of the so-called virulent bubo but the probability is that its occurrence is due to secondary infection from the chancroidal ulcer.

Symptoms of Bubo.- The symptoms of ordinary inguinal adenitis 
are those of inflammatory involvement of the glands elsewhere or from any other cause in this same region, namely: pain, swelling and tenderness. The more distinctive feature of the chancroidal bubo is the periglandular swelling composing an irregular brawny mass of a bluish, turbid appearance which may produce a sufficient amount of pain and systemic disturbance to interfere with the patient's movements and cause confinement to bed. Suppuration may be preceded by chill. The diagnosis is self-evident. In rare instances, it has been confused with strangulated hernia but this is a far fetched possibility. The outcome is favorable under modern methods of antisepsis and surgical measures. Bubo may occur at any time in the course of a chancroid before complete cicatrization.

Treatment of Chancroid and Its Complications. Prophylaxis.-The same general remarks as to the question of personal prophylaxis as in the case of gonorrhea (p. 212) apply in the consideration of chancroid. The proper means of personal prevention are extreme cleanliness and the use of an antiseptic wash such as bichloride of mercury, 1 to 1000 or carbolic acid, 2 per cent., following which a mild calomel ointment may be applied. The prevention of the development of complications in connection with the already-existing chancroid, requires the adoption of the proper measures of treatment, to be hereinafter given, and the observance of precautions against physical fatigue and strain and protection of the adjacent parts by cleanliness.

Abortive Treatment.-This involves complete destruction of the ulcers by cauterization, transforming virulent sores into healthy, granulating ulcers which follow an uninterrupted course of repair. The earlier in the course of chancroid cauterization treatment is applied, the more effective the result. To be completely curative, each and every sore must be reached and thoroughly destroyed otherwise even one small undestroyed area may reinfect the entire granulating surface of the cauterized area. The caustic treatment should always be radical and therefore one of the strongest cauterizing agents employed (fuming nitric acid) has proved to be most effective in the greatest number of cases. When applied without previous anesthetization of the parts, it is exceedingly painful.

It is worse than useless to cauterize chancroids surrounding the circumference of an unretracted prepuce without reaching any that may preëxist within, as reinfection is bound to take place and the recurring sores will be more extensive than the original ones.

The following method of cauterization I have found effective and relatively free from pain as compared with the use of nitric acid alone.

Requirements.-Fuming nitric acid, pure carbolic acid, cocaine or 
novocain in powder form, petroleum jelly (vaseline), small glass rod, small wooden applicators or tooth-picks with cotton twisted on the ends, some strips of blotting or filter paper.

The parts should be thoroughly cleansed and dried and all secretions removed from the surface of the sores. This presupposes that the prepuce can be thoroughly retracted and all the sores reached. Following the cleansing, it is desirable to apply a small amount of petroleum (vaseline) by means of a cotton-tipped wooden applicator around the circumference of the sores but not upon the sores themselves. Next, apply a small quantity of the pure cocaine or novocain to each sore. After this has remained in contact for from 3 to 5 minutes, remove with plain sterile or antiseptic solution and again dry thoroughly. Pure carbolic acid is then applied with cotton applicator. Remove excess with filter or blotting paper so as to prevent overflow upon the healthy surface. After one or two minutes' contact with the pure carbolic acid, remove moisture with filter or blotting paper and now with glass rod apply, carefully, fuming nitric acid to one sore at a time so as to avoid orerflow. The nitric acid is left in contact with each sore until cauterization is complete which generally takes from two to three minutes and the moisture is then removed with the absorbent paper, after which the sores are dressed with a dry antiseptic powder (such as iodicle of thymol) or with an antiseptic wash - the official black wash (lotio nigra) or bichloride of mercury, 1 to 4000 . When this cauterization has been applied early in the course of chancroid, it may produce the desired result, but when the sores have been allowed to run for some time, its effect is only temporary and a second or third application may be required with continual local antiseptic treatment until healing finally results.

The antiseptic and expectant treatment of chancroid will lead to successful healing after the application of cauterization, and in many instances without such abortive measures. Such a course is conducted in simple and uncomplicated lesions and in cases where all the sores cannot be reached for cauterization. The treatment involves careful cleansing of the parts, several times daily, followed by the application of an antiseptic lotion or other local medicament, as may be selected. For this purpose one of the following lotions may be used: black wash (lotio nigra); solution of bichloride of mercury 1 to 3000 to 1 to 1000 ; potassium permanganate 1 to 1000 to 1 to 500 ; carbolic acid 1 or 2 per cent. In employing one or the other of these lotions, the parts should be immersed in the same for five or ten minutes each time, following which, a wet dressing of the same, with gauze or absorbent cotton, should be applied in a water-proof bag or piece of oil silk or 
rubber tissue; or after the immersion, an antiseptic powder may be used such as the following: iodoform which, on account of its objectionable odor, in spite of its great value is usually discarded; one of the iodoform substitutes such as iodide of thymol (aristol), subgallate of bismuth (dermatol), dioxy-iodo gallate of bismuth (airol), or nosophen. In the condition where the chancroid cannot be reached by the direct methods of treatment described, the cavity of the prepuce must be frequently washed out with one or the other of the lotions named, by means of a long nozzle or duckbill syringe. In such instances it may be necessary to expose the glans and affected area by means of a bilateral or dorsal incision (see p. 340); complete cauterization of the sores may be then accomplished and the regular antiseptic method of treatment carried on thereafter. The question as to the advisability of opening the prepuce by incision in the case of interior chancroid may be decided by the condition of the parts and the action of treatment. Sometimes the ulcers will take a favorable course and the swelling reduce as a result of the intrapreputial injection, so that the prepuce can eventually be retracted. In other cases, however, the tension becomes so severe and the swelling so great that incision is deemed expedient to prevent serious destruction of the parts involved. After incision, while the resulting condition has an exceedingly intense and inflamed appearance, the ultimate result is sure to be favorable under the constant application of the proper treatment.

The treatment of chancroid, generally speaking, is varied according to the period of its existence and also with respect to its location; thus, chancroid of the margin of the prepuce, there being no ulcers within, may be treated by cauterization, which is not the case when sores coexist inside the prepuce.

Chancroid undermining the frenum and perforating from one side to the other, calls for division of the frenum (see p. 342) to hasten the cure and avoid the possibility of bleeding.

Extragenital chancroid requires the same method of treatment as genital ulcer.

Treatment of Complications. The Mixed Sore.--Treatment of the mixed sore is the same as that for chancroid until the diagnosis of the coexistence of syphilis has been established when the treatment of the latter disease is in order $(q . v$.).

Inflamed Chancroid.-Treatment of the inflammatory condition of chancroid varies according to the nature and extent of the inflammation. Rest in bed and elevation of the parts with the application of wet dressings as already given and, in instances of severe pain, anodynes and local analgesics may be required. Internally, codeine 
may be given, $0.03 \mathrm{gm}$. (about $\frac{1}{2} \mathrm{gr}$.), two or three times a day, and locally, powdered anesthesine, applied by means of a blower inside the prepuce or dusted directly on the sores, may give some temporary relief from pain.

Lymphangitis.-Mild cases require no especial care beyond the application of the wet dressings ordinarily used in the treatment of the underlying condition. In active cases, continuous rest and quiet in bed are required.

Phagedena calls for thorough cauterization, under general anesthesia, sometimes with the actual cautery, of the entire area involved. Later, the wound is treated according to methods suggested for nonphagedenic ulcerations.

Phimosis requires primary incision, dorsal or bilateral and later circumcision (see p. 340).

Hot Air Treatment of Chancroid.-The treatment of phagedenic chancroid by means of the thermal effect of dry hot air has been recommended ${ }^{1}$ and this method has proved successful with a specially designed oven.

In any case of extensive chancroidal ulceration, with severe inflammation, I find it is useful to utilize this principle, when it is not essential to have a specially manufactured oven but an improvised contrivance of simple design. It is necessary that the patient be confined to bed and therefore cases of severe and obstinate character only are treated in this manner. An ordinary wire frame cage, such as is generally used to keep the bed clothes from the body in surgical cases, is placed over the patient. To this is suspended an incandescent electric lamp of thirty candle-power surrounded by an asbestos cone. The adjacent part of the body and the penis, excepting the region of the ulceration, are protected by sheet asbestos. The lamp is then so placed that the radiated dry heat will be constantly in contact with the chancroid as close to the body as the patient can tolerate. By this method, the tendency to spread seems to be controlled and healing is more rapid. Daily cleansing and disinfection with phenol or sublimate lotion are also kept up.

\footnotetext{
1 J. Wood Ruggles (Trans. Am. Assoc. G.-U. Surgeons, 1909) reported "a new method of treatment of phagedenic chancroid and chancre by means of hot air," describing cases and special apparatus for same.
} 


\section{CHAPTER XXXIX}

\section{SYPHILIS}

\section{DEFINITION, ORIGIN AND CAUSE, GENERAL PATHOLOGY-THE PRIMARY SORE}

Definition.-Syphilis is a general, constitutional disease of specific origin propagated by inheritance (congenital syphilis) or by inoculation (acquired syphilis). The acquired form of syphilis is characterized by the appearance of a typical sore at the point of entry, the site of inoculation, which is called the chancre or primary lesion. Within a few weeks generalized symptoms are exhibited upon the skin and mucous membranes which manifest the constitutional character of the malady. They are the secondary lesions. Following this stage, months or years may pass without further evidence of the disease; moreover, it may cease entirely, spontaneously or under favorable therapeutic measures; but notwithstanding such measures, there may develop deep-seated lesions in the form of granulomatous growths of the skin, the muscles, the bones or the viscera. These are the tertiary lesions. Finally, after a period of quiescence during which there has occurred an apparent cure, or consecutive to the previous stages, this octopus of diseases may expend its ruthless influence and lay low its victim long years after the beginning of the malady in the degenerative forms of cerebrospinal luetic lesions, chief of which are locomotor ataxia and paresis.

It is not within the scope of this work to discuss the question of the primeval origin of syphilis. The subject has given rise to much controversy and no absolute conclusion has been reached. However, it was not recognized as a specific and individual malady before the end of the fifteenth century when it is thought that it was brought to Spain by the sailors of Columbus landing from Hayti and Central America.

So great an investigator as John Hunter in his day clogged the wheels of scientific progress by a serious error in teaching to the effect that gonorrhea, chancre, chancroid and syphilis were all one and the same disease. It was Ricord who cleared the atmosphere of its confusion and recognized the distinction between syphilis and the other purely local venereal infections.

Microbiology.-Metchnikoff and Roux in 1903 found that syphilis was transmissible to monkeys, and Chaudinn in 1905 discovered 
the spirocheta pallida, the undoubted specific organism of syphilis. This spirillum is seen in the primary, secondary and tertiary lesions, and has also been found in the late degenerative so-called parasyphilitic invasions. It is found extensively in the tissues of the hereditarily diseased infant.

The spirocheta pallida occurs in regular spires, from eight to fourteen in number, four to fourteen millimeters in length, one-quarter to one-half millimeter in width (Fig. 277). While this particularly narrow width renders it difficult to detect them, the regularity of the spires affords a marked distinction. There is some variation of the spiral form; they are somewhat shorter when obtained from lesions

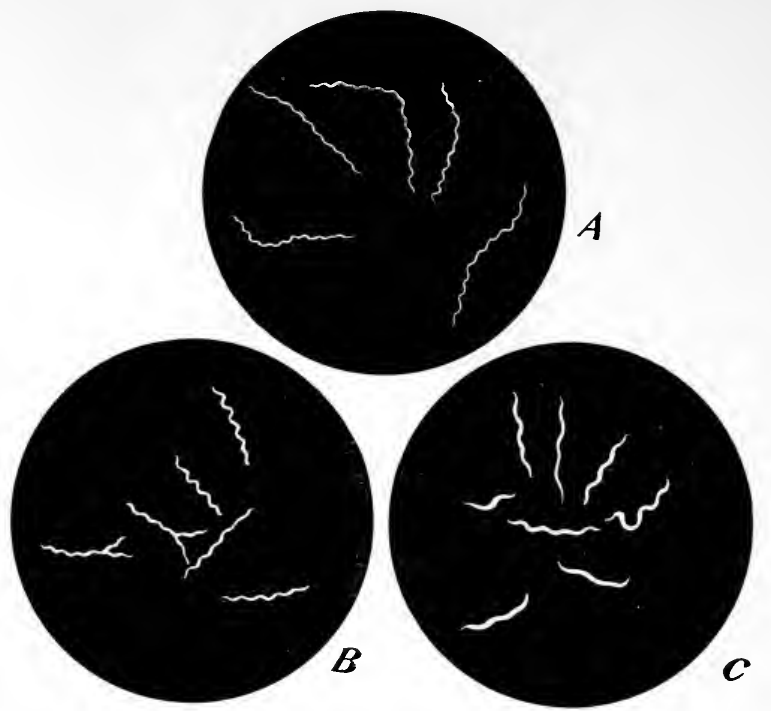

FIG. 277.-Spirilla with dark field illuminator. $A$, Spirocheta pallida. $B$, S. refringens. $C$, S. gracilis.

that have been treated with mercury, and are more likely to be longer in recent untreated lesions.

The dark field illuminator is employed to recognize the living spirochæta or they are examined after staining in smears.

The dark field illuminator is especially useful to reveal the presence of the organisms, and is an attachment furnished for the microscope which is substituted for the condenser, and allows no rays of light to pass through the field except those that outline the delicate translucent spirilla forms of these parasites (Fig. 277).

Examination by Dark Field Illumination.-The lesion is first cleansed with sterile water, and is then compressed laterally until 
there is caused to exude a certain amount of lymph from the surface of the sore. This is collected upon a clean slide with a drop of normal salt solution and covered with a cover-glass. A drop of cedar oil is then placed upon the cover-glass and another on the opposite side of the slide. In placing under the microscope, focus is made upon the white corpuscles and the spirochetes are then revealed. It will be noted that the pallida variety invariably keep their spiral shape, while other varieties, from which they must be distinguished, such as that of Vincent's Angina are larger and much more rapid in their motion, and when motion ceases the spires straighten out, which is not the case with the pallida.

Spirochetes pallida may also be found in the lymph aspirated from the nearest lymphatic glands associated with the primary sore.

Examination for the spirochetes in smears is much slower and more difficult.

Giemsa Stain.-A smear is obtained from the exudate of the sore after previously cleansing; the smear is dried thoroughly and fixed with alcohol or ether; a mixture of ten drops of giemsa in 10 c.c. of distilled water and ten drops of 1-100 sodium carbonate solution is employed as a stain. A few drops of this are applied to the smear, which is heated until vapor forms. This is repeated three times. The smear is then washed in sterile water and dried. The spirochetes are stained violet red. Other stains have been used successfully, but on account of the delicate and sinewy structure of the spires they are difficult to see.

Burri's India-ink Stain.-The specimen to be examined is taken from the sore in the usual manner with a platinum loop and placed upon a slide. With this is mixed in equal amount one or two drops of Chinese ink, and distilled water, and spread in a thin smear over the slide, upon which it is allowed to dry. It is then examined with an oil emersion lens, and when the organisms are present, the ink forms a black background to the colorless outline of the organisms.

\section{General Pathology of Syphilis}

There exists an individuality in the morbid changes that belong to syphilis which occur in all stages of the disease, and are revealed as a tendency to the formation of new connective tissue of cellular proliferation and finally, to the formation of fibrous and caseous tumors. The connective tissue hyperplasia plays the most constant rôle in the pathology of syphilis and while these pathogenic lesions are simple and uniform, the symptoms to which they give rise are numerous and varied on account of the fact that practically 
none of the organs are exempt from the far-reaching spread of these morbid changes.

The chancre or primary sore is first a cutaneous lesion but later extends into the substance of the subcutaneous tissue (see p. 701).

The eruptive stage of a typical case of syphilis is ushered in by the so-called roseola which is largely congestive or erythematous. In the papule, which is slightly elevated, there is a cellular infiltration as well; in the vesicle there is beneath the epidermis an exudate of serum, which eventually either dries up or becomes pustular. There may be various combinations of these types of skin lesions (polymorphous) and they may fuse together forming a mixed variety such as the squamous and papulo-squamous patches, or they may develop into ulcerations which appear alone and in combination with the other forms. The cutaneous manifestations of syphilis that occur later than the above as a rule are gummatous and appear either as single or multiple tumors beneath the skin which usually contain caseous material and which, if not removed by therapeutic intervention, tend to break down extensively and leave permanent scars as a result of superficial necrosis.

Gummata elsewhere may be soft or hard - that is to say, they may contain granulomatous material which shows a tendency to soften or they may be fibrotic and again they may be composed of a combination of these two elements. As the feature in the tertiary lesions is the formation of fibrous tissue, there is not the same tendency to resolution as in the primary and secondary manifestations.

Disease of the arteries is the important late manifestation of the syphilitic process. The tendency may be either toward gummatous or fibrous changes. Obliterating endarteritis is the most serious development of syphilitic arterial disease. Occlusion of the lumen of the arterial tube is caused by callous and fibrous induration of the internal coat which affects the circulation of the area to which the particular artery is distributed. Other secondary effects are also produced on account of the impairment of elasticity of the vascular walls, leading to rupture and hemorrhage and possibly pressure upon the vital nerve centres. In fact, it may be stated that the arterial system bears the most important relation to all syphilitic manifestations as they take their origin from and are distributed about the bloodvessels.

The primary and secondary manifestations of syphilis appear mostly on the surface; the tertiary lesions are found practically in all parts both inside and out, and attack all organs. While the skin is a favorite site of the latter, the central nervous system, the bones and the viscera are 
commonly attacked. These direct manifestations of the disease are due to the effect upon the blood and cellular structures exerted by the specific organism (the spirocheta), or the syphilitic toxin, giving rise to symptoms according to the particular region attacked; and later is produced a more permanent influence upon the cells of the body, which is a process of degeneration, ending in the development of the so-called "parasyphilitic" affections already mentioned-the terminal state of this far-reaching disease.

\section{The Transmission of Syphilis}

Syphilis is most commonly transmitted from the chancre and the secondary.lesions of the mucous membranes. During the early stages of the disease the blood is in its most virulent state and, therefore, is a source of contagion as are all of the inflammatory products of any active lesions. It is staged upon reasonable evidence that the physiological secretions - the sweat, saliva and semen-are not carriers of contagion unless they are contaminated by contact with an open lesion or by the admixture of blood. It is probable that only the moist secondary lesions are clinically infectious.

Modes of Infection.- The common method of transmission is by sexual contact, yet the disease is not essentially venereal since there are numerous other avenues of infection, and any conditions which bring together the virus from one source, be it in the blood or an inflammatory secretion, and the blood of another will produce the disease.

Accidental Infection.-Before a complete body resistance or active immunity has become established it is probable that the chancre is auto-inoculable, which is, roughly speaking, within the first two weeks. During this period it has been noticed also that the complement fixation test of the blood is negative; a fact that has been used as an argument in favor of total excision of the chancre as soon as it appears. While this has been practised by some, modern therapy has rendered its consideration superfluous.

Extragenital chancres may occur on any part of the body, but are more common upon the regions susceptible to exposure, such as the finger tips, the mouth, etc. The method of conveyance may be by direct contact or through some intermediary such as a drinking cup, feeding utensil, or surgical instrument.

Hereditary Transmission.-When both parents are diseased the off-spring is most likely to be syphilitic unless perhaps the disease in the mother has become well nigh exhausted. 
Maternal transmission is certain to ensue when the disease is active in the mother, exceptions being when there has occurred an apparent cessation, during which period a healthy child may be born, to be followed later by another syphilitic infant. According to Colles law the mother who has borne a syphilitic child is positively immune even though she shows no outward evidence of the disease. This is demonstrated in the case of a child born with active syphilis, perhaps with lesions of the mouth; such a child, it is held, will promptly infeet a healthy wet-nurse but not its mother. The blood of the fetus has evidently conveyed to the mother its own active immunity.

Paternal independent of maternal transmission is much less common than the reverse, as it is possible for a syphilitic father to beget a healthy child - his physiological secretion (semen) being free of contamination. The commonest method of hereditary transmission is from the father through the mother to their offspring, although it would seem from careful observation that the disease may pass from father to child without infecting the mother.

Profeta's law contains the interesting fact that a healthy child born of a syphilitic mother may be suckled by a syphilitic wet-nurse without danger of infection. It is probable here also that antibodies obtained from the blood of the mother produce an active immunity.

Placental Transmission. - A pregnant woman may become infected with syphilis, in which case the child may or may not inherit the disease. As a rule it escapes if infection takes place after the seventh month of pregnancy.

As to the duration of inoculability there is basis for the belief that the power of transmission does not survive more than three or fou years after infection, but there are undoubtedly individual variations.

Acquired Immunity. - Wide experience has demonstrated that a patient who has had syphilis once is not prone to suffer a second siege thereof. It is not unlikely that what has been considered immunity in the past is in reality due to a latent existence of the disease as our present facility of examining the blood by the complement fixation test has demonstrated, in many instances long after the commencement of the disease, that the patient may be negative as to symptoms while positive as to the blood. On account of the great infrequency of second attacks the insensitiveness of a patient who has once had syphilis is considerable but not absolute. Since the introduction of the modern salvarsan treatment cases have been reported as giving a negative blood examination following the treatment by salvarsan and have subsequently acquired an initial syphilitic lesion. 
Transmission to the third generation is, at the present time, not proven. The facts bearing upon this question are that the liability of transmitting syphilis as already stated, diminishes with the lapse of time from the date of infection, that inherited syphilis is not a permanent protection against the acquired disease, and that parents with inherited syphilis do have perfectly normal children.

\section{The Primary Lesion of Syphilis, The Chancre}

Incubation.-Following the exposure and previous to the appearance of the chancre at the site of inoculation there is an intervening period of incubation which averages between twenty and thirty days, and varies from two weeks to three months.

Number.-Although usually single, the primary lesion may be multiple.

Development and Course. - The chancre is a granulomatous lesion composed of an infiltration of round cells and proliferation of connective tissue cells.

An endarteritis of the small blood-vessels with sclerosis and an obliteration of some of them produce a surrounding edema and firm induration that especially characterize most chancres upon the mucous membranes. This feature is peculiarly persistent and may last for months after the chancre has healed. The color of the chancre is at first red and later becomes a bright coppery hue. There is little pain or tenderness associated with its development; such symptoms are produced only by inflammation and by stretching or pressure of the parts.

Varieties of Chancre.-The eroded chancre is the most common form of syphilitic sore (Fig. 278). It is found in both sexes on the skin as well as upon the mucous or semi-mucous surfaces. The size varies from that of a split pea to a beefy patch as large as the thumb nail. The surface is generally flat and raw, yielding a

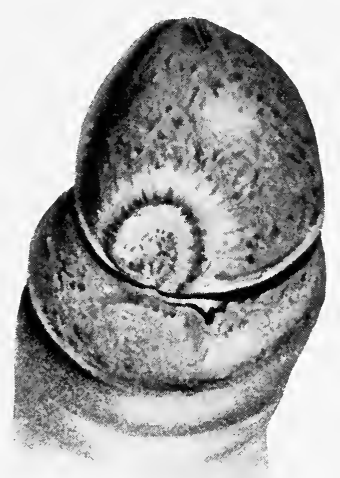
slight secretion of bloody serum. It is oval or irregularly rounded in shape and may follow the course of one of the natural folds of the covering of the penis when it has two opposing surfaces, as it has also in the "split pea" variety. The surrounding edema causes the 
sore to have an elevated appearance but the central plain is slightly punched-out by erosion with edges sloping toward the centre.

The Ulcerated Chancre.-This is known as the "Hunterian chancre" and resembles the eroded form with the addition of ulceration as though the underlying induration had become excavated (Fig. 279, A). The edges, however, are not undermined but thickened.

The dry papular form of true chancre is a hard dry papule of

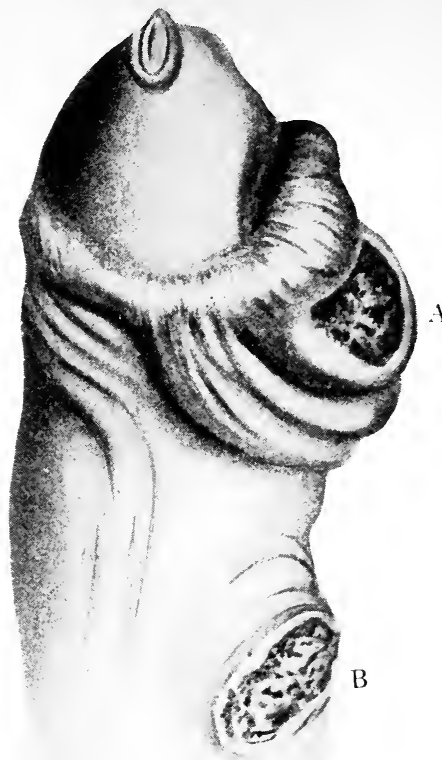
large dimensions which usually appears upon the skin.

The mixed chancre represents a combined infection of chancroid with syphilis (see Chancroid).

The Re-indurated Chancre.-A relapsing induration which resembles in appearance the original sore, sometimes appears at the site of a chancre after the latter has healed, and is generally due to insufficient treatment.

Site of Chancre.-Chancres are designated according to their site genital and extragenital.

Genital Chancres. - The largest number of these sores develop upon the mucous membrane of the prepuce behind the corona, or upon the glans penis and present the characteristic appearances above described. They may be found also in the orifice of the urethra, upon the integument of the

Fig. 279.-A, Hunterian chancre. B, Chancre of integument of penis. (Johnston's Atlas.) penis, at the extreme end of the prepuce, upon any part of the scrotum, and in the groin.

Chancre of the glans penis is at first generally flat and raw, but later becomes depressed and surrounded by an indurated area.

Chancre of the integument of the penis appears in the beginning as an abrasion or papule. It is usually circular in form with hardened edges. It may attain considerable size, becomes eroded upon the surface, but is not always ulcerated (Fig. 279, B).

Chancre of the scrotum resembles the previous form with a greater degree of erosion and sometimes ulceration.

Chancre of the end of the prepuce is irregular in outline with smooth and shiny surface surrounded with induration, giving it a marked 
prominence. It occurs sometimes underneath the prepuce when it may be invisible on account of phimosis.

Chancre of the urethral orifice may be on the outside and resemble a chancre on any other portion of the glans, or it may be hidden within the lips of the meatus when, on account of the extensive accompanying edema, it may be mistaken for an infection of one of the urethral follicles (see p. 242). An inspection of the exterior surface of the sore and the character of the induration should lead to its recognition. It may be deep down in the urethra.

Chancres of the peri-genital regions may also develop, including chancre of the anus and of the groin.

In the female the primary syphilitic sores are discovered around the mucous membrane of the vagina and upon the labia. They may not be appreciably indurated but appear as flat excoriations and raised mucous tubercles. Upon the cervix uteri, the chancre appears upon the anterior lip as an elevated or flattened erosion having a red areola and covered with a membranous secretion.

Extragenital Chancres. - Chancre of the integument appears as a large flattened papule or elevated tubercle and sometimes as an indurated ulcer. The induration thereof is sometimes less abrupt in outline than in the case of genital chancre, being more like an extensive edema.

Chancre of the lip resembles chancre of the prepuce and glans penis. It becomes very prominent and feels like a cartilaginous mass beneath the surface, and is excoriated or ulcerated exteriorly.

Chancre of the tongue resembles the raw erosion found upon the genitals; it is generally on the dorsal surface but may be on the sides or tip. Its surface is red and shiny with swollen margins. The submaxillary glands are always enlarged.

Chancre of the tonsil is characterized by a brawny induration and an ulcerated surface covered with whitish or greenish exudate. The submaxillary glands, and sometimes those under the sterno-mastoid are enlarged.

Chancre of the fingers resembles the sore upon other portions of the integument, accompanied by an extensive amount of thickening with a raw shiny surface (Fig. 280); when upon the dorsum of the terminal phalanx the appearance is not unlike that seen with a paronychia in the declining state. The epitrochlear and axillary glands are enlarged.

Other extragenital chancres occur in different portions of the body but without any specially significant features.

The course of chancre is sometimes insidious and usually prolonged 
unless controlled by active treatment. There is at first an erythematous patch that soon develops into a papule; later it commonly becomes eroded and finally ulcerated. Upon the mucous membrane an excoriation or small ulcer is practically the first appearance. The erosion, which from irritation has become an ulcer, increases in size for a varying period and assumes one of the different forms already described.

Induration is the striking characteristic of most syphilitic chancres, so much so that they are distinguished by the name of "hard chancre." This induration is sometimes lacking; sometimes it is late in coming, at other times it is the first appearance of the sore. The most common

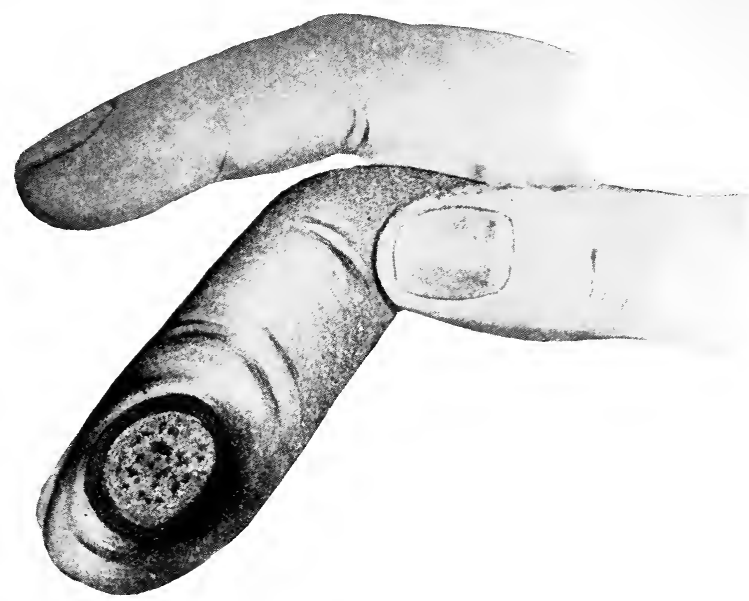

FIG. 280.-C'hanere of finger. (Johnston's Atlas.)

form is a parchment-like hardness that underlies the ulcer or erosion, best appreciated by being felt between the thumb and index-finger. The "split pea" variety is where two opposing surfaces, generally upon the prepuce, are involved by the ulcer with a symmetrical cartilaginous nodular formation underlying both sides. A different form of induration is that which, instead of being confined to the adjacent portions of the ulcer, is more excessive and invades the surrounding tissues for some distance; as when the whole prepuce or a large portion of it is invaded by a wood-like hardness; or an irregular area surrounding a skin lesion produces an inflammatory edema of large proportions compared with the size of the lesion. Absorption of the induration may commence to take place at the end of three weeks and it may disappear at the end of eight. It is not accompanied by pain or itching. 
Complications of Chancre.-Inflammation of the sore and adjacent area may cause the ordinary chancre to change its appearance, leading to swelling, pain, and suppuration. This may be due to too strong applications or uncleanliness. Inflammation is more a characteristic of chancroid and herpetic ulcers, and it is the occurrence of this condition which may render a differential diagnosis somewhat difficult.

Phagedena may also invade a syphilitic chancre as well as chancroid, leading to a chronic and indolent course, unless checked by treatment.

Lymphangitis and Adenitis.-Within one or two weeks after the appearance of chancre the lymphatic vessels become involved; sometimes those that extend from the region of the sore may be felt. The associated glands-in the case of genital sore those of the groin-form the typical syphilitic bubo. These enlarged and indurated glands may be single or multiple. In certain regions they are more likely to be single, as under the chin, at the elbow, and in the axilla. They are usually not painful but are felt as firm, hard nodes, freely movable beneath the skin, especially in the groin, and differing from the bubo of chancroid which is surrounded by brawny swelling due to periglandular edema.

As the disease progresses toward the secondary period it is not uncommon to feel lymphatic enlargement in many different parts of the body signifying the lymphogenous distribution of the syphilitic virus.

Diagnosis of Chancre.-The diagnosis is made upon the clinical appearances already described, and is more difficult in the early stages or when it is complicated by inflammation. Principal reliance, in the clinical diagnosis, is to be placed upon the characteristic appearance of the sore, with or without induration, the glandular enlargement, the history of exposure and period of incubation. Of especial importance is the modern bacteriologic method of examination for the spirocheta or specific organism which, if demonstrated in the secretion beyond question, is sufficient evidence of the disease (see p. 696).

The Wasserman or complement fixation test (see p. 106) is not available until about six weeks after the first appearance of the sore, but, when a positive reaction is obtained, furnishes conclusive proof.

Constitutional treatment exercises controlling influence upon the chancre but should not be resorted to until a positive diagnosis is reached.

The prognosis of chancre is unexceptionally good when treatment is instituted; resulting deformity and undue destruction of tissue occur only when treatment is neglected or delayed. 
An attempt is made to find a relation between the later course of the disease and the character of the original chancre to the effect that more serious eruptions follow an ulcerated lesion than a dry papule and that a brief incubation period is an index of a severe constitutional course. Such a relationship is not always borne out.

Treatment of Chancre.-Prophylaxis here, as in other venereal disease, is a primary consideration; cleanliness and the avoidance of injury or abrasion of the skin is of first importance. Following exposure, gentle washing and disinfection should be resorted to, but a disinfectant of too irritant a character should be avoided as a possible means of excoriating the surface and hence engendering inoculation instead of preventing it. A calomel ointment (Calomelol) is a safe and expeditious manner of protecting the surface. This may be applied after the previous cleansing and disinfecting wash already directed.

Excision of the chancre has its advocates. It seems reasonable to believe that it is necessary that the chancre be but a few hours old, with no glandular involvement and no diffused extension beyond the actual area involved, to expect a radical result following excision; such limitations are rare and therefore it would follow that this expedient has little to recommend it.

The local treatment of chancre should not be irritating; over-stimulating or caustic applications will only serve to transform a simple lesion into an inflammatory one. The best course consists in keeping the parts clean with a mild antiseptic lotion such as boric solution or weak bichloride (1-5000), and if a dry dressing is preferred, a mild dusting powder composed of calomel one part, to from five to ten of nosophen, iodide of thymol (aristol), or dioxy-iodo-bismuth (airol). If there is a tendency to suppuration a wet dressing may prove more satisfactory, in which case the parts may be kept immersed with a mild solution of corrosive sublimate, or with the officinal lotio-nigra. Generally speaking, ointments are undesirable.

The effect of mercury upon the spirochæta is specific. This has been demonstrated in the laboratory as well as clinically.

In complicated cases the treatment must be adopted according to the existing circumstances; for balanitis the applications usually employed in this disorder are resorted to, p. 173.

If phimosis exists it is usually necessary to perform bilateral incision (see p. 340). In extremely inflamed conditions or phagedena enforced confinement may become necessary with the constant application of hot antiseptic dressings; or the employment of dry heat continuously, as in the case of chancroid (see p.694). 


\section{CHAPTER XL}

\section{THE CONSTITUTIONAL DISEASE-CLINICAL COURSE- REGIONAL SYPHILIS}

The clinical course of syphilis, although theoretically divided into several consecutive stages and intermediate periods, is seldom stereotype throughout its course the same in any two cases. Its development is full of surprises and irregular occurrences; symptoms may be absent during the regular course of the disease, or they may appear out of time. Tertiary manifestations in precocious cases may ensue a few months after the chancre, while erythematous scaly patches may crop out or persist long after the regular time for the tertiary stage to supervene. There is, however, in spite of such variations, a similarity in cases that marks the identity of the disease and in all of the pathological changes and clinical manifestations a strong individuality exists. The division of the malady into primary, secondary, and tertiary stages refers to the clinical symptoms which are peculiar to these respective stages.

In the first stage the infection appears to be purely local, the chancre and the glandular involvement comprise the primary group of symptoms. This stage extends from the beginning of the local sore to the onset of constitutional symptoms. Antedating this stage there is, as already stated, a period of incubation of several weeks, and succeeding it there is sometimes, but not always, a period of secondary incubation. During the primary stage there is no disturbance of the general health.

The secondary stage occurs six weeks or more after the first appearance of the primary lesion and marks the time when the general system has succumbed to the invasion of the disease. The length of time of the secondary incubation or period between the primary and secondary manifestation varies and is influenced by the patient's constitution. The duration of secondary syphilis, like that of the other stages, varies so greatly that it is impossible to give to it definite boundaries. In some cases the symptoms of the secondary stage merge into those of the first and last from two to five years, whereas in many cases the same lesions continue to crop out year after year for an almost indefinite period. These variations have happened both with and without what has seemed to be a fair amount of treatment. In other cases the secondary manifestation may cease for an extended 
period, and the disease may break out at a later day in some more deepseated lesion.

The peculiar symptoms to the secondary stage give to syphilis a resemblance to the acute infectious eruptive fevers, but with a much longer clinical duration. Thus the symptoms of the secondary stage, after a preliminary period of incubation, exhibit general constitutional disturbance, elevation of temperature, headache, pains in different parts of the body - generally worse at night - and the appearance of an eruption upon the skin and mucous membranes.

The clinical symptoms attending the constitutional invasion of the disease, which is the beginning of the secondary stage, are not uniform. The fever may appear coincidentally with the skin eruption or before it. The type of fever may be remittent or intermittent; it may be a sudden short outbreak or last several days. It is generally accompanied by pains in the joints and in the bones and under the sternum. Associated with the systemic outbreak of syphilis there are many manifestations characteristic of the secondary stage; some of these may be absent, and others unduly emphasized. The description of these different phases of the disease belongs to that of regional syphilis $(q \cdot v$.). The most characteristic symptoms besides the lesions of the skin are glandular involvement; the falling of the hair; the throat symptoms, of which mucous patches and tonsillar involvement are common occurrences; and rheumatic or bone pains, one the manifestation of a general toxemia, and the other a localized periostitis.

The Tertiary Stage.-There is no distinct line of demarcation between the secondary and tertiary stages, but like that of the other stages the individuality of the latter is marked by characteristic symptoms and lesions. These lesions are infiltrative, ulcerating, and destructive. They may affect any portion of the body by extensive connective tissue and vascular changes or in circumscribed deposits (gumma) which may appear on the outside of the body or involve the viscera.

The quaternary stage is the last chapter of syphilis in which occur the parasyphilitic or degenerative affections which, though not so closely associated with the inoculation of the disease, as they often follow years after its activity apparently has ceased, are the development of a chronic process due to the prolonged viability of the infecting organisms in the cerebro-spinal system. Most important of these are locomotor ataxia and paresis.

\section{SYPHILIS OF THE SKIN}

The cutaneous lesions of syphilis are spoken of as "syphilides" and are most important manifestations of the secondary stage of the 
disease. One of the prominent characteristics of the syphilitic eruption is polymorphism, that is to say, embracing nearly every known type of skin eruption; and while these types are described separately, as though appearing in sequence, they are very commonly found at the same time upon the same surface of the body. These different types embrace the macule, the papule, the vesicle, the pustule, the scaly patch, and pigmented spots. Generally speaking they may be divided for convenience into two classes, (1) the early syphilides, and (2) the late syphilides. Characteristic of the early group are (1) erythematous (roseolar or macular), (2) papular, (3) papulo-squamous, (4) pustular, (5) pigmentary, and (6) vesicular forms.

The late class includes (1) the tubercular or nodular, (2) the gummatous, both of which may or may not undergo secondary changes involving softening and ulceration.

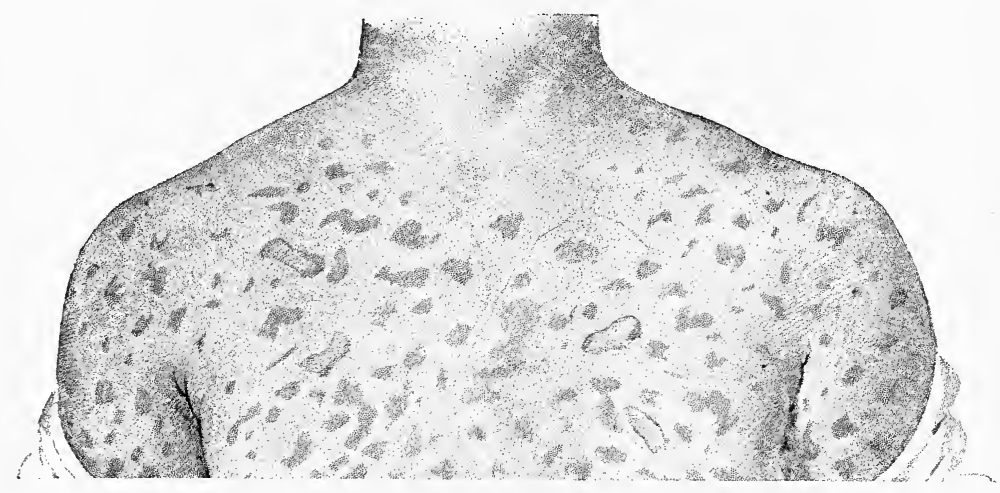

FIG. 281.-Erythematous syphilide-roseola.

\section{Early Syphilides}

The erythematous syphilides, which is the earliest eruption of syphilis, is known as the roseolar or macular syphilide (Fig. 281). It commonly appears on the front of the arm and upon the trunk, notably the abdomen and chest. The spots or blotches are reddishbrown in color and profusely distributed.

This eruption makes its appearance at the onset of the constitu. tional stage of the disease and at the same time as the general lym. phatic enlargement. It may appear within a month after the beginning of the chancre, but generally six weeks or two months thereafter and sometimes later, especially when delayed by treatment. Besides its common appearance upon the arms, chest and abdomen, it may in- 
volve other parts of the body, including the face and neck. A very hot bath will sometimes serve to bring out its appearance. The patches, which are flat in the beginning later are covered with minute elevations sometimes becoming vesicles and occasionally pustules. At first pressure causes the mottling to disappear entirely; later a slight livid staining remains behind after removal of the pressure. Finally, when the eruption is fading and has acquired a slight coppery hue, pressure has no effect upon it. The eruption is never confluent, there is always normal skin between the macules, or other lesions of a different variety scattered here and there.

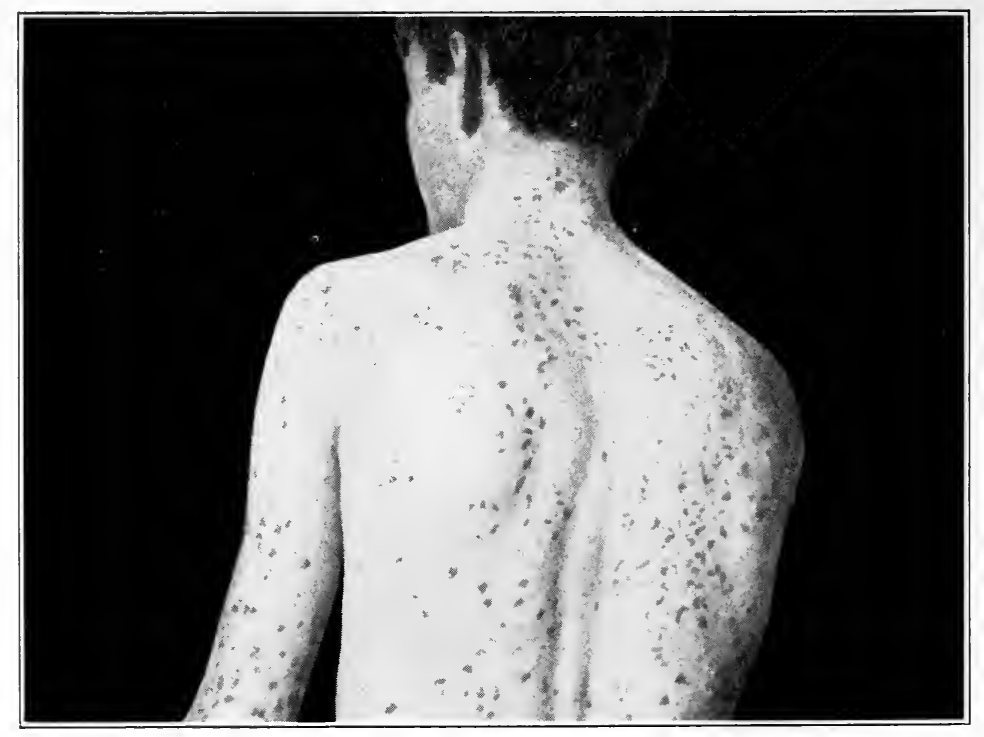

Fig. 282.- Papular syphilide.

The duration of the roseola is from two days to six weeks. It rapidly disappears under treatment, but relapses frequently during the intervals thereof. This eruption is easily recognized, as it is thoroughly characteristic, and the accompanying history of glandular engorgement, preëxisting chancre, throat symptoms, etc., make the roseola one of the easiest of all syphilitic lesions to diagnose clinically.

The Papular Syphilide.-Also called in different stages "syphilitic lichen" and "syphilitic psoriasis." Numerous varieties of the papular syphilide occur, including the flat papular and the raised form, large and small (Fig. 282). This eruption may be combined with, or 
follow the roseola (Fig. 283), or it may appear as the first skin lesion of the disease, when its date of appearance is about the same as that of the roseola.

The characteristic flat papule may be small or large; it grows in circumference but not in height; it is firm and smooth on the surface, pink or red in color at the beginning, but later assumes a coppery hue. The surface soon becomes dried up, the superficial skin cracks at the areolar border and falls away in whole or in part, leaving a characteristic encrusted appearance. Many papules in different stages of development may be found distributed over the skin. On the palm of the hand the papules often are encrusted in appearance approaching

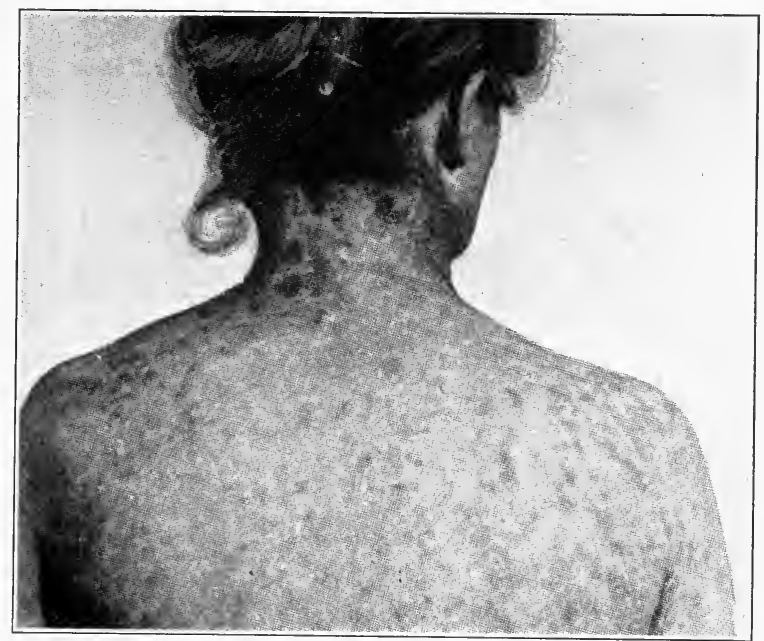

FIG. 283.-Papular syphilide, combined with roseola.

the papulo-squamous variety. The general surface appears yellowish and when the top drops off there is left a punched-out circular area not unlike an uncovered blister surrounded by a reddish areola. These spots show a greater tendency to get well than the later syphilides of the palm, the true papulo-squamous.

Other types of papular syphilides are the small elevated variety, in which are irregularly distributed with those of the flat non-elevated type. They pass through the same stages of evolution and like them disappear leaving a pigmented margin which may persist after the eruption has gone. The flat and raised papule occurs commonly upon the moist surfaces. It is many times larger than the commoner variety of this syphilide, and while it may appear on different parts of the body, it is 
usually found in muco-cutaneous areas, such as the prepuce, and in the region of the anus where it is known as the condylomata-lata, and should not be confused with the papillary growth of venereal warts or vegetations. The duration of the papular syphilide is variable. It may occur as stated, as the first eruption, alone or mixed with the roseola, and continue for a few weeks to several months. The papules sometimes run together and form patches when they become more obstinate.

The Diagnosis of the papular syphilide should be easy in typical cases. Difficulty may be encountered when there are only a few

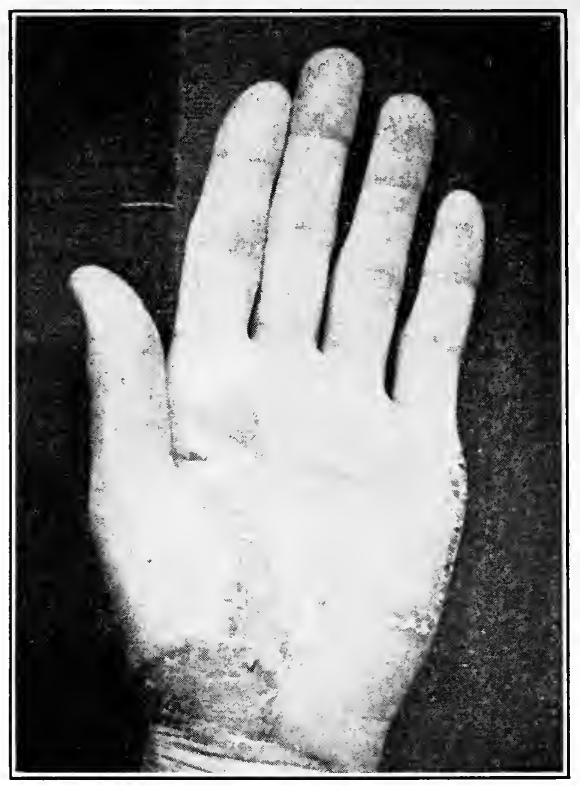

FIG. 281.-Papulo-squamous palmar syphilide.

discrete lesions here and there. Formerly in obscure cases some trouble was experienced in distinguishing it from types of acne and lichen planus, but at the present day with the confirmatory means of sero-diagnosis (see p. 105) there is no reason to take the risk of delay in the diagnosis or treatment.

Papulo-Squamous Syphilide is a variety of the papular eruption that occurs after the first year or two of syphilis, and it may appear long after the tertiary period has arrived. It is one of the most persistent forms of skin lesion. It may invade any portion of the body; favorite sites are the face, the hands and feet (Fig. 284) palmar and plantar 
surfaces-and the scrotum. It occurs in lesions of variable size and shape from scaly papules to rounded or irregular patches covering an extended area (Fig. 285). The surface of the skin invaded by the eruption appears thickened, livid in color and covered by a fine, scaly coating. It usually disappears without leaving a scar, but, if the patch has been unusually infiltrated, like the tuberculous form of eruption; round scars are likely to result which remain permanently after the lesion has healed.

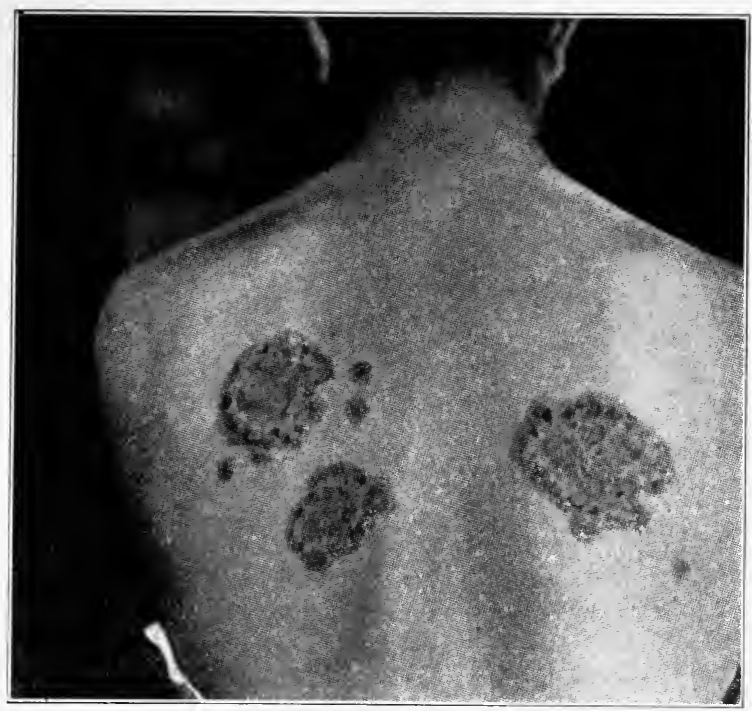

FIG. 285.-Papulo-squamous syphilide of back.

The circinate form of this eruption develops either late or early in the disease and attacks almost any part of the body, but most com. monly the skin of the genitals in either sex. It commences in the form of a circle or segment of a circle resembling the appearance of ordinary ringworm, but does not show a tendency to increase in size from the original circumference (Fig. 286). Some of the segments may overlap each other forming a festooned arrangement. The skin in the centre of the circle usually remains sound. The border is elevated and the patch is sometimes moist and at other times dry.

Diagnosis.-The color and general arrangement of the patches of papulo-squamous syphilide are often confused with psoriasis and a single patch upon the face may resemble lupus of the erythematous variety. The circinate variety should not be mistaken for ringworm, inasmuch as the circle does not grow by circumferential extension: 
The palmar and plantar papulo-squamous syphilide is a common and obstinate type of this form of eruption. They are so characteristic and striking that their appearance immediately suggests the nature of the disease. They may or may not be accompanied by other types of eruption upon the rest of the body. An ordinary eczematous patch may be encountered in one or the other of these regions, but it is apt to itch and the edges thereof are thinner and tend to taper off into the surrounding integument.

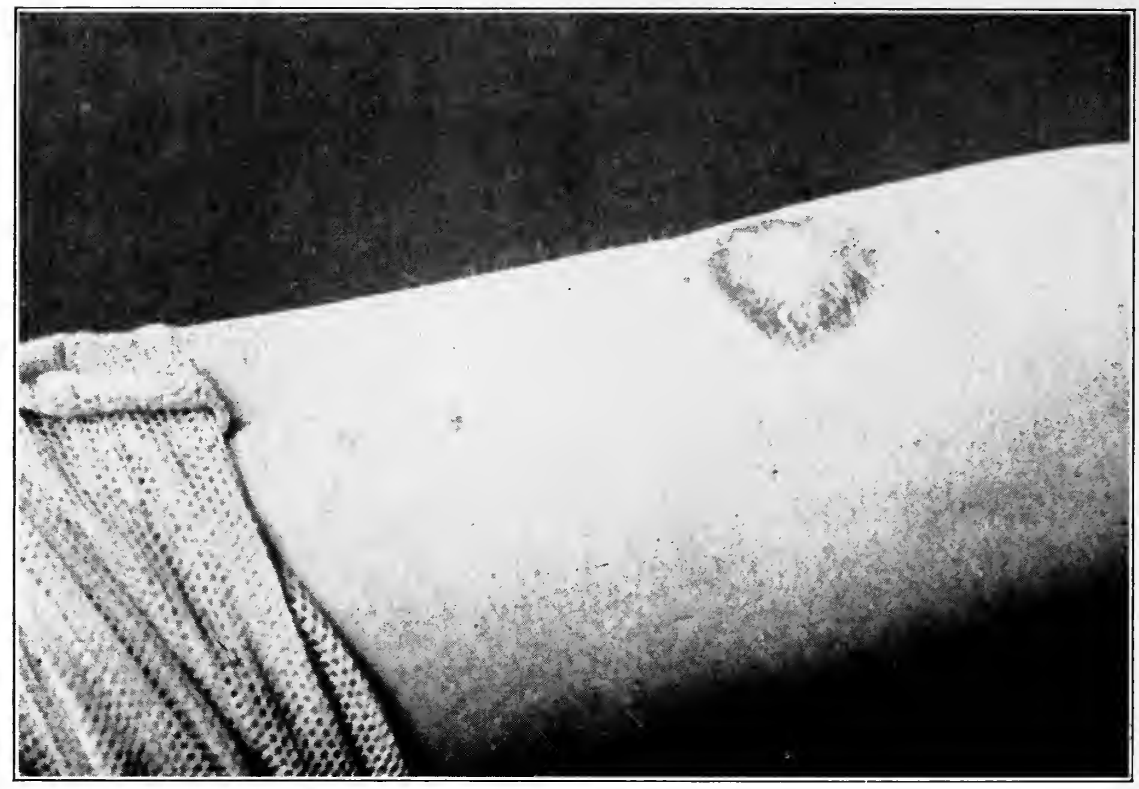

FIG. 286.-Circinate papulo-squamous syphilide.

Pustular Syphilide.-Also termed the syphilide acneiform, syphilitic impetigo, ecthymatous syphilide, etc.

The pustules occur in small and large size scattered about different portions of the body, mainly upon the trunk (Fig. 287) and may be distributed among a number of the other types of lesion, namely, vesicles, papules and erythematous spots. A diffused and exclusive pustular eruption usually occurs over the entire body, including the scalp and face, as well as the fingers and palm. Their tendency is to break and form a scab which dries down, producing a crust. When the lesions run together, there is formed as a result, a more or less extended patch. Sometimes the pustules are umbilicated and show a strong resemblance to the eruption of smallpox. 
The diagnosis is not difficult in connection with characteristic clinical symptoms and history. The peculiar color of the eruption, namely, the areola of coppery hue, is strongly suggestive, and when there is an extensive distribution of this type of lesion over the entire body, there is always a suspicion that it is dependent upon the disease in question.

This type appears in an exaggerated form as the ecthymatous syphilide, in which case it is more extensive, extends more deeply into the tissues, is suggestive of a later stage of the disease, well nigh the tertiary period, and is, therefore, an intermediate form between the ustular and gummatous lesions.

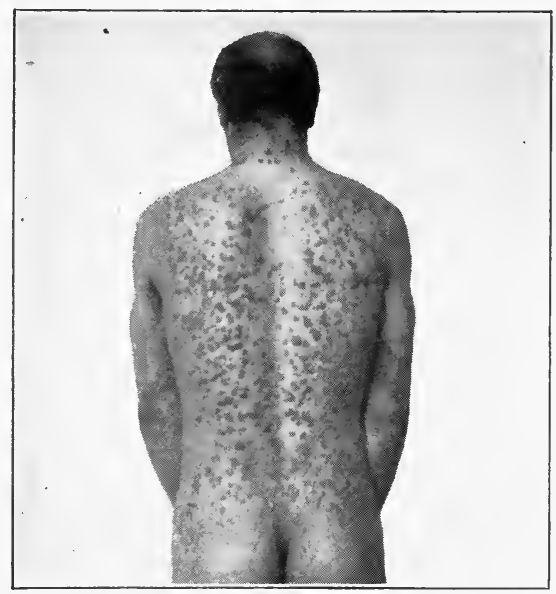

Fig. 287.-Pustular syphilide.

The pigmentary syphilide is an interesting but unusual manifestation. It is a pigmentation of the skin of a brownish hue, sometimes mottled. It is generally secondary to the roseolar type of eruption, is unattended by any local symptoms and usually uninfluenced by treatment. It has been seen in a complete collar form encircling the neck and in leucodermic areas of varying distribution.

The vesicular syphilide, also spoken of as herpetiform syphilide, is a rare variety and appears in the early secondary stage. The vesicles are of different sizes and generally occur upon the trunk, singly or in clusters. They are likely to accompany other lesions forming one of the elements of the polymorphous eruption. The individual lesions may be surrounded by a bronzed areola during the process of healing 
and they may dry up, forming scales, or suppurate beneath a scab. Sometimes they are umbilicated.

\section{Late Syphilides}

The different varieties of syphilides, excepting the squamous, the deep ecthymatous and the rupia, may be considered characteristic of early syphilis, and while there is no distinct line of demarcation between early and late syphilitic eruptions, the distribution of the tertiary lesions is not as a rule symmetrical; they are less numerous and attack the deeper skin layers. They are, therefore, more destructive in their tendency, resulting in ulcerations and scars.

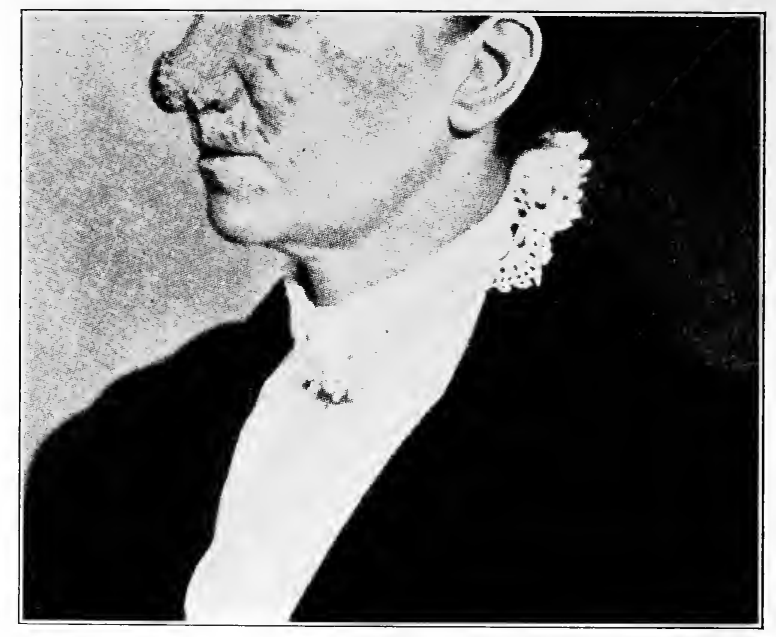

FIG. 288.-Nodular syphilide of face.

Tubercular or Nodular Syphilide.-This form is a species of gumma of the skin. The separate lesions may resemble the large papular secondary lesions, but there is a greater tendency toward ulceration in the tertiary form, while the secondary lesions are more likely to disappear without such a course. They may appear in any part of the skin, but there is a decided predilection for the face, especially around the nose and the forehead (Fig. 288). Other common sites are the back of the neck, the shoulders and elbow. They are composed of an infiltrated nodule measuring 0.3 to $1.2 \mathrm{~cm}$. (1/8 to $1 / 2 \mathrm{in}$.) in diameter elevated above the surface of the skin, with clean cut edges firm and smooth in feeling and rounded or flattened on top. In color they are either bronze or purplish hue. They are characterized by 
indolence and a tendency to ulcerate. In some cases they coalesce and form irregular or circular ulcers covered by a dark membrane and scaly crust. The arrangement of the tubercles is usually in a semicircular form, spreading from the extremities in a more or less serpentine distribution (Fig. 289), which gives a most characteristic appearance.

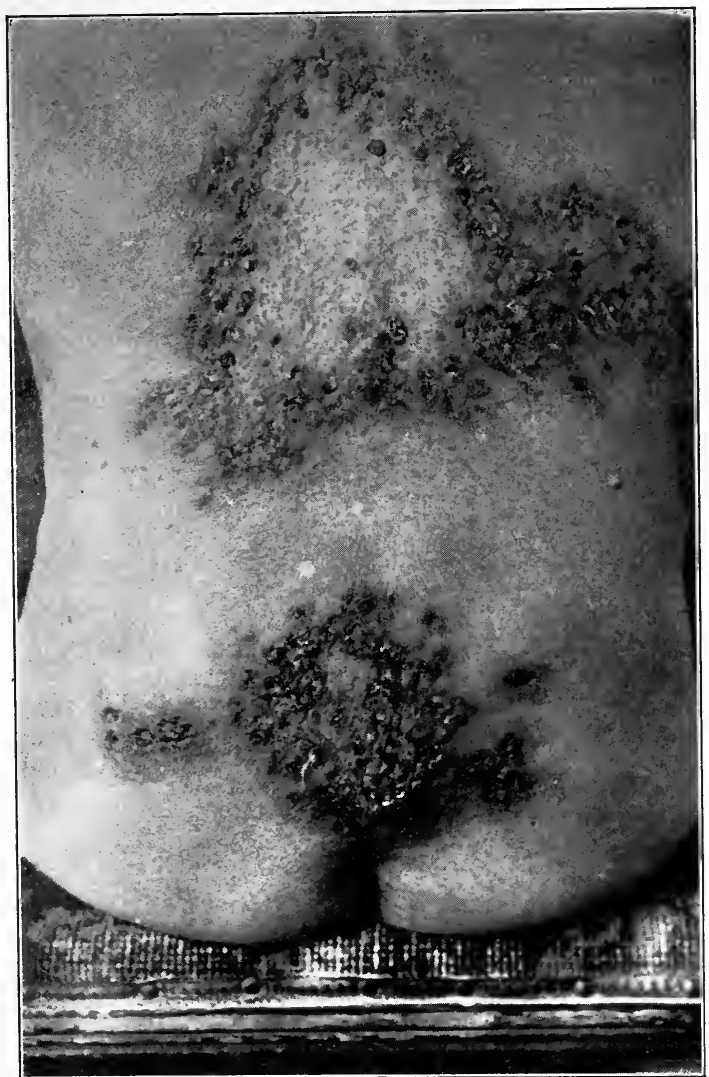

Frg. 289.-Serpiginous syphilide.

When ulceration has occurred in one or more of the tubercles and there is shown a heaping up of the crusts, so as to produce an elevated ulcer from 1.2 to $0.6 \mathrm{~cm}$. ( $1 / 4$ to $1 / 2 \mathrm{in}$.) in thickness, the lesion is known as rupia (Fig. 290), which is a thoroughly pronounced tertiary skin lesion.

The gummatous syphilide is, like the previous lesion, characteristic of the tertiary stage. At first the gumma is found as a subcutaneous 
nodule, varying in size from a pea to a marble. The shape is round, oval or flat. In the beginning the skin is not involved, being freely movable, but later it becomes red or purplish, due to pressure from beneath, as the growth increases in size, which may reach that of a hen's egg. Sometimes ulceration of the surface does not occur and absorption takes place. More often the gumma softens and breaks down (Fig. 291).

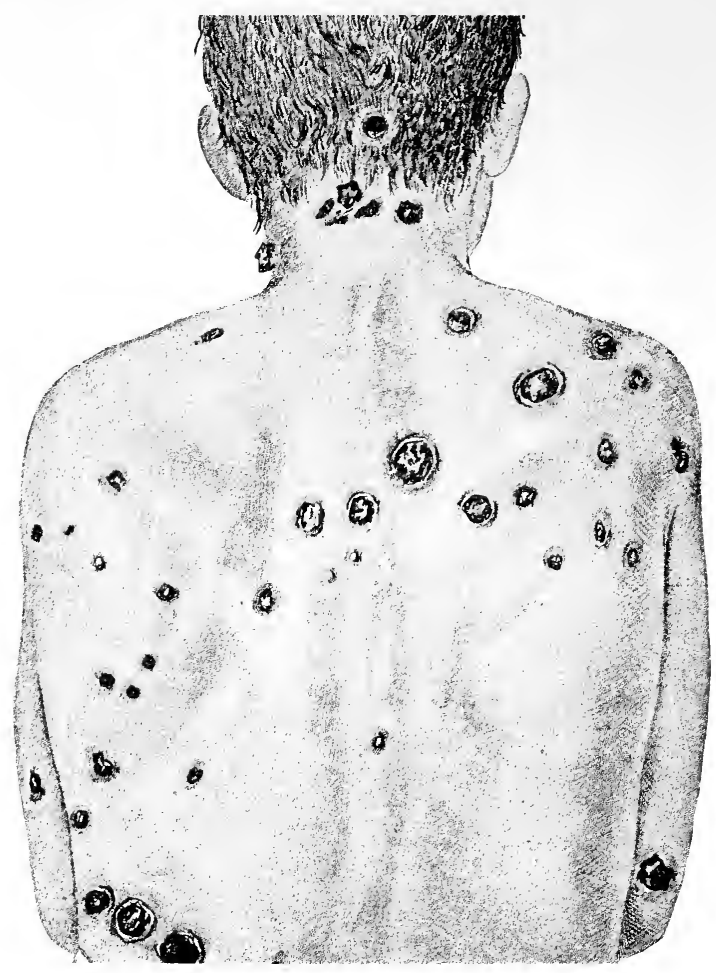

FIG. 290.-Rupial syphilide.

Gummata as a rule are few in number. They may involve almost any area and seem to select by choice the lower extremities (Fig. 292). They are not usually accompanied by pain or constitutional symptoms, although extensive destruction of tissue by ulceration is naturally accompanied by severe cachexia (Fig. 293).

The diagnosis of the various skin lesions already described is usually accomplished by clinical examination, especially when a definite history renders such diagnosis possible. At the present time, however, in all cases, recourse is had to the complement fixation test 
of the blood, which in most instances affords the desired confirmation and renders the diagnosis positive (see p. 106).

The treatment of these lesions is that of the stage in which they occur (see treatment, p. 765). Local treatment is usually not necessary except in the severe types, in which case the white precipitate ointment (hydrarg. ammoniat.) one part to four or six of zinc oxide is a good preparation for inflamed and ulcerated syphilides, and the red oxide

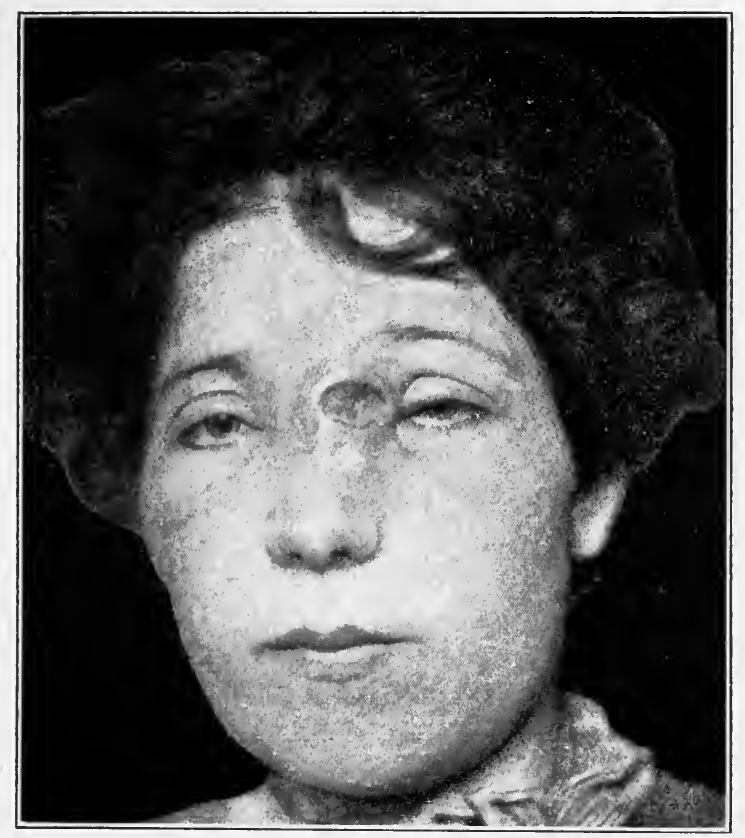

Fig. 291.-Gummatous ulcer of face.

(hydrarg. oxid. rubri) one part to two or four of petrolatum may be used for rebellious squamous and crustaceous patches.

Syphilis of the Hair and Nails.-Alopecia is a common feature of the disease. It varies greatly in degree, is usually partial and localized, although it may be general and complete. The most common occurrence is a generalized thinning of the hair all over the scalp in the early secondary stage, which usually grows again rapidly under treatment. Later in the disease there occur scattered or isolated patches of alopecia on the scalp or upon any of the hairy parts of the body (alopecia areata). While the treatment of the general disease is carried on, it is customary to use a stimulating lotion in the form of 


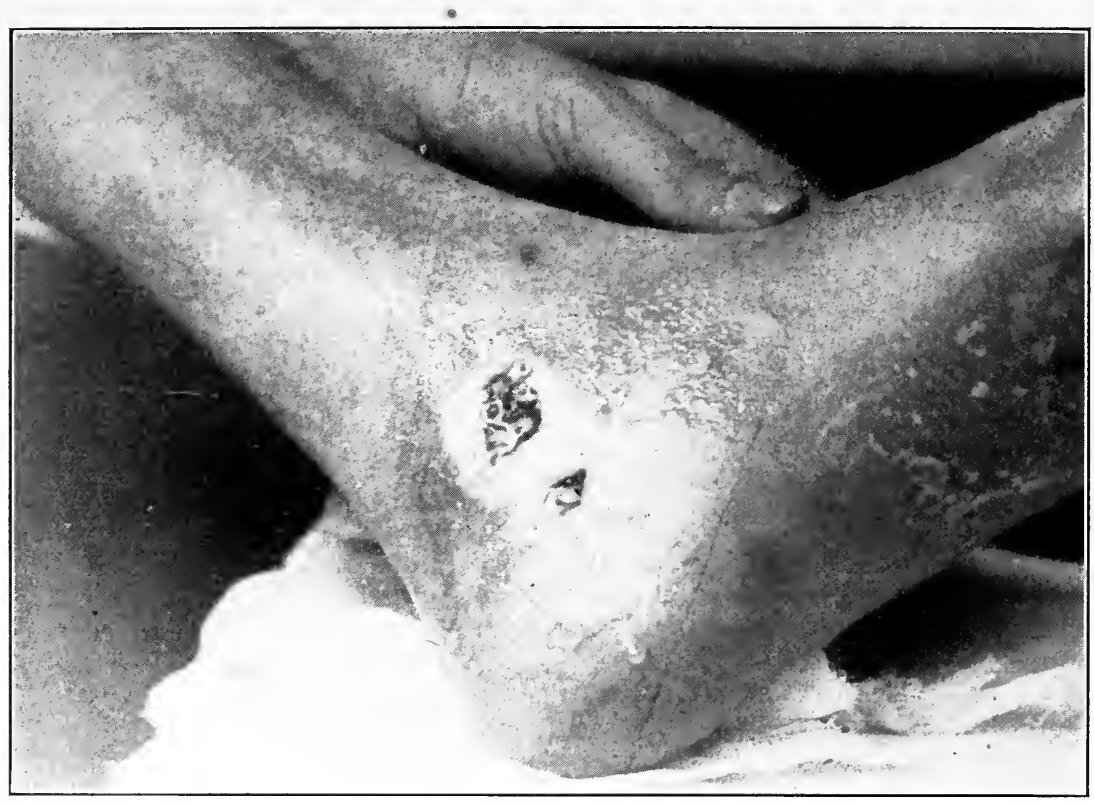

Fig. 292.-Gummatous ulceration of foot.

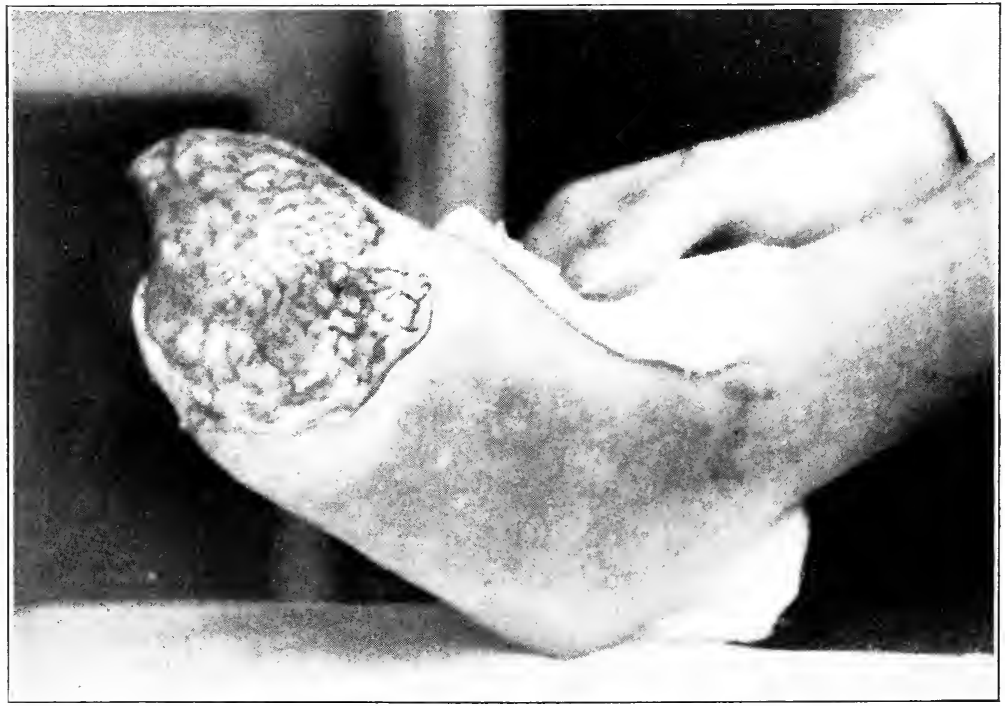

FIg. 293.-Extensive syphilitic destruction of foot. 
a sublimate solution 1-1000 to 1-500. The outlook is favorable for both types of this manifestation; it is aided by the administration of a general tonic such as the tartrate of iron and potash.

Syphilis of the nails is somewhat analogous to that of the hair, being apparently caused by the disturbance in general nutrition. The involvement of the nails occurs in the form of an onychia or paronychia due to infiltration of the matrix or of the skin around the nails. In the former variety the nail becomes dry, brittle and rough, having many deep furrows and irregularities. In some cases the nail is shed. Similar changes may occur with paronychia accompanied by more or less dry thickening of the integument around the nail. Both of these affections may appear in the secondary or in the tertiary stage of the disease.

The diagnosis is not always easy from a clinical standpoint, on account of the close resemblance of syphilitic onychia to the similar condition produced by eczema and psoriasis.

The treatment is that of the disease (see p. 765) in addition to which are employed such surgical measures as may be necessary for the local condition, involving wet dressing and the evacuation of pus when it occurs.

\section{Syphilides of the Mucous Membranes}

The most common site upon the mucous surfaces is the buccal membranes of both sexes, and in the vagina of the female; but they may also occur in the nose, the external auditory meatus, etc. From the standpoint of the clinical diagnosis of the disease they are of utmost importance, and also because they are a prevalent source of contagion. Their pathology is similar to that of the cutaneous lesions of the same stage and they appear simultaneously with them.

Simple erythema of the mucous membrane is the mildest form of eruption that appears and usually is seen upon the fauces.

The more common variety of mucous lesion is customarily spoken of as the mucous patch, and this assumes various phases from a simple erosion leading up to a definite ulceration, ${ }^{1}$ characteristic of different stages of development. They may appear first as an erosion, later as an eroded papule; under conditions of irritation and uncleanliness, they become thickened and inflamed, and finally a definite ulceration occurs.

Mucous Patches of the Mouth and Throat involve the angles of the mouth, the lips, the inner surface of the cheeks, the dorsal surface of the tongue, as well as the palate, tonsils and adjoining parts of the

${ }^{1}$ Fournier takes exception to the general use of the term mucous patch and has classified the mucous syphilide in four groups. 1. Erosive syphilides, 2. Papulo-erosive syphilides, 3. Papulohypertrophic syphilides, 4 . Ulcerated syphilides. 
pillars of the fauces. Men are more prone to these lesions on account of the use of tobacco and alcohol. The most frequent form of mucous patch in the mouth is a small, painless, slightly elevated patch of a pearl gray color irregular or rounded in shape. Mild types of these mucous lesions appear as excoriations upon the lips or throat and at the tip of the tongue. They are produced by a collection of thickened epithelial cells that covers the surface of the papillæ. In long standing cases these patches, at times, form fissures in the folds of the mucous membrane which are resistant to treatment.

A very common occurrence after the lapse of several years is an irregular redness and excoriation of the sides of the tongue far back on both sides. The chronic lesions, if not subdued by treatment, invite the development of epithelioma of the tongue late in life.

The diagnosis of lingual, faucial and buccal lesions should be made with care and, as in the case of skin lesions, is not difficult in the presence of a positive clinical history. They are to be distinguished from simple fissures, herpes and non-specific leucoplakia. After a careful and deliberate clinical diagnosis, the blood examination is resorted to.

Treatment.--The important consideration with relation to these lesions is the danger of producing contagion. The patient should be constantly warned of this and the frequent use of cleansing mouth washes should be recommended, such as chlorate of potash, thymol and mild carbolic solutions. The individual spots may be touched with chromic acid, 5 per cent., or better still, with the officinal acid nitrate of mercury solution.

Mucous Lesions of the Larynx.--Erythema of the larynx is evidenced by slight hoarseness at the beginning. Later a chronic inflammation may be developed with thickening of the laryngeal membrane, and ulcerations occur on different portions of the surface sometimes involving the vocal chords. In the mild stages, the constitutional treatment is all that is necessary to control these lesions. In the more active form where ulceration has occurred, the application of chromic acid, nitrate of silver or the acid nitrate of mercury may be called for.

Mucous Syphilides of the Genitals and Anus.- The erosive type is the commonest form and appears upon the prepuce, the glans and in the meatus. Around the anus all varieties of mucous syphilides are to be found. The true condylomata (broad papules) and flat moist papules are a common variety in this region and resemble vegetations or warts.

Local cleanliness of these parts is of great importance to avoid extensive excoriation and ulceration. The lesions should be kept clean 
and continually dry and dusted with calomel or other disinfectant powder.

Late Syphilides of the Mucous Membranes.-Tertiary lesions also invade the various mucous surfaces in the form of edematous infiltration and nodules and the regions thus affected have a tendency to break down and ulcerate. The syphilitic leucoplakia is a form of lesion which invades a large surface and transforms the normal pliable and pink membrane into a hardened and white plaque which may crack in the centre or on the sides and give rise to much pain. Any mucous surface may be thus affected; the tongue and the mucous membrane of the mouth are the most frequent sites. A serious feature of this lesion is the tendency it offers to the development of malignant disease.

Gummata may develop in the mucous membrane itself or in the submucous tissues. The mucous gummata soften and ulcerate and occur in the soft and hard palate as favorite sites. Diffuse infiltration then invades these parts, ulcerations occur and perforation takes place, producing a small or large sinus between the floor of the nose and the roof of the mouth. As a result of this destructive invasion, swallowing becomes difficult, fluids regurgitate through the nose and the voice has a characteristic nasal sound.

The tongue has already been mentioned as a common site of secondary manifestations. It is also the seat of tertiary involvement in the form of sclerosing glossitis characterized by infiltration and swelling. Gummatous glossitis occurs from four to six years after infection, and is a localized tertiary glossal lesion which has its greatest importance from the fact that it may be confounded with cancer of the tongue, and unless a correct diagnosis is made, a serious and dangerous operation may be performed. The differential diagnosis may in some cases be difficult, clinically; the characteristics of cancer are probably more distinctive than those of gumma; repeated blood examinations are the proper means of clearing up a doubtful situation.

Treatment.-As a means of preventing the occurrence of late specific lesions in the mouth, every possible source of irritation should be avoided, and strict cleanliness observed. Smoking is objectionable, except in moderation, and should be omitted entirely when the mouth is in an unsatisfactory stage. Spirits are usually excluded throughout the treatment, and are especially to be avoided in the event of mouth lesions. To the specific treatment is added such constitutional measures as may be suggested by the general condition. The local treatment depends upon the nature of any existing lesion. Ulcerations are best treated by the application of the acid nitrate of mercury solution. 
Cracks and fissures in the early stages may be treated with the application of 5 per cent. solution of chromic acid, or the daily use of peroxide of hydrogen solution as a mouth wash.

\section{SYPHILIS OF MUSCLES, TENDONS, APONEUROSES, AND BURS $Æ$}

The first manifestation of the constitutional disease in the form of the skin eruption, etc., is accompanied by pains in the muscles and joints. This disturbance develops sometimes before the healing of the primary sore. The muscles of the extremities are most commonly affected, and the pain has its maximum severity as night comes on, varying from a slight soreness to a severity so great as to incapacitate. The affection is known as myosalgia, or syphilitic rheumatism. Although the ordinary means of treatment adopted for the relief of kindred pains may cause some measure of relief, constitutional treatment for the disease proper furnishes the only effectual cure.

Other changes in the muscular system occurring early in the disease are a gradual depreciation in muscular strength and slow atrophy. which occur as a part of general constitutional impairment.

In the later stages, the disease attacks the muscles in different ways. There may be an inflammatory infiltration, a chronic myositis, in which case a muscle frequently involved is the biceps, or several muscles may be involved at the same time. The muscles may also be invaded by gummatous tumors of variable size. Softening of these tumors takes place and ulcers are apt to form as a result.

The tendons and their sheaths are not infrequently involved at an early period in the form of a tenosynovitis, in which case the pain is severe. Later in the course, the tendon becomes thickened and even calcified as a result of the disease, and in the tertiary stage gummatous nodules may develop in the substance of the tendons.

In like manner, a syphilitic bursitis may occur in the early stages and be confounded with inflammatory rheumatism, and later in the disease gummatous infiltration may attack any of the bursæ, especially those prone to injury.

The prognosis of all of these affections of the muscles, tendons or bursæ, is favorable, provided they are subjected to appropriate constitutional treatment $(q . v$.).

\section{SYPHILIS OF JOINTS AND BONES}

Neuralgic pains of the joints and bones have already been referred to as a feature of the disease in the first few weeks of the secondary 
stage. This symptom is also spoken of as arthralgia. The pain may be general or localized, and is always more severe at night. There is as a rule no sign of inflammation; the larger joints are more often affected.

Synovitis occurs as a prominent secondary manifestation in association with other symptoms, under which circumstances there is a distinct effusion of serum in one of the joints, such as the elbow, wrist or knee; the swelling may not be great, but the pain is severe, and always grows more so at night.

The more chronic form of synovitis which occurs later in the disease, involves the same condition of the joints, but with substantially no subjective symptoms whatever, so that attention may be called to its existence by accident. Gummatous changes involve the synovial membrane, either beginning in the synovial sheaths or starting in the articular end of the bones and by the destructive process, extending into the joints.

Syphilitic involvement of the joints therefore may occur as a synovitis, acute or chronic, and as a gummatous arthritis; in the last case, there is a chance of its being confused with tubercular affections of the joints. The essential point of difference is the absence of any tendency to suppuration and of much disturbance with the function of the joint except after the condition has existed for a long period and considerable advancement in the process has taken place.

These affections of the joints and bones call for prompt measures of treatment to prevent the extension of the disease and to obviate impairment of the function. It is usually possible with such prompt action to gain a satisfactory restoration of the parts, unless the process has extended so far as to produce permanent structural changes.

During the adoption of constitutional treatment appropriate local measures, such as dry heat and bandaging, elevation and rest and the application of a proper splint, are of value in some cases.

Dactylitis.-This process affects primarily the bones and periosteum of the fingers and toes, or it may commence in the subcutaneous tissues thereof; commonly two or more phalanges of the hand or foot are attacked, or the disease may be confined to one only. A common site is the proximal phalanx of a finger or toe which slowly enlarges, with little or no pain, and the skin becomes stretched over the swollen part (Fig. 294). Sometimes the whole finger or toe becomes affected. After persisting for some time, absorption takes place, generally with a resulting deformity of the bone, which is left shortened and thickened (Fig. 295). Gummatous infiltration may originate in the subcutaneous structure of the phalanx or the entire digit, producing a swelling 
that ends abruptly. It is not accompanied by pain. If this condition extends, it may lead to total destruction of the neighboring joint.

The cartilages which compose the different joints are likely to be destroyed by the action of the disease when the joint is involved. The cartilages in the other portions of the body, such as that of the ear, the larynx and the trachea, may also be attacked by syphilitic inflammation, and occasionally gummatous deposits impair their vitality.

Syphilis of the Bones.-Syphilitic periostitis occurs in the early secondary period in some instances, and in other cases it does not develop until the sixth or ninth month of the disease. It may be

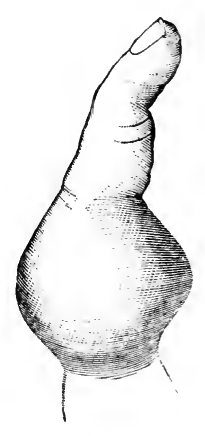

FIG. 294.--Syphilitic dactylitis involving phalangeal joint.

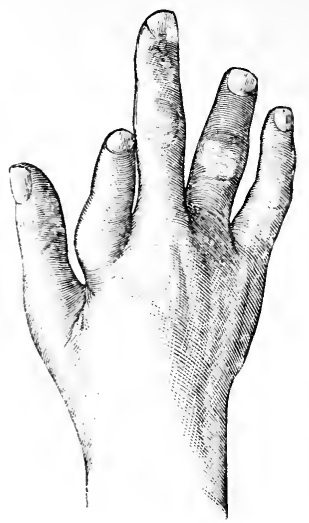

FIG. 295.-Syphilitic dactylitis in volving index-finger.

localized in area, or diffuse. The localized form frequently develops in the subcutaneous bones, notably the shin, clavicle, etc., on account of exposure to injury (Fig. 296). Such localized areas of inflammation are spoken of as nodes. Pain in these swellings is sometimes so severe that sleep is impossible. Such periosteal swelling and thickening are revealed on a radiographic plate. Diffuse periostitis occurs more frequently in the hereditary than in the acquired form of the disease. Osteocopic pains are a common symptom of early syphilis, and of the late period of the disease as well. The bones of the skull, the sternum, ribs and clavicle are all affected. The pain is greatest at night and is of a boring character. It is probably due to subperiosteal swelling which explains its splitting character.

Osteitis and osteomyelitis may be either localized or diffused. These conditions are tertiary manifestations of the disease. They are accompanied by periostitis and in this case the inflammation, instead of confining itself to the periosteum, extends into the bone itself. The 
localized form of gummatous osteitis occurs in the condyle of one of the long bones, the femur or humerus, as a localized affection, or associated with gummatous synovitis. Gumma may also occur in the skull bones, the sternum, ribs or clavicle, and sometimes appear as precocious manifestations of the secondary stage. Pain is usually severe, and the bone is likely to undergo structural alteration involving impairment of motion in the adjacent joint.

Diffuse osteitis occurs as a sclerosis of the bones, and involves the skull, or the shafts of the long bones. The process produces a transformation of the soft or cellular bony structure into dense tissue. It

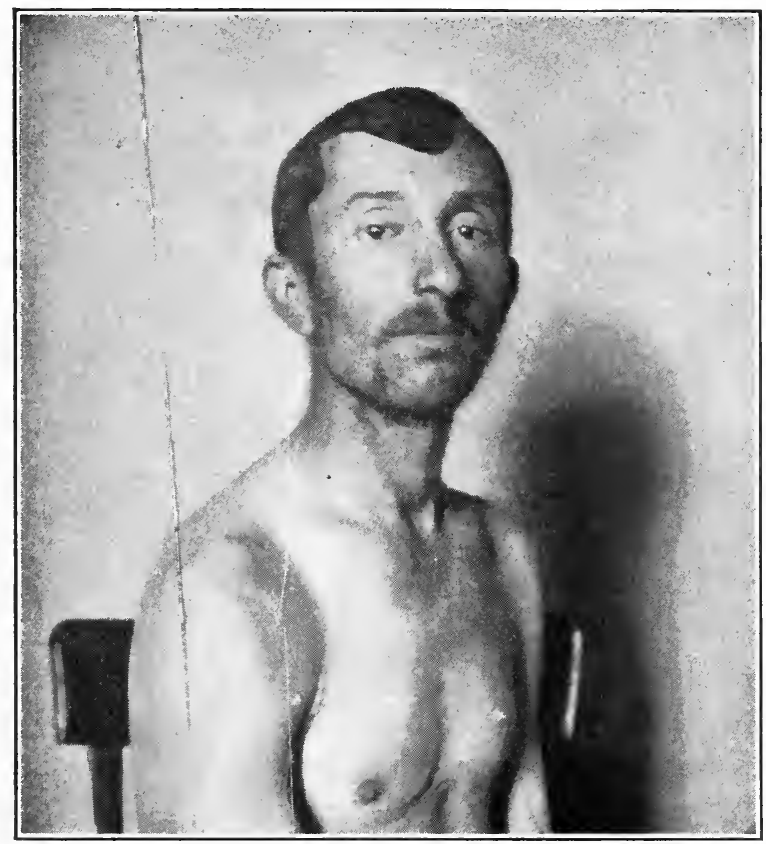

Fig. 296.-Bony node of clavicle.

is more common in the inherited than in the acquired form of disease.

Osteomyelitis is an extensive inflammation of the entire bony structure involving the medulla, the periosteum and the compact tissue of the bone, with a predilection for those bones in which the cancellous tissue predominates, as, for instance, the skull and face bones.

Radiographs of syphilitic bones show distinctive conditions (Fig. 297): an increase in the compact tissue and a thickening of the periosteal covering as well. The epiphyses are enlarged and translucent, while in the case of tuberculous bone none of these features is so pronounced. 
Syphilitic inflammation of the vertebral column more frequently attacks the cervical vertebræ, the affection being known as syphilitic spondylitis. There also occurs an inflammation of the lamellæ, "lamellites," as a result of which are produced symptoms due to pressure upon the efferent spinal nerves. The symptoms are more or less obscure. An indirect result of destruction of the vertebræ may be a paralysis of the extremities, due to pressure or implication of the

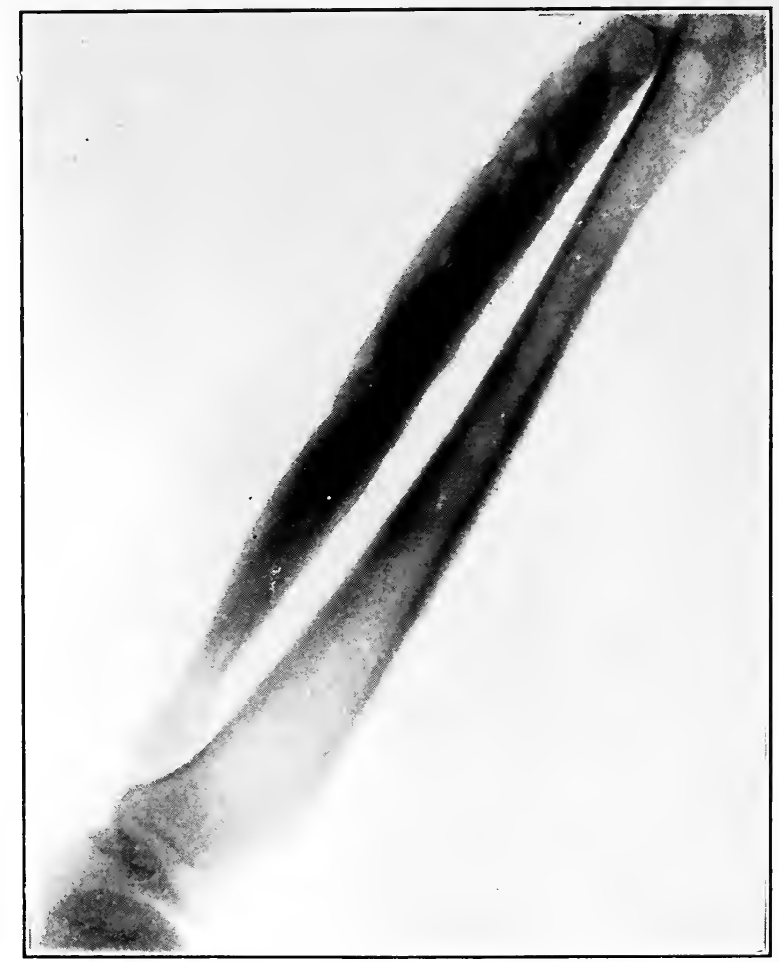

FIG. 297.-Radiograph of syphilitic osteitis.

spinal cord. In this class of cases there is usually a prompt response to treatment, be that the old specific form of medication-mercurial and iodide, or the more modern course of salvarsan, which often exerts its almost miraculous influence upon these lesions. An important preliminary thereto are the findings of a careful sero-diagnosis.

\section{SYPHILIS OF THE RESPIRATORY TRACT}

Both the primary and secondary manifestations of syphilis may be located in the nasal cavity. Chancre in this situation is rare and 
when it has appeared, has been mistaken for a malignant growth. Such an occurrence of malignancy, however, is likely to be more destructive and extensive.

Erythema and mucous patches occur not infrequently as secondary manifestations and are accompanied by evidences of nasal catarrh, soreness and scabs that may be recognized by intranasal examination.

The most important syphilitic nasal affections occur in the tertiary stage, in the form of gumma, ulceration, perichondritis, necrosis and atrophic rhinitis. One or the other of these affections produces the so-called syphilitic ozena or catarrh of a more or less purulent character. The odor of the breath is foul and the ultimate outcome, unless checked by treatment, is likely to be most serious, producing the striking deformity of "saddle nose" a permanent depression of the bridge of the nose, due to destruction of the nasal cartilages and bone, that presents a characteristic appearance.

Abscess of the lachrymal sac accompanied by conjunctivitis and sometimes necrosis of the lachrymal bone, may lead to inflammation of the middle ear and result in deafness. In the progressive forms of violent tendency, the roof of the nasal cavity may become implicated, and it may extend thence to the base of the brain and end fatally.

In all such conditions of nasal involvement, syphilis is likely to be suspected as the cause thereof and prompt general and local treatment is called for, always preceded by blood examination. Iodide of potash has been the time honored remedy, with mercurial inunctions pushed to the full limit of tolerance, but at the present time, here as elsewhere, recourse is had to the more modern method of salvarsan, which by intravenous injection and repeated dosage has proved to be a most rapid means of immediately controlling the ravages of this disease in almost every quarter.

The Pharynx.- The occurrence of the primary lesion upon the tonsil has already been mentioned. Secondary manifestations of faucial lesions have also been described $(q . v$.).

Tertiary syphilis of the pharynx occurs in the form of gumma, or of chronic inflammation and ulceration. In the one form there is a gummy tumor which encroaches upon the normal structures and may soften and cause destruction, unless treatment is instituted; in the second form there is a gummatous infiltration of more diffuse character, and in the last condition, an ulceration of more or less serpiginous character.

The symptoms vary according to the nature of the lesion, its extent and location. The treatment is that of the disease. Local treatment is secondary and entails the use of cleansing and disinfectant lotions, 
according to the individual requirements. When extensive scar tissues occur, after the healing of the lesion, operation is unwise unless demanded on account of some pronounced functional difficulty.

The Larynx. - Primary syphilis of the larynx is rare. Secondary involvement is more common, and occurs in the form of mucous patch or erythema, producing a laryngeal catarrh generally accompanied by

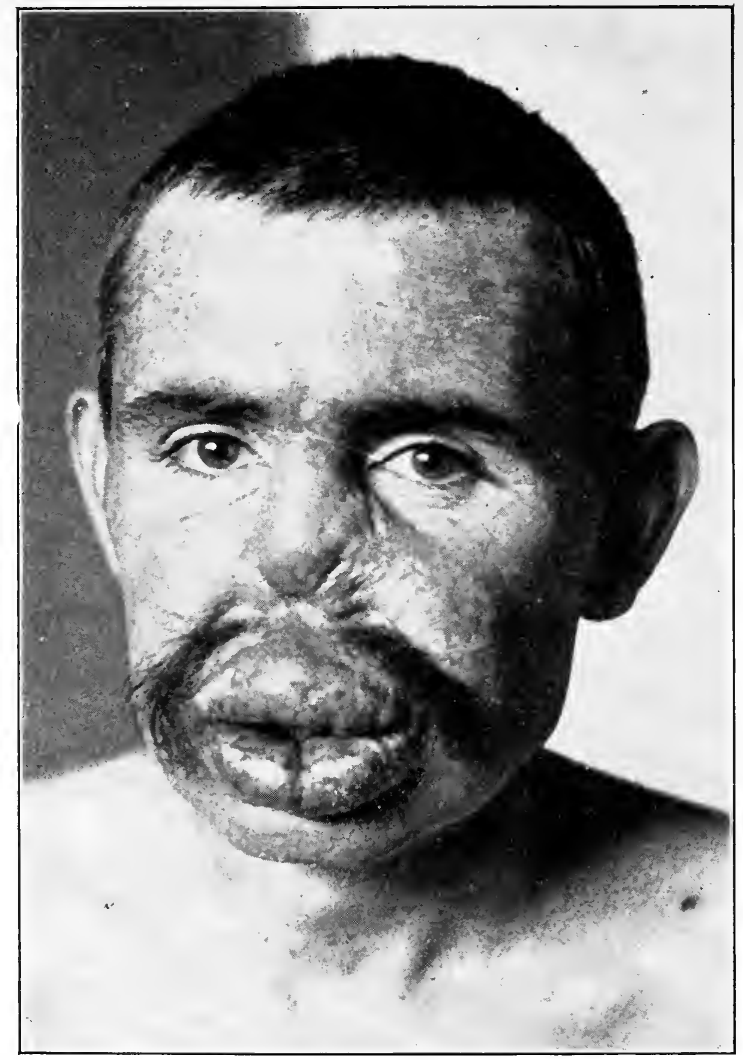

FIG. 298.-Tertiary syphilitic infiltration of lip.

secondary symptoms of the adjacent regions which aid in the clinical diagnosis of the condition.

Tertiary syphilis of the larynx occurs in the form of gumma, ulceration and perichondritis, with the secondary results of necrosis and cicatricial contraction. The symptoms accompanying such involvement of the larynx are those of laryngeal catarrh, characterized by a hoarseness of the voice of a husky or squeaky quality, and a 
certain amount of irritable cough. Laryngoscopic examination may show suspicious evidences of the disease. The condition is very chronic and must not be confused with tuberculous or malignant disease. In the former, the lungs are usually also involved and in suspected malignancy, a microscopic section will reveal the nature of the lesion, if this has not been satisfactorily decided by the complement fixation test of the blood. The prognosis depends upon the promptness with which treatment is instituted. The immediate effect of treatment is usually most favorable, especially with salvarsan, and the ultimate result thereafter depends upon the persistency and activity of the treatment.

The Trachea, Bronchi and Lungs.-The trachea and larger bronchi are subject to the same lesions as the larynx, but less commonly. Tracheal stenosis in the absence of an injury or burn by way of explanation, is likely to be due to syphilis. The condition is usually discovered by Kilian's method of direct tracheoscopy and bronchoscopy. Inasmuch as the condition is insidious, stenosis usually occurs before it can be prevented by active treatment of the existing lesions.

Pulmonary syphilis is very rare, although pathologists have discovered it in several forms on postmortem examinations, namely, gummata, sclerosis and syphilitic phthisis. The clinical aspect is likely to be identical with that of pulmonary tuberculosis and the diagnosis must rest upon the exclusion of the latter by examination and the establishment of the presence of syphilis by serum diagnosis.

The favorable influence of specific treatment is not necessarily conclusive of the existence of syphilis, inasmuch as phthisical patients have been most favorably affected by hypodermic mercurial injections, notably the succinimide of mercury.

\section{SYPHILIS OF THE ALIMENTARY TRACT}

The Mouth.-The occurrence of extragenital chancre of the lips has already been described, as well as the mucous lesions of the buccal membrane. In the later stages of the disease, inflammation and ulceration, also previously referred to, may take place, and sloughing phagedena is one of the extreme types (Fig. 298). Syphilis of the hard and soft palate has also been considered.

The Tongue.-Primary and secondary syphilis and the different types of glossitis as results of tertiary syphilis, have already been described.

Syphilitic Involvement of Esophagus, Stomach and Intestines is rare. The rectum on the other hand is often affected. 
Gummata and ulcers of the esophagus have been observed by direct esophageal inspection and stenosis from cicatricial contraction may result.

Acute and diffuse syphilitic gastritis has been reported, as well as gummata in this region. Ulcerations of syphilitic character have occurred in the intestinal tract, more frequently in the jejunum with a resulting obstruction or perforation, followed by peritonitis.

In all of these conditions, the character of which is necessarily obscure, the diagnosis must be made by the elimination of other causes and the establishment of a positive serum diagnosis for syphilis.

Syphilis of the Rectum.- - The most frequent lesion in this region is ulceration, as a result of which there may occur much thickening of the rectal walls and secondary stricture. In all cases presenting these symptoms the existence of syphilis as a causative agency should be considered. The favorable response to treatment in syphilitic affections here, as in other parts of the alimentary tract, furnishes evidence that is lacking when the same conditions exist from other causes.

Syphilis of the Liver, Spleen, Pancreas and Peritoneum.-Syphilis of the liver appears in a variety of forms: diffuse hepatitis, perihepatitis, gummata, amyloid degeneration. In diffuse hepatitis, there is an intralobular new growth caused by greatly increased connective tissue. In perihepatitis, there is extensive cellular proliferation on the outer surface of the liver.

Gummata may be single or multiple, varying in size, and composed of the usual elements of gummatous construction, namely, fibrous tissue with a necrotic centre of cheesy material. As the result of gummatous invasion, numerous cicatricial areas may be found in different portions of the liver substance, causing contraction and distortion of its shape. Taking it all in all, the liver is more commonly affected by syphilis than any of the other viscera. The symptoms of syphilitic disease of the liver vary according to the stage of the disease. Jaundice has already been spoken of as an early manifestation and is probably then due to a catarrhal condition. Enlargement and tenderness of the liver may be elicited by physical examination, when it is the seat of late syphilitic disease, resembling a cirrhosis and accompanied by indigestion, some jaundice, ascites and decrease in weight.

Some conditions of syphilitic involvement of the liver are promptly and effectively cleared up by constitutional treatment. In other cases of long duration where there is decided enlargement of the organ, and the syphilitic changes have involved extensive destruction of the normal structure, no radical curative result can be expected. 
The Spleen.-Enlargement of this organ sometimes occurs in the secondary stage, due to hyperemia and engorgement, in which case it may be a part of a generalized condition including also the liver.

The most common lesion affecting the spleen is the gumma, which resembles those in the liver, and may be single or multiple. The other changes are also similar, namely, acute splenitis, perisplenitis, and amyloid degeneration.

The Pancreas.-Chronic and interstitial pancreatitis occurs as a result of syphilis; gummata and cicatrices have also been observed; the resulting symptoms of pancreatic involvement are indigestion, emaciation and fatty stools.

Peritoneum.-Invasion of the peritoneum in syphilis usually occurs on account of involvement of the liver, spleen and intestinal tract by gummatous changes, resulting in perforation, under which circumstances the peritoneum becomes thickened and adherent, the same as in inflammatory conditions from other causes.

\section{SYPHILIS OF THE CIRCULATORY SYSTEM}

The cardio-vascular changes occurring as a result of syphilis are the most important of its various morbid lesions and the secondary effects resulting therefrom are the most dangerous and far reaching, inasmuch as they are accountable for the late degenerative cerebrospinal affections and may produce that common circulatory depreciation that impairs the health and shortens the life of the individual, namely, hardening of the arteries.

Syphilis of the Heart.-There may occur a fibrous pericarditis as a result of syphilis and syphilitic endocarditis is also observed.

Myocarditis occurs either as a fibrous or gummatous infiltration of the left ventricle. The symptoms are those of cardiac enlargement, accompanied by high tension of pulse, tachycardia, dyspnea and vertigo. Sudden death may occur from rupture of a gumma or embolism. The diagnosis is made upon the clinical history and the response to treatment, which it is needless to say in any case under suspicion, should be pressed with the greatest degree of activity. The iodides have always been a favorite remedy. There is some question as to the propriety of using salvarsan in cardiac conditions, but where such condition is frankly and definitely due to the disease itself, it would seem that the careful and deliberate use of the remedy is not contraindicated, but in cases of doubt, it is safer to begin with the time honored remedies and wait until the dangerous symptoms have succumbed before having recourse to salvarsan medication. 
Syphilis of the Arteries. - Syphilis is considered an important causative factor of arteriosclerosis; and with the occurrence of aneurism, careful investigation of the clinical history is always made with a view of finding evidence of a syphilitic infection. Syphilis involves the arteries in the nature of atheroma. Spirochætæ have been demonstrated in the lesions of arteries. In the large arteries, areas of degeneration are observed, consisting of fibrous thickening and sometimes calcareous deposits. In the small and large arteries the entire circumference is often involved in the deposit of connective tissue. Obliterating endarteritis may develop as a result of the above-mentioned changes, but gummata are more commonly found. In the larger arteries, gummatous foci are encountered here and there, and as in other portions of the body, these lesions may undergo softening and perforation, which may lead to serious consequences, such as severe hemorrhage, resulting blood clot with dangerous pressure in the vital nerve centres, and secondary inflammation, when rupture occurs in one of the important carities, such as the peritoneum, the pleura or the pericardium. The symptoms of syphilitic arteritis are found in manifestations of paralysis or hemiplegia and such focal symptoms as would naturally occur as a result of a lesion localized in that portion of the brain or spinal cord from whence the nerve control of the particular region involved is derived (see Syphilis of the Nervous System). Angina pectoris follows implication of the coronary artery. As already mentioned, hemorrhage into the various organs and secondary inflammation may be a sequel to arterial disease.

The constitutional treatment usually followed favors a preponderance of the iodide. On account of the chronicity of the character of the lesions salvarsan may be of value in the acute active manifestations but for the chronic changes and secondary condition resulting therefrom, namely, arteriosclerosis, rupture of the blood-vessels and attendant conditions, little expectation of brilliant therapeutic action can be entertained.

\section{SYPHILIS OF THE GENITO-URINARY ORGANS}

Urethro-penile syphilis may occur at any stage of the disease. The glans penis is the principal site of the primary lesion and with the resulting lymphangitis represents the extent of the disease at this most frequent point of entry (see p. 701). Certain possible sequels of the endo-urethral chancre have an importance in the domain of genito-urinary surgery, namely: cicatricial stricture and resulting urethral fistula. Such a stricture develops rapidly during the repara- 
tive stage of the sore, and shows little response to dilatation or urethrotomy, a plastic operation being sometimes required. The fistula when it occurs, appears on the under surface and may be so resistant to the unusual treatment as to demand operation. (See p. 297.)

In the secondary stage, the cutaneous eruptions, mucous patches, and ulcerations occur within the preputial cavity, but rarely in the urethra. These secondary ulcerations are sometimes, in the absence of a previous clinical history, mistaken for a primary syphilitic lesion.

Gummata attack this region in the tertiary stage from five to ten years after infection. They generally occur primarily in the external coverings of the glans and may eventually invade the urethra. Gummata of the corpora eavernosa are of rare occurrence and should not be confused with chronic circumscribed inflammation (fibrosis) of these bodies. The latter condition never softens, whereas the gumma is likely to undergo caseous degeneration. When the gumma extends its growth so as to invade the urethra, marked deformity may result and fistula form. The gumma may be single or multiple, and, if a correct diagnosis is not made, the process may continue for years, and be ascribed to tuberculosis or carcinoma. It is not likely, however, at the present day that the condition will be overlooked with the modern methods of blood examination. In doubtful cases unsettled by blood examination, it is wise to institute treatment either in the form of the older specific remedies or the more recent salvarsan therapy.

The Prostate.-There is little knowledge of syphilis of the prostate, and it is certainly rare. In any case with other syphilitic manifestations, in which by rectal examination the prostate is found to be hard and nodular with no definite etiology obtainable, and accompanied by such symptoms as dysuria, discharge, and perhaps retention, it is proper that the blood be examined and specific treatment tried.

The Testicles.-These organs may be affected at any stage of the disease; while the epididymis may contain the main focus of trouble, more frequently the body of the testicle is principally involved (Fig. 299).

Epididymitis.-Nodules of different sizes, free from any tenderness, develop in the head of the epididymis; the remaining portion may undergo a general chronic infiltration surmounting the body of the testicle, and give to it a characteristic clam-shell conformation and hardness; it is insidious of onset, and this together with the beginning localization and absence of pain differentiates it from acute epididymitis of urethral origin; again gonorrheal epididymitis commences in the 
tail of the epididymis, and rapidly invades the other portion, accompanied by increasing pain and general constitutional disturbance.

In tuberculosis of the epididymis, softening of the parts is rapid, and the vas deferens is likely to be involved, which is not so often the case with syphilis. In sarcoma of the testicle, the nodules are larger, the growth is more rapid, and is likely to be accompanied from the beginning with a fluid, generally bloody, in the tunica vaginalis.

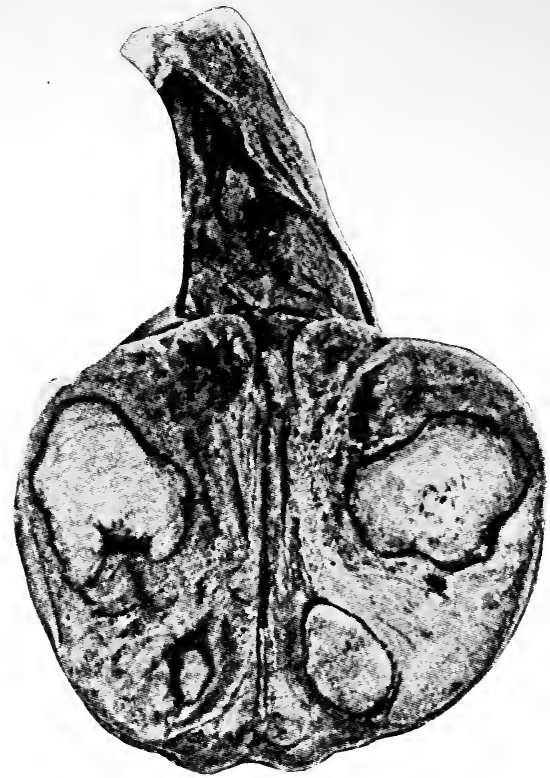

FIG. 299.-Syphilis of testicle.

Cysts of the epididymis should not be misleading; they are more elastic in feeling and the fluid content is readily detected by aspiration.

The prognosis is favorable, both as to the patient's general condition and the disappearance of the lesions, when prompt treatment is instituted, either by mercury and iodide of potash or, preferably, by an efficient salvarsan course (see p. 780).

Orchitis. - The testicle as a whole is attacked by syphilis in two forms: localized gummatous growths and a generally diffused involvement. In the latter form, the deposit of new connective tissue occurs in abundant masses, causing an obliteration of the seminal tubules; the organ is uniformly enlarged and indurated, the hardness is of a woody character, and the natural sensitiveness is decreased; hydrocele may be present, and the outline of the epididymis may be lost and become a portion of the symmetrical enlargement of the organ, or 
there may be a separate implication of epididymis surmounting that of the rest of the gland.

In the gummatous type superficial nodules are distinctly felt, giving to the organ an irregular contour; it is generally smaller than the diffusely inflamed condition and is likely to be a later development than the latter variety.

Diagnosis.-The chief conditions to be differentiated are tuberculosis and malignancy. In tuberculosis, the body of the testicle may be but slightly involved, and the epididymis more commonly bears the brunt of the trouble; whereas in syphilis the body of the testicle rather than the epididymis is more likely to be implicated. The vas deferens is frequently involved in tuberculosis, while in syphilis this is uncommon, and when the latter disease extends this far it generally involves the cord in toto. The seminal vesicles and prostate are often attacked early in tuberculosis, which is not the case in syphilis; that is, they give no local evidence of being so affected. Ulceration and resulting abscess are far more common in tuberculosis. The painless condition of the testicle may he a feature of either of these maladies.

Sarcoma of the testicles may be confused with syphilis, and the possibility of its existence must always be considered in the differential diagnosis. The scrotal veins in sarcoma commonly show severe congestion; the testicle is liable to be cystic, and as has been stated in the case of such lesion of the epididymis, there is likely to be present a bloody fluid in the tunica vaginalis. The spread of the malignant process is prone to be rapid and involve the cord from below upward. In any case, a doubtful diagnosis may be cleared up by the usual blood examination.

The prognosis of syphilitic testes is likely to be favorable unless the disease has progressed so far that the resulting fibrous infiltration has caused an atrophy of the normal substance, and, if both organs are so involved, sterility will be the natural result.

The treatment by the old remedies of mercury and iodide of potash has always been the means of producing a prompt cessation in the activity of the process and removal of the lesions of syphilitic testicles; this may be expected with still greater assurance in the use of salvarsan treatment.

Syphilis of the Bladder. - This condition is generally considered to be very rare which may be due to the fact that existing specific lesions have gone undetected. Recent cases have been reported of syphilis of the bladder recognized by cystoscopic examination which have occurred in the form of circumscribed areas of inflammatory infiltration involving the interior of the bladder that have been accompanied 
by a positive blood examination and have disappeared under iodide medication or salvarsan infusion.

Renal Syphilis. - While it is difficult to determine the frequency of renal syphilis, its occurrence is rare. It is believed that during the secondary stage both kidneys may play a part in the general constitutional invasion of the disease, and that the toxins of syphilis may cause inflammation of these organs similar to that caused by other toxins. More commonly the tertiary form of the disease is found in these organs producing such lesions as gumma, amyloid degeneration and chronic nephritis of different types. The gumma are of various sizes and number and appear in the cortex as well as in the medulla.

A sclerotic degeneration associated with an amyloid lesion similar in character to that in the liver may affect the kidney and produce a misshapen organ. The various types of nephritis are found as a result of syphilitic infection and the lesions are those that are characteristic of the different stages of Bright's disease. The entire kidney may atrophy from arteriosclerosis and other causes besides syphilis may be at work in producing the renal lesions, such as alcohol, excessive amounts of iodide of potash, etc. In the latter instance it may be determined by discontinuing the medication, that an improvement in the kidney condition is noted. In advanced forms of syphilitic kidney disease as in similar conditions from other causes, it will be found by the functional urinary tests that the elimination of methylene blue and phenolsulphonephthalein is retarded (see Functional Renal Diagnosis).

In the early stage the disease will yield to antisyphilitic medication, and while it is generally taught that kidney disease is a contraindication to the employment of salvarsan, if it can be determined that the lesion is entirely of syphilitic origin and is not too far advanced, treatment by this method may produce a promptly beneficial result. In doubtful cases it is better to begin with the older remedies and when improvement has become erident and the kidney is apparently less susceptible, subsequent salvarsan medication may be adopted. Serere advanced cases are beyond medical aid of any kind.

\section{OCULAR SYPHILIS}

Syphilis may attack the eye in any of its stages. The occurrence of an extragenital primary sore in this region is a possibility, notably upon the conjunctiva. Its appearance under these conditions is characteristic of the primary lesion in other locations.

Hyperemia, mucous patches and ulceration attack the mucous membrane of the eye during the secondary stage. 
Iritis is a common ocular manifestation of the secondary stage. The inflammation usually involves the other structures of the eye besides the iris: namely, the cornea, ciliary body and other coats of the orbit. The resulting symptoms are diffuse haziness of the cornea and a characteristic injection of the ciliary body. The iris loses its lustre, reacts sluggishly and sometimes shows adhesions. There are a dimness of vision, pain, and sensitiveness to light to a greater or less degree. The diagnosis is suggested by the clinical history and blood reaction, and confirmed by competent ophthalmoscopic examination. The course of this condition is tedious and the function of the eye is not infrequently impaired by resulting adhesions.

Treatment entails the adoption of the appropriate course for the general disease and especial attention to the ocular condition, which requires the expert management of one properly trained in this line of work in order to gain as much as possible from the treatment, and at the same time to exercise wise judgment that such treatment is not being pushed to the extent of producing an irritating rather than a controlling influence. Generally speaking, it may be stated that there is no contraindication to the employment of salvarsan in such ocular lesions, although sometimes there will occur as a result of its use a sharp conjunctival congestion, which will promptly pass away.

Retinitis as a result of syphilis occurs as a secondary manifestation. The most frequent site of such involvement is in the region of the optic nerve. One eye is usually affected at a time. It occurs in combination with choroiditis (choroido-retinitis).

The symptoms are night blindness, micropsia or metamorphopsia and defective vision. Scotomata may be present. The retina loses its transparency and appears dull. Further intraocular appearances, including opacities in the vitreous, yellowish spots and pigment deposits in the choroid, are recognized by competent ophthalmoscopic examination. It is important that diagnosis be made early as complete recovery depends upon prompt treatment.

The treatment is that of the stage of the disease and the complicating condition. Mercury by inunction has been a favorite method in the beginning of the trouble, and iodide of potash in the later course thereof. To-day, the possibility of any ocular damage being eliminated, the objection to the use of salvarsan has been removed, and this treatment can be resorted to with benefit at any stage of the affection. In addition to this, however, competent management of the local condition, entailing complete rest and protection from light and appropriate measures for guarding against intraocular adhesions, should be adopted. 
In the tertiary stage of the disease gummata may attack the different portions of the eye: namely, the lids, the conjunctiva, the choroid, and the sclera. An extensive gummatous ulceration of the face frequently invades the exterior orbital region (Fig. 300). Involvement of the lachrymal sac by a gumma has already been mentioned, and a secondary stricture of the nasal duct may result. Chronic periostitis and gumma of the orbital cavity also occur. The treatment of these various forms of late syphilitic disease consists in the use of antisyphilitic remedies and the proper protection of the eye

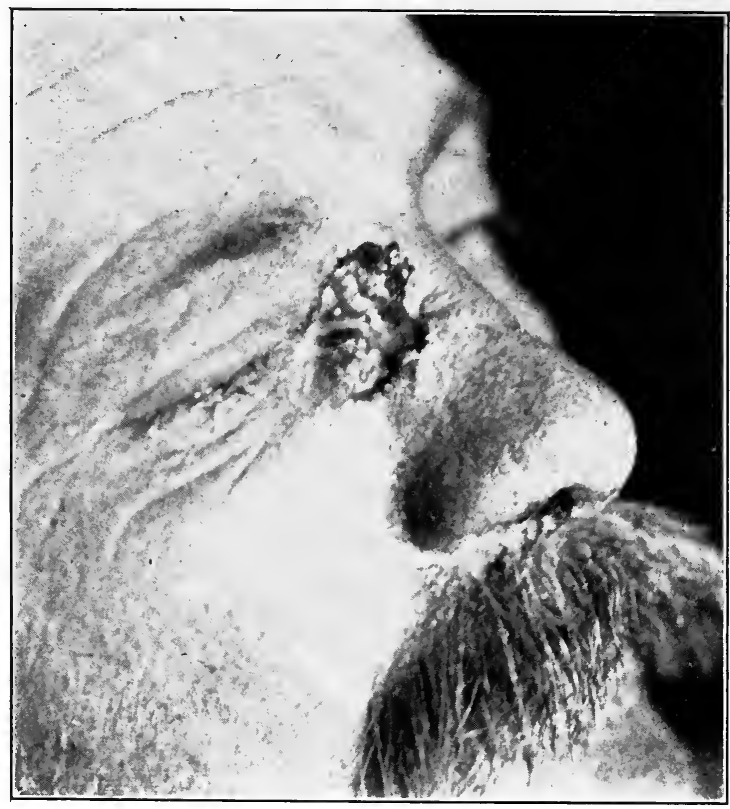

FIG. 300.-Gumma of face, involving eye.

under expert management (see General Treatment of Syphilis, p. 765). Optic neuritis is usually an indication of intracranial disease such as gumma or syphilitic meningitis. In this case the clinical history is an important element in the diagnosis as the symptoms are similar to those of any other intracranial tumor. Serological and spinal fluid examination should both be adopted to settle the question; antisyphilitic treatment will usually exercise a prompt beneficial effect upon the syphilitic disease. The outlook is not generally favorable so far as complete recovery is concerned, as sight is often impaired 
before the effect of treatment is established. Some cases end in complete atrophy of the optic nerve.

The treatment is that of the disease, and while the iodides have always been the favorite form of medication, some brilliant instances of the cessation of this condition under salvarsan are recorded.

\section{AURAL SYPHILIS}

Almost any part of the auditory system may be attacked by the disease, and in any stage. Extragenital chancre may occur in the region of the external ear. The secondary eruptions appear upon the external portion of the ear in the form of papular and squamous patches. Gummatous ulceration also attacks this part.

The middle ear has been infected by surgical instruments, notably the eustachian tube. The various lesions of the nasopharynx may terminate in catarrh, as a result of which condition there may be suppuration in this region; this may arise either as an indirect consequence of the disease, or on account of a lesion of the middle ear and may lead to impairment of hearing.

In addition to the constitutional treatment of the disease itself, the requirements for treatment call for special management of the condition in the middle ear.

The Internal Ear.-Disease of the labyrinth develops as a manifestation of the secondary stage, and may occur as a part of the syphilitic process extending from the nasopharynx. This condition will produce impairment of the hearing, which will promptly disappear under appropriate treatment.

The more severe form of internal ear involvement is represented in one of the well-recognized neuro-recurrences of the disease, when the auditory nerve is affected; such involvement of this nerve may be peripheral, in the form of neuritis, or central, in the form of a basal gummatous meningitis that involves the nerve at its point of origin. Deafness may occur in either case.

The prognosis depends upon the early recognition of the cause of the lesion, the extent to which the destructive change has extended and the prompt and vigorous employment of treatment.

The treatment of syphilitic disease of the labyrinth and auditory nerve has usually called for the active administration of mercury and the iodides; mercury by hypodermic injection and iodides in large doses by mouth. Salvarsan treatment has the same value here as in other active syphilitic manifestations, and while it cannot be expected to restore to the normal condition tissues that have undergone destruc- 
tive changes, it certainly exercises the most rapid means of causing a cessation of the activity of the syphilitic process, and it is not believed that direct damage to the auditory apparatus is produced by its action. Those cases in which deafness has been ascribed to the employment of the remedy are no doubt due to an active syphilitic process within the labyrinth or at the origin of the auditory nerve. 


\section{CHAPTER XLI}

\section{SYPHILIS OF THE NERVOUS SYSTEM-THE HEREDIT- ARY DISEASE}

Nervous Syphilis.- Under this division are embraced all forms of syphilis of the nervous system, both inflammatory and degenerative, inasmuch as the causative relation of the spirochete in these two phases of the disease is an undoubted one.

The date of onset of syphilitic nervous symptoms is recognized as being earlier than was formerly believed; in fact in precocious cases manifestations that properly belong to the late tertiary stage are sometimes observed in the early secondary period, and represent the sole development of the disease immediately following its inception.

As to the variety of nervous diseases that are encountered, there is hardly a type that is not found within the category of syphilis. Therefore, in all organic affections of the nervous system, and in fact, in any serious nervous condition, it is customary to consider the possibility of a luetic history, and to examine the blood. Furthermore, it is usual that victims of this disease are more prone to give themselves concern about the possible development of such nervous phenomena than about any other consideration pertaining to their malady.

The affections of the brain, spinal cord, cranial and spinal nerves are many, and head the list of tertiary lesions. Any one of these tertiary manifestations may be of grave and serious character, and when they reach the degenerative stage, the outlook becomes exceedingly gloomy, especially in the much-feared types of locomotor ataxia and paresis. There are likely to be, although not always, warning signs of cerebral syphilis. Persistent and severe headache as a symptom should always be taken account of and investigated. Formerly it was quite customary, in cases of doubt, to give a course of mercurial medication, as a possible means of clearing up the question. At the present time, however, recourse is had to the modern method of blood examination and if there continues to be a doubt, the still more modern practice of salvarsan treatment is resorted to, on account of the prompt and brilliant results that follow its use during an active inflammatory stage. It has been stated that salvarsan works marvels in the case of 
syphilitic skin affections and that it is accompanied with pitfalls and uncertainties in nervous lesions. This is believed to be more the case when a degenerative stage has set in or when an adequate amount of the drug has not been given, or a sufficiently prolonged course followed, as a result of which defect, instead of producing complete destruction of the morbid process, a diminished resistance ensues.

Causation. - It is generally considered that the hereditary nervous temperament affords a predisposition to the occurrence of late nervous syphilitic affections. In an examination of the histories of many cases, it will be found that some individuals who have taken a very small amount of treatment may not develop such symptoms, whereas those who have taken an apparently ample course are among the victims of nervous disease. Nevertheless, it is a generally accepted fact that the more prolonged and efficient is the treatment, the less likelihood there should be of the development of those after-occurrences; but when, the predisposition to nervous excitability is exceptionally strong or when an excessive amount of treatment has been carried out to the extent of maintaining a depreciation in the quality of the blood, and therefore a diminished nerve resistance, a greater tendency to some form of neuro-recurrence is engendered.

Excesses of any kind are doubtless strong exciting factors. The abuse of alcohol, undue worry and anxiety, prolonged fatigue and any cause of great constitutional depreciation may play a part in the causation. The active demands of business life to-day, with the continuous competition and struggle for supremacy, throw a great burden upon the nerrous system and increase the susceptibility thereof. Treatment exercises a strong preventative influence, but is not a certain protection. ${ }^{1}$

It has been observed that in such locations as Central Africa, when syphilis is introduced among the natives of a certain district, while it runs a most malignant course, in some instances causing rapid destruction and death, these late manifestations of profound nervous lesions, particularly the degenerative forms, are practically unknown and this is probably due to the absence of all of the malign nervous influences that accompany life in a cirilized community and from which the natives of a wild and savage existence are entirely free.

Pathology.-A study of the microbiology reveals that the disease is due to inoculation with a specific organism (spirochete-pallida) which has been discovered and isolated and more recently cultured

\footnotetext{
${ }^{1}$ Fournier, in studying a group of cases, determined that spinal cord disease occurred in only 5 per cent. of those thoroughly treated, contrasted with 95 per cent. of those whose treatment was neglected.
} 
(Noguchi). And while these parasites, formerly, were found only in the primary and secondary stages of the disease, they are also present in the tertiary period, in gummata and lesions of the arteries and in the degenerative lesions of the brain and spinal cord, the spirochæte have been found, most recently, isolated in the lymph spaces almost beyond reach or detection.

The different pathologic lesions characteristic of lues of the nervous system are embraced in meningitis, arteritis and neoplasms. In many cases, all of these conditions occur simultaneously.

The organisms and toxins of the disease exert their pernicious influence directly upon the tissues of the nervous system, or indirectly, through the blood-vessels, by impairment of the circulation, rupture, blood-clot and softening.

The tissue changes in the case of the direct lodgment of the spirochætæ in the lymph spaces, radiate around these organisms and consist of a hyperplasia of the connective-tissue cells and a proliferation from the blood-vessels of round cells. The walls of the blood-vessels, as well as the connective tissue proper, are the seat of these changes. It is natural to believe that the disease lays its mark upon the portion which, in a sense, is the point of least resistance, but what exactly is the cause and explanation of the existing weakness may not be evident. In a word, lues produces the formation of new tissue as evidenced in the development of endarteritis, chronic infiltration, peri-arteritis, gummatous meningitis and gummata; and secondary changes caused by obliteration of the blood-vessels beginning in the smaller arteries and extending to the large ones.

Brain-lues.-Gummatous invasion of the brain occurs in circumscribed and diffused form. The starting-point thereof may be a preëxistent luetic disease of the bone, an osteitis of the skull or vertebræ, or the growth may start primarily in the meningeal covering of the brain and thence extend outward to the neighboring bone. Most commonly, the gumma of meningeal origin is derived directly from the blood-vessels in contact with the meninges. The dura is more frequently attacked upon the summit of the cerebrum and the arachnoid at the base.

Circumscribed gummata more often involve the base of the brain (Fig. 301). The cranial nerves may be implicated and almost any other part of the brain or spinal cord. These gummata may be single or multiple. They are composed of a mass of cells, crowded in the interspaces of the connective tissue, exerting harmful pressure upon the blood-vessels which leads to central necrosis (caseation). According as the brain tissue is destroyed or pressed upon, there is 
a corresponding loss of function, which may also occur as a result of destruction of the nutrient artery supplying that particular portion. The diffuse gummatous infiltrate may involve all other coverings of the brain or spinal cord or confine itself to the arachnoid and pia. The pathological process may extend itself from one end of the spinal canal to the other and this may be accompanied by a distribution of gummatous foci throughout the brain, a characteristic form of cerebrospinal meningitis. The most common form of luetic meningitis is

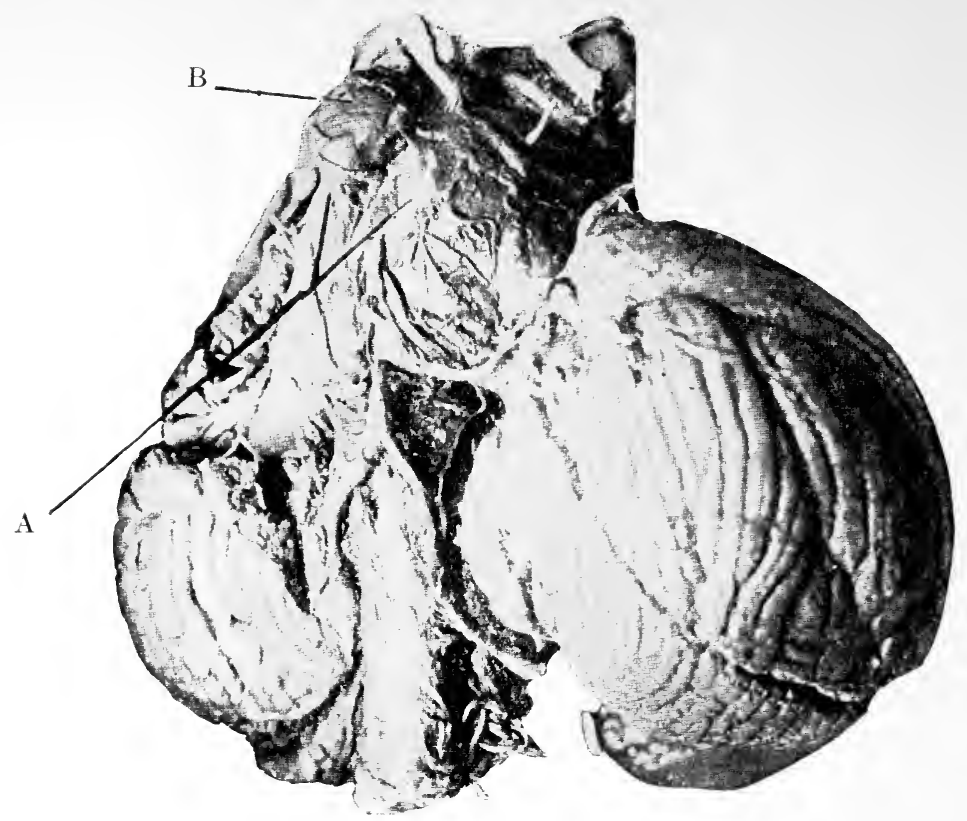

FIG. 301.-Cerebral syphilis of base. A, Basilar meningitis. B, Erosion of basilar artery. (Bailey.)

diffuse leptomeningitis of the base of the brain. Involvement of the cranial nerves may or may not accompany this condition.

Symptoms of Brain-lues. - Inasmuch as the pathological process of cerebral lues has a more or less serpentine distribution, and characteristically undergoes a cessation in one part when it invades another, the symptoms accompanying that process are correspondingly variegated, that is to say, the symptoms present different phases, sometimes improving and relapsing alternately. This combination of shifting focal symptoms with a persistent headache is characteristically suggestive. Otherwise there may be a solitary symptom as an evidence of the disease, such as transitory aphasia; there may develop 
a chronic muscular disability, or the symptom-complex may contain a combination of these symptoms referable to the brain and the spinal cord. In such generalized condition the patient appears heavy and dull, headache is a prominent symptom with increased severity at night. The pupils are of ten irregular in size, although both of them respond to light; sometimes the Argyll Robertson pupil is present. The ordinary symptoms of meningeal irritation such as dizziness, vomiting and epileptiform seizures are also manifested.

A further description will embrace a designation of the mosi common clinical types.

Basal Meningitis. - There is a variation in the clinical manifestation of the disease when it attacks the base of the brain on account of the complex structures composing this region. One of the most constant of these symptoms is, as stated, a common accompaniment of all brain lesions, severe and paroxysmal headache, and, inasmuch as it is almost invariably more severe at night, it is accompanied by marked insomnia. Then come a general failing in health, constant or intermittent attacks of dizziness and sometimes a staggering and even total loss of muscular control which may be transitory in character. Temperature as a rule remains normal, although there may be an elevation in the beginning. Vomiting attacks often occur and sometimes epileptiform seizures. The dulness or somnolent condition is due to cerebral anemia and this may progress to a comatose state. Other symptoms, that occur to a lesser or greater degree in different cases, are polydipsia and polyuria; diabetes mellitus and ocular symptoms of various sorts are frequently present. These ocular lesions are recognized by visual and ophthalmoscopic examination and consist of faulty vision, due to changes involving the optic tracts, without involvement of the fundus, hemianopsia, concentric limitation of the visual field, or diminution in the acuteness of the central vision.

With implication of the cranial nerves, resulting symptoms are produced. The third nerve is most commonly affected, which is evidenced by a difference in the size of the pupils and an absence of pupillary contraction. In certain cases ptosis follows paralysis of the levator palpebræ muscle.

As motor paralysis of the ocular apparatus is a frequent symptom of cerebral lues, it becomes an important manifestation to be taken account of in reaching a diagnosis, and in this connection it is noteworthy that transitory paralysis occurs more frequently in tabes than in cerebral lues.

The olfactory nerve is sometimes, but rarely, affected. Paralysis of the fourth nerve is also unusual. Involvement of the sixth nerve 
is far more frequent. When the fifth nerve is affected, which occurs about as frequently as the fourth, the affection is unilateral and the sensory are more marked than the motor symptoms, neuralgia being a prominent disturbance; and, while there is liable to be hyperesthesia at first, there may be complete anesthesia later on. With involvement of the motor fibres of the fifth nerve, there is shown a decrease in the muscular power of mastication. The seventh and eighth nerves may be involved together, the resulting symptoms being facial paralysis and deafness. Deafness has been considered a very hopeless symptom, but some striking cures have occurred since the introduction of the salvarsan method. Facial paralysis usually yields to treatment.

Involvement of the ninth and tenth nerves is exceptional but has been reported. The eleventh and twelfth are not uncommonly affected, producing paralysis of the soft palate and vocal cord, and motor paralysis with, sometimes, atrophy of the tongue.

When one of the cerebral peduncles is implicated, there may be a partial loss of power of one of the limbs, in which case the arm or leg is unwieldy, but not completely useless. These symptoms may also be produced by the pressure of a gumma or by arterial disease. The symptoms occur on the opposite side of the body with peduncular disease due to decussation of the nerve fibres, whereas where the cranial nerves are affected, the symptoms occur on the side on which the pressure is exerted upon the fibres outside of the brain.

The general course of basal meningitis is chronic, although in some rapid and severe cases, death may occur within a few weeks. Cerebral apoplexy may occur at any time throughout the course due to thrombosis of the basilar and middle cerebral arteries.

Meningitis of the Cerebral Cortex.-Involvement of the summit of the brain alone may occur, although a disease of the base is liable to be associated with it. Cortical meningitis is either circumscribed or diffuse. An injury to the head may be the beginning of such trouble. It may commence as an extension from an active bony lesion, or start in the membranes and may thence extend into the brain structure, producing an encephalitis.

Here again, as in other brain lesions, the peculiar headache is a constant symptom. The pain is often extreme, and extended over a large or confined to a small area; it is intensified by pressure. Other symptoms occur to indicate the location of the area involved. Epileptiform convulsions, or monoplegia and hemiplegia, signify that the motor area is the seat of trouble. It has been noted that, in this connection, specific remedies do not have the same effect upon the disappearance of the symptoms when the substance of the brain is 
encroached upon as when the meninges or bony structure is involved. This is probably due to permanent loss of tissue. While epileptic convulsions are associated with involvement of the motor area, they may also be caused by arterial disease, and in some instances are congenital in origin, or due to involvement of areas adjacent to, but not in, the motor tract. The epilepsy occurs in the two usual types, grand mal and petit mal. The attacks may occur at regular intervals: once, twice and three times a month. There is usually a terrific headache, but other premonitory symptoms are wanting. The clinical history will aid in the diagnosis, and syphilitic epilepsy will be favorably affected by specific treatment, be that along the old line of mercury and potash or the most recent method of salvarsan, whereas the non-specific type of the malady will be unaffected by such a course. The blood and spinal fluid should be examined and may establish the diagnosis.

Syphilitic Arteritis.-The symptoms of arteritis develop either slowly, in the obliterating form, or suddenly, when thrombosis occurs. In the latter instance the appearance is that of apoplexy. The symptoms of arteritis of the brain and spinal cord occur in connection with and as a sequence to meningitis, or they may precede it. The headache is not as severe as in meningitis but is worse at night. The other symptoms of giddiness, insomnia, mental apathy, may occur and various focal symptoms, such as spastic seizures, aphasia and paralysis are all produced.

Lues of the Spinal Cord.-As in the case of cerebral disease, so it is with disease of the spinal cord: 75 per cent. of the cases owe their origin to syphilis. The occurrence may take place, exceptionally, within a few months after the infection, but as a rule they appear in about three years or later, and there may be an advance of the disease of two decades before such development occurs. The predisposing causes are those that have been mentioned as predisposing to nervous lues in general, such as excesses of various kinds, mental stress and overexertion, susceptibility of temperament, and neglect of treatment. Men are more commonly affected than women and it is less frequent than the cerebral form of disease.

Symptoms.- Often the symptoms are so varied and numerous that they cannot be ascribed to a single lesion. This is partially explained by the variable distribution of the diseased process, there being in some cases a meningitis of the spinal canal and a basal cerebral meningitis as well. It may begin as a meningeal gumma, giving rise first to the pressure symptoms of a tumor, and then those of meningitis (Fig. 302), followed by changes in the cord, such as sclerosis or 
gummatous arteritis, followed by softening, with a changing array of symptoms, and finally a permanent disability, inasmuch as the lost structure cannot be replaced.

Diagnosis.-The presence or absence of the lesion of the cord is

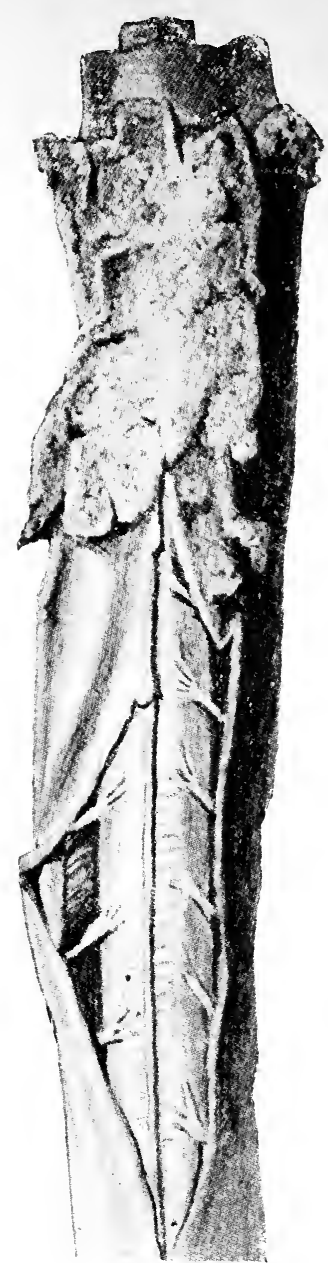

Fig. 302.-Gumma of dura of spinal cord. (Bailey.) the earliest possible date, thus insuring for the patient the best chances of success. To this end indispensable information is obtained by resort to the diagnostic investigation of the blood serum and spinal fluid. When the condition is recognized early, good nants of the lesions which have gone before and the determination of the complement fixation reaction of the blood, and when this is nonconclusive the same test is applied to the cerebrospinal fluid, which will sometimes give the positive result when the blood serum does not. An important point to be relied upon in the diagnosis is the invariable lymphocytosis of the cerebrospinal fluid in organic disease of the nervous system, be it tuberculous or luetic. A favorable effect of treatment is also a means of illuminating the diagnosis.

Prognosis of Cerebrospinal Lues.-This depends upon various factors. Early treatment is of immense advantage; especially may this be stated of salvarsan, more especially in the inflammatory than in the degenerative stages. Symptoms of hemiplegia and hemianopsia are grave signs, indicating arterial clisease and the more serious forms of cerebarl lues dependent upon endarteritis, on account of the great danger of rupture, thrombosis and destruction of tissue. Before these secondary effects are produced, the possibilities of treatment are great. Many cases, therefore, of cerebral disease are never completely restored, but there is always an expectation of relative relief and the important aim is to arrest the further progress of the disease.

Treatment of Cerebrospinal Syphilis.-It is important that the first manifestation of syphilis of the nerrous system be detected, in order that treatment may be instituted at determined by the previous history, the rem- 
results have usually been obtained by following the old and tried line of treatment with mercury and iodide of potash. The mercury is administered by inunctions or by hypodermic injections, and is given over a period of five or six weeks, accompanied by the internal administration of iodide of potash, or is followed by a sharp course of the latter remedy. The iodide should be pushed to the full point of tolerance during the inflammatory stage of the malady. It is proper, however, to carefully watch the effect of these remedies, notably their influence upon the kidneys and the spinal centres, as in some instances there seems to be incurred a direct irritating effect after the prolonged use thereof or when specially large doses are employed (for further directions and Intra-spinous Treatment see p. 797).

Special conditions attending the different forms of nervous lesions demand special treatment. When the patient is discovered with incipient nervous syphilis and it seems to succumb to treatment under the older specific measures it is deemed advisable to administer a renewed course of treatment at regular intervals. At the present day with the modern laboratory method of sero-diagnosis it is customary to precede the treatment by a preliminary blood test and then check off the result by repeated blood examinations at different intervals following the cessation of treatment for two to four months and also in most cases examination of the spinal fluid. This is especially applicable to the treatment by salvarsan, and following a vigorous and sufficient course by this remedy, often determines whether the same shall be renewed (see Salvarsan Treatment, p. 779).

In general, patients with nervous manifestations should be managed with a view of combating the same unfavorable influences that are recognized as predisposing causes to their condition. This entails a regularity of living, freedom from excitement and excesses of all kinds and abundant night's rest.

Paralytic symptoms, after the activity of the disease is controlled, are sometimes favorably influenced by passive motion, massage, electricity, and strychnine internally. Severe pains must be relieved when necessary by sedatives (bromides) or if needed anodynes, morphine or codeine. In conditions indicating acute cerebral or spinal irritation, as evidenced by distressing pain and focal signs of pressure, the local treatment by blister or cupping may prove of benefit.

Finally, in all cases, when symptoms of beginning nervous syphilis are recognized a great vigilance is called for in order that the incipient condition which in the majority of instances is amenable to successful treatment, may be satisfactorily controlled, and thus, perhaps, forestall the further progress of the disease or the ultimate development of the gravest and often hopeless form of general paresis. 


\section{QUATERNARY SYPHILIS}

This phase of syphilis is summed up under the titles of the two maladies, general paralysis (paresis) and tabes dorsalis (locomotor ataxia). And while these two conditions are usually considered independently, in many respects they are pathologically identical and are often different stages of the same morbid process. With regard to their close relationship to syphilis there can be little question. That over 90 per cent. of all cases are referable to this malady has long been recognized, and the compliment fixation test of the blood reveals a positive result in a high percentage of these cases while examination of the spinal fluid, when the serological test of the blood has failed to give a positive reaction, serves to attest the almost unexceptional etiological factor of this disease and the finding of spirochete in the isolated lymph spaces of the brain further confirms this fact.

\section{GENERAL PARALYSIS}

On account of the mental strain and anxiety of modern business life, which acts as a predisposing factor to this as well as the earlier nervous manifestations, it seems that men, more than women, are prone to the development of this malady, notably between the ages of thirty and fifty-five. One of the chief causes, however, is negligent treatment and lack of care during the early course of the disease. Whatever has been stated in the general consideration of nervous syphilis with respect to the predisposing causes applies equally here, as in all other forms of nervous syphilis.

Symptoms.-Generally speaking the course is chronic and progressive. There is usually a preliminary stage, characterized by symptoms of variable degree, sometimes insignificant, which require the closest inspection to be detected. These evidences generally amount to a definite alteration in temperament or character, as for instance, boasting and egotism in an individual of naturally modest and unassuming character, the sudden development of irritability and other acts not at all in keeping with the patient's disposition or the immediate occasion therefor. There may be defects of memory or a suggestion of moral perversion. Thus, if a careful watch is not instituted, the insidious progress of the disease may in the beginning pass unnoticed. On the other hand, there is an exception to this mode of development in the occasional occurrence of a sudden seizure of apoplectic or epileptic attacks which ushers in the appearance of the disease.

Certain minor motor symptoms may identify the preliminary stage. 
These involve a slight tremor of the muscles of the lips or tongue, accompanied sometimes by a hesitancy in speech and a confusing of words. There may be also at this period an irregularity of the pupils, or the failure of one or both to contract to light-the Argyll Robertson pupil.

The second stage of the disease exhibits more clearly defined and pronounced symptoms. This may be signalized by periods of great excitement, grandiose delusions or attacks of profound melancholia. The motor symptoms become increased and more evident, affecting both the lingual and facial muscles to a marked degree. The kneejerks are generally altered, being in this stage increased in activity, although they may be absent on one or both sides. Other muscular symptoms are evidenced by alteration in the gait, which in some cases is spastic and in some tabetic. Further muscular depreciation is evidenced by bladder and rectal symptoms, which may commence as irritability and frequency of urination and later assume the form of incontinence of both the urine and feces. This usually occurs at a late stage when the patient's mental condition has so completely failed that he becomes totally oblivious to the natural demand of these functions. The patient finally becomes hopelessly demented and is subject at any time to sudden outbreaks of violence or the occurrence of a comatose condition which closes the scene. In the majority of cases of general paralysis affection of the spinal cord follows the mental symptoms; but they may occur simultaneously, or cases may begin with a pretabetic stage and later develop definite symptoms of tabes, during which period they may show signs of developing paresis, and finally the condition may merge in to that of the latter malady.

The prognosis is usually bad; the most hopeful are the cases discovered in the preliminary period, before they become potentially paretic; that advancement may be restrained by active treatment has been practically demonstrated.

Treatment.-During the preliminary stage the treatment is that of the general disease by preventive measures and the proper regulation of the patient's life with respect to the avoidance of excesses of all kinds. When the malady has actually appeared, the older remedies of mercury and iodide of potash alone seem to exert no beneficial influence, while the latest treatment of salvarsan should be given in intensive doses, if the patient tolerates it at all (p. 797). The direct application of salvarsan or of salvarsanized serum to the spinal canal and even to the cerebral ventricles has been tried (p. 798). In the last stages careful nursing and confinement to a sanitarium are the only resources at hand, when the other measures have failed. 


\section{TABES DORSALIS (LOCOMOTOR ATAXIA)}

This condition is brought about by luetic invasion of the posterior columns of the spinal cord causing a sclerosis thereof. As already stated, it is believed by many that the pathogeny of this malady is similar to that of paresis, the brain being chiefly affected in the case of the latter, and the spinal cord in tabes. These affections are often associated in the same patient at the same time or occur in sequence. The etiology of syphilis in the large percentage of cases is again demonstrated in tabes, as in general paralysis, by sero-diagnosis (see p. 106). Further diagnostic laboratory data are obtained by examination of the cerebrospinal fluid which in the case of both these conditions reveals a lymphocytosis.

The predisposing and exciting causes in the presence of the syphilitic contamination are the same as in all nervous conditions, namely, excessive physical strain, nervous exhaustion, exposure, sexual excesses and trauma.

Symptoms.-Tabes presents a complex tissue of symptoms variable in degree and progressive in course; it is therefore divided into stages, namely, the pretabetic and the tabetic or ataxic stage.

The pretabetic stage is characterized by so-called lightning pains. They are sharp and abrupt in nature and felt in different portions of the body, the upper and lower extremities, as well as the trunk, being subject to these transitory pains, which come and go at varying intervals with different degrees of severity. Romberg's sign, or swaying of the body with the eyes closed, may be an early symptom of developing tabes.

Eye symptoms are also significant of this period of the malady. There may be inequality of the pupils or the typical non-contractile Argyll Robertson pupils. Ophthalmoscopic examination may detect optic atrophy. Paralysis of the external orbital muscle may produce strabismus, double vision or ptosis. At first the reflexes may be exaggerated, and later a loss of the reflex on one or both sides may be found. Urinary disturbances are exhibited by bladder irritability or sluggishness; and gastric crises occur in the form of gastralgia, colic, indigestion, etc.

The period of time that elapses between the development of tabes and infection is usually ten years or more, and although tabes has appeared after shorter periods, the progress is generally slow. The most prominent evidences of the developing malady are the ocular symptoms, namely, the Argyll Robertson pupil, ocular motor paralysis and sometimes atrophy of the optic nerve; loss of knee jerks, the 
occurrence of lightning pains and bladder symptoms, together with lymphocytosis and a positive Wassermann of the spinal fluid, and a positive blood reaction in a large percentage of cases.

Ataxic Stage.-At this period the motor symptoms are increased, as evidenced particularly by the muscular disability which is strikingly characteristic of the disease and amounts to an incoördination of the muscles of locomotion. There is little loss of muscular power, but an inability to properly direct motion when walking, and thus is developed gradually the peculiar ataxic gait. The lightning pains sometimes persist with a greater severity; at other times they grow less. There is likely to be a retardation of tactile sensibility, therefore an impairment of localization of sensibility, as well as the muscular sense, with regard to the perception of the actual position of the extremities in one position or another. Romberg's sign is most marked.

Severe paroxysms of pain may occur in the form of crises associated with gastric or laryngeal spasms. Deafness may develop as a result of involvement of the auditory nerve. Sexual power is generally impaired and eventually lost.

Trophic changes are characterized by localized vasomotor disorders, affecting the nails and producing herpetic skin eruptions, joint effusions and muscular atrophy. Paralysis may develop in the early course of the disease or after it has existed for years. The slowly progressive course of the malady gradually produces a bedridden condition and the patient is usually carried away by the intercurrence of some acute malady affecting the lungs or the kidneys, to which he readily succumbs.

The diagnosis in a well-developed case is not difficult. It is important to distinguish it from pseudo-tabes, due to acute inflammation or meningomyelitis. The latter condition may present some of the signs peculiar to tabes, but there is an absence of the complete diagnostic picture: of the optic atrophy, loss of knee-jerks and motor ocular paralysis. The active response to treatment on the part of meningomyelitis and the complete disappearance of symptoms, are further evidence of the character of the malady. In the earlier stage of tabes the lightning pains, impaired reflexes and ocular symptoms are strongly suggestive, and it is at this stage that vigorous measures of treatment should be adopted for the purpose of preventing the further progress of the disease. For upon this the prognosis depends, and in favorable cases considerable benefit may be obtained, and complete cessation is not rare. When, however, the disease has attained a well-developed state and the ataxic stage is 
reached, the outlook is unfavorable, although in many instances the progress is exceedingly slow and depreciation gradual.

Treatment.-During the preliminary stage, as in all conditions of impending implication of the nerve centres, an energetic preventative course of treatment should be instituted. When the older remedies of mercury and iodide of potash are used, they should be pushed persistently and given in several vigorous and interrupted courses. Mercury is employed by inunction or hypodermic method and the iodide of potash in large and increasing doses. Of important consideration, at the present time, during the pretabetic stage, is the adoption of a vigorous course of salvarsan treatment under the most careful conditions. It is imperative in this respect to realize that salvarsan, if given in sufficient amount and over a proper period, can be the means of totally destroying the active agents and thus of producing a cessation of the disease. On the other hand, an insufficient quantity may be the means of undermining the body resistance so that the spirochætæ present in the cerebrospinal fluid may act as a greater irritant to the cord and tend to induce, rather than retard, neuro-recurrence.

When the disease is fully developed the treatment becomes that of the symptomatic management of the patient's condition: the functions of the bowels and bladder should be properly looked after, and the general health improved by dietary and tonic measures. Special symptoms require suitable treatment. Lightning pains are treated, when necessary, with sedatives, for which purpose the Cachet El Zernac will be found serviceable. When cystitis supervenes, on account of infection of the bladder in connection with the use of the catheter when necessitated for the relief of retention, the treatment of that condition by irrigations of boric solution and nitrate of silver is demanded (see Cystitis, p. 427). As to the use of antisyphilitic treatment during the advanced condition of tabes, cases differ. It has been noted that when treatment is of little avail it is sometimes irritating. The modern methods of examining the blood for the complement fixation reaction and in addition to that, examination of the cerebrospinal fluid for lymphocytosis and the Wassermann reaction as well, are taken as factors determining whether or not to employ such treatment, it being believed that the expectation of beneficial results is more likely to be realized in those cases in which there is a positive reaction, and also where the lymphocytosis of the spinal fluid is markedly diminished by treatment. With respect to the use of salvarsan in these cases, different reports and results are recorded. Generally speaking, what has been stated in regard to the treatment by other specific measures applies to the use of salvarsan. 
So long as evidence is obtained of the continued activity of the disease in the blood or the spinal fluid there is always reason for a persistent effort to eliminate the spirochætæ and prevent further havoc, even though, in the degenerative stage improvement is beyond hope. The selection of treatment in such cases lies between intensive intravenous salvarsan infusion and direct intraspinal treatment (see pp. 797 and 798).

\section{INHERITED SYPHILIS}

The transmission of syphilis by inheritance from maternal and paternal sources has already been referred to (see p. 699). The terms inherited, congenital and infantile are used to signify different periods of transmission to the child; such a distinction is not essential, however. A subdivision according to clinical manifestations is more practical.

Hereditary syphilis produces a high degree of infantile mortality, and the earlier in pregnancy it is acquired, the more serious are its consequences. During the earlier months syphilitic abortion is common; later on premature birth is often a sequel; and during the earlier weeks of the new-born child, the fatality of the malady is apparent. In other cases hereditary syphilis does not develop until the victim has grown to adolescence, when a lesion of more or less seriousness may present itself.

Syphilis is so common a cause of frequent miscarriages that the occurrence of such invariably leads to suspicion of the hereditary disease.

The severity of syphilis propagated by inheritance becomes decreased with each subsequent pregnancy; beginning with abortion, then a still-born fetus, finally a child is born in apparently good physical condition, but which may later develop symptoms of the hereditary taint. It is furthermore possible that eventually, after the mother has recovered her health, perfectly healthy children may be born.

Morbid Anatomy.-When a dead fetus is retained in the uterus, maceration takes place, the amniotic liquid becomes cloudy and putrid and septicemia of the mother is threatened.

Pathological changes in the bones and viscera of the still-born fetus are similar to those developing in children born with the disease. The characteristic lesions of acquired syphilis of all the organs of the body are seen in the congenital form, the skin, the glands, the bones, the vascular system and the viscera being included. The different types of morbid changes seen are the acute and chronic 
inflammatory (exudative and infiltrating) and gummatous tumors. The principal viscera, the kidneys and the liver, are the seat of cell proliferation which causes an arrest of development. The spirochætæ and the syphilitic toxin present in the cerebrospinal fluid cause central irritation and secondary changes that interfere with the normal development of these parts. Lymphocytosis and positive Wassermann reaction of the spinal fluid are diagnostic evidences associated with the pathological changes in the central nervous system in the congenital, as in the acquired form of the disease.

Symptoms of Inherited Syphilis.-A syphilitic child born at full term may show no syphilitic manifestations at the time of birth. The disease will then develop at various periods in the infantile existence or early youth. ${ }^{1}$

In a few instances, cases of late hereditary syphilis occur long after birth.

When the child with inherited syphilis appears healthy at the time of birth, the disease puts in its appearance later, generally in a few weeks. The first appearance may be snuffles and a rash on different parts of the body or over a large part of its surface. The eruption may be a diffuse or erythematous rash, accompanied by fissures, scaly patches and moist papules in the muco-cutaneous regions. When the eruption appears at birth it may assume the form of a pemphigus which occurs in large bullous patches with a predilection for the palms of the hands and soles of the feet. This form of skin eruption usually occurs in the more severe and frequently, although not necessarily, fatal types of the malady.

The fissures develop in the folds of the skin and mucous membranes and are the source of an infectious secretion.

The hair of the hereditary syphilitic may be thick, but more commonly there is alopecia involving the eyebrows as well as the scalp.

The nails may show evidence of faulty development and deformity, and syphilitic onychia is not uncommon.

Laryngitis soon follows the symptom of snuffles and the voice becomes hoarse. The morbid condition may be purely inflammatory,

\footnotetext{
first appearance of the disease in 158 cases:

86 show the disease in less than 1 month after birth.

45 show the disease in less than 2 months after birth.

15 show the disease in less than 3 months after birth.

7 show the disease in less than 4 months after birth.

1 shows the disease in less than 5 months after birth.

1 shows the disease at 6 months.

1 shows the disease at 8 months.

1 shows the disease at 1 year.

1 shows the disease at 2 years.
}

${ }_{1}^{1}$ Didays (quoted from Power taking various authorities) gives the following periods for the 
with swelling of the mucous membrane of the nasopharynx (syphilitic rhinitis). In this case there is a laryngeal and nasal catarrh, accompanied by some difficulty in breathing and coryza, that produces a nasal discharge of more or less abundance, serous or bloody and sometimes of foul odor. With later development there may be ulceration and gummatous infiltration, in which case the pharynx as well as the larynx, including the hard and soft palate, may become implicated. Ulceration may lead to necrosis of the nasal cartilages or bone and cause the deformity of "saddle nose," or perforations of the hard and soft palate may result.

The bones and joints present characteristic changes. The bones sometimes become thickened and show surface irregularity or nodes. Chronic periostitis is not rare and attacks particularly the long bones. When this condition develops as a late manifestation it is to be distinguished from periosteal sarcoma. Other affections of these structures are found in delayed development at the articular ends of the bone, producing the so-called pseudosyphilitic paralysis, due to the degeneration of the cartilages, and changes in the epiphyseal junction with resulting thickening and deformity.

Dactylitis is one of the forms of inherited syphilis, attacking the bones of the hands and feet, notably the articular ends, and invading the joints, in which case the deformity produced is similar to that observed in the acquired form of the disease (see p. 725).

The flat bones, especially those of the skull, are sometimes attacked, and give evidence of periosteal thickening or nodes.

The visceral manifestations of congenital syphilis develop in most of the organs.

The liver may be the seat of the same lesions that characterize the acquired disease in the form of syphilitic hepatitis, amyloid degeneration and gummata.

The kidneys present early and late changes which are likely to be associated with lesions of the other organs. All types of renal disease may be simulated, including parenchymatous inflammation and amyloid degeneration. The evidence of such lesions may be pronounced, and in the form of acute exacerbations, or of insidious development.

The pancreas, the stomach and intestines, may be the seat of interstitial fibrous changes similar to those in acquired syphilis.

The spleen is attacked by various lesions, including amyloid degeneration, interstitial splenitis and gummata. On account of the marked attendant leucocytosis, the syphilitic spleen is mistaken for leukemia. 
The testicle presents changes as the result of disease in early childhood, although it is not infrequent that such changes do not appear until puberty and early adult life. The condition then assumes one of the forms peculiar to the acquired disease, producing a large and hardened organ which may be a nodular formation or include the entire gland in one mass. If the disease continues unitreated, the resulting fibrosis is likely to lead to atrophy of the testicle.

The mouth and teeth show characteristic evidences in the form of ulcerations and mucous patches of the buccal membrane. Glossitis is sometimes observed.

The teeth present characteristic deformity. The upper front incisors are separated from the others by a wide space. The cutting edge is narrower, producing a peg shape, and this edge is characteristically notched; instead of the sharp angular corners, there is a rounding of the lateral ends (Fig. 303). In contradistinction from this

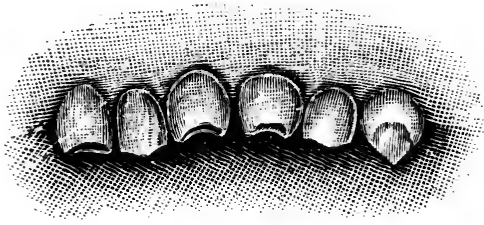

FIG. 303.-Hereditary syphilitic teeth.

condition is that produced by the effect of excessive mercurial medication, which effect is seen in the loss of portions of the surface enamel and consequent caries on the crown of the tooth as well as the tusk.

The eyes are the common seat of lesions of congenital syphilis. As a part of early syphilitic rhinitis there may be an attendant catarrhal affection of the conjunctiva.

In the early months of life iritis may develop and gumma may attack different portions of the eyes.

Parenchymatous or interstitial keratitis may affect the eyes of young children after several years of life, at the time when the second teeth appear.

Affections of the choroid occur early and may impair the vision seriously. Lesions of the choroid and the vitreous may accompany those of the brain.

A less common condition is amaurosis, in which the child pays no attention to light. The outcome of this form is very uncertain as there may be loss of sight entirely, or death may occur owing to the advanced condition of the disease. 
The outlook in the other ocular conditions may be favorable, but the prognosis should be guarded on account of the invisible changes that may take place, and, therefore, treatment requires the supervision of an oculist.

The ear may present trouble soon after birth, but in a larger number of cases the affection does not appear until early youth. The earliest cases may become deaf mutes when the condition is irremedial. The later development is usually the outcome of an early nasopharyngeal catarrh with otorrhea which, being neglected in the early treatment, leads to progressive changes in the internal ear and later syphilitic deafness. When-the condition reaches this stage the outlook is most unfavorable.

The Nervous System.--Hereditary syphilis may, by early implication of the brain, impede its development, which may result in the production of idiocy; on the other hand, the disease may cause an inflammation of the brain in the form of gummata and meningitis of both cerebral and spinal origin, with resulting hydrocephalus, epilepsy and spastic palsies.

General paralysis may manifest itself in early youth, generally assuming the type of early dementia.

Tabes dorsalis is recognized as being due to congenital syphilis, when it develops in early adult life.

The outlook of nervous syphilitic disease of congenital origin is of variable character and depends upon the gravity of the affection. Sometimes, as in the acquired form, following prompt diagnosis by the modern method of examination of the blood and cerebrospinal fluid, active treatment may produce brilliant results.

The diagnosis of congenital syphilis is of great importance, not only to safeguard the child, but to be aware of a dangerous source of contagion. Suspicion will be aroused by the existence of pronounced physical defect, such as the hard and enlarged testicle and the enlargement and deformities of the bones. The ocular symptoms are likewise significant; iritis is an almost unmistakable evidence of early congenital syphilis. The peg-shaped and notched teeth, nasopharyngeal catarrh and otorrhea of more than ordinary persistence and the characteristic skin eruption compose the means of reaching a clinical diagnosis; whereas the examination of the blood of the child and the cerebrospinal fluid, as well as the blood of the mother, will furnish conclusive evidence, when a positive result is obtained.

Other more generalized symptoms of congenital syphilis are marked debility, jaundice, and infantile hemorrhages of gastrointestinal origin. Hemorrhages may also occur beneath the skin. Various 
other signs of impaired nutrition, such as a cachectic appearance, emaciation and wrinkled skin, give to the subject a wizened appearance. The syphilitic child who appears healthy and well nourrished at birth soon shows a tendency to waste away under the pernicious influence of its malady.

Prognosis.-Infantile mortality in the form of still-births and miscarriages is of great frequency, and, when the child is not totally destroyed before or after birth the resulting permanent stigmata are numerous, among which are defective eyesight and blindness, deafness, juvenile paralysis and mental impairment from lack of development. While there is no hope of curing any of these permanent defects, many of the active manifestations succumb to the influence of early treatment, if such treatment be prompt and persistent. Even in favorable cases, howerer, relapses may occur, and, when the disease has been temporarily controlled in infancy, it may manifest itself again in late childhood in the characteristic ocular or auditory lesions, leading to functional defects, or there may then develop gummata of the bones or viscera.

Treatment.-The most important therapeutic consideration relating to congenital syphilis involves the adoption of preventive measures, by the adequate treatment of the syphilitic mother. When this course is followed early enough in the existence of the developing child, it is possible to ward off the impending disease. When it is known that the mother is syphilitic, in view of her previous history, even though she shows no signs of syphilis at the time, a blood examination should be made and careful antisyphilitic treatment instituted as early as possible in the pregnancy. After the fifth month, there is little hope of a favorable outcome. The older course of treatment involves the employment of mercurial and iodide of potash medication. This is given in the usual effective manner by hypodermic injection of mercury and iodide of potash or one of its substitutes (iodipin or iodival) internally (see p. 774). This treatment should be kept up continuously for a period of two months or more, followed by an intervening period of from two to four weeks' rest, then another period of treatment, to be followed or not by a period of rest, according to the effect of treatment upon the mother and the condition of the general health, it being desirable to give as much treatment as is consistent with satisfactory general condition and an absence of a resulting depressing influence therefrom.

Salvarsan has been administered to the pregnant mother with brilliant results, and its use under this condition is advocated in the same manner as in the active form of the disease (see p. 797). 
Generally speaking, treatment of congenital syphilis does not differ from that of the acquired form. Even if the child is apparently healthy, if born of syphilitic parents, it is considered a wise precaution to administer an antisyphilitic course of treatment. With the present possibilities of serum diagnosis, if the disease in the parents is not recent, the result of such an examination may be a guide to action in the absence of symptoms, together with a careful vigilance for any developments.

Mercurial inunction treatment is employed by preference. The mercurial ointment or the oleate of mercury, 4 c.c. (3i) of the former or a lesser amount of the latter, is used each day by manual friction, or by applying the same with a binder and allowing absorption to occur through the agency of the movements of the body. The constitutional effects of the drug and its influence upon the lesions are closely watched and jointly afford the basis of increasing or diminishing the amount employed. The treatment should be prolonged with reasonable intermissions, and should be continued for eighteen months or two years in this manner and thereafter the development of the child carefully watched. It has been customary to renew this treatment again at the period of early youth, regardless of requirements. The blood examination may now be employed as a means of guidance.

For internal administration, the gray powder has been used, $0.065 \mathrm{gm}$. (gr. 1) three times a day; and for hypodermic medication the bi-iodide or succinimide of mercury $0.0032 \mathrm{gm}$. $(1 / 20 \mathrm{gr}$.) of one or the other of these preparations for a child one or two years old. For the control of the tertiary type of symptoms, the iodide of potash is resorted to as an added remedy, being administered in appropriate doses and increasing amount, beginning with 0.065 to $0.2 \mathrm{gm}$. ( 1 to $3 \mathrm{gr}$.) either suspended in a milk curd, in a draught of milk, or by rectal injection.

The use of salvarsan in congenital syphilis has been followed by brilliant results and the introduction of this treatment has in many instances, revolutionized the method adopted and taken the place of the older remedies to a considerable degree. It has been found that syphilitic children bear the treatment very well, in spite of the earlier expectations to the contrary. Cases are reported without number that have presented all the characteristic evidences of early congenital syphilis, including the nasopharyngeal symptoms, skin lesions, enlargément of the viscera and bones, in which the effect of treatment has been well-nigh magical. A careful observance of all the requirements of surgical technic, the grading of dosage and the continuation of treatments should be followed, as in the case of the acquired form of the disease (see p. 787). 
Instances are reported of hereditary syphilis beneficially treated by salvarsan administration to the mother, the child receiving its treatment through nursing, and symptoms of the latter under this practice have totally disappeared. Inasmuch as the examination of the mother's milk for arsenic failed to reveal its presence, the explanation has been suggested that the maternal antitoxins reached the syphilitic nursling and exercised their curative influence through the agency of the mother's milk. 


\section{CHAPTER XLII}

\section{THE GENERAL TREATMENT OF SYPHILIS}

\section{INTERNAL ADMINISTRATION-INUNCTIONS-HYPODERMIC METHOD-THE IODIDES-THE MODERN SYNTHETIC PREPARATIONS-SALVARSAN}

In giving this subject the attention demanded, it must first be appreciated that at the present day, on account of the modern innovation of salvarsan therapy and the more recent chemical substitutes, the entire method of treatment in all phases has practically undergone a complete transformation. It may be truly said that when any case of the disease is encountered, in whatever stage it happens to be, the indication for the administration of salvarsan and the most effective manner of applying this agent should be considered. Notwithstanding this fact, the older and time-honored remedies have not been entirely misplaced. Extensive experience in all directions seems to favor a combination of the old and modern treatments in many cases, and even finds a preference for the old in some instances. It is therefore necessary that a full knowledge of the different methods should be acquired.

The prophylaxis of syphilis is an important consideration. This question has already been considered in connection with the description of the primary lesion, so far as venereal prophylaxis is concerned; since, however, the origin of syphilis is not necessarily venereal, the means of its prevention have a more extensive sanitary importance than is attached to a purely venereal disease. In this respect, it is desirable that a knowledge of the gravity and prevalence of the disease should be disseminated, as well as the possibilities of transmission through immediate and intermediate channels. Sterilization and prophylactic measures should be enforced upon those whose vocations render them particularly dangerous as a source of transmission; among such are included not only medical practitioners, but dentists, barbers, manicurists and chiropodists as well. The menace of the common drinking cup is now well understood, and the promiscuous use of common eating utensils, and of all articles that come in contact with the exterior or mucous surfaces of the body are a fruitful means of contagion, especially among the poor and ignorant classes, who are more likely to be exposed to this danger. 
It is necessary to treat all cases of syphilis._- An exceptional case is reported now and again that has received no treatment of any kind and has not seemed to suffer serious consequences thereby. There is a grave danger in this course, however, and there is no question that the majority of cases that have undergone no treatment pay for their imprudence sooner or later in the development of serious tertiary manifestations.

As to the duration of syphilis, there is no certain and reliable standard by which the virulence and probable duration of a case can be judged, as there has always been an unknown element which has shrouded the disease with uncertainty. At the present day, however, we are much more enlightened with respect to latent forms and reputed cures by means of the information afforded by examination of the serum and spinal fluid. This important technic is relied upon to determine the presence or absence of the Treponema pallidum in the blood of suspects, in clemonstrating in a large percentage of cases, after the disease has apparently spent its force, the continued activity of the virus; and in cases of paresis and tabes dorsalis, a positive reaction when obtained, may determine the adoption of antisyphilitic treatment which otherwise might be considered useless. It is believed that by serum diagnosis, blood and spinal fluid, the activity of the etiological factor in the late meta-syphilitic manifestations may be detected.

The general treatment is considered under the headings of hygienic and specific medication.

The hygienic medication entails an intelligent appreciation of the requirements of each individual case. The observance of the ordinary laws of hygiene is most important in the treatment of this disease. Cleanliness is an essential requirement, and its necessity should be insisted upon in every respect. Attention should be particularly given to the cleanliness of the mouth, and all mucous surfaces, as the collection thereupon of irritating secretions tends to invite the development of active lesions. Smoking is objectionable, but it is difficult to enforce its complete prohibition; this should be insisted upon, howerer, when the mouth is the seat of mucous patches or other sores. A smoker should always be impressed with the extra necessity of frequently availing himself of disinfectant mouth washes.

From the very beginning, the teeth should be inspected and thoroughly cared for throughout the entire course of treatment.

Excesses of all kinds must be interdicted; alcohol is particularly objectionable, and is better excluded entirely. In the case of mercurial medication by the stomach, it seems to exert a direct counter- 
acting influence upon the action of the remedy. Regularity of living, diet and exercise, and an adequate amount of sleep, are especially demanded, to afford the disease the least encouragement, and to support the treatment with the most favorable conditions.

Tonics, too, have their place, and should be appropriately selected, according to conditions and circumstances.

\section{THE SPECIFIC TREATMENT OF SYPHILIS}

Specific medication aims first, to control promptly the symptoms; and, second, to eradicate completely the specific virus from the system.

For these two purposes, medicine has been most fortunate in having at its command well-tried and powerful specifics for several hundred years, in the older form of medication, the mercurials and iodides, and now, more recently, the more rapid direct spirochetecidal action of salvarsan.

There is no abortive treatment of the disease, properly speaking; but that constitutional treatment should be commenced as promptly as possible, as soon as the diagnosis is made, admits of no argument. The more quickly treatment is instituted, the more certain the cure of the malady.

The Mercurial Treatment.-Mercury is of undoubted value in all stages of syphilis. It has less control over the gummatous deposits than over the other lesions, and while the iodides are generally given for their greater activity in reaching these later lesions of the disease, it has always been considered necessary to resort to the mercurials, after the iodides have vanquished the symptoms.

The administration of mercury is believed to exert a specific destructive influence upon the syphilitic bodies, the spirochæte pallidæ themselves; in other words, it is curative, for its effect is not exerted alone upon the symptoms, but upon the disease and the future development thereof. This preventive action is shown in its effect upon the secondary manifestations, as patients treated from the inception of the disease frequently exhibit only superficial and benign secondary symptoms. Experience has also proved its value in prohibiting the development of tertiary lesions. Finally, the most notable preventive action of the mercurial treatment is seen in its influence upon inherited syphilis, when the effect upon infant mortality is compared between treated and untreated maternal cases.

Generally speaking, the different systems followed in the mercurial treatment are the continuous tonic course; the vigorous, interruptive or alterative method. 
The mercurials are given in soluble and insoluble preparations. Of the former, the most generally used are the biniodide, bichloride, protoiodide (only slightly soluble), oxycyanide, and bi-bromide succinimide.

The common insoluble preparations are the metallic mercury, or mercury mass (massa hydrarg.); the mercury with chalk (hydrarg. cum cret.) ; tannate of mercury ; salicylate of mercury and mild chloride of mercury (calomel). The different methods of administration of these various preparations are by internal use, by inunction, and by hypodermic injection.

The Internal Use of Mercury.-It has been fully substantiated that the mercury, when given in sufficiently moderate doses, has a tendency to increase the number of red blood cells, and is therefore, under such conditions, a tonic. This has given to the treatment of the disease with small doses of mercury the name of the tonic treatment of syphilis (Keyes). A great many cases have undoubtedly been satisfactorily and successfully treated by a prolonged course of medication thus administered.

The alternative method is to administer the drug in as large a quantity and dosage as the individual may be able to sustain; such a course may be called the alterative method.

For the tonic treatment of syphilis, the favorite preparation has been the protoiodicle of mercury, either the preparation of Garnier and Lamoureux, pills of protoiodide, $0.01 \mathrm{gm}$. each, or the same dose, in tablet form of other manufacture.

It is customary to begin with the dose of $0.01 \mathrm{gm}$. $(1 / 7 \mathrm{gr}$.) three times daily, and to increase the aggregate daily dosage $0.01 \mathrm{gm}$. continuously until the maximum amount of toleration is reached, which is evidenced by the marked physiological effects produced in the form of colic, diarrhea, soreness of the gums, etc. This may be anywhere from 10 to 15 doses per diem, of $0.01 \mathrm{gm}$. each. When this point is reached, the total dosage is decreased one-half, which is regarded as the appropriate tonic dose for that individual patient. According to this method, this amount is continued more or less indefinitely and uninterruptedly for a period of two or three years. Intercurrently, if the system rebels against the continuous use of this preparation during this period, or, if, in spite of its continuation, symptoms occur, the supplementary use of hypodermic injections of one of the other preparations is resorted to as a temporary expedient.

Instead of this system of tonic treatment, any of the preparations of the salts of mercury may be employed for internal use, giving the average or full dose from the start. The protoiodide of mercury may 
be given in $.016 \mathrm{gm}$. (1/4 gr.) doses, three times a day, and increased or diminished, according to the effect: The biniodide, .005 to $.016 \mathrm{gm}$. (1/12 to $1 / 4 \mathrm{gr}$.) three times daily; bichloride of mercury, .0065 to $.008 \mathrm{gm}$. (1/10 to $1 / 8 \mathrm{gr}$.) three times daily; or one of these preparations may be given for a certain period and another substituted at the expiration thereof, the treatment being kept up continuously or interruptedly according to individual judgment or preference.

The insoluble preparations of mercury may be given in place of the soluble preparations, or interchangeably with them, for internal administration. The mass mercury seems to suit some patients better than the salts of mercury, and may be given in doses of 0.065 to 0.13 gm. ( 1 to $2 \mathrm{gr}$.) three times a day. When this preparation seems to exercise an irritating effect upon the bowels, it is desirable to combine it with an equal dosage of iron, and the following pill is a satisfactory one for administration:

\section{R. Blue Mass.}

Reduced Iron............. of each 0.065 gm. (gr. i.)

One or two pills, three times daily.

The mercury with chalk (hydrarg. cum cret.) is a very mild form of mercurial medication, and may be given in doses of 0.13 or $0.19 \mathrm{gm}$. ( 2 or $3 \mathrm{gr}$.) three times a day; this preparation is especially suitable in the case of patients suffering from indigestion, and for children. The tannate of mercury is the least irritating of all the preparations in doses of 0.065 to $0.19 \mathrm{gm}$. ( 1 to $3 \mathrm{gr}$.) after meals.

Inunctions of mercury are a favorite means of obtaining the effect of mercury upon the system, on account of the absence of any digestive disturbances attendant upon their use. This form of treatment is suitably applied at sanatoria and spas, where the patient sojourns especially for treatment, in view of the inconvenience and uncleanliness attendant upon this method. It is furthermore, more especially used in the late stages of the disease - the nervous manifestations-for which it seems to have special efficacy. More recently, however, the hypodermic means of medication has gained favor, and has more or less supplanted the inunction method.

Inunctions are given by means of mercurial ointment, or of special preparations thereof manufactured, alleged to be more readily absorbed by friction. The aim of the inunction method is to obtain the full physiological effect of the mercury (mercurialization) without pushing this effect to the extreme of salivation. To begin with, 4 c.c.(3i) of the mercurial ointment, or the equivalent in one of the substitutes are rubbed into the sensitive parts of the skin, generally up and down the spinal column and the inner surfaces of the thighs, or around the chest, 
for a period of one-quarter to one-half hour each day, increasing the daily amount until the beginning physiological action is exhibited by soreness of the gums, looseness of the bowels, etc. When the full physiological effect is experienced, which it is generally aimed shall be reached in about three weeks, the treatment is discontinued, and an interval of rest is allowed, or a course of iodides is substituted, according to the stage of the disease and the symptoms.

In place of the mercurial ointment or its substitutes, calomel ointment has been used as an inunction in the same manner, for which purpose a preparation of calomel paste (calomelol) is a convenient form.

\section{Hypodermic Medication}

The hypodermic administration of mercury is a favorite and effective method of treatment, and the preparations used are either insoluble or soluble. The soluble preparations are necessarily more quickly absorbed by the system, and as quickly eliminated. They must, therefore, be administered in relatively small doses, and more frequently.

The insoluble preparations are less quickly absorbed, and consequently can be given in larger dosage, and at longer intervals.

The soluble preparations are bichloride, biniodide, oxycyanide, succinimide and bi-bromide. With each one of these preparations there may be combined some form of analgesic to lessen the pain produced by the injection. For this purpose, tablet triturates are manufactured, containing a small amount of cocaine or other anesthetic. Bichloride of mercury is given in dose of from .008 to $.016 \mathrm{gm} .(1 / 8$ to $1 / 4 \mathrm{gr}$.) and is administered daily, or three or four times a week, according to the effect produced. A bi-bromide preparation is put up in ampules, $.01 \mathrm{gm}$. each; and any one of these soluble salts may be combined with cocaine or novocain, being administered daily for about three weeks, or 2 or 3 times per week for a similar number of doses. Oxycyanide of mercury is given in dosage of from .0065 to $.008 \mathrm{gm}$. (1/10 to $1 / 8 \mathrm{gr}$.) for a similar period. Succinimide of mercury is a valuable soluble preparation for hypodermic use, and seems to exercise an especially beneficial influence upon symptoms in the late secondary and tertiary stages of syphilis. For rapid and effective control, this form, or the biniodide, is especially useful, and are to be recommended when it is desired to conduct a short and active course of treatment. The former is given in doses of .013 to $.026 \mathrm{gm}$. (1/5 or $2 / 5 \mathrm{gr}$.$) , and the latter, from .01$ to $.02 \mathrm{gm}$. (1/6 to $1 / 3 \mathrm{gr}$.), every one, two or three days according to the effect, for a period of two or three weeks, to be repeated after an interval, as the situation demands, often to supplement the salvarsan treatment. 
The Hypodermic Use of the Insoluble Mercurials.-These preparations include the salicylate, metallic mercury and calomel. Calomel was one of the first mercurials to be used hypodermically, but it is practically superseded by the other two preparations, both of which are much less irritating when used intramuscularly.

The Salicylate of Mercury is, on the whole, the most satisfactory insoluble preparation for hypodermic use. It possesses the advantage, in common with the other insoluble mercurials, of not being immediately absorbed, and therefore it may be used at intervals of four or five days to a week. The average dose is $.049 \mathrm{gm}$. $(3 / 4 \mathrm{gr})$. In the case of the existence of an unusually severe lesion, especially in the tertiary stage, the size of this dose may be doubled, and the administration of it continued at intervals of 48 hours, for as long as it is possible to exercise a controlling influence on the activity of the symptoms and avoid marked mercurial irritation.

A favorite and satisfactory preparation of the salicylate for hypodermic use is the combination of this mercurial with liquid petroleum (albolene or benzoinol) in the proportion of $0.1 \mathrm{gm}$. to 4 c.c. (gr. iss-3i); of this, the average dose is 2 c.c. ( $m \times x x)$. With regard to the amount of pain attendant upon the injection of this particular combination, it may be stated that it is relatively small as compared with other preparations, when the injection is made intramuscularly so that there is no leakage outside of the deep fascia. The site selected is in the soft parts above the gluteal fold. There is usually a small amount of pain immediately following the injection; the subsequent pain depends upon the resultant swelling, which is sometimes insignificant, and at other times marked. It is important that the injection should be made under strictly antiseptic precautions. The salicylate may be given also in suspension in sterilized distilled water, in same proportion, and with good result.

The pain accompanying Salicylate Injections is variable, being more in some instances than in others, without apparent explanation. Different means have been tried to limit the amount of this pain. I have found that a satisfactory expedient, when the petroleum mixture is used, consists in the addition to the contents of each hypodermic of 3 or 4 drops of an essential oil of pine (apinol); and according to the reports of patients, this combination materially lessens the attendant pain.

Hypodermic Injections of Metallic Mercury are employed in the same manner and for the same indications as the salicylate. The preparation known as gray oil contains about 40 per cent. of mercury, finely suspended in liquid fat $(0.04 \mathrm{gm}$. $(6 / 10 \mathrm{gr}$.) to 1 c.c.) which is the 
average dose. This combination has been improved upon in special preparations of gray oil which are composed of metallic mercury with lanoline, and more recently with palmitin, the latter being considered the best vehicle, on account of its close resemblance in chemical composition to that of the tissues. The percentage of mercury may be the same or greater than that of the older preparation of gray oil, and to this is added some form of analgesic; absolute creosote and camphoric acid have been used in a formula consisting of metallic mercury 10 gms., absolute creosote and camphoric acid, each 10 c.c., palmitin to make 100 c.c. Dose, $0.065 \mathrm{gm}$. (1 gr.) of mercury or 0.6 c.c. $(\mathrm{mx})$ of this mixture. ${ }^{1}$

As in the case of salicylate enough of the metallic mercury is given hypodermically to control the symptoms; at the same time caution is exercised to avoid salivation. If an average amount, not exceeding $0.1 \mathrm{gm}$. to $0.13 \mathrm{gm}$. (1 1/2 to $2 \mathrm{grs}$.) a week, is given, no fear need be experienced in this regard.

The advantages of this form of administration are the small bulk of the amount injected, and, like the salicylate, its slow absorption; but the pain produced is often fully as much as that attending the use of the salicylate, and there is no advantage over the latter preparation in therapeutic effect. The salicylate of mercury, therefore, seems to be entitled to the first place among the insoluble mercurials for hypodermic use.

The hypodermic use of mercury may be extended over an indefinite period to the extent of full toleration, for interrupted courses of treatment of two or three months each-eight to twelve injections in a course; or it may be used continuously, in such amount as may be comfortably sustained by the patient and exercise a controlling influence upon the symptoms, for a similar period to that adopted for internal medication. Again it may be used interchangeably with the internal course, or as an accessory measure, when symptoms occur in spite of the latter form of treatment. Finally, the hypodermic method affords a ready means of combating serious symptoms at any time throughout the progress of the disease; and in connection with the modern salvarsan therapy, it furnishes a suitable medium for supplementing this treatment, when it is deemed advisable to combine the two methods.

Intramuscular Injection.-A satisfactory method of intramuscular injection is best conducted by observing carefully certain details of technic. For the soluble preparations, a syringe of 2 c.c. capacity, with a fine, sharp needle, and for the insoluble preparations, a somewhat

\footnotetext{
${ }^{2}$ F. J. Lambkin, Syphilis, London, 1910.
} 
larger syringe, with a much larger needle (about 19 gauge and $4 \mathrm{~cm}$. (about $11 / 2$ in.) in length) (Fig. 304). The injection should be made deep in the tissues, beneath the intermuscular planes. The gluteal region is preferable, using each side alternately, and the area lying between the gluteal fold below, and a horizontal line passing above the margin of the trochanter. Care should be employed to avoid the same site for consecutive injections. This is occasionally accomplished by schematically mapping out the area, and using a new site each time; but this does not always work out, as some injections cause greater swell ing than others, and the proper site may be chosen with greater satisfaction by palpation than otherwise. The exterior surface is prepared by cleansing with alcohol and an application of tincture of iodine. The needle is thrust perpendicularly deep into the tissue, and the contents of the syringe slowly injected. One of the disagreeable features that sometimes accompany intramuscular injection of insoluble preparations is the occurrence of mild pulmonary embolism, from the injection

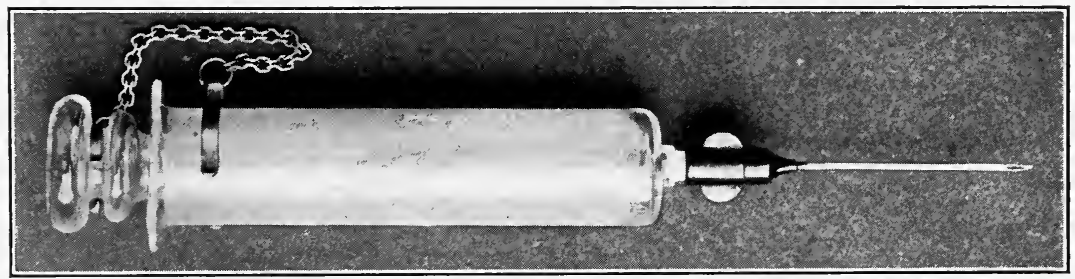

FIG. 304.-Glass syringe with needle for insoluble mercurial injections.

directly into the vein of a large part of the preparation. The untoward effect of such an occurrence is not serious, and usually amounts to a mild bronchial cough, with some dyspnea and, possibly, thoracic oppression. The condition passes away in from one to several hours. The most practical means of avoiding this unfavorable reaction is to exercise the precaution of uncoupling the syringe from the needle, after penetration of the latter, so as to decide whether or not the end of the needle seems to be within the lumen of the vessel by the presence or absence of a return flow of blood.

Intraspinal Administration of Mercury.-Following the example of the Swift-Ellis method of obtaining the localized therapeutic effect of arsenic upon the central nervous system by injecting the patient's salvarsanized serum into the spinal canal, a similar technic has been resorted to for the direct application of small doses of mercury with the same medium, namely, human blood serum. ${ }^{1}$

${ }^{1}$ Byrnes, Jour. A.M.A., Dec. 19, 1914. 
It would seem to be equally true with regard to this form of treatment as of the intravenous salvarsan method, that where the examination of the blood shows a high degree of activity of the disease, the indication is rather for the diffuse and intensive means of reaching it, through the agency of the blood and its more extensive distribution. But when examination of the blood shows a low degree of activity, while the spinal fluid indicates a centralized area of activity, it may be more effective to attack this region directly, by the spinal application of the specific therapeutic agent, either in conjunction with the intravenous or general treatment, or after the latter has failed. Thus far, salvarsan has been used more extensively than mercury by this method of administration (p. 798).

The preparation of mercury for spinal injection requires careful laboratory technic. ${ }^{1}$ The dosage is 150 gr. mercuric chloride in the medium composed of blood serum and normal salt solution, which is given by lumbar puncture, in the same manner as that employed for the Swift-Ellis treatment (p. 798). Twenty-four or forty-eight hours' confinement in bed is required. The number of treatments is determined by the clinical symptoms and examination of the spinal fluid. The number usually given in one case has been only a few-five or six -but it is suggested that the administration might be continued over a longer period, if desired. Ampules containing $1.3 \mathrm{mg}$. $(1 / 50 \mathrm{gr}$.) and $1.6 \mathrm{mg}$. (1,25 gr.) mercuric chloride are obtainable. In the technic of administration the bulk of the preparation, in the vehicle of serum and normal salt solution, is equal to 30 c.c. for each dose. It is introduced by gravity, at body temperature. The lumbar puncture is made with an intraspinal needle, the patient lying upon the edge of the bed, with the thighs flexed upon the abdomen. The amount of reaction accompanying the injections has not been pronounced. The objection to popularizing this method of treatment is that it tends to encourage a too generalized adoption thereof, the value of which must necessarily be confined to properly selected cases, which selection demands experienced observation and painstaking analysis. If such a course is not pursued, many cases are treated that would do as well, and perhaps better, by other methods, less difficult to conduct, and the deductions obtained are of far less value.

Salivation is the ordinary pathological effect of excessive dosage of mercury; with the modern method of treatment this seldom occurs. The evidence of mercurial saturation is generally seen in the form of a stomatitis, which is rarely severe. The most important prophylactic factor is the observation of strict cleanliness of the mouth, as already

${ }^{1}$ Byrnes, Jour. A.M.A., Dec. 19, 1914. 
suggested under hygienic treatment. Any excessive or unusual complications in this respect are due to special idiosyncrasies.

The irritations of the gastrointestinal tract are part of the physiological effects of mercury, and are usually controlled by the cessation of treatment or by the administration of a mild digestive tonic, such as a combination of peptenzyme and nux vomica. These symptoms are due to the direct irritating influences of mercury, when administered internally, or by the elimination into the bowel, after inunction or hypodermic injection. They consist of gastric pain, diarrhea and colic. If this condition is allowed to go unchecked, debility and anemia may result, and the mercurial treatment, instead of exercising a sustaining and tonic effect, may have a contrary influence, leading to undermining of the general health of the individual and, in consequence, a lessened resistance to the disease.

The effect of Mercurial medication upon the Kidneys is not usually unfavorable, except after prolonged use or in subjects peculiarly susceptible, on account of antecedent disease or developing kidney irritability. In many cases that have been regarded as a consequence of mercurial poisoning, the effect thereof is more than likely an acute secondary syphilitic nephritis. The existence of an albuminuria is not a contraindication to the use of mercury, but should lead to caution in the regulation of the dosage.

Cutaneous sequelæ, due to the effect of Mercury, sometimes occur; they are not common, and are more or less idiosyncrasies. The symptoms consist of urticaria or acne, and in some cases generalized eruption.

\section{THE ADMINISTRATION OF IODIDES}

The Antisyphilitic effect of Iodine has long been recognized, and while it cannot be substituted for mercury, it must be considered as a valuable aid in the treatment of syphilis. It has no effect upon the early manifestations except such as, in reality, belong to the tertiary period. It is therefore in the chronic or late stages that this chemical agent is particularly beneficial. It was at one time a matter of common practice to begin treatment of the disease by mercury, and later to combine the mercury and iodides in the form of mixed treatment; and, finally, to finish the treatment with the iodides alone. Such a routine practice, however, can hardly be regarded as suitable to all cases, and it may be properly stated that of these two remedies, the mercury more nearly acts as a curative agent, while the iodides are relied upon as a supplementary means of reaching some of the rebellious symptoms. Generally speaking, these symptoms 
belong to the ulcerative and infiltrative forms of the secondary lesions and all phases of tertiary manifestations. While it is believed that mercury destroys the syphilitic toxin, iodides tend to bring about absorption of connective tissue that is not entirely developed; fibrous or scar tissue is little affected. It is known that the iodides act upon the mononuclear leucocytes, but the exact manner in which absorption is aided by this remedy is not understood.

During the administration of the iodides, the patient should be encouraged to drink freely of fluids, to aid elimination by way of the different secretions, in all of which iodine may be found when introduced into the system.

Preparations.-The Iodide of Potash is the form of iodide most commonly used. Other preparations are the iodide of sodium, ammonium, strontium and rubidium. In some cases the iodides of potassium, sodium and ammonium are combined, it being believed that the addition of a small amount of ammonium renders the combination more readily absorbable.

The Mixed Treatment, in which the iodide and mercury are combined, may be given in a pharmaceutical preparation containing these products, or the mercury may be given separately, in tablet form, followed an hour or two later by potassium iodide in solution. The internal administration of iodide is sometimes combined with mercurial inunction; in such instances, the iodide is taken before meals, and the inunction independently, possibly at bedtime. Again, iodide is administered by mouth in combination with injections of mercury. Either of these methods is suitable in cases of visceral syphilis, when the object is to spare the alimentary tract as much as possible. When the mixed treatment is given in a mixture, the best preparation of mercury combined with the iodide is the biniodide. The following is a satisfactory example of this combination:

Mercury biniodide............ $0.13 \mathrm{gm}$. to $5 \mathrm{gm}$. (gr. ii-viiss)

Potassium Iodide............ 12 to 24 gm. (3iii-vi.)

Water

Syrup sarsaparilla compound, equal parts to make. ............ (气 120 c.c.

Sig. one teaspoonful in $\frac{1}{2}$ tumbler of water, 3 times a day, after meals.

The mixed treatment seems to be particularly useful for certain phases of the disease in the secondary stage, among which may be mentioned the dry tubercular syphilide, iritis, choroiditis, testicular enlargements and periosteitis.

The iodides alone are best administered in solution, in which case the following preparation is ordered: 
Potassium iodide or sodium, or strontium............ 15 c.c. ( 5 ss.)

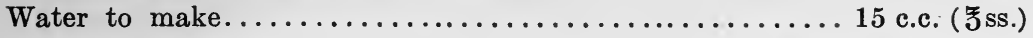

Of these three, the strontium is the most soluble, being soluble in half the quantity of water. It is usually customary, however, to have 0.06 c.c. ( $\mathrm{mi}$ ) of the solution equal to $0.065 \mathrm{gm}$. ( $1 \mathrm{gr}$.) of the drug: 0.3 to 0.6 c.c. $(\mathrm{mv}-\mathrm{x})$ of the above preparation are administered in ordinary cases, in a half tumbler of water, three times a day. When the stomach is susceptible, the iodide may be given in milk, or in junket, or combined with elixir of lactopeptin. A rapid means of preparation in junket as a vehicle is to combine the saturated solution with a tablespoonful of warm milk and add thereto a few drops of essence of pepsin. The dose is then administered in gelatinous mass and swallowed as a raw oyster.

Iodine of rubidium is one of the less commonly used alkaline salts of iodine, but is more soluble than sodium and potassium iodide and in some instances peculiarly effective. It seems to be readily absorbed and generally speaking much less irritating to the intestinal canal. The dosage is the same as that of the other alkaline preparations.

Dosage.-When the iodides are given for early manifestations, 0.3 to $0.6 \mathrm{gm}$. (5 to $10 \mathrm{grs}$.), three times daily, is the ordinary amount administered; and this may be kept up or increased $0.3 \mathrm{gm}$. ( 5 grs.) every second or third day, until the symptoms have disappeared, when it is usual to resort to the mercurial treatment.

In the late stages of the disease, especially when deep-seated lesions threaten important organs or nerve centers, it has been customary, and even considered imperative, that progressively increasing doses, sometimes appallingly large, be given. In exceptional cases, the amount required may reach 12 to $16 \mathrm{gm}$. (3iii to iv), three times a day, and as high as $30 \mathrm{gm}$. ( $3 \mathrm{i})$, three times a day, have been administered.

Such a sharp iodide course should never last longer than a fortnight, and as the iodine is rapidly absorbed and the system quickly accommodates itself to its presence, the administration should be repeated as often as may be necessary, following intervening periods of about one week. This applies more particularly to those conditions of the tertiary disease in which the iodides are especially indicated, when, if the symptoms are not controlled by treatment, some vital and permanent effect will ensue.

The precautions to be observed in the administration of this remedy are proper dilution, interrupted courses, and a careful vigilance as regards the possible irritating effects upon the organs of excretion, notably the kidneys, and upon the nerve centers, as in some instances 
it has been found that this remedy has exercised a detrimental influence upon one or the other.

Certain contraindications to the internal use of Iodides arise, such as severe throat lesions, on account of direct local irritation, and digestive disturbances. In this case, the iodide may be given in solution, by rectal enema, 2 to $3 \mathrm{gm}$. (30 to $45 \mathrm{grs}$.) of potassium iodide being dissolved in 100 to 125 c.c. (䒓ii to iv) of water or milk to which a few drops of laudanum have been added, and inserted into the sigmoid by means of a syringe and soft-rubber catheter. This may be repeated daily, or as often as may be deemed necessary in the course of the treatment.

There are various substitute preparations for the alkaline iodides, among them Iodopin, Sajodin, Iodival, and Riodine, which are suitable for the administration of a moderate amount of iodine, but not for the maximum dose.

Iodopin, a combination of iodine and oil of sesame, is alleged to be more slowly absorbed and less rapidly eliminated than the iodide of potassium. It is also less disturbing to the digestion, is absorbed in the small intestine, and may be administered by the mouth, by rectal enema, or by subcutaneous injection. It is prepared in two strengths, 10 and 25 per cent.; 2 c.c. of the stronger mixture are ordinarily given three times a day, in gelatine capsule form, or in milk. The hypodermic injection of this preparation is employed in the case of irritable digestion, from 15 to 20 c.c. being given at a time, every day for ten days. It is necessary to use a large syringe and a needle with a sufficient bore to allow the passage of this viscid mixture, which must be warmed before being injected. It is given the same as the mercurials, in the loose tissue of the buttocks. On account of the large quantity required for hypodermic injection, this method is considered disadvantageous, but it is useful in refractory cases, in which the iodide cannot be administered by the mouth over the required period.

Sajodin.-This preparation has been employed as a substitute for the alkaline iodides on account of its slow elimination and the absence of any irritating effects. It is therefore considered by some to be more suitable for prolonged usage. It is usually administered in doses of 2 to $3 \mathrm{gms}$. daily; $1 \mathrm{gm}$. two or three times a day.

Iodival is a pharmaceutical iodide substitute which is stated to contain as high as 47 per cent. of iodine. On account of the fact that it is digested in the intestinal tract and not in the stomach, it is less likely to cause irritation of the latter. Its absorption is gradual and elimination slow. It is administered in tablet form in a total amount of from 1 to $2 \mathrm{gms}$. daily in divided doses. 
Riodine is an organic iodide preparation in an oily vehicle put up in capsule form, which I have found to possess moderate chemical and favorable therapeutic action in suitable chronic cases, where a mild iodide is indicated. The dose is one or more capsules three times daily. Each capsule represents $0.19 \mathrm{gm}$. (grs. iii) of alkaline iodide.

There are many other substitutes for the alkaline iodides which are given for a mild and continuous iodide effect, but none of them has the potency of the iodide of potash or other alkaline salts or is subject to the increasing dosage in urgent cases.

The toxic effect of the Iodides is summed up in what is known as "iodism." This embraces a train of symptoms which sometimes follows the administration of this remedy in small, as well as in large quantity. Certain patients show a special idiosyncrasy, and in some instances, strangely enough, patients who seem to show marked signs of irritability from a small dosage, acquire toleration when the amount is substantially increased.

This toxic condition is expressed by a metallic coppery taste, the symptoms of influenza, sometimes with edema of the pharynx and laryngitis; cuneiform skin eruptions, gastrointestinal disturbance, neurasthenia and depression. The more malign influence of this drug is experienced with extremely large doses, when dangerous edema of the throat, inflammation of the kidneys, and cerebrospinal irritation may be produced.

The treatment of this condition is governed by the severity of the symptoms. When they are slight, the administration is continued, and the system becomes accommodated to the remedy. When they are severe, the iodides must be stopped, and elimination encouraged by diuretics, and careful dietary restriction instituted.

\section{The Synthetic Preparations for the Treatment of Syphilis}

Scientific experimentation in chemotherapy has resulted in the discovery that certain drugs have specific influence upon certain parasites. Ehrlich of Frankfort, working along this line, has been able to reveal synthetic combinations of great potency, so far as the destruction in the body of parasitic microörganisms is concerned, without doing damage to the tissue cells of the body; that is to say, "parasitotropic" without being "organotropic."

Arsenic has been employed for years in the treatment of syphilis, not only on account of its markedly tonic effect, but also for its direct influence on certain chronic skin lesions; Fowler's and Donovan's solutions of arsenous acid have been favorite remedies. 
Atoxyl is the sodium salt of arsenous acid, containing over 25 per cent. of arsenic. It belongs to the classification of arylarsonates, another of which is soamin.

Atoxyl is readily soluble in hot water, and about 1 to 6 parts of cold water. It has been employed as an antisyphilitic treatment, especially for ulcerations of the mucous membranes and for condylomatous eruption; it has been used also as a substitute or alternative course of treatment in connection with the mercurial medication, but there is no proof that atoxyl exercises any curative effect upon the syphilitic disease. It is administered hypodermically, and causes little pain. The dose for injection is $0.4 \mathrm{gm}$. (gr. vi), dissolved in sterile water, continued every second day for ten days, and repeated after an interval of two weeks.

Some toxic symptoms have followed the use of this agent, such as irregular pains, general weakness, jaundice, vomiting, and blindness from non-discoverable retinal changes. Whether these changes are due to the impurities of the remedy or not, their occurrence suggests the necessity for great care and watchfulness in its administration.

Soamin is another arylarsonate, containing a slightly smaller amount of arsenic than atoxyl. It is soluble in five parts of cold water, and is said to be staple, pure and free from toxic effects. It is given in intramuscular injection, in doses of $0.65 \mathrm{gm}$. (gr. $\mathrm{x}$ ) every second day, until $6.65 \mathrm{gm}$. (100 grs.) have been given. After the expiration of the interval of a month, the course is repeated. It must be freshly prepared before each injection, and is therefore kept in tablet form. Some observers claim excellent results from this preparation, especially in the early eruptions and ulcerations of the mouth or tongue, also later, for long-standing and obstinate leukoplakia. Marked improvement in the general health accompanies its administration in some cases.

The culmination of these various experiences along chemotherapeutical lines, involving the use of one or the other of these arsenical compositions, was reached in the discovery by Ehrlich of his preparation, the so-called " 606 ," or salvarsan. And so great have been the achievements attained by the use of this agent, and of the later product, neosalvarsan, that the consideration in comparison therewith of such kindred products as those already mentioned becomes insignificant and unimportant.

\section{SALVARSAN}

In the development of this most valuable remedy, it has been noted by Ehrlich and his co-workers that small doses of a powerful 

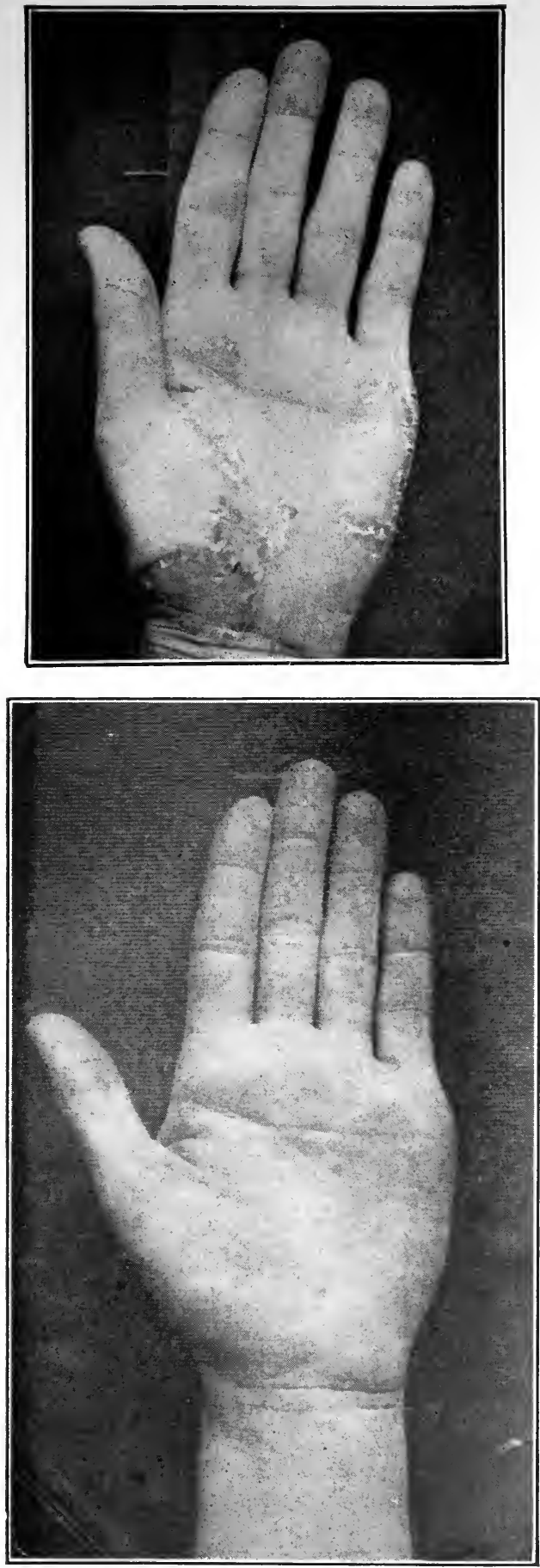

FIG. 305, A (above) and B (below).-Palmar syphilide, before (A) and after (B) salvarsan injection. 
chemical agent, continuously repeated, are less effective than a number of large doses, as the invading parasites acquire a tolerance when the amount administered is small.

Salvarsan is a yellow crystalline powder, containing about one-third of its weight of arsenic. Following a large experience in animal experiments, and now extensive use in the human subject, it has demonstrated a specific destructive action upon the pathogenic spirilla of syphilis, with a comparatively mild degree of toxicity. These two facts give to salvarsan its indisputable preëminence in the modern treatment of syphilis; and the successful results attained have accumulated to such an extent that it may be truly stated that the present treatment of the disease has become revolutionized by the introduction of this product.

Neosalvarsan (Salvarsan-new) is a still more modern product, derived from the laboratory of Ehrlich, representing a condensation of salvarsan with sodium formaldehyd sulpholoxylate. The general properties of this preparation are very similar to those of salvarsan, with some decided advantages.

The standardized dosage ampules of these two substances and their respective equivalents are as follows:

\begin{tabular}{|c|c|c|c|c|}
\hline EQUIVALENT & 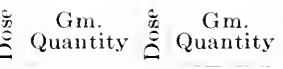 & 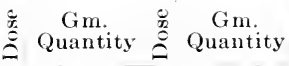 & $\begin{array}{l}\text { Gm. } \\
\stackrel{\tilde{t}}{\varrho} \\
\stackrel{G}{\varrho}\end{array}$ & $\begin{array}{c}\text { Gm. } \\
\stackrel{\text { ڤnd }}{\varrho} \text { Quantity }\end{array}$ \\
\hline Salvarsan & $\mathrm{II}=0.2$ & $\mathrm{III}=0.3$ & $\mathrm{~V}=0.5$ & $\mathrm{VI}=0.6$ \\
\hline Neosalvarsan. & $\mathrm{I}=0.15$ & $\mathrm{III}=0.45 \quad \mathrm{IV}=0.6$ & $V=0.75$ & $\mathrm{VI}=0.9$ \\
\hline
\end{tabular}

The aim of Salvarsan Treatment of Syphilis is epitomized in the phrase emanating from Ehrlich, "therapia sterilisans magna," which signifies a complete destruction of all of the parasites at one time. This may have been accomplished in a certain number of instances, but in most cases, the expectation of such, with a single dose, has not been realized in practice. There is, however, a radical difference between the actions of salvarsan and mercury. The former destroys a great number of the spirochete at one time, whereas mercury serves only to kill a few of these parasites, and therefore must be taken continuously over a much longer period with a greater likelihood, in consequence, of a tolerance being produced toward the remedy on the part of the organisms.

Salvarsan (Ehrlich), being the original, is always referred to as the standard preparation of this important modern system of specific therapy; and neosalvarsan is a later development from the same source with almost identical therapeutic properties, but having certain chemical transformation which renders it easier to administer-more soluble 
and less bulky. The latter, therefore, is more universally employed. Substitutes of the same chemical combination and aimed to have the same therapeutic value as salvarsan, old and new, naturally enough are being introduced; especially when the original foreign preparations have been difficult to obtain.

Arsenobenzol and diarsenol, of these newer remedies, have had sufficiently widespread and extensive trial to warrant at least preliminary conclusions with respect to their relative merits. It is well to keep in mind that all of these later substitutes are made as equivalents to the standard salvarsan, old or new, and the dosage, number of treatments and method of administration are substantially similar. Both of these preparations are marketed as satisfactory substitutes for salvarsan (old), they both, like the latter, dissolve with difficulty and make strongly acid solutions requiring the addition of an alkali before administration (see p. 787).

On the whole, it must be allowed that neither of these drugs is able to take the place of the older and original preparation. And while they are acceptable as useful substitutes, which have won praise from reliable source for favorable results obtained under their administration, yet the number of such cases have been fewer, and the essential property of low toxicity, combined with high therapeutic potency is by no means so satisfactorily demonstrated.

One dose of salvarsan has demonstrated indisputably its influence in overcoming and controlling the symptoms during the most active early stage, and of causing a cessation thereof for periods of varying duration (Fig. 305, A and B). A single dose has demonstrated its ability to remove promptly the obstinate chronic lesions upon the skin and mucous membranes (Fig. 306, A and B), and to cause enlargements of the organs and of the bones to disappear in a short period. It has exercised an immediate restraint upon what seemed to be a rapidly destructive late syphilitic process in various parts of the body: and all of this in cases in which mercury has either failed to attain the desired result, or where such result could have been accomplished only by the prolonged and continuous use thereof. It may, therefore, be reasonably stated that one dose of salvarsan is the equivalent of several months' course of mercurial treatment; and taking into account the knowledge that a single dose of this remedial agent will destroy a large percentage of the parasitic organisms of the disease, the natural development in the direction of treatment is the continuation of the warfare against the remaining organisms, either with a progressive course of treatment by mercury, or, better still, by the continued use of salvarsan. To this end, the tendency is to organ- 
ize a system of administration aimed to obtain the full desired effect by repeated and progressive doses, and then, as a matter of expediency, to follow this treatment with mercurial medication, or to allow an interval to elapse, during which the closest vigilance is kept up by
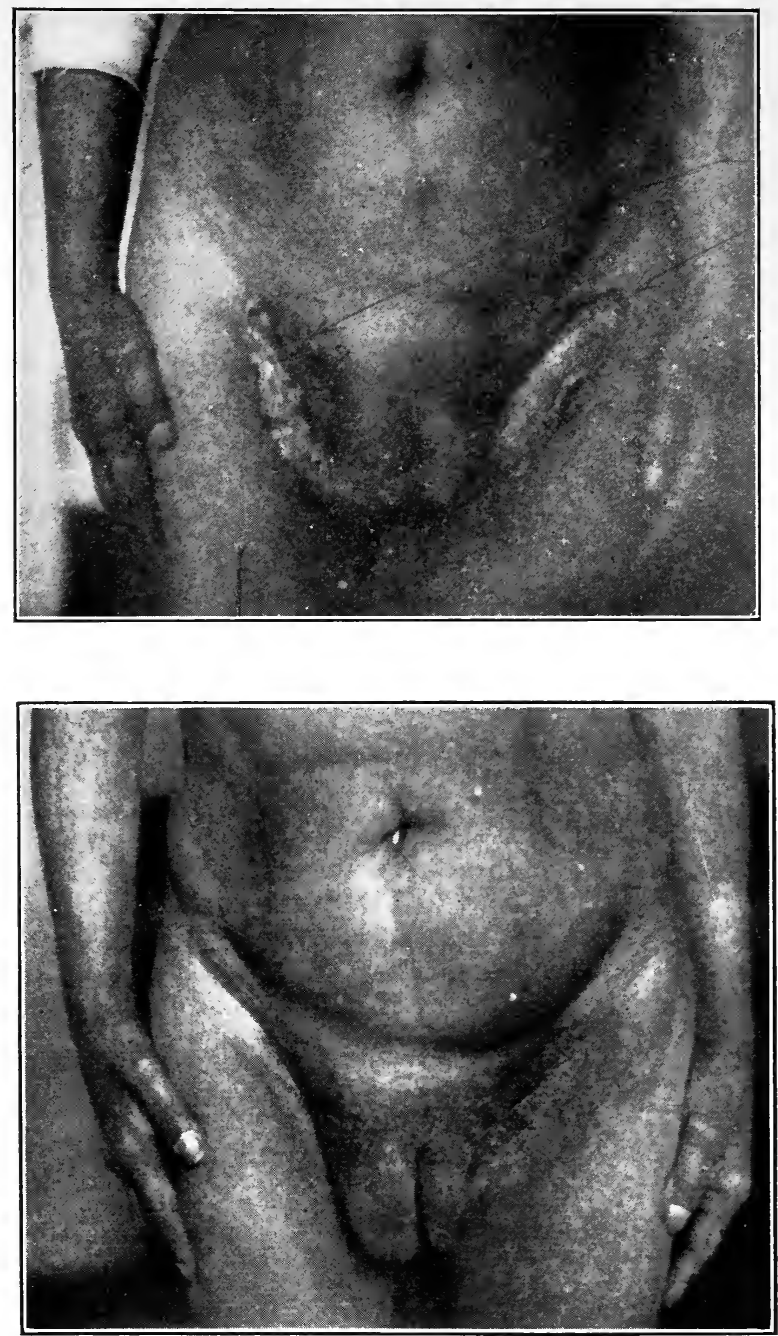

FIG. 306, A (above) and B (below).-Condylomatous syphilide, before (A) and after (B) salvarsan injection.

repeated blood examinations which will determine the future course, it being aimed in every instance to establish and maintain a condition of negative (minus) symptoms and negative blood. 
The elimination of salvarsan begins within half an hour after the intravenous injection, and reaches a maximum in 36 to 48 hours. This is the common course; in exceptional cases there is an accumulation of the drug in the excretory organs, particularly the liver.

The absorption of salvarsan occurs, no matter whether it be administered by intramuscular or intravenous injection. Following the intramuscular injection, its absorption is at first rapid, but on account of the sclerosis surrounding the point of injection, this is soon interfered with, and cases are reported in which nodes have remained at the site of injection, and being aspirated eighteen months thereafter, have yielded 10 per cent. of the amount of arsenic originally injected. Intravenous injections, on the other hand, are accompanied by regular and prompt absorption.

The abortive action of salvarsan has been demonstrated in a number of syphilitic cases, notably those at the onset of the malady, which have remained clinically free of all symptoms, and have shown a negative blood reaction continuously thereafter. In most instances this result has not been realized following a single injection. When the remedy, in the old or new form, is applied in repeated dosage over a certain period, and, after an interval is resumed for another period alternated with or followed by a concomitant course of mercurial treatment, a favorable result is produced in a large percentage of cases, as evidenced by the demonstration of negative Wassermann reactions and total absence of symptoms, which is the ordinary proof resorted to of the complete elimination of the disease. It may be generally stated that, other things being equal, the earlier in the course of the disease such a method of attack with salvarsan is undertaken, the more likely is the desired result to be obtained. Therefore, in the primary and the early secondary stages, the percentage of successful results should be high; in the late secondary and tertiary stages, the outlook may not be so favorable, and the addition of continuous after-treatment with the older remedies may be more frequently called for, whereas, in the quaternary or parasyphilitic stage, satisfactory results are fewer, and depend upon whether or not degenerative changes have occurred leaving irreparable damage. And if there is any value in selecting cases for treatment in this terminal stage of the malady, it should be those in which, as a result of an examination of the blood and spinal fluid there is shown a continued activity of the invading parasites.

The toxicity of salvarsan is revealed by its local and general effects The local effects follow the intramuscular or subcutaneous injection, or an unintentional leakage around the point of intravenous puncture. These local effects are those of an exceedingly strong chemical irritant, 
amounting to exudative inflammation, sclerosis and thickening, sometimes followed by necrosis. The successful intravenous injection is followed by no such irritating effect, unless accompanied by leakage into the subcutaneous tissue, when there are pain and swelling caused by infiltration and edema. These may be little in the case of a slight amount of leakage, or excessive when the exudation is much. With proper technic this should not occur.

The general untoward effects of salvarsan are expressed by a prompt systemic reaction in from one to three hours, which occurs in all grades of severity. There may be none at all, and this is the rule, or the symptoms may be those of slight gastric irritation with temperature elevation; or a severe and active gastric disturbance, with high temperature elevation, copious and loose stools, great general weakness and vertigo. A few cases of epileptic convulsions have been reported, following the treatment, after three or four days' interval. Some of these have disappeared without further difficulties; others have continued progressively to coma and death. In a small percentage of cases, death has followed with the symptoms of arsenic poisoning. ${ }^{1}$ The untoward effects accompanying administration are minimized by the observance of all the necessary precautions in the preparation of the patient and the salvarsan solution. In the use of neosalvarsan, while some reaction occurs in certain instances, the percentage of such is much less than with the older salvarsan. Other advantages of the newer remedy are associated with the technic of its preparation ( $p$. $788)$.

The Late Effects of Salvarsan on the System.-Irritation of the kidneys may be experienced during the process of elimination. In most instances there is nothing more than a transitory albuminuria, but in exceptional cases there has been produced an active acute nephritis, with attendant symptoms of gastro-intestinal irritability. This condition usually passes away, as the elimination of salvarsan is only temporary, and the kidneys gradually regain their former state. Of greatest importance are the so-called neuro-recurrences, which are ascribed to the salvarsan treatment. At first there was a tendency to attribute these manifestations to the direct action of the drug, and as important among these symptoms are those of the auditory and ocular nerves, it was stated that salvarsan exercised a direct influence upon the integrity of these cranial nerves, producing deafness, and in some cases blindness. This was actively disputed,

\footnotetext{
${ }^{1}$ Some of these deaths may be ascribed to defective technic; others to the fact that the remedy was administered after the onset of advanced degenerative lesions of the nervous system, possibly undetected, and in cases of organic diseases of the vital organs. A few cases have been reported in young, apparently sound individuals.
} 
and it was claimed that the symptoms affecting both of these regions were due to the disease itself. It has more recently developed that this latter statement is true, and it has been further revealed that involvement of these organs of special sense, as well as other neurorecurrences, while due to the disease, are indirectly, and not directly, the result of the administration of salvarsan. This has been explained by the fact that during the active stage of the disease there are spirochæte pallida in the cerebrospinal fluid, and that when the amount of salvarsan introduced into the system is insufficient to exterminate these organisms, it renders the spinal column and nerve centers more generally susceptible to their action by a lowered power of resistance. Hence the conclusion is that an adequate course of salvarsan therapy is essential, following the initial dose, to complete the work that has been begun, and to effect the destruction of all the remaining active organisms.

Administration of Salvarsan.-The intramuscular method of injecting salvarsan is still employed by some, notwithstanding the great superiority of the intravenous administration and its general adoption by best authorities. The amount of pain alone attending hypodermic injection is a very objectionable feature; the relatively smaller doses call for constant repetition, and add to the likelihood of an insufficient aggregate quantity being given. The hypodermic preparations have been made up with iodopin and almond oil, and a specially prepared mixture may be obtained put up in hermetically sealed ampules, usually $0.1 \mathrm{gm}$. each. These are given over a prolonged period, about once a week, to obtain an aggregate dosage of 2 to 3 grams -20 to 30 weeks. The technic of intravenous injection is simple and effective, but it is maintained by some, and perhaps with some truth, that the more continuous effect of salvarsan may be obtained by small doses hypodermatically. In cases where, in spite of repeated efforts with intravenous injections, the blood remains positive this is one of the alternative courses to pursue.

The preparation of Salvarsan (old) for intravenous injection is as follows:

The end of the glass capsule is opened by means of a file and the contained powder emptied into a glass graduate. About 100 c.c. of very hot, freshly distilled water are poured upon the powder, allowed to stand for a short time and then mixed with a glass rod (Fig. 307,A). The resulting solution is strongly acid, and it is desirable to have it neutral or alkaline; therefore a solution of 15 per cent. sodium hydroxide is added, drop by drop, until the bulk of the preparation becomes turbid by the precipitation of the arsenic salt (Fig. 307,B). 
The alkaline solution is continuously added, drop by drop, until the mixture becomes perfectly clear again (Fig. 307,C). Enough cold distilled water is then added to bring the total amount up to about 125 c.c. in the case of $0.6 \mathrm{gm}$. of the powder, a proportionate amount being used for a smaller or larger dose of salvarsan. The mixture thus prepared may contain more or less fine sediment and should be carefully filtered through a glass funnel with sterile cotton or gauze (Fig. 307,D), when
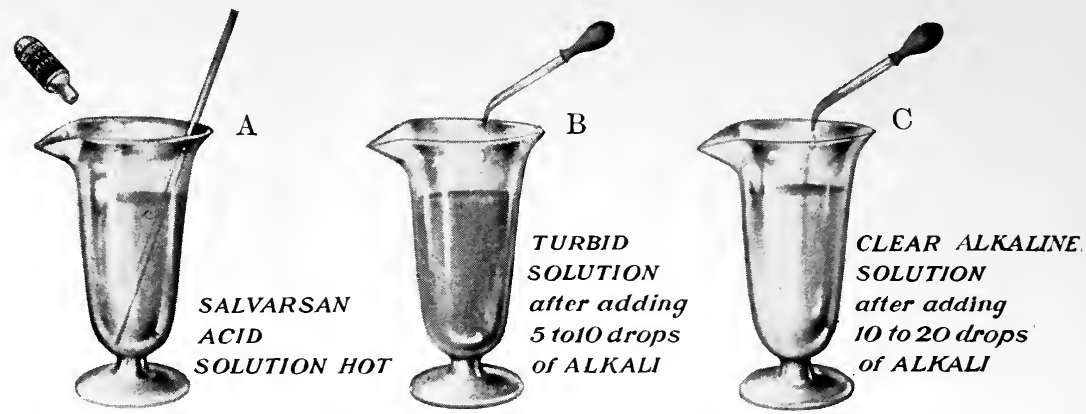

PREPARATION OF OLD SALVARSAN
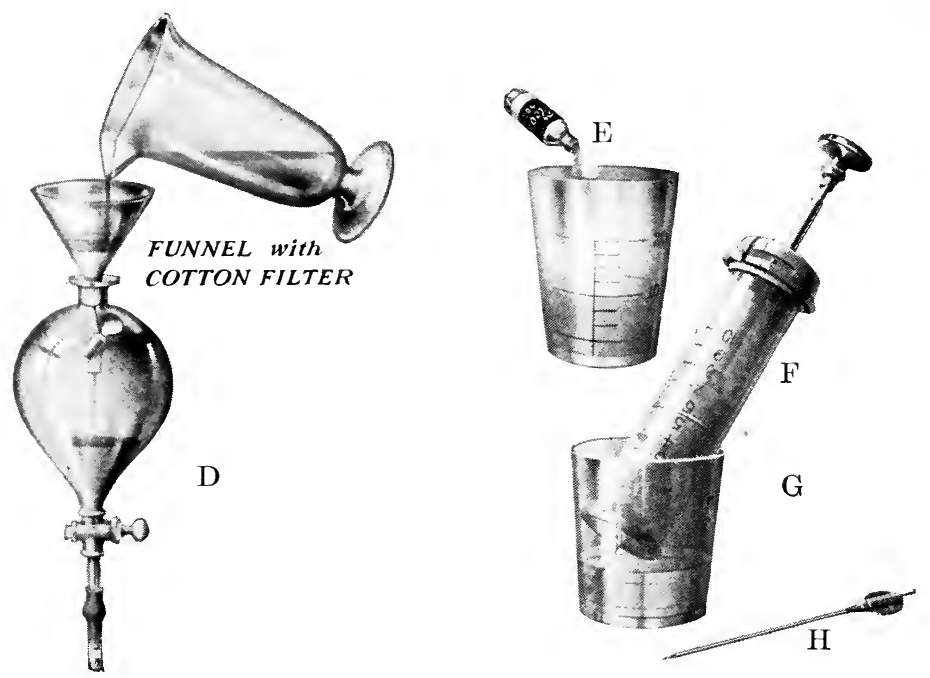

Fig. 307.

it is poured into the container ready for injection, the temperature being about $40.5^{\circ} \mathrm{C}$. $\left(105^{\circ} \mathrm{F}\right.$.).

The preparation of Neosalvarsan for intravenous use is much simpler than that of salvarsan. It is more easily dissolved in water at room temperature and requires far less bulk of fluid for the solution and no alkali to be added, being neutral in reaction. After carefully 
filing the end of glass ampule, avoiding splintering in so doing, the contents are emptied on the top of about 10 c.c. of freshly distilled and carefully sterilized water (Fig. $307, \mathrm{~F}$ ) at room temperature, $20-22^{\circ} \mathrm{C}$. $\left(68\right.$ to $71.6^{\circ} \mathrm{F}$.). This is gently stirred with a glass rod, or by drawing. into and expelling from the syringe, when it will be found that a perfectly clear solution is promptly obtained-generally speaking, 10 c.c. of water for each full dose of $0.9 \mathrm{gm}$. is employed and smaller dosage in same proportion. The solution should not be allowed to stand long nor the temperature thereof materially changed.

Neosalvarsan is generally better tolerated than salvarsan and less frequently accompanied by reaction. Therefore it is believed that it can be repeated at shorter intervals and a greater amount administered within a stated period, affording greater assurance of the destruction of the parasitic organisms of the disease. Finally, its efficiency is at least as great as that of salvarsan

The water employed for making up the solution of Salvarsan for intravenous injection is a most important consideration. In the earlier experiences it was a common practice to use normal salt solution, made up with distilled water. On account of the apparent hypertonic effect of throwing into the circulation so large a volume of fluid of high specific gravity, in most instances this practice is abandoned at the present time, although in some cases it is quite permissible to employ the normal salt solution in mixing either the salvarsan or neosalvarsan, especially in such cases where, owing to cachexia or anemia, the sustaining effect during the course of the administration may be indicated.

In the largest number of cases it is customary to employ freshly distilled water as the vehicle of the salvarsan and two requisites in connection with the preparation thereof are most essential. First the water should be freshly distilled, preferably within one or two hours of the time of its use, and secondly, it must be subjected to most careful sterilization, such as cannot be obtained by ordinary boiling, and therefore should be provided by means of an autoclave or highpressure sterilizer. When the second of these requirements is effectually carried out, water that has been distilled at any time within twenty-four hours may be used.

The importance of this provision cannot be overestimated and its discovery has considerably reduced the occurrence of reaction dependent upon the presence of fungi and saprophytic bacteria which have not been destroyed by the ordinary methods of sterilization, and which it is almost impossible to eliminate except by fresh distillation and sterilization. 
The Technic of the Intravenous Injection of Salvarsan (Old) is as follows: The arm of the patient is prepared at the bend of the elbow by cleansing, all the requirements of a thoroughly aseptic operation being provided for. The essentials include salvarsan needle (Figs. 307,H or 308) and container, graduate glasses, stirring rod, funnel, and rubber tourniquet. Enough distilled water is poured into the container to displace the air in the outlet tube, after which the prepared salvarsan solution is filtered into the container (Fig. 307,D), which is held by an assistant. A rubber tourniquet is placed around the arm tight enough to retard the return circulation; the salvarsan needle is thrust within the lumen of one or the other of the superficial veins," at the bend of the elbow (Fig. 309,A); the successful accomplishment of this feat is revealed by the free exit of blood from the needle. The outlet tube from the receptacle is then coupled with the needle, and the entire quantity of the solution is allowed to enter the

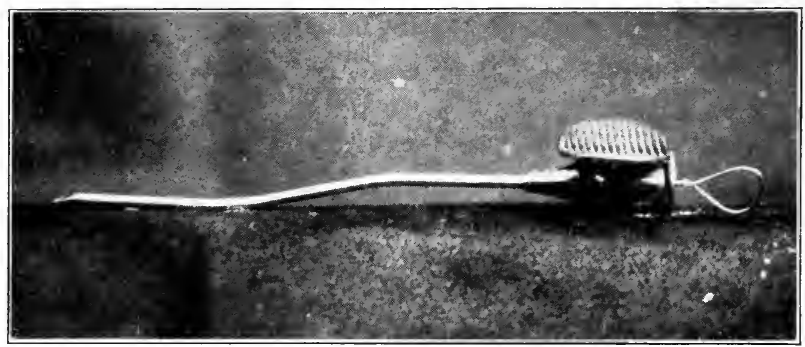

Fig. 308.-Author's salvarsan needle.

vein (Fig. 309,A). In order to insure the entrance of the total amount, the last portion of the solution is followed up with a small quantity of distilled water.

The intravenous method is simple in most cases, although in some instances it may be found difficult to puncture the vein without exposure thereof through an open incision. Indeed it is much better to resort to the latter means of reaching the vessel in difficult cases rather than run risk of infiltrating the tissues. Therefore when the attempt to puncture is unsuccessful, the vein should be laid bare at the same or another site and, after elevation in the wound, or the exposed vein may be punctured with the salvarsan needle without opening (Fig. 310), or it may be opened and a canula inserted through the aperture (in the latter case the vein must be ligated when the canula is withdrawn); the operation is then continued as with the closed method of injection. 
The Technic of Intravenous Injection of Salvarsan (New) is, like its preparation therefore, much simpler than the old remedy. The

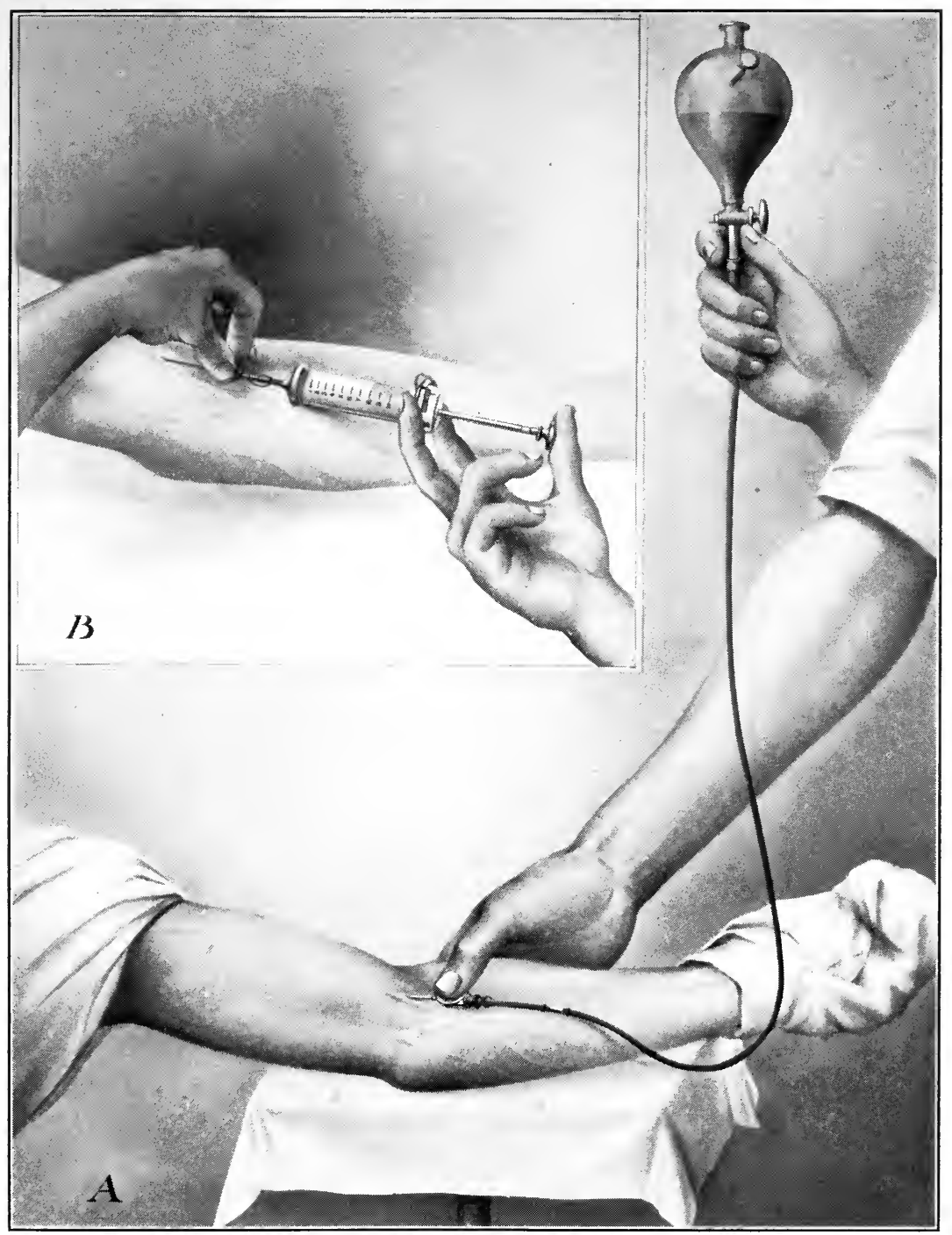

FIG. 309.-Closed method of intravenous administration of salvarsan, old (A) and new (B).

bulk of the solution being so small (10 c.c.) it is convenient to make use of a 10 c.c. piston syringe as a rapid and easy means of introduction. 
After inserting the needle into the lumen of the vein, as for the other method, the syringe is coupled while the needle is held firmly in place and the solution is injected deliberately $(309, \mathrm{~B})$. If puncture of the vein is difficult, for any reason the open method may be resorted to as in the case of old salvarsan (Fig. 310).

Confinement in the house for the first twelve to twenty-four hours following the intravenous injection is always desirable. This does not necessarily mean hospital treatment, provided all the requirements of careful technic and preparation can be carried out in the patient's

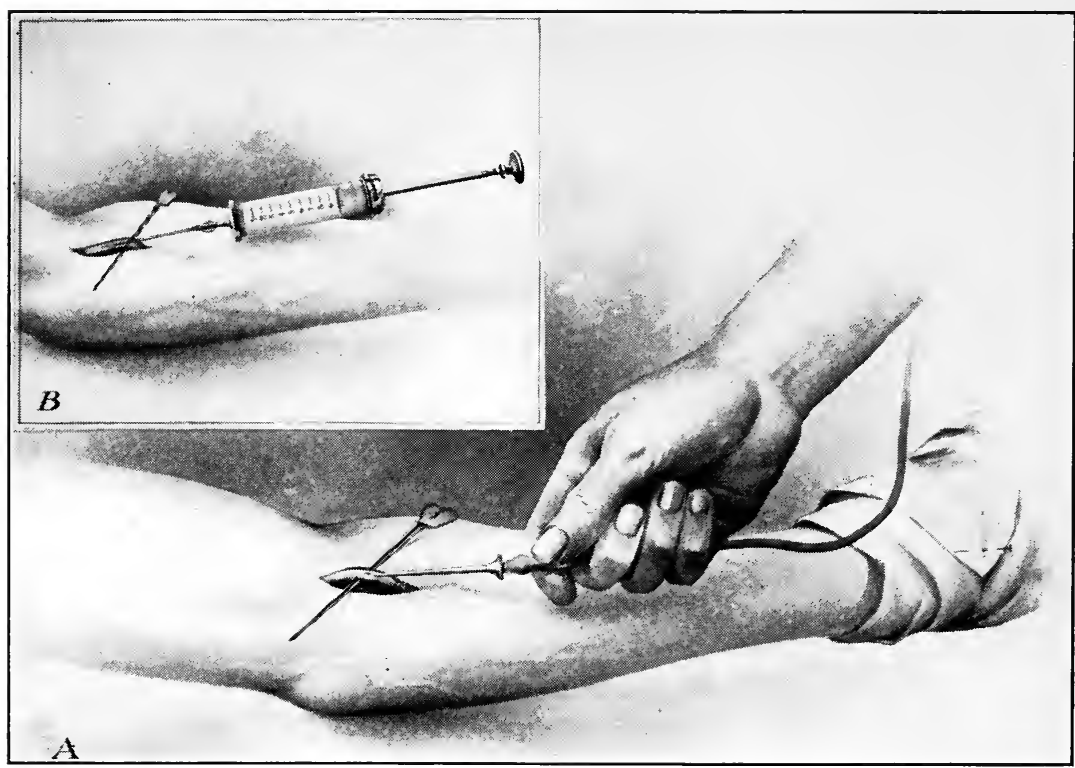

FIG. 310.-Open method of intravenous administration of salvarsan, old (A) and new (B).

house or in the physician's office. In the latter case, the patient is allowed to travel a short distance following the treatment, and is instructed to remain in the house until the next day, after which there is no further necessity for confinement, provided there has been no severe reaction.

Dosage and Repetition of Treatments.-The administration of salvarsan therapy is based upon one of two indications, viz.- - the presence or absence of spirochete in the system of the individual and the presence or absence of active manifestations of the disease, which considerations, in general respects, govern the size of the dose and the continuation of treatment. A single full dose may temporarily 
change the Wassermann blood reaction from positive to negative, and cause a complete disappearance of symptoms. This effect is likely to be only temporary, unless it is followed up by the continued administration of one or the other forms of salvarsan in repeated doses till an aggregate amount is reached of from two to four grams, which usually requires subsequent mercurial treatments, and other salvarsan administrations or duplicate courses. A single full dose of salvarsan, therefore, can be regarded only as a symptomatic dose, and one which may be the means of confirming the clinical diagnosis. The repeated dosage, as at present laid down, either with or without the addition of mercurial treatment is recognized as the routine form of treatment, and the most likely to afford a satisfactory result; while the same or almost double the regular dosage in half the period of administration, or about two weeks, is called the intensive course of treatment.

Finally, a small dose of salvarsan, about half the ordinary dose, may be employed for diagnosis instead of treatment, when the Wassermann blood reaction is negative, and more especially following an interval of several months after a course of salvarsan treatment. This last procedure is based upon the fact that a small or inadequate dose of salvarsan will tend to increase the activity of the few remaining parasites in the system, and be the means of bringing about a positive reaction which the number of the organisms were too few to produce without this expedient. In doubtful cases, and after a prolonged course, this expedient is resorted to by some, when the other means at hand fail to carry conviction.

Salvarsan treatment is employed in all stages of the disease; an inadequate amount of the remedy may exercise an unfavorable influence upon the later development of the malady. A single dose is seldom used, repetition being regarded as important in every instance.

The average single dose of salvarsan is $0.6 \mathrm{gm}$. for men and $0.5 \mathrm{gm}$. for women, to be repeated for three or four consecutive injections, at weekly intervals. The later the stage of the disease, the greater the necessity of repeated treatments. In addition to such a course being adopted, it is important to follow up the salvarsan injections by a vigorous and efficient course of mercurials.

It is sometimes permissible to double the initial dose in cases of severe and threatening lesions. I have done this successfully in a number of instances, and while it should not be practised indiscriminately, there may be some cases in which the percentage of risk is no greater, if as great, as that threatened by the disease itself.

Neosalvarsan is put up in six different doses, from $0.15 \mathrm{gm}$. to 0.9 gm. The proportion of arsenic contained in the two preparations is 
relatively greater in the old than in the new, which governs the size of the dose, and three parts of neosalvarsan is equivalent to two parts of salvarsan (see p. 782).

The average single dose of neosalvarsan for men is 0.75 to $0.9 \mathrm{gm}$.; for women 0.6 to $0.75 \mathrm{gm}$. As in the case of salvarsan, in exceptionally severe cases, it is permissible to double this amount with the initial dosage, when conditions of impending serious damage to the vital tissues of the body warrant prompt and energetic action. In the ordinary run of cases, however, it is not necessary to begin with such large doses. The general purpose being to administer an aggregate of from. $2.5 \mathrm{gm}$. to $4 \mathrm{gm}$. within a period of from three to six weeks, the first dose may be from $0.6 \mathrm{gm}$. to $0.9 \mathrm{gm}$. and the amount varied thereafter from $0.45 \mathrm{gm}$. to $0.9 \mathrm{gm}$. at intervals of three to seven days, and sometimes longer, according to the activity of the disease, and the effect of treatment. It is important to distinguish, if possible, between the reaction from the medication, and the increased activity of the invading parasites of the disease. In one instance increased dosage and renewed attack are demanded, in the other, an interruption of treatment, until the irritating influence of the chemical agent upon the system has subsided. The administration is regulated much the same as is the treatment by raccines and serum, and it has been suggested that there occurs in the course of the treatment a kind of anaphylaxis similar to that accompanying the extreme negative phase of the over sensitized subject following serum injection, and this period should be allowed to subside before reintroducing the activating substance, in this instance salvarsan. Generally speaking, in ordinary cases, the average dose is given to begin with, and repetitions may be administered in moderate doses, at intervals of two or three days, or in full dosage at intervals of a week, until an aggregate of from 2.5 to 4 $\mathrm{gm}$. have been employed in a period of from three to six weeks. The best results, as already stated, are obtained in the early stages, when from four to six average doses, in a period of four to six weeks will often accomplish the desired end of causing a total disappearance of clinical symptoms and in some instances a negative reaction of the blood, with or without the supplementary action of a mercurial course. In the late stages of the disease, a greater amount and more prolonged treatment are demanded, and intermediate courses of mercurial medication with subsequent courses of treatment by salvarsan. From past experience in the most severe cases, I am not favorably impressed with the plan of testing the effect of mild doses before proceeding with the treatment. In fact it has seemed that reaction in the nature of anaphylaxis has occurred as readily following small doses as from 
large, and that in serious cases the severity of the situation demands the immediate administration of a large dose. When special contraindications exist in the general condition of the patient, or of his vital organs, it may be preferable to begin with a preliminary course of the older remedies, mercury and iodide of potash, and later to follow with the salvarsan treatment, rather than begin with a too small and insufficient dose of the latter remedy.

Before commencing treatment, at suitable intervals during its administration, and following the cessation thereof, the systematic examination of the blood is resorted to, and thereby the indication for and result of treatment are determined. In those cases with positive clinical history, which give a negative Wassermann reaction of the blood, the Noguchi Luetin reaction may be resorted to or, of greater importance, in cases with physical signs of cerebro-spinal involvement, determination of the cellular content and complement fixation reaction of the spinal fluid, and no case should be considered as properly exempt from the necessity of further treatment until after it has been possible to obtain repeated negative blood reactions, and total absence of symptoms, during consecutive periods of three, six, and twelve months following the last treatment.

In late and rebellious cases, clinically active, where it is impossible to obtain the assistance of the blood reaction in confirmation thereof, the treatment should be administered as though the same were not lacking, and followed by a vigorous course of mercurials and iodide.

Indications and Contraindications.-To give all the indications for the use of the salvarsan treatment in syphilis is to mention every stage of the disease and each phase thereof. As has already been stated, there is no question of its immediate effect in removing promptly all clinical manifestations of the disease in whichever stage they occur; there is a difference, however, in the permanency of the result, which seems to be governed first by the period of the disease when treatment is commenced; second, by the resistance displayed by the parasitic organisms to the action of the chemical agent; and third, by the continuation of treatment.

In the primary stage, the chancre is made to disappear with a single dose of salvarsan, and when followed by three or four additional doses with or without a supplemental mercurial course, a permanent absence of symptoms will often result. When the effectiveness of the treatment is incomplete, as evidenced later, after the expiration of several months, by a pcsitive blood examination or recurring symptoms, it is then necessary to renew the attack with salvarsan, increasing 
the amount of dosage and total amount employed, and then to follow with an active course of mercurial medication.

In the secondary stage, when the constitutional disease is at its greatest activity, it is necessary to push the treatment to a higher degree of intensity, and by way of comparison, where three or four full doses may produce the desired result in the primary stage, double this number are required, in the average case, during the active secondary stage being repeated several months later on; and likewise subsequent mercurial treatment with or independent of the renewed salvarsan courses.

In the tertiary stage of the disease, when as is known, the morbid process strikes deeply into the tissues and organs, here again some of the most brilliant results so far as the immediate presence of symptoms is concerned, are obtained by a single dose. Such initial dose must always be followed by repeated treatments until the maximum aggregate amount is given, supplemented by active mercurial medication, in many instances; then after an interval of weeks or months, another perhaps even more intensive course of similar character may be required. Notwithstanding these general rules, there are many exceptions, and a single dose in the well-advanced stage of the disease has been known to effect a permanent result, whereas a number of doses in the primary stage have failed. With respect to the first of these exceptions, such a result is more likely to follow in those cases which have been subjected to a thorough and continuous early course of mercurial treatment, in spite of which the disease has persisted; and pertaining to the other exception, renewed treatment along the lines laid down will usually ultimately result in a successful outcome.

Children of seven years and upwards may be given $0.3 \mathrm{gm}$. salvarsan, and $0.5 \mathrm{gm}$. neosalvarsan, and the same proportion to the adult dosage followed with respect to the total amount.

Infants suffering from congenital syphilis, may be treated in two ways: by direct administration or by treatment of the mother. The direct administration of salvarsan intravenously, in dosage of 0.01 $\mathrm{gm}$. to $0.02 \mathrm{gm}$., may be cautiously repeated as judgment dictates with respect to the action of the drug upon the malady and its effect upon the organism. Some risk must be assumed in these cases, as the situation is at best unfavorable. The infant may also be benefited through the agency of the mother's milk while the latter is receiving the salvarsan treatment. The exact explanation of this effect is not understood, as the presence of arsenic in the mother's milk has been sought and not found, and therefore it is suggested that antibodies from the blood of the mother are transmitted through the medium 
of the milk to the infant. Be that as it may, the effect of such treatment is symptomatic and not permanent, and therefore the subject should receive in addition direct treatment.

Pregnant women who have the clinical history or reveal symptoms of the disease, may be submitted to the salvarsan treatment intravenously, and such treatment repeated at varying intervals, care being exercised to avoid too severe a reaction. The intervals between treatments may be longer than in the ordinary run of cases, and the course made to cover a period of four to six months.

The foregoing directions embody what is considered the routine practice of Salvarsan administration. In exceptional instances of great severity departure is made in favor of a much more active and vigorous course.

The Intensive Method of Treatment involves pushing the remedy to the limit. Instead of giving the average full dosage in 4 to 6 administrations in about the same number of weeks, reaching an aggregate of 4 or 5 grams in all, double the amount is given in a much shorter period either by halving the interval or by doubling the dosage. Thus we may start with the full single dose (No. vi see p. 781) and in a few days double same, thereafter making the interval as short as is consistent with avoiding severe reaction, and in the stated reduced period endeavor to reach about double the ordinary aggregate amount or 8 grams. It is expedient meanwhile to watch for evidence of acute arsenical poisoning as well as the more cumulative disturbances.

It is in the most severe forms of tertiary syphilis, when the integrity of the vital tissues is endangered, the brain, spinal cord, and cranial nerves threatened, that it is necessary to obtain the full potency of the remedy, and it is in these instances that the question of administering at once a double dosage and a potentially intensive course will arise. In my own experience, I have seen excellent results follow this course, and am inclined to discourage the employment of the small or under average dosage in such crises, with the greater possible chance of failure, and of over sensitizing the organism to the surviving invading parasites. As an alternative course, it is far better in my belief to refrain from the salvarsan treatment entirely, and to push the older remedies of mercury and potash until a vitally dangerous crisis has yielded under such measures.

The same rules for the persistence in and renewal of treatments and the constant investigation of the blood reaction and spinal fluid content, apply with even greater import to the severe forms than to the average type of cases. 
The contraindications to the use of salvarsan, so far as the disease itself is concerned, are only those above referred to, in which the situation is so dangerous with respect to the organs of special sense, the cerebrospinal axis, or other vital organs, that some hesitancy is felt in pushing the remedy to the extent that is demanded by a precarious situation. This question must be weighed in each individual case, and decided upon its merits, the patient taking the risk, with a full understanding, as in the event of an important surgical operation.

In the parasyphilitic disease, the degenerative forms of tabes and paresis, when it has been proven beyond doubt, by careful study of the spinal fluid with respect to the cellular content, differential and quantitative, as well as the complement fixation test, that the luetic proccess no longer survives, the salvarsan treatment is not only ineffective, but its use is unwarranted, and likely to do harm. There are many types however of these maladies, which have not reached an irremedial terminal stage and which may be favorably influenced by treatment, be that the intensive method of procedure as above, or a combination of intravenous and intraspinous salvarsan medication.

Intraspinal Administration of Salvarsan.-This method of introducing salvarsan into the system is intended to be confined to cases of advanced disease of the central nervous system; and when certain facts that are known are carefully considered, it should be stated that it is only applicable to special types of syphilis and certain stages of cerebro-spinal involvement, as indicated by investigation and comparison of the cerebro-spinal fluid and the blood serum. When the disease is generalized and active, whether this be evidenced by pronounced clinical manifestations or strong blood reaction, the indication is all in favor of intravenous treatment of an average or highly intensive degree, according to the activity of the symptoms. Such treatment should be succeeded by repeated administrations, until the blood serum and spinal fluid yield results indicative of diminished activity of the disease. If this change occurs in the blood serum, and only to a lesser degree in the spinal fluid, the question of the indication for intraspinous treatment must be considered. In a word, when the remaining parasitic contamination seems to be localized in the cerebrospinal system intradurally, the direct application of the remedial agent may be more successful in reaching the organisms than by the more indirect and impeded route of the blood stream.

The different methods of introducing salvarsan directly into the spinal canal are: 1. The use of blood removed from the patient one hour after the intravenous injection of salvarsan (Swift-Ellis). ${ }^{1}$

${ }^{1}$ N. Y. Medical Journal, July 13, 1912. 
2. Intraspinous injections of solution of neosalvarsan; 3. Intraspinous injections of serum salvarsanized in vitro, of standardized strength (Ogilvie). ${ }^{1}$

1. The Swift-Ellis method is the original technic, and more generally adopted. It is carried out as follows: one hour after the intravenous administration of a full dose of salvarsan, 40 c.c. of blood are withdrawn, allowed to clot, and then centrifugalized until perfectly clear serum is obtained, which is kept on ice for twenty-four hours; 12 c.c. of this serum are combined with 18 c.c. of normal salt solution, and heated to $56^{\circ} \mathrm{C}$. This amount of 30 c.c. is introduced by lumbar puncture with an intraspinal needle, by gravity, following the removal of sufficient spinal fluid to reduce the intraspinous pressure to $30 \mathrm{~mm}$. There generally follows a reaction, varying from a sharp headache to pains of variable degree in the extremities and lateral spinal regions, accompanied by loss of muscular control, together with bladder and rectal disturbance. The objection raised to this method is the inability to standardize the amount of specific treatment that is administered, it being impossible to know how much of the arsenical preparation is contained in the blood serum of each individual case. Furthermore, on account of the intravenous introduction of the salvarsan prior to the removal of the blood, there is the question as to how much the credit of the resultant action is to be given to the intravenous application in every instance that this method of procedure is followed.

2. The introduction of neosalvarsan in the spinal canal in any form does not seem to be warranted, judging by the experience reported of its use in this manner. It seems to be chemically unsuited to spinal application, and has proved to be a great irritant, even in small doses, being followed by sharp local and general reactions.

3. The Ogilvie technic ${ }^{1}$ of injecting serum salvarsanized in vitro of standardized strength is an alternative for the Swift-Ellis method. It has the advantage that knowledge is afforded as to exactly how much of the drug is administered. Furthermore, the system devised for its preparation provides for favorable biological changes that seem to occur in the salvarsanized serum in vivo. This is maintained outside the body by incubation at a temperature of $56^{\circ} \mathrm{C}$. for 30 minutes. The technic of preparation is scientifically devised, and every step thereof being essential, should be carefully carried out. ${ }^{2}$

When prepared according to detailed directions, to 15 c.c. is added the dosage of salvarsan to be given, which dosage has been determined,

${ }^{1}$ Jour. A.M.A., Nov. 28, 1914 and Med. Record, June 26, 1915.

${ }^{2}$ Jour. A.M.A., Nov. 28, 1914. 
as safest for repeated usagè, to be from $0.25 \mathrm{mg}$. to $0.5 \mathrm{mg}$. The lumbar puncture is made in the usual way, with the patient on the edge of the bed, thighs flexed on the abdomen. About 15 c.c. of spinal fluid is withdrawn, previous to the introduction of the salvarsanized serum, which should be done by gravity.

The intraspinous treatments, by both the Swift-Ellis and Ogilvie methods, are repeated at intervals of one, two and three weeks, according to the reaction and response therefrom, and number from six to twelve or more treatments, according to the severity of the case and the result obtained.

As to the indications for this delicate and direct method of application, in general terms, it may be stated that the method is more particularly suited to those late forms of parasyphilitic invasion, notably tabes and tabo-paresis, wherein the specific organisms have become entrenched in the cerebro-spinal canal, beyond the reach of the ordinary method of intravenous medication. In selecting these cases, it should be remembered that certain cerebro-spinal lesions of syphilitic origin are dependent upon vascular disturbance and involvement of the meninges. These cases are often the most active forms, can generally be reached by the blood stream, and are therefore more suitable for intravenous than intraspinal medication. They are represented by the types of syphilitic meningitis and cerebro-spinal arteritis. In such cases the blood stream is likely to be very active in reaction and whereas the spinal fluid may also show active reaction and a high cell count, it is possible for it to be normal.

That class of cases of cerebro-spinal syphilis in which the nerve tissue itself is attacked, is represented pathologically by the lesions of myelitis, infiltrating gummas, and the characteristic changes of tabes dorsalis and paresis, which last two, in the quarternary or terminal stage are degenerative, and in this respect, beyond repair. Therefore in the selection of cases, it may be stated that in the active inflammatory ones, notably, of the meningeal and arterial types, the indication is for the sharp, intensive course of intravenous salvarsan; in the more advanced cases of paresis and tabes, when the blood is less active, and the spinal fluid gives the characteristic evidence of these maladies, the specific indication for the intraspinous treatment is the most positive, either alone, or in conjunction with the intravenous method. In the late degenerative stages, which have heretofore been considered almost hopeless, the intraspinous treatment is warranted, but the attendant result is only comparatively favorable, and dependent in all likelihood upon the extent of irreparable damage, which cannot be remedied by any treatment whatever; yet there is no accurate way of measuring 
the degree of such damage, and therefore, in some apparently hopeless instances, the response may be favorable beyond expectation.

Both the Swift-Ellis and Ogilvie methods have strong adherents. Their relative value is not satisfactorily decided. More extensive experience, under careful and reliable observation, is necessary to determine the question (see also Intraspinous Treatment with Mercury, p. 773).

Generally speaking, there is no contraindication to the use of this remedy for attacking the active syphilitic process affecting any of the organs of the body, including the ocular and auditory apparatus. Some cases having previously shown a positive Wassermann reaction, become promptly negative after treatment, and, if the treatment is not successful, subsequently the blood becomes positive again; other cases remain positive in spite of energetic administration, to yield eventually to repeated dosage and still more intensive course. Some cases, it is alleged, retain the positive reaction in the blood despite the clinical cessation of the disease, and nothing serves to efface this reaction. In the lght of our present knowledge, it is not possible to state the correctness of this view or to offer satisfactory explanation; but all that has been written applies equally to the administration of salvarsan (old), and any one of its substitutes as well. 
TABLES GIVING COMPARATIVE EQUIVALENTS OF INCHES AND CENTIMETERS, MINIMS, DRAMS, OUNCES AND CUBIC CENTIMETERS, GRAINS AND GRAMS

$\begin{array}{cr}\text { Inches } & \text { Centimeter } \\ & \\ 1 / 4 & 6.40 \\ 1 / 2 & 12.70 \\ 1 & 2.54 \\ 2 & 5.10 \\ 3 & 7.60 \\ 4 & 10.20 \\ 5 & 12.70 \\ 6 & 15.20 \\ 7 & 17.50 \\ 8 & 20.30 \\ 9 & 22.90 \\ 10 & 25.40 \\ 11 & 27.90 \\ 12 & 30.50\end{array}$

$3.28 \mathrm{ft} .=$ one meter

Minims Cubic Centimeters
0.06
0.12
0.18
0.24
0.30
0.37
0.43
0.50
0.55
0.61
0.92
1.23
1.54
1.85
2.15
2.46
2.77
3.08
3.04

one dram $=$ (about) 4 c.c.

one ounce $=$ (about) 30 c.c.

one pint $=$ (about) 500 c.c.

one quart $=$ (about) 1000 c.c. $=1$ liter

$\begin{array}{ll}\text { Grains } & \text { Grams } \\ 1 / 64 & 0.0010 \\ 1 / 32 & 0.0020 \\ 1 / 16 & 0.0040 \\ 1 / 10 & 0.0065 \\ 1 / 8 & 0.0081 \\ 1 / 6 & 0.0108 \\ 1 / 4 & 0.0162 \\ 1 / 2 & 0.0324 \\ 3 / 4 & 0.0486 \\ 7 / 8 & 0.0567\end{array}$

Grains
$\begin{aligned} & 1 \\ & 11 / 2 \\ & 2 \\ & 3 \\ & 4 \\ & 5 \\ & 6 \\ & 7 \\ & 8 \\ & 9 \\ & 10\end{aligned}$

Grams

15 grs. = (about) 1 gram 


\section{INDEX}

Abscess, of Cowper's glands, 244

of lacrymal sac, 729

of prostate, 372

periprostatic, 373

peri-urethral, 243, 296

prevesical, 431

renal, 599

retrorenal, 627

Acetone in urine, 89

Adenectomy, inguinal, 346

Adenitis, 241

inguinal, 690

in syphilis, 705

Agglutinins, 105

Albarran cystoscope, 129

Albumin in urine, 86

quantitative estimation of, 88

tests for, 87

Alimentary tract, syphilis of, 731

Alypin, for anesthetizing the urethra, 47

American scale of urethral instruments, 31

Ammonio-magnesium phosphate crystals in urine, 100

Ammonium urates, in urine, 100

Amorphous phosphate crystals in urine, 101

urates in urine, 100

Amyloid casts in urine, 98

Anaphylaxis, 110

Anastomosis, ureteral, 651, 652

Anesthesia, local, for external urethrotomy, 500

for operations upon external genitals, 499, 500

lower urinary tract, 499

scrotum, 501

testicles, 501

for suprapubic cystotomy, 500

perineal, 500

Anesthetic jelly, 47

Anesthetization of urethra, 46

Anodynes for use in acute urethritis, 209
Anomalies of kidney, 520

of penis, 184

of spermatic cord, 304

of testicle, 302, 303

of ureter, 524,525

Anorchism, 302

Anterior urethra, injection of, 32 irrigation of, 36

Antibodies, 105

Antigens, polyvalent, 108

Antisepsis, of instruments, 54

of patient, 53

of surgeon's hands, 53

Antiseptic cabinet for instruments, 55

Antiseptics, internal, in acute urethritis, 209

Antitoxins, 105

Anuria, 503

calculous, 572

Anus, mucous syphilides of, 722

Argyrol, in acute urethritis, 215, 220, 221

in chronic urethritis, 239

Armamentarium, 29

Arsenic, preparations of, in treatment of syphilis, 779

Arteries, syphilis of, 734

Arteritis, syphilitic, 749

Arthritis, gonorrheal, 249

Aspermatism, 515

Atony of bladder, 432, 505

Atoxyl, in treatment of syphilis, 780

Aural, see Ear.

Autogenous vaccines, 111

Azoospermia, 313, 516

Bacilli in urine, 102

Bacillus coli in urine, 102

Bacteria in urine, 79

Bacterial casts in urine, 98

extracts, 109, 113

filtrates, 113, 114

Bacteriology of urine, 101

Bacteriolysins, 105 
Bacteriotropins, 105

Bacteriuria, 78, 79

Balanitis, 173 erosive and gangrenous, 176

Balanoposthitis, 173

Bangs' canula, 34

Banks' bougie, 43, 46

Basal meningitis, syphilitic, 747

Basins, sterilization of, 59

Beck's operation for hypospadias, 278, 279,280

Béniqué catheter, 51, 72

curved obturators of Guvon, 51

scale of urethral instruments, 31

shaped catheter directors, 51

sounds, 41, 43

Bevan's operation for undescended testicle, 359

Bigelow's evacuating tube, 494

evacuator, 496

lithotrite, 493

washer, 496

Bile acids in urine, 90

pigments in urine, 90

Bladder, and see Vesical.

abnormal conditions of, cystoscopy in, 148

absence of, 415

anatomy of, 5

atony of, 432,505

atrophy of, 416

blood from, in urine, 81

calculus of, see Vesical calculus.

capacity of, 6,61

carcinoma of, 450

chronic inflammation of, eystoscopy in, 148

congenital malformations of, 415

continuous irrigation of, 460

contracture of neck of, 435

Bottini's operation for, 439

causation of, 435

diagnosis of, 437

pathology of, 436

perineal-galvano prostatomy for, 440

symptoms of, 436

treatment of, 438

cystoscopic appearance of, 141

landmarks of, 142
Bladder, cystoscopic pictures of, 133

cysts of, 450

deformity of, 416

dermoid cysts of, 450

diverticulum of, 416

eystoscopy in, 149

treatment of, 470

drainage of, by electric suction apparatus, 489

through perineal opening, 460

embryology of, 26

epithelium from, in urine, 94

examination of, 75

exploration of, 75

exstrophy of, 415

Maydl's operation for, 471

Peter's operation for, 471

treatment of, 471

fibroma of, 450

hernia of, 418

hypertrophy of, 416

inflammation of, 420 , and see Cystitis.

injuries of, 418

in prostatic hypertrophy, 400

irritable, 504

lavage of, 39

multiple, 415

myoma of, 450

myxoma of, 450

operations on, 458, 492, 497

Bottini's, 498

transurethral, 492

Young's, 498

palpation of, 7.5

papilloma of, 450

paresis of, 505

partial resection of, 465

pus from, in urine, 79

relations of, 6

removal of, 455

rupture of, 419

treatment, 470

sareoma of, 452

stone in, 441, and see Vesical calculus.

structure of, 8

suprapubic puncture of, 471

syphilis of, 737

transurethral operations upon, 492 
Bladder, tuberculosis of, 665

cystoscopy in, 667

diagnosis of, 667

morbid changes in, 665

prognosis of, 668

symptoms of, 665

treatment of, 668,670

tuberculous inflammation of, cystoscopy in, 149

tumors of, 449

cystoscopy in, 149

diagnosis of, 453

etiology of, 452

pathology of, 452

removal of, 464

symptoms of, 453

treatment of, 454, 456, 457, 464

varieties of, 449

varices of, cystoscopy in, 148

Blizzard knife, for perineal section, 289

Blood casts, in urine, 98

corpuscles, red, in urine, 92

Blood, in urine, 63, 80, 90, 92

tests for, 90

Bottini's operation, 439, 498

Bougie, 29, and see Instruments.

à boule, 44

armée, 45

Banks', 43, 46

bulbous, 42,44

filiform, 42,44

olivary, 42

Philip's, 43,46

sterilization of, 57,58

tortillée, 44

woven conical, 44

Brain, gumma of, 745

lues of, 745

Bright's disease, 535, and see Nephritis.

Bronchi, syphilis of, 731

Brown-Buerger cystoscope, 130, 136

Bubo, 690

chancroidal, 690

double, 690

varieties of, 690

virulent, 690

Buerger cysto-urethroscope, 124 urethroscope, 121

Bulb ejector, 35

Bulbous syringe, 32

Bursitis, gonorrheal, 251
Cabot, Hugh, resection of urethra, drainage following, 295

Calcium oxalate crystals, in urine, 100

Calculous anuria, 572

Calculus, in seminal vesicles, 331

in urethra, urethroscopy in, 127, 128

prostatic, 369

renal, 562

causation of, 563

course of, 566

diagnosis of, 566

morbid changes in kidney in, 564

primary, 563

secondary, 563

symptoms of, 565, 566

treatment of, 568,569

roentgenography in diagnosis of, 162

ureteral, 569

cystoscopy in, 571

diagnosis of, 571

symptoms of, 570

treatment of, 571

vesical, 441, and see Vesical calculus.

Camera attachment for cystoscope, 132

Cancer, and see Carcinoma.

chimney-sweeps', 302

of penis, 183

Canula, Bangs', 34

Guyon's, 34

Carcinoma, and see Cancer.

of bladder, 450

of kidney, 553

of prostate, 366

of scrotum, 302

Casper cystoscope, 129

Castration, 358

Casts in urine, 96, 97, 98

Catheters, 29, 47

Béniqué curve, 51 .

directors, 51

elbowed, 51

essential qualities of, 52

filiform, 43, 44

glass, 47

indwelling, 53

introduction of, 72

Mercier, 50, 51

Nélaton, 48 
Catheters, prostatic, 50

retained, 53

retainer, 52

soft rubber, 48

"bout coupé," 49

elbow, 49

staff, 290

sterilization of, 57

ureteral, 147

care of, 58

drainage by, 154

sterilization of, 57

treatment by, 154

woven olivary, 49

double elbow (bi-coudé), 50

olive-tipped elbow, 50

single elbow, 50

Catheterization, 72

in prostatic hypertrophy, 397, 408

of ureters, 147

retrograde, 274

Cavernous tuberculosis of kidney, 655

Cellulitis of scrotum, 299

Centrifuge, 91

Cerebrospinal syphilis, 743

causation of, 744

diagnosis of, 750

pathology of, 744

prognosis of, 750

treatment of, 750

Chancre, 698, 701

complications of, 705

course of, 701, 703

development of, 701

diagnosis of, 705

eroded, 701

extragenital, 699, 703

genital, 702

hard, 681

incubation period of, 701

induration of, 704

mixed, 681, 687, 702

treatment of, 693

number of, 701

of urethra, 194, 703

papular, 702

perigenital, 703

prognosis of, 705

re-indurated, 702

site of, 702

soft, 681, and see Chancroid.
Chancre, treatment of, 706

by salvarsan, 795

ulcerated, 702

varieties of, 701

Wassermann reaction for, 106

Chancroid, 681

abortive treatment of, 691

bacteriology of, 683

causation of, 681

complications of, 687

treatment of, 693

diagnosis of, 685

duration of, 685

expectant treatment of, 692

follicular, 684

frequency of, 683

hot air treatment of, 694

inflamed, 687

treatment of, 693

inoculability of, 682

number of, 681

pathology of, 683

phagedenic, 683

prophylaxis, 691

site of, 681

symptoms of, 684

treatment of, 691

Charrière scale of urethral instruments, 31

Chetwood's cut-off, 29,36

cystoscope, 136, 137

double current rectal tube, 382

galvano-cautery prostatic incisor, 477

galvano-prostatotomy, 477

irrigating apparatus, 224

method of anterior urethral irrigation, 36

modification of Isaac's table, 138

operation of prostatotomy, 477, 478

salvarsan needle, 790

urethral clamp, 29, 33, 36, 44

endoscope and speculum, 121

nozzles, 121

Chill, urethral, 75

Chimney-sweeps' cancer, 302

Chismore's lithotrite and evacuator, 494

Chlorides in urine, 84

Chordee, breaking the, 171

Chylocele, 324 
Chyluria, 90

Circumcision, 342

Clamp, irrigation, Chetwood's, 36 urethral, 33, 44

Chetwood's, 29, 33, 36, 44

Cocaine, for anesthetizing the urethra, 46

Colic, renal, 568

Collargol, in acute urethritis, 220, 221

Colon bacillus, in urine, 102

Colorimetric test of renal insufficiency, 157

Complement fixation test, 106

for echinococcus disease, 109

for gonorrhea, 108

for renal tuberculosis, 109

for syphilis, 106

method of obtaining blood for, 108

Concretions in seminal vesicles, 331

Condylomata lata, 712

Corpora cavernosa, fibrous plaques of, 172

Corpus spongiosum, rupture of, 171

Cowperitis, 244

Cowper's glands, abscess of, 244

inflammation of, 244

palpation of, 67

Crises, Dietl's, 592

renal, 592

Cryoscopy, 146

Cryptorchism, 303

Crystalline casts in urine, 98

Crystals in urine, 99, 100

Cultures, fluid, diagnosis with, 109

Cystic kidney, 560

testicle, 328

Cystitis, acute, 420, 422

diagnosis of, 424

pathology of, 422

symptoms of, 423

treatment of, 424

urine in, 423

causation of, 420

chronie, 426

pathology of, 426

symptoms of, 426

treatment of, 427

complicating stricture, 267

in prostatic hypertrophy, 409

varieties of, 421

Cystocele, 418
Cystography, 168

Cystoma of testicle, 328

Cystoscope, 129

Brown-Buerger, 130, 136

camera attachment for, 132

Chetwood's 136, 137

component parts of, 132

direct, 129, 130, 132

indirect, 129, 132

lens system of, 132

Nitze's, 135

Otis', 130

principles of, 129

sterilization of, 58

treatment through, 153

types of, 130, 131

Cystoscopic landmarks of bladder, 142

Cystoscopy, 129

anesthetization for, 47

antisepsis for, 54

by direct electric illumination, 129

general requirements for, 138

in abnormal conditions of bladder, 148

in abnormalities of ureteral orifices, 150

in diverticula of bladder, 149

in inflammation of bladder, 148

in prostatic hypertrophy, 149, 401

in pyonephrosis, 623

in renal calculus, 567

tuberculosis, 660

in tuberculosis of bladder, 148

in tumors of bladder, 149

in ulcerations of ureteral orifice, 150

in ureteral calculus, 150

in varices of bladder, 148

in vesical calculus, 148

tuberculosis, 667

landmarks of bladder in, 142

position of patient for, 138

proficiency in, 141

technic of, 138

Cystostomy, 474

Cystotomy, for prostatic hypertrophy, 411

perineal, 458

local anesthesia in, 500

suprapubic, 460

extraperitoneal, 461

local anesthesia in, 500 
Cystotomy, transperitoneal, 467

Cysto-urethroscope, 121

Cysto-urethrotomy, retrograde, 472

Cysts, of bladder, 450

of kidney, 560

of penis, 183

of scrotum, 302

of seminal vesicles, 331

Dactylitis, syphilitic, 725

Decapsulation of kidney, 638

Diacetic acid, in urine, 89

Dietl's crises, 592

Dilatation for stricture, 269

progressive, 269

rapid, 271

Dilating instruments, 40

Dilators, Kollmann's, 47 sterilization of, 58

Diverticulum of bladder, 416 symptoms of, 417 treatment of, 418,470

Doremus' ureometer, 83

Dressings, sterilization of, 59

DuCrey-Unna bacillus, 683

Dystopia of kidney, 522

Dysuria, 62

in prostatic hypertrophy, 393

Ear, syphilis of, 741

Echinococcus disease, complement fixation test for, 109

Eczema of scrotum, 300

Edebohls' operation for decapsulation, 638

Ejaculatory duct, anatomy of, 18

embryology of, 26

Ejector bulb, 35

Electricity, destruction of vesical growths by, 153

Electrolysis, treatment of stricture by, 268

Elephantiasis of scrotum, 300

Emasculation, complete, 362

Emissions, nocturnal, 507

Emphysema of scrotum, 299

Endoscopes, and see Cystoscope, Urethroscope.

sterilization of, 58

English scale of urethral instruments, 31
Enuresis, 506

Epididymectomy, 358

Epididymis, anatomy of, 20

inflammation of, 307, 310, and see Epididymitis.

operations on, 356,357

syphilis of, 735, 736

tuberculosis of, 674

tumors of, 327

Epididymitis, 307, 310

chronic, 312

complicating stricture, 265

morbid changes in, 310

sequels of, 312

subacute, 312

symptoms of, 311

syphilitic, 735, 736

treatment of, 313

Epididymotomy, 356

Epididymo-vasotomy, 357

Epispadias, 187

operation for, 280

Epithelial casts, in urine, 97

Epithelium, in urine, 92

from bladder, 94

from kidney, 95

from urethra, 94

Erection centers, 511

Erosions of urethra, urethroscopy in, 125

Erythrema intertrigo, of scrotum, 301

Esbach's test for albumin, 88

Eucaine, for anesthetizing the urethra, 47

Evacuating tubes, for litholapaxy, 494

Evacuation, instruments for, 47

Examination of patient, oral, 60 physical, 64

of urine, chemical, 82

clinical, 76

microscopical, 91

Examining room, 30

table, 30

Exploration, instrumental, 68

of bladder, 75

urethral, 68

Exploratory instruments, 40

External genitals, anatomy of, 1 anesthesia of, 499

operations on, 340

Exstrophy of bladder, 415 
Extravasation of urine, complicating stricture, 266

Eye, syphilis of, 738

False casts in urine, 98 passages, 73

Fat in urine, 90

Fatty casts in urine, 98

Fehling's test for glucose, 89

Fenger's operation (pyeloplasty), 587

Fever, urinary, 75

Fibrous plaques of corpora cavernosa, 172

Filiform bougies, 42,44 catheters, 43,44

Fingers, chancre of, 703

Fistula, of ureter, 531

perineal, operation for, 296

perineo-urethral, 190

operation for, 298

urethral, 189

operation for, 296

urethro-rectal, 190

urinary, complicating stricture, 267

Folliculitis, 241, 242

Foreign bodies in urethra, urethroscopy for, 127

Formaldehyde fumigation for instruments, 55

French scale of urethral instruments, 31

Frenum, 2

division of, 342

Frequency of urination, 61

Functional impotence, 378, 510, 512

renal diagnosis, 155

urinary tests, 155, 157, 161

as a means of diagnosis, 155

colorimetric test, 157

indigo-carmine test, 160

methods employed, 157, 161

phenolsulphonephthalein test, 157, 160

phlorizin test, 161

technic of, 157

Galactocele, 324

Galvano-cautery operation for prostatic hypertrophy, 411

Galvano-prostatotomy, perineal, 477

Gangrene of testicle, 306

Gangrenous ulceration of penis, 176
Genitals, external, anatomy of, 1

anesthesia for operations on, 499, 500

mucous syphilides of, 722

Genital tract, embryology of, 23 tuberculosis, 671

Gentil's syringe, 29

Gibson's method of reaching the lower end of the ureter, 647

Glandular changes in urethra, urethroscopy in, 125

Glans penis, 1

chancre of, 702

inflammation of, 173

Glucose in urine, tests for, 88

Glycosuria, 88

Gmelin's test for bile pigment in urine, 90

Goldschmidt urethroscope, 119, 122, 499

Gonococcus, 195

in urine, 103

infections, complement fixation tests for, 108, 214

staining of, 197

Gonorrhea, 191, and see Urethritis.

abortive treatment of, 221

acute, complications of, 117 vaccine therapy in, 117

local treatment of, 216

prophylaxis of, 212,214

bacteriology of, 195

chronic, infectiousness of, 232

sequels of, 118

complement fixation test in, 106, 108,214

duration of, 206

extragenital complications of, 246

incubation period of, 200

local treatment of, 216

pathology of, 198

prophylaxis of, 212,214

serotherapy in, 115

stricture due to, 257

symptoms of, 201

treatment of, 207, 212, 214, 216, 221,223

Gonorrheal arthritis, 249

bursitis, 251

endocarditis, 251

filtrates, 114 
Gonorrheal myositis, 251

ophthalmia, 247

osteoarthritis, 251

osteomyelitis, 251

periostitis, 251

phylacogen, 114

proctitis, 246

rheumatism, 117, 249

rhinitis, 246

serum, 115

stomatitis, 246

tenosynovitis, 251

toxemia, acute, serotherapy in, 116

Gowns, sterilization of, 59

Gram's method of staining, 197

Granular casts in urine, 97

Gubernaculum testis, 26

Gumma, 723, 727

of brain, 745

of scrotum, 302

Guyon canula, 34

curved obturators (Béniqué), 51

instillator, 35

sound, 29, 43

with conductor, 43

Hagner's operation, 316, 356

Hayden's modification of Otis urethroscope, 120

Heart, syphilis of, 733

Hedgehog crystals in urine, 100

Heller's test for albumin in urine, 87

for blood in urine, 90

for indican in urine, 89

Hematocele, 321 treatment of, 322

Hematoma of testicle, 321

Hematuria, 63

in prostatic hypertrophy, 394, 401

in renal tumors, 656

in vesical calculus, 445

renal, 546

Hemoglobinuria, 90

Hemolysins, 105

Hemolysis, 105

Herpes progenitalis, 176

Herpetic vesicles, 176

Hydrocele, 317

acute, 318

chronic, 318

clinical history of, 320
Hydrocele, congenital, 317, 322

closed operation for, 350

encysted, 317

infantile, 322

of spermatic cord, 317,323

open operation for, 352

pathology of, 319

treatment of, 318,321

varieties of, 317,318

Hydronephrosis, 575

acquired, 578

calculous, 581

causation of, 575,577

clinical features of, 583

congenital, 577

diagnosis of, 584

differential diagnosis of, 586

intermittent, 580

morbid changes in, 582

prognosis of, 586

traumatic, 581

treatment of, 586

Hypernephroma, 551

Hypertrophy of prostate, 385, and see Prostatic hypertrophy.

Hypodermic syringe, sterilization of, 59

Hypospadias, 185

operations for, $277,278,280$

Immunity, 105, 110

active, 111

passive, 115

Immunization with dead bacteria, 111

Impotence, 510

atonic, 510

functional, $378,510,512$

organic, 510, 511

paralytic, 510

psychic, 510

relative, 510

symptomatic, 511

Impotentia, 510

coeundi, 510

generandi, 514

Indican in urine, 89

tests for, 89

Indigo-carmine test of renal insufficiency, 160

Indwelling catheter, 53

Infarction of kidney, 543, 611 
Infarcts, unilateral septic, of kidney, 611

acute type of, 614

causation of, 613

clinical course of, 614

diagnosis of, 615

morbid changes in, 613

prognosis of, 618

subacute type of, 615

treatment of, 619

Inflammation of bladder, 420, and see Cystitis.

of Cowper's glands, 244

of epididymis, 307, 310, and see Epididymitis.

of inguinal glands, 690

of kidney, see Nephritis.

of penis, 170, 172, 173

of prostate, 370 , and see Prostatitis.

of seminal vesicles, 332 , and see

Seminal vesiculitis.

of testicle, 307, and see Orchitis.

of ureter, 532

of urethra, 191, and see Urethritis.

of urethral follicles, 241, 242

peri-urethral, 242

Inguinal adenectomy, 346

glands, excision of, 346

incision of, 346

inflammation of, 690

region, lipoma in, 329

Inherited syphilis, 757

diagnosis of, 761

morbid anatomy of, 757

pathological changes in, 757

prognosis of, 762

symptoms of, 758

transmission of, 757

treatment of, 762

Injections, in acute urethritis, 222

in chronic urethritis, 236

of anterior urethra, 32

of posterior urethra, 34

Injectors, urethral, 32

Inspection, 64

Instillation, 7

in chronic urethritis, 237

Instillator, Guyon's, 35

Keyes-Ultzmann's, 33, 34

Instrumental exploration, 68

Instruments, antisepsis of, 54
Instruments, antiseptic cabinet for, 55

dilating, 40

evacuating, 47

exploratory, 40

introduction of, into urethra, 71

lubrication of, 31

special, 30

sterilization of, 54

Intramuscular injection of salvarsan 787

Intraspinal injection of Salvarsan, 798

Intravenous injection of phenolsulphonephthalein, 160

of salvarsan, 787, 790, 791

Iodides, in treatment of syphilis, 775

Iodism, 779

Iritis, syphilitic, 739

Irrigation in urethritis, acute, 219, 223, 224

chronic, 236

clamp, Chetwood's, 36

of anterior urethra, 36

of posterior urethra, 39

Irrigators, 29, 36

sterilization of, 59

Irritable bladder, 504

Janet's method of urethral irrigation, 223

syringe, 29, 33

Keyes evacuating tube, 495

lithotrite, 493

modification of Reverdin's needle, 347

Keyes-Ultzmann instillator, 29, 33, 34

Kidney, and see Renal.

absence of, 522

adenoma of, 554

anatomy of, 12

anemia of, 534

angioma of, 554

anomalies of, 520

anomalous, predisposed to infection, 602

arteriosclerosis of, 542

atrophied, 522

blood from, in urine, 80

carcinoma of, 553

calculus in, 562, and see Calculus, renal. 
Kidney, circulatory changes in, 534 congenital displacement of, 522 congestion of, 535

contracted, 542

cystic, 560

eysts of, 560

decapsulation of, 638

dermoids of, 554

drainage of, by ureteral catheter, 154

dystopia of, 522

embryology of, 25

embryonic tumor of, $\mathbf{5 5 4}$

epithelioma of, 555

epithelium from, in urine, 95

fetal, 521

fibroma of, 554

floating, 523

functional test of, 155, 157, 161

gouty, 542

granular red, 542

horseshoe, 521

hyperemia of, active, 534

passive, 535

infarction of, 543, 611

inflammation of, see Nephritis.

bacterial, 599

diffused hematogenous non-suppurative, 535

injuries of, 527

diagnosis of, 529

pathological conditions in, 527

symptoms of, 527

treatment of, 530

large white, 539

lipoma of, 554

metastatic infection of, 611

misplaced, 523

mixed tumors of, 554

movable, 523, 588, and see Nephroptosis.

non-suppurative infaretion of, 543

operations on, 632

palpation of, 65

pelvic, 523

pelvis of, measurements of, 151

percussion of, 68

polycystic, 560

prolapse of, 588, and see Nephroptosis.

pus from, in urine, 79
Kidney, red granular, 542

relations of, 12,14

relative functional capacity of, 161

rhabdomyoma of, 554

roentgenography in diseases of, 162,661

rudimentary, 522

rupture of, 527

sarcoma of, 553

senile, 542

supernumerary, 522

suture of, in nephrotomy, 636

syphilis of, 738

tuberculosis of, 653,654

causation of, 654

clinical history of, 658

cystoscopy in, 660

diagnosis of, 660

morbid changes in, 654

prognosis of, 662

roentgenography in, 661

symptoms of, 658

treatment of, 664

tumors of, 550

causation of, 5.50

classification of, 550

clinical features of, 556

diagnosis of, 557

of pelvis of, 554

prognosis of, 559

treatment of, 559

unilateral septic infarcts of, 611

Koenig's incision in nephrotomy, 633

Kollmann's urethral dilators, 30,47

Larynx, syphilis of, 722,730

Lavage of posterior urethra and bladder, 39

Lilienthal's technic for removal of ureter, 643

Lip, chancre of, 703

Lipoma in inguinal region, 329

Lipuria, 90

Litholapaxy, 492

Lithotomy forceps, 458, 464

medial perineal, 459

suprapubic, 463

Lithotrites, 493

Liver, syphilis of, 732

Locomotor ataxia, 754

Lubricants, 31 
Lubrication of instruments, 31

Lues of brain, 745 of spinal cord, 749

Luetin reaction, in syphilis, 109

Lungs, syphilis of, 731

Luys' urine separator, 151

Lymphangitis, 241, 705

Lymph scrotum, 300

Lysis, 106

Maisonneuve urethrotome, 283, 284, 287

Martin's operation, 357

Masseur, prostatic, 338

Masturbation, 509

Maydl's operation for exstrophy of bladder, 471

Meatotomy, 281, 283

Meatus urinarius, 1, 4

Meningitis, syphilitic, 747, 748

Mercier catheter, 29, 30, 50, 51

Mercury, in treatment of syphilis, 767 effect of, on kidneys, 775

hypodermic use of, 770,771

internal use of, 768

intramuscular injections of, 772

intraspinal administration of, 773 inunctions of, 769

Mixed infection vaccines, 113 treatment, in syphilis, 776

Molluscum contagiosum, of scrotum, 302

Morning drop, 230, 264

Mouth, syphilis of, 721, 731

Mucous patches, 721

Müllerian duct, 25

Myocarditis, syphilitic, 733

Myositis, gonorrheal, 251

Necrospermia, 518

Needles, sterilization of, 59

Nélaton catheter, 48

Neosalvarsan, in treatment of syphilis, 782 , and see Salvarsan.

dosage of, 793, 794

intravenous injection of, 791

preparations of, for intravenous injection, 788

toleration of, 789

untoward effects of, 786
Nephrectomy, 640

complete, 640

difficulties and dangers of, 645

extracapsular, 640

intracapsular, 641

partial, 646

removal of ureter with, 643

subcapsular, 641

transperitoneal, 644

Nephritis, actue diffuse, 536

causes of, 536

clinical features of, 537

morbid changes in, 536

prognosis of, 538

treatment of, 538

varieties of, 537

chronic, 539

causation of, 539

clinical features of, 540

diagnosis of, 541

morbid changes in, 539

prognosis of, 541

treatment of, 542

varieties of, 542

chronic diffuse, 539

desquamative, 539

parenchymatous, 539

glomerular, 537

interstitial, 537

parenchymatous, 537

suppurative, 599, and see Pyelonephritis.

treatment of, 610

tuberculous, 654

tubular, 537

Nephrolithotomy, 634, 636

Nephropexy, 597, 637

Nephroptosis, 588

anatomical considerations, 588

causation of, 588

clinical features of, 591

diagnosis of, 593

hygienic treatment of, 596

mechanical treatment of, 596

morbid changes in, 590

operative treatment of, 597

preventive treatment of, 596

traumatic, 592

treatment of, 595

Nephrostomy, 639

double, 639 
Nephrotomy, 632

incisions for, 632

involving incision of kidney, 634

lumbar, 632

suture of kidney, 636

Nervous system, syphilis of, 743

Neuralgia, vesical, 504

Neuroses, sexual, 507

urinary, 502

Nitrogen, in urine, estimation of, 83, 155

output of, 155, 161

Nitze's cystoscope, 129, 135

Nocturnal emissions, 507

Noguchi's modification of Wassermann reaction, 107

Nové-Josserand's operation for epispadias, 281

operation for hypospadias, 279 , 280,281

Novocaine for anesthetizing the urethra, 47

Nozzles, 32

Chetwood's urethral, 36

Obturators for catheters, 51

Ogilvie's method of administering salvarsan, 799

Oligospermia, 516

Ophthalmia, gonorrheal, 247 non-virulent, 248

rheumatic, 248

treatment of, 248

Opsonic index, 111

Opsonins, 105, 111

Optic neuritis, syphilitic, 740

Oral examination of patient, 60

Orchidectomy, 358

Orchitis, 307

chronic, 309

etiology of, 307

operation for, 316

pathology of, 308

primary, 308

secondary, 308

symptoms of, 308

syphilitic, 736

treatment of, 309

Organisms, in urine, 102

Osteitis, syphilitic, 726, 727

Osteocopic pains, 726
Osteomyelitis, 726, 727 gonorrheal, 251

Otis' cystoscope, 130 urethroscope, 120 urethrotome, 283, 284

Otis-Leiter urethroscope, 119

Oxaluria, 80, 100, 504

Pain, as a symptom, 62

Painful urination, 62, 63

Pains, osteocopic, 726

Palpation, 64

of bladder, 75

of kidneys, 65

per rectum, 65

Pancreas, syphilis of, 733

Papillary tuberculosis of kidney, 655

Papilloma, of penis, 178

of ureter, 554

of urethra, urethroscopy in, 126, 127,128

Paralysis, general, 752

Paraphimosis, 180

complicating chancroid, 688

Pararenal tumors, 555

Parasites, in urine, 102

Parasyphilis, salvarsan in, 798, 800

Patent urachus, 418

Patient, asepsis, of, 53

examination of, 60,64

Pediculi pubis, 301

Penis, absence of, 184

amputation of, conservative, 360

anatomy of, 1

anomalies of, 184

cancer of, 183

carcinoma of, 183

cellulitis of, 172

chancre of, 702

chancroid of, 681

contusions of, 170

eysts of, 183

double, 184

dressings for, in urethritis, 225

elephantiasis of, 173

emasculation, complete, 362

embryology of, 28

epithelioma of, 183

fibrosis of, 172

fracture of, 171

gangrenous ulceration of, 176 
Penis, growths on, 170

herpetic vesicles on, 176

horny growths on, 183

inflammation of, $170,172,173$

injuries of, 170

lipoma of, 183

luxation of, 171

lymphangitis of, 173

maladies of, 170

operations on, 340

papilloma of, 178

phlebitis of, 172

sarcoma of, 183, 184

tumors of, 182

vegetations of, 178

venereal warts on, 178

verrucæ on, 178

wounds of, 170

Percussion, 68

Pericowperitis, 244

Perigenital chancres, 703

Perineo-urethral fistula, 190 operation for, 298

Perinephritis, 624 bacteriology of, 626

causation of, 625

clinical course of, 628

diagnosis of, 629

morbid changes in, 627

suppurative, 627

treatment of, 630

Perineum, anatomy of, 21

fistula of, operation for, 297

Periostitis, gonorrheal, 251

syphilitic, 726

Periprostatic abscess, 373

Peritoneum, syphilis of, 733

Periurethral abscess, 243

operation for, 296

inflammation, 241, 242

Peter's operation for exstrophy of bladder, 471

Pharynx, syphilis of, 729

Phenolsulphonephthalein test, 156, 157, 160

Philip's bougie, 43, 46

Phimosis, 180

clamp, 344

operation for, 340

Phlorizin test of renal sufficiency, 161

Phosphaturia, 80, 100
Phosphoric acid in urine, 84

Phylacogens, 113

gonorrheal, 114

mixed, 114

Physical examination of patient, 64

Plaques, fibrous, of corpora cavernosa, 172

Pneumoscrotum, 299

Pollakiuria, 503

Polycystic kidney, 560

Polypi of urethra, urethroscopy in, 126

Polyuria, 502

Polyvalent antigens, 108

Posterior urethra, injection of, 34 lavage of, 39

Potassium permanganate, in acute gonorrhea, 221, 224

Precipitins, 105

Prepuce, 2

bilateral incision of, 340

chancre of, 702

inflammation of, 173

Prevesical abscess, 431

Proctitis, gonorrheal, 246

Prostate, abscess of, 372

absence of, 365

anatomy of, 16

atrophy of, 365

calculi of, 369

carcinoma of, 366

diagnosis of, 368

duration of, 367

radical removal of, 490

symptoms of, 367

treatment of, 368

congestion of, 370

eysts of, 365

embryology of, 27

fibroma of, 366

hypertrophy of, 385, and see Prostatic hypertrophy.

inflammation of, 370, and see Prostatitis.

injuries of, 366

operations on, $474,490,492$

transurethral, 492

palpation of, per rectum, 66

radical removal of, 490 ,

retention cysts of, 365

sarcoma of, 369

syphilis of, 735 
Prostate, tuberculosis of, 671 tumors of, 366

Prostatectomy, 412, 479 after treatment, 413 perineal, 479 extra-urethral, 480 intra-urethral, 479 suprapubic, 485

Prostatic calculi, 369 catheter, 50

enlargement, cystoscopy in, 149, 401

hypertrophy, 385

after-treatment, 413

catheterization in, 397

causation of, 385

changes in urethra in, 389

clinical symptoms of, 392

complete retention of urine in, 409

complications of, 406

constitutional symptoms of, 396 course of, 394

cystoscopy in, 149, 401

cystotomy in, 411

diagnosis of, 397

dysuria in, 393

frequent urination in, 392

galvano-cautery operation for, 411

gross appearances of, 387

hematuria in, 394,401

hygienic treatment of, 407

incomplete retention of urine in, 410

infection and cystitis in, 409

local treatment in, 408

medical treatment in, 407

operative treatment of, 410

palliative treatment of, 407

pathology of, 386

prognosis of, 405

progress of, 394

prostatectomy for, 412

rectal examination in, 397

residual urine in, 398

retention of urine in, 404, 409

secondary changes due to, 390

svmptoms of, 394

treatment of, 407

types of, 402
Prostatic hypertrophy, urine in, 395

masseur, 338

retractors, 482

tractor, 482

Prostatism, 397

without enlargement of prostate, 436

Prostatitis, 370

acute, 370

diagnosis of, 373

symptoms of, 372

treatment of, 374

chronic, 375

diagnosis of, 378

discharge in, 379

symptoms of, 377

treatment of, 381

vaccine therapy in, 384

follicular, 371

parenchymatous, 371

Prostatotomy, 474

extra-urethral, 476

galvano-perineal, 440, 477

intra-urethral, 474

Prostatorrhea, 376

Pruritus of scrotum, 301

Pus in urine, $77,78,92$

casts in urine, 98

tubes, in the male, 339

Pyelitis, 599, and see Pyelonephritis.

catarrhal, 606

causation of, 599, 601

chronic, 606

in children, 601

method of invasion, 599

morbid changes in, 603

suppurative, 604

treatment of, 606

through ureteral catheters, 154

varieties of, 605

Pyelography, 168

Pyelometry, 151

Pyelonephritis, 599, and see Pyelitis.

catarrhal, 605

causation of, 601

clinical course of, 605

morbid changes in, 603

suppurative, $606,607,608$

diagnosis of, 609

symptoms of, 608

treatment of, 609,610 
Pyelonephritis, treatment of, 610 varieties of, 605

Pyeloplasty, 587

Pyonephrosis, 620 causation of, 620

clinical course of, 622

cystoscopy in, 623

diagnosis of, 623

morbid changes in, 621

prognosis of, 623

symptoms of, 622

treatment of, 624

tuberculous, 655

Pyuria, 77, 78, 92

Quaternary syphilis, 752

Rectal palpation, 65

Rectal tube, double current, 382

Rectum, palpation by, 65 syphilis of, 732

Reliquet lithotrite, 493

Renal, and see Kidney. abscess, 599

calculus, 562, and see Calculus, renal

casts, 96

colic, 565,568

treatment of, 568

crises, 592

epithelium, 95

hematuria, 546

clinical symptoms of, 548

diagnosis of, 548

essential, 546

idiopathic, 546

treatment of, 548

pelvis, 10

measurements of, 151

operations on, 646

tumors of, 554

sufficiency, tests of, 155,157

syphilis, 738

tuberculosis, 653, 654, and see Kidney, tuberculosis of.

complement fixation test in, 109

Resection of urethra, 294

Residual urine, in prostatic hypertrophy, 398

Respiratory tract, syphilis of, 728
Retained catheter, 53

testicle, 303

Retinitis, syphilitic, 739

Retrograde catheterism, 274

cysto-urethrotomy, 472

Retrorenal abscess, 627

Rheumatism, gonorrheal, 117, 249

Rochet operation for hypospadias, 280 , 282

Roentgenographic diagnosis, 162

Roentgenography, 162

in renal calculus, 163

tuberculosis, 661

in ureteral calculus, 164

in vesical calculus, 165

Rosenbach's test for bile pigment in urine, 90

Rupia, 717

Rupture of corpus spongiosum, 171

of kidney, 527

of urethra, 188

complicating stricture, 266

operation for, 293

Salvarsan, 780

abortive action of, 785

absorption of, 785

administration of, 787

aim of, 782

continued use of, 784

contraindications to, 795,798

death from use of, 786

dosage of, 792,793

elimination of, 785

in acquired syphilis, 780

in inherited syphilis, 763, 796

in parasyphilis, 798

in primary stage of syphilis, 795

in secondary stage of syphilis, 796

in tertiary stage of syphilis, 796, 797

indications for, 795

intensive method, 797

intramuscular injection of, 787

intraspinal injection of, 798

intravenous injection of, 787, 790, 791

late effects of, 786

needle for, Chetwood's, 790

neuro occurrences due to, 786 
Salvarsan, Ogilvie's method of administering, 799

indications for, 800

preparations of, for intravenous injection, 787

repetition of, 792

standard preparations of, 782

Swift-Ellis method of administering, 798, 799

indications for, 800

toxicity of, 785

untoward effect of, 786

water used to make solution, 789

Sarcoma of bladder, 452

of kidney, 553

of penis, 183, 184

of prostate, 369

of scrotum, 302

Scabies of scrotum, 301

Scale of urethral instruments, 30

Scrotum, anatomy of, 1

anesthesia, local, for operations on, 501

carcinoma of, 302

cellulitis of, 299

chancre of, 702

chimney-sweep's cancer of, 302

contusions of, 299

cysts of, 302

deformities of, 299

eczema of, 300

elephantiasis of, 173, 300

emphysema of, 299

epithelioma of, 302

erythrema intertrigo of, 301

gangrene of, 300

gumma of, 302

injuries of, 299

lymph, 300

molluscum contagiosum of, 302

operations on, 340

pediculi of, 301

pruritus of, 301

sarcoma of, 302

scabies of, 301

tumors of, 301, 302

wounds of, 299

Searcher, for strictures, 44

Thompson's, 75, 447

Sediments in urine, summary of, 101

Seminal vesicles, 330
Seminal vesicles, abnormalities of, 330 anatomy of, 19

calculi in, 331

concretions in, 331

cysts of, 331

embryology of, 26

inflammation of, 332, and see Seminal vesiculitis.

injuries of, 330

operations on, 355, 491

tuberculosis of, 332, 673

tumors of, 332

Seminal vesiculitis, 332

acute, 332

chronic, 334

operation for, 355

Separator, Luys, 151

Serodiagnosis, 105

Serotherapy, 105, 110

Serum, general indications for use of, 115

gonorrheal, 115

therapy, 110

Sexual neurasthenia, in varicocele, 325 neuroses, 507

Sheets, sterilization of, 59

Silver compounds, in treatment of chronic cystitis, 429

nitrate, in acute urethritis, 218, 220,221

in chronic urethritis, 238

preparations in treatment of gonorrhea, 220

Sinclair's operation of suprapubic puncture and cystoscopy, 472

Skin, chancre of, 703

Smegma bacillus differentiated from tubercle bacillus, 103

Soamin, in treatment of syphilis, 780

Sodium chloride, output of, 155

Soft chancre, 681, and see Chancroid.

Solutions, preparation of, 59

Sounds, 29, 40

Béniqué, 43

Guyon, 43

with conductor, 43

introduction of, 72

sterilization of, 58

Spasm, urethra!, 252

vesical, 505

Spermatic artery, shortening of, 303 
Spermatic cord, absence of, 305 anatomy of, 19 anomalies of, 304 fusion of, 305 hydrocele of, 323 operations on, 340 torsion of, 306 tumors of, 329

Spermatocele, 323, 324 operation for, 352

Spermato-cystitis, 332, 335

Spermatorrhea, 379, 508

Spinal cord, lues of, 749

Spirochæta pallida, 696

Spleen, syphilis of, 733

Squier's modification of Otis' urethroscope, 120

Staphylococcus pyogenes, in urine, 103

Sterility, 514

Sterilization of instruments, 54

Stone, see Calculus.

Streptococcus pyogenes, in urine, 103

Stricture of ureter, 532

of urethra, 252

acquired, 252, 257

after treatment of, 274

causes of, 257

classification of, 261

clinical symptoms of, 263

complications of, 265

constitutional symptoms of, 266

diagnosis of, 265

morbid changes in, 259

prognosis of, 267

sequelæ of, 265

treatment of, 268

by electrolysis, 268

by external perineal urethrotomy, 273

by internal urethrotomy, 273

by progressive dilatation, 269

by rapid and prolonged dilatation, 271

by resection of urethra, 294

by retrograde catheterization, 274

annular, 256, 259, 260, 269

callous, 260
Stricture of urethra, congenital, 252, 256

causes of, 256

symptoms of, 257

treatment of, 257

cylindrical, 256

detection of, 70

diaphragmatic, 256

due to bands or spurs, 256

electrolysis for, 268

hard, 269

impassable, 271

inflammatory, 252

irritable, 269

linear, 259, 260

muscular, 252

nodular, 269

organic, 252,256

resilient, 267

searchers for, 44

soft, 269

spasmodic, 252, and see Urethral

spasm.

tortuous, 260,261

traumatic, 257

true, 252,256

urethroscopy in, 123,128

valvular, 256

Sugar in urine, 88

Suprapubic cystotomy, local anesthesia for, 500

puncture and drainage, 471

Sinclair's method of, 472

Surgeon's hands, antisepsis of, 53

Suture of urethra, 293

$\sim$ Swift-Ellis intraspinal administration of salvarsan, 798, 799

Swinburne's interrupter, 36,37

urethroscope, 120, 124

Synovitis, 725

Syphilides, 708

diagnosis of, 715,718

early, 709

erythematous, 709

gummatous, 717

late, 716,723

mucous, 722, 723

nodular, 716

papular, 710, 712

papulo-squamous, 712, 713

pigmentary, 715 
Syphilides, pustular, 714, 715

serpiginous, 717

treatment of, 719

tubercular, 716

vesicular, 715

Syphilis, 695

acquired immunity to, 700

adenitis in, 705

alterative treatment of, 768

arsenic, in treatment of, 779

atoxyl, in treatment of, 780

cerebrospinal, 743

clinical course of, 707, 708

complement fixation test for, 106

congenital, 757

duration of, 766

eruptive stage of, 698

first stage of, 707

hygienic treatment of, 766

inherited, 755, and see Inherited syphilis.

intensive method of treatment, 797

intraspinal treatment of, 773,798

iodides, in treatment of, 775

luetin reaction for, 109

mereury, in treatment of, 767

effect of, on kidneys, 775

hypodermic use of, 770,771

internal use of, 768

intramuscular injection of, 772

intraspinal administration of, 773

inunctions of, 769

salivation from, 774

microbiology of, 695

mixed treament in, 776

modes of infection in, 699

neosalvarsan, in treatment of, 782, and see Neosalvarsan.

nodes, 726

Noguchi's modification of Wassermann reaction for, 107

of alimentary tract, 731

of aponeuroses, 724

of arteries, 734

of bladder, 737

of bones, 724,726

of brain, 745

of bronchi, 731

of bursæ, 724

of circulatory system, 733
Syphilis of ear, 741

of esophagus, 731

of eye, 738

of genito-urinary organs, 734

of hair, 719

of heart, 733

of intestines, 731

of joints, 724

of kidney, 738

of larynx, 722, 730

of liver, 732

of lungs, 731

of mouth, 721, 731

of mucous membrane, 721

of muscles, 724

of nails, 721

of nervous system, 743

of nose, 728

of pancreas, 733

of peritoneum, 733

of pharynx, 729

of prostate, 735

of rectum, 732

of respiratory tract, 728

of skin, 708, and see Syphilides.

of spleen, 733

of stomach, 731

of tendons, 724

of testicles, 735

of throat, 721

of tongue, 723, 731

of trachea, 731

of vertebral column, 728

pathology of, 697

polymorphous, 698

primary lesions of, 698, 701, and see

Chancre.

manifestations of, 698

prophylaxis of, 765

quaternary, 708, 752

salvarsan, in treatment of, 780 , and see Salvarsan.

second stage of, 707

secondary manifestations of, 698

soamin, in treatment of, 780

specific treatment of, 767

synthetic preparations for treatment of, 779

tertiary stage of, 708

tonic treatment of, 768

transmission of, 699,700 
Syphilis, treatment of, 765 urethro-penile, 734

Wassermann reaction in, 106

Syringes, 29, 32, 33, 34, 35

sterilization of, 33,59

Tabes dorsalis, 754

Teeth, in hereditary syphilis, 760

Tenosynovitis, gonorrheal, 251

Testicle, absence of, 302

anatomy of, 20

anesthetic, local, for operations upon, 501

anomalies of, 302, 303

atrophy of, 303

carcinoma of, 327

contusion of, 306

cystoma of, 328

descent of, 28

dislocation of, 306

ectopia of, 304

embryology of, 26,28

enlargement of, 303

gangrene of, 306

hematoma of, 321

inflammation of, 307, and see Orchitis.

injuries of, 305

luxation of, 306

operations on, 340

puncture of, 306

retained, 303

sarcoma of, 327

syphilis of, 735

teratoma of, 329

tuberculosis of, 674

clinical history of, 675

diagnosis of, 676

morbid changes in, 675

prognosis of, 677

treatment of, 678

tumors of, 327

undescended, 303, 304

operation for, 359

wounds of, 305, 306

Thompson searcher, 75,447

Tilden-Brown cystoscope, 129

Tongue, chancre of, 703

syphilis of, 723,731

Tonsil, chancre of, 703

Trachea, syphilis of, 731
Transperitoneal cystotomy, 467

Trigone, 8 cystoscopic picture of, 143

Triple phosphates, in urine, 100

Tubercle bacillus, in urine, 103 differentiated from smegma bacillus, 103

Tuberculin diagnosis of tuberculosis, 110

therapy, 113

Tuberculosis, genital, 671

of bladder, 665

of epididymis, 674

of kidney, 653, 654

of prostate, 671

of seminal vesicles, 332,673

of testicle, 674

of urethra, 679

of urogenital tract, 653

of vas deferens, 674

tuberculin diagnosis of, 110 therapy of, 113

Tuberculous nephritis, 654

pyonephrosis, 655

Tumors, of bladder, 449

cystoscopy in, 149

of epididymis, 327

of kidney, 550, 554

of penis, 182

of prostate, 366

of scrotum, 301, 302

of seminal vesicles, 332

of spermatic cord, 329

of testicle, 327

of ureter, 555

pararenal, 555

Typhoid bacilli, in urine, 102

Ulceration, of penis, gangrenous, 176 of urethra, urethroscopy in, 125

Undescended testicle, 304

operation for, 359

Urachus, 6

embryology of, 26

patent, 418

Urates, in urine, 80,100

Urea, estimation of, $82,83,155$

output of, 155,161

Ureometer, Doremus', 83

Ureter, anastomosis of, 651, 652 end to end, 651 
Ureter, anastomosis of, intestinal, 652 side to side, 651,652 uretero-vesical, 652 anatomy of, 9 anomalies of, 524,525 calculus in, 569 catheterization of, 147 division and reimplantation of, for hydronephrosis, 587

double, 525

exposure of, 646

fistula of, 531

incision of, 649

inflammation of, 532

injuries of, 531

invagination of, 651

kinks of, 526

malformations of, 526

operations on, 646

papilloma of, 554

removal of, with nephrectomy, 643

resection of, 650

roentgenography of, 162,661

stricture of, 526,532

suture of, 651

tumors of, 555

ulceration of orifice, cystoscopy in, 150

vesical implantation of, 651

Ureteral calculi, 569, and see Calculus, ureteral.

catheter, 147, and see Catheter, ureteral.

orifice, abnormalities of, cystoscopy in, 144,150

cystoscopic picture of, 143

Ureteritis, 532

Uretero-vesical anastomosis, 652

Urethra, absence of, 185

anatomy of, 2

anesthetization of, 46

bands in, 256

blood from, in urine, $\$ 1$

bridles in, 256

calculi in, urethroscopy for, 127 , 128

calibre of, 4,68

chancre of, 194, 703

curves of, 3

double, 185

embryology of, 27
Urethra, epithelium from, in urine, 94 erosions of, 125

exploration of, 68

false passages in, 73

fissure of, 185

fistula of, 189

operation for, 296,297

foreign bodies in, urethroscopy for, 127

glandular changes in, 125

inflammation of, 191, and see Urethritis.

injecting, 32

introduction of instruments into, 71

lavage of, 39

length of, 68

lesions of, urethroscopy in, 125, 127

local anesthesia of, 46

malformations of, 185

narrow parts of, 262

obliteration of, 185

operations on, 276

local anesthesia for, 276, 499

preparations for, 276,277

papillomata of, 126

polypi of, 126

pus from, in urine, 79

relations of, 3

resection of, 294

rupture of, 188

complicating stricture, 266

operation for, 293

spurs in, 256

stricture of, 252, and see Stricture of urethra.

suture of, 293

tuberculosis of, 679

ulceration of, 125

wounds of, 187

Urethral chill, 75

clamp, 29, 33, 36, 44

discharge, in stricture, 264

endoscope, Chetwood's, 121

endoscopic syringe, 127

follicles, inflammation of, 242

injectors, 32

irrigation, 36, 39

in urethritis, 223

nozzles, Chetwood's, 36

spasm, 252

causes of, 254 
Urethral spasm, location of, 254 result of, 255 treatment of, 255 syringes, 32

Urethritis, acute, 191 bacterial filtrate in, 115 complications of, 241 expectant treatment of, 211 hygienic treatment of, 207 internal treatment of, 208 local treatment of, 215 pathology of, 198 serum treatment of, 115 treatment of, 207 urine in, 204 vaccine treatment of, 115 bacteriology of, 195 chronic, 227

causes of, 227

clinical course of, 230

complications of, 241

diagnosis of, 230

forms of, 228

internal treatment of, 234

irrigations, in treatment of, 236

local treatment of, 235

location of lesion in, 231

pathology of, 229

posterior, 310

treatment of, 233

urethroscopy in, 125

clinical course of, 198

complicating stricture, 265

diagnosis of, 203

diathetic, 194

dressing of penis in, 225

duration of non-specific, 206

eruptive, 194

herpetic, 194

irrigations in, 223

location of, 191

non-specific, 191

causation of, 191

duration of, 206

pathology of, 198

symptoms of, 200

pathology of, 198

posterior, 202

symptoms of, 203

treatment of, 224

prophylaxis of, 193
Urethritis, simple, 192 specific, see Gonorrhea. symptoms of, 200 traumatic, 193 urethroscopy in, 125

Urethro-cystitis, 422

Urethrometer, 262

Urethro-rectal fistula, 189

Urethrorrhœa ex libidine, 379

Urethroscope, 119

Buerger's, 121, 124

Goldschmidt's, 119, 121, 122

Swinburne's, 120, 124

treatment of urethral lesions through, 127, 128

Urethroscopy, 119

anterior, 119, 122

position of patient in, 122

posterior, 119, 126

purpose of, 123, 126

technic of, 122, 126

Urethrotome. Maisonneuve's, 283, 284, 287

Otis', 284

sterilization of, 56

Urethrotomy, external, 273, 287, 292

after treatment of, 274, 290

local anesthesia for, 500

internal, 272, 283, 287, 292

Uric acid, in urine, 84,99

Urinary extravasation, operation for, 296

fever, 75

fistula, complicating stricture, 267

neuroses, 502

stream, 62

in stricture, 263

tests, functional, 155, 157, 161

tract, embryology of, 23

local anesthesia for lower, 499

roentgenography in diagnosis of diseases of, 162

Urination, frequency of, 61

in prostatic hypertrophy, 392

in vesical calculi, 445

painful, 62,63

Urine, abnormal ingredients in, 82,86

acetone in, 89

acid deposits in, 99

albumin in, 86

alkaline deposits in, 99 
Urine, ammonio-magnesium phosphates in, 100

ammonium urate in, 100

amorphous phosphates in, 101

urates in, 100

bacteria in, 78, 79, 101

bacteriology of, 101

bile acids in, 90

pigment in, 90

blood in, 63, 80, 90, 92

calcium oxalate in, 100

casts in, 96, 97, 98

chlorides in, 84, 155

chyle in, 90

collection of, for examination, 76

colon bacillus in, 102

color of, 77

colorimetric estimation of, 157

condition of, in prostatic hypertrophy, 395

daily quantity of, 61

diacetic acid in, 89

electrical conductivity of, 128

epithelium in, 92

examination of, chemical, 82

clinical 76

microscopical 91

extravasation of, complicating stricture, 266

fat in, 90

freezing point of, 156

functional tests of $155,157,161$

glucose in, 88

gonococcus in, 103

hemoglobin in, 90

in acute urethritis, 204

in prostatic hypertrophy, 395, 398, 404, 409, 410

in stricture, 264

indican in, 89

indigo carmine test, 160

inspection of, 77

nitrogen and urea estimation in, $55,155,161$

normal constituents of, 82

odor of, 78

organisms in, 102

oxalates in, 80,100

parasites in, 102

phenolsulphonephthalein test of, $156,157,160$
Urine, phlorizin test, 161

phosphates in, 80, 100

phosphoric acid in, 84

preservation of specimen of, 77

pus in, 77, 78, 92

quantity, 61,77

reaction of, 84

residual, in prostatic hypertrophy, 398

retention of, in prostatic hypertrophy, 404, 409, 410

suprapubic puncture for, 471

sediments in, 101

to obtain, 91

separator, Luys, 151

smegma bacillus in, 103

sodium chloride in, 155

specific gravity of, 85

staphylococcus pyogenes in, 103

streptococcus pyogenes in, 103

sugar in, 88

triple phosphates in, 100

tubercle bacilli in, 103

typhoid bacilli in, 102

urates in, 80, 100

urea in, estimation of, $82,155,161$

uric acid in, 84, 99

Urogenital tract, tuberculosis of, 653

Vaccine therapy, 110

Vaccines, autogenous, 111

general indications for use of, 115

mixed infection, 113

Valentine's interrupter, 36,37

Varicocele, 324

causation of, 324

clinical history of, 325

operation for, 347,348

pathology of, 325

subcutaneous ligation for, 347

symptoms of, 326

treatment of, 326

Vas deferens, anatomy of, 19

embryology of, 26

ligation and resection of, 355

shortening of, 303

tuberculosis of, 674

Vasectomy, 355

Vasotomy, 354

scrotal, 354

Vegetations, venereal, 178 
Venereal prophylaxis, 212, 214, 765 warts, 178

Verrucæ, 178

Vertebral column, syphilitic inflammation of, 728

Vesical, and see Bladder. calculus, 441 cystoscopy in, 148

diagnosis of, 446

form and consistence of, 444 nucleus of, 442

primary, 441

roentgenography in, 162

secondary, 441

site of, 444

size of, 443

symptoms of, 445

treatment of, 448

growths, destruction of by electricity, 153

treated through cystoscope, 153 neuralgia, 504
Vesical spasm, 505 tuberculosis, 665, and see Bladder, tuberculosis of.

Vesiculectomy, 491

Vesiculitis, see Seminal vesiculitis.

Vesiculotomy, 491

Warts, in urethra, 127 venereal, 178

Wassermann reaction, 106 Noguchi's modification of, 107

Watson's double nephrostomy, 639 nephropexy, 638

Waxy casts, in urine, 98

Wolffian body, 25 duct, 25

Young's lithotrite and evacuator, 495 operation on vesical orifice, 498 prostatic retractors, 482 punch operation, 498 







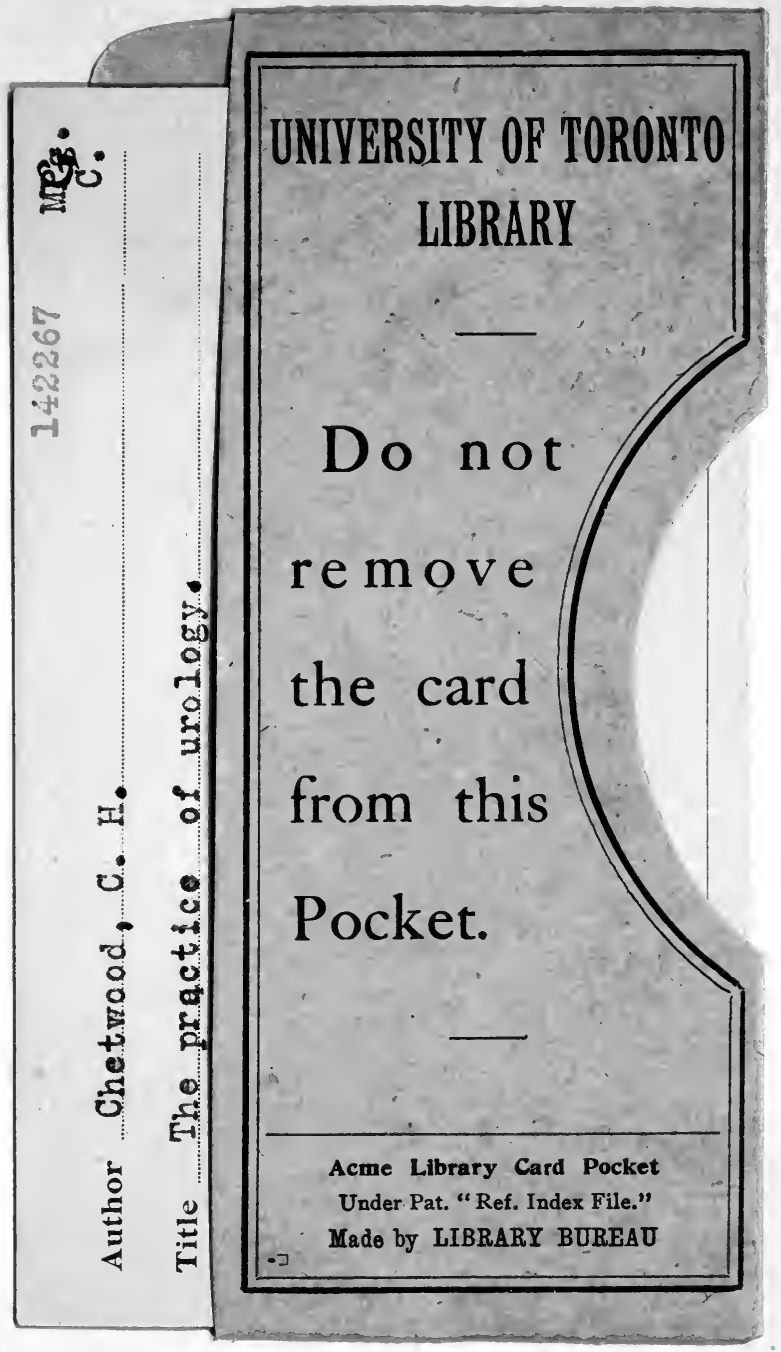


